Water-Supply and Irrigation Paper No. 192 Series $\left\{\begin{array}{l}\text { H, Forestry, } 14 \\ \text { L, Quality of Water, } 18 \\ \text { M, General Hydrographic } \\ \text { Investigations, } 20\end{array}\right.$

DEPARTMENT OF THE INTERFOR

UNITED STATES GEOLOGICAL SURVEY

CHARLES D. WALCOTT, DIRECTOR

\title{
THE POTOMAC RIVER BASIN
}

GEOGRAPHIC HISTORY-RAINFALL AND STREAM FLOW-POLLUTION, TYPHOID FEVER, AND CHARACTER OF WATER-RELATION OF SOILS AND FOREST COVER TO QUALITY AND QUANTITY OF SURFACE WATEREFFECT OF INDUSTRLAL WASTES ON FISHES

BY

HORATIO N. PARKER, BAILEY WILLIS, R. H. BOLSTER W. W. ASHE, AND M. C. MARSH

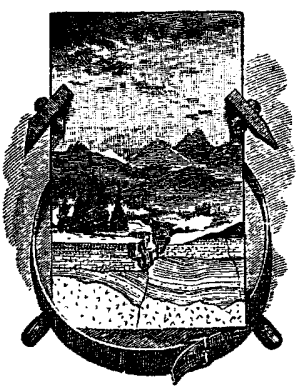

WASHINGTON

GOVERNMENT PRINTING OFFIC F

1907 



\section{CONTENTS.}

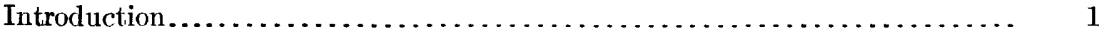

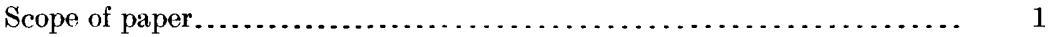

Acknowledgments........................................... 2

Historical sketch of the Potomac basin, by Horatio N. Parker............ 2

Geographic history of Potomac River, by Bailey Willis.................... 7

General description of basin................................. 7

Development of the river system . ............................. 9

Stream flow in the Potomac basin, by R. H. Bolster................... 23

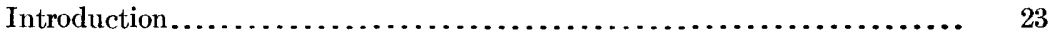

Methods of work....................................... 23

Field methods...................................... 23

Office methods........................................ 24

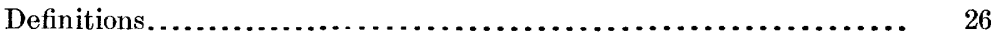

Fxplanation of tables.................................... 27

Accuracy of estimates of stream flow....................... 28

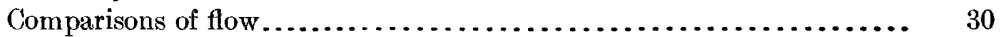

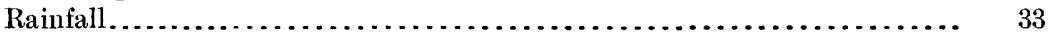

Comparison of rainfall and run-off............................. 40

Gaging stations........................................... 42

North Branch of Potomac River basin.......................... 43

General description.............................. 43

Savage River at Bloomington, Md.................. 43

North Branch of Potomac River at Piedmont, W. Va....... 46

Georges Creek at Westernport, Md.................... 55

Wills Creek at Cumberland, Md.................... 58

North Branch of Potomac River at Cumberland, Md........ 60

Miscellaneous discharge measurements................ $\quad 65$

South branch of Potomac River basin........................ $\quad 66$

General description................................ 66

South Branch of Potomac River near Springfield, W. Va..... $\quad 66$

Miscellaneous discharge measurements................ $\quad 77$

Potomac River basin between mouth of South Branch and Shenandoah River.................................... 78

Potomac River at Great Cacapon, W. Va............... 78

Opequon Creek near Martinsburg, W. Va................ 78

Tuscarora Creek at Martinsburg, W. Va............... 81

Antietam Creek near Sharpsburg, Md................ $\quad 82$

Miscellaneous discharge measurements................ $\quad 90$

Shenandoah River basin................................. 91

South Fork of Shenandoah River basin................... 91

South River basin.................................. 91

South River at Basic City, Va................... 91

South River at Port Republic, Va................... 94 
Stream flow in the Potomac basin-Continued.

Shenandoah River basin-Continued.

South Fork of Shenandoah River basin-Continued.

North River basin.................................. 98

Cooks Creek at Mount Crawford, Va................ 98

Lewis Creek near Staunton, Va.................... 101

North River at Port Republic, Va................. 103

Miscellaneous discharge measurements............... 108

South Fork of Shenandoah River below Port Republic, Va...... 108

General description........................... 108

Elk Run at Elkton, Va........................ 110

Hawksbill Creek near Luray, Va.................... 112

South Fork of Shenandoah River near Front Royal......... 115

Miscellaneous discharge measurements............... 123

North Fork of Shenandoah River basin ................... 124

Passage Creek at Buckton, Va...................... 124

North Fork of Shenandoah River near Riverton, Va....... 125

Miscellaneous discharge measurements............... 135

Shenandoah River basin below North and South forks .......... 135

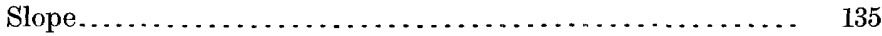

Shenandoah River at Millville, W. Va.............. 135

Miscellaneous discharge measurements............... 147

Potomac River basin below Shenandoah River.................. 148

Potomac River at Point of Rocks, Md................ 148

Monocacy River near Frederick, Md................. 161

Potomac River at Great Falls, Md., and Chain Bridge, Dis-

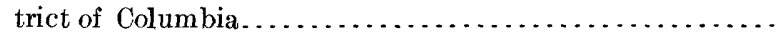

Rock Creek at Lyon's Mill and Zoological Park, District of

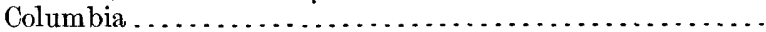

Miscellaneous discharge measurements..$\ldots \ldots \ldots \ldots \ldots \ldots$

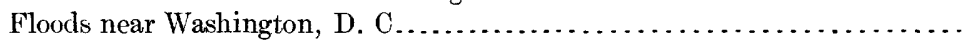

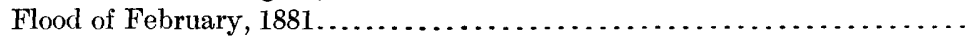

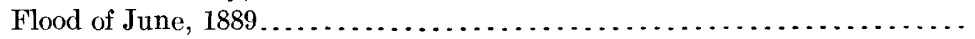

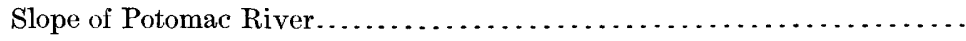

The Chesapeake and Ohio canal, by Horatio N. Parker.................

Stream pollution, occurrence of typhoid fever, and character of surface waters

in Potomac basin, by Horatio N. Parker........................

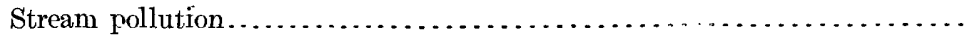

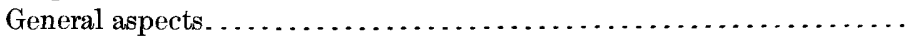

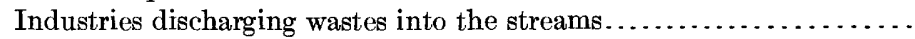

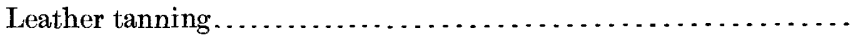

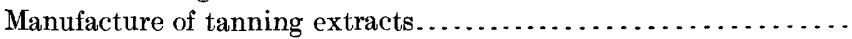

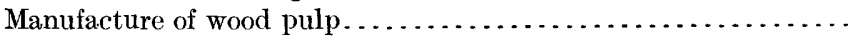

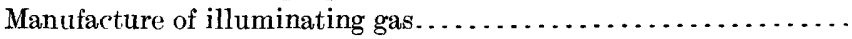

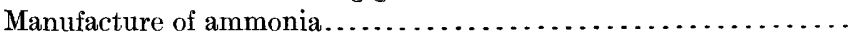

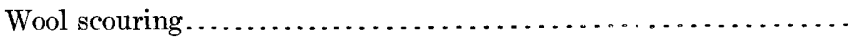

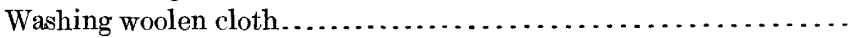

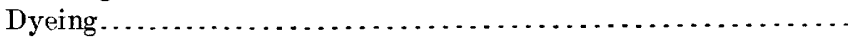

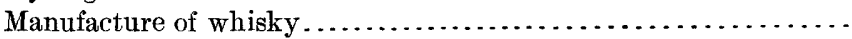

North Branch of Potomac River basin........................ 213

General description.............................. 213

North Branch of Potomac River from Wilsonia to Georges

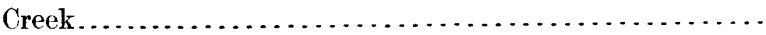

Georges Creek............................... 
Stream pollution, occurrence of typhoid fever, etc.-Continued.

Page.

Stream pollution-Continued.

North Branch of Potomac River basin-Continued.

North Branch of Potomac River from Georges Creek to Wills Creek

Wills Creek and Cumberland.......................... 218

North Branch of Potomac River below Wills Creek........ 223

South Branch of Potomac River basin......................... 223

Potomac River basin between mouth of South Branch and Shenandoah

River

Potomac River from mouth of South Branch to Pawpaw......

Great Cacapon River.

Potomac River from Great Cacapon-River to Conocorheague Creek.

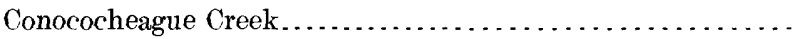

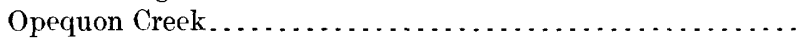

Potomac River from Opequon ('reek to Antietam Creek.....

Antietam Creek...................................

Potomac River from Antietam Creek to Shenandoah River ..

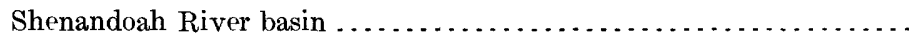

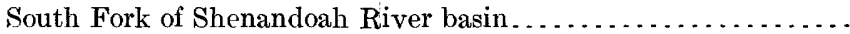

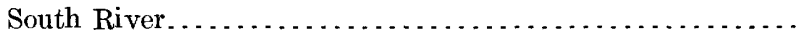

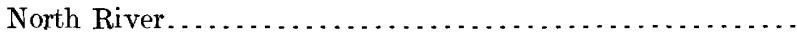

South Fork of Shenandoah River below Riverton............

North Fork of Shenandoah River basin...................

Shenandoah River basin below North and South forks..........

Potomac River basin below Shenandoah River..................

Potomac River from Shenandoah River to Monocacy River..

Monocacy River basin............................

Potomac River from Monocacy River to Great Falls .........

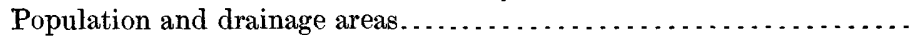

Occurrence of typhoid fever................................... 254

Causes of typhoid fever.................................... 254

Typhoid fever at Washington, Cumberland, and Mount Savage...... 270

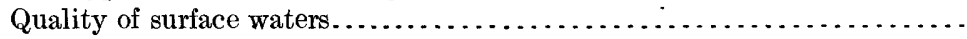

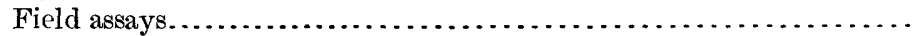

Sanitary and mineral analyses, by Raymond Outwater..............

Relation of soils and forest cover to quality and quantity of surface water in the

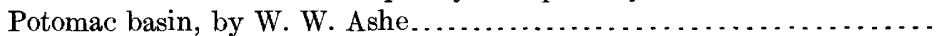

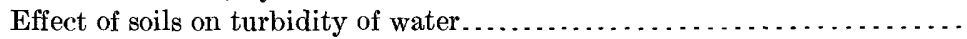

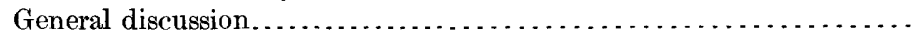

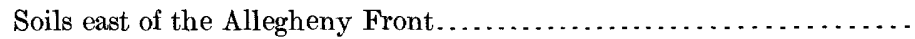

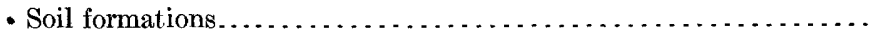

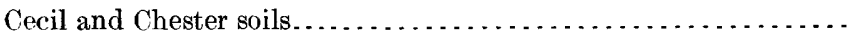

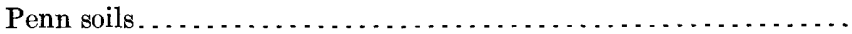

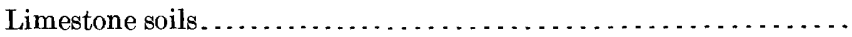

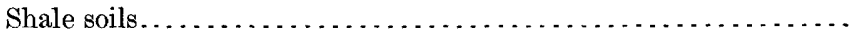

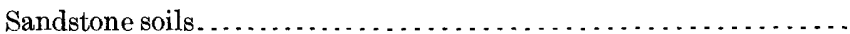

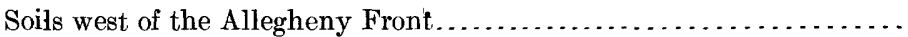

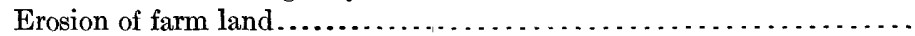

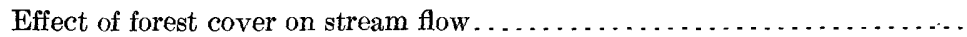

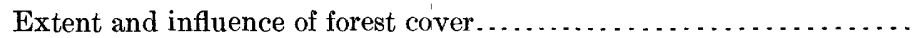

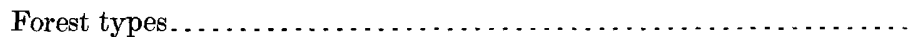

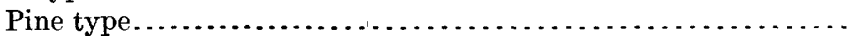


Relation of soils and forest cover to quality and quantity of surface water in Page. the Potomac basin-Continued.

Effect of forest cover on stream flow-Continued.

Forest types-Continued.

Chestnut oak - white oak type...................... 321

Chestnut type..................................... 322

Birch-basswood-red oak type........................ 323

Beech-hard maple-hemlock type........................ 324

Spruce type..................................... 324

Melting of snow..................................... 325

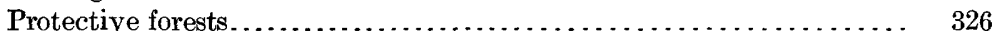

Extension of cleared area............................... 327

Turbidity in reservoirs at Washington, D. C................... 329

The effect of some industrial wastes on fishes, by M. C. Marsh............ $\quad 337$

Introduction.............................................. 337

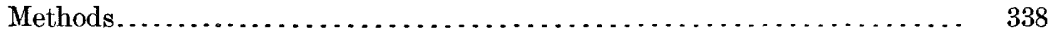

Paper and pulp mill wastes............................. 340

Tannery wastes.......................................... 343

Dye wastes from knitting mills............................. 345

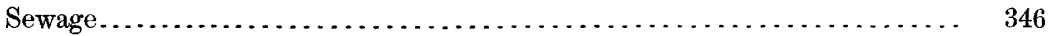

Wastes from manufacture of illuminating gas.................. 346

Water-gas process...................................... 346

Coal-gas process.......................................... 347

Wastes from both water and coal-gas processes................ 348

Summary.......................................... 348

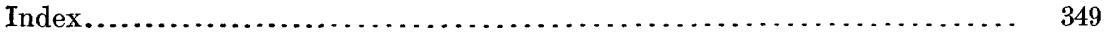

\section{LLUSTRATIONS.}

Plate I. Topographic and rainfall map of the Potomac drainage basin....... Pocket.

II. Drainage map of Potomac basin........................... 8

III. Profile of Shenandoah River and South Fork of Shenandoah River from Harpers Ferry, W. Va., to Port Republic. Va ................ 134

IV. Great Falls of the Potomac................................ 180

V. Plan and profile of North Branch of Potomac River and Potomac River from Cumberland, Md., to Williamsport, Md................

VI. Plan and profile of Potomac River from Williamsport, Md., to George-

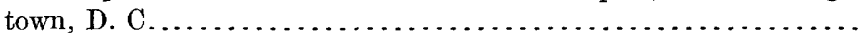

VII. $A$, Chesapeake and Ohio Canal above Williamsport, Md.; $B$, Potomac River and Chesapeake and Ohio Canal at Dam No. $5 \ldots \ldots \ldots . . . . .$.

VIII. $A$, Wills Creek from Market Street Bridge, Cumberland, Md.; $\dot{B}$, Pollution of Potomac River by wastes from the mechanical wood-pulp mill at Harpers Ferry, W. Va........................

IX. Diagram showing relation of stream flow to cases of typhoid fever in the District of Columbia................................ 278

X. Forestry map of the Potomac drainage basin ............... 316

Fig. 1. Discharge, mean-velocity, and area curves for Potomac River at Point of

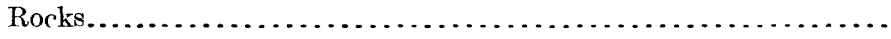

2. Elevation of north bank of Jennings Run, showing course of drainage... 


\section{.THE POTOMAC RIVER BASIN.}

By Horatio N. Parker, Bailey Willis, R. H. Bolster, W. W. Ashe, and M. C. Marsh.

\section{INTRODUCTION.}

\section{SCOPE OF THE PAPER.}

Hardly a river basin in the country is of more importance from the point of view of the utilization of water resources than that of the Potomac. The water power developed in this area drives the wheels of many mills, and the waters of the streams are used in the processes of diverse industries. The beauty of the streams and the supply of fish have made a large portion of the basin a recreation ground for thousands of people, while the Potomac itself furnishes drinking water for the National Capital. In order to obtain definite information on the character of the water supply an extensive investigation was undertaken jointly by the Geological Survey, the Bureau of Forestry, and the Bureau of Fisheries. The result of this work is the present paper, in which are described all the conditions that affect the economic utilization of the water resources. The scope of the paper is best shown by enumerating the principal features of the investigation, which are as follows:

1. A study of the geographic history of the basin.

2. The determination of the amount of water flowing in the principal streams, a compilation of all data relating to the quantity of water, and a study of the distribution of the rainfall.

3. A complete reconnaissance of the drainage area with respect to sources of pollution, a study of the prevalence of typhoid fever in the District of Columbia and at other points, and an investigation of the quality of the surface water as shown by field assays and sanitary and mineral analyses of water taken at many points.

4. A study by the Bureau of Forestry of the effect of the soils and forest cover on the turbidity of the water and the flow of the streams, and the preparation of a map showing the forest conditions.

5. A study by the Bureau of Fisheries of the effect of industrial wastes on fishes. 


\section{ACKNOWLEDGMENTS.}

The lively interest shown by many citizens of the Potomac basin in this report and the help they have given in its preparation are much appreciated. Especial recognition is due to W. D. Bryon '\& Sons, the United States Leather Company, J. R. Cover \& Sons, the Hambleton Leather Company, the West Virginia Pulp and Paper Company, the Potomac Pulp Company, the Blue Ridge Knitting C mpany, the Washington Gas Light Company, and the Clapp Ammonia C.smpany for furnishing samples of the effluents from their factories. 'i his cooperation on their part has added materially to our knowledge of the effect of industrial wastes on fish life.

Acknowledgment should be made to the United Stairs Weather Bureau for rainfall da'a; to the Chief of Engineers of the United States Army for profilcs and elevations along certain portions of the river, and to the Maryland Geological Survey for the maintenance of the gages on Monocacy River and Antietam Creek.

\section{HISTORICAT, SKFTCH OF THE PO'TOMAC BASIN.}

\section{By Horatio N. Parker.}

The Potomac became of moment in English annals with the settlement of Jamestown, Va. Capt. John Smith discovered the river (Patawomek, as he spelled it) June 16, 1608, and sailed upstream about 30 miles to a point where, after having met with a hostile reception from the Indians, he landed on the Virginia shore. From this place, probably Nomini Bay, he continued up the river, touching at various points, until he had passed the present site of Washington, "having gone up as high as they could in a boat." Here they were met by savages in canoes loaded with the flesh of deer, bears, and other animals, of which they obtained-a portion. On their return journey they met with many adventures, but reached Jamestown in safety. In early colonial iimes the name Potomac was applied to the river from its mouth to its junction with the Shenandoah at Harpers Ferry. The portion of the river from that point to its source at the headwaters of North Branch was called the Cohongoruton, a name said to be a corruption of the Indian Kohonk-on-roo-ta, or "wild goose stream," from the great number of wild geese that inhabited it, the "ko-honk! ko-honk!" of the bird suggesting the term.

Lord Fairfax in his land grants on this part of the watercourse designated it Potomac, by which means it gradually lost its anciient name. Shenandoah River was first called Gerando, then Sherandoah, and finally Shenandoah. For a long time after the settlement of Jamestown the colonists, terrified by the gloomy forests of the interior, clung to the coast; but in 1716 Governor Spottswood led an expedition to 
the Blue Ridge and reached its summit, probably near Swift Run Gap. He descended into the valley, crossed the river, which he named Euphrates, and took possession of the country in the name of the King of England. There were no direct results from the expedition, but it had the good effect of dispelling the mystical terror with which the colonists had invested the region.

Prior to its occupation by the settlers the valley of Virginia was a hunting ground for various Indian tribes, who burned the grass every fall before going into winter quarters in order to keep down the forests. Consequently the only timber was along the streams and well back in the mountains. The forests that now exist have sprung up since those times. The trails followed by the colonists through the mountains were established by the buffaloes and other large game and were well worn by the Indians. The valley, as has been said, was a hunting ground rather than a permanent abode of the aborigines. Hence the few villages in it were of a temporary nature and had a fitful existence. The game consisted of buffalo, elk, deer, bear, panther, and wild cats, besides beavers, wolves, foxes, and other animals. The Indians welcomed the Pennsylvania colonists because of the trust they had in William Penn, but they showed great hostility toward the settlers from tide water, whom they called "The Long Knives," and whom they hated. In 1753 emissaries from west of the Alleghenies came among the valley Indians and invited them to cross the mountains, which they did in 1754. Their sudden exodus caused much uneasiness among the Virginia colonists, who feared that the action foreboded impending hostilities. This proved true enough, for it was probably French influence that coaxed the Indians away, and after Braddock's defeat they terrorized the valleys of South Branch and the Shenandoah, committing many outrages, and not being driven back until the close of the French and Indian war.

The upper and lower portions of the valley of Virginia were settled at about the same time. The colonists of the tide-water region made their way up the lowland rivers and finally passed over the mountains into the valley, and at the same time, or a few years before, the region toward the Potomac was settled by Scotch-Irish and Germans from Pennsylvania. The Scotch-Irish were the pioneers and established homesteads along Opequon Creek from the Potomac to what is now Winchester. The Germans followed. Joist Hite, in 1732, obtained a grant of 40,000 acres and with 16 families moved from Pennsylvania, cutting the road from York, crossing the Cohongoruton 2 miles above Harpers Ferry, and settling on Opequon Creek 5 miles south of Winchester. His followers built Strasburg and other towns along Massanutten Mountain. In 1733 Jacob Stover took a grant for 5,000 acres of land on South Fork of the Shenandoah, and in 1734 settlers from Monocacy, Md., located on North Fork of the Shenandoah, 12 miles 
south of Woodstock. Two cabins, erected in 1738 mear Shawnee Springs, were the beginning of the town of Winchester, long a frontier post of the colony in that quarter. John Lewis brought over from Ireland and Scotland 100 families and settled near what is now Staunton, Augusta.County. Conococheague Creek was settled at Greencastle, Pa., in 1734, the place being first known as the Conococheague Settlements. In 1734 Richard Morgan obtained a tract of land near Shepherdstown, the oldest town in West Virginia. Romney, W. Va., was laid out by Lord Fairfax in 1742 and is the second oldest town in the State. In 1748 Robert Harper, an English millwright, came to Harpers Ferry. Benjamin Allen, Riley Moore, and William White built homes on the Monocacy prior to 1734, and in 1735 the Schleys, with about 100 families from Germany, Switzerland, and France, established themselves on the Monocacy, the first house in Frederick being erected by Thomas Schley in 1735. By 1748 the German immigrants had taken possession of many valuable tracts along Monocacy River and Catoctin Creek. At an early period many immigrants became occupants of the Cacapon and Lost River valleys and numerous settlements were made on Back and Cedar creeks. In 1741 Col. Thomas Cresap, with his own and several other families, located at "Shewaneese" Oldtown, on North Branch of the Potomac.

The first settlers on the Wappatomaka, as South Branch of the Potomac was called, located in 1734 or 1735 . They failed to secure title to their lands, and so became involved in a dispute with Lord Fairfax, who, they felt, dealt harshly with them. There is a tradition that Lord Fairfax became interested in his Virginia venture through meeting John Howard, who, with his son, is said to have explored the valley of Virgimia prior to its settlement and to have discovered the valley of South Branch, crossed the Allegheny Mountains, and gone down Ohio and Mississippi rivers to New Orleans, where they were arrested as suspicious characters and sent to Paris; thence, no cause being found for holding them, they went to London, where the meeting with Lord Fairfax is said to have occurred. Lord Fairfax came to Virginia in 1742 and opened an office in Fairfax County for granting land warrants. A few years later he moved to what he called Greenway Court, 12 or 14 miles southeast of Winchester, where he kept his office until he died in 1781. His surveyors decided that North Branch was the main stream of the Potomac and located the "Fairfax Stone" at its head October 17, 1746. This action was greatly to his advantage, for had South Branch been chosen as the "first fountain" the Fairfax holdings would have been much reduced. Later the States of Virgimia and Maryland became involved in a dispute as to the location of the boundary lime between them, and though the question has never been settled Virginia has been able to maintain the North Branch as the boundary, basing her claim on the location of the "Fairfax Stone." 
In 1725 John Van Metre, a trader from Hudson River, traversed the lower Shenandoah, upper Potomac, and South Branch valleys, and at Hanging Rocks witnessed a bloody battle between two parties of Indians. He returned home much impressed with the richness of the South Branch region and advised his sons to move there, which they subsequently did. The earliest settlers found a natural clearing in the woods at Oldfields and built a fort there, which was the scene of many fights with the savages.

Lands on Patterson Creek began to attract the pioneers a little before Fort Cumberland was completed in the winter of 1754-55. In 1728 there was an Indian town known as Caiuc-tu-cuc on the ground between Wills Creek, or, as it was then known, Caiuc-tu-cuc Creek, and North Branch; it was located for the most part upon the site of the west side of what is now Cumberland. The Indian village was abandoned and in its place a settlement of whites slowly grew up. The last Indian to remain and have authority was known as Will, and the town for a long time was known as Will's Town, the creek as Will's Creek, and the mountain where he had his home as Will's Mountain. His rights in the country appear to have been recognized, for the early settlers always made him a present when they took up land. The first comer to Cumberland of whom there is record was an Englishman named Evitt, who led the life of a recluse in his cabin on top of Evitts Mountain, where he died before 1749.

Georges Creek took its name from an Indian, George, who had his hunting lodge on the present site of Lonaconing. He was a favorite of and lived with Col. Thomas Cresap, of Oldtown, who had employed his father, Nemacolin, to mark out the road from Cumberland to Brownsville, on the Monongahela. General Braddock followed the path and the national road varies but little from it. This testifies to the excellent manner in which the Indian did his work.

Cumberland was long the outpost of civilization in the Potomac Valley. The last refuge of the Indians was on Savage Mountain; hence its name. The first settlers on Georges Creek came from New Jersey and Virginia. Prior to 1830 there were not more than 30 houses in Georges Creek valley. North Branch above Westernport seems to have been well known at an early date. Washington, on his return from the trip to Ohio in 1784, crossed the stream and mentions in his journal for September 26 that he was told by Joseph Logston, who had hunted along the river, that there was no fall in it, and that from Fort Cumberland to the mouth of Savage River the water was frequently made use of in its natural condition for canoes, and that from thence upward it was rapid only in places, with loose rocks which could be easily removed.

September 27 Washington crossed Stony River, which he speaks of as appearing larger than North Branch. On his return to Mount Vernon he made a map of the country he had visited, on which was 
shown North Branch with the tributaries Difficult Creek, Stony River, Abrams Creek, New Creek, Georges Creek, Savage River, and the head of Patterson Creek. A map by Joseph Shriver, published in 1824, shows North Branch from Westernport to its source, the only town above Westernport being Paddytown, now Keyser, W. Va.

Coal seems to have been known to the earliest settlers. In 1804 it was discovered near the present site of Frostburg. In 1810 a tremendous freshet stripped the earth from the banks of Guinea Run, displaying the coal on what is known as the Barton property. People came from miles around to see "the mountain of coal." For some time is was mined with mattocks and the ore was hauled to Winchester and Romney for blacksmithing purposes. In 1814 or 1815, while the national road was being made, coal was found at Eckhart Mines and was hauled in wagons to Cumberland and Baltimore. Three or four bateaux arrived at Washington April 20, 1826, laden with coal from the rich mines at Cumberland. Up to 1842 merchants, laborers, and others engaged in various pursuits in the summer and worked in the mines or coal banks, as they were called, in the winter, some as teamsters, some as boat builders, and some as miners. The coal was hauled to the river bank and piled there in large quantities. In the spring freshets the boats, which hauled from 1,000 to 1,500 bushels, were sent down the river to the purchasers. The flatboats were not returned, but occasionally a keel boat laden with supplies was laboriously poled back. From 50 to 60 boats, carrying an aggregate of 75,000 bushels of coal, comprised the total shipment each year previous to the completion of the Baltimore and Ohio Railroad in 1842 . As the coal business was conducted up to that time, it was hazardous to capital and destructive to the lives of those engaged in carrying it on, many boats being wrecked on the rocks in the river. Hence few mines were worked, the chief being the old Eckhart mine, 9 miles west of Cumberland. The Georges Creek Coal and Iron Company was the first to develop mines west of Frostburg. It began excavations for its iron furnace in 1836. Coal was first shipped on the Chesapeake and Ohio Canal in 1850. The coal fields of North Branch above Piedmont were described by Prof. W. B. Rogers in 1839 in his report on the geology of Virginia.

The orderly development of the Potomac Valley proceeded until the outbreak of the civil war, when the arts of peace were suspended and this battle ground of the Indian became that of the white man. The great battles of Antietam and Gettysburg were fought within the valley's borders, as were a host of other no less bravely contested engagements. For four years the work of destruction went on, but with the advent of peace in due time came prosperity, which has continued, until to-day the growth of the industries and population in the valley is healthy and vigorous. 


\title{
GEOGRAPHIC HISTORY OF POTOMAC RIVER.
}

\author{
By Bailey Willis.
}

\section{GENERAL DESCRIPTION OF BASIN.}

The Potomac, rising among the Allegheny Plateaus and Appalachian Ranges, ${ }^{a}$ gathers its waters in a main channel which crosses the grain of the country in a southeasterly course. Its mouth is an estuary, a branch of Chesapeake Bay. Washington is situated at the head of tide water, where the estuary receives the river proper. The stretch from Washington upstream to Cumberland, a distance of 108 miles in a direct line and 186 miles by the river, is the trunk channel. The Shenandoah, Great Cacapon, and South Branch are its principal feeders. They enter from the southwest. North Branch is the actual head of the river. The tributaries from the northeast are relatively short, Wills Creek, Conococheague Creek, and Monocacy River being the principal ones.

Although the Potomac watershed is a mountainous region, characterized by ranges of notable height and continuity, it is not limited by the greater elevations. We are apt to think of the basin of a river as an area surrounded by a high or at least obvious divide, but that is not true of the Potomac. Its trunk channel cuts across the ranges; its branches embrace them; its headwaters in North Branch invade even the plateau whose bold scarp suggests an unbroken divide. The principal streams rise in valleys which extend with undiminished width and without change of the gentle slope beyond the head springs. In their continuation other springs and brooks gather and flow in a direction opposite to that taken by the waters of the Potomac. The parting streams are opponents, which compete for territory. The basin which the Potomac may drain is limited by its competitors. The Susquehanna holds the valley of Pennsylvania, the James is entrenched in southern Virginia, and the Big Kanawha and Monongahela contest the western plateau region.

The shape of the Potomac drainage basin west of the Blue Ridge is oval; its length, northeast to southwest, being 160 miles and its width

a Powell, J. W., Physiographic regions of the United States: National |Geographic Monographs, vol. 1, No. 3, 1895, map. 
but 80 miles. In consequence of the great length of the southern tributaries, the trunk channel crosses the northern part of the basin and leaves the oval at its northeast corner, where it and the brooks that join it constitute a triangular expansion of the watershed.

The arrangement of streams within the watershed deserves notice. By a study of the outline map (Pl. II) it will be seen that there is a peculiar parallelism among the many rivers flowing to the northeast or southwest, and also a marked tendency to courses which for short distances are at right angles to the general direction. The arrangement is a common one in certain regions, and a stream system thus developed is known as "trellised drainage," from the resemblance which the rivers bear to the stems of a vine on a trellis. While a trellised plan exists in much of the Potomac basin, it does not extend throughout. Another plan is to be noted, for example, in the Monocacy, Goose Creek, headwaters of the Shenandoah, and highest forks of North Branch. This is a plan characterized by acutely branching streams which divide as do the limbs of an elm tree.

Trellised drainage of the Potomac is restricted to the Appalachian Ranges and results from the grain of the country; that is to say, from the fact that the rocks are arranged in layers which show their edges at the surface and thus extend long distances in one direction. Some are hard (sandstones) and some are soft (shales and limestones). Ridges persist on the hard belts as valleys develop on the soft rocks between, and the streams for the most part follow the grain. There are conditions, however, under which they must cross from one valley to another, which they do in a gap at right angles to the sandstone ridge; hence the short, transverse courses at right angles to the longitudinal ones.

Where the rocks which appear at the surface are of the same texture and solubility over a considerable area, the streams find no belts especially adapted to the development of valleys; neither are there any harder layers peculiarly competent to maintain ridges; and in engraving the bas-relief of hills and ravines, the streams grow according to minor accidents of the surface, as gullies grow in a field.

Specific names have been given to the various patterns which river systems assume. Where the valleys are developed on belts of soft rocks, and ridges are maintained by hard rocks, the streams are said to be "adjusted." Trellised drainage is adjusted. Where the branches diverge upstream from one another like the gullies in a field or the branches of an elm, they are called "self-grown," or "autogenous." The Monocacy presents an example of the autogenous pattern.

We have thus far considered the plan of the Potomac system as it appears on a map. The vertical profile and cross section also present significant peculiarities. 


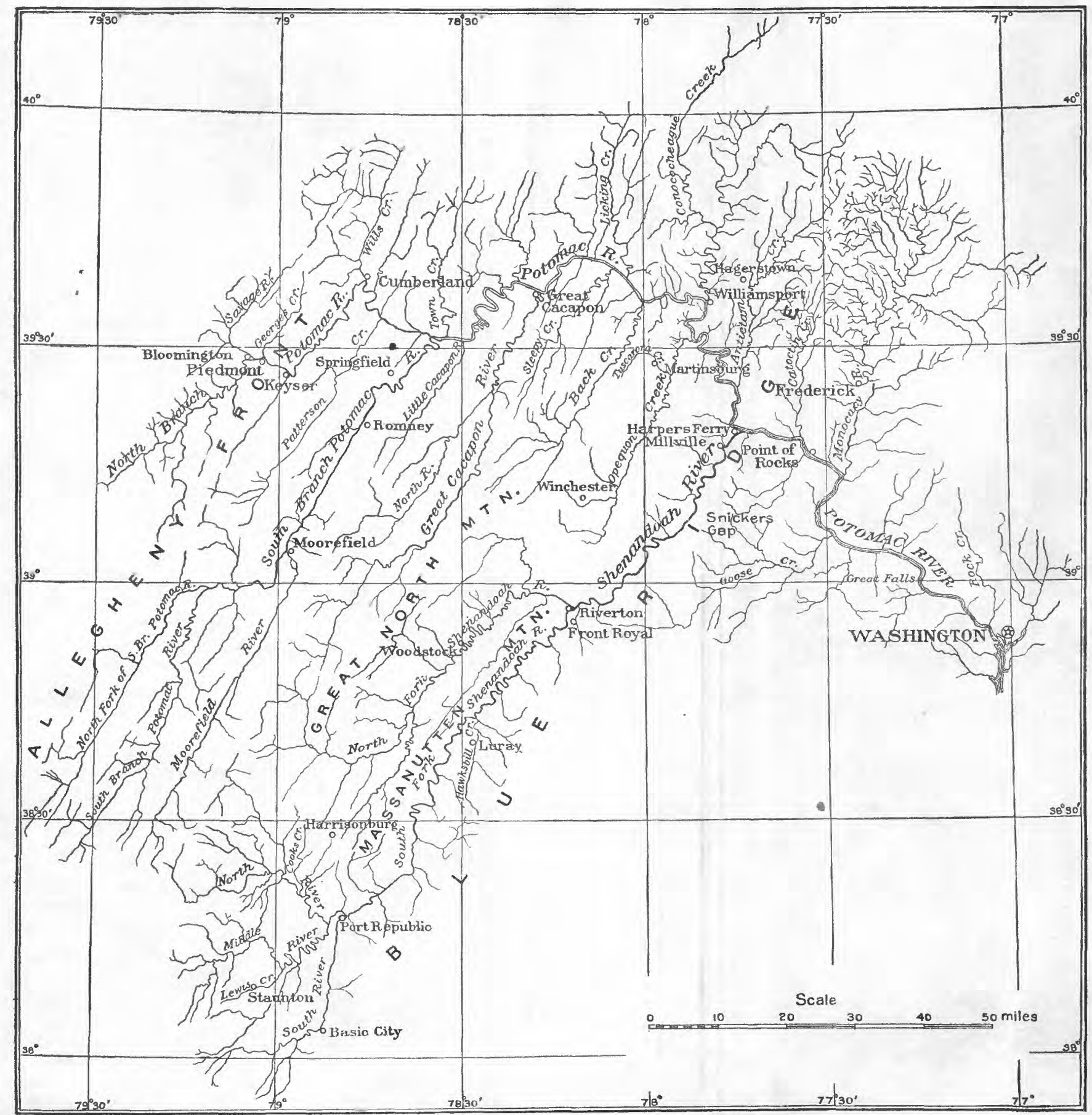

DRAINAGE MAP OF THE POTOMAC BASIN. 


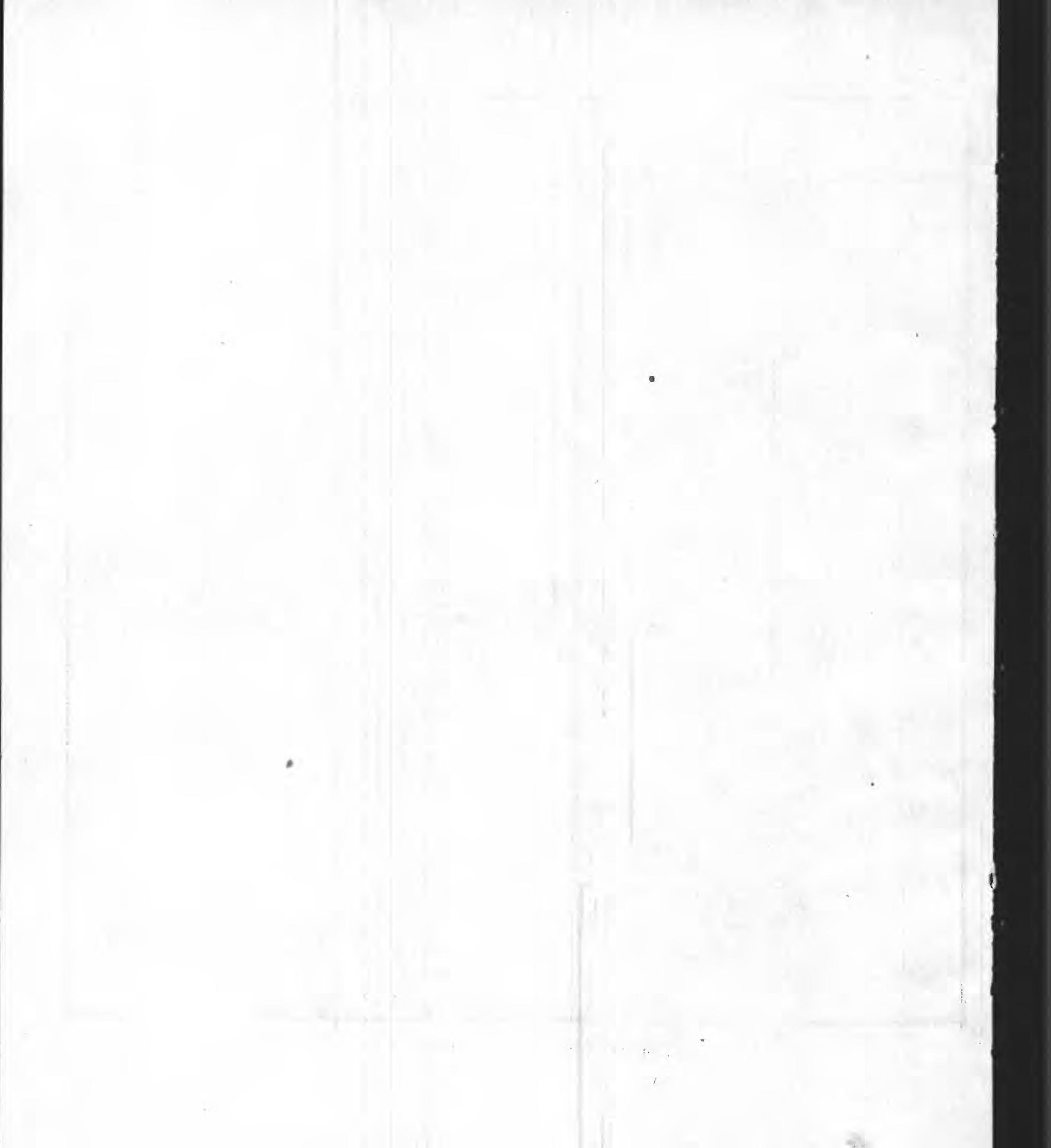


An ideal river profile is a curve which descends sharply near the source, becomes flatter and flatter, and at the mouth is a straight line, tangent to the level of discharge. The ideal curve is concave upward from source to mouth. The Potomac departs very decidedly from this ideal." The trunk channel from Cumberland to Washington is interrupted by rapids, which separate long flat reaches; at each rapid the profile is broken by a sharp bend, which is convex on the upper side-the reverse of the ideal. Near the very mouth of the river is Great Falls, over which the waters plunge to a series of lesser rapids that descend sharply to tide water. This is not at all the normal tangent. (See profiles, Pls. V and VI, p. 182.)

The tributaries exhibit profiles possessing similar irregularities, and it is particularly noticeable throughout the system that wherever a smaller stream enters a larger one a rapid or cascade marks the final descent of the smaller.

The ideal cross section of a river valley is, like the ideal profile, a curve which is concave upward and flattens from the divide to the stream. In this respect also the Potomac and its tributaries depart from the normal. The cross sections of the valleys are made up of steep slopes and flats, which constitute an irregular curve. Descending steeply from a divide, the traveler comes upon a flat or plain, which may extend for several miles. Although the surface of the flat is as a rule deeply cut by brooks, the journey may be continued nearly at a level by following the spurs between them. But wherever a stream, large or small, is approached, it is necessary to descend sharply into a trench. Along the lower Potomac, below Great Falls, this trench is a picturesque canyon 220 feet deep. The flat on each side of it is an outer valley several miles across. Along the Shenandoah similar features are found, the river flowing at the bottom of a ravine, while the broad plain of the great valley of Virginia stretches away with nearly level though dissected surface to the Blue Ridge and Massanutten Mountain.

\section{DEVELOPMEN'T OF THE RIVER SYSTEM.}

Enough has been suggested in the preceding description to show that the Potomac and its tributaries are regarded as an individual stream system which has developed from some previous condition to its present proportions. It has been limited in growth by competition with neighboring rivers. Its development has been directed along lines of least resistance and its branches have extended in belts of weak rocks. It has sculptured the surface, its rills, rivulets, brooks, creeks, and branches everywhere constantly taking some material in solution or as sediment and delivering it to the trunk stream, which carried it away. The features which the river has modeled are the channel or inner canyon in which it flows, the 
broader valley that expands at a higher level, and the steep slopes of the ridges that rise within and around the basin. But these are the features of the entire landscape, except perhaps the highest parts of the ridges; and they, too, owe their long level crests to the activity of the river, as will be better understood when the history is traced.

We recognize that the Potomac has been, and indeed is, a working, growing system. Its task is to excavate its basin, to erode valleys and mountains till no elevations remain. Its power depends on its volume, its fall, and a just proportion of sand with which to cut away the hard rocks in its course.

The trunk channel being deepened, the tributary channels have also been cut down, but not so speedily; hence the rapids near their mouths. The deepening, spreading from the main stream to large branches, from the large branches to their forks, and from each fork to the smallest rivulets, has extended outward over the entire basin. It proceeds immediately from an elevation of the land. Its limit is the lowest level to which the main stream can cut its channel at its mouth-the level of discharge, from which when the work of channel cutting is done the profile will rise in the long ideal concave curve. A stream that has reached that stage is said to be graded. It is evident that the Potomac has much work to do before it can be called a graded stream.

The channel of the main river will usually become graded before those of its tributaries, and the next step is the grading of the valley slopes. Each brook, rivulet, and rill goes through the same process as the main stream. The effect is reduction of the slopes to the inclination on which the waters flow but do not cut. As the grade extends to the higher divides, even they are reduced, and in time the lowest possible slope is established over the entire surface of a river basin.

Anyone familiar with the mountains among which the Potomac flows may well pause to ask if such a leveling of their heights can ever be accomplished; but the student of the river's history learns not only that in time they must be leveled, but also that in times past the river has had the work then before it much more nearly accomplished than now. It now runs in a canyon which it is deepening. It once flowed on the level of the outer valley, which it had cut to that level and widened to an extensive grade. Indeed, long before that it had taken its course over a plain which coincided with the tops of the present ridges and which it had graded from still older mountains.

The history of the river's work has been one of successive gradings in consequence of successive elevations of the land. Let us attempt to follow its major outlines.

Age is a subject not usually discussed with reference to rivers and 
mountains. They all appear very old. But some are older than others, and among the rivers of North America the Potomac and its neighbors are of an older generation. The Appalachian Ranges, on the other hand, are relatively young; and so it happens that the Potomac is older than the mountains in which it rises and across which it flows. It may, however, be compared to a tree of which the trunk is aged, while the branches and branchlets are younger, some of them very young. The careful student of physiography will some day search out the history of the system as a whole and of each branch separately - a complex study, for which the data are not yet available; but we can indicate the principal facts and, where our present knowledge halts, point out the problem to be attacked.

Before there was a Potomac, in the age of the coal deposits of the Carboniferous, streams flowed southwestward from New York, easterm Pennsylvania, and eastern Virginia toward the interior sea that lingered over the Southwestern States. We feel confident of this, because the relative positions of land and coal marsh and sea are recorded in the rocks laid down at the time, but we can not identify the position of any particular river. There were then no mountains where the Appalachians now extend, but ranges began to develop in the next succeeding epoch, during what is known as the Appalachian revolution. Very great changes occurred in the relative positions of land and water, and the movement of the earth's crust was such that a belt of strata 100 miles or more in width, extending from New York to Alabama, and from 10,000 to 30,000 feet thick, was folded so as to produce arches and troughs. The effects were no doubt of gradual development, but in all probability they were such that the arches attained the height of notable mountains, and the troughs became open valleys or inclosed basins. The previously existing streams were more or less checked and diverted by folding of the strata, and we suppose that they were so effectually changed that a new river system was substituted for them. A portion of that system flowed or a surface above the Potomac basin, and the Potomac is probably descended from it.

The geologic age referred to in the last paragraph is the Permian, an age during which aridity was a common, if not a general, condition of the climate of several continents. It is possible that the climate of the Appalachian province was for a longer or shorter epoch so arid that rivers ceased to flow, but there is no direct evidence of the condition.

We suppose that the oldest rivers, which developed courses on the surface of the folded strata, flowed along the troughs and across from trough to trough, between and across the arches that stood as mountain ridges. The courses were essentially parallel to those of the trellised system of the present time, but the trunk channel may

IRR $192-07-2$ 
have led the waters westward toward the interior sea, instead of southeastward to the Atlantic, as is now the case. ${ }^{a}$

The surface was then several thousand feet above the present surface. Even the mountain tops which we now see were then deeply buried beneath solid rock and lay below sea level. A swèlling of the earth's crust has since raised the mass of the Appalachian Mountains.

Thus the earliest rivers with which the Potomac may be related are those which developed in consequence of the folding that occurred in the Appalachian region during the Permian age. Their courses are supposed to have been determined by the troughs or valleys which resulted from folding, and they are therefore called "consequent."

Consequent streams are those which flow in the direction of slope that is due to folding or warping of the surface. They differ from adjusted streams in that they take their courses along a low line or down a slope instead of working out a valley in soft rocks. But in a region like that of the Potomac, where beds of hard and soft rocks occur in long parallel folds, a consequent system becomes an adjusted system at an early stage of valley cutting.

The folded structure of the Appalachian Ranges has been carefully studied, and we are able to locate the lines which were the bottoms of troughs in the Permian surface. Though high above the present surface, the deeper troughs closely corresponded in position with Massanutten Mountain, Great North Mountain, and South Branch Mountain. Rivers which occupied them flowed parallel to the present streams, but along and above the now existing mountain tops. The old valleys have become mountain ridges. This change is of frequent occurrence in the process of adjustment, as streams sink their channels through alternate hard and soft strata, ${ }^{b}$ and there is no difficulty in understanding how the rivers that now flow by the sides of the former troughs, or even in valleys along the crests of arches which correspond with former mountains, are related to the old consequent system.

Another trough which should be mentioned is the valley of Georges Creek and North Fork above Bloomington. It is one of the deepest troughs in the Potomac basin, and we need not doubt that it was occupied by a branch of the consequent drainage, but on a valley bottom high above the present surface.

Following the line of thought suggested in the preceding paragraphs, we may state the simplest outline of the history of the Potomac in the following way: The Potomac is the descendant of a consequent drainage system which developed on the Permian surface during or after the Appalachian folding. Being established in a region which presents an alternation of decidedly hard and soft

a Davis, W. M., Rivers and valleys of Pennsylvania: Nat. Geog. Mag., vol. 1, 1889, pp. 222 et seg.

6 Willis, Bailey, Topography and structure of the Bays Mountains: School of Mines Quarterly, vol. 8, 1887, pp. 242-252. 
rocks arranged in long, narrow belts, the streams have become adjusted to the softer strata. In sinking their channels down through several thousand feet of varying rock they have indeed become so thoroughly adjusted that stretches beneath the old valleys have become mountain ridges capped with hard sandstone, and valleys are developed on either side, in places even along the tops of arches.

Granting this statement the advantage of being probably true, we may compare it with one that we are descended from Adam. Many links are omitted and much is unaccounted for. It is not enough to know the structure of a river basin and the adjustment of the river system to it. We need to know also the profiles and cross sections of the valleys and the deposits which the river from time to time in the course of its long existence has made in them, as well. Furthermore, we need to look over the mountain tops to ascertain what remnants of old surfaces are there visible.

To pursue the subject more closely it is necessary to digress to the history of the mountains before the elevation of the ranges which we now see.

The Permian Appalachians are known to have been greatly elevated in the process of folding. It is possible that elevation progressed so slowly that erosion nearly kept pace with it in wearing down the heights, and if so, the mountains never attained great altitude; but it is more likely that the elevation went on with comparative rapidity and was attended by the development of conspicuous heights. This inference, rests, however, on geologic reasoning. There are no great mountains to which one, looking abroad over the Appalachian Ranges, can point as Permian mountains. On the contrary, he who looks across from Massanutten to Great North and from Great North to the Allegheny Front sees long, even-crested ridges, which suggest a plain. If the valleys were filled to the rim with the material which the streams have carried away, the region would become a plain; and above such a surface stood the mountains of Permian time. They are no longer there.

In the lowlands of New Jersey, eastern Pennsylvania, Maryland, and Virginia there are deposits of red sandstone and mud rock, the materials of which were derived from adjacent areas, in large part from the district of the Permian mountains. The strata are Triassic, slightly more recent than the Permian, and are of such volume that if restored en masse to the place of their origin they would form a considerable mountain chain. They no doubt represent a part of the Permian mountains which wasted away under attacks of eroding agents.

It is a somewhat surprising conclusion that the Permian Appalachian Mountains not only wasted to low hills, but disappeared so 
completely that a plain extended from sea level across much of the region where they previously stood; yet that such was the fact we are led to believe by two lines of reasoning. Spread over the Atlantic Coastal Plain are deposits of gravel, sand, and clay washed from the region to the west during the epochs succeeding that of the strata which represent part of the waste of the Permian Appalachians. Geologists class the epochs as Jurassic and Cretaceous. The deposits are small in amount, and if restored to the watersheds of the streams which carried them away would not materially increase the altitude of the surface. As there is no mass of sediments of that time equivalent to a mountain range in volume, we reason that there was no range. The only escape from the conclusion is through the assumption that thicker deposits lie buried out to sea; but well borings show that the strata which do exist there are of fine calcareous material, chiefly marine sediment, which does not represent the immediate waste of mountains.

In corroboration, if we look over the Appalachian Ranges for remnants of highlands which may have existed during Jurassic and Cretaceous times, we find them of slight extent. The principal summits of the Blue Ridge, scattered heights of the Allegheny Plateaus, and the big balds of the Great Smoky Mountains were then low, rounded hills. ${ }^{a}$ They still possess that form. Extending from them at a lower level are the long, even crests of the Appalachian Ranges, which, if the valleys between them were filled, would correspond with the surface of a plain. Once nearly level, this plain is so no longer. In West Virginia it lies at an altitude of 4,000 feet above the present sea level, but west of Washington it sinks to 1,000 feet, and near the city passes under the surface, being buried by the gravels and clays of the so-called Potomac formation, which is at the base of the Jurassic and Cretaceous sediments above referred to. The topographic features of the time are thus distinguished from those of later epochs by the fact that in the existing mountains they possess peculiar roundness and flatness and occur at high altitudes, whereas along the Coastal Plain the representative surface passes beneath the strata of later age.

The recognition of the ancient plain which characterized the eastern United States and also Canada during the Jurassic and Cretaceous ages was a most important step in the understanding of the history of the mountains and rivers. From its conspicuous character in the crest of Schooley Mountain, New Jersey, it has been named the Schooley peneplain. ${ }^{b}$

$a$ Hayes, C. W., and Campbell, M. R., Geomorphology of the southern Appalachians: Nat. Geog. Mag., vol. 6, 1894, pp. 63-126, Pl. V.

$b$ Peneplain is a technical term meaning almost plain. It is used to a void the suggestion of a perfectly plain surface. It is consistently applied to a region of wide valleys among low hills, or to a true plain, the degree of unevenness being indeterminate; but it carries by definition the implication that the surface has been planed by the ordinary processes of atmospheric erosion. 
We may now return to the Potomac, to discover, if possible, its course on the Schooley peneplain and to trace its further development.

It has already been stated that the consequent drainage of the Permian Appalachians probably joined in a trunk channel and flowed to the southwest. The Potomac above Harpers Ferry could not then have existed, except perhaps as a stream rising in the Blue Ridge and pursuing a course toward Cumberland. The Shenandoah, South Branch, and other large tributaries, which are now adjusted to the valleys in limestone and shale, were then represented by streams flowing along the troughs produced by folding. By the time the Permian Appalachians had wasted to a peneplain still having pronounced relief the adjustment of the branches was accomplished and they were probably established along the lines of their present valleys, but near the level of the now existing sandstone ridges. The trunk channel may still have descended westward. East of the Blue Ridge there were rivers that carried down sediment to the Coastal Plain and spread it there. Part of it constitutes the base of the Potomac formation, and consists of coarse pebbles and bowlders of quartz and quartzite derived from ledges in the Blue Ridge. It was distributed by streams meandering over the eroded surface of the ancient gneisses, with the sands of which the cobbles are mingled. A river corresponding with the Potomac below Harpers Ferry probably had a more or less important share in this work, which was accomplished during the later part of the Jurassic age. It is possible that the river even then rose west of the Blue Ridge.

When the Schooley peneplain had been eroded to very low relief, conditions were favorable for extension of drainage lines on the part of strong streams at the expense of weaker ones. The processes by which such extension is accomplished are complex and subject to many qualifying conditions. They can not be detailed here, but in general there are three principal factors which affect the result.

A river of large volume is commonly stronger than one of less volume. One which has rapid fall-that is, one which takes a short course to a low point of discharge-is advantageously situated. Finally, one which is developing a channel in soft rocks is likely to reach a low level sooner than one which is working in hard rocks, and may thus develop a steep fall near its head, which gives it a local advantage.

In attempting to understand how the consequent drainage that initially flowed westward became reversed, so that the present direction of flow was established, we find that the item of relatively short course and steeper fall appears to have been the determining factor. Whether the divide be assumed at the Blue Ridge or at any other point within the Potomac basin, the course to tide level near Washington is much shorter than that toward the southwest, in w'ich 
direction there was then, so far as we know, no sea nearer than the Gulf of Mexico, if as near. The eastern course, being shorter, was steeper, and streams pursuing it attacked the divide between themselves and western rivers more vigorously than the latter did. The rocks of the Blue Ridge are hard and no doubt formed a height which long resisted the work of the gnawing brooks that ran down its eastern slope; but inasmuch as it was leveled to a low ridge by the very slow process of general denudation, it must have yielded sooner to the more effective abrading action of running water and sand.

At some time, probably early in the development of the Schooley peneplain, the Blue Ridge was thus cut through from the east. This rcsult follows directly from its geographic position in relation to tide water, but it may have been accelerated by elevation of the western or depression of the eastern region in such a way as to increase the advantage of the eastward course. Davis, who first recognized the reversal, ${ }^{a}$ suggests that it occurred when the basin in which the Triassic sediments from the Permian Appalachians were deposited was developed, in which case the present course of the Potomac has been established since Triassic time instead of only since late Jurassic or early Cretaceous.

The Potomac at Harpers Ferry was not the only stream which succeeded in crossing the ridge. Each of the several gaps that notch the Blue Ridge, as, for example, Snickers Gap at the head of Goose Creek, though a wind gap now, was a water gap then, and was occupied by the successful stream. The Blue Ridge being cut through, the eastern waters were divided only by limestone from the rivers which drained the Great Valley, and having gained ground in the contest for the main divide, they were able to continue doing so; but as the hard rocks of the Blue Ridge lay across their upper courses their progress beyond was probably slow at first, until they had cut the gaps below the general level of the peneplain on the limestone. That they should eventually expand in the Great-Valley and capture the streams which still formed the headwaters of the westward-flowing main river was an inevitable result of their shorter course to the sea. The development of several systems, among which the basin of the present Potomac was divided, was a natural result.

The preceding explanation of the growth of the Potomac across the Blue Ridge and beyond to the Allegheny Front is based on a wellknown action by which streams grow at their heads as a tree grows at the tips of twigs. It is technically known as "headwater" erosion or "retrogressive" erosion.

A somewhat different account of the development of the Potomac may be based on what is known as a "superimposed" course. If it be assumed that the Schooley peneplain was covered with alluvium to a

a Davis, W. M., Rivers and valleys of Pennsylvania: Nat. Geog. Mag., vol. 1, 1889, p. 229. 
sufficient depth to bury the lowest parts of sandstone ridges, then it is probable that streams would become established across the ridges as well as between them. Transverse channels would develop rapidly if the plain were so warped as to increase the declivity toward the east. In the progress of warping the deepening channel would be cut down to hard rocks, but the river would then be intrenched and could not depart from its established course across the grain. This process implies very uniform planation of the surface, and might have led to a less direct course than that which the Potomac has; but it may have played some part in the river's early history, as it probably did in some later episodes. ${ }^{a}$

Leaving the problem of the exact manner of development to the careful investigation which it merits, we may consider the course of the Potomac from Cumberland to Harpers Ferry as having been established on the Schooley peneplain. The trunk channel was then fed by tributaries which entered it as the principal branches now do, and the system was one which may fairly be called the "Potomac." It did not, however, have the expansion of watershed which it now has, but was probably much more restricted toward the south, the Shenandoah, South Branch, and others on that side being at the time comparatively short. The northern branches, on the other hand, may have been longer.

In the preceding discussion one important fact has been tacitly passed over-the altitude of the Schooley peneplain at the time of its development with reference to sea level. The evenness of the plain is attributed to planation by streams, which are able to produce such a surface only when they have cut their channels down to the lowest possible grade - that is, to a slope which is tangent with the sea level or with some other fixed level of discharge. A barrier of hard rock, a dam, for instance, may for a time constitute a local level of this sort. The Schooley peneplain is so extensive that no local level can have sufficed to fix it. Sea level alone could determine the grade common to many streams draining thousands of square miles. We reason accordingly, from the laws of river action and the extent of the pencplain, that the surface of the land rose gradually from sea level to a very moderate altitude only. This was in the Jurassic and Cretaceous periods.

At the present time the Schooley peneplain in West Virginia lies at an altitude of 4,000 feet and its surface has the form of a very broad, somewhat uneven dome, sloping from the greatest height in that region to a position below the Coastal Plain on the east and to one nearly as low in the Mississippi Valley on the west. It is a warped

a Willis, Bailey, The northern Appalachians: Physiography of the United States: National Geographic Monographs, vol. 1, No. 6, 1896, p. 190. 
surface, raised on a gentle swelling of the underlying rocky crust from the low grade at which it was developed to its present form., The consequences of elevation where streams flow upon swelling surfaces are increased velocity of flow and deeper cutting of the channels. Large rivers may do so nearly or quite as rapidly as the mass beneath them rises, and may thus maintain a relatively low grade at the bottom of a canyon; but smaller streams do not keep pace and acquire steep profiles. At their headwaters the branches tend to grow as their channels deepen; competition is renewed between opponent brooks across a divide; and if the changed conditions favor one more than another the favored one grows accordingly. Furthermore, rivers flowing across rock masses which consist of alternate hard and soft layers sooner or later cut down to a change of rock, from soft to hard, or vice versa, and thus become favored or retarded in the process of deepening their channels. The advantages thus gained or lost lead to readjustments of watersheds--a kind of natural gerrymander, to borrow a political phrase-and to the diversion of streams from one course to another by the process known as stream capture.

The growth which the Potomac and its tributaries had in consequence of the doming of the Schooley peneplain resulted in the existing arrangement, which probably differs notably from that of the older river. The detailed changes within the Potomac basin escape our present knowledge, but they may be more or less closely traced by study of the wind gaps, which represent abandoned channels, and by investigation of the relations which streams had to the underlying rocks during the process of sinking their valleys from the level of the mountain tops to their present position.

One fact is, however, so striking that it stands out clearly-the great length of the southern tributaries of the.Potomac as compared with the opponent streams that flow to the James. The headwaters of the Shenandoah, for example, in Augusta County, 120 miles from the Potomac at Harpers Ferry, are but 25 miles from the James atBalcony Falls. A sufficient reason is found in the fact that the warped surface of the Schooley peneplain slopes toward the Potomac. It is highest above the region where the divide extends between the Shenandoah and South River (the opposing tributary of the James), ${ }^{a}$ and the long course of the Shenandoah corresponds with the long slope of the old surface. The inference is that the Shenandoah grew to its present dimensions because, when it was a much smaller river, its fall was increased by the northward tilting of the surface. Having a low point of discharge it extended its basin by headwater erosion, capturing in succession the heads of those streams which rose in the Great Valley and flowed eastward across the Blue Ridge. Their

\footnotetext{
$a$ Hayes, C. W., and Cımpbell, M. R., Geomorphology of the southern Appatachians: Nat. Geog.; Mag., vol. 6, 1894, Pl. V.
} 
abandoned gaps, such as Snickers Gap, remain as evidence of their former existence. In the course of its conquests the Shenandoah became opposed by the tributaries of the James, but it continued to push the divide southward until an equilibrium was established between the opponents across the area where the Schooley peneplain was most elevated.

The northern tributaries of the Potomac are short as compared with the southern branches of the Susquehanna opposed to them. They were at a disadvantage, as their fall southward was lessened by the rise of the northward slope of the peneplain, and they lost ground to the Susquehanna, as the James did to the Potomac.

The doming of the Schooley peñeplain has been a gradual process, involving in the Virginias a maximum change of level of about 3,500 feet. As the uplift progressed the Potomac developed a canyon which in due process widened to a valley. Had the uplift been accomplished and ceased long ago, the valleys would be very wide, especially along the master stream, and much of the region would be eroded to grade. Had the upward movement been continuous, the river would exhibit a simple profile and the valleys simple cross sections, generally concave upward and broken only by hard beds of rock, which would project above the average slope. Neither of these cases corresponds with the facts. There are wide valleys, but within them are narrow canyons. The greater width was developed when the stream had worked down to grade during a pause in the elevation; the narrower channel was sunk when the activity of the river was renewed by renewed uplift. Thus it is apparent that warping has been an intermittent process.

At every stage of sculpture through which the surface passed, the Potomac and other streams bore to their lower courses the sediment taken from upper districts and spread it upon the Coastal Plain or delivered it to tidal waters in estuaries or the open sea. The volume of sediment and its character, whether coarse or fine, varied with the rate of uplift. The strata are thus a record of the river's work and indirectly of the height of land. Something may be inferred from them regarding the rate of warping. There is, however, another factor which complicates the problem--variation of climate, according to which the river's volume, and consequently its ability to carry sediment, changed from time to time. Though probably subordinate to uplift, it is not negligible. Bearing in mind that there are two factors which have determined the river's action, the careful student may investigate the sediments on the one hand and the valley profiles on the other and work out a more detailed history than we now possess. At present we are not able to describe the successive stages accurately, but certain marked ones stand out clearly with such 
decided character that we can with confidence attribute them to the more effective of the two variable factors, the progress of uplift.

The wide valley of the Shenandoah marks the earliest pause in excavation of which there is record in the sculptured surface. No general view of it can be had from the river, which, near its mouth, in consequence of later cutting, runs 350 feet below the valley level, but it may be inspected from any of the numerous low shale hills that diversify the former valley plain. It is not difficult, when looking down on the river's turbid flood, to realize that it has sunk its channel among the hills. It is but another step to recognize that if we could restore what the river and its branches have carried away the hills would be joined together by the fills and the whole wide valley would present a plain. That it once did have such a plain surface, which was worked out to the grade of the river, is not questioned, and the laws of river action lead directly to the conclusion that the level of discharge which the river then had was the level of the plain near its mouth.

From its characteristic development in the Shenandoah Valley the valley plain has come to be known as the Shenandoah. It is not, however, a local feature, but a surface which is present throughout the Appalachian Mountains wherever the rocks are soft shale or the even less resistant limestone.

As the Shenandoah plain is thus a general fact of sculpture, to a greater or less extent worked out by all the rivers of the region, its grade could have been determined only by a common level of discharge- sea level-and a plain of such wide development as it exhibits. could not have been sculptured while the level of discharge was changing in course of uplift, but only during a prolonged interval of constant level. We divide the uplift and erosion of the mountains accordingly into an earlier cycle, during which valleys were sunk 1,000 to 2,000 feet below the Schooley peneplain in the Potomac region and the Shenandoah plain was eroded over all the areas of softer rocks, and the later cycles, during which the lower features of the valleys have been cut.

During the earlier or Shenandoah cycle the Potomac and its southern branches grew very nearly or qui e to their present lengths; the northern branches diminished as they gave ground to the Susquehanna; and thus the competing streams established the watersheds that now exist. North Branch of the Potomac held a very advantageous position in opposition to the western streams on the platean, as ii reached a relatively low level on soft rocks in a much shorter distance than they. It was therefore able to extend such branches as Savage River and Crabtree into their territory, and it is still doing so. 
The Shenandoah plain (recently rechristened the Harrisburg peneplain $^{a}$ ) no longer exists as a continuous surface. Cut by the larger rivers and their branches, even out to the smallest, it is represented only by hilltops that approach its level. Its altitude near Harpers Ferry is about 600 feet above the sea; about the headwaters of the Shenandoah it is 1,200 feet. Between it and the channel of the river, 200 to 350 feet below, are sculptured the terraces and slopes of later development. Among these is a lower valley level, about 100 feet below the Shenandoah plain, which apparently corresponds to a surface that extends about Somerville, N. J., and is known as the Somerville peneplain. ${ }^{b}$ It is eroded on the limestones or very soft Triassic sandstones and represents a shorter pause in the progress of uplift than did the Shenandoah.

The Shenandoah and Somerville plains are not everywwere distinguishable one from the other, being represented in some places by one extensive surface. Toward the close of their development, in the epoch known to geologists as the late Tertiary or Pliocene, they became covered by a widespread deposit of gravel and loam, which is called the Lafayette formation.

The Lafayette covers the outer slopes from the Appalachian Mountains toward the Atlantic and Gulf coasts and the Mississippi Valley with an almost continuous mantle. It is represented in the districts of the Appalachian Ranges and Allegheny Plateaus by deposits of gravel that now cap terraces and hills. It is composed throughout of alluvial material, carried, sorted, and deposited by streams in the first instance and to some extent rearranged by marine waters about the margins. What part is fluviatile and what part marine is to be determined only by further studies; but it is probable that the activity of rivers in spreading the material has been underestimated and that the degree of marine submergence has been correspondingly overestimated. The Potomac, like other rivers of the Lafayette epoch, flowed in a wide alluvial plain, which coalesced with those of adjacent rivers in the lower courses.

The epoch of low, level, and wide-spreading plains was followed by one during which the land was again elevated and the rivers incised the channels they had assumed. It is probable that the elevation was not constant, especially in the outer Coastal Plain, for there is evidence that the lower valleys were at times submerged after having been eroded. ${ }^{c}$ Other influences were, however, almost if not quite as important. It was the time called Pleistocene, the time of the

\footnotetext{
a Campbell, M. R., Geographic d vclopment cf north:m Pennsylvania and southern New York: Bull. Geol. Soc. America, vcl. 14, 19:3, pp. $277-\approx 86$.

$b$ Davis, W. M., and Wood, J. W., jr., Geographic devclopment of northern New Jersay: Proc. Boston Soc. Nat. Hist., vol. 24, 1890, pp. 391-392.

c Darton, N. H., Washington folio: Geologic atlas U. S., folio 70, U. S. Geol. Survey, 1901.
} 
glacial and interglacial epochs, when the climate varied from temperate to semiarctic and back to temperate again; the rivers changed their activities accordingly and alternately cut their channels or partially filled them. The minor gorges that characterize all the valleys, the cascades that beautify the rivers, and the wide rocky shallows that are peculiar features in streams so large as the Potomac and Susquehanna resulted from these activities, as did also the later gravel and silt deposits constituting the Columbia formation, which are extensively spread upon terraces along the Potomac, especially in the vicinity of Washington.

As we approach the present, the seeming importanee of details increases. The deposition of the Columbia formation, for example, marks an episode which seems to compare with the erosion of the old Permian mountains, though it is indeed a relatively insignificant fact. But every detail of the river's course or profile or deposits is significant of some past circumstance, if we can but understand.

Old as the Potomac is and varied as have been the activities affecting its development, a new agent has appeared in its watershed withif the last three hundred years and is acting as the chill climate of the ice age acted to denude the surface and load the river with sediment. Throughout the Tertiary age, when the Schooley peneplain was cut away, when the Shenandoah plain was graded, and when the inner canyons were sculptured, the region constantly bore a luxuriant deciduous forest, in which the tulip tree and the magnolia appeared at an early date and the more modern oaks and maples and many others found place later. With the advent of the ice age the climate changed from semitropic to temperate, and then to that of the Barren Grounds of the Far North to-day. Vegetation died; the surface was bared; rain or waters from melting snow swept away the frostloosened earth; winds carried the dust in eddying clouds; the rivers were surcharged with sediment, and the Columbia deposits resulted. The new agent in his own peculiar way is preparing another such deposit. He has bared the surface almost as effectually as did the blasts of the ice age, but with an ax only, and he is causing a new record to be made in the hills that are scored with gullies and in the lowlands that are buried beneath deposi is of gravel and mud.

The Potomac's long history has been influenced by great forcesthe internal energy of the earth, that has shaped the surface on which the river grew; the attraction of gravitation, that has caused the river to flow; the external force of the sun, that has set the atmospheric agents to work. They were sometimes conservative, sometimes destructive. The new force has demonstrated his capacity to destroy; in his own interest he needs to acquire the art to protect. The future of the Potomac and the fitness of its watershed to be a home for man depend on his intelligent use of what the ages have fashioned. 


\title{
S'TREAM FLOW IN THE PO'TOMAC BASIN.
}

\author{
By R. H. Bolster.
}

\section{INTRODUCTION.}

\section{METHODS OF WORK.}

\section{FIELD METHODS.}

The methods by which the records of stream discharge have been made are those in common use in the United States Geological Survey. They are described in detail in Water-Supply and Irrigation Papers Nos. 94 and 95 and briefly in the anmual progress reports for 1904 to 1906. An outline of the method used in the Potomac River drainage basin is given below, to assist in making clear the data which follow.

A gage for observing the stage of the river is established at a bridge or other place where the record of flow is to be made. This gage is a vertical staff or some other device by which the height of water may be observed, and is read each day by a person living near by. The average of the gage readings, if more than one, in any day is used as the mean gage height for that day.

At various stages of the river one of the hydrographers of the Survey visits the station and measures with a current meter the amount of water flowing. This meter is primarily an instrument for measuring the velocity of moving water, and consists essentially of a wheel with vanes, which may be shaped like those of a owindmill or of a screw, or with cups like those of an anemometer, the necessary qualification being that moving water shall readily cause the wheel of the meter to turn. Each meter is rated before use. The rating is done by moving the meter through still water at various observed speeds to determine the relation between the velocity with which the meter moves through the water and the revolutions of the wheel. This relation having been determined, the meter is used in running water, the revolutions per unit of time noted, and the velocity of the water computed.

Observations of depth of water are also made, and from them the area in cross section of each portion of the stream is computed; each 
partial area multiplied by the mean velocity of that area gives a partial discharge. The sum of the partial discharges is the total discharge of the stream.

\section{OFFICE METHODS.}

Measurements of flow as outlined above are made covering a considerable range of gage height. They are then plotted on coordinate paper, with gage heights for ordinates and discharges for abscissas, and a smooth curve, called the rating curve, is drawn through the points. From this curve a rating table is made which shows the discharge of the stream for any gage height.

The data necessary for the construction of a rating table for a gaging station as just stated are the results of the discharge measurements, which include the record of stage of the river at the time of measurement, the area of the cross section, the mean velocity of the current and the quantity of water flowing; and a thorough knowledge of the conditions at and in the vicinity of the station.

The construction of the rating table depends on the following laws of flow for open permanent channels: (1) the discharge will remain constant se long as the conditions at and near the gaging station remain constant; (2) the change of slope due to the rise and fall of the stream being neglected, the discharge will be the same whenever the stream is at a given stage; (3) the discharge is a function of, and increases gradually with, the stage.

The plotting of results of the various discharge measurements, using gage heights as ordinates and discharge, mean velocity, and area as abscissas, will define curves which show the discharge, mean velocity, and area corresponding to any gage height. For the development of these curves there should be, therefore, a sufficient number of discharge measurements to cover the range of the stage of the stream. Fig. 1 shows a typical rating curve with its corresponding mean velocity and area curves.

As the discharge is the product of two factors, the area and the mean velocity, any change in either factor alone will produce a corresponding change in the discharge. Their curves are therefore constructed in order to study each independently of the other.

The area curve can be definitely determined from accurate soundings extending to the limits of high water. It is always concave toward the horizontal axis or on a straight line unless the banks of the stream are overhanging.

The form of the mean velocity curve depends chiefly on the surface slope, the roughness of the bed, and the cross section of the stream. Of these the slope is the principal factor. In accordance with the relative change of these factors the curve may be either a straight line, a curve convex or concave toward either axis, or a 


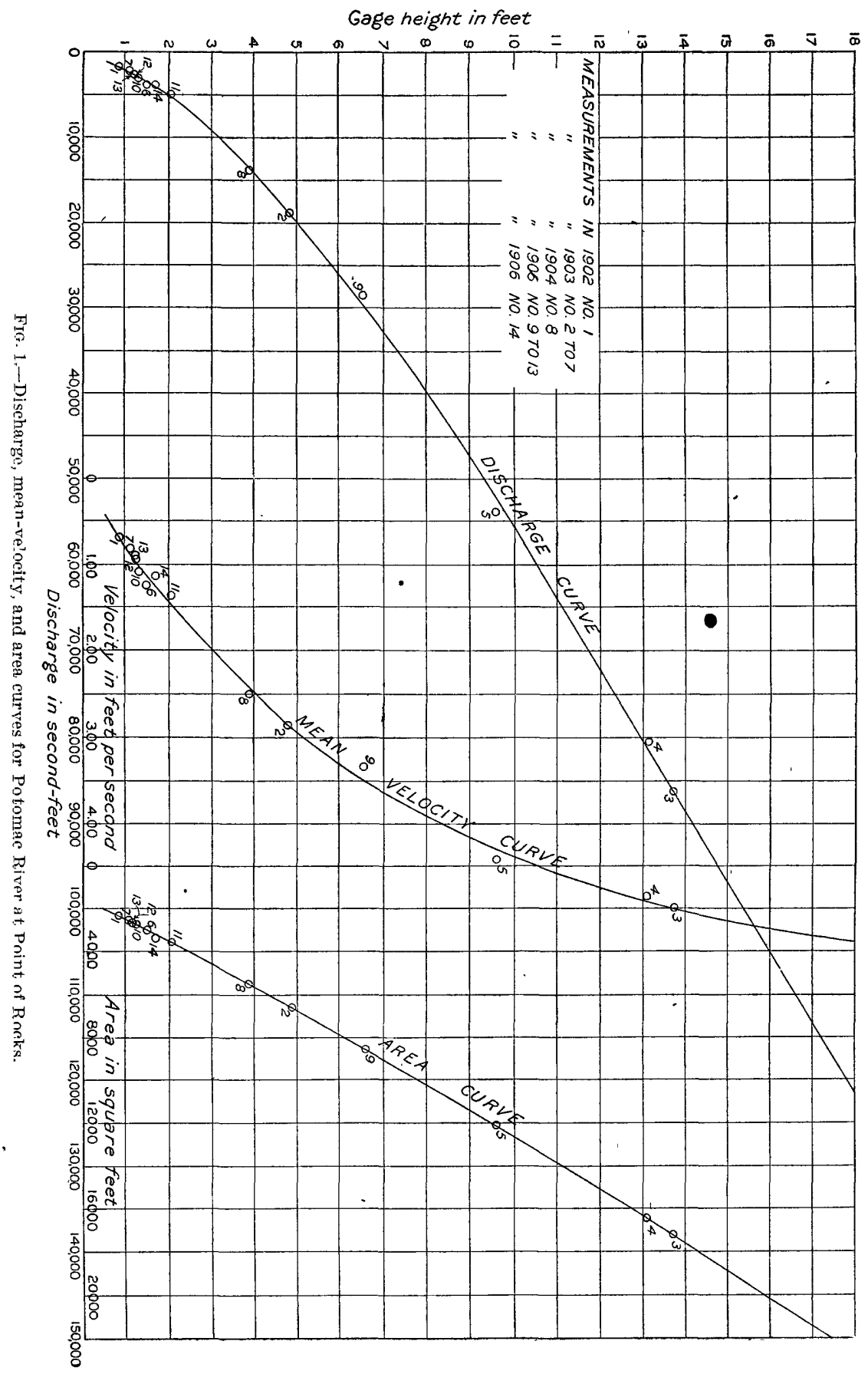


combination of the three. From careful study of the conditions at any gaging station the form which the vertical velocity curve will take can be predicted, and it may be extended with reasonable certainty to stages beyond the limits of actual measurements. It is used principally in connection with the area curve in locating errors in discharge measurements and in constructing the rating table.

The discharge curve is defined primarily by the measurements of discharge, which are studied and weighted in accordance with the local conditions existing at the time of each measurement. The curve may, however, be best located between and beyond the measurements by means of the curves of area and mean velocity. This curve under normal conditions is concave toward the horizontal axis and is generally parabolic in form.

In the preparation of the rating table the discharge for each tenth on the gage is taken from the curve. The differences between successive discharges are then taken and adjusted according to the law that they shall either be constant or increasing.

\section{DEFINITIONS.}

The volume of water flowing in a stream, the "run-off," is expressed in various terms, each of which is associated with a certain class of work. These terms may be divided into two classes: Those which represent a rate of flow, as second-foot, gallons per minute, and run-off in second-feet per square mile; and those which represent actual quantities of water, as run-off in depth in inches. They may be defined as follows:

"Second-foot" is an abbreviation for cubic foot per second, and is the rate of discharge of water flowing in a stream 1 foot wide 1 foot deep at the rate of 1 foot per second. It is generally used as a fundamental unit from which the others are computed.

"Gallons per minute" is generally used in connection with pumping and city water supply.

"Second-feet per square mile" is the average number of cubic feet of water flowing per second from each square mile of area drained, on the assumption that the run-off is distributed uniformly both as regards time and area.

"Run-off in inches" is the depth to which the drainage area would be covered if all the water flowing from it in a given period were conserved and uniformly distributed over the surface. It is used for comparing run-off with rainfall, which is usually expressed in depth in inches. 
EXPLANATION OF TABLES.

For each regular station are given, as far as available, the following data:

1. Description of station.

2. List of discharge measurements.

3. Gage-height tables.

4. Rating tables.

5. Tables of estimated monthly and yearly discharges, run-off, and precipitation, based on all the facts available to date.

The descriptions of stations give such general information about the locality and equipment as would enable the reader to find and use the station, and, as far as possible, a complete history of all the changes that have occurred since the establishment of the station that would affect the use of the data collected. They also give statements concerning the probable percentage of error of the estimates. The probable errors have been based principally on the errors of the discharge measurements with reference to the rating curves.

The discharge-measurement table gives the results of the discharge measurements made during each year, and includes the date, the gage height, and the discharge in second-feet.

The tables of daily gage heights give for each day the height of the surface of the river above the zero of gage, as found from the mean of the gage readings taken on that day.

The rating tables give discharges in second-feet corresponding to each stage of the river as given by the gage heights and statements concerning the measurements on which it has been based and the portion of the curve which is well defined.

In the tables of estimated monthly discharges the column headed "Maximum" gives the mean flow for the day when the mean gage height was highest; this is the flow as given in the rating table for that mean gage height. As the gage height is the mean for the day, there might have been short periods when the water. was higher and the corresponding discharge larger than given in this column. Likewise in the column headed "Minimum," the quantity given is the mean flow for the day when the mean gage height was lowest. The column headed "Mean" is the average flow for each second during the month.

On this, the computations for the columns under the general heading "Run-off" are based. The mean precipitation, which has been entered in the column headed "Precipitation in inches," for gaging stations which have been maintained for a series of years has been determined from the United States Weather Bureau records. The mean precipitation has been determined from well-distributed rainfall stations in the drainage basin above the gaging station.

IRR $192-07-3$ 
From the precipitation in inches and the run-off in depth in inches the run-off in per cent of precipitation has been determined, also the loss of precipitation in inches or the amount which for several causes does not flow past the gaging section.

\section{ACCURACY OF ESTIMATES OF STREAM FLOW.}

The description of each gaging station is followed by a statement indicating the probable percentage of error in the values for mean monthly flow. This percentage is only approximate, as no refinement has been attempted in its determination. The probable errors have been based principally on the errors of the discharge measurements with reference to the rating curves and the known conditions of the flow in the vicinity of the gaging section. It is impossible to estimate closely the errors caused by temporary or gradual changes in the conditions of flow, unreliability or ignorance of the observers, changes in wire or chain length, or ice conditions.

Errors due to changes in conditions of flow are relatively small for the large streams except at a few stations. On small streams, however, a temporary obstruction at or below the gaging section, causing a change in area of cross section or in velocity of the current, may cause large errors in daily estimates of discharge. These changes as a rule do not occur frequently and are usually of a temporary character; for example, the lodging of driftwood on the controlling point below the gage reduces the velocity, and hence the discharge for a given gage height. A few days later a sudden rise in the stream may clear the channel and restore normal flow. Unless the hydrographer has chanced to make a measurement of discharge during the period of abnormal conditions, an error has been introduced into the monthly estimates. Owing to the limited appropriation for stream gaging and the large number and wide separation of the gaging stations, it is impossible for the hydrographers to make measurements frequently enough to eliminate all errors arising from these abnormal conditions. It has further been found impracticable to so instruct the observers that they will correctly report unusual conditions.

Gradual changes in the conditions which affect the flow can be estimated and corrected more readily than temporary changes. Here again the hydrographer is often handicapped by inability to make sufficient measurements to show properly the varying rate of change in channel conditions. In such cases the estimates are obtained either by an indirect method which is based on the assumption of a constant rate of change from day to day between measurements or by a series of rating curves.

Observers are as a rule conscientious in reading the gages, but with few exceptions they are wholly unfamiliar with engineering work of any description. The observers' records, however, are examined and 
checked by hydrographers, and large errors are thus eliminated. The observers are usually instructed to read the gage to the nearest tenth or half tenth of a foot twice each day, and at times of floods several times a day. At high and medium stages the errors in reading the gage are thus negligible; but at low stages, when a difference of one or two hundredths in the stage of the river or slight fluctuations during the day cause errors of several per cent it is evident that the regular method of observation is inadequate. Hence, monthly minimums may be considerably in error; but in general the monthly means for months of low flow are good, owing to the tendency of positive and negative errors to offset each other.

Prior to the fall of 1903 wire gages were used at many of the stations . for observing the stage of the river. The correct length of gages of this type was difficult to maintain on account of the stretching of the wire. Small changes of length took place frequently, making necessary the application of corrections to the observed gage heights at the station. In some instances the magnitude of the corrections and the time over which they should have been applied were not recorded, and the proper adjustments are therefore somewhat in doubt. In such instances, if the data warranted it, the gage heights were corrected by the amount that the measurements of the period in question were vertically above or below the curve. It is believed that by the use of corrected gage heights reasonably accurate estimates of discharge have been made for all the rivers described.

The extent of frozen periods at many of the stations is very uncertain. All ice notes are from observers' gage-height records, but as the observers' notes prior to 1904 are very incomplete their absence does not always imply open-channel conditions. Estimates for ice periods have been made as if open-channel conditions existed except as noted. This method involves errors for the relatively short ice periods of a few to 40 per cent.

The errors which are described above are not to be considered as applying to every station. They have been fully described here in order to call to the attention of the reader the possible sources of error and the limitations of engineering work of this kind. Although the resulting error may seem large, it should be remembered that stream-gaging data and estimates of flow are used mainly as a basis for predicting the maximum, minimum, and mean discharge which may be expected in future years. Since the mean annual flow of a stream may be several times larger one year than it is the next, it is evident that for records of short duration an estimate which involves an error as great as 50 per cent is not without value. On the other hand, it is a waste of money and needless refinement-indeed, virtually impossible - to obtain estimates much closer than 3 per cent in ordinary current-meter work. 
Special emphasis is laid on the fact that the value of stream-gaging data is determined mainly by the number of years during which the record has been maintained and not so much by the degree of accuracy of the discharge for each year. That is, the longer the record the more nearly does it indicate the maximum, minimum, and mean flow which may be expected in the future.

Monthly means which are stated in the descriptions to be within 5 per cent of the true flow are considered to be very good, and those within 10 per cent are considered close enough for all practical purposes. Errors in estimates which are greater than 15 per cent are due either to an insufficient number of measurements, or to poor natural conditions which could not be avoided, or to changes at the gaging station which could not be foreseen at the time of its establishment. The larger errors in daily discharge values occur at the highest stages, which continue only for a few days, and hence the effect on the accuracy of the monthly mean is not so great as might at first appear. Also by far the greater number of gage heights are for medium stages, at which the error of the rating curve is seldom as great as 10 per cent and is usually much less than 5 per cent. The errors in the daily discharge values are often considerable, owing to fluctuation of the river height. The values for the maximum and the minimum flows for the month may also contain an additional error, because they are based on the extreme low or high part of the rating curve, which is usually not so well defined as the intermediate portion. In the case of the mean monthly flow, for which the estimates of accuracy are made, the error is reduced to a very small amount by reason of the compensation of variable negative and positive errors.

\section{COMPARISONS OF FLOW.}

The figures in the following table have been brought together for the purpose of comparing the flow from a partial drainage area with the flow from the total drainage area over a relatively long period of time.

The totals show that the ratio of the run-off from the tributaries to the run-off from the total basin is 6 per cent greater than the ratio of their respective drainage areas. This is entirely reasonable and just what should be expected, for during medium and especially during high stages the run-off is greater on the tributaries, owing to somewhat greater rainfall and more precipitate slopes.

In the comparison of the run-off from month to month it should be remembered that there is a considerable time interval between the stations. For example, a flood on the tributaries occurring at the end of a month does not reach the Point of Rocks station until the following month. It is believed that this accounts for most of the larger deviations of discharge from the normal for the individual months. 
Mean monthly discharge in second-feet, Potomac River basin.

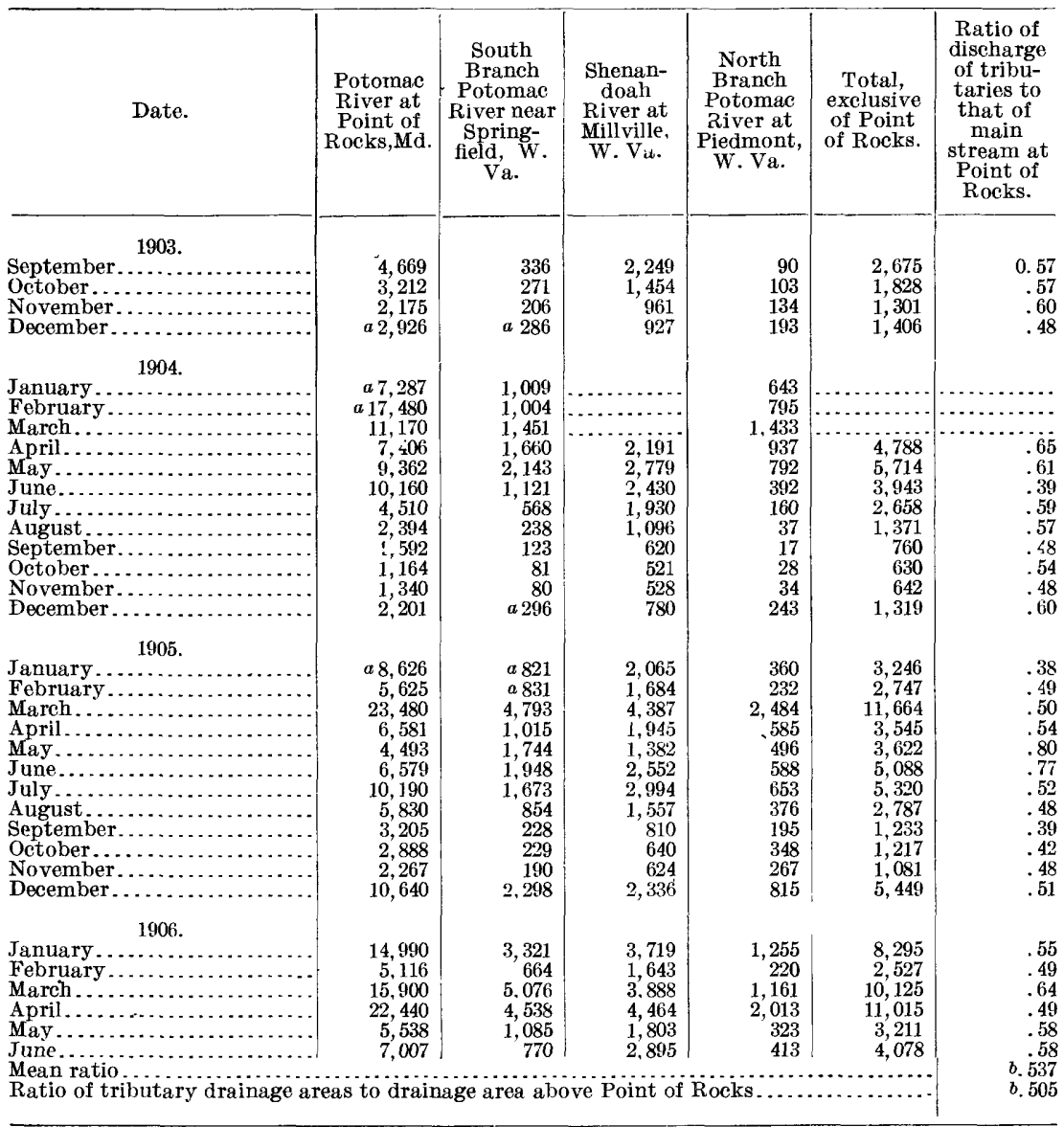

As a practical application of such comparisons as have been given above, the following may be of interest and value.

1. The observers' gage heights for the Point of Rocks station from January 1 to June 18,1896, were corrected 0.7 by the hydrographer at that time; 0.4 was due to change of datum, but there are no data available to show why the correction for the remaining 0.3 was made. There has been a good deal of doubt in the mind of the writer whether it should have been made at all. The following comparisons strengthen this doubt still further. The mean daily discharge for the total period from January 1 to February 29 and from May 11 to June $17,1896,^{a}$ was found at all stations, except that an allowance of four days was made for flow from Cumberland and Spring- 
field to Point of Rocks. The estimate of the discharge at the Springfield station for May and June, during which there was no record was based on a comparison of Springfield and Millville estimates from May, 1895, to February, 1896, inclusive.

Discharge at various stations, January 1 to February 29 and May 11 to June 1\%, 1896.

Cumberland ...................................... second-feet.. 882

Millville................................................ 2, 635

Springfield........................................... 725

4,242

Mean daily discharge at Point of Rocks, 0.7 correction being used..... do. . . 5, 510 Ratio of discharge at upper stations to that at Point of Rocks 0.7 correc-

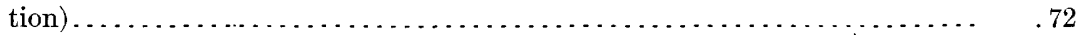

Ratio of drainage area at upper stations to that of Point of Rocks.......... . .55

If the 0.3 correction had not been made the approximate mean - daily discharge for the Point of Rocks station would have been 7,270 second-feet and the ratio of discharge of the upper stations to Point of Rocks would have been .58 .

2. During 1897 the gage length was greatly in error, the final error recorded in January, 1898, being 1.8 feet. These gage heights were corrected by varying amounts for several periods. The amounts of the corrections were based on the gage-height distance that the several measurements of 1897 plotted above or below the rating curve. That this gave essentially correct results for the year is borne out by the following figures:

Discharge at various stations, December 28, 1896, to November 24, 1897.

Mean daily discharge at Cumberland December 28,a 1896, to November 20,

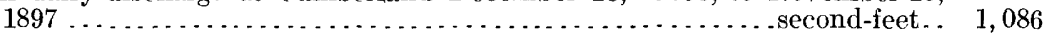

Mean daily discharge at Millville January 1 to November $24,1897 \ldots \ldots$. . . . . 3,058

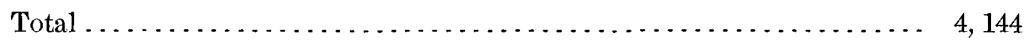

Mean daily discharge at Point of Rocks January 1 to November 24,

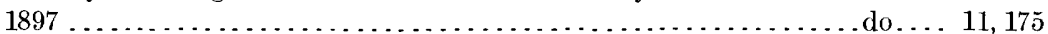

Ratio of discharge at upper stations to that at Point of Rocks........... $\quad .37$

Ratio of drainage area at upper stations to that at Point of Rocks........ $\quad .40$

The ratio of discharge is thus about 92 per cent of that of the drainage area. An allowance of 20,000 second-feet per day was made for omitted gage heights at Millville February 7, 8, and 9. There is every reason to believe that about 30,000 second-feet each day should also have been added for February 23 and 24, first, because it was known that the observer recorded gage heights above the top of the 10-foot gage on those days as 10.0 feet, and second, because of the flood at Point of Rocks the latter part of February, which shows a discrepancy of about this amount by a comparison of the relative drainage basins. If this is done the difference of the ratios is about 4 
per cent. However, this still leaves a negative error of approximately 10 per cent, since the ratio of the discharges should be about 6 per cent greater than that of the drainage areas, according to the comparisons presented in the table (p. 31).

\section{RAINFALL.}

Probably no phenomenon has so important a bearing on the development of the country as rainfall. A study of this phenomenon, although an essential part of the hydrography of a district, is difficult, owing to the numerous and various conditions which regulate it. The fluctuations of both the yearly and monthly precipitation are great, and it is only by having a long series of records at many welldistributed points over an area that even a fair estimate of the conditions prevailing may be made.

The United States Weather Bureau has for a number of years regularly maintained rainfall stations well distributed throughout the United States.. On the data collected at about 40 such stations, located either in or near the Potomac drainage basin, the accompanying discussion, tables, and map have been based.

The map (Pl. I, pocket) shows, by means of lines of equal rainfall, the average annual distribution of precipitation over the basin of the Potomac during the ten years from 1896 to 1905.

At many of the stations the data were missing for some portions of the period, and in order to complete the records for such stations the missing values were obtained by comparison with other near-by stations by the method of interpolation and extrapolation. This is made possible by the fact that the ratio between the precipitation at, two adjacent stations remains fairly constant, although there is considerable variation in the actual amounts.

Since the prevailing wind directions are as important as the topographic surroundings in determining the precipitation at any given pair of stations, it seemed desirable to compute the ratios for many of the individual months as well as for the whole year. This proceeding involved more labor than would have been required to determine the ratios for whole years only; but it seems to have increased the accuracy of the results, especially in those cases where an interpolation of only a few months was required to fill out an otherwise complete series. This calculation of monthly ratios also makes it possible to obtain an approximately true annual ratio for two stations, one of which has many scattered monthly records, but few or no complete annual records. Another valuable feature of these ratios is that they make it possible to readily detect and eliminate errors in the rainfall records due to changes in gage exposure or to errors in recording or computing. This is illustrated by the ratios for the pair 
of stations, Point of Rocks and Harpers Ferry, where an excessive ratio of 7.43 was found for October, 1895, as against an average ratio of 0.98 for that month.

In preparing the map of the Potomac basin the annual means as given on pages 34 to 40 were first plotted; then with the aid of several approximate means not given on the map, all points having the same precipitation were connected by meandering lines. Inspection shows that these lines, called "isohyets," follow closely the surface contours of the base map, while it will at once strike the reader that nearly all the stations are located in the valleys. Only a few stations in western Maryland and one station on the Blue Ridge of Virginia (Mount Weather) can be classed as mountain stations. Consequently we have at present no sufficient basis for calculating the rate of increase of precipitation with altitude in this basin. On this account also the course of the isohyet of 40 inches is, with few exceptions, to be regarded as hypothetical, although wherever possible it has been made to accord with such scanty and imperfect records as are obtainable.

Although the net of rainfall stations is not so finely meshed as is desirable for an area of the importance of the Potomac basin, yet the map shows distinctly that the rainfall of the lowlands decreases upstream from Washington. The lower-lying portions of the valley of the Shenandoah and its continuation, the Cumberland Valley of Maryland and Pennsylvania, were characterized by an annual fall of 35 to 40 inches of rain and melted snow. Generally the smaller amount is found along the Potomac itself, but the driest portion of * the great valley lies in that section drained by Opequon Creek.

There seems to have been an exception to the rule just stated about the headwaters of South Branch of the Potomac. In that region there is a considerable area, inclosed by the isohyet of 35 inches, which has a rainfall of less than that amount. The two stations inclosed by the curve show amounts of 33.7 and 34.7 inches. This is apparently the dryest portion of the whole Potomac basin and, to judge from the neighboring portions of other river basins, it is but a portion of a relatively arid district which embraces the whole valley occupied by Bull Pasture River and South Branch of the Potomac.

Mean precipitation, in inches, at stations in drainage basin of Potomac River.

BACHMAN VALLEY, MD., AITITUDE 860 FEET.

\begin{tabular}{|c|c|c|c|c|c|c|c|c|c|c|c|c|c|}
\hline & Jan. & Feb. & Mar. & Apr. & May. & June. & July. & Aug. & Scpt. & Oct. & Nov. & Dec. & Annual. \\
\hline $\begin{array}{l}1896-1900 \ldots \ldots \ldots \\
1901-1905 \ldots \ldots\end{array}$ & $\begin{array}{l}2.77 \\
4.65\end{array}$ & $\begin{array}{l}5.92 \\
4.17\end{array}$ & $\begin{array}{l}5.46 \\
6.30\end{array}$ & $\begin{array}{l}2.80 \\
4.21\end{array}$ & $\begin{array}{l}6.26 \\
3.57\end{array}$ & $\begin{array}{l}3.67 \\
6.87\end{array}$ & $\begin{array}{l}4.01 \\
7.76\end{array}$ & $\begin{array}{l}3.92 \\
7.35\end{array}$ & $\begin{array}{l}3.19 \\
4.93\end{array}$ & $\begin{array}{l}3.01 \\
5.26\end{array}$ & $\begin{array}{r}5.16 \\
a 2.51\end{array}$ & $\begin{array}{l}\text { 3. } 36 \\
\text { 6. } 28\end{array}$ & $\begin{array}{l}49.56 \\
63.93\end{array}$ \\
\hline 10-year mean... & 3. 70 & 5.04 & 5.88 & 3.50 & 4.91 & 5. 27 & 5.88 & 5.63 & 4.06 & 4. 13 & 3.83 & 4.82 & 56.74 \\
\hline
\end{tabular}

a 1 year interpolated, based on observations at Taneytown. 
Mean precipitation at stations in drainage basin of Potomac River-Continued.

BAYARD, W. VA., ALTITUDE 2,500 FEET.

\begin{tabular}{|c|c|c|c|c|c|c|c|c|c|c|c|c|c|}
\hline & Jan. & Feb. & Mar. & Apr. & May. & June. & July. & Aug. & Sept. & Oet. & Nov. & Dec. & Annual. \\
\hline $\begin{array}{l}1896-1900 \ldots \\
1901-1905 \ldots\end{array}$ & & & & & & & & & & & & $\mid \begin{array}{c}\mid \cdots \\
\cdots \cdots\end{array}$ & $\begin{array}{c}a[45.02[ \\
b 43.84\end{array}$ \\
\hline 10-year mean. & & & & & & & & & & & & & \\
\hline
\end{tabular}

BOETTCHERVILLE, MD., ALTITUDE 780 FEE'T.

\begin{tabular}{|c|c|c|c|c|c|c|c|c|c|c|c|c|c|}
\hline $\begin{array}{l}1896-1900 \ldots \ldots \ldots . \\
1901-1905 \ldots \ldots \ldots \ldots\end{array}$ & $\begin{array}{l}1.96 \\
2.39\end{array}$ & $\begin{array}{l}2.59 \\
2.48\end{array}$ & $\begin{array}{l}4.05 \\
3.5 .5\end{array}$ & $\begin{array}{l}1.86 \\
3.86\end{array}$ & $\begin{array}{l}4.21 \\
3.72\end{array}$ & $\begin{array}{l}3.41 \\
3.71\end{array}$ & $\begin{array}{l}3.60 \\
3.50\end{array}$ & $\begin{array}{l}2.58 \\
4.91\end{array}$ & $\begin{array}{l}3.01 \\
1.95\end{array}$ & $\begin{array}{l}2.35 \\
2.43\end{array}$ & $\begin{array}{l}3.07 \\
1.49\end{array}$ & $\begin{array}{l}1.99 \\
3.18\end{array}$ & $\begin{array}{l}34.73 \\
37.18\end{array}$ \\
\hline 10-year mean... & 2.17 & 2.53 & 3.80 & 2.86 & 3.96 & 3.56 & 3.55 & 3.74 & 2.48 & 2.39 & 2.28 & 2.58 & 35.95 \\
\hline
\end{tabular}

BURLINGTON, W. VA., ALTITUDE 875 FEET.

\begin{tabular}{r|c|c|c|c|c|c|c|c|c|c|c|c|c|c}
\hline $1896-1900 \ldots \ldots \ldots .$. & 2.15 & 3.85 & 3.58 & $c 1.99$ & 4.30 & 2.94 & 4.05 & 2.93 & 3.08 & 2.14 & 2.91 & 2.10 & 36.05 \\
$1901-1905 \ldots \ldots \ldots \ldots$ & 2.53 & 2.21 & 3.11 & 3.04 & 3.87 & 4.21 & 4.23 & 4.25 & 2.21 & 2.08 & 1.52 & 3.39 & 36.67 \\
\hline $10-$-year mean... & 2.34 & 3.03 & 3.34 & 2.51 & 4.08 & 3.57 & 4.14 & 3.59 & 2.64 & 2.11 & 2.21 & 2.74 & 36.36 \\
\hline
\end{tabular}

CHAmbersbURG, PA., ÁLTitUde 1,000 FEET.

\begin{tabular}{l|l|l|l|l|l|l|l|l|l|l|l|l|l|l}
\hline $1896-1900 \ldots \ldots \ldots$ & 1.86 & 3.31 & 4.06 & 1.65 & 4.20 & 3.57 & 3.62 & 4.75 & 2.44 & 2.55 & 3.56 & 1.70 & 37.28 \\
\hline
\end{tabular}

CHEWSVILLE, MD., ALTITUDE 530 FEET.

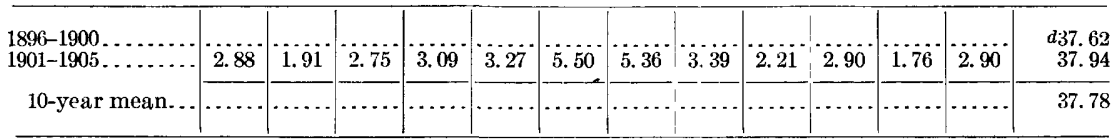

CLEARSPRING, MD., ALTITUDE 500 FEET.

\begin{tabular}{|c|c|c|c|c|c|c|c|c|c|c|c|c|c|}
\hline $\begin{array}{l}1896-1900 \ldots \ldots \ldots \\
1901-1905 \ldots \ldots \ldots\end{array}$ & 3. 52 & 2.21 & 73.89 & 93.49 & 3.55 & 3.75 & 4.86 & 3.90 & 2.88 & 2.60 & i. 73 & 4. 37 & $\begin{array}{r}e 37.83 \\
40.65\end{array}$ \\
\hline 10-year mean & & & & & & & & & & & & & 39.24 \\
\hline
\end{tabular}

CUMBERLAND, MD., ALTITUDE 700 FEET.

\begin{tabular}{|c|c|c|c|c|c|c|c|c|c|c|c|c|c|}
\hline $\begin{array}{l}1896-1900 \ldots \ldots \ldots \\
1901-1905 \ldots \ldots \ldots\end{array}$ & $\begin{array}{l}2.51 \\
2.70\end{array}$ & $\begin{array}{l}\text { 3. } 32 \\
2.13\end{array}$ & $\begin{array}{l}3.91 \\
3.14\end{array}$ & $\begin{array}{l}2.06 \\
3.48\end{array}$ & $\begin{array}{l}\text { 4. } 06 \\
\text { 2. } 46\end{array}$ & $\begin{array}{l}3.02 \\
3.52\end{array}$ & $\begin{array}{l}3.11 \\
3.13\end{array}$ & $\begin{array}{l}2.60 \\
3.80\end{array}$ & $\begin{array}{l}2.77 \\
1.94\end{array}$ & $\begin{array}{l}\text { 2. } 61 \\
2.11\end{array}$ & $\begin{array}{l}3.41 \\
1.35\end{array}$ & $\begin{array}{l}2.29 \\
3.41\end{array}$ & $\begin{array}{l}35.90 \\
33.20\end{array}$ \\
\hline 10-year mean.. & 2. 60 & 2. 72 & 3. 52 & 2. 77 & 3.26 & 3. 27 & 3.12 & 3. 20 & 2.35 & 2. 36 & 2. 38 & 2.85 & 34. 55 \\
\hline
\end{tabular}

DALE ENTERPRISE, VA., ALTITUDE 1,350 FEET.

\begin{tabular}{r|c|c|c|c|c|c|c|c|c|c|c|c|c}
\hline $1896-1900 \ldots \ldots \ldots$. & 2.15 & 3.62 & 3.54 & 1.74 & 3.62 & 4.55 & 4.54 & 3.28 & 3.62 & 2.86 & 2.51 & 1.89 & 37.92 \\
$1901-1905 . \ldots \ldots \ldots$ & 2.86 & 2.40 & 3.51 & 2.95 & 3.61 & 7.47 & 5.54 & 4.63 & 2.48 & 2.24 & 1.43 & 3.44 & 42.58 \\
$10-$ year mean. & 2.50 & 3.01 & 3.52 & 2.34 & 3.61 & 6.01 & 5.04 & 3.95 & 3.05 & 2.55 & 1.97 & 2.66 & 40.25 \\
\hline
\end{tabular}

a 5 years interpolated, based on observations at Westernport. $b 2$ years interpolated, based on observations at Westernport. c 1 year interpolated, based on observations at Romney. $d 21$ years interpolated, based on observations at Hagerstown.

$e 3$ years 5 months interpolated, based on observations at Greenspring Furnace.

$f 2$ years interpolated, based on observations at Greenspring Furnace.

$g 1$ year interpolated, based on observations at Greenspring Furnace. 
Mean precipitation at stations in drainage basin of Potomac River-Continued.

DEER PARK, MD., ALTITUDE 2,457 FEET.

\begin{tabular}{|c|c|c|c|c|c|c|c|c|c|c|c|c|c|}
\hline & Jan. & Feb. & Mar. & Apr. & May. & June. & July. & Aug. & Sept. & Oct. & Nov. & Dec. & Annual. \\
\hline $\begin{array}{l}1896-1900 . . \\
1901-1905 .\end{array}$ & $\begin{array}{l}3.14 \\
4.29\end{array}$ & $\begin{array}{r}3.73 \\
a 3.16\end{array}$ & $\begin{array}{r}4.56 \\
b 2.42\end{array}$ & $\begin{array}{l}2.42 \\
3.95\end{array}$ & $\begin{array}{l}4.84 \\
4.58\end{array}$ & $\begin{array}{l}\text { 4. } 38 \\
5.26\end{array}$ & $\begin{array}{l}5.81 \\
4.65\end{array}$ & $\begin{array}{r}3.86 \\
a 3.14\end{array}$ & $\begin{array}{l}2.46 \\
1.74\end{array}$ & $\begin{array}{l}1.97 \\
2.66\end{array}$ & $\begin{array}{r}3.56 \\
a 2.16\end{array}$ & $\begin{array}{r}3.37 \\
a 3.67\end{array}$ & $\begin{array}{l}44.10 \\
41.70\end{array}$ \\
\hline 10-year me & 3.71 & 3.44 & 3.47 & 3. 18 & 4. 71 & 4.82 & 5.23 & 3.50 & 2.10 & 2.31 & 2.86 & 3.52 & 42.90 \\
\hline
\end{tabular}

DISTRIBUTING RESERVOIR, D. C., ALTITUDE 120 FEET.

\begin{tabular}{|c|c|c|c|c|c|c|c|c|c|c|c|c|c|}
\hline $\begin{array}{l}1896-1900 \\
1901-1905\end{array}$ & $\begin{array}{r}2.26 \\
c 3.05\end{array}$ & $\begin{array}{r}4.26 \\
c 2.00\end{array}$ & $\begin{array}{l}2.84 \\
3.68\end{array}$ & $\begin{array}{l}\text { 1. } 64 \\
3.31\end{array}$ & $\begin{array}{l}3.44 \\
2.87\end{array}$ & $\begin{array}{l}2.71 \\
3.91\end{array}$ & $\begin{array}{l}3.32 \\
5.47\end{array}$ & $\begin{array}{l}3.83 \\
4.17\end{array}$ & $\begin{array}{l}2.82 \\
2.86\end{array}$ & $\begin{array}{l}2.00 \\
3.05\end{array}$ & $\begin{array}{l}2.44 \\
1.62\end{array}$ & $\begin{array}{l}c .06 \\
c 4.40\end{array}$ & $\begin{array}{l}33.64 \\
40.42\end{array}$ \\
\hline 10-year mean... & 2.65 & 3. 13 & 3. 17 & 1.65 & 3.10 & 3.31 & 4. 39 & 4. 00 & 2.84 & 2.52 & 2.03 & 3.23 & 37.03 \\
\hline
\end{tabular}

FREDERICK, MD., ALTITUDE 345 FEET.

\begin{tabular}{r|c|c|c|c|c|c|c|c|c|c|c|c|c|c}
\hline $1896-1900 \ldots \ldots \ldots \ldots$ & 2.52 & 4.47 & 3.69 & 1.63 & 3.09 & 2.61 & 4.06 & 2.92 & 2.39 & 2.71 & 3.36 & 2.49 & 35.99 \\
$1901-1905 . \ldots \ldots \ldots$. & 3.31 & 2.74 & 3.81 & 3.33 & 2.78 & 2.85 & 5.17 & 4.28 & 2.69 & 2.85 & 1.98 & 4.35 & 43.17 \\
\hline 10-year mean... & 2.91 & 3.60 & 3.80 & 2.48 & 2.93 & 4.23 & 4.61 & 3.60 & 2.54 & 2.78 & 2.67 & 3.42 & 39.58 \\
\hline
\end{tabular}

GETTYEBURG, PA.

\begin{tabular}{|c|c|c|c|c|c|c|c|c|c|c|c|c|c|}
\hline $\begin{array}{l}1896-1900 \ldots \\
1891-1905 \ldots\end{array}$ & & & & & & & & & & & & & $\begin{array}{l}d 44.75 \\
\text { e46. } 76\end{array}$ \\
\hline 10-year mean. & 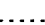 & & & & & & & & & & & & 45.75 \\
\hline
\end{tabular}

GRANTSVILLE, MD, ALTITUDE 2,400 FEET.

\begin{tabular}{r|c|c|c|c|c|c|c|c|c|c|c|c|c|c|c}
\hline $1896-1900 \ldots \ldots \ldots \ldots$ & 3.21 & 4.30 & 5.00 & 2.53 & 4.05 & 3.84 & 6.57 & 3.59 & 2.85 & 2.56 & 4.16 & 2.88 & 45.59 \\
$1901-1905 . \ldots \ldots \ldots . .5$ & 3.51 & 2.98 & 3.83 & 3.79 & 3.96 & 4.24 & 3.50 & 3.32 & 2.12 & 2.63 & 1.78 & 4.11 & 39.81 \\
\hline 10 -year mean... & 3.36 & 3.64 & 4.41 & 3.16 & 4.00 & 4.05 & 5.03 & 3.45 & 2.48 & 2.59 & 2.97 & 3.49 & 42.70 \\
\hline
\end{tabular}

GREAT FALLS, MD., ALTITUDE 150 FEET.

\begin{tabular}{|c|c|c|c|c|c|c|c|c|c|c|c|c|c|}
\hline $\begin{array}{l}1896-1900 \ldots \ldots \ldots \\
1901-1905 \ldots \ldots \ldots\end{array}$ & $\begin{array}{l}2.46 \\
2.89\end{array}$ & $\begin{array}{l}\text { 4. } 35 \\
2.20\end{array}$ & $\begin{array}{l}\text { 3. } 04 \\
3.65\end{array}$ & $\begin{array}{l}\text { 1. } 68 \\
\text { 3. } 27\end{array}$ & $\begin{array}{l}3.45 \\
2.34\end{array}$ & $\begin{array}{l}2.19 \\
4.84\end{array}$ & $\begin{array}{l}\text { 3. } 52 \\
6.29\end{array}$ & $\begin{array}{l}2.73 \\
3.24\end{array}$ & $\begin{array}{l}\text { 2. } 71 \\
\text { 3. } 10\end{array}$ & $\begin{array}{l}\text { 1. } 90 \\
\text { 3. } 39\end{array}$ & $\begin{array}{l}2.91 \\
1.47\end{array}$ & $\begin{array}{r}1.91 \\
f 4.61\end{array}$ & $\begin{array}{l}32.87 \\
41.32\end{array}$ \\
\hline 10-year mean... & 2.67 & 3.27 & 3. 34 & 2.47 & 2.84 & 3. 51 & 4. 90 & 2. 98 & 2.90 & 2.64 & 2. 19 & 3. 26 & 37.09 \\
\hline
\end{tabular}

GREENSPRING FURNACE, MD., ALTITUDE 500 FEET.

\begin{tabular}{|c|c|c|c|c|c|c|c|c|c|c|c|c|c|}
\hline $\begin{array}{l}1896-1900 . \ldots . \\
1901-1905 . \ldots .\end{array}$ & $\begin{array}{l}2.65 \\
2.98\end{array}$ & $\begin{array}{r}g 3.80 \\
2.27\end{array}$ & $\begin{array}{l}3.43 \\
3.18\end{array}$ & $\begin{array}{l}\text { 1. } 63 \\
\text { 3. } 32\end{array}$ & $\begin{array}{l}\text { 4. } 12 \\
\text { 3. } 13\end{array}$ & $\begin{array}{l}2.85 \\
4.61\end{array}$ & $\begin{array}{l}\text { 3. } 52 \\
5.04\end{array}$ & $\begin{array}{l}\text { 3. } 39 \\
\text { 3. } 87\end{array}$ & $\begin{array}{l}2.69 \\
2.37\end{array}$ & $\begin{array}{l}\text { 2. } 21 \\
\text { 2. } 53\end{array}$ & $\begin{array}{l}\text { 3. } 11 \\
\text { 1. } 82\end{array}$ & $\begin{array}{l}\text { 2. } 16 \\
\text { 3. } 64\end{array}$ & $\begin{array}{l}35.71 \\
38.81\end{array}$ \\
\hline 10-year mean... & 2.81 & 3. 03 & 3. 30 & 2. 47 & 3. 62 & 3. 73 & 4. 28 & 3. 63 & 2. 53 & 2.37 & 2. 46 & 2.90 & 37.26 \\
\hline
\end{tabular}

HAGERSTOWN, MD., ALTITUDE 550 FEET.

\begin{tabular}{|c|c|c|c|c|c|c|c|c|c|c|c|c|c|}
\hline $\begin{array}{l}1896-1900 \ldots \ldots \\
1901-1905 \ldots \ldots\end{array}$ & 2.27 & 4. 28 & 4. 13 & 1. 31 & h3. 02 & $i 3.54$ & j3. 73 & j3. 85 & i2. 56 & i2. 37 & 3. 25 & 2.21 & $\begin{array}{r}36.52 \\
k 33.89\end{array}$ \\
\hline 10-year mean. & & & & & & & & & & & & & 35.20 \\
\hline
\end{tabular}

$a 1$ year interpolated, based on ohservations at Oakland. $b 2$ years intei polated, based on observations at Oakland.

$c 1$ year interpolated, based on observations at $W$ ashington.

d 5 years inte polated, based on observations at Mount St. Mary College.

$e 2$ years interpolated, based on observations at Mount St. Mary College.

$f 2$ years interpolated, based on observations at Washington.

$g 1$ year interpolated, based on observations at Clear Spring.

$h 1$ year interpolated, based on observations,at Chewsville and Sharpsburg.

$i 3$ years interpolated, based on observations at Chewsville and Sharpsburg.

i 2 years interpolated, based on observations at Chew sville and Sharpsburg.

$k 4$ years interpolated, based on observations at Chewsville and Sharpsburg. 
Mean precipitation at stations in drainage basin of Potomac River-Continued.

HANCOCK, MD., ALTITUDE 455 FEET.

\begin{tabular}{|c|c|c|c|c|c|c|c|c|c|c|c|c|c|}
\hline & Jan. & Feb. & Mar. & Apr. & May. & June. & July. & Aug. & Sept. & Oet. & Nov. & Dec. & Annual. \\
\hline $\begin{array}{l}1896-1900 \ldots \\
1901-1905 \ldots\end{array}$ & 2.76 & 1. 83 & 3.17 & 3.79 & 2.67 & 5. 27 & 4. 71 & 3. 81 & $b 2.16$ & $b 2.45$ & b1. 48 & $b 3.41$ & $\begin{array}{r}a 33.74 \\
37.54\end{array}$ \\
\hline 10-year mean. & & & & & & & & & & & & & $a 35.64$ \\
\hline
\end{tabular}

HARNEY, MD., ALTITUDE 500 FEET.

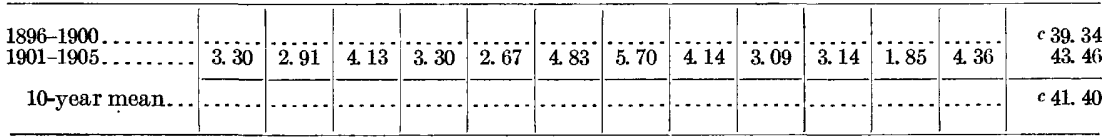

HARPERS FERRY, W. VA., ALTITUDE 277 FEET.

\begin{tabular}{r|c|c|c|c|c|c|c|c|c|c|c|c|c|c}
\hline $1896-1900 . \ldots . \ldots$. & 2.64 & 4.18 & 3.60 & 1.66 & 4.63 & 3.12 & 3.74 & 3.80 & 2.75 & 2.91 & 3.58 & 2.76 & 39.37 \\
$1901-1905 . \ldots \ldots \ldots$. & 3.19 & 1.95 & 4.27 & 3.75 & 3.42 & 4.50 & 5.14 & 3.21 & 3.16 & 2.76 & 1.89 & 3.89 & 41.13 \\
\hline 10-year mean... & 2.91 & 3.06 & 3.93 & 2.70 & 4.02 & 3.81 & 4.44 & 3.50 & 2.95 & 2.83 & 2.73 & 3.32 & 40.25 \\
\hline
\end{tabular}

LINCOLN, VA., ALTITUDE 500 FEET.

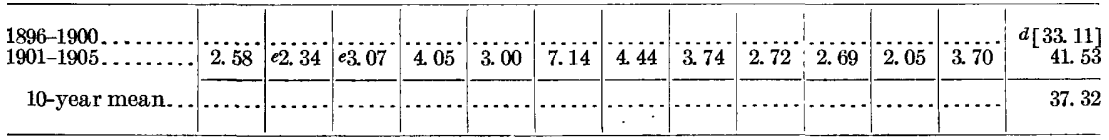

MARION, PA., ALTITUDE 640 FEET.

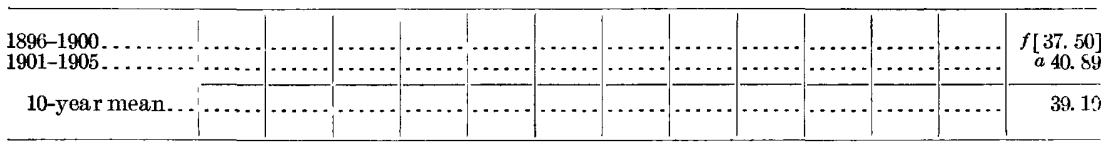

MARTINSBURG, W. VA., ALTITUdE 435 FEET.

\begin{tabular}{r|c|c|c|c|c|c|c|c|c|c|c|c|c|c}
\hline $1896-1900 \ldots . . . . .$. & 1.92 & 3.60 & 3.13 & 1.65 & 3.25 & 3.82 & 3.27 & 3.28 & 2.78 & 2.09 & 3.05 & 2.16 & 34.02 \\
$1901-1905 . . . \ldots . .$. & 2.72 & 1.70 & 3.46 & 3.82 & 4.24 & 4.99 & 6.18 & 3.69 & 2.55 & 2.70 & 2.20 & 3.01 & 41.24 \\
\cline { 2 - 9 } & 2.32 & 2.15 & 3.29 & 2.73 & 3.74 & 4.40 & 4.72 & 3.48 & 2.66 & 2.39 & 2.62 & 2.53 & 37.65 \\
\hline
\end{tabular}

MOUNT ST. MARY COLLEGE, MD., ALTITUDE 720 FEET.

\begin{tabular}{|c|c|c|c|c|c|c|c|c|c|c|c|c|c|}
\hline $\begin{array}{l}1896-1900 \ldots \\
1901-1905 \ldots\end{array}$ & $\begin{array}{l}2.28 \\
3.65\end{array}$ & $\begin{array}{l}\text { 3. } 87 \\
2.48\end{array}$ & $\begin{array}{l}\text { 3. } 89 \\
\text { 4. } 47\end{array}$ & $\begin{array}{l}\text { 1. } 67 \\
\text { 4. } 36\end{array}$ & $\begin{array}{r}\text { 94. } 31 \\
3.17\end{array}$ & $\begin{array}{l}3.35 \\
5.12\end{array}$ & $\begin{array}{l}\text { 4. } 66 \\
\text { 4. } 74\end{array}$ & $\begin{array}{r}4.42 \\
g 3.81\end{array}$ & $\begin{array}{r}3.01 \\
g 2.85\end{array}$ & $\begin{array}{l}\text { 3. } 06 \\
\text { 3. } 38\end{array}$ & $\begin{array}{l}3.95 \\
2.00\end{array}$ & $\begin{array}{l}\text { 2. } 57 \\
4.02\end{array}$ & $\begin{array}{l}\text { 41. } 07 \\
44.06\end{array}$ \\
\hline 10-yea $r$ mean... & 2. 96 & 3. 17 & 4. 18 & 3. 01 & 3.74 & 4. 23 & 4. 70 & 4. 11 & 2.93 & 3. 72 & 2.97 & 3.29 & 42. 56 \\
\hline
\end{tabular}

NEW MARKET, MD., ALTITUDE 550 FEET.

\begin{tabular}{|c|c|c|c|c|c|c|c|c|c|c|c|c|c|}
\hline $\begin{array}{l}1896-1900 \ldots \ldots \ldots \\
1901-1905 \ldots \ldots \ldots\end{array}$ & $\begin{array}{l}\text { 2. } 62 \\
\text { 3. } 61\end{array}$ & $\begin{array}{l}4.48 \\
3.00\end{array}$ & $\begin{array}{r}h 4.26 \\
4.12\end{array}$ & $\begin{array}{l}1.99 \\
3.53\end{array}$ & $\begin{array}{l}3.74 \\
2.72\end{array}$ & $\begin{array}{l}2.76 \\
6.86\end{array}$ & $\begin{array}{l}\text { 5. } 13 \\
6.44\end{array}$ & $\begin{array}{l}\text { 3. } 36 \\
\text { 4. } 82\end{array}$ & $\begin{array}{l}2.82 \\
2.91\end{array}$ & $\begin{array}{l}2.58 \\
\text { 3. } 50\end{array}$ & $\begin{array}{l}4.04 \\
2.13\end{array}$ & $\begin{array}{l}2.37 \\
4.75\end{array}$ & $\begin{array}{l}40.18 \\
48.65\end{array}$ \\
\hline 10-year mean... & 3.11 & 3. 74 & 4. 19 & 2. 76 & 3. 23 & 4.81 & 5. 78 & 409 & 2.86 & 3.04 & 3.08 & 3.56 & 44. 41 \\
\hline
\end{tabular}

a 2 years interpolated, based on observations at Greenspring Furnace.

$b 1$ year interpolated, based on observations at Greenspring Furnace.

c 3 years interpolated, based on observations at Mount St Mary College.

d 5 years interpolated, based on observations at Frederick.

$\ell 1$ year interpolated, based on observations at Frederick.

$f 5$ years interpolated, based on observations at Greenspring Furnace.

$g 1$ year interpolated, based on observations at Harney.

$h 1$ year interpolated, based on observations at Frederick. 
Mean precipitation at stations in drainage basin of Potomac River-Continued.

OLD FIELD, W. VA., ALTITUDE 800 FEET.

\begin{tabular}{|c|c|c|c|c|c|c|c|c|c|c|c|c|c|}
\hline & Jan. & Feb. & Mar. & Apr. & May. & June. & July. & Aug. & Sept. & Oct. & Nov. & Dec. & Annual. \\
\hline $\begin{array}{l}1896-1900 \ldots \ldots \ldots \\
1901-1905 . \ldots \ldots \ldots\end{array}$ & $\begin{array}{l}2.16 \\
\cdots \ldots\end{array}$ & 3. 73 & $\begin{array}{l}2.74 \\
\cdots \cdots\end{array}$ & 1.57 & 4. 00 & $\begin{array}{l}3.10 \\
\cdots\end{array}$ & 3.89 & $\begin{array}{l}3.74 \\
\cdots \cdots\end{array}$ & 2.85 & $\begin{array}{l}2.12 \\
\cdots \cdots\end{array}$ & $\begin{array}{l}a 2.19 \\
\cdots \ldots . .\end{array}$ & $\left|\begin{array}{l}a 1.77 \\
\cdots . . .\end{array}\right|$ & $\begin{array}{r}33.87 \\
b 33.64\end{array}$ \\
\hline 10-year mean. . & & & & & & & & & & & & & 33. 75 \\
\hline
\end{tabular}

POINT OF ROCKS, MD., ALTITUDE 235 (?) FEET.

\begin{tabular}{|c|c|c|c|c|c|c|c|c|c|c|c|c|c|}
\hline $\begin{array}{l}1896-1900 \ldots \ldots \ldots \ldots \\
1901-1905 \ldots \ldots \ldots\end{array}$ & $\begin{array}{l}2.39 \\
2.68\end{array}$ & $\begin{array}{l}3.66 \\
2.15\end{array}$ & $\begin{array}{l}\text { 3. } 55 \\
\text { 3. } 27\end{array}$ & $\begin{array}{l}\text { 1. } 79 \\
\text { 3. } 43\end{array}$ & $\begin{array}{l}\text { 3. } 98 \\
\text { 3. } 57\end{array}$ & $\begin{array}{l}\text { 3. } 38 \\
\text { 4. } 91\end{array}$ & $\begin{array}{l}3.82 \\
4.48\end{array}$ & $\begin{array}{l}\text { 3. } 30 \\
\text { 3. } 69\end{array}$ & $\begin{array}{l}2.98 \\
2.31\end{array}$ & $\begin{array}{r}2.54 \\
\text { c2. } 19\end{array}$ & $\begin{array}{r}2.97 \\
d 1.46\end{array}$ & $\begin{array}{r}2.11 \\
d 3.32\end{array}$ & $\begin{array}{l}36.69 \\
37.30\end{array}$ \\
\hline 10-year mean. & 2. 53 & 2. 90 & 3. 41 & 2. 61 & 3. 77 & 4.14 & 4. 15 & 3.49 & 2.64 & 2.36 & 2.21 & 2.71 & 36.99 \\
\hline
\end{tabular}

RECEIVING RESERVOIR, MD., ALTITUDE 160 FEET.

\begin{tabular}{|c|c|c|c|c|c|c|c|c|c|c|c|c|c|}
\hline $\begin{array}{l}1896-1900 \ldots \ldots \\
1900-1905 . \ldots \ldots\end{array}$ & $\begin{array}{r}2.44 \\
e 3.01\end{array}$ & $\begin{array}{l}\text { 4. } 05 \\
2.01\end{array}$ & $\begin{array}{l}3.04 \\
3.61\end{array}$ & $\begin{array}{l}\text { 1. } 77 \\
\text { 3. } 73\end{array}$ & $\begin{array}{l}\text { 3. } 40 \\
\text { 2. } 70\end{array}$ & $\begin{array}{l}\text { 3. } 16 \\
\text { 3. } 82\end{array}$ & $\begin{array}{l}4.03 \\
6.08\end{array}$ & $\begin{array}{l}\text { 4. } 74 \\
\text { 3. } 85\end{array}$ & $\begin{array}{l}3.26 \\
2.92\end{array}$ & $\begin{array}{l}2.01 \\
3.44\end{array}$ & $\begin{array}{l}3.04 \\
1.65\end{array}$ & $\begin{array}{l}e 2.13 \\
e 4.76\end{array}$ & $\begin{array}{l}37.05 \\
41.61\end{array}$ \\
\hline 10.year mean. . & 2.72 & 3.03 & 3.32 & 2.75 & $\overline{3.05}$ & 3.49 & 5.05 & 4.29 & 3.09 & 2.72 & 2.34 & 3. 44 & 39.33 \\
\hline
\end{tabular}

RIVERTON, VA., ALTITUDE 493 FEET.

\begin{tabular}{|c|c|c|c|c|c|c|c|c|c|c|c|c|c|}
\hline $\begin{array}{l}1896-1900 \ldots \ldots \\
1901-1905 \ldots \ldots\end{array}$ & $\begin{array}{ll}\cdots & \ldots \\
\cdots & \ldots . . .\end{array}$ & 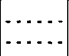 & $\left|\begin{array}{ll}\cdots & \cdots \\
\cdots & \ldots\end{array}\right|$ & $\begin{array}{ll}\cdots & \ldots \\
\cdots & \ldots\end{array}$ & $\left|\begin{array}{lll}\cdots & \cdots & \ldots \\
\cdots & \ldots & \ldots\end{array}\right|$ & $\mid \begin{array}{ll}\cdots & \ldots \\
\cdots & \ldots\end{array}$ & ….... & $\left|\begin{array}{lll}\cdots & \cdots & \\
\cdots & \cdots & \cdots\end{array}\right|$ & 2.01 & 2.28 & 1.35 & 3.40 & $\begin{array}{r}f[32.50] \\
g 32.58\end{array}$ \\
\hline 10-year mean... & ..... & $\ldots$ & & $\ldots$ & $\ldots$ & $\ldots$. & . & $\ldots$ & & & . . . . & ...... & 32.54 \\
\hline
\end{tabular}

ROMNEY, W. VA., ALTITUDE 824 FEET.

\begin{tabular}{|c|c|c|c|c|c|c|c|c|c|c|c|c|c|}
\hline $\begin{array}{l}1896-1900 \ldots \ldots \ldots \\
1901-1905 \ldots \ldots \ldots\end{array}$ & 2.29 & h1. 78 & 3. 04 & 3. 13 & 3. 77 & 3. 78 & h4. 34 & h3. 99 & 1. 59 & 2. 36 & 1.80 & 2.91 & $\begin{array}{r}h 35.40 \\
34.79\end{array}$ \\
\hline 10-year mean. & & & & & & & & & & & & & 35.09 \\
\hline
\end{tabular}

SHARPSBURG, MD., ALTITUDE 440 FEET.

\begin{tabular}{|c|c|c|c|c|c|c|c|c|c|c|c|c|c|}
\hline $\begin{array}{l}1896-1900 \ldots \ldots . \\
1900-1905 \ldots \ldots\end{array}$ & $\begin{array}{l}2.56 \\
\ldots \ldots\end{array}$ & $\begin{array}{l}3.83 \\
\ldots \ldots\end{array}$ & $\begin{array}{l}3.26 \\
\ldots \ldots\end{array}$ & $\mid \begin{array}{l}d 1.46 \\
\ldots \ldots\end{array}$ & $\begin{array}{l}d 3.94 \\
\ldots \ldots\end{array}$ & $\begin{array}{c}3.22 \\
\ldots \ldots\end{array}$ & $\begin{array}{c}3.78 \\
\ldots\end{array}$ & $\begin{array}{c}3.16 \\
\ldots \ldots\end{array}$ & $\left\{\begin{array}{l}2.62 \\
\ldots \ldots\end{array}\right.$ & $\begin{array}{l}a 2.29 \\
\ldots \ldots\end{array}$ & $\begin{array}{c}3.07 \\
\ldots \ldots\end{array}$ & $\mid \begin{array}{c}2.16 \\
\ldots \ldots\end{array}$ & $\begin{array}{r}35.37 \\
i 38.11\end{array}$ \\
\hline 10-year mean... & & & & & & & & & & & *.. & $\cdots$ & 36. 74 \\
\hline
\end{tabular}

SHENANDOAH, VA., ALTITUDE 937 FEET.

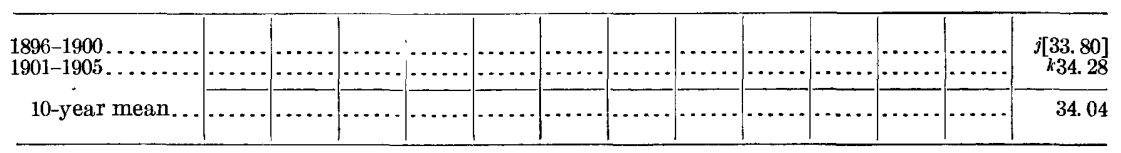

a 1 year interpolated, based on observations at Romney.

$b 5$ years interpolated, based on observations at Romney.

c 2 years interpolated, based on observations at Harpers Ferry.

$d 1$ year interpolated, based on observations at Harpers Ferry.

$\epsilon 1$ year interpolated, based on observations at Washington.

$f 5$ years interpolated, based on observations at Stephen City.

$g 1$ year interpolated, based on observations at Stephen City.

$h 1$ year interpolated, based on observations at Burlington.

$i$ years interpolated, based on observations at Harpers Ferry.

$j 5$ years interpolated, based on observations at Staunton.

$k 1$ year interpolated, based on observations at Staunton. 
Mean precipitation at stations in drainage basin of Potomac River-Continued.

SOMERSET, PA., ALTITUDE 2,250 FEET.

\begin{tabular}{|c|c|c|c|c|c|c|c|c|c|c|c|c|c|}
\hline & Jan. & Feb. & Mar. & Apr. & May. & June. & July. & Aug. & Sept. & Oct. & Nov. & Dec. & Annual. \\
\hline $\begin{array}{l}1896-1900 \ldots \ldots \ldots \\
1901-1905 \ldots \ldots \ldots\end{array}$ & $\begin{array}{l}3.84 \\
4.86\end{array}$ & $\begin{array}{l}4.82 \\
\text { 3. } 95\end{array}$ & $\begin{array}{l}5.80 \\
5.71\end{array}$ & $\begin{array}{l}3.48 \\
5.95\end{array}$ & $\begin{array}{l}4.04 \\
4.30\end{array}$ & $\begin{array}{l}5.48 \\
6.01\end{array}$ & $\begin{array}{l}5.81 \\
5.55\end{array}$ & $\begin{array}{l}\text { 4. } 73 \\
4.71\end{array}$ & $\begin{array}{l}3.50 \\
2.38\end{array}$ & $\begin{array}{l}2.67 \\
3.06\end{array}$ & $\begin{array}{l}4.25 \\
2.31\end{array}$ & $\begin{array}{l}4.02 \\
4.56\end{array}$ & $\begin{array}{l}52.48 \\
53.37\end{array}$ \\
\hline 10-year mean.. & 4.35 & 4. 38 & 5. 75 & 4. 71 & 4.17 & 5. 74 & 5.68 & 4. 72 & 2.94 & 2.86 & 3.28 & 4.29 & 52.87 \\
\hline
\end{tabular}

STAUNTON, VA., ALTITUDE 1,380 FEET.

\begin{tabular}{r|c|c|c|c|c|c|c|c|c|c|c|c|c}
\hline $1896-1900 \ldots \ldots \ldots \ldots$ & 2.56 & 3.32 & 3.93 & 1.95 & 4.30 & 3.59 & 3.62 & 3.83 & 3.71 & 3.57 & 2.53 & 1.90 & 38.85 \\
$1901-1905 \ldots \ldots \ldots .$. & 2.69 & 2.95 & 3.16 & 3.95 & 4.09 & 5.45 & 4.32 & 3.55 & 2.62 & 1.99 & 1.68 & 3.53 & 40.02 \\
\hline $10-$ year mean... & 2.62 & 3.13 & 3.54 & 2.95 & 4.19 & 4.52 & 3.77 & 3.69 & 3.16 & 2.78 & 2.10 & 2.71 & 39.43
\end{tabular}

STEPHENS CITY, VA., ALTITUDE 710 FEET.

\begin{tabular}{|c|c|c|c|c|c|c|c|c|c|c|c|c|c|}
\hline $\begin{array}{l}1896-1900 \ldots \ldots \ldots \\
1901-1905 \ldots \ldots \ldots\end{array}$ & 2.39 & $\begin{array}{c}4.16 \\
\cdots \cdots\end{array}$ & $\begin{array}{l}3.69 \\
3.64\end{array}$ & 1.57 & 4.22 & 3.90 & 4.01 & 4. 11 & $\begin{array}{l}2.99 \\
2.85\end{array}$ & $\begin{array}{l}\text { 3. } 17 \\
2.34\end{array}$ & 2. 51 & $\begin{array}{l}2.41 \\
\ldots . . .\end{array}$ & $\begin{array}{r}39.17 \\
a 39.95\end{array}$ \\
\hline 10-year mean. & ....... & ....... & 3.66 & & & & & & 2.92 & 2.75 & & & 39.56 \\
\hline
\end{tabular}

SUNNYSIDE, MD., ALTITUDE 2,500 FEET.

\begin{tabular}{|c|c|c|c|c|c|c|c|c|c|c|c|c|c|}
\hline $\begin{array}{l}1896-1900 \ldots \ldots \ldots \\
1901-1905 \ldots \ldots \ldots\end{array}$ & $\begin{array}{r}4.73 \\
\ldots \ldots \\
\end{array}$ & 5.78 & $\begin{array}{r}6.46 \\
\ldots . . \\
\end{array}$ & $\begin{array}{r}3.49 \\
\cdots \cdots \\
\end{array}$ & 5.68 & 5.69 & $\begin{array}{r}7.04 \\
\cdots \cdots \\
\end{array}$ & $\begin{array}{r}4.63 \\
\ldots \ldots \\
\end{array}$ & $\begin{array}{c}3.99 \\
\cdots \cdots \\
\end{array}$ & $\begin{array}{c}3.01 \\
\ldots \ldots \\
\end{array}$ & $\begin{array}{r}5.44 \\
\cdots \cdots \\
\end{array}$ & 4. 42 & $\begin{array}{r}60.38 \\
b 51.32 \\
\end{array}$ \\
\hline 10-year mean & & & & & & & & & & & & & 55.85 \\
\hline
\end{tabular}

TAKOMA PARK, MD., ALTITUDE 250 FEET.

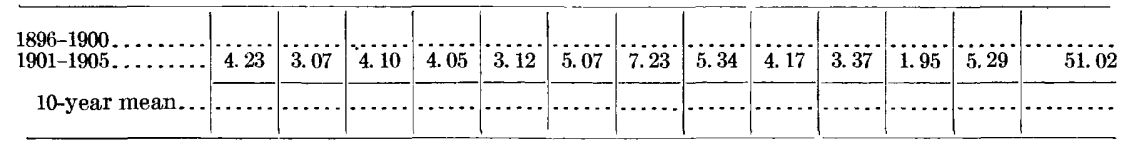

TANEYTOWN, MD., ALTITUDE 490 FEET.

\begin{tabular}{|c|c|c|c|c|c|c|c|c|c|c|c|c|c|}
\hline $\begin{array}{l}1896-1900 \ldots \ldots \ldots \\
1901-1905 \ldots \ldots \ldots\end{array}$ & $\mid \begin{array}{l}c 2.15 \\
\cdots \ldots\end{array}$ & \begin{tabular}{l} 
c4.14 \\
\hdashline$\ldots \ldots$ \\
\end{tabular} & $\begin{array}{l}c 3.19 \\
\cdots \ldots\end{array}$ & c1.82 & $\begin{array}{c}c 3.91 \\
\cdots\end{array}$ & $\begin{array}{l}c 3.22 \\
\cdots \cdots\end{array}$ & $\begin{array}{r}4.56 \\
\cdots \cdots \\
\end{array}$ & 3.96 & 3.00 & 2.26 & $\begin{array}{r}3.66 \\
\cdots \cdots . \\
\end{array}$ & $\begin{array}{r}2.52 \\
\ldots \ldots \\
\end{array}$ & $\begin{array}{r}38.39 \\
d 45.08 \\
\end{array}$ \\
\hline 0 & & & & & & & & & & & & & 41.73 \\
\hline
\end{tabular}

UPPER TRACT, W. VA., ALTITUDE 1,230 FEET.

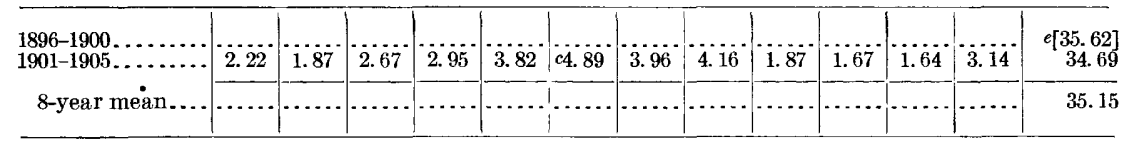

WASHINGTON, D. C., ALTITUDE 112 FEET.

\begin{tabular}{r|c|c|c|c|c|c|c|c|c|c|c|c|c|c}
\hline $1896-1900 \ldots \ldots \ldots$. & 2.76 & 4.97 & 3.67 & 1.94 & 3.87 & 3.94 & 3.93 & 4.16 & 3.27 & 2.33 & 2.58 & 2.28 & 39.73 \\
$1901-1905 \ldots \ldots . .$. & 3.40 & 2.97 & 3.69 & 3.70 & 2.95 & 4.39 & 5.81 & 4.64 & 3.06 & 3.37 & 1.96 & 4.69 & 44.67 \\
\hline 10-year mean... & 3.08 & 3.97 & 3.68 & 2.82 & 3.41 & 4.16 & 4.87 & 4.40 & 3.16 & 2.85 & 2.27 & 3.48 & 42.20 \\
\hline
\end{tabular}

$a 1$ year interpolated, based on observations at Riverton.

$b 3$ years interpolated, based on observations at Deer Park and Oakland.

$c 1$ year interpolated, based on observations at Bachman Valley.

$d 3$ years interpolated, based on observations at Bachman Valley.

$e$ Mean for $1898-1900$. 
Mean precipitation at stations in drainage basin of Potomac River-Continued.

WESTERNPOR'T, MD., ALTITUDE 1,000 FEET.

\begin{tabular}{r|c|c|c|c|c|c|c|c|c|c|c|c|c}
\hline & Jan. & Feb. & Mar. & Apr. & May. & June. & July. & Aug. & Sept. & Oct. & Nov. & Dec. & Annual. \\
\hline $1896-1900 \ldots \ldots \ldots \ldots$ & 2.35 & 3.10 & 3.52 & 2.41 & 4.08 & 3.51 & 4.10 & 3.10 & 2.81 & 2.35 & 2.98 & 1.70 & 36.02 \\
$1901-1905 . \ldots \ldots \ldots$. & 2.28 & 1.93 & 2.92 & 3.08 & 3.68 & 4.76 & 4.71 & 3.14 & 2.33 & 1.92 & 1.17 & 2.89 & 34.81 \\
10-year mean... & 2.31 & 2.51 & 3.22 & 2.74 & 3.88 & 4.13 & 4.40 & 3.12 & 2.57 & 2.13 & 2.07 & 2.29 & 35.41 \\
\hline
\end{tabular}

WOODSTOCK, VA., ALTITUdE 927 FEET.

\begin{tabular}{|c|c|c|c|c|c|c|c|c|c|c|c|c|c|}
\hline $\begin{array}{l}1896-1900 \ldots \ldots \ldots . \\
1901-1905 . \ldots \ldots \ldots\end{array}$ & 2.59 & 1.77 & 2.99 & 3.25 & 3.57 & 5.71 & 3.03 & 4.04 & $\begin{array}{l}3.12 \\
2.23\end{array}$ & $\begin{array}{l}2.92 \\
2.06\end{array}$ & $\begin{array}{l}2.11 \\
1.41\end{array}$ & $\begin{array}{l}1.80 \\
3.00\end{array}$ & $\begin{array}{r}a 35.15 \\
\quad 35.67\end{array}$ \\
\hline 10-year mean. & & & & & & & & & 2.67 & 2.49 & 1. 76 & 2.40 & 35.41 \\
\hline
\end{tabular}

$a 1$ year interpolated, based on observations at Stephens City.

\section{COMPARISON OF RAINFALC AND RUN-OFF.}

At eight river-measurement stations in the Potomac drainage basin the records of run-off were of sufficient extent for the comparison of rainfall and run-off. These stations are as follows:

Potomac at Point of Rocks, Md.

Monocacy near Frederick, Md.

Antietam Creek, near Sharpsburg, Md.

South Branch of Potomac near Springfield, W. Va.

North Branch of Potomac near Piedmont, W. Va.

South Branch of Shenandoah near Front Royal, Va.

North Branch of Shenandoah near Riverton, Va.

Shenandoah at Millville, W. Va.

The rainfall stations in the areas above these stations are so distributed as to represent fairly well the conditions over the various areas. It has been assumed that for any area the mean rainfall for a given month is the mean of the monthly rainfall at the various stations in that area. Based on this assumption, the monthly and yearly rainfall for the years for which run-off records are available has been determined. These values are included in the tables of mean monthly run-off given for each gaging-station in other parts of this report. When these monthly rainfall tables were prepared some of the data given on pages 34-40 were not available.

From the monthly rainfall and the monthly run-off the run-off in per cent of rainfall has been determined and also the loss of rainfall. 
In the comparison of run-off and rainfall the following facts must be kept in mind. First, run-off is a resultant quantity, being the amount of water left after evaporation, vegetation, seepage, etc., in the area have been satisfied; second, a rain gage shows only the rain that falls on a few square inches of surface. There should be many rain gages in order to ascertain the average precipitation over a watershed, and, as maximum precipitation often occurs in comparatively small areas, it may not be recorded. Third, ground and surface storage modify the relation between, precipitation and run-off. The run-off for a month in early spring may be much larger than the precipitation for that month, on account of melting snow that falls during the winter months. Again, in the early fall, when the ground water is low, a small rainfall yields a much smaller run-off than it would during the spring, when the ground is nearly saturated with water. Fourth, heavy rainfall frequently occurs at the end of the month, and as the run-off data are computed for the calendar months, the results from such rainfall may appear in the following month's record. Fifth, data are not available relative to snow storage, which produces the high percentages of run-off usually obtained for the late winter and early spring. To account fully for this storage, a sample of snow extending from top to bottom should be melted at the end of each month, in order to determine the total amount of water stored on the ground. The quantity available for run-off during the following month would be the amount thus determined plus the precipitation during the following month minus the amount left in snow storage at the end of that month plus or minus the change in ground water. These and other causes make the monthly ratios of run-off to rainfall appear very erratic. A month is too short a period for the comparison of these quantities. A year is a better period, but not entirely satisfactory, especially if the calendar year is taken, as the snow and ground-water storage are not in the same conditions at the end of each year.

Notwithstanding the above facts, the rainfall and stream data and the comparison between the two are very consistent from year to year and from station to station, and are of great interest both for comparative purposes and to show the general conditions existing in the drainage. 


\section{GAGING STATIONS.}

In the following table are listed the stations in the Potomac basin at which stream measurements have been made by the United States Geological Survey:

Gaging stations of the United States Geological Survey in the Potomac basin.

\begin{tabular}{|c|c|}
\hline Station. & Period of observations. \\
\hline 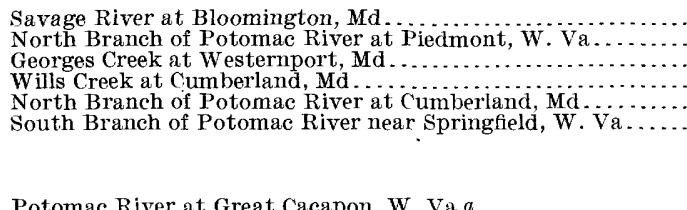 & $\begin{array}{l}\text { May } 3,1905, \text { to July } 15,1906 . \\
\text { June } 27,1899 \text {, to July } 15,1906 . \\
\text { May 4, } 1905 \text {, to July } 15,1906 . \\
\text { May 6, 1905, to July } 14,1906 . \\
\text { June } 11,1894 \text {, to Nov. } 20,1897 \text {. } \\
\text { June } 3,1894 \text {, to Oct. } 20,1894 ; \text { Apr. } 11 \text {, } \\
\text { 1895, to Feb. } 29,1896 ; \text { June } 26,1899 \text {, } \\
\text { to Feb. } 24,1902 ; \text { Aug. } 28,1903 \text {, to } \\
\text { July } 14,1906 .\end{array}$ \\
\hline 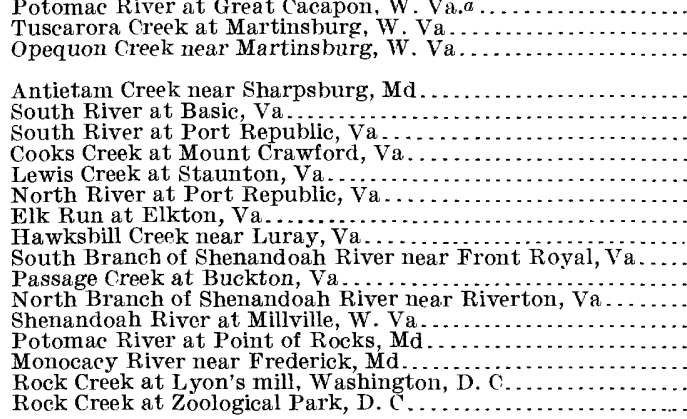 & $\begin{array}{l}\text { May } 8 \text { to Dec. } 30,1905 \\
\text { May } 9 \text { to June } 4,1905 ; \text { Oct. } 8,1905 \text {, to } \\
\text { July } 15,1906 . \\
\text { July } 1,1897 \text {, to Aug. } 25,1905 . \\
\text { June } 29,1905 \text {, to July } 15,1906 . \\
\text { Aug. } 6,1895 \text {, to Apr. } 1,1899 . \\
\text { July } 1,1905 \text {, to July } 15,1906 . \\
\text { Do } \\
\text { Aug. } 6,1895 \text {. to Apr. } 1,1899 . \\
\text { June } 28,1905 \text {, to July } 15,1906 . \\
\text { June } 27,1905 \text {, to July } 15,1906 . \\
\text { June } 26,1899 \text {, to July } 16,1906 . \\
\text { Oct. } 26,1905 \text {, to July } 15,1906 . \\
\text { June } 26,1899 \text {, to July } 14,1906 . \\
\text { Since Apr. } 15,1895 . \\
\text { Since Feb. } 17,1895 . \\
\text { Since Aug. 4, } 1896 \text {. } \\
\text { Aug. 18, } 1892, \text { to Nov. } 30,1894 . \\
\text { Jan. } 18,1897 \text {, to Nov. } 10,1900 \text {. }\end{array}$ \\
\hline
\end{tabular}

a Gage-height records were obtained from June 21, 1894, to March 7, 1896, but are not republished on account of their unreliahility.

Daily gage-height records are at present maintained by the Weather Bureau in the Potomac drainage basin at Cumberland, Md.; Riverton, Va., and Harpers Ferry, W. Va. General information and tables of gage heights at these stations can be found in the Weather Bureau Report of Daily River Stages of the Principal Rivers of the United States.

The Cumberland station on North Branch of Potomac River was established September 1, 1901, at the dam just below the mouth of Wills Creek. The range of stage sinee the date of establishment has been from 0 to 11.5 feet. The danger line is at 8 feet. The datums of the Weather Bureau and Geological Survey gages have not been connected by level at this point. It is not considered advisable to attempt to obtain additional estimates at Cumberland based on the Weather Bureau records, because no discharge measurements have been made there since 1898 .

The Riverton station on Shenandoah River was established September 1, 1901, at the Norfolk and Western Railway bridge below the junction of North and South branches. Gage heights were also obtained at this point during 1892 to 1894 . The highest water was 
47 feet September 30 and October 1, 1870; the lowest since 1900 was -1.7 feet. The danger line is at 22 feet.

The Harpers Ferry station on Potomac River was established in 1882 at the Baltimore and Ohio Railroad bridge. November 1, 1901, the gage datum was lowered 2.00 feet. The highest water was 36 feet, June 1, 1889; the high water of November, 1877, was 29.2 feet; the lowest on various dates -3 feet. The danger line is at 18 feet. Elevations have been reduced to new datum.

Records were also kept of the elevation of the water surface at high tide at Long Bridge, Washington, D. C., from June 1, 1891, to April 15, 1893. For flood stages see page 181. The danger point is 8 feet on the gage, or about 7 feet above mean sea level. Daily gage heights at Great Falls have likewise been published from 1890 to 1892 inclusive.

\section{NORTH BRANCH OF POTOMAC RIVER BASIN.}

GENERAL DESCRIPTION.

The general features of the basin of North Branch of Potomac River are described on page 213 . The country drained comprises steep mountain slopes and narrow valleys. The run-off is rapid and fluctuations in flow are great. The fall of the stream both above and below Cumberland is large. Facilities for dams are excellent. The tributaries of North Branch are comparatively small and the fall of many of them is rapid.

\section{SAVAGE RIVER AT BLOOMINGTON, MD.}

Savage River rises on Bog and Little Savage mountains, in the northeastern part of Garrett County, Md., and flows in a general southwesterly direction for about 20 miles, then turns to the southeast, and enters North Branch of Potomac River near Bloomington. The drainage area is practically uninhabited and the watershed is given over to lumbering. The river receives the waters of a number of small runs. The gaging station on Savage River was established May 3, 1905, and was discontinued July 16, 1906. It is located at a highway bridge about 800 feet above the junction of Savage River with North Branch of the Potomac.

The channel is straight for 200 feet above and below the station. The current is swift. The right bank is low and clean and has an overflow channel at high water. The left bank is high and does not overflow. The bed of the stream is rocky, very irregular, and permanent. There is but one channel at low and ordinary stages; during high-water stages there are two channels.

Discharge measurements were made from the downstream side of the steel bridge to which the gage is fastened. The initial point for soundings is the center of the bridge pier at the left abutment, downstream side.

IRR $192-07-4$ 
A standard chain gage is attached to the downstream side of the bridge near the left abutment. The length of the chain from the end of the weight to the marker is 21.15 feet. The gage was read twice each day by F. S. Cline. Bench mark No. 1 is a chiseled cross on the downstream corner of the left abutment. Its elevation is 16.49 feet above gage datum. Bench mark No. 4 is the top of the pulley wheel of the gage. Its elevation was 20.82 feet above gage datum March 16,1906 .

Estimates are considered to be within 5 per cent of the true discharge for stages above 30 second-feet. Below this limit the probable error may be somewhat greater. The estimates do not include the flow in the 10-inch pipe which carries the Piedmont water supply. This pipe rests upon the river bottom at the bridge and in low water carries an appreciable percentage of the total flow of the river. Ice conditions did not affect the flow at this station during the winter of $1905-6$.

Discharge measurements of Savage River at Bloomington, Md.

\begin{tabular}{|c|c|c|c|c|c|}
\hline Date. & $\begin{array}{c}\text { Gage } \\
\text { height. }\end{array}$ & Discharge. & Date. & $\begin{array}{c}\text { Gage } \\
\text { height. }\end{array}$ & Discharge. \\
\hline 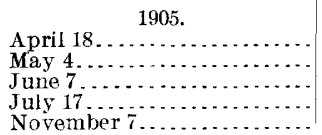 & $\begin{array}{l}\text { Feet. } \\
2.96 \\
2.68 \\
2.88 \\
2.47 \\
2.59\end{array}$ & \begin{tabular}{r|} 
Second-feet. \\
89 \\
72 \\
81 \\
37 \\
66
\end{tabular} & 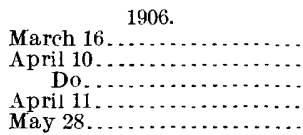 & $\begin{array}{l}\text { Feet. } \\
2.95 \\
5.60 \\
5.40 \\
4.79 \\
3.25\end{array}$ & $\begin{array}{r}\text { Second-feet. } \\
87 \\
1,139 \\
1.027 \\
685 \\
141\end{array}$ \\
\hline
\end{tabular}

Daily gage height, in feet, of Savage River at Bloomington, Md.

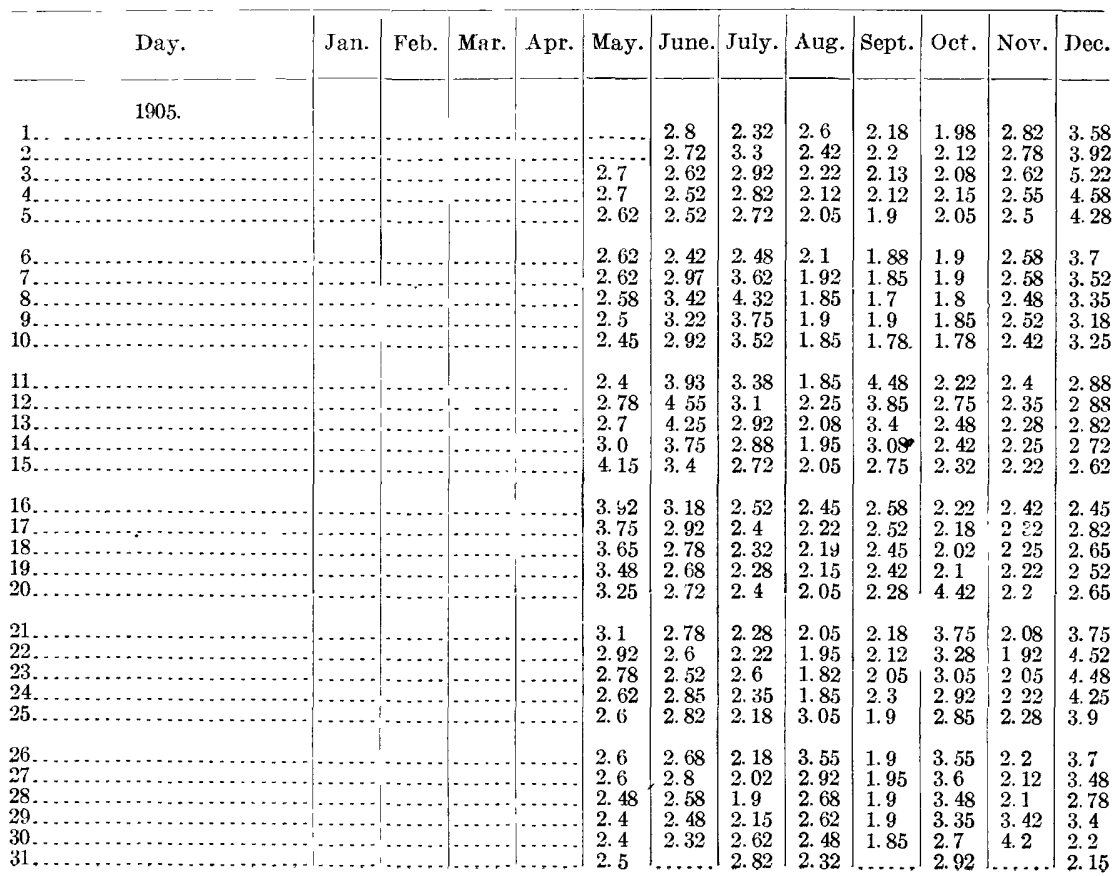


Daily gage height, in feet, of Savage River at Bloomington, Md.-Continued.

\begin{tabular}{|c|c|c|c|c|c|c|c|c|c|c|c|c|}
\hline Day. & Jan. & Feb. & Mar. & Apr. & May. & June & July. & Aug. & Sept & Oct. & Nov. & Dec. \\
\hline $\begin{array}{l} \\
1 \ldots \ldots \ldots \\
2 \ldots \ldots \ldots \ldots \\
3 \ldots \ldots \ldots \\
4 \ldots \ldots \ldots \\
5 \ldots \ldots \ldots \ldots\end{array}$ & $\begin{array}{l}2.8 \\
2.75 \\
3.05 \\
4.28 \\
4.52\end{array}$ & $\begin{array}{l}2.75 \\
2.55 \\
2.65 \\
3.03 \\
2.75\end{array}$ & $\begin{array}{l}2.53 \\
2.43 \\
2.70 \\
4.45 \\
3.83\end{array}$ & $\begin{array}{l}5.32 \\
482 \\
4.98 \\
5.22 \\
5.88\end{array}$ & $\begin{array}{l}3.22 \\
3.18 \\
3.32 \\
3.22 \\
3.0\end{array}$ & 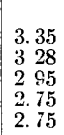 & $\begin{array}{l}\text { 2. } 48 \\
238 \\
2.28 \\
2.2 \\
2.12\end{array}$ & & & & & \\
\hline $\begin{array}{r}6 \ldots \\
7 \ldots \ldots \\
8 \ldots \ldots \\
9 \ldots \ldots \ldots \\
10 \ldots \ldots\end{array}$ & $\begin{array}{l}4.15 \\
3.85 \\
3.6 \\
3.3 \\
3.12\end{array}$ & $\begin{array}{l}2.6 \\
2.67 \\
2.6 \\
2.53 \\
2.6\end{array}$ & $\begin{array}{l}3.5 \\
3.37 \\
3.25 \\
3.17 \\
3.15\end{array}$ & $\begin{array}{l}\text { 5. } 92 \\
4.88 \\
4.48 \\
4.42 \\
5.95\end{array}$ & $\begin{array}{l}3.0 \\
2.92 \\
2.82 \\
2.82 \\
2.8\end{array}$ & $\begin{array}{l}3.35 \\
4.62 \\
5.15 \\
105 \\
3.6\end{array}$ & $\begin{array}{l}1.98 \\
1.88 \\
1.88 \\
1.98 \\
1.88\end{array}$ & & & & & \\
\hline 3 & $\begin{array}{l}3.18 \\
3.2 \\
2.75 \\
3.18 \\
3.02\end{array}$ & $\begin{array}{l}2.37 \\
2.47 \\
2.5 \\
2.47 \\
2.37\end{array}$ & $\begin{array}{l}2.97 \\
3.05 \\
2.87 \\
2.93 \\
3.2\end{array}$ & $\begin{array}{l}4.9 \\
4.35 \\
3.95 \\
3.8 \\
4.78\end{array}$ & $\begin{array}{r}2.72 \\
2.68 \\
2.58 \\
\cdot 2.52 \\
2.52\end{array}$ & $\begin{array}{l}3.32 \\
3.05 \\
2.88 \\
3.05 \\
2.85\end{array}$ & $\begin{array}{l}1.92 \\
1.88 \\
1.88 \\
1.92 \\
1.78\end{array}$ & & & & & $\cdots$ \\
\hline $\begin{array}{l}18 . \\
19 .\end{array}$ & $\begin{array}{ll}3 & 25 \\
3 & 52 \\
3 & 6 \\
4 & 02 \\
4 & 0\end{array}$ & $\begin{array}{l}2.4 \\
2.47 \\
2.27 \\
2.33 \\
2.33\end{array}$ & $\begin{array}{l}2.93 \\
2.88 \\
2.74 \\
2.78 \\
2.95\end{array}$ & $\begin{array}{l}4.6 \\
4.3 \\
4.05 \\
3.75 \\
3.55\end{array}$ & $\begin{array}{ll}2 . & 48 \\
2 & 38 \\
2 & 32 \\
2 & 42 \\
2 . & 32\end{array}$ & \begin{tabular}{|l}
2.65 \\
2.55 \\
2.38 \\
2.3 \\
2.52
\end{tabular} & & & & & & $\cdots$ \\
\hline $\begin{array}{l}21 \ldots \ldots \ldots \\
22, \ldots \ldots \ldots \\
23, \ldots \ldots \\
24 \ldots \ldots \\
25, \ldots \ldots \\
\end{array}$ & $\begin{array}{l}\text { 4. } 15 \\
4.32 \\
7.08 \\
55 \\
4.55\end{array}$ & $\begin{array}{l}2.47 \\
2.63 \\
2.63 \\
2.35 \\
2.47\end{array}$ & $\begin{array}{ll}2 & 9 \\
2 & 98 \\
2.75 \\
3.18 \\
3.05\end{array}$ & $\begin{array}{l}3.52 \\
3.4 \\
3.48 \\
3.4 \\
3.52\end{array}$ & $\begin{array}{ll}2 . & 3 \\
2 & 3 \\
2 & 25 \\
2 . & 2 \\
2 . & 2\end{array}$ & $\begin{array}{l}298 \\
2.82 \\
2.78 \\
2.78 \\
2.62\end{array}$ & & & & & & $\begin{array}{l}\cdots \\
\cdots \\
\cdots \\
\cdots\end{array}$ \\
\hline $\begin{array}{l}26 \ldots \ldots \ldots \\
27 \ldots \ldots \\
28 \ldots \ldots \ldots \\
29 \ldots \ldots \\
31 \ldots \ldots \ldots \\
31 \ldots \ldots\end{array}$ & $\begin{array}{l}4.1 \\
3.6 \\
3.52 \\
3.48 \\
3.2 \\
318\end{array}$ & $\begin{array}{l}237 \\
2.23 \\
2.25 \\
\cdots \\
\cdots \\
\ldots\end{array}$ & $\begin{array}{l}3.05 \\
485 \\
65 \\
6.0 \\
7.15 \\
605\end{array}$ & $\begin{array}{l}3.62 \\
3.65 \\
3.58 \\
3.48 \\
3.35\end{array}$ & $\begin{array}{ll}2 & 2 \\
2 & 42 \\
3 . & 4 \\
3 . & 35 \\
2 & 95 \\
2 & 82\end{array}$ & $\begin{array}{ll}2 & 65 \\
2 & 78 \\
2.75 \\
2 & 55 \\
2 . & 42\end{array}$ & … & & & & & $\cdots$ \\
\hline
\end{tabular}

Rating table for Savage River at Bloomington, Md., from May 3, 1905, to July 15, 1906.a

\begin{tabular}{|r|r|r|r|r|r|r|r|}
\hline $\begin{array}{c}\text { Gage } \\
\text { height. }\end{array}$ & Discharge. & $\begin{array}{c}\text { Gage } \\
\text { height. }\end{array}$ & Discharge. & $\begin{array}{c}\text { Gage } \\
\text { height. }\end{array}$ & Discharge. & $\begin{array}{c}\text { Gage } \\
\text { height. }\end{array}$ & Discharge. \\
\cline { 1 - 5 } Feet. & Second-feet. & Feet. & Second-fect. & Feet. & Second-feet. & Fect. & Sfcond-fect. \\
1.70 & 8 & 2.90 & 84 & 4.00 & 344 & 5.20 & 912 \\
1.80 & 10 & 3.00 & 98 & 4.10 & 380 & 5.40 & 1,030 \\
1.90 & 12 & 3.10 & 114 & 4.20 & 418 & 5.60 & 1,150 \\
2.00 & 15 & 3.20 & 132 & 4.30 & 453 & 5.80 & 1,280 \\
2.10 & 19 & 3.30 & 152 & 4.40 & 500 & 6.00 & 1,415 \\
2.20 & 23 & 3.40 & 174 & 4.50 & 544 & 6.20 & 1,555 \\
2.30 & 28 & 3.50 & 197 & 4.60 & 591 & 6.40 & 1,695 \\
2.40 & 34 & 3.60 & 222 & 4.70 & 640 & 6.60 & 1,840 \\
2.50 & 41 & 3.70 & 249 & 4.80 & 691 & 6.80 & 1,990 \\
2.60 & 50 & 3.80 & 278 & 4.90 & 744 & 7.00 & 2,140 \\
2.70 & 60 & 3.90 & 310 & 5.00 & 799 & 7.20 & 2,300 \\
2.80 & 71 & & & & & & \\
\hline
\end{tabular}

$a$ This table is strictly applicable only for open-channel conditions. It is based upon eight discharge measurements made during 1905 and 1906. It is well defined between gage heights 2.4 feet and 5.6 feet. 
Estimated monthly discharge of Savage River at Bloomington, $M d$.

[Drainage area, 120 square miles.]

\begin{tabular}{|c|c|c|c|c|c|}
\hline \multirow[b]{2}{*}{ Month. } & \multicolumn{3}{|c|}{ Discharge in second-feet. } & \multicolumn{2}{|c|}{ Run-off. } \\
\hline & Maximum. & Minimum. & Mean. & $\begin{array}{c}\text { Second-feet } \\
\text { per square } \\
\text { mile. }\end{array}$ & $\begin{array}{l}\text { Depth in } \\
\text { inches. }\end{array}$ \\
\hline May $3-31 \ldots \ldots \ldots \ldots \ldots$ & & & & & \\
\hline $\begin{array}{l}\text { May } \\
\text { June }\end{array}$ & $\begin{array}{l}399 \\
568\end{array}$ & $\begin{array}{l}34 \\
29\end{array}$ & 118 & $\begin{array}{r}0.815 \\
.983\end{array}$ & $\begin{array}{l}0.879 \\
1.10\end{array}$ \\
\hline July........... & 466 & 12 & 85.0 & .708 & .816 \\
\hline August......... & 210 & 10 & 34.0 & .283 & .326 \\
\hline September...... & 535 & 8 & 56.0 & .467 & .521 \\
\hline October... & 509 & 10 & 82.1 & .684 & .789 \\
\hline November. & 418 & 13 & 51.1 & .426 & .475 \\
\hline December... & 924 & 21 & 220 & 1.83 & 2.11 \\
\hline January ........ & 2,204 & 66 & 330 & 2.75 & \\
\hline February. & 103 & 24 & 43. 8 & .365 & .380 \\
\hline March..... & 2,260 & 36 & 348 & 2.90 & $\begin{array}{l}3.34 \\
4.84\end{array}$ \\
\hline $\begin{array}{l}\text { April. } \\
\text { May.. }\end{array}$ & $\begin{array}{r}1,380 \\
174\end{array}$ & $\begin{array}{r}163 \\
23\end{array}$ & $\begin{array}{l}521 \\
69.9\end{array}$ & $\begin{array}{l}4.34 \\
582\end{array}$ & $\begin{array}{l}4.84 \\
.671\end{array}$ \\
\hline June. & 884 & 28 & 138 & 1.15 & 1.28 \\
\hline July $1-1$ & 40 & 10 & 17.8 & .148 & .082 \\
\hline
\end{tabular}

NORTH BRANCH OF POTOMAC RIVER AT PIEDMONT, W, VA.

This gaging station was established June 27, 1899, by E. G. Paul, and was discontinued July 16, 1906. It is located at the iron highway bridge connecting Luke, Md., with Piedmont, W. Va.

The channel is straight for 1,200 feet above and 600 feet below the station. The current has a moderate velocity. The right bank is high and rocky and does not overflow. The left bank is low and liable to overflow, but all water passes beneath the bridge. The bed of the stream is composed of gravel and cobblestones, overlain in the left channel and in part of the right channel by refuse from pulp mills above the bridge. It is free from vegetation. Discharge measurements were made from the downstream side of the bridge to which the gage is attached. The initial point for soundings is the face of the pier on the right bank.

The standard chain gage is attached to the hand rail on the lower side of the bridge in the second span from the right end. . The length of the chain from the end of the weight to the marker is 38.87 feet. The gage was read twice each day by Charles H. Beck. The bench mark is the top of a small shoulder in the face of the sandstone ledge which forms the right abutment of the bridge. It is about 4 feet above the ground and 10 feet downstream from the bridge. The point is indicated by an arrow cut in the vertical face of the ledge. Its elevation is 20.40 feet above gage datum.

The plotting of the discharge measurements made during 1899 to 1906 indicates that the channel has been gradually filling in at the control below the gaging section, and also to some extent at the gaging section itself, thus causing a gradually diminishing discharge for a given gage height. This apparently is due to refuse discharged into 
the river from pulp mills immediately above the bridge. The amount of refuse affecting the flow does not increase at a constant rate, and at times of high water part of it may be washed away. Owing to these changing conditions of flow all estimates at this station are somewhat uncertain, there being a varying error of from 5 to 20 per cent. The larger percentages of error occur at low-gage heights, especially during periods when no measurements were made. The three rating curves prior to 1906 have been united at about gage height 4.5 feet. This is not strictly correct, but the percentage of error involved is relatively small. Ice conditions at this station probably do not greatly affect the discharge. No corrections were made in estimates for ice periods. Estimates for the period from June 27, 1899, to December 31, 1903, previously published have been revised. Estimates for 1904 and 1905, as published in the 1905 report, have not been changed.

A summary of the records furnishes the following results: Maximum discharge for twenty-four hours, 13,450 second-feet; minimum discharge for twenty-four hours, 6 second-feet; mean annual discharge for six years, 687 second-feet; mean annual rainfall for seven years, 38.66 inches.

Discharge measurements of North Branch of Potomac River at Piedmont, W. Va.

\begin{tabular}{|c|c|c|c|c|c|}
\hline Date. & $\begin{array}{c}\text { Gage } \\
\text { height. }\end{array}$ & Discharge. & Date. & $\begin{array}{c}\text { Gage } \\
\text { height. }\end{array}$ & Discharge. \\
\hline January $27 . \ldots$. & $\begin{array}{l}\text { Feet. } \\
\quad 3.00\end{array}$ & \begin{tabular}{|} 
Second-feet. \\
350
\end{tabular} & September $9 \ldots$ & $\begin{array}{l}\text { Feet. } \\
1.99 \\
1.99\end{array}$ & $\begin{array}{r}\text { Second-feet } \\
14.7 \\
20\end{array}$ \\
\hline $\begin{array}{c}1900 . \\
\text { February } 22 \ldots \ldots\end{array}$ & 3. 75 & 735 & 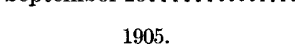 & & \\
\hline $\begin{array}{l}\text { June } 20 \\
\text { September } 12\end{array}$ & 4.40 & 1,249 & March $9 \ldots \ldots \ldots$ & 6.70 & 4,516 \\
\hline 1901. & & 97 & March 29. & $\begin{array}{l}. .46 \\
4.25 \\
3.46\end{array}$ & $\begin{array}{r}0,096 \\
1,096 \\
416\end{array}$ \\
\hline $\begin{array}{l}\text { July } 24, \ldots . . . \\
\text { November } 7 . . .\end{array}$ & $\begin{array}{l}2.90 \\
2.10\end{array}$ & $\begin{array}{r}275 \\
39\end{array}$ & April $24 .$. & $\begin{array}{l}\text { 3. } 63 \\
\text { 3. } 22\end{array}$ & $\begin{array}{l}526 \\
326\end{array}$ \\
\hline $\begin{array}{r}1902 . \\
\text { August } 19 \ldots \ldots \ldots\end{array}$ & 2. 14 & 50 & $\begin{array}{l}\text { June } 7, \ldots \ldots . . \\
\text { July } 17, \ldots . . \\
\text { November } 7 . . .\end{array}$ & $\begin{array}{l}3.44 \\
3.15 \\
3.38\end{array}$ & $\begin{array}{l}441 \\
280 \\
439\end{array}$ \\
\hline $\begin{array}{r}1903 . \\
\text { August } 31 . \ldots \ldots\end{array}$ & 2.80 & 208 & March $16 \ldots \ldots$ & 3.51 & 412 \\
\hline $\begin{array}{r}1904 . \\
\text { July } 8 a \ldots \ldots \ldots\end{array}$ & 2.80 & 116 & $\begin{array}{l}\text { April } 11 . . . \\
\text { May } 28 . . .\end{array}$ & $\begin{array}{l}5.64 \\
3.49\end{array}$ & $\begin{array}{r}2,589 \\
391\end{array}$ \\
\hline
\end{tabular}

a Measurement unreliable, owing to defective meter. 
Daily gage height, in feet, of North Branch of Potomac River at Piedmont, W. Va.

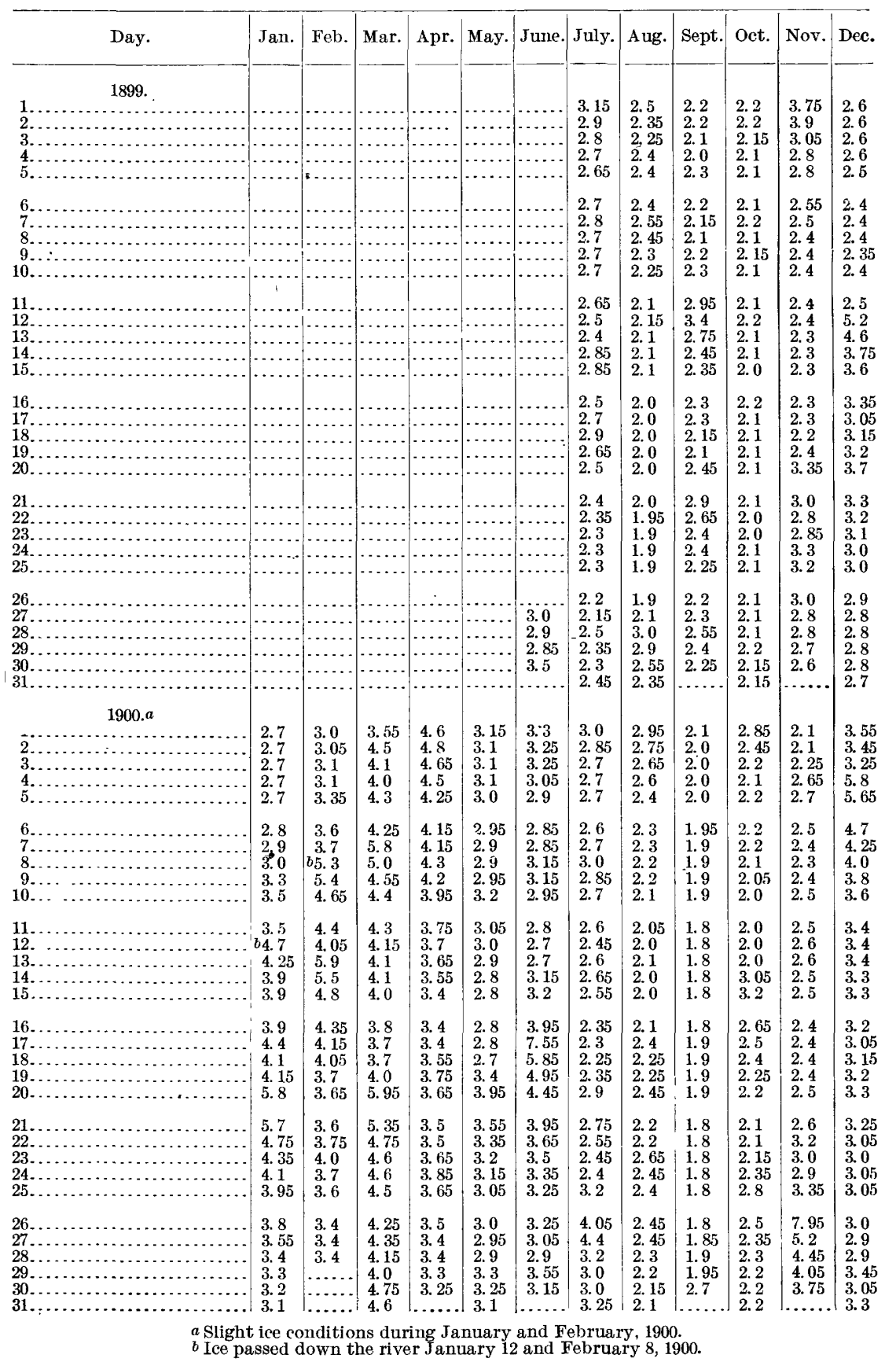


Daily gage height, in feet, of North Branch of Potomac River at Piedmont, W. Va.Continued.

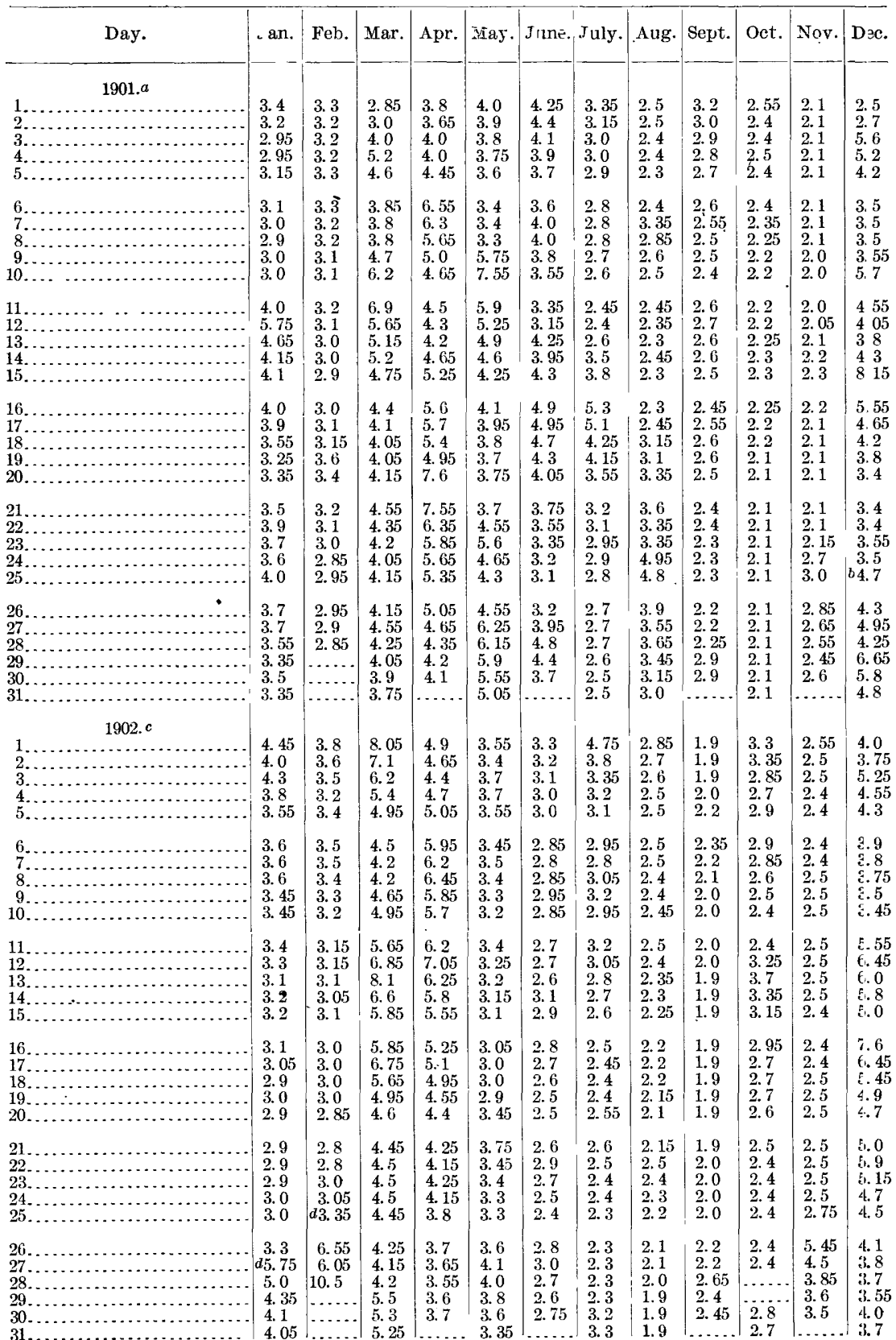

a Slight ice conditions during December, 1901.

$b$ Ice passed down the river December $25,1901$.

$c$ Ice conditions January and February, 1902.

$d$ Ice passed down the river January 27 and February $25,1902$. 
Daily gage height, in feet, of North Branch of Potomac River at Predmont, W. Va.Continued.

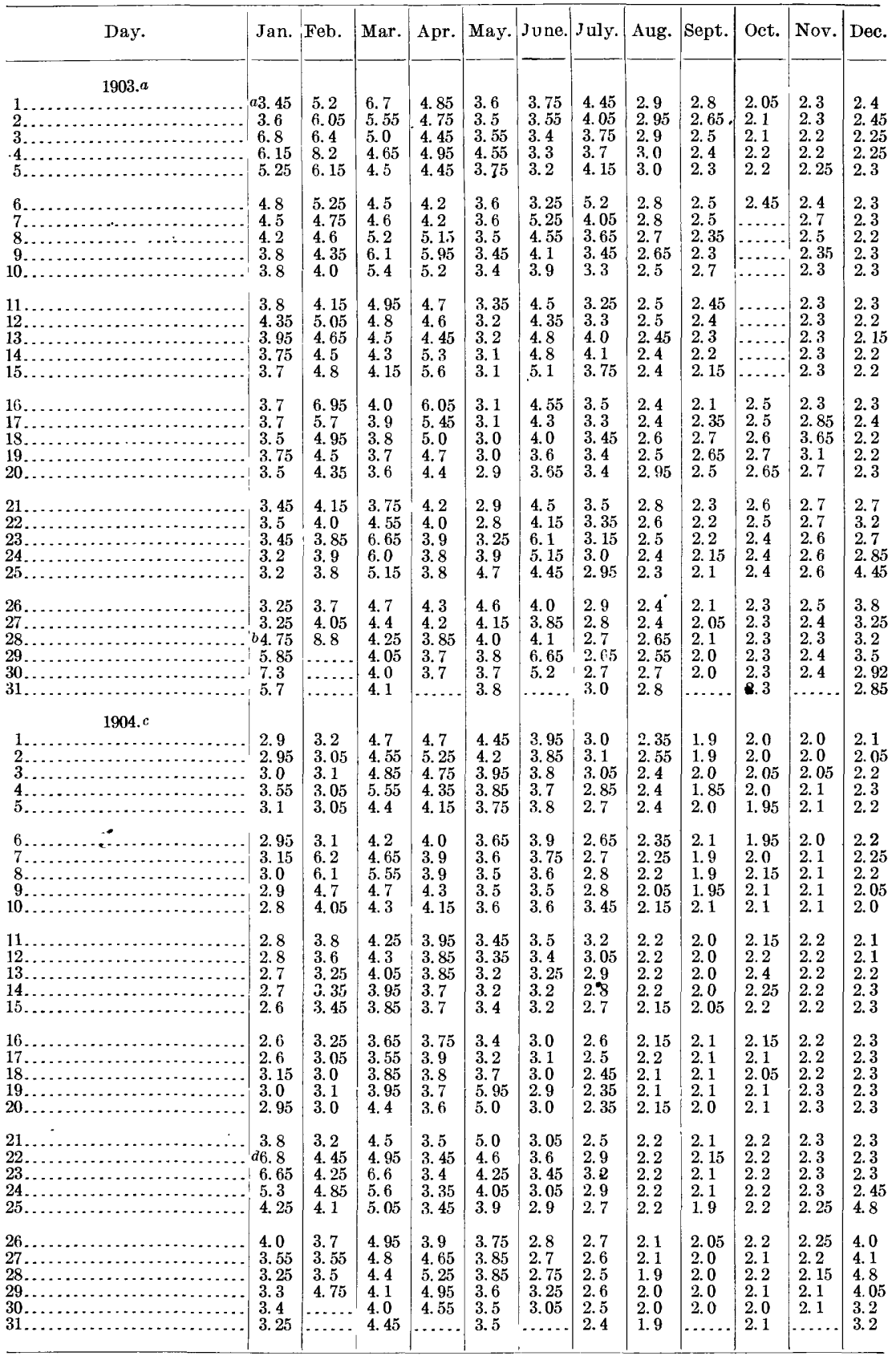

$a$ Ice conditions J anuary, 1903.

$b$ Ice passed down the river January 28, 1903.

$c$ Ice conditions January, 1904.

$d$ Ice passed down the river January 22.1904. 
Daily gage height, in feet, of North Branch of Potomac River at Predmont, W. Va.Continued.

\begin{tabular}{|c|c|c|c|c|c|c|c|c|c|c|c|c|}
\hline Day. & Jan. & Feb & Mar. & Apr. & May. & June. & July. & Aug. & Sept. & Oct. & Nov. & Dec. \\
\hline 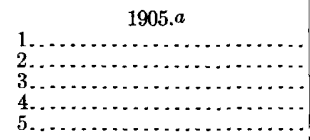 & $\begin{array}{l}3.05 \\
3.05 \\
3.05 \\
2.95 \\
2.95\end{array}$ & $\begin{array}{l}2.75 \\
2.7 \\
2.65 \\
2.55 \\
2.55\end{array}$ & $\begin{array}{r}a 3.35 \\
3.25 \\
3.15 \\
3.15 \\
3.35\end{array}$ & $\begin{array}{l}3.85 \\
3.7 \\
3.6 \\
3.55 \\
3.55\end{array}$ & $\begin{array}{r}\text { 4. } 5 \\
3.3 \\
3.3 \\
3.2 \\
3.2\end{array}$ & $\begin{array}{l}4.75 \\
4.0 \\
3.65 \\
3.4 \\
3.3\end{array}$ & $\begin{array}{l}3.3 \\
3.85 \\
3.55 \\
3.4 \\
4.05\end{array}$ & $\begin{array}{l}3.85 \\
3.45 \\
3.2 \\
3.1 \\
3.5\end{array}$ & $\begin{array}{l}2.8 \\
2.8 \\
2.8 \\
2.8 \\
2.7\end{array}$ & $\begin{array}{l}2.3 \\
2.3 \\
2.4 \\
2.45 \\
2.4\end{array}$ & $\begin{array}{l}3.2 \\
3.1 \\
3.05 \\
3.0 \\
3.0\end{array}$ & $\begin{array}{l}\text { 3. } 9 \\
\text { 3. } 75 \\
\text { 5. } 75 \\
\text { 4. } 9 \\
4.4\end{array}$ \\
\hline 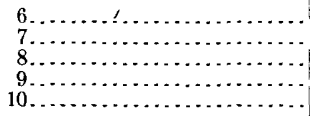 & $\begin{array}{l}2.95 \\
2.9 \\
2.8 \\
2.75 \\
2.65\end{array}$ & $\begin{array}{l}2.55 \\
2.55 \\
2.55 \\
2.55 \\
3.15\end{array}$ & $\begin{array}{l}4.15 \\
b .5 \\
5.0 \\
7.05 \\
7.45\end{array}$ & $\begin{array}{l}3.8 \\
3.95 \\
3.95 \\
3.95 \\
4.0\end{array}$ & $\begin{array}{l}3.2 \\
3.3 \\
3.2 \\
3.1 \\
3.1\end{array}$ & $\begin{array}{l}3.2 \\
3.55 \\
4.15 \\
3.65 \\
3.45\end{array}$ & $\begin{array}{l}4.2 \\
4.3 \\
5.45 \\
4.55 \\
4.05\end{array}$ & $\begin{array}{l}3.15 \\
3.0 \\
2.85 \\
2.8 \\
2.8\end{array}$ & $\begin{array}{l}2.6 \\
2.5 \\
2.5 \\
2.5 \\
2.45\end{array}$ & $\begin{array}{l}2.35 \\
2.3 \\
2.2 \\
2.2 \\
2.2\end{array}$ & $\begin{array}{l}3.15 \\
3.36 \\
3.2 \\
3.2 \\
\text { 3. } 05\end{array}$ & $\begin{array}{l}3.95 \\
3.85 \\
3.7 \\
3.65 \\
3.6\end{array}$ \\
\hline 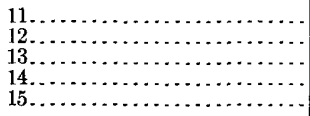 & $\begin{array}{l}2.75 \\
2.8 \\
5.95 \\
4.45 \\
3.65\end{array}$ & $\begin{array}{l}3.1 \\
3.05 \\
3.05 \\
\text {.. } 4 \\
\text { 3. } 3\end{array}$ & $\begin{array}{l}\text { 6. } 3 \\
5.7 \\
\text { 5. } 65 \\
5.3 \\
5.0\end{array}$ & $\begin{array}{l}3.95 \\
3.95 \\
3.85 \\
3.75 \\
3.65\end{array}$ & $\begin{array}{l}3.15 \\
3.5 \\
3.9 \\
\text { 3. } 65 \\
5.02\end{array}$ & $\begin{array}{l}3.85 \\
4.2 \\
4.15 \\
\text { 3. } 75 \\
\text { 3. } 45\end{array}$ & $\begin{array}{l}4.1 \\
4.0 \\
3.95 \\
\text { 3. } 5 \\
\text { 3. } 35\end{array}$ & $\begin{array}{l}3.2 \\
3.25 \\
3.0 \\
2.8 \\
2.85\end{array}$ & $\begin{array}{l}4.2 \\
4.15 \\
3.6 \\
3.25 \\
3.05\end{array}$ & $\begin{array}{l}2.65 \\
3.6 \\
3.05 \\
2.75 \\
2.6\end{array}$ & $\begin{array}{l}3.0 \\
2.9 \\
2.9 \\
2.9 \\
2.9\end{array}$ & $\begin{array}{l}\text { 3. } 3 \\
3.25 \\
\text { 3. } 35 \\
\text { 3. } 25 \\
\text { 3. } 0\end{array}$ \\
\hline $\begin{array}{l}16 \ldots \ldots \\
17{ }_{1} \ldots \ldots \\
18 \ldots \ldots \ldots \\
190 \ldots \ldots\end{array}$ & $\begin{array}{l}3.3 \\
3.25 \\
3.25 \\
3.25 \\
3.25\end{array}$ & $\begin{array}{l}3.05 \\
2.95 \\
2.95 \\
2.85 \\
2.85\end{array}$ & $\begin{array}{l}4.85 \\
6.1 \\
6.45 \\
6.75 \\
6.7\end{array}$ & $\begin{array}{l}3.55 \\
3.5 \\
3.5 \\
3.45 \\
3.75\end{array}$ & $\begin{array}{l}4.4 \\
4.55 \\
4.05 \\
\text { 3. } 95 \\
3.75\end{array}$ & $\begin{array}{l}3.25 \\
3.05 \\
3.65 \\
3.45 \\
3.65\end{array}$ & $\begin{array}{l}3.3 \\
3.15 \\
3.05 \\
2.95 \\
3.35\end{array}$ & $\begin{array}{l}4.15 \\
3.5 \\
3.15 \\
3.0 \\
2.95\end{array}$ & $\begin{array}{l}2.9 \\
2.8 \\
2.8 \\
2.8 \\
2.7\end{array}$ & $\begin{array}{l}2.55 \\
2.5 \\
2.45 \\
3.8 \\
4.45\end{array}$ & $\begin{array}{l}2.85 \\
3.05 \\
3.0 \\
2.9 \\
2.9\end{array}$ & $\begin{array}{l}\text { 2. } 95 \\
3.3 \\
\text { 3. } 2 \\
\text { 3. } 1 \\
\text { 3. } 1\end{array}$ \\
\hline $\begin{array}{l}21 \ldots \ldots \ldots \\
22 \ldots \ldots \ldots \ldots \\
23 \ldots \ldots \ldots \\
24 \ldots \ldots \ldots \\
25 \ldots \ldots \ldots\end{array}$ & $\begin{array}{l}3.25 \\
3.3 \\
3.15 \\
3.05 \\
2.85\end{array}$ & $\begin{array}{l}2.85 \\
2.95 \\
3.4 \\
3.3 \\
3.25\end{array}$ & $\begin{array}{l}8.1 \\
6.75 \\
5.8 \\
5.25 \\
5.65\end{array}$ & $\begin{array}{l}3.85 \\
3.9 \\
3.85 \\
3.7 \\
3.6\end{array}$ & $\begin{array}{l}3.55 \\
3.5 \\
3.4 \\
3.2 \\
3.1\end{array}$ & $\begin{array}{l}3.65 \\
3.65 \\
3.4 \\
3.45 \\
3.65\end{array}$ & $\begin{array}{l}3.3 \\
3.1 \\
3.65 \\
3.6 \\
3.25\end{array}$ & $\begin{array}{l}2.9 \\
2.75 \\
2.65 \\
2.5 \\
4.35\end{array}$ & $\begin{array}{l}2.6 \\
2.55 \\
2.5 \\
2.4 \\
2.45\end{array}$ & $\begin{array}{l}\text { 4. } 0 \\
\text { 3. } 4 \\
\text { 3. } 15 \\
3.0 \\
2.95\end{array}$ & $\begin{array}{l}2.9 \\
2.75 \\
2.75 \\
2.85 \\
2.8\end{array}$ & $\begin{array}{l}4.0 \\
5.4 \\
4.9 \\
4.9 \\
4.05\end{array}$ \\
\hline $\begin{array}{l}26 \\
27 \\
28 \ldots \\
29 \\
30 \ldots \\
31 \ldots \ldots \\
31 \ldots \ldots\end{array}$ & $\begin{array}{l}2.55 \\
2.55 \\
2.75 \\
2.75 \\
2.85 \\
2.75\end{array}$ & $\begin{array}{l}3.25 \\
3.55 \\
3.45 \\
\cdots \\
\cdots \\
\cdots\end{array}$ & $\begin{array}{l}\text { 5. } 05 \\
4.9 \\
4.6 \\
4.3 \\
4.15 \\
4.0\end{array}$ & $\begin{array}{l}3.5 \\
2.8 \\
2.9 \\
3.65 \\
3.5 \\
-.\end{array}$ & $\begin{array}{l}3.1 \\
3.1 \\
3.0 \\
2.9 \\
2.9 \\
3.15\end{array}$ & $\begin{array}{l}3.45 \\
4.4 \\
3.65 \\
3.35 \\
3.2 \\
-1.2\end{array}$ & $\begin{array}{l}3.05 \\
2.9 \\
2.8 \\
2.9 \\
5.05 \\
4.2\end{array}$ & $\begin{array}{l}4.9 \\
\text { 3. } 7 \\
\text { 3. } 35 \\
\text { 3. } 15 \\
\text { 3. } 0 \\
3.0\end{array}$ & $\begin{array}{l}2.4 \\
2.4 \\
2.3 \\
2.3 \\
2.3 \\
\cdots\end{array}$ & $\begin{array}{l}4.85 \\
4.4 \\
3.9 \\
3.6 \\
3.45 \\
3.3\end{array}$ & $\begin{array}{l}2.8 \\
2.8 \\
2.8 \\
3.65 \\
4.55 \\
\cdots\end{array}$ & $\begin{array}{l}\text { 4. } 0 \\
3.85 \\
\text { 3. } 65 \\
\text { 3. } 8 \\
\text { 4. } 15 \\
\text { 3. } 65\end{array}$ \\
\hline 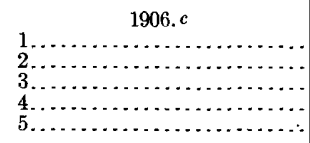 & $\begin{array}{l}3.55 \\
3.35 \\
3.65 \\
5.65 \\
5.3\end{array}$ & $\begin{array}{l}3.65 \\
3.55 \\
3.2 \\
3.4 \\
3.5\end{array}$ & $\begin{array}{l}\text { 2. } 75 \\
\text { 3. } 1 \\
\text { 3. } 3 \\
\text { 5. } 15 \\
\text { 4. } 35\end{array}$ & $\begin{array}{l}6.05 \\
5.55 \\
5.7 \\
6.0 \\
6.15\end{array}$ & $\begin{array}{l}4.0 \\
3.9 \\
4.0 \\
3.8 \\
3.7\end{array}$ & $\begin{array}{l}\text { 3. } 25 \\
\text { 3. } 45 \\
3.3 \\
\text { 3. } 1 \\
\text { 3. } 3\end{array}$ & $\begin{array}{l}3.0 \\
2.9 \\
2.8 \\
2.8 \\
3.1\end{array}$ & 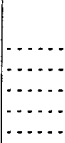 & & & & \\
\hline 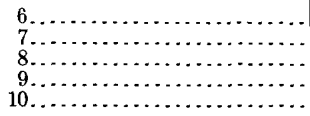 & $\begin{array}{l}\text { 4. } 6 \\
3.9 \\
4.05 \\
3.65 \\
3.55\end{array}$ & $\begin{array}{l}\text { 3. } 0 \\
\text { 3. } 1 \\
\text { 3. } 1 \\
\text { 3. } 1 \\
2.9\end{array}$ & $\begin{array}{l}3.95 \\
3.8 \\
3.8 \\
3.7 \\
3.6\end{array}$ & $\begin{array}{l}6.8 \\
5.7 \\
5.05 \\
5.15 \\
6.45\end{array}$ & $\begin{array}{l}3.6 \\
3.7 \\
3.6 \\
3.5 \\
3.5\end{array}$ & $\begin{array}{l}\text { 4. } 6 \\
\text { 5. } 25 \\
\text { 4. } 7 \\
\text { 4. } 1 \\
\text { 3. } 65\end{array}$ & $\begin{array}{l}2.85 \\
2.75 \\
2.6 \\
2.6 \\
2.6\end{array}$ & & & & & \\
\hline $\begin{array}{l}11 \\
12 \ldots \ldots \\
13 \\
14 \\
15 \\
15 \ldots \ldots\end{array}$ & $\begin{array}{l}3.55 \\
3.7 \\
3.95 \\
3.8 \\
3.8\end{array}$ & $\begin{array}{l}2.75 \\
2.85 \\
3.0 \\
3.05 \\
3.0\end{array}$ & $\begin{array}{l}3.4 \\
3.6 \\
3.5 \\
3.5 \\
3.5\end{array}$ & $\begin{array}{l}5.5 \\
5.45 \\
4.55 \\
4.45 \\
6.15\end{array}$ & $\begin{array}{l}3.4 \\
3.4 \\
3.3 \\
3.2 \\
3.2\end{array}$ & $\begin{array}{l}\text { 3. } 35 \\
3.1 \\
3.25 \\
3.1 \\
3.0\end{array}$ & $\begin{array}{l}2.5 \\
2.65 \\
2.6 \\
2.6 \\
2.55\end{array}$ & & & & & \\
\hline $\begin{array}{l}16 \ldots \ldots \\
17 \ldots \ldots \\
18 \ldots \ldots \\
19 \ldots \ldots \\
20 \ldots \ldots \ldots\end{array}$ & $\begin{array}{l}3.95 \\
4.15 \\
4.1 \\
4.7 \\
4.35\end{array}$ & $\begin{array}{l}2.75 \\
2.8 \\
2.9 \\
2.8 \\
2.95\end{array}$ & $\begin{array}{l}3.5 \\
3.4 \\
\text { 3. } 15 \\
3.4 \\
4.05\end{array}$ & $\begin{array}{l}5.45 \\
4.85 \\
4.45 \\
4.25 \\
4.1\end{array}$ & $\begin{array}{l}\text { 3. } 1 \\
\text { 3. } 1 \\
\text { 3. } 0 \\
\text { 3. } 0 \\
2.9\end{array}$ & $\begin{array}{l}3.0 \\
2.9 \\
2.9 \\
2.9 \\
2.95\end{array}$ & & & & $\ldots \ldots$ & & \\
\hline $\begin{array}{l}21 \ldots \ldots \\
22 \ldots \ldots \\
23 \ldots \ldots \\
24 \ldots \ldots \\
25 \ldots \ldots \ldots\end{array}$ & $\begin{array}{l}4.5 \\
4.85 \\
8.2 \\
6.4 \\
5.1\end{array}$ & $\begin{array}{l}\text { 3. } 0 \\
\text { 3. } 2 \\
\text { 3. } 1 \\
\text { 3. } 05 \\
\text { 3. } 1\end{array}$ & $\begin{array}{l}3.8 \\
3.7 \\
\text { 3. } 7 \\
\text { 3. } 75 \\
\text { 3. } 55\end{array}$ & $\begin{array}{l}3.95 \\
4.0 \\
4.0 \\
4.0 \\
4.1\end{array}$ & $\begin{array}{l}2.9 \\
2.8 \\
2.8 \\
2.7 \\
2.7\end{array}$ & $\begin{array}{l}3.1 \\
3.3 \\
\text { 3. } 2 \\
\text { 3. } 1 \\
3.0\end{array}$ & ...... & & & & & \\
\hline $\begin{array}{l}26 \ldots \ldots \\
27 \ldots \ldots \\
28 \ldots \ldots \\
29 \ldots \ldots \\
30 \ldots \ldots \\
31 \ldots \ldots\end{array}$ & $\begin{array}{l}\text { 4. } 6 \\
4.35 \\
4.3 \\
4.15 \\
3.95 \\
3.8\end{array}$ & 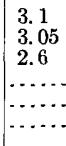 & $\begin{array}{l}3.6 \\
5.05 \\
7.3 \\
6.25 \\
7.4 \\
7.4\end{array}$ & $\begin{array}{c}6.5 \\
5.4 \\
4.65 \\
4.35 \\
4.15 \\
\ldots . . .\end{array}$ & $\begin{array}{l}2.7 \\
2.7 \\
3.35 \\
3.45 \\
3.2 \\
3.0\end{array}$ & $\begin{array}{l}3.0 \\
3.6 \\
3.35 \\
3.25 \\
3.0\end{array}$ & & & & & & \\
\hline
\end{tabular}

$a$ Ice conditions during January, February, and March, 1905.

$b$ Ice passed down the river March 7,1905 .

$c$ No ice during the winter of $1905-6$. 
Rating tabies for North Branch of Potomac River at Piedmont, W. Va.

JUNE 27, 1899, TO AUGUST 25, 1901. $a$

\begin{tabular}{|c|c||c|c|c|c||c|c|}
\hline $\begin{array}{c}\text { Gage } \\
\text { height. }\end{array}$ & Discharge. & $\begin{array}{c}\text { Gage } \\
\text { height. }\end{array}$ & Discharge. & $\begin{array}{c}\text { Gage } \\
\text { height. }\end{array}$ & Discharge. & $\begin{array}{c}\text { Gage } \\
\text { height. }\end{array}$ & Discharge. \\
\hline Feet. & Second-feet. & Feet. & Second-feet. & Feet. & Second-feet. & Feet. & Second-feet. \\
1.80 & 34 & 2.60 & 210 & 3.30 & 490 & 4.00 & 910 \\
1.90 & 50 & 2.70 & 240 & 3.40 & 540 & 4.10 & 985 \\
2.00 & 67 & 2.80 & 275 & 3.50 & 595 & 4.20 & 1,070 \\
2.10 & 85 & 2.90 & 310 & 3.60 & 650 & 4.30 & 1,160 \\
2.20 & 105 & 3.00 & 350 & 3.70 & 710 & 4.40 & 1,250 \\
2.30 & 128 & 3.10 & 395 & 3.80 & 775 & 4.50 & 1,345 \\
2.40 & 153 & 3.20 & 440 & 3.90 & 840 & 4.60 & 1,445 \\
2.50 & 180 & & & & & & \\
\hline
\end{tabular}

AUGUST 26, 1901, TO DECEMBER 31, 1903.b

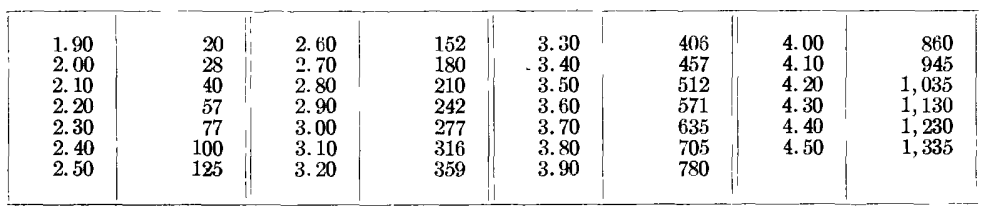

JANUARY 1, 1904, TO DECEMBER 31, 1905.c

\begin{tabular}{|l|r|r|r||l|l|l|l|}
\hline 2.00 & 15 & 3.30 & 347 & 4.60 & 1,440 & 6.80 & 4,720 \\
2.10 & 24 & 3.40 & 397 & 4.70 & 1,550 & 7.00 & 5,100 \\
2.20 & 36 & 3.50 & 453 & 4.80 & 1,660 & 7.20 & 5,500 \\
2.30 & 51 & 3.60 & 515 & 4.90 & 1,775 & 7.40 & 5,910 \\
2.40 & 68 & 3.70 & 584 & 5.00 & 1,890 & 7.60 & 6,330 \\
2.50 & 88 & 3.80 & 660 & 5.20 & 2,130 & 7.80 & 6,760 \\
2.60 & 110 & 3.90 & 742 & 5.40 & 2,390 & 8.00 & 7,200 \\
2.70 & 134 & 4.00 & 830 & 5.60 & 2,670 & 8.50 & 8,350 \\
2.80 & 161 & 4.10 & 023 & 5.80 & 2,970 & 9.00 & 9,550 \\
2.90 & 191 & 4.20 & 1,020 & 6.00 & 3,290 & 1.50 & 10,800 \\
3.00 & 224 & 4.30 & 1,120 & 6.20 & 3,630 & 10.00 & 12,100 \\
3.10 & 261 & 4.40 & 1,225 & 6.40 & 3,980 & 10.50 & 13,450 \\
3.20 & 302 & 4.50 & 1,330 & 6.60 & 4,340 & & \\
\hline
\end{tabular}

JANUARY 1 TO JULY 15, 1906.d

\begin{tabular}{|l|r|r|r|r|r|r|r|}
\hline 2.50 & 70 & 3.60 & 45. & 4.70 & 1,430 & 6.40 & 3,785 \\
2.60 & 88 & 3.70 & 519 & 4.80 & 1,540 & 6.60 & 4,140 \\
2.70 & 109 & 3.80 & 587 & 4.90 & 1,655 & 6.80 & 4,515 \\
2.80 & 133 & 3.90 & 661 & 5.00 & 1,770 & 7.00 & 4,900 \\
2.90 & 160 & 4.00 & 740 & 5.20 & 2,015 & 7.20 & 5,300 \\
3.00 & 190 & 4.10 & 825 & 5.40 & 2,270 & 7.40 & 5,710 \\
3.10 & 224 & 4.20 & 915 & 5.60 & 2.535 & 7.60 & 6,130 \\
3.20 & 262 & 4.30 & 1,010 & 5.80 & 2,820 & 7.80 & 6,570 \\
3.30 & 304 & 4.40 & 1,110 & 6.00 & 3,125 & 8.00 & 7,030 \\
3.40 & 350 & 4.50 & 1,215 & 6.20 & 3,450 & 8.20 & 7,500 \\
3.50 & 401 & 4.60 & 1,320 & & & & \\
\hline
\end{tabular}

$a$ This table is strictly applicable only for open-channel conditions. It is based on four discharge measurements made during 1899 and 1900 . It is fairly well defined hetween gage heights 1.8 feet and 4.5 feet. As applied to 1901 gage heights it is liable to give estimates several per cent too high, owing to changes in the condition of flow at and below the section during 1901. Above gage height 4.60 feet the table is the same as the 1904-5 table.

$b$ This table is strictly applicable only for open-channel conditions. It is based on three discharge measurements made during 1901-1903 and on the form of other curves at this station. It is fairly well defined. Above gage height 4.50 feet the table is the same as the 1904-5 table.

$c$ This table is strictly applicable only for open-channel conditions. - It is based on discharge measurements made during 1904-1905. It is fairly well defined between gage heights 3.0 feet and 7.5 feet. Below 3 feet the table is based on two measurements at 2 feet. The above table as applied to 1904 gage heights is liable to give estimates several per cent too low above gage height 2.5 feet. As applied to 1905 gage heights it is liable to give estimates several per cent too high below gage height 2.5 feet.

$d$ This table is strictly applicable only for open-channel conditions. It is based on three discharge measurements made during 1906 and the form of previous curves at this station. It is well defined between gage heights 3.5 feet and 5.5 feet. 
Estimated monthly discharge of North Branch of Potomac River at Piedmont, W. Va. a

[Drainage area, 410 square miles.]

\begin{tabular}{|c|c|c|c|c|c|c|c|c|}
\hline \multirow[b]{2}{*}{ Month. } & \multicolumn{3}{|c|}{ Discharge in second-feet. } & \multicolumn{3}{|c|}{ Run-off. } & \multicolumn{2}{|c|}{ Precipitation. } \\
\hline & Maximum. & Minimum. & Mean. & $\begin{array}{l}\text { Second-feet } \\
\text { per square } \\
\text { mile. }\end{array}$ & $\begin{array}{l}\text { Depth in } \\
\text { inches. }\end{array}$ & $\begin{array}{l}\text { Per cent } \\
\text { of precip- } \\
\text { itation. }\end{array}$ & $\begin{array}{c}\text { In } \\
\text { inches. }\end{array}$ & $\begin{array}{l}\text { Loss in } \\
\text { inches. }\end{array}$ \\
\hline 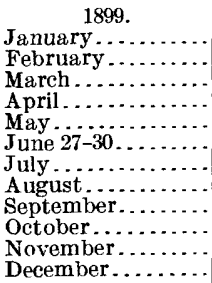 & 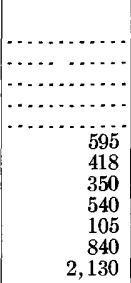 & $\begin{array}{r}292 \\
95 \\
50 \\
67 \\
67 \\
105 \\
140\end{array}$ & $\begin{array}{l}387 \\
209 \\
119 \\
157 \\
88.4 \\
275 \\
421\end{array}$ & $\begin{array}{r}0.944 \\
.510 \\
.290 \\
.383 \\
.216 \\
.671 \\
1.03\end{array}$ & $\begin{array}{c}0.140 \\
.588 \\
.334 \\
.427 \\
.249 \\
.749 \\
1.19\end{array}$ & $\begin{array}{c} \\
\ldots \ldots \\
22 \\
16 \\
11 \\
11 \\
43 \\
49\end{array}$ & $\begin{array}{r}2.01 \\
3.85 \\
5.08 \\
1.93 \\
6.60 \\
b 4.81 \\
2.64 \\
2.11 \\
3.91 \\
2.29 \\
1.76 \\
2.44\end{array}$ & \begin{tabular}{|l}
$\ldots \ldots$ \\
$\cdots \ldots$ \\
$\cdots \ldots \ldots$ \\
$\cdots \ldots$ \\
$\ldots \ldots$ \\
$\cdots \ldots$ \\
2.05 \\
1.78 \\
3.48 \\
2.04 \\
1.01 \\
1.25
\end{tabular} \\
\hline 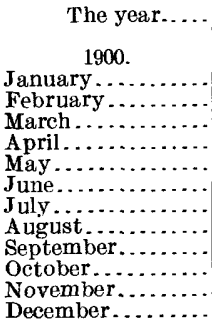 & $\begin{array}{r}2,970 \\
3,130 \\
3,210 \\
1,660 \\
875 \\
6,225 \\
1,250 \\
330 \\
240 \\
440 \\
7,090 \\
2,970\end{array}$ & $\begin{array}{r}240 \\
350 \\
622 \\
465 \\
240 \\
240 \\
116 \\
67 \\
34 \\
67 \\
81 \\
310\end{array}$ & $\begin{array}{c}838 \\
1,032 \\
1,306 \\
821 \\
392 \\
804 \\
308 \\
136 \\
53.6 \\
143 \\
577 \\
692\end{array}$ & $\begin{array}{l}2.04 \\
2.02 \\
3.19 \\
2.00 \\
.956 \\
1.96 \\
.751 \\
.332 \\
.131 \\
.349 \\
1.41 \\
1.69\end{array}$ & $\begin{array}{l}2.35 \\
2.62 \\
3.68 \\
2.23 \\
1.10 \\
2.19 \\
.866 \\
.383 \\
.146 \\
.402 \\
1.57 \\
1.95\end{array}$ & $\begin{array}{r}137 \\
73 \\
109 \\
170 \\
50 \\
49 \\
20 \\
14 \\
20 \\
19 \\
29 \\
79\end{array}$ & $\begin{array}{l}39.43 \\
\\
1.72 \\
3.59 \\
3.37 \\
1.31 \\
2.22 \\
4.47 \\
4.24 \\
2.64 \\
.74 \\
2.07 \\
5.46 \\
2.46\end{array}$ & $\begin{array}{r} \\
-0.63 \\
. .97 \\
-.31 \\
-.92 \\
1.12 \\
2.28 \\
3.37 \\
2.26 \\
.59 \\
1.67 \\
3.89 \\
.51\end{array}$ \\
\hline 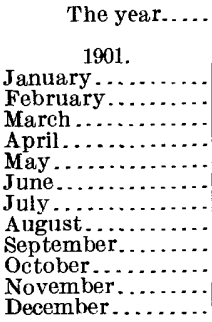 & $\begin{array}{r}2,895 \\
650 \\
4,910 \\
6,330 \\
6,225 \\
1,832 \\
2,260 \\
1,832 \\
359 \\
138 \\
277 \\
7,545\end{array}$ & $\begin{array}{r}310 \\
292 \\
292 \\
680 \\
490 \\
395 \\
153 \\
128 \\
57 \\
40 \\
28 \\
125\end{array}$ & $\begin{array}{c}705 \\
407 \\
1,387 \\
2,234 \\
1,681 \\
925 \\
490 \\
409 \\
149 \\
63.5 \\
70.3 \\
1,472\end{array}$ & $\begin{array}{l}1.72 \\
.993 \\
3.38 \\
5.45 \\
4.10 \\
2.26 \\
1.20 \\
1.00 \\
.363 \\
.155 \\
.171 \\
3.59\end{array}$ & $\begin{array}{l}\text { i. } 98 \\
1.03 \\
3.90 \\
6.08 \\
4.73 \\
2.52 \\
1.38 \\
1.15 \\
.405 \\
.179 \\
.191 \\
4.14\end{array}$ & $\begin{array}{r}54 \\
79 \\
114 \\
102 \\
67 \\
72 \\
51 \\
24 \\
19 \\
28 \\
7 \\
72\end{array}$ & $\begin{array}{l}3.68 \\
1.30 \\
3.42 \\
5.93 \\
7.03 \\
3.52 \\
2.69 \\
4.89 \\
2.09 \\
.65 \\
2.69 \\
5.73\end{array}$ & $\begin{array}{r}1.70 \\
.27 \\
-.48 \\
-.15 \\
2.30 \\
1.00 \\
1.31 \\
3.74 \\
1.69 \\
.47 \\
2.50 \\
1.59\end{array}$ \\
\hline The year... & 7,545 & 28 & 831 & 2.03 & 27.68 & 63 & 43.62 & 15.94 \\
\hline 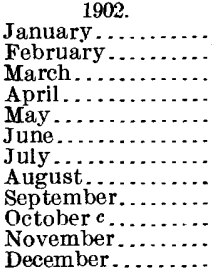 & $\begin{array}{r}2,895 \\
13,450 \\
7,430 \\
5,200 \\
945 \\
406 \\
1,605 \\
226 \\
166 \\
635 \\
2,460 \\
6,330\end{array}$ & $\begin{array}{r}242 \\
210 \\
990 \\
542 \\
242 \\
100 \\
77 \\
20 \\
20 \\
100 \\
100 \\
484\end{array}$ & $\begin{array}{c}644 \\
1,082 \\
2,578 \\
1,980 . \\
483 \\
219 \\
265 \\
85.8 \\
40.9 \\
213 \\
288 \\
1,764\end{array}$ & $\begin{array}{l}1.57 \\
2.64 \\
6.29 \\
4.83 \\
1.18 \\
.534 \\
.646 \\
.209 \\
.100 \\
.520 \\
.702 \\
4.30\end{array}$ & $\begin{array}{l}1.81 \\
2.75 \\
7.25 \\
5.39 \\
1.36 \\
.596 \\
.745 \\
.241 \\
.112 \\
.600 \\
.783 \\
4.96\end{array}$ & $\begin{array}{r}57 \\
72 \\
173 \\
130 \\
42 \\
14 \\
21 \\
11 \\
4 \\
20 \\
34 \\
94\end{array}$ & $\begin{array}{l}3.19 \\
3.83 \\
4.18 \\
4.14 \\
3.26 \\
4.34 \\
3.62 \\
2.14 \\
2.84 \\
3.00 \\
2.32 \\
5.29\end{array}$ & $\begin{array}{r}1.38 \\
1.08 \\
-3.07 \\
-1.25 \\
1.90 \\
3.74 \\
2.87 \\
1.90 \\
2.73 \\
2.40 \\
1.54 \\
.33\end{array}$ \\
\hline The year..... & 13,450 & 20 & 804 & 1.96 & 26. 60 & 63 & 42.15 & 15.55 \\
\hline
\end{tabular}

$a$ See description and footnotes to the rating tables.

$b$ Precipitation for complete month June, 1899.

c Discharge interpolated October 28 and $29,1902$. 
Estimated monthly discharge of North Branch of Potomac River at Piedmont, W. Va.Continued.

\begin{tabular}{|c|c|c|c|c|c|c|c|c|}
\hline \multirow[b]{2}{*}{ Month. } & \multicolumn{3}{|c|}{ Discharge in second-feet. } & \multicolumn{3}{|c|}{ Run-off. } & \multicolumn{2}{|c|}{ Psecipitation. } \\
\hline & Maximum. & Minimum. & Mean. & $\begin{array}{c}\text { Second-feet } \\
\text { per square } \\
\text { mile. }\end{array}$ & $\begin{array}{l}\text { Depth in } \\
\text { inches. }\end{array}$ & $\begin{array}{l}\text { Per cent } \\
\text { of precip- } \\
\text { itation. }\end{array}$ & $\underset{\text { inches. }}{\text { In }}$ & $\begin{array}{c}\text { Loss in } \\
\text { inches. } \\
.\end{array}$ \\
\hline 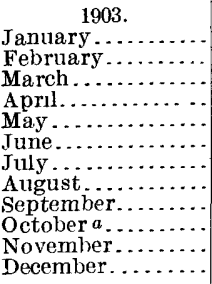 & $\begin{array}{r}5,700 \\
9,070 \\
4,530 \\
3,375 \\
1,550 \\
4,435 \\
2,130 \\
277 \\
210 \\
180 \\
603 \\
1,282\end{array}$ & $\begin{array}{r}359 \\
635 \\
571 \\
635 \\
210 \\
359 \\
166 \\
77 \\
28 \\
34 \\
57 \\
48\end{array}$ & $\begin{array}{c}1,292 \\
2,223 \\
1,662 \\
1,506 \\
580 \\
1,292 \\
570 \\
163 \\
90.5 \\
103 \\
134 \\
193\end{array}$ & $\begin{array}{l}3.15 \\
5.42 \\
4.05 \\
3.67 \\
1.41 \\
3.15 \\
1.39 \\
.398 \\
.221 \\
.251 \\
.327 \\
.471\end{array}$ & $\begin{array}{l}3.63 \\
5.64 \\
4.67 \\
4.10 \\
1.63 \\
3.51 \\
1.60 \\
.459 \\
.247 \\
.289 \\
.365 \\
.543\end{array}$ & $\begin{array}{r}102 \\
103 \\
122 \\
113 \\
51 \\
54 \\
32 \\
14 \\
14 \\
12 \\
20 \\
37\end{array}$ & $\begin{array}{l}3.57 \\
5.50 \\
3.82 \\
3.64 \\
3.18 \\
6.50 \\
4.96 \\
3.18 \\
1.77 \\
2.36 \\
1.78 \\
1.47\end{array}$ & $\begin{array}{r}-0.06 \\
-\quad .14 \\
-.85 \\
-.46 \\
1.55 \\
2.99 \\
3.36 \\
2.72 \\
1.52 \\
2.07 \\
1.42 \\
.93\end{array}$ \\
\hline The year... & 9.070 & 28 & 817 & 1.99 & 26.68 & 64 & 41.73 & 15.05 \\
\hline 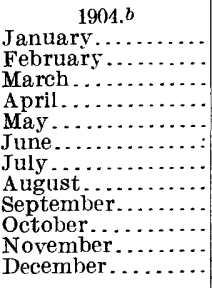 & $\begin{array}{r}4,720 \\
3,630 \\
4,340 \\
2,195 \\
3,210 \\
786 \\
425 \\
99 \\
30 \\
68 \\
51 \\
1,660\end{array}$ & $\begin{array}{r}110 \\
224 \\
484 \\
372 \\
302 \\
134 \\
60 \\
9 \\
6 \\
12 \\
15 \\
15\end{array}$ & $\begin{array}{r}643 \\
795 \\
1,433 \\
937 \\
792 \\
392 \\
160 \\
37 \\
17 \\
28 \\
34 \\
243\end{array}$ & $\begin{array}{l}1.58 \\
1.96 \\
3.53 \\
2.31 \\
1.95 \\
.966 \\
.394 \\
.091 \\
.042 \\
.069 \\
.084 \\
.599\end{array}$ & $\begin{array}{l}1.82 \\
2.11 \\
4.07 \\
2.58 \\
2.25 \\
1.08 \\
.454 \\
.105 \\
.047 \\
.080 \\
.094 \\
.691\end{array}$ & $\begin{array}{r}59 \\
108 \\
163 \\
98 \\
70 \\
27 \\
12 \\
6 \\
3 \\
5 \\
17 \\
24\end{array}$ & $\begin{array}{l}3.07 \\
1.96 \\
2.50 \\
2.62 \\
3.20 \\
3.95 \\
3.69 \\
1.96 \\
1.81 \\
1.76 \\
.54 \\
2.92\end{array}$ & $\begin{array}{r}1.25 \\
-.15 \\
-1.57 \\
.04 \\
.95 \\
2.87 \\
3.24 \\
1.85 \\
1.76 \\
1.68 \\
.45 \\
2.23\end{array}$ \\
\hline The year.... & 4,720 & 6 & 459 & 1.13 & 15.38 & 51 & 29.98 & 14.60 \\
\hline 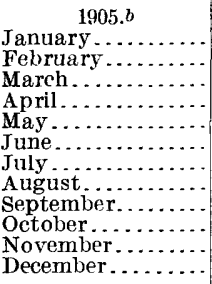 & $\begin{array}{r}3,210 \\
484 \\
7,420 \\
830 \\
1,914 \\
i, 605 \\
2,460 \\
1,775 \\
1,020 \\
1,718 \\
1,385 \\
2,895\end{array}$ & $\begin{array}{r}99 \\
99 \\
282 \\
161 \\
191 \\
242 \\
161 \\
88 \\
51 \\
36 \\
148 \\
208\end{array}$ & $\begin{array}{r}360 \\
232 \\
2,484 \\
585 \\
496 \\
588 \\
653 \\
376 \\
195 \\
348 \\
267 \\
815\end{array}$ & $\begin{array}{c}.887 \\
.571 \\
6.12 \\
1.44 \\
1.22 \\
1.45 \\
1.61 \\
.926 \\
.480 \\
.857 \\
.658 \\
2.01\end{array}$ & $\begin{array}{c}1.02 \\
.595 \\
7.06 \\
1.61 \\
1.41 \\
1.62 \\
1.86 \\
1.07 \\
.536 \\
.988 \\
.734 \\
2.32\end{array}$ & $\begin{array}{r}33 \\
34 \\
201 \\
82 \\
49 \\
40 \\
28 \\
28 \\
25 \\
24 \\
31 \\
74\end{array}$ & $\begin{array}{l}3.13 \\
1.75 \\
3.52 \\
1.97 \\
2.87 \\
4.03 \\
6.55 \\
3.88 \\
2.16 \\
4.08 \\
2.36 \\
3.13\end{array}$ & $\begin{array}{r}2.11 \\
1.16 \\
-3.54 \\
.36 \\
1.46 \\
2.41 \\
4.69 \\
2.81 \\
1.62 \\
3.09 \\
1.63 \\
.81\end{array}$ \\
\hline The year & 7,420 & 36 & 617 & 1.52 & 20.82 & 53 & 39.43 & $\overline{18.61}$ \\
\hline 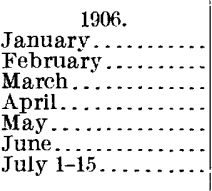 & $\begin{array}{r}7,500 \\
488 \\
5,710 \\
4,515 \\
740 \\
2,078 \\
224\end{array}$ & $\begin{array}{r}327 \\
88 \\
121 \\
700 \\
109 \\
160 \\
70\end{array}$ & $\begin{array}{r}1,255 \\
220 \\
1,161 \\
2,013 \\
323 \\
413 \\
120\end{array}$ & $\begin{array}{c}3.06 \\
.537 \\
2.83 \\
4.91 \\
.788 \\
1.01 \\
.293\end{array}$ & $\begin{array}{l}3.53 \\
.559 \\
3.26 \\
5.48 \\
.908 \\
1.13 \\
.163\end{array}$ & $\begin{array}{l}\ldots \ldots \\
\ldots \ldots \\
\ldots \ldots \\
\ldots \ldots \\
\ldots \ldots \\
\ldots \ldots\end{array}$ & $\cdots$ & $\begin{array}{l}\cdots \\
\cdots \\
\cdots \\
\cdots \\
\cdots \\
\cdots\end{array}$ \\
\hline
\end{tabular}

$a$ Discharge interpolated October 7-15. 1903.

$b$ Drainage area of 406 square miles used to obtain 1904 and 1905 run-off 
Georges Creek rises on Mount Savage, in the northwestern part of Allegany County, Md., and flows southwestward into North Branch of Potomac River at Westernport. Its length is about 15 miles.

The gaging station was established May 4, 1905, and was discontinued July 16, 1906. It is located at a highway bridge in Westernport, Md., about one-half mile above the mouth of the creek.

Above the station the channel is straight for about 50 feet and then makes a sharp bend. Below the station it is straight for 300 feet. The current is swift. Both banks are fairly high, clean, and do not overflow. The bed of the stream is rocky and shifting. There is but one channel at all stages.

Discharge measurements were made from the upstream side of the bridge to which the gage is attached. The initial point for soundings is the inside face of the left abutment, upstream side.

A standard chain gage is attached to the middle of the upstream side of the bridge. The length of the chain from the end of the weight to the marker is 16.23 feet. The gage was read twice each day by G. A. Biggs. Bench mark No. 1 is the top of the downstream bedplate at the left abutment, on the downstream corner toward the railroad track. Its elevation is $\mathbf{1 1 . 5 8}$ feet above the datum of the gage. Bench mark No. 3 is the top of the pulley wheel of the gage. Its elevation was 15.88 feet above the datum of the gage May 4, 1905.

The bed of Georges Creek at the gaging section is rather unstable and subject to frequent changes on account of the high velocity of the current. The estimates are therefore subject to varying errors of from 5 to 20 per cent of the true flow. Ice conditions did not affect the estimates during the winter of 1905-6.

Discharge measurements of Georges Creek at Westernport, Md.

\begin{tabular}{|c|c|c|c|c|c|}
\hline Date. & $\begin{array}{c}\text { Gage } \\
\text { height. }\end{array}$ & Discharge. & Date. & $\begin{array}{c}\text { Gage } \\
\text { height. }\end{array}$ & Discharge. \\
\hline 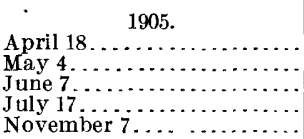 & $\begin{array}{l}\text { Feet. } \\
1.60 \\
1.35 \\
1.98 \\
1.58 \\
1.35\end{array}$ & \begin{tabular}{r|} 
Second-feet. \\
53 \\
33 \\
131 \\
43 \\
45
\end{tabular} & $\begin{array}{l}1906 . \\
\text { Merch } 16 \ldots \ldots \ldots \\
\text { March } 30 \ldots \ldots \ldots \\
\text { April } 10 \ldots \ldots \ldots \\
\text { May } 28 \ldots \ldots \ldots\end{array}$ & $\begin{array}{l}\text { Feet. } \\
\text { 1. } 49 \\
3.38 \\
2.50 \\
2.25\end{array}$ & $\begin{array}{r}\text { Second-fert. } \\
65 \\
1,230 \\
476 \\
353\end{array}$ \\
\hline
\end{tabular}


Daily gage height, in feet, of Georges Creek at Westernport, Md.

\begin{tabular}{|c|c|c|c|c|c|c|c|c|c|c|c|c|}
\hline Day. & Jan. & Feb. & Mar. & Apr. & May. & June. & July. & Aug. & Sept. & Oct. & Nov. & Dec. \\
\hline 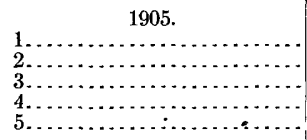 & & & 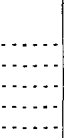 & $\cdots$ & \begin{tabular}{l}
$\cdots$ \\
$\cdots$ \\
\hdashline 1.45 \\
1.4
\end{tabular} & $\begin{array}{l}1.45 \\
1.45 \\
1.38 \\
1.3 \\
1.25\end{array}$ & $\begin{array}{l}\text { 1. } 38 \\
\text { 1. } 58 \\
\text { 1. } 32 \\
\text { 1. } 48 \\
\text { 1. } 52\end{array}$ & $\begin{array}{l}1.22 \\
1.12 \\
1.1 \\
1.1 \\
1.08\end{array}$ & $\begin{array}{l}1.05 \\
1.05 \\
1.08 \\
1.12 \\
1.1\end{array}$ & $\begin{array}{l}0.95 \\
1.28 \\
1.18 \\
1.1 \\
1.05\end{array}$ & $\begin{array}{l}1.5 \\
1.5 \\
1.45 \\
1.38 \\
1.4\end{array}$ & $\begin{array}{l}1.62 \\
1.8 \\
2.35 \\
2.22 \\
2,12\end{array}$ \\
\hline 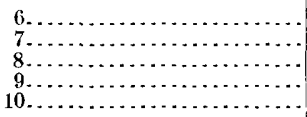 & & & $\ldots$ & & \begin{tabular}{l|}
1.42 \\
1.5 \\
1.4 \\
1.35 \\
1.38
\end{tabular} & $\begin{array}{l}\text { 1. } 2 \\
\text { 1. } 98 \\
\text { 1. } 58 \\
\text { 1. } 42 \\
1.35\end{array}$ & $\begin{array}{l}1.48 \\
3.45 \\
3.38 \\
2.65 \\
2.15\end{array}$ & $\begin{array}{l}1.05 \\
1.05 \\
1.05 \\
1.05 \\
1.05\end{array}$ & $\begin{array}{l}1.1 \\
1.05 \\
1.0 \\
1.0 \\
1.28\end{array}$ & $\begin{array}{l}1.02 \\
1.0 \\
1.0 \\
1.0 \\
.98\end{array}$ & $\begin{array}{l}1.52 \\
1.4 \\
1.32 \\
1.25 \\
1.2\end{array}$ & $\begin{array}{l}2.55 \\
2.35 \\
2.22 \\
2.12 \\
2.02\end{array}$ \\
\hline $\begin{array}{l}11 \ldots \ldots \\
12 \ldots \ldots \\
13 \ldots \ldots \\
14 \ldots \ldots\end{array}$ & & & & $\begin{array}{l}- \\
\cdots \\
\cdots \\
-\end{array}$ & $\begin{array}{l}1.38 \\
1.5 \\
1.38 \\
1.48 \\
1.72\end{array}$ & $\begin{array}{l}2.05 \\
1.98 \\
1.85 \\
1.72 \\
1.62\end{array}$ & $\begin{array}{l}2.05 \\
1.98 \\
1.92 \\
1.8 \\
1.7\end{array}$ & $\begin{array}{l}1.08 \\
1.12 \\
1.15 \\
1.12 \\
1.28\end{array}$ & $\begin{array}{l}2.55 \\
1.9 \\
1.65 \\
1.5 \\
1.42\end{array}$ & $\begin{array}{l}1.75 \\
1.55 \\
1.25 \\
1.12 \\
1.08\end{array}$ & $\begin{array}{l}1.12 \\
1.1 \\
1.05 \\
1.0 \\
.95\end{array}$ & $\begin{array}{l}1.9 \\
1.88 \\
1.82 \\
1.78 \\
1.42\end{array}$ \\
\hline 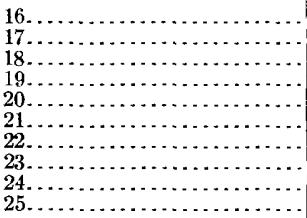 & & & & & $\begin{array}{l}1.68 \\
1.65 \\
1.62 \\
1.58 \\
1.52 \\
1.5 \\
1.45 \\
1.4 \\
1.38 \\
1.28\end{array}$ & $\begin{array}{l}\text { 1. } 52 \\
1.5 \\
\text { 1. } 48 \\
\text { 1. } 4 \\
\text { 1. } 55 \\
\text { 1. } 48 \\
\text { 1. } 42 \\
1.38 \\
\text { 1. } 5 \\
\text { 1. } 55\end{array}$ & \begin{tabular}{l|}
1.62 \\
1.52 \\
1.42 \\
1.32 \\
1.4 \\
1.38 \\
1.35 \\
1.7 \\
1.48 \\
1.22
\end{tabular} & $\begin{array}{l}1.25 \\
1.12 \\
1.05 \\
1.02 \\
1.0 \\
1.0 \\
1.0 \\
.98 \\
.92 \\
1.72\end{array}$ & $\begin{array}{l}\text { 1. } 32 \\
1.38 \\
1.28 \\
\text { 1. } 22 \\
\text { 1. } 18 \\
1.12 \\
1.1 \\
1.05 \\
1.05 \\
1.0\end{array}$ & $\begin{array}{l}1.05 \\
1.0 \\
1.0 \\
1.18 \\
2.3 \\
1.65 \\
1.52 \\
1.48 \\
1.42 \\
1.52\end{array}$ & $\begin{array}{l}.98 \\
1.0 \\
1.0 \\
1.02 \\
1.02 \\
1.0 \\
1.0 \\
1.0 \\
1.0 \\
1.08\end{array}$ & $\begin{array}{l}1.45 \\
1.3 \\
1.3 \\
1.35 \\
1.4 \\
3.4 \\
3.08 \\
2.2 \\
2.2 \\
2.12\end{array}$ \\
\hline 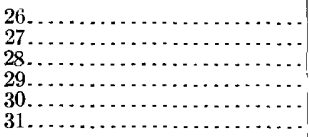 & & & & & $\begin{array}{l}1.28 \\
1.25 \\
1.3 \\
1.3 \\
1.28 \\
1.32\end{array}$ & $\begin{array}{l}1.4 \\
1.32 \\
1.25 \\
1.22 \\
1.2 \\
\cdots\end{array}$ & $\begin{array}{l}1.18 \\
1.15 \\
1.15 \\
1.2 \\
1.45 \\
1.38\end{array}$ & $\begin{array}{l}1.65 \\
1.45 \\
1.25 \\
1.1 \\
1.12 \\
1.08\end{array}$ & $\begin{array}{l}1.0 \\
1.0 \\
1.0 \\
.95 \\
.95 \\
\ldots\end{array}$ & $\begin{array}{l}2.02 \\
1.85 \\
1.72 \\
1.62 \\
1.52 \\
1.5\end{array}$ & $\begin{array}{l}1.05 \\
1.08 \\
1.1 \\
1.55 \\
1.65 \\
\cdots \cdots\end{array}$ & $\begin{array}{l}2.15 \\
1.98 \\
1.95 \\
2.08 \\
1.88 \\
1.75\end{array}$ \\
\hline 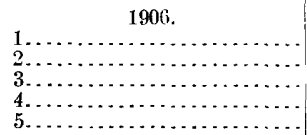 & $\begin{array}{l}1.68 \\
1.55 \\
2.3 \\
2.58 \\
2.45\end{array}$ & $\begin{array}{l}1.8 \\
1.8 \\
1.75 \\
1.72 \\
1.68\end{array}$ & $\begin{array}{l}1.6 \\
1.78 \\
2.05 \\
2.1 \\
2.02\end{array}$ & $\begin{array}{l}3.18 \\
2.98 \\
2.88 \\
2.92 \\
2.85\end{array}$ & $\begin{array}{l}\text { 1. } 68 \\
\text { 1. } 72 \\
\text { 1. } 62 \\
\text { 1. } 52 \\
1.5\end{array}$ & $\begin{array}{l}1.65 \\
1.55 \\
1.55 \\
1.32 \\
1.6\end{array}$ & $\begin{array}{l}1.18 \\
1.15 \\
1.15 \\
1.1 \\
1.1\end{array}$ & $\begin{array}{l}\ldots \ldots \\
\cdots \ldots \\
\ldots \ldots\end{array}$ & - & $\cdots$ & & 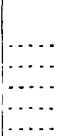 \\
\hline 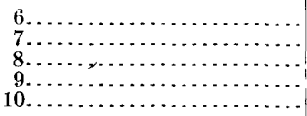 & $\begin{array}{l}2.38 \\
2.2 \\
2.2 \\
1.88 \\
1.95\end{array}$ & $\begin{array}{l}1.65 \\
1.6 \\
1.55 \\
1.52 \\
1.5\end{array}$ & $\begin{array}{l}1.98 \\
1.8 \\
1.75 \\
1.68 \\
1.6\end{array}$ & $\begin{array}{l}252 \\
2.32 \\
2.32 \\
2.55 \\
2.58\end{array}$ & $\begin{array}{l}1.55 \\
1.5 \\
1.5 \\
1.45 \\
1.4\end{array}$ & $\begin{array}{l}1.68 \\
2.22 \\
2.25 \\
1.95 \\
1.8\end{array}$ & $\begin{array}{l}1.1 \\
1.05 \\
1.05 \\
1.05 \\
1.1\end{array}$ & $\begin{array}{l}\cdots \\
\cdots \\
\cdots\end{array}$ & $\cdots$ & & $\because$ & $\begin{array}{c}\cdots \\
\cdots \\
\cdots \\
\cdots \\
\cdots \\
\cdots\end{array}$ \\
\hline $\begin{array}{l}11 \ldots \ldots \ldots \\
12 \ldots \ldots \ldots \\
13 \ldots \ldots \ldots \\
14 \ldots \ldots \ldots \\
15 .{ }_{1} \ldots \ldots \ldots\end{array}$ & $\begin{array}{l}1.95 \\
2.0 \\
1.95 \\
1.88 \\
1.78\end{array}$ & $\begin{array}{l}1.45 \\
1.45 \\
1.42 \\
1.4 \\
1.4\end{array}$ & $\begin{array}{l}1.55 \\
1.52 \\
1.5 \\
1.5 \\
1.5\end{array}$ & $\begin{array}{l}2.32 \\
2.32 \\
2.22 \\
2.22 \\
2.2\end{array}$ & $\begin{array}{l}1.4 \\
1.4 \\
1.4 \\
1.42 \\
.4\end{array}$ & $\begin{array}{l}\text { 1. } 72 \\
\text { 1. } 75 \\
\text { 1. } 75 \\
\text { 1. } 7 \\
\text { 1. } 62\end{array}$ & $\begin{array}{l}1.1 \\
1.1 \\
1.1 \\
1.05 \\
1.02\end{array}$ & $\cdots$ & $\cdots$ & $\begin{array}{l}\cdots \\
\cdots \\
\cdots\end{array}$ & & $\begin{array}{l}\cdots \\
\cdots \\
\cdots \\
\cdots \\
\cdots\end{array}$ \\
\hline 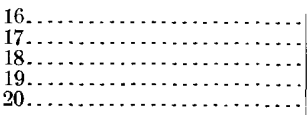 & $\begin{array}{l}1.82 \\
2.0 \\
2.05 \\
2.12 \\
2.15\end{array}$ & $\begin{array}{l}\text { 1. } 4 \\
\text { 1. } 4 \\
\text { 1. } 4 \\
\text { 1. } 4 \\
1.45\end{array}$ & $\begin{array}{l}1.45 \\
1.32 \\
1.32 \\
1.38 \\
1.5\end{array}$ & $\begin{array}{l}2.22 \\
2.12 \\
2.12 \\
2.22 \\
2.0\end{array}$ & $\begin{array}{l}\text { 1. } 3 \\
\text { 1. } 32 \\
\text { 1. } 32 \\
\text { 1. } 25 \\
1.22\end{array}$ & $\begin{array}{l}\text { 1. } 55 \\
1.25 \\
1.2 \\
1.2 \\
1.35\end{array}$ & $\begin{array}{l}\ldots \\
\cdots \\
\cdots \\
\cdots\end{array}$ & $\ldots \ldots$ & $\ldots$ & & & $\begin{array}{l}\ldots \\
\ldots \\
\cdots \\
\cdots \\
\cdots\end{array}$ \\
\hline $\begin{array}{l}21 \ldots \ldots \ldots \\
22 \ldots \ldots \\
23 \ldots \ldots \\
24 \ldots \\
25 \ldots \ldots\end{array}$ & $\begin{array}{l}2.18 \\
2.22 \\
\text { 3. } 28 \\
\text { 3. } 08 \\
\text { 2. } 68\end{array}$ & $\begin{array}{l}1.45 \\
1.4 \\
1.35 \\
1.32 \\
1.3\end{array}$ & $\begin{array}{l}1.55 \\
1.58 \\
1.55 \\
1.58 \\
1.6\end{array}$ & $\begin{array}{l}2.05 \\
1.9 \\
1.88 \\
1.88 \\
1.82\end{array}$ & $\begin{array}{l}1.2 \\
1.2 \\
1.15 \\
1.15 \\
1.1\end{array}$ & $\begin{array}{l}1.35 \\
1.35 \\
1.38 \\
1.4 \\
1.3\end{array}$ & $\mid \begin{array}{l}\cdots \\
\cdots \\
\cdots \\
\cdots\end{array}$ & & & & & $\begin{array}{l}\cdots \\
\cdots \\
\cdots \\
\cdots \\
\cdots\end{array}$ \\
\hline 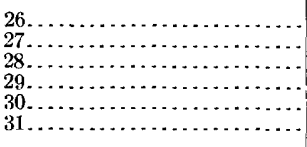 & $\begin{array}{l}2.2 \\
2.12 \\
2.1 \\
2.05 \\
1.98 \\
1.9\end{array}$ & $\begin{array}{l}1.3 \\
1.38 \\
1.42 \\
\ldots \ldots \\
\ldots \ldots . \\
\ldots \ldots \ldots\end{array}$ & $\begin{array}{l}1.68 \\
2.62 \\
3.2 \\
3.2 \\
3.42 \\
3.45\end{array}$ & $\begin{array}{l}1.9 \\
1.85 \\
1.75 \\
1.7 \\
1.72 \\
\cdots \cdots\end{array}$ & $\begin{array}{l}1.1 \\
2.02 \\
2.08 \\
2.2 \\
1.95 \\
1.88\end{array}$ & $\begin{array}{l}1.32 \\
1.38 \\
1.25 \\
1.2 \\
1.2\end{array}$ & & & & & & . \\
\hline
\end{tabular}


Rating tables for Georges Creek at Westernport, $M d$.

MAY 4,1905 , TO JUNE $6,1905 . a$

\begin{tabular}{|c|r|r|r|}
$\begin{array}{c}\text { Gage } \\
\text { height. }\end{array}$ & Discharge. & $\begin{array}{c}\text { Gage } \\
\text { height. }\end{array}$ & Discharge. \\
\hline Feet. & Second-feet. & Feet. & Second-feet. \\
1.20 & 22 & 1.60 & 66 \\
1.30 & 29 & 1.70 & 87 \\
1.40 & 38 & 1.80 & 112 \\
1.50 & 50 & & \\
\hline
\end{tabular}

JUNE 7 TO SEPTEMBER 10, 1905.b

\begin{tabular}{|c|c|c|c|c|c|c|c|}
\hline $\begin{array}{l}\text { Gage } \\
\text { height. }\end{array}$ & Discharge. & $\begin{array}{l}\text { Gage } \\
\text { height. }\end{array}$ & Discharge. & $\begin{array}{c}\text { Gage } \\
\text { height. }\end{array}$ & Discharge. & $\begin{array}{c}\text { Gage } \\
\text { height. }\end{array}$ & Discharge. \\
\hline $\begin{array}{r}\text { Feet. } \\
0.90 \\
1.00 \\
1.10 \\
1.20 \\
1.30 \\
1.40 \\
1.50\end{array}$ & $\begin{array}{l}\text { Second-feet. } \\
5 \\
8 \\
11 \\
15 \\
20 \\
27 \\
36\end{array}$ & $\begin{array}{r}\text { Feet. } \\
1.60 \\
1.70 \\
1.80 \\
1.90 \\
2.00 \\
2.10 \\
2.20\end{array}$ & \begin{tabular}{|c} 
Second-feet \\
48 \\
64 \\
84 \\
108 \\
137 \\
170 \\
208
\end{tabular} & $\begin{array}{r}\text { Feet. } \\
2.30 \\
2.40 \\
2.50 \\
2.60 \\
2.70 \\
2.80 \\
2.90\end{array}$ & \begin{tabular}{|c|} 
Second-feet. \\
252 \\
301 \\
355 \\
415 \\
480 \\
550 \\
625
\end{tabular} & $\begin{array}{l}\text { Feet. } \\
3.00 \\
3.10 \\
3.20 \\
3.30 \\
3.40 \\
3.50\end{array}$ & $\begin{array}{r}\text { Second-feet. } \\
700 \\
780 \\
865 \\
955 \\
1,045 \\
1,140\end{array}$ \\
\hline
\end{tabular}

SEPTEMBER 11, 1905, TO JULY 15, 1906.c

\begin{tabular}{|l|l||l|l||l|l|l|l|}
\hline 0.90 & 10 & 1.70 & 115 & 2.50 & 495 & 3.30 & 1,150 \\
1.00 & 15 & 1.80 & 145 & 2.60 & 560 & 3.40 & 1,250 \\
1.10 & 21 & 1.90 & 180 & 2.70 & 630 & 3.50 & 1,350 \\
1.20 & 29 & 2.00 & 220 & 2.80 & 705 & 3.60 & 1,455 \\
1.30 & 39 & 2.10 & 265 & 2.90 & 785 & 3.70 & 1,560 \\
1.40 & 52 & 2.20 & 315 & 3.00 & 870 & 3.80 & 1,670 \\
1.50 & 69 & 2.30 & 370 & 3.10 & 960 & 3.90 & 1,780 \\
1.60 & 90 & 2.40 & 430 & 3.20 & 1,055 & 4.00 & 1,895 \\
\hline
\end{tabular}

a This table is based on one discharge measurement made during 1905 and the form of the 1906 curve. It is not well defined.

$b$ This table is based on three discharge measurements made during 1905 and the form of the 1906 curve. $1 \mathrm{t}$ is fairly well defined between gage heights 1.5 feet and 2.0 feet.

c This table is strictly applicable only for open-channel conditions. It is based on five discharge measurements made during 1905 and 1906 . It is fairly well defined between gage heights 1.3 feet and 3.5 feet.

Estimated monthly discharge of Georges Creek at Westernport, Md.

[Drainage area, 76 square miles.]

\begin{tabular}{|c|c|c|c|c|c|}
\hline \multirow[b]{2}{*}{ - Month. } & \multicolumn{3}{|c|}{ Discharge in second-feet. } & \multicolumn{2}{|c|}{ Run-off. } \\
\hline & Maximum. & Minimum. & Mean. & $\begin{array}{c}\text { Second-feet } \\
\text { per } \\
\text { square mile. }\end{array}$ & $\begin{array}{l}\text { Depth in } \\
\text { inches. }\end{array}$ \\
\hline May 4-31,.. & 92 & 26 & 44.8 & 0.589 & \\
\hline $\begin{array}{l}\text { May } 4-\delta 1 \ldots \\
\text { June . . . . . }\end{array}$ & $\begin{array}{r}92 \\
154\end{array}$ & $\begin{array}{l}20 \\
15\end{array}$ & $\begin{array}{l}44.8 \\
45.7\end{array}$ & $\begin{array}{r}0.089 \\
.601\end{array}$ & $\begin{array}{r}0.613 \\
.670\end{array}$ \\
\hline July .. & 1,092 & 13 & 127 & 1.67 & 1.92 \\
\hline August ..... & 68 & 6 & 15.1 & .199 & .229 \\
\hline September.. & 528 & 8 & 46.4 & .611 & .682 \\
\hline October. & 370 & 12 & 65.8 & .866 & 998 \\
\hline November.... & 102 & 12 & 33.8 & .445 & .496 \\
\hline December..... & 1,250 & 39 & 267 & 3.51 & 4. 05 \\
\hline 1906. & & & & & \\
\hline January....... & 1,131 & 80 & 320 & 4. 21 & 4.85 \\
\hline $\begin{array}{l}\text { February ...... } \\
\text { March...... }\end{array}$ & $\begin{array}{r}145 \\
300\end{array}$ & $\begin{array}{l}39 \\
42\end{array}$ & 260 & .937 & .976 \\
\hline April......... & $\begin{array}{l}1,200 \\
1,036\end{array}$ & 115 & $\begin{array}{l}200 \\
378\end{array}$ & $\begin{array}{l}3.42 \\
4.97\end{array}$ & $\begin{array}{l}\text { 5. } 94 \\
5.54\end{array}$ \\
\hline May.. & 315 & 21 & 83.0 & 1.09 & 1.26 \\
\hline June. & 342 & 29 & 91.4 & 1.20 & 1. 34 \\
\hline July $1-$ & 27 & 16 & 20.8 & .274 & .153 \\
\hline
\end{tabular}


WILLS CREEK AT CUMBERLAND, MD.

Wills Creek rises on the western slope of Savage Mountain, in the southeastern part of Somerset County, Pa., and flows northwestward to Mance, eastward to Hyndman, Pa., and southward to Cumberland, Md., where it enters North Branch of Potomac River. Its length is about 25 miles.

The gaging station was established May 5, 1905, and was discontinued.July 15,1906 . It is located at the highway bridge at the upper end of "The Narrows," Cumberland, Md.

The channel is straight for 200 feet above and 500 feet below the station. The current is fairly swift. Both banks are high and do not overflow. The bed of the stream is rocky, very rough, and permanent. There are two channels at all but very low stages.

Discharge measurements were made from the downstream side of the two-span bridge to which the gage is fastened. The initial point for soundings is the face of the right abutment.

A standard chain gage is fastened to the downstream side of the bridge, near the middle of the right span. The length of the chain from the end of the weight to the marker is 26.98 feet. The gage was read twice each day by $\mathrm{H}$. E. McKenzie. Bench mark No. 1 is a square chisel draft on the top of the bridge-seat stone at the downstream side of the right abutment. Its elevation is 21.88 feet above the datum of the gage. Bench mark No. 2 is the top of the pulley wheel of the gage. Its elevation was 26.65 feet above the datum of the ga'ge March 17, 1906.

Estimates are considered to be within 5 per cent of the true flow. Ice conditions probably did not affect the flow during the winter of $1905-6$.

Discharge measurements of Wills Creek at Cumberland, Md.

\begin{tabular}{|c|c|c|c|c|c|}
\hline Date. & $\begin{array}{c}\text { Gage } \\
\text { height. }\end{array}$ & Discharge. & Date. & $\begin{array}{c}\text { Gage } \\
\text { height. }\end{array}$ & Discharge. \\
\hline 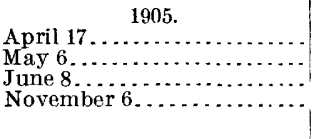 & $\begin{array}{l}\text { Feet. } \\
4.20 \\
\quad 3.72 \\
\quad 4.22 \\
3.68\end{array}$ & \begin{tabular}{r|} 
Second-feet. \\
236 \\
130 \\
248 \\
130
\end{tabular} & 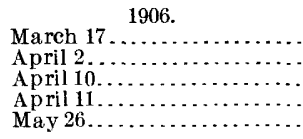 & $\begin{array}{l}\text { Feet. } \\
4.01 \\
6.32 \\
6.05 \\
5.88 \\
3.27\end{array}$ & $\begin{array}{r}\text { Second-feet. } \\
175 \\
1,486 \\
1,200 \\
1,066 \\
55\end{array}$ \\
\hline
\end{tabular}


Daily gage height, in feet, of Wills Creek at Cumberland, Md.

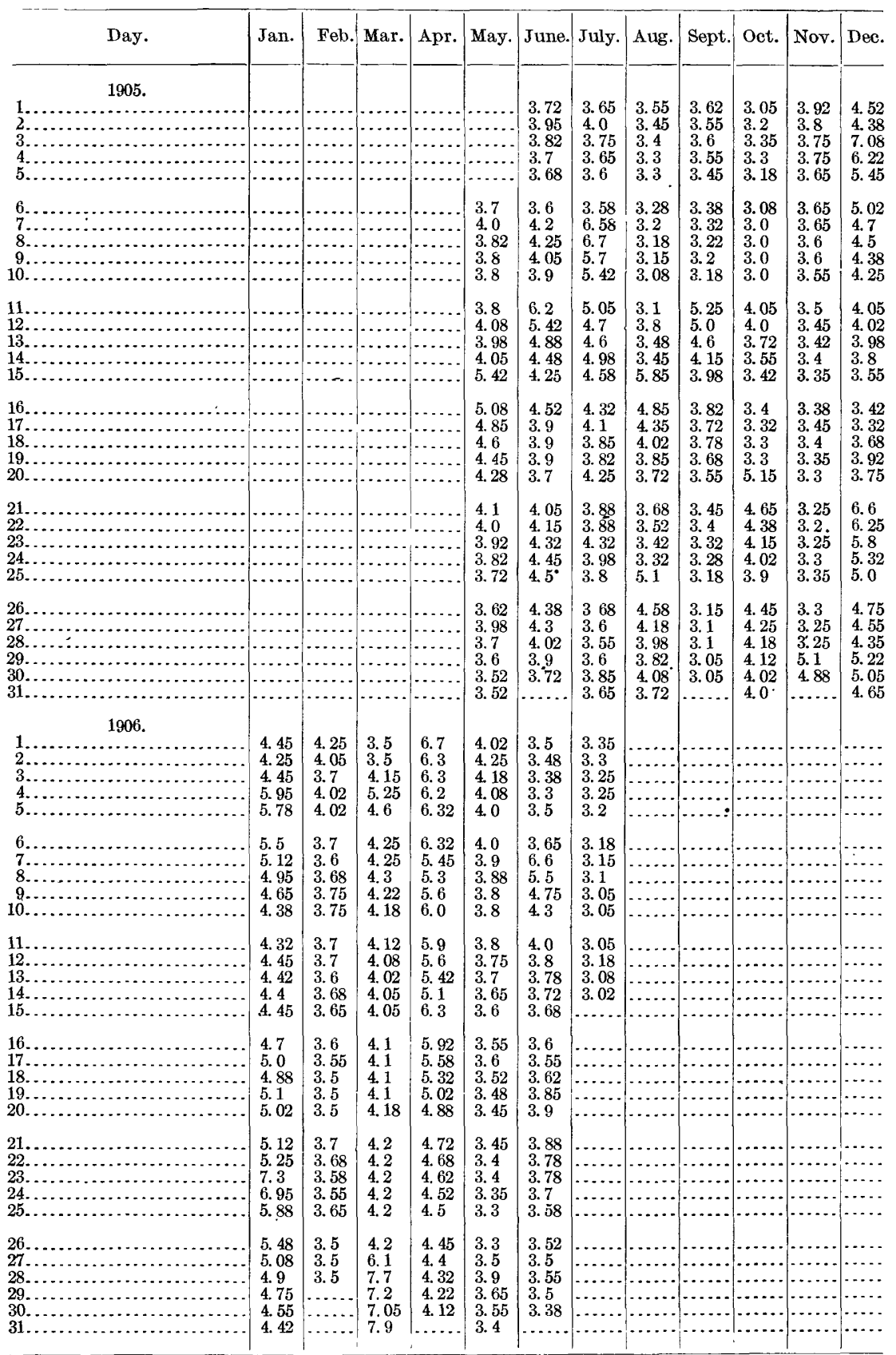

IRR $192-() 7--5$ 
Rating table for Wills Creek at Cumberland, Md., from May 6, 1905, to july 14, 1906.a

\begin{tabular}{|r|r||r|r|r|r||r|r|}
\hline $\begin{array}{c}\text { Gage } \\
\text { height. }\end{array}$ & Discharge. & $\begin{array}{c}\text { Gage } \\
\text { height. }\end{array}$ & Discharge. & $\begin{array}{c}\text { Gage } \\
\text { height. }\end{array}$ & Discharge. & $\begin{array}{c}\text { Gage } \\
\text { height. }\end{array}$ & Discharge. \\
\hline Feet. & Second-feet. & Feet. & Second-feet. & Feet. & Second-feet. & $\begin{array}{r}\text { Feet. } \\
3.00\end{array}$ \\
3.10 & 30 & 4.30 & 270 & 5.60 & 860 & 6.80 & Second-feet. \\
3.20 & 48 & 4.40 & 301 & 5.70 & 930 & 6.90 & 2,160 \\
3.30 & 60 & 4.60 & 334 & 5.80 & 1,005 & 7.00 & 2,295 \\
3.40 & 73 & 4.70 & 368 & 5.90 & 1,085 & 7.10 & 2,435 \\
3.50 & 88 & 4.80 & 404 & 6.00 & 1,170 & 7.20 & 2,575 \\
3.60 & 104 & 4.90 & 482 & 6.10 & 1,260 & 7.30 & 2,720 \\
3.70 & 122 & 5.00 & 525 & 6.20 & 1,355 & 7.40 & 2,870 \\
3.80 & 142 & 5.10 & 570 & 6.40 & 1,455 & 7.50 & 3,020 \\
3.90 & 164 & 5.20 & 620 & 6.50 & 1,560 & 7.60 & 3,170 \\
4.00 & 188 & 5.30 & 675 & 6.60 & 1,785 & 7.70 & 3,320 \\
4.10 & 214 & 5.40 & 735 & 6.70 & 1,905 & 7.90 & 3,480 \\
4.20 & 241 & 5.5 & 795 & & & & \\
& & & & & & & \\
\hline
\end{tabular}

a This table is strictly applicable only for open-channel conditions. It is based on nine discharge measurements made during 1905 and 1906. It is well defined between gage heights 3.0 feet and 6.5 feet.

Estimated monthly discharge of Wills Creek at Cumberland, $M d$.

[Drainage area, 240 square miles.]

\begin{tabular}{|c|c|c|c|c|c|}
\hline \multirow[b]{2}{*}{ Month. } & \multicolumn{3}{|c|}{ Discharge in second-feet. } & \multicolumn{2}{|c|}{ Run-off. } \\
\hline & Maximum. & Minimum. & Mean. & $\begin{array}{c}\text { Second-feet } \\
\text { per square } \\
\text { mile. }\end{array}$ & $\begin{array}{l}\text { Depth in } \\
\text { inches. }\end{array}$ \\
\hline May $6-31$ & & & & & \\
\hline May $6-31 \ldots \ldots \ldots \ldots \ldots$ & $\begin{array}{r}747 \\
1.355\end{array}$ & $\begin{array}{r}91 \\
104\end{array}$ & $\begin{array}{l}221 \\
272\end{array}$ & 0.921 & $\begin{array}{l}0.891 \\
1.26\end{array}$ \\
\hline July... & $\begin{array}{l}1,000 \\
1,905\end{array}$ & 96 & 353 & 1.47 & 1.70 \\
\hline August. & 1,045 & 36 & 174 & .725 & .836 \\
\hline September.. & 647 & 34 & 128 & .533 & .595 \\
\hline October..... & 595 & 30 & 149 & .621 & .716 \\
\hline November. . & 570 & 48 & 115 & .479 & .534 \\
\hline December........ & 2,407 & 63 & 529 & 2.20 & 2.54 \\
\hline 1906. & & 256 & 637 & 265 & 3.06 \\
\hline 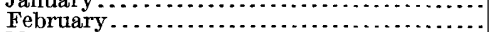 & 256 & 88 & 122 & .508 & $\begin{array}{l}.00 \\
.529\end{array}$ \\
\hline Mareh..... & 3,640 & 88 & 626 & 2.61 & 3.01 \\
\hline April...... & 1,905 & 219 & 806 & 3.36 & 3.75 \\
\hline May. & 256 & 60 & 126 & .525 & .605 \\
\hline June. & 1,785 & 60 & 207 & .862 & .962 \\
\hline July 1-14. & & & 44.6 & .186 & .097 \\
\hline
\end{tabular}

NORTH BRANCH OF POTOMAC RIVER AT CUMBERLAND, MD, $a$

Gage-height records were obtained at this station from June 11, 1894, to November 20,1897. The gage was located about 1,000 feet below the mouth of Wills Creek and consisted of a vertical rod 10 feet long, bolted to the east side of the abutment of the head-gate of the eastern feeder of the Chesapeake and Ohio Canal, just above the diversion dam. The top of the rod, or the 10.00-foot mark, was 5.40 feet below the top of the abutment. The crest of the dam was at elevation about 2.65 feet above the datum of the gage, hence for stages below that point no water passed the gaging section, which was below the dam, all flow being diverted to the canal. Discharge measurements of the river were made from the West Virginia Central Railroad bridge, about 200 yards below the dam. The channel is straight both above

$a$ For description of Weather Bureau station maintained at this point see p. 42 . 
and below the bridge. The left bank does not overflow; the right bank is liable to overflow at times of high water. The bed of the stream is composed of bowlders and loose rocks and is not liable to change. At high water the section is fairly smooth and the velocity high. At low water rocks, riffles, and angular currents appear, making it difficult to obtain accurate discharge measurements. Measurements of the canal feeders were also made near the hèad-gates.

All estimates previously published for this station have been revised. The monthly discharge as given in the accompanying table is for the section at the bridge only, the flow diverted to the canal feeders not being included, as it is an uncertain function of the total discharge of the river. (See discharge measurements.) No statement of the runoff is given, because the flow in the canal is a large percentage of the total run-off of the drainage basin above Cumberland.

Owing to the poor conditions at this station the measurements plot somewhat erratically, but since the rating curve is defined by a large number of measurements the monthly estimates which are based on it are probably within 10 per cent of the true results for normal conditions of flow above gage height 3.0 feet. Estimates for stages below 3.0 feet are somewhat uncertain. Ice conditions at this station were not recorded by the observer. It is probable, however, that ice collecting at the crest of the dam may at times have affected the gage heights, and hence the estimates.

Discharge measurements of North Branch of Potomac River at Cumberlaind, Md.

\begin{tabular}{|c|c|c|c|c|c|c|c|}
\hline Date. & $\begin{array}{c}\text { Gage } \\
\text { height. }\end{array}$ & $\begin{array}{l}\text { Discharge } \\
\text { of river. }\end{array}$ & $\begin{array}{l}\text { Discharge } \\
\text { of canal } \\
\text { feeders. }\end{array}$ & Date. & $\begin{array}{c}\text { Gage } \\
\text { height. }\end{array}$ & $\begin{array}{c}\text { Discharge } \\
\text { of river. }\end{array}$ & $\begin{array}{l}\text { Di scharge } \\
\text { of canal } \\
\text { feeders. }\end{array}$ \\
\hline $\begin{array}{r}1894 . \\
\text { May } 24 \ldots . .\end{array}$ & Feet. & $\begin{array}{r}\text { Second-feet. } \\
3,037\end{array}$ & Second-feet. & $\begin{array}{c}1896 . \\
\text { June } 24 \ldots \ldots \ldots \\
\text { August } 6 \ldots \ldots\end{array}$ & $\begin{array}{l}\text { Feet. } \\
\text { 3. } 31 \\
3.30\end{array}$ & $\begin{array}{r}\text { Second-feet } \\
696 \\
552\end{array}$ & $\begin{array}{r}\text { Second-feet. } \\
126 \\
54\end{array}$ \\
\hline $\begin{array}{r}1895 . \\
\text { March } 30 \ldots\end{array}$ & 4.50 & 3,446 & & November $18 .$. & $\begin{array}{l}\text { D. } 30 \\
3.38\end{array}$ & 548 & 04 \\
\hline April $10 \ldots$ & 5. 40 & 6,054 & (n...... & 1897. & & & \\
\hline April 25. & 3. 30 & 630 & ...... & February $10 \ldots$ & 3. 75 & 1,307 & \\
\hline May 3. & 3.75 & 1,728 & ....... & March 27 & 3.93 & 1,971 & 24 \\
\hline May 9. & 3. 40 & 777 & ........ & June 25 . & 3. 00 & 289 & 136 \\
\hline May 23.. & 3.40 & 831 & 45 & September $1 \ldots$ & 2.60 & 0 & 86 \\
\hline June 5. & 2.95 & 216 & 40 & September 22. & 2.70 & 7 & 85 \\
\hline June 6 . & 3.10 & 530 & 79 & & & & \\
\hline July 1 & $\begin{array}{l}0.00 \\
3.05\end{array}$ & $\begin{array}{l}149 \\
266\end{array}$ & $\begin{array}{l}50 \\
79\end{array}$ & May $12 \ldots 9$. & 3.80 & 1,659 & 170 \\
\hline
\end{tabular}


Daily gage height, in feet, of North Branch of Potomac River at Cumberland, Md.

\begin{tabular}{|c|c|c|c|c|c|c|c|c|c|c|c|c|}
\hline D & an. & eb. & Mar. & Apr. & [ay. & June. & July. & Aug. & Sept. & Oct. & Nov. & Dec. \\
\hline $\begin{array}{l}1 \\
2 \ldots \ldots \ldots \\
3 . \ldots \ldots \\
4 \ldots \ldots \\
5 \ldots \ldots\end{array}$ & & & & & & $\cdots$ & $\begin{array}{l}2.9 \\
2.9 \\
2.9 \\
2.8 \\
2.8\end{array}$ & $\begin{array}{l}3.0 \\
3.0 \\
3.1 \\
3.1 \\
2.9\end{array}$ & $\begin{array}{r}0.7 \\
.5 \\
.6 \\
.6 \\
.5\end{array}$ & $\begin{array}{l}1.7 \\
3.0 \\
2.8 \\
2.7 \\
2.5\end{array}$ & $\begin{array}{l}3.5 \\
3.5 \\
3.3 \\
3.0 \\
2.9\end{array}$ & $\begin{array}{l}2.9 \\
3.1 \\
3.1 \\
3.3 \\
3.2\end{array}$ \\
\hline $\begin{array}{l}7 . . \\
8 .- \\
9 . \\
10 .\end{array}$ & & & & & & & $\begin{array}{l}2.8 \\
2.8 \\
2.8 \\
2.8 \\
2.8\end{array}$ & $\begin{array}{l}2.8 \\
2.8 \\
2.6 \\
2.5 \\
2.1\end{array}$ & $\begin{array}{l}1.0 \\
1.0 \\
1.1 \\
1.1 \\
1.1\end{array}$ & $\begin{array}{l}2.3 \\
1.7 \\
1.5 \\
1.6 \\
1.9\end{array}$ & $\begin{array}{l}3.0 \\
3.0 \\
3.0 \\
3.1 \\
3.1\end{array}$ & $\begin{array}{l}3.2 \\
3.1 \\
3.1 \\
3.0 \\
3.0\end{array}$ \\
\hline 5. & & & & & & $\begin{array}{l}3.5 \\
3.4 \\
3.4 \\
3.3 \\
3.3\end{array}$ & $\begin{array}{l}2.7 \\
2.6 \\
2.6 \\
2.7 \\
2.6\end{array}$ & $\begin{array}{l}2.0 \\
2.1 \\
2.0 \\
3.0 \\
3.0\end{array}$ & $\begin{array}{l}1.0 \\
1.0 \\
1.2 \\
1.2 \\
1.3\end{array}$ & $\begin{array}{l}2.0 \\
3.0 \\
2.9 \\
2.9 \\
2.9\end{array}$ & $\begin{array}{l}3.2 \\
3.1 \\
3.1 \\
3.1 \\
3.0\end{array}$ & $\begin{array}{l}3.2 \\
3 . \\
4 . \\
4 . \\
3 .\end{array}$ \\
\hline 19. & & & & & & $\begin{array}{l}\text { 3. } 3 \\
\text { 3. } 3 \\
3.3 \\
3.3 \\
3.2\end{array}$ & $\begin{array}{l}2.5 \\
2.4 \\
2.3 \\
2.2 \\
2.0\end{array}$ & $\begin{array}{l}2.9 \\
2.8 \\
2.7 \\
2.0 \\
1.9\end{array}$ & $\begin{array}{l}1.4 \\
1.7 \\
2.9 \\
2.9 \\
3.1\end{array}$ & $\begin{array}{l}2.9 \\
2.9 \\
2.9 \\
2.8 \\
2.8\end{array}$ & $\begin{array}{l}3.0 \\
3.0 \\
3.0 \\
2.3 \\
2.3\end{array}$ & $\begin{array}{l}3.2 \\
3.2 \\
3.1 \\
3.1 \\
3.0\end{array}$ \\
\hline $\begin{array}{l}21 . \\
22 . \\
23 . \\
24 . \\
25 .\end{array}$ & & & & & & $\begin{array}{l}\text { 3. } 1 \\
\text { 3. } 1 \\
\text { 3. } 4 \\
\text { 3. } 4 \\
\text { 3. } \\
\end{array}$ & $\begin{array}{l}1.9 \\
1.5 \\
2.0 \\
3.0 \\
3.0\end{array}$ & $\begin{array}{l}1.9 \\
2.5 \\
3.0 \\
2.8 \\
2.8\end{array}$ & $\begin{array}{l}3.2 \\
3.2 \\
2.9 \\
2.9 \\
2.8\end{array}$ & $\begin{array}{l}2.7 \\
2.7 \\
2.7 \\
2.2 \\
1.9\end{array}$ & $\begin{array}{l}3.1 \\
3.0 \\
3.0 \\
3.0 \\
3.2\end{array}$ & $\begin{array}{l}3.1 \\
3 . \\
2.9 \\
3.1 \\
3 .\end{array}$ \\
\hline $\begin{array}{l}26 . \\
27 . \\
28 . \\
29 . \\
30 .\end{array}$ & & & & & & $\begin{array}{l}\text { 3. } 3 \\
\text { 3. } 3 \\
\text { 3. } 3 \\
\text { 3. } 2 \\
\text { 3. } 0\end{array}$ & $\begin{array}{l}2 . \\
2 . \\
2 . \\
2 .\end{array}$ & $\begin{array}{r}2.5 \\
1.9 \\
1.8 \\
1.3 \\
1.0 \\
.9\end{array}$ & $\begin{array}{l}2.7 \\
2.7 \\
2.5 \\
2.0 \\
1.8\end{array}$ & $\begin{array}{l}1 . \\
1 . \\
1 . \\
1 . \\
1 .\end{array}$ & $\begin{array}{l}\text { 3. } 1 \\
3.0 \\
3.0 \\
3.0 \\
3.0\end{array}$ & $\begin{array}{l}\text { 3. } \\
3.0 \\
3.1 \\
3.1 \\
3.1 \\
3.1\end{array}$ \\
\hline $\begin{array}{l}1 \\
2 \ldots \ldots \\
3 . \ldots \ldots \\
4 . \ldots \ldots \\
5 . \ldots \ldots \\
\end{array}$ & $\begin{array}{l}3.0 \\
3.0 \\
3.0 \\
3.0 \\
3.0\end{array}$ & $\begin{array}{l}3.6 \\
3.6 \\
3.5 \\
3.5 \\
3.5\end{array}$ & $\begin{array}{l}6.0 \\
5.7 \\
4.9 \\
4.6 \\
4.2\end{array}$ & $\begin{array}{l}3.9 \\
3.8 \\
4.4 \\
3.9 \\
3.8\end{array}$ & $\begin{array}{l}3.4 \\
3.8 \\
3.8 \\
3.6 \\
3.6\end{array}$ & $\begin{array}{l}2.9 \\
2.9\end{array}$ & $\begin{array}{l}\text { 3. } 3 \\
\text { 3. } 1 \\
\text { 3. } 1\end{array}$ & $\begin{array}{l}2.7 \\
2.7 \\
2.7\end{array}$ & $\begin{array}{r}1.0 \\
1.0 \\
1.0 \\
.9 \\
.9\end{array}$ & 2 & & $\begin{array}{l}\cdots \cdots \\
\cdots \cdots \\
\cdots \cdots \\
\cdots\end{array}$ \\
\hline $\begin{array}{r}6 \ldots \ldots \\
7 \ldots \ldots \\
8 \ldots \ldots \\
9 \ldots \ldots \\
10 \ldots .\end{array}$ & $\begin{array}{l}3.1 \\
4.8 \\
6.0 \\
4.8 \\
4.7\end{array}$ & $\begin{array}{l}3.5 \\
3.5 \\
3.5 \\
3.5 \\
3.5\end{array}$ & $\begin{array}{l}4 . \\
3.8 \\
3.8 \\
4 . \\
4 .\end{array}$ & $\begin{array}{l}3 . \\
3 . \\
3 . \\
5 . \\
5 .\end{array}$ & $\begin{array}{l}3.4 \\
3.3 \\
3.2 \\
3.4 \\
3.2\end{array}$ & 3.0 & $\begin{array}{l}3.2 \\
3.2 \\
3.3 \\
3.7\end{array}$ & $\begin{array}{l}2 . \\
2 . \\
2 . \\
2 .\end{array}$ & $\begin{array}{l}.9 \\
.8 \\
.8 \\
.7 \\
.5\end{array}$ & $\cdots$ & & $\begin{array}{l}\cdots \ldots \\
\cdots \\
\cdots \\
\cdots \\
\cdots \\
\cdots\end{array}$ \\
\hline $\begin{array}{l}\cdots \\
\cdots \\
\cdots\end{array}$ & $\begin{array}{l}4.1 \\
3.8 \\
3.5 \\
3.5 \\
3.5\end{array}$ & $\begin{array}{l}3.5 \\
3.4 \\
3.4 \\
3.4 \\
3.4\end{array}$ & $\begin{array}{l}4 . \\
3.8 \\
3.8 \\
4 . \\
4 .\end{array}$ & $\begin{array}{l}4.7 \\
4.7 \\
3.9 \\
3.8 \\
3.8\end{array}$ & $\begin{array}{l}3.2 \\
3.7 \\
3.7 \\
3.7 \\
3.5\end{array}$ & $\begin{array}{l}\text { 3. } 3 \\
\text { 3. } 3\end{array}$ & $\begin{array}{l}3.2 \\
3.2 \\
3.3 \\
3.3\end{array}$ & $\begin{array}{l}2.4 \\
2.4 \\
2.3 \\
2.2\end{array}$ & $\begin{array}{r}.5 \\
.6 \\
1.1 \\
1.2 \\
1.0\end{array}$ & ... & $\ldots$. & $\begin{array}{l}\cdots \cdot \\
\cdots \cdots \\
\cdots \cdots \\
\cdots \\
\cdots \\
\cdots\end{array}$ \\
\hline …… & $\begin{array}{l}3.9 \\
3.9 \\
3.9 \\
3.8 \\
3.8\end{array}$ & $\begin{array}{l}3.3 \\
3.4 \\
3.4 \\
3.4 \\
3.4\end{array}$ & $\begin{array}{l}5 . \\
4 . \\
4 . \\
4 . \\
4 .\end{array}$ & $\begin{array}{l}3.8 \\
3.7 \\
3.6 \\
3.5\end{array}$ & $\begin{array}{l}3.4 \\
3.4 \\
3.4 \\
3.3\end{array}$ & $\begin{array}{l}\text { 3. } 0 \\
3.0\end{array}$ & $\begin{array}{l}3.0 \\
3.0 \\
2.9 \\
2.9\end{array}$ & $\begin{array}{l}2.0 \\
1.9 \\
1.8 \\
1.7\end{array}$ & $\begin{array}{l}.9 \\
.9 \\
.8 \\
.7 \\
.5\end{array}$ & $\cdots$ & & $\begin{array}{l}\cdots \\
\cdots \\
\cdots \\
\cdots\end{array}$ \\
\hline $\begin{array}{l}23 \ldots \ldots \\
24 \ldots \ldots \\
25 \ldots \ldots\end{array}$ & $\begin{array}{l}3.9 \\
4.6 \\
4.0 \\
3.7 \\
3.6\end{array}$ & $\begin{array}{l}3.4 \\
3.4 \\
3.4 \\
3.4 \\
3.4\end{array}$ & $\begin{array}{l}3.8 \\
3 . \\
3 . \\
3.7 \\
3 .\end{array}$ & $\begin{array}{l}3.4 \\
3.4 \\
3.3 \\
3.3 \\
3.2\end{array}$ & $\begin{array}{l}3.4 \\
3.4 \\
3.4 \\
3.3\end{array}$ & $\begin{array}{l}3.0 \\
3.0 \\
2.9\end{array}$ & $\begin{array}{l}2.7 \\
2.7 \\
2.7 \\
2.7\end{array}$ & $\begin{array}{l}1.0 \\
1.3 \\
1.2 \\
1.1 \\
1.0\end{array}$ & $\begin{array}{l}.4 \\
.3 \\
.2 \\
.1 \\
\text { (a) }\end{array}$ & $\ldots$ & & $\begin{array}{l}(a) \\
3.0 \\
3.2 \\
2.9 \\
2.9\end{array}$ \\
\hline $\begin{array}{l}26 \ldots \\
27 \ldots \\
28 \ldots \\
29 \ldots \\
30 \ldots \\
30\end{array}$ & $\begin{array}{l}3.5 \\
3.5 \\
3.4 \\
3.4 \\
3.7 \\
3.7\end{array}$ & $\begin{array}{c}3.6 \\
4.1 \\
5.0 \\
\cdots \cdots\end{array}$ & $\begin{array}{l}4.5 \\
4.3 \\
4.3 \\
5.1 \\
4.5 \\
4.5\end{array}$ & $\begin{array}{l}3.3 \\
3.3 \\
3.3 \\
3.3 \\
3.4\end{array}$ & $\begin{array}{l}3.3 \\
3.2 \\
3.1 \\
3.1 \\
3.1\end{array}$ & \begin{tabular}{l|}
3.0 \\
3.3 \\
3.2 \\
3.0
\end{tabular} & $\begin{array}{l}2.7 \\
2.8 \\
2.9 \\
2.9 \\
2.8\end{array}$ & $\begin{array}{l}.9 \\
.9 \\
.9 \\
.9 \\
.8 \\
.9\end{array}$ & $\begin{array}{l}\cdots \\
\cdots \\
\cdots\end{array}$ & $\cdots$ & $\cdots$ & $\begin{array}{l}3.0 \\
2.9 \\
2.9 \\
2.9 \\
3.1 \\
3.0\end{array}$ \\
\hline
\end{tabular}

$a$ Water surface below gage zero September 25 to December 21, 1895. 
Daily gage height, in feet, of North Branch of Potomac River at Cumberland, Md.-Cont'd.

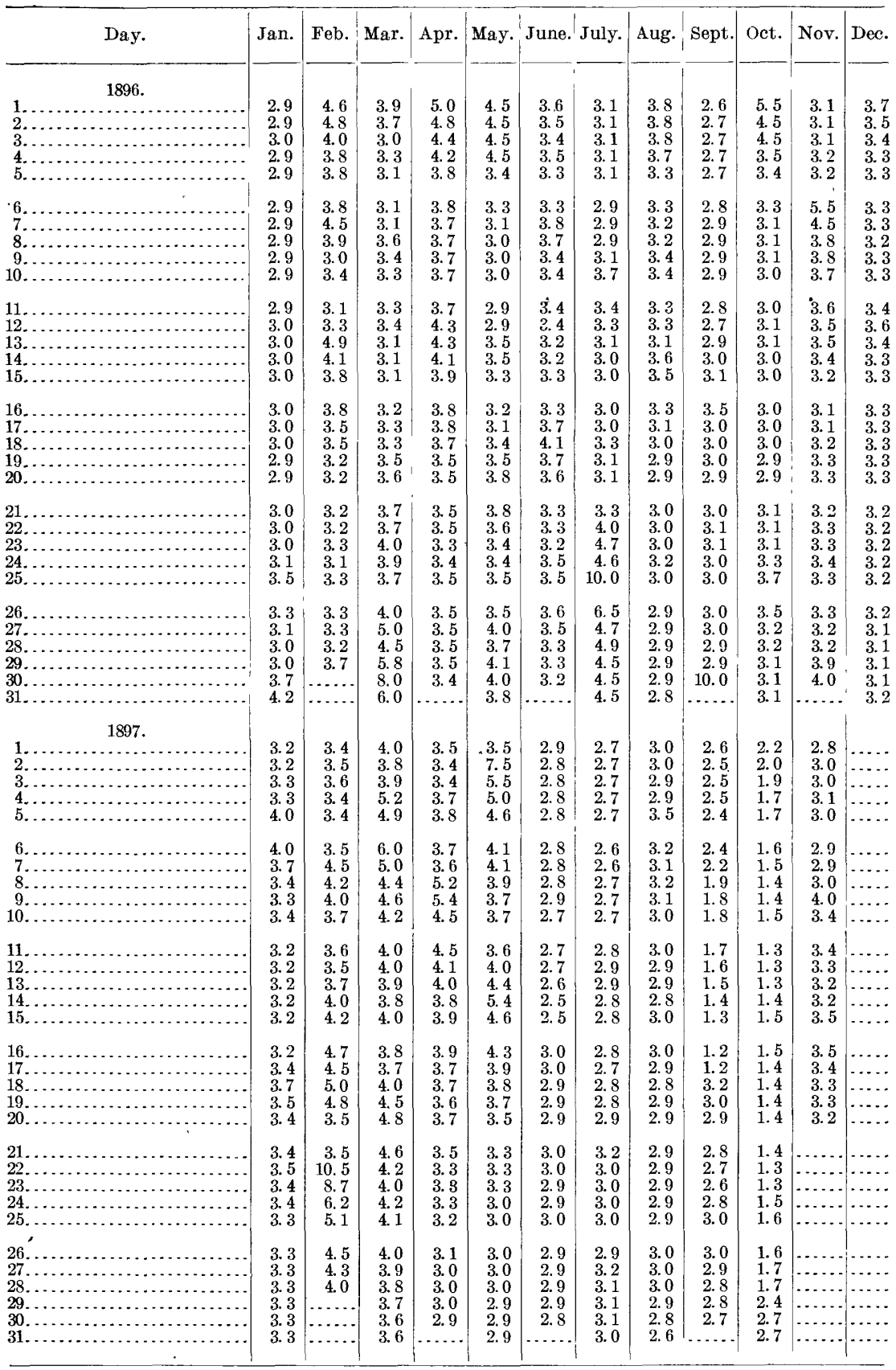


Rating table for North Branch of Potomac River at Cumberland, Md., from June 11, 189.4, to November $20,1897 . a$

\begin{tabular}{|c|r||r|r|r|r|r|r|}
\hline $\begin{array}{c}\text { Gage } \\
\text { height. }\end{array}$ & Discharge. & $\begin{array}{c}\text { Gage } \\
\text { height. }\end{array}$ & Discharge. & $\begin{array}{c}\text { Gage } \\
\text { height. }\end{array}$ & Discharge. & $\begin{array}{c}\text { Gage } \\
\text { height. }\end{array}$ & Discharge. \\
\hline Feet. & Second-feet. & Feet. & Second-feet. & Feet. & Second-feet. & Feet. & Second-feet. \\
2.65 & 0 & 3.70 & 1,420 & 4.80 & 4,280 & 5.90 & 7,590 \\
2.70 & 7 & 3.80 & 1,650 & 4.90 & 4,570 & 6.00 & 7,900 \\
2.80 & 50 & 3.90 & 1,890 & 5.00 & 4,860 & 6.50 & 9,500 \\
2.90 & 135 & 4.00 & 2,130 & 5.10 & 5,150 & 7.00 & 11,150 \\
3.00 & 235 & 4.10 & 2,380 & 5.20 & 5,450 & 7.50 & 12,850 \\
3.10 & 350 & 4.20 & 2,610 & 5.30 & 5,750 & 8.00 & 14,600 \\
3.20 & 480 & 4.30 & 2,900 & 5.40 & 6,050 & 8.50 & 16,400 \\
3.30 & 630 & 4.40 & 3,170 & 5.50 & 6,350 & 9.00 & 18,250 \\
3.40 & 800 & 4.50 & 3,440 & 5.60 & 6,660 & 9.50 & 20,150 \\
3.50 & 490 & 4.60 & 3,720 & 5.70 & 6,970 & 10.00 & 22,100 \\
3.60 & 1,200 & 4.70 & 4,000 & 5.80 & 7,280 & 10.50 & 24,100 \\
\hline
\end{tabular}

a This table is strictly applicable only for open-channel conditions. It is based on 20 discharge measurements made during 1895-1898. It is fairly well defined between gage heights 3.0 feet and 5.5 feet. Below 3.0 feet it is uncertain. The extension above 5.5 feet is probahly fairly accurate.

Estimated monthly discharge of North Branch of Potomac River at Cumberland, Md.a

\begin{tabular}{|c|c|c|c|c|c|c|c|}
\hline \multirow{2}{*}{ Month. } & \multicolumn{3}{|c|}{ Discharge in second-feet. } & \multirow{2}{*}{ Month. } & \multicolumn{3}{|c|}{ Discharge in second-fect. } \\
\hline & Maximum. & Minimum. & Mean. & & Maximum. & Minimum. & Mean. \\
\hline $\begin{array}{r}1894 . \\
\text { June } 11-30 .\end{array}$ & $9 \subset 0$ & 350 & 632 & 1896. & 2,640 & 135 & 357 \\
\hline July ....... & 235 & $\begin{array}{rl}0 & 0 \\
0 & 0\end{array}$ & 56.5 & February & $\begin{array}{l}4,570 \\
4,570\end{array}$ & 350 & $\begin{array}{r}508 \\
1,426\end{array}$ \\
\hline August.. & 350 & 0 & 77.5 & March .... & 14,600 & 235 & 2,024 \\
\hline September & 480 & 0 & 63.8 & April .. & 4,860 & 630 & 1,709 \\
\hline October ... & 235 & 0 & 47.0 & May. & $3,4: 0$ & 135 & 1,275 \\
\hline November & 990 & 0 & 323 & June... & $2,3 \varepsilon 0$ & 480 & 927 \\
\hline December. . & 4,000 & 135 & 547 & July .... & 22,100 & 135 & 2,224 \\
\hline 1895. & & & & $\begin{array}{l}\text { August ..... } \\
\text { September. }\end{array}$ & $\begin{array}{r}1,650 \\
22,100\end{array}$ & $\begin{array}{r}50 \\
0\end{array}$ & $\begin{array}{l}568 \\
916\end{array}$ \\
\hline January ... & 7,900 & 235 & 1,669 & October. & 6,350 & 135 & 820 \\
\hline February & 4,860 & 630 & 1,099 & November & 6,350 & 350 & 1,073 \\
\hline March ... & 7,900 & 1,420 & 3,094 & December. & 1,420 & 350 & 627 \\
\hline April .. & 6,660 & 480 & 1,810 & & & & \\
\hline May. & 1,650 & 350 & 853 & The year.. & 22,100 & 0 & 1,162 \\
\hline June.. & 630 & 50 & 267 & & & $==$ & \\
\hline July . & 1,420 & 7 & 348 & 1897. & & & \\
\hline August & 50 & 0 & 2.5 & January ... & 2.130 & 480 & 801 \\
\hline September & 0 & 0 & 0 & February & 24,100 & 800 & 3,770 \\
\hline October. & 0 & 0 & 0 & March .... & 7,900 & 1,200 & 2,704 \\
\hline November & 0 & 0 & 0 & April .... & 6,050 & 135 & 1,554 \\
\hline December & 480 & 0 & 71. 3 & May .... & 12,850 & 135 & 2,156 \\
\hline The year. & 7,900 & 0 & 768 & July ..... & $\begin{array}{l}235 \\
480\end{array}$ & $\begin{array}{l}0 \\
0\end{array}$ & $\begin{array}{l}103 \\
132\end{array}$ \\
\hline & & & & September... & $\begin{array}{l}990 \\
480\end{array}$ & $\begin{array}{l}0 \\
0\end{array}$ & $\begin{array}{r}215 \\
55.6\end{array}$ \\
\hline & & & & October ......... & $\begin{array}{r}7 \\
2130\end{array}$ & $\begin{array}{r}0 \\
50\end{array}$ & $572^{.45}$ \\
\hline & & & & er $1-20$. & & & \\
\hline
\end{tabular}

a These estimates do not include flow in canal feeders. 
MISCELLANEOUS DISCHARGE MEASUREMENTS IN NORTH BRANCH OF POTOMAC RIVER BASIN.

The following miscellaneous discharge measurements have beem made in the basin of North Branch of Potomac River:

Miscellaneous discharge measurements in North Branch of Potomac River drainage basin.

\begin{tabular}{|c|c|c|c|c|c|c|}
\hline Date. & Stream. & Locality. & Width. & $\begin{array}{l}\text { Irea of } \\
\text { section. }\end{array}$ & $\begin{array}{l}\text { Mean } \\
\text { veloc- } \\
\text { ity. }\end{array}$ & $\begin{array}{c}\text { Dis- } \\
\text { charge. }\end{array}$ \\
\hline Sept. 24, 1897 & Buffalo Creek......... & At mouth near Bayard, W. & Feet. & $\begin{array}{c}\text { Square } \\
\text { feet. } \\
12\end{array}$ & $\begin{array}{c}\text { Feet } \\
\text { per sec. } \\
1.92\end{array}$ & $\begin{array}{l}\text { Second- } \\
\text { feet. } \\
\text { a } 23\end{array}$ \\
\hline Sept. 23,1897 & $\begin{array}{l}\text { North Branch of Po- } \\
\text { tomac River. }\end{array}$ & Near Gormania, W. Va ...... & 61 & 74 & .73 & 54 \\
\hline July 17,1905 & ‥do $\ldots$................. & ....do................... & 67 & 65 & .58 & 38 \\
\hline July 16,1905 & Difficult Creek. & $\begin{array}{l}\text { At mouth, } 4 \text { miles below Gor- } \\
\text { mania, W. Va. }\end{array}$ & 12 & 9 & 1. 11 & 10 \\
\hline Sept. 25,1897 & Stony River. & $\begin{array}{l}200 \text { yards above mouth near } \\
\text { Schell, W. Va. }\end{array}$ & 29 & 30 & 1.27 & 38 \\
\hline July. 16,1905 & .....do. & $\begin{array}{l}500 \text { feet above bridge near } \\
\text { Gormania, W. Va. }\end{array}$ & 45 & 57 & 1.37 & 78 \\
\hline Sept 25,1897 & $\begin{array}{l}\text { North Branch of Po- } \\
\text { tomac River. }\end{array}$ & $\begin{array}{l}200 \text { yards above mouth of } \\
\text { Laurel Run near Schell, } \\
\text { W. Va. }\end{array}$ & 44 & 100 & 1.36 & 136 \\
\hline Sept. 25,1897 & Abram Creek. & $\begin{array}{l}100 \text { yards above railroad } \\
\text { bridge near Harrison, } W . \\
\text { Va. }\end{array}$ & 13 & 13 & .39 & 7.4 \\
\hline Sept. 27,1897 & $\begin{array}{l}\text { North Branch of Po- } \\
\text { tomac River. }\end{array}$ & $\begin{array}{l}100 \text { yards above mouth of } \\
\text { Savage River near Bloom- } \\
\text { ington, Md. }\end{array}$ & 36 & 62 & 1. 97 & 122 \\
\hline Oct. $\quad 27,1897$ & ....do. & $\begin{array}{l}\text { Above mouth of S a vage } \\
\text { River and below Balti- } \\
\text { more and Ohio R. R. } \\
\text { bridge near Bloomington, } \\
\text { Md. }\end{array}$ & 45 & 64 & 1.59 & 102 \\
\hline Sept. 271897 & Savage River... & $\begin{array}{l}\text { A bove junction with North } \\
\text { Branch of Potomac River } \\
\text { near Bloomington, Md. }\end{array}$ & 20 & 7.5 & .93 & $b 7$ \\
\hline Oct. $\quad 27,1897$ & ...do. & $\begin{array}{l}\frac{1}{2} \text { mile above mouth and } \\
\text { above Piedmont water sup- } \\
\text { ply intake near Blooming- } \\
\text { ton, Md. }\end{array}$ & 13 & 12 & .92 & 11 \\
\hline Sept. 28,1897 & Georges Creek. & $\begin{array}{l}\text { At Cumberland and Pennsyl- } \\
\text { vania R. R. bridge, } \\
\text { Westernport, Md. }\end{array}$ & 9 & 13 & .46 & 6 \\
\hline Sept. 291897 & New Creek.... & $\begin{array}{l}150 \text { yards above mouth near } \\
\text { Keyser. W. Va. }\end{array}$ & 9 & 4. 9 & .71 & a 3.5 \\
\hline Oct. $\quad 28,1897$ & $\begin{array}{l}\text { North Branch of } \mathrm{P}^{\prime} \mathrm{O}- \\
\text { tomac River. }\end{array}$ & Near Twenty-first, Md...... & 80 & 110 & 1.15 & 126 \\
\hline $\begin{array}{ll}\text { Oct. } & 281897 \\
\text { Sept. } & 28,1897\end{array}$ & 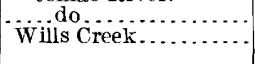 & $\begin{array}{l}\text { Near Gerstell, } \mathrm{Md} \text {......... } \\
\text { Above paper mill near cum- } \\
\text { berland, } \mathrm{M} d .\end{array}$ & $\begin{array}{r}187 \\
22\end{array}$ & $\begin{array}{r}268 \\
10\end{array}$ & $\begin{array}{r}.52 \\
1.20\end{array}$ & $\begin{array}{r}138 \\
12\end{array}$ \\
\hline May 12,1898 & $\ldots$. do & $\begin{array}{l}\text { Pulp - mill- bridge, Cumber- } \\
\text { land, } \mathbf{M} \text { d. }\end{array}$ & 86 & 262 & 1. 45 & 381 \\
\hline May 13,1898 & & $\because \ldots$ & 86 & 262 & 1. 40 & 368 \\
\hline July 18,1905 & Town Dra & $\begin{array}{l}\text { Mechanics street bridge, } \\
\text { Cumberland, Md. }\end{array}$ & 20 & 12 & 1.50 & $b 18$ \\
\hline Sept. 25,1897 & Evitts Creek. & $\begin{array}{l}200 \text { yards above railroad near } \\
\text { Cumberland, Md. }\end{array}$ & 12 & 9 & 2. 33 & 21 \\
\hline Sept. 28,1897 & Patterson Creek. & $\begin{array}{l}\text { Baltimore and ohio R. R } \\
\text { bridze near Patterson De- } \\
\text { pot, W. Va. }\end{array}$ & 14 & 5 & 2. 40 & 12 \\
\hline
\end{tabular}

$a$ Increased discharge caused by rain Sept. 23, 1897.

$b$ Discharge does not include Piedmont water supply.

c Discharge does not inslude water pumped to Baltimore and Ohio R. R. car shops arid two mills where it is used for hoiler feed. Creek stated to be exceptionally low.

$d$ Measured at $7 \mathrm{a} . \mathrm{m}$., when flow was 'argely house sewage. 


\section{SOUTH BRANCH OF POTOMAC RIVER BASIN.}

GENERAL DESCRIPTION. $a$

The bed of South Branch of the Potomac is mostly coarse gravel, the banks are of loose sediment, and on account of the sudden and local swells to which the river is subject the channel is continually changing. At no places are there falls of any magnitude. The slope of the stream is as follows:

Slope of South Branch of Potomac River.

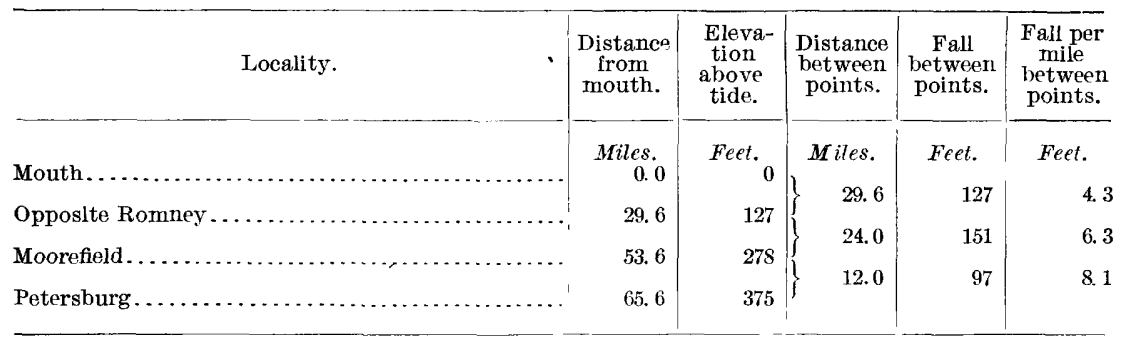

SOUTH BRANCH OF POTOMAC RIVER NEAR SPRINGFIELD, W, VA.

A gaging station was first established at the Baltimore and Ohio Railroad bridge 3 miles southwest of Springfield, by C. C. Babb, June 3,1894 . The chanmel above and below the station is straight and the water rather swift. The banks are liable to overflow at times of high water. The bed of the stream is composed of rock and gravel and is probably permanent. A wire gage 34.00 feet long was used. April 10, 1895, the gage datum was raised 1.00 foot. The bench mark was a cross cut in a broad capstone of the lower wall of the north abutment of the bridge. Its elevation was 28.18 feet above gage datum. February 29, 1896, this station was discontinued on account of difficulty in obtaining an observer.

June 26,1899 , a station was established by E. G. Paul at an iron highway bridge one-fourth mile from Grace Station and $1 \frac{1}{2}$ miles southwest of Springfield. The channel of the stream at this point is curved and the current too sluggish to make satisfactory discharge measurements, and they were therefore made at the railroad bridge where the station was originally located, $1 \frac{1}{2}$ miles above. A wire gage was used to determine the stages of the river. February 2, 1902, the highway bridge and the gage were carried away by ice.

August 28, 1903, a station was established by E. G. Paul at the steel highway bridge $2 \frac{1}{2}$ miles east of Springfield. It was discontinued July 15, 1906.

The channel is straight for several hundred feet above and below the station. Both banks are liable to overflow at very high stages of 
the river. The bed of the stream is of gravel and probably subject to some changes in conditions of flow from time to time. The bridge has two spans of 150 feet each. During high water the river flows beneath both spans, but at low stages beneath the left span only. There is a small island just above and also one below the station.

Discharge measurements were made from the bridge, to which the gage is attached. The initial point for soundings is the river face of the left abutment at the downstream side of the bridge.

A standard chain gage is located in the center of the left span on the downstream side of the bridge. The length of the chain from the end of the weight to the marker is 37.59 feet. The gage was read twice each day by James R. Blue. Bench mark No. 1 is a nail in a large sycamore tree 15 feet downstream from the left approach to the bridge. The nail is in the side of the tree away from the river and about 6 feet above the ground. Its elevation is 18.80 feet above gage datum.

The estimates given below for the station at the railroad bridge during 1894 to 1896 are essentially the same as previously published, the same rating curve being used. Some slight changes were necessary, however, on account of corrections made in the gage heights. No estimates have heretofore been made for stages below gage height 2.5 feet, but the accompanying table was extended to include a discharge of 80 second-feet at gage height 2.0 feet (the minimum gage height in 1895), on the assumption that the minimum discharge of 1895 was the same as the minimum discharge of 1904 . Comparisons of 1895 and 1904 minimums at other stations in the Potomac River basin indicate that this assumption is very nearly correct. Estimates for 1894 to 1896 are considered to be within 10 per cent of the true discharge for normal conditions of flow.

Estimates for the station at the highway bridge during 1899 to 1902, as previously published, have been revised. Estimates for stages between 4.0 feet and 8.0 feet are probably within 10 per cent of the true discharge for normal conditions of flow. At gage height 3.0 feet the probable error may be as high as 20 per cent. Estimates for stages above 8.0 feet are somewhat uncertain, especially for the winter months, owing to occasional ice gorging and backwater effects at this station. (See measurement made December 31,1901 .) However, it is considered that the probable error is less than 25 per cent at gage height 20 feet.

Estimates of flow corrected for the effect of ice conditions during ice periods from 1896 to 1902 have been made. They were based on a comparison of the flow at this station with the flow at other stations in the Potomac River basin, and should be reasonably close.

Estimates for 1903 to 1906 are considered to be within 5 per cent of the true discharge below gage height 5.0 feet. At 11.0 feet the 
error may be as high as 20 per cent. They are the same as those published in the 1905 report. Estimates for 1903 to 1906 were not corrected for ice conditions.

A summary of the records gives the following results: Maximum discharge for twenty-four hours, 19,350 second-feet; minimum discharge for twenty-four hours, 78 second-feet; mean annual discharge for four years, 1,311 second-feet; mean annual rainfall for seven years, 33.94 inches.

Discharge measurements of South Branch of Potomac River near Springfield, W. Va.

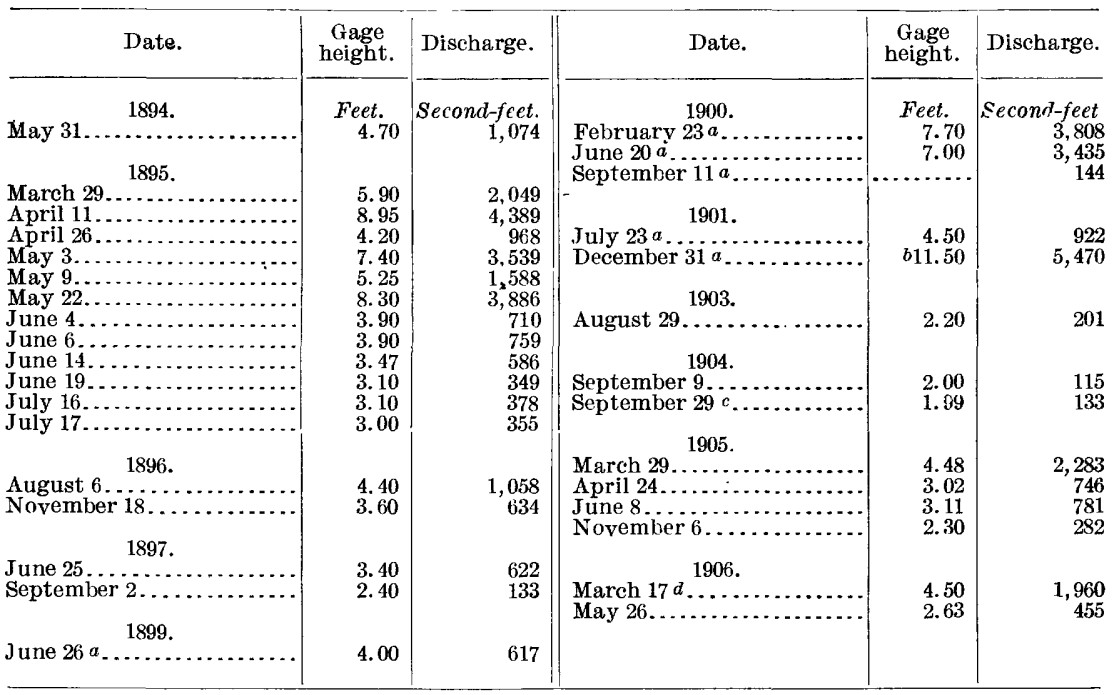

a Measurement made at railroad bridge; gage height taken from gage at highway bridge near Grace Station.

$b$ Owing to ice jam below the station, this gage height is about 2.6 feet higher than for normal conditions of flow.

$c$ By wading below station.

$d$ Discharge may be small on account of ice about the meter pivot.

Daily gage height, in feet, of South Branch of Potomac River near Springfield, W. Va.

\begin{tabular}{|c|c|c|c|c|c|c|c|c|c|c|c|}
\hline Day. & June. & July. & Aug. & Sept. & Oct. & Day. & June. & July. & Aug. & Sept. & Oct. \\
\hline 1894. $a$ & & & & & & 1894. $a$ & & & & & \\
\hline $1 \ldots$ & $\cdots$ & 3.1 & 3.2 & 2.7 & 2.6 & 17. & 3.6 & & 2.5 & 2.6 & \\
\hline & & 3.1 & 3.1 & 2.7 & 4.1 & & 3.65 & & 2.5 & 3.6 & 3.2 \\
\hline $3 \ldots$ & 4.4 & 3.1 & 2.8 & 2.6 & 3.8 & 19. & 3.9 & $\ldots$ & 2.8 & 5.7 & 3.1 \\
\hline $4 \ldots$ & 4.2 & 3.0 & 2.8 & 2.6 & 3.5 & 20. & 4.2 & $\ldots$ & 2.7 & 5.1 & 3.1 \\
\hline & 4.1 & 3.0 & 2.7 & 2.6 & 3.3 & & 3.65 & & & 4.0 & - \\
\hline 6. & 4.0 & 3.0 & 2.6 & 2.6 & 3.1 & 22 . & \begin{tabular}{|l|}
3.6 \\
\end{tabular} & 2.6 & 2.65 & 3.6 & \\
\hline & 4.5 & 3.0 & 2.5 & 2.5 & 3.0 & 23. & $\begin{array}{l}0.0 \\
3.5\end{array}$ & 3.0 & 2.0 & 3.3 & |...... \\
\hline 8. & 5.4 & 3.0 & 2.5 & 3.0 & 2.9 & 24 . & 3.4 & 3.0 & 2.6 & 3.1 & $\ldots$ \\
\hline 9. & 5.0 & 3.0 & 2.5 & 3.0 & 2.9 & 25 . & 3.4 & 2.9 & 2.6 & 3.1 & $\ldots$ \\
\hline 10. & 4.7 & 2.9 & 2.5 & 2.9 & 2.8 & 26 . & 3.3 & 2.8 & 2.6 & 3.0 & $\cdots$ \\
\hline 11. & 4.3 & 2.8 & 2.5 & 2.8 & 4.2 & 27 & 3.4 & 2.8 & 2.6 & 2.9 & .. \\
\hline 12. & 4.1 & 2.8 & 2.5 & 2.8 & 4.1 & 28 . & 3.4 & 2.8 & 2.7 & 2.9 & 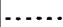 \\
\hline 13. & 3.9 & 2.8 & 3.0 & 2.7 & 3.8 & 29 . & 3.25 & 2.8 & 2.7 & 2.7 & ... \\
\hline $14 .$. & 3.85 & 2.7 & 2.8 & 2.7 & 3.6 & 30 . & 3.2 & 2.7 & 2.7 & 2.6 & $\ldots$ \\
\hline $15 \ldots$ & 3.8 & ..... & 2.7 & 2.6 & 3.5 & $31 .$. & ...... & 3.8 & 2.7 & & \\
\hline 16.. & 3.8 & & 2.6 & 2.6 & 3.4 & & & & & & \\
\hline
\end{tabular}

a 1894 gage heights have been reduced 1.00 foot to the new datum established April 10, 1895 . 
Daily gage height, in feet, of South Branch of Polomac River near Springfield, W. Va.Continued.

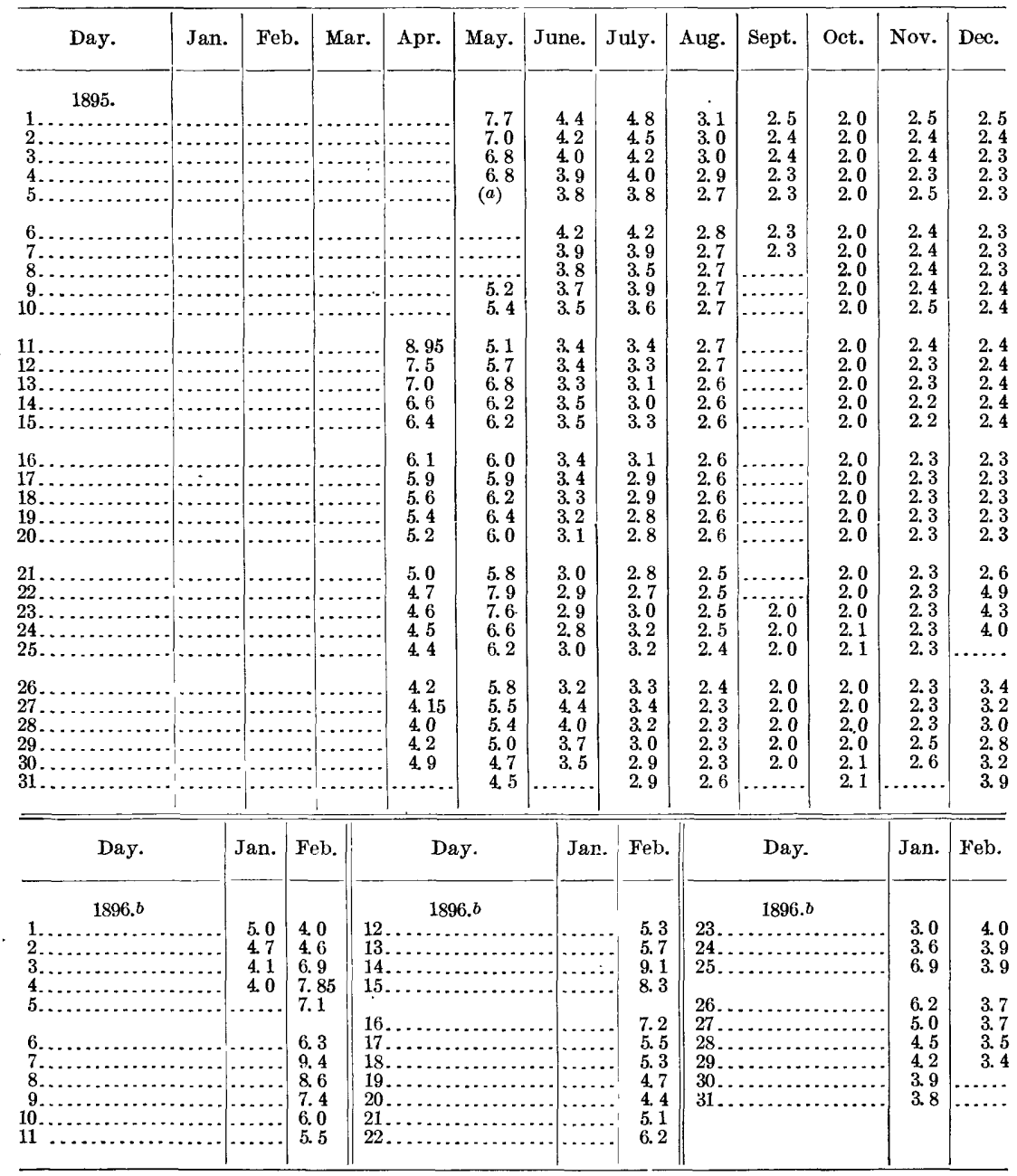

$a$ Repairing bridge; record lost May 5-8, 1895.

$b$ River frozen January 4-23, 1896. 
Daily gage height, in feet, of South Branch of Potomac River near Springfield, W. Va.Continued.

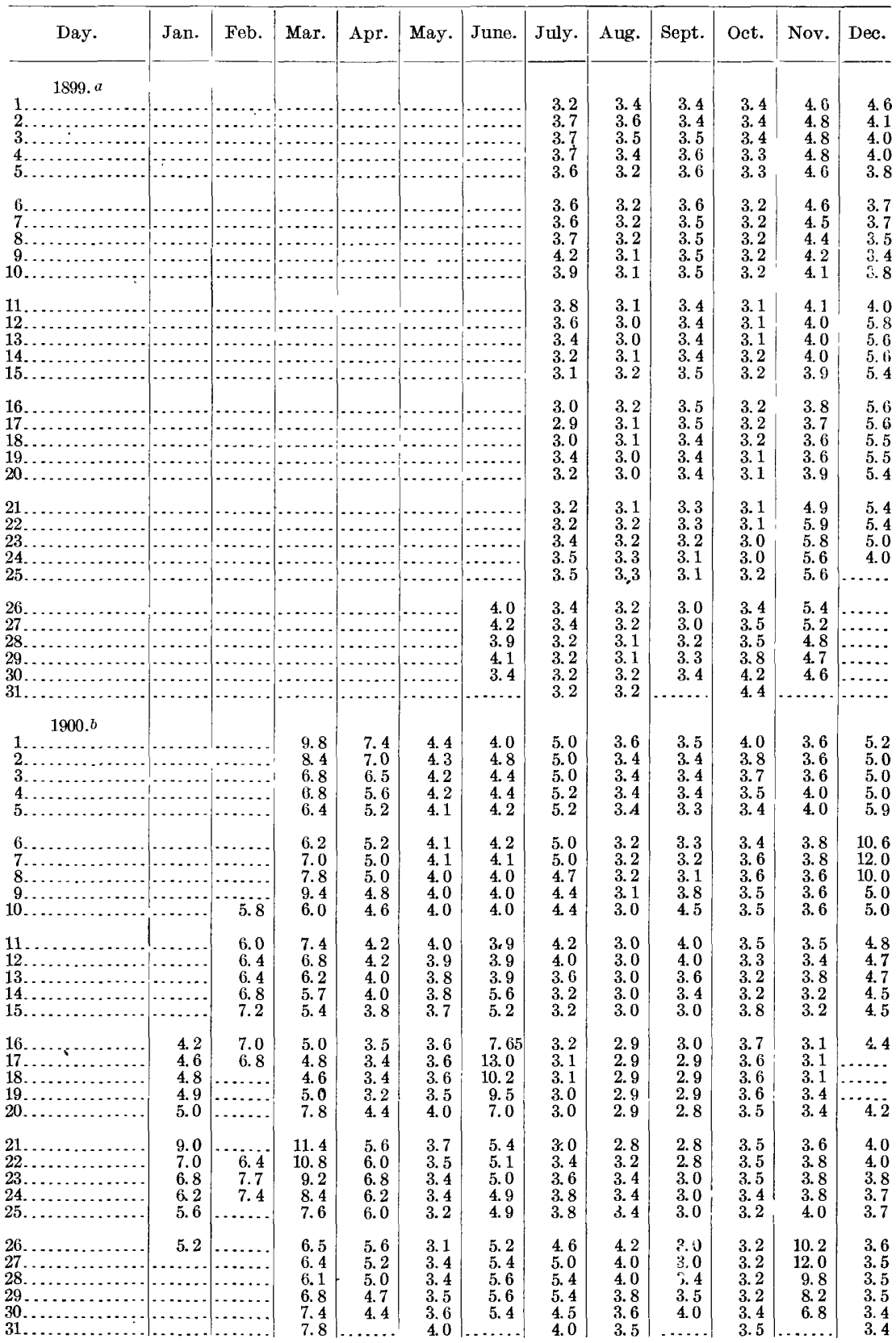

$a$ Ice conditions December 25-31, 1899. 1900 .

$b$ Ice conditions January 1-15, January 27 to February 9, February 18-21, 25-28, and December 17-19, 
STREAM FLOW: SOUTH BRANCH OF POTOMAC.

Daily gage height, in feet, of South Branch of Potomac River near Springfield, W. Va.Continued.

\begin{tabular}{|c|c|c|c|c|c|c|c|c|c|c|c|c|}
\hline Day. & Jan. & Feb. & Mar. & Apr. & May. & June. & July. & Aug. & Sept. & Oct. & Nov. & Dec. \\
\hline 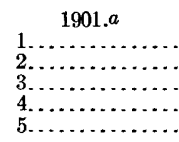 & $\begin{array}{r}3.4 \\
3.4 \\
\ldots \ldots . \\
\ldots . . \\
\ldots . . \\
\end{array}$ & $\begin{array}{l}4.0 \\
4.0\end{array}$ & $\begin{array}{l}3.8 \\
3.8 \\
5.0\end{array}$ & $\begin{array}{l}4.8 \\
4.5 \\
4.2 \\
8.2 \\
8.6\end{array}$ & $\begin{array}{l}5.8 \\
5.6 \\
5.4 \\
5.5 \\
5.0\end{array}$ & $\begin{array}{l}7.6 \\
6.8 \\
6.2 \\
5.8 \\
5.8\end{array}$ & $\begin{array}{l}5.0 \\
5.0 \\
5.4 \\
5.6 \\
5.6\end{array}$ & $\begin{array}{l}3.6 \\
3.4 \\
3.1 \\
3.1 \\
3.1\end{array}$ & $\begin{array}{l}\text { 7. } 25 \\
4.6 \\
4.4 \\
4.4 \\
4.2\end{array}$ & $\begin{array}{l}4.0 \\
4.2 \\
4.6 \\
3.8 \\
3.6\end{array}$ & $\begin{array}{r}3.2 \\
3.4 \\
3.4 \\
3.3 \\
.3 .3\end{array}$ & $\begin{array}{l}3.4 \\
3.4 \\
3.4 \\
7.0 \\
6.4\end{array}$ \\
\hline $\begin{array}{r}6 \ldots \\
7 \ldots \\
8 \ldots \\
9 \ldots \\
10 \ldots\end{array}$ & $\begin{array}{l}3.6 \\
3.6\end{array}$ & & $\begin{array}{l}4.5 \\
4.6 \\
4.8 \\
4.8\end{array}$ & $\begin{array}{l}9.4 \\
8.6 \\
8.0 \\
7.6 \\
6.8\end{array}$ & $\begin{array}{r}4.9 \\
4.5 \\
4.8 \\
6.1 \\
16.5\end{array}$ & $\begin{array}{l}6.0 \\
6.4 \\
6.2 \\
5.4 \\
5.0\end{array}$ & $\begin{array}{l}5.3 \\
5.8 \\
5.8 \\
5.4 \\
5.0\end{array}$ & $\begin{array}{l}3.6 \\
4.2 \\
4.0 \\
3.6 \\
3.2\end{array}$ & $\begin{array}{l}\text { 4. } 2 \\
4.1 \\
4.1 \\
4.1 \\
4.0\end{array}$ & $\begin{array}{l}3.6 \\
3.5 \\
3.5 \\
3.5 \\
3.4\end{array}$ & $\begin{array}{l}3.2 \\
3.2 \\
3.2 \\
3.2 \\
3.2\end{array}$ & $\begin{array}{r}6.0 \\
-4.8 \\
4.6 \\
4.5\end{array}$ \\
\hline $\begin{array}{l}11 \ldots \ldots \\
12 \ldots \ldots \\
13 \ldots \ldots \\
14 \ldots \ldots \\
15 \ldots \ldots\end{array}$ & $\begin{array}{l}3.8 \\
7.6 \\
9.2 \\
8.0 \\
6.4\end{array}$ & & $\begin{array}{r}12.2 \\
11.4 \\
9.4 \\
8.2 \\
7.4\end{array}$ & $\begin{array}{r}6.4 \\
5.8 \\
5.6 \\
6.4 \\
11.2\end{array}$ & $\begin{array}{r}12.1 \\
8.2 \\
7.4 \\
6.5 \\
6.0\end{array}$ & $\begin{array}{l}4.8 \\
4.5 \\
4.4 \\
5.3 \\
5.6\end{array}$ & $\begin{array}{l}4.8 \\
4.2 \\
4.0 \\
4.0 \\
6.0\end{array}$ & $\begin{array}{l}3.8 \\
3.8 \\
3.6 \\
3.6 \\
3.5\end{array}$ & $\begin{array}{l}4.0 \\
4.0 \\
5.2 \\
5.4 \\
5.0\end{array}$ & $\begin{array}{l}3.4 \\
3.4 \\
3.3 \\
3.3 \\
3.2\end{array}$ & $\begin{array}{l}3.1 \\
3.1 \\
3.1 \\
3.1 \\
\text { 3. } 1\end{array}$ & $\begin{array}{r}4.5 \\
4.5 \\
4.8 \\
5.0 \\
20.0\end{array}$ \\
\hline $\begin{array}{l}16 \ldots \ldots \ldots \\
17 \ldots \ldots \ldots \\
18 \ldots \ldots \\
19 \ldots \ldots \\
20 \ldots \ldots\end{array}$ & \begin{tabular}{l|}
5.6 \\
5.4 \\
5.4 \\
5.2 \\
....
\end{tabular} & $\ldots$ & $\begin{array}{l}6.0 \\
5.8 \\
5.4 \\
5.2 \\
5.2\end{array}$ & $\begin{array}{r}10.0 \\
9.2 \\
9.0 \\
8.6 \\
10.5\end{array}$ & $\begin{array}{l}5.8 \\
5.6 \\
5.2 \\
5.0 \\
5.0\end{array}$ & $\begin{array}{r}12.4 \\
10.2 \\
8.1 \\
7.8 \\
7.0\end{array}$ & $\begin{array}{l}6.2 \\
6.5 \\
6.8 \\
5.4 \\
5.0\end{array}$ & $\begin{array}{l}3.5 \\
3.4 \\
3.4 \\
3.4 \\
3.8\end{array}$ & $\begin{array}{l}4.4 \\
3.8 \\
3.6 \\
3.6 \\
3.6\end{array}$ & $\begin{array}{l}3.2 \\
3.2 \\
3.2 \\
3.2 \\
3.2\end{array}$ & $\begin{array}{l}3.1 \\
3.1 \\
3.1 \\
3.1 \\
3.1\end{array}$ & $\begin{array}{r}15.0 \\
11.2 \\
6.8 \\
5.7\end{array}$ \\
\hline $\begin{array}{l}21 \ldots \ldots \\
22 \ldots \ldots \\
23 \ldots \ldots \\
24 \ldots \\
25 \ldots \ldots \\
\end{array}$ & $\begin{array}{c}\dddot{4} .4 \\
4.4\end{array}$ & & $\begin{array}{l}5.0 \\
6.8 \\
7.0 \\
7.0 \\
6.8\end{array}$ & $\begin{array}{r}18.4 \\
16.0 \\
12.2 \\
8.9 \\
8.4\end{array}$ & $\begin{array}{r}4.8 \\
11.5 \\
9.0 \\
7.6 \\
5.4\end{array}$ & $\begin{array}{l}6.1 \\
5.6 \\
5.4 \\
5.4 \\
5.2\end{array}$ & $\begin{array}{l}4.2 \\
4.0 \\
4.5 \\
4.0 \\
3.8\end{array}$ & $\begin{array}{l}4.0 \\
4.2 \\
4.5 \\
3.8 \\
3.8\end{array}$ & \begin{tabular}{l|l}
3.5 \\
3.5 \\
3.5 \\
3.4 \\
3.4
\end{tabular} & $\begin{array}{l}3.1 \\
3.1 \\
3.1 \\
3.1 \\
3.1\end{array}$ & $\begin{array}{l}3.1 \\
3.0 \\
3.0 \\
\text { 3. } \\
5.2\end{array}$ & $\begin{array}{l}\cdots \cdots \\
\cdots \cdots \\
\cdots \cdots \\
\cdots \cdots \\
\cdots \cdots \\
\cdots \cdots\end{array}$ \\
\hline $\begin{array}{l}26 \ldots \\
27 \ldots \\
28 \ldots \\
29 \ldots \\
30 \ldots \\
31 \ldots \\
\end{array}$ & $\begin{array}{r}4.2 \\
4.2 \\
4.2 \\
\cdots \\
\cdots\end{array}$ & & $\begin{array}{l}6.5 \\
6.2 \\
6.0 \\
5.6 \\
5.4 \\
5.2\end{array}$ & $\begin{array}{l}7.6 \\
7.2 \\
6.8 \\
6.4 \\
6.0\end{array}$ & $\begin{array}{r}5.0 \\
6.5 \\
8.7 \\
12.2 \\
11.6 \\
8.4\end{array}$ & $\begin{array}{l}5.6 \\
6.4 \\
6.0 \\
6.1 \\
5.0\end{array}$ & \begin{tabular}{l|}
4.2 \\
3.6 \\
3.4 \\
3.0 \\
3.0 \\
3.4
\end{tabular} & $\begin{array}{l}3.5 \\
3.5 \\
3.8 \\
4.5 \\
4.5 \\
4.4\end{array}$ & \begin{tabular}{l|}
3.4 \\
3.4 \\
3.3 \\
3.3 \\
3.2 \\
$\cdots .$.
\end{tabular} & $\begin{array}{l}3.1 \\
3.1 \\
3.1 \\
3.1 \\
3.1 \\
3.1\end{array}$ & $\begin{array}{l}\text { 4. } 6 \\
4.0 \\
3.8 \\
3.7 \\
3.5\end{array}$ & $\begin{array}{r}\cdots \cdots \\
\cdots \cdots \\
\cdots .6 \\
14.6 \\
12.2\end{array}$ \\
\hline Day. & Jan. & & Day. & & Tan. & $\mathrm{Da}$ & & Jan. & & Day. & & Jan. \\
\hline $\begin{array}{c}1902 . b \\
1 \ldots \ldots \ldots \\
2 \ldots \ldots \ldots \\
3 \ldots \ldots \ldots \\
4 \ldots \ldots \ldots \\
5 \ldots \ldots \ldots \\
6 \ldots \ldots \ldots \\
7 \ldots \ldots \ldots \\
8 \ldots \ldots \ldots \\
9 \ldots \ldots \ldots \\
\end{array}$ & $\begin{array}{l}8.2 \\
6.8 \\
6.4 \\
6.0\end{array}$ & $\begin{array}{l}11 . \\
12 . \\
13 . \\
14 . \\
15 . \\
16 . \\
17 .\end{array}$ & $\begin{array}{c}1902 . b \\
\ldots \ldots \ldots\end{array}$ & & $\begin{array}{l}8.6 \\
5.6 \\
4.5\end{array}$ & $\begin{array}{l}\quad 190 \\
18 \ldots \ldots \\
19 \ldots \ldots \\
20 \ldots \ldots \\
21 \ldots \ldots \\
22 \ldots \ldots \\
23 \ldots \ldots \\
24 \ldots \ldots\end{array}$ & & & $\begin{array}{l}25 . \\
26 . \\
27 . \\
28 . \\
29 . \\
30 . \\
31 .\end{array}$ & 1902. & & $\begin{array}{l}5.2 \\
7.6 \\
7.8\end{array}$ \\
\hline
\end{tabular}

$a$ Ice conditions January 3- $\varepsilon, 20-23$, January 29 to Fobruary 3, February 6 to March 2, March 6, December $7,20-28,1901$.

$b$ Ice conditions January 5-8, 13-26, and January 30 to February 24, 1932. Bridge and gage carried away by flood February 24,1902 . 
Daily gage height, in feet, of South Branch of Potomac River near Springfield, W. Va.Continued.

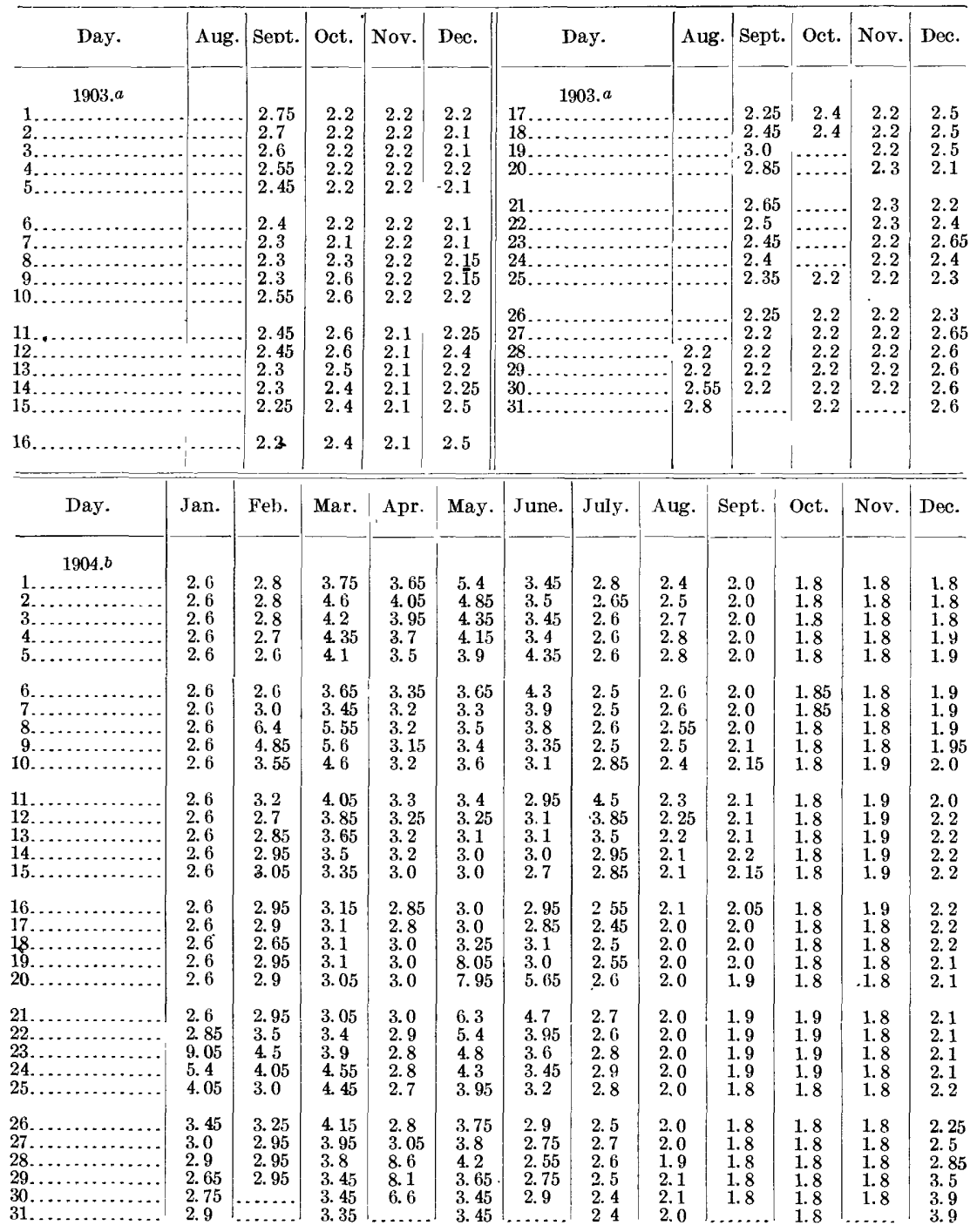

$a$ Ice conditions December 4-31, 1903 .

$b$ River frozen over January 1-22, 1904; river clear January 23-27, 1904; ice conditions January 28 to February 7, 1904; river frozen over December 19-31, 1904. 
Daily gage height, in feet, of South Branch of Potomac River near Springfield, W. Va.-Continued.

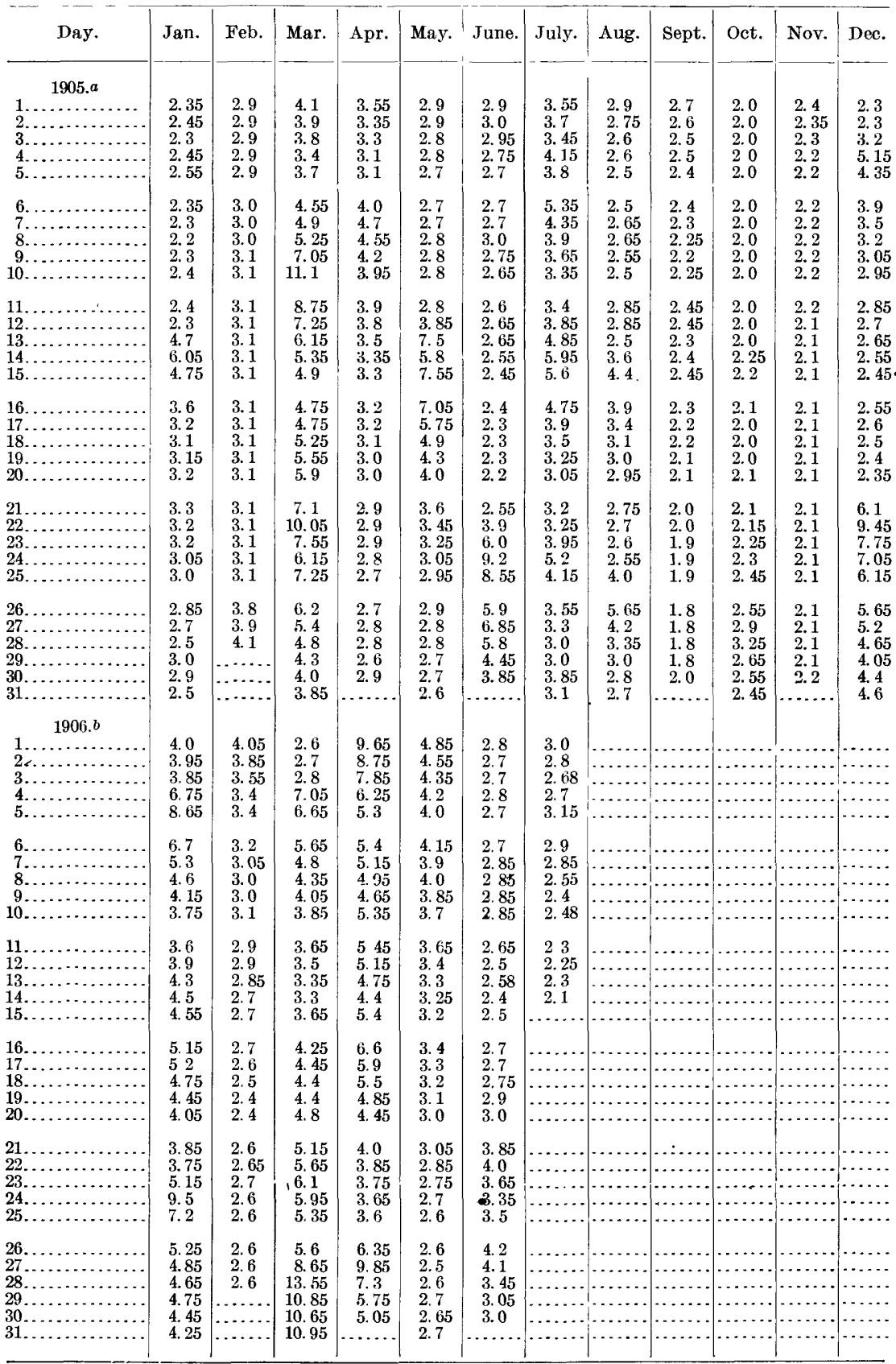


Rating tables for South Branch of Potomac River, near Springfield, W. Va.

JUNE 3, 1894, TO FE'BRUARY 29, 1896.a

\begin{tabular}{|c|c|c|c|c|c|c|c|}
\hline $\begin{array}{c}\text { Gage } \\
\text { height. }\end{array}$ & Discharge. & $\begin{array}{c}\text { Gage } \\
\text { height. }\end{array}$ & Discharge. & $\begin{array}{c}\text { Gage } \\
\text { height. }\end{array}$ & Discharge. & $\begin{array}{c}\text { Gage } \\
\text { height. }\end{array}$ & Discharge. \\
\hline $\begin{array}{l}\text { Feet. } \\
2.00 \\
2.10 \\
2.20 \\
2.30 \\
2.40 \\
2.50 \\
2.60 \\
2.70 \\
2.80\end{array}$ & \begin{tabular}{|c|} 
Second-feet \\
80 \\
96 \\
113 \\
132 \\
154 \\
180 \\
210 \\
240 \\
270
\end{tabular} & $\begin{array}{r}\text { Fect. } \\
2.90 \\
3.00 \\
3.10 \\
3.20 \\
3.30 \\
3.40 \\
3.50 \\
3.60 \\
3.70\end{array}$ & \begin{tabular}{|c|} 
Second-feet. \\
300 \\
330 \\
360 \\
400 \\
450 \\
500 \\
550 \\
600 \\
650
\end{tabular} & $\begin{array}{r}\text { Feet. } \\
3.80 \\
3.90 \\
4.00 \\
4.20 \\
4.40 \\
4.60 \\
4.80 \\
5.00 \\
5.20\end{array}$ & \begin{tabular}{|c} 
Second-feet. \\
700 \\
750 \\
800 \\
910 \\
1,030 \\
1,160 \\
1,300 \\
1,440 \\
1,580
\end{tabular} & $\begin{array}{r}\text { Feet. } \\
5.40 \\
5.60 \\
5.80 \\
6.00 \\
7.00 \\
8.00 \\
9.00 \\
10.00\end{array}$ & $\begin{array}{c}\text { Second-fect. } \\
1,720 \\
1,860 \\
2,000 \\
2,145 \\
2,895 \\
3,645 \\
4,395 \\
5,145\end{array}$ \\
\hline
\end{tabular}

JUNE 26, 1899, TO JANUARY 29, 1902.b

\begin{tabular}{|r|r|r|r|r|r|r|r|}
\hline 2.70 & 85 & 4.00 & 615 & 5.60 & 1,785 & 8.00 & 4,370 \\
2.80 & 110 & 4.10 & 670 & 5.80 & 1,965 & 8.50 & 4,975 \\
2.90 & 140 & 4.20 & 730 & 6.00 & 2,150 & 9.00 & 5,600 \\
3.00 & 170 & 4.30 & 790 & 6.20 & 2,345 & 10.00 & 6,850 \\
3.10 & 205 & 4.40 & 855 & 6.40 & 2,545 & 11.00 & 8,100 \\
3.20 & 240 & 4.50 & 920 & 6.60 & 2,755 & 12.00 & 9,350 \\
3.30 & 280 & 4.60 & 990 & 6.80 & 2,975 & 13.00 & 10,600 \\
3.40 & 320 & 4.70 & 1.060 & 7.00 & 3,200 & 14.00 & 11,850 \\
3.50 & 365 & 4.80 & 1,135 & 7.20 & 3,430 & 15.00 & 13,100 \\
3.60 & 410 & 4.90 & 1,210 & 7.40 & 3,660 & 16.00 & 14,350 \\
3.70 & 460 & 5.00 & 1,285 & 7.60 & 3,890 & 18.00 & 16,850 \\
3.80 & 510 & 5.20 & 1,445 & 7.80 & 4,130 & 20.00 & 19,350 \\
3.90 & 560 & 5.40 & 1,615 & & & & \\
\hline
\end{tabular}

AUGUST 28, 1903, TO JULY 14, 1906.c

\begin{tabular}{|l|r||l|l||l|l|l|r|r|}
\hline 1.80 & 78 & 3.00 & & 702 & 4.10 & 1.790 & 5.40 & 3,690 \\
1.90 & 96 & 3.10 & 778 & 4.20 & 1,912 & 5.60 & 4,030 \\
2.00 & 125 & 3.20 & 860 & 4.30 & 2,038 & 5,80 & 4,380 \\
2.10 & 163 & 3.30 & 947 & 4.40 & 2,168 & 6.00 & 4,745 \\
2.20 & 210 & 3.40 & 1,039 & 4.50 & 2,302 & $\mathbf{6 . 5 0}$ & 5,750 \\
2.30 & 261 & 3.50 & 1,135 & 4.60 & 2,440 & 7.00 & 6,890 \\
2.40 & 315 & 3.60 & 1,235 & 4.70 & 2,582 & 7.50 & 8,120 \\
2.50 & 372 & 3.70 & 1,339 & 4.80 & 2,728 & 8.00 & 9,370 \\
2.60 & 432 & 3.80 & 1,447 & 4.90 & 2,879 & 9.00 & 12,000 \\
2.70 & 495 & 3.90 & 1,558 & 5.00 & 3,035 & 10.00 & 15,000 \\
2.80 & 561 & 4.00 & 1,672 & 5.20 & 3,355 & 11.00 & 18,000 \\
2.90 & 630 & & & & & & \\
\hline
\end{tabular}

$a$ This table is strictly applicable only for open-channel conditions. It is based on discharge measurements made during 1894-1896. It is fairly well defined between gage heights 3.0 feet and 10.5 feet. Above gage height 5.9 feet the rating curve is a tangent, the difference being 75 per tenth.

$b$ This tahle is strictly applicable only for open-channel conditions. It is based on four discharge measurements made during 1899-1901. It is not well defined. Above gage height 8.4 feet the rating curve is a tangent, the difference being 125 per tenth. For high stages a tangent is considered to give the best results, owing to conditions below the station, which cause a backwater efiect at the gage.

$c$ This table is strictly applicable only for open-channel conditions. It is based on eight discharge measurements made during 1903-1906. It is fairly well defined between gage heights 2 feet and 4.5 feet. Above 6 feet gage height the discharge is approximate. 
Estimated monthly discharge of South Branch of Potomac River near Spring field, W. Va.

[Drainage area, 1,440 to 1,475 square miles.] $a$

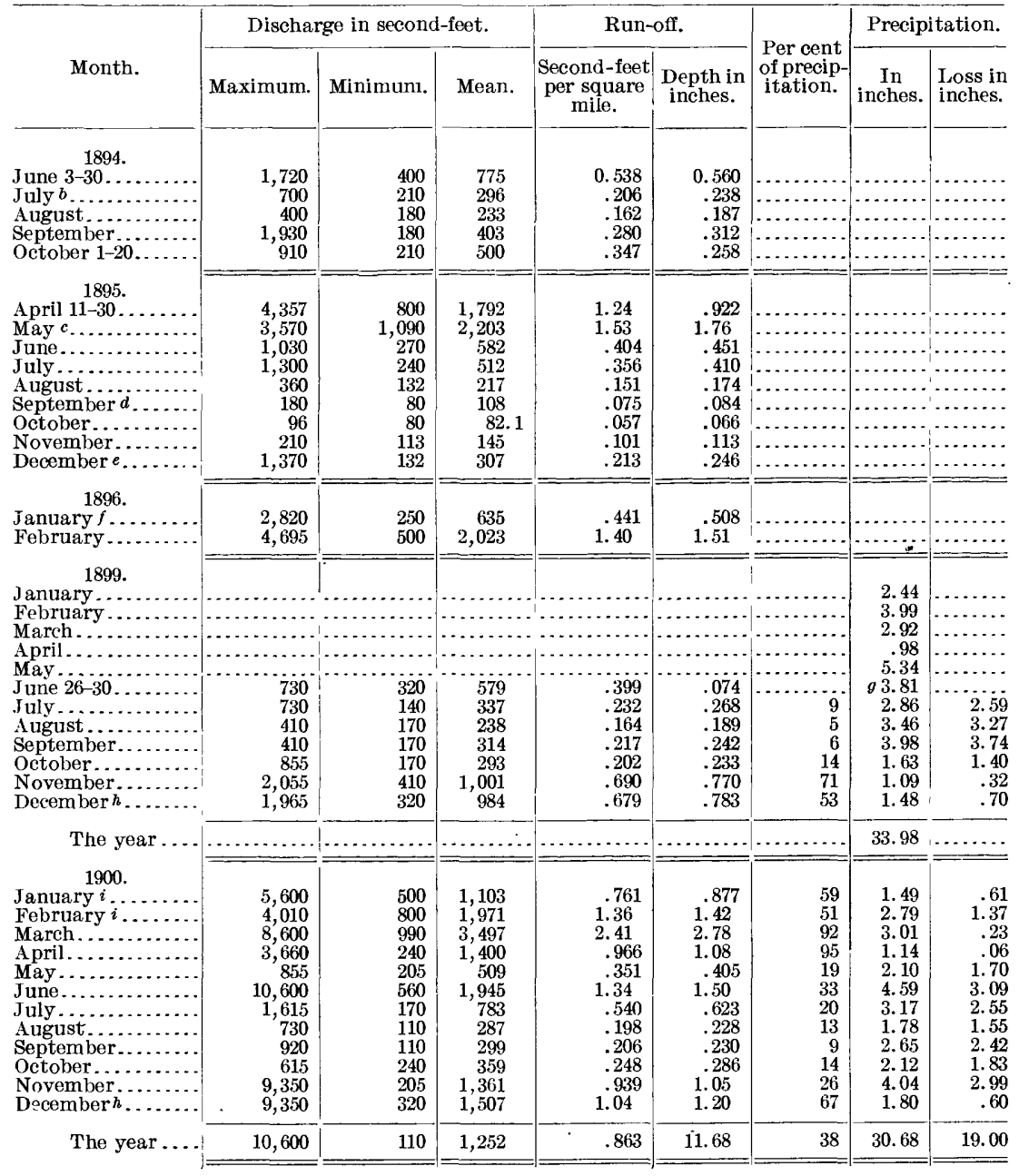

$a$ Various drainage areas are used to obtain run-off on account of changes in location of stations.

$b$ Discharge interpolated July 15-21, 1894

$c$ Discharge interpolated May 5-8, 1895.

d Discharge interpolated September 8-22, 1895.

$e$ Discharge interpolated December $25,1895$.

$f$ River frozen January 4-23, 1896; discharge corrected for ice conditions, the discharge of Shenandoah River at Millville, W. Va., being used as a. basis.

$g$ Precipitation for complete month June, 1899.

$h$ Ice conditions December 25-31, 1899; discharge corrected for this period, the daily discharges at Piedmont, Riverton, and Millville being used as a basis.

$i$ Ice conditions January 1-15, January 27 to February 9 , February 18-21, 25-28, and December 17-19, 1900. Discharge corrected for these periods, the daily discharges at Piedmont, Riverton, and Millville being used as a basis.

IRR $192-07-6$ 
Estimated monthly discharge of South Branch of Potomac River, etc.-Continued.

\begin{tabular}{|c|c|c|c|c|c|c|c|c|}
\hline \multirow[b]{2}{*}{ Month. } & \multicolumn{3}{|c|}{ Discharge in second-feet. } & \multicolumn{2}{|c|}{ Run-off. } & \multirow{2}{*}{$\begin{array}{l}\text { Per cent } \\
\text { of precip- } \\
\text { itation. }\end{array}$} & \multicolumn{2}{|c|}{ Precipitation. } \\
\hline & Maximum. & Minimum. & Mean. & $\begin{array}{l}\text { Second-feet } \\
\text { per square } \\
\text { mile. }\end{array}$ & $\begin{array}{l}\text { Depth in } \\
\text { inches. }\end{array}$ & & $\begin{array}{c}\text { In } \\
\text { inches. }\end{array}$ & $\begin{array}{l}\text { Loss in } \\
\text { inches. }\end{array}$ \\
\hline 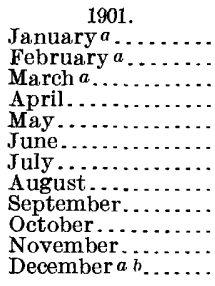 & $\begin{array}{r}5,850 \\
615 \\
9,600 \\
17,350 \\
9,600 \\
9,850 \\
2,975 \\
920 \\
3,488 \\
990 \\
1,445 \\
19,350\end{array}$ & $\begin{array}{l}300 \\
400 \\
400 \\
730 \\
920 \\
855 \\
170 \\
205 \\
240 \\
205 \\
170 \\
320\end{array}$ & $\begin{array}{r}1,119 \\
426 \\
2,423 \\
4,987 \\
3,631 \\
2,560 \\
1,185 \\
484 \\
721 \\
322 \\
326 \\
3,326\end{array}$ & $\begin{array}{l}.772 \\
.294 \\
1.67 \\
3.44 \\
2.50 \\
1.77 \\
.817 \\
.334 \\
.497 \\
.222 \\
.225 \\
2.29\end{array}$ & $\begin{array}{c}.890 \\
.306 \\
1.92 \\
3.84 \\
2.88 \\
1.98 \\
.942 \\
.385 \\
.554 \\
.256 \\
.251 \\
2.64\end{array}$ & $\begin{array}{r}52 \\
115 \\
82 \\
62 \\
44 \\
81 \\
32 \\
6 \\
27 \\
137 \\
11 \\
50\end{array}$ & $\begin{array}{l}1.70 \\
.27 \\
2.33 \\
6.20 \\
6.54 \\
2.44 \\
2.92 \\
6.60 \\
2.02 \\
.19 \\
2.25 \\
5.31\end{array}$ & $\begin{array}{r}.81 \\
-.04 \\
.41 \\
2.36 \\
3.66 \\
.46 \\
1.98 \\
6.22 \\
1.47 \\
-.07 \\
2.00 \\
2.67\end{array}$ \\
\hline The year. & 19,350 & 170 & 1,792 & 1.24 & 16.84 & 43 & 38.77 & 21.93 \\
\hline 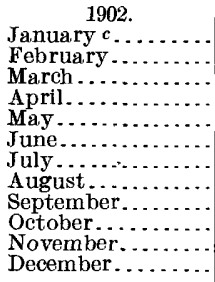 & 5,350 & $\begin{array}{c}800 \\
\cdots \ldots \\
\cdots \ldots\end{array}$ & 1,810 & $\begin{array}{l}1.25 \\
\cdots \cdots\end{array}$ & 1.44 & $\begin{array}{l}\cdots \\
\cdots \\
\cdots\end{array}$ & $\begin{array}{l}2.19 \\
3.83 \\
4.34 \\
2.77 \\
2.38 \\
3.67 \\
2.32 \\
2.37 \\
1.95 \\
2.84 \\
2.93 \\
3.87\end{array}$ & 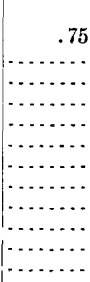 \\
\hline The yes & $\cdots$ & $\cdots$ & & & ${ }^{2}$ & $\begin{array}{lll}\ldots \ldots \\
\end{array}$ & 35.46 & $\ldots \ldots$ \\
\hline 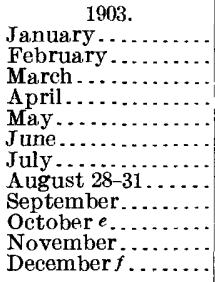 & $\begin{array}{l}561 \\
702 \\
432 \\
261 \\
463\end{array}$ & $\begin{array}{l}210 \\
210 \\
163 \\
163 \\
163\end{array}$ & $\begin{array}{l}346 \\
336 \\
271 \\
206 \\
286\end{array}$ & $\begin{array}{l}.235 \\
.228 \\
.184 \\
.140 \\
.194\end{array}$ & $\begin{array}{l}.035 \\
.254 \\
.212 \\
.156 \\
.224\end{array}$ & $\begin{array}{r}73 \\
8 \\
20 \\
55\end{array}$ & $\begin{array}{r}2.76 \\
2.85 \\
2.98 \\
3.02 \\
3.17 \\
5.33 \\
3.79 \\
d 3.89 \\
1.91 \\
2.78 \\
.79 \\
.40\end{array}$ & 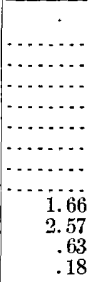 \\
\hline The year ... & ..... & $\cdots$ & & $\cdots$ & $\ldots$ & & 33.67 & - . . . . . \\
\hline 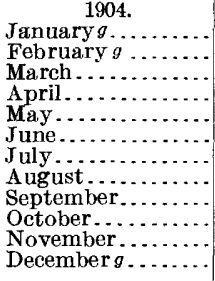 & $\begin{array}{r}12,150 \\
5,540 \\
4,030 \\
10,930 \\
9,500 \\
4,118 \\
2,302 \\
561 \\
210 \\
96 \\
96 \\
1,558\end{array}$ & \begin{tabular}{r|}
432 \\
432 \\
740 \\
495 \\
702 \\
402 \\
315 \\
96 \\
78 \\
78 \\
78 \\
78
\end{tabular} & $\begin{array}{c}1,009 \\
1,004 \\
1,451 \\
1,660 \\
2,143 \\
1,121 \\
568 \\
238 \\
123 \\
80.9 \\
79.9 \\
296\end{array}$ & $\begin{array}{c}.684 \\
.681 \\
.984 \\
1.13 \\
1.45 \\
.760 \\
.385 \\
.161 \\
.083 \\
.055 \\
.054 \\
.200\end{array}$ & $\begin{array}{l}.789 \\
.734 \\
1.13 \\
1.26 \\
1.67 \\
.848 \\
.444 \\
.186 \\
.093 \\
.063 \\
.060 \\
.231\end{array}$ & $\begin{array}{r}48 \\
74 \\
61 \\
51 \\
56 \\
16 \\
8 \\
10 \\
5 \\
5 \\
8 \\
9\end{array}$ & $\begin{array}{l}1.66 \\
.99 \\
1.84 \\
2.45 \\
3.01 \\
5.47 \\
5.58 \\
1.95 \\
1.83 \\
1.14 \\
.76 \\
2.64\end{array}$ & $\begin{array}{r}.87 \\
.26 \\
.71 \\
1.19 \\
1.34 \\
4.62 \\
5.14 \\
1.76 \\
1.74 \\
1.08 \\
.70 \\
2.41\end{array}$ \\
\hline The year.. & 12,150 & 78 & 814 & .552 & 7.50 & 26 & 29.32 & 21.82 \\
\hline
\end{tabular}

a Ice conditions January 3-8, 20-23, January 29 to February 3, February 6 to March 2, March 6 , December 7, 20-28, 1901 . Discharge corrected for these periods, the daily discharge at Piedmont, Riverton, and Millville being used as a basis.

$b$ There was backwater at the gage December 29-31, 1901, owing to an ice jam, but since the discharge when applied to the gage heights as observed is relatively small as compared with that at Riverton and Millville, it was considered best to allow them to stand without reduction for backwater effect.

$c$ Ice conditions January 5-8, 13-26, and January 30 to February 24, 1902. Discharge corrected for January, the daily discharges at Piedmont, Riverton. and Millville being used as a basis.

$d$ Precipitation for complete month August, 1903.

e Dis?harge interpolated October 19-24, 1903.

$f$ Ice conditions December 4-31, 1903 . No correction made in estimates.

$g$ Ice conditions Jan. 1 to 22 , Jan, 28 to Feb. 7, and December 19-31, 1904; no correction nade in estimates. 
Estimated monthly discharqe of South Branch of Potomac River, etc.-Continued.

\begin{tabular}{|c|c|c|c|c|c|c|c|c|}
\hline \multirow[b]{2}{*}{ Month. } & \multicolumn{3}{|c|}{ Discharge in second-feet. } & \multicolumn{2}{|c|}{ Run-off. } & \multirow{2}{*}{$\begin{array}{l}\text { Per cent } \\
\text { of precip- } \\
\text { itation. }\end{array}$} & \multicolumn{2}{|c|}{ Precipitation. } \\
\hline & Maximum. & Minimum. & Mean. & $\begin{array}{l}\text { Second-feet } \\
\text { per square } \\
\text { aile. }\end{array}$ & $\begin{array}{l}\text { Depth in } \\
\text { inches. }\end{array}$ & & $\begin{array}{c}\text { In } \\
\text { inches. }\end{array}$ & $\begin{array}{l}\text { Loss in } \\
\text { inches. }\end{array}$ \\
\hline 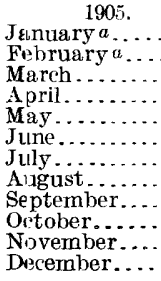 & $\begin{array}{r}4,840 \\
1,790 \\
18,300 \\
2,582 \\
8,245 \\
12,600 \\
4,652 \\
4,118 \\
495 \\
904 \\
315 \\
13,350\end{array}$ & $\begin{array}{r}210 \\
630 \\
1,039 \\
432 \\
432 \\
210 \\
702 \\
372 \\
78 \\
125 \\
163 \\
261\end{array}$ & $\begin{array}{r}821 \\
831 \\
4,793 \\
1,015 \\
1,744 \\
1,948 \\
1,673 \\
854 \\
228 \\
229 \\
190 \\
2,298\end{array}$ & $\begin{array}{c}.557 \\
.563 \\
3.25 \\
.688 \\
1.18 \\
1.32 \\
1.13 \\
.579 \\
.155 \\
.155 \\
.129 \\
1.56\end{array}$ & $\begin{array}{c}.642 \\
.586 \\
3.75 \\
.768 \\
1.36 \\
1.47 \\
1.30 \\
.668 \\
.173 \\
.179 \\
.144 \\
1.80\end{array}$ & $\begin{array}{r}27 \\
48 \\
162 \\
63 \\
37 \\
25 \\
21 \\
14 \\
15 \\
6 \\
13 \\
59\end{array}$ & $\begin{array}{l}2.34 \\
1.22 \\
2.32 \\
1.23 \\
3.64 \\
5.78 \\
6.26 \\
4.63 \\
1.12 \\
3.02 \\
1.10 \\
3.06\end{array}$ & $\begin{array}{r}1.70 \\
.63 \\
-1.43 \\
.46 \\
2.28 \\
4.31 \\
4.96 \\
3.96 \\
.95 \\
2.84 \\
.96 \\
1.26\end{array}$ \\
\hline The year & 18,300 & 78 & 1,385 & .939 & 12.84 & 36 & 35.72 & 22.88 \\
\hline $\begin{array}{l}\quad 1900 . \\
\text { January .... } \\
\text { February... } \\
\text { March ..... } \\
\text { April ...... } \\
\text { Mayy ....... } \\
\text { June ...... } \\
\text { July } 1-14 \ldots .\end{array}$ & $\begin{array}{r}13,500 \\
1,731 \\
25,650 \\
14,550 \\
2,804 \\
1,912 \\
819\end{array}$ & $\begin{array}{r}1,235 \\
315 \\
432 \\
1,235 \\
372 \\
315 \\
163\end{array}$ & $\begin{array}{r}3,321 \\
664 \\
5,076 \\
4,538 \\
1,085 \\
770 \\
449\end{array}$ & $\begin{array}{l}2.26 \\
.452 \\
3.45 \\
3.09 \\
.738 \\
.524 \\
.305\end{array}$ & $\begin{array}{l}2.61 \\
.471 \\
3.98 \\
3.45 \\
.851 \\
.585 \\
.159\end{array}$ & & & $\begin{array}{l}\cdots \\
\cdots \\
\cdots\end{array}$ \\
\hline
\end{tabular}

$a$ Ice conditions January and February, 1905; no correction made in estimates.

\section{MISCELLANEOUS DISCHARGE MEASUREMENTS IN SOUTH BRANCH OF POTOMAC RIVER} BASIN.

The following miscellaneous discharge measurements have been made in the basin of South Branch of Potomac River:

Miscellareous discharge measurements in South Branch of Potomac River drainage basin.

\begin{tabular}{|c|c|c|c|c|c|c|}
\hline Date. & Stream. & Locality. & Width. & $\begin{array}{c}\text { Area of } \\
\text { sec- } \\
\text { tion. }\end{array}$ & $\begin{array}{l}\text { Mean } \\
\text { veloc- } \\
\text { ity. }\end{array}$ & $\begin{array}{c}\text { Dis- } \\
\text { charge. }\end{array}$ \\
\hline $\begin{array}{l}1897 . \\
\text { Sejptember } 24 . .\end{array}$ & $\begin{array}{l}\text { North Fork of South } \\
\text { Branch of Potomac } \\
\text { River. }\end{array}$ & $\begin{array}{l}500 \text { feet above mouth near } \\
\text { Petersburg, W. Va. }\end{array}$ & $\begin{array}{r}\text { Feet. } \\
38\end{array}$ & $\begin{array}{c}\text { Square } \\
\text { fert. } \\
33\end{array}$ & $\begin{array}{l}\text { Ft. per } \\
\text { second. } \\
0.52\end{array}$ & $\begin{array}{r}\text { Second- } \\
\text { feet. } \\
17\end{array}$ \\
\hline Do. & $\begin{array}{l}\text { South Branch of Poto- } \\
\text { mac River. }\end{array}$ & $\begin{array}{l}300 \text { yards above mouth of } \\
\text { North Fork, near Peters- }\end{array}$ & 80 & 58 & 1.65 & 96 \\
\hline Do........ & $\begin{array}{l}\text { South Fork of South } \\
\text { Branch of Potomac } \\
\text { River. }\end{array}$ & Near Moorefield, W. Va .... & 24 & 23 & 1.48 & 34 \\
\hline September $26 .$. & Mill Creek. ............. & Near mouth near Romney & & & & 3 \\
\hline Do. & $\begin{array}{l}\text { South Branch of Poto- } \\
\text { mac River. }\end{array}$ & $\begin{array}{l}\text { A short distance above } \\
\text { Romney bridge near } \\
\text { Romney, w. Va. }\end{array}$ & 65 & 91 & 2.25 & 205 \\
\hline
\end{tabular}




\section{POTOMAC RIVER BASIN BETWEEN MOUTH OF SOUTH BRANCH AND SHENANDOAH RIVER.}

POTOMAC RIVER AT GREAT CACAPON, W, VA.

Gage-height records were obtained of Potomac River at Dam No. 6 of the Chesapeake and Ohio Canal near Great Cacapon, from June 21, 1894, to March 7, 1896. The gage was located above the dam. Discharge measurements were made of the main river and also of the canal feeder by wading at a point about 1,000 feet below the diversion dam. The channel at this point is rocky and the current swift. The crest of the dam is not level and no data are available for determining the point on the gage at which zero flow over the dam occurs. No estimates of discharge have been made, as the number of measurements is insufficient. The gage heights are not considered of enough value for republication.

Discharge measurements of Potomac River at Great Cacapon, W. Va.

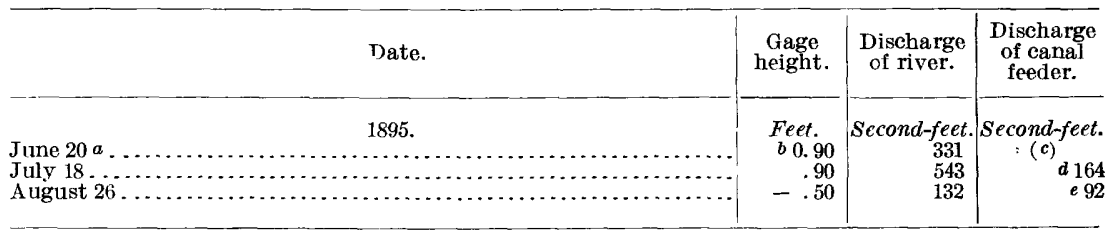

$a$ No water flowing over the West Virginia end of the dam for about one-eighth of the total length.

$b$ Gage height not considered accurate.

$c$ Water flowing in feeder, but no measurement made.

$d$ Feeder gate open 3.0 feet.

$e$ Feeder gate wide open.

OPEQUON CREEK NEAR MARTINSBURG, W. VA, $a$

The gaging station was established May 8, 1905, and was discontinued July 16, 1906. It is located at the highway bridge known as "Rileys Ford Bridge," about 4 miles southeast of Martinsburg, W. Va.

The channel is straight for about 300 feet above and below the station. The current is swift. Both banks are clean. The right bank is high and does not overflow. The left bank has a flood plain extending 600 feet across a level meadow. The bed of the stream is rocky, very rough, free from vegetation, and permanent. There is but one channel at ordinary stages. The stream is liable to extreme fluctuations at high water, covering the entire flood plain on the west.

Discharge measurements were made from the downstream side of the steel bridge to which the gage is attached. The initial point for soundings is the top face of the left abutment, downstream side.

A standard chain gage is fastened to the downstream side of the bridge near the left abutment. The length of the chain from the end of the weight to the marker is 19.90 feet. The gage was read twice 
each day by Frank Mose. Bench mark No: 1 is a cross chiseled on the upstream left abutment. Its elevation is 18.44 feet above the datum of the gage. Bench mark No. 2 is the top of the pulley wheel of the gage. Its elevation was 19.55 feet above the datum of the gage March 18, 1906.

Low-water measurements at this station plot very erratically, and since the section at the gaging station has remained unchanged it is assumed that the trouble lies with the controlling point below the gage. This assumption is borne out by the fact that it is known that refuse matter is thrown into the creek from the bridge and collects on a bar a short distance below. It has seemed best to determine the discharge by means of three rating curves of the same general form.

Estimates based on the first two curves may be 10 to 20 per cent in error. Estimates based on the third curve are probably within 5 to 10 per cent of the true flow for normal conditions. The 1905-6 estimates are not affected by ice conditions.

Discharge measurements of Opequon Creek near Martinsburg, W. Va.

\begin{tabular}{|c|c|c|c|c|c|}
\hline Date. & $\begin{array}{c}\text { Gage } \\
\text { height. }\end{array}$ & Discharge. & Date. & $\begin{array}{c}\text { Gage } \\
\text { height. }\end{array}$ & Discharge. \\
\hline $\begin{array}{c}1905 . \\
\text { May } 7 \ldots \ldots \ldots \ldots \ldots \ldots \\
\text { November } 8 . \ldots \ldots \ldots \ldots \ldots \ldots \\
1906 . \\
\text { March } 18 \ldots \ldots \ldots \ldots \ldots \ldots\end{array}$ & $\begin{array}{l}\text { Feet. } \\
\quad 1.33 \\
\quad 1.25 \\
\\
1.51\end{array}$ & $\begin{array}{r}\text { Second-feet. } \\
141 \\
63\end{array}$ & 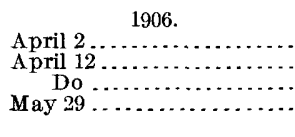 & $\begin{array}{l}\text { Feet. } \\
3.32 \\
2.57 \\
2.56 \\
1.57\end{array}$ & $\begin{array}{r}\text { Second-feet. } \\
600 \\
338 \\
332 \\
152\end{array}$ \\
\hline
\end{tabular}

Daily gage height, in feet, of Opequon Creek near Martinsburg, W. Va.

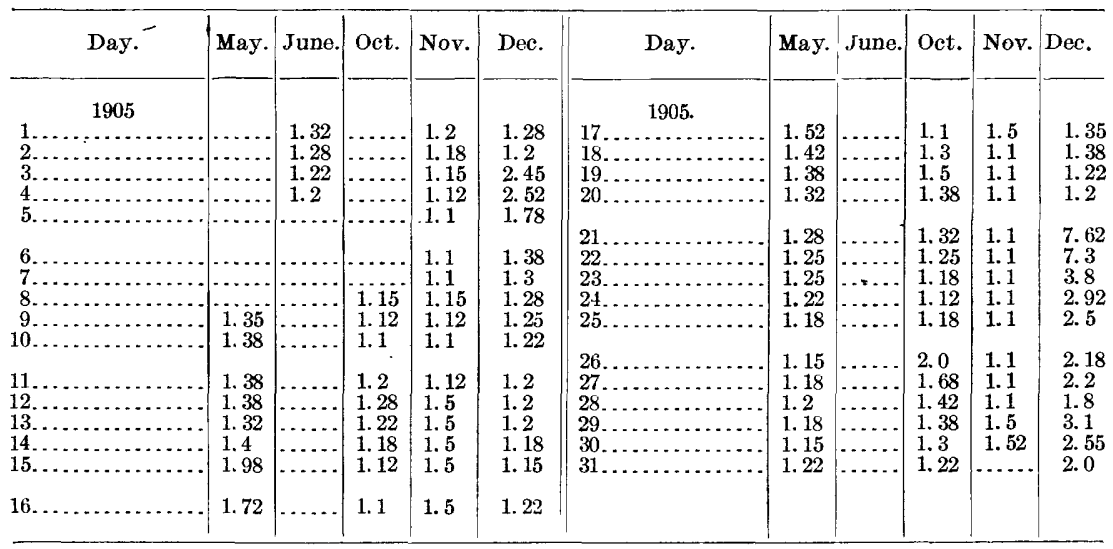


Daily gage height, in feet, of Opequon Creek near Martinsburg, W. Va.-Continued.

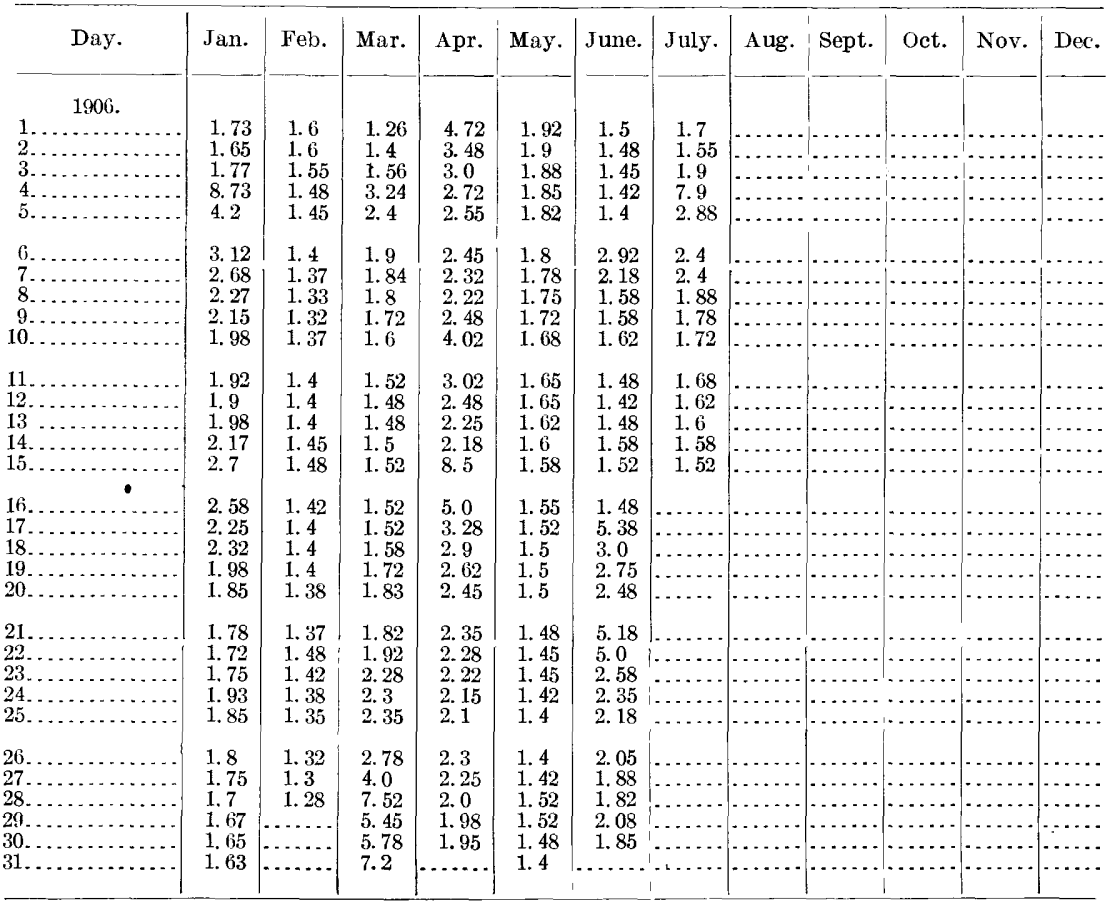

Rating tables for Opequon Creek near Martinsburg, W. Va.

MAY 9 TO JUNE 4, 1905.a

\begin{tabular}{|c|c|c|c|c|c|c|c|}
\hline $\begin{array}{r}\text { Gage . } \\
\text { height. }\end{array}$ & Discharge. & $\begin{array}{c}\text { Gage } \\
\text { height. }\end{array}$ & Discharge. & $\begin{array}{c}\text { Gage } \\
\text { height. }\end{array}$ & Discharge. & $\begin{array}{c}\text { Gage } \\
\text { height. }\end{array}$ & Discharge. \\
\hline $\begin{array}{l}\text { Feet. } \\
1.15 \\
1.20 \\
1.30\end{array}$ & $\begin{array}{c}\text { Second-feet } \\
116 \\
123 \\
137\end{array}$ & $\begin{array}{l}\text { Feet. } \\
1.40 \\
1.50 \\
1.60\end{array}$ & $\begin{array}{c}\text { Second-feet } \\
152 \\
167 \\
183\end{array}$ & $\begin{array}{l}\text { Feet. } \\
1.70 \\
1.80\end{array}$ & $\begin{array}{c}\text { Second-feet. } \\
200 \\
218\end{array}$ & $\begin{array}{l}\text { Feet. } \\
1.90 \\
2.00\end{array}$ & $\begin{array}{c}\text { Second-feet. } \\
237 \\
257\end{array}$ \\
\hline
\end{tabular}

OCTOBER 8 TO DECEMBER $20,1905 . b$

\begin{tabular}{|l|l|l|l|l|l|l|l|}
\hline 1.10 & 50 & 1.50 & 88 & 1.90 & 140 & 2.30 & 205 \\
1.20 & 58 & 1.60 & 100 & 2.00 & 155 & 2.40 & 223 \\
1.30 & 67 & 1.70 & 113 & 2.10 & 171 & 2.50 & 242 \\
1.40 & 77 & 1.80 & 126 & 2.20 & 188 & \\
\hline
\end{tabular}

DECEMBER 21, 1905, TO JULY 15, 1906.c

\begin{tabular}{|l|r||c|c|c|c|c|c|}
\hline 1.20 & 97 & 2.20 & 251 & 3.20 & 549 & 4.40 & 1,092 \\
1.30 & 109 & 2.30 & 271 & 3.30 & 589 & 4.60 & 1,193 \\
1.40 & 122 & 2.40 & 293 & 3.40 & 630 & 4.80 & 1,295 \\
1.50 & 135 & 2.50 & 317 & 3.50 & 672 & 5.00 & 1,399 \\
1.60 & 149 & 2.60 & 343 & 3.60 & 715 & 5.20 & 1,504 \\
1.70 & 164 & 2.70 & 371 & 3.70 & 759 & 5.40 & 1,610 \\
1.80 & 180 & 2.80 & 402 & 3.80 & 804 & 5.60 & 1,717 \\
1.90 & 197 & 2.90 & 436 & 3.90 & 850 & 5.80 & 1,825 \\
2.00 & 214 & 3.00 & 472 & 4.00 & 897 & 6.00 & 1,935 \\
2.10 & 232 & 3.10 & 510 & 1.20 & 993 & & \\
\hline
\end{tabular}

$a$ The rating curve on which this table is based has been drawn through one measurement made May, 1905 , and has the form of the 1906 curve.

$b$ This table is strictly applicable only for open-channel conditions. The rating curve has been drawn through one measurement made November, 1905, and has the form of the 1906 curve.

$c$ This table is strictly applicable only for open-channel conditions. It is baser on five discharge measurements made during 1906 . It is fairly well defined between gage heights 1.5 feet and 3.4 feet. A bove gage height 6.0 feet the rating curve is a tangent, the difference being 55 per tenth. 
Estimated monthly discharge of Opequon Creek near Martinsburg, W. Va.

[Drainage area, 275 square miles.]

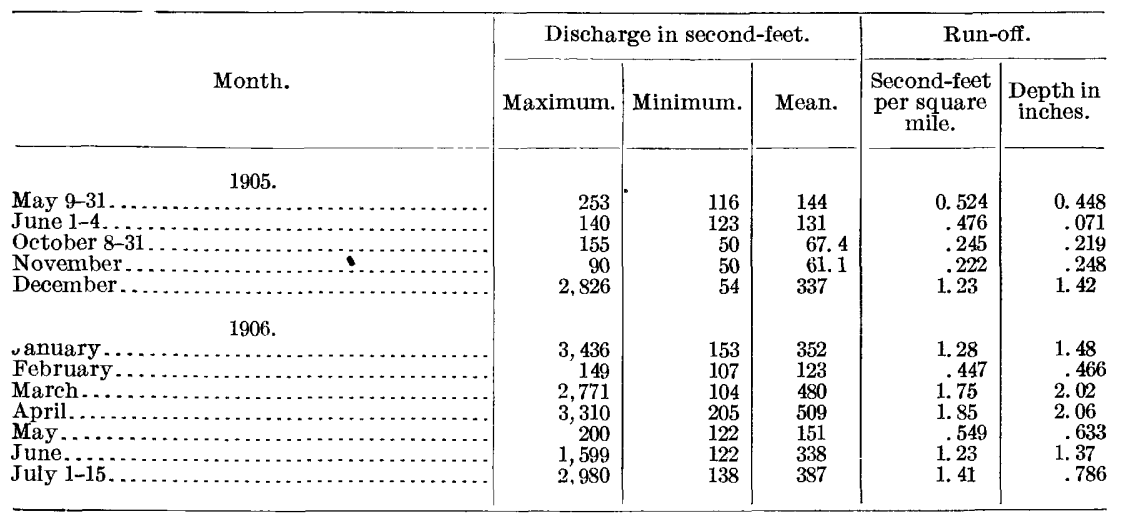

TUSCARORA CREEK AT MARTINSBURG, W, VA.

Tuscarora Creek rises in Little North Mountain, in the western part of Berkeley County, W. Va., and flows southeastward into Opequon Creek about 2 miles southeast of Martinsburg.

- The gaging station was established May 8, 1905, by F. H. Tillinghast. It is located at the dam formerly used to impound water for the use of the city of Martinsburg.

The channel is curved for 20 feet above the dam and straight for 200 feet beyond this point. The channel below is a steep race way from the crest of the dam, paved with riprap. The current is swift. Both banks are low, clean, and not liable to overflow. The bed of the stream above the dam is fairly uniform and shallow, with a mud bottom. There is but one channel at all stages. The stream is liable to small fluctuations, owing to the varying demands of factories above the station. The water level at the gage is somewhat unsteady. The velocity of approach is high.

It was intended to determine the discharge by applying the weir formula to the flow over the crest of the dam.

A vertical staff gage is bolted to the upstream face of the left abutment, the zero being set at the level of the floor of the spillway. The gage was read twice each day by B. N. Martin. Bench mark No. 1 is the crest of the dam at the left corner; elevation, 0.04 foot below the gage datum. Bench mark No. 2 is the crest of the dam at the right corner; elevation, 0.04 foot above the gage datum.

A current-meter measurement, made May 9, 1905, at gage height 0.78 foot, gave a discharge of 25.2 second-feet.

Owing to poor conditions and especially to the collection of debris on the crest of the dam, rendering estimates based on the gage heights of little value, this station was abandoned December 31, 1905. 
Daily gage height, in feet, of Tuscarora Creek at Martinsburg, W. Va.

\begin{tabular}{|c|c|c|c|c|c|c|c|c|}
\hline Day. & May. & June. & July. & Aug. & Sept. & Oct. & Nov. & Dec. \\
\hline 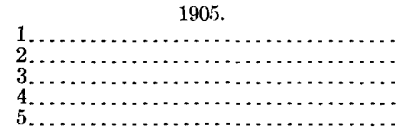 & & $\begin{array}{l}0.72 \\
.64 \\
.62 \\
.65 \\
.62\end{array}$ & $\begin{array}{l}0.6 \\
.7 \\
.72 \\
.75 \\
.68\end{array}$ & $\begin{array}{l}1.55 \\
1.75 \\
1.65 \\
1.7 \\
1.75\end{array}$ & $\begin{array}{l}0.68 \\
.75 \\
.85 \\
.75 \\
.65\end{array}$ & $\begin{array}{r}0.75 \\
.82 \\
.70 \\
.85 \\
.75\end{array}$ & $\begin{array}{l}0.85 \\
.9 \\
.88 \\
.88 \\
.8\end{array}$ & $\begin{array}{r}0.55 \\
.65 \\
1.45 \\
.85 \\
.65\end{array}$ \\
\hline $\begin{array}{r}6 \\
7 \ldots \ldots \\
8 \\
9 \\
10 \ldots \ldots\end{array}$ & $\begin{array}{c}0.75 \\
.8 \\
.8\end{array}$ & $\begin{array}{l}.61 \\
.9 \\
.68 \\
.62 \\
.62\end{array}$ & $\begin{array}{l}.85 \\
.82 \\
.72 \\
.78 \\
.78\end{array}$ & $\begin{array}{l}1.75 \\
1.3 \\
1.4 \\
1.3 \\
1.25\end{array}$ & $\begin{array}{l}.6 \\
.58 \\
.7 \\
.55 \\
.6\end{array}$ & $\begin{array}{l}.68 \\
.75 \\
.78 \\
.68 \\
.65\end{array}$ & $\begin{array}{l}.85 \\
.88 \\
.6 \\
.58 \\
.55\end{array}$ & $\begin{array}{l}.55 \\
.55 \\
.55 \\
.55 \\
.55\end{array}$ \\
\hline $\begin{array}{l}11 \ldots \ldots \\
12 \ldots \ldots \\
13 \ldots \ldots \\
15 \ldots \ldots\end{array}$ & $\begin{array}{l}.74 \\
.75 \\
.72 \\
.75 \\
.82\end{array}$ & $\begin{array}{l}.6 \\
.18 \\
.5 \\
.7 \\
.68\end{array}$ & $\begin{array}{l}.85 \\
\text { 1. } 45 \\
2.65 \\
3.0 \\
2.35\end{array}$ & $\begin{array}{l}.88 \\
.88 \\
.85 \\
.78 \\
.92\end{array}$ & $\begin{array}{l}.58 \\
.7 \\
.65 \\
.55 \\
.55\end{array}$ & $\begin{array}{l}.55 \\
.55 \\
.78 \\
.68 \\
.65\end{array}$ & $\begin{array}{l}.58 \\
.58 \\
.55 \\
.55 \\
.58\end{array}$ & $\begin{array}{l}.58 \\
.55 \\
.58 \\
.58 \\
.65\end{array}$ \\
\hline $\begin{array}{l}16 \ldots \\
17 \ldots \ldots \\
19 \ldots \ldots \\
20 \ldots \ldots\end{array}$ & $\begin{array}{l}.76 \\
.8 \\
.78 \\
.75 \\
.72\end{array}$ & $\begin{array}{l}.72 \\
.65 \\
.75 \\
.78 \\
.72\end{array}$ & $\begin{array}{r}1.15 \\
.85 \\
.82 \\
.78 \\
.78\end{array}$ & $\begin{array}{l}.78 \\
.8 \\
1.25 \\
1.02 \\
1.05\end{array}$ & $\begin{array}{l}.55 \\
.65 \\
.55 \\
.55 \\
.6\end{array}$ & $\begin{array}{l}.70 \\
.78 \\
.68 \\
.72 \\
.65\end{array}$ & $\begin{array}{l}.6 \\
.52 \\
.55 \\
.5 \\
.52\end{array}$ & $\begin{array}{l}.7 \\
.58 \\
.68 \\
.65 \\
.75\end{array}$ \\
\hline $\begin{array}{r}21 \\
22 \\
23 \\
23 \\
24 \\
25 \\
25\end{array}$ & $\begin{array}{l}.72 \\
.74 \\
.72 \\
.74 \\
.68\end{array}$ & $\begin{array}{l}.78 \\
.8 \\
.72 \\
.28 \\
.14\end{array}$ & $\begin{array}{l}.68 \\
.7 \\
1.02 \\
1.45 \\
1.25\end{array}$ & $\begin{array}{l}1.02 \\
1.1 \\
1.08 \\
1.5 \\
1.15\end{array}$ & $\begin{array}{l}.65 \\
.6 \\
. .55 \\
.55 \\
.55\end{array}$ & $\begin{array}{l}.58 \\
.75 \\
.75 \\
.68 \\
.58\end{array}$ & $\begin{array}{l}.55 \\
.52 \\
.58 \\
.5 \\
.58\end{array}$ & $\begin{array}{l}.88 \\
2.15 \\
1.8 \\
1.25 \\
1.0\end{array}$ \\
\hline $\begin{array}{l}26 \ldots \ldots \ldots \\
27 \ldots \ldots \ldots \\
28 \ldots \ldots \\
29 \ldots \ldots \\
30 \ldots \ldots \\
31 \ldots \ldots \\
\ldots \ldots \ldots \\
\end{array}$ & $\begin{array}{l}.62 \\
.6 \\
.6 \\
.62 \\
.59 \\
.78\end{array}$ & $\begin{array}{l}.42 \\
.72 \\
.75 \\
.72 \\
.68\end{array}$ & $\begin{array}{l}1.1 \\
1.6 \\
1.5 \\
1.85 \\
1.55 \\
1.55\end{array}$ & $\begin{array}{l}.98 \\
1.35 \\
2.0 \\
.85 \\
.8 \\
.7\end{array}$ & $\begin{array}{l}.48 \\
.48 \\
.75 \\
.65 \\
.75\end{array}$ & $\begin{array}{l}.65 \\
.68 \\
.58 \\
.85 \\
.92 \\
.82\end{array}$ & $\begin{array}{l}.55 \\
.62 \\
.65 \\
.55 \\
.5\end{array}$ & $\begin{array}{l}.75 \\
.65 \\
.7 \\
.75 \\
.7\end{array}$ \\
\hline
\end{tabular}

ANTIETAM CREEK NEAR SHARPSBURG, MD.

Antietam Creek drains a rolling, fertile country with uniform declivity and is uninterrupted by natural falls or rapids. It is utilized to a considerable extent to run mills of various kinds.

The gaging station was established June 24, 1897, by A. P. Davis, and was discontinued August 26, 1905. It is located 1 mile east of Sharpsburg, a few hundred feet below the bridge on the toll road from Sharpsburg to Keedysville, Md. There is an old dam, not now in use, just below the bridge.

The channel is straight for 300 feet above and below the station. It has a width at ordinary stages of about 90 feet, and is shallow and unobstructed. There is a good measurable velocity at all stages. The right bank is low and liable to overflow; the left bank is high and rocky; both are fringed with trees. The bed of the stream is composed of gravel, is free from vegetation, and is permanent. There is but one channel at all stages.

Discharge measurements were made from a steel-wire cable, which is supported by the forks of a sycamore tree on each bank and is anchored to timbers set in the ground.

The gage was a vertical rod driven into the gravel of the stream bed and spiked to a tree on the left bank near the cable. It was 
read once each day. The bench mark is a copper bolt set in a ledge of rock on the left bank at a point about 125 feet above the cable. Its elevation is 16.34 feet above gage datum.

All estimates previously published have been revised. Estimates for stages below gage height 2.0 feet are probably within 10 per cent of the true discharge for normal conditions of flow; estimates between gage heights 2.0 feet and 4.0 feet are within 5 per cent, and estimates above gage height 4.0 feet are within 10 per cent. Allowance has been made for the discharge of the overflow section. The flow is probably affected to a greater or less extent by ice conditions.

A summary of the records gives the following results: Maximum discharge for twenty-four hours, 6,835 second-feet; minimum discharge for twenty-four hours, 59 second-feet; mean annual discharge for five years, 350 second-feet; mean annual rainfall for seven years, 37.56 inches.

Discharge measurements of Antietam Creek near Sharpsburg, Md.

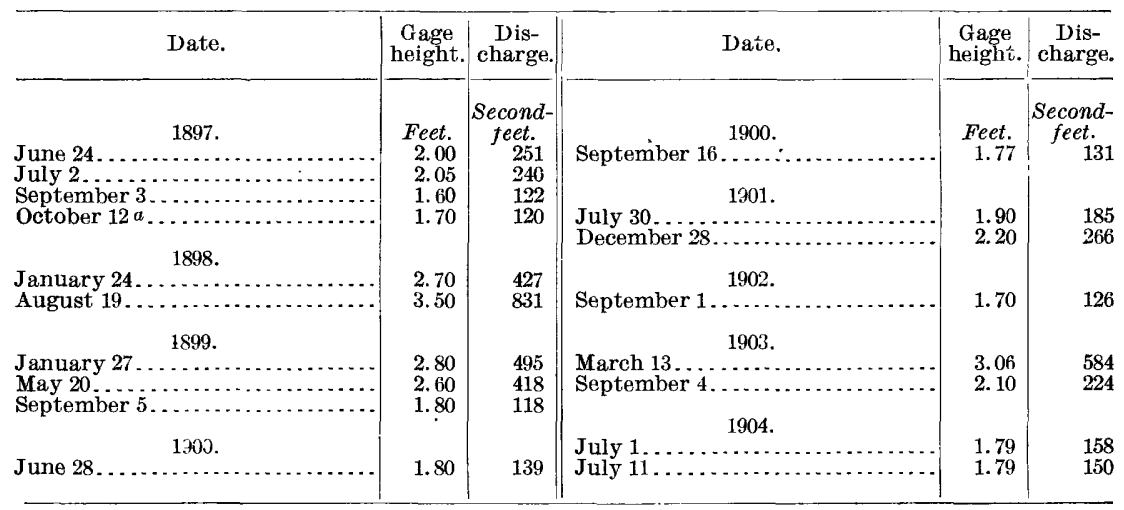

$a$ Measurementmade near mou $h$ of stream.

Daily gage height, in feet, of Antietam Creek near Sharpsburg, Md.

\begin{tabular}{|c|c|c|c|c|c|c|c|c|c|c|c|c|c|}
\hline Day. & July. & Aug. & Sept. & Oct. & Nov. & Dec. & Day. & July. & Aug. & Sept. & Oct. & Nov. & Dec. \\
\hline $\begin{array}{c}1897 . \\
1 \ldots \ldots \ldots \\
2 \ldots \ldots \\
3 \ldots \ldots \\
5 \ldots \ldots \ldots\end{array}$ & $\begin{array}{l}1.9 \\
2.1 \\
1.85 \\
1.7 \\
1.75\end{array}$ & $\begin{array}{l}1.5 \\
1.7 \\
1.7 \\
1.75 \\
1.6\end{array}$ & $\begin{array}{l}1.7 \\
1.7 \\
1.6 \\
1.6 \\
1.5\end{array}$ & $\begin{array}{l}1.5 \\
1.6 \\
1.4 \\
1.6 \\
1.7\end{array}$ & $\begin{array}{l}1.45 \\
2.7 \\
2.1 \\
1.9 \\
1.6\end{array}$ & $\begin{array}{l}1.7 \\
1.6 \\
1.6 \\
1.7 \\
2.25\end{array}$ & $\begin{array}{c}1897 . \\
16 \ldots \ldots \\
17 \ldots \ldots \\
18 \ldots \ldots \\
19 \ldots \ldots \\
20 \ldots \ldots\end{array}$ & $\begin{array}{l}1.8 \\
1.75 \\
1.7 \\
1.75 \\
1.75\end{array}$ & $\begin{array}{l}1.9 \\
1.8 \\
1.7 \\
1.6 \\
1.5\end{array}$ & $\begin{array}{l}1.7 \\
1.6 \\
1.6 \\
1.6 \\
1.5\end{array}$ & $\begin{array}{l}1.65 \\
1.6 \\
1.4 \\
1.5 \\
1.7\end{array}$ & \begin{tabular}{l|}
1.75 \\
1.7 \\
1.6 \\
1.5 \\
1.6
\end{tabular} & $\begin{array}{l}2.4 \\
2.2 \\
2.1 \\
2.0 \\
2.1\end{array}$ \\
\hline $\begin{array}{r}6 \ldots \ldots \\
7 \ldots \ldots \\
8 \ldots \ldots \\
9 . \ldots \\
10 \ldots .\end{array}$ & $\begin{array}{l}1.8 \\
1.7 \\
1.7 \\
1.7 \\
1.7\end{array}$ & $\begin{array}{l}1.7 \\
1.7 \\
1.5 \\
1.4 \\
1.7\end{array}$ & $\begin{array}{l}1.7 \\
1.7 \\
1.6 \\
1.65 \\
1.6\end{array}$ & $\begin{array}{l}1.6 \\
1.7 \\
1.6 \\
1.5 \\
1.4\end{array}$ & $\begin{array}{l}1.6 \\
1.7 \\
1.75 \\
1.8 \\
1.6\end{array}$ & $\begin{array}{l}2.45 \\
2.05 \\
2.0 \\
1.8 \\
1.9\end{array}$ & $\begin{array}{l}21 \ldots \\
22 \ldots \\
23 \ldots \\
24 \ldots \\
25 \ldots\end{array}$ & $\begin{array}{l}1.85 \\
1.85 \\
1.7 \\
1.7 \\
1.55\end{array}$ & $\begin{array}{l}1.5 \\
1.55 \\
1.6 \\
3.0 \\
2.9\end{array}$ & $\begin{array}{l}1.6 \\
1.6 \\
1.7 \\
1.9 \\
1.8\end{array}$ & $\begin{array}{l}1.7 \\
1.6 \\
1.6 \\
1.65 \\
1.65\end{array}$ & $\begin{array}{l}1.7 \\
1.6 \\
1.5 \\
1.7 \\
1.8\end{array}$ & $\begin{array}{l}2.0 \\
2.0 \\
2.1 \\
2.5 \\
2.2\end{array}$ \\
\hline $\begin{array}{l}11 \ldots \\
12 \ldots \\
13 \ldots \\
14 \ldots \\
15 \ldots\end{array}$ & $\begin{array}{l}1.55 \\
1.75 \\
1.95 \\
1.8 \\
1.8\end{array}$ & $\begin{array}{l}1.9 \\
1.85 \\
1.6 \\
1.7 \\
2.45\end{array}$ & $\begin{array}{l}1.6 \\
1.5 \\
1.4 \\
1.5 \\
1.6\end{array}$ & $\begin{array}{l}1.5 \\
1.6 \\
1.7 \\
1.5 \\
1.6\end{array}$ & $\begin{array}{l}1.55 \\
1.5 \\
1.6 \\
1.4 \\
1.5\end{array}$ & $\begin{array}{l}1.9 \\
1.9 \\
2.05 \\
2.0 \\
2.35\end{array}$ & $\begin{array}{l}26 \ldots \\
27 \ldots \\
28 \ldots \\
29 \ldots \\
30 \ldots \\
31 \ldots\end{array}$ & $\begin{array}{l}1.65 \\
1.75 \\
1.8 \\
1.85 \\
1.8 \\
1.7\end{array}$ & $\begin{array}{l}2.0 \\
1.75 \\
1.7 \\
1.55 \\
1.8 \\
1.7\end{array}$ & $\begin{array}{l}1.5 \\
1.7 \\
1.6 \\
1.6 \\
1.5\end{array}$ & $\begin{array}{l}1.8 \\
1.7 \\
1.6 \\
1.6 \\
1.6 \\
1.5\end{array}$ & $\begin{array}{l}1.8 \\
1.9 \\
2.0 \\
1.9 \\
1.8\end{array}$ & $\begin{array}{l}2.0 \\
2.0 \\
1.9 \\
1.8 \\
1.8 \\
1.9\end{array}$ \\
\hline
\end{tabular}


Daily gage height, in feet, of Antietam Creek near Sharpsburg, Md.-Continued.

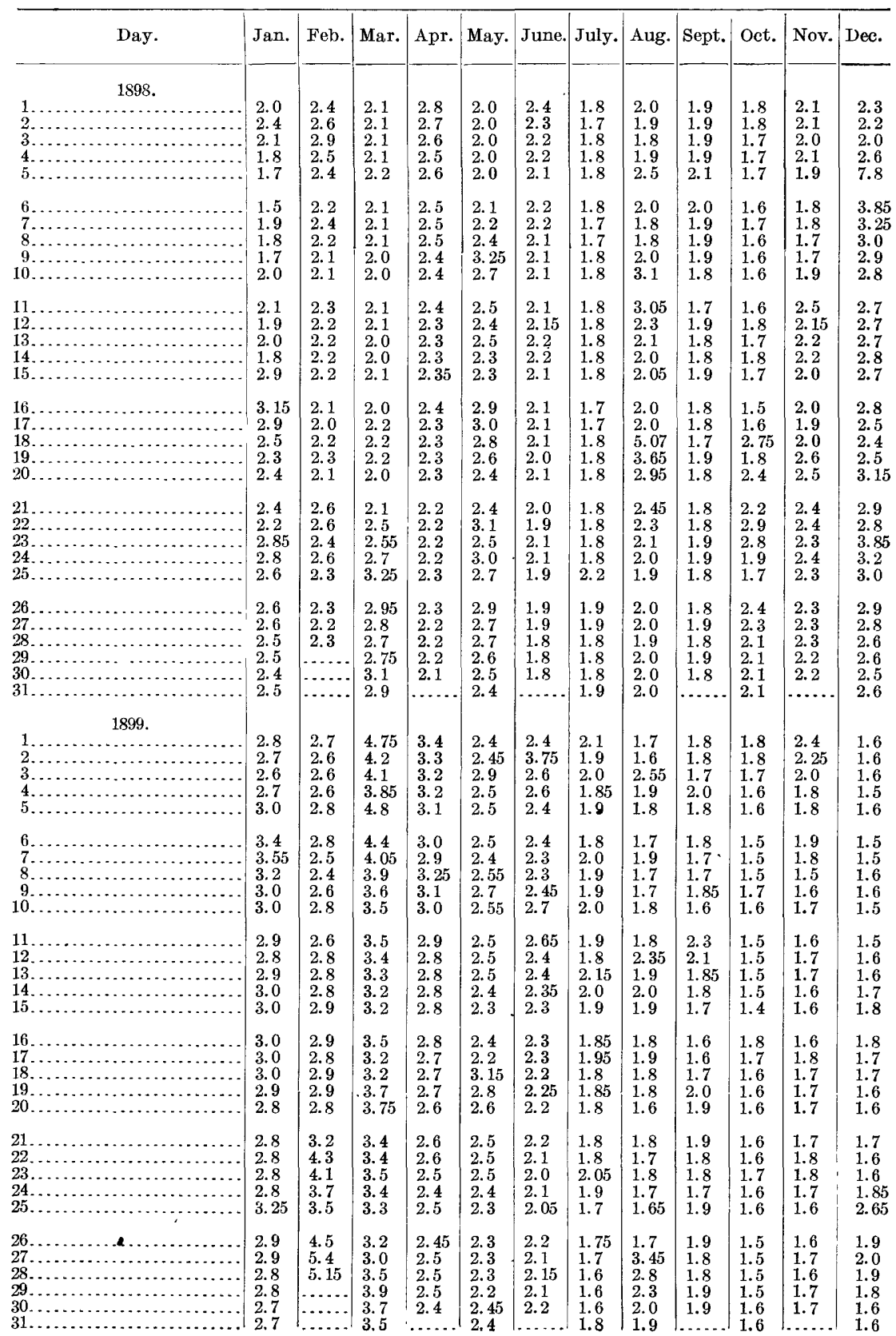


STREAM FLOW: ANTIETAM CREEK.

Daily gage height, in feet, of Antietam Creek near Sharpsburg, Md.-Continued.

\begin{tabular}{|c|c|c|c|c|c|c|c|c|c|c|c|c|}
\hline Day. & Jan. & Feb. & Mar. & Apr. & May. & June. & July. & Aug. & Sept. & Oct. & Nov. & Dec. \\
\hline $\begin{array}{l}1 \ldots \ldots \\
2 \ldots \ldots \\
3 \ldots \ldots \\
4 \ldots \ldots \\
5 \ldots \ldots\end{array}$ & $\begin{array}{l}1.5 \\
1.5 \\
1.5 \\
1.6 \\
1.6\end{array}$ & $\begin{array}{l}1.6 \\
1.7 \\
1.6 \\
1.5 \\
1.5\end{array}$ & $\begin{array}{l}3.8 \\
3.6 \\
3.0 \\
2.8 \\
2.7\end{array}$ & $\begin{array}{l}2.5 \\
2.5 \\
2.4 \\
2.3 \\
2.3\end{array}$ & $\begin{array}{l}2.1 \\
2.1 \\
2.0 \\
2.1 \\
2.0\end{array}$ & $\begin{array}{l}2.0 \\
1.9 \\
1.9 \\
2.0 \\
1.9\end{array}$ & $\begin{array}{l}1.7 \\
1.7 \\
1.6 \\
1.5 \\
1.7\end{array}$ & $\begin{array}{l}1.4 \\
1.4 \\
1.5 \\
1.5 \\
1.4\end{array}$ & $\begin{array}{l}1.6 \\
1.6 \\
1.4 \\
1.6 \\
1.7\end{array}$ & $\begin{array}{l}1.4 \\
1.5 \\
1.4 \\
1.4 \\
1.5\end{array}$ & $\begin{array}{l}1.7 \\
1.5 \\
1.5 \\
1.5 \\
1.5\end{array}$ & $\begin{array}{l}1.55 \\
1.55 \\
1.5 \\
2.3 \\
2.4\end{array}$ \\
\hline $\begin{array}{r}6 \ldots \ldots \\
7 \ldots \ldots \\
8 . \ldots \\
9 \ldots \ldots \\
10 \ldots\end{array}$ & $\begin{array}{l}1.7 \\
1.7 \\
1.8 \\
1.9 \\
1.8\end{array}$ & $\begin{array}{l}1.6 \\
1.5 \\
2.0 \\
2.2 \\
2.0\end{array}$ & $\begin{array}{l}2.7 \\
2.75 \\
2.6 \\
2.5 \\
2.5\end{array}$ & $\begin{array}{l}2.3 \\
2.4 \\
2.3 \\
2.3 \\
2.2\end{array}$ & $\begin{array}{l}1.9 \\
2.1 \\
2.0 \\
2.0 \\
1.9\end{array}$ & $\begin{array}{l}1.9 \\
1.9 \\
1.9 \\
1.9 \\
1.7\end{array}$ & $\begin{array}{l}1.7 \\
1.6 \\
1.6 \\
1.7 \\
1.6\end{array}$ & $\begin{array}{l}1.4 \\
1.4 \\
1.4 \\
1.45 \\
1.5\end{array}$ & $\begin{array}{l}1.6 \\
1.5 \\
1.5 \\
1.4 \\
1.4\end{array}$ & $\begin{array}{l}1.4 \\
1.4 \\
1.3 \\
1.5 \\
1.6\end{array}$ & $\begin{array}{l}1.4 \\
1.5 \\
1.6 \\
1.6 \\
1.6\end{array}$ & $\begin{array}{l}2.0 \\
1.6 \\
1.7 \\
1.7 \\
1.6\end{array}$ \\
\hline 5 & $\begin{array}{l}1.8 \\
1.8 \\
1.7 \\
1.7 \\
1.8\end{array}$ & $\begin{array}{l}1.9 \\
2.0 \\
2.7 \\
2.7 \\
2.4\end{array}$ & $\begin{array}{l}2.5 \\
2.5 \\
2.4 \\
2.4 \\
2.4\end{array}$ & $\begin{array}{l}2.2 \\
2.3 \\
2.3 \\
2.3 \\
2.2\end{array}$ & $\begin{array}{l}1.8 \\
1.9 \\
1.8 \\
1.8 \\
2.0\end{array}$ & $\begin{array}{l}2.0 \\
1.9 \\
1.8 \\
1.7 \\
1.7\end{array}$ & $\begin{array}{l}1.5 \\
1.55 \\
1.5 \\
1.55 \\
1.6\end{array}$ & $\begin{array}{l}1.4 \\
1.5 \\
1.5 \\
1.4 \\
1.7\end{array}$ & $\begin{array}{l}1.4 \\
1.4 \\
1.5 \\
1.6 \\
1.6\end{array}$ & $\begin{array}{l}1.6 \\
1.5 \\
1.5 \\
2.05 \\
1.9\end{array}$ & $\begin{array}{l}1.5 \\
1.4 \\
1.7 \\
1.5 \\
1.7\end{array}$ & $\begin{array}{l}1.6 \\
1.5 \\
1.5 \\
1.5 \\
1.4\end{array}$ \\
\hline $0 \ldots \ldots$ & $\begin{array}{l}1.7 \\
1.7 \\
1.7 \\
1.8 \\
2.1\end{array}$ & $\begin{array}{l}2.4 \\
2.2 \\
2.1 \\
2.2 \\
2.2\end{array}$ & $\begin{array}{l}2.4 \\
2.3 \\
2.25 \\
2.4 \\
2.9\end{array}$ & $\begin{array}{l}2.3 \\
2.2 \\
2.3 \\
2.35 \\
2.3\end{array}$ & $\begin{array}{l}1.9 \\
2.0 \\
2.0 \\
2.4 \\
3.1\end{array}$ & $\begin{array}{l}1.85 \\
2.1 \\
2.2 \\
2.55 \\
2.1\end{array}$ & $\begin{array}{l}1.45 \\
1.5 \\
1.5 \\
1.5 \\
1.6\end{array}$ & $\begin{array}{l}1.6 \\
1.6 \\
1.6 \\
1.6 \\
1.7\end{array}$ & \begin{tabular}{c}
1.8 \\
\hdashline 1.4 \\
1.5 \\
1.5
\end{tabular} & $\begin{array}{l}1.7 \\
1.6 \\
1.4 \\
1.6 \\
1.6\end{array}$ & $\begin{array}{l}1.5 \\
1.5 \\
1.4 \\
1.5 \\
1.5\end{array}$ & $\begin{array}{l}1.3 \\
1.5 \\
1.4 \\
1.5 \\
1.5\end{array}$ \\
\hline $\begin{array}{l}24 . \\
24 . \\
25 .\end{array}$ & $\begin{array}{l}2.5 \\
2.0 \\
1.9 \\
1.8 \\
1.7\end{array}$ & $\begin{array}{l}2.3 \\
4.15 \\
3.75 \\
3.05 \\
3.8\end{array}$ & $\begin{array}{l}3.1 \\
2.8 \\
2.7 \\
2.7 \\
2.6\end{array}$ & $\begin{array}{l}2.7 \\
2.3 \\
2.4 \\
2.3 \\
2.2\end{array}$ & $\begin{array}{l}2.4 \\
2.2 \\
2.1 \\
2.1 \\
2.1\end{array}$ & $\begin{array}{l}2.0 \\
1.9 \\
1.8 \\
1.7 \\
1.8\end{array}$ & $\begin{array}{l}1.5 \\
1.4 \\
1.9 \\
1.85 \\
1.65\end{array}$ & $\begin{array}{l}1.8 \\
1.8 \\
1.7 \\
1.7 \\
1.8\end{array}$ & $\begin{array}{l}1.5 \\
1.4 \\
1.4 \\
1.3 \\
1.4\end{array}$ & $\begin{array}{l}1.4 \\
1.5 \\
1.5 \\
1.8 \\
1.7\end{array}$ & $\begin{array}{l}1.6 \\
1.5 \\
1.5 \\
1.5 \\
1.5\end{array}$ & $\begin{array}{l}1.5 \\
1.5 \\
1.5 \\
1.4 \\
1.3\end{array}$ \\
\hline $\begin{array}{l}26 . \\
27 . \\
28 . \\
29 . \\
30 . \\
31 .\end{array}$ & $\begin{array}{l}2.3 \\
2.2 \\
2.0 \\
1.8 \\
1.6 \\
1.6\end{array}$ & \begin{tabular}{l}
3.3 \\
3.0 \\
3.0 \\
$\cdots \cdots$ \\
\hdashline$\ldots .$. \\
$\ldots .$.
\end{tabular} & $\begin{array}{l}2.5 \\
2.6 \\
2.5 \\
2.5 \\
2.6 \\
2.6\end{array}$ & $\begin{array}{c}2.2 \\
2.1 \\
2.1 \\
2.1 \\
2.0 \\
\ldots \ldots\end{array}$ & $\begin{array}{l}1.9 \\
1.9 \\
2.0 \\
1.9 \\
2.1\end{array}$ & $\begin{array}{c}1.8 \\
1.9 \\
1.8 \\
1.8 \\
1.7 \\
\cdots \cdots\end{array}$ & $\begin{array}{l}2.0 \\
1.9 \\
1.8 \\
1.55 \\
1.4 \\
1.4\end{array}$ & $\begin{array}{l}1.7 \\
1.7 \\
2.0 \\
1.9 \\
1.8 \\
1.7\end{array}$ & $\begin{array}{l}1.4 \\
1.4 \\
1.4 \\
1.5 \\
1.6 \\
\ldots \ldots\end{array}$ & $\begin{array}{l}1.5 \\
1.5 \\
1.4 \\
1.3 \\
1.4 \\
1.6\end{array}$ & $\begin{array}{l}2.5 \\
2.1 \\
1.8 \\
1.7 \\
1.6 \\
\ldots \ldots\end{array}$ & $\begin{array}{l}1.6 \\
1.5 \\
1.5 \\
1.5 \\
1.4 \\
1.5\end{array}$ \\
\hline $\begin{array}{l} \\
1 \ldots \ldots \\
2 \ldots \ldots \\
3 \ldots \\
4 \ldots \\
5 \ldots\end{array}$ & $\begin{array}{l}1.5 \\
1.5 \\
1.4 \\
1.4 \\
1.4\end{array}$ & $\begin{array}{l}1.5 \\
1.4 \\
1.4 \\
1.4 \\
1.5\end{array}$ & $\begin{array}{l}1.5 \\
1.5 \\
1.4 \\
1.5 \\
1.6\end{array}$ & \begin{tabular}{|l|}
2.8 \\
2.7 \\
3.3 \\
5.25 \\
3.65
\end{tabular} & $\begin{array}{l}2.5 \\
2.5 \\
2.4 \\
2.4 \\
2.4\end{array}$ & $\begin{array}{l}2.8 \\
2.8 \\
2.6 \\
2.5 \\
2.5\end{array}$ & $\begin{array}{l}2.2 \\
2.1 \\
2.1 \\
2.0 \\
2.1\end{array}$ & $\begin{array}{l}1.9 \\
1.8 \\
1.7 \\
1.7 \\
1.8\end{array}$ & $\begin{array}{l}2.5 \\
2.3 \\
2.1 \\
2.0 \\
2.0\end{array}$ & $\begin{array}{l}2.0 \\
1.9 \\
2.2 \\
2.0 \\
1.8\end{array}$ & $\begin{array}{l}1.6 \\
1.6 \\
1.4 \\
1.5 \\
1.5\end{array}$ & $\begin{array}{l}\cdots \cdot . \\
\cdots \cdots \\
\cdots \cdots \\
\cdots \cdots \\
\cdots \cdots\end{array}$ \\
\hline $\begin{array}{r}6 . \\
7 . \\
8 . \\
9 . \\
10 .\end{array}$ & $\begin{array}{l}1.4 \\
1.4 \\
1.4 \\
1.4 \\
1.5\end{array}$ & $\begin{array}{l}1.6 \\
1.6 \\
1.5 \\
1.5 \\
1.4\end{array}$ & $\begin{array}{l}1.5 \\
1.8 \\
1.7 \\
1.6 \\
1.6\end{array}$ & \begin{tabular}{|l|}
3.4 \\
3.3 \\
3.2 \\
3.1 \\
2.85
\end{tabular} & $\begin{array}{l}2.2 \\
2.3 \\
2.3 \\
2.2\end{array}$ & $\begin{array}{l}2.0 \\
2.8 \\
2.7 \\
2.6 \\
2.4\end{array}$ & $\begin{array}{l}2.1 \\
2.1 \\
2.1 \\
2.0 \\
2.0\end{array}$ & $\begin{array}{l}2.1 \\
2.6 \\
2.4 \\
2.2 \\
2.0\end{array}$ & $\begin{array}{l}1.8 \\
1.7 \\
1.6 \\
1.6\end{array}$ & $\begin{array}{l}1.8 \\
1.8 \\
1.7 \\
1.7 \\
1.6\end{array}$ & $\begin{array}{l}1.5 \\
1.6 \\
1.6 \\
1.6 \\
1.6\end{array}$ & $\left.\begin{array}{l}\cdots \\
\cdots \\
\cdots \\
\cdots\end{array}\right]$ \\
\hline $\begin{array}{l}13 \\
14 \\
15\end{array}$ & $\begin{array}{l}1.5 \\
1.7 \\
1.6 \\
1.6 \\
1.5\end{array}$ & $\begin{array}{l}1.4 \\
1.4 \\
1.3 \\
1.5 \\
1.4\end{array}$ & \begin{tabular}{|l|}
5.35 \\
3.35 \\
2.45 \\
2.2 \\
2.3
\end{tabular} & $\begin{array}{l}2.7 \\
2.6 \\
2.5 \\
2.7 \\
4.3\end{array}$ & $\begin{array}{l}2.3 \\
2.2 \\
2.2 \\
2.2\end{array}$ & $\begin{array}{l}2.3 \\
2.3 \\
2.2 \\
2.2 \\
2.4\end{array}$ & $\begin{array}{l}2.1 \\
2.2 \\
2.1 \\
2.1 \\
2.1\end{array}$ & $\begin{array}{l}1.9 \\
1.8 \\
1.8 \\
1.7 \\
3.9\end{array}$ & $\begin{array}{l}1.6 \\
1.7 \\
1.7 \\
1.6 \\
2.6\end{array}$ & $\begin{array}{l}1.6 \\
1.6 \\
1.6 \\
1.7 \\
1.7\end{array}$ & $\begin{array}{l}1.6 \\
1.6 \\
1.5 \\
1.5 \\
1.5\end{array}$ & $\begin{array}{l} \\
\cdots \\
\cdots \\
\cdots\end{array}$ \\
\hline $\begin{array}{l}18 \\
19 \\
20\end{array}$ & $\begin{array}{l}1.5 \\
1.6 \\
1.5 \\
1.4 \\
1.4\end{array}$ & $\begin{array}{l}1.6 \\
1.4 \\
1.5 \\
1.6 \\
1.6\end{array}$ & $\begin{array}{l}2.2 \\
2.2 \\
2.1 \\
2.1 \\
2.0\end{array}$ & \begin{tabular}{|l}
3.45 \\
2.9 \\
2.7 \\
2.6 \\
2.95
\end{tabular} & $\begin{array}{l}2.2 \\
2.2 \\
2.2 \\
2.2 \\
2.15\end{array}$ & $\begin{array}{l}2.8 \\
2.6 \\
2.5 \\
2.4 \\
2.3\end{array}$ & $\begin{array}{l}2.2 \\
2.5 \\
2.2 \\
2.1 \\
2.0\end{array}$ & $\begin{array}{l}2.2 \\
2.1 \\
2.0 \\
1.9 \\
1.8\end{array}$ & $\begin{array}{l}2.0 \\
2.1 \\
2.0 \\
1.8 \\
1.7\end{array}$ & $\begin{array}{l}1.6 \\
1.6 \\
1.5 \\
1.5 \\
1.6\end{array}$ & $\begin{array}{l}1.4 \\
\ldots . . \\
\cdots . .\end{array}$ & . \\
\hline $\begin{array}{l}23 . \\
24 . \\
25 .\end{array}$ & $\begin{array}{l}1.5 \\
1.5 \\
1.6 \\
1.6 \\
1.6\end{array}$ & $\begin{array}{l}1.7 \\
1.7 \\
1.8 \\
1.6 \\
1.3\end{array}$ & $\begin{array}{l}2.45 \\
2.4 \\
2.2 \\
2.2 \\
2.1\end{array}$ & \begin{tabular}{|l|}
3.6 \\
3.6 \\
3.2 \\
3.0 \\
2.9
\end{tabular} & $\begin{array}{l}2.1 \\
4.8 \\
6.3 \\
3.7 \\
3.85\end{array}$ & $\begin{array}{l}2.3 \\
2.3 \\
2.3 \\
2.3 \\
2.2\end{array}$ & $\begin{array}{l}2.5 \\
2.2 \\
2.1 \\
2.1 \\
2.5\end{array}$ & $\begin{array}{l}1.9 \\
1.8 \\
1.9 \\
2.0 \\
2.0\end{array}$ & $\begin{array}{l}1.7 \\
1.7 \\
1.7 \\
1.7 \\
1.6\end{array}$ & $\begin{array}{l}1.6 \\
1.5 \\
1.5 \\
1.5 \\
1.5\end{array}$ & & $\begin{array}{l}\cdots \cdot . \\
\cdots \cdots \\
\cdots \cdots \\
\cdots \cdots\end{array}$ \\
\hline $\begin{array}{l}26 . \\
27 . \\
28 . \\
29 . \\
30 . \\
31 .\end{array}$ & $\begin{array}{l}1.6 \\
1.4 \\
1.4 \\
1.5 \\
1.5 \\
1.5\end{array}$ & $\begin{array}{c}1.4 \\
1.5 \\
1.5 \\
\ldots . .\end{array}$ & $\begin{array}{l}2.25 \\
2.2 \\
2.2 \\
2.1 \\
2.2\end{array}$ & $\begin{array}{l}2.8 \\
2.9 \\
2.8 \\
2.7 \\
2.6\end{array}$ & $\begin{array}{l}3.4 \\
3.1 \\
3.0 \\
3.5 \\
3.0\end{array}$ & $\begin{array}{l}2.2 \\
2.3 \\
2.3 \\
2.35 \\
2.2\end{array}$ & $\begin{array}{l}2.1 \\
2.1 \\
2.0 \\
1.9 \\
1.9 \\
1.8\end{array}$ & $\begin{array}{l}2.0 \\
1.9 \\
1.7 \\
1.7 \\
1.7 \\
2.05\end{array}$ & $\begin{array}{l}1.6 \\
1.7 \\
1.7 \\
1.6 \\
2.15\end{array}$ & $\begin{array}{l}1.8 \\
1.7 \\
1.7 \\
1.6 \\
1.7 \\
1.7\end{array}$ & $\ldots$ & $\begin{array}{l}\ldots . . \\
\ldots 2.2 \\
4.7 \\
4.5 \\
3.3\end{array}$ \\
\hline
\end{tabular}


Daily gage height, in feet, of Antietam Creek near Sharpsburg, Md.-Continued.

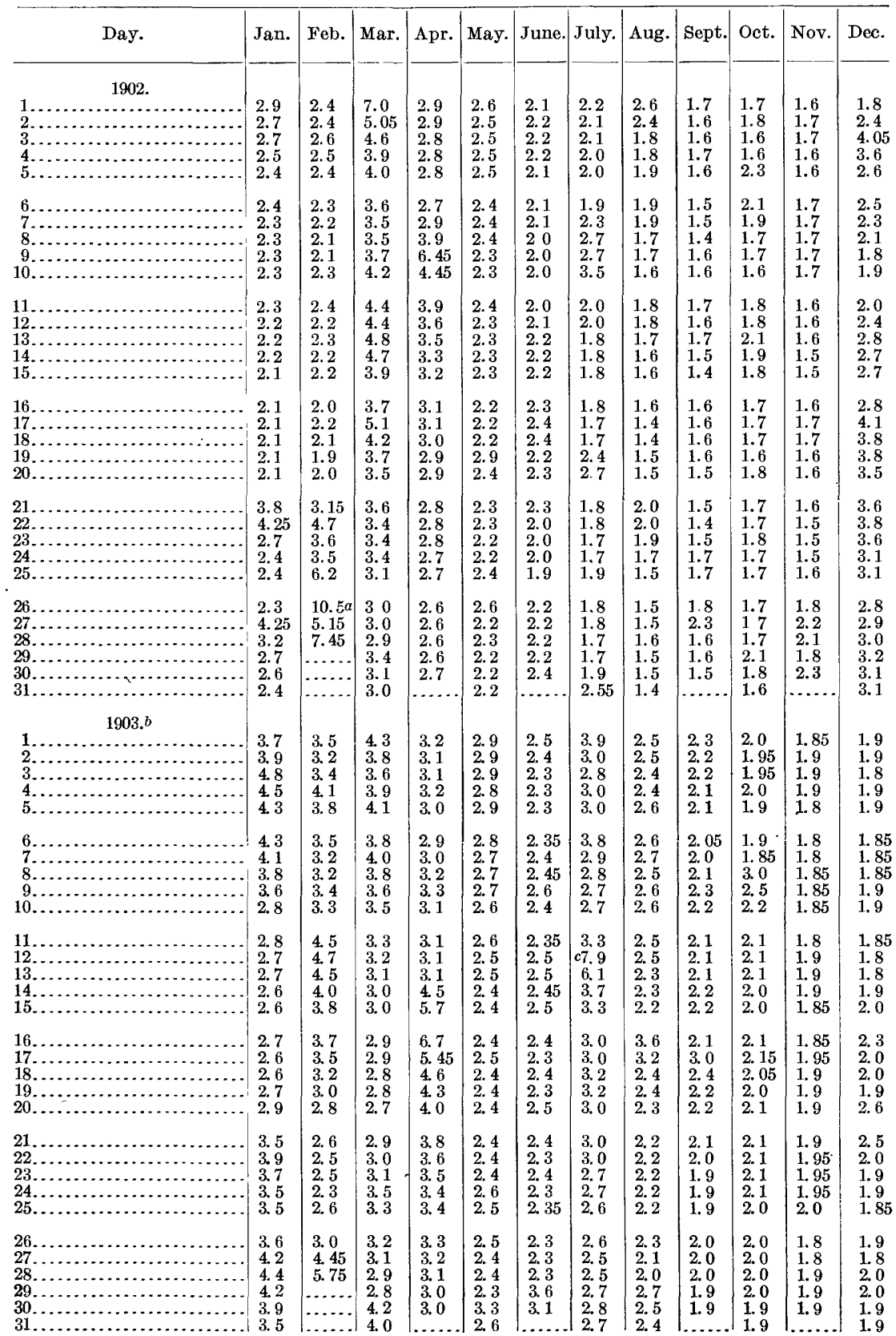


Daily gage height, in feet, of Antietam Creek near Sharpsburg, Md.-Continued.

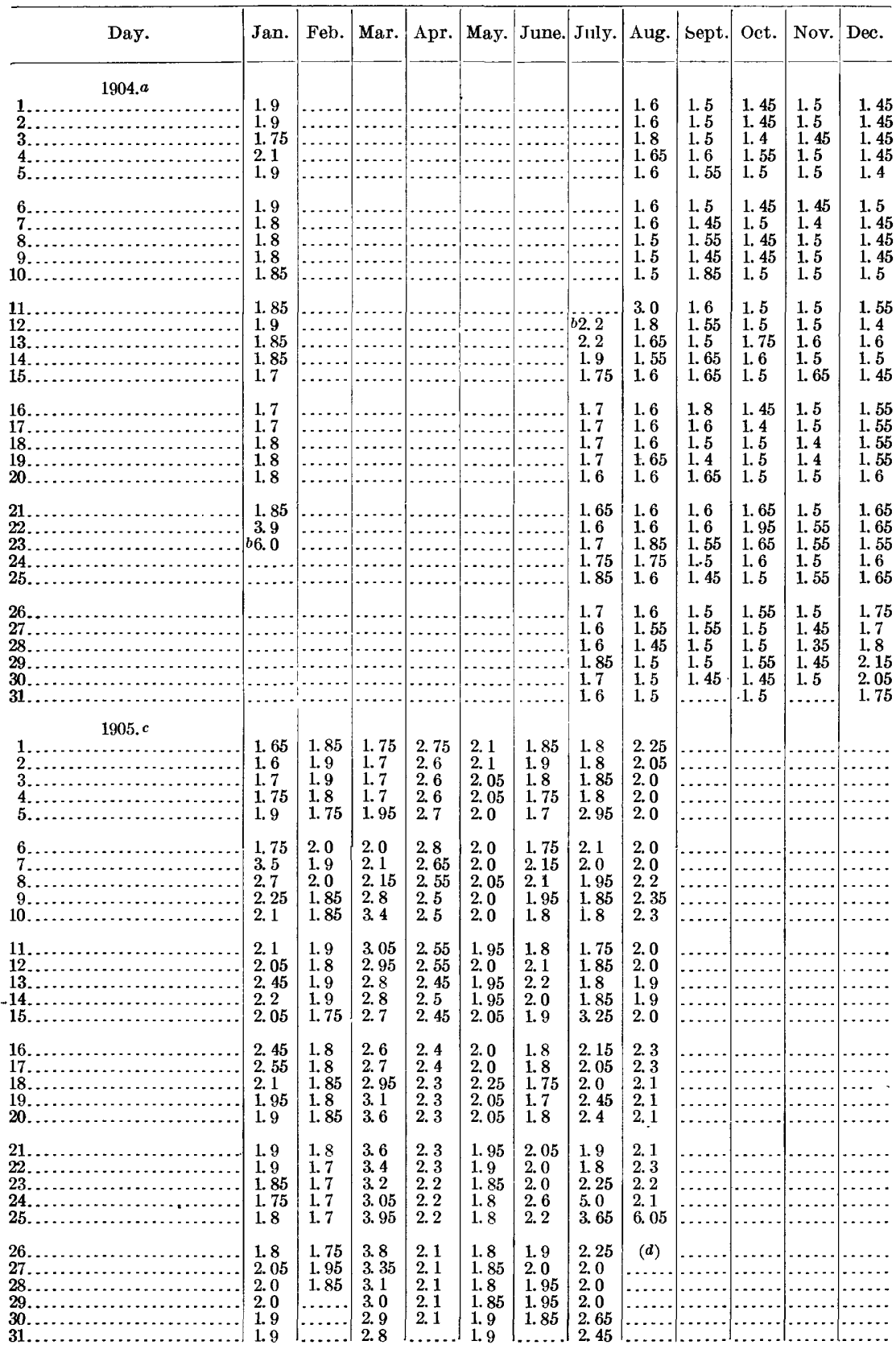

$a$ Ice conditions during January, 1904.

$b$ Ice carried gage away January 23, 1904; reestablished July 12, 1904

$c$ Ice conditions during part of February, 1905.

d Gage washed out August 26, 1905. 
Rating table for Antietam Creek near Sharpsburg, Md., from June 24, 1897, to August 25, 1905.a

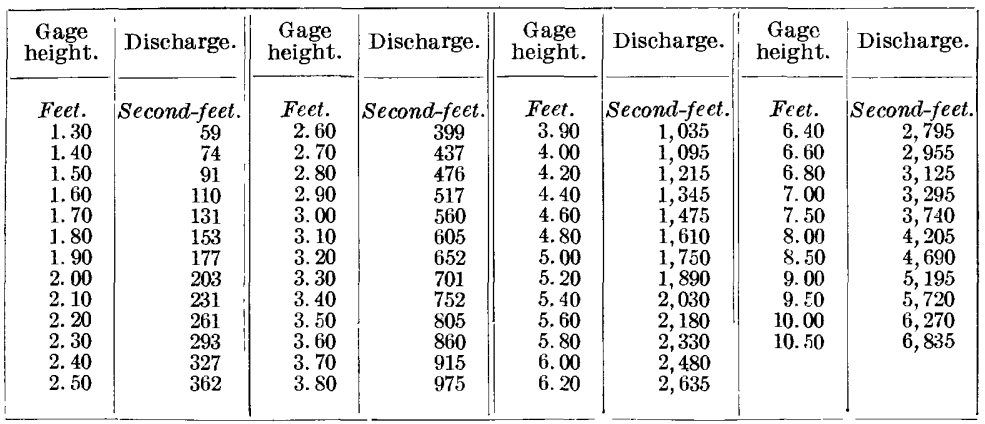

a This table is strictly applicable only for open-channel conditions. It is based on discharge measurements made durin 7 1897-1904. It is well defined between gage heights 1.7 feet and 3.5 feet. Estimates above 3.5 feet and below 1.7 feet are based on the extension of the area and velocity curves. Overflow at this section begins at about gage height 6.0 feet. It was assumed to amount to 300 second-feet at gage height 10.0 feet, or a mean velocity in the overflow portion of about one-half the velocity in the main channel.

\section{Estimated monthly discharge of Antietam Creek near Sharpsburg, Md.}

.[Drainage area, 295 square miles.]

\begin{tabular}{|c|c|c|c|c|c|c|c|c|}
\hline \multirow[b]{2}{*}{ Month. } & \multicolumn{3}{|c|}{ Discharge in second-feet. } & \multicolumn{2}{|c|}{ Run-off. } & \multirow{2}{*}{$\begin{array}{l}\text { Per cent } \\
\text { of pre- } \\
\text { cipita- } \\
\text { tion. }\end{array}$} & \multicolumn{2}{|c|}{ Precipitation. } \\
\hline & Maximum. & Minimum. & Mean. & $\begin{array}{c}\text { Second- } \\
\text { feet per } \\
\text { square } \\
\text { mile. }\end{array}$ & $\begin{array}{l}\text { Depth in } \\
\text { inches. }\end{array}$ & & $\underset{\text { inches. }}{\text { In }}$ & $\begin{array}{l}\text { Loss in } \\
\text { inehes. }\end{array}$ \\
\hline 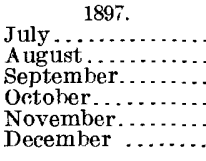 & $\begin{array}{l}231 \\
560 \\
177 \\
153 \\
437 \\
362 \\
\end{array}$ & $\begin{array}{r}100 \\
74 \\
74 \\
74 \\
74 \\
110\end{array}$ & $\begin{array}{l}146 \\
161 \\
114 \\
109 \\
140 \\
210\end{array}$ & $\begin{array}{r}0.495 \\
.546 \\
.386 \\
.369 \\
.475 \\
.712 \\
\end{array}$ & $\begin{array}{r}0.571 \\
.630 \\
.431 \\
.425 \\
.530 \\
.821 \\
\end{array}$ & & $\cdots$ & 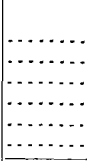 \\
\hline 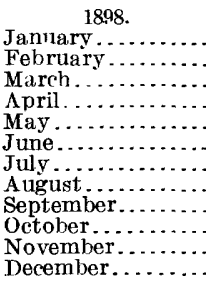 & $\begin{array}{r}628 \\
517 \\
676 \\
476 \\
676 \\
327 \\
261 \\
1,799 \\
231 \\
456 \\
399 \\
4,015\end{array}$ & $\begin{array}{r}91 \\
203 \\
203 \\
231 \\
203 \\
153 \\
131 \\
153 \\
131 \\
91 \\
131 \\
203\end{array}$ & $\begin{array}{l}303 \\
302 \\
312 \\
316 \\
375 \\
226 \\
159 \\
322 \\
167 \\
200 \\
249 \\
598\end{array}$ & $\begin{array}{c}1.03 \\
1.02 \\
1.06 \\
1.07 \\
1.27 \\
.766 \\
.539 \\
1.09 \\
.566 \\
.678 \\
.844 \\
2.03\end{array}$ & $\begin{array}{c}1.19 \\
1.06 \\
1.22 \\
1.19 \\
1.46 \\
.855 \\
.621 \\
1.26 \\
6.32 \\
.782 \\
.942 \\
2.34\end{array}$ & $\cdots$ & & $\begin{array}{l}\cdots \\
\cdots \\
\cdots \\
\cdots \\
\cdots \\
\cdots \\
\cdots\end{array}$ \\
\hline The year. & 4,015 & 91 & 294 & .997 & 13.55 & 1 & & \\
\hline 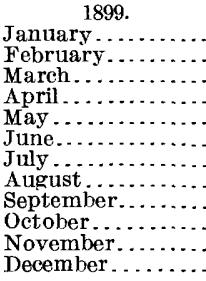 & $\begin{array}{r}832 \\
2,030 \\
1,610 \\
752 \\
517 \\
945 \\
246 \\
778 \\
293 \\
153 \\
327 \\
418\end{array}$ & $\begin{array}{r}399 \\
327 \\
560 \\
327 \\
261 \\
203 \\
110 \\
110 \\
110 \\
74 \\
91 \\
91\end{array}$ & $\begin{array}{l}529 \\
682 \\
897 \\
483 \\
358 \\
317 \\
169 \\
200 \\
160 \\
109 \\
144 \\
133\end{array}$ & $\begin{array}{l}1.79 \\
2.31 \\
3.04 \\
1.64 \\
1.21 \\
1.07 \\
.572 \\
.678 \\
.542 \\
.369 \\
.488 \\
.451\end{array}$ & $\begin{array}{c}2.06 \\
2.40 \\
3.50 \\
1.83 \\
1.40 \\
1.19 \\
.660 \\
.782 \\
.605 \\
.425 \\
.544 \\
.520\end{array}$ & $\begin{array}{r}103 \\
56 \\
88 \\
193 \\
28 \\
28 \\
60 \\
20 \\
13 \\
26 \\
22 \\
23\end{array}$ & $\begin{array}{l}2.00 \\
4.30 \\
4.00 \\
.95 \\
5.08 \\
4.19 \\
1.10 \\
3.85 \\
4.68 \\
1.63 \\
2.46 \\
2.26\end{array}$ & $\begin{array}{r}-0.06 \\
1.90 \\
.50 \\
-.88 \\
3.68 \\
3.00 \\
.44 \\
3.07 \\
4.07 \\
1.20 \\
1.92 \\
1.74\end{array}$ \\
\hline The year & 2,030 & 74 & 349 & 1. 18 & 15.92 & 44 & 36.50 & 20.58 \\
\hline
\end{tabular}


Estimated monthly discharge of Antietam Creek near Sharpsburg, Md.-Continued.

\begin{tabular}{|c|c|c|c|c|c|c|c|c|}
\hline \multirow[b]{2}{*}{ Month. } & \multicolumn{3}{|c|}{ Di scharge in second-feet. } & \multicolumn{2}{|c|}{ Run-off. } & \multirow[b]{2}{*}{$\begin{array}{c}\text { Per cent } \\
\text { of pre- } \\
\text { cipita- } \\
\text { tion. }\end{array}$} & \multicolumn{2}{|c|}{ Precipitation. } \\
\hline & Maximum. & Minimum. & Mean. & $\begin{array}{c}\text { Second- } \\
\text { feet per } \\
\text { square } \\
\text { mile. }\end{array}$ & $\begin{array}{l}\text { Depth in } \\
\text { inches. }\end{array}$ & & $\underset{\text { inches. }}{\text { In }}$ & $\begin{array}{l}\text { Loss in } \\
\text { inches. }\end{array}$ \\
\hline 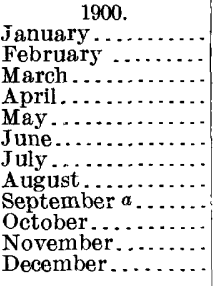 & $\begin{array}{r}362 \\
1,185 \\
975 \\
362 \\
605 \\
380 \\
203 \\
203 \\
153 \\
217 \\
362 \\
327\end{array}$ & $\begin{array}{r}91 \\
91 \\
277 \\
203 \\
153 \\
131 \\
74 \\
74 \\
74 \\
59 \\
74 \\
59\end{array}$ & $\begin{array}{c}158 \\
372 \\
432 \\
291 \\
222 \\
181 \\
115 \\
112 \\
91.7 \\
99.1 \\
114 \\
110\end{array}$ & $\begin{array}{l}.536 \\
1.26 \\
1.46 \\
.986 \\
.753 \\
.614 \\
.390 \\
.380 \\
.311 \\
.336 \\
.386 \\
.373\end{array}$ & $\begin{array}{l}.618 \\
1.31 \\
1.68 \\
1.10 \\
.868 \\
.685 \\
.450 \\
.438 \\
.347 \\
.387 \\
.431 \\
.430\end{array}$ & $\begin{array}{l}26 \\
34 \\
62 \\
80 \\
34 \\
15 \\
10 \\
15 \\
16 \\
28 \\
14 \\
27\end{array}$ & $\begin{array}{l}2.34 \\
3.82 \\
2.71 \\
1.38 \\
2.52 \\
4.52 \\
4.34 \\
3.00 \\
2.25 \\
1.37 \\
3.07 \\
1.59\end{array}$ & $\begin{array}{r}1.72 \\
2.51 \\
1.03 \\
.28 \\
1.65 \\
3.84 \\
3.89 \\
2.56 \\
1.90 \\
.98 \\
2.64 \\
1.16 \\
\end{array}$ \\
\hline The year & 1,185 & 59 & 192 & .649 & 8.75 & 27 & 32.91 & 24. 16 \\
\hline 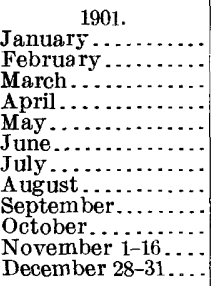 & $\begin{array}{r}131 \\
153 \\
1,995 \\
1,925 \\
2,715 \\
476 \\
362 \\
1,035 \\
399 \\
261 \\
110 \\
1,540\end{array}$ & $\begin{array}{r}74 \\
59 \\
74 \\
362 \\
231 \\
261 \\
153 \\
131 \\
110 \\
91 \\
74 \\
261\end{array}$ & $\begin{array}{c}90.5 \\
92.4 \\
285 \\
632 \\
523 \\
341 \\
238 \\
218 \\
171 \\
130 \\
98.4 \\
978\end{array}$ & $\begin{array}{l}.307 \\
.313 \\
.966 \\
2.14 \\
1.77 \\
1.16 \\
.807 \\
.739 \\
.580 \\
.441 \\
.334 \\
3.32\end{array}$ & $\begin{array}{l}.354 \\
.326 \\
1.11 \\
2.39 \\
2.04 \\
1.29 \\
.930 \\
.852 \\
.647 \\
.508 \\
.199 \\
.494\end{array}$ & $\begin{array}{r}16 \\
52 \\
28 \\
42 \\
36 \\
29 \\
25 \\
20 \\
26 \\
57 \\
\cdots \\
\end{array}$ & $\begin{array}{r}2.16 \\
.63 \\
3.92 \\
5.73 \\
5.71 \\
4.43 \\
3.77 \\
4.33 \\
2.51 \\
.89 \\
b 2.36 \\
b 5.83\end{array}$ & \begin{tabular}{r}
1.81 \\
.30 \\
2.81 \\
3.34 \\
3.67 \\
3.14 \\
2.84 \\
3.48 \\
1.86 \\
.38 \\
\hdashline .3. \\
\end{tabular} \\
\hline The y & 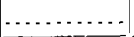 & . & 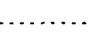 & .... & 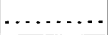 & & 42.27 & $\ldots . . .1$. \\
\hline 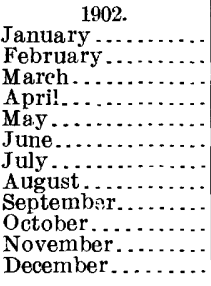 & $\begin{array}{r}1,248 \\
6,835 \\
3,295 \\
2,835 \\
517 \\
327 \\
805 \\
399 \\
293 \\
293 \\
293 \\
1,155\end{array}$ & $\begin{array}{r}231 \\
177 \\
517 \\
399 \\
261 \\
177 \\
131 \\
74 \\
74 \\
110 \\
91 \\
153\end{array}$ & $\begin{array}{r}410 \\
871 \\
1,068 \\
662 \\
313 \\
249 \\
232 \\
141 \\
113 \\
150 \\
131 \\
570\end{array}$ & $\begin{array}{l}1.39 \\
2.95 \\
3.62 \\
2.24 \\
1.06 \\
.844 \\
.786 \\
.478 \\
.383 \\
.508 \\
.444 \\
1.93\end{array}$ & $\begin{array}{c}1.60 \\
3.07 \\
4.17 \\
2.50 \\
1.22 \\
.942 \\
.906 \\
5.51 \\
.427 \\
.586 \\
.495 \\
2.22\end{array}$ & $\begin{array}{r}48 \\
69 \\
105 \\
98 \\
97 \\
22 \\
29 \\
60 \\
26 \\
16 \\
20 \\
56\end{array}$ & $\begin{array}{l}3.30 \\
4.44 \\
3.98 \\
2.54 \\
1.26 \\
4.28 \\
3.09 \\
.91 \\
1.64 \\
3.73 \\
2.48 \\
3.98\end{array}$ & $\begin{array}{r}1.70 \\
1.37 \\
-.19 \\
.04 \\
.04 \\
3.34 \\
2.18 \\
.36 \\
1.21 \\
3.14 \\
1.99 \\
1.76\end{array}$ \\
\hline The year.... & 6,835 & 74 & 409 & 1. 39 & 18.69 & 52 & 35.63 & 16. 94 \\
\hline $\begin{array}{l}\text { January } \\
\text { February } \\
\text { March... } \\
\text { April... } \\
\text { May.... } \\
\text { June.... } \\
\text { July.... } \\
\text { August. } \\
\text { Septemb } \\
\text { October. } \\
\text { Novemb } \\
\text { Decembe }\end{array}$ & $\begin{array}{r}1,610 \\
2,292 \\
1,280 \\
3,040 \\
701 \\
860 \\
4,110 \\
860 \\
560 \\
560 \\
203 \\
399\end{array}$ & $\begin{array}{l}399 \\
293 \\
437 \\
517 \\
293 \\
293 \\
362 \\
203 \\
177 \\
165 \\
153 \\
153\end{array}$ & $\begin{array}{l}843 \\
849 \\
750 \\
926 \\
398 \\
350 \\
738 \\
354 \\
242 \\
226 \\
172 \\
195\end{array}$ & $\begin{array}{l}2.86 \\
2.88 \\
2.54 \\
3.14 \\
1.35 \\
1.19 \\
2.50 \\
1.20 \\
.820 \\
.766 \\
.583 \\
.661\end{array}$ & $\begin{array}{c}2.93 \\
3.50 \\
1.56 \\
1.33 \\
2.88 \\
1.38 \\
.915 \\
.883 \\
.650 \\
.762\end{array}$ & $\begin{array}{r}84 \\
109 \\
91 \\
94 \\
36 \\
23 \\
40 \\
27 \\
57 \\
27 \\
47 \\
69\end{array}$ & $\begin{array}{l}3.95 \\
2.74 \\
3.23 \\
3.73 \\
4.28 \\
5.86 \\
7.20 \\
5.10 \\
1.60 \\
3.21 \\
1.38 \\
1.11\end{array}$ & $\begin{array}{r}0.65 \\
-.26 \\
.30 \\
.23 \\
2.72 \\
4.53 \\
4.32 \\
3.72 \\
.68 \\
2.33 \\
.73 \\
.35\end{array}$ \\
\hline The year. . & 4,110 & 153 & 504 & 1. 71 & 23.09 & 53 & 43.39 & 20.30 \\
\hline
\end{tabular}

a Discharge interpolated September 17, 1900

$b$ Precipitation for complete month, November and December, 1901.

c ice conditions during part of December, 1903; no correction made in estimates. 
Estimated monthly discharge of Antietam Creek near Sharpsburg, Md.-Continued.

\begin{tabular}{|c|c|c|c|c|c|c|c|c|}
\hline \multirow[b]{2}{*}{ Month. } & \multicolumn{3}{|c|}{ Discharge in second-feet. } & \multicolumn{2}{|c|}{ Run-off. } & \multirow[b]{2}{*}{$\begin{array}{c}\text { Per cent } \\
\text { of pre- } \\
\text { cipita- } \\
\text { tion. }\end{array}$} & \multicolumn{2}{|c|}{ Precipitation. } \\
\hline & Maximum. & Minimum. & Mean. & $\begin{array}{c}\text { Second- } \\
\text { feet per } \\
\text { square } \\
\text { mile. }\end{array}$ & $\begin{array}{l}\text { Depth in } \\
\text { inches. }\end{array}$ & & $\begin{array}{c}\text { In } \\
\text { inehes. }\end{array}$ & $\begin{array}{l}\text { Loss iu } \\
\text { inches. }\end{array}$ \\
\hline & & & & & & & & \\
\hline $\begin{array}{l}\text { January } 1-23 \text { a } \ldots . . \\
\text { February }\end{array}$ & 2,480 & 131 & 300 & 1.02 & .872 & & b 2.42 & \\
\hline & $\cdots$ & $\cdots$ & $\ldots \ldots$ & 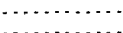 & $\cdots+$ & & $\begin{array}{l}1.08 \\
1.98\end{array}$ & \\
\hline April... & & $\cdots$ & 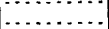 & $\cdots$ & $\ldots$ & & 1.95 & . \\
\hline May... & 1 & $\ldots$ & |- & . & $\ldots$. & & 2.73 & \\
\hline June. . & & & 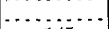 & $\ldots$ & $\ldots$. & & 5.54 & \\
\hline July 12-31. & 261 & 110 & 145 & .492 & .366 & & b 4.05 & \\
\hline August... & 560 & 82 & 126 & .427 & .492 & 19 & 2.54 & 2. 05 \\
\hline September........ & 165 & 74 & 101 & .342 & .382 & 13 & 2.86 & 2.48 \\
\hline October.......... & 190 & 74 & 96.7 & .328 & .378 & 15 & 2.53 & 2. 15 \\
\hline November........ & 120 & 66 & 92.8 & .315 & .351 & 41 & .85 & .50 \\
\hline December....... & 246 & 74 & 110 & .373 & .430 & 17 & 2.46 & 2.03 \\
\hline The year. ... & & $\cdots$ & & & & & 30.99 & \\
\hline 1905. & & & & & & & & \\
\hline January .... & 805 & 110 & 229 & .776 & .895 & 22 & 4.00 & 3. 10 \\
\hline February $c .$. & 203 & 131 & 161 & .546 & .569 & 31 & 1.86 & 1. 29 \\
\hline March. - & 1,065 & 131 & 508 & 1. 72 & 1.98 & 79 & 2.50 & .52 \\
\hline April... & 476 & 231 & 332 & 1. 13 & 1.26 & 68 & 1.84 & .58 \\
\hline May.. & 231 & 153 & 196 & .664 & .766 & 33 & 2. 31 & 1.54 \\
\hline June... & 399 & 131 & 190 & .644 & .718 & 12 & 5.84 & 5. 12 \\
\hline July.. & 1,750 & 142 & 311 & 1.06 & 1.22 & 18 & 6.84 & 5.62 \\
\hline August 1-25. & 2,518 & 177 & 326 & 1.11 & 1.03 & & d 4.84 & $\ldots . .$. \\
\hline September... & & & 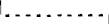 & $\ldots$ & & ... & 2.11 & $\ldots \ldots$ \\
\hline October.......... & . & & & & 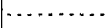 & $\cdots$ & 3.71 & $\ldots \ldots$ \\
\hline November......... & - & & - & & & $\ldots$ & $\begin{array}{l}1.92 \\
3.48\end{array}$ & $\ldots \ldots$ \\
\hline December ......... & & & & & & & & 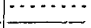 \\
\hline The year. & & & & & & & 41.25 & $\ldots$ \\
\hline
\end{tabular}

$a$ Ice conditions during January, 1904; no correction made in estimates.

$b$ Precipitation for complete month, January and July, 1904.

$c$ Ice conditions during part of February, 1905 ; no correction made in estimates.

$d$ Precipitation for complete month, August, 1905.

MISCELLANEOUS DISCHARGE MEASUREMENTS IN POTOMAC RIVER BASIN BETWEEN MOUTH OF SOUTH BRANCH AND SHENANDOAH RIVER.

The following miscellaneous discharge measurements have been made in the drainage basin of Potomac River between the mouth or South Branch and Shenandoah River:

Muscellaneous discharge measurements in Potomac River basin between mouth of South Branch and Shenandoah Rivers.

\begin{tabular}{|c|c|c|c|c|c|c|}
\hline Date. & Stream. & Locality. & Width. & $\begin{array}{l}\text { Area of } \\
\text { section. }\end{array}$ & $\begin{array}{l}\text { Mean } \\
\text { veloc- } \\
\text { ity. }\end{array}$ & $\begin{array}{l}\text { Dis- } \\
\text { charge. }\end{array}$ \\
\hline $\begin{array}{l}1897 . \\
\text { September } 25 .\end{array}$ & Town Creek. & $\begin{array}{l}200 \text { yards above Chesapeake } \\
\text { and Ohio Canal,and } 3 \text { miles } \\
\text { below junction of North } \\
\text { and South branches of Po- } \\
\text { tomac River. }\end{array}$ & $\begin{array}{l}\text { Feet. } \\
22\end{array}$ & $\begin{array}{l}\text { Square } \\
\text { feet. } \\
11\end{array}$ & $\begin{array}{l}\text { Ft. per } \\
\text { second. } \\
\quad 1.00\end{array}$ & $\begin{array}{c}\text { Sec.-ft. } \\
11\end{array}$ \\
\hline $\begin{array}{l}\text { September } 29 . \\
\text { September } 26 .\end{array}$ & $\begin{array}{l}\text { Little Cacapon River. } \\
\text { Purslane Run........ }\end{array}$ & $\begin{array}{l}\text { Near Little Cacapon, W. Va. } \\
\text { Near Pawpaw, W. Va....... }\end{array}$ & 4 & $\begin{array}{c}1.8 \\
\cdots\end{array}$ & $\begin{array}{l}.89 \\
\cdots\end{array}$ & $\begin{array}{r}1.6 \\
.5\end{array}$ \\
\hline $\begin{array}{l}\text { Do } \\
\text { Do }\end{array}$ & $\begin{array}{l}\text { Fifteenmile Creek } . . . \\
\text { Sideling Hill Creek... }\end{array}$ & Near Little Orleans, Md & 9 & 2.1 & .67 & $\begin{array}{r}.5 \\
1.4\end{array}$ \\
\hline September 29. & Great Cacapon River. & One-half mile above mouth, & 64 & 123 & .66 & a. 81 \\
\hline $\begin{array}{l}\text { October } 11 . \\
\text { Do.... } \\
\text { Do.... }\end{array}$ & $\begin{array}{l}\text { Sir Johns Run... } \\
\text { Tonoloway Creek } \\
\text { Potomae River.. }\end{array}$ & $\begin{array}{l}\text { Near Sir Johns Run, W. Va.. } \\
\text { Near Hancock, Md......... } \\
\text { At Hancock, Md........... }\end{array}$ & $\begin{array}{r}2.5 \\
8 \\
180\end{array}$ & $\begin{array}{l}3.55 \\
364\end{array}$ & $\begin{array}{r}1.63 \\
1.68 \\
.56\end{array}$ & $\begin{array}{r}5.9 \\
202\end{array}$ \\
\hline
\end{tabular}

$a$ Residents state river very low. 
Miscellaneous discharge measurements in Potomac River basin between mouth of South Branch and Shenandoah Rivers-Continued.

\begin{tabular}{|c|c|c|c|c|c|c|}
\hline Date. & Stream. & Locality. & Width. & $\begin{array}{l}\text { Area of } \\
\text { section. }\end{array}$ & $\begin{array}{l}\text { Mean } \\
\text { veloc- } \\
\text { ity. }\end{array}$ & charge. \\
\hline $\begin{array}{c}1897 . \\
\text { September } 30 .\end{array}$ & Warm Spring Run. & Below Baltimore and ohio & $\begin{array}{l}\text { Feet. } \\
6\end{array}$ & $\begin{array}{c}\text { Square } \\
\text { feet. } \\
2.1\end{array}$ & $\begin{array}{l}\text { Ft. per } \\
\text { second. } \\
1.28\end{array}$ & $\begin{array}{r}\text { Sec.-ft. } \\
2.7\end{array}$ \\
\hline October $1^{-}$ & $\begin{array}{l}\text { Great } \\
\text { Creek. }\end{array}$ & $\begin{array}{l}\text { R. R., near Hancock, Md. } \\
\text { Short distance above Chesa- } \\
\text { peake and Ohio Canal aque }\end{array}$ & 9 & 4 & 1.40 & 5. 6 \\
\hline Do... & Sleepy Creek. & $\begin{array}{l}\text { One-fourth mile above Bal- } \\
\text { timore and ohio R. R. } \\
\text { bridge, near Munson, } \\
\text { W.Va. }\end{array}$ & 8 & 3 & .77 & 2. 3 \\
\hline Do. & Licking Creek... & $\begin{array}{l}\text { Short distance above Chesa- } \\
\text { peake and Ohio Canal aque- }\end{array}$ & 33 & - 36 & .61 & 22 \\
\hline October 7 . & Back Creek. & $\begin{array}{l}\text { Near Baltimore and ohio } \\
\text { R. R. bridge, above North } \\
\text { Mountain, W. Va. }\end{array}$ & 29 & 11 & .59 & 6.5 \\
\hline October 8 . & ....do.. & Near mouth, near North & & & & 50 \\
\hline October 7 & Big Spring Run. & At Charles Mills, near Big & 4 & 7.2 & .53 & 3. 2 \\
\hline Do. & $\begin{array}{l}\text { Little Conococheague } \\
\text { Creek. }\end{array}$ & $\begin{array}{l}\text { At Charles Mills, near Four } \\
\text { locks, Md. }\end{array}$ & 2.5 & 1.2 & 2.08 & 2.5 \\
\hline Octoher $2 .$. & Conococheague Creek. & $\begin{array}{l}500 \text { feet below northeast } \\
\text { turnpike bridge, near Wil- } \\
\text { liamsport, Md. }\end{array}$ & 125 & 124 & 1.60 & 198 \\
\hline October 8 . & Opequon Creek. & Near mouth, near Bedington & 58 & 65 & .77 & 50 \\
\hline October 9. & $\begin{array}{l}\text { Marsh Run (tribu- } \\
\text { tary to Antietam } \\
\text { Creek). }\end{array}$ & $\begin{array}{l}\text { Street crossing, below south } \\
\text { end drain, Hagerstown, } \\
\text { Md. }\end{array}$ & 4 & 3.1 & 1.16 & 3.6 \\
\hline October & Antietam Creek & $\begin{array}{l}\text { At Stonebreaker's paper } \\
\text { mill, near Hagerstown, } \\
\text { Md. }\end{array}$ & 68 & 68 & 1.03 & a 70 \\
\hline July $2 \ldots . .$. & Potomac River.. & At Harpers Ferry, W. Va.... & & & & $b 1,223$ \\
\hline $\begin{aligned} 1897 . \\
\text { October } 13\end{aligned}$ & & & & & & $c 377$ \\
\hline
\end{tabular}

$a$ Gage height on Antietam Creek at Sharpsburg, Md., 1.8 feet.

$b$ Gage height on Potomac River at Harpers Ferry, W. Va., 2.52 feet.

c 77 second-feet in river, and 300 second-feet in Pulp Company's canal.

SHENANDOAH RIVER BASIN.

SOUTH FORK OF SHENANDOAH RIVER BASIN.

SOUTH RIVER BASIN.

SOUTH RIVER AT BASIO GITY, VA.

South River rises near Greenville, in the southern part of Augusta County, Va., and flows eastward and northward to Port Republic, Rockingham County, where it unites with North River to form South Fork of the Shenandoah. Its drainage area is 245 square miles. It is fed by numerous springs and is utilized to a considerable extent by small mills.

The gaging station was established June 29, 1905, by N. C. Grover, in connection with the investigation of stream pollution in the Shenandoah Valley. It was discontinued July 16, 1906. It is located at the highway bridge one-half mile below the Chesapeake and Ohio Railway bridge at Basic City, Va.

IRR 192-07-7 
The channel is straight for 300 feet above and 500 feet below the station. The current is sluggish at ordinary stages. Both banks are subject to overflow, the right bank only during very high water. The bed of the stream is composed of rocks and mud and is liable to change after floods. The approximate depth of water is 3 to 4 feet at medium stage. Gage-height observations and measurements are affected by flour mills above the station, which cause rapid fluctuations in the gage height at times.

Discharge measurements were made from the upstream side of the single span bridge to which the gage is fastened. The initial point for soundings is the face of the right abutment.

A standard chain gage is fastened to the upstream hand rail of the bridge. The length of the chain from the end of the weight to the outer edge of the ring is 20.84 feet. The gage was read once each day by F. J. Bates. Bench mark No. 1 is the upstream corner of the lowest step of the wing wall of the bridge at the right bank, nearest the river. It is marked with red paint. Its elevation is 13.97 feet above the datum of the gage.

Estimates at this station above gage height 2.4 feet are considered to be within 5 per cent of the true discharge for normal conditions of flow. Below 2.4 feet the probable error is from 5 to 20 per cent. The flow during the winter of 1905-6 was probably unaffected by ice conditions.

Discharge measurements of South River at Basic City, Va.

\begin{tabular}{|c|c|c|c|c|c|c|}
\hline Date. & $\begin{array}{c}\text { Gage } \\
\text { height. }\end{array}$ & Discharge. & Date. & . & $\begin{array}{c}\text { Gage } \\
\text { height. }\end{array}$ & Discharge. \\
\hline $\begin{aligned} & 1895 . \\
\text { August } 5 \text { a. } & \ldots \ldots\end{aligned}$ & $\begin{array}{l}\text { Feet. } \\
\text { Fe. }\end{array}$ & $\left|\begin{array}{r}\text { Second-feet } \\
72\end{array}\right|$ & $\begin{array}{r}1905 . \\
\text { December } 29 \ldots\end{array}$ & & $\begin{array}{l}\text { Feet. } \\
\quad 4.05\end{array}$ & Second-feet. \\
\hline \begin{tabular}{l}
\multicolumn{1}{c}{1905.} \\
June $11 \ldots \ldots \ldots \ldots$ \\
June $29 \ldots \ldots \ldots \ldots$ \\
September $16 \ldots \ldots \ldots \ldots$
\end{tabular} & $\begin{array}{l}2.59 \\
2.62 \\
2.52\end{array}$ & $\begin{array}{l}64 \\
90 \\
63\end{array}$ & $\begin{array}{l}1906 . \\
\text { April } 11 \ldots \ldots \\
\text { June } 14 \ldots \ldots\end{array}$ & & $\begin{array}{l}3.25 \\
269\end{array}$ & $\begin{array}{r}221 \\
86\end{array}$ \\
\hline
\end{tabular}

$a$ At rapids, 200 feet above iron bridge. 
Daily gage height, in feet, of South River at Basic City, Va.

\begin{tabular}{|c|c|c|c|c|c|c|c|c|c|c|c|c|}
\hline Day. & Jan. & Feb. & Mar. & Apr. & May. & June. & July. & Ang. & Sept. & Oct. & Nov. & Dec. \\
\hline 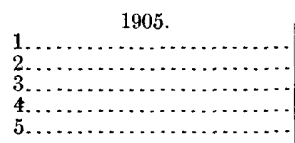 & $\ldots$ & $\ldots$ & $\cdots$ & $\ldots$ & $\ldots$ & 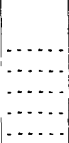 & \begin{tabular}{l|}
2.6 \\
2.5 \\
2.7 \\
2.6 \\
2.55
\end{tabular} & $\begin{array}{l}2.6 \\
2.7 \\
2.5 \\
2.5 \\
2.5\end{array}$ & $\begin{array}{l}2.5 \\
2.5 \\
2.5 \\
2.5 \\
2.6\end{array}$ & $\begin{array}{l}2.4 \\
2.3 \\
2.5 \\
2.3 \\
2.2\end{array}$ & $\begin{array}{l}2.3 \\
2.1 \\
2.2 \\
2.1 \\
2.2\end{array}$ & $\begin{array}{l}2.2 \\
2.3 \\
2.4 \\
2.9 \\
2.9\end{array}$ \\
\hline 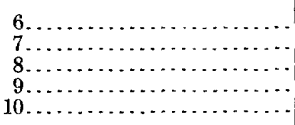 & $\begin{array}{l}\cdots \\
\cdots \\
\cdots \\
\cdots\end{array}$ & & & & & & $\begin{array}{l}2.6 \\
3.0 \\
2.8 \\
2.7 \\
3.0\end{array}$ & $\begin{array}{l}2.5 \\
2.5 \\
2.5 \\
2.5 \\
2.6\end{array}$ & $\begin{array}{l}2.3 \\
2.2 \\
2.3 \\
2.4 \\
2.5\end{array}$ & $\begin{array}{l}2.2 \\
2.4 \\
2.4 \\
2.5 \\
2.4\end{array}$ & \begin{tabular}{l|}
2.3 \\
2.3 \\
2.3 \\
2.3 \\
2.3
\end{tabular} & $\begin{array}{l}2.8 \\
2.6 \\
2.5 \\
2.5 \\
2.5\end{array}$ \\
\hline $\begin{array}{l}11 \\
12 \\
13 \\
14 \\
14 \\
15 \\
15 \ldots \ldots\end{array}$ & $\begin{array}{l}\cdots \\
\cdots \\
\cdots \\
\cdots \\
\cdots\end{array}$ & $\begin{array}{l}\cdots \\
\cdots \\
\cdots \\
\cdots \\
\cdots\end{array}$ & - & $\ldots$ & $\mid \begin{array}{l}\cdots \cdots \\
\cdots \cdots \\
\cdots \\
\cdots \cdots\end{array}$ & $\ldots$ & $\begin{array}{l}2.7 \\
4.25 \\
4.3 \\
5.0 \\
3.5\end{array}$ & $\begin{array}{l}2.6 \\
2.6 \\
2.6 \\
2.7 \\
2.7\end{array}$ & $\begin{array}{l}2.5 \\
2.5 \\
2.2 \\
2.2 \\
2.2\end{array}$ & $\begin{array}{l}2.5 \\
2.7 \\
2.3 \\
2.2 \\
2.4\end{array}$ & $\begin{array}{l}2.1 \\
2.3 \\
2.4 \\
2.3 \\
2.2\end{array}$ & $\begin{array}{l}2.5 \\
2.6 \\
2.3 \\
2.4 \\
2.3\end{array}$ \\
\hline 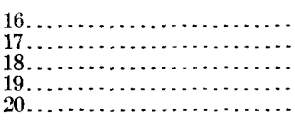 & $\ldots$ & $\begin{array}{l}\cdots \\
\cdots\end{array}$ & $\ldots$ & $\begin{array}{l}\cdots \cdots \\
\cdots \cdots \\
\cdots \cdots \\
\cdots \cdots\end{array}$ & $\mid \begin{array}{l}\cdots \cdots \\
\cdots \\
\cdots \\
\cdots \\
\cdots \cdots\end{array}$ & $\mid \begin{array}{l}\cdots \cdots \\
\cdots \\
\cdots \cdots \\
\cdots \cdots \\
\cdots \cdots\end{array}$ & $\begin{array}{l}3.2 \\
3.0 \\
2.9 \\
2.9 \\
2.8\end{array}$ & $\begin{array}{l}2.9 \\
2.8 \\
2.7 \\
2.7 \\
2.7\end{array}$ & $\begin{array}{l}2.2 \\
2.6 \\
3.2 \\
2.8 \\
2.7\end{array}$ & $\begin{array}{l}2.4 \\
2.2 \\
2.6 \\
2.6 \\
2.4\end{array}$ & $\begin{array}{l}2.1 \\
2.4 \\
2.2 \\
2.3 \\
2.3\end{array}$ & $\begin{array}{l}2.3 \\
2.5 \\
2.5 \\
2.5 \\
2.5\end{array}$ \\
\hline $\begin{array}{l}21 \ldots \ldots \ldots \ldots \\
22 \ldots \ldots \ldots \ldots \ldots \\
23 \ldots \ldots \ldots \\
24 \ldots \ldots \ldots \ldots \\
25 \ldots \ldots \ldots \ldots\end{array}$ & & & & & $\cdots$ & $\cdots$ & $\begin{array}{l}2.8 \\
2.7 \\
2.4 \\
2.5 \\
2.9\end{array}$ & $\begin{array}{l}2.6 \\
2.6 \\
2.5 \\
2.5 \\
2.5\end{array}$ & $\begin{array}{l}2.7 \\
2.6 \\
2.4 \\
2.5 \\
2.6\end{array}$ & $\begin{array}{l}2.3 \\
2.4 \\
2.5 \\
2.4 \\
2.1\end{array}$ & $\begin{array}{l}2.1 \\
2.3 \\
2.3 \\
2.3 \\
2.1\end{array}$ & $\begin{array}{l}5.5 \\
4.1 \\
3.9 \\
3.7 \\
3.4\end{array}$ \\
\hline 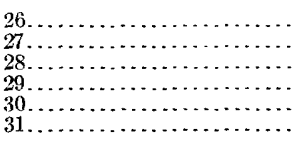 & & $\begin{array}{l}\cdots \\
\cdots \\
\cdots\end{array}$ & & & $\mid \begin{array}{c}\ldots \\
\ldots \\
\ldots\end{array}$ & $\begin{array}{l}\cdots 5 \\
2.5 \\
\cdots . .\end{array}$ & $\begin{array}{l}2.8 \\
2.8 \\
2.7 \\
2.8 \\
2.7 \\
2.6\end{array}$ & $\begin{array}{l}2.8 \\
2.6 \\
2.5 \\
2.5 \\
2.3 \\
2.3\end{array}$ & $\begin{array}{l}2.5 \\
2.6 \\
2.6 \\
2.4 \\
2.4 \\
2 . .\end{array}$ & $\begin{array}{l}2.4 \\
2.5 \\
2.3 \\
2.4 \\
2.4 \\
2.6\end{array}$ & $\begin{array}{c}2.3 \\
2.1 \\
2.0 \\
2.2 \\
2.3 \\
\ldots . .\end{array}$ & $\begin{array}{l}3.2 \\
3.1 \\
2.9 \\
3.7 \\
3.3 \\
3.2\end{array}$ \\
\hline 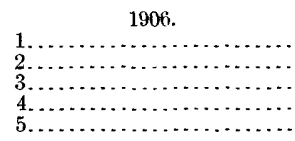 & $\begin{array}{l}3.0 \\
2.9 \\
3.2 \\
4.4 \\
3.7\end{array}$ & $\begin{array}{l}3.4 \\
3.3 \\
3.2 \\
3.2 \\
3.2\end{array}$ & $\begin{array}{l}2.8 \\
2.8 \\
5.3 \\
4.9 \\
4.0\end{array}$ & $\begin{array}{l}3.6 \\
3.6 \\
3.5 \\
3.4 \\
3.2\end{array}$ & $\begin{array}{l}2.9 \\
2.9 \\
2.8 \\
2.9 \\
2.9\end{array}$ & $\begin{array}{l}2.75 \\
2.7 \\
2.6 \\
2.6 \\
2.5\end{array}$ & $\begin{array}{l}2.6 \\
2.6 \\
2.6 \\
2.7 \\
2.6\end{array}$ & $\begin{array}{l}\cdots \\
\cdots \\
\cdots \\
\cdots \\
\cdots \\
\cdots \\
\cdots\end{array}$ & $\begin{array}{l}\ldots . \\
\ldots \ldots \\
\ldots \ldots \\
\ldots \ldots \\
\ldots \ldots\end{array}$ & $\cdots$ & $\begin{array}{l}\cdots \ldots \\
\cdots \ldots \\
\cdots \ldots . \\
\cdots \ldots \\
\cdots \ldots\end{array}$ & $\begin{array}{l}\ldots \ldots \\
\cdots \\
\cdots \\
\cdots \\
\cdots \\
\cdots\end{array}$ \\
\hline 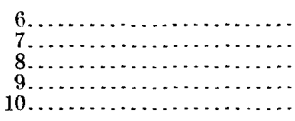 & $\begin{array}{l}3.4 \\
3.2 \\
3.0 \\
2.9 \\
2.8\end{array}$ & $\begin{array}{l}3.1 \\
3.1 \\
3.0 \\
2.9 \\
2.9\end{array}$ & $\begin{array}{l}3.8 \\
3.5 \\
3.5 \\
3.3 \\
3.0\end{array}$ & $\begin{array}{l}3.3 \\
3.2 \\
3.1 \\
3.1 \\
3.2\end{array}$ & $\begin{array}{l}2.8 \\
3.0 \\
3.0 \\
2.8 \\
2.85\end{array}$ & $\begin{array}{l}2.4 \\
2.6 \\
2.6 \\
2.5 \\
2.5\end{array}$ & $\begin{array}{l}2.65 \\
2.45 \\
2.55 \\
2.65 \\
2.6\end{array}$ & $\begin{array}{l}\cdots \cdots \\
\cdots \cdots \\
\cdots \cdots \\
\cdots \cdots \\
\cdots \cdots\end{array}$ & $\begin{array}{c}\cdots \\
\cdots \ldots \\
\cdots \\
\cdots\end{array}$ & $\begin{array}{l}\cdots \\
\cdots \\
\cdots \\
\cdots \\
\cdots\end{array}$ & $\begin{array}{l}\ldots \ldots \\
\cdots \\
\cdots \\
\cdots\end{array}$ & $\begin{array}{c}\ldots \ldots \\
\cdots \\
\cdots \\
\cdots \\
\cdots \\
\cdots\end{array}$ \\
\hline $\begin{array}{l}11, \ldots \ldots \\
12 \ldots \ldots \\
13 \ldots \ldots \\
14 \ldots \ldots \\
15 \\
15 \ldots \ldots\end{array}$ & $\begin{array}{l}2.7 \\
2.8 \\
3.1 \\
3.1 \\
3.1\end{array}$ & $\begin{array}{l}2.9 \\
2.9 \\
2.9 \\
2.9 \\
2.9\end{array}$ & $\begin{array}{l}\text { 3. } 1 \\
3.1 \\
\text { 3. } 1 \\
\text { 3. } 0 \\
\text { 3. } 1\end{array}$ & $\begin{array}{l}3.25 \\
3.2 \\
3.15 \\
3.1 \\
4.5\end{array}$ & $\begin{array}{l}2.85 \\
2.7 \\
2.8 \\
2.8 \\
2.7\end{array}$ & $\begin{array}{l}2.4 \\
2.5 \\
2.6 \\
2.7 \\
2.6\end{array}$ & $\begin{array}{l}2.3 \\
2.4 \\
2.55 \\
2.35 \\
2.4\end{array}$ & $\begin{array}{l}\cdots \\
\cdots \\
\cdots \\
\cdots\end{array}$ & $\begin{array}{l} \\
\ldots \ldots \\
\ldots \ldots \\
\ldots \ldots\end{array}$ & & $\begin{array}{l}\ldots \ldots \\
\ldots \ldots \\
\cdots \cdots \\
\cdots \cdots\end{array}$ & $\mid \begin{array}{l}\cdots \\
\cdots \\
\cdots \\
\ldots \\
\ldots\end{array}$ \\
\hline $\begin{array}{l}16 \ldots \ldots \ldots \\
17 \ldots \ldots \\
18 \ldots \ldots \\
19 \ldots \ldots \ldots \\
20 \ldots \ldots\end{array}$ & $\begin{array}{l}3.0 \\
3.0 \\
3.1 \\
3.2 \\
\text { 3. } 2\end{array}$ & $\begin{array}{l}2.8 \\
27 \\
2.7 \\
2.7 \\
2.7\end{array}$ & $\begin{array}{l}\text { 3. } 2 \\
\text { 3. } 3 \\
\text { 3. } 3 \\
\text { 3. } 3 \\
\text { 3. } 2\end{array}$ & $\begin{array}{l}4.1 \\
3.75 \\
3.55 \\
3.4 \\
3.25\end{array}$ & $\begin{array}{ll}2 & 75 \\
2 & 8 \\
2.65 \\
2.7 \\
2.7\end{array}$ & $\begin{array}{l}2.6 \\
3.2 \\
2.5 \\
2.8 \\
5.45\end{array}$ & 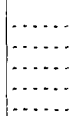 & $\begin{array}{l}\cdots \cdots \\
\cdots \ldots \\
\cdots \\
\cdots \\
\cdots \\
\cdots\end{array}$ & $\begin{array}{l}\ldots \ldots \\
\cdots \\
\cdots\end{array}$ & $\cdots$ & $\mid \begin{array}{c}\cdots \\
\cdots \\
\cdots \\
\cdots\end{array}$ & $\begin{array}{l}\ldots \ldots \\
\cdots \\
\cdots \\
\cdots \\
\cdots \\
\cdots\end{array}$ \\
\hline 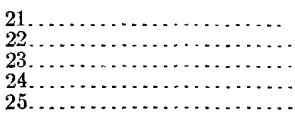 & $\begin{array}{l}\text { 3. } 1 \\
3.1 \\
4.9 \\
4.4 \\
\text { 3. } 9\end{array}$ & $\begin{array}{l}2.7 \\
2.8 \\
2.8 \\
2.8 \\
2.8\end{array}$ & $\begin{array}{l}\text { 3. } 3 \\
3.2 \\
\text { 3. } 2 \\
\text { 3. } 2 \\
\text { 3. } 1\end{array}$ & $\begin{array}{l}3.25 \\
3.2 \\
3.2 \\
3.1 \\
3.0\end{array}$ & $\begin{array}{l}2.6 \\
2.6 \\
2.6 \\
2.5 \\
2.6\end{array}$ & $\begin{array}{l}4.9 \\
3.45 \\
3.2 \\
3.0 \\
2.9\end{array}$ & $\begin{array}{l}\cdots \\
\cdots \\
\cdots \\
\cdots\end{array}$ & & & & $\ldots$ & $\begin{array}{l}\cdots \\
\cdots \\
\cdots \\
\cdots\end{array}$ \\
\hline 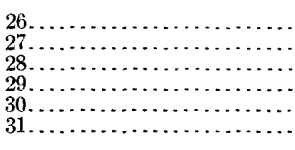 & $\begin{array}{l}3.6 \\
3.6 \\
3.7 \\
3.7 \\
3.6 \\
3.5\end{array}$ & $\begin{array}{r}2.8 \\
2.8 \\
2.8 \\
\cdots \\
\cdots \\
\cdots\end{array}$ & $\begin{array}{l}3.2 \\
3.5 \\
3.7 \\
3.7 \\
3.5 \\
3.8\end{array}$ & $\begin{array}{l}3.1 \\
3.05 \\
3.0 \\
3.0 \\
2.9 \\
\cdots\end{array}$ & $\begin{array}{l}2.6 \\
2.65 \\
2.75 \\
3.0 \\
2.7 \\
2.75\end{array}$ & $\begin{array}{l}3.2 \\
2.95 \\
2.85 \\
2.8 \\
2.7\end{array}$ & $\begin{array}{l}- \\
\cdots\end{array}$ & $\cdots$ & & & .. & {$\left[\begin{array}{c}\cdots \\
\cdots \\
\cdots \\
\cdots \\
\cdots \\
\ldots\end{array}\right.$} \\
\hline
\end{tabular}


Rating table for South River at Basic City, Va., from June 29, 1905, to July 15, 1906.a

\begin{tabular}{|r|r|r|r|r|r|r|r|}
\hline $\begin{array}{c}\text { Gage } \\
\text { height. }\end{array}$ & Discharge & $\begin{array}{c}\text { Gage } \\
\text { height. }\end{array}$ & Discharge. & $\begin{array}{c}\text { Gage } \\
\text { height. }\end{array}$ & Discharge. & $\begin{array}{c}\text { Gage } \\
\text { height. }\end{array}$ & Discharge. \\
\hline Feet. & Second-feet. & Feet. & Second-feet. & Feet. & Second-feet. & Feet. & Second-feet. \\
2.00 & 7 & 2.90 & 135 & 3.30 & 377 & 4.70 & 678 \\
2.10 & 12 & 3.00 & 159 & 3.90 & 407 & 4.80 & 714 \\
2.20 & 19 & 3.10 & 183 & 4.00 & 438 & 4.90 & 750 \\
2.30 & 29 & 3.20 & 208 & 4.10 & 470 & 5.00 & 786 \\
2.40 & 41 & 3.30 & 234 & 4.20 & 503 & 5.10 & 323 \\
2.50 & 55 & 3.40 & 261 & 4.30 & 537 & 5.20 & 860 \\
2.60 & 72 & 3.50 & 289 & 4.40 & 572 & 5.30 & 897 \\
2.70 & 91 & 3.60 & 318 & 4.50 & 607 & 5.40 & 934 \\
2.80 & 112 & 3.70 & 347 & 4.60 & 642 & 5.50 & 972 \\
\hline
\end{tabular}

a This table is strictly applicable only for open-channel conditions. It is based on six discharge measurements made during 1905-6. It is fairly well defined between gage heights 2.5 feet and 4.1 feet

Estimated monthly discharge of South Ricer at Basic City, Va.

[Drainage area, 142 square iniles.]

\begin{tabular}{|c|c|c|c|c|c|}
\hline \multirow[b]{2}{*}{ Month. } & \multicolumn{3}{|c|}{ Discharge in second-feet. } & \multicolumn{2}{|c|}{ Run-off. } \\
\hline & Maxinum. & Minimum. & Mean. & $\begin{array}{l}\text { Second-feet } \\
\text { per square } \\
\text { mile. }\end{array}$ & $\begin{array}{l}\text { Depth in } \\
\text { inches. }\end{array}$ \\
\hline 1905. & & & & & \\
\hline 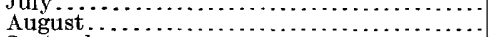 & $\begin{array}{l}786 \\
135\end{array}$ & $\begin{array}{l}41 \\
29\end{array}$ & $\begin{array}{r}159 \\
70.9\end{array}$ & $\begin{array}{l}1.12 \\
.499\end{array}$ & $\begin{array}{l}1.29 \\
.575\end{array}$ \\
\hline September..... & 208 & 19 & 58.2 & .410 & .457 \\
\hline October.... & 91 & 12 & 42.2 & .297 & .342 \\
\hline November.. & 41 & 7 & 23.4 & .165 & .184 \\
\hline December........ & 972 & 19 & 160 & 1.13 & 1. 30 \\
\hline January ............... & 750 & 91 & 258 & 1.82 & 2.10 \\
\hline February $\ldots \ldots$ & 261 & 91 & $\begin{array}{l}258 \\
14 \mathrm{i}\end{array}$ & $\begin{array}{l}1.82 \\
.993\end{array}$ & $\begin{array}{l}2.10 \\
1.03\end{array}$ \\
\hline March........ & 897 & 112 & 276 & 1.94 & 2. 24 \\
\hline April........ & 607 & 135 & 241 & 1. 70 & 1.90 \\
\hline May..... & 159 & 55 & 106 & .746 & .860 \\
\hline June... & 953 & 41 & 154 & 1.08 & 1.20 \\
\hline July $1-15$. & 91 & 29 & 62.5 & .440 & .245 \\
\hline
\end{tabular}

SOUTH RIVER AT PORT REPUBLIC, VA.

This station was established August 6, 1895, and discontinued April 1, 1899. It was located at the highway bridge about 300 feet above the junction of this river with North River. The banks are high and not subject to overflow. Part of the flow of the river is diverted and used above the bridge in a power plant. This water flows under the bridge in the tailrace, and was always included in the measurements. It did not, however, affect the observed gage height. The bed of the river is composed of gravel and cobblestones and is permanent. The current is swift at all stages and normal to the bridge.

Estimates previously published for South River at Port Republic have not been revised. As based on the plotting of the discharge measurements, they are probably within 15 per cent of the true flow for low stages. This estimate of accuracy is founded on the assumption that the flow in the power canal is practically constant. For high stages the estimates are probably too low and may be in error 20 to 25 per cent. 
Daily gage height, in feet, of South River at Purt Republic, Va.-Continued.

\begin{tabular}{|c|c|c|c|c|c|c|c|c|c|c|c|c|}
\hline Day & Jan. & Feb. & Mar. & Apr. & May. & June. & July. & Aug. & Sept. & Oct. & Nov. & Dec. \\
\hline $\begin{array}{l}1 \ldots \ldots \\
2 \ldots \ldots \\
3 \ldots \\
4 \ldots \ldots \\
5 \ldots \ldots\end{array}$ & \begin{tabular}{|l}
1.45 \\
1.45 \\
1.45 \\
1.45 \\
1.45
\end{tabular} & $\begin{array}{l}1.85 \\
1.85 \\
1.85 \\
1.85 \\
1.85\end{array}$ & $\begin{array}{l}2.6 \\
2.5 \\
2.4 \\
2.4 \\
2.35\end{array}$ & $\begin{array}{l}2.0 \\
2.0 \\
2.0 \\
2.0 \\
2.0\end{array}$ & $\begin{array}{l}1.75 \\
9.3 \\
4.5 \\
3.3 \\
3.0\end{array}$ & $\begin{array}{l}1.85 \\
1.8 \\
1.75 \\
1.75 \\
1.9\end{array}$ & $\begin{array}{l}1.6 \\
1.65 \\
1.65 \\
1.6 \\
1.55\end{array}$ & $\begin{array}{l}1.5 \\
1.5 \\
1.5 \\
1.5 \\
1.5\end{array}$ & $\begin{array}{l}1.2 \\
1.2 \\
1.2 \\
1.2 \\
1.2\end{array}$ & $\begin{array}{l}1.3 \\
1.3 \\
1.3 \\
1.3 \\
1.3\end{array}$ & $\begin{array}{l}1.75 \\
2.8 \\
2.5 \\
2.1 \\
2.0\end{array}$ & $\begin{array}{l}1.5 \\
1.5 \\
1.5 \\
1.5 \\
1.5\end{array}$ \\
\hline $\begin{array}{r}6 \ldots \\
7 \ldots \\
8 \ldots \\
9 \ldots \\
10 \ldots\end{array}$ & $\begin{array}{l}1.45 \\
1.45 \\
1.45 \\
1.45 \\
1.45\end{array}$ & $\begin{array}{l}1.95 \\
5.0 \\
4.1 \\
3.2 \\
3.05\end{array}$ & $\begin{array}{l}2.3 \\
2.3 \\
2.3 \\
2.25 \\
2.25\end{array}$ & $\begin{array}{l}2.1 \\
2.1 \\
2.1 \\
2.1 \\
2.1\end{array}$ & $\begin{array}{l}2.8 \\
2.5 \\
2.5 \\
2.4 \\
2.3\end{array}$ & \begin{tabular}{|l}
1.75 \\
1.7 \\
1.65 \\
1.6 \\
1.55
\end{tabular} & $\begin{array}{l}1.55 \\
1.55 \\
1.55 \\
1.55 \\
1.55\end{array}$ & $\begin{array}{l}1.5 \\
1.5 \\
1.45 \\
1.4 \\
1.4\end{array}$ & $\begin{array}{l}1.2 \\
1.2 \\
1.2 \\
1.2 \\
1.2\end{array}$ & $\begin{array}{l}1.3 \\
1.3 \\
1.3 \\
1.3 \\
1.3\end{array}$ & $\begin{array}{l}2.0 \\
1.8 \\
1.75 \\
1.7 \\
1.7\end{array}$ & $\begin{array}{l}1.5 \\
1.5 \\
1.5 \\
1.5 \\
1.5\end{array}$ \\
\hline $\begin{array}{l}13 \ldots \\
15 \ldots\end{array}$ & $\begin{array}{l}1.45 \\
1.45 \\
1.45 \\
1.45 \\
1.45\end{array}$ & $\begin{array}{l}2.95 \\
2.9 \\
3.15 \\
\text { 3. } 15 \\
3.15\end{array}$ & $\begin{array}{l}2.25 \\
2.25 \\
2.2 \\
2.3 \\
2.4\end{array}$ & $\begin{array}{l}2.0 \\
2.0 \\
1.9 \\
1.9 \\
1.9\end{array}$ & $\begin{array}{l}2.3 \\
2.3 \\
3.2 \\
4.8 \\
3.2\end{array}$ & $\begin{array}{l}1.5 \\
1.5 \\
1.65 \\
1.65 \\
1.65\end{array}$ & $\begin{array}{l}1.55 \\
1.55 \\
1.5 \\
1.5 \\
1.5\end{array}$ & $\begin{array}{l}1.4 \\
1.4 \\
1.4 \\
1.4 \\
1.35\end{array}$ & $\begin{array}{l}1.2 \\
1.3 \\
1.3 \\
1.3 \\
1.3\end{array}$ & $\begin{array}{l}1.3 \\
1.3 \\
1.45 \\
1.45 \\
1.45\end{array}$ & $\begin{array}{l}1.7 \\
1.65 \\
1.65 \\
1.65 \\
1.65\end{array}$ & $\begin{array}{l}1.5 \\
1.5 \\
1.5 \\
1.5 \\
1.45\end{array}$ \\
\hline $\begin{array}{l}18 \ldots \\
19 \\
20\end{array}$ & $\begin{array}{l}1.45 \\
\text { 1. } 45 \\
\text { 1. } 45 \\
1.45 \\
1.45\end{array}$ & $\begin{array}{l}3.4 \\
3.4 \\
3.2 \\
3.2 \\
3.1\end{array}$ & $\begin{array}{l}2.6 \\
2.6 \\
2.6 \\
2.6 \\
2.6\end{array}$ & $\begin{array}{l}1.9 \\
1.9 \\
1.85 \\
1.85 \\
1.8\end{array}$ & $\begin{array}{l}2.85 \\
2.7 \\
2.5 \\
2.4 \\
2.35\end{array}$ & $\begin{array}{l}1.65 \\
1.6 \\
1.6 \\
1.6 \\
1.65\end{array}$ & $\begin{array}{l}1.5 \\
1.5 \\
1.5 \\
1.7 \\
1.6\end{array}$ & $\begin{array}{l}1.35 \\
1.35 \\
1.3 \\
1.25 \\
1.2\end{array}$ & $\begin{array}{l}1.3 \\
1.3 \\
1.3 \\
1.3 \\
1.3\end{array}$ & $\begin{array}{l}1.45 \\
\text { 1. } 45 \\
\text { 1. } 45 \\
\text { 1. } 45 \\
1.45\end{array}$ & $\begin{array}{l}1.65 \\
1.65 \\
1.65 \\
1.65 \\
1.65\end{array}$ & $\begin{array}{l}2.4 \\
2.0 \\
1.9 \\
1.9 \\
1.85\end{array}$ \\
\hline $\begin{array}{l}21 \ldots \\
22 \ldots \\
23 \ldots \\
24 \ldots \\
25 \ldots\end{array}$ & $\begin{array}{l}1.45 \\
1.45 \\
1.45 \\
1.65 \\
1.85\end{array}$ & \begin{tabular}{|l}
3.1 \\
3.6 \\
6.75 \\
4.1 \\
3.3
\end{tabular} & \begin{tabular}{|l}
2.7 \\
2.5 \\
2.3 \\
2.35 \\
2.25
\end{tabular} & $\begin{array}{l}1.8 \\
1.8 \\
1.8 \\
1.8 \\
1.8\end{array}$ & $\begin{array}{l}2.3 \\
2.25 \\
2.2 \\
2.2 \\
2.2\end{array}$ & $\begin{array}{l}1.8 \\
1.7 \\
1.65 \\
1.6 \\
1.6\end{array}$ & $\begin{array}{l}1.55 \\
1.5 \\
1.5 \\
1.5 \\
1.45\end{array}$ & $\begin{array}{l}1.2 \\
1.2 \\
1.2 \\
1.2 \\
1.2\end{array}$ & $\begin{array}{l}1.3 \\
1.3 \\
1.3 \\
1.3 \\
1.3\end{array}$ & $\begin{array}{l}1.5 \\
1.5 \\
1.5 \\
1.5 \\
1.5\end{array}$ & $\begin{array}{l}1.6 \\
1.55 \\
1.55 \\
1.55 \\
1.5\end{array}$ & $\begin{array}{l}1.8 \\
1.8 \\
1.8 \\
1.8 \\
1.8\end{array}$ \\
\hline $\begin{array}{l}26 \ldots \\
27 \ldots \\
28 \ldots \\
29 \ldots \\
30 \ldots \\
31 \ldots\end{array}$ & $\begin{array}{l}1.85 \\
1.85 \\
1.85 \\
1.85 \\
1.85 \\
1.85\end{array}$ & \begin{tabular}{|c}
3.2 \\
3.1 \\
2.8 \\
$\cdots$.
\end{tabular} & $\begin{array}{l}2.15 \\
2.05 \\
2.1 \\
2.1 \\
2.1 \\
2.0\end{array}$ & $\begin{array}{l}1.8 \\
1.8 \\
1.8 \\
1.8 \\
1.75 \\
\cdots . .\end{array}$ & $\begin{array}{l}2.15 \\
2.15 \\
2.1 \\
2.1 \\
2.0 \\
1.95\end{array}$ & $\begin{array}{l}1.6 \\
1.6 \\
1.6 \\
1.6 \\
1.6 \\
\cdots . . .\end{array}$ & $\begin{array}{l}1.4 \\
1.4 \\
1.4 \\
1.4 \\
1.4 \\
1.4\end{array}$ & $\begin{array}{l}1.2 \\
1.2 \\
1.2 \\
1.2 \\
1.2 \\
1.2\end{array}$ & \begin{tabular}{l}
1.3 \\
1.3 \\
1.3 \\
1.3 \\
1.3 \\
\hdashline...
\end{tabular} & $\begin{array}{l}1.5 \\
1.5 \\
2.0 \\
1.9 \\
1.85 \\
1.75\end{array}$ & $\begin{array}{l}1.5 \\
1.5 \\
1.5 \\
1.5 \\
1.5 \\
1.5\end{array}$ & $\begin{array}{l}1.8 \\
1.8 \\
1.8 \\
1.8 \\
1.8 \\
1.8\end{array}$ \\
\hline $\begin{array}{l} \\
1 \ldots \ldots \ldots \\
2 \ldots \ldots \ldots \\
3 \ldots \ldots \\
4 \ldots \ldots \\
5 \ldots \ldots \ldots\end{array}$ & $\begin{array}{l}1.8 \\
1.8 \\
1.8 \\
1.75 \\
1.75\end{array}$ & $\begin{array}{l}2.0 \\
2.0 \\
2.0 \\
2.0 \\
2.0\end{array}$ & $\begin{array}{l}1.5 \\
1.5 \\
1.6 \\
1.6 \\
1.6\end{array}$ & $\begin{array}{l}2.25 \\
2.25 \\
2.25 \\
2.2 \\
2.15\end{array}$ & $\begin{array}{l}2.1 \\
2.05 \\
2.0 \\
2.0 \\
1.9\end{array}$ & $\begin{array}{l}1.75 \\
1.65 \\
1.6 \\
1.6 \\
1.6\end{array}$ & $\begin{array}{l}1.6 \\
1.6 \\
1.55 \\
1.55 \\
1.55\end{array}$ & $\begin{array}{l}2.1 \\
2.0 \\
2.0 \\
2.0 \\
4.4\end{array}$ & $\begin{array}{l}1.6 \\
1.5 \\
1.5 \\
1.5 \\
1.5\end{array}$ & $\begin{array}{l}1.35 \\
1.35 \\
1.35 \\
1.35 \\
1.75\end{array}$ & $\begin{array}{l}2.5 \\
2.35 \\
2.2 \\
2.15 \\
2.1\end{array}$ & $\begin{array}{l}1.9 \\
1.8 \\
1.8 \\
2.5 \\
4.0\end{array}$ \\
\hline $\begin{array}{r}6 \\
7 \\
8 \ldots \ldots \\
9 \ldots \ldots \\
10 \ldots \ldots \\
10 \ldots\end{array}$ & $\begin{array}{l}1.65 \\
1.65 \\
1.6 \\
1.6 \\
1.6\end{array}$ & $\begin{array}{l}1.95 \\
1.9 \\
1.85 \\
1.8 \\
1.75\end{array}$ & $\begin{array}{l}1.6 \\
1.6 \\
1.6 \\
1.6 \\
1.6\end{array}$ & $\begin{array}{l}2.15 \\
2.15 \\
2.1 \\
2.0 \\
2.1\end{array}$ & $\begin{array}{l}2.2 \\
4.1 \\
5.0 \\
4.1 \\
3.5\end{array}$ & $\begin{array}{l}1.6 \\
1.6 \\
1.6 \\
1.55 \\
1.55\end{array}$ & $\begin{array}{l}1.55 \\
1.55 \\
1.55 \\
1.55 \\
1.5\end{array}$ & $\begin{array}{l}3.0 \\
2.4 \\
2.4 \\
2.3 \\
6.4\end{array}$ & $\begin{array}{l}1.5 \\
1.5 \\
1.5 \\
1.5 \\
1.5\end{array}$ & $\begin{array}{l}3.0 \\
2.9 \\
2.6 \\
2.3 \\
2.15\end{array}$ & $\begin{array}{l}2.1 \\
2.05 \\
2.0 \\
2.0 \\
2.0\end{array}$ & $\begin{array}{l}3.0 \\
2.9 \\
2.7 \\
2.5 \\
2.4\end{array}$ \\
\hline $\begin{array}{l}13 . \\
14 . \\
15 .\end{array}$ & $\begin{array}{l}1.6 \\
1.55 \\
1.55 \\
1.55 \\
1.55\end{array}$ & $\begin{array}{l}1.7 \\
1.7 \\
1.65 \\
1.6 \\
1.55\end{array}$ & $\begin{array}{l}1.6 \\
1.6 \\
1.6 \\
1.6 \\
1.6\end{array}$ & $\begin{array}{l}2.05 \\
2.0 \\
2.0 \\
2.0 \\
2.75\end{array}$ & $\begin{array}{l}3.1 \\
2.9 \\
2.6 \\
2.6 \\
2.5\end{array}$ & $\begin{array}{l}1.85 \\
1.75 \\
1.65 \\
1.55\end{array}$ & $\begin{array}{l}1.45 \\
1.4 \\
1.4 \\
1.4 \\
1.6\end{array}$ & $\begin{array}{l}6.6 \\
\text { 3. } \\
3.0 \\
3.0 \\
2.8\end{array}$ & $\begin{array}{l}1.5 \\
1.5 \\
1.5 \\
1.5 \\
1.5\end{array}$ & $\begin{array}{l}2.0 \\
1.95 \\
1.9 \\
1.8 \\
1.8\end{array}$ & $\begin{array}{l}2.0 \\
2.0 \\
2.0 \\
1.9 \\
1.9\end{array}$ & $\begin{array}{l}2.35 \\
2.3 \\
2.3 \\
2.25 \\
2.2\end{array}$ \\
\hline $\begin{array}{l}18 . \\
19 . \\
20 .\end{array}$ & $\begin{array}{l}1.55 \\
1.55 \\
1.55 \\
1.55 \\
1.55\end{array}$ & $\begin{array}{l}1.5 \\
1.5 \\
1.5 \\
1.5 \\
1.5\end{array}$ & $\begin{array}{l}1.6 \\
1.85 \\
1.85 \\
1.85 \\
1.8\end{array}$ & $\begin{array}{l}3.0 \\
2.8 \\
2.7 \\
2.5 \\
2.4\end{array}$ & $\begin{array}{l}2.4 \\
2.4 \\
2.3 \\
2.2 \\
2.1\end{array}$ & $\begin{array}{l}1.75 \\
1.75 \\
1.75 \\
1.75 \\
1.75\end{array}$ & $\begin{array}{l}1.6 \\
1.6 \\
1.6 \\
1.6 \\
1.6\end{array}$ & $\begin{array}{l}2.6 \\
2.4 \\
2.3 \\
2.3 \\
2.2\end{array}$ & $\begin{array}{l}1.5 \\
1.5 \\
1.5 \\
1.5 \\
1.5\end{array}$ & $\begin{array}{l}1.8 \\
1.75 \\
1.75 \\
7.5 \\
3.8\end{array}$ & $\begin{array}{l}1.9 \\
1.9 \\
1.85 \\
1.85 \\
2.15\end{array}$ & $\begin{array}{l}2.15 \\
2.1 \\
2.1 \\
2.1 \\
2.0\end{array}$ \\
\hline $\begin{array}{l}21 . \\
22 . \\
23 . \\
24 .\end{array}$ & $\begin{array}{l}1.55 \\
1.55 \\
2.0 \\
2.0 \\
2.0\end{array}$ & $\begin{array}{l}1.5 \\
1.5 \\
1.5 \\
1.5 \\
1.5\end{array}$ & $\begin{array}{l}1.8 \\
1.8 \\
1.8 \\
1.9 \\
1.9\end{array}$ & $\begin{array}{l}2.25 \\
2.15 \\
2.1 \\
2.1 \\
2.2\end{array}$ & $\begin{array}{l}2.0 \\
2.6 \\
2.0 \\
2.0 \\
1.9\end{array}$ & $\begin{array}{l}1.75 \\
1.75 \\
1.7 \\
1.7 \\
1.7\end{array}$ & $\begin{array}{l}1.6 \\
1.6 \\
1.6 \\
1.7 \\
1.7\end{array}$ & $\begin{array}{l}2.2 \\
2.2 \\
2.2 \\
2.1 \\
2.0\end{array}$ & $\begin{array}{l}1.5 \\
1.5 \\
1.65 \\
1.65 \\
1.6\end{array}$ & $\begin{array}{l}3.8 \\
7.6 \\
4.5 \\
4.0 \\
3.1\end{array}$ & $\begin{array}{l}2.1 \\
2.1 \\
2.1 \\
2.1 \\
2.1\end{array}$ & $\begin{array}{l}2.0 \\
2.0 \\
2.6 \\
2.6 \\
2.5\end{array}$ \\
\hline $\begin{array}{l}26 \ldots \\
27 \ldots \\
28 \ldots \\
29 \ldots \\
30 \ldots \\
31 \ldots\end{array}$ & $\begin{array}{l}2.0 \\
2.0 \\
2.0 \\
2.0 \\
2.0 \\
2.0\end{array}$ & $\left\{\begin{array}{l}1.5 \\
1.5 \\
1.5\end{array}\right.$ & $\begin{array}{l}1.9 \\
2.0 \\
2.0 \\
2.0 \\
2.0 \\
2.2\end{array}$ & $\begin{array}{l}2.5 \\
2.3 \\
2.25 \\
2.2 \\
2.1 \\
. . .\end{array}$ & $\begin{array}{l}1.9 \\
1.85 \\
1.8 \\
1.8 \\
1.8 \\
1.8\end{array}$ & $\begin{array}{l}1.7 \\
1.65 \\
1.6 \\
1.6 \\
1.6 \\
\cdots . . .\end{array}$ & $\begin{array}{l}1.7 \\
1.7 \\
1.7 \\
1.7 \\
1.7 \\
2.1\end{array}$ & $\begin{array}{l}1.9 \\
1.8 \\
1.7 \\
1.6 \\
1.6 \\
1.6\end{array}$ & $\begin{array}{l}\text { 1. } 5 \\
\text { 1. } 45 \\
\text { 1. } 4 \\
1.35 \\
1.35\end{array}$ & $\begin{array}{l}3.0 \\
2.8 \\
2.6 \\
2.5 \\
2.5 \\
2.5\end{array}$ & $\begin{array}{l}2.1 \\
2.1 \\
2.1 \\
2.1 \\
2.0 \\
. . . .\end{array}$ & $\begin{array}{l}2.45 \\
2.4 \\
2.35 \\
2.3 \\
2.3 \\
2.3\end{array}$ \\
\hline
\end{tabular}


Daily gage height, in feet, of South River at Port Republic, Va.-Continued.

\begin{tabular}{|c|c|c|c|c|c|c|c|c|c|}
\hline Day. & Jan. & Feb. & Mar. & Apr. & Day. & Jan. & Feb. & Mar. & Apr. \\
\hline 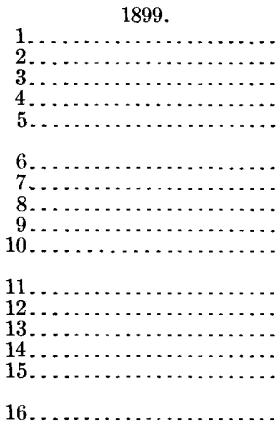 & $\begin{array}{l}2.5 \\
2.5 \\
2.4 \\
2.3 \\
2.3 \\
\\
3.4 \\
5.0 \\
3.8 \\
3.6 \\
3.0 \\
2.9 \\
2.75 \\
2.6 \\
2.6 \\
2.6 \\
2.6\end{array}$ & $\begin{array}{l}2.1 \\
2.1 \\
2.1 \\
2.1 \\
2.1 \\
2.1 \\
2.1 \\
2.2 \\
2.2 \\
2.3 \\
2.3 \\
2.3 \\
2.3 \\
2.4 \\
2.5 \\
2.6\end{array}$ & \begin{tabular}{|r|}
.0 \\
3.6 \\
3.5 \\
5.1 \\
12.0 \\
\\
6.7 \\
5.5 \\
4.0 \\
3.6 \\
3.9 \\
\\
3.7 \\
3.6 \\
3.4 \\
3.3 \\
3.4 \\
\\
3.3
\end{tabular} & $\begin{array}{c}2.5 \\
\cdots \cdots \\
\cdots \cdots \\
\cdots \cdots \\
\cdots \cdots\end{array}$ & 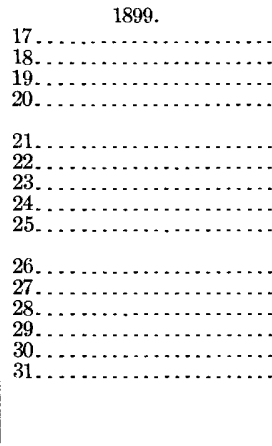 & $\begin{array}{l}2.5 \\
2.4 \\
2.2 \\
2.2 \\
2.2 \\
2.2 \\
2.2 \\
2.2 \\
2.2 \\
2.2 \\
2.2 \\
2.2 \\
2.2 \\
2.2 \\
2.1\end{array}$ & \begin{tabular}{|l}
2.6 \\
2.6 \\
2.8 \\
3.0 \\
3.5 \\
5.0 \\
5.6 \\
4.1 \\
3.5 \\
4.4 \\
6.5 \\
5.3 \\
$\cdots$ \\
$\cdots$ \\
$\cdots .$. \\
\end{tabular} & $\begin{array}{l}3.2 \\
2.9 \\
3.2 \\
3.7 \\
\\
3.5 \\
3.2 \\
3.1 \\
2.9 \\
2.8 \\
2.8 \\
2.8 \\
2.7 \\
2.7 \\
2.6 \\
2.5\end{array}$ & 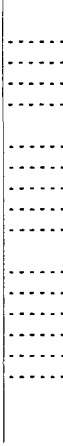 \\
\hline
\end{tabular}

Rating table for South River at Port Republic, Va., from August 5, 1895, to April 1, 1899.a

\begin{tabular}{|c|c|c|c|c|c|c|c|}
\hline $\begin{array}{c}\text { Gage } \\
\text { height. }\end{array}$ & Discharge. & $\begin{array}{l}\text { Gage } \\
\text { height: }\end{array}$ & Discharge. & $\begin{array}{c}\text { Gage } \\
\text { height. }\end{array}$ & Discharge. & $\begin{array}{c}\text { Gage } \\
\text { height. }\end{array}$ & Discharge. \\
\hline $\begin{array}{r}\text { Feet. } \\
1.3 \\
1.4 \\
1.5 \\
1.6 \\
1.7 \\
1.8 \\
1.9 \\
2.0\end{array}$ & \begin{tabular}{|c|} 
Second-feet \\
95 \\
105 \\
120 \\
140 \\
160 \\
180 \\
210 \\
250
\end{tabular} & $\begin{array}{r}\text { Feet. } \\
2.2 \\
2.4 \\
2.6 \\
2.8 \\
3.0 \\
3.2 \\
3.4 \\
3.6\end{array}$ & \begin{tabular}{|r} 
Second-feet. \\
350 \\
495 \\
645 \\
795 \\
945 \\
1,090 \\
1,240 \\
1,390
\end{tabular} & $\begin{array}{r}\text { Feet. } \\
3.8 \\
4.0 \\
4.2 \\
4.4 \\
4.6 \\
4.8 \\
5.0\end{array}$ & \begin{tabular}{|c} 
Second-feet. \\
1,535 \\
1,685 \\
1,835 \\
1,980 \\
2,125 \\
2,275 \\
2,425
\end{tabular} & $\begin{array}{r}\text { Feet. } \\
5.2 \\
5.4 \\
5.6 \\
5.8 \\
6.0 \\
6.5 \\
7.0\end{array}$ & \begin{tabular}{|c} 
Second-feet. \\
2,575 \\
2,720 \\
2,870 \\
3,015 \\
3,165 \\
3,535 \\
3,905
\end{tabular} \\
\hline
\end{tabular}

$a$ This rating table is strictly applicable only for open-channel conditions. It is not well defined.

Estimated monthly discharge of South River at Port Republic, Va.

[Drainage area, 246 square miles.]

\begin{tabular}{|c|c|c|c|c|c|}
\hline \multirow[b]{2}{*}{ Month. } & \multicolumn{3}{|c|}{ Discharge in second-feet. } & \multicolumn{2}{|c|}{ Run-off. } \\
\hline & Maxinum. & Minimum. & Mean. & $\begin{array}{l}\text { Second-feet } \\
\text { per square } \\
\text { mile. }\end{array}$ & $\begin{array}{l}\text { Depth } \\
\text { in inches. }\end{array}$ \\
\hline 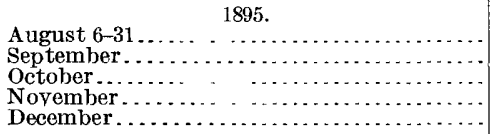 & $\begin{array}{r}105 \\
112 \\
85 \\
95 \\
290\end{array}$ & $\begin{array}{l}70 \\
85 \\
85 \\
85 \\
95\end{array}$ & $\begin{array}{r}80 \\
94 \\
85 \\
93 \\
136\end{array}$ & $\begin{array}{r}0.33 \\
.38 \\
.35 \\
.38 \\
.55\end{array}$ & $\begin{array}{r}0.32 \\
.43 \\
.40 \\
.43 \\
.63\end{array}$ \\
\hline 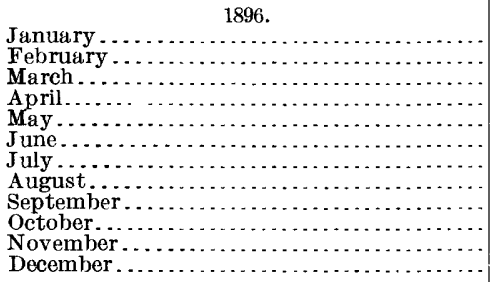 & $\begin{array}{r}2,350 \\
1,390 \\
1,905 \\
1,090 \\
945 \\
570 \\
2,275 \\
250 \\
9,200 \\
2,425 \\
1,760 \\
720\end{array}$ & $\begin{array}{r}85 \\
250 \\
195 \\
160 \\
140 \\
120 \\
120 \\
105 \\
90 \\
140 \\
140 \\
112\end{array}$ & $\begin{array}{l}305 \\
517 \\
473 \\
375 \\
218 \\
255 \\
322 \\
123 \\
597 \\
362 \\
376 \\
208\end{array}$ & $\begin{array}{r}1.24 \\
2,10 \\
1.11 \\
1.52 \\
.89 \\
1.04 \\
1.31 \\
.50 \\
2.43 \\
1.47 \\
1.53 \\
.85\end{array}$ & $\begin{array}{r}1.43 \\
2.19 \\
1.28 \\
1.70 \\
1.02 \\
1.16 \\
1.51 \\
.58 \\
2.71 \\
1.69 \\
1.71 \\
.98\end{array}$ \\
\hline The year. & 9,200 & 85 & 344 & 1.33 & 17.96 \\
\hline
\end{tabular}


Estimated monthly discharge of Suth River at Port Republic, Va.-Continued.

\begin{tabular}{|c|c|c|c|c|c|}
\hline \multirow[b]{2}{*}{ Month. } & \multicolumn{3}{|c|}{ Discharge in second-feet. } & \multicolumn{2}{|c|}{ Run-off. } \\
\hline & Maximum. & Minimum. & Mean. & $\begin{array}{l}\text { Second-feet } \\
\text { per square } \\
\text { mile. }\end{array}$ & $\begin{array}{l}\text { Depth } \\
\text { in inches. }\end{array}$ \\
\hline 189 & & & & & \\
\hline January ........ & 195 & 112 & 131 & .53 & .61 \\
\hline February.. & 3,717 & 195 & 1,072 & 4.36 & 4. 54 \\
\hline March.. & 720 & 250 & 459 & 1.87 & 2.16 \\
\hline $\begin{array}{l}\text { April... } \\
\text { May ... }\end{array}$ & 290 & $\begin{array}{l}170 \\
170\end{array}$ & 211 & $\begin{array}{r}.86 \\
3.24\end{array}$ & $\begin{array}{r}.95 \\
3.74\end{array}$ \\
\hline $\begin{array}{l}\text { May... } \\
\text { June.. }\end{array}$ & $\begin{array}{r}5,607 \\
210\end{array}$ & $\begin{array}{l}170 \\
120\end{array}$ & 152 & $\begin{array}{r}.24 \\
.62\end{array}$ & $\begin{array}{r}0.44 \\
.69\end{array}$ \\
\hline July... & 160 & 105 & 125 & .51 & .59 \\
\hline August. & 120 & 85 & 103 & .42 & .48 \\
\hline September. & 95 & 85 & 91 & .37 & .41 \\
\hline October...... & 250 & 95 & 119 & .48 & .55 \\
\hline November. & 795 & 120 & 192 & .78 & .87 \\
\hline December... & 495 & 112 & 166 & .67 & .77 \\
\hline The year. & 5,607 & 85 & 301 & 1. 23 & 16.36 \\
\hline 1898. & & & & & \\
\hline January .... & 250 & 130 & 175 & .71 & .82 \\
\hline Februar & 250 & 120 & 162 & .66 & .69 \\
\hline March.. & 350 & 120 & 177 & .72 & .83 \\
\hline April... & 945 & 250 & 408 & 1.66 & 1.85 \\
\hline May & 2,425 & 180 & 561 & 2. 28 & 2.63 \\
\hline June. & 195 & 120 & 153 & .62 & .69 \\
\hline July. . & 290 & 105 & 142 & .58 & .67 \\
\hline August.. & 3,605 & 140 & 690 & 2.80 & 3.23 \\
\hline September. & 150 & 100 & 121 & .49 & .55 \\
\hline October. & 4,345 & 100 & 835 & $\begin{array}{l}3.39 \\
1.5\end{array}$ & 3.91 \\
\hline Novem & 570 & 195 & 282 & 1.15 & 1. 28 \\
\hline December. & 1,685 & 180 & 484 & 1.97 & 2.27 \\
\hline The year. & 4,345 & 100 & 349 & 1.42 & 19.42 \\
\hline Tonuers & & & & & \\
\hline $\begin{array}{l}\text { January............ } \\
\text { February ....... }\end{array}$ & $\begin{array}{l}2,425 \\
3,535\end{array}$ & 290 & $\begin{array}{l}638 \\
964\end{array}$ & $\begin{array}{l}2.59 \\
3.92\end{array}$ & $\begin{array}{l}2.99 \\
4.08\end{array}$ \\
\hline March.. & 7,700 & 570 & 1,525 & 6.20 & 7.15 \\
\hline
\end{tabular}

NORTH RIVER BASIN.

COOKS CREEK AT MOUNT GRAWFORD, VA.

Cooks Creek rises near Harrisonburg, in Rockingham County, Va., and flows southwestward and southeastward into North River near Mount Crawford.

The gaging station was established July 1, 1905, by N. C. Grover, in connection with the investigation of stream pollution in the Shenandoah Valley. It was discontinued July 16, 1906. It is located at the upper highway bridge across Cooks Creek, three-fourths of a mile from Mount Crawford.

The channel is straight for 200 feet above and 100 feet below the station. The current is very sluggish at low water. Both banks are low and liable to overflow during high water, but all the water passes beneath the bridge. The bed of the stream is composed of mud and gravel. The stream is polluted by tanneries.

Discharge measurements were made from the side of the bridge to which the gage is attached, or by wading a short distance below, at a point where the velocity is greater. 
A standard chain gage is fastened to the outside of the downstream guard rail of the bridge. The length of the chain from the end of the weight to the outer edge of the ring is 14.38 feet. The gage was read once each day by S. H. Craun. Bench mark No. 1 is a nail driven vertically into a root on the downstream side of a large tree 150 feet below the gage, on the left bank. Its elevation is 4.24 feet above the gage datum.

Estimates at this station are within 10 per cent of the true flow between gage heights 1.9 and 2.7 feet. Above and below these stages the estimates are liable to error of 10 to 25 per cent. The flow may have been slightly affected by ice conditions during the winter of $1905-6$.

Discharge measurements of Cooks Creek at Mount Crawford, Va.

\begin{tabular}{|c|c|c|c|c|c|}
\hline Date. & $\begin{array}{c}\text { Gagi } \\
\text { height. }\end{array}$ & Discharge. & Date. & $\begin{array}{c}\text { Gage } \\
\text { height. }\end{array}$ & Discharge. \\
\hline $\begin{array}{l}1905 . \\
\text { July } 1 \ldots \ldots \ldots \ldots \\
\text { December } 30 a \ldots \ldots \ldots \ldots \ldots\end{array}$ & $\begin{array}{l}\text { Feet. } \\
\quad 2.10 \\
\quad 2.08\end{array}$ & $\mid \begin{array}{c}\text { Second-feet. } \\
20 \\
22.6\end{array}$ & April 10. 1906. & $\begin{array}{l}\text { Feet. } \\
2.32\end{array}$ & $\begin{array}{r}\text { Second-feet. } \\
32.4\end{array}$ \\
\hline
\end{tabular}

$a$ Measurement made by wading.

Daily gage height, in feet, of Cooks Creek at Mount Crawford, Va.

\begin{tabular}{|c|c|c|c|c|c|c|c|c|c|c|c|c|c|}
\hline Day. & July & Aug. & Sept. & Oct. & Nov. & Dec. & Day. & July. & Aug. & Sept. & Oct. & Nor. & Dec. \\
\hline $\begin{array}{r}1905 . \\
1 \ldots \ldots \ldots \\
2 \ldots \ldots \\
3 \ldots \ldots \\
4 \ldots \ldots \\
5 \ldots \ldots \ldots \\
\end{array}$ & $\begin{array}{l}2.1 \\
2.1 \\
2.0 \\
2.0 \\
5.5\end{array}$ & $\begin{array}{l}2.2 \\
2.2 \\
2.1 \\
2.1 \\
2.1\end{array}$ & $\begin{array}{l}1.8 \\
1.9 \\
1.9 \\
1.8 \\
1.8\end{array}$ & $\begin{array}{l}1.8 \\
1.8 \\
1.8 \\
1.8 \\
1.8\end{array}$ & $\begin{array}{l}1.7 \\
1.7 \\
1.7 \\
1.7 \\
1.7\end{array}$ & $\begin{array}{l}1.8 \\
1.8 \\
2.0 \\
1.8 \\
1.8\end{array}$ & $\begin{array}{l}1905 . \\
17 \ldots \ldots \\
18 \ldots \ldots \\
19 \ldots \ldots \\
20 \ldots \ldots \\
\end{array}$ & $\begin{array}{l}\text { 2.3 } \\
2.2 \\
\text { 2.2 } \\
2.4\end{array}$ & $\begin{array}{l}1.9 \\
2.0 \\
1.9 \\
2.0\end{array}$ & $\begin{array}{l}1.8 \\
1.8 \\
1.8 \\
1.8\end{array}$ & $\begin{array}{l}1.7 \\
1.7 \\
1.8 \\
1.7\end{array}$ & $\begin{array}{l}1.7 \\
1.7 \\
1.8 \\
1.8\end{array}$ & $\begin{array}{l}1.7 \\
1.7 \\
1.7 \\
1.7\end{array}$ \\
\hline & $\begin{array}{l}\text { 3. } 3 \\
2.9 \\
2.4 \\
2.2 \\
\text { 2. } 1\end{array}$ & $\begin{array}{l}2.1 \\
2.0 \\
2.0 \\
2.0 \\
2.0\end{array}$ & $\begin{array}{l}1.8 \\
1.7 \\
1.8 \\
1.8 \\
1.8\end{array}$ & $\begin{array}{l}1.8 \\
1.7 \\
1.7 \\
1.7 \\
1.7\end{array}$ & $\begin{array}{l}1.7 \\
1.7 \\
1.7 \\
1.7 \\
1.7\end{array}$ & $\begin{array}{l}1.8 \\
1.8 \\
1.8 \\
1.8 \\
1.8\end{array}$ & $\begin{array}{l}22 . \\
23 . \\
24 . \\
25 .\end{array}$ & $\begin{array}{r}2.1 \\
2.2 \\
2.4 \\
2.3 \\
2.2\end{array}$ & $\begin{array}{l}1.0 \\
1.9 \\
1.8 \\
1.9\end{array}$ & $\begin{array}{l}1.8 \\
1.8 \\
1.8 \\
1.7\end{array}$ & $\begin{array}{l}1.7 \\
1.7 \\
1.7 \\
1.7 \\
1.8\end{array}$ & $\begin{array}{l}1.8 \\
1.8 \\
1.8 \\
1.8 \\
1.8\end{array}$ & $\begin{array}{r}2.9 \\
2.6 \\
2.4 \\
2.3 \\
2.1\end{array}$ \\
\hline & $\begin{array}{l}2.5 \\
2.1 \\
2.3 \\
2.2 \\
2.8\end{array}$ & $\begin{array}{l}2.0 \\
2.1 \\
2.1 \\
2.1 \\
2.0\end{array}$ & $\begin{array}{l}1.8 \\
1.8 \\
1.8 \\
1.8 \\
1.8\end{array}$ & $\begin{array}{l}1.7 \\
1.7 \\
1.7 \\
1.7 \\
1.7\end{array}$ & $\begin{array}{l}1.7 \\
1.8 \\
1.8 \\
1.7 \\
1.7\end{array}$ & $\begin{array}{l}1.8 \\
1.8 \\
1.7 \\
1.7 \\
1.7\end{array}$ & $\begin{array}{l}26 . \\
27 . \\
28 . \\
29 . \\
30 . \\
31 .\end{array}$ & $\begin{array}{l}2.2 \\
2.2 \\
2.0 \\
2.0 \\
3.1 \\
2.4\end{array}$ & $\begin{array}{l}2.1 \\
2.0 \\
2.0 \\
2.0 \\
1.9 \\
1.9\end{array}$ & $\begin{array}{l}1.7 \\
1.8 \\
1.7 \\
1.7 \\
1.8\end{array}$ & $\begin{array}{l}2.0 \\
1.8 \\
1.8 \\
1.8 \\
1.7 \\
1.7\end{array}$ & $\begin{array}{l}1.8 \\
1.8 \\
1.8 \\
1.8 \\
1.8\end{array}$ & $\begin{array}{l}2.0 \\
2.0 \\
2.0 \\
2.3 \\
2.1 \\
2.2\end{array}$ \\
\hline 16 & 2.4 & 2.0 & 1.8 & 1.7 & 1.7 & 1.7 & & & & & & & \\
\hline
\end{tabular}


Daily gage height, in feet, of Cooks Creek at Mount Crawford, Va.-Continued.

\begin{tabular}{|c|c|c|c|c|c|c|c|c|c|c|c|c|}
\hline Day. & Jan. & Feb. & Mar. & Apr. & May. & June. & July. & Aug. & Sept. & Oct. & Nov. & Dec. \\
\hline 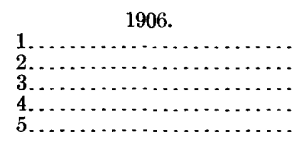 & $\begin{array}{l}2.05 \\
2.05 \\
2.05 \\
3.0 \\
2.5\end{array}$ & $\begin{array}{l}2.0 \\
2.0 \\
1.8 \\
2.0 \\
2.0\end{array}$ & $\begin{array}{l}2.0 \\
2.0 \\
2.0 \\
2.4 \\
2.2\end{array}$ & $\begin{array}{l}2.5 \\
2.4 \\
2.3 \\
2.3 \\
2.2\end{array}$ & $\begin{array}{l}2.1 \\
2.1 \\
2.1 \\
2.1 \\
2.1\end{array}$ & $\begin{array}{l}2.0 \\
2.0 \\
2.0 \\
2.0 \\
2.0\end{array}$ & $\begin{array}{l}2.1 \\
2.1 \\
2.1 \\
2.1 \\
2.0\end{array}$ & & & & & $\cdots$ \\
\hline $\begin{array}{r}6 \\
7 \ldots \\
8 \ldots \ldots \\
9 \\
10\end{array}$ & $\begin{array}{l}2.3 \\
2.3 \\
2.2 \\
2.2 \\
2.1\end{array}$ & $\begin{array}{l}2.1 \\
2.0 \\
2.0 \\
2.0 \\
2.0\end{array}$ & $\begin{array}{l}2.1 \\
2.1 \\
2.1 \\
2.1 \\
2.1\end{array}$ & $\begin{array}{l}2.3 \\
2.2 \\
2.2 \\
2.3 \\
2.3\end{array}$ & $\begin{array}{l}2.1 \\
2.2 \\
2.2 \\
2.1 \\
2.1\end{array}$ & $\begin{array}{l}1.9 \\
1.9 \\
1.9 \\
1.9 \\
1.9\end{array}$ & $\begin{array}{l}2.0 \\
2.0 \\
2.0 \\
2.0 \\
2.0\end{array}$ & & & & & 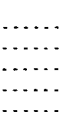 \\
\hline $\begin{array}{l}11 \ldots \ldots \ldots \ldots \\
12 \ldots \ldots \ldots \\
13 \ldots \ldots \ldots \\
14 \ldots \ldots \ldots\end{array}$ & $\begin{array}{l}2.1 \\
2.1 \\
2.1 \\
2.3 \\
2.3\end{array}$ & $\begin{array}{l}2.1 \\
2.0 \\
2.0 \\
2.0 \\
2.0\end{array}$ & $\begin{array}{l}2.1 \\
2.1 \\
2.05 \\
2.4 \\
2.6\end{array}$ & $\begin{array}{l}2.2 \\
2.2 \\
2.2 \\
2.2 \\
2.5\end{array}$ & $\begin{array}{l}2.1 \\
2.0 \\
2.1 \\
2.1 \\
2.0\end{array}$ & $\begin{array}{l}1.9 \\
1.9 \\
1.8 \\
1.8 \\
1.8\end{array}$ & $\begin{array}{l}3.0 \\
2.1 \\
2.0 \\
2.0 \\
2.0\end{array}$ & & & & & . \\
\hline $\begin{array}{l}16 \ldots \ldots \\
17 \ldots \ldots \ldots \\
18 \ldots \ldots \\
19 \ldots \ldots \\
20 \ldots \ldots \\
\end{array}$ & $\begin{array}{l}2.2 \\
2.2 \\
2.2 \\
2.1 \\
2.1\end{array}$ & $\begin{array}{l}1.9 \\
2.0 \\
2.0 \\
1.9 \\
1.9\end{array}$ & $\begin{array}{l}2.5 \\
2.4 \\
2.3 \\
2.4 \\
2.3\end{array}$ & $\begin{array}{l}2.4 \\
2.3 \\
2.2 \\
2.2 \\
2.2\end{array}$ & $\begin{array}{l}2.0 \\
2.0 \\
2.0 \\
2.0 \\
2.0\end{array}$ & $\begin{array}{l}1.8 \\
2.0 \\
2.0 \\
2.0 \\
2.5\end{array}$ & $\begin{array}{l}\ldots \ldots \\
\cdots \cdots \\
\cdots \cdots\end{array}$ & & & & & $\ldots$ \\
\hline $\begin{array}{l}21 \ldots \ldots \ldots \\
22 \ldots \ldots \\
23 \ldots \ldots \\
24 \ldots \ldots \\
25 \ldots \ldots \\
\end{array}$ & $\begin{array}{l}2.1 \\
2.1 \\
2.3 \\
2.3 \\
2.2\end{array}$ & $\begin{array}{l}2.0 \\
2.0 \\
2.0 \\
1.8 \\
2.0\end{array}$ & $\begin{array}{l}2.3 \\
2.3 \\
2.3 \\
2.3 \\
2.3\end{array}$ & $\begin{array}{l}2.2 \\
2.2 \\
2.2 \\
2.1 \\
2.1\end{array}$ & $\begin{array}{l}1.9 \\
1.9 \\
1.9 \\
2.0 \\
2.0\end{array}$ & $\begin{array}{l}2.1 \\
2.2 \\
2.1 \\
2.0 \\
2.0\end{array}$ & 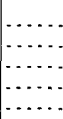 & & & & & 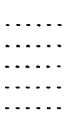 \\
\hline $\begin{array}{l}26 \ldots \\
27 \ldots \\
28 \ldots \\
29 \ldots \\
30 \ldots \\
31 \ldots\end{array}$ & $\begin{array}{l}2.1 \\
2.1 \\
2.2 \\
2.2 \\
2.1 \\
2.1\end{array}$ & $\begin{array}{r}2.0 \\
2.0 \\
2.0 \\
\cdots \cdots \\
\cdots \\
\cdots\end{array}$ & $\begin{array}{l}2.3 \\
2.6 \\
2.5 \\
2.5 \\
2.4 \\
2.5\end{array}$ & $\begin{array}{l}2.2 \\
2.2 \\
2.1 \\
2.1 \\
2.1\end{array}$ & $\begin{array}{l}2.0 \\
2.0 \\
2.0 \\
2.0 \\
2.0 \\
2.0\end{array}$ & $\begin{array}{l}2.1 \\
3.0 \\
2.0 \\
2.2 \\
2.1\end{array}$ & & & & & & $\cdots$ \\
\hline
\end{tabular}

Rating table for Cooks Creek at Mount Crauford, Va., from July 1, 1905, to July 15, 1906. a

\begin{tabular}{|c|c||c|r|r|r||r|r|}
\hline $\begin{array}{c}\text { Gage } \\
\text { height. }\end{array}$ & Discharge. & $\begin{array}{c}\text { Gage } \\
\text { height. }\end{array}$ & Discharge. & $\begin{array}{c}\text { Gage } \\
\text { height. }\end{array}$ & Discharge. & $\begin{array}{c}\text { Gage } \\
\text { height. }\end{array}$ & Discharge. \\
\hline & & & & & \\
Feet. & Second-feet. & Feet. & Second-feet. & Feet. & Second-feet. & Feet. & Second-feet. \\
1.70 & 10 & 2.70 & 52 & 3.70 & 126 & 4.70 & 235 \\
1.80 & 13 & 2.80 & 58 & 3.80 & 135 & 4.80 & 248 \\
1.90 & 16 & 2.90 & 64 & 3.90 & 144 & 4.90 & 261 \\
2.00 & 19 & 3.00 & 71 & 4.00 & 154 & 5.00 & 274 \\
2.10 & 23 & 3.10 & 78 & 4.10 & 164 & 5.10 & 288 \\
2.20 & 27 & 3.20 & 85 & 4.20 & 175 & 5.20 & 302 \\
2.30 & 31 & 3.30 & 92 & 4.30 & 186 & 5.30 & 316 \\
2.40 & 36 & 3.40 & 100 & 4.40 & 198 & 5.40 & 331 \\
2.50 & 41 & 3.50 & 108 & 4.50 & 210 & 5.50 & 346 \\
2.60 & 46 & 3.60 & 117 & 4.60 & 222 & & \\
\hline
\end{tabular}

$a$ This table is strictly applicable only for open-channel conditions. It is hased on two discharge measurements made during 1905-6. It is not well defined. Estimates based on this table are only roughly approximate. 
Estimated monthly discharge of Cooks Creek at Mount Crawford, Va.

[Drainage area, 41 square miles.]

\begin{tabular}{|c|c|c|c|c|c|}
\hline \multirow[b]{2}{*}{ Month. } & \multicolumn{3}{|c|}{ Discharge in second-feet. } & \multicolumn{2}{|c|}{ Run-off. } \\
\hline & Maximum. & Minimum. & Mean. & $\begin{array}{l}\text { Second-feet } \\
\text { per square } \\
\text { mile. }\end{array}$ & $\begin{array}{l}\text { Depth in } \\
\text { inches. }\end{array}$ \\
\hline 1905. & & & & & \\
\hline July ........................ & 346 & 19 & 43. 8 & 1.07 & 1.23 \\
\hline August $\ldots \ldots$ & 27 & 13 & 19.7 & .480 & .553 \\
\hline 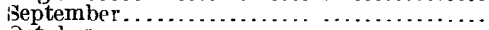 & 16 & 10 & 12. 7 & .310 & .346 \\
\hline Jetober $\ldots \ldots \ldots$ & 19 & 10 & 11.4 & .278 & .320 \\
\hline 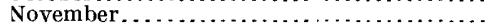 & 13 & 10 & 11. 4 & .278 & .310 \\
\hline 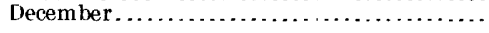 & 64 & 10 & 18.7 & .456 & .526 \\
\hline 1906. & & & & & \\
\hline January . . . . . . & 71 & 21 & 27.5 & .671 & .774 \\
\hline February........ & 23 & 13 & 18.5 & .451 & .470 \\
\hline$\ldots \ldots \ldots \ldots$ & 46 & 19 & 30.6 & .746 & .860 \\
\hline 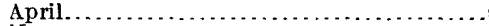 & 41 & 23 & 28. 7 & .700 & .781 \\
\hline 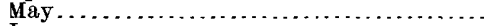 & 27 & 16 & 20.6 & .502 & .579 \\
\hline 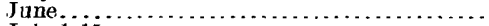 & 71 & 13 & 21.0 & .512 & .571 \\
\hline 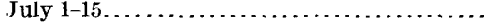 & 71 & 19 & 23.8 & .580 & .324 \\
\hline
\end{tabular}

LEWIS GREEK NEAR STAUNTON, VA.

Lewis Creek rises in the central part of Augusta County, Va., about 4 miles southwest of Staunton, and flows northeastward into Middle River.

The gaging station was established June 30,1905 , by N. C. Grover, in connection with the investigation of stream pollution in the Shenandoah Valley. It was discontinued July 16, 1906. It is located at the private bridge across Lewis Creek, on the property of William Glenn, 2 miles from Stauntion.

The chanel is st:aight for 300 feet above and below the station. The current is sluggish. Both banks are about 5 feet high and do not overflow, except during very high water. The bed of the stream is composed of soft miud. There is but one channel at all stages. The stream is composed almost wholly of sewage from the city of Staunton, and is very shallow at ordinary stages.

Discharge measurements were made from the downstream side of the bridge, the initial point for soundings being the gatepost near the left end of the bridge.

A vertical staff gage, graduated to feet and tenths, is fastened to a tree 6 feet downstream from the bridge. The gage was read once each day by Ashby Glenn. Bench mark No. 1 is a nail in the locust tree to which the gage is attached, 1 foot above the ground, on the upstream side. Its elevation is 7.56 feet above the zero of the gage.

Prior to about May 27, 1906, the estimates for this station are accurate within 5 to 10 per cent for gage heights 0.45 to 0.9 foot. Above and below these stages the error may be as high as 15 per cent. Estimates after about May 27, 1906, may be in error 15 to 20 per cent. The flow was probably not affected by ice conditions during 1905-6. 
Discharge measurements of Lewis Creek near Staunton, Va.

\begin{tabular}{|c|c|c|c|c|c|}
\hline Date. & $\begin{array}{c}\text { Gage } \\
\text { height. }\end{array}$ & Discharge. & Date. & $\begin{array}{c}\text { Gage } \\
\text { height. }\end{array}$ & Discharge. \\
\hline \begin{tabular}{l}
\multicolumn{1}{c}{1905.} \\
June $30 \ldots \ldots \ldots \ldots \ldots \ldots$
\end{tabular} & $\begin{array}{l}\text { Feet. } \\
0.51 \\
.63\end{array}$ & \begin{tabular}{|} 
Second-feet. \\
3.8 \\
6.2
\end{tabular} & 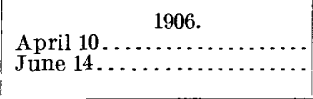 & $\begin{array}{l}\text { Feet. } \\
0.83 \\
.55\end{array}$ & $\begin{array}{r}\text { Second-feet. } \\
12.5 \\
6.6\end{array}$ \\
\hline
\end{tabular}

Daily gage height, in feet, of Lewis Creek near Staunton, Va.

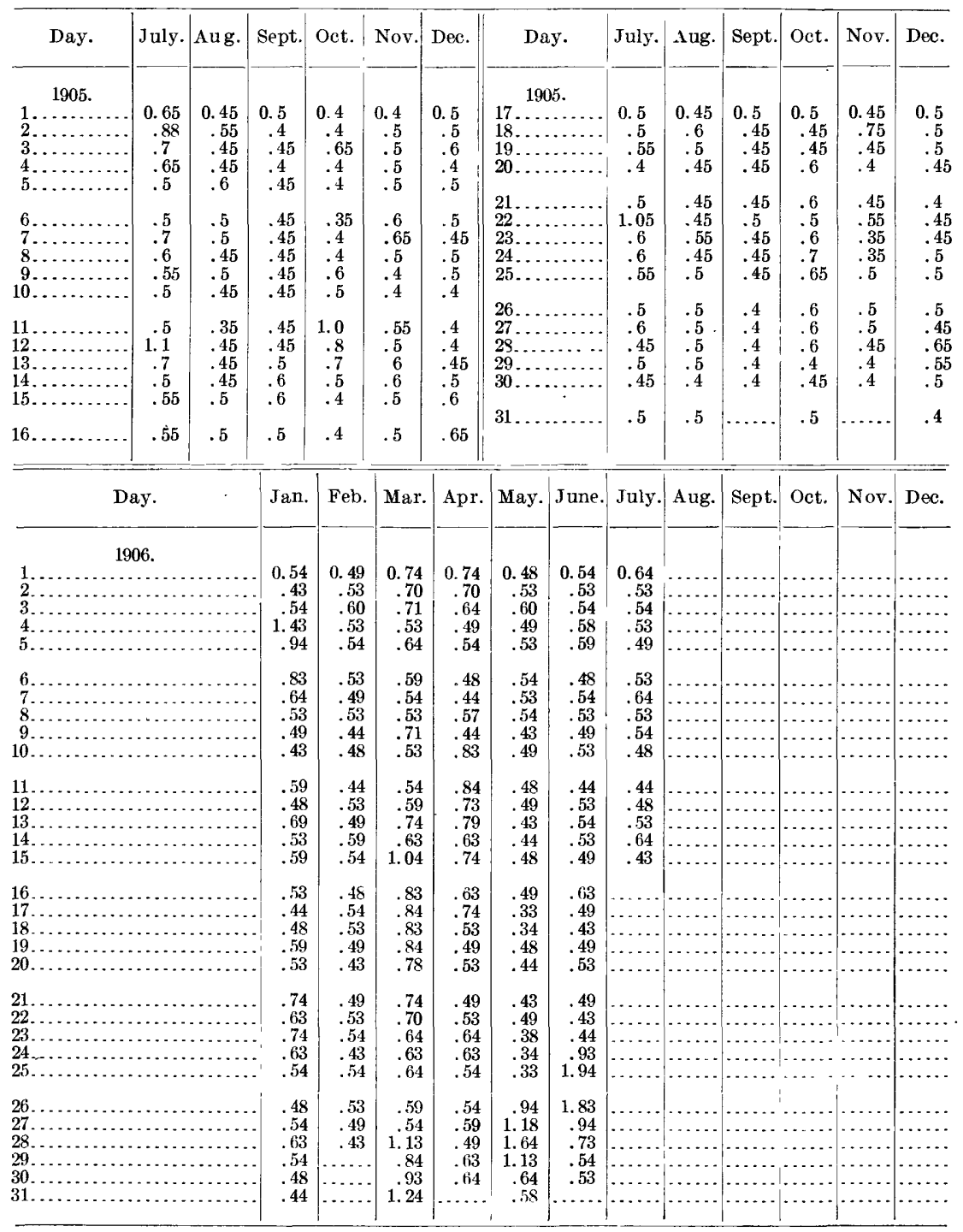


Rating tables for Lewis Creek near Staunton, Va.

JULY 1, 1905, TO MAY $27,1906 . a$

\begin{tabular}{|c|c|c|c|c|c|c|c|}
\hline $\begin{array}{c}\text { Gage } \\
\text { height. }\end{array}$ & Discharge. & $\begin{array}{c}\text { Gage } \\
\text { height. }\end{array}$ & Discharge. & $\begin{array}{c}\text { Gage } \\
\text { height. }\end{array}$ & Discharge. & $\begin{array}{c}\text { Gage } \\
\text { height. }\end{array}$ & Discharge. \\
\hline $\begin{array}{r}\text { Feet. } \\
0.30 \\
.35 \\
.40 \\
.45 \\
.50 \\
.55 \\
.60\end{array}$ & \begin{tabular}{|c|} 
Second-feet. \\
1.0 \\
1.4 \\
1.9 \\
2.6 \\
3.4 \\
4.4 \\
5.5
\end{tabular} & $\begin{array}{r}\text { Feet. } \\
065 \\
.70 \\
.75 \\
.80 \\
.85 \\
.90 \\
.95\end{array}$ & \begin{tabular}{|r|} 
Second-feet. \\
6.8 \\
8.2 \\
9.7 \\
11.3 \\
13.0 \\
14.9 \\
16.8
\end{tabular} & $\begin{array}{l}\text { Feet. } \\
1.00 \\
1.05 \\
1.10 \\
1.15 \\
1.20 \\
1.25 \\
1.30\end{array}$ & \begin{tabular}{|c|} 
Second-feet. \\
18.9 \\
21.0 \\
23.5 \\
26.0 \\
29.0 \\
32.0 \\
35.5
\end{tabular} & $\begin{array}{l}\text { Feet. } \\
1.35 \\
1.40 \\
1.45 \\
1.50 \\
1.55 \\
1.60 \\
1.65\end{array}$ & \begin{tabular}{|c} 
Second-feet. \\
39.0 \\
42.5 \\
46.5 \\
50.5 \\
54.5 \\
59.0 \\
63.5
\end{tabular} \\
\hline
\end{tabular}

MAY 28, 1906, TO JULY 15, 1906.b

\begin{tabular}{|l|l|l|l|l|l|l|l|}
\hline 0.25 & 1.3 & 0.60 & 7.9 & 0.95 & 20.5 & 1.50 & 55 \\
.30 & 1.8 & .65 & 9.4 & 1.00 & 23.0 & 1.60 & 63 \\
.35 & 2.5 & .70 & 11.0 & 1.10 & 28.5 & 1.70 & 71 \\
.40 & 3.3 & .75 & 12.7 & 1.20 & 64.5 & 1.80 & 79 \\
.45 & 4.2 & .80 & 14.5 & 1.30 & 41 & 1.90 & 88 \\
.50 & 5.3 & .85 & 16.3 & 1.40 & 48 & 2.00 & 97 \\
.55 & 6.5 & .90 & 18.3 & & & & \\
\hline
\end{tabular}

$a$ This table is strictly applicable only for open-channel conditions. It is based on three discharge measurements made during 1905 . It is fairly well defined between gage hejghts 0.5 and 0.8 foot.

$b$ This table is based on one discharge measurement made during 1906 and on the form of the preceding curve.

Estimated monthly discharge of Lewis Creek near Staunton, Va.

[Drainage area, 20 square miles.]

\begin{tabular}{|c|c|c|c|c|c|}
\hline \multirow[b]{2}{*}{ Month. } & \multicolumn{3}{|c|}{ Discharge in second--eet. } & \multicolumn{2}{|c|}{ Run-off. } \\
\hline & Maximum. & Minimum. & Mean. & $\begin{array}{c}\text { Second-feet } \\
\text { per square } \\
\text { mile. }\end{array}$ & $\begin{array}{l}\text { Depth in } \\
\text { inches. }\end{array}$ \\
\hline 1905. & & & & & \\
\hline $\begin{array}{l}\text { July } \\
\text { August }\end{array}$ & $\begin{array}{c}24 \\
5.5\end{array}$ & $\begin{array}{l}1.9 \\
1.4\end{array}$ & $\begin{array}{l}5.98 \\
3.15\end{array}$ & $\begin{array}{r}0.299 \\
.158\end{array}$ & $\begin{array}{r}0.345 \\
.182\end{array}$ \\
\hline September.... & 5.5 & 1.9 & 2.76 & .138 & .154 \\
\hline October. & 19 & 1.4 & 4.58 & .229 & .264 \\
\hline November... & 9.7 & 1.4 & 3.46 & $: 173$ & .193 \\
\hline December... & 6.8 & 1.9 & 3.34 & .167 & .192 \\
\hline January 1906. & & & & & \\
\hline $\begin{array}{l}\text { January ................ } \\
\text { February............. }\end{array}$ & 45 & $\begin{array}{l}2.3 \\
2.3\end{array}$ & $\begin{array}{l}6.58 \\
3.61\end{array}$ & $\begin{array}{r}.329 \\
.180\end{array}$ & $\begin{array}{l}.379 \\
.187\end{array}$ \\
\hline March..... & 31 & 4.0 & 9.70 & .485 & .559 \\
\hline April. & 13 & 2.3 & 6.06 & .303 & .338 \\
\hline May......... & 66 & 1.2 & 7.41 & .370 & .427 \\
\hline June. & 92 & 3.8 & 12.3 & .615 & .686 \\
\hline Iuly 1-15 & 9.1 & 3.8 & 6.17 & .308 & .172 \\
\hline
\end{tabular}

NORTH RIVER AT PORT REPUBLIC, VA.

North River rises in the Shenandoah Mountains in the northwestern part of Augusta County, Va., and flows in a general easterly and southeasterly direction to Port Republic, Rockingham County, Va., where it unites with South River to form South Fork of the Shenandoah. Its drainage area is 805 square miles. It is fed by numerous springs and is utilized to a considerable extent by mills of various kinds.

An important tributary is Middle River, which has been considered by some authorities as the main stream, North River being regarded 
as the tributary. Middle River rises on Little North Mountain, in the southern part of Augusta County, and flows in a general northeasterly direction, uniting with North River 4 miles above Port Republic. Its drainage area is 365 square miles. It is fed by numerous springs and is utilized to a considerable extent by mills of various kinds.

The gaging station at Port Republic was established August 6, 1895, and was discontinued April 1, 1899. It was located at the highway bridge, about 500 feet above the junction of North River with South River. Measurements were made from the downstream side of the bridge. The banks are high and not subject to overflow. The bed is very rough, but permanent. The current is broken and uneven at low stages. A dam about 200 feet above the bridge controls the flow at low stages.

Estimates previously published for North River at Port Republic have not been revised. They are probably within 15 per cent of the true flow. Ice conditions probably do not affect the flow at this station.

Discharge measurements of North River at Port Republic, Va.

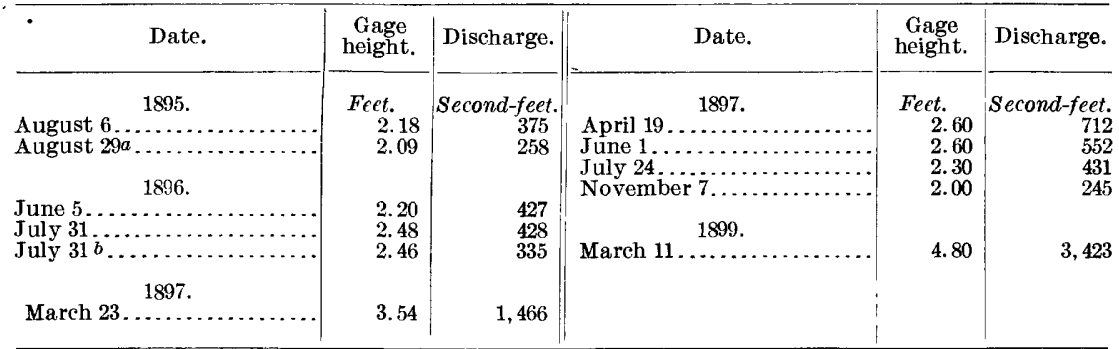

$a$ Result obtained by deducting the discharge of South River, 87 second-feet, from total discharge of South Fork of Shenandoah River, 345 second-feet, measured below the junction.

$b$ Result obtained as on August 29,1895 , by deducting 139 second-feet, discharge of South River, from 474 second-feet, discharge of South Fork.

Daily gage height, in feet, of North River at Port Republic, Va.

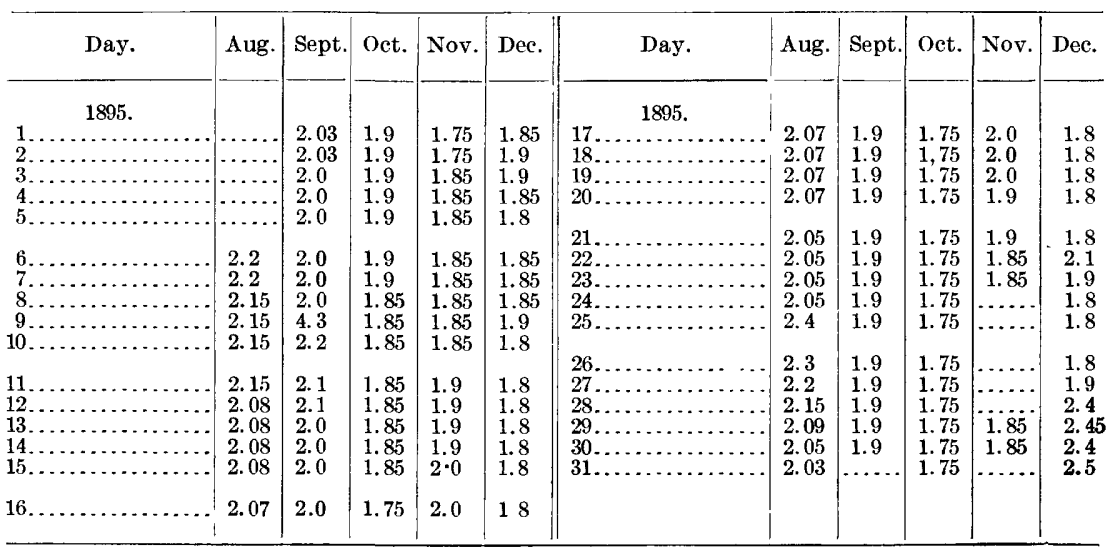


STREAM FLOW: NORTH RIVER.

Daily gage height, in feet, of North River at Port Republic, Va.-Continued.

\begin{tabular}{|c|c|c|c|c|c|c|c|c|c|c|c|c|}
\hline Day. & Jan. & Feb. & Mar. & Apr. & May. & June. & July. & Aug. & Sept. & Oct. & Nov. & Dec. \\
\hline $\begin{array}{l}1 \ldots \\
2 \ldots \\
3 \ldots \\
4 . . \\
5 \ldots\end{array}$ & $\begin{array}{l}3.0 \\
2.7 \\
2.5 \\
2.4 \\
2.4\end{array}$ & $\begin{array}{l}2.5 \\
2.5 \\
2.8 \\
3.8 \\
3.8\end{array}$ & $\begin{array}{l}2.6 \\
2.6 \\
2.6 \\
2.6 \\
2.6\end{array}$ & $\begin{array}{l}5.4 \\
5.0 \\
4.6 \\
4.2 \\
4.0\end{array}$ & $\begin{array}{l}2.32 \\
2.52 \\
2.52 \\
3.32 \\
3.32\end{array}$ & $\begin{array}{l}2.42 \\
2.32 \\
2.32 \\
2.22 \\
2.2\end{array}$ & $\begin{array}{l}2.8 \\
2.7 \\
2.7 \\
2.6 \\
2.6\end{array}$ & $\begin{array}{l}2.43 \\
2.43 \\
2.55 \\
2.45 \\
2.4\end{array}$ & $\begin{array}{l}2.0 \\
1.95 \\
1.9 \\
1.9 \\
1.9\end{array}$ & $\begin{array}{l}7.5 \\
4.5 \\
4.0 \\
3.5 \\
3.3\end{array}$ & $\begin{array}{l}2.35 \\
2.35 \\
2.35 \\
2.35 \\
4.7\end{array}$ & $\begin{array}{l}4.4 \\
3.5 \\
3.3 \\
3.3 \\
3.25\end{array}$ \\
\hline $\begin{array}{r}6 \ldots \\
7 \ldots \\
8 \ldots \\
9 . \\
10 . \\
\end{array}$ & $\begin{array}{l}2.4 \\
2.4 \\
2.3 \\
2.3 \\
2.3\end{array}$ & $\begin{array}{l}5.0 \\
5.7 \\
4.5 \\
4.1 \\
3.7\end{array}$ & $\begin{array}{l}2.5 \\
2.5 \\
2.5 \\
2.5 \\
2.5\end{array}$ & $\begin{array}{l}3.6 \\
3.5 \\
3.5 \\
3.4 \\
3.3\end{array}$ & $\begin{array}{l}3.32 \\
3.12 \\
2.92 \\
2.72 \\
2.72\end{array}$ & $\begin{array}{l}2.2 \\
4.5 \\
3.0 \\
2.6 \\
2.5\end{array}$ & $\begin{array}{l}2.8 \\
2.8 \\
2.8 \\
7.5 \\
5.3\end{array}$ & $\begin{array}{l}2.4 \\
2.3 \\
2.3 \\
2.3 \\
2.25\end{array}$ & $\begin{array}{l}1.85 \\
1.85 \\
1.8 \\
1.8 \\
1.75\end{array}$ & $\begin{array}{l}3.2 \\
3.1 \\
3.0 \\
2.9 \\
2.8\end{array}$ & $\begin{array}{l}6.0 \\
4.3 \\
3.6 \\
3.3 \\
3.3\end{array}$ & $\begin{array}{l}3.1 \\
3.05 \\
2.9 \\
2.8 \\
2.7\end{array}$ \\
\hline $\begin{array}{l}11 . . \\
12 . \\
13 . \\
14 . \\
15 . .\end{array}$ & $\begin{array}{l}2.3 \\
2.3 \\
2.3 \\
2.2 \\
2.2\end{array}$ & $\begin{array}{l}3.5 \\
3.3 \\
3.3 \\
5.2 \\
4.7\end{array}$ & $\begin{array}{l}2.4 \\
2.4 \\
2.4 \\
2.4 \\
2.6\end{array}$ & $\begin{array}{l}\text { 3. } \\
\text { 3. } \\
\text { 3.2 } \\
3.0 \\
\text { 3. } 0\end{array}$ & $\begin{array}{l}2.72 \\
2.62 \\
2.52 \\
5.82 \\
3.82\end{array}$ & $\begin{array}{l}2.3 \\
2.3 \\
2.2 \\
2.2 \\
2.2\end{array}$ & $\begin{array}{l}3.9 \\
3.6 \\
3.3 \\
3.0 \\
3.3\end{array}$ & & $\begin{array}{l}1.7 \\
1.7 \\
1.7 \\
1.7 \\
2.0\end{array}$ & $\begin{array}{l}2.65 \\
255 \\
2.5 \\
2.5 \\
2.5\end{array}$ & $\begin{array}{l}3.2 \\
3.0 \\
2.8 \\
2.8 \\
2.7\end{array}$ & $\begin{array}{l}2.65 \\
2.6 \\
2.55 \\
2.55 \\
2.5\end{array}$ \\
\hline $\begin{array}{l}16 \ldots \\
17 \ldots \\
18 \ldots \\
19 \ldots \\
20 \ldots\end{array}$ & $\begin{array}{l}2.1 \\
2.1 \\
2.0 \\
2.0 \\
2.0\end{array}$ & $\begin{array}{l}3.8 \\
3.6 \\
3.1 \\
3.0 \\
2.7\end{array}$ & $\begin{array}{l}2.6 \\
2.8 \\
3.2 \\
4.8 \\
7.2\end{array}$ & & $\begin{array}{l}3.22 \\
3.02 \\
2.82 \\
2.62 \\
2.62\end{array}$ & $\begin{array}{l}2.2 \\
2.5 \\
2.4 \\
2.4 \\
3.3\end{array}$ & $\begin{array}{l}2.7 \\
2.7 \\
2.7\end{array}$ & & $\begin{array}{l}1.9 \\
1.8 \\
1.8 \\
1.8 \\
1.7\end{array}$ & $\begin{array}{l}2.5 \\
2.5 \\
2.45 \\
2.45 \\
2.4\end{array}$ & $\begin{array}{l}2.7 \\
2.65 \\
2.65 \\
2.6 \\
2.6\end{array}$ & $\begin{array}{l}2.5 \\
2.45 \\
2.45 \\
2.45 \\
2.45\end{array}$ \\
\hline $\begin{array}{l}21 \ldots \\
22 \ldots \\
23 \ldots \\
24 \ldots \\
25 \ldots\end{array}$ & $\begin{array}{l}2.0 \\
2.0 \\
2.1 \\
6.15 \\
5.3\end{array}$ & $\begin{array}{l}2.7 \\
2.7 \\
2.7 \\
2.7 \\
2.7\end{array}$ & $\begin{array}{l}5.0 \\
4.8 \\
4.0 \\
3.8 \\
3.5\end{array}$ & & $\begin{array}{l}2.62 \\
2.62 \\
2.62 \\
2.52 \\
2.52\end{array}$ & $\begin{array}{l}3.0 \\
2.9 \\
2.5 \\
2.4 \\
2.4\end{array}$ & & & $\begin{array}{l}1.7 \\
1.7 \\
1.7 \\
1.65 \\
1.65\end{array}$ & & $\begin{array}{l}2.55 \\
2.5 \\
2.5 \\
2.45 \\
2.4\end{array}$ & $\begin{array}{l}2.45 \\
2.45 \\
2.45 \\
2.45 \\
2.4\end{array}$ \\
\hline $\begin{array}{l}26 \ldots \\
27 \ldots \\
28 \ldots \\
29 \ldots \\
30 \ldots \\
31 \ldots\end{array}$ & $\begin{array}{l}4.0 \\
3.3 \\
3.0 \\
2.8 \\
2.6 \\
2.5\end{array}$ & $\begin{array}{l}2.6 \\
2.6 \\
2.6 \\
2.6\end{array}$ & $\begin{array}{l}3.5 \\
3.5 \\
3.5 \\
4.4 \\
6.5 \\
5.9\end{array}$ & $\begin{array}{l}2.22 \\
2.22 \\
2.22\end{array}$ & $\begin{array}{l}2.52 \\
2.52 \\
2.42 \\
2.42 \\
2.42 \\
2.42\end{array}$ & $\begin{array}{l}3 . \\
3 . \\
3 . \\
2 . \\
2 .\end{array}$ & $\begin{array}{l}2.5 \\
2.45\end{array}$ & $\begin{array}{l}2.2 \\
2.15 \\
2.15 \\
2.1 \\
2.1\end{array}$ & $\begin{array}{c}1.65 \\
1.65 \\
1.65 \\
7.2 \\
18.0\end{array}$ & $\begin{array}{r}2.35 \\
2.35 \\
2.35 \\
2.35 \\
2.35\end{array}$ & $\begin{array}{l}2.4 \\
2.35 \\
2.35 \\
3.0 \\
5.5\end{array}$ & $\begin{array}{l}2.4 \\
2.4 \\
2.4 \\
2.4 \\
2.4 \\
2.35\end{array}$ \\
\hline $\begin{array}{l} \\
1 \ldots \\
2 \ldots \\
3 \ldots \\
4 \ldots \\
5 \ldots\end{array}$ & $\begin{array}{l}2.35 \\
2.35 \\
2.35 \\
2.35 \\
2.35\end{array}$ & $\begin{array}{l}2.35 \\
2.35 \\
2.35 \\
2.35 \\
2.35\end{array}$ & \begin{tabular}{|l|}
3.6 \\
3.5 \\
3.4 \\
3.4 \\
- 3.35
\end{tabular} & $\begin{array}{l}2.7 \\
2.7 \\
2.6 \\
2.6 \\
2.6\end{array}$ & $\begin{array}{c}2.4 \\
11.5 \\
7.3 \\
5.15 \\
4.3\end{array}$ & $\begin{array}{l}2.6 \\
2.55 \\
2.5 \\
2.5 \\
3.0\end{array}$ & $\begin{array}{l}2.35 \\
2.45 \\
2.45 \\
2.4 \\
2.35\end{array}$ & $\begin{array}{l}2.15 \\
2.15 \\
2.15 \\
2.15 \\
2.15\end{array}$ & $\begin{array}{l}2.0 \\
2.0 \\
2.0 \\
2.0 \\
1.95\end{array}$ & $\begin{array}{l}1.8 \\
1.8 \\
1.8 \\
1.8 \\
1.8\end{array}$ & $\begin{array}{l}2.2 \\
2.0 \\
2.6 \\
2.0 \\
2.0\end{array}$ & $\begin{array}{l}1.85 \\
1.85 \\
1.85 \\
1.85 \\
2.0\end{array}$ \\
\hline $\begin{array}{r}6 \ldots \\
7 \ldots \\
8 \ldots \\
9 \ldots \\
10 \ldots\end{array}$ & $\begin{array}{l}2.3 \\
2.3 \\
2.3 \\
2.3 \\
2.3\end{array}$ & $\begin{array}{l}2.55 \\
7.0 \\
5.5 \\
4.8 \\
4.3\end{array}$ & $\begin{array}{l}3.3 \\
3.3 \\
3.3 \\
3.25 \\
3.25\end{array}$ & $\begin{array}{l}2.9 \\
2.9 \\
2.9 \\
2.9 \\
3.0\end{array}$ & $\begin{array}{l}3.8 \\
3.5 \\
3.3 \\
3.1 \\
3.0\end{array}$ & $\begin{array}{l}2.85 \\
2.6 \\
2.55 \\
2.5 \\
2.5\end{array}$ & $\begin{array}{l}2.25 \\
2.2 \\
2.4 \\
2.3 \\
2.2\end{array}$ & $\begin{array}{l}2.15 \\
2.1 \\
2.1 \\
2.1 \\
2.1\end{array}$ & $\begin{array}{l}1.95 \\
1.9 \\
1.9 \\
1.9 \\
1.85\end{array}$ & 1. & $\begin{array}{l}2.0 \\
2.0 \\
1.95 \\
1.9 \\
1.9\end{array}$ & $\begin{array}{l}1.95 \\
1.95 \\
1.9 \\
1.85\end{array}$ \\
\hline $14 \ldots$ & $\begin{array}{l}2.3 \\
2.3 \\
2.3 \\
2.2 \\
2.25\end{array}$ & $\begin{array}{l}4.0 \\
3.9 \\
5.45 \\
4.7 \\
5.0\end{array}$ & $\begin{array}{l}3.25 \\
3.25 \\
3.2 \\
3.3 \\
3.5\end{array}$ & $\begin{array}{l}3.0 \\
3.0 \\
2.9 \\
2.9 \\
2.9\end{array}$ & $\begin{array}{l}3.0 \\
3.0 \\
4.9 \\
7.5 \\
5.2\end{array}$ & $\begin{array}{l}2.43 \\
2.4 \\
2.4 \\
2.4 \\
2.4\end{array}$ & \begin{tabular}{l|}
2.15 \\
2.5 \\
2.4 \\
2.4 \\
2.3
\end{tabular} & $\begin{array}{l}2.00 \\
2.05 \\
2.05 \\
2.05 \\
2.0\end{array}$ & $\begin{array}{l}1.85 \\
1.85 \\
1.85 \\
1.85 \\
1.85\end{array}$ & $\begin{array}{l}1.0 \\
1.8 \\
2.0 \\
2.0 \\
2.0\end{array}$ & $\begin{array}{l}1.9 \\
1.85 \\
1.85 \\
1.8 \\
1.8\end{array}$ & $\begin{array}{l}1 . \\
1 . \\
1 . \\
2 .\end{array}$ \\
\hline $\begin{array}{l}16 \ldots \\
17 \ldots \\
18 \ldots \\
19 \ldots \\
20 \ldots\end{array}$ & $\begin{array}{l}2.25 \\
2.25 \\
2.25\end{array}$ & $\begin{array}{l}5.3 \\
5.2 \\
4.6 \\
4.5 \\
4.3\end{array}$ & $\begin{array}{l}3.5 \\
3.7 \\
3.7 \\
3.7 \\
3.7\end{array}$ & $\begin{array}{l}2.9 \\
2.9 \\
2.8 \\
2.65 \\
2.6\end{array}$ & $\begin{array}{l}4.4 \\
4.0 \\
3.6 \\
3.45 \\
3.25\end{array}$ & $\begin{array}{l}2.4 \\
2.4 \\
2.4 \\
2.4 \\
2.5\end{array}$ & $\begin{array}{l}2.2 \\
2.15 \\
3.5 \\
3.5\end{array}$ & $\begin{array}{l}2.0 \\
2.0 \\
2.0 \\
2.0 \\
2.0\end{array}$ & $\begin{array}{l}1.85 \\
1.85 \\
1.85 \\
1.8 \\
1.8\end{array}$ & & $\begin{array}{l}1.9 \\
1.9 \\
1.9 \\
1.9\end{array}$ & $\begin{array}{l}2.45 \\
2.25 \\
2.1 \\
2.25 \\
2.25\end{array}$ \\
\hline $\begin{array}{l}21 \ldots \\
22 \ldots \\
23 \ldots \\
24 \ldots \\
25 \ldots\end{array}$ & $\begin{array}{l}2.25 \\
2.25 \\
2.55 \\
2.25 \\
2.25\end{array}$ & $\begin{array}{l}4.2 \\
4.8 \\
9.85 \\
6.7 \\
5.4\end{array}$ & $\begin{array}{l}3.9 \\
3.6 \\
3.35 \\
3.4 \\
3.3\end{array}$ & $\begin{array}{l}2.55 \\
2.5 \\
2.5 \\
2.5 \\
2.5\end{array}$ & $\begin{array}{l}3.15 \\
3.1 \\
3.0 \\
2.95 \\
2.9\end{array}$ & $\begin{array}{l}2.45 \\
2.4 \\
2.4 \\
2.4 \\
2.4\end{array}$ & $\begin{array}{l}2.5 \\
2.4 \\
2.35 \\
2.35 \\
2.3\end{array}$ & $\begin{array}{l}2.0 \\
2.0 \\
2.0 \\
2.0\end{array}$ & $\begin{array}{l}1.8 \\
1.8 \\
1.8 \\
1.8 \\
1.8\end{array}$ & $\begin{array}{l}2.0 \\
2.0 \\
2.0 \\
2.0 \\
2.0\end{array}$ & $\begin{array}{l}1.85 \\
1.85 \\
1.85 \\
1.85 \\
1.85\end{array}$ & $\begin{array}{l}2.25 \\
2.25 \\
2.25 \\
2.25 \\
2.25\end{array}$ \\
\hline $\begin{array}{l}26 \ldots \\
27 \ldots \\
28 \ldots \\
29 \ldots \\
30 \ldots \\
31 \ldots\end{array}$ & $\begin{array}{l}2.25 \\
2.25 \\
2.25 \\
2.25 \\
2.25 \\
2.35\end{array}$ & $\begin{array}{l}4.5 \\
4.4 \\
3.8\end{array}$ & $\begin{array}{l}3.2 \\
3.1 \\
3.0 \\
2.9 \\
2.8 \\
2.7\end{array}$ & $\begin{array}{l}2.5 \\
2.45 \\
2.45 \\
2.45 \\
2.4\end{array}$ & $\begin{array}{l}2.85 \\
2.8 \\
2.75 \\
2.7 \\
2.65 \\
2.6\end{array}$ & $\begin{array}{l}2.4 \\
2.35 \\
2.35 \\
2.35 \\
2.35 \\
\ldots . . .\end{array}$ & $\begin{array}{l}2.25 \\
2.2 \\
2.15 \\
2.15 \\
2.15 \\
2.15\end{array}$ & $\begin{array}{l}2.0 \\
2.0 \\
2.0 \\
2.0 \\
2.0 \\
2.0\end{array}$ & $\begin{array}{l}1.8 \\
1.8 \\
1.8 \\
1.8 \\
1.8\end{array}$ & $\begin{array}{l}2.0 \\
2.0 \\
2.1 \\
2.05 \\
1.95 \\
1.95\end{array}$ & $\begin{array}{l}1.85 \\
1.85 \\
1.85 \\
1.85 \\
1.85\end{array}$ & $\begin{array}{l}2.25 \\
2.25 \\
2.25 \\
2.25 \\
2.2 \\
2.15\end{array}$ \\
\hline
\end{tabular}


Daily gage height, in feet, of North River at Port Republic, Ta.-Continued.

\begin{tabular}{|c|c|c|c|c|c|c|c|c|c|c|c|c|}
\hline Day. & Jan. & Feb. & Mar. & Apr. & r. May. & June. & July. & Aug. & sept. & Oct. & Nov. & Dec. \\
\hline $\begin{array}{l}1 \\
1 \ldots \\
2 \ldots \\
3 \ldots \\
4 \ldots \\
5 \ldots\end{array}$ & $\begin{array}{l}2.15 \\
2.15 \\
2.15 \\
2.1 \\
2.1\end{array}$ & $\begin{array}{l}2.7 \\
2.7 \\
2.7 \\
2.7 \\
2.7\end{array}$ & $\begin{array}{l}2.1 \\
2.1 \\
2.1 \\
2.1 \\
2.1\end{array}$ & $\begin{array}{l}3.8 \\
3.5 \\
3.3 \\
3.1 \\
3.0\end{array}$ & $\begin{array}{l}2.65 \\
2.6 \\
2.5 \\
2.45 \\
2.4\end{array}$ & $\begin{array}{l}2.35 \\
2.3 \\
2.15 \\
2.15 \\
2.15\end{array}$ & $\begin{array}{l}2.2 \\
2.2 \\
2.2 \\
2.2 \\
2.2\end{array}$ & $\begin{array}{l}4.25 \\
4.25 \\
4.0 \\
4.5 \\
8.0\end{array}$ & $\begin{array}{l}2.6 \\
2.5 \\
2.5 \\
2.5 \\
2.5\end{array}$ & $\begin{array}{l}2.4 \\
2.4 \\
2.4 \\
2.4 \\
3.0\end{array}$ & $\begin{array}{l}3.0 \\
2.8 \\
2.65 \\
2.6 \\
2.6\end{array}$ & $\begin{array}{l}2.4 \\
2.3 \\
2.3 \\
3.0 \\
7.0\end{array}$ \\
\hline $\begin{array}{r}6 \ldots \\
7 \ldots \\
8 \ldots \\
9 \ldots \\
10 \ldots\end{array}$ & $\begin{array}{l}2.1 \\
2.05 \\
2.05 \\
2.0 \\
2.6\end{array}$ & $\begin{array}{l}2.65 \\
2.65 \\
2.6 \\
2.5 \\
2.4\end{array}$ & $\begin{array}{l}2.1 \\
2.1 \\
2.1 \\
2.1 \\
2.1\end{array}$ & $\begin{array}{l}2.9 \\
2.9 \\
2.8 \\
2.6 \\
2.7\end{array}$ & $\begin{array}{l}3.0 \\
6.4 \\
7.25 \\
6.3 \\
5.0\end{array}$ & $\begin{array}{l}2.1 \\
2.1 \\
2.1 \\
2.1 \\
2.0\end{array}$ & $\begin{array}{l}2.2 \\
2.2 \\
2.2 \\
2.2 \\
2.15\end{array}$ & $\begin{array}{l}5.0 \\
5.0 \\
4.6 \\
4.0 \\
9.0\end{array}$ & $\begin{array}{l}2.5 \\
2.5 \\
2.5 \\
2.5 \\
2.5\end{array}$ & $\begin{array}{l}2.9 \\
2.8 \\
2.6 \\
2.4 \\
2.25\end{array}$ & $\begin{array}{l}2.6 \\
2.5 \\
2.5 \\
2.45 \\
2.4\end{array}$ & $\begin{array}{l}5.0 \\
3.6 \\
3.3 \\
3.0 \\
2.9\end{array}$ \\
\hline $\begin{array}{l}11 \ldots \\
12 \ldots \\
13 \ldots \\
14 \ldots \\
15 . \ldots\end{array}$ & $\begin{array}{l}2.55 \\
2.5 \\
2.45 \\
2.45 \\
2.45\end{array}$ & $\begin{array}{l}2.3 \\
2.2 \\
2.2 \\
2.15 \\
2.15\end{array}$ & $\begin{array}{l}2.1 \\
2.1 \\
2.1 \\
2.1 \\
2.1\end{array}$ & $\begin{array}{r}2.7 \\
2.7 \\
2.7 \\
2.7 \\
4.1\end{array}$ & $\begin{array}{l}4.0 \\
3.6 \\
3.3 \\
3.2 \\
\text { 3. } 0\end{array}$ & $\begin{array}{l}2.0 \\
2.6 \\
2.5 \\
2.35 \\
2.2\end{array}$ & $\begin{array}{l}2.1 \\
2.1 \\
2.0 \\
2.0 \\
2.1\end{array}$ & $\begin{array}{l}9.0 \\
7.0 \\
6.0 \\
6.0 \\
6.0\end{array}$ & $\begin{array}{l}2.5 \\
2.5 \\
2.5 \\
2.5 \\
2.5\end{array}$ & $\begin{array}{l}2.1 \\
2.1 \\
2.1 \\
2.0 \\
2.0\end{array}$ & $\begin{array}{l}2.4 \\
2.4 \\
2.35 \\
2.35 \\
2.3\end{array}$ & $\begin{array}{l}2.8 \\
2.7 \\
2.65 \\
2.6 \\
2.55\end{array}$ \\
\hline $\begin{array}{l}16 \ldots \ldots \\
17 \ldots \ldots \\
18 \ldots \\
19 \ldots \\
20 \ldots\end{array}$ & $\begin{array}{l}2.45 \\
2.45 \\
2.45 \\
2.55 \\
2.55\end{array}$ & $\begin{array}{l}2.1 \\
2.1 \\
2.1 \\
2.1 \\
2.1\end{array}$ & $\begin{array}{l}2.1 \\
2.2 \\
2.8 \\
2.9 \\
2.8\end{array}$ & $\begin{array}{l}5.2 \\
4.5 \\
4.0 \\
3.5 \\
3.2\end{array}$ & $\begin{array}{l}2.8 \\
2.8 \\
2.7 \\
2.6 \\
2.6\end{array}$ & $\begin{array}{l}4.2 \\
3.4 \\
3.0 \\
3.0 \\
3.0\end{array}$ & $\begin{array}{l}2.1 \\
2.25 \\
2.5 \\
2.4 \\
2.3\end{array}$ & $\begin{array}{l}4.5 \\
4.2 \\
4.0 \\
4.0 \\
3.6\end{array}$ & $\begin{array}{l}2.5 \\
2.5 \\
2.5 \\
2.5 \\
2.5\end{array}$ & $\begin{array}{l}2.0 \\
2.0 \\
2.0 \\
9.5 \\
5.0\end{array}$ & $\begin{array}{l}2.3 \\
2.25 \\
2.2 \\
2.15 \\
2.6\end{array}$ & $\begin{array}{l}2.5 \\
2.5 \\
2.4 \\
2.3 \\
2.3\end{array}$ \\
\hline $\begin{array}{l}21 \ldots \ldots \\
22 \ldots \ldots \\
23 \ldots \\
24 \ldots \\
25 \ldots\end{array}$ & $\begin{array}{l}2.55 \\
2.55 \\
2.7 \\
2.8 \\
2.8\end{array}$ & $\begin{array}{l}2.1 \\
2.1 \\
2.1 \\
2.1 \\
2.1\end{array}$ & $\begin{array}{l}2.7 \\
2.7 \\
2.7 \\
2.7 \\
2.8\end{array}$ & $\begin{array}{l}3.0 \\
2.9 \\
2.7 \\
2.7 \\
2.7\end{array}$ & $\begin{array}{l}2.6 \\
\text { 3. } 35 \\
\text { 3. } 3 \\
\text { 3. } 2 \\
\text { 3. } 0\end{array}$ & $\begin{array}{l}3.0 \\
2.9 \\
2.8 \\
2.65 \\
2.5\end{array}$ & $\begin{array}{l}2.3 \\
2.3 \\
2.3 \\
3.0 \\
2.8\end{array}$ & $\begin{array}{l}3.3 \\
3.2 \\
3.1 \\
3.1 \\
3.0\end{array}$ & $\begin{array}{l}2.5 \\
2.5 \\
2.7 \\
2.7 \\
2.6\end{array}$ & \begin{tabular}{l|r} 
& 5.0 \\
& 10.0 \\
5 & 6.5 \\
5 & 5.0 \\
5 & 3.8
\end{tabular} & $\begin{array}{l}2.6 \\
2.6 \\
2.6 \\
2.6 \\
2.6\end{array}$ & $\begin{array}{l}2.3 \\
2.3 \\
4.0 \\
4.3 \\
3.6\end{array}$ \\
\hline $\begin{array}{l}26 \ldots \\
27 \ldots \\
28 \ldots \\
29 \ldots \\
30 \ldots \\
31 \ldots\end{array}$ & $\begin{array}{l}2.8 \\
2.8 \\
2.7 \\
2.7 \\
2.7 \\
2.7\end{array}$ & $\begin{array}{l}2.1 \\
2.1 \\
2.1 \\
\ldots . .\end{array}$ & $\begin{array}{l}2.9 \\
3.0 \\
3.0 \\
3.0 \\
3.3 \\
4.2\end{array}$ & $\begin{array}{l}2.7 \\
2.7 \\
2.7 \\
2.6 \\
2.6\end{array}$ & $\begin{array}{l}2.9 \\
2.75 \\
2.65 \\
2.65 \\
2.5 \\
2.4\end{array}$ & $\begin{array}{l}2.5 \\
2.5 \\
2.4 \\
2.4 \\
2.4\end{array}$ & $\begin{array}{l}2.7 \\
3.5 \\
4.75 \\
5.0 \\
5.0 \\
4.5\end{array}$ & $\begin{array}{l}2.9 \\
2.8 \\
2.7 \\
2.7 \\
2.6 \\
2.6\end{array}$ & $\begin{array}{l}2.6 \\
2.6 \\
2.6 \\
2.5 \\
2.4\end{array}$ & $\begin{array}{l}\text { 3. } 65 \\
\text { 3. } 5 \\
\text { 3. } 3 \\
\text { 3. } 15 \\
\text { 3. } 15 \\
\text { 3. } 15\end{array}$ & $\begin{array}{l}2.6 \\
2.55 \\
2.5 \\
2.5 \\
2.5\end{array}$ & $\begin{array}{l}3.3 \\
3.0 \\
2.8 \\
2.6 \\
2.6 \\
2.6\end{array}$ \\
\hline & $\mathrm{Ja}$ & & & Mar. & Apr. & & & & & Feb. & Mar. & Apr. \\
\hline $\begin{array}{c} \\
1899 . \\
1 \ldots \ldots \\
2 \ldots \ldots \\
3 \ldots \ldots \\
4 \ldots \ldots \\
5 \ldots \ldots\end{array}$ & $\begin{array}{l}2 . \\
2 . \\
2 . \\
2 . \\
2 .\end{array}$ & & $\begin{array}{l}2.3 \\
2.3 \\
2.3 \\
2.3 \\
2.3\end{array}$ & $\begin{array}{r}7.1 \\
5.5 \\
5.0 \\
7.4 \\
14.0\end{array}$ & 3.0 & $\begin{array}{l}17 \ldots \\
18 \ldots \\
19 \ldots \\
20 . \\
21 .\end{array}$ & 99. & & $\begin{array}{l}2.7 \\
2.7 \\
2.6 \\
2.6 \\
2.6\end{array}$ & $\begin{array}{l}3.0 \\
3.0 \\
3.4 \\
4.0 \\
5.5\end{array}$ & $\begin{array}{l}4.0 \\
3.7 \\
4.0 \\
4.3 \\
3.9\end{array}$ & $\cdots$ \\
\hline $\begin{array}{c}6 \ldots \\
7 \ldots \\
8 \ldots \\
9 \ldots \\
10 \ldots\end{array}$ & $\begin{array}{l}5.4 \\
6.7 \\
5.0 \\
4.0 \\
3.6\end{array}$ & & $\begin{array}{l}2.3 \\
2.3 \\
2.4 \\
2.4 \\
2.5\end{array}$ & $\begin{array}{l}8.9 \\
7.5 \\
5.8 \\
4.8 \\
5.3\end{array}$ & & $\begin{array}{l}22 . \\
23 . \\
24 . \\
25 . \\
26 .\end{array}$ & & & $\begin{array}{l}2.6 \\
2.6 \\
2.6 \\
2.6 \\
2.6\end{array}$ & $\begin{array}{l}7.0 \\
7.6 \\
6.0 \\
4.9 \\
4.9\end{array}$ & $\begin{array}{l}3.6 \\
3.4 \\
3.2 \\
3.1 \\
3.1\end{array}$ & 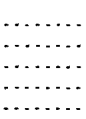 \\
\hline $\begin{array}{l}11 \ldots \\
12 \ldots \\
13 \ldots \\
14 \ldots \\
15 \ldots \\
16 \ldots\end{array}$ & $\begin{array}{l}3.4 \\
3.2 \\
3.0 \\
2.9 \\
2.8 \\
2.8\end{array}$ & & $\begin{array}{l}2.5 \\
2.5 \\
2.5 \\
2.7 \\
2.8 \\
3.0\end{array}$ & $\begin{array}{l}\text { 4. } 8 \\
\text { 4. } 6 \\
\text { 4. } 0 \\
\text { 3. } 8 \\
4.5 \\
4.2\end{array}$ & & $\begin{array}{l}27 \ldots \\
28 \ldots \\
29 \ldots \\
30 \ldots \\
31 \ldots\end{array}$ & & & $\begin{array}{l}2.6 \\
2.6 \\
2.5 \\
2.5 \\
2.4\end{array}$ & $\begin{array}{c}6.0 \\
9.0 \\
\cdots . .\end{array}$ & $\begin{array}{l}\text { 3. } 0 \\
\text { 3. } 0 \\
3.0 \\
\text { 3. } 0 \\
\text { 3. } 0\end{array}$ & $\begin{array}{l} \\
\ldots \ldots \\
\cdots \\
\ldots \ldots\end{array}$ \\
\hline
\end{tabular}

Rating table for North River at Port Republic, Va., from August 5, 1895, to Aprit 1. 1899.a

\begin{tabular}{|r|r|r|r|r|r|r|r|}
\hline $\begin{array}{c}\text { Gage } \\
\text { height. }\end{array}$ & Discharge. & $\begin{array}{c}\text { Gage } \\
\text { height. }\end{array}$ & Discharge. & $\begin{array}{r}\text { Gage } \\
\text { height. }\end{array}$ & Discharge. & $\begin{array}{r}\text { Gage } \\
\text { height. }\end{array}$ & Discharge. \\
\cline { 2 - 6 } Feet. & Second-feet. & Feet. & Second-feet. & Feet. & Second-feet. & Feet. & Second-feet. \\
1.6 & 165 & 3.2 & 1,050 & 4.8 & 3,410 & 8.5 & 9,145 \\
1.8 & 215 & 3.4 & 1,290 & 5.0 & 3,720 & 9.0 & 9,920 \\
2.0 & 275 & 3.6 & 1,550 & 5.5 & 4,495 & 9.5 & 10,695 \\
2.2 & 350 & 3.8 & 1,860 & 6.0 & 5,270 & 10.0 & 11,470 \\
2.4 & 445 & 4.0 & 2,170 & 6.5 & 6,045 & 10.5 & 12,245 \\
2.6 & 555 & 4.2 & 2,480 & 7.0 & 6,820 & 11.0 & 13,020 \\
2.8 & 700 & 4.4 & 2,790 & 7.5 & 7.595 & & \\
3.0 & 865 & 4.6 & 3,100 & 8.0 & 8,370 & & \\
\hline
\end{tabular}

a This table is strictly applicable only for open-channel conditions. It is not well defined. 
Estimated monthly discharge of North River at Port Republic, Va.

[Drainage area, 804 square miles.]

\begin{tabular}{|c|c|c|c|c|c|}
\hline \multirow[b]{2}{*}{ Month. } & \multicolumn{3}{|c|}{ Discharge in second-feet. } & \multicolumn{2}{|c|}{ Run-off. } \\
\hline & Maximum. & Minimum. & Mean. & $\begin{array}{l}\text { Second-feet } \\
\text { per square } \\
\text { mile. }\end{array}$ & $\begin{array}{l}\text { Depth in } \\
\text { inches. }\end{array}$ \\
\hline 1895. & & & & & \\
\hline $\begin{array}{l}\text { August } 6-31 \ldots \ldots \\
\text { Sipptember....... }\end{array}$ & $\begin{array}{r}445 \\
2,635\end{array}$ & $\begin{array}{l}292 \\
345\end{array}$ & $\begin{array}{l}319 \\
346\end{array}$ & 0.40 & 0.39 \\
\hline October.............. & 245 & 202 & $\begin{array}{l}0+0 \\
219\end{array}$ & .27 & .31 \\
\hline November $a$. & 275 & 202 & 239 & .30 & .33 \\
\hline December..... & 500 & 215 & 251 & .31 & .36 \\
\hline January . . & & & & & \\
\hline February. & $\begin{array}{l}5,502 \\
4,805\end{array}$ & $\begin{array}{l}275 \\
500\end{array}$ & $\begin{array}{r}799 \\
1,488\end{array}$ & $\begin{array}{r}.99 \\
1.85\end{array}$ & $\begin{array}{l}1.14 \\
1.93\end{array}$ \\
\hline March .... & 7,130 & 445 & 1,653 & 2.05 & 2.37 \\
\hline A pril 1-15, 28-30. & 4,340 & 350 & 1,594 & 1.98 & 1.33 \\
\hline May............ & 4,960 & 395 & 824 & 1.02 & 1.18 \\
\hline June......... & 2,945 & 350 & $\begin{array}{r}642 \\
1\end{array}$ & .80 & .89 \\
\hline July . . . . . . & 7,595 & 472 & 1,085 & 1.35 & 1.56 \\
\hline August....... & $\begin{array}{r}1,550 \\
23,870\end{array}$ & 310 & 458 & .57 & .66 \\
\hline $\begin{array}{l}\text { September... } \\
\text { October.... }\end{array}$ & $\begin{array}{r}23,870 \\
7,595\end{array}$ & $\begin{array}{l}177 \\
420\end{array}$ & $\begin{array}{r}1,224 \\
931\end{array}$ & $\begin{array}{l}1.52 \\
1.16\end{array}$ & 1.34 \\
\hline & $\begin{array}{l}7,595 \\
5,270\end{array}$ & $\begin{array}{l}420 \\
420\end{array}$ & $\begin{array}{r}931 \\
1,088\end{array}$ & 1.35 & 1.51 \\
\hline December... & 2,790 & 420 & 699 & .87 & 1.00 \\
\hline 1897 & & & & & \\
\hline $\begin{array}{l}\text { January ...... } \\
\text { Fiabruary }\end{array}$ & $\begin{array}{r}420 \\
11,240\end{array}$ & $\begin{array}{l}372 \\
420\end{array}$ & $\begin{array}{r}388 \\
138\end{array}$ & .48 & .55 \\
\hline March. & $\begin{array}{r}11,240 \\
2,015\end{array}$ & $\begin{array}{l}420 \\
625\end{array}$ & $\begin{array}{l}3,138 \\
1,250\end{array}$ & $\begin{array}{l}3.90 \\
1.55\end{array}$ & $\begin{array}{l}4.06 \\
1.79\end{array}$ \\
\hline April....... & 865 & 445 & 642 & .80 & .89 \\
\hline May... & 13,790 & 445 & 2,212 & 2.75 & 3.17 \\
\hline June.... & 865 & 420 & 489 & .61 & .68 \\
\hline July... & 1,420 & 330 & 463 & .58 & .67 \\
\hline August. & 330 & 275 & 292 & . .36 & .41 \\
\hline Stptember. . . . & 275 & 215 & 234 & .29 & .32 \\
\hline $\begin{array}{l}\text { Ostober......... } \\
\text { Noyember }\end{array}$ & 310 & 215 & 250 & .31 & .35 \\
\hline $\begin{array}{l}\text { November....... } \\
\text { December...... }\end{array}$ & $\begin{array}{l}555 \\
590\end{array}$ & $\begin{array}{l}215 \\
230\end{array}$ & $\begin{array}{l}256 \\
319\end{array}$ & $\begin{array}{l}.32 \\
.39\end{array}$ & $\begin{array}{l}.36 \\
.45\end{array}$ \\
\hline & & & & & \\
\hline The year.. & 13,790 & 215 & 829 & 1.03 & 13.70 \\
\hline 1898. & & & & & \\
\hline Fibbruary........... & $\begin{array}{l}700 \\
625\end{array}$ & $\begin{array}{l}275 \\
310\end{array}$ & $\begin{array}{l}491 \\
414\end{array}$ & $\begin{array}{l}.61 \\
.51\end{array}$ & .70 \\
\hline March ... & 2,480 & 310 & 581 & .72 & .80 \\
\hline April.. & 4,030 & 555 & 1,110 & 1.38 & 1.54 \\
\hline May.... & 7,207 & 44 & 1,430 & 1.78 & 2.05 \\
\hline June. . & 2,480 & 27 & 585 & .73 & .81 \\
\hline Julv.... & 3,720 & 27 & 822 & 1.02 & 1.18 \\
\hline A lagust. . & 9,920 & 555 & 3,030 & 3.77 & 4.35 \\
\hline September. - & 662 & 445 & 520 & .65 & .72 \\
\hline $\begin{array}{l}\text { Ototober.................. } \\
\text { November }\end{array}$ & 11,470 & $\begin{array}{r}275 \\
330\end{array}$ & $\begin{array}{r}1,814 \\
507\end{array}$ & 2.26 & $\begin{array}{r}2.61 \\
70\end{array}$ \\
\hline $\begin{array}{l}\text { November . . . . . . } \\
\text { December...... }\end{array}$ & $\begin{array}{r}865 \\
6,820\end{array}$ & 395 & $\begin{array}{r}1,089 \\
1,0\end{array}$ & 1.35 & 1.56 \\
\hline The year.. & 11,470 & 275 & 1,032 & 1.28 & 17.55 \\
\hline Tspularp & & & & & \\
\hline $\begin{array}{l}\text { Jenuary.. } \\
\text { February. }\end{array}$ & $\begin{array}{l}6,35 E \\
9,920\end{array}$ & $\begin{array}{l}445 \\
395\end{array}$ & $\begin{array}{l}1,126 \\
2,132\end{array}$ & $\begin{array}{l}1.40 \\
2.65\end{array}$ & $\begin{array}{l}1.61 \\
2.76\end{array}$ \\
\hline March.. & 17,670 & 865 & 3,452 & 4.29 & 4.95 \\
\hline
\end{tabular}

a Discharge interpolated November 24-28, 1895.

IRR $192-07-8$ 
MISCELLANEOUS DISGHARGE MEASUREMENTS IN NORTH RIVER BASIN.

The following miscellaneous measurements have been made in the basin of North River:

Miscellaneous discharge measurements in North River basin.

\begin{tabular}{|c|c|c|c|c|c|c|}
\hline Date. & Stream. & Locality. & Width. & $\begin{array}{l}\text { Area of } \\
\text { section. }\end{array}$ & $\begin{array}{c}\text { Mean } \\
\text { velocity. }\end{array}$ & $\begin{array}{c}\text { Dis- } \\
\text { charge. }\end{array}$ \\
\hline $\begin{array}{r}1897 . \\
\text { March } 22 .\end{array}$ & Middle River. & At mouth near Mount Me- & Feet. & $\begin{array}{c}\text { Square } \\
\text { feet. } \\
396\end{array}$ & $\begin{array}{l}\text { Feet per } \\
\text { second. } \\
1.58\end{array}$ & $\begin{array}{r}\text { Second- } \\
\text { feet. } \\
625\end{array}$ \\
\hline April $19 \ldots$ & ..... do. & $\begin{array}{l}\text { malan, va. } \\
\text {....do....... }\end{array}$ & & 228 & 1.00 & 229 \\
\hline $\begin{array}{l}\text { November } 7 \\
\text { March } 22 \ldots\end{array}$ & ... do .... & …do do & 90 & 151 & .61 & 92 \\
\hline March $22 \ldots$ & North River.. & $\begin{array}{l}\text { Above junction with Mid- } \\
\text { dle River near Mount } \\
\text { Meridian, Va. }\end{array}$ & & & & 841 \\
\hline November $7 \ldots$ & do. & .....do........... & & & & 153 \\
\hline
\end{tabular}

SOUTH FORK OF SHENANDOAH RIVER BASIN BELOW PORT REPUBLIC, VA.

GENERAL DESCRIPTION.

South Fork of Shenandoah River is formed at Port Republic, Rockingham County, Va.; by the union of North and South rivers, and flows northeastward to Riverton, Va., where it unites with North Fork to form the main Shenandoah. Its length by river course is about 96 miles.

South Fork of the Shenandoah is to a certain extent navigable for small boats, and works have at various times been executed for improving it. The basin is traversed by the Norfolk and Western Railway, and nearly every part of it is accessible. Theoretically a large amount of power is available, but very little is utilized. The bed and banks are favorable for the construction of dams, but space for canals and buildings is in places small.

The river is subject to frequent and rapidly rising freshets. The stream flows alternately in pools of comparatively slack water and over ledges and shoals, forming rapids and falls, the pools being shorter and the ledges more numerous in the upper reaches than in the lower.

In August, 1899, a reconnaissance survey of South Fork of Shenandoah River between Port Republic and Riverton, Va., was made by F. H. Anschutz, under the direction of D. C. Humphreys, for the purpose of obtaining a profile of the river and a map showing the location of the mills, the unused falls, and the localities where water power might póssibly be developed.

The results of the work are shown by the profile (Pl. III, p. 134). The distances along the profile and map are measured along the center line of the stream, the initial point being the forks of the river at Port Republic. 
The developed and undeveloped power of the river at the time of the survey was briefly stated, as follows:

At Port Republic a combined sawmill and gristmill utilizes a fall of 10 feet in South River, which is obtained by a dam about a quarter of a mile above the mouth.

On North River, in the first 1,500 feet above the forks, there is a fall of 6 feet which was once used for power and which could easily be developed agąin.

At Shendun, 4 miles above Port Republic, on South River, an excellent power was partially developed during what is known as "boom times" in 1890. The fall is said to be about 20 feet.

Two miles downstream from the forks there is a gristmill utilizing about 6 feet fall, chtained by a brush dam and a long race.

At 6 miles there is a fall of 4.5 feet, but the banks are low.

At $7 \frac{1}{2}$ miles there is a small gristmill with a 3 -foot timber dam.

Between the $7 \frac{1}{2}$-mile point and the next mill, at 11 miles, there is a total fall of 25 feet, but the river banks are much broken and generally are low.

At 11 miles there is a small gristmill using about 6 feet fall.

At $12 \frac{1}{2}$ miles there is a gristmill using $5 \frac{1}{2}$ feet fall.

At 17 miles, opposite Elkton, there is a combined gristmill and sawmill, with a 4 -foot clam, using 6 feet fall.

In the neighborhood of Shenandoah the fall is rapid, but the banks are low.

At $31 \frac{1}{2}$ miles there is an old mill and a timber dam 4 feet high, the first course of timkers of which is missing.

At $32 \frac{1}{2}$ miles, Grove Hill, there is a gristmill and a good dam of timber and loose rocks, 4 feet high.

At $37 \frac{1}{2}$ miles, Kemple Falls, there is a fall of 15 feet in three-fourths of a mile. The kank on the left side is fairly good, but on the right side of the main group of channels the river spreads out over and runs through a large area of bowlders. and the bank for a. considerable distance back is but little above the surface of the stream.

At 38 miles, Newport, there is a combined sawmill and gristmill that uses about 6 feet fall. The dam is about 4 feet high, loosely made of timber and stonework.

At 41 miles is Manks' mill, a gristmill with a dam $3 \frac{1}{2}$ feet high, rudely constructed of brush and loose stone. The fall below the mill is good and about 7 feet are utilized.

At 50 miles there is an old mill site, nothing being left except the foundation of the clam.

At 55 miles, Schuler, there is a gristmill, with a dam $3 \frac{1}{2}$ feet high.

At $66 \frac{1}{2}$ miles is Goode's mill, the dam for which is 3 feet high, of timber and planking backed by loose rock. The mill uses a fall of about 6 feet.

At $79 \frac{1}{2}$ miles is Hazard's mill, on the left bank of the stream, at a point where the river splits and runs around a large island. The dam is in the left branch and is substantially constructed of timber; it is $4 \frac{1}{2}$ feet high.

At $97 \frac{1}{2}$ miles is Blackmore's dam. It was a stoutly built dam of timber and dry raasonry, but it is broken at each end and part of the upper course is missing at the raiddle. The mill is no longer standing.

At 101 miles, Riverton, are the Riverton mills. The dam is 6 feet high, $a$ of timber and is well constructed.

a See description of station on North Fork of Shenandoah River near Riverton, Va., pp. 125-126. 
ELK RUN AT ELKTON, VA.

Elk Run rises in the Blue Ridge in the eastern part of Rockingham County, Va., and flows northwestward into•South Fork of Shenandoah River near Elkton.

The gaging station was established June 28,1905 , by N. C. Grover, in connection with the investigation of stream pollution in the Shenandoah Valley. It was discontinued July 16, 1906. It is located at the highway bridge 500 feet south of the railroad station at Elkton, Va.

The channel is straight for 100 feet above and 200 feet below the station. The current is sluggish at the gage. Both banks are low and overflow during high water. All the water passes beneath the bridge, except during extreme floods. The bed of the stream is composed of gravel and is permanent. The stream is highly polluted by waste from tanneries along its banks.

Discharge measurements were made at ordinary stages at a footbridge 1,000 feet downstream from the bridge to which the gage is fastened. During high water discharge measurements were made from the highway bridge.

A standard chain gage is fastened to a floor beam on the downstream side of the bridge, near the right end. The length of the chain from the end of the weight to the marker is 8.74 feet. The gage was read once each day by C. L. Gooden. Bench mark No. 2 is the underside of the coping at the southwest corner of the first railwaybridge pier on the right bank of the creek, marked with red paint "U. S. G. S. B. M." Its elevation is 11.96 feet above the datum of the gage.

Unsatisfactory conditions of flow prevail at this station. The discharge measurements plot very erratically, owing probably to changes at the controlling point below the station. It was necessary to obtain the discharge by an indirect method based on the assumption of a gradual change in channel conditions between successive measurements. The estimates can probably be considered accurate within only 20 per cent of the true flow. Ice conditions probably affect the flow somewhat.

Discharge measurements of Elk Run at Elkton, Va.

\begin{tabular}{|c|c|c|c|c|c|}
\hline Date. & $\begin{array}{c}\text { Gage } \\
\text { height. }\end{array}$ & Discharge. & Date. & $\begin{array}{c}\text { Gage } \\
\text { height. }\end{array}$ & Discharge. \\
\hline $\begin{array}{r}1905 . \\
\text { May } 22 \ldots \ldots \ldots \ldots\end{array}$ & Feet. & \multirow{2}{*}{$\begin{array}{r}\text { Second-feet. } \\
4.9 \\
4.0 \\
17.4 \\
3.4 \\
11.0\end{array}$} & \multirow[t]{2}{*}{$\begin{array}{rr} & 1906 . \\
\text { April } 11\end{array}$} & \multirow{2}{*}{$\begin{array}{l}\text { Feet. } \\
2.78 \\
2.57\end{array}$} & \multirow{2}{*}{$\begin{array}{r}\text { Second-feet. } \\
15.7 \\
6.5\end{array}$} \\
\hline $\begin{array}{l}\text { Done } \\
\text { June } 28 \\
\text { July } 20 \ldots \ldots \\
\text { December } 28 \ldots \ldots\end{array}$ & $\begin{array}{l}2.80 \\
2.10 \\
2.78\end{array}$ & & & & \\
\hline
\end{tabular}


Daily gage height, in feet, of Elk Run at Elkton, Va.

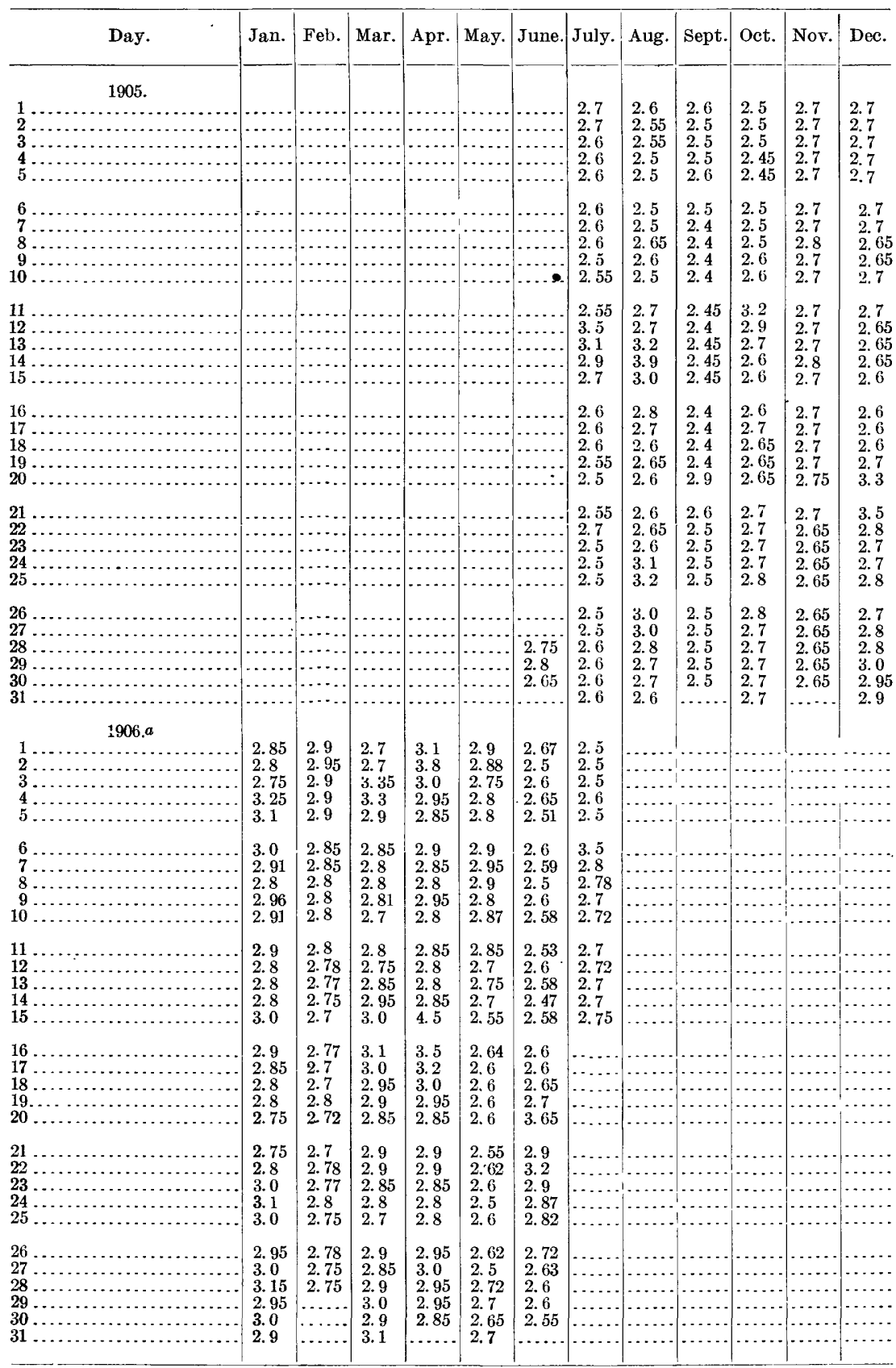

$a$ Slight ice conditions during the winter season. 
Estimated monthly discharge of Elk Run at Elkton, Va.

[Drainage area, 15.8 square miles.]

\begin{tabular}{|c|c|c|c|c|c|}
\hline \multirow[b]{2}{*}{ Month. } & \multicolumn{3}{|c|}{ Discharge in second-feet. . } & \multicolumn{2}{|c|}{ Run-off. } \\
\hline & Maximum. & Minimum. & Mean. & $\begin{array}{l}\text { Second-feet } \\
\text { per square } \\
\text { mile. }\end{array}$ & $\begin{array}{l}\text { Depth in } \\
\text { inches. }\end{array}$ \\
\hline 1905. & 57 & 7.0 & 11.9 & 0.753 & 0.868 \\
\hline August..... & 85 & 7.0 & $\begin{array}{l}11.9 \\
14.1\end{array}$ & $\begin{array}{r}0.700 \\
.892\end{array}$ & $\begin{array}{l}0.808 \\
1.03\end{array}$ \\
\hline September... & 15 & 5.5 & 6.73 & .426 & .475 \\
\hline October... & 27 & 6.0 & 9.39 & .594 & .685 \\
\hline November. & 12 & 8.5 & 5.40 & .595 & .664 \\
\hline December. & 46 & 7.5 & 11.8 & .747 & .861 \\
\hline 1906. & & & & & \\
\hline $\begin{array}{l}\text { January } \ldots \ldots \ldots \ldots \\
\text { February } \ldots \ldots \ldots\end{array}$ & $\begin{array}{l}29 \\
18\end{array}$ & $\begin{array}{l}10 \\
11\end{array}$ & $\begin{array}{l}15.8 \\
13.5\end{array}$ & $\begin{array}{l}1.00 \\
.854\end{array}$ & $\begin{array}{l}1.15 \\
.889\end{array}$ \\
\hline March............ & 42 & 11 & 19.3 & 1. 22 & 1.41 \\
\hline April... & 132 & 15 & 27.0 & 1.71 & 1.91 \\
\hline May. & 20 & 6.5 & 11.5 & .728 & .839 \\
\hline June. & 54 & 5.5 & 9.9 & .627 & .700 \\
\hline July 1-15. & 44 & 6 & 10.7 & .677 & .378 \\
\hline
\end{tabular}

HAWKSBILL GREEK NEAR LURAY, VA.

Hawksbill Creek rises in the Blue Ridge in the southeastern part, of Page County, Va., and flows northward into South Fork of Shenandoah River about 4 miles north of Luray.

The gaging station was established June 27, 1905, by N. C. Grover, in connection with the investigation of stream pollution in the Shenandoah River valley. It was discontinued July 16, 1906. It is located a short distance above the mouth of Dry Run, $1 \frac{1}{2}$ miles north of Luray.

The channel is straight for 500 feet above and 200 feet below the station. The current is moderate above and swift below the station. Rapids below the gage prevent backwater influence from Dry Run, except in case of extreme floods. From well-defined marks the highest stage known was found to be 19.55 feet above the zero of the gage. This stage occurred October 13, 1893. The right bank is high, rocky, and wooded, and does not overflow. The left bank is low and subject to overflow during high water. The bed of the stream is composed of gravel, is free from vegetation, and is permanent. There is but one channel at all stages. The approximate depth of the water at the bridge is 2 to 3 feet.

Discharge measurements were made from the footbridge in front of the observer's house. The initial point for soundings is the edge of rock at the right end of the bridge.

A staff gage in two sections, the lower one inclined and the upper vertical, graduated to feet and tenths, is fastened to the left bank 100 feet above the footbridge. The gage was read once each day by J. S. Miller. Bench mark No. 2 is the top of the stone under the vertical post on the upstream side of door frame in the old dairy building, 200 feet from the left end of the footbridge. Its elevation is 15.10 feet above the zero of the gage. 
Estimates at this station are within 5 per cent of the true flow for normal conditions between gage heights 1.4 and 2.0 feet. Above and below these stages the probable error is 10 per cent. The flow was probably unaffected by ice conditions during the winter of 1905-6.

Discharge measurements of Hawksbill Creek near Luray, Va.

\begin{tabular}{|c|c|c|c|c|c|}
\hline Date. & $\begin{array}{c}\text { Gage } \\
\text { height. }\end{array}$ & Discharge. & Date. & $\begin{array}{c}\text { Gage } \\
\text { height. }\end{array}$ & Discharge. \\
\hline $\begin{array}{l}1905 . \\
\text { May } 21 \ldots \ldots \ldots \ldots \ldots \ldots \ldots \ldots \\
\text { June } 27 \ldots \ldots \ldots \ldots \ldots \ldots \ldots \ldots \\
\text { December } 2 \ldots_{\ldots} \ldots \ldots \ldots \ldots \ldots\end{array}$ & $\begin{array}{l}\text { Feet. } \\
1.44 \\
1.74 \\
1.81\end{array}$ & $\mid \begin{array}{r}\text { Second-feet. } \\
35 \\
64 \\
72\end{array}$ & $\begin{array}{c}1906 . \\
\text { April } 12 \ldots \ldots \ldots \ldots \ldots \ldots \ldots \\
\text { June } 15 a_{\ldots} \ldots \ldots \ldots \ldots \ldots\end{array}$ & $\begin{array}{l}\text { Feet. } \\
1.86 \\
1.50\end{array}$ & $\begin{array}{r}\text { Second-feet. } \\
75 \\
36\end{array}$ \\
\hline
\end{tabular}

a Measurement made by wading below footbridge.

Daily gage height, in feet, of Hawksbill Creek near Luray, Va.

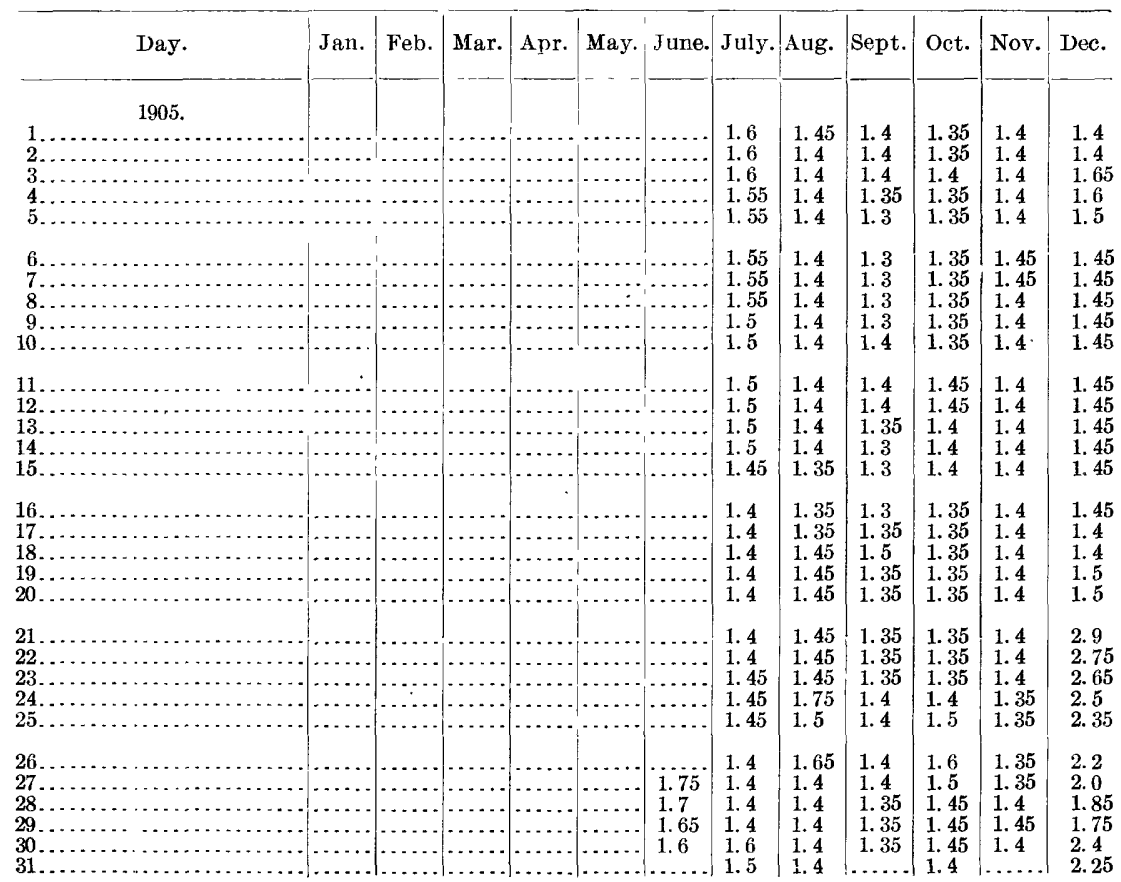


Daily gage height, in feet, of Hawksbill Cretk near Luray, Va.-Continued.

\begin{tabular}{|c|c|c|c|c|c|c|c|c|c|c|c|c|}
\hline Day. & Jan. & Feb. & Mar. & Apr. & May. & June. & July. & Aug. & Sept. & Oct. & Nov. & Dec. \\
\hline 1906 & & & & & & & & & & & & \\
\hline . & 2.0 & 1.9 & 1. 55 & 2.3 & 1.9 & 1. 6 & 1. 6 & .... & $\ldots$ & $-\theta_{0}$ & ... & $\ldots .$. \\
\hline$\ldots \ldots \ldots \ldots \ldots$ & 1.9 & 1.9 & 1. 55 & 2.2 & 1.85 & 1.6 & 1.55 & $\ldots .$. & $\ldots$ & - & $\cdots$ & $\ldots \ldots$ \\
\hline$\ldots \ldots \ldots \ldots \ldots \ldots \ldots$ & 3.3 & 1.9 & 1.8 & 2.1 & 1.85 & 1. 55 & 1.55 & $\ldots \ldots$ & $\cdots$ & . & $\ldots .$. & $\ldots .$. \\
\hline . & 2.7 & 1.85 & 2.0 & 2.05 & 1.85 & 1. 55 & 1.55 & (...... & $\cdots$ & & 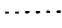 & $\ldots . .$. \\
\hline $5 \ldots \ldots \ldots \ldots \ldots \ldots \ldots \ldots$ & 2.45 & 1.85 & & & & & & - $\cdots$ & $\cdots$ & & $\cdots$ & $\cdots \cdots$ \\
\hline 6. & 2.25 & 1. 75 & 1.85 & 1.95 & 1. 75 & 1. 5 & 1.5 & |- & & & & $\ldots$ \\
\hline 7. & 2.2 & 1.7 & 1.85 & 1.95 & 1.75 & 1.5 & 1.5 & $\ldots$ & $\ldots$ & . & $\cdots$ & $\because$ \\
\hline 8. & 2.1 & 1. 7 & 1.85 & 1.9 & 1.75 & 1.5 & 1.5 & $\ldots .$. & $\ldots$. & . & & \\
\hline 9 & 2.0 & 1. 7 & 1.8 & 1.9 & 1.75 & 1.5 & 1.5 & $\ldots \ldots$ & $\cdots$ & & & \\
\hline 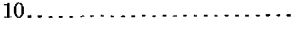 & 2.05 & 1.65 & 1.8 & 1.95 & 1.75 & 2.0 & 1.5 & & & & & - \\
\hline $11 \ldots$ & 2.0 & 1.65 & 1.75 & 1.9 & 1. 75 & 1. 8 & 1.8 & & & & & $\ldots$ \\
\hline 12. & 1.9 & 1.65 & 1. 7 & 1.9 & 1.7 & 1.6 & 1.6 & & & & $\ldots$ & $\ldots$ \\
\hline . & 1.8 & 1.65 & 1. 7 & 1.8 & 1. 7 & 1.55 & 1.55 & & & & & $\cdots$ \\
\hline 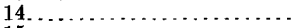 & 1.8 & 1.7 & 1.7 & 1.8 & 1.65 & 1.55 & 1.55 & - & & & & 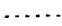 \\
\hline 15 . & 1.8 & 1.65 & 1. 75 & a3. 35 & 1.65 & 2.65 & 1.55 & - & & & $\cdots$ & \\
\hline 16. & 1.8 & 1. 6 & 1.8 & 3.05 & 1.65 & 1.95 & & & & & & $\ldots$ \\
\hline 17. & 1.75 & 1. 6 & 1.75 & 2. 55 & 1. 65 & 2.5 & $\ldots$ & & & & $\cdots$ & $\ldots \ldots$ \\
\hline 18. & 1.75 & 1. 6 & 1.75 & 2.35 & 1. 6 & 1.8 & $\ldots$ & $\cdots$ & - & & $\ldots .$. & \\
\hline 19. & 1. 75 & 1.55 & 1.85 & 2.25 & 1.6 & 1.6 & & & $\ldots$ & $\ldots$. & $\ldots . .$. & $\cdots$ \\
\hline $20 \ldots \ldots$ & 1. 75 & 1.55 & 1.9 & 2.2 & 1.6 & 1.6 & $\ldots .$. & & $\cdots$ & & $\cdots$ & $\cdots$ \\
\hline 21. & 1.7 & 1. 6 & 1.9 & 2.1 & 1.6 & 2. 45 & & & & & & $\cdots$ \\
\hline & 1. 65 & 1.55 & 1.9 & 2.0 & 1.55 & 1.75 & & & & & & \\
\hline 23. & 1.75 & 1.55 & 1.9 & 1.85 & 1.5 & 1.7 & $\ldots \ldots$ & $\cdots$ & & & & $\cdots$ \\
\hline $24 \ldots \ldots \ldots \ldots$ & 1.75 & 1.5 & 1. 9 & 1.85 & 1.5 & 1.7 & $\ldots$ & & & & & $\ldots$ \\
\hline 25 . & 1.8 & 1.55 & 1.9 & 1.85 & 1.5 & 1.7 & - & & & & & $\cdots$ \\
\hline 26. & 1.8 & 1.55 & 1.9 & 2.1 & 1.5 & 1.8 & & & & & & $\cdots$ \\
\hline & 1. 9 & 1.55 & 2.15 & 2.05 & 1. 5 & 1.8 & & & & & & $\ldots \ldots$ \\
\hline 28. & 1. 9 & 1.55 & 2.4 & 2.0 & 1. 6 & 1.75 & & & & &. & $\cdots \cdots$ \\
\hline & 1. 9 & $\ldots$. & 2.35 & 1. 9 & 1. 6 & 1. 7 & & & & & $\cdots$ & $\cdots$ \\
\hline & 1.9 & & 2.3 & 1.9 & 1.55 & 1.65 & & & & & $\cdots \cdots$ & $\cdots \cdots$ \\
\hline & 1.9 & & 2.4 & $\ldots$ & 1.5 & $\cdots \cdots$ & & & & & & $\cdots \cdots$ \\
\hline
\end{tabular}

$a$ Gage height estimated Apr. 15, 1906.

Rating table for Hawksbill Creek near Luray, Va., from June 27,1905 , to July 15, 1906.a

\begin{tabular}{|c|r|r|r|r||r|r|r|}
\hline $\begin{array}{c}\text { Gage } \\
\text { height. }\end{array}$ & Discharge. & $\begin{array}{c}\text { Gage } \\
\text { height. }\end{array}$ & Discharge. & $\begin{array}{c}\text { Gage } \\
\text { height. }\end{array}$ & Discharge. & $\begin{array}{c}\text { Gage } \\
\text { height. }\end{array}$ & Discharge. \\
\cline { 3 - 6 } & & & & & & \\
Feet. & Second-feet. & Feet. & Second-feet. & Feet. & Second-feet. & Feet. & Second-feet. \\
1.30 & 24 & 1.90 & 83 & 2.50 & 196 & 3.00 & 326 \\
1.40 & 30 & 2.00 & 98 & 2.60 & 220 & 3.10 & 355 \\
1.50 & 37 & 2.10 & 115 & 2.70 & 245 & 3.20 & 384 \\
1.60 & 46 & 2.20 & 133 & 2.80 & 271 & 3.30 & 414 \\
1.70 & 57 & 2.30 & 152 & 2.90 & 298 & 3.40 & 445 \\
1.80 & 69 & 2.40 & 173 & & & & \\
\hline
\end{tabular}

$a$ This table is strictly applicable only for open-channel conditions. It is based on five discharge measurements made during 1505-6. It is fairly well defined between gage heights 1.4 feet and 2.0 feet. 
Estimated monthly discharge of Hawksbill Creek near Luray, Va.

[Drainage area, 52 square miles.]

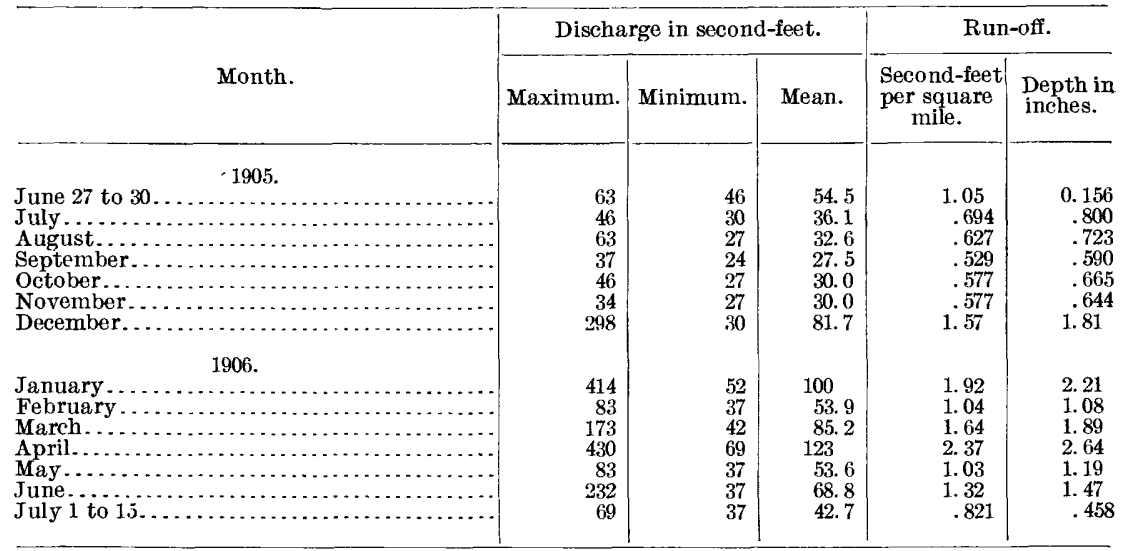

SOUTH FORK OF SHENANDOAH RIVER NEAR FRONT ROYAL, VA.

South Fork of Shenandoah River is formed at Port Republic, Rockingham County, Va., by the union of North and South rivers, and flows northeastward to Riverton, Va., where it unites with North Fork to form the main Shenandoah. Its length by river course is about 96 miles.

The gaging station at Front Royal was established June 26, 1899, by A. P. Davis, and was discontinued July 17, 1906. It is located about 1 mile above the bridge, which is near the Norfolk and Western Railway station.

The channel is straight for 600 feet above and below the station, and the current is sluggish. The railroad follows the right bank of the stream, and the railroad embankment, a few feet back from the river, is overflowed at extreme flood stages only. The bed of the stream is composed of bed rock and is very uneven; in places the rock is overlain by silt, and this is liable to shift.

Discharge measurements were made from a cable, which has a span of 300 feet and is suspended over the branches of two large sycamore trees, with its right end fastened to the tree and its left anchored in the ground. The imitial point for soundings is on the main cable 0.5 foot from the tree on the left bank.

The gage is a vertical timber spiked to a large sycamore tree on the left bank, about 800 feet upstream from the cable. A high-water gage, reading from 14 to 26 feet, was established September 18, 1905. It is a vertical board spiked to the shore side of a large sycamore tree, 325 feet upstream from the regular gage. The gage was read twice each day by Miss Brentie Johnson. The bench mark is a headless spike on the river side of an elm tree on the left bank, 8 feet downstream from the gage. It is 1.5 feet above the ground and has. an elevation of 10.49 feet above the zero of the gage. 
Estimates for this station are within 5 per cent of the true discharge for normal conditions of flow for gage heights below 9.0 feet. Above gage height 9.0 feet the probable error of the extension of the curve may be as much as 15 to 20 per cent. Estimates for the winter months are affected by ice conditions. All estimates published prior to 1905 have been revised.

A summary of the records furnishes the following results: Maximum discharge for twenty-four hours, 76,800 second-feet; minimum discharge for twenty-four hours, 305 second-feet; mean annual discharge for five years, 2,238 second-feet; mean annual rainfall for seven years, 37.45 inches.

Discharge measurements of South Fork of Shenandoah River near Front Royal, Va.

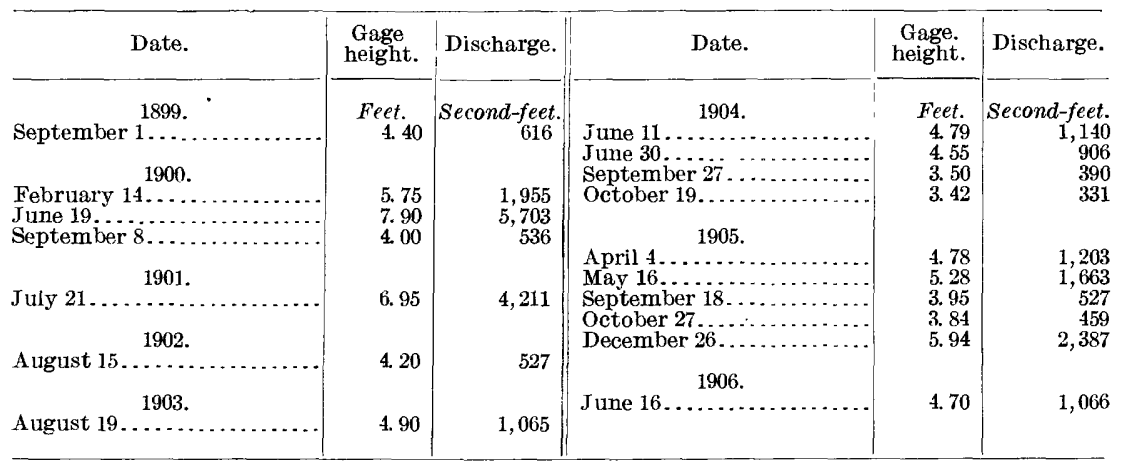

Dally gage height, in feet, of South Fork of Shenandoah Rivier near Front Royal, Va.

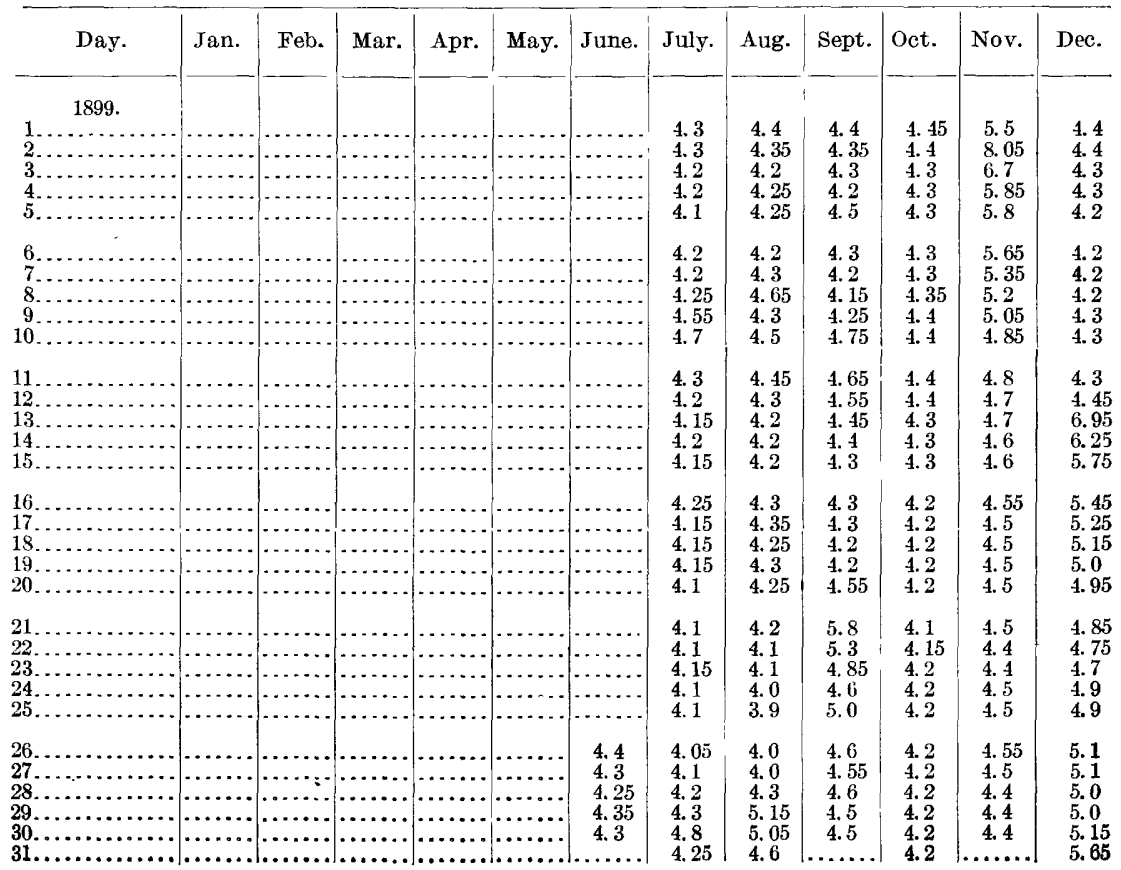


Daily gage height, in feet, of South Fork of Shenandoah River near Front Royal, Va.Continued.

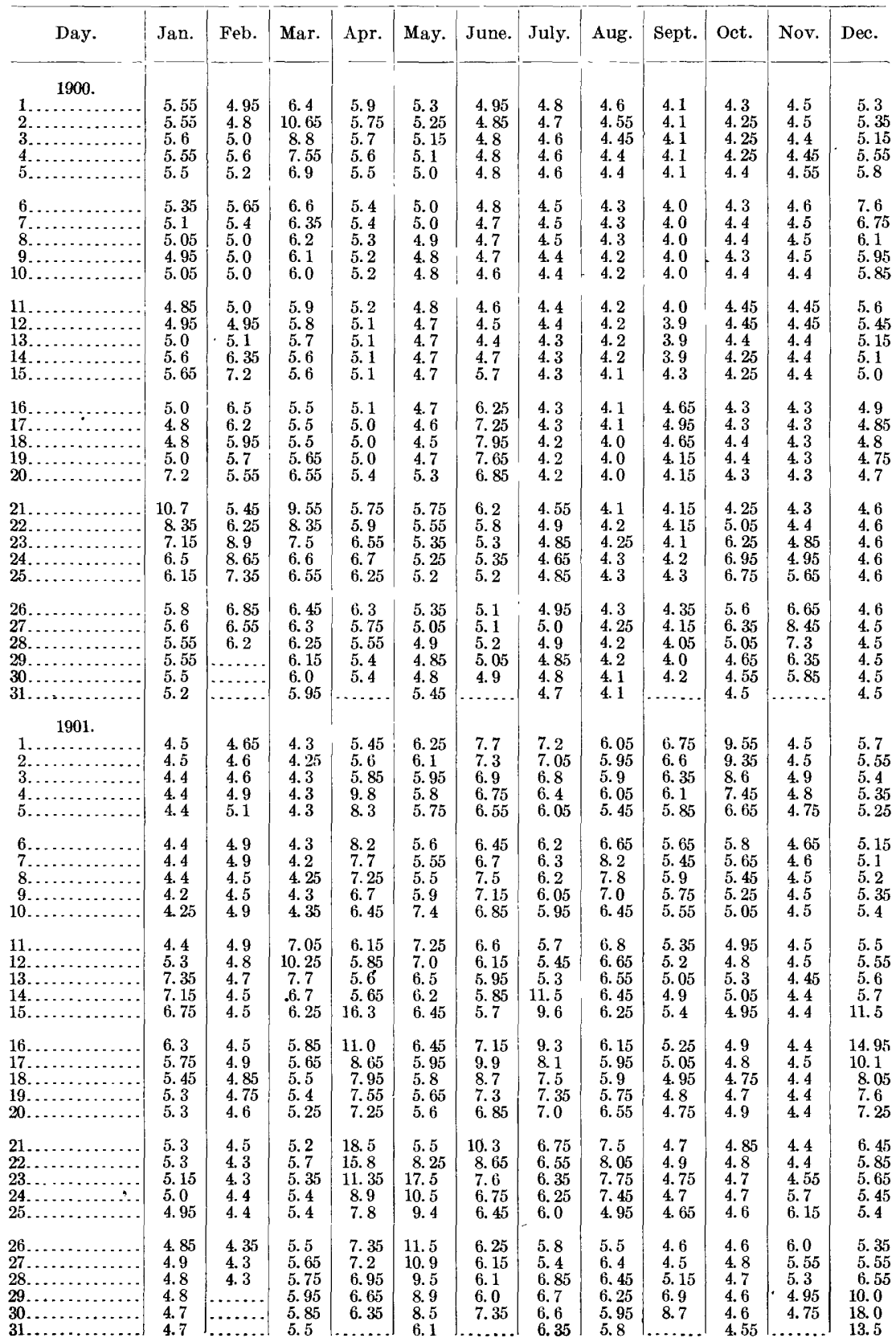


Daily gage height, in feet, of South Fork of Shenandoah River near Front Royal, Va.Continued.

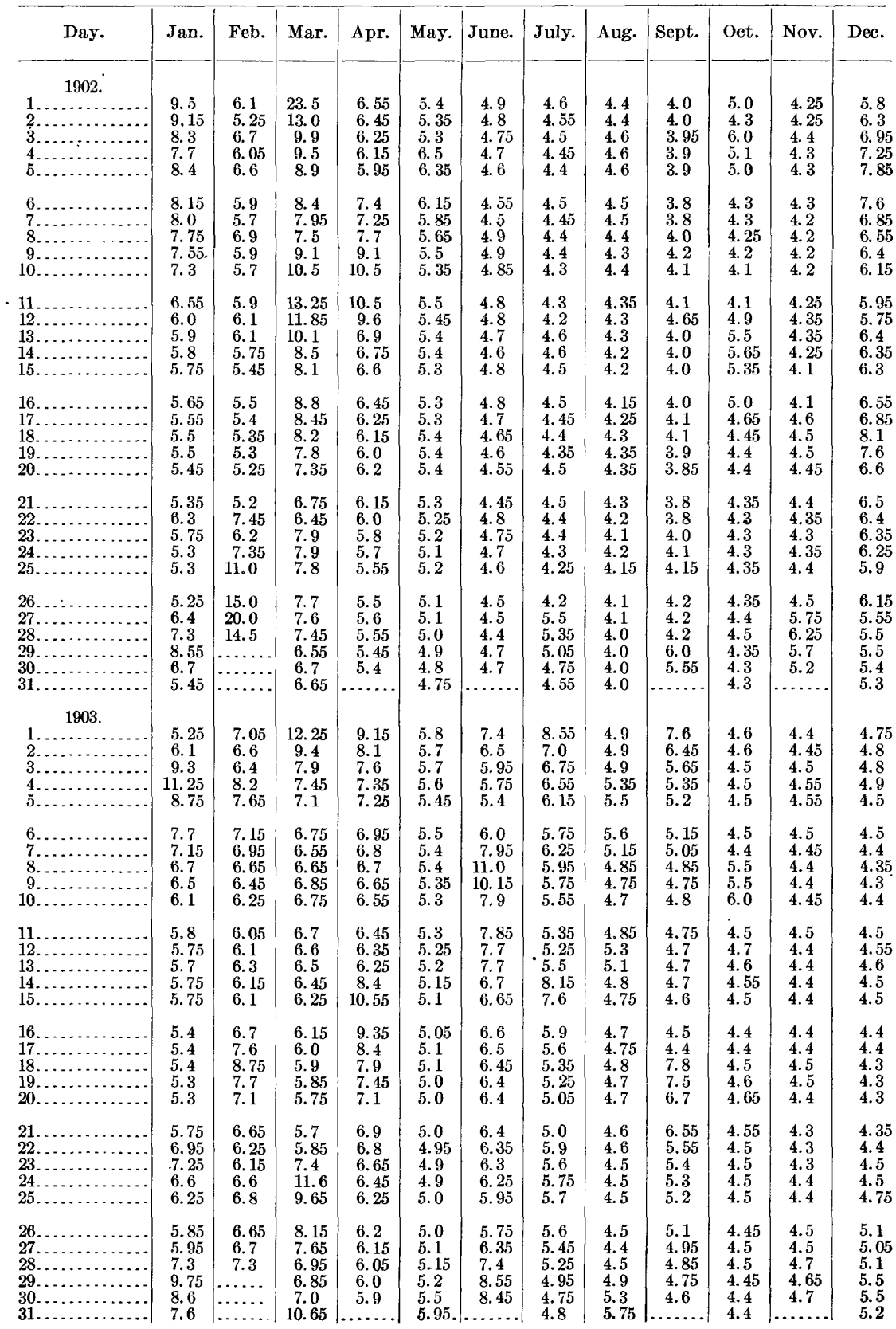


Daily gage height, in feet, of South Fork of Shenandoah River near Front Royal, Va.Continued.

\begin{tabular}{|c|c|c|c|c|c|c|c|c|c|c|c|c|}
\hline Day. & Jan. & Feb. & Mar. & Apr. & May. & June. & July. & Aug. & Sept. & Oct. & Nov. & Dec. \\
\hline $\begin{array}{l}1 \ldots \ldots \\
2 \ldots \\
3 \ldots \\
4 \ldots \\
5 \ldots\end{array}$ & $\begin{array}{l}4.95 \\
4.75 \\
4.75 \\
5.05 \\
5.05\end{array}$ & $\begin{array}{l}4.2 \\
4.1 \\
4.15 \\
4.25 \\
4.25\end{array}$ & $\begin{array}{l}4.85 \\
4.85 \\
5.05 \\
4.95 \\
5.05\end{array}$ & $\begin{array}{l}5.2 \\
5.05 \\
4.85 \\
4.7 \\
4.65\end{array}$ & $\begin{array}{l}6.4 \\
5.85 \\
5.45 \\
5.15 \\
5.1\end{array}$ & $\begin{array}{l}4.95 \\
5.05 \\
5.3 \\
6.2 \\
6.45\end{array}$ & $\begin{array}{l}4.5 \\
4.4 \\
4.3 \\
4.2 \\
4.1\end{array}$ & $\begin{array}{l}4.1 \\
4.05 \\
4.1 \\
4.3 \\
4.4\end{array}$ & $\begin{array}{l}3.6 \\
3.6 \\
3.6 \\
3.6 \\
3.6\end{array}$ & $\begin{array}{l}3.4 \\
3.4 \\
3.4 \\
3.4 \\
3.4\end{array}$ & $\begin{array}{l}3.42 \\
3.4 \\
3.4 \\
3.4 \\
3.42\end{array}$ & $\begin{array}{l}3.45 \\
3.4 \\
3.5 \\
3.5 \\
3.5\end{array}$ \\
\hline $\begin{array}{r}6 . . \\
7 . . \\
8 . . \\
9 . . \\
10 . .\end{array}$ & $\begin{array}{l}4.8 \\
4.7 \\
4.6 \\
4.55 \\
4.45\end{array}$ & $\begin{array}{l}4.0 \\
4.05 \\
4.85 \\
4.65 \\
4.85\end{array}$ & $\begin{array}{l}5.0 \\
5.05 \\
7.3 \\
7.4 \\
6.7\end{array}$ & $\begin{array}{l}4.6 \\
4.6 \\
4.6 \\
4.7 \\
5.25\end{array}$ & $\begin{array}{l}5.0 \\
5.0 \\
5.0 \\
5.15 \\
5.7\end{array}$ & $\begin{array}{l}6.5 \\
6.0 \\
5.4 \\
5.05 \\
4.95\end{array}$ & $\begin{array}{l}4.1 \\
4.05 \\
4.0 \\
4.2 \\
5.5\end{array}$ & $\begin{array}{l}4.5 \\
4.7 \\
4.45 \\
4.4 \\
4.75\end{array}$ & $\begin{array}{l}3.65 \\
3.7 \\
3.7 \\
3.7 \\
3.7\end{array}$ & $\begin{array}{l}3.4 \\
3.4 \\
3.4 \\
3.4 \\
3.4\end{array}$ & $\begin{array}{l}3.4 \\
3.42 \\
3.45 \\
3.5 \\
3.42\end{array}$ & $\begin{array}{l}3.45 \\
3.42 \\
3.45 \\
3.45 \\
3.52\end{array}$ \\
\hline $14 \ldots$ & $\begin{array}{l}4.4 \\
4.5 \\
4.55 \\
4.55 \\
4.4\end{array}$ & $\begin{array}{l}4.85 \\
4.7 \\
4.6 \\
4.5 \\
4.4\end{array}$ & $\begin{array}{l}6.0 \\
5.75 \\
5.55 \\
5.2 \\
5.1\end{array}$ & $\begin{array}{l}5.75 \\
5.7 \\
5.35 \\
5.15 \\
4.95\end{array}$ & $\begin{array}{l}5.85 \\
5.65 \\
5.35 \\
5.25 \\
4.9\end{array}$ & $\begin{array}{l}4.75 \\
4.7 \\
4.65 \\
4.55 \\
4.5\end{array}$ & $\begin{array}{l}7.95 \\
7.45 \\
5.4 \\
4.75 \\
4.55\end{array}$ & $\begin{array}{l}4.6 \\
4.5 \\
4.4 \\
4.2 \\
4.15\end{array}$ & $\begin{array}{l}3.7 \\
3.7 \\
3.7 \\
3.65 \\
4.15\end{array}$ & $\begin{array}{l}3.4 \\
3.45 \\
3.55 \\
3.45 \\
3.4\end{array}$ & $\begin{array}{l}3.4 \\
3.4 \\
3.4 \\
3.55 \\
3.5\end{array}$ & $\begin{array}{l}4.15 \\
4.4 \\
4.6 \\
4.8 \\
3.95\end{array}$ \\
\hline $\begin{array}{l}16 \ldots \\
17 \ldots \\
18 \ldots \\
19 \ldots \\
20 \ldots\end{array}$ & $\begin{array}{l}4.25 \\
4.25 \\
4.3 \\
4.4 \\
4.35\end{array}$ & $\begin{array}{l}4.4 \\
4.35 \\
4.4 \\
4.6 \\
4.75\end{array}$ & $\begin{array}{l}5.0 \\
4.9 \\
4.9 \\
4.9 \\
4.7\end{array}$ & $\begin{array}{l}4.85 \\
4.8 \\
4.65 \\
4.55 \\
4.5\end{array}$ & $\begin{array}{l}4.9 \\
4.9 \\
5.3 \\
5.7 \\
7.6\end{array}$ & $\begin{array}{l}4.4 \\
4.6 \\
4.65 \\
4.4 \\
4.55\end{array}$ & $\begin{array}{l}4.4 \\
4.35 \\
4.3 \\
4.35 \\
4.25\end{array}$ & $\begin{array}{l}4.1 \\
4.0 \\
4.0 \\
3.9 \\
3.8\end{array}$ & $\begin{array}{l}3.95 \\
3.7 \\
3.6 \\
3.6 \\
3.6\end{array}$ & $\begin{array}{l}3.4 \\
3.4 \\
3.4 \\
3.4 \\
3.45\end{array}$ & $\begin{array}{l}3.52 \\
3.5 \\
3.45 \\
3.45 \\
3.4\end{array}$ & $\begin{array}{l}4.1 \\
4.1 \\
4.2 \\
3.9 \\
3.9\end{array}$ \\
\hline $\begin{array}{l}21 \ldots \\
22 \ldots \\
23 . \\
24 \\
25 \ldots \\
\end{array}$ & $\begin{array}{r}4.35 \\
4.9 \\
6.75 \\
8.15 \\
b 5.75\end{array}$ & \begin{tabular}{|l}
4.85 \\
5.4 \\
6.9 \\
7.9 \\
$b 7.7$
\end{tabular} & $\begin{array}{l}4.7 \\
4.7 \\
4.95 \\
4.95 \\
4.95\end{array}$ & $\begin{array}{l}4.5 \\
4.4 \\
4.4 \\
4.3 \\
4.4\end{array}$ & $\begin{array}{l}6.75 \\
5.9 \\
5.45 \\
5.25 \\
5.1\end{array}$ & $\begin{array}{l}5.0 \\
5.15 \\
5.05 \\
4.75 \\
4.4\end{array}$ & $\begin{array}{l}4.1 \\
4.1 \\
4.0 \\
4.3 \\
4.4\end{array}$ & $\begin{array}{l}3.8 \\
3.75 \\
3.65 \\
3.6 \\
3.6\end{array}$ & $\begin{array}{l}3.5 \\
3.5 \\
3.5 \\
3.5 \\
3.5\end{array}$ & $\begin{array}{l}3.55 \\
3.5 \\
3.45 \\
3.4 \\
3.4\end{array}$ & $\begin{array}{l}3.42 \\
3.4 \\
3.42 \\
3.42 \\
3.42\end{array}$ & $\begin{array}{l}3.7 \\
3.7 \\
3.88 \\
3.95 \\
3.9\end{array}$ \\
\hline $\begin{array}{l}26 \ldots \\
27 \ldots \\
28 \ldots \\
29 \ldots \\
30 \ldots \\
31 \ldots \\
\end{array}$ & $\begin{array}{l}5.05 \\
4.8 \\
4.65 \\
4.45 \\
4.35 \\
4.25\end{array}$ & $\begin{array}{l}7.15 \\
6.35 \\
5.75 \\
5.2 \\
\ldots \ldots\end{array}$ & $\begin{array}{l}5.05 \\
5.1 \\
5.1 \\
5.0 \\
5.25 \\
5.3\end{array}$ & $\begin{array}{l}4.45 \\
5.5 \\
6.25 \\
6.8 \\
6.65\end{array}$ & $\begin{array}{l}5.0 \\
4.95 \\
4.9 \\
4.9 \\
4.8 \\
4.8\end{array}$ & $\begin{array}{l}4.3 \\
4.35 \\
4.4 \\
4.7 \\
4.5\end{array}$ & $\begin{array}{l}4.3 \\
4.2 \\
4.35 \\
4.15 \\
4.2 \\
4.2\end{array}$ & $\begin{array}{l}3.55 \\
3.5 \\
3.5 \\
3.5 \\
3.55 \\
3.6\end{array}$ & $\begin{array}{l}3.5 \\
3.4 \\
3.4 \\
3.4 \\
3.4\end{array}$ & $\begin{array}{l}3.4 \\
3.42 \\
3.4 \\
3.4 \\
3.4 \\
3.4\end{array}$ & $\begin{array}{l}3.4 \\
3.4 \\
3.4 \\
3.4 \\
3.4\end{array}$ & $\begin{array}{l}3.8 \\
3.95 \\
3.9 \\
4.5 \\
4.2 \\
4.0\end{array}$ \\
\hline $\begin{array}{l}\quad 19 \\
1 \ldots \ldots \\
2 \ldots \ldots \\
3 \ldots \ldots \\
4 \ldots \ldots \\
5 \ldots \ldots\end{array}$ & $\begin{array}{l}3.7 \\
3.85 \\
3.95 \\
4.0 \\
4.0\end{array}$ & $\begin{array}{l}4.15 \\
4.05 \\
4.0 \\
4.15 \\
4.35\end{array}$ & 6.15 & $\begin{array}{l}5.4 \\
5.4 \\
5.28 \\
5.2 \\
4.75\end{array}$ & $\begin{array}{l}4.3 \\
4.2 \\
4.2 \\
4.0 \\
4.2\end{array}$ & $\begin{array}{l}4.35 \\
4.25 \\
4.15 \\
4.3 \\
4.25\end{array}$ & $\begin{array}{l}4.85 \\
4.7 \\
4.55 \\
4.5 \\
6.25\end{array}$ & $\begin{array}{l}4.85 \\
4.7 \\
4.6 \\
4.5 \\
4.4\end{array}$ & $\begin{array}{l}4.3 \\
4.38 \\
4.85 \\
4.65 \\
4.5\end{array}$ & $\begin{array}{l}3.7 \\
3.7 \\
3.7 \\
3.6 \\
3.6\end{array}$ & $\begin{array}{l}3.82 \\
3.7 \\
3.8 \\
3.78 \\
3.75\end{array}$ & $\begin{array}{l}3.7 \\
3.8 \\
3.8 \\
3.75 \\
3.8\end{array}$ \\
\hline $\begin{array}{r}6 \ldots . \\
7 \ldots \ldots \\
8 \ldots \\
9 \ldots \\
10 \ldots\end{array}$ & $\begin{array}{l}4.0 \\
4.6 \\
5.15 \\
5.2 \\
4.6\end{array}$ & $\begin{array}{l}4.25 \\
4.2 \\
4.3 \\
4.3 \\
4.5\end{array}$ & $\begin{array}{l}5.85 \\
5.4 \\
5.05 \\
5.5 \\
6.65\end{array}$ & $\begin{array}{l}5.15 \\
5.3 \\
5.15 \\
5.1 \\
5.1\end{array}$ & $\begin{array}{l}4.15 \\
4.2 \\
4.1 \\
4.05 \\
4.0\end{array}$ & $\begin{array}{l}4.2 \\
4.1 \\
4.1 \\
4.0 \\
4.0\end{array}$ & $\begin{array}{l}5.7 \\
5.35 \\
5.05 \\
4.95 \\
4.8\end{array}$ & $\begin{array}{l}4.4 \\
4.35 \\
4.25 \\
4.2 \\
4.1\end{array}$ & $\begin{array}{l}4.4 \\
4.35 \\
4.3 \\
4.15 \\
4.0\end{array}$ & $\begin{array}{l}3.6 \\
3.55 \\
3.6 \\
3.6 \\
3.6\end{array}$ & $\begin{array}{l}3.8 \\
3.75 \\
3.7 \\
3.72 \\
3.72\end{array}$ & $\begin{array}{l}3.82 \\
3.88 \\
4.25 \\
4.18 \\
4.05\end{array}$ \\
\hline $\begin{array}{l}11 . . \\
12 . \\
13 . \\
14 . \\
15 .\end{array}$ & $\begin{array}{l}4.45 \\
4.85 \\
4.85 \\
5.1 \\
5.55\end{array}$ & $\begin{array}{l}4.45 \\
4.4 \\
4.35 \\
4.4 \\
4.45\end{array}$ & $\begin{array}{l}8.25 \\
7.4 \\
6.9 \\
6.52 \\
6.15\end{array}$ & $\begin{array}{l}5.0 \\
4.8 \\
4.9 \\
4.8 \\
4.8\end{array}$ & $\begin{array}{l}4.05 \\
4.15 \\
4.0 \\
5.45 \\
5.2\end{array}$ & $\begin{array}{l}4.0 \\
4.0 \\
4.0 \\
3.9 \\
3.9\end{array}$ & $\begin{array}{l}4.65 \\
5.15 \\
6.2 \\
7.45 \\
6.8\end{array}$ & $\begin{array}{l}4.1 \\
4.0 \\
4.25 \\
4.4 \\
5.25\end{array}$ & $\begin{array}{l}3.9 \\
3.88 \\
3.8 \\
3.9 \\
4.0\end{array}$ & $\begin{array}{l}3.6 \\
3.65 \\
3.6 \\
3.7 \\
3.72\end{array}$ & $\begin{array}{l}3.7 \\
3.8 \\
3.72 \\
3.7 \\
3.6\end{array}$ & $\begin{array}{l}4.0 \\
4.12 \\
4.05 \\
3.95 \\
4.05\end{array}$ \\
\hline $\begin{array}{l}16 \ldots \\
17 \ldots \ldots \\
18 \ldots \ldots \\
19 \ldots \ldots \\
20 \ldots\end{array}$ & $\begin{array}{l}5.2 \\
5.05 \\
4.9 \\
4.9 \\
-4.45\end{array}$ & $\begin{array}{l}4.35 \\
4.35 \\
4.4 \\
4.3 \\
4.3\end{array}$ & $\begin{array}{l}6.0 \\
5.85 \\
5.8 \\
5.8 \\
5.75\end{array}$ & $\begin{array}{l}4.7 \\
4.7 \\
4.6 \\
4.5 \\
4.4\end{array}$ & $\begin{array}{l}5.28 \\
5.55 \\
5.55 \\
5.2 \\
4.45\end{array}$ & $\begin{array}{l}3.8 \\
3.8 \\
3.8 \\
3.8 \\
4.2\end{array}$ & $\begin{array}{l}7.6 \\
6.45 \\
5.7 \\
5.25 \\
5.0\end{array}$ & $\begin{array}{l}6.2 \\
6.28 \\
5.65 \\
5.1 \\
4.78\end{array}$ & $\begin{array}{l}3.95 \\
3.9 \\
3.9 \\
3.9 \\
3.9\end{array}$ & $\begin{array}{l}3.8 \\
3.7 \\
3.7 \\
3.75 \\
3.75\end{array}$ & $\begin{array}{l}3.65 \\
3.58 \\
3.65 \\
3.7 \\
3.7\end{array}$ & $\begin{array}{l}4.15 \\
4.15 \\
4.25 \\
4.12 \\
4.0\end{array}$ \\
\hline $\begin{array}{l}21 \ldots \\
22 \ldots \\
23 \ldots \\
24 \ldots \\
25 \ldots\end{array}$ & $\begin{array}{l}4.32 \\
4.22 \\
4.28 \\
4.1 \\
3.95\end{array}$ & $\begin{array}{l}4.4 \\
4.4 \\
4.75 \\
4.95 \\
5.7\end{array}$ & $\begin{array}{l}5.95 \\
7.0 \\
7.6 \\
6.9 \\
6.75\end{array}$ & $\begin{array}{l}4.35 \\
4.3 \\
4.3 \\
4.3 \\
4.42\end{array}$ & $\begin{array}{l}4.65 \\
4.45 \\
4.4 \\
4.35 \\
4.3\end{array}$ & $\begin{array}{l}5.25 \\
4.95 \\
5.05 \\
6.2 \\
9.35\end{array}$ & $\begin{array}{l}4.85 \\
4.6 \\
5.8 \\
6.9 \\
6.55\end{array}$ & $\begin{array}{l}4.45 \\
4.3 \\
4.25 \\
4.2 \\
4.15\end{array}$ & $\begin{array}{l}3.9 \\
3.8 \\
3.82 \\
3.82 \\
3.75\end{array}$ & $\begin{array}{l}3.72 \\
3.72 \\
3.6 \\
3.5 \\
3.6\end{array}$ & $\begin{array}{l}3.75 \\
3.68 \\
3.68 \\
3.62 \\
3.6\end{array}$ & $\begin{array}{l}5.5 \\
8.32 \\
8.0 \\
6.75 \\
6.4\end{array}$ \\
\hline $\begin{array}{l}26 \ldots \ldots \\
27 \ldots \\
28 \ldots \\
29 \ldots \\
30 \ldots \\
31 \ldots\end{array}$ & $\begin{array}{l}4.15 \\
4.15 \\
4.1 \\
4.3 \\
4.25 \\
4.05\end{array}$ & $\begin{array}{l}6.45 \\
8.0\end{array}$ & $\begin{array}{l}5.55 \\
6.35 \\
6.25 \\
5.95 \\
5.65 \\
5.55\end{array}$ & $\begin{array}{l}4.4 \\
4.5 \\
4.45 \\
4.3 \\
4.3\end{array}$ & $\begin{array}{l}4.25 \\
4.2 \\
4.3 \\
4.15 \\
4.55 \\
4.55\end{array}$ & $\begin{array}{l}7.3 \\
6.1 \\
5.65 \\
5.25 \\
4.9\end{array}$ & $\begin{array}{l}6.35 \\
6.2 \\
6.05 \\
5.5 \\
5.15 \\
5.0\end{array}$ & $\begin{array}{l}4.1 \\
4.1 \\
4.3 \\
4.4 \\
4.35 \\
4.3\end{array}$ & $\begin{array}{l}3.7 \\
3.7 \\
3.78 \\
3.8 \\
3.68 \\
\cdots . .\end{array}$ & $\begin{array}{l}3.65 \\
3.78 \\
3.82 \\
3.8 \\
3.8 \\
3.82\end{array}$ & $\begin{array}{l}3.65 \\
3.7 \\
3.7 \\
3.65 \\
3.62\end{array}$ & $\begin{array}{l}5.95 \\
5.55 \\
5.25 \\
5.25 \\
5.45 \\
5.85\end{array}$ \\
\hline
\end{tabular}

$a$ Ice conditions during January (ice about 8 inches thick) and December (ice about 3 inches thick), 1904.

$b$ Gage heights January 25 to February 25, 1904, approximate. Gage not in correct position.

$c$ Ice conditions during January and February, 1905 
Daily gage height, in feet, of South Fork of Shenandoah River near Front Royal, Va.Continued.

\begin{tabular}{|c|c|c|c|c|c|c|c|c|c|c|c|c|}
\hline Day. & Jan. & Feb. & Mar. & Apr. & May. & June. & July. & Aug. & Sept. & Oet. & Nov. & Dec. \\
\hline $\begin{array}{c}1906 . \\
1 \ldots \ldots \\
2 \ldots \ldots \ldots \\
3 \ldots \ldots \ldots \\
4 \ldots \ldots \\
5 \ldots \ldots \ldots\end{array}$ & $\begin{array}{l}5.65 \\
5.45 \\
5.25 \\
6.13 \\
8.47\end{array}$ & $\begin{array}{l}5.43 \\
5.3 \\
5.2 \\
5.1 \\
4.85\end{array}$ & $\begin{array}{l}4.2 \\
4.17 \\
4.23 \\
5.05 \\
7.02\end{array}$ & $\begin{array}{l}7.05 \\
6.8 \\
6.45 \\
6.25 \\
5.85\end{array}$ & $\begin{array}{l}5.22 \\
5.1 \\
5.0 \\
4.95 \\
4.9\end{array}$ & $\begin{array}{l}4.7 \\
4.62 \\
4.5 \\
4.42 \\
4.38\end{array}$ & $\begin{array}{l}5.45 \\
5.28 \\
5.25 \\
5.25 \\
4.9\end{array}$ & $\left.\mid \begin{array}{l}\ldots \ldots \\
\ldots \\
\ldots\end{array}\right]$ & $\left.\mid \begin{array}{c}\cdots \\
\cdots \\
\cdots \\
\cdots\end{array}\right]$ & $\begin{array}{c}\ldots \ldots \\
\ldots \\
\ldots \\
\ldots \\
\ldots \\
\ldots\end{array}$ & $\mid \begin{array}{l}\ldots \ldots \\
\ldots \ldots \\
\ldots \ldots \\
\ldots \ldots \\
\ldots \ldots\end{array}$ & $\begin{array}{l}\ldots \ldots \\
\ldots \\
\ldots \\
\cdots \\
\cdots \\
\cdots \\
\cdots\end{array}$ \\
\hline $\begin{array}{r}6 \ldots \ldots \ldots \\
7 \ldots \ldots \ldots \\
8 \ldots \ldots \ldots \\
9 \ldots \ldots \ldots \ldots \ldots \\
10 \ldots \ldots \ldots \ldots\end{array}$ & $\begin{array}{l}7.22 \\
6.4 \\
6.0 \\
5.65 \\
5.38\end{array}$ & $\begin{array}{l}4.82 \\
4.9 \\
4.73 \\
4.65 \\
4.5\end{array}$ & $\begin{array}{l}6.33 \\
5.8 \\
5.47 \\
5.15 \\
5.15\end{array}$ & $\begin{array}{l}5.68 \\
5.52 \\
5.4 \\
5.32 \\
5.3\end{array}$ & $\begin{array}{l}4.85 \\
4.88 \\
5.0 \\
5.1 \\
5.05\end{array}$ & $\begin{array}{l}4.3 \\
4.22 \\
4.2 \\
4.18 \\
4.1\end{array}$ & $\begin{array}{l}4.45 \\
4.65 \\
4.62 \\
4.4 \\
4.28\end{array}$ & $\begin{array}{l}\ldots \ldots \\
\cdots \\
\cdots \\
\cdots \\
\cdots\end{array}$ & $\begin{array}{l}\cdots \\
\cdots \\
\cdots \\
\cdots\end{array}$ & $\begin{array}{l}\cdots \\
\cdots \\
\cdots \\
\cdots\end{array}$ & $\begin{array}{c}\ldots \\
\ldots \\
\cdots \\
\cdots\end{array}$ & $\left.\begin{array}{l}\cdots \\
\cdots \\
\cdots\end{array}\right]$ \\
\hline $\begin{array}{l}11 \ldots \ldots \\
12 \ldots \ldots \\
13 \ldots \ldots \\
14 \ldots \ldots \\
15 \ldots \ldots\end{array}$ & $\begin{array}{l}5.17 \\
5.0 \\
5.0 \\
5.0 \\
5.05\end{array}$ & $\begin{array}{l}4.52 \\
4.45 \\
4.4 \\
4.4 \\
4.43\end{array}$ & $\begin{array}{l}5.05 \\
4.9 \\
4.83 \\
4.7 \\
4.8\end{array}$ & $\begin{array}{l}5.4 \\
5.35 \\
5.28 \\
5.2 \\
6.35\end{array}$ & $\begin{array}{l}5.0 \\
4.9 \\
4.8 \\
4.78 \\
4.6\end{array}$ & $\begin{array}{l}4.1 \\
4.15 \\
4.22 \\
4.2 \\
4.4\end{array}$ & $\begin{array}{l}4.3 \\
4.38 \\
5.32 \\
4.65 \\
4.4\end{array}$ & $\begin{array}{l}\cdots \\
\cdots \\
\cdots \\
\cdots \\
\cdots \\
\cdots\end{array}$ & $\begin{array}{c}\cdots \\
\cdots \\
\cdots\end{array}$ & $\left\{\begin{array}{l}\cdots \\
\ldots \\
\ldots \\
\ldots \\
\ldots \\
\cdots \\
\cdots\end{array}\right.$ & & $\mid \begin{array}{l}\ldots \ldots \\
\ldots \ldots \\
\ldots \\
\ldots\end{array}$ \\
\hline $\begin{array}{l}16 \ldots \ldots \\
17 \ldots \ldots \\
18 \ldots \ldots \\
19 \ldots \ldots \\
20 \ldots \ldots\end{array}$ & $\begin{array}{l}5.3 \\
5.3 \\
5.3 \\
5.2 \\
5.12\end{array}$ & $\begin{array}{l}4.32 \\
4.4 \\
4.33 \\
4.3 \\
4.3\end{array}$ & $\begin{array}{l}5.25 \\
5.95 \\
5.77 \\
5.8 \\
5.75\end{array}$ & $\begin{array}{l}7.75 \\
6.8 \\
6.38 \\
6.08 \\
5.8\end{array}$ & $\begin{array}{l}4.62 \\
4.52 \\
4.55 \\
4.45 \\
4.4\end{array}$ & $\begin{array}{l}4.65 \\
5.45 \\
5.75 \\
5.35 \\
5.35\end{array}$ & 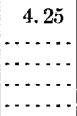 & $\left.\begin{array}{l}\cdots \\
\cdots \\
\cdots \\
\cdots \\
\ldots \ldots\end{array}\right]$ & $\left.\mid \begin{array}{l}\cdots \\
\cdots \\
\cdots \\
\cdots\end{array}\right]$ & & $\begin{array}{c}\ldots \ldots \\
\ldots \\
\cdots \\
\ldots \ldots \\
\ldots \ldots\end{array}$ & $\left.\begin{array}{l}\ldots \ldots \\
\cdots \\
\cdots\end{array}\right]$ \\
\hline $\begin{array}{l}21 \ldots \ldots \\
22 \\
23 \ldots \\
24 \ldots \\
25 \ldots \ldots\end{array}$ & $\begin{array}{l}5.0 \\
4.9 \\
4.93 \\
6.8 \\
6.85\end{array}$ & $\begin{array}{l}4.3 \\
4.25 \\
4.22 \\
4.28 \\
4.25\end{array}$ & $\begin{array}{l}5.8 \\
6.1 \\
5.9 \\
5.8 \\
5.6\end{array}$ & $\begin{array}{l}5.55 \\
5.45 \\
5.35 \\
5.25 \\
5.05\end{array}$ & $\begin{array}{l}4.35 \\
4.3 \\
4.28 \\
4.2 \\
4.2\end{array}$ & $\begin{array}{l}6.2 \\
6.9 \\
6.05 \\
5.5 \\
5.35\end{array}$ & & & & & & $\left.\begin{array}{|c|}\cdots \\
\cdots \\
\cdots \\
\cdots\end{array}\right]$ \\
\hline $\begin{array}{l}26 \ldots \\
27 \ldots \\
28 \ldots \\
29 \ldots \\
30 \ldots \\
31 \ldots\end{array}$ & $\begin{array}{l}6.22 \\
5.75 \\
5.63 \\
5.6 \\
5.55 \\
5.55\end{array}$ & $\begin{array}{r}4.25 \\
4.12 \\
4.25 \\
. . .\end{array}$ & $\begin{array}{l}5.58 \\
5.77 \\
6.75 \\
7.35 \\
7.1 \\
6.9\end{array}$ & $\begin{array}{l}5.1 \\
5.35 \\
5.45 \\
5.4 \\
5.3 \\
. . . .\end{array}$ & $\begin{array}{l}4.2 \\
4.22 \\
4.3 \\
4.65 \\
4.4 \\
4.72\end{array}$ & $\begin{aligned} 5.28 \\
5.72 \\
6.15 \\
5.5 \\
5.35 \\
. . .\end{aligned}$ & 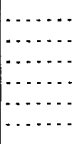 & & …… & $\cdots$ & $\ldots \ldots$ & 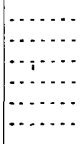 \\
\hline
\end{tabular}

Rating tables for South Fork of Shenandoah River near Front Royal, Va.

JUNE 26, 1899, TO DECEMBER 31, 1903, a

\begin{tabular}{|c|c|c|c|c|c|c|c|}
\hline $\begin{array}{c}\text { Gage } \\
\text { height. }\end{array}$ & Discharge. & $\begin{array}{c}\text { Gage } \\
\text { height. }\end{array}$ & Discharge. & $\begin{array}{l}\text { Gage } \\
\text { height. }\end{array}$ & Discharge. & $\begin{array}{c}\text { Gage } \\
\text { height. }\end{array}$ & Discharge. \\
\hline $\begin{array}{r}\text { Feet. } \\
3.80 \\
3.90 \\
4.00 \\
4.10 \\
4.20 \\
4.30 \\
4.40 \\
4.50 \\
4.60 \\
4.70 \\
4.80 \\
4.90 \\
5.00\end{array}$ & \begin{tabular}{|r} 
Second-feet \\
305 \\
350 \\
400 \\
455 \\
515 \\
580 \\
650 \\
720 \\
795 \\
870 \\
950 \\
1,030 \\
1,120
\end{tabular} & $\begin{array}{c}\text { Feet. } \\
5.10 \\
5.20 \\
5.30 \\
5.40 \\
5.50 \\
5.60 \\
5.70 \\
5.80 \\
5.90 \\
6.00 \\
6.10 \\
6.20 \\
6.30\end{array}$ & \begin{tabular}{|c|} 
Second-feet. \\
1,220 \\
1,330 \\
1,440 \\
1,560 \\
1,680 \\
1,810 \\
1,940 \\
2,080 \\
2,220 \\
2,370 \\
2,520 \\
2,670 \\
2,830
\end{tabular} & $\begin{array}{r}\text { Feet. } \\
6.40 \\
6.50 \\
6.60 \\
6.70 \\
6.80 \\
7.00 \\
7.20 \\
7.40 \\
7.60 \\
7.80 \\
8.00 \\
9.00 \\
10.00\end{array}$ & \begin{tabular}{|} 
Second-feet. \\
2,990 \\
3,150 \\
3,320 \\
3,490 \\
3,660 \\
4,010 \\
4,370 \\
4,750 \\
5,150 \\
5,570 \\
6,000 \\
8,300 \\
10,910
\end{tabular} & $\begin{array}{l}\text { Feet. } \\
11.00 \\
12.00 \\
13.00 \\
14.00 \\
15.00 \\
16.00 \\
17.00 \\
18.00 \\
19.00 \\
20.00 \\
22.00 \\
24.00\end{array}$ & \begin{tabular}{|c} 
Second-feet. \\
13,610 \\
16,590 \\
$\mathbf{2 0}, 000$ \\
24,000 \\
28,500 \\
33,300 \\
38,300 \\
43,500 \\
48,900 \\
54,700 \\
66,900 \\
80,200
\end{tabular} \\
\hline
\end{tabular}

a This table is strictly applicable only for open-channel conditions. It is based on five discharge measurements made during 1899-1903. It is well defined between gage heights 4.2 feet and 6.0 feet. Above gage height 8.0 feet estimates are based on a discharge curve which is the product of a welldefined area curve and an approximate extension of the velocity curve. The discharge eurves for the periods 1899-1905, inclusive, are the same above gage height 10.6 feet. 
Rating tables for South Fork of Shenandoah River near Front Royal, Va.-Continued. JANUARY 1, 1904, TO JULY 16, 1906.a

\begin{tabular}{|c|c|c|c|c|c|c|c|}
\hline $\begin{array}{c}\text { Gage } \\
\text { height. }\end{array}$ & Discharge. & $\begin{array}{c}\text { Gage } \\
\text { height. }\end{array}$ & Discharge. & $\begin{array}{c}\text { Gage } \\
\text { height. }\end{array}$ & Discharge. & $\begin{array}{c}\text { Gage } \\
\text { height. }\end{array}$ & Discharge. \\
\hline $\begin{array}{c}\text { Feet. } \\
3.40 \\
3.50 \\
3.60 \\
3.70 \\
3.80 \\
3.90 \\
4.00 \\
4.10 \\
4.20 \\
4.30 \\
4.40\end{array}$ & \begin{tabular}{|c} 
Second-feet. \\
320 \\
350 \\
385 \\
425 \\
465 \\
510 \\
560 \\
610 \\
670 \\
740 \\
810
\end{tabular} & $\begin{array}{r}\text { Feet. } \\
4.50 \\
4.60 \\
4.70 \\
4.80 \\
4.90 \\
\mathbf{5 . 0 0} \\
\mathbf{5 . 1 0} \\
\mathbf{5 . 2 0} \\
\mathbf{5 . 3 0} \\
\mathbf{5 . 4 0} \\
\mathbf{5 . 5 0}\end{array}$ & \begin{tabular}{|c} 
Second-feet. \\
890 \\
970 \\
1,060 \\
1,150 \\
1,250 \\
1,350 \\
1,460 \\
1,570 \\
1,690 \\
1,810 \\
1,940
\end{tabular} & $\begin{array}{r}\text { Feet. } \\
5.60 \\
5.70 \\
5.80 \\
5.90 \\
6.00 \\
6.10 \\
6.20 \\
6.30 \\
6.40 \\
6.50 \\
6.60\end{array}$ & \begin{tabular}{|c} 
Second-feet. \\
2,070 \\
2,210 \\
2,350 \\
2,490 \\
2,640 \\
2,790 \\
2,940 \\
3,100 \\
3,260 \\
3,430 \\
3,600
\end{tabular} & $\begin{array}{r}\text { Feet. } \\
6.70 \\
6.80 \\
6.90 \\
7.00 \\
7.20 \\
7.40 \\
7.60 \\
7.80 \\
8.00 \\
9.00 \\
10.00\end{array}$ & \begin{tabular}{|r} 
Second-feet. \\
3,770 \\
3,940 \\
4,120 \\
4,300 \\
4,680 \\
5,080 \\
5,480 \\
5,900 \\
6,320 \\
8,550 \\
10,980
\end{tabular} \\
\hline
\end{tabular}

$a$ This table is strictly applicable only for open-channel conditions. It is based on 9 discharge measurements made during 1904 to 1906. It is well defined between gage heights 3.4 feet and 5.3 feet. Above gage height 8.0 feet estimates are based upon a discharge curve which is the product of a well defined area curve and an approximate extension of the velocity curve. Above gage height 10.6 feet both rating tables are the same.

Estimated monthly discharge of South Fork of Shenandoah River near Front Royal, Va.

[Drainage area, 1,570 square miles.a]

\begin{tabular}{|c|c|c|c|c|c|c|c|c|}
\hline \multirow[b]{2}{*}{ Month. } & \multicolumn{3}{|c|}{ Discharge in second-feet. } & \multicolumn{3}{|c|}{ Run-off. } & \multicolumn{2}{|c|}{ Precipitation. } \\
\hline & Maximum. & Minimum. & Mean. & $\begin{array}{c}\text { Second- } \\
\text { feet per } \\
\text { souare } \\
\text { mile. }\end{array}$ & $\begin{array}{l}\text { Depth in } \\
\text { inches. }\end{array}$ & $\begin{array}{l}\text { Per cent } \\
\text { of precip- } \\
\text { itation. }\end{array}$ & $\begin{array}{l}\text { In } \\
\text { inches. }\end{array}$ & $\begin{array}{l}\text { Loss in } \\
\text { inches. }\end{array}$ \\
\hline $\begin{array}{r}1899 . \\
\text { January.... }\end{array}$ & & & & & & & 2 $5 ?$ & \\
\hline February. & & & & & & & $\begin{array}{l}2.02 \\
4.95\end{array}$ & \\
\hline March. & $\cdots$ & & & & & & 5. 12 & \\
\hline April. & & & & & & $\cdots$ & 1.16 & $\therefore$... \\
\hline May $\ldots \ldots$ & & & & & & & 4. 95 & ..... \\
\hline June 26-30. & 650 & 547 & 594 & .378 & .070 & & $b 2.09$ & $\cdots$ \\
\hline July ....... & $\begin{array}{r}950 \\
975\end{array}$ & 428 & 538 & $\begin{array}{l}.343 \\
382\end{array}$ & .395 & 16 & 2. 51 & 2.11 \\
\hline August ..... & $\begin{array}{l}1,275 \\
2,080\end{array}$ & $\begin{array}{l}350 \\
485\end{array}$ & $\begin{array}{l}599 \\
759\end{array}$ & .484 & $\begin{array}{r}.440 \\
.540\end{array}$ & 11 & $\begin{array}{l}3.94 \\
3\end{array}$ & $\begin{array}{l}3.50 \\
3.24\end{array}$ \\
\hline $\begin{array}{l}\text { September. } \\
\text { October... }\end{array}$ & $\begin{array}{r}2,080 \\
685\end{array}$ & $\begin{array}{l}480 \\
455\end{array}$ & 559 & $\begin{array}{l}.304 \\
.356\end{array}$ & 410 & $\begin{array}{l}14 \\
18\end{array}$ & $\begin{array}{l}3.78 \\
2.34\end{array}$ & $\begin{array}{l}3.24 \\
1.93\end{array}$ \\
\hline $\begin{array}{l}\text { Notoper.. } \\
\text { November }\end{array}$ & 6.110 & 650 & 1,236 & .788 & .879 & 109 & .81 & $\begin{array}{r}1.00 \\
-.07\end{array}$ \\
\hline December & 3,920 & 515 & 1,121 & .714 & .823 & 61 & 1. 34 & .52 \\
\hline The year & . & $\cdots$ & $\cdots$ & $\cdots$ & & $\cdots$ & 35. 51 & \\
\hline 1900. & & & & & & & & \\
\hline $\begin{array}{l}\text { January.. } \\
\text { February }\end{array}$ & 12,800 & 950 & 2,265 & 1. 44 & 1.66 & 65 & 2. 57 & $\therefore 97$ \\
\hline March. & 12,680 & 1,680 & $\begin{array}{l}2,492 \\
3,551\end{array}$ & $\begin{array}{l}1.99 \\
2.26\end{array}$ & 2. 61 & 70 & 3. 72 & 1.14 \\
\hline April.. & 3,490 & 1,120 & 1,755 & 1.12 & 1.25 & 66 & 1.89 & .67 \\
\hline May. & 2,010 & 720 & 1,160 & .739 & .852 & 35 & 2.42 & 1.57 \\
\hline June. & 5,890 & 65 & 1,708 & 1.09 & 1.22 & 0 & 5.98 & 4. 76 \\
\hline July - & 1,120 & 515 & 776 & .495 & .571 & 15 & 3.76 & 3. 19 \\
\hline August. & 795 & 400 & 538 & .343 & .395 & 21 & 1.93 & 1. 53 \\
\hline Septembe & 1,070 & 350 & 499 & .318 & .355 & 11 & & 2. \\
\hline October. & 3,920 & 547 & 1,042 & .664 & .765 & 24 & 3. 19 & 2. 43 \\
\hline Novembe & 7,005 & 580 & 1,285 & .819 & .914 & 31 & 2. 94 & 2. 03 \\
\hline December. & 5,150 & 720 & 1,417 & .903 & 1. 04 & 57 & 1.83 & .79 \\
\hline The year. & 12,800 & 350 & 1,540 & .982 & 13. 29 & 36 & 37.10 & 23.81 \\
\hline
\end{tabular}

$a$ Drainage area of 1,570 square miles used to obtain the run-off for 1906, and 1,569 used for all other years.

b Precipitation for complete month, June, 1899. 
Estimated monthly discharge of South Fork of Shenanäoah River near Front Royal, Va.-Continued.

\begin{tabular}{|c|c|c|c|c|c|c|c|c|}
\hline \multirow[b]{2}{*}{ Month. } & \multicolumn{3}{|c|}{ Discharge in second-feet. } & \multicolumn{3}{|c|}{ Run-off. } & \multicolumn{2}{|c|}{ Precipitation. } \\
\hline & Maximum. & Minimum. & Mean. & $\begin{array}{l}\text { Second- } \\
\text { feet per } \\
\text { square } \\
\text { mile }\end{array}$ & $\begin{array}{l}\text { Depth in } \\
\text { inches. }\end{array}$ & $\begin{array}{l}\text { Per cent } \\
\text { of precip- } \\
\text { itation. }\end{array}$ & $\begin{array}{c}\operatorname{lin} \\
\text { inches. }\end{array}$ & $\begin{array}{l}\text { Loss in } \\
\text { inches. }\end{array}$ \\
\hline 1901. & 4,655 & 515 & 1366 & 871 & 1.00 & 43 & 2,31 & \\
\hline February.. & $\begin{array}{l}4,052 \\
1,220\end{array}$ & $\begin{array}{l}515 \\
580\end{array}$ & $\begin{array}{r}1,560 \\
818\end{array}$ & .871 & $\begin{array}{l}1.00 \\
.542\end{array}$ & $\begin{array}{r}40 \\
164\end{array}$ & $\begin{array}{r}2.01 \\
.33\end{array}$ & $\begin{array}{r}1.01 \\
-.21\end{array}$ \\
\hline March... & $\begin{array}{l}1,220 \\
11,580\end{array}$ & 515 & $\begin{array}{r}1,975 \\
\end{array}$ & 1. 26 & 1. 45 & 38 & 3. 80 & 2. 35 \\
\hline April... & 46,200 & 1,810 & 8,374 & 5.34 & 5. 96 & 95 & 6. 24 & .28 \\
\hline May.. & 40,900 & 1,680 & 5,768 & 3. 68 & 4. 24 & 73 & 5. 82 & 1. 58 \\
\hline June: & 11,720 & 1,940 & $\begin{array}{l}\text { 4, } 302 \\
402\end{array}$ & $\begin{array}{l}2.74 \\
2.74\end{array}$ & 3. 06 & 41 & 7.54 & 4.48 \\
\hline July . . & 15,070 & 1,440 & 3,912 & 2. 49 & 2.87 & 65 & 438 & 1.51 \\
\hline August. & 6,440 & 1,075 & 3,204 & 2.04 & 2. 35 & 40 & 5.92 & 3.57 \\
\hline September & 7,580 & 720 & 1,825 & 1. 16 & 1. 29 & 34 & 3. 78 & 2.49 \\
\hline October. & 9,730 & 758 & 2,020 & 1. 29 & $\begin{array}{l}1.49 \\
\text { 1. }\end{array}$ & 216 & .69 & -.80 \\
\hline Novembe & 2,595 & 650 & 966 & .616 & .687 & 34 & 2.01 & 1. 32 \\
\hline December. & 43,500 & 1,220 & 5,984 & 3.81 & 4. 39 & 72 & 6. 12 & 1.73 \\
\hline The yed & 46,200 & 515 & 3,319 & 2.11 & 29. 33 & 60 & 48. 94 & 19. 61 \\
\hline 1902 & & & & & & & & \\
\hline January.. & 9,600 & 1,385 & 3,730 & 2. 38 & 2.74 & 102 & 2. 69 & -.05 \\
\hline February. & 54,700 & 1,330 & 6,439 & 4. 10 & 4. 27 & 96 & 4.46 & \\
\hline March & 76,800 & 3,070 & 9.871 & 6. 29 & 7.25 & 201 & 3. 61 & -3.64 \\
\hline April.. & 12,260 & 1,560 & 3,826 & 2. 44 & 2. 72 & 122 & 2. 23 & -.49 \\
\hline May.. & 3,150 & 910 & 1,579 & 1. 01 & 1. 16 & 44 & 2.64 & 1. 48 \\
\hline June. & 1,030 & 650 & 861 & .549 & .612 & 21 & 2.93 & 2. 32 \\
\hline July. & 1,680 & 515 & 754 & .481 & .554 & 25 & 2.17 & 1. 62 \\
\hline August. & 795 & 400 & 571 & .364 & .420 & 19 & 2.22 & 1. 80 \\
\hline Septemb & 2,370 & 305 & 531 & .338 & .377 & 15 & 2. 59 & 2. 21 \\
\hline October & 2,370 & 455 & 848 & .540 & .623 & 17 & 3. 65 & 3. 03 \\
\hline November & 2,750 & 455 & 784 & .500 & .558 & 17 & 3. 21 & 2.65 \\
\hline December & 6,220 & 1,440 & 3,040 & & 2.24 & & 3. 38 & 1.14 \\
\hline The year & 76,800 & 305 & 2,736 & 1.74 & 23. 52 & 66 & 35.78 & 12.26 \\
\hline 1908 & & & & & & & & \\
\hline January. & 14,320 & 1,385 & 3,859 & 2. 46 & 2.84 & 70 & 4. 08 & 1.24 \\
\hline February & 7,700 & 2,445 & 3,768 & 2. 40 & 2. 50 & 72 & 3. 49 & .99 \\
\hline March. & 17,390 & 1,940 & 5,043 & 3.21 & 3. 70 & 89 & 4. 15 & .45 \\
\hline April.. & 12,400 & 2,220 & 4,494 & 2. 86 & 3. 19 & 88 & 3. 62 & .43 \\
\hline $\begin{array}{l}\text { May .... } \\
\text { June... }\end{array}$ & $\begin{array}{r}2,295 \\
3,610\end{array}$ & 1,560 & $\begin{array}{l}1,411 \\
4,308\end{array}$ & $\begin{array}{r}.903 \\
2.74\end{array}$ & 1.04 & $\begin{array}{l}39 \\
40\end{array}$ & $\begin{array}{l}2.69 \\
7.63\end{array}$ & 1.65 \\
\hline $\begin{array}{l}\text { June..... } \\
\text { July.... }\end{array}$ & $\begin{array}{r}13,610 \\
7,225\end{array}$ & $\begin{array}{r}1,500 \\
910\end{array}$ & $\begin{array}{l}4,000 \\
2,338\end{array}$ & $\begin{array}{l}\text { 2. } 44 \\
\text { 1. } 49\end{array}$ & $\begin{array}{l}3.00 \\
1.72\end{array}$ & 56 & $\begin{array}{l}1.00 \\
3.06\end{array}$ & $\begin{array}{l}\text { 4. } 57 \\
1.34\end{array}$ \\
\hline August. & 2,010 & 650 & 1,046 & .667 & .769 & 22 & $\begin{array}{l}3.50 \\
3.53\end{array}$ & 2.76 \\
\hline Septe & 5,570 & 650 & 1,738 & 1. 11 & 1. 24 & 51 & 2. 42 & 1. 18 \\
\hline Octo & 2,370 & 650 & 842 & .537 & .619 & 26 & 2.39 & 1.77 \\
\hline Nove & 870 & 580 & 691 & .440 & .491 & 60 & .82 & .33 \\
\hline Decembe & 1,680 & 580 & 853 & .54 & .627 & 66 & .96 & .33 \\
\hline The year. & 17,390 & 580 & 2,533 & 1. 61 & 21.80 & 56 & 38.84 & 17.04 \\
\hline 19 & & & & & & & & \\
\hline $\begin{array}{l}\text { January } a b \\
\text { February } b\end{array}$ & $\begin{array}{l}6,650 \\
6,110\end{array}$ & $\begin{array}{l}705 \\
560\end{array}$ & 1,293 & .824 & $\begin{array}{l}.950 \\
1.13\end{array}$ & $\begin{array}{l}53 \\
90\end{array}$ & 1.80 & .85 \\
\hline Marc & 5,080 & 1,060 & 1,746 & 1.11 & 1.28 & 62 & 2. 08 & .80 \\
\hline April & 3,940 & 740 & 1,486 & .947 & 1. 06 & 40 & 2. 64 & 1. 58 \\
\hline May. & 5,480 & 1,150 & 1,875 & 1.20 & 1. 38 & 40 & 3. 43 & 2.05 \\
\hline Jun & 3,430 & 740 & 1,375 & .876 & .977 & 18 & 5. 57 & 4. 59 \\
\hline July. & 6,215 & 560 & 1.117 & .712 & .821 & 16 & 5. 04 & 4. 22 \\
\hline & 1,105 & 350 & 613 & .391 & .451 & 18 & 2. 53 & 2. 08 \\
\hline Septe & 640 & 320 & 395 & .252 & .281 & 14 & 1.95 & 1.67 \\
\hline Octobe & 368 & 320 & 326 & .208 & .240 & 20 & 1.20 & .96 \\
\hline Novem & 368 & 320 & 329 & .210 & .234 & 24 & .95 & .72 \\
\hline Decemb & 1,150 & 320 & 530 & .338 & .390 & 16 & 2. 46 & 2.07 \\
\hline The year. & 6,650 & 320 & 1,061 & .676 & 9. 19 & 30 & 30.91 & 21.72 \\
\hline
\end{tabular}

a lce conditıons during January and December, 1904; no correction made in estimates. $b$ Estimates January 25 to February 25, 1904, approximate. 
Estimated monthly discharge of South Fork of Shenandoah River near Front Royal, Va.Continued.

\begin{tabular}{|c|c|c|c|c|c|c|c|c|c|c|}
\hline \multirow[b]{2}{*}{ Month. } & \multicolumn{3}{|c|}{ Discharge in second-feet. } & \multicolumn{5}{|c|}{ Run-off. } & \multicolumn{2}{|c|}{ Precipitation. } \\
\hline & Maximum. & Mirimum. & Mean. & $\begin{array}{c}\text { Second- } \\
\text { feet per } \\
\text { square } \\
\text { mile. }\end{array}$ & $\begin{array}{c}\text { Del } \\
\text { in }\end{array}$ & $\begin{array}{l}\text { h in } \\
\text { hes. }\end{array}$ & & $\begin{array}{l}\text { c cent } \\
\text { orecip- } \\
\text { tion. }\end{array}$ & $\underset{\text { inches }}{\mathrm{Hn}}$ & $\begin{array}{l}\text { Loss in } \\
\text { inches. }\end{array}$ \\
\hline 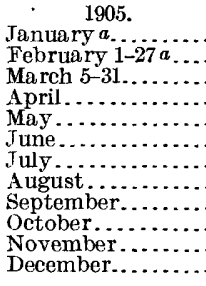 & $\begin{array}{r}2,005 \\
6,320 \\
6,870 \\
1,810 \\
2,005 \\
9,380 \\
5,480 \\
3,068 \\
1,200 \\
474 \\
474 \\
7,024\end{array}$ & $\begin{array}{r}425 \\
560 \\
1,405 \\
740 \\
560 \\
465 \\
890 \\
560 \\
417 \\
350 \\
378 \\
425\end{array}$ & $\begin{array}{r}917 \\
1,140 \\
3,104 \\
1,143 \\
920 \\
1,372 \\
2,236 \\
1,014 \\
595 \\
417 \\
425 \\
1,476\end{array}$ & $\begin{array}{c}.584 \\
.726 \\
1.98 \\
.728 \\
.586 \\
.874 \\
1.42 \\
.646 \\
.379 \\
.266 \\
.271 \\
.941\end{array}$ & & $\begin{array}{c}.673 \\
.729 \\
1.99 \\
.812 \\
.676 \\
.975 \\
1.64 \\
.745 \\
.423 \\
.307 \\
.302 \\
1.08\end{array}$ & & $\begin{array}{c}24 \\
\cdots \\
339 \\
20 \\
23 \\
26 \\
25 \\
25 \\
12 \\
36 \\
28\end{array}$ & $\begin{array}{r}2.79 \\
\text { b2.11 } \\
\text { b } 2.25 \\
2.08 \\
3.45 \\
4.30 \\
6.23 \\
2.90 \\
1.68 \\
2.65 \\
.83 \\
3.81\end{array}$ & \begin{tabular}{r}
2.12 \\
$\cdots$ \\
\hdashline 1.27 \\
2.77 \\
3.32 \\
4.59 \\
2.16 \\
1.26 \\
2.34 \\
.53 \\
2.73
\end{tabular} \\
\hline The & & & & & & $\cdots$ & & & 35. 08 & \\
\hline \multirow{2}{*}{\multicolumn{3}{|c|}{ Month. }} & \multicolumn{5}{|c|}{ Discharge in second-feet. } & \multicolumn{3}{|c|}{ Run-off. } \\
\hline & & & Maximum. & \multicolumn{2}{|c|}{ Minimum. } & Mean & \multicolumn{3}{|c|}{$\begin{array}{l}\text { Second-feet } \\
\text { per square } \\
\text { mile. }\end{array}$} & $\begin{array}{l}\text { Depth in } \\
\text { inches. }\end{array}$ \\
\hline $\begin{array}{l}\text { January.... } \\
\text { February. } \\
\text { March .... } \\
\text { April...... } \\
\text { May ....... } \\
\text { June ....... } \\
\text { July } 1-16 . .\end{array}$ & $\begin{array}{c}1906 . \\
\ldots \ldots \ldots\end{array}$ & - & $\begin{array}{l}7,354 \\
1,849 \\
4,980 \\
5,795 \\
1,594 \\
4,120 \\
1,875\end{array}$ & \multicolumn{2}{|c|}{$\begin{array}{r}1,250 \\
622 \\
652 \\
1,405 \\
670 \\
610 \\
705\end{array}$} & \multicolumn{2}{|c|}{$\begin{array}{r}2,283 \\
957 \\
2,233 \\
2,393 \\
1,049 \\
1,464 \\
1,139\end{array}$} & \multicolumn{2}{|r|}{$\begin{array}{l}1.45 \\
.610 \\
1.42 \\
1.52 \\
.668 \\
.932 \\
.725\end{array}$} & $\begin{array}{l}1.67 \\
.635 \\
1.64 \\
1.70 \\
.770 \\
1.04 \\
.431\end{array}$ \\
\hline
\end{tabular}

$a$ Ice conditions during January and February, 1905; no correction made in estimates.

$b$ Precipitation for complete month, February and March, 1905.

\section{MISCELLANEOUS DISCHARGE MEASUREMENTS IN SOUTH FORK OF SHENANDOAH RIVER} BASIN BELOW PORT REPUBLIC.

The following miscellaneous discharge measurements were made in the basin of South Fork of Shenandoah River below Port Republic:

Miscellaneous discharge measurements in South Fork of Shenandoah River basin below Port Republic.

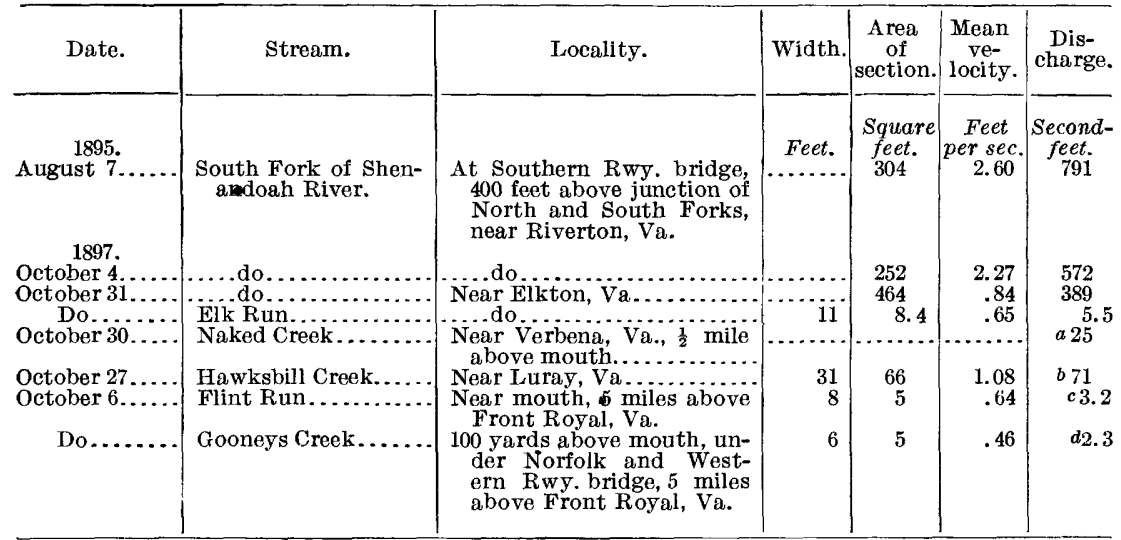

$a$ Includes 11 second-feet in tail race below mill.

$b$ Discharge probably increased by rains of preceding week. $c$ Flint Run fed by large springs.

a Flow stated to be exceptionally low, IRR $192-07-9$ 


\section{NORTH FORK OF SHENANDOAH RIVER BASIN.}

PASSAGE CREEK AT BUCKTON, VA.

Passage Creek rises on Massanutten Mountain, in the western part of Page County, Va., flows northeastward, and joins North Fork of Shenandoah River about 5 miles above Riverton, Va.

The gaging station was established October 26, 1905, by Robert Follansbee and was discontinued July 16, 1906. It is located about 700 feet above the mouth of the creek, at the trestle of the Southern Railway at Buckton, which is a siding 1 mile east of Waterlick, Va.

The channel is straight for 200 feet above and 100 feet below the station. The current is moderate at the measuring section, but from a point 100 feet below the section to the mouth of the creek the velocity is rapid, the fall in that distance being from 6 to 8 feet. The banks above the bridge are fairly high, wooded, and not liable to overflow. Below the bridge they are low and liable to overflow during very high water, the flood plain being several hundred feet wide at such times. The channel between abutments is broken by seven trestle bents, and there are from three to eight channels, according to the stage of the river.

Discharge measurements at ordinary and low stages were made from the railway trestle or by wading a short distance above at a point wherè conditions are better. High-water measurements can be made from the highway bridge 2 miles upstream. This latter is not a good section at ordinary stages, as the current is too sluggish.

A staff gage, which is read once each day and oftener in floods by Nehemiah Messick, is nailed vertically to the third trestle bent from the left abutment.

The bench mark is the head of a nail 4 feet above ground, driven horizontally in a blaze on a tree situated 15 feet downstream from the lower end of the wing wall on the right bank; elevation, 5.41 feet above gage datum.

No estimates of flow have been made for this station, as the number of measurements is insufficient.

Discharge measurements of Passage Creek at Buckton, Va.

\begin{tabular}{|c|c|c|c|c|c|}
\hline Date. & $\begin{array}{c}\text { Gage } \\
\text { height. }\end{array}$ & $\begin{array}{c}\text { Dis- } \\
\text { charge. }\end{array}$ & Date. & $\begin{array}{c}\text { Gage } \\
\text { height. }\end{array}$ & $\begin{array}{l}\text { Dis- } \\
\text { charge. }\end{array}$ \\
\hline $\begin{array}{c}1905 . \\
\text { October } 26 \ldots \ldots \ldots \ldots \ldots \\
\text { December } 27 \ldots \ldots \ldots \ldots \cdots \cdots\end{array}$ & $\begin{array}{l}\text { Feet. } \\
\quad 1.23 \\
1.25\end{array}$ & $\mid \begin{array}{r}\text { Second-feet. } \\
40 \\
50\end{array}$ & $\begin{array}{c}1906 . \\
\text { April } 13 . \ldots \ldots \ldots \ldots \ldots \ldots \ldots \ldots \ldots \\
\text { June } 16 . \ldots \ldots \ldots \ldots \ldots \ldots \ldots\end{array}$ & $\begin{array}{l}\text { Feet. } \\
1.40 \\
1.72\end{array}$ & $\begin{array}{r}\text { Second-feet } \\
91 \\
132\end{array}$ \\
\hline
\end{tabular}


Daily gage height, in feet, of Passage Creek at Buckton, Va.

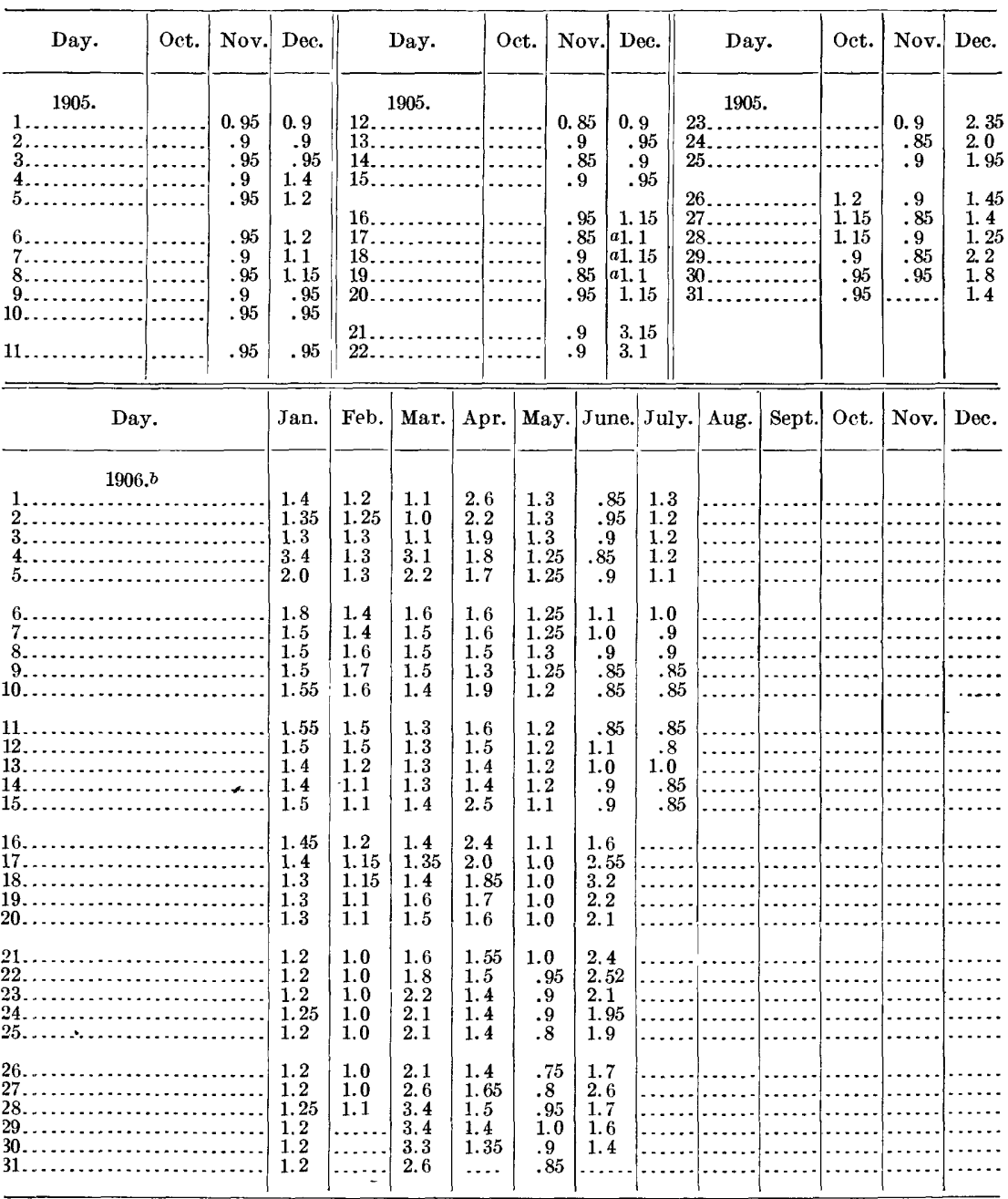

$a$ Creek frozen December 17-19, 1905.

b Ice conditions February 4-13, 1906.

NORTH FORK OF SHENANDOAH RIVER NEAR RIVERTON, VA.

North Fork of Shenandoah River rises in the northern part of Rockingham County, Va., and flows in a very tortuous southeastward and 1ortheastward course to Riverton, Va., where it unites with South Fork to form the main Shenandoah. Its drainage area is 1,040 square miles.

The flow of this stream is rather variable, although the discharge of some of its tributaries, especially in the upper part of the valley, is sonstant. The mills on North Fork are small, and the dams are gensally of wood or brush. 
The gaging station was established June 26, 1899, by A. P. Davis, and was discontinued July 15, 1906. It is located about 2 miles above Riverton, on the farm owned by L. W. Burke. It is most easily reached by driving from Front Royal.

The channel is straight for 600 feet above and below the station. The current has a moderate velocity. Both banks are low and liable to overflow and are fringed with trees. The bed of the stream is composed of rock and mud and shifts somewhat, but the flow is controlled by the dam at Riverton, 2 miles below. There is but one channel at all stages.

Discharge measurements were made by means of a cable, car, and tagged wire just above the ford. The cable has a span of 260 feet, is supported by timbers, and is anchored in the ground at each end. The initial point for soundings is 0.5 foot from the timber which supports the tag wire on the left bank.

The original gage was a vertical timber bolted to a large sycamore tree on the right bank. On September 10,1900, the gage was removed to the left bank, and its datum was lowered 1 foot, causing all readings to be increased by 1 foot. The gage at this station was washed out in the flood of February 22, 1902, and the station was abandoned until August 17, 1902, when it was reestablished, the zero of the new gage being at the same elevation as the zero of the preceding gage. The vertical gage rod is spiked to a sycamore tree on the left bank 100 feet above the cable station. The gage was read twice each day by L. W. Burke. The bench mark is a wire nail driven into a pear tree, located near a fence, 150 feet from the left bank of the river. Its elevation above the zero of the gage is 26.75 feet.

All estimates published prior to 1905 have been revised. The plotting of the discharge measurements gives three distinct curves. The change from the first to the second curve occurred some time between June 20,1901, and August 16, 1902. It was due, to a slight extent, to changes at the cable section, but chiefly to a radical change at some controlling point below the cable. The change from the second to the third curve was due to an increase in the height of the dam at Riverton from 8 feet to 10 feet during August, 1904. Estimates below gage height 6.5 feet as based on the first two curves are considered to be within 5 per cent and as based on the third curve within 5 per cent or less of the true discharge for normal conditions of flow. Estimates above gage height 6.5 feet may be in error from 10 to 15 per cent. Estimates for June 20, 1901, to August 16, 1902, are also liable to errors as high as 20 to 50 per cent owing to lack of information concerning the exact date when the change in conditions of flow took place. Estimates for August, 1904, are also liable to considerable error from the same cause. Ice conditions at and below the gage affect the flow during the winter months. 
Daily gage height, in feet, of North Fork of Shenandoah River near Riverton, Va.Continued.

\begin{tabular}{|c|c|c|c|c|c|c|c|c|c|c|c|c|}
\hline $\mathrm{Da}$ & Jan. & Feb. & Mar. & Apr. & May. & June & July & Aug. & Sept. & Oet. & Nov. & Dec. \\
\hline $\begin{array}{l} \\
1 \ldots \\
2 \ldots \\
3 \ldots \\
4 \ldots \\
5 \ldots\end{array}$ & $\begin{array}{l}4.0 \\
4.0 \\
4.0 \\
4.0 \\
4.0\end{array}$ & $\begin{array}{l}5.0 \\
5.0 \\
5.0 \\
5.0 \\
4.9\end{array}$ & $\begin{array}{l}5.05 \\
7.85 \\
6.55 \\
5.4 \\
5.1\end{array}$ & $\begin{array}{l}4.7 \\
4.58 \\
4.52 \\
4.48 \\
4.4\end{array}$ & $\begin{array}{l}4.25 \\
4.17 \\
4.15 \\
4.1 \\
4.05\end{array}$ & $\begin{array}{l}3.8 \\
3.8 \\
3.8 \\
3.9 \\
3.88\end{array}$ & $\begin{array}{l}3.9 \\
3.9 \\
3.85 \\
3.78 \\
3.72\end{array}$ & $\begin{array}{l}3.72 \\
3.65 \\
3.6 \\
3.55 \\
3.52\end{array}$ & $\begin{array}{l}3.6 \\
3.6 \\
3.62 \\
3.62 \\
3.58\end{array}$ & $\begin{array}{l}3.8 \\
3.8 \\
3.75 \\
3.7 \\
3.7\end{array}$ & $\begin{array}{l}3.7 \\
3.72 \\
3.7 \\
3.75 \\
3.7\end{array}$ & $\begin{array}{l}4.1 \\
3.9 \\
4.0 \\
4.4 \\
6.1\end{array}$ \\
\hline $\begin{array}{r}6 . . \\
7 . . \\
8 . \\
9 . \\
10 .\end{array}$ & $\begin{array}{l}4.0 \\
4.0 \\
4.0 \\
4.0 \\
4.0\end{array}$ & $\begin{array}{l}4.5 \\
4.38 \\
4.1 \\
4.0 \\
3.95\end{array}$ & $\begin{array}{l}4.88 \\
4.82 \\
4.75 \\
4.68 \\
4.6\end{array}$ & $\begin{array}{l}4.32 \\
4.28 \\
4.25 \\
4.2 \\
4.15\end{array}$ & $\begin{array}{l}4.05 \\
4.0 \\
3.98 \\
3.95 \\
3.92\end{array}$ & $\begin{array}{l}3.82 \\
3.8 \\
3.8 \\
3.8 \\
3.8\end{array}$ & $\begin{array}{l}3.72 \\
3.7 \\
3.7 \\
3.68 \\
3.68\end{array}$ & $\begin{array}{l}3.5 \\
3.5 \\
3.55 \\
3.58 \\
3.6\end{array}$ & $\begin{array}{l}3.6 \\
3.6 \\
3.58 \\
3.6 \\
3.6\end{array}$ & $\begin{array}{l}3.7 \\
3.78 \\
3.8 \\
3.7 \\
3.7\end{array}$ & $\begin{array}{l}3.7 \\
3.7 \\
3.7 \\
3.65 \\
3.62\end{array}$ & $\begin{array}{l}5.7 \\
5.1 \\
4.7 \\
4.4 \\
4.4\end{array}$ \\
\hline $\begin{array}{l}11 . . \\
12 . \\
13 . \\
14 . \\
15 . .\end{array}$ & $\begin{array}{l}4.0 \\
4.0 \\
4.0 \\
3.98 \\
3.92\end{array}$ & $\begin{array}{l}4.0 \\
3.98 \\
4.12 \\
5.1 \\
5.2\end{array}$ & $\begin{array}{l}4.5 \\
4.42 \\
4.38 \\
4.35 \\
4.35\end{array}$ & $\begin{array}{l}4.15 \\
4.15 \\
4.15 \\
4.15 \\
4.1\end{array}$ & $\begin{array}{l}3.9 \\
3.9 \\
3.9 \\
3.9 \\
3.9\end{array}$ & $\begin{array}{l}3.75 \\
3.72 \\
3.7 \\
3.72 \\
3.75\end{array}$ & $\begin{array}{l}3.62 \\
3.62 \\
3.62 \\
3.65 \\
3.62\end{array}$ & $\begin{array}{l}3.6 \\
3.58 \\
3.5 \\
3.5 \\
3.5\end{array}$ & $\begin{array}{l}3.6 \\
3.6 \\
3.58 \\
3.6 \\
3.6\end{array}$ & $\begin{array}{l}3.68 \\
3.65 \\
3.7 \\
3.7 \\
3.7\end{array}$ & $\begin{array}{l}3.7 \\
3.6 \\
3.6 \\
3.68 \\
3.65\end{array}$ & $\begin{array}{l}4.3 \\
4.0 \\
3.9 \\
3.9 \\
4.0\end{array}$ \\
\hline $\begin{array}{l}16 \ldots \\
17 \ldots \\
18 \ldots \\
19 \ldots \\
20 \ldots\end{array}$ & $\begin{array}{l}3.9 \\
3.8 \\
3.8 \\
3.92 \\
4.92\end{array}$ & $\begin{array}{l}4.87 \\
4.62 \\
4.42 \\
4.35 \\
4.35\end{array}$ & $\begin{array}{l}4.35 \\
4.3 \\
4.25 \\
4.5 \\
6.95\end{array}$ & $\begin{array}{l}4.1 \\
4.1 \\
4.1 \\
4.2 \\
4.55\end{array}$ & $\begin{array}{l}3.82 \\
3.8 \\
3.8 \\
3.8 \\
3.8\end{array}$ & $\begin{array}{l}3.78 \\
4.45 \\
5.75 \\
5.75 \\
4.98\end{array}$ & $\begin{array}{l}3.6 \\
3.68 \\
3.62 \\
3.6 \\
3.6\end{array}$ & $\begin{array}{l}3.55 \\
3.6 \\
3.6 \\
3.55 \\
3.55\end{array}$ & $\begin{array}{l}3.7 \\
3.68 \\
3.65 \\
3.68 \\
3.6\end{array}$ & $\begin{array}{l}3.7 \\
3.78 \\
3.8 \\
3.75 \\
3.7\end{array}$ & $\begin{array}{l}3.6 \\
3.6 \\
3.62 \\
3.62 \\
3.65\end{array}$ & $\begin{array}{l}3.9 \\
4.0 \\
3.9 \\
3.9 \\
3.7\end{array}$ \\
\hline $\begin{array}{l}21 \ldots \\
22 \ldots \\
23 \ldots \\
24 \ldots \\
25 \ldots\end{array}$ & $\begin{array}{l}7.3 \\
5.65 \\
4.9 \\
4.55 \\
4.25\end{array}$ & $\begin{array}{l}4.25 \\
4.75 \\
6.98 \\
6.3 \\
5.63\end{array}$ & $\begin{array}{l}8.2 \\
6.7 \\
5.9 \\
5.42 \\
5.18\end{array}$ & $\begin{array}{l}4.6 \\
4.6 \\
5.22 \\
5.4 \\
5.1\end{array}$ & $\begin{array}{l}3.8 \\
3.8 \\
3.8 \\
3.8 \\
3.8\end{array}$ & $\begin{array}{l}4.62 \\
4.3 \\
4.2 \\
4.15 \\
4.08\end{array}$ & $\begin{array}{l}3.62 \\
3.7 \\
3.82 \\
5.28 \\
4.32\end{array}$ & $\begin{array}{l}3.5 \\
3.62 \\
3.6 \\
3.6 \\
3.6\end{array}$ & $\begin{array}{l}3.6 \\
3.58 \\
3.58 \\
3.6 \\
3.6\end{array}$ & $\begin{array}{l}3.7 \\
3.7 \\
3.7 \\
3.75 \\
3.78\end{array}$ & $\begin{array}{l}3.7 \\
3.62 \\
3.6 \\
3.68 \\
3.7\end{array}$ & $\begin{array}{l}3.7 \\
3.6 \\
3.8 \\
3.7 \\
3.7\end{array}$ \\
\hline $\begin{array}{l}26 \ldots \\
27 \ldots \\
28 \ldots \\
29 \ldots \\
30 \ldots \\
31 \ldots\end{array}$ & $\begin{array}{l}4.02 \\
4.0 \\
4.0 \\
4.5 \\
5.0 \\
5.0\end{array}$ & $\begin{array}{l}5.15 \\
4.9 \\
4.8\end{array}$ & $\begin{array}{l}5.0 \\
5.0 \\
4.9 \\
4.9 \\
4.85 \\
4.8\end{array}$ & $\begin{array}{l}4.75 \\
4.62 \\
4.5 \\
4.42 \\
4.32\end{array}$ & \begin{tabular}{l|}
3.8 \\
3.8 \\
3.8 \\
3.8 \\
3.8 \\
3.75
\end{tabular} & $\begin{array}{l}4.0 \\
3.92 \\
3.9 \\
3.9 \\
3.9\end{array}$ & $\begin{array}{l}4.22 \\
4.0 \\
3.92 \\
3.82 \\
4.08 \\
3.8\end{array}$ & $\begin{array}{l}3.6 \\
3.55 \\
3.6 \\
3.62 \\
3.6 \\
3.62\end{array}$ & $\begin{array}{l}3.6 \\
3.6 \\
3.62 \\
3.72 \\
3.95\end{array}$ & $\begin{array}{l}3.85 \\
3.9 \\
3.8 \\
3.75 \\
3.72 \\
3.7\end{array}$ & $\begin{array}{l}4.65 \\
7.0 \\
5.25 \\
5.35 \\
4.45\end{array}$ & $\begin{array}{l}3.7 \\
3.8 \\
3.7 \\
3.7 \\
3.7 \\
3.7\end{array}$ \\
\hline \begin{tabular}{c}
\multicolumn{1}{c}{1901.} \\
$1 . \ldots \ldots$ \\
$2 \ldots \ldots$ \\
$3 \ldots \ldots$ \\
$4 \ldots \ldots$ \\
$5 \ldots \ldots$
\end{tabular} & $\begin{array}{l}3.72 \\
3.75 \\
3.8 \\
3.78 \\
3.8\end{array}$ & $\begin{array}{l}3.95 \\
3.9 \\
3.85 \\
3.78 \\
3.8\end{array}$ & $\begin{array}{l}3.7 \\
3.7 \\
3.7 \\
3.8 \\
3.78\end{array}$ & $\begin{array}{l}4.38 \\
4.4 \\
6.0 \\
7.65 \\
6.6\end{array}$ & $\begin{array}{l}4.98 \\
4.9 \\
4.8 \\
4.72 \\
4.65\end{array}$ & $\begin{array}{l}6.1 \\
5.65 \\
5.15 \\
5.48 \\
4.85\end{array}$ & $\begin{array}{l}4.95 \\
4.75 \\
4.7 \\
4.7 \\
4.5\end{array}$ & $\begin{array}{l}4.2 \\
4.28 \\
4.1 \\
4.05 \\
4.05\end{array}$ & $\begin{array}{l}5.25 \\
5.55 \\
5.2 \\
4.92 \\
4.75\end{array}$ & $\begin{array}{l}5.02 \\
4.65 \\
4.4 \\
4.35 \\
4.3\end{array}$ & $\begin{array}{l}3.8 \\
3.8 \\
3.8 \\
3.8 \\
3.8\end{array}$ & $\begin{array}{l}3.9 \\
3.8 \\
3.9 \\
4.4 \\
4.4\end{array}$ \\
\hline $\begin{array}{r}6 \ldots \\
7 \ldots \\
8 . . . \\
9 \ldots \\
10 \ldots\end{array}$ & $\begin{array}{l}3.85 \\
3.75 \\
3.8 \\
3.72 \\
3.7\end{array}$ & $\begin{array}{l}3.9 \\
3.95 \\
3.88 \\
3.82 \\
3.82\end{array}$ & $\begin{array}{l}3.8 \\
3.85 \\
3.85 \\
3.8 \\
3.85\end{array}$ & $\begin{array}{l}6.25 \\
6.25 \\
5.9 \\
5.4 \\
5.1\end{array}$ & $\begin{array}{l}4.6 \\
4.5 \\
4.42 \\
5.15 \\
6.25\end{array}$ & $\begin{array}{l}5.95 \\
6.6 \\
7.1 \\
5.9 \\
\mathbf{5 . 4}\end{array}$ & $\begin{array}{l}4.8 \\
5.5 \\
4.3 \\
4.45 \\
4.35\end{array}$ & $\begin{array}{l}4.3 \\
5.8 \\
5.5 \\
4.8 \\
4.45\end{array}$ & $\begin{array}{l}4.4 \\
4.25 \\
4.22 \\
4.1 \\
4.05\end{array}$ & $\begin{array}{l}4.22 \\
4.1 \\
4.15 \\
4.1 \\
3.95\end{array}$ & $\begin{array}{l}3.8 \\
3.8 \\
3.8 \\
3.8 \\
3.8\end{array}$ & $\begin{array}{l}4.5 \\
4.3 \\
4.2 ! \\
4.38 \\
4.3\end{array}$ \\
\hline $\begin{array}{l}11 . . \\
12 . . \\
13 . . \\
14 . \\
15 . .\end{array}$ & $\begin{array}{l}4.15 \\
5.3 \\
5.75 \\
5.1 \\
4.85\end{array}$ & $\begin{array}{l}3.82 \\
3.8 \\
3.9 \\
4.0 \\
3.95\end{array}$ & $\begin{array}{c}11.25 \\
9.05 \\
6.5 \\
5.68 \\
5.35\end{array}$ & $\begin{array}{c}4.85 \\
4.7 \\
4.6 \\
7.45 \\
12.6\end{array}$ & $\begin{array}{l}6.62 \\
6.25 \\
5.65 \\
5.3 \\
4.85\end{array}$ & $\begin{array}{l}5.0 \\
4.78 \\
5.1 \\
5.3 \\
4.95\end{array}$ & $\begin{array}{l}4.4 \\
4.7 \\
4.7 \\
6.4 \\
6.9\end{array}$ & $\begin{array}{l}4.3 \\
5.2 \\
5.55 \\
4.7 \\
4.4\end{array}$ & $\begin{array}{l}4.2 \\
4.1 \\
4.02 \\
4.0 \\
4.0\end{array}$ & $\begin{array}{l}3.95 \\
4.0 \\
3.95 \\
3.9 \\
3.9\end{array}$ & $\begin{array}{l}3.7 \\
3.6 \\
3.6 \\
3.7 \\
3.7\end{array}$ & $\begin{array}{r}4.4 \\
4.4 \\
4.3 \\
4.4 \\
10.6\end{array}$ \\
\hline $\begin{array}{l}16 \ldots \\
17 \ldots \\
18 \ldots \\
19 \ldots \\
20 \ldots\end{array}$ & $\begin{array}{l}4.68 \\
4.45 \\
4.3 \\
4.3 \\
5.55\end{array}$ & $\begin{array}{l}3.88 \\
3.8 \\
3.8 \\
3.82 \\
3.78\end{array}$ & $\begin{array}{l}4.98 \\
4.75 \\
4.62 \\
4.52 \\
4.2\end{array}$ & $\begin{array}{l}8.3 \\
6.9 \\
6.25 \\
5.85 \\
7.4\end{array}$ & $\begin{array}{l}4.68 \\
4.6 \\
4.72 \\
4.55 \\
4.52\end{array}$ & $\begin{array}{l}12.15 \\
11.1 \\
7.5 \\
6.25 \\
5.9\end{array}$ & $\begin{array}{l}6.82 \\
7.1 \\
6.48 \\
6.95 \\
6.1\end{array}$ & $\begin{array}{l}4.6 \\
4.58 \\
4.35 \\
4.55 \\
4.4\end{array}$ & $\begin{array}{l}4.15 \\
4.15 \\
4.15 \\
4.1 \\
4.05\end{array}$ & $\begin{array}{l}3.82 \\
3.8 \\
3.8 \\
3.8 \\
3.82\end{array}$ & $\begin{array}{l}3.8 \\
3.8 \\
3.8 \\
3.7 \\
3.7\end{array}$ & $\begin{array}{l}9.1 \\
6.2 \\
5.5 \\
5.1 \\
4.9\end{array}$ \\
\hline $\begin{array}{l}21 \ldots \\
22 \ldots \\
23 \ldots \\
24 \ldots \\
25 \ldots \\
\end{array}$ & $\begin{array}{l}4.95 \\
4.35 \\
4.05 \\
3.98 \\
4.0\end{array}$ & $\begin{array}{l}3.8 \\
4.0 \\
3.98 \\
3.95 \\
3.92\end{array}$ & $\begin{array}{l}4.9 \\
4.9 \\
4.9 \\
4.82 \\
4.7\end{array}$ & $\begin{array}{c}16.5 \\
12.45 \\
8.5 \\
6.8 \\
6.65\end{array}$ & $\begin{array}{c}4.65 \\
7.05 \\
14.7 \\
8.7 \\
7.15\end{array}$ & $\begin{array}{l}6.95 \\
6.75 \\
6.2 \\
5.75 \\
5.25\end{array}$ & $\begin{array}{l}5.3 \\
4.95 \\
4.75 \\
4.55 \\
4.5\end{array}$ & $\begin{array}{l}4.1 .5 \\
4.05 \\
4.05 \\
4.2 \\
4.6\end{array}$ & $\begin{array}{l}3.95 \\
.3 .88 \\
3.88 \\
3.8 \\
3.8\end{array}$ & $\begin{array}{l}3.78 \\
3.75 \\
3.7 \\
3.7 \\
3.72\end{array}$ & $\begin{array}{l}3.7 \\
3.8 \\
3.85 \\
5.25 \\
4.85\end{array}$ & $\begin{array}{l}8.0 \\
7.5 \\
7.5 \\
7.5 \\
7.5\end{array}$ \\
\hline 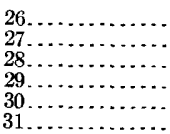 & $\begin{array}{l}3.98 \\
4.0 \\
4.0 \\
3.95 \\
3.92 \\
3.9\end{array}$ & $\begin{array}{l}3.85 \\
3.82 \\
3.72\end{array}$ & $\begin{array}{l}4.62 \\
4.75 \\
4.75 \\
4.55 \\
4.5 \\
4.45\end{array}$ & $\begin{array}{l}6.1 \\
5.7 \\
5.3 \\
5.1 \\
5.0\end{array}$ & $\begin{array}{l}6.55 \\
6.65 \\
7.05 \\
7.2 \\
6.7 \\
6.2\end{array}$ & $\begin{array}{l}5.3 \\
5.55 \\
4.7 \\
5.4 \\
5.6\end{array}$ & \begin{tabular}{l|}
4.3 \\
4.35 \\
4.3 \\
4.15 \\
4.65 \\
4.35
\end{tabular} & $\begin{array}{l}4.75 \\
4.5 \\
4.25 \\
4.3 \\
4.5 \\
4.9\end{array}$ & $\begin{array}{l}3.7 \\
3.65 \\
3.65 \\
5.15 \\
5.9\end{array}$ & $\begin{array}{l}3.8 \\
3.8 \\
3.8 \\
3.82 \\
3.82 \\
3.8\end{array}$ & $\begin{array}{l}4.5 \\
4.25 \\
4.35 \\
4.05 \\
4.0\end{array}$ & $\begin{array}{r}7.5 \\
7.5 \\
6.9 \\
8.2 \\
13.1 \\
8.25\end{array}$ \\
\hline
\end{tabular}

a All gage heights June 26, 1893, to September 10, 1900, refer to 1906 datum. Datum lowered 1.00 foot September 10,1900 .

$c$ River frozen January 1-14 and January 30 to February 6, 1900.

d Ice conditions during part of January and February, 1901; river frozen December 21 to 28, 1901. 
Daily gage height, in feet, of North Fork of Shenandoah River near Riverton, Va.-Cont'd.

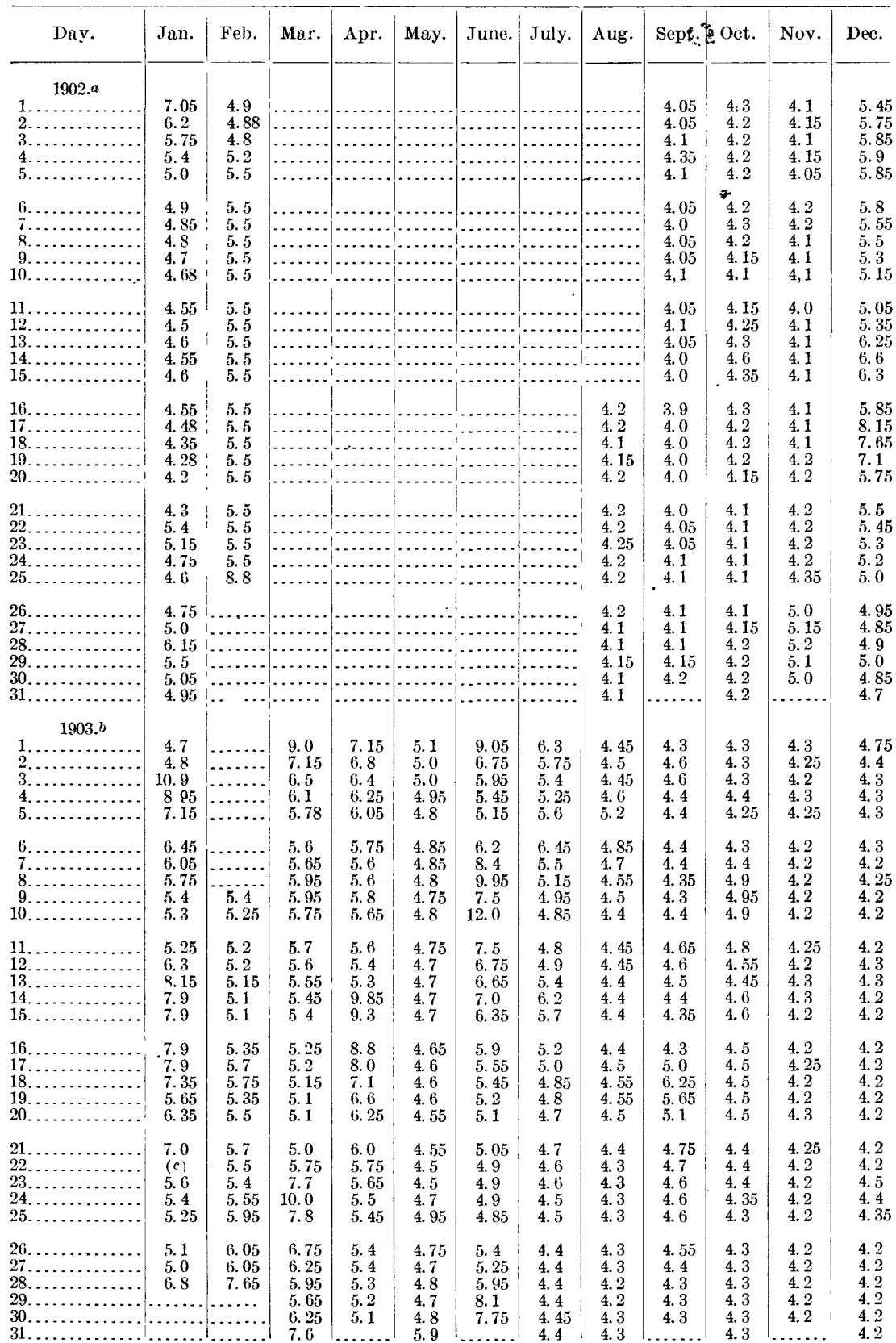


Daily gage height, in feet, of North Fork of Shenandoah River near Ricerton, Va.-Cont'd.

\begin{tabular}{|c|c|c|c|c|c|c|c|c|c|c|c|c|}
\hline Day. & Jan. & Feb. & Mar. & Apr. & May. & June. & July. & Ang. & Sapt. & Oct. & Nov. & Dec. \\
\hline $\begin{array}{r}1904 \\
1 \ldots \ldots \\
2 \ldots \ldots \\
3 \ldots \ldots \\
4 \ldots \ldots \\
5 \ldots \ldots \\
\ldots \ldots\end{array}$ & $\begin{array}{l}4.2 \\
4.2 \\
4.2 \\
4.2 \\
4.2\end{array}$ & & 4.7 & $\begin{array}{l}4.88 \\
5.0 \\
4.98 \\
4.88 \\
4.8\end{array}$ & $\begin{array}{l}5.85 \\
5.7 \\
5.4 \\
5.1 \\
5.05\end{array}$ & $\begin{array}{l}5.65 \\
5.05 \\
4.85 \\
4.8 \\
4.85\end{array}$ & $\begin{array}{l}\text { 4. } 72 \\
\text { 4. } 5 \\
\text { 4. } 38 \\
\text { 4. } 3 \\
\text { 4. } 35\end{array}$ & $\begin{array}{l}4.3 \\
4.45 \\
4.4 \\
4.5 \\
4.5\end{array}$ & $\begin{array}{l}4.1 \\
4.05 \\
4.15 \\
4.15 \\
4.1\end{array}$ & $\begin{array}{l}4.05 \\
4.0 \\
3.95 \\
4.0 \\
4.1\end{array}$ & $\begin{array}{l}4.0 \\
4.0 \\
4.05 \\
4.05 \\
4.0\end{array}$ & $\begin{array}{l}4.02 \\
4.05 \\
4.1 \\
4.1 \\
4.1\end{array}$ \\
\hline $\begin{array}{r}6 \ldots \\
7 \ldots \\
8 \ldots \\
9 \ldots \\
10 \ldots\end{array}$ & \begin{tabular}{l|}
4.2 \\
4.2 \\
4.2 \\
4.2 \\
4.2
\end{tabular} & $\cdots$ & $\begin{array}{l}\text { 4. } 65 \\
4.7 . \\
6.2 \\
6.2 \\
5.6\end{array}$ & $\begin{array}{l}4.72 \\
4.72 \\
4.7 \\
4.75 \\
5.1\end{array}$ & $\begin{array}{l}5.0 \\
4.9 \\
4.9 \\
4.9 \\
5.0\end{array}$ & $\begin{array}{l}6.05 \\
6.7 \\
5.3 \\
4.95 \\
4.8\end{array}$ & $\begin{array}{l}\text { 4. } 35 \\
\text { 4. } 32 \\
\text { 4. } 35 \\
\text { 4. } 4 \\
6.15\end{array}$ & $\begin{array}{l}4.8 \\
4.5 \\
4.5 \\
4.45 \\
4.38\end{array}$ & $\begin{array}{l}4.05 \\
4.05 \\
4.1 \\
4.05 \\
4.1\end{array}$ & $\begin{array}{l}4.1 \\
4.0 \\
4.05 \\
4.0 \\
4.0\end{array}$ & $\begin{array}{l}4.05 \\
4.05 \\
4.0 \\
4.0 \\
4.1\end{array}$ & $\begin{array}{l}4.2 \\
4.2 \\
4.1 \\
4.1 \\
4.15\end{array}$ \\
\hline & $\begin{array}{l}4.2 \\
4.2 \\
4.2 \\
4.2 \\
4.2\end{array}$ & . & $\begin{array}{l}\text { 5. } 15 \\
5.0 \\
4.9 \\
4.8 \\
4.78\end{array}$ & $\begin{array}{l}5.38 \\
5.15 \\
5.0 \\
4.9 \\
4.8\end{array}$ & $\begin{array}{l}\text { 4. } 95 \\
\text { 4. } 9 \\
4.8 \\
\text { 4. } 8 \\
4.75\end{array}$ & $\begin{array}{l}4.72 \\
4.6 \\
4.5 \\
4.5 \\
4.4\end{array}$ & $\begin{array}{l}6.82 \\
6.0 \\
5.75 \\
5.1 \\
4.85\end{array}$ & $\begin{array}{l}4.4 \\
4.35 \\
4.35 \\
4.3 \\
4.2\end{array}$ & \begin{tabular}{l|}
4.1 \\
4.1 \\
4.05 \\
4.2 \\
4.2
\end{tabular} & $\begin{array}{l}4.0 \\
4.1 \\
4.0 \\
4.05 \\
4.0\end{array}$ & \begin{tabular}{l|}
4.1 \\
4.05 \\
4.1 \\
4.1 \\
4.05
\end{tabular} & $\begin{array}{l}4.2 \\
4.5 \\
4.5 \\
4.5 \\
4.5\end{array}$ \\
\hline & $\begin{array}{l}\text { 4. } 2 \\
\text { 4. } \\
\text { 4. } 2 \\
\text { 4. } \\
\text { 4. } 2\end{array}$ & $\cdots$ & $\begin{array}{l}4.7 \\
4.7 \\
4.68 \\
4.6 \\
4.52\end{array}$ & $\begin{array}{l}4.72 \\
4.65 \\
4.55 \\
4.58 \\
4.5\end{array}$ & $\begin{array}{l}4.8 \\
4.7 \\
4.7 \\
4.78 \\
6.45\end{array}$ & $\begin{array}{l}\text { 4. } 45 \\
\text { 4. } 45 \\
\text { 4. } 42 \\
\text { 4. } 5 \\
\text { 4. } 75\end{array}$ & $\begin{array}{l}4.7 \\
4.55 \\
4.55 \\
4.5 \\
4.7\end{array}$ & $\begin{array}{l}4.3 \\
4.2 \\
4.22 \\
4.2 \\
4.22\end{array}$ & $\begin{array}{l}4.15 \\
4.15 \\
4.1 \\
4.1 \\
4.05\end{array}$ & $\begin{array}{l}4.05 \\
4.05 \\
4.05 \\
4.0 \\
4.05\end{array}$ & $\begin{array}{l}4.1 \\
4.1 \\
4.02 \\
4.0 \\
4.0\end{array}$ & $\begin{array}{l}4.5 \\
4.5 \\
4.5 \\
4.5 \\
4.5\end{array}$ \\
\hline & $\begin{array}{l}4.2 \\
4.2 \\
4.2 \\
(b)\end{array}$ & & $\begin{array}{l}\text { 4. } 55 \\
\text { 4. } 52 \\
\text { 4. } 65 \\
\text { 4. } 75 \\
\text { 4. } 8\end{array}$ & $\begin{array}{l}4.45 \\
4.48 \\
4.4 \\
4.4 \\
4.35\end{array}$ & $\begin{array}{l}5.7 \\
5.35 \\
5.15 \\
4.95 \\
4.78\end{array}$ & $\begin{array}{l}\text { 5. } 25 \\
5.3 \\
4.85 \\
\text { 4. } 62 \\
\text { 4. } 45\end{array}$ & $\begin{array}{l}\text { 4. } 55 \\
\text { 4. } 4 \\
\text { 4. } 38 \\
\text { 4. } 35 \\
4.5\end{array}$ & $\begin{array}{l}4.2 \\
4.2 \\
4.3 \\
4.35 \\
4.2\end{array}$ & $\begin{array}{l}4.25 \\
4.1 \\
4.05 \\
4.1 \\
4.0\end{array}$ & $\begin{array}{l}4.05 \\
4.0 \\
4.1 \\
4.1 \\
4.0\end{array}$ & \begin{tabular}{l|}
4.0 \\
4.1 \\
4.1 \\
4.1 \\
4.02
\end{tabular} & $\begin{array}{l}4.5 \\
4.5 \\
4.5 \\
4.5 \\
4.5\end{array}$ \\
\hline & & & $\begin{array}{l}4.8 \\
4.8 \\
4.78 \\
4.7 \\
4.7 \\
4.78\end{array}$ & $\begin{array}{l}4.5 \\
4.6 \\
6.0 \\
7.05 \\
6.4\end{array}$ & $\begin{array}{l}5.15 \\
4.95 \\
4.8 \\
4.7 \\
4.6 \\
4.8\end{array}$ & $\begin{array}{l}4.4 \\
4.35 \\
4.4 \\
4.38 \\
4.75\end{array}$ & $\begin{array}{l}4.5 \\
4.4 \\
4.4 \\
4.65 \\
4.55 \\
4.45\end{array}$ & $\begin{array}{l}4.25 \\
4.2 \\
4.2 \\
4.1 \\
4.1 \\
4.15\end{array}$ & $\begin{array}{l}4.0 \\
3.98 \\
4.05 \\
4.05 \\
4.1\end{array}$ & $\begin{array}{l}4.1 \\
4.05 \\
4.05 \\
4.1 \\
4.02 \\
4.05\end{array}$ & $\begin{array}{l}\text { 4. } 0 \\
4.05 \\
4.0 \\
4.05 \\
4.1\end{array}$ & $\begin{array}{l}\text { 4. } 55 \\
\text { 4. } 6 \\
4.65 \\
\text { 4. } 45 \\
4.45 \\
\text { 4. } 35\end{array}$ \\
\hline $\begin{array}{l}19 \\
1 \ldots \ldots \\
2 \ldots \ldots \\
3 \ldots \ldots \\
4 \ldots \ldots \\
5 \ldots \ldots\end{array}$ & $\begin{array}{l}4.28 \\
4.2 \\
4.35 \\
5.55 \\
5.9\end{array}$ & $\begin{array}{l}5.5 \\
5.5 \\
5.5 \\
5.5 \\
5.5\end{array}$ & $\begin{array}{l}\text { 5. } 5 \\
5.5 \\
5.5 \\
5.5\end{array}$ & $\begin{array}{l}5.45 \\
5.3 \\
5.2 \\
5.15 \\
5.15\end{array}$ & $\begin{array}{l}4.6 \\
4.7 \\
4.6 \\
4.6 \\
4.52\end{array}$ & $\begin{array}{l}\text { 4. } 6 \\
\text { 5. } 05 \\
\text { 4. } 85 \\
\text { 4. } 68 \\
\text { 4. } 55\end{array}$ & $\begin{array}{l}5.3 \\
5.45 \\
5.25 \\
5.05 \\
5.3\end{array}$ & $\begin{array}{l}5.05 \\
4.8 \\
4.65 \\
4.6 \\
4.6\end{array}$ & $\begin{array}{l}4.4 \\
4.4 \\
4.35\end{array}$ & $\begin{array}{l}4.2 \\
4.2 \\
4.15 \\
4.25 \\
4.2\end{array}$ & $\begin{array}{l}4.35 \\
4.4 \\
4.3\end{array}$ & $\begin{array}{l}\text { 4. } 22 \\
4.15 \\
4.45 \\
4.7 \\
5.05\end{array}$ \\
\hline & $\begin{array}{l}5.9 \\
5.9\end{array}$ & $\begin{array}{l}5.5 \\
5.5 \\
5.5 \\
5.5 \\
5.5\end{array}$ & $\begin{array}{l}5.8 \\
5.75 \\
6.0 \\
6.4 \\
7.65\end{array}$ & $\begin{array}{l}5.6 \\
5.8 \\
5.65 \\
5.5 \\
5.4\end{array}$ & $\begin{array}{l}4.6 \\
\text { 4. } 6 \\
\text { 4. } 52 \\
4.55 \\
4.5\end{array}$ & $\begin{array}{l}4.5 \\
4.9 \\
\text { 4. } 95 \\
4.7 \\
4.5\end{array}$ & $\begin{array}{l}6.9 \\
5.75 \\
5.5 \\
5.7 \\
5.3\end{array}$ & $\begin{array}{l}4.48 \\
4.4 \\
4.35 \\
4.38 \\
4.4\end{array}$ & $\begin{array}{l}4.45 \\
4.4 \\
4.4 \\
4.35 \\
4.3\end{array}$ & $\begin{array}{l}4.15 \\
4.15 \\
4.15 \\
4.15 \\
4.05\end{array}$ & $\begin{array}{l}4.35 \\
4.35 \\
4.3 \\
4.22 \\
4.32\end{array}$ & $\begin{array}{l}4.9 \\
4.75 \\
4.7 \\
4.55 \\
4.5\end{array}$ \\
\hline & & $\begin{array}{l}5.5 \\
5.5 \\
5.5 \\
5.5 \\
5.5\end{array}$ & $\begin{array}{l}7.45 \\
6.75 \\
6.4 \\
5.95 \\
\mathbf{5 . 7 5}\end{array}$ & $\begin{array}{l}5.25 \\
5.3 \\
5.25 \\
5.15 \\
5.1\end{array}$ & $\begin{array}{l}4.5 \\
\text { 4. } 5 \\
\text { 4. } 5 \\
\text { 4. } 55 \\
\text { 5. } 45\end{array}$ & $\begin{array}{l}\text { 4. } 42 \\
4.25 \\
4.65 \\
4.4 \\
4.4\end{array}$ & $\begin{array}{l}5.35 \\
6.15 \\
5.75 \\
6.9 \\
7.1\end{array}$ & $\begin{array}{l}4.4 \\
4.4 \\
4.32 \\
5.1 \\
4.7\end{array}$ & $\begin{array}{l}\text { 4. } 28 \\
4.3 \\
4.3 \\
4.3 \\
4.28\end{array}$ & $\begin{array}{l}4.25 \\
4.35 \\
4.25 \\
4.2 \\
4.2\end{array}$ & $\begin{array}{l}4.38 \\
4.32 \\
4.28 \\
4.15 \\
4.22\end{array}$ & $\begin{array}{l}4.42 \\
4.42 \\
4.42 \\
4.48 \\
4.38\end{array}$ \\
\hline & & $\begin{array}{l}5.5 \\
5.5 \\
5.5 \\
5.5 \\
5.5\end{array}$ & $\begin{array}{l}5.6 \\
5.5 \\
5.4 \\
5.4 \\
5.4\end{array}$ & $\begin{array}{l}5.05 \\
4.98 \\
4.92 \\
4.9 \\
4.9\end{array}$ & $\begin{array}{l}\text { 6. } 15 \\
\text { 5. } 65 \\
5.35 \\
5.2 \\
4.55\end{array}$ & $\begin{array}{l}4.3 \\
4.2 \\
4.2 \\
4.25 \\
4.2\end{array}$ & $\begin{array}{l}6.1 \\
5.5 \\
5.2 \\
5.05 \\
4.92\end{array}$ & $\begin{array}{l}5.0 \\
4.8 \\
4.7\end{array}$ & $\begin{array}{l}\text { 4. } 32 \\
4.32 \\
4.4 \\
4.32 \\
4.32\end{array}$ & $\begin{array}{l}4.25 \\
4.25 \\
4.25 \\
4.2 \\
4.25\end{array}$ & $\begin{array}{l}4.2 \\
4.18 \\
4.1 \\
4.18 \\
4.2\end{array}$ & $\begin{array}{l}4.4 \\
4.55 \\
4.7 \\
4.62 \\
4.6\end{array}$ \\
\hline & $\begin{array}{l}4.75 \\
5.5 \\
5.5\end{array}$ & $\begin{array}{l}5.5 \\
5.5 \\
5.5 \\
5.5 \\
5.5\end{array}$ & $\begin{array}{l}5.95 \\
6.4 \\
6.5 \\
6.05 \\
7.45\end{array}$ & $\begin{array}{l}4.8 \\
4.8 \\
4.75 \\
4.7 \\
4.7\end{array}$ & $\begin{array}{l}\text { 4. } 92 \\
4.8 \\
4.7 \\
4.62 \\
4.6\end{array}$ & $\begin{array}{c}6.2 \\
6.95 \\
7.5 \\
10.75 \\
9.05\end{array}$ & $\begin{array}{l}4.8 \\
4.8 \\
5.55 \\
5.95 \\
5.4\end{array}$ & $\begin{array}{l}4.6 \\
4.4 \\
4.4 \\
4.35 \\
5.0\end{array}$ & $\begin{array}{l}4.28 \\
4.25 \\
4.3 \\
4.2 \\
4.2\end{array}$ & $\begin{array}{l}4.2 \\
4.2 \\
4.15 \\
4.1 \\
4.2\end{array}$ & $\begin{array}{l}4.15 \\
4.18 \\
4.2 \\
4.2 \\
4.22\end{array}$ & $\begin{array}{l}\text { 6. } \\
6.5\end{array}$ \\
\hline $26 \ldots$ & $\begin{array}{l}5.5 \\
5.5 \\
5.5 \\
5.5 \\
5.5 \\
5.5\end{array}$ & $\begin{array}{l}5.5 \\
5.5 \\
5.5\end{array}$ & $\begin{array}{l}6.95 \\
6.5 \\
6.2 \\
5.9 \\
5.7 \\
5.55\end{array}$ & $\begin{array}{l}4.7 \\
4.7 \\
4.8 \\
4.8 \\
4.7\end{array}$ & $\begin{array}{l}\text { 4. } 55 \\
4.4 \\
4.5 \\
\text { 4. } 42 \\
\text { 4. } 4 \\
4.45\end{array}$ & $\begin{array}{l}7.0 \\
6.2 \\
6.2 \\
5.7 \\
5.4\end{array}$ & $\begin{array}{l}5.15 \\
4.92 \\
4.78 \\
4.75 \\
5.6 \\
5.7\end{array}$ & $\begin{array}{l}6.1 \\
5.45 \\
4.9 \\
4.75 \\
4.6 \\
4.5\end{array}$ & $\begin{array}{l}4.15 \\
4.2 \\
4.2 \\
4.2 \\
4.2\end{array}$ & $\begin{array}{l}\text { 4.5 } \\
4.48 \\
4.35 \\
4.3 \\
4.32 \\
4.42\end{array}$ & $\begin{array}{l}4.22 \\
4.18 \\
4.12 \\
4.2 \\
4.2\end{array}$ & $\begin{array}{l}5.95 \\
5.55 \\
5.4 \\
5.7 \\
0.28 \\
6.0\end{array}$ \\
\hline
\end{tabular}

$a$ River frozen January 1-23, and December 12-26, 1904

$b$ Gage removed January 23, 1904, to protect from ice; replaced March 5, 1904.

$c$ River frozen January 5 to March 4, 1905. 
Daily gage height, in feet, of North Fork of Shenandoah River near Riverton, Va.-Cont'd.

\begin{tabular}{|c|c|c|c|c|c|c|c|c|c|c|c|c|}
\hline Day. & Jan. & Feb. & Mar. & Apr. & May. & June. & July. & Aug. & Sept. & Oct. & Nov. & Dec. \\
\hline $\begin{array}{c}1906 . \\
1 \ldots \ldots \ldots \\
2 \ldots \ldots \ldots \\
3 \ldots \ldots \ldots \\
4 \ldots \ldots \ldots \\
5 \ldots \ldots \ldots\end{array}$ & $\begin{array}{l}5.7 \\
5.45 \\
5.4 \\
8.6 \\
7.75\end{array}$ & \begin{tabular}{|l}
5.3 \\
5.2 \\
5.1 \\
a 5.5 \\
5.0
\end{tabular} & $\begin{array}{l}4.55 \\
4.5 \\
4.75 \\
6.45 \\
6.55\end{array}$ & \begin{tabular}{l|}
7.9 \\
7.1 \\
6.7 \\
6.32 \\
6.1
\end{tabular} & $\begin{array}{l}5.65 \\
5.5 \\
5.4 \\
5.35 \\
5.25\end{array}$ & $\begin{array}{l}4.62 \\
4.7 \\
4.58 \\
4.52 \\
4.65\end{array}$ & $\begin{array}{l}5.05 \\
4.88 \\
4.85 \\
6.12 \\
5.52\end{array}$ & $\begin{array}{l}- \\
\cdots\end{array}$ & . & & & - $\ldots$ \\
\hline $\begin{array}{r}6 \ldots \ldots \ldots \\
7 \ldots \ldots \\
8 \ldots \ldots \\
9 \ldots \ldots \ldots \\
10 \ldots \ldots \ldots \\
\ldots \ldots\end{array}$ & $\begin{array}{l}6.83 \\
6.2 \\
5.85 \\
5.58 \\
5.35\end{array}$ & $\begin{cases}\text { a } & 5.98 \\
a & 5.6 \\
a & 5.5 \\
a & 5.25\end{cases}$ & $\begin{array}{l}\text { 5. } 9 \\
5.55 \\
5.45 \\
5.35 \\
5.25\end{array}$ & $\begin{array}{l}5.92 \\
5.75 \\
5.6 \\
5.52 \\
6.1\end{array}$ & $\begin{array}{l}5.32 \\
5.32 \\
5.48 \\
5.45 \\
5.3\end{array}$ & $\begin{array}{l}4.62 \\
4.55 \\
4.75 \\
4.62 \\
4.6\end{array}$ & $\begin{array}{l}5.2 \\
4.95 \\
4.82 \\
4.7 \\
4.6\end{array}$ & & & & & \\
\hline $\begin{array}{l}11 \ldots \ldots \\
12 \ldots \ldots \\
13 \ldots \ldots \\
14 \ldots \ldots \\
15 \ldots \ldots \ldots\end{array}$ & $\begin{array}{l}5.37 \\
5.27 \\
5.25 \\
5.3 \\
5.4\end{array}$ & \begin{tabular}{|l} 
a \\
5.45 \\
4.9 \\
4.68 \\
4.6 \\
4.68
\end{tabular} & $\begin{array}{l}5.15 \\
5.0 \\
5.0 \\
5.0 \\
5.05\end{array}$ & $\begin{array}{l}5.95 \\
5.75 \\
5.62 \\
5.28 \\
6.4\end{array}$ & $\begin{array}{l}5.22 \\
5.2 \\
5.05 \\
5.0 \\
4.9\end{array}$ & $\begin{array}{l}4.85 \\
4.78 \\
4.65 \\
4.58 \\
5.05\end{array}$ & $\begin{array}{l}4.6 \\
4.6 \\
4.65 \\
4.62 \\
. \ldots .\end{array}$ & & & & & \\
\hline $\begin{array}{l}16 \ldots \ldots \\
17 \ldots \ldots \ldots \\
18 \ldots \ldots \ldots \\
19 \ldots \ldots \ldots \\
20 \ldots \ldots \ldots \ldots\end{array}$ & $\begin{array}{l}\text { 5. } 48 \\
\text { 5. } 4 \\
5.38 \\
\text { 5. } 33 \\
\text { 5. } 27\end{array}$ & $\begin{array}{l}4.65 \\
4.8 \\
4.55 \\
4.52 \\
4.6\end{array}$ & $\begin{array}{l}5.2 \\
5.45 \\
5.58 \\
5.8 \\
5.9\end{array}$ & $\begin{array}{l}6.98 \\
6.4 \\
6.05 \\
5.88 \\
5.7\end{array}$ & $\begin{array}{l}4.88 \\
4.82 \\
4.8 \\
4.8 \\
4.72\end{array}$ & $\begin{array}{l}5.25 \\
5.5 \\
5.85 \\
5.9 \\
5.65\end{array}$ & $\begin{array}{l}\cdots \ldots \\
\ldots \ldots \\
\cdots \\
\cdots\end{array}$ & & & & & \\
\hline $\begin{array}{l}21 \ldots \ldots \ldots \\
22 \ldots \ldots \ldots \\
23 \ldots \ldots \\
24 \ldots \ldots \\
25 \ldots \ldots\end{array}$ & $\begin{array}{l}5.2 \\
5.1 \\
5.05 \\
5.7 \\
5.9\end{array}$ & $\begin{array}{l}4.6 \\
4.6 \\
4.6 \\
4.6 \\
4.53\end{array}$ & $\begin{array}{l}5.95 \\
6.2 \\
6.55 \\
6.45 \\
6.2\end{array}$ & $\begin{array}{l}\text { 5. } 58 \\
5.48 \\
5.4 \\
5.28 \\
5.2\end{array}$ & $\begin{array}{l}4.62 \\
4.6 \\
4.6 \\
4.55 \\
4.55\end{array}$ & $\begin{array}{l}6.85 \\
7.25 \\
6.6 \\
5.8 \\
5.55\end{array}$ & $\because$ & & & & & 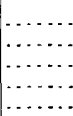 \\
\hline $\begin{array}{l}26 \ldots \ldots \ldots \\
27 \ldots \ldots \\
28 \ldots \ldots \\
29 \ldots \ldots \\
30 \ldots \ldots \\
31 \ldots \ldots \ldots \\
\end{array}$ & $\begin{array}{l}5.55^{\circ} \\
5.33 \\
5.3 \\
5.37 \\
5.43 \\
5.35\end{array}$ & $\begin{array}{r}4.55 \\
4.52 \\
4.58 \\
\cdots \cdots \\
\cdots \cdots \\
\cdots\end{array}$ & $\begin{array}{l}6.1 \\
7.5 \\
9.4 \\
8.45 \\
8.35 \\
8.1\end{array}$ & $\begin{array}{l}5.2 \\
7.48 \\
6.7 \\
6.15 \\
5.85 \\
\cdots \cdots\end{array}$ & $\begin{array}{l}4.52 \\
4.52 \\
5.08 \\
4.9 \\
4.75 \\
4.65\end{array}$ & $\begin{array}{l}5.3 \\
6.1 \\
6.2 \\
5.7 \\
5.35\end{array}$ & . & & & . & - & \\
\hline
\end{tabular}

$a$ Backwater due to ice conditions February 4, 7-11, and 17, 1906.

Rating tables for North Fork of Shenandoah River near Riverton, $V a$.

JUNE 26, 1899, TO FEBRUARY 25, 1902,a

\begin{tabular}{|c|c|c|c|c|c|c|c|}
\hline $\begin{array}{c}\text { Gage } \\
\text { height. }\end{array}$ & Discharge. & $\begin{array}{c}\text { Gage } \\
\text { height. }\end{array}$ & Discharge. & $\begin{array}{c}\text { Gage } \\
\text { height. }\end{array}$ & Discharge. & $\begin{array}{c}\text { Gage } \\
\text { height. }\end{array}$ & Discharge. \\
\hline $\begin{array}{l}\text { Feet. } \\
3.50 \\
3.60 \\
3.70 \\
3.80 \\
3.90 \\
4.00 \\
4.10 \\
4.20 \\
4.30 \\
4.40\end{array}$ & \begin{tabular}{|c} 
Second-feet. \\
90 \\
140 \\
195 \\
255 \\
320 \\
390 \\
465 \\
545 \\
625 \\
710
\end{tabular} & $\begin{array}{r}\text { Feet. } \\
4.50 \\
4.60 \\
4.70 \\
4.80 \\
4.90 \\
5.00 \\
5.10 \\
5.20 \\
5.30\end{array}$ & $\begin{array}{c}\text { Second-feet. } \\
800 \\
895 \\
990 \\
1,090 \\
1,190 \\
1,300 \\
1,410 \\
1,520 \\
1,640\end{array}$ & $\begin{array}{l}\text { Feet. } \\
5.40 \\
5.50 \\
5.60 \\
5.70 \\
5.80 \\
5.90 \\
6.00 \\
6.10 \\
6.20\end{array}$ & \begin{tabular}{|c} 
Second-feet. \\
1,760 \\
1,880 \\
2,000 \\
2,130 \\
2,260 \\
2,390 \\
2,520 \\
2,650 \\
2,780
\end{tabular} & $\begin{array}{l}\text { Feet. } \\
6.30 \\
6.40 \\
6.50 \\
6.60 \\
6.70 \\
6.80 \\
6.90 \\
7.00 \\
8.00\end{array}$ & \begin{tabular}{|c} 
Second-feet \\
2,910 \\
3,050 \\
3,190 \\
3,330 \\
3,470 \\
3,610 \\
3,750 \\
3,890 \\
5,380
\end{tabular} \\
\hline
\end{tabular}

$a$ This table is strictly applicable only for open-channel conditions. It is based on six discharge measurements made during 1899-1901. It is fairly well defined between gage heights 3.5 feet and 6.5 feet. Estimates above gage height 8.0 feet were determined directly from the curve; no rating table was prepared. 
Rating tables for North Fork of Shenandoah River near Riverton, Va:-Continued.

AUGUST 16, 1902, TO AUGUST 15, 1904.a

\begin{tabular}{|c|c|c|c|c|c|c|c|}
\hline $\begin{array}{c}\text { Gage } \\
\text { height. }\end{array}$ & Discharge. & $\begin{array}{c}\text { Gage } \\
\text { height. }\end{array}$ & Discharge. & $\begin{array}{c}\text { Gage } \\
\text { height. }\end{array}$ & Discharge. & $\begin{array}{c}\text { Gage } \\
\text { height. }\end{array}$ & Discharge. \\
\hline $\begin{array}{l}\text { Feet. } \\
3.90 \\
4.00 \\
4.10 \\
4.20 \\
4.30 \\
4.40 \\
4.50 \\
4.60 \\
4.70 \\
4.80 \\
4.90\end{array}$ & \begin{tabular}{|c|} 
Second-feet. \\
165 \\
190 \\
220 \\
255 \\
295 \\
340 \\
390 \\
440 \\
495 \\
550 \\
610
\end{tabular} & $\begin{array}{l}\text { Feet. } \\
5.00 \\
5.10 \\
5.20 \\
5.30 \\
5.40 \\
5.50 \\
5.60 \\
5.70 \\
5.80 \\
5.90 \\
6.00\end{array}$ & \begin{tabular}{|r|} 
Second-feet. \\
670 \\
735 \\
800 \\
870 \\
940 \\
1,015 \\
1,090 \\
1,170 \\
1,255 \\
1,340 \\
1,430
\end{tabular} & $\begin{array}{l}\text { Feet. } \\
6.10 \\
6.20 \\
6.30 \\
6.40 \\
6.50 \\
6.60 \\
6.70 \\
6.80 \\
6.90 \\
7.00 \\
7.10\end{array}$ & \begin{tabular}{|c|} 
Second-feet. \\
1,520 \\
1,610 \\
1,700 \\
1,790 \\
1,890 \\
1,990 \\
2,090 \\
2,190 \\
2,300 \\
2,410 \\
2,520
\end{tabular} & $\begin{array}{l}\text { Feet. } \\
7.20 \\
7.30 \\
7.40 \\
7.50 \\
7.60 \\
7.70 \\
7.80 \\
7.90 \\
8.00\end{array}$ & \begin{tabular}{|c} 
Second-feet. \\
2,640 \\
2,760 \\
2,880 \\
3,010 \\
3,140 \\
3,270 \\
3,400 \\
3,540 \\
3,680
\end{tabular} \\
\hline
\end{tabular}

AUGUS'T 16, 1904, TO JULY 14, 1906.b

\begin{tabular}{|c|c|c|c|c|c|c|c|}
\hline $\begin{array}{l}4.00 \\
4.10 \\
4.20 \\
4.30 \\
4.40 \\
4.50 \\
4.60 \\
4.70 \\
4.80 \\
4.90 \\
5.00\end{array}$ & $\begin{array}{l}100 \\
125 \\
155 \\
190 \\
230 \\
275 \\
325 \\
375 \\
425 \\
480 \\
535\end{array}$ & $\begin{array}{l}5.10 \\
5.20 \\
5.30 \\
5.40 \\
5.50 \\
5.60 \\
5.70 \\
5.80 \\
5.90 \\
6.00\end{array}$ & $\begin{array}{r}595 \\
655 \\
720 \\
785 \\
855 \\
925 \\
1,000 \\
1,075 \\
1,155 \\
1,235\end{array}$ & $\begin{array}{l}6.10 \\
6.20 \\
6.30 \\
6.40 \\
6.50 \\
6.60 \\
6.70 \\
6.80 \\
6.90 \\
7.00\end{array}$ & $\begin{array}{l}1,320 \\
1,405 \\
1,495 \\
1,585 \\
1,680 \\
1,775 \\
1,870 \\
1,970 \\
2,070 \\
2,170\end{array}$ & $\begin{array}{l}7.10 \\
7.20 \\
7.30 \\
7.40 \\
7.50 \\
7.60 \\
7.70 \\
7.80 \\
7.90 \\
8.00\end{array}$ & $\begin{array}{l}2,270 \\
2,380 \\
2,490 \\
2,600 \\
2,720 \\
2,840 \\
2,960 \\
3,080 \\
3,210 \\
3,340\end{array}$ \\
\hline
\end{tabular}

a This table is strictly applicable only for open-channel conditions. It is based on four discharge measurements made during 1902,1903, and the first half of 1904. It is fairly well defined between gage heights 4.2 feet and 4.8 feet. Estimates above gage height 8.0 feet were determined directly from the curve.

$b$ This table is strictly applicable only for open-channel conditions. It is based on seven discharge measurements made during the latter part of 1904 and 1905-1906, after the dam had been raised. It is well defined between gage heights 4.0 feet and 5.5 feet. Estimates above gage height 8.0 feet were determined directly from the curve. During August, 1904, the dam below the station was raised 2 feet. The exact date is uncertain, so the table is assumed to apply from August 16, 1904.

Estimated monthly discharge of North Fork of Shenandoah River near Riverton, Va.

[Drainage area, 1,040 square miles.a]

\begin{tabular}{|c|c|c|c|c|c|c|c|c|}
\hline \multirow[b]{2}{*}{ Month. } & \multicolumn{3}{|c|}{ Discharge in second-feet. } & \multicolumn{3}{|c|}{ Run-off. } & \multicolumn{2}{|c|}{ Precipitation.' } \\
\hline & Maximum. & Minimum. & Mean. & $\begin{array}{l}\text { Second- } \\
\text { feet per } \\
\text { square } \\
\text { mile. }\end{array}$ & $\begin{array}{l}\text { Depth in } \\
\text { inches. }\end{array}$ & $\begin{array}{c}\text { Per cent } \\
\text { of pre- } \\
\text { cipita- } \\
\text { tion. }\end{array}$ & $\begin{array}{c}\text { In } \\
\text { inches. }\end{array}$ & $\begin{array}{l}\text { Loss in } \\
\text { inches. }\end{array}$ \\
\hline $\begin{array}{r}1899 . \\
\text { January .... }\end{array}$ & & & & & & & & \\
\hline Febre & & & & & & & 4.95 & \\
\hline March. & & & $\cdots$ & 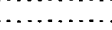 & & & 5.12 & - \\
\hline April... & & & & & & & 1.16 & \\
\hline $\begin{array}{l}\text { May - } 26-30 \\
\text { June } 20-\end{array}$ & 307 & 25 & 2 & .282 & & & $b 2$. & . \\
\hline July .... & 481 & 13 & & .2 & .2 & 10 & & 2.26 \\
\hline A ugu & 390 & 15 & 22 & .212 & .244 & 6 & 3. & 3.70 \\
\hline Septemb & 355 & 14 & 22 & .21 & .2 & 6 & 3.78 & 3.54 \\
\hline Octol & 225 & 140 & 18 & .17 & .2 & 9 & 2.34 & 2.14 \\
\hline Novem! & 54 & & & .230 & .2 & 32 & .81 & .55 \\
\hline Decemb & 390 & 140 & 255 & .246 & .284 & 21 & 1.34 & 1.06 \\
\hline The year & & & & & $\ldots \ldots$ & $\ldots$ & 35.51 & \\
\hline
\end{tabular}

a Drainage area of 1,040 square miles used to obtain run-off for $1906 ; 1,037$ used for all other years. $b$ Precipitation for complete month, June, 1899.

c River frozen December 26-31, 1899; no correction made in estimates. 
Estimated monthly discharge of North Fork of Shenandoah River ncar Riverton, Va.Continued.

\begin{tabular}{|c|c|c|c|c|c|c|c|c|}
\hline \multirow[b]{2}{*}{ Month. - } & \multicolumn{3}{|c|}{ Discharge in second-feet. } & \multicolumn{3}{|c|}{ Run-off. } & \multicolumn{2}{|c|}{ Precipitation. } \\
\hline & Maximum. & Minimum. & Mean. & $\begin{array}{c}\text { Second- } \\
\text { feet per } \\
\text { square } \\
\text { mile. }\end{array}$ & $\begin{array}{l}\text { Depth in } \\
\text { inches. }\end{array}$ & $\begin{array}{c}\text { Per cent } \\
\text { of pre- } \\
\text { eipita- } \\
\text { tion. }\end{array}$ & $\begin{array}{c}\text { In } \\
\text { inches. }\end{array}$ & $\begin{array}{l}\text { Loss in } \\
\text { inches. }\end{array}$ \\
\hline 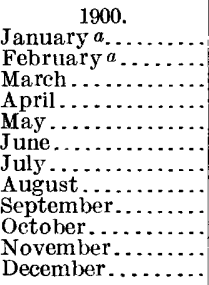 & $\begin{array}{r}4,330 \\
3,862 \\
5,700 \\
1,760 \\
585 \\
2,195 \\
1,616 \\
207 \\
355 \\
320 \\
3,890 \\
2,650\end{array}$ & $\begin{array}{r}255 \\
355 \\
585 \\
465 \\
225 \\
195 \\
140 \\
90 \\
130 \\
168 \\
140 \\
140\end{array}$ & $\begin{array}{r}702 \\
1,128 \\
1,620 \\
765 \\
324 \\
504 \\
294 \\
128 \\
155 \\
219 \\
441 \\
548\end{array}$ & $\begin{array}{l}0.677 \\
1.09 \\
1.56 \\
.738 \\
.312 \\
.486 \\
.284 \\
.123 \\
.149 \\
.211 \\
.425 \\
.528\end{array}$ & $\begin{array}{l}0.780 \\
1.14 \\
1.80 \\
.823 \\
.360 \\
.542 \\
.327 \\
.142 \\
.166 \\
.243 \\
.474 \\
.609\end{array}$ & $\begin{array}{r}30 \\
31 \\
48 \\
43 \\
15 \\
9 \\
9 \\
7 \\
5 \\
8 \\
16 \\
33\end{array}$ & $\begin{array}{l}2.57 \\
3.73 \\
3.72 \\
1.89 \\
2.42 \\
5.98 \\
3.76 \\
1.93 \\
3.14 \\
3.19 \\
2.94 \\
1.83\end{array}$ & $\begin{array}{l}1.79 \\
2.59 \\
1.92 \\
1.07 \\
2.06 \\
5.44 \\
3.43 \\
1.79 \\
2.97 \\
2.95 \\
2.46 \\
1.22\end{array}$ \\
\hline The year..... & 5,700 & 90 & 569 & .549 & 7.41 & 20 & 37.10 & 29.69 \\
\hline 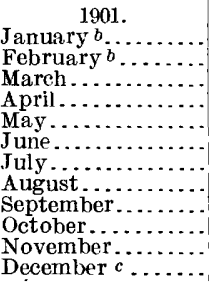 & $\begin{array}{r}2,195 \\
390 \\
10,960 \\
21,630 \\
17,850 \\
12,680 \\
4,030 \\
2,260 \\
2,390 \\
1,322 \\
1,580 \\
14,580\end{array}$ & $\begin{array}{l}195 \\
207 \\
195 \\
693 \\
728 \\
990 \\
505 \\
428 \\
168 \\
195 \\
140 \\
268\end{array}$ & $\begin{array}{r}627 \\
299 \\
1,391 \\
4,034 \\
2,653 \\
2,842 \\
1,499 \\
862 \\
684 \\
396 \\
363 \\
3,091\end{array}$ & $\begin{array}{l}.605 \\
.288 \\
1.34 \\
3.89 \\
2.56 \\
2.74 \\
1.44 \\
.831 \\
.660 \\
.382 \\
.350 \\
2.98\end{array}$ & $\begin{array}{l}.698 \\
.300 \\
1.54 \\
4.34 \\
2.95 \\
3.06 \\
1.66 \\
.958 \\
.736 \\
.440 \\
.390 \\
3.44\end{array}$ & $\begin{array}{l}30 \\
91 \\
41 \\
70 \\
51 \\
41 \\
38 \\
16 \\
20 \\
64 \\
19 \\
56\end{array}$ & $\begin{array}{l}2.31 \\
.33 \\
3.80 \\
6.24 \\
5.82 \\
7.54 \\
4.38 \\
5.92 \\
3.78 \\
.69 \\
2.01 \\
6.12\end{array}$ & $\begin{array}{l}1.61 \\
.03 \\
2.26 \\
1.90 \\
2.87 \\
4.48 \\
2.72 \\
4.96 \\
3.05 \\
.25 \\
1.62 \\
2.68\end{array}$ \\
\hline The year... & 21,630 & 140 & 1,562 & 1.50 & 20.51 & 42 & 48.94 & 28.43 \\
\hline 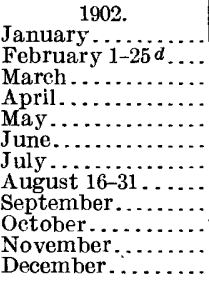 & 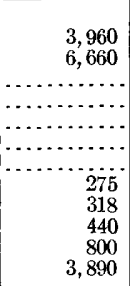 & $\begin{array}{r}220 \\
165 \\
220 \\
190 \\
495\end{array}$ & $\begin{array}{r}243 \\
211 \\
259 \\
317 \\
1,228\end{array}$ & $\begin{array}{l}.234 \\
.204 \\
.250 \\
.306 \\
1.18\end{array}$ & $\begin{array}{l}1.45 \\
1.77 \\
\ldots \ldots \ldots \\
\ldots \ldots \ldots \\
\ldots \ldots \ldots \\
\ldots \ldots \ldots \\
.139 \\
.228 \\
.288 \\
.341 \\
1.36\end{array}$ & 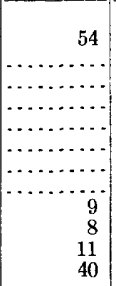 & $\begin{array}{r}2.69 \\
e .46 \\
3.61 \\
2.23 \\
2.64 \\
2.93 \\
2.17 \\
e 2.22 \\
2.59 \\
3.65 \\
3.21 \\
3.38\end{array}$ & 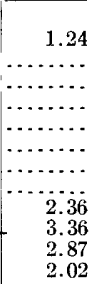 \\
\hline - The year.. & & & & & & $\ldots \ldots \ldots$ & 35.78 & $\cdots$ \\
\hline 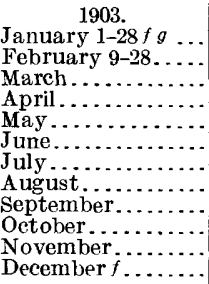 & $\begin{array}{r}8,390 \\
3,205 \\
6,860 \\
6,605 \\
1,340 \\
10,360 \\
1,840 \\
800 \\
1,655 \\
640 \\
295 \\
522\end{array}$ & $\begin{array}{l}495 \\
735 \\
670 \\
735 \\
390 \\
580 \\
340 \\
255 \\
295 \\
275 \\
255 \\
255\end{array}$ & $\begin{array}{r}2,225 \\
1,127 \\
1,750 \\
1,848 \\
549 \\
2,208 \\
736 \\
371 \\
464 \\
373 \\
265 \\
284\end{array}$ & $\begin{array}{l}2.15 \\
1.09 \\
1.69 \\
1.78 \\
.529 \\
2.13 \\
.710 \\
.358 \\
.447 \\
.360 \\
.256 \\
.274\end{array}$ & $\begin{array}{l}2.24 \\
.811 \\
1.95 \\
1.99 \\
.610 \\
2.38 \\
.819 \\
.413 \\
.499 \\
.415 \\
.286 \\
.316\end{array}$ & $\begin{array}{|cc|}\cdots \cdots & \ldots \\
& 47 \\
& 55 \\
& 23 \\
31 \\
\\
27 \\
12 \\
21 \\
18 \\
35 \\
33\end{array}$ & 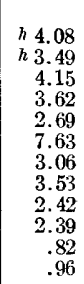 & $\begin{array}{r}. . . \\
2.20 \\
1.63 \\
2.08 \\
5.25 \\
2.24 \\
3.12 \\
1.92 \\
1.97 \\
.53 \\
.64\end{array}$ \\
\hline The year.. & & & & & & $\cdots$ & 38.84 & $\cdots$ \\
\hline
\end{tabular}

a River frozen January 1-14 and January 30 to February 6, 1900; no correction made in estimates.

$b$ Ice conditions during part of January and February, 1901; no correction made in estimates.

$c$ River frozen December 21-28, 1901; no correction made in estimates.

$d$ River frozen February 4-24, 1902; no correction made in estimates.

$e$ Precipitation for complete months, February and August, 1902

$f$ River frozen January 12-28, December 16-23, 26-31, 1903; no correction made in estimates.

$g$ Freshet January 22, 1903 . Gage pushed over by ice; discharge estimated.

$h$ Precipitation for complete months, January and February, 1903. 
Estimated monthly discharge of North Fork of Shenandoah River near Riverton, Va.Continued.

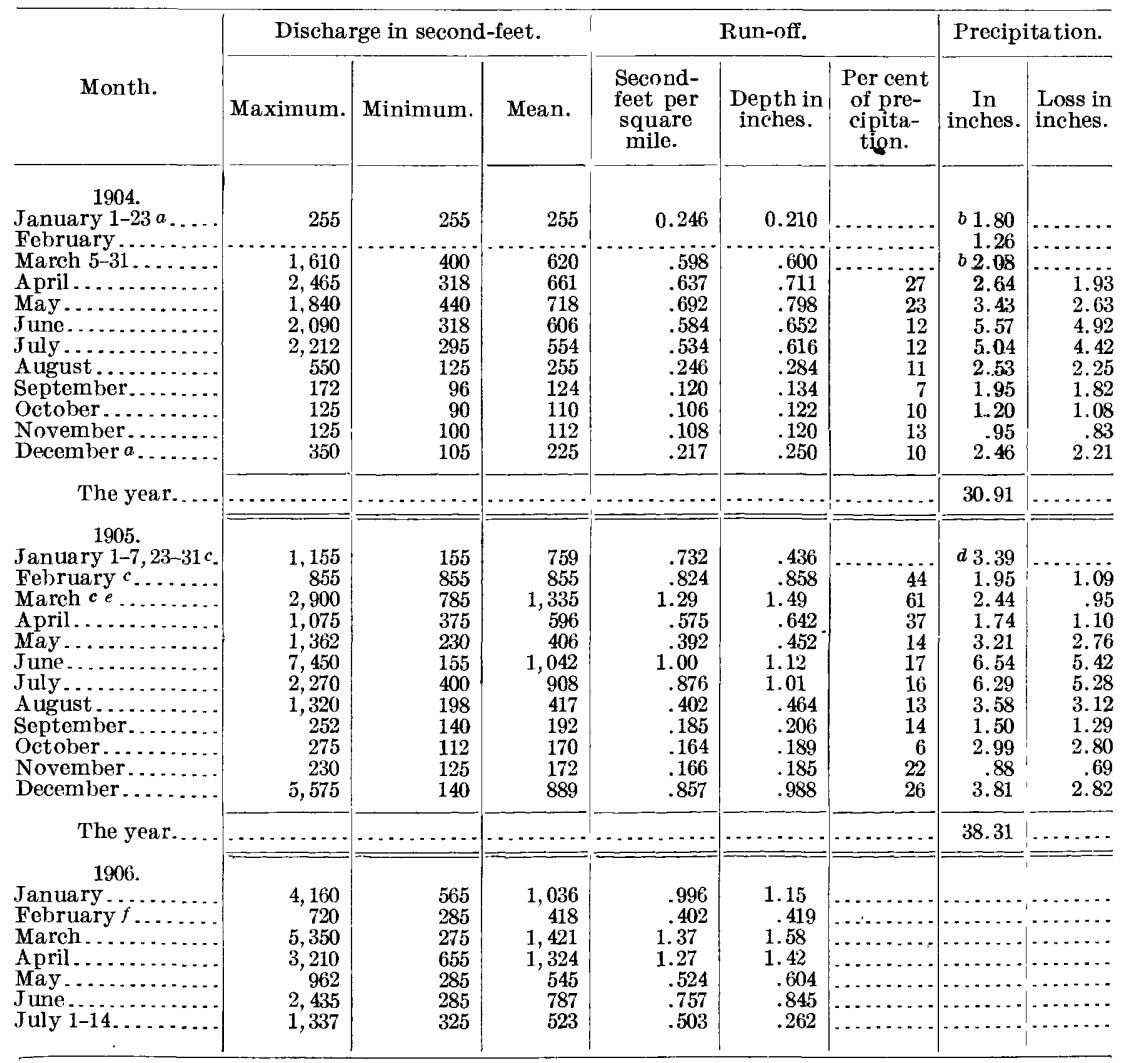

$a$ River frozen January 1-23, and December 12-26, 1904; no correction made in estimates. $b$ Precipitation for complete months, January and March, 1904.

$c$ River frozen January 5 to March 4, 1905; no correction made in estimates.

d Precipitation for complete month, January, 1905.

$e$ Estimate March 5, 1905, interpolated.

$f$ Backwater due to ice conditions February 4, 7-11, and 17, 1906; discharge corrected. 


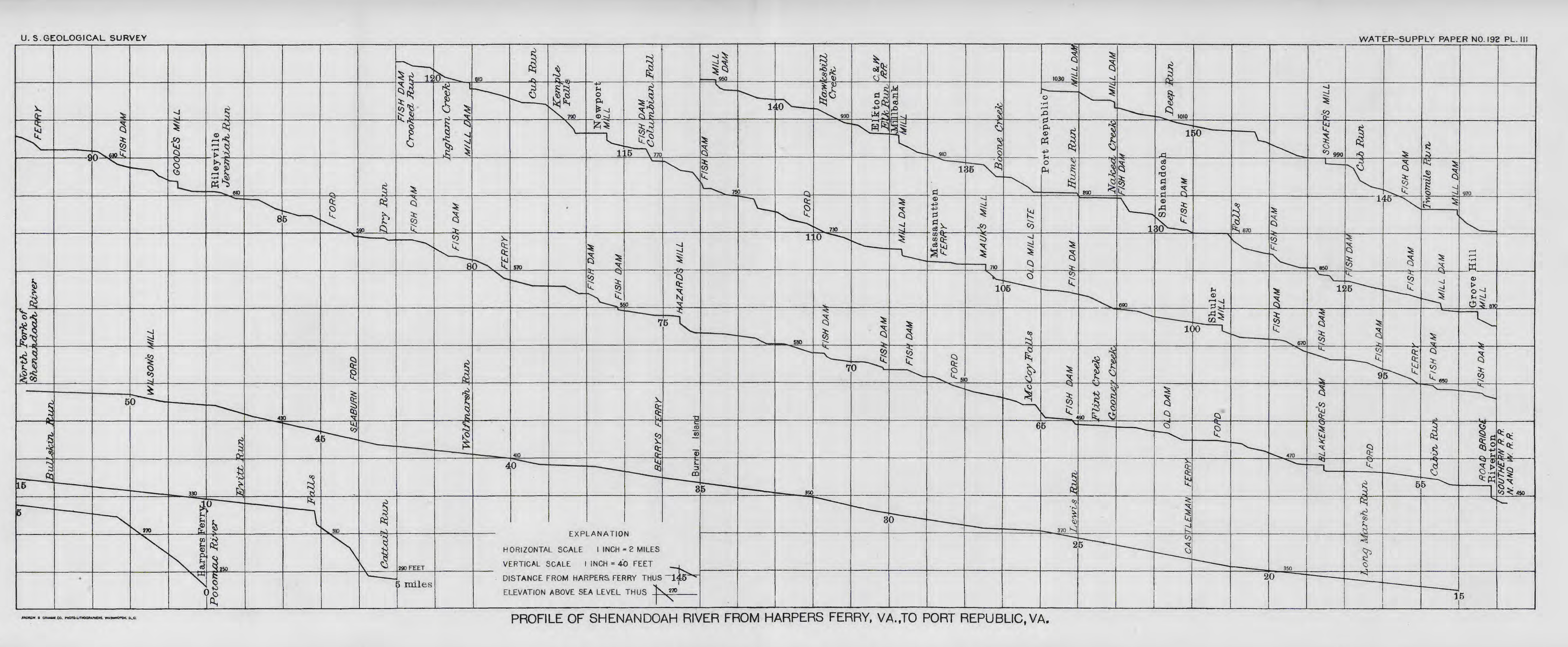




\section{MISCELLANEOUS DISCHARGE MEASUREMENTS IN NORTH FORK OF SHENANDOAH RIVER} BASIN.

The following miscellancous discharge measurements have been made in the basin of North Fork of Shenandoah River:

Miscellaneous discharge measurements in North Fork of Shenandoah River Basin.

\begin{tabular}{|c|c|c|c|c|c|c|}
\hline Date. & Stream. & Locality. & Width. & $\begin{array}{l}\text { Area } \\
\text { of sec- } \\
\text { tion. }\end{array}$ & $\begin{array}{l}\text { Mean } \\
\text { veloc- } \\
\text { ity. }\end{array}$ & $\begin{array}{l}\text { Dis- } \\
\text { charge. }\end{array}$ \\
\hline $\begin{array}{c}1895 . \\
\text { August } 7 \ldots . . .\end{array}$ & $\begin{array}{l}\text { North Fork of Shen- } \\
\text { andoah River. }\end{array}$ & $\begin{array}{l}300 \text { feet below dam at county } \\
\text { bridge and } \frac{2}{3} \text { mile above } \\
\text { junction with South Fork } \\
\text { of Shenandoah River, near } \\
\text { Riverton, Va. }\end{array}$ & Feet. & $\begin{array}{l}\text { Square } \\
\text { feet. } \\
210\end{array}$ & $\begin{array}{l}\text { Feet } \\
\text { per sec. } \\
1.72\end{array}$ & $\begin{array}{l}\text { Second- } \\
\text { feet. } \\
362\end{array}$ \\
\hline $\begin{array}{c}1897 . \\
\text { October } 4 \ldots \\
\text { Do....... }\end{array}$ & Happy Creek. & 200 feet above mouth, near & $\begin{array}{c}150 \\
3.5\end{array}$ & $\begin{array}{l}100 \\
2.1\end{array}$ & $\begin{array}{l}1.40 \\
1.47\end{array}$ & $\begin{array}{l}140 \\
3.1\end{array}$ \\
\hline October 12. & Cedar Creek.. & $\frac{1}{2}$ mile above mouth, near & 18 & 16 & 1.69 & $a_{27}$ \\
\hline October 15 . & Passage Creek. & At entrance to Fort Valley, & 8 & 3 & .2 .00 & 6 \\
\hline October 7 .. & Crooked Run.. & $\begin{array}{l}\text { Near highway bridge, near } \\
\text { Riverton and on road to } \\
\text { Cedarville, Va. }\end{array}$ & 6 & 6 & .58 & 3.4 \\
\hline
\end{tabular}

$a$ Discharge increased by heavy rains of preceding night.

\section{SHENANDOAH RIVER BASIN BELOW NORTH AND SOUTH FORKS.}

SLOPE.

The slope of the Shenandoah and South Fork from Harpers Ferry to Port Republic, Va., is shown on Pl. III. The following table shows the elevations above tide of a number of points on Shenandoah River:

Slope of Shenandoah River.

[Pl. III.]

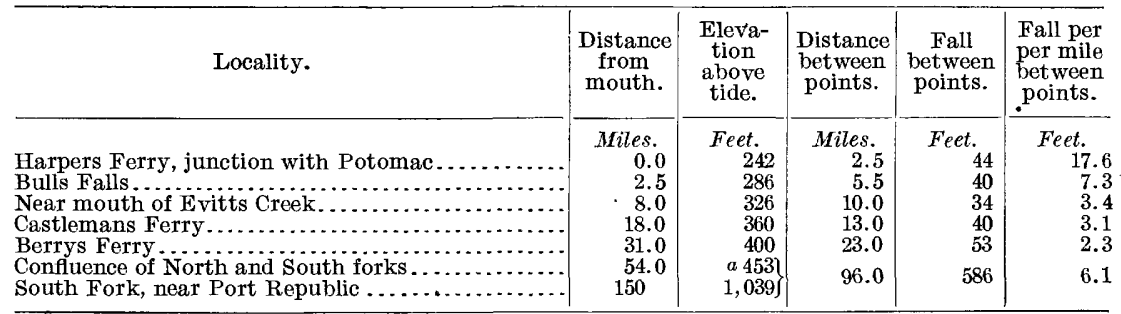

$a$ Profiles of the Norfolk and Western Railway give this elevation as 445 feet.

SHENANDOAH RIVER AT MILIVILLE, w, VA.

The Millville station was established April 15, 1895, by C. C. Babb. It is located about one-fourth mile above the Baltimore and Ohio Railroad station at Millville, W. Va. The highway runs within a few rods of the stream at the gaging station. The station is best reached by driving from Harpers Ferry, W. Va.

The channel is straight for several hundred feet above and below the station, and the current is swift and unobstructed. Both banks are low and liable to overflow. There is but one channel at all stages. The bed of the stream is composed of mud and rocks. 
Discharge measurements are made from a $\frac{3}{4}$-inch cable, from which is suspended a car. The cable, which is suspended over the branches of two large sycamore trees and is securely anchored to the bank at both ends, has a total span of 500 feet. The initial point for soundings is the side of the tree to which the cable is attached on the left bank.

The vertical gage is spiked to a large sycamore tree on the left bank a few hundred feet downstream from the cable. The gage is read once each day by W. R. Nicewarner, the railroad station agent. The bench mark is a copper plug in the upstream side of the base of the second tree downstream from the gage. Its elevation is 6.68 feet above the zero of the gage.

All estimates published prior to 1905 have been revised. For normal conditions of flow the estimates are probably within 5 to 10 per cent of the true discharge up to gage height 6.0 feet. Above gage height 6.0 feet the probable error is from 10 to 15 per cent, or even greater at extreme flood stages. Ice conditions affect the flow at this station during the winter months.

A summary of the records furnishes the following results: Maximum discharge for twenty-four hours, 139,700 second-feet; minimum discharge for twenty-four hours, 480 second-feet; mean annual discharge for eight years, 3,352 second-feet; mean annual rainfall for eleven years, 38.05 inches.

Discharge measurements of Shenandoah River at Milliille, W. Va.

\begin{tabular}{|c|c|c|c|c|c|}
\hline Date. & $\begin{array}{c}\text { Gage } \\
\text { height. }\end{array}$ & Discharge. & Date. & $\begin{array}{c}\text { Gage } \\
\text { height. }\end{array}$ & Discharge. \\
\hline April 24 1895. & Feet. & Second-feet. & $\begin{array}{c}1900 . \\
\text { February } 24\end{array}$ & Feet. & Second-feet. \\
\hline May 1 a....... & 7.50 & 19,710 & June $19 \ldots \ldots \ldots$ & 4.55 & $\begin{array}{r}12,9 \times 0 \\
9,132\end{array}$ \\
\hline May $2 a$. & 6.80 & 15,860 & September $15 .$. & .40 & 500 \\
\hline May $4 .$. & 5.20 & 10,980 & & & \\
\hline May 8.. & 3.08 & 4,311 & 1901. & & \\
\hline May 24. & 3.60 & 5.745 & Iuly $22 \ldots \ldots \ldots$ & 3.30 & 5,419 \\
\hline June $8 \ldots$ & 1.50 & 1,516 & December $27 . .$. & 2.35 & 3,291 \\
\hline Jume $15 \ldots \ldots \ldots \ldots \ldots$ & 2.35 & 3,044 & 1902 & & \\
\hline $\begin{array}{l}\text { June } \\
\text { July } 12, \ldots \ldots \ldots \ldots\end{array}$ & $\begin{array}{l}1.10 \\
1.30\end{array}$ & $\begin{array}{l}1,126 \\
1,150\end{array}$ & August $17 \ldots . .$. & .70 & 811 \\
\hline 1896. & & & 1903. & & \\
\hline June $22 \ldots \ldots$ & 1.99 & 2,513 & August $26 . . .$. . & 1.00 & 1,107 \\
\hline 1897. & & & 1904. & & \\
\hline June 24 . & 1.20 & 1,371 & June $13 \ldots \ldots$. & 1.70 & 1,883 \\
\hline July & 1.82 & 2,791 & July 4 . & 1.20 & 1,137 \\
\hline September $4 . .$. & .52 & 632 & July 10 & 1.34 & 1,371 \\
\hline October $25 \ldots . . . \ldots$. & .72 & 814 & September $28 \ldots \ldots \ldots$ & .50 & 564 \\
\hline 1898. & & & 20. & & \\
\hline January $24 \ldots . .$. & 2.20 & 3,001 & 1905. & & \\
\hline August $16 \ldots \ldots$ & 64.30 & 7,834 & April $22 \ldots . .$. & 1.40 & 1,595 \\
\hline October $1 \ldots$. & .90 & 1,001 & September $20 .$. & .84 & 799 \\
\hline 1899. & & & 1906. & & \\
\hline $\begin{array}{l}\text { January } 27 \ldots \ldots \\
\text { March } 10 . . . .\end{array}$ & $\begin{array}{l}2.40 \\
5.00\end{array}$ & $\begin{array}{r}3,156 \\
10,840\end{array}$ & May $29 . . . .$. & 1.58 & 1,790 \\
\hline May $16 \ldots \ldots$ & 2.10 & 2,753 & & & \\
\hline September 3. & .90 & 1,086 & & & \\
\hline October 29. . & .60 & 766 & & & \\
\hline
\end{tabular}

$a$ The data for these measurements were partly estimated. They were not considered in preparing the rating tables.

$b$ Gage height is uncertain. 
Daily gage height, in feet, of Shenandoah River at Millville, W. Va.

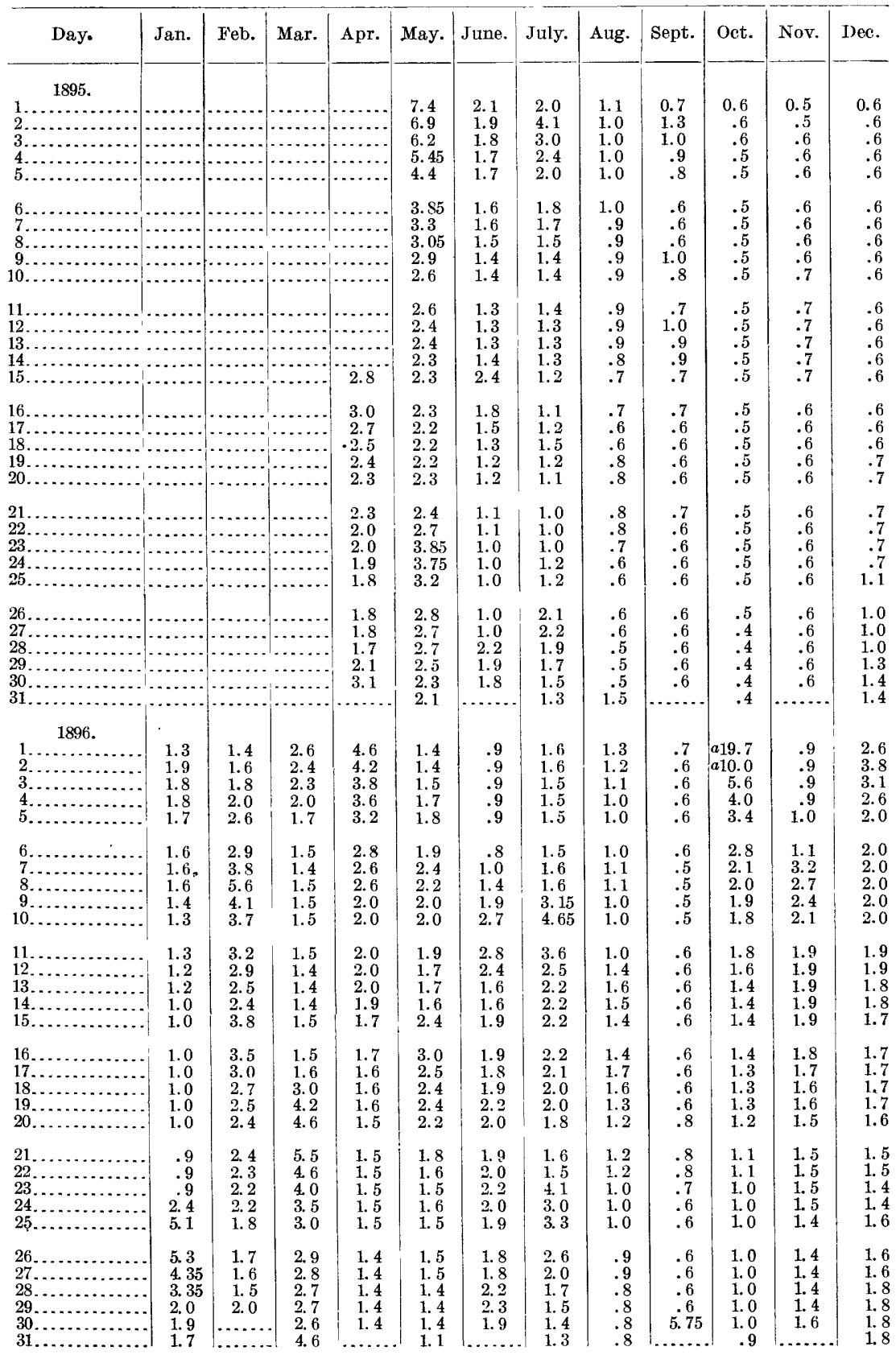

$a$ Gage heights estimated October 1 and 2, 1896. 
Daily gage height, in feet, of Shenandoah River at Millville, W. Va.-Continued.

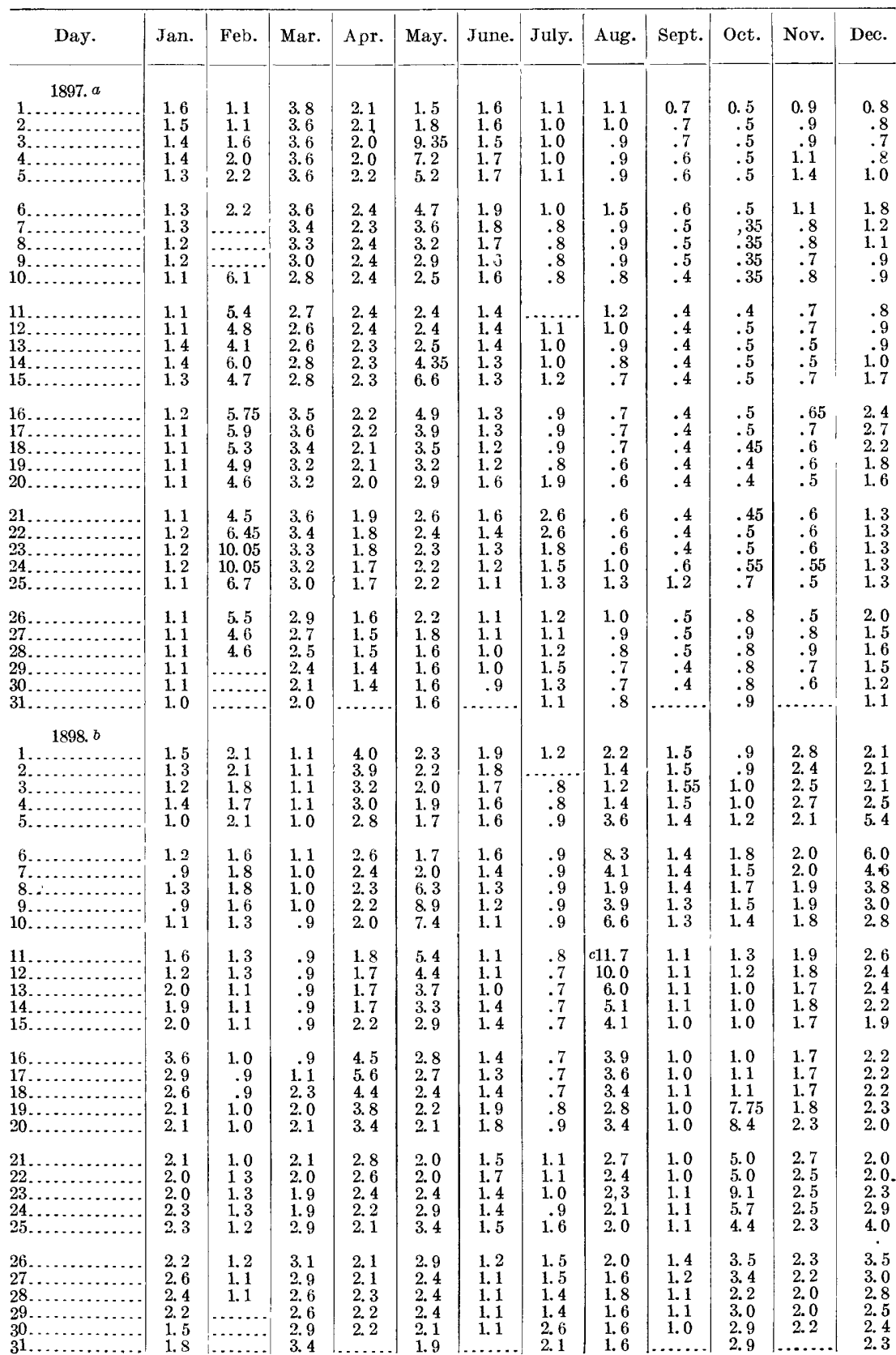

a Tee conditions during January and December 24-31, 1897.

$b$ Ice conditions during February, 1898.

$c$ Gage height estimated August 11, 1898. 
Daily gage height, in feet, of Shenandoah River at Millville, 'W. Va.-Continued.

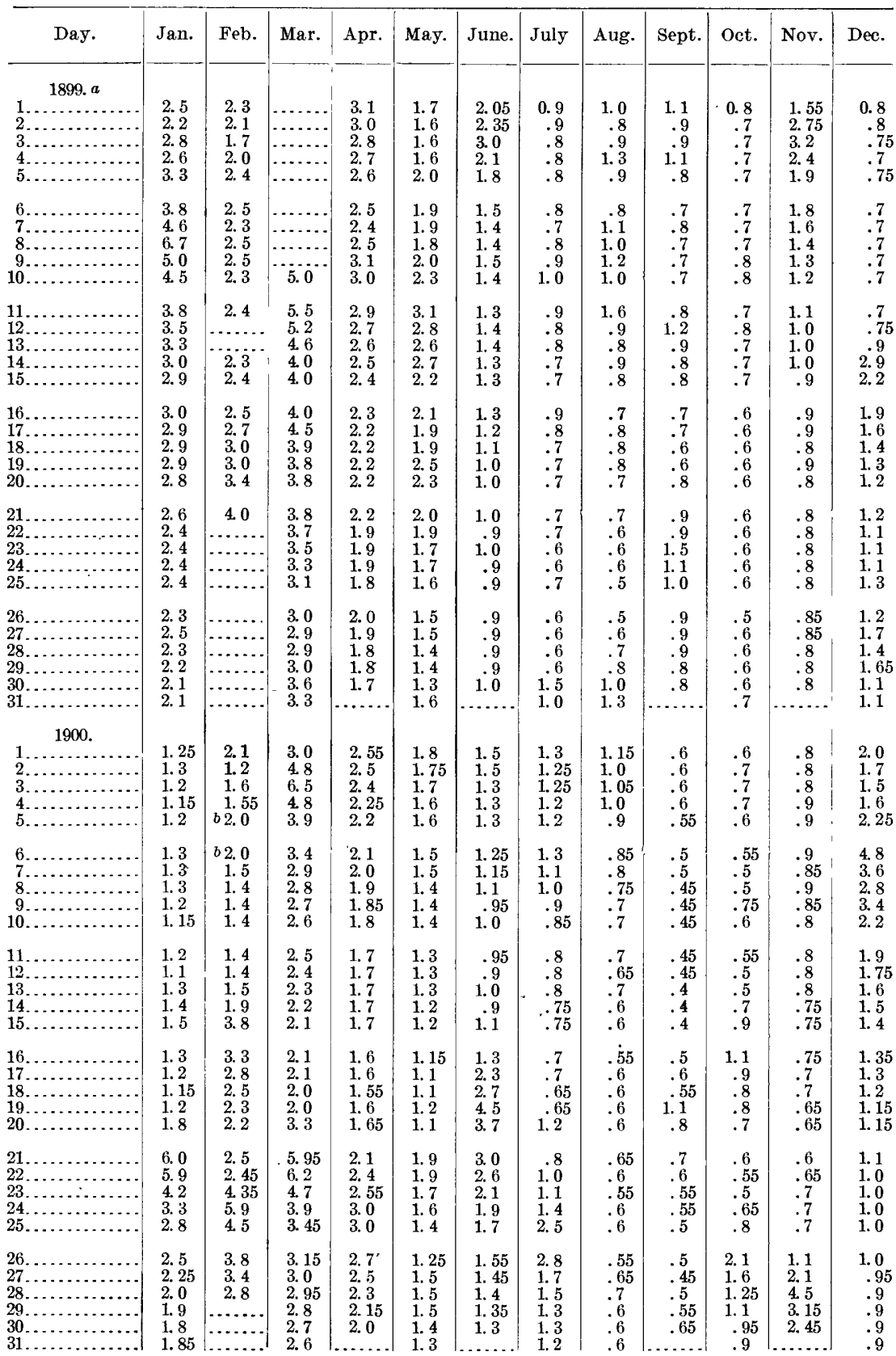

$a$ Ice conditions January 1 to February 21, 1899. River frozen February 22 to March 9, 1899; no readings taken.

Backwater from ice February 5-6, 1900.

IRR $192-07-10$ 
Daily gage height, in feet, of Shenanaoah River at Millville, W. Va.-Continued.

\begin{tabular}{|c|c|c|c|c|c|c|c|c|c|c|c|c|}
\hline Day. & Jan. & Feb. & Mar. & Apr. & May. & June. & July. & Aug. & Seṇt. & Oct. & Nov. & Dee. \\
\hline $\begin{array}{l}1 \ldots \\
1 \ldots \\
2 \ldots \\
3 \ldots \\
4 \ldots \\
5 \ldots\end{array}$ & $\begin{array}{l}0.9 \\
.9 \\
.9 \\
.95 \\
.7\end{array}$ & $\begin{array}{l}1.5 \\
1.6 \\
1.1 \\
1.0 \\
1.2\end{array}$ & $\begin{array}{l}0.7 \\
.7 \\
.7 \\
.75 \\
.75\end{array}$ & $\begin{array}{l}2.0 \\
1.95 \\
2.5 \\
4.7 \\
5.7\end{array}$ & $\begin{array}{l}3.0 \\
2.8 \\
2.7 \\
2.5 \\
2.4\end{array}$ & $\begin{array}{l}4.7 \\
4.2 \\
3.7 \\
3.35 \\
3.3\end{array}$ & $\begin{array}{l}3.5 \\
2.7 \\
2.4 \\
2.2 \\
1.15\end{array}$ & $\begin{array}{l}1.8 \\
1.6 \\
1.5 \\
1.45 \\
1.4\end{array}$ & $\begin{array}{l}2.8 \\
3.3 \\
3.0 \\
2.8 \\
2.3\end{array}$ & $\begin{array}{l}3.7 \\
2.8 \\
2.4 \\
2.1 \\
1.8\end{array}$ & $\begin{array}{l}0.95 \\
.95 \\
.9 \\
.9 \\
.9\end{array}$ & $\begin{array}{l}1.3 \\
1.2 \\
1.25 \\
1.5 \\
1.7\end{array}$ \\
\hline & $\begin{array}{l}.9 \\
.7 \\
.8 \\
.7 \\
.8\end{array}$ & $\begin{array}{l}2.3 \\
1.6 \\
1.6 \\
1.2 \\
1.2\end{array}$ & $\begin{array}{l}.75 \\
.8 \\
.7 \\
.8 \\
.8\end{array}$ & $\begin{array}{l}4.6 \\
4.2 \\
4.15 \\
3.6 \\
3.2\end{array}$ & $\begin{array}{l}2.3 \\
2.2 \\
2.1 \\
2.1 \\
5.5\end{array}$ & $\begin{array}{l}3.6 \\
4.1 \\
4.9 \\
4.5 \\
3.7\end{array}$ & $\begin{array}{l}2.0 \\
2.5 \\
2.6 \\
2.0 \\
1.9\end{array}$ & $\begin{array}{l}1.7 \\
2.1 \\
5.1 \\
3.8 \\
2.7\end{array}$ & $\begin{array}{l}2.0 \\
1.9 \\
1.8 \\
1.6 \\
1.5\end{array}$ & $\begin{array}{l}1.7 \\
1.5 \\
1.5 \\
1.5 \\
1.4\end{array}$ & $\begin{array}{l}.9 \\
.85 \\
.85 \\
.85 \\
.85\end{array}$ & $\begin{array}{l}1.8 \\
1.9 \\
1.8 \\
1.7 \\
1.7\end{array}$ \\
\hline & $\begin{array}{l}.9 \\
2.1 \\
2.3 \\
3.5 \\
2.9\end{array}$ & $\begin{array}{l}1.2 \\
.9 \\
1.2 \\
1.3 \\
1.3\end{array}$ & $\begin{array}{l}1.7 \\
7.3 \\
5.3 \\
3.9 \\
3.2\end{array}$ & $\begin{array}{c}2.85 \\
2.6 \\
2.4 \\
3.0 \\
10.25\end{array}$ & $\begin{array}{l}3.25 \\
4.2 \\
3.5 \\
3.0 \\
2.7\end{array}$ & $\begin{array}{l}3.1 \\
2.8 \\
2.6 \\
3.5 \\
2.9\end{array}$ & $\begin{array}{l}1.8 \\
1.85 \\
2.1 \\
3.0 \\
4.3\end{array}$ & $\begin{array}{l}2.2 \\
2.3 \\
3.1 \\
3.4 \\
2.6\end{array}$ & $\begin{array}{l}1.45 \\
1.5 \\
1.9 \\
1.6 \\
1.5\end{array}$ & $\begin{array}{l}\text { 1. } 4 \\
\text { 1. } 35 \\
\text { 1. } 35 \\
\text { 1. } 4 \\
\text { 1. } 35\end{array}$ & $\begin{array}{l}.85 \\
.85 \\
.85 \\
.8 \\
.8\end{array}$ & $\begin{array}{l}1.7 \\
1.7 \\
2.0 \\
2.0 \\
7.35\end{array}$ \\
\hline & $\begin{array}{l}2.4 \\
2.1 \\
1.9 \\
1.8 \\
1.4\end{array}$ & $\begin{array}{r}1.5 \\
1.0 \\
.9 \\
.8 \\
.8\end{array}$ & $\begin{array}{l}2.75 \\
2.4 \\
2.2 \\
2.0 \\
1.8\end{array}$ & $\begin{array}{l}9.6 \\
6.1 \\
4.9 \\
4.3 \\
4.6\end{array}$ & $\begin{array}{l}2.5 \\
2.3 \\
2.25 \\
2.2 \\
2.15\end{array}$ & $\begin{array}{r}5.8 \\
10.1 \\
5.7 \\
4.6 \\
3.9\end{array}$ & $\begin{array}{l}5.8 \\
5.2 \\
4.2 \\
4.3 \\
3.9\end{array}$ & $\begin{array}{l}2.2 \\
2.75 \\
2.6 \\
2.6 \\
2.6\end{array}$ & $\begin{array}{l}1.6 \\
1.5 \\
1.5 \\
1.45 \\
1.4\end{array}$ & $\begin{array}{l}\text { 1. } 3 \\
1.2 \\
1.1 \\
1.1 \\
1.1\end{array}$ & $\begin{array}{l}.8 \\
.8 \\
.8 \\
.8 \\
.8\end{array}$ & $\begin{array}{r}10.5 \\
6.2 \\
4.7 \\
3.8 \\
3.2\end{array}$ \\
\hline & $\begin{array}{l}1.4 \\
1.5 \\
1.4 \\
1.3 \\
1.3\end{array}$ & $\begin{array}{l}.8 \\
.9 \\
.9 \\
.8 \\
.9\end{array}$ & $\begin{array}{l}2.0 \\
2.2 \\
2.4 \\
2.55 \\
2.3\end{array}$ & $\begin{array}{c}10.55 \\
(b) \\
8.5 \\
6.5 \\
5.3\end{array}$ & $\begin{array}{r}2.0 \\
2.5 \\
11.1 \\
8.4 \\
6.3\end{array}$ & $\begin{array}{l}3.5 \\
5.7 \\
5.5 \\
3.8 \\
3.2\end{array}$ & $\begin{array}{l}3.5 \\
3.2 \\
2.7 \\
2.4 \\
2.2\end{array}$ & $\begin{array}{l}2.2 \\
2.1 \\
2.0 \\
1.9 \\
2.2\end{array}$ & $\begin{array}{l}1.3 \\
1.25 \\
1.2 \\
1.2 \\
1.15\end{array}$ & $\begin{array}{l}1.0 \\
1.0 \\
1.0 \\
1.1 \\
1.0\end{array}$ & $\begin{array}{r}.8 \\
.8 \\
1.0 \\
2.4 \\
2.7\end{array}$ & $\begin{array}{l}2.8 \\
1.2 \\
1.2 \\
2.5 \\
2.4\end{array}$ \\
\hline & $\begin{array}{l}1.3 \\
1.3 \\
1.3 \\
1.5 \\
1.4 \\
1.2\end{array}$ & $\begin{array}{l}.6 \\
.8 \\
.8\end{array}$ & $\begin{array}{l}2.1 \\
2.1 \\
2.1 \\
2.6 \\
2.4 \\
2.15\end{array}$ & $\begin{array}{l}4.8 \\
4.3 \\
3.9 \\
3.5 \\
3.2\end{array}$ & $\begin{array}{l}6.0 \\
6.7 \\
6.2 \\
6.3 \\
5.6 \\
4.9\end{array}$ & $\begin{array}{l}2.9 \\
2.9 \\
3.1 \\
2.7 \\
3.0\end{array}$ & $\begin{array}{l}2.0 \\
2.1 \\
1.85 \\
1.8 \\
1.8 \\
1.85\end{array}$ & $\begin{array}{l}2.1 \\
2.2 \\
2.5 \\
2.6 \\
2.3 \\
3.0\end{array}$ & $\begin{array}{l}1.15 \\
1.1 \\
1.1 \\
1.3 \\
5.3\end{array}$ & $\begin{array}{l}1.0 \\
1.0 \\
1.0 \\
1.0 \\
1.0 \\
1.0\end{array}$ & $\begin{array}{l}2.3 \\
1.8 \\
1.7 \\
1.5 \\
1.3\end{array}$ & $\begin{array}{r}1.8 \\
2.3 \\
2.7 \\
4.0 \\
10.8 \\
(b)\end{array}$ \\
\hline & $\begin{array}{l}7.4 \\
5.3 \\
4.5 \\
4.0 \\
3.5\end{array}$ & $\begin{array}{l}3.1 \\
2.85 \\
3.0 \\
3.0 \\
2.8\end{array}$ & $\begin{array}{r}14.5 \\
9.0 \\
7.2 \\
5.7 \\
5.0\end{array}$ & $\begin{array}{l}3.75 \\
3.4 \\
3.2 \\
3.1 \\
2.9\end{array}$ & $\begin{array}{l}2.2 \\
2.15 \\
2.2 \\
2.25 \\
2.1\end{array}$ & $\begin{array}{l}145 \\
1.4 \\
1,35 \\
1.3 \\
1.3\end{array}$ & $\begin{array}{l}1.3 \\
1.2 \\
1.15 \\
1.1 \\
1.05\end{array}$ & $\begin{array}{l}.9 \\
1.5 \\
1.9 \\
1.7 \\
1.3\end{array}$ & $\begin{array}{l}.65 \\
.65 \\
.6 \\
.6 \\
.6\end{array}$ & $\begin{array}{l}.9 \\
.85 \\
.8 \\
.7 \\
.9\end{array}$ & $\begin{array}{l}.8 \\
.8 \\
.8 \\
.8 \\
.75\end{array}$ & $\begin{array}{l}2.7 \\
2.45 \\
3.1 \\
3.2 \\
4.5\end{array}$ \\
\hline & $\begin{array}{l}3.25 \\
3.0 \\
2.85 \\
2.75 \\
2.6\end{array}$ & $\begin{array}{l}2.5 \\
2.8 \\
2.7 \\
2.2 \\
2.2\end{array}$ & $\begin{array}{l}4.5 \\
4.2 \\
4.2 \\
5.5 \\
7.8\end{array}$ & $\begin{array}{l}2.8 \\
2.6 \\
2.8 \\
6.0 \\
6.9\end{array}$ & $\begin{array}{l}2.05 \\
2.0 \\
4.0 \\
2.7 \\
2.4\end{array}$ & $\begin{array}{l}1.3 \\
1.4 \\
1.25 \\
1.2 \\
1.2\end{array}$ & $\begin{array}{l}1.1 \\
1.1 \\
1.05 \\
1.05 \\
1.0\end{array}$ & $\begin{array}{l}1.2 \\
1.1 \\
1.05 \\
1.0 \\
.9\end{array}$ & $\begin{array}{l}.65 \\
.6 \\
.55 \\
.6 \\
.55\end{array}$ & $\begin{array}{c}1.0 \\
.8 \\
.75 \\
1.1 \\
1.0\end{array}$ & $\begin{array}{l}.75 \\
.7 \\
.7 \\
.7 \\
.65\end{array}$ & $\begin{array}{l}3.4 \\
3.7 \\
3.2 \\
2.9 \\
2.65\end{array}$ \\
\hline & $\begin{array}{l}2.5 \\
2.4 \\
2.25 \\
2.4 \\
2.2\end{array}$ & $\begin{array}{l}2.1 \\
2.2 \\
2.1 \\
2.0 \\
2.1\end{array}$ & $\begin{array}{l}8.0 \\
7.4 \\
7.2 \\
7.0 \\
6.0\end{array}$ & $\begin{array}{l}7.2 \\
6.2 \\
5.6 \\
5.0 \\
4.4\end{array}$ & $\begin{array}{l}2.1 \\
2.0 \\
2.0 \\
1.9 \\
1.9\end{array}$ & $\begin{array}{l}1.15 \\
1.1 \\
1.1 \\
1.0 \\
1.1\end{array}$ & $\begin{array}{l}.9 \\
.9 \\
.9 \\
.9 \\
.8\end{array}$ & $\begin{array}{l}.8 \\
.8 \\
.8 \\
.8 \\
.8\end{array}$ & $\begin{array}{l}.55 \\
.55 \\
.5 \\
.5 \\
.55\end{array}$ & $\begin{array}{l}.9 \\
\text { 1. } 0 \\
1.2 \\
1.75 \\
\text { 1. } 55\end{array}$ & $\begin{array}{l}.65 \\
.6 \\
.65 \\
.7 \\
.65\end{array}$ & $\begin{array}{l}2.6 \\
2.35 \\
2.8 \\
3.4 \\
3.4\end{array}$ \\
\hline & $\begin{array}{l}2.1 \\
2.0 \\
1.9 \\
1.85 \\
1.8\end{array}$ & $\begin{array}{l}1.5 \\
1.9 \\
2.0 \\
2.0 \\
1.7\end{array}$ & $\begin{array}{l}5.0 \\
5.2 \\
5.9 \\
5.0 \\
4.4\end{array}$ & $\begin{array}{l}3.9 \\
3.6 \\
3.4 \\
3.2 \\
3.0\end{array}$ & $\begin{array}{l}1.8 \\
1.7 \\
1.7 \\
1.75 \\
1.8\end{array}$ & $\begin{array}{l}\text { 1. } 2 \\
\text { 1. } 2 \\
\text { 1. } 1 \\
\text { 1. } 6 \\
\text { 1. } 4\end{array}$ & $\begin{array}{l}.8 \\
.8 \\
.8 \\
.75 \\
.75\end{array}$ & $\begin{array}{l}.75 \\
.7 \\
.7 \\
.85 \\
.8\end{array}$ & $\begin{array}{l}.55 \\
.5 \\
.5 \\
.5 \\
.55\end{array}$ & $\begin{array}{l}1.3 \\
1.15 \\
1.0 \\
.95 \\
.9\end{array}$ & $\begin{array}{l}.6 \\
.65 \\
.8 \\
.8 \\
.9\end{array}$ & $\begin{array}{l}3.4 \\
4.4 \\
4.3 \\
4.0 \\
3.5\end{array}$ \\
\hline & $\begin{array}{l}1.9 \\
3.2 \\
3.4 \\
2.9 \\
2.5\end{array}$ & $\begin{array}{l}1.0 \\
2.4 \\
4.5 \\
3.9 \\
4.7\end{array}$ & $\begin{array}{r}4.1 \\
3.8 \\
3.5 \\
3.2 \\
3.1\end{array}$ & $\begin{array}{l}2.8 \\
2.7 \\
2.6 \\
2.5 \\
2.4\end{array}$ & $\begin{array}{l}1.75 \\
1.7 \\
1.65 \\
1.6 \\
1.6\end{array}$ & $\begin{array}{l}1.2 \\
1.2 \\
1.1 \\
1.1 \\
1.2\end{array}$ & $\begin{array}{l}.8 \\
.8 \\
.8 \\
.75 \\
.75\end{array}$ & $\begin{array}{l}.8 \\
.8 \\
.8 \\
.8 \\
.75\end{array}$ & $\begin{array}{l}.6 \\
.6 \\
.6 \\
.55 \\
.55\end{array}$ & $\begin{array}{l}.85 \\
.8 \\
.75 \\
.7 \\
.7\end{array}$ & $\begin{array}{r}.8 \\
.9 \\
1.0 \\
1.0 \\
1.0\end{array}$ & $\begin{array}{l}3.1 \\
2.9 \\
2.7 \\
2.5 \\
2.3\end{array}$ \\
\hline …6. & $\begin{array}{l}2.3 \\
2.5 \\
3.6 \\
4.4 \\
3.6 \\
3.3\end{array}$ & $\begin{array}{r}(d) \\
\stackrel{d}{d}) \\
11.0\end{array}$ & $\begin{array}{l}2.95 \\
2.8 \\
2.75 \\
3.0 \\
3.8 \\
3.9\end{array}$ & $\begin{array}{l}2.3 \\
2.25 \\
2.2 \\
2.2 \\
2.2\end{array}$ & $\begin{array}{l}1.6 \\
2.2 \\
1.75 \\
1.6 \\
1.55 \\
1.5\end{array}$ & $\begin{array}{l}1.2 \\
1.15 \\
1.1 \\
1.3 \\
1.4\end{array}$ & $\begin{array}{r}.8 \\
1.0 \\
.9 \\
1.3 \\
1.3 \\
1.4\end{array}$ & $\begin{array}{l}.75 \\
.7 \\
.7 \\
.7 \\
.65 \\
.65\end{array}$ & $\begin{array}{l}.6 \\
.6 \\
.6 \\
.65 \\
1.0\end{array}$ & $\begin{array}{l}.7 \\
.65 \\
.9 \\
.85 \\
.8 \\
.8\end{array}$ & $\begin{array}{l}1.2 \\
2.0 \\
2.5 \\
2.4 \\
2.1\end{array}$ & $\begin{array}{l}2.2 \\
2.1 \\
2.1 \\
2.1 \\
1.9 \\
1.8\end{array}$ \\
\hline
\end{tabular}

$a$ Water backed by ice during part of January and February, 1901. $b$ No reading; river out of banks April 22 and December 31, 1901, $c$ Ice conditions during part of February, 1902. d. Flood; no observation February 26-27, 1902, 
Daily gage height, in feet, of Shenandoah River at Millville, W. Va.-Continued

\begin{tabular}{|c|c|c|c|c|c|c|c|c|c|c|c|c|}
\hline Day. & Janl. & Feb. & Mar. & Apr. & May. & June. & July. & Aug. & Sept. & Oct. & Nor. & Dec. \\
\hline $\begin{array}{l} \\
1 \ldots \\
2 \ldots \\
3 \ldots \\
4 \ldots \\
5 \ldots\end{array}$ & $\begin{array}{l}1.7 \\
1.7 \\
6.1 \\
8.7 \\
5.9\end{array}$ & $\begin{array}{l}4.0 \\
3.6 \\
3.3 \\
3.3 \\
3.8\end{array}$ & $\begin{array}{l}7.2 \\
6.5 \\
5.0 \\
4.2 \\
3.8\end{array}$ & $\begin{array}{l}6.4 \\
5.0 \\
4.4 \\
4.1 \\
3.9\end{array}$ & $\begin{array}{l}2.5 \\
2.4 \\
2.3 \\
2.2 \\
2.15\end{array}$ & $\begin{array}{l}4.4 \\
3.8 \\
3.2 \\
2.5 \\
2.2\end{array}$ & $\begin{array}{l}4.6 \\
4.2 \\
3.0 \\
2.75 \\
2.5\end{array}$ & $\begin{array}{l}1.3 \\
1.35 \\
1.3 \\
1.5 \\
1.6\end{array}$ & $\begin{array}{l}1.2 \\
2.2 \\
2.7 \\
2.05 \\
1.7\end{array}$ & $\begin{array}{l}1.1 \\
1.1 \\
1.1 \\
1.05 \\
1.05\end{array}$ & $\begin{array}{l}0.9 \\
.9 \\
.9 \\
.9 \\
.95\end{array}$ & $\begin{array}{l}0.7 \\
.7 \\
.7 \\
.65 \\
.6\end{array}$ \\
\hline $\begin{array}{r}6 . \\
7 . \\
8 . \\
9 . \\
10 .\end{array}$ & $\begin{array}{l}4.75 \\
4.0 \\
3.5 \\
3.1 \\
2.8\end{array}$ & $\begin{array}{l}4.2 \\
3.7 \\
3.3 \\
2.7 \\
2.5\end{array}$ & $\begin{array}{l}3.5 \\
3.25 \\
3.2 \\
3.6 \\
3.4\end{array}$ & $\begin{array}{l}3.7 \\
3.5 \\
3.3 \\
3.3 \\
3.3\end{array}$ & $\begin{array}{l}2.1 \\
2.1 \\
2.05 \\
2.0 \\
1.9\end{array}$ & $\begin{array}{l}2.0 \\
3.9 \\
9.2 \\
6.5 \\
8.9\end{array}$ & $\begin{array}{l}3.4 \\
4.65 \\
3.7 \\
2.3 \\
2.0\end{array}$ & $\begin{array}{l}1.9 \\
2.0 \\
1.7 \\
1.5 \\
1.55\end{array}$ & $\begin{array}{l}1.55 \\
1.45 \\
1.3 \\
1.25 \\
1.2\end{array}$ & $\begin{array}{l}1.0 \\
1.0 \\
1.8 \\
2.1 \\
2.0\end{array}$ & $\begin{array}{r}1.0 \\
.9 \\
.9 \\
.9 \\
.9\end{array}$ & $\begin{array}{l}.6 \\
.6 \\
.55 \\
.6 \\
.8\end{array}$ \\
\hline $\begin{array}{l}14 \ldots \\
15 \ldots\end{array}$ & $\begin{array}{l}2.7 \\
2.7 \\
2.2 \\
2.3 \\
2.0\end{array}$ & $\begin{array}{l}2.3 \\
2.1 \\
2.3 \\
2.7 \\
2.6\end{array}$ & $\begin{array}{l}3.3 \\
3.2 \\
3.2 \\
3.0 \\
2.95\end{array}$ & $\begin{array}{l}3.2 \\
3.0 \\
3.1 \\
5.4 \\
7.8\end{array}$ & $\begin{array}{l}1.85 \\
1.8 \\
1.75 \\
1.7 \\
1.7\end{array}$ & $\begin{array}{l}4.9 \\
4.6 \\
4.0 \\
4.3 \\
3.6\end{array}$ & $\begin{array}{l}1.95 \\
1.9 \\
2.7 \\
2.6 \\
2.8\end{array}$ & $\begin{array}{l}1.4 \\
1.4 \\
1.35 \\
1.3 \\
1.3\end{array}$ & $\begin{array}{l}1.2 \\
1.3 \\
1.2 \\
1.2 \\
1.2\end{array}$ & $\begin{array}{l}1.8 \\
1.65 \\
1.55 \\
1.5 \\
1.4\end{array}$ & $\begin{array}{l}.9 \\
.9 \\
.9 \\
.85 \\
.85\end{array}$ & $\begin{array}{l}.7 \\
.7 \\
.7 \\
.8 \\
.8\end{array}$ \\
\hline $\begin{array}{l}19 \ldots \\
20 \ldots\end{array}$ & $\begin{array}{l}2.3 \\
2.2 \\
2.35 \\
2.3 \\
1.95\end{array}$ & $\begin{array}{l}2.6 \\
3.3 \\
4.6 \\
4.1 \\
3.6\end{array}$ & $\begin{array}{l}2.8 \\
2.7 \\
2.6 \\
2.55 \\
2.5\end{array}$ & $\begin{array}{l}6.6 \\
6.1 \\
5.0 \\
4.4 \\
3.9\end{array}$ & $\begin{array}{l}1.65 \\
1.65 \\
1.6 \\
1.55 \\
1.55\end{array}$ & $\begin{array}{l}3.1 \\
2.8 \\
2.6 \\
2.4 \\
2.1\end{array}$ & $\begin{array}{l}2.7 \\
2.2 \\
2.0 \\
1.9 \\
1.8\end{array}$ & $\begin{array}{l}1.25 \\
1.25 \\
1.2 \\
1.5 \\
1.45\end{array}$ & $\begin{array}{l}1.1 \\
1.3 \\
2.1 \\
4.6 \\
3.1\end{array}$ & $\begin{array}{l}1.3 \\
1.3 \\
1.25 \\
1.2 \\
1.2\end{array}$ & $\begin{array}{l}.85 \\
.9 \\
.9 \\
.85 \\
. .85\end{array}$ & $\begin{array}{r}a 1.1 \\
.5 \\
.7 \\
.8 \\
.9\end{array}$ \\
\hline $\begin{array}{l}21 \ldots \\
22 \ldots \\
23 \ldots \\
24 \ldots \\
25 \ldots\end{array}$ & $\begin{array}{l}2.2 \\
3.5 \\
6.0 \\
3.4 \\
2.8\end{array}$ & $\begin{array}{l}3.4 \\
3.3 \\
3.0 \\
3.0 \\
3.2\end{array}$ & $\begin{array}{l}2.5 \\
2.5 \\
4.1 \\
7.3 \\
7.6\end{array}$ & $\begin{array}{l}3.7 \\
3.6 \\
3.3 \\
3.1 \\
3.0\end{array}$ & $\begin{array}{l}1.5 \\
1.5 \\
1.5 \\
1.5 \\
1.5\end{array}$ & $\begin{array}{l}2.0 \\
2.0 \\
1.9 \\
1.9 \\
1.85\end{array}$ & $\begin{array}{l}1.7 \\
1.65 \\
1.6 \\
1.55 \\
1.5\end{array}$ & $\begin{array}{l}1.3 \\
1.25 \\
1.2 \\
1.1 \\
1.05\end{array}$ & $\begin{array}{l}2.7 \\
2.2 \\
1.8 \\
1.6 \\
1.5\end{array}$ & $\begin{array}{l}1.2 \\
1.15 \\
1.1 \\
1.05 \\
1.0\end{array}$ & $\begin{array}{l}.85 \\
.85 \\
.8 \\
.8 \\
.8\end{array}$ & $\begin{array}{l}1.1 \\
1.0 \\
1.2 \\
1.4 \\
1.2\end{array}$ \\
\hline $\begin{array}{l}26 \ldots \\
27 \ldots \\
28 \ldots \\
29 \ldots \\
30 \ldots \\
31 \ldots\end{array}$ & $\begin{array}{l}2.6 \\
2.3 \\
3.35 \\
6.9 \\
5.2 \\
4.5\end{array}$ & $\begin{array}{l}3.5 \\
3.5 \\
4.0\end{array}$ & $\begin{array}{l}5.7 \\
4.3 \\
3.8 \\
3.4 \\
3.4 \\
5.5\end{array}$ & $\begin{array}{l}2.9 \\
2.8 \\
2.7 \\
2.65 \\
2.55\end{array}$ & $\begin{array}{l}1.8 \\
1.8 \\
1.7 \\
1.7 \\
1.85 \\
2.05\end{array}$ & $\begin{array}{l}1.8 \\
2.65 \\
3.2 \\
5.2 \\
5.7 \\
\ldots . .\end{array}$ & $\begin{array}{l}1.5 \\
1.45 \\
1.35 \\
1.3 \\
1.25 \\
1.25\end{array}$ & $\begin{array}{l}1.0 \\
.95 \\
1.1 \\
1.8 \\
1.25 \\
1.2\end{array}$ & $\begin{array}{l}1.4 \\
1.3 \\
1.25 \\
1.2 \\
1.15\end{array}$ & $\begin{array}{l}1.0 \\
1.0 \\
.95 \\
.95 \\
.95 \\
.95\end{array}$ & $\begin{array}{l}.8 \\
.75 \\
.7 \\
.6 \\
.6\end{array}$ & $\begin{array}{r}.8 \\
.9 \\
1.0 \\
.9 \\
.8 \\
.8\end{array}$ \\
\hline $\begin{array}{l} \\
1 \\
1 \ldots \\
2 \ldots \\
3 \ldots \\
4 \ldots \\
5 \ldots\end{array}$ & $\begin{array}{l}.8 \\
.85 \\
1.2 \\
1.2 \\
1.0\end{array}$ & & $\ldots$ & $\begin{array}{l}1.8 \\
1.8 \\
1.75 \\
1.7 \\
1.6\end{array}$ & $\begin{array}{l}3.3 \\
3.3 \\
2.7 \\
2.4 \\
2.15\end{array}$ & $\begin{array}{l}4.0 \\
2.6 \\
2.3 \\
2.1 \\
2.6\end{array}$ & $\begin{array}{l}1.5 \\
1.4 \\
1.3 \\
1.2 \\
1.1\end{array}$ & $\begin{array}{l}1.15 \\
1.05 \\
1.1 \\
1.25 \\
1.4\end{array}$ & $\begin{array}{l}.6 \\
.6 \\
.6 \\
.6 \\
.6\end{array}$ & $\begin{array}{l}.45 \\
.4 \\
.4 \\
.4 \\
.4\end{array}$ & $\begin{array}{l}.45 \\
.45 \\
.4 \\
.5 \\
.45\end{array}$ & $\begin{array}{l}.45 \\
.45 \\
.5 \\
.5 \\
.5\end{array}$ \\
\hline $\begin{array}{r}6 \ldots \\
7 \ldots \\
8 \ldots \\
9 \ldots \\
10 \ldots\end{array}$ & $\begin{array}{l}1.0 \\
1.1 \\
1.1 \\
1.3 \\
1.2\end{array}$ & & $\begin{array}{l}2.3 \\
4.9 \\
3.5\end{array}$ & $\begin{array}{l}1.5 \\
1.5 \\
1.5 \\
1.6 \\
1.7\end{array}$ & $\begin{array}{l}2.1 \\
1.95 \\
2.0 \\
2.0 \\
2.3\end{array}$ & $\begin{array}{l}2.85 \\
4.0 \\
2.9 \\
2.4 \\
2.15\end{array}$ & $\begin{array}{l}1.2 \\
1.15 \\
1.3 \\
1.25 \\
1.35\end{array}$ & $\begin{array}{l}1.4 \\
1.7 \\
1.8 \\
1.45 \\
1.4\end{array}$ & $\begin{array}{l}.6 \\
.6 \\
.6 \\
.55 \\
.55\end{array}$ & $\begin{array}{l}.4 \\
.45 \\
.45 \\
.4 \\
.4\end{array}$ & $\begin{array}{l}.45 \\
.45 \\
.45 \\
.4 \\
.4\end{array}$ & $\begin{array}{l}.55 \\
.5 \\
.5 \\
.5 \\
.5\end{array}$ \\
\hline $\begin{array}{l}11 \ldots \\
12 \ldots \\
13 \ldots \\
14 \ldots \\
15 \ldots\end{array}$ & $\begin{array}{l}1.2 \\
1.1 \\
1.1 \\
1.0 \\
1.0\end{array}$ & & $\begin{array}{l}3.0 \\
2.5 \\
2.3 \\
2.0 \\
1.9\end{array}$ & $\begin{array}{l}2.45 \\
2.4 \\
2.2 \\
2.0 \\
1.75\end{array}$ & $\begin{array}{l}2.5 \\
2.45 \\
2.3 \\
2.05 \\
1.9\end{array}$ & $\begin{array}{l}2.0 \\
1.85 \\
1.75 \\
1.5 \\
1.4\end{array}$ & $\begin{array}{l}5.6 \\
3.7 \\
2.8 \\
2.1 \\
1.75\end{array}$ & $\begin{array}{l}1.35 \\
1.4 \\
1.35 \\
1.3 \\
1.1\end{array}$ & $\begin{array}{l}.5 \\
.5 \\
.6 \\
.6 \\
.9\end{array}$ & $\begin{array}{l}.4 \\
.5 \\
.5 \\
.55 \\
.5\end{array}$ & $\begin{array}{l}.5 \\
.45 \\
.5 \\
.6 \\
.6\end{array}$ & $\begin{array}{l}.85 \\
.5 \\
.65 \\
.65 \\
.75\end{array}$ \\
\hline $\begin{array}{l}16 \ldots \\
17 \ldots \\
18 \ldots \\
19 \ldots \\
20 \ldots\end{array}$ & $\begin{array}{l}1.0 \\
1.0 \\
1.0 \\
1.1 \\
1.1\end{array}$ & & $\begin{array}{l}1.8 \\
1.75 \\
1.7 \\
1.6 \\
1.5\end{array}$ & $\begin{array}{l}1.65 \\
1.6 \\
1.55 \\
1.45 \\
1.45\end{array}$ & $\begin{array}{l}1.85 \\
1.8 \\
1.8 \\
1.9 \\
2.3\end{array}$ & $\begin{array}{l}1.35 \\
1.4 \\
1.65 \\
1.4 \\
1.35\end{array}$ & $\begin{array}{l}1.5 \\
1.4 \\
1.3 \\
1.3 \\
1.2\end{array}$ & $\begin{array}{l}1.05 \\
1.0 \\
1.0 \\
.95 \\
.9\end{array}$ & $\begin{array}{l}.9 \\
.8 \\
.75 \\
.7 \\
.65\end{array}$ & $\begin{array}{l}.5 \\
.5 \\
.5 \\
.5 \\
.45\end{array}$ & $\begin{array}{l}.5 \\
.5 \\
.5 \\
.55 \\
.5\end{array}$ & $\begin{array}{l}.6 \\
1.65 \\
1.8 \\
1.55 \\
.8\end{array}$ \\
\hline $\begin{array}{l}21 \ldots \\
22 \ldots \\
23 \ldots \\
24 \ldots \\
25 \ldots\end{array}$ & $\begin{array}{l}1.3 \\
1.6 \\
2.3 \\
2.6\end{array}$ & & $\begin{array}{l}1.45 \\
1.5 \\
1.6 \\
1.6 \\
1.65\end{array}$ & $\begin{array}{l}1.3 \\
1.25 \\
1.2 \\
1.15 \\
1.1\end{array}$ & $\begin{array}{l}3.5 \\
2.95 \\
2.45 \\
2.15 \\
2.0\end{array}$ & $\begin{array}{l}1.6 \\
1.7 \\
1.9 \\
1.6 \\
1.45\end{array}$ & $\begin{array}{l}1.2 \\
1.2 \\
1.15 \\
1.1 \\
1.2\end{array}$ & $\begin{array}{l}.9 \\
.85 \\
.9 \\
.8 \\
.9\end{array}$ & $\begin{array}{l}.6 \\
.65 \\
.6 \\
.55 \\
.5\end{array}$ & $\begin{array}{l}.6 \\
.6 \\
.6 \\
.55 \\
.5\end{array}$ & $\begin{array}{l}.5 \\
.5 \\
.5 \\
.5 \\
.5\end{array}$ & $\begin{array}{l}.7 \\
.7 \\
.7 \\
.7 \\
.8\end{array}$ \\
\hline $\begin{array}{l}26 \ldots \\
27 \ldots \\
28 \ldots \\
29 \ldots \\
30 \ldots \\
31 \ldots\end{array}$ & & & $\begin{array}{l}1.8 \\
1.85 \\
1.8 \\
1.75 \\
1.7 \\
1.7\end{array}$ & $\begin{array}{l}1.1 \\
1.3 \\
2.5 \\
4.3 \\
3.85 \\
\ldots . . .\end{array}$ & $\begin{array}{l}1.95 \\
2.3 \\
1.95 \\
1.7 \\
1.7 \\
1.55\end{array}$ & $\begin{array}{l}1.3 \\
1.2 \\
1.15 \\
1.1 \\
1.6\end{array}$ & $\begin{array}{l}1.3 \\
1.2 \\
1.1 \\
1.1 \\
1.25 \\
1.1\end{array}$ & $\begin{array}{l}.8 \\
.75 \\
.7 \\
.6 \\
.55 \\
.6\end{array}$ & $\begin{array}{l}.5 \\
.5 \\
.5 \\
.5 \\
.45\end{array}$ & $\begin{array}{l}.5 \\
.45 \\
.4 \\
.4 \\
.4 \\
.4\end{array}$ & $\begin{array}{r}.45 \\
.45 \\
.45 \\
.45 \\
.45 \\
. . .\end{array}$ & $\begin{array}{l}1.15 \\
1.1 \\
1.3 \\
2.25 \\
1.2 \\
1.1\end{array}$ \\
\hline
\end{tabular}

$a$ Backwater from ice December 16, 1903. $\quad b$ Backwater from ice during part of December, 1904. 
Daily gage height, in feet, of Shenandoah River at Millville, W. Va.-Continued.

\begin{tabular}{|c|c|c|c|c|c|c|c|c|c|c|c|c|}
\hline Day. & Jan. & Feb. & Mar. & Apr. & May. & IJune. & July. & Aug. & Sept. & Oct. & Nov. & Dec. \\
\hline 4 & $\begin{array}{c}1.0 \\
.95 \\
.85 \\
1.4 \\
1.6\end{array}$ & $\begin{array}{l}1.4 \\
1.2 \\
1.3 \\
1.3 \\
1.2\end{array}$ & $\begin{array}{l}3.35 \\
2.75 \\
2.7 \\
2.8 \\
2.8\end{array}$ & $\begin{array}{l}2: 2 \\
2.05 \\
1.9 \\
1.8 \\
1.8\end{array}$ & $\begin{array}{l}1.2 \\
1.15 \\
1.15 \\
1.1 \\
1.1\end{array}$ & $\begin{array}{l}1.2 \\
1.2 \\
1.5 \\
1.2 \\
1.1\end{array}$ & $\begin{array}{l}1.9 \\
1.75 \\
1.75 \\
1.6 \\
1.6\end{array}$ & $\begin{array}{l}2.1 \\
1.7 \\
1.4 \\
1.3 \\
1.2\end{array}$ & $\begin{array}{c}1.15 \\
1.0 \\
.95 \\
.9 \\
1.0\end{array}$ & $\begin{array}{l}0.6 \\
.55 \\
.5 \\
.5 \\
.5\end{array}$ & $\begin{array}{c}0.7 \\
.7 \\
.65 \\
.65 \\
.65\end{array}$ & $\begin{array}{c}0.6 \\
.6 \\
.65 \\
.6 \\
.6\end{array}$ \\
\hline $\begin{array}{r}6 . \\
7 . \\
8 . \\
9 \ldots \\
10 .\end{array}$ & $\begin{array}{l}1.55 \\
2.1 \\
2.8 \\
2.5 \\
2.0\end{array}$ & $\begin{array}{l}1.2 \\
1.2 \\
1.25 \\
1.25 \\
1.3\end{array}$ & \begin{tabular}{|r}
$a 4.1$ \\
2.5 \\
2.5 \\
2.8 \\
3.7
\end{tabular} & $\begin{array}{l}2.0 \\
2.45 \\
2.45 \\
2.3 \\
2.1\end{array}$ & $\begin{array}{l}1.0 \\
1.0 \\
1.0 \\
.95 \\
.95\end{array}$ & $\begin{array}{l}1.0 \\
1.15 \\
2.0 \\
1.2 \\
1.0\end{array}$ & $\begin{array}{l}1.6 \\
2.3 \\
2.1 \\
2.3 \\
2.1\end{array}$ & $\begin{array}{l}1.1 \\
1.1 \\
1.1 \\
1.1 \\
1.05\end{array}$ & $\begin{array}{l}1.05 \\
1.0 \\
1.0 \\
.95 \\
.8\end{array}$ & $\begin{array}{l}.5 \\
.5 \\
.5 \\
.5 \\
.5\end{array}$ & $\begin{array}{l}.65 \\
.65 \\
.65 \\
.65 \\
.65\end{array}$ & $\begin{array}{l}.8 \\
1.35 \\
1.2 \\
1.1 \\
1.0\end{array}$ \\
\hline & $\begin{array}{l}1.8 \\
1.5 \\
2.0 \\
2.6 \\
2.9\end{array}$ & $\begin{array}{l}1.35 \\
1.45 \\
1.4 \\
1.25 \\
1.25\end{array}$ & $\begin{array}{l}4.75 \\
4.3 \\
3.3 \\
3.2 \\
2.9\end{array}$ & $\begin{array}{l}2.0 \\
1.95 \\
1.9 \\
1.8 \\
1.7\end{array}$ & $\begin{array}{l}1.0 \\
1.0 \\
1.0 \\
.95 \\
2.0\end{array}$ & $\begin{array}{r}1.0 \\
1.1 \\
.9 \\
.8 \\
.8\end{array}$ & $\begin{array}{l}1.9 \\
2.0 \\
2.8 \\
2.6 \\
3.8\end{array}$ & $\begin{array}{c}1.05 \\
1.0 \\
.95 \\
.95 \\
1.1\end{array}$ & $\begin{array}{l}.8 \\
.85 \\
.8 \\
.8 \\
.8\end{array}$ & $\begin{array}{l}.5 \\
.55 \\
.7 \\
.7\end{array}$ & $\begin{array}{l}.6 \\
.6 \\
.6 \\
.6 \\
.6\end{array}$ & $\begin{array}{l}.95 \\
.9 \\
.85 \\
.8 \\
.8\end{array}$ \\
\hline & $\begin{array}{l}2.5 \\
2.0 \\
2.0 \\
2.0 \\
1.7\end{array}$ & $\begin{array}{l}1.3 \\
1.55 \\
1.55 \\
1.5 \\
1.6\end{array}$ & $\begin{array}{l}2.6 \\
2.4 \\
2.3 \\
2.2 \\
2.1\end{array}$ & $\begin{array}{l}\mathbf{1 . 7} \\
1.65 \\
1.6 \\
1.5 \\
1.45\end{array}$ & $\begin{array}{l}2.0 \\
2.1 \\
2.3 \\
2.1 \\
1.8\end{array}$ & $\begin{array}{l}.75 \\
.75 \\
.7 \\
.7 \\
.7\end{array}$ & $\begin{array}{l}5.3 \\
3.3 \\
2.7 \\
2.3 \\
2.0\end{array}$ & $\begin{array}{l}1.1 \\
3.2 \\
2.3 \\
1.9 \\
1.5\end{array}$ & $\begin{array}{l}.75 \\
.8 \\
.8 \\
.85 \\
.8\end{array}$ & $\begin{array}{l}.7 \\
.65 \\
.65 \\
.65 \\
.65\end{array}$ & $\begin{array}{l}.6 \\
.6 \\
.6 \\
.55 \\
.55\end{array}$ & $\begin{array}{l}.8 \\
.8 \\
.8 \\
.9 \\
.9\end{array}$ \\
\hline & $\begin{array}{l}1.6 \\
1.5 \\
1.4 \\
1.3 \\
1.6\end{array}$ & $\begin{array}{l}1.55 \\
1.5 \\
1.55 \\
1.6 \\
2.0\end{array}$ & $\begin{array}{l}2.4 \\
2.7 \\
4.0 \\
3.4 \\
3.2\end{array}$ & $\begin{array}{l}1.4 \\
1.4 \\
1.4 \\
1.35 \\
1.3\end{array}$ & $\begin{array}{l}1.7 \\
1.5 \\
1.4 \\
1.3 \\
1.3\end{array}$ & $\begin{array}{l}.9 \\
3.3 \\
3.2 \\
3.5 \\
5.9\end{array}$ & $\begin{array}{l}1.8 \\
1.8 \\
1.8 \\
2.8 \\
2.4\end{array}$ & $\begin{array}{l}1.4 \\
1.3 \\
1.2 \\
1.1 \\
1.1\end{array}$ & $\begin{array}{l}. .8 \\
.8 \\
.8 \\
.8 \\
.75\end{array}$ & $\begin{array}{l}.6 \\
.6 \\
.6 \\
.6 \\
.6\end{array}$ & $\begin{array}{l}.6 \\
.6 \\
.6 \\
.6 \\
.6\end{array}$ & $\begin{array}{l}2.3 \\
4.1 \\
5.3 \\
4.25 \\
3.6\end{array}$ \\
\hline & $\begin{array}{l}1.6 \\
1.85 \\
1.6 \\
1.5 \\
1.6 \\
1.5\end{array}$ & $\begin{array}{l}2.2 \\
2.6 \\
3.0\end{array}$ & $\begin{array}{l}3.6 \\
3.2 \\
2.9 \\
2.7 \\
2.5 \\
2.3\end{array}$ & $\begin{array}{l}1.2 \\
1.2 \\
1.2 \\
1.3 \\
1.3\end{array}$ & $\begin{array}{l}1.2 \\
.9 \\
1.0 \\
1.0 \\
1.0 \\
1.1\end{array}$ & $\begin{array}{l}4.8 \\
3.5 \\
3.0 \\
2.5 \\
2.1\end{array}$ & $\begin{array}{l}2.2 \\
1.9 \\
1.6 \\
1.4 \\
1.9 \\
2.4\end{array}$ & $\begin{array}{l}1.9 \\
2.1 \\
1.8 \\
1.5 \\
1.25 \\
1.1\end{array}$ & $\begin{array}{l}.6 \\
.6 \\
.6 \\
.55 \\
.55\end{array}$ & $\begin{array}{l}.65 \\
.8 \\
.9 \\
.85 \\
.85 \\
.85\end{array}$ & $\begin{array}{l}.6 \\
.6 \\
.6 \\
.6 \\
.6\end{array}$ & $\begin{array}{l}3.2 \\
2.75 \\
2.4 \\
2.2 \\
2.3 \\
2.3\end{array}$ \\
\hline & $\begin{array}{l}2.7 \\
2.45 \\
2.25 \\
3.4 \\
5.3\end{array}$ & $\begin{array}{r}2.3 \\
2.2 \\
2.0 \\
b 2.4 \\
2.0\end{array}$ & $\begin{array}{l}1.1 \\
1.1 \\
1.2 \\
2.2 \\
2.5\end{array}$ & $\begin{array}{l}4.75 \\
4.2 \\
3.8 \\
3.4 \\
3.1\end{array}$ & $\begin{array}{l}2.3 \\
2.2 \\
2.1 \\
2.0 \\
1.95\end{array}$ & $\begin{array}{l}1.5 \\
1.5 \\
1.4 \\
1.3 \\
1.2\end{array}$ & $\begin{array}{l}2.0 \\
1.8 \\
1.6 \\
1.3 \\
2.15\end{array}$ & $\begin{array}{l}1.3 \\
1.4 \\
1.8 \\
1.4 \\
2.5\end{array}$ & $\begin{array}{l}3.55 \\
3.8 \\
2.7 \\
2.2 \\
2.2\end{array}$ & $\begin{array}{l}1.45 \\
1.45 \\
1.4 \\
1.7 \\
2.5\end{array}$ & $\begin{array}{l}2.7 \\
2.5 \\
2.4 \\
2.3 \\
2.2\end{array}$ & $\begin{array}{l}1.85 \\
1.8 \\
1.75 \\
1.7 \\
1.65\end{array}$ \\
\hline & $\begin{array}{l}4.5 \\
3.6 \\
3.0 \\
2.7 \\
2.45\end{array}$ & $\begin{array}{l}1.95 \\
1.9 \\
1.8 \\
1.6 \\
1.6\end{array}$ & $\begin{array}{l}3.5 \\
2.9 \\
2.5 \\
2.3 \\
2.1\end{array}$ & $\begin{array}{l}2.9 \\
2.7 \\
2.6 \\
2.4 \\
2.45\end{array}$ & $\begin{array}{l}1.9 \\
1.85 \\
1.8 \\
2.1 \\
2.05\end{array}$ & $\begin{array}{l}2.1 \\
1.3 \\
1.2 \\
1.2 \\
1.65\end{array}$ & $\begin{array}{l}1.7 \\
1.55 \\
1.6 \\
11.5 \\
1.35\end{array}$ & $\begin{array}{l}2.3 \\
2.1 \\
2.0 \\
2.2 \\
2.3\end{array}$ & $\begin{array}{l}2.0 \\
1.9 \\
1.8 \\
1.65 \\
1.6\end{array}$ & $\begin{array}{l}3.5 \\
3.1 \\
2.6 \\
2.3 \\
2.05\end{array}$ & $\begin{array}{l}2.1 \\
2.05 \\
2.0 \\
1.9 \\
1.85\end{array}$ & $\begin{array}{l}1.7 \\
1.65 \\
1.6 \\
1.55 \\
1.5\end{array}$ \\
\hline & $\begin{array}{l}2.3 \\
2.15 \\
2.0 \\
2.0 \\
2.0\end{array}$ & $\begin{array}{l}1.75 \\
1.5 \\
1.45 \\
1.3 \\
1.35\end{array}$ & $\begin{array}{l}2.0 \\
1.9 \\
1.75 \\
1.7 \\
1.7\end{array}$ & $\begin{array}{l}2.6 \\
2.6 \\
2.45 \\
2.3 \\
3.2\end{array}$ & $\begin{array}{l}1.9 \\
1.85 \\
1.8 \\
1.7 \\
1.6\end{array}$ & $\begin{array}{l}1.9 \\
1.5 \\
1.2 \\
1.2 \\
1.2\end{array}$ & $\begin{array}{l}1.3 \\
1.3 \\
1.3 \\
1.3 \\
1.4\end{array}$ & $\begin{array}{l}2.0 \\
2.0 \\
3.0 \\
3.4 \\
3.8\end{array}$ & $\begin{array}{l}1.5 \\
1.5 \\
1.45 \\
1.9 \\
1.6\end{array}$ & $\begin{array}{l}1.8 \\
1.8 \\
1.65 \\
1.5 \\
1.4\end{array}$ & $\begin{array}{l}1.8 \\
1.8 \\
1.75 \\
1.75 \\
1.75\end{array}$ & $\begin{array}{l}1.6 \\
1.65 \\
1.55 \\
1.5 \\
1.4\end{array}$ \\
\hline $\begin{array}{l}16 . . \\
17 . \\
18 . \\
19 . \\
20 .\end{array}$ & $\begin{array}{l}2.2 \\
2.3 \\
2.25 \\
2.2 \\
2.1\end{array}$ & $\begin{array}{l}1.3 \\
1.3 \\
1.2 \\
1.2 \\
1.2\end{array}$ & $\begin{array}{l}1.8 \\
1.9 \\
2.6 \\
2.7 \\
2.7\end{array}$ & $\begin{array}{l}4.7 \\
4.2 \\
3.6 \\
3.2 \\
2.95\end{array}$ & $\begin{array}{l}1.55 \\
1.4 \\
1.35 \\
1.45 \\
1.4\end{array}$ & $\begin{array}{l}2.0 \\
2.6 \\
2.1 \\
3.8 \\
2.4\end{array}$ & $\begin{array}{l}1.3 \\
1.3 \\
1.3 \\
1.4 \\
1.5\end{array}$ & $\begin{array}{l}3.9 \\
3.2 \\
4.1 \\
3.35 \\
4.2\end{array}$ & $\begin{array}{l}1.5 \\
1.35 \\
1.3 \\
1.35 \\
1.3\end{array}$ & $\begin{array}{r}1.4 \\
1.4 \\
1.5 \\
4.1 \\
11.3\end{array}$ & $\begin{array}{l}1.7 \\
1.65 \\
1.6 \\
1.8 \\
2.25\end{array}$ & $\begin{array}{l}1.45 \\
1.7 \\
4.2 \\
4.5 \\
4.0\end{array}$ \\
\hline & $\begin{array}{l}2.0 \\
1.9 \\
1.8 \\
1.8 \\
3.8\end{array}$ & $\begin{array}{l}1.2 \\
1.2 \\
1.2 \\
1.2 \\
1.15\end{array}$ & $\begin{array}{l}2.7 \\
2.9 \\
3.15 \\
3.1 \\
2.9\end{array}$ & $\begin{array}{l}2.7 \\
2.5 \\
2.4 \\
2.25 \\
2.1\end{array}$ & $\begin{array}{l}1.3 \\
1.25 \\
12 \\
1.2 \\
1.15\end{array}$ & $\begin{array}{l}2.7 \\
4.8 \\
3.7 \\
2.9 \\
2.5\end{array}$ & $\begin{array}{l}1.4 \\
1.3 \\
1.2 \\
2.0 \\
1.6\end{array}$ & $\begin{array}{l}3.9 \\
3.4 \\
3.3 \\
4.0 \\
4.5\end{array}$ & $\begin{array}{l}1.25 \\
1.2 \\
1.2 \\
1.2 \\
1.1\end{array}$ & $\begin{array}{r}12.8 \\
8.3 \\
6.5 \\
5.7 \\
4.9\end{array}$ & $\begin{array}{l}4.3 \\
3.8 \\
3.85 \\
2.9 \\
2.7\end{array}$ & $\begin{array}{l}3.5 \\
3.6 \\
3.4 \\
3.0 \\
2.6\end{array}$ \\
\hline $\begin{array}{l}26 \ldots \ldots \ldots \\
27 \ldots \ldots \ldots \\
28 \ldots \ldots \ldots \\
29 \ldots \ldots \\
30 \ldots \ldots \\
31 \ldots \ldots\end{array}$ & $\begin{array}{l}3.2 \\
2.7 \\
2.5 \\
2.4 \\
2.4 \\
2.5\end{array}$ & $\begin{array}{l}1.1 \\
1.1 \\
1.1\end{array}$ & $\begin{array}{l}2.9 \\
3.0 \\
4.8 \\
4.9 \\
4.6 \\
4.6\end{array}$ & $\begin{array}{l}2.0 \\
2.0 \\
3.4 \\
2.9 \\
2.5\end{array}$ & $\begin{array}{l}1.1 \\
1.2 \\
1.3 \\
1.5 \\
1.4 \\
1.4\end{array}$ & $\begin{array}{l}2.15 \\
2.4 \\
3.5 \\
3.25 \\
2.4\end{array}$ & $\begin{array}{l}1.5 \\
1.4 \\
1.4 \\
1.4 \\
1.5 \\
1.3\end{array}$ & $\begin{array}{l}4.5 \\
3.8 \\
5.4 \\
4.7 \\
5.0 \\
4.2\end{array}$ & $\begin{array}{l}1.25 \\
1.25 \\
1.25 \\
1.25 \\
1.35\end{array}$ & $\begin{array}{l}4.2 \\
3.8 \\
3.5 \\
3.2 \\
3.0 \\
2.8\end{array}$ & $\begin{array}{l}2.7 \\
2.25 \\
2.15 \\
2.1 \\
2.0\end{array}$ & $\begin{array}{l}2.2 \\
2.0 \\
2.4 \\
2.4 \\
2.25 \\
2.2\end{array}$ \\
\hline
\end{tabular}


Rating tables for Shenandoah River at Millville, W. Va.

APRIL 15, 1895, TO FEBRUARY 6, 1897, AND MARCH 8, 1994, TO DECEMBER 31, 1906.a

\begin{tabular}{|c|r|r|r|r|r|r|r|}
\hline $\begin{array}{c}\text { Gage } \\
\text { height. }\end{array}$ & Discharge. & $\begin{array}{c}\text { Gage } \\
\text { height. }\end{array}$ & Discharge. & $\begin{array}{c}\text { Gage } \\
\text { height. }\end{array}$ & Discharge. & $\begin{array}{c}\text { Gage } \\
\text { height. }\end{array}$ & Discharge. \\
\cline { 1 - 5 } Feet. & Second-feet. & Feet. & Second-feet. & Feet. & Second-feet. & Feet. & Second-feet. \\
0.30 & 430 & 2.30 & 2,940 & 4.60 & 8,860 & 8.40 & 26,400 \\
.40 & 480 & 2.40 & 3,130 & 4.80 & 9,540 & 8.60 & 27,700 \\
.50 & 540 & 2.50 & 3,330 & 5.00 & 10,260 & 8.80 & 29,000 \\
.60 & 610 & 2.60 & 3,530 & 5.20 & 11,020 & 9.00 & 30,400 \\
.70 & 690 & 2.70 & 3,730 & 5.40 & 11,780 & 9.20 & 31,300 \\
.80 & 780 & 2.80 & 3,940 & 5.60 & 12,580 & 9.40 & 33,200 \\
.90 & 880 & 2.90 & 4,150 & 5.80 & 13,400 & 9.60 & 34,600 \\
1.00 & 980 & 3.00 & 4,370 & 6.00 & 14,240 & 9.80 & 36,000 \\
1.10 & 1,090 & 3.10 & 4,600 & 6.20 & 15,120 & 10.00 & 37,500 \\
1.20 & 1,200 & 3.20 & 4,840 & 6.40 & 16,000 & 11.00 & 45,100 \\
1.30 & 1,320 & 3.30 & 5,080 & 6.60 & 16,920 & 12.00 & 53,500 \\
1.40 & 1,450 & 3.40 & 5,320 & 6.80 & 17,840 & 13.00 & 62,700 \\
1.50 & 1,580 & 3.50 & 5,570 & 7.00 & 18,800 & 14.00 & 72,700 \\
1.60 & 1,720 & 3.60 & 5,830 & 7.20 & 19,780 & 15.00 & 83,200 \\
1.70 & 1,870 & 3.70 & 6,100 & 7.40 & 20,780 & 16.00 & 94,300 \\
1.80 & 2,030 & 3.80 & 6,380 & 7.60 & 21,820 & 17.00 & 105,900 \\
1.90 & 2,200 & 3.90 & 6,670 & 7.80 & 22,880 & 18.00 & 118,000 \\
2.00 & 2,380 & 4.00 & 6,960 & 8.00 & 24,020 & 19.00 & 130,600 \\
2.10 & 2,560 & 4.20 & 7,560 & 8.20 & 25,200 & 20.00 & 143,700 \\
2.20 & 2,750 & 4.40 & 8,200 & & & & \\
\hline
\end{tabular}

FEBRUARY 10, 1897, TO JANUARY 24, 1904.b

\begin{tabular}{|r|r||l|l|l|l|l|l|}
\hline 0.20 & 470 & 2.10 & 2,770 & 4.00 & 7,380 & 6.80 & 18,300 \\
.30 & 520 & 2.20 & 2,960 & 4.10 & 7,700 & 7.00 & 19,220 \\
.40 & 580 & 2.30 & 3,150 & 4.20 & 8,020 & 7.20 & 20,140 \\
.50 & 650 & 2.40 & 3,350 & 4.30 & 8,340 & 7.40 & 21,100 \\
.60 & 730 & 2.50 & 3,560 & 4.40 & 8,680 & 7.60 & 22,060 \\
.70 & 820 & 2.60 & 3,770 & 4.50 & 9,020 & 7.80 & 23,060 \\
.80 & 910 & 2.70 & 3,990 & 4.60 & 9,360 & 8.00 & 24,060 \\
.90 & 1,010 & 2.80 & 4,210 & 4.70 & 9,700 & 8.20 & 25,100 \\
1.00 & 1,120 & 2.90 & 4,440 & 4.80 & 10,060 & 8.40 & 26,200 \\
1.10 & 1,230 & 3.00 & 4,670 & 4.90 & 10,420 & 8.60 & 27,300 \\
1.20 & 1,350 & 3.10 & 4,910 & 5.00 & 10,800 & 8.80 & 28,500 \\
1.30 & 1,480 & 3.20 & 5,150 & 5.20 & 11,560 & 9.00 & 29,800 \\
1.40 & 1,620 & 3.30 & 5,400 & 5.40 & 12,320 & 9.20 & 31,200 \\
1.50 & 1,760 & 3.40 & 5,650 & 5.60 & 13,120 & 9.40 & 32,700 \\
1.60 & 1,910 & 3.50 & 5,910 & 5.80 & 13,940 & 9.60 & 34,200 \\
1.70 & 2,070 & 3.60 & 6,180 & 6.00 & 14,780 & 9.80 & 35,800 \\
1.80 & 2,240 & 3.70 & 6,460 & 6.20 & 15,640 & 10.00 & 37,500 \\
1.90 & 2,410 & 3.80 & 6,760 & 6.40 & 16,520 & & \\
2.00 & 2,590 & 3.90 & 7,060 & 6.60 & 17,400 & & \\
\hline
\end{tabular}

a This table is strictly applicable only for open-channel conditions. It is based on 14 discharge measurements made during 1895 and 1904. It is well defined between gage heights 0.4 feet and 5.2 feet. Estimates above 5.0 feet are based on a discharge curve which is the product of a well-defined area curve and a fairly accurate extension of the velocity curve. The discharge curves 1895-1906 are the same above gage height 10.0 feet.

Th s table is strictly applicable only for open-channel conditions. It is based on 14 discharge measments made during $1897-1903$. It is well defined between gage heights 0.5 foot and 5.0 feet. The discharge curves 1895-1906 are the same above gage height, 10.0 feet. 
Estimated monthly discharge of Shenandoah River at Millville, W. Va.

[Drainage area, 3,000 square miles. $a$ ]

\begin{tabular}{|c|c|c|c|c|c|c|c|c|}
\hline \multirow[b]{2}{*}{ Month. } & \multicolumn{3}{|c|}{ Discharge in second-feet. } & \multicolumn{3}{|c|}{ Run-off. } & \multicolumn{2}{|c|}{ Precipitation. } \\
\hline & Maximum. & Minimum. & Mean. & $\begin{array}{l}\text { Second- } \\
\text { feet per } \\
\text { square } \\
\text { mile. }\end{array}$ & $\begin{array}{l}\text { Depth } \\
\text { in inches. }\end{array}$ & $\begin{array}{l}\text { Per cent } \\
\text { of pre- } \\
\text { cipita- } \\
\text { tion. }\end{array}$ & $\underset{\text { inches. }}{\text { In }}$ & $\begin{array}{c}\text { Loss } \\
\text { of, in } \\
\text { inches. }\end{array}$ \\
\hline 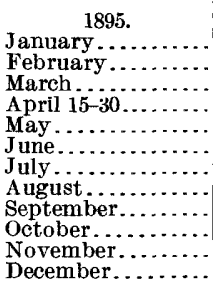 & $\begin{array}{r}4,600 \\
20,780 \\
3,130 \\
7,260 \\
1,580 \\
1,320 \\
610 \\
690 \\
1,450\end{array}$ & $\begin{array}{r}1,870 \\
2,560 \\
980 \\
980 \\
540 \\
610 \\
480 \\
540 \\
610\end{array}$ & $\begin{array}{r}\ldots, 904 \\
5,570 \\
1,613 \\
1,880 \\
806 \\
722 \\
537 \\
621 \\
754\end{array}$ & $\begin{array}{l}0.970 \\
1.86 \\
.539 \\
.628 \\
.269 \\
.241 \\
.179 \\
.207 \\
.252\end{array}$ & $\begin{array}{l}0.576 \\
2.14 \\
.601 \\
.724 \\
.310 \\
.269 \\
.206 \\
.231 \\
.290\end{array}$ & $\begin{array}{r}65 \\
14 \\
16 \\
17 \\
41 \\
20 \\
15 \\
9\end{array}$ & $\begin{array}{r}5.54 \\
1.34 \\
2.73 \\
\text { b } 3.78 \\
3.28 \\
4.14 \\
4.44 \\
1.82 \\
.66 \\
1.06 \\
1.57 \\
3.09\end{array}$ & $\begin{array}{r}3.14 \\
3.54 \\
3.72 \\
1.51 \\
.39 \\
.85 \\
1.34 \\
2.80\end{array}$ \\
\hline The year..... & $\ldots$ & & & & & $\ldots$. & 33.45 & \\
\hline 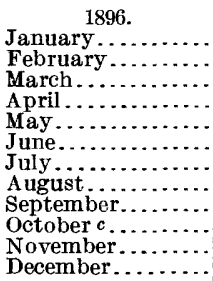 & $\begin{array}{r}11,400 \\
12,580 \\
12,180 \\
8,860 \\
4,370 \\
3,940 \\
9,030 \\
1,870 \\
13,190 \\
139,700 \\
4,840 \\
6,380\end{array}$ & $\begin{array}{r}880 \\
1,450 \\
1,450 \\
1,450 \\
1,090 \\
780 \\
1,320 \\
780 \\
540 \\
880 \\
880 \\
1,450\end{array}$ & $\begin{array}{l}2,434 \\
3,709 \\
3,842 \\
2,807 \\
2,127 \\
2,056 \\
2,858 \\
1,150 \\
1,042 \\
7,768 \\
1,835 \\
2,296\end{array}$ & $\begin{array}{l}.813 \\
1.24 \\
1.28 \\
.937 \\
.710 \\
.686 \\
.954 \\
.384 \\
.348 \\
2.59 \\
.613 \\
.767\end{array}$ & $\begin{array}{c}.937 \\
1.34 \\
1.48 \\
1.05 \\
.819 \\
.765 \\
1.10 \\
.443 \\
.388 \\
2.99 \\
.684 \\
.884\end{array}$ & $\begin{array}{r}38 \\
35 \\
35 \\
85 \\
36 \\
13 \\
18 \\
14 \\
5 \\
623 \\
16 \\
383\end{array}$ & $\begin{array}{l}2.49 \\
3.78 \\
\text { 4. } 25 \\
\text { 1. } 24 \\
\text { 2. } 28 \\
\text { 5. } 71 \\
\text { 6. } 21 \\
3.20 \\
\text { 7. } 22 \\
.48 \\
4.16 \\
.23\end{array}$ & $\begin{array}{r}1.55 \\
2.44 \\
2.77 \\
.19 \\
1.46 \\
4.95 \\
5.11 \\
2.76 \\
6.83 \\
-2.51 \\
3.48 \\
-.65\end{array}$ \\
\hline The year.... & 139,700 & 540 & 2,827 & .944 & 12.88 & 31 & 41.25 & 28.37 \\
\hline 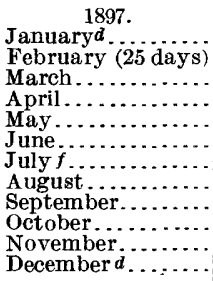 & $\begin{array}{r}1,720 \\
37,870 \\
6,760 \\
3,350 \\
30,620 \\
2,410 \\
3,770 \\
1,760 \\
1,350 \\
1,010 \\
1,620 \\
3,990\end{array}$ & $\begin{array}{r}980 \\
1,090 \\
2,590 \\
1,620 \\
1,760 \\
1,010 \\
910 \\
730 \\
580 \\
550 \\
650 \\
820\end{array}$ & $\begin{array}{r}1,220 \\
11,680 \\
4,946 \\
2,671 \\
6,243 \\
1,632 \\
1,426 \\
987 \\
664 \\
692 \\
859 \\
1,619\end{array}$ & $\begin{array}{l}.407 \\
3.90 \\
1.65 \\
.892 \\
2.08 \\
.545 \\
.476 \\
.330 \\
.222 \\
.231 \\
.287 \\
.541\end{array}$ & $\begin{array}{l}.469 \\
3.63 \\
1.90 \\
.995 \\
2.40 \\
.608 \\
.549 \\
.380 \\
.248 \\
.266 \\
.320 \\
.624\end{array}$ & $\begin{array}{l}33 \\
81 \\
53 \\
47 \\
19 \\
13 \\
27 \\
25 \\
10 \\
12 \\
18\end{array}$ & $\begin{array}{r}4.41 \\
\text { e. } 68 \\
2.34 \\
1.90 \\
5.13 \\
3.18 \\
4.14 \\
1.41 \\
1.01 \\
2.59 \\
2.59 \\
3.51\end{array}$ & $\begin{array}{r}.94 \\
.44 \\
.90 \\
2.73 \\
2.57 \\
3.59 \\
1.03 \\
.76 \\
2.32 \\
2.27 \\
2.89\end{array}$ \\
\hline The year.... & & ... & & -- & $\because$ & $\ldots$ & 35.49 & \\
\hline 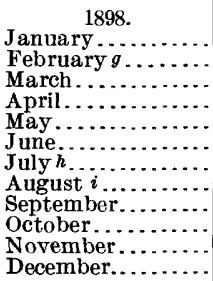 & $\begin{array}{r}6,180 \\
2,770 \\
5,650 \\
13,120 \\
29,150 \\
2,410 \\
3,770 \\
50,900 \\
1,835 \\
30,500 \\
4,210 \\
14,780\end{array}$ & $\begin{array}{r}1,010 \\
1,010 \\
1,010 \\
2,070 \\
2,070 \\
1,120 \\
820 \\
1,350 \\
1,120 \\
1,010 \\
2,070 \\
2,410\end{array}$ & $\begin{array}{l}2,435 \\
1,612 \\
2,227 \\
4,353 \\
5,711 \\
1,647 \\
1,249 \\
8,164 \\
1,360 \\
5,684 \\
2,827 \\
4,498\end{array}$ & $\begin{array}{l}.813 \\
.538 \\
.744 \\
1.45 \\
1.91 \\
.550 \\
.417 \\
2.73 \\
.454 \\
1.90 \\
.944 \\
1.50\end{array}$ & $\begin{array}{l}.937 \\
.560 \\
.858 \\
1.62 \\
2.20 \\
.614 \\
.481 \\
3.15 \\
.506 \\
2.19 \\
1.05 \\
1.73\end{array}$ & $\begin{array}{l}31 \\
75 \\
23 \\
61 \\
42 \\
21 \\
11 \\
41 \\
26 \\
31 \\
59 \\
55\end{array}$ & $\begin{array}{l}3.05 \\
.75 \\
3.73 \\
2.66 \\
5.21 \\
2.86 \\
4.18 \\
7.73 \\
1.97 \\
7.10 \\
1.79 \\
3.13\end{array}$ & $\begin{array}{l}2.11 \\
.19 \\
2.87 \\
1.04 \\
3.01 \\
2.25 \\
3.70 \\
4.58 \\
1.46 \\
4.91 \\
.74 \\
1.40\end{array}$ \\
\hline The year..... & 50,900 & 820 & 3,481 & 1.16 & 15. 90 & 36 & 44. 16 & 28.26 \\
\hline
\end{tabular}

$a 3,000$ square iniles used to obtain run-off for 1906; 2,995 used for all other years.

$b$ Precipitation for complete month, April, 1895 .

c Estimates October 1-2, 1896, approximate.

$d$ Ice conditions during January and December 24-31, 1897. No correction made in estimates.

$e$ Precipitation for whole month, February, 1897.

$f$ Estimate July 11, 1897, interpolated.

$g$ Ice conditions during February, 1898. No correction made in estimates.

$h$ Estimate July 2, 1898, interpolated.

$i$ Gage height A ugust 11, 1898, estimated. Discharge approxımate. 
Estimated monthly discharge of Shenandoah River at Millville, W. Va.-Continued.

\begin{tabular}{|c|c|c|c|c|c|c|c|c|}
\hline \multirow[b]{2}{*}{ Month. } & \multicolumn{3}{|c|}{ Discharge in second-feet. } & \multicolumn{3}{|c|}{ Run-off. } & \multicolumn{2}{|c|}{ Precipitation. } \\
\hline & Maximum. & Minimum. & Mean. & $\begin{array}{l}\text { [Second } \\
\text { feet per } \\
\text { square } \\
\text { mile. }\end{array}$ & $\begin{array}{c}\text { Depth } \\
\text { in inches. }\end{array}$ & $\begin{array}{c}\text { Per cent } \\
\text { of pre- } \\
\text { cipita- } \\
\text { tion. }\end{array}$ & $\begin{array}{c}\text { In } \\
\text { inches. }\end{array}$ & $\begin{array}{l}\text { Loss } \\
\text { of, in } \\
\text { inches. }\end{array}$ \\
\hline 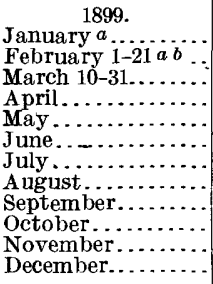 & $\begin{array}{r}17,840 \\
7,380 \\
12,720 \\
4,910 \\
4,910 \\
4,670 \\
1,760 \\
1,910 \\
1,760 \\
910 \\
5,150 \\
4,440\end{array}$ & $\begin{array}{r}2,770 \\
2,070 \\
4,440 \\
2,070 \\
1,480 \\
1,010 \\
730 \\
650 \\
730 \\
650 \\
910 \\
820\end{array}$ & $\begin{array}{r}5,116 \\
3,675 \\
7,065 \\
3,289 \\
2,533 \\
1,607 \\
877 \\
992 \\
989 \\
785 \\
1,403 \\
1,398\end{array}$ & $\begin{array}{l}1.71 \\
1.23 \\
2.36 \\
1.10 \\
.846 \\
.537 \\
.293 \\
.331 \\
.330 \\
.262 \\
.468 \\
.467\end{array}$ & $\begin{array}{l}1.97 \\
.960 \\
1.93 \\
1.23 \\
.975 \\
.599 \\
.338 \\
.382 \\
.368 \\
.302 \\
.522 \\
.538\end{array}$ & \begin{tabular}{|r|} 
\\
78 \\
\hdashline 106 \\
20 \\
29 \\
14 \\
10 \\
10 \\
13 \\
64 \\
40
\end{tabular} & $\begin{array}{r}2.52 \\
c 4.95 \\
c 5.12 \\
1.16 \\
4.95 \\
2.09 \\
2.51 \\
3.94 \\
3.78 \\
2.34 \\
.81 \\
1.34\end{array}$ & \begin{tabular}{r}
0.55 \\
$\ldots$ \\
\hdashline .07 \\
3.97 \\
1.49 \\
2.17 \\
3.56 \\
3.41 \\
2.04 \\
.29 \\
.80
\end{tabular} \\
\hline The y & & $\cdots$ & & & & & 35.51 & \\
\hline $\begin{array}{l}\text { January.... } \\
\text { January } \\
\text { February... } \\
\text { March....... } \\
\text { April....... } \\
\text { May......... } \\
\text { June......... } \\
\text { July........ } \\
\text { August..... } \\
\text { September... } \\
\text { October.... } \\
\text { November.. } \\
\text { December... }\end{array}$ & $\begin{array}{r}14,780 \\
14,360 \\
16,960 \\
4,670 \\
2,410 \\
9,020 \\
4,210 \\
1,290 \\
1,230 \\
2,770 \\
9,020 \\
10,060 \\
\end{array}$ & $\begin{array}{r}1,230 \\
1,350 \\
2,590 \\
1,835 \\
1,230 \\
1,010 \\
775 \\
690 \\
580 \\
650 \\
730 \\
1,010 \\
\end{array}$ & $\begin{array}{r}2,970 \\
3,833 \\
5,866 \\
2,803 \\
1,689 \\
2,249 \\
1,374 \\
829 \\
696 \\
947 \\
1,453 \\
2,232 \\
\end{array}$ & $\begin{array}{l}.992 \\
1.28 \\
1.96 \\
.936 \\
.564 \\
.751 \\
.459 \\
.277 \\
.232 \\
.316 \\
.485 \\
.745 \\
\end{array}$ & $\begin{array}{l}1.14 \\
1.33 \\
2.26 \\
1.04 \\
.650 \\
.838 \\
.529 \\
.319 \\
.259 \\
.364 \\
.541 \\
.859 \\
\end{array}$ & $\begin{array}{r}44 \\
36 \\
61 \\
55 \\
27 \\
14 \\
14 \\
17 \\
8 \\
11 \\
18 \\
47 \\
\end{array}$ & $\begin{array}{l}2.57 \\
3.73 \\
3.72 \\
1.89 \\
2.42 \\
5.98 \\
3.76 \\
1.93 \\
3.14 \\
3.19 \\
2.94 \\
1.83 \\
\end{array}$ & $\begin{array}{r}1.43 \\
2.40 \\
1.46 \\
.85 \\
1.77 \\
5.14 \\
3.23 \\
1.61 \\
2.88 \\
2.83 \\
2.40 \\
.97\end{array}$ \\
\hline The year. & 16,960 & 580 & 2,245 & .750 & 10.13 & 27 & 37.10 & 26.97 \\
\hline 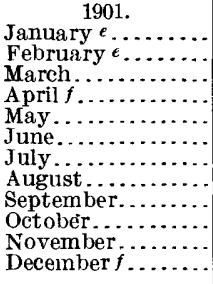 & $\begin{array}{r}5,910 \\
3,150 \\
20,620 \\
50,000 \\
45,+20 \\
38,240 \\
13,940 \\
11,180 \\
11,940 \\
6,460 \\
3,990 \\
50,000\end{array}$ & $\begin{array}{r}820 \\
730 \\
820 . \\
2,500 \\
2,590 \\
3,770 \\
1,290 \\
1,620 \\
1,230 \\
1,120 \\
910 \\
1,350\end{array}$ & $\begin{array}{r}1,812 \\
1,315 \\
3,376 \\
12,840 \\
8,704 \\
8,225 \\
4,437 \\
3,528 \\
2,496 \\
1,769 \\
1,341 \\
8,124\end{array}$ & $\begin{array}{l}.605 \\
.439 \\
1.13 \\
4.29 \\
2.91 \\
2.75 \\
1.48 \\
1.18 \\
.833 \\
.591 \\
.448 \\
2.71\end{array}$ & $\begin{array}{c}.698 \\
.457 \\
1.30 \\
4.79 \\
3.36 \\
3.07 \\
1.71 \\
1.36 \\
.929 \\
.681 \\
.500 \\
3.12\end{array}$ & $\begin{array}{r}30 \\
139 \\
34 \\
77 \\
58 \\
41 \\
39 \\
23 \\
25 \\
99 \\
.25 \\
51\end{array}$ & $\begin{array}{l}2.31 \\
.33 \\
3.80 \\
6.24 \\
5.82 \\
7.54 \\
4.38 \\
5.92 \\
3.78 \\
.69 \\
2.01 \\
6.12\end{array}$ & $\begin{array}{r}1.61 \\
-.13 \\
2.50 \\
1.45 \\
2.46 \\
4.47 \\
2.67 \\
4.56 \\
2.85 \\
.01 \\
1.51 \\
3.00\end{array}$ \\
\hline The year.... & 50,000 & 730 & 4,831 & 1.61 & 21.98 & 45 & 48.94 & 26. 96 \\
\hline 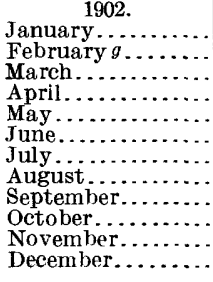 & $\begin{array}{r}21,100 \\
50,000 \\
77,900 \\
20,140 \\
7,380 \\
1,910 \\
1,620 \\
2,410 \\
1,120 \\
2,155 \\
3,560 \\
9,020\end{array}$ & $\begin{array}{r}2,240 \\
1,120 \\
4,100 \\
2,960 \\
1,760 \\
1,120 \\
865 \\
775 \\
650 \\
775 \\
730 \\
2,240\end{array}$ & $\begin{array}{r}5,176 \\
8,611 \\
13,880 \\
6,785 \\
2,606 \\
1,402 \\
1,097 \\
1,062 \\
724 \\
1,069 \\
1,157 \\
4,728\end{array}$ & $\begin{array}{l}1.73 \\
2.88 \\
4.63 \\
2.27 \\
.870 \\
.468 \\
.366 \\
.355 \\
.242 \\
.357 \\
.386 \\
1.58\end{array}$ & $\begin{array}{c}1.99 \\
3.00 \\
5.34 \\
2.53 \\
1.00 \\
.522 \\
.422 \\
.409 \\
.270 \\
.412 \\
.431 \\
1.82\end{array}$ & $\begin{array}{r}74 \\
67 \\
148 \\
113 \\
38 \\
18 \\
19 \\
18 \\
10 \\
11 \\
13 \\
54\end{array}$ & $\begin{array}{l}2.69 \\
4.46 \\
3.61 \\
2.23 \\
2.64 \\
2.93 \\
2.17 \\
2.22 \\
2.59 \\
3.65 \\
3.21 \\
3.38\end{array}$ & $\begin{array}{r}.70 \\
1.46 \\
-1.73 \\
-.30 \\
1.64 \\
2.41 \\
1.75 \\
1.81 \\
2.32 \\
3.24 \\
2.78 \\
1.56\end{array}$ \\
\hline The year. & 77,900 & 650 & 4,025 & 1.34 & 18.15 & 51 & 35.78 & 17.63 \\
\hline
\end{tabular}

$a$ Ice conditions during January and February, 1899. No correction made in estimates.

b Estimates February 12-13, 1899, interpolated.

c Precipitation for complete months, February and March, 1899.

d Backwater from ice February 5-6, 1900 . Discharge cnrrected.

$e$ Slight backwater from ice during part of January and February, 1901; no correction made in estimates.

$f$ April 22 and December 31, 1901, river out of banks, discharge estimated 50,000 second-feet.

$g$ Ice conditions during part of February. 1902; no correction made in estimates. February 26-27, 1902, river out of banks; discharge estimated 50,000 second-feet. 
Estimated monthly discharge of Shenandoah River at Millville, W. Va.-Continued.

\begin{tabular}{|c|c|c|c|c|c|c|c|c|}
\hline \multirow{2}{*}{ Mohth. } & \multicolumn{3}{|c|}{ Discharge in second-feet. } & \multicolumn{3}{|c|}{ Run-off. } & \multicolumn{2}{|c|}{ Precipitation. } \\
\hline & Maximum. & Minimum. & Mean. & $\begin{array}{l}\text { Second- } \\
\text { feet per } \\
\text { square } \\
\text { mile. }\end{array}$ & $\begin{array}{l}\text { Depth } \\
\text { in inches. }\end{array}$ & $\begin{array}{l}\text { Per cent } \\
\text { of pre- } \\
\text { cipita- } \\
\text { tion. }\end{array}$ & $\underset{\text { inches. }}{\text { In }}$ & $\begin{array}{c}\text { Loss } \\
\text { of, in } \\
\text { inches. }\end{array}$ \\
\hline 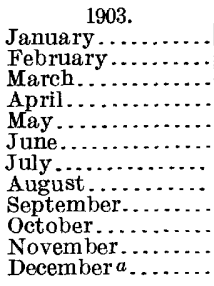 & $\begin{array}{r}27,900 \\
9,360 \\
22,060 \\
23,060 \\
3,560 \\
31,200 \\
9,530 \\
2,590 \\
9,360 \\
2,770 \\
1,120 \\
1,620\end{array}$ & $\begin{array}{l}2,070 \\
2,770 \\
3,560 \\
3,665 \\
1,760 \\
2,240 \\
1,415 \\
1,065 \\
1,230 \\
1,065 \\
730 \\
650\end{array}$ & $\begin{array}{r}6,802 \\
5,448 \\
7,907 \\
7,880 \\
2,321 \\
7,448 \\
3,453 \\
1,590 \\
2,249 \\
1,454 \\
961 \\
927\end{array}$ & $\begin{array}{l}2.27 \\
1.82 \\
2.64 \\
2.63 \\
.775 \\
2.49 \\
1.15 \\
.531 \\
.751 \\
.485 \\
.321 \\
.310\end{array}$ & $\begin{array}{l}2.62 \\
1.90 \\
3.04 \\
2.93 \\
.894 \\
2.78 \\
1.33 \\
.612 \\
.838 \\
.559 \\
.358 \\
.357\end{array}$ & $\begin{array}{l}64 \\
54 \\
73 \\
81 \\
33 \\
36 \\
43 \\
17 \\
35 \\
23 \\
44 \\
38\end{array}$ & $\begin{array}{l}4.08 \\
3.49 \\
4.15 \\
3.62 \\
2.69 \\
7.63 \\
3.06 \\
3.53 \\
2.42 \\
2.39 \\
.82 \\
.96\end{array}$ & $\begin{array}{l}1.46 \\
1.59 \\
1.11 \\
.69 \\
1.80 \\
4.85 \\
1.73 \\
2.92 \\
1.58 \\
1.83 \\
.46 \\
.60\end{array}$ \\
\hline The yea & 31,200 & 650 & 4,037 & 1. 35 & 18. 22 & 47 & 38.84 & 20.62 \\
\hline 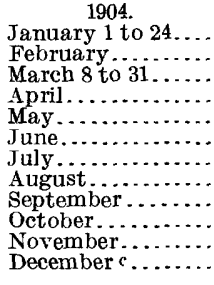 & $\begin{array}{r}3,770 \\
\cdots 9,900 \\
7,880 \\
5,570 \\
6,960 \\
12,580 \\
2,030 \\
880 \\
610 \\
610 \\
2,845 \\
\end{array}$ & \begin{tabular}{r}
910 \\
\hdashline 1,515 \\
1,090 \\
1,650 \\
1,090 \\
1,090 \\
575 \\
510 \\
480 \\
480 \\
510
\end{tabular} & \begin{tabular}{r}
1,428 \\
\hdashline 2,546 \\
2,191 \\
2,779 \\
2,430 \\
1,930 \\
1,096 \\
620 \\
521 \\
528 \\
.780
\end{tabular} & $\begin{array}{l}.477 \\
.850 \\
.732 \\
.928 \\
.811 \\
.644 \\
.366 \\
.207 \\
.174 \\
.176 \\
.260 \\
\end{array}$ & $\begin{array}{c}.426 \\
\cdots .759 \\
.817 \\
1.07 \\
.905 \\
.742 \\
.422 \\
.231 \\
.201 \\
.196 \\
.300\end{array}$ & $\begin{array}{l}31 \\
31 \\
16 \\
15 \\
17 \\
12 \\
17 \\
21 \\
12\end{array}$ & $\begin{array}{r}b 1.80 \\
1.26 \\
b 2.08 \\
2.64 \\
3.43 \\
5.57 \\
5.04 \\
2.53 \\
1.95 \\
1.20 \\
.95 \\
2.46\end{array}$ & $\begin{array}{r}1.82 \\
2.36 \\
4.67 \\
4.30 \\
2.11 \\
1.72 \\
1.00 \\
2.75 \\
2.16\end{array}$ \\
\hline The year. & $\cdots$ & . . . . . & 年 & . & ...... & & 30.91 & 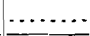 \\
\hline 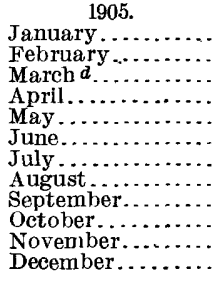 & $\begin{array}{r}4,150 \\
4,370 \\
9,370 \\
3,230 \\
2,940 \\
13,820 \\
11,400 \\
4,840 \\
1,145 \\
880 \\
690 \\
11,400\end{array}$ & $\begin{array}{r}830 \\
1,200 \\
2,560 \\
1,200 \\
880 \\
690 \\
1,450 \\
930 \\
575 \\
540 \\
575 \\
610\end{array}$ & $\begin{array}{r}2,065 \\
1,684 \\
4,387 \\
1,945 \\
1,382 \\
2,552 \\
2,994 \\
1,557 \\
810 \\
640 \\
624 \\
2,336\end{array}$ & $\begin{array}{l}.689 \\
.562 \\
1.46 \\
.649 \\
.461 \\
.852 \\
1.00 \\
.520 \\
.270 \\
.214 \\
.208 \\
.780\end{array}$ & $\begin{array}{c}.794 \\
.585 \\
1.68 \\
.724 \\
.531 \\
.950 \\
1.15 \\
.600 \\
.301 \\
.247 \\
.232 \\
.899\end{array}$ & $\begin{array}{r}28 \\
27 \\
69 \\
38 \\
14 \\
17 \\
19 \\
19 \\
18 \\
9 \\
26 \\
24\end{array}$ & $\begin{array}{l}2.86 \\
2.14 \\
2.44 \\
1.90 \\
3.93 \\
5.50 \\
5.98 \\
3.19 \\
1.65 \\
2.84 \\
.90 \\
3.82\end{array}$ & $\begin{array}{l}2.06 \\
1.56 \\
.76 \\
1.18 \\
3.40 \\
4.55 \\
4.83 \\
2.59 \\
1.35 \\
2.59 \\
.67 \\
2.92\end{array}$ \\
\hline The year.... & 13,820 & 540 & 1,915 & .639 & 8.69 & 23 & 37.15 & 28.46 \\
\hline 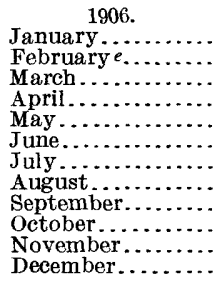 & $\begin{array}{r}11,400 \\
2,940 \\
9,900 \\
9,370 \\
2,940 \\
9,540 \\
2,655 \\
11,780 \\
6,380 \\
60,820 \\
7,880 \\
8,520\end{array}$ & $\begin{array}{l}2,030 \\
1,090 \\
1,090 \\
2,380 \\
1,090 \\
1,200 \\
1,200 \\
1,320 \\
1,090 \\
1,450 \\
1,720 \\
1,450\end{array}$ & $\begin{array}{l}3,719 \\
1,643 \\
3,888 \\
4,464 \\
1,803 \\
2,895 \\
1,578 \\
5,220 \\
1,980 \\
8,248 \\
3,021 \\
3,052\end{array}$ & $\begin{array}{l}1.24 \\
.548 \\
1.30 \\
1.49 \\
.601 \\
.965 \\
.527 \\
1.74 \\
.660 \\
2.75 \\
1.01 \\
1.02\end{array}$ & $\begin{array}{l}1.43 \\
.571 \\
1.50 \\
1.66 \\
.693 \\
1.08 \\
.608 \\
2.01 \\
.736 \\
3.17 \\
1.13 \\
1.18\end{array}$ & & $\begin{array}{c}\cdots \\
\cdots \cdots \\
\cdots \cdots\end{array}$ & 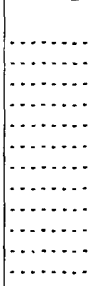 \\
\hline The year. & 60,820 & 1,090 & 3,459 & 1.15 & 15.77 & & $\cdots$ & $\cdots$ \\
\hline
\end{tabular}

a Backwater December 16, 1903, discharge corrected.

$b$ Preeipitation for complete months, January and March, 1904.

$c$ Backwater during part of December, 1904, discharge corrected.

$d$ Backwater March 6, 1905; discharge corrected.

e Backwater February 4, 1906; discharge corrected. 
The following table gives the horsepower, 80 per cent efficiency per foot of fall, that may be developed at different rates of discharge, and shows the number of days on which the flow and the corresponding horsepower were respectively less than the amounts given in the columns for "discharge" and "horsepower."

Discharge and horsepower table for Shenandoah River at Millville, W. Va., for 1895 to 1906.

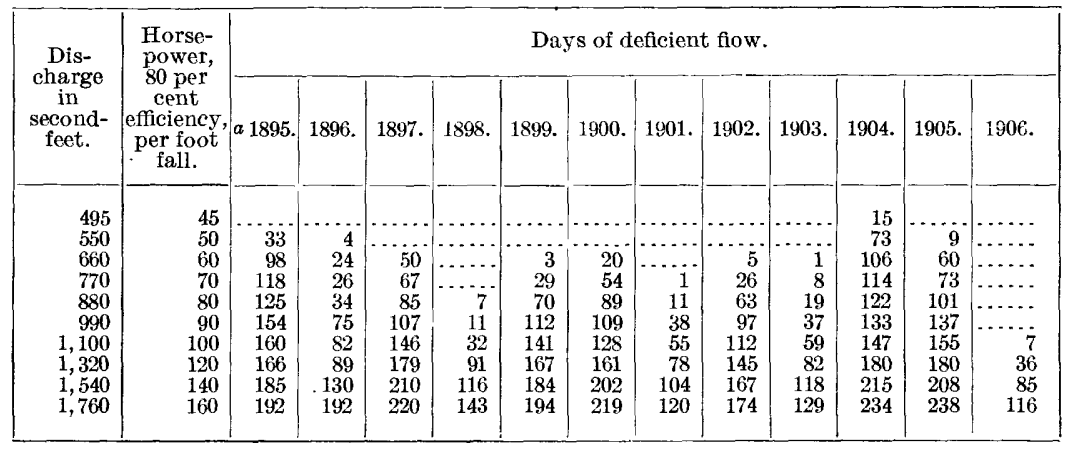

a April 15 to December 31, 1895.

Note.-The minimum flow during the period covered by the above table was 480 second-feet, giving 44 horsepower per foot of fall, for fifteen days during October and November, 1904.

\section{MISCELLANEOUS DISCHARGE MEASUREMENTS IN SHENANDOAH RIVER BASIN BELOW NORTH AND SOUTH FORKS.}

The following miscellaneous discharge measurements have been made in the basin of Shenandoah River below the junction of North and South forks.

Miscellaneous discharge measurements in Shenandoah River basin below North and South Jorks.

\begin{tabular}{|c|c|c|c|c|c|c|}
\hline Date. & Stream. & Locality. & Width. & $\begin{array}{l}\text { Area of } \\
\text { section. }\end{array}$ & $\begin{array}{l}\text { Mean } \\
\text { veloc. } \\
\text { ity. }\end{array}$ & $\begin{array}{l}\text { Dis- } \\
\text { charge. }\end{array}$ \\
\hline $\begin{aligned} & 1897 . \\
& \text { October } 7 .\end{aligned}$ & Wappan Run. & Near mouth near Linden, Va. & Feet. & $\begin{array}{c}\text { Square } \\
\text { feet. } \\
1.4\end{array}$ & $\begin{array}{c}\text { Feet } \\
\text { per sec. } \\
0.79\end{array}$ & $\begin{array}{c}\text { Second- } \\
\text { feet } . \\
\text { a } 1.1\end{array}$ \\
\hline $\begin{array}{l}\text { October } 9 . \\
\text { Do }\end{array}$ & Stonebridge Run & Near Milldale, Va... & & & & $b 3$ \\
\hline Do $\because .$. & $\begin{array}{l}\text { Parker Creek. } \\
\text { Crystal Run... }\end{array}$ & Near Millwood. Va.......... & & & 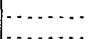 & $\begin{array}{r}4 \\
b 3\end{array}$ \\
\hline October 2. & Bullskin Run. & $\begin{array}{l}\text { At Johnson's factory, at } \\
\text { mouth near Kabletown, }\end{array}$ & 8 & 12 & .62 & c 7.5 \\
\hline October 1. & Evitt Run. . & At bridge near mouth near & 16 & 13 & .92 & 12 \\
\hline October 25 . & Flowing Run. & Near Millville, w. va... & 6 & 7.2 & .63 & 4.5 \\
\hline $\begin{array}{l}1894 . \\
\text { July } 6 \ldots \ldots\end{array}$ & Shenandoah River... & Harpers Ferry, W. Va & & 467 & 2.61 & 1,218 \\
\hline
\end{tabular}

$a$ Low discharge; it may be due to storage of water at dam above.

$b$ Stonebridge Run and Crystal Run fed by large springs.

c Water considered very low. 


\section{POTOMAC RIVER BASIN BELOW SHENANDOAH RIVER.}

POTOMAC RIVER AT POINT OF ROCKS, MD.

This station was established by C. C. Babb February 17, 1895 . It is located at the steel highway bridge at Point of Rocks, Md.

The channel is straight for 500 feet above and 200 feet below the station. It is 1,300 feet wide, broken by seven bridge piers. Both banks overflow only at extremely high water, and are not wooded. In the two right spans the bed is composed of mud, and is subject to some change; in the other spans the bed is composed of gravel and cobblestones and is permanent. The current does not flow at right angles with the bridge in all of the spans.

Discharge measurements are made from the eight-span steel toll bridge, to which the gage is attached. The initial point for soundings is the left end of the lower guard rail, 0.4 foot beyond the center of the end pin, on the downstream side of the bridge.

As originally placed the gage was located in the third span of the bridge from the left bank. The length of the wire from the end of the weight to the marker was 48.0 feet. The zero of the gage was 40.9 feet below bench mark No. 1, described below. June 18, 1896, a new wire gage was placed on the lower side of the first span of the bridge. The zero of the gage was changed to 41.3 feet below bench mark No. 1, the wire length being 44.19 feet. During 1896 and 1897 the wire became rusted and broke frequently; the changes in wire length were not recorded. January 25, 1898, a new wire was put in, the length being 44.22 feet, and this length has been maintained since that date, the datum elevation continuing at 41.3 feet below bench mark No. 1.

During the period between the measurements of April 16 and July 29, 1901, a large quantity of earth excavated from the canal was thrown into the river along its left bank, changing the section and possibly affecting the flow of the river. The shifting of this material necessitated moving the gage farther from the shore. This was done by the observer, who attempted to install a temporary gage to read the same as the old one. The indications are, however, that the gage was not set to read exactly the same, and the gage heights between April 16, 1901, and September 2, 1902, are somewhat in error.

A standard chain gage was installed at this station September 2, 1902. It is bolted to the hand rail on the lower side of the bridge on the first span from the left bank. The length of the chain is 44.22 feet, the same length as had been previously used. In placing the box, however, the gage datum was lowered 0.45 foot, making elevation of the bench mark above gage datum 41.75 feet, instead of 41.3 feet. The gage is read once each day by George H. Hickman. Bench mark No. 1, established November 16,1896, is a copper bolt in a large 
capstone on the lower wing wall of the north abutment, about 10 feet from the north end of the first iron truss and 41.75 feet above the datum of the gage.

All estimates published in Progress Reports prior to 1905 have been revised. Estimates do not include the discharge of the Chesapeake and Ohio Canal, which forms an appreciable percentage of the total flow of the river at low stages. Uncertain length of gage wire or chain and ice conditions are the chief sources of error at this station. Two rating curves are necessary on account of the change in gage datum made September 2, 1902. For normal conditions of flow and correct wire or chain length the estimates as published below are probably within 5 per cent of the true discharge of the river section.

For the period January 1 to June 17, 1896, when the gage-height records are in doubt, a comparison of the Point of Rocks estimates was made with the Cumberland, Springfield, and Millville estimates. This comparison seems to indicate that the Point of Rocks estimates for this period may be as much as 20 per cent too low.

During 1897 the gage-wire length was greatly in error. The proper correction to be applied to the gage heights was determined by the amount that the discharge measurements of that year plotted above or below the rating curve. A comparison of the Point of Rocks estimates with the Cumberland and Millville estimates indicates that the former are well within 10 per cent of the true discharge for 1897.

It is believed that estimates from April 16 to December 31, 1901, can be considered within 10 per cent of the correct discharge. Although the difference between the gage zero and the datum plane was not known with certainty, it is believed to have been very small, because the measurements for that period plot on the first rating curve, and because the hydrographer's and the observer's gage-height observations on the dates of the measurements agree.

It is believed that the second rating curve, which is applied after March 31, 1902, gives estimates correctly within 10 per cent from June 22 to September 1, 1902, because the measurement of June 22 plots on this rating curve and the gage height agrees with the observer's gage height. There are no data available, however, for determining the exact date on which the change should be made from the first to the second rating curve. The date was arbitrarily chosen as April 1, 1902 , which is about halfway between the measurements of December 27, 1901, and June 22, 1902. It should be noticed that during the two most doubtful months-March and April-much high water exists, and hence the percentage error during those months is not large. But if the date for changing rating tables was not chosen correctly there is liability of errors from 15 to 40 per cent during the remainder of the doubtful period. 
A summary of the records furnishes the following discharge data: Maximum discharge for twenty-four hours, 218,700 second-feet; minimum discharge for twenty-four hours, 900 second-feet; mean annual discharge, 1897 to 1906, inclusive, 10,575 second-feet; mean annual rainfall for eleven years, 36.21 inches.

Discharge measurements of Potomac River at Point of Rocks, Md.

\begin{tabular}{|c|c|c|c|c|c|}
\hline Date. & $\begin{array}{c}\text { Gage } \\
\text { height.a }\end{array}$ & Discharge. & Date. & $\begin{array}{c}\text { Gage } \\
\text { height. } a\end{array}$ & Discharge. \\
\hline 1895. & $\begin{array}{l}\text { Feet. } \\
3.05\end{array}$ & $\begin{array}{c}\text { Second-feet } \\
10,520\end{array}$ & June $28 \quad 1900$. & $\begin{array}{l}\text { Feet. } \\
1.50\end{array}$ & Second-feet. \\
\hline $\begin{array}{l}\text { March } 25 . . . \\
\text { April } 5 . . .\end{array}$ & $\begin{array}{l}3.05 \\
3.42\end{array}$ & $\begin{array}{l}10,520 \\
14,030\end{array}$ & October $2 d$ & $\begin{array}{r}1.50 \\
.30\end{array}$ & $\begin{array}{l}5,212 \\
1,259\end{array}$ \\
\hline April $13 \ldots$ & 4.27 & 17,520 & September 19. & $b .30$ & 2,277 \\
\hline April 23. & 2.10 & 7,371 & & & \\
\hline May $1 \ldots$ & 4.35 & 21,070 & 1901. & & \\
\hline May 7 & 3.10 & 12,480 & April $16 \ldots . .$. & 14.30 & 100,800 \\
\hline May $17 \ldots$ & 2.35 & 8,918 & July $29 .$. & 1.85 & 6,530 \\
\hline May 28. & 2.45 & 9,189 & December 27 . & 2.89 & 11,410 \\
\hline June 3. & 1.53 & 4,536 & & & \\
\hline June $17 \ldots$ & 1.30 & 4,233 & 1902. & & \\
\hline July $10, \ldots$. & 1.50 & 4,695 & June $22 \ldots$ & 1.25 & 2,921 \\
\hline November 6 . & .40 & 1,202 & September 2 . & .87 & 1,717 \\
\hline 1896. & & & 1903. & & \\
\hline June $23 \ldots \ldots \ldots$ & 1.82 & 6,462 & March $12 \ldots \ldots$ & 4.84 & 18,880 \\
\hline August 4. & 1.86 & 7,057 & April $17 \ldots \ldots$ & 13.70 & 86,420 \\
\hline Noveniber 16. & 1.60 & 6,109 & .... Do ........... & 13.10 & 80,520 \\
\hline 1897. & & & April $18 \ldots$ & $\begin{array}{l}9.60 \\
1.50\end{array}$ & $\begin{array}{r}54,080 \\
3,770\end{array}$ \\
\hline February $9 . . .$. & $b 7.95$ & 40,650 & November 9. & 1.12 & 2,140 \\
\hline February $23 \mathrm{c}$ & $b 21.70$ & 154,800 & & & \\
\hline March $8 .$. & 5.70 & 27,380 & 1904. & & \\
\hline March 27. & 4.05 & 17,120 & July $11 \ldots$ & 3.87 & 13,750 \\
\hline July $23 \ldots$ & $b 2.85$ & 8,130 & & & \\
\hline October 29 & $b .20$ & 2,141 & 1905. & & \\
\hline April $12 \ldots$ & $b 5.00$ & 20,620 & March $13 \ldots \ldots$. & 6.56 & $\begin{array}{r}28,640 \\
2,997\end{array}$ \\
\hline 1898. & & & October $30 \ldots \ldots$ & $\begin{array}{l}1.29 \\
2.05\end{array}$ & 4,889 \\
\hline January $25 .$. & 6.50 & 33,340 & November $9 \ldots$. & 1.20 & 2,531 \\
\hline A ugust 19 . & 3.30 & 14,310 & ....DDo ....... & 1.20 & 2,467 \\
\hline October $3 \ldots$ & .65 & $\mathbf{1}, 939$ & 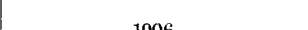 & & \\
\hline 1899. & & & May $30 \ldots . . .$. & 1.70 & 3,892 \\
\hline January $28 \ldots$ & 3.80 & 17,330 & Dec. $7 \ldots$ & 1.76 & 4,450 \\
\hline May $20 \ldots \ldots$. & 8.15 & 45,990 & & & \\
\hline $\begin{array}{l}\text { September } 5 \ldots \\
\text { October } 29 \ldots\end{array}$ & $\begin{array}{l}.80 \\
.50\end{array}$ & $\begin{array}{l}2,360 \\
1,628\end{array}$ & & & \\
\hline
\end{tabular}

a All gage heights refer to datum 41.30 feet below bench mark No. 1, 1895 to 1901 , melusive, and 41.75 feet below the same bench mark, 1902 to 1906 .

$b$ Gage height inaccurate.

c Measurement recomputed, 1905

d Measurement made by wading.

Discharge measurements of Chesapeake and Ohio Canal at Point of Rocks, Md.

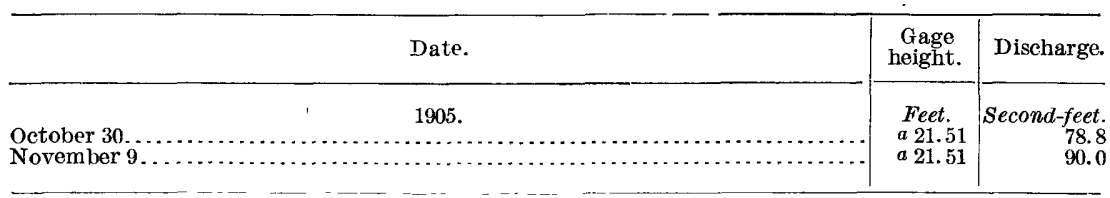

a Elevation of water surface of canal above Potomac River gage datum determined with a level. 
Daily gage height, in feet, of Potomac River at Point of Rocks, Md.

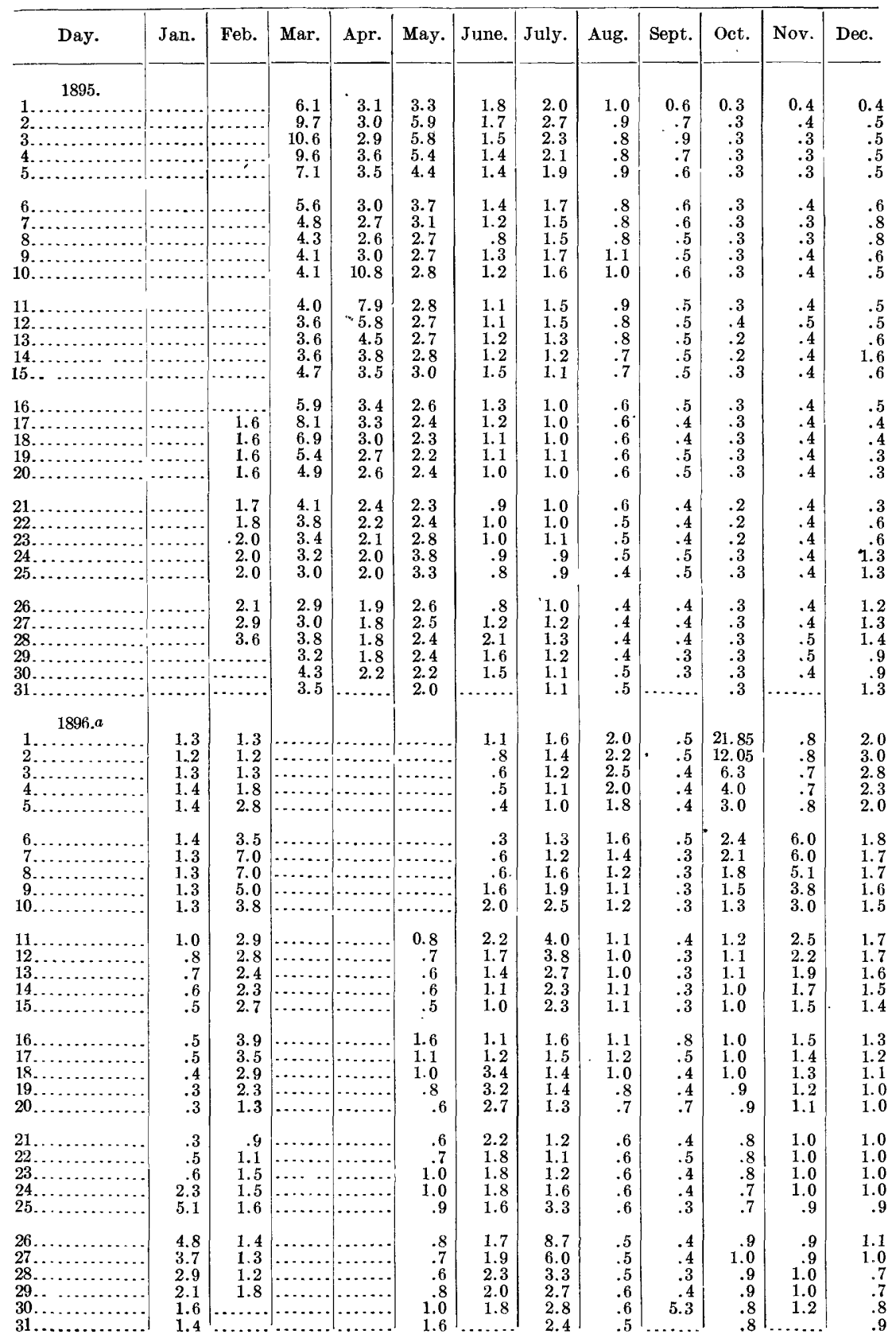

$a$ Gage heights January 1 to June 17, 1896, approximate. "See Introduction, page 31.

NoTE.-Sce description of station in regard to datum of gage. 
Daily gage height, in feet, of Potomac'River at Point of Rocks, Md.-Continued.

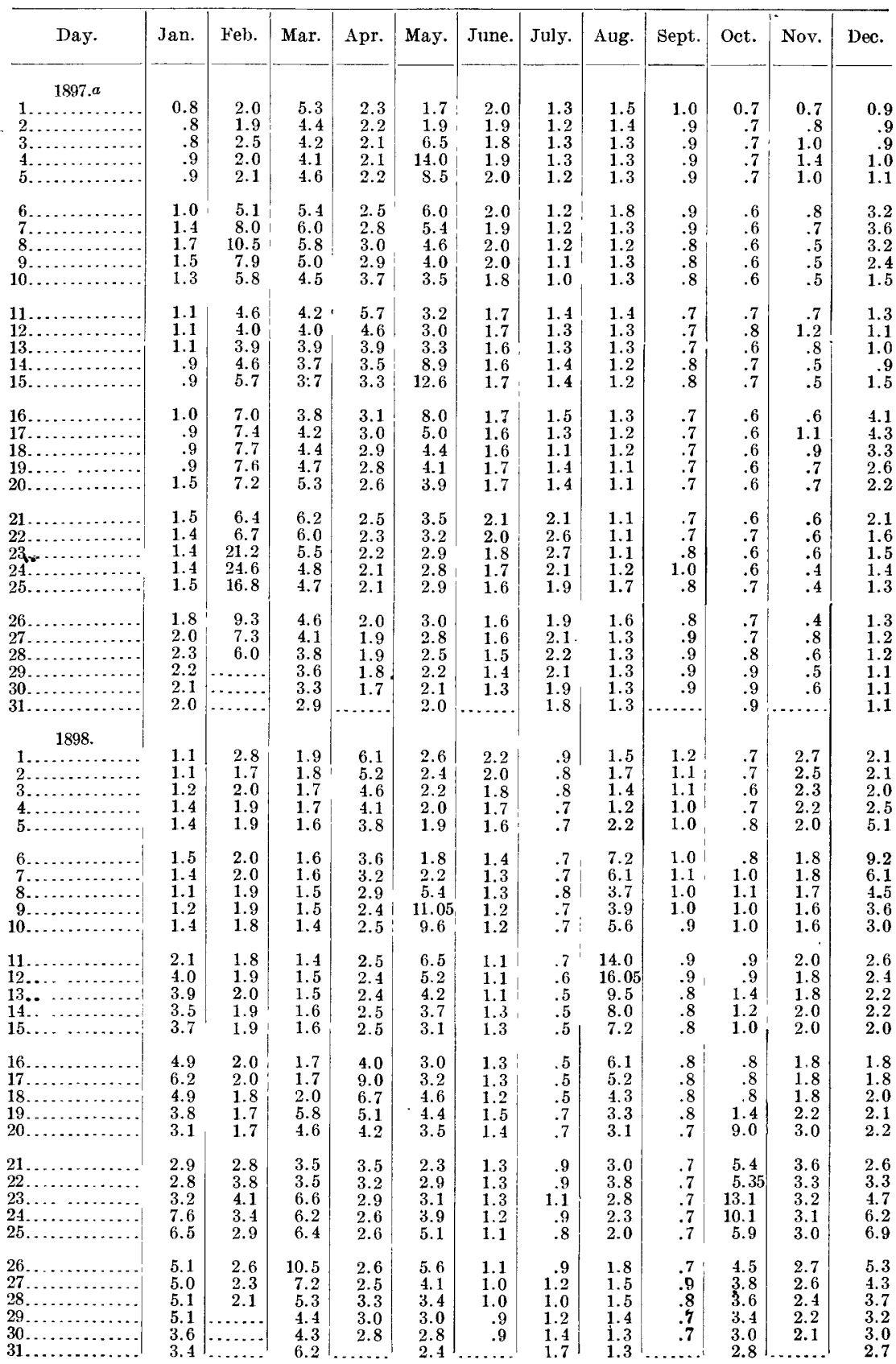


STREAM FLOW: POTOMAC RIVER.

Daily gage height, in feet, of Potomac River at Point of Rocks, Md.-Continued.

\begin{tabular}{|c|c|c|c|c|c|c|c|c|c|c|c|c|}
\hline $\mathrm{D}$ & Jan. & Feb. & Mar. & Apr. & May. & June. & July. & Ạug. & Sept. & Oct. & Nov. & Dec. \\
\hline $\begin{array}{l}1 \ldots \\
2 \ldots \\
3 \ldots \\
4 \ldots \\
5 \ldots\end{array}$ & $\begin{array}{l}3.0 \\
3.4 \\
2.9 \\
2.7 \\
2.3\end{array}$ & $\begin{array}{l}2.4 \\
2.0 \\
2.1 \\
2.1 \\
2.0\end{array}$ & $\begin{array}{r}11.9 \\
9.2 \\
8.2 \\
7.6 \\
8.5\end{array}$ & $\begin{array}{l}5.3 \\
4.5 \\
4.0 \\
3.6 \\
3.3\end{array}$ & $\begin{array}{l}1.6 \\
1.6 \\
2.1 \\
1.7 \\
1.7\end{array}$ & $\begin{array}{l}2.0 \\
2.4 \\
3.8 \\
2.6 \\
2.1\end{array}$ & $\begin{array}{r}1.0 \\
1.0 \\
1.1 \\
1.0 \\
.9\end{array}$ & $\begin{array}{r}0.7 \\
.7 \\
.7 \\
.9 \\
1.0\end{array}$ & $\begin{array}{r}0.9 \\
.9 \\
.8 \\
.8 \\
.8\end{array}$ & $\begin{array}{r}0.7 \\
.6 \\
.6 \\
.6 \\
.6\end{array}$ & $\begin{array}{l}0.8 \\
1.5 \\
2.5 \\
2.0 \\
1.8\end{array}$ & $\begin{array}{r}0.8 \\
.8 \\
.8 \\
.8 \\
.8\end{array}$ \\
\hline $\begin{array}{r}6 \ldots \\
7 \ldots \\
8 \ldots \\
9 \ldots \\
10 \ldots\end{array}$ & $\begin{array}{l}4.1 \\
6.9 \\
8.1 \\
6.8 \\
5.4\end{array}$ & $\begin{array}{l}2.0 \\
2.0 \\
2.3 \\
2.3 \\
3.6\end{array}$ & $\begin{array}{c}16.55 \\
12.9 \\
10.0 \\
8.1 \\
6.0\end{array}$ & $\begin{array}{l}3.0 \\
2.8 \\
3.0 \\
3.5 \\
3.4\end{array}$ & $\begin{array}{l}2.1 \\
2.0 \\
1.9 \\
2.0 \\
2.4\end{array}$ & $\begin{array}{l}1.8 \\
1.6 \\
1.5 \\
1.4 \\
1.7\end{array}$ & $\begin{array}{r}1.0 \\
2.0 \\
1.0 \\
1.0 \\
.9\end{array}$ & $\begin{array}{l}1.1 \\
1.1 \\
1.1 \\
1.0 \\
1.0\end{array}$ & $\begin{array}{l}.7 \\
.7 \\
.6 \\
.6 \\
.6\end{array}$ & $\begin{array}{l}.6 \\
.6 \\
.7 \\
.7 \\
.6\end{array}$ & $\begin{array}{l}1.4 \\
1.4 \\
1.3 \\
1.2 \\
1.0\end{array}$ & $\begin{array}{l}.7 \\
.7 \\
.7 \\
.7 \\
.7\end{array}$ \\
\hline $\begin{array}{l}11 \ldots \\
12 \ldots \\
13 \ldots \\
14 \ldots \\
15 \ldots\end{array}$ & $\begin{array}{l}4.6 \\
3.9 \\
3.6 \\
3.4 \\
3.4\end{array}$ & $\begin{array}{l}4.2 \\
4.2 \\
4.0 \\
3.9 \\
3.8\end{array}$ & $\begin{array}{l}5.8 \\
5.6 \\
5.0 \\
4.8 \\
4.5\end{array}$ & $\begin{array}{l}4.2 \\
4.5 \\
3.4\end{array}$ & $\begin{array}{l}4.5 \\
3.8 \\
3.4 \\
3.2 \\
2.8\end{array}$ & $\begin{array}{l}1.8 \\
1.7 \\
1.7 \\
1.6 \\
1.5\end{array}$ & $\begin{array}{r}.9 \\
.8 \\
.8 \\
.8 \\
.9\end{array}$ & $\begin{array}{l}.9 \\
.8 \\
.6 \\
.6 \\
.6\end{array}$ & $\begin{array}{r}.6 \\
.8 \\
1.2 \\
1.1 \\
1.0\end{array}$ & $\begin{array}{l}.6 \\
.6 \\
.6 \\
.5 \\
.5\end{array}$ & $\begin{array}{l}.9 \\
.9 \\
.8 \\
.8 \\
.8\end{array}$ & $\begin{array}{r}.7 \\
.8 \\
.8 \\
1.2 \\
3.0\end{array}$ \\
\hline $\begin{array}{l}16 \ldots \\
17 \ldots \\
18 \ldots \\
19 \ldots \\
20 \ldots\end{array}$ & $\begin{array}{l}4.0 \\
5.7 \\
5.2 \\
4.7 \\
4.4\end{array}$ & $\begin{array}{l}4.0 \\
4.5 \\
5.0 \\
5.2 \\
5.4\end{array}$ & $\begin{array}{l}4.6 \\
4.7 \\
4.4 \\
5.2 \\
5.4\end{array}$ & $\begin{array}{l}2.8 \\
2.7 \\
2.6 \\
2.5 \\
2.4\end{array}$ & \begin{tabular}{l|}
2.5 \\
2.3 \\
2.4 \\
8.55 \\
6.3
\end{tabular} & $\begin{array}{l}1.5 \\
1.4 \\
1.4 \\
1.3 \\
1.3\end{array}$ & $\begin{array}{r}1.1 \\
.9 \\
.7 \\
.7 \\
.7\end{array}$ & $\begin{array}{l}.7 \\
.7 \\
.6 \\
.6 \\
.6\end{array}$ & $\begin{array}{l}.9 \\
.8 \\
.7 \\
.7 \\
.9\end{array}$ & $\begin{array}{l}.5 \\
.5 \\
.5 \\
.5 \\
.5\end{array}$ & $\begin{array}{l}.8 \\
.8 \\
.7 \\
.7 \\
.7\end{array}$ & $\begin{array}{l}2.1 \\
1.7 \\
1.5 \\
1.6 \\
1.5\end{array}$ \\
\hline $\begin{array}{l}21 \ldots \\
22 \ldots \\
23 \ldots \\
24 \ldots \\
25 \ldots\end{array}$ & $\begin{array}{l}4.0 \\
3.5 \\
3.3 \\
3.2 \\
3.3\end{array}$ & $\begin{array}{r}6.0 \\
8.5 \\
14.8 \\
13.7 \\
9.0\end{array}$ & $\begin{array}{l}5.3 \\
4.7 \\
4.2 \\
4.0 \\
3.9\end{array}$ & $\begin{array}{l}2.2 \\
2.1 \\
2.0 \\
2.0 \\
2.0\end{array}$ & 5.0 & $\begin{array}{l}1.2 \\
1.2 \\
1.1 \\
1.1 \\
1.0\end{array}$ & $\begin{array}{l}.7 \\
.7 \\
.7 \\
.6 \\
.6\end{array}$ & $\begin{array}{l}.6 \\
.5 \\
.5 \\
.5 \\
.5\end{array}$ & $\begin{array}{l}.7 \\
.8 \\
.9 \\
.9 \\
.9\end{array}$ & $\begin{array}{l}.5 \\
.5 \\
.5 \\
.5 \\
.5\end{array}$ & $\begin{array}{l}.7 \\
.7 \\
.8 \\
.8 \\
.9\end{array}$ & $\begin{array}{l}1.3 \\
1.1 \\
1.0 \\
1.2 \\
1.8\end{array}$ \\
\hline $\begin{array}{l}26 \ldots \\
27 \ldots \\
28 \ldots \\
29 \ldots \\
30 \ldots \\
31 \ldots\end{array}$ & $\begin{array}{l}4.8 \\
4.7 \\
3.9 \\
3.2 \\
2.8 \\
2.6\end{array}$ & $\begin{array}{c}6.0 \\
9.25 \\
13.9 \\
\cdots \ldots . . \\
\cdots \ldots . .\end{array}$ & $\begin{array}{l}3.7 \\
3.5 \\
3.4 \\
4.6 \\
8.6 \\
7.0\end{array}$ & $\begin{array}{l}1.9 \\
1.9 \\
1.8 \\
1.7 \\
1.7\end{array}$ & $\begin{array}{l}1.9 \\
1.7 \\
1.7 \\
1.8\end{array}$ & $\begin{array}{l}1.0 \\
1.0 \\
1.2 \\
1.1 \\
1.0\end{array}$ & $\begin{array}{l}.6 \\
.6 \\
.6 \\
.5 \\
.8 \\
.8\end{array}$ & $\begin{array}{l}.5 \\
1.2 \\
1.1 \\
1.1 \\
1.0 \\
1.0\end{array}$ & $\begin{array}{r}1.0 \\
.8 \\
.7 \\
.7 \\
.8\end{array}$ & $\begin{array}{l}.5 \\
.5 \\
.5 \\
.5 \\
.5 \\
.6\end{array}$ & $\begin{array}{l}.9 \\
.8 \\
.8 \\
.8 \\
.8\end{array}$ & $\begin{array}{l}2.1 \\
1.6 \\
1.5 \\
1.6 \\
1.6 \\
1.6\end{array}$ \\
\hline $\begin{array}{c}1 \\
1 \ldots \\
2 \ldots \\
3 \ldots \\
4 \ldots \\
5 \ldots\end{array}$ & $\begin{array}{l}1.5 \\
1.5 \\
1.4 \\
1.5 \\
1.5\end{array}$ & $\begin{array}{l}1.0 \\
1.6 \\
2.0 \\
2.1 \\
2.2\end{array}$ & $\begin{array}{l}3.5 \\
4.5 \\
6.6 \\
5.9 \\
4.4\end{array}$ & $\begin{array}{l}4.8 \\
3.8 \\
3.4 \\
3.1 \\
2.8\end{array}$ & & $\begin{array}{l}1.3 \\
1.3 \\
1.3 \\
1.4 \\
1.6\end{array}$ & $\begin{array}{r}1.2 \\
1.1 \\
1.0 \\
1.0 \\
.9\end{array}$ & $\begin{array}{r}1.2 \\
1.1 \\
1.0 \\
.9 \\
.9\end{array}$ & $\begin{array}{l}.7 \\
.7 \\
.6 \\
.6 \\
.5\end{array}$ & $\begin{array}{l}.3 \\
.3 \\
.3 \\
.3 \\
.3\end{array}$ & $\begin{array}{l}.5 \\
.5 \\
.5 \\
.5 \\
.5\end{array}$ & $\begin{array}{l}2.4 \\
1.9 \\
1.6 \\
1.6 \\
2.0\end{array}$ \\
\hline $\begin{array}{r}8 \ldots \\
9 \ldots \ldots \\
10 \ldots\end{array}$ & $\begin{array}{l}1.4 \\
1.4 \\
1.3 \\
1.3 \\
1.2\end{array}$ & $\begin{array}{l}1.9 \\
1.8 \\
1.5 \\
1.8 \\
2.3\end{array}$ & $\begin{array}{l}4.0 \\
3.6 \\
3.9 \\
4.5 \\
4.0\end{array}$ & $\begin{array}{l}2.7 \\
2.6 \\
2.5 \\
2.4 \\
2.3\end{array}$ & 14 & $\begin{array}{l}1.6 \\
1.5 \\
1.4 \\
1.4 \\
1.3\end{array}$ & $\begin{array}{r}1.3 \\
1.0 \\
.9 \\
.8 \\
.7\end{array}$ & $\begin{array}{l}.8 \\
.8 \\
.7 \\
.6 \\
.6\end{array}$ & $\begin{array}{l}.6 \\
.5 \\
.4 \\
.4 \\
.3\end{array}$ & $\begin{array}{l}.3 \\
.3 \\
.3 \\
.3 \\
.3\end{array}$ & $\begin{array}{l}.4 \\
.4 \\
.4 \\
.5 \\
.6\end{array}$ & $\begin{array}{l}4.1 \\
5.9 \\
3.7 \\
3.0 \\
2.5\end{array}$ \\
\hline & $\begin{array}{l}1.3 \\
1.4 \\
1.4 \\
1.5 \\
1.8\end{array}$ & $\begin{array}{l}3.0 \\
2.5 \\
3.0 \\
4.5 \\
5.3\end{array}$ & $\begin{array}{l}3.6 \\
3.2 \\
2.9 \\
2.8 \\
2.7\end{array}$ & $\begin{array}{l}2.1 \\
2.1 \\
2.0 \\
1.9 \\
1.9\end{array}$ & & $\begin{array}{r}1.2 \\
1.1 \\
1.0 \\
.9 \\
1.3\end{array}$ & $\begin{array}{l}.6 \\
.6 \\
.5 \\
.5 \\
.5\end{array}$ & $\begin{array}{l}.5 \\
.5 \\
.4 \\
.4 \\
.4\end{array}$ & $\begin{array}{l}.4 \\
.3 \\
.3 \\
.4 \\
.4\end{array}$ & $\begin{array}{l}.3 \\
.3 \\
.3 \\
.3 \\
.9\end{array}$ & $\begin{array}{l}.5 \\
.4 \\
.4 \\
.4 \\
.4\end{array}$ & $\begin{array}{l}2.1 \\
1.8 \\
1.6 \\
1.5 \\
1.4\end{array}$ \\
\hline 19 & $\begin{array}{l}2.1 \\
1.9 \\
1.7 \\
1.6 \\
2.2\end{array}$ & $\begin{array}{l}4.5 \\
3.8 \\
3.1 \\
2.6 \\
2.3\end{array}$ & $\begin{array}{l}2.6 \\
2.4 \\
2.3 \\
2.4 \\
2.6\end{array}$ & $\begin{array}{l}1.8 \\
1.8 \\
1.7 \\
1.7 \\
1.6\end{array}$ & & $\begin{array}{l}1.0 \\
1.8 \\
2.8 \\
8.5 \\
6.2\end{array}$ & $\begin{array}{r}.4 \\
.4 \\
.4 \\
.5 \\
1.4\end{array}$ & $\begin{array}{l}.4 \\
.4 \\
.4 \\
.4 \\
.4\end{array}$ & $\begin{array}{l}.4 \\
.3 \\
.3 \\
.3 \\
.3\end{array}$ & $\begin{array}{l}.5 \\
.4 \\
.3 \\
.3 \\
.3\end{array}$ & $\begin{array}{l}.4 \\
.4 \\
.3 \\
.2 \\
.2\end{array}$ & $\begin{array}{l}1.4 \\
1.5 \\
1.2 \\
1.1 \\
1.1\end{array}$ \\
\hline 23 & $\begin{array}{l}3.6 \\
6.8 \\
5.5 \\
4.0 \\
3.3\end{array}$ & $\begin{array}{l}1.9 \\
3.0 \\
6.9 \\
7.1 \\
5.6\end{array}$ & $\begin{array}{l}5.8 \\
8.7 \\
6.6 \\
5.4 \\
5.0\end{array}$ & $\begin{array}{l}1.7 \\
2.2 \\
2.5 \\
2.8 \\
3.0\end{array}$ & $\begin{array}{l}1.7 \\
1.5\end{array}$ & $\begin{array}{l}4.6 \\
3.6 \\
2.7 \\
2.3 \\
2.1\end{array}$ & $\begin{array}{r}1.0 \\
.9 \\
.8 \\
1.8 \\
1.6\end{array}$ & $\begin{array}{l}.4 \\
.4 \\
.4 \\
.4 \\
.5\end{array}$ & $\begin{array}{l}.3 \\
.3 \\
.3 \\
.3 \\
.3\end{array}$ & $\begin{array}{l}.3 \\
.5 \\
.4 \\
.4 \\
.4\end{array}$ & $\begin{array}{l}.3 \\
.4 \\
.4 \\
.4 \\
.4\end{array}$ & $\begin{array}{r}1.0 \\
.9 \\
.9 \\
.8 \\
.8\end{array}$ \\
\hline $\begin{array}{l}26 \\
27 \\
28\end{array}$ & $\begin{array}{l}2.8 \\
2.5 \\
2.1 \\
1.8 \\
1.3 \\
1.2\end{array}$ & $\begin{array}{l}3.9 \\
3.3 \\
3.0\end{array}$ & $\begin{array}{l}4.1 \\
3.7 \\
3.5 \\
3.3 \\
3.2\end{array}$ & $\begin{array}{l}2.8 \\
2.5 \\
2.3 \\
2.1 \\
2.0\end{array}$ & $\begin{array}{l}1.4 \\
1.3 \\
1.2 \\
1.3 \\
1.3\end{array} \mid$ & $\begin{array}{l}2.0 \\
1.8 \\
1.5 \\
1.4 \\
1.3\end{array}$ & $\begin{array}{l}2.0 \\
1.4 \\
1.2 \\
1.0 \\
1.3 \\
1.3\end{array}$ & $\begin{array}{r}.6 \\
.7 \\
.9 \\
.9 \\
.8 \\
.8\end{array}$ & $\begin{array}{l}.3 \\
.3 \\
.2 \\
.3 \\
.3\end{array}$ & $\begin{array}{l}.4 \\
.5 \\
.5 \\
.5 \\
.5 \\
.5\end{array}$ & $\begin{array}{l}1.2 \\
3.3 \\
8.2 \\
5.4 \\
3.2 \\
\ldots . .\end{array}$ & $\begin{array}{l}.8 \\
.8 \\
.8 \\
.8 \\
.8\end{array}$ \\
\hline
\end{tabular}


Daily gage height, in feet, of Potomac River at Point of Rocks, Md.-Continued.

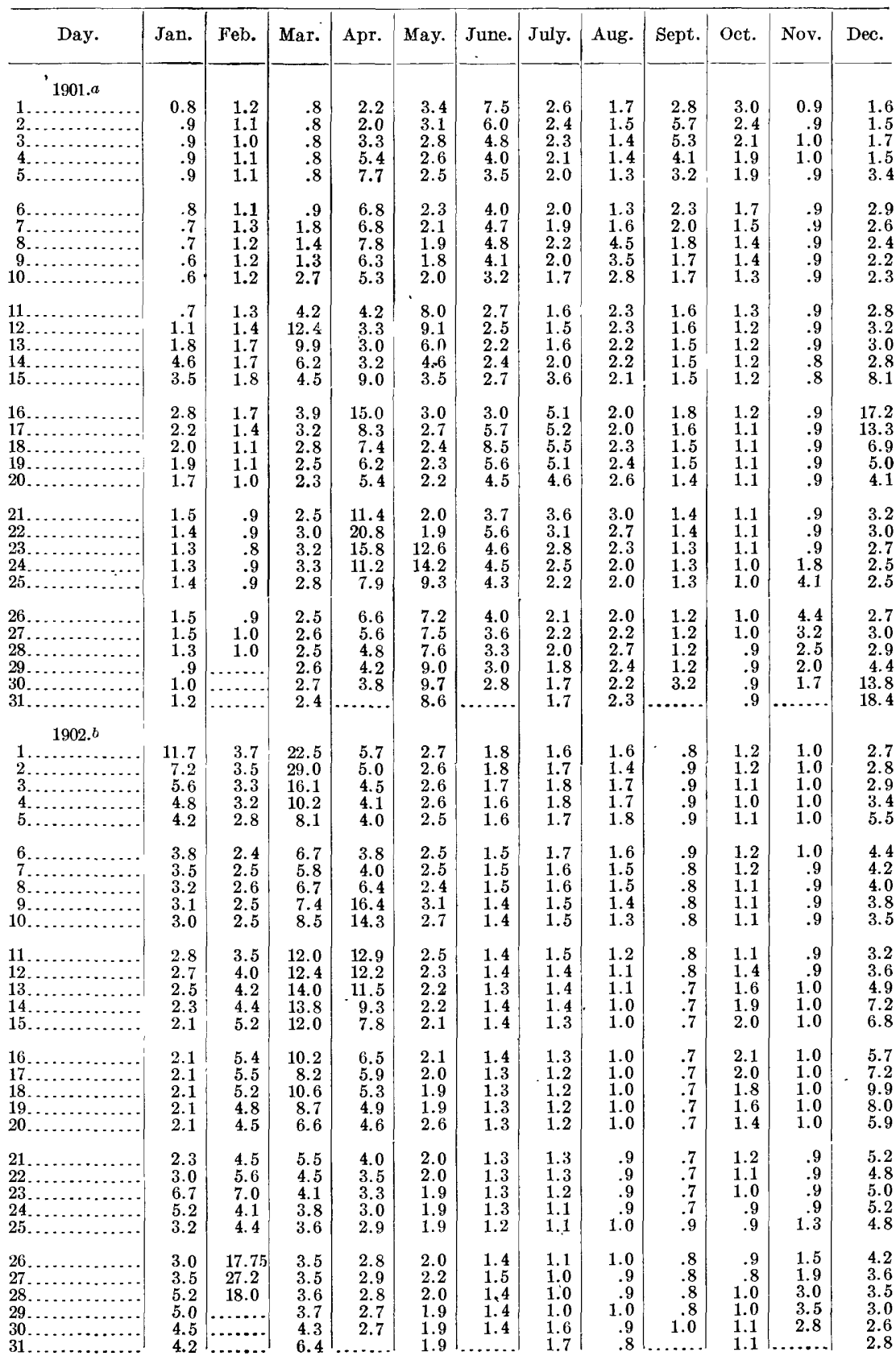

a Gage heights April 16 to December 31, 1901, are somewhat in error on account of uncertainty of the gage datum.

$b$ Gage heights January 1 to September 1, 1902, somewhat in error; datum not known. Gage heights September 2 to December 31, 1902, refer to datum 41.75 feet below bench mark No. 1 . Rise in gage February $11-23,1902$, due to ice gorging. 
Daily gage height, in feet, of Potomac River at Point of Rocks, Md.-Continued.

\begin{tabular}{|c|c|c|c|c|c|c|c|c|c|c|c|c|}
\hline Da & Jan. & Feb. & Mar. & Apr. & May. & June. & July. & Aug. & Sept. & Oct. & Nov. & Dec. \\
\hline $\begin{array}{l}\quad 1 \\
1 \ldots . \\
2 \ldots \\
3 \ldots \\
4 \ldots \\
5 \ldots\end{array}$ & $\begin{array}{r}2.6 \\
2.4 \\
4.9 \\
11.6 \\
10.3\end{array}$ & $\begin{array}{l}6.5 \\
6.1 \\
6.0 \\
6.3 \\
8.2\end{array}$ & $\begin{array}{r}14.2 \\
15.3 \\
8.9 \\
6.6 \\
5.7\end{array}$ & $\begin{array}{l}7.7 \\
7.0 \\
6.1 \\
5.0 \\
4.9\end{array}$ & $\begin{array}{l}3.0 \\
2.9 \\
2.8 \\
2.8 \\
2.8\end{array}$ & $\begin{array}{l}3.7 \\
4.9 \\
4.0 \\
3.4 \\
3.0\end{array}$ & $\begin{array}{l}8.7 \\
5.8 \\
4.5 \\
4.0 \\
3.5\end{array}$ & $\begin{array}{l}1.7 \\
1.7 \\
1.6 \\
1.7 \\
1.8\end{array}$ & $\begin{array}{l}2.0 \\
2.1 \\
2.2 \\
2.1 \\
2.0\end{array}$ & $\begin{array}{l}1.2 \\
1.1 \\
1.1 \\
1.1 \\
1.0\end{array}$ & $\begin{array}{l}1.1 \\
1.1 \\
1.0 \\
1.0 \\
1.0\end{array}$ & $\begin{array}{l}1.1 \\
1.1 \\
1.1 \\
1.1 \\
1.1\end{array}$ \\
\hline $\begin{array}{r}6 \ldots \ldots \\
7 \ldots \ldots \\
8 \ldots \\
9 \ldots \\
10 \ldots\end{array}$ & $\begin{array}{l}7.6 \\
5.7 \\
4.8 \\
4.2 \\
3.5\end{array}$ & $\begin{array}{l}9.2 \\
6.7 \\
6.3 \\
5.2 \\
4.5\end{array}$ & $\begin{array}{l}5.3 \\
5.0 \\
4.8 \\
4.6 \\
4.8\end{array}$ & $\begin{array}{l}4.6 \\
4.3 \\
4.1 \\
4.5 \\
6.1\end{array}$ & $\begin{array}{l}2.7 \\
2.7 \\
2.6 \\
2.5 \\
2.4\end{array}$ & $\begin{array}{l}2.6 \\
2.4 \\
6.4 \\
8.2 \\
7.4\end{array}$ & $\begin{array}{l}4.7 \\
8.2 \\
5.8 \\
4.0 \\
3.4\end{array}$ & $\begin{array}{l}1.9 \\
2.3 \\
2.3 \\
2.2 \\
2.0\end{array}$ & \begin{tabular}{l|}
1.9 \\
1.8 \\
1.7 \\
1.6 \\
1.5
\end{tabular} & $\begin{array}{l}1.0 \\
1.0 \\
1.1 \\
2.3 \\
2.0\end{array}$ & $\begin{array}{l}1.0 \\
1.0 \\
1.1 \\
1.1 \\
1.1\end{array}$ & $\begin{array}{l}1.1 \\
1.1 \\
1.1 \\
1.0 \\
1.0\end{array}$ \\
\hline $\begin{array}{l}11 . . \\
12 . . \\
13 . . \\
14 . . \\
15 . .\end{array}$ & $\begin{array}{l}3.0 \\
2.8 \\
2.7 \\
2.6 \\
2.7\end{array}$ & $\begin{array}{l}4.1 \\
3.7 \\
3.7 \\
3.9 \\
3.9\end{array}$ & $\begin{array}{l}5.4 \\
5.0 \\
4.6 \\
4.3 \\
4.0\end{array}$ & $\begin{array}{r}5.6 \\
5.4 \\
5.3 \\
5.6 \\
14.4\end{array}$ & $\begin{array}{l}2.3 \\
2.3 \\
2.2 \\
2.1 \\
2.1\end{array}$ & $\begin{array}{l}6.5 \\
5.1 \\
5.3 \\
6.0 \\
5.6\end{array}$ & $\begin{array}{l}3.0 \\
3.5 \\
5.6 \\
3.9 \\
3.5\end{array}$ & $\begin{array}{l}2.9 \\
2.8 \\
2.5 \\
2.3 \\
2.0\end{array}$ & $\begin{array}{l}1.5 \\
1.4 \\
1.4 \\
1.5 \\
1.5\end{array}$ & $\begin{array}{l}1.9 \\
1.8 \\
1.8 \\
1.7 \\
1.7\end{array}$ & $\begin{array}{l}1.0 \\
1.0 \\
1.0 \\
1.0 \\
1.0\end{array}$ & $\begin{array}{l}1.0 \\
1.0 \\
1.0 \\
1.0 \\
1.1\end{array}$ \\
\hline $\begin{array}{l}16 \ldots \\
17 \ldots \\
18 \ldots \\
19 \ldots \\
20 \ldots\end{array}$ & $\begin{array}{l}2.8 \\
2.9 \\
2.8 \\
2.8 \\
2.8\end{array}$ & $\begin{array}{l}4.0 \\
6.8 \\
7.4 \\
6.0 \\
4.9\end{array}$ & $\begin{array}{l}3.8 \\
3.6 \\
3.4 \\
3.2 \\
3.1\end{array}$ & $\begin{array}{r}15.1 \\
14.0 \\
10.4 \\
7.8 \\
6.7\end{array}$ & $\begin{array}{l}2.0 \\
2.0\end{array}$ & $\begin{array}{l}4.5 \\
4.0 \\
3.6 \\
3.2 \\
3.0\end{array}$ & $\begin{array}{l}3.2 \\
3.0 \\
2.8 \\
2.8 \\
3.1\end{array}$ & $\begin{array}{l}2.0 \\
1: 9 \\
1.8 \\
1.8 \\
1.7\end{array}$ & $\begin{array}{l}1.4 \\
1.5 \\
2.6 \\
4.5 \\
2.9\end{array}$ & $\begin{array}{l}1.6 \\
1.5 \\
1.5 \\
1.5 \\
1.6\end{array}$ & $\begin{array}{l}1.0 \\
1.1 \\
1.1 \\
1.1 \\
1.1\end{array}$ & $\begin{array}{l}1.2 \\
1.7 \\
1.5 \\
1.4 \\
1.5\end{array}$ \\
\hline $\begin{array}{l}21 . . \\
22 . . \\
23 . . \\
24 . \\
25 . .\end{array}$ & $\begin{array}{l}2.9 \\
3.6 \\
4.4 \\
3.8 \\
3.3\end{array}$ & $\begin{array}{l}4.5 \\
4.2 \\
4.1 \\
3.8 \\
4.2\end{array}$ & $\begin{array}{r}3.0 \\
3.1 \\
5.1 \\
7.0 \\
12.1\end{array}$ & $\begin{array}{l}5.5 \\
5.0 \\
4.5 \\
4.1 \\
3.8\end{array}$ & $\begin{array}{l}1.8 \\
1.8 \\
1.8 \\
1.9 \\
2.0\end{array}$ & $\begin{array}{l}2.8 \\
2.6 \\
2.4 \\
2.5 \\
3.3\end{array}$ & $\begin{array}{l}2.3 \\
2.1 \\
2.0\end{array}$ & $\begin{array}{l}1.7 \\
1.6 \\
1.6 \\
1.5 \\
1.4\end{array}$ & $\begin{array}{l}2.4 \\
2.0 \\
1.9 \\
1.7 \\
1.6\end{array}$ & $\begin{array}{l}1.6 \\
1.5 \\
1.5 \\
1.4 \\
1.4\end{array}$ & $\begin{array}{l}1.1 \\
1.1 \\
1.1 \\
1.2 \\
1.1\end{array}$ & $\begin{array}{l}1.6 \\
1.7 \\
1.7 \\
1.6 \\
1.6\end{array}$ \\
\hline $\begin{array}{l}26 \ldots \\
27 \ldots \\
28 \ldots \\
29 \ldots \\
30 \ldots \\
31 \ldots\end{array}$ & $\begin{array}{l}3.1 \\
2.8 \\
3.6 \\
6.2 \\
7.3 \\
7.0\end{array}$ & $\begin{array}{r}4.4 \\
4.5 \\
5.4 \\
\cdots \cdots\end{array}$ & $\begin{array}{l}8.6 \\
6.6 \\
5.3 \\
4.7 \\
4.5 \\
5.1\end{array}$ & $\begin{array}{l}3.6 \\
3.5 \\
3.4 \\
3.2 \\
3.1\end{array}$ & $\begin{array}{l}2.0 \\
2.1 \\
2.3 \\
2.4 \\
2.6 \\
2.8\end{array}$ & $\begin{array}{r}3.6 \\
3.2 \\
2.7 \\
6.2 \\
12.2 \\
\ldots \ldots . .\end{array}$ & $\begin{array}{l}2.0 \\
1.9 \\
1.9 \\
1.8 \\
2.0 \\
1.8\end{array}$ & $\begin{array}{l}1.3 \\
1.3 \\
1.5 \\
2.1 \\
2.5 \\
2.1\end{array}$ & $\begin{array}{r}1.4 \\
1.3 \\
1.3 \\
1.2 \\
1.2 \\
\ldots \ldots\end{array}$ & $\begin{array}{l}1.3 \\
1.3 \\
1.2 \\
1.2 \\
1.1 \\
1.1\end{array}$ & $\begin{array}{l}1.0 \\
1.0 \\
1.3 \\
1.1 \\
1.1\end{array}$ & $\begin{array}{l}1.6 \\
1.6 \\
1.6 \\
1.7 \\
1.7 \\
1.6\end{array}$ \\
\hline 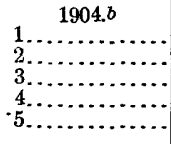 & $\begin{array}{l}1.5 \\
1.5 \\
2.0 \\
2.3 \\
1.8\end{array}$ & $\begin{array}{l}3.0 \\
2.9 \\
2.9 \\
3.3 \\
4.8\end{array}$ & $\begin{array}{l}5.3 \\
4.4 \\
4.0 \\
3.8 \\
4.0\end{array}$ & $\begin{array}{l}2.4 \\
2.6 \\
3.0 \\
3.5 \\
3.1\end{array}$ & $\begin{array}{l}5.6 \\
4.0 \\
3.6 \\
3.0 \\
2.8\end{array}$ & $\begin{array}{l}4.6 \\
4.1\end{array}$ & $\begin{array}{l}1.4 \\
1.3 \\
1.3 \\
1.3 \\
1.2\end{array}$ & $\begin{array}{l}1.4 \\
1.3 \\
1.2 \\
1.2 \\
1.2\end{array}$ & $\begin{array}{l}.9 \\
.8 \\
.8 \\
.8 \\
.9\end{array}$ & $\begin{array}{l}.6 \\
.6 \\
.6 \\
.6 \\
.6\end{array}$ & $\begin{array}{l}.6 \\
.6 \\
.7 \\
.7 \\
.7\end{array}$ & $\begin{array}{l}.8 \\
.8 \\
.8 \\
.8 \\
.8\end{array}$ \\
\hline $\begin{array}{c}6 \ldots \\
7 \ldots \\
8 \ldots \\
9 \ldots \\
10 \ldots\end{array}$ & $\begin{array}{l}1.5 \\
1.5 \\
1.5 \\
1.6 \\
1.6\end{array}$ & $\begin{array}{l}4.9 \\
4.9 \\
3.3 \\
3.5 \\
5.7\end{array}$ & $\begin{array}{l}4.2 \\
4.5 \\
5.0 \\
4.8 \\
5.4\end{array}$ & $\begin{array}{l}3.5 \\
3.0 \\
2.4 \\
2.3 \\
2.3\end{array}$ & $\begin{array}{l}2.6 \\
2.5 \\
2.8 \\
2.7 \\
2.6\end{array}$ & $\begin{array}{l}4.0 \\
3.6 \\
3.2\end{array}$ & $\begin{array}{l}1.5 \\
1.5 \\
1.6 \\
1.6 \\
1.7\end{array}$ & $\begin{array}{l}1.2 \\
1.3 \\
1.4 \\
1.5 \\
1.4\end{array}$ & $\begin{array}{r}1.0 \\
.9 \\
.8 \\
.7 \\
.7\end{array}$ & $\begin{array}{l}.6 \\
.6 \\
.6 \\
.5 \\
.5\end{array}$ & $\begin{array}{l}.7 \\
.7 \\
.7 \\
.7 \\
.7\end{array}$ & $\begin{array}{l}.8 \\
.8 \\
.8 \\
.8 \\
.9\end{array}$ \\
\hline $14, \ldots \ldots$ & $\begin{array}{l}1.6 \\
1.7 \\
1.6 \\
1.6 \\
1.6\end{array}$ & $\begin{array}{l}5.0 \\
3.9 \\
3.6 \\
3.5 \\
3.4\end{array}$ & $\begin{array}{l}4.5 \\
3.8 \\
3.3 \\
2.8 \\
2.6\end{array}$ & $\begin{array}{l}2.6 \\
3.1 \\
2.8 \\
2.6 \\
2.5\end{array}$ & $\begin{array}{l}2.5 \\
2.5 \\
2.4 \\
2.3 \\
2.2\end{array}$ & $\begin{array}{l}2.8 \\
2.6 \\
2.5 \\
2.4 \\
2.2\end{array}$ & $\begin{array}{l}2.9 \\
2.6 \\
3.4 \\
3.1 \\
3.0\end{array}$ & $\begin{array}{l}1.3 \\
1.3 \\
1.2 \\
1.2 \\
1.1\end{array}$ & $\begin{array}{r}.7 \\
.8 \\
1.0 \\
.9 \\
.9\end{array}$ & $\begin{array}{l}.5 \\
.6 \\
.7 \\
.7 \\
.6\end{array}$ & $\begin{array}{l}.7 \\
.7 \\
.7 \\
.8 \\
.8\end{array}$ & $\begin{array}{l}.9 \\
.9 \\
.9 \\
.9 \\
.9\end{array}$ \\
\hline $19 \ldots \ldots \ldots$ & $\begin{array}{l}1.5 \\
1.5 \\
1.5 \\
1.5 \\
1.5\end{array}$ & $\begin{array}{l}3.8 \\
3.8 \\
3.9 \\
3.9 \\
3.8\end{array}$ & $\begin{array}{l}2.4 \\
2.4 \\
2.2 \\
2.1 \\
2.2\end{array}$ & $\begin{array}{l}2.2 \\
2.1 \\
2.1 \\
2.0 \\
1.9\end{array}$ & $\begin{array}{l}2.2 \\
2.1 \\
2.2 \\
2.3 \\
3.1\end{array}$ & & $\begin{array}{l}2.8 \\
2.4 \\
2.0 \\
1.8 \\
1.6\end{array}$ & $\begin{array}{l}1.1 \\
1.1 \\
1.0 \\
1.0 \\
1.0\end{array}$ & $\begin{array}{r}1.0 \\
1.0 \\
.9 \\
.8 \\
.7\end{array}$ & $\begin{array}{l}.6 \\
.5 \\
.5 \\
.5 \\
.6\end{array}$ & $\begin{array}{l}.8 \\
.7 \\
.8 \\
.8 \\
.8\end{array}$ & $\begin{array}{r}.9 \\
.9 \\
.9 \\
.9 \\
1.0\end{array}$ \\
\hline $\begin{array}{l}21 \\
22 \\
23 \\
24 \ldots \ldots \\
25 \ldots \ldots \ldots \\
25 \ldots \ldots \ldots \\
\end{array}$ & $\begin{array}{l}1.5 \\
2.0 \\
4.0 \\
5.2 \\
7.4\end{array}$ & $\begin{array}{l}3.8 \\
4.5 \\
5.6 \\
6.0 \\
6.6\end{array}$ & $\begin{array}{l}2.3 \\
2.4 \\
2.5 \\
2.7 \\
3.7\end{array}$ & $\begin{array}{l}1.9 \\
1.8 \\
1.7 \\
1.7 \\
1.6\end{array}$ & $\begin{array}{l}6.2 \\
4.7 \\
4.0 \\
3.6 \\
3.1\end{array}$ & $\begin{array}{l}1.5 \\
2.5 \\
3.0 \\
2.8 \\
2.5\end{array}$ & $\begin{array}{l}1.4 \\
1.4 \\
1.3 \\
1.3 \\
1.3\end{array}$ & $\begin{array}{r}1.0 \\
.9 \\
.9 \\
1.0 \\
1.0\end{array}$ & $\begin{array}{r}.8 \\
1.0 \\
.9 \\
.8 \\
.8\end{array}$ & $\begin{array}{r}1.0 \\
.9 \\
.8 \\
.7 \\
.7\end{array}$ & $\begin{array}{l}.7 \\
.7 \\
.7 \\
.7 \\
.7\end{array}$ & $\begin{array}{l}1.0 \\
1.0 \\
1.0 \\
1.0 \\
1.1\end{array}$ \\
\hline $\begin{array}{l}26 . \\
27 \\
28\end{array}$ & $\begin{array}{l}5.1 \\
3.6 \\
3.3 \\
3.2 \\
3.0 \\
3.0\end{array}$ & $\begin{array}{l}6.6 \\
7.6 \\
6.6 \\
6.0\end{array}$ & $\begin{array}{l}3.4 \\
3.0 \\
2.8 \\
2.6 \\
2.5 \\
2.4\end{array}$ & $\begin{array}{l}1.5 \\
1.6 \\
2.0 \\
3.7 \\
6.3\end{array}$ & \begin{tabular}{l|}
2.8 \\
2.5 \\
2.4 \\
2.3 \\
2.2 \\
2.4
\end{tabular} & $\begin{array}{l}2.1 \\
1.7 \\
1.5 \\
1.5 \\
1.4\end{array}$ & $\begin{array}{l}1.4 \\
1.4 \\
1.4 \\
1.5 \\
1.5 \\
1.5\end{array}$ & $\begin{array}{l}1.2 \\
1.1 \\
1.1 \\
1.0 \\
1.0 \\
.9\end{array}$ & $\begin{array}{l}.8 \\
.7 \\
.7 \\
.7 \\
.7\end{array}$ & $\begin{array}{l}.7 \\
.7 \\
.7 \\
.6 \\
.6 \\
.6\end{array}$ & $\begin{array}{l}.7 \\
.7 \\
.7 \\
.8 \\
.8\end{array}$ & $\begin{array}{l}1.4 \\
1.5 \\
1.8 \\
1.8 \\
1.9 \\
2.0\end{array}$ \\
\hline
\end{tabular}

a Ice conditions December 17 to $31,1903$.

$b$ Ice conditions during part of January and February, 1904. 
Daily gage height, in feet, of Potomac River at Point of Rocks, Md.-Continued.

\begin{tabular}{|c|c|c|c|c|c|c|c|c|c|c|c|c|}
\hline Day. & Jan. & Feb. & Mar. & Apr. & May. & June. & July. & Aug. & Sept. & Oct. & Nov. & Dec. \\
\hline $\begin{array}{l}1 \\
1 \ldots \\
2 \ldots \\
3 \ldots \ldots \\
4 \ldots \\
5 \ldots\end{array}$ & $\begin{array}{l}2.0 \\
2.4 \\
2.5 \\
2.8 \\
3.0\end{array}$ & $\begin{array}{l}2.4 \\
2.4 \\
2.3 \\
2.2 \\
2.2\end{array}$ & $\begin{array}{l}2.4 \\
2.5 \\
2.6 \\
2.8 \\
3.4\end{array}$ & $\begin{array}{l}3.2 \\
3.0 \\
2.7 \\
2.5 \\
2.6\end{array}$ & $\begin{array}{l}1.6 \\
1.6 \\
1.6 \\
1.5 \\
1.5\end{array}$ & $\begin{array}{l}1.5 \\
1.4 \\
1.4 \\
1.4 \\
1.3\end{array}$ & $\begin{array}{l}2.1 \\
1.8 \\
1.6 \\
1.5 \\
3.0\end{array}$ & $\begin{array}{l}2.9 \\
2.7 \\
2.4 \\
2.2 \\
2.0\end{array}$ & $\begin{array}{l}2.2 \\
2.0 \\
1.8 \\
1.7 \\
1.6\end{array}$ & $\begin{array}{l}1.0 \\
1.0 \\
1.0 \\
1.0 \\
1.0\end{array}$ & $\begin{array}{l}1.6 \\
1.5 \\
1.5 \\
1.4 \\
1.4\end{array}$ & $\begin{array}{l}1.6 \\
1.6 \\
2.5 \\
4.6 \\
4.0\end{array}$ \\
\hline $\begin{array}{c}6 . . \\
7 . \\
8 . . \\
9 \\
10 .\end{array}$ & $\begin{array}{l}3.2 \\
4.4 \\
4.6 \\
4.0 \\
3.4\end{array}$ & $\begin{array}{l}2.2 \\
2.2 \\
2.1 \\
2.1 \\
2.0\end{array}$ & $\begin{array}{l}4.0 \\
4.4 \\
5.1 \\
6.0 \\
6.4\end{array}$ & $\begin{array}{l}2.7 \\
2.8 \\
3.0 \\
3.0 \\
2.9\end{array}$ & $\begin{array}{l}1.5 \\
1.4 \\
1.4 \\
1.4 \\
1.4\end{array}$ & $\begin{array}{l}1.3 \\
1.5 \\
1.6 \\
1.9 \\
2.0\end{array}$ & $\begin{array}{l}3.5 \\
4.0 \\
4.5 \\
4.0 \\
3.6\end{array}$ & $\begin{array}{l}1.8 \\
1.6 \\
1.5 \\
1.5 \\
1.4\end{array}$ & $\begin{array}{l}1.5 \\
1.5 \\
1.4 \\
1.4 \\
1.3\end{array}$ & $\begin{array}{l}1.0 \\
1.0 \\
1.0 \\
1.0 \\
.9\end{array}$ & $\begin{array}{l}13 \\
1.3 \\
1.3 \\
1.2 \\
1.2\end{array}$ & $\begin{array}{l}3.4 \\
3.0 \\
2.4 \\
2.3 \\
2.2\end{array}$ \\
\hline & $\begin{array}{l}3.0 \\
2.8 \\
2.6 \\
2.5 \\
2.5\end{array}$ & $\begin{array}{l}2.0 \\
2.0 \\
2.0 \\
2.0 \\
2.0\end{array}$ & $\begin{array}{r}11.0 \\
10.1 \\
6.9 \\
6.0 \\
5.9\end{array}$ & $\begin{array}{l}2.8 \\
2.8 \\
2.9 \\
2.8 \\
2.6\end{array}$ & $\begin{array}{l}1.4 \\
1.4 \\
1.4 \\
1.4 \\
3.0\end{array}$ & $\begin{array}{l}1.9 \\
1.9 \\
2.3 \\
2.2 \\
2.0\end{array}$ & $\begin{array}{l}2.8 \\
2.3 \\
3.2 \\
4.1 \\
5.0\end{array}$ & $\begin{array}{l}1.4 \\
1.3 \\
1.3 \\
1.3 \\
1.6\end{array}$ & $\begin{array}{l}1.3 \\
1.4 \\
1.4 \\
2.0 \\
1.7\end{array}$ & $\begin{array}{r}.9 \\
1.2 \\
1.1 \\
1.4 \\
1.6\end{array}$ & $\begin{array}{l}1.0 \\
1.0 \\
1.0 \\
1.0 \\
1.0\end{array}$ & $\begin{array}{l}2.1 \\
2.0 \\
1.9 \\
1.8 \\
1.8\end{array}$ \\
\hline & $\begin{array}{l}2.9 \\
3.8 \\
3.2 \\
2.8 \\
2.6\end{array}$ & $\begin{array}{l}2.0 \\
2.1 \\
2.1 \\
2.1 \\
2.2\end{array}$ & $\begin{array}{l}5.5 \\
5.0 \\
45 \\
4.4 \\
5.0\end{array}$ & $\begin{array}{l}2.4 \\
2.3 \\
2.2 \\
2.1 \\
2.0\end{array}$ & $\begin{array}{l}2.8 \\
2.7 \\
2.6 \\
2.5 \\
2.4\end{array}$ & $\begin{array}{l}2.0 \\
1.9 \\
1.8 \\
1.6 \\
1.4\end{array}$ & $\begin{array}{l}5.4 \\
4.2 \\
4.0 \\
3.6 \\
3.3\end{array}$ & $\begin{array}{l}2.0 \\
2.9 \\
3.4 \\
2.5 \\
2.1\end{array}$ & $\begin{array}{l}1.5 \\
1.4 \\
1.4 \\
1.4 \\
1.3\end{array}$ & $\begin{array}{l}1.5 \\
1.3 \\
1.2 \\
10 \\
1.2\end{array}$ & $\begin{array}{r}1.0 \\
1.0 \\
.9 \\
.9 \\
.9\end{array}$ & $\begin{array}{l}1.7 \\
1.6 \\
1.5 \\
1.5 \\
1.5\end{array}$ \\
\hline $\begin{array}{l}3 . \\
4\end{array}$ & $\begin{array}{l}2.6 \\
2.7 \\
2.8 \\
2.4 \\
2.1\end{array}$ & $\begin{array}{l}2.2 \\
2.2 \\
2.2 \\
2.2 \\
2.2\end{array}$ & $\begin{array}{l}5.9 \\
7.5 \\
8.4 \\
6.7 \\
5.8\end{array}$ & $\begin{array}{l}2.0 \\
1.9 \\
1.9 \\
1.9 \\
1.9\end{array}$ & $\begin{array}{l}2.3 \\
2.2 \\
22 \\
2.0 \\
1.8\end{array}$ & $\begin{array}{l}1.3 \\
1.5 \\
2.5 \\
3.6 \\
5.2\end{array}$ & $\begin{array}{l}3.0 \\
2.8 \\
2.6 \\
4.0 \\
3.5\end{array}$ & $\begin{array}{l}1.8 \\
1.7 \\
1.5 \\
1.5 \\
20\end{array}$ & $\begin{array}{l}1.3 \\
1.2 \\
1.2 \\
1.2 \\
1.2\end{array}$ & $\begin{array}{l}1.4 \\
1.8 \\
1.6 \\
1.4 \\
1.4\end{array}$ & $\begin{array}{l}.9 \\
.9 \\
.9 \\
.9 \\
.9\end{array}$ & $\begin{array}{l}2.8 \\
7.6 \\
7.5 \\
6.1 \\
5.5\end{array}$ \\
\hline $\begin{array}{l}28 \\
29 \\
30\end{array}$ & $\begin{array}{r}1.9 \\
a 3.0 \\
2.6 \\
2.6 \\
25 \\
2.5\end{array}$ & $\begin{array}{l}2.3 \\
2.3 \\
2.4\end{array}$ & $\begin{array}{l}6.9 \\
5.9 \\
52 \\
4.6 \\
4.0 \\
36\end{array}$ & $\begin{array}{l}1.8 \\
1.8 \\
1.7 \\
1.7 \\
1.7\end{array}$ & $\begin{array}{l}1.7 \\
16 \\
1.5 \\
1.5 \\
1.4 \\
1.3\end{array}$ & $\begin{array}{l}7.0 \\
5.0 \\
3.6 \\
3.0 \\
2.6\end{array}$ & $\begin{array}{l}3.0 \\
2.6 \\
2.3 \\
2.0 \\
1.8 \\
25\end{array}$ & $\begin{array}{l}2.9 \\
3.4 \\
3.9 \\
2.9 \\
2.8 \\
2.5\end{array}$ & $\begin{array}{ll}1 & 1 \\
1.1 \\
1.1 \\
1.1 \\
1 & 0\end{array}$ & $\begin{array}{l}1.5 \\
1.6 \\
1.7 \\
2.1 \\
2.0 \\
1.8\end{array}$ & $\begin{array}{r}.9 \\
.9 \\
.9 \\
.9 \\
1.3\end{array}$ & $\begin{array}{l}4.8 \\
4.1 \\
3.4 \\
3.1 \\
3.6 \\
3.2\end{array}$ \\
\hline 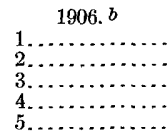 & $\begin{array}{l}3.6 \\
3.4 \\
3.3 \\
3.7 \\
8.0\end{array}$ & $\begin{array}{l}3.0 \\
2.9 \\
2.7 \\
2.5 \\
2.4\end{array}$ & $\begin{array}{l}1.9 \\
1.8 \\
1.8 \\
2.0 \\
4.0\end{array}$ & $\begin{array}{r}11.1 \\
9.6 \\
7.7 \\
6.5 \\
5.5\end{array}$ & $\begin{array}{l}3.3 \\
3.1 \\
2.8 \\
2.8 \\
2.6\end{array}$ & $\begin{array}{l}1.8 \\
1.7 \\
1.7 \\
1.6 \\
1.5\end{array}$ & $\begin{array}{l}2.2 \\
1.9 \\
2.0 \\
2.9 \\
2.6\end{array}$ & $\begin{array}{l}1.6 \\
1.5 \\
1.5 \\
4.3 \\
3.6\end{array}$ & $\begin{array}{l}3.7 \\
3.1 \\
2.8 \\
2.6 \\
2.3\end{array}$ & $\begin{array}{l}1.2 \\
1.2 \\
1.4 \\
1.5 \\
2.2\end{array}$ & $\begin{array}{l}2.6 \\
2.5 \\
2.3 \\
2.3 \\
2.1\end{array}$ & $\begin{array}{l}1.9 \\
1.8 \\
1.9 \\
1.7 \\
1.8\end{array}$ \\
\hline & $\begin{array}{l}8.6 \\
7.0 \\
4.8 \\
4.0 \\
3.5\end{array}$ & $\begin{array}{l}2.2 \\
2.2 \\
2.0 \\
1.9 \\
1.8\end{array}$ & $\begin{array}{l}5.45 \\
4.5 \\
3.6 \\
3.0 \\
2.7\end{array}$ & $\begin{array}{l}5.1 \\
4.8 \\
4.8 \\
4.3 \\
4.2\end{array}$ & $\begin{array}{l}2.5 \\
2.6 \\
2.5 \\
2.4 \\
2.4\end{array}$ & $\begin{array}{l}1.7 \\
1.9 \\
1.7 \\
2.4 \\
3.0\end{array}$ & $\begin{array}{l}2.4 \\
2.2 \\
2.0 \\
1.9 \\
1.7\end{array}$ & $\begin{array}{l}2.8 \\
2.4 \\
2.6 \\
2.8 \\
3.2\end{array}$ & \begin{tabular}{l|}
2.1 \\
1.9 \\
1.8 \\
1.8 \\
1.7
\end{tabular} & $\begin{array}{l}2.5 \\
2.7 \\
2.6 \\
2.5 \\
2.2\end{array}$ & $\begin{array}{l}2.1 \\
2.1 \\
2.0 \\
2.0 \\
1.9\end{array}$ & $\begin{array}{l}1.8 \\
1.85 \\
1.7 \\
1.75 \\
1.8\end{array}$ \\
\hline $15 .$. & $\begin{array}{l}3.1 . \\
2.9 \\
2.8 \\
3.0 \\
3.3\end{array}$ & $\begin{array}{l}1.8 \\
1.9 \\
1.8 \\
1.8 \\
1.9\end{array}$ & $\begin{array}{l}2.6 \\
2.5 \\
2.3 \\
2.3 \\
2.3\end{array}$ & $\begin{array}{l}5.5 \\
5.6 \\
4.9 \\
4.0 \\
\text { 5. } 75\end{array}$ & $\begin{array}{l}2.4 \\
2.2 \\
2.3 \\
2.2 \\
2.0\end{array}$ & $\begin{array}{l}2.5 \\
.2 \\
1.8 \\
1.6 \\
1.6\end{array}$ & $\begin{array}{l}1.5 \\
1.4 \\
1.3 \\
1.2 \\
1.3\end{array}$ & $\begin{array}{l}7.6 \\
5.7 \\
4.3 \\
3.7 \\
3.0\end{array}$ & $\begin{array}{l}1.7 \\
1.6 \\
1.6 \\
1.5 \\
1.5\end{array}$ & $\begin{array}{l}2.0 \\
1.9 \\
1.6 \\
1.5 \\
1.6\end{array}$ & $\begin{array}{l}1.8 \\
1.8 \\
1.8 \\
1.7 \\
1.8\end{array}$ & $\begin{array}{l}2.0 \\
2.2 \\
3.5 \\
3.1 \\
2.65\end{array}$ \\
\hline & $\begin{array}{l}3.6 \\
3.7 \\
4.1 \\
3.9 \\
3.5\end{array}$ & \begin{tabular}{l|}
1.8 \\
1.7 \\
1.6 \\
1.6 \\
1.6
\end{tabular} & $\begin{array}{l}2.4 \\
2.5 \\
2.5 \\
2.6 \\
2.9\end{array}$ & $\begin{array}{l}8.45 \\
7.5 \\
6.3 \\
5.1 \\
4.5\end{array}$ & 1.8 & $\begin{array}{l}1.6 \\
1.6 \\
2.5 \\
3.2 \\
2.9\end{array}$ & $\begin{array}{l}1.2 \\
1.2 \\
1.8 \\
1.8 \\
1.7\end{array}$ & $\begin{array}{l}5.1 \\
4.8 \\
4.2 \\
3.5 \\
4.4\end{array}$ & $\begin{array}{l}1.4 \\
\text { 1. } 6 \\
1.5 \\
\text { 1. } 4 \\
1.4\end{array}$ & $\begin{array}{r}1.4 \\
1.4 \\
1.5 \\
1.7 \\
12.5\end{array}$ & \begin{tabular}{l|}
1.7 \\
1.8 \\
1.8 \\
1.9 \\
1.9
\end{tabular} & $\begin{array}{r}2.7 \\
2.8 \\
4.7 \\
10.0 \\
7.2\end{array}$ \\
\hline $5 \ldots \ldots$ & $\begin{array}{l}3.4 \\
3.3 \\
3.2 \\
3.1 \\
6.35\end{array}$ & $\begin{array}{l}1.7 \\
1.8 \\
1.9 \\
2.0 \\
2.0\end{array}$ & $\begin{array}{l}2.9 \\
3.1 \\
3.4 \\
3.9 \\
3.9\end{array}$ & $\begin{array}{l}4.1 \\
3.6 \\
3.3 \\
3.1 \\
2.9\end{array}$ & $\begin{array}{l}1.6 \\
1.6 \\
1.6 \\
1.4 \\
1.5\end{array}$ & \begin{tabular}{l|}
3.1 \\
5.0 \\
4.3 \\
3.6 \\
3.1
\end{tabular} & $\begin{array}{l}1.5 \\
1.4 \\
1.5 \\
2.0 \\
2.1\end{array}$ & $\begin{array}{l}4.0 \\
5.3 \\
4.8 \\
3.7 \\
4.1\end{array}$ & $\begin{array}{l}1.4 \\
1.3 \\
1.3 \\
1.3 \\
1.3\end{array}$ & $\begin{array}{c}16.1 \\
11.65 \\
8.5 \\
6.6 \\
5.4\end{array}$ & $\begin{array}{l}3.0 \\
4.2 \\
4.0 \\
3.3 \\
3.0\end{array}$ & $\begin{array}{l}5.6 \\
5.2 \\
4.6 \\
3.8 \\
3.2\end{array}$ \\
\hline $\begin{array}{l}26 \ldots \\
27 \ldots \\
28 \ldots \\
29 \ldots \\
30 \ldots \\
31 \ldots\end{array}$ & $\begin{array}{l}5.1 \\
4.2 \\
3.6 \\
3.4 \\
3.2 \\
3.1\end{array}$ & $\begin{array}{l}1.9 \\
1.8 \\
2.0\end{array}$ & $\begin{array}{r}3.9 \\
3.7 \\
7.8 \\
12.9 \\
10.5 \\
10.9\end{array}$ & $\begin{array}{l}2.9 \\
2.8 \\
5.1 \\
5.0 \\
4.3\end{array}$ & $\begin{array}{l}1.4 \\
1.3 \\
1.9 \\
1.9 \\
1.7 \\
1.7\end{array}$ & $\begin{array}{l}2.6 \\
2.4 \\
2.3 \\
3.1 \\
3.0\end{array}$ & $\begin{array}{l}1.9 \\
1.7 \\
1.8 \\
1.9 \\
2.0 \\
1.7\end{array}$ & $\begin{array}{l}4.8 \\
4.6 \\
6.7 \\
6.0 \\
5.3 \\
4.4\end{array}$ & $\begin{array}{l}1.3 \\
1.2 \\
1.2 \\
1.2 \\
1.2\end{array}$ & $\begin{array}{l}4.5 \\
4.1 \\
3.6 \\
3.3 \\
3.1 \\
2.8\end{array}$ & $\begin{array}{l}2.7 \\
2.6 \\
2.2 \\
2.1 \\
2.1\end{array}$ & $\begin{array}{l}2.8 \\
2.85 \\
2.9 \\
3.0 \\
3.1 \\
3.2\end{array}$ \\
\hline
\end{tabular}


Rating tables for Potomac River at Point of Rocks, $M d$.

FEBRUARY 17, 1895, TO MARCH 31, 1902.a

\begin{tabular}{|r|r|r|r|r|r|r|r|}
\hline $\begin{array}{c}\text { Gage } \\
\text { height. }\end{array}$ & Discharge. & $\begin{array}{c}\text { Gage } \\
\text { height. }\end{array}$ & Discharge. & $\begin{array}{c}\text { Gage } \\
\text { height. }\end{array}$ & Discharge. & $\begin{array}{c}\text { Gage } \\
\text { height. }\end{array}$ & Discharge. \\
\hline Feet. & Second-feet. & Feet. & Second-feet. & Feet. & Second-feet. & Feet. & Second-feet. \\
0.20 & 1,040 & 2.00 & 7,120 & 3.80 & 16,380 & 7.20 & 38,560 \\
.30 & 1,180 & 2.10 & 7,580 & 3.90 & 16,940 & 7.40 & 40,080 \\
.40 & 1,340 & 2.20 & 8,060 & 4.00 & 17,520 & 7.60 & 41,600 \\
.50 & 1,540 & 2.30 & 8,540 & 4.20 & 18,680 & 7.80 & 43,160 \\
.60 & 1,760 & 2.40 & 9,020 & 4.40 & 19,840 & 8.00 & 44,720 \\
.70 & 2,000 & 2.50 & 9,500 & 4.60 & 21,040 & 8.50 & 48,700 \\
.80 & 2,280 & 2.60 & 10,000 & 4.80 & 22,240 & 9.00 & 52,700 \\
.90 & 2,600 & 2.70 & 10,500 & 5.00 & 23,480 & 10.00 & 61,000 \\
1.00 & 2,940 & 2.80 & 11,000 & 5.20 & 24,740 & 11.00 & 69,300 \\
1.10 & 3,300 & 2.90 & 11,520 & 5.40 & 26,020 & 12.00 & 77,600 \\
1.20 & 3,680 & 3.00 & 12,040 & 5.60 & 27,340 & 13.00 & 85,900 \\
1.30 & 4,080 & 3.10 & 12,560 & 5.80 & 28,660 & 14.00 & 94,200 \\
1.40 & 4,480 & 3.20 & 13,080 & 6.00 & 30,020 & 15.00 & 102,500 \\
1.50 & 4,900 & 3.30 & 13,620 & 6.20 & 31,380 & 16.00 & 110,800 \\
1.60 & 5,320 & 3.40 & 14,160 & 6.40 & 32,780 & 17.00 & 119,100 \\
1.70 & 5,760 & 3.50 & 14,700 & 6.60 & 34,180 & 18.00 & 127,400 \\
1.80 & 6,200 & 3.60 & 15,260 & 6.80 & 35,620 & 19.00 & 135,700 \\
1.90 & 6,660 & 3.70 & 15,820 & 7.00 & 37,080 & 20.00 & 144,000 \\
\hline
\end{tabular}

APRIL 1, 1902, TO DECEMBER 31, 1906.b

\begin{tabular}{|r|r|r|r|r|r|r|r|r|}
\hline 0.50 & 900 & 2.30 & 6,130 & 4.20 & 15,150 & 7.80 & 38,500 \\
.60 & 1,090 & 2.40 & 6,520 & 4.40 & 16,270 & 8.69 & 39,980 \\
.70 & 1,295 & 2.50 & 6,920 & 4.60 & 17,430 & 8.50 & 43,740 \\
.80 & 1,515 & 2.60 & 7,330 & 4.80 & 18,610 & 9.00 & 47,600 \\
.90 & 1,750 & 2.70 & 7,750 & 5.00 & 19,820 & 9.50 & 51,560 \\
1.00 & 2,000 & 2.80 & 8,180 & 5.20 & 21,060 & 10.00 & 55,600 \\
1.10 & 2,260 & 2.90 & 8,620 & 5.40 & 22,300 & 11.00 & 63,900 \\
1.20 & 2,530 & 3.00 & 9,070 & 5.60 & 23,560 & 12.00 & 72,200 \\
1.30 & 2,810 & 3.10 & 9,530 & 5.80 & 24,840 & 13.00 & 80,500 \\
1.40 & 3,100 & 3.20 & 10,000 & 6.00 & 26,140 & 14.00 & 88,800 \\
1.50 & 3,400 & 3.30 & 10,480 & 6.20 & 27,460 & 15.00 & 97,100 \\
1.60 & 3,700 & 3.40 & 10,970 & 6.40 & 28,780 & 16.00 & 105,400 \\
1.70 & 4,010 & 3.50 & 11,470 & 6.60 & 30,100 & 17.00 & 113,700 \\
1.80 & 4,330 & 3.60 & 11,980 & 6.80 & 31,460 & 18.00 & 122,000 \\
1.90 & 4,670 & 3.70 & 12,490 & 7.00 & 32,820 & 19.00 & 130,300 \\
2.00 & 5,020 & 3.80 & 13,010 & 7.20 & 34,220 & 20.00 & 138,600 \\
2.10 & 5,380 & 3.90 & 13,530 & 7.40 & 35,620 & & \\
2.20 & 5,750 & 4.00 & 14,070 & 7.60 & 37,060 & & \\
\hline
\end{tabular}

$a$ The above table is strictly applicable only for open-channel conditions. It is based on 23 discharge measurements made during 1895-1901, inclusive. It is well defined between gage heights 0.3 foot and 14.0 feet. Above 9.00 feet the rating curve is a tangent, the difference being 830 per tenth.

$b$ This table is strictly applicable only for open-channel conditions. It is based on discharge measurements made during 1902-1906, inclusive. It is fairly well defined between gage heights 1.0 foot and 14.0 feet. Above gage height 10.0 feet the rating curve is a tangent, the difference being 830 per tenth. 
Estimated monthly discharge of Potomac River at Point of Rocks, Md.

[Drainage area, 9,650 square miles. $a$ ]

\begin{tabular}{|c|c|c|c|c|c|c|c|c|}
\hline \multirow[b]{2}{*}{ Month. } & \multicolumn{3}{|c|}{ Discharge in second-feet. } & \multicolumn{3}{|c|}{ Run-off. } & \multicolumn{2}{|c|}{ Precipitation. } \\
\hline & Maximum. & Minimum. & Mean. & $\begin{array}{l}\text { Second- } \\
\text { feet per } \\
\text { square } \\
\text { mile. }\end{array}$ & $\begin{array}{l}\text { Depth in } \\
\text { inches. }\end{array}$ & $\begin{array}{l}\text { Per cent } \\
\text { of pre- } \\
\text { cipita- } \\
\text { tion. }\end{array}$ & $\underset{\text { inches. }}{\text { In }}$ & $\begin{array}{l}\text { Loss in } \\
\text { inches. }\end{array}$ \\
\hline 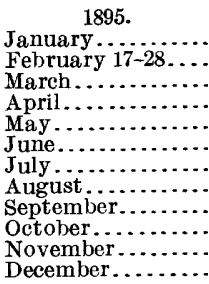 & $\begin{array}{r}15,260 \\
65,980 \\
67,640 \\
29,340 \\
7,580 \\
10,500 \\
3,300 \\
2,600 \\
1,340 \\
1,540 \\
5,320\end{array}$ & $\begin{array}{r}5,320 \\
11,520 \\
6,200 \\
7,120 \\
2,280 \\
2,600 \\
1,340 \\
1,180 \\
1,040 \\
1,180 \\
1,180\end{array}$ & $\begin{array}{r}7,413 \\
24,560 \\
14,500 \\
12,540 \\
4,033 \\
4,443 \\
1,997 \\
1,565 \\
1,163 \\
1,333 \\
2,259\end{array}$ & $\begin{array}{l}0.768 \\
2.54 \\
1.50 \\
1.30 \\
.418 \\
.460 \\
.207 \\
.162 \\
.120 \\
.138 \\
.234\end{array}$ & $\begin{array}{l}0.343 \\
2.93 \\
1.67 \\
1.50 \\
.466 \\
.530 \\
.239 \\
.181 \\
.138 \\
.154 \\
.270\end{array}$ & $\begin{array}{r}122 \\
71 \\
61 \\
12 \\
18 \\
11 \\
21 \\
12 \\
11 \\
10\end{array}$ & $\begin{array}{r}3.25 \\
b 1.16 \\
2.41 \\
2.37 \\
2.46 \\
3.88 \\
3.03 \\
2.10 \\
.85 \\
1.19 \\
1.37 \\
2.59\end{array}$ & $\begin{array}{r}r .52 \\
.70 \\
.96 \\
3.41 \\
2.50 \\
1.86 \\
.67 \\
1.05 \\
1.22 \\
2.32\end{array}$ \\
\hline The year..... & & & & & & & 26.66 & \\
\hline 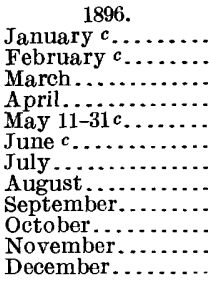 & $\begin{array}{r}24,100 \\
37,080 \\
\ldots \ldots \ldots \\
5,320 \\
14,160 \\
50,300 \\
9,500 \\
25,380 \\
159,400 \\
30,020 \\
12,040\end{array}$ & $\begin{array}{l}1,540 \\
1,180 \\
2,940 \\
1,540 \\
1,180 \\
2,000 \\
2,000 \\
2,000\end{array}$ & $\begin{array}{r}5,257 \\
10,470 \\
\ldots \\
2 . \\
2,560 \\
5,429 \\
9,283 \\
3,449 \\
2,175 \\
12,490 \\
6,928 \\
4,723\end{array}$ & $\begin{array}{c}.545 \\
1.08 \\
\ldots \ldots \ldots \\
\ldots . .265 \\
.562 \\
.962 \\
.357 \\
.225 \\
1.29 \\
.718 \\
.489\end{array}$ & $\begin{array}{c}.628 \\
1.16 \\
\mathbf{3} \ldots . . \\
.2 . .20 \\
.207 \\
.627 \\
1.11 \\
.412 \\
.251 \\
1.49 \\
.801 \\
.564\end{array}$ & 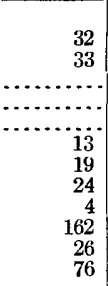 & $\begin{array}{r}1.97 \\
3.48 \\
3.55 \\
1.61 \\
a 2.91 \\
5.01 \\
5.79 \\
1.69 \\
6.09 \\
.92 \\
3.02 \\
.74\end{array}$ & \begin{tabular}{r}
1.34 \\
2.32 \\
$\ldots \ldots .$. \\
\hdashline$\ldots . .$. \\
\hdashline .38 \\
4.68 \\
1.27 \\
5.84 \\
-.57 \\
2.22 \\
.18
\end{tabular} \\
\hline The year..... & & & & $\cdots$ & & & 36.78 & . \\
\hline 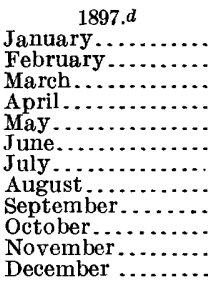 & $\begin{array}{r}8,540 \\
182,200 \\
31,380 \\
28,000 \\
94,200 \\
7,580 \\
10,500 \\
6,200 \\
2,940 \\
2,600 \\
4,480 \\
19,260\end{array}$ & $\begin{array}{r}2,280 \\
6,660 \\
11,520 \\
5,760 \\
5,760 \\
4,080 \\
2,940 \\
3,300 \\
2,000 \\
1,760 \\
1,340 \\
2,600\end{array}$ & $\begin{array}{r}4,284 \\
42,660 \\
20,850 \\
10,830 \\
22,950 \\
5,997 \\
5,315 \\
4,092 \\
2,337 \\
1,968 \\
2,096 \\
6,579\end{array}$ & $\begin{array}{l}.444 \\
4.42 \\
2.16 \\
1.12 \\
2.38 \\
.621 \\
.551 \\
.424 \\
.242 \\
.204 \\
.217 \\
.681\end{array}$ & $\begin{array}{l}.512 \\
4.60 \\
2.49 \\
1.25 \\
2.74 \\
.693 \\
.635 \\
.489 \\
.270 \\
.235 \\
.242 \\
.785\end{array}$ & $\begin{array}{r}33 \\
78 \\
104 \\
55 \\
57 \\
28 \\
16 \\
17 \\
17 \\
17 \\
6 \\
24\end{array}$ & $\begin{array}{l}\text { 1. } 55 \\
5.88 \\
2.40 \\
2.28 \\
4.77 \\
2.43 \\
4.08 \\
2.93 \\
1.60 \\
1.43 \\
4.10 \\
3.22\end{array}$ & $\begin{array}{r}1.04 \\
1.28 \\
-.09 \\
1.03 \\
2.03 \\
1.74 \\
3.44 \\
2.44 \\
1.33 \\
1.19 \\
3.86 \\
2.44\end{array}$ \\
\hline The year.... & 182,200 & 1,340 & 10,830 & 1.12 & 14. 94 & 41 & 36.68 & 21.74 \\
\hline 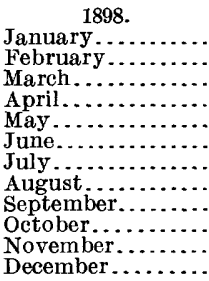 & $\begin{array}{r}41,600 \\
18,100 \\
65,150 \\
52,700 \\
69,720 \\
8,060 \\
5,760 \\
111,400 \\
3,680 \\
86,730 \\
15,260 \\
54,360\end{array}$ & $\begin{array}{l}3,300 \\
5,760 \\
4,480 \\
9,020 \\
6,200 \\
2,600 \\
1,540 \\
3,680 \\
2,000 \\
1,760 \\
5,320 \\
6,200\end{array}$ & $\begin{array}{r}14,660 \\
8,339 \\
15,470 \\
15,970 \\
18,060 \\
4,178 \\
2,418 \\
22,140 \\
2,497 \\
13,580 \\
8,557 \\
15,330\end{array}$ & $\begin{array}{l}1.52 \\
.864 \\
1.60 \\
1.65 \\
1.87 \\
.433 \\
.250 \\
2.29 \\
.259 \\
1.41 \\
.886 \\
1.59\end{array}$ & $\begin{array}{c}1.75 \\
.900 \\
1.84 \\
1.84 \\
2.16 \\
.483 \\
.288 \\
2.64 \\
.289 \\
1.63 \\
.988 \\
1.83\end{array}$ & $\begin{array}{r}46 \\
84 \\
41 \\
78 \\
45 \\
27 \\
9 \\
38 \\
22 \\
25 \\
41 \\
63\end{array}$ & $\begin{array}{l}3.78 \\
1.07 \\
4.44 \\
2.36 \\
4.76 \\
1.81 \\
3.21 \\
7.00 \\
1.32 \\
6.41 \\
2.41 \\
2.91\end{array}$ & $\begin{array}{r}2.03 \\
.17 \\
2.60 \\
.52 \\
2.60 \\
1.33 \\
2.92 \\
4.36 \\
1.03 \\
4.78 \\
1.42 \\
1.08\end{array}$ \\
\hline The & 111,400 & 1,540 & 11,780 & 1.22 & 16.64 & 40 & 41.48 & 24.84 \\
\hline
\end{tabular}

a 9,650 square miles used to obtain run-off for 1906: 9,654 used for all other years.

$b$ Precipitation for complete month, February, 1895, and May, 1896.

c Estimates January 1 to June 17, 1896, liable to considerable error, owing to possible error in gage heights. See Introduction, page 31 .

$d 1897$ estimates may be only approximate, owing to large errors in the wire length during the year. See Introduction, page 32. 
STREAM FLOW : POTOMAC RIVER.

Estimated monthly descharge of Potomac River at Point of Rocks, Md.-Continued.

\begin{tabular}{|c|c|c|c|c|c|c|c|c|}
\hline \multirow[b]{2}{*}{ Month. } & \multicolumn{3}{|c|}{ Discharge in second-feet. } & \multicolumn{3}{|c|}{ Run-off. } & \multicolumn{2}{|c|}{ Precipitation. } \\
\hline & Maximum. & Minimum. & Mean. & $\begin{array}{l}\text { Second- } \\
\text { feet per } \\
\text { square } \\
\text { mile. }\end{array}$ & $\begin{array}{l}\text { Depth in } \\
\text { inches. }\end{array}$ & $\begin{array}{c}\text { Per cent } \\
\text { of pre- } \\
\text { cipita- } \\
\text { tion. }\end{array}$ & inches. & $\begin{array}{l}\text { Loss in } \\
\text { inches. }\end{array}$ \\
\hline 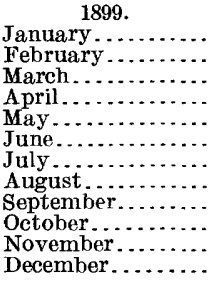 & $\begin{array}{r}45,500 \\
100,800 \\
115,400 \\
25,380 \\
49,140 \\
16,380 \\
7,120 \\
3,680 \\
3,680 \\
2,000 \\
9,500 \\
12,040\end{array}$ & $\begin{array}{r}8,540 \\
7,120 \\
14,160 \\
5,760 \\
5,320 \\
2,940 \\
1,540 \\
1,540 \\
1,760 \\
1,540 \\
2,000 \\
2,000\end{array}$ & $\begin{array}{r}20,870 \\
28,130 \\
35,240 \\
11,750 \\
11,600 \\
5,314 \\
2,519 \\
2,335 \\
2,345 \\
1,663 \\
3,171 \\
4,068\end{array}$ & $\begin{array}{l}2.16 \\
2.91 \\
3.65 \\
1.22 \\
1.20 \\
.550 \\
.261 \\
.242 \\
.243 \\
.172 \\
.328 \\
.421\end{array}$ & $\begin{array}{l}2.49 \\
3.03 \\
4.21 \\
1.36 \\
1.38 \\
.614 \\
.301 \\
.279 \\
.271 \\
.198 \\
.366 \\
.485\end{array}$ & $\begin{array}{r}98 \\
70 \\
106 \\
99 \\
27 \\
18 \\
13 \\
10 \\
7 \\
11 \\
22 \\
25\end{array}$ & $\begin{array}{l}2.54 \\
4.44 \\
3.96 \\
1.37 \\
5.15 \\
3.47 \\
2.28 \\
2.84 \\
3.94 \\
1.88 \\
1.69 \\
1.94\end{array}$ & $\begin{array}{r}0.05 \\
1.41 \\
-.25 \\
.01 \\
3.77 \\
2.86 \\
1.98 \\
2.56 \\
3.67 \\
1.68 \\
1.32 \\
1.46\end{array}$ \\
\hline The year. & 115,400 & 1,540 & 10,750 & 1.11 & 14. 98 & 42 & 35.50 & 20.52 \\
\hline 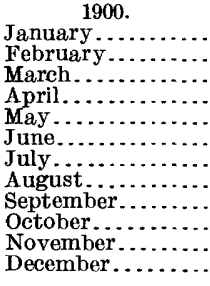 & $\begin{array}{r}35,620 \\
37,820 \\
50,300 \\
22,240 \\
7,120 \\
48,700 \\
7,120 \\
3,680 \\
2,000 \\
2,600 \\
46,300 \\
29,340 \\
\end{array}$ & $\begin{array}{l}3,680 \\
2,940 \\
8,540 \\
5,320 \\
2,940 \\
2,600 \\
1,340 \\
1,340 \\
1,040 \\
1,180 \\
1,040 \\
2,280\end{array}$ & $\begin{array}{r}8,166 \\
13,340 \\
18,470 \\
9,295 \\
4,466 \\
8,394 \\
3,008 \\
1,917 \\
1,344 \\
1,333 \\
4,570 \\
6,218\end{array}$ & $\begin{array}{l}.846 \\
1.38 \\
1.91 \\
.963 \\
.463 \\
.869 \\
.312 \\
.199 \\
.139 \\
.138 \\
.473 \\
.644\end{array}$ & $\begin{array}{l}.975 \\
1.44 \\
2.20 \\
1.07 \\
.534 \\
.970 \\
.360 \\
.229 \\
.155 \\
.159 \\
.528 \\
.742\end{array}$ & $\begin{array}{r}47 \\
42 \\
64 \\
80 \\
23 \\
23 \\
10 \\
11 \\
8 \\
8 \\
15 \\
42 \\
\end{array}$ & $\begin{array}{l}2.10 \\
3.44 \\
3.42 \\
1.34 \\
2.32 \\
4.19 \\
3.74 \\
2.08 \\
1.95 \\
2.08 \\
3.62 \\
1.77 \\
\end{array}$ & $\begin{array}{l}1.12 \\
2.00 \\
1.22 \\
.27 \\
1.79 \\
3.22 \\
3.38 \\
1.85 \\
1.79 \\
1.92 \\
3.09 \\
1.03\end{array}$ \\
\hline The y & 50,300 & 1,040 & 6,710 & .695 & 9.36 & 29 & 32.04 & 22.68 \\
\hline 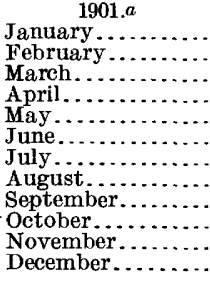 & $\begin{array}{r}21,040 \\
6,200 \\
80,920 \\
150,600 \\
95,860 \\
48,700 \\
26,680 \\
20,440 \\
28,000 \\
12,040 \\
19,840 \\
130,700\end{array}$ & $\begin{array}{l}1,760 \\
2,280 \\
2,280 \\
7,120 \\
6,200 \\
8,060 \\
4,900 \\
4,080 \\
3,680 \\
2,600 \\
2,280 \\
4,480\end{array}$ & $\begin{array}{r}4,929 \\
3,649 \\
13,800 \\
39,750 \\
26,920 \\
18,840 \\
10,720 \\
8,337 \\
7,636 \\
4,303 \\
4,648 \\
25,610\end{array}$ & $\begin{array}{l}.511 \\
.378 \\
1.43 \\
4.12 \\
2.79 \\
1.95 \\
1.11 \\
.864 \\
.791 \\
.446 \\
.481 \\
2.65\end{array}$ & $\begin{array}{l}.589 \\
.394 \\
1.65 \\
4.60 \\
3.22 \\
2.18 \\
1.28 \\
.996 \\
.882 \\
.514 \\
.537 \\
3.06\end{array}$ & $\begin{array}{r}30 \\
85 \\
48 \\
76 \\
50 \\
48 \\
34 \\
16 \\
28 \\
90 \\
22 \\
54\end{array}$ & $\begin{array}{l}1.95 \\
.46 \\
3.46 \\
6.05 \\
6.47 \\
4.56 \\
3.82 \\
6.23 \\
3.15 \\
.57 \\
2.50 \\
5.71\end{array}$ & $\begin{array}{l}1.36 \\
.07 \\
1.81 \\
1.45 \\
3.25 \\
2.38 \\
2.54 \\
5.23 \\
2.27 \\
.06 \\
1.96 \\
2.65\end{array}$ \\
\hline The & 150,600 & 1,760 & 14,100 & 1.46 & 19.90 & 44 & 44. 93 & 25.03 \\
\hline 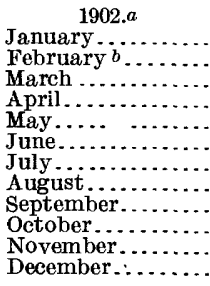 & $\begin{array}{r}75,110 \\
203,800 \\
218,700 \\
108,700 \\
9,530 \\
4,330 \\
4,330 \\
4,330 \\
2,000 \\
5,380 \\
11,470 \\
\mathbf{5 4 , 7 8 0}\end{array}$ & $\begin{array}{r}7,580 \\
9,020 \\
14,700 \\
7,750 \\
4,670 \\
2,530 \\
2,000 \\
1,515 \\
1,295 \\
1,515 \\
1,750 \\
7,330\end{array}$ & $\begin{array}{r}17,520 \\
32,520 \\
54,410 \\
28,760 \\
5,973 \\
3,186 \\
3,086 \\
2,464 \\
1,490 \\
2,767 \\
2,837 \\
18,970\end{array}$ & $\begin{array}{c}1.81 \\
3.37 \\
5.64 \\
2.99 \\
.619 \\
.330 \\
.320 \\
.255 \\
.154 \\
.287 \\
.294 \\
1.96\end{array}$ & $\begin{array}{l}2.09 \\
3.51 \\
6.50 \\
3.34 \\
.714 \\
.368 \\
.369 \\
.294 \\
.172 \\
.331 \\
.328 \\
2.26\end{array}$ & $\begin{array}{r}75 \\
90 \\
149 \\
104 \\
36 \\
11 \\
13 \\
13 \\
8 \\
10 \\
13 \\
50\end{array}$ & $\begin{array}{l}2.78 \\
3.88 \\
4.35 \\
3.20 \\
1.97 \\
3.45 \\
2.75 \\
2.16 \\
2.01 \\
3.36 \\
2.49 \\
4.50\end{array}$ & $\begin{array}{r}.69 \\
.37 \\
-2.15 \\
-.14 \\
1.26 \\
3.08 \\
2.38 \\
1.87 \\
1.84 \\
3.03 \\
2.16 \\
2.24\end{array}$ \\
\hline The & 218,700 & 1,295 & 14,500 & 1.50 & 20.28 & 55 & 36.90 & 16.62 \\
\hline
\end{tabular}

$a$ Estimates April 16, 1901, to September 1, 1902, liable to some error on account of uncertainty of the gage datum.

$b$ Ice gorge February 11-23, 1902; no correction made in estimates. 
Estimated monthly discharge of Potomac River at Pont of Rocks, Md.--Continued.

\begin{tabular}{|c|c|c|c|c|c|c|c|c|}
\hline \multirow[b]{2}{*}{ Month. } & \multicolumn{3}{|c|}{ Discharge in second-feet. } & \multicolumn{3}{|c|}{ Run-off. } & \multicolumn{2}{|c|}{ Precipitation. } \\
\hline & Maximum. & Minimum. & Mean. & $\begin{array}{c}\text { Second- } \\
\text { feet per } \\
\text { square } \\
\text { mile. }\end{array}$ & $\begin{array}{l}\text { Depth in } \\
\text { inches. }\end{array}$ & $\begin{array}{l}\text { Per cent } \\
\text { of pre- } \\
\text { cipita- } \\
\text { tion. }\end{array}$ & $\begin{array}{c}\text { In } \\
\text { inches. }\end{array}$ & $\left\{\begin{array}{l}\text { Loss in } \\
\text { inches. }\end{array}\right.$ \\
\hline 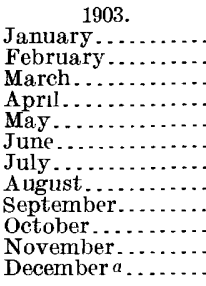 & $\begin{array}{r}68,880 \\
49,160 \\
99,590 \\
97,930 \\
9,070 \\
73,860 \\
45,260 \\
8,620 \\
16,850 \\
6,130 \\
2,810 \\
4,010\end{array}$ & $\begin{array}{r}6,520 \\
12,490 \\
9,070 \\
9,530 \\
4,330 \\
6,520 \\
4,330 \\
2,810 \\
2,530 \\
2,000 \\
2,000 \\
2,000\end{array}$ & $\begin{array}{r}17,200 \\
22,190 \\
26,730 \\
28,900 \\
6,212 \\
17,970 \\
12,760 \\
4,826 \\
4,669 \\
3,212 \\
2,175 \\
2,926\end{array}$ & $\begin{array}{l}1.78 \\
2.30 \\
2.77 \\
2.99 \\
.643 \\
1.86 \\
1.32 \\
.500 \\
484 \\
.333 \\
.225 \\
.303\end{array}$ & $\begin{array}{l}2.05 \\
2.40 \\
3.19 \\
3.34 \\
.741 \\
2.08 \\
1.52 \\
.576 \\
.540 \\
.384 \\
.251 \\
.349\end{array}$ & $\begin{array}{l}58 \\
69 \\
89 \\
89 \\
25 \\
32 \\
33 \\
16 \\
24 \\
15 \\
27 \\
45\end{array}$ & $\begin{array}{l}3.51 \\
3.49 \\
3.59 \\
3.75 \\
2.92 \\
6.57 \\
4.67 \\
3.67 \\
2.29 \\
2.57 \\
.91 \\
.78\end{array}$ & $\begin{array}{r}1.46 \\
1.09 \\
.40 \\
.41 \\
2.18 \\
4.49 \\
3.15 \\
3.09 \\
1.75 \\
2.19 \\
.66 \\
.43\end{array}$ \\
\hline The ye & 99,590 & 2,000 & 12,480 & 1.29 & 17.42 & 45 & 38.72 & 21.30 \\
\hline 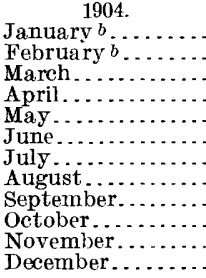 & $\begin{array}{r}35,620 \\
37,060 \\
22,300 \\
28,120 \\
27,460 \\
38,500 \\
10,970 \\
3,400 \\
2,000 \\
2,000 \\
1,515 \\
5,020\end{array}$ & $\begin{array}{r}3,400 \\
8,620 \\
5,380 \\
3,400 \\
5,380 \\
3,100 \\
2,530 \\
1,750 \\
1,295 \\
900 \\
1,090 \\
1,515\end{array}$ & $\begin{array}{r}7,287 \\
17,480 \\
11,170 \\
7,406 \\
9,362 \\
10,160 \\
4,510 \\
2,394 \\
1,592 \\
1.164 \\
1,340 \\
2,201\end{array}$ & $\begin{array}{l}.755 \\
1.81 \\
1.16 \\
.767 \\
.970 \\
1.05 \\
.467 \\
.248 \\
.165 \\
.121 \\
.139 \\
.228\end{array}$ & $\begin{array}{l}.870 \\
1.95 \\
1.34 \\
.856 \\
1.12 \\
1.17 \\
.538 \\
.286 \\
.184 \\
.140 \\
.155 \\
.263\end{array}$ & $\begin{array}{r}42 \\
163 \\
-64 \\
35 \\
33 \\
24 \\
12 \\
12 \\
8 \\
8 \\
19 \\
10\end{array}$ & $\begin{array}{l}2.05 \\
1.20 \\
2.08 \\
2.46 \\
3.43 \\
4.78 \\
4.54 \\
2.32 \\
2.25 \\
1.66 \\
.79 \\
2.52\end{array}$ & $\begin{array}{r}1.18 \\
-.75 \\
.74 \\
1.60 \\
2.31 \\
3.61 \\
4.00 \\
2.03 \\
2.07 \\
1.52 \\
.64 \\
2.26\end{array}$ \\
\hline The year. & 38,500 & 900 & 6,339 & .657 & 8.87 & 30 & 30.08 & $2] .21$ \\
\hline 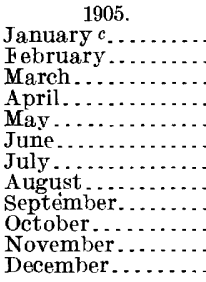 & $\begin{array}{r}17,430 \\
6,520 \\
63,900 \\
10,000 \\
9,070 \\
32,820 \\
22,300 \\
13,530 \\
5,750 \\
5,380 \\
3,700 \\
37,060\end{array}$ & $\begin{array}{l}4,670 \\
5,020 \\
6,520 \\
4,010 \\
2,810 \\
2,810 \\
3,400 \\
2,810 \\
2,000 \\
1,750 \\
1,750 \\
3,400\end{array}$ & $\begin{array}{r}8,626 \\
5,625 \\
23,480 \\
6,581 \\
4,493 \\
6,579 \\
10,190 \\
5,830 \\
3,205 \\
2,888 \\
2,267 \\
10,640\end{array}$ & $\begin{array}{c}.894 \\
.583 \\
2.43 \\
.682 \\
.465 \\
.681 \\
1.06 \\
.604 \\
.332 \\
.299 \\
.235 \\
1.10\end{array}$ & $\begin{array}{c}1.03 \\
.607 \\
2.80 \\
.761 \\
.536 \\
.760 \\
1.22 \\
.696 \\
.370 \\
.345 \\
.262 \\
1.27\end{array}$ & $\begin{array}{l}33 \\
35 \\
97 \\
\mathbf{4 4} \\
18 \\
15 \\
18 \\
17 \\
20 \\
10 \\
21 \\
36\end{array}$ & $\begin{array}{l}3.12 \\
1.73 \\
2.89 \\
1.72 \\
3.05 \\
5.23 \\
6.63 \\
4.08 \\
1.86 \\
3.47 \\
1.22 \\
3.52\end{array}$ & 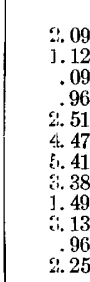 \\
\hline The year... & 63,900 & 1,750 & 7,534 & .780 & 10.66 & 28 & 38.52 & $2 \pi .86$ \\
\hline $\begin{array}{l}\quad 1906 . \\
\text { January ..... } \\
\text { February .... } \\
\text { March...... } \\
\text { April........ } \\
\text { May......... } \\
\text { June......... } \\
\text { July......... } \\
\text { August..... } \\
\text { September... } \\
\text { October.... } \\
\text { November... } \\
\text { December... }\end{array}$ & $\begin{array}{r}44,500 \\
9,070 \\
79,670 \\
64,730 \\
10,480 \\
19,820 \\
8,620 \\
37,060 \\
12,490 \\
106,200 \\
15,150 \\
55,600\end{array}$ & $\begin{array}{l}8,180 \\
3,700 \\
4,330 \\
8,180 \\
2,810 \\
3,400 \\
2,530 \\
3,400 \\
2,530 \\
2,530 \\
4,010 \\
4,010\end{array}$ & $\begin{array}{r}14,990 \\
5,116 \\
15,900 \\
22,440 \\
5,538 \\
7,007 \\
4,381 \\
15,200 \\
4,275 \\
16,310 \\
6,341 \\
11,000\end{array}$ & $\begin{array}{l}1.55 \\
.530 \\
1.65 \\
2.33 \\
.574 \\
.726 \\
.454 \\
1.58 \\
.444 \\
1.69 \\
.657 \\
1.14\end{array}$ & $\begin{array}{l}1.79 \\
.552 \\
1.90 \\
2.60 \\
.662 \\
.810 \\
.523 \\
1.82 \\
.495 \\
1.95 \\
.733 \\
1.31\end{array}$ & & $\cdots$ & $\begin{array}{c}\cdots \\
\cdots\end{array}$ \\
\hline The year. & 106,200 & 2,530 & 10,710 & 1.11 & 15. 14 & & & . \\
\hline
\end{tabular}

a Ice conditions December 17-31, 1903; no correction made in estimates.

$b$ Ice conditions during portions of J anuary and February, 1904; no correction made in estimates,

$c$ Ice gorge January 27, 1905: no correction made in estimate.

The following table gives the horsepower, 80 per cent efficiency per foot of fall, that may be developed at different rates of discharge and shows the number of days on which the flow and the corresponding 
horsepower were respectively less than the amounts given in the columns for "discharge" and "horsepower."

Discharge and horsepower table for Potomac River at Point of Rocks, Md., from 1895 to 1906.

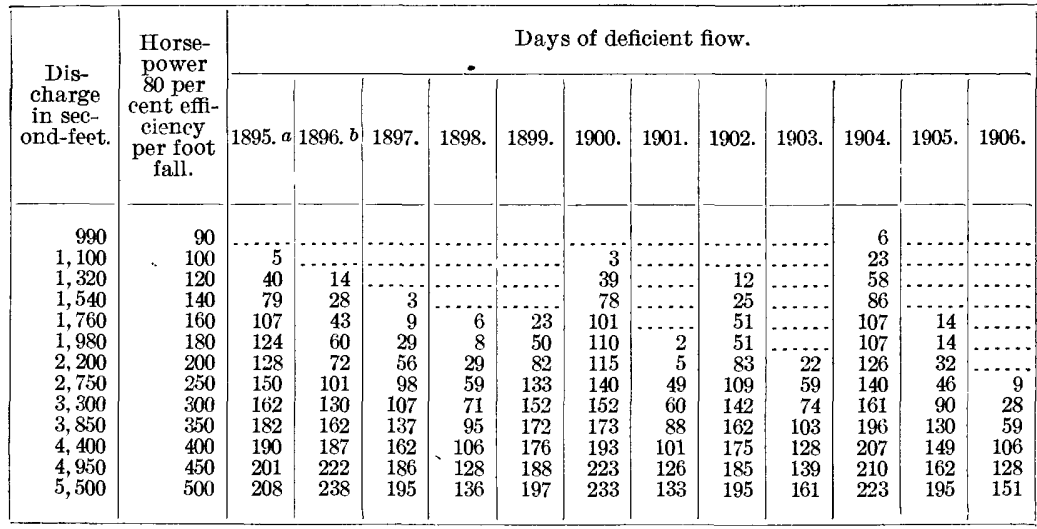

a February 17 to December $31,1895$.

$b$ Missing days estimated from Millville records.

Note.-The minimum flow during the period covered by the above table was 900 second-feet, giving 82 horsepower per foot of fall for two three-day periods during October, 1904.

\section{MONOCACY RIVER NEAR FREDERICK, MD.}

Monocacy River rises in Adams County, Pa., flows somewhat west of south, and enters Potomac River in the southeastern part of Frederick County, Md. Its length below the confluence of Rock and Marsh creeks is 55 miles. Its drainage area is 940 square miles. It has a number of tributaries on which small mills are located.

The gaging station was established August 4, 1896, by E. G. Paul. It is located at the county bridge on the toll road leading from Frederick to Mount Pleasant, Md. It is 4 miles northeast of Frederick, about 2,000 feet above the mouth of Israel Creek and 3,000 feet below the mouth of Tuscarora Creek.

The channel is straight for 300 feet above and 100 feet below the bridge. Both banks are low, liable to overflow, and covered with a fringe of trees, but all water passes beneath the bridge. The bed is composed of gravel and cobblestones, except near the banks, where it is composed of silt and is subject to change.

Discharge measurements are made from the two-span highway bridge, which has a total span of 310 feet. The channel at this point is divided by a small, low island, which serves as a foundation for the pier of the bridge. The right channel is measured from the lower and the left from the upper side of the bridge, as the results are better than would be furnished by a continuous section on either side of the bridge. The pier and island obstruct the flow to some extent, causing 
dead water for 20 feet to the right of the pier at low water and eddies at high water. The initial point for soundings is a crosscut in the face of the parapet wall on the lower wing of the right abutment.

September 3, 1902, the original wire gage was replaced by a standard chain gage which is located in the middle of the first span from the right bank and is attached to the bridge floor on the lower side of the bridge. The length of the chain from the end of the weight to the marker is 35.04 feet. The gage is read twice each day by E. L. Derr. The bench mark is a hole drilled in the top of a coping stone on the lower wing of the right abutment, about 100 feet back from the initial point for soundings. Its elevation is 29.17 feet above gage datum. On October 31, 1905, the elevation of the top of the pulley was found to be 25.47 feet above gage datum.

Estimates published for 1896 to 1903 have been revised; 1904 and 1905 estimates, as previously published, have not been changed. Two rating curves have been used to determine the discharge of the river. The estimates are probably well within 10 per cent of the true discharge for normal conditions of flow. Owing to gorging below the bridge at high stages, the tangent has been considered to give the best results above gage height 12.0 feet. Ice conditions probably affect the discharge to a considerable-extent.

A summary of the records gives the following results: Maximum discharge for twenty-four hours, 20,460 second-feet; minimum discharge for twenty-four hours, 49 second-feet; mean annual discharge for ten years, 1,130 second-feet; mean annual rainfall for ten years, 45.04 inches.

Discharge measurements of Monocacy River near Frederick, Md.

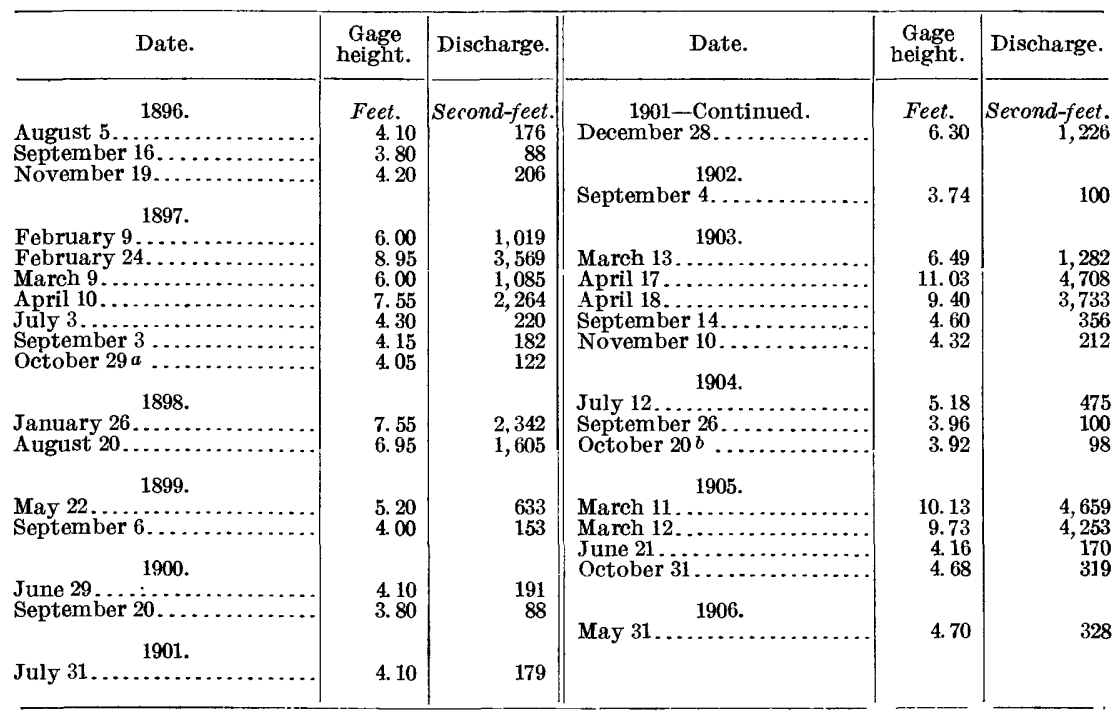


Daily gage height, in feet, of Monocacy River near Frederick, $M d$.

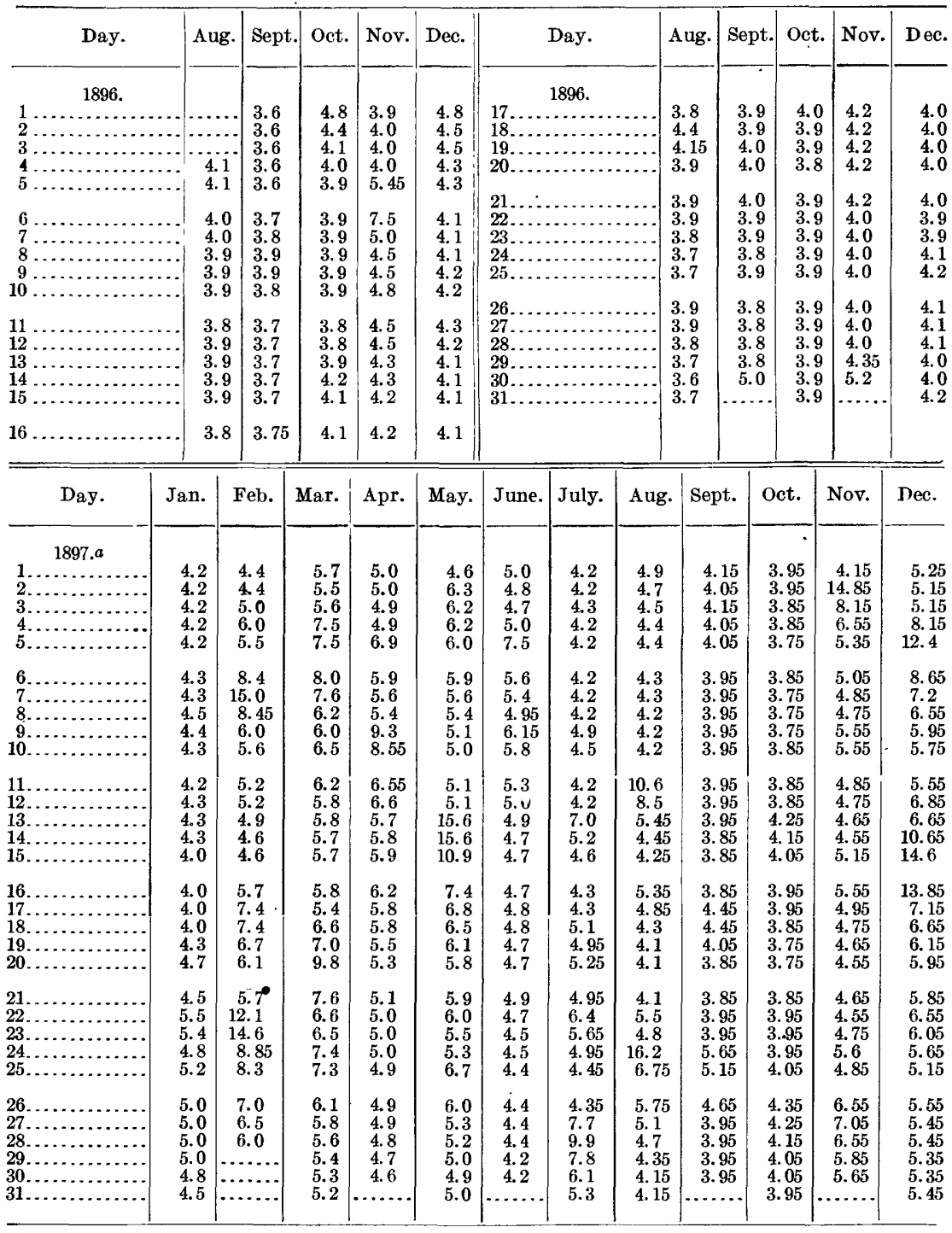

$a$ River frozen at the gage January 25 to February 2, 1897. All gage heights from July 3 to December 31,1897 , are liable to errors of one or two tenths owing to incorrect wire length. 
Daily gage height, in feet, of Monocacy River near Frederick, Md.-Continued.

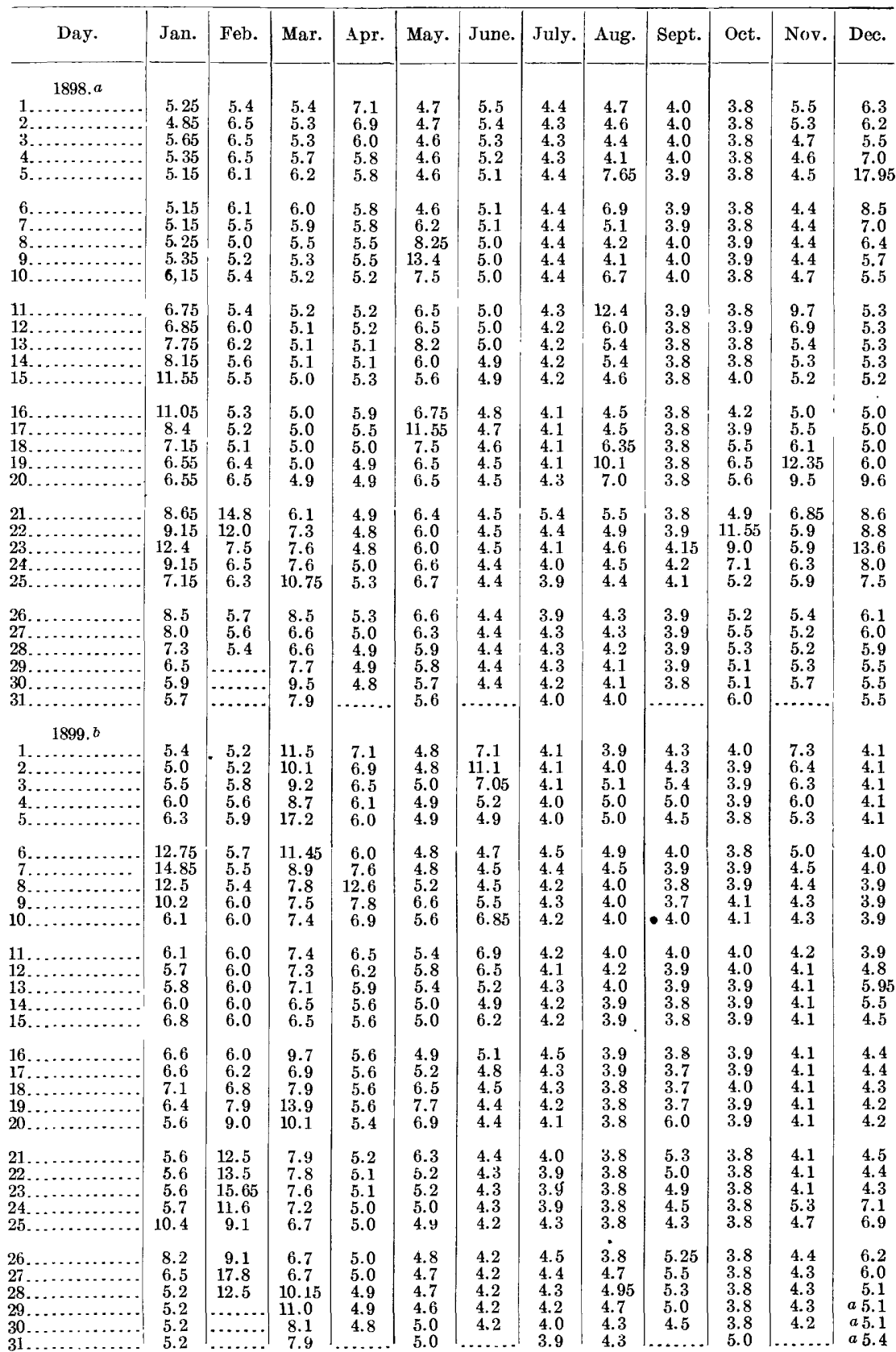

$a$ River frozen February 2-9, 1898.

$b$ River frozen at the gage January 2, February 9-21, and December 28-31, 1899. 
Daily gage height, in feet, of Monocacy River near Frederick, Md.-Continued.

\begin{tabular}{|c|c|c|c|c|c|c|c|c|c|c|c|c|}
\hline $\mathrm{Da}$ & Jan. & Feb. & Mar. & Apr. & May. & June. & July. & Aug. & Sept. & Oct. & Nov. & Dec. \\
\hline $\begin{array}{l}1 \ldots \\
2 \ldots \\
3 \ldots \\
4 \ldots \\
5 \ldots\end{array}$ & $\begin{array}{l}5.4 \\
5.4 \\
5.4 \\
5.2 \\
5.0\end{array}$ & $\begin{array}{l}5.1 \\
5.1 \\
5.1 \\
5.1 \\
5.2\end{array}$ & $\begin{array}{c}13.45 \\
12.0 \\
9.5 \\
8.2 \\
7.3\end{array}$ & $\begin{array}{l}6.4 \\
5.7 \\
5.5 \\
5.5 \\
5.5\end{array}$ & $\begin{array}{l}4.7 \\
4.7 \\
4.7 \\
4.9 \\
4.8\end{array}$ & $\begin{array}{l}4.4 \\
4.4 \\
4.4 \\
4.4 \\
4.4\end{array}$ & $\begin{array}{l}4.0 \\
4.0 \\
3.9 \\
3.9 \\
3.9\end{array}$ & $\begin{array}{l}3.95 \\
3.85 \\
3.85 \\
3.75 \\
3.65\end{array}$ & $\begin{array}{l}3.85 \\
3.75 \\
3.75 \\
3.6 \\
3.6\end{array}$ & $\begin{array}{l}3.9 \\
3.8 \\
3.8 \\
3.8 \\
3.8\end{array}$ & $\begin{array}{l}3.7 \\
3.7 \\
3.7 \\
4.1 \\
4.1\end{array}$ & $\begin{array}{r}5.2 \\
4.3 \\
4.3 \\
7.2 \\
12.1\end{array}$ \\
\hline $\begin{array}{r}6 . . \\
7 . . \\
8 . \\
9 \\
10 .\end{array}$ & $\begin{array}{l}4.9 \\
4.8 \\
4.5 \\
4.5 \\
4.9\end{array}$ & $\begin{array}{l}5.1 \\
5.3 \\
6.9 \\
7.55 \\
6.9\end{array}$ & $\begin{array}{l}7.1 \\
8.0 \\
7.5 \\
6.5 \\
6.3\end{array}$ & $\begin{array}{l}5.4 \\
5.3 \\
5.2 \\
5.1 \\
5.1\end{array}$ & $\begin{array}{l}4.6 \\
4.5 \\
4.4 \\
4.4 \\
4.4\end{array}$ & $\begin{array}{l}4.3 \\
4.2 \\
4.2 \\
4.2 \\
4.1\end{array}$ & $\begin{array}{l}3.9 \\
3.8 \\
3.8 \\
3.7 \\
3.7\end{array}$ & $\begin{array}{l}3.65 \\
3.65 \\
3.65 \\
3.65 \\
3.65\end{array}$ & $\begin{array}{l}3.65 \\
3.65 \\
3.65 \\
3.6 \\
3.6\end{array}$ & $\begin{array}{l}3.7 \\
3.7 \\
3.7 \\
3.7 \\
3.7\end{array}$ & $\begin{array}{l}4.0 \\
4.0 \\
4.0 \\
3.9 \\
3.9\end{array}$ & $\begin{array}{l}6.0 \\
5.5 \\
5.2 \\
4.9 \\
4.5\end{array}$ \\
\hline $\begin{array}{l}11 . . \\
12 . \\
13 . \\
14 . \\
15 .\end{array}$ & $\begin{array}{r}5.0 \\
10.2 \\
9.2 \\
5.4 \\
4.9\end{array}$ & $\begin{array}{c}5.3 \\
5.5 \\
15.1 \\
12.85 \\
7.0\end{array}$ & $\begin{array}{l}6.3 \\
5.9 \\
5.7 \\
5.6 \\
5.5\end{array}$ & $\begin{array}{l}5.0 \\
5.0 \\
5.0 \\
5.0 \\
5.0\end{array}$ & $\begin{array}{l}4.4 \\
4.4 \\
4.3 \\
4.3 \\
4.3\end{array}$ & $\begin{array}{l}4.1 \\
4.1 \\
4.1 \\
4.1 \\
4.4\end{array}$ & $\begin{array}{l}3.7 \\
3.7 \\
3.7 \\
3.7 \\
3.7\end{array}$ & $\begin{array}{l}3.55 \\
3.55 \\
3.45 \\
3.45 \\
3.45\end{array}$ & $\begin{array}{l}3.55 \\
3.55 \\
3.55 \\
3.5 \\
3.5\end{array}$ & $\begin{array}{l}3.7 \\
3.7 \\
3.7 \\
3.9 \\
4.1\end{array}$ & $\begin{array}{l}3.9 \\
3.9 \\
3.9 \\
3.9 \\
3.9\end{array}$ & $\begin{array}{l}4.3 \\
4.2 \\
4.0 \\
4.0 \\
4.0\end{array}$ \\
\hline $20 \ldots$ & $\begin{array}{l}4.8 \\
4.6 \\
4.6 \\
4.9 \\
9.0\end{array}$ & $\begin{array}{l}6.5 \\
5.9 \\
5.9 \\
5.8 \\
5.5\end{array}$ & $\begin{array}{r}5.4 \\
5.4 \\
5.4 \\
5.4 \\
13.5\end{array}$ & $\begin{array}{l}4.9 \\
4.9 \\
5.1 \\
5.2 \\
5.1\end{array}$ & $\begin{array}{l}4.3 \\
4.3 \\
4.3 \\
6.5 \\
7.0\end{array}$ & $\begin{array}{l}4.8 \\
5.0 \\
5.8 \\
6.0 \\
5.1\end{array}$ & $\begin{array}{l}3.6 \\
3.6 \\
3.6 \\
3.7 \\
4.55\end{array}$ & $\begin{array}{l}3.45 \\
3.55 \\
3.85 \\
3.75 \\
3.75\end{array}$ & $\begin{array}{l}3.85 \\
3.85 \\
3.85 \\
3.8 \\
3.8\end{array}$ & $\begin{array}{l}4.1 \\
4.1 \\
3.9 \\
3.8 \\
3.7\end{array}$ & $\begin{array}{l}3.9 \\
3.9 \\
3.8 \\
3.8 \\
3.8\end{array}$ & $\begin{array}{l}4.0 \\
4.0 \\
4.0 \\
3.9 \\
3.9\end{array}$ \\
\hline $\begin{array}{l}21 \ldots \\
22 \ldots \\
23 \ldots \\
24 \ldots \\
25 \ldots\end{array}$ & $\begin{array}{l}9.5 \\
7.05 \\
6.5 \\
6.0 \\
5.7\end{array}$ & $\begin{array}{r}5.5 \\
20.8 \\
15.0 \\
10.5 \\
8.5\end{array}$ & $\begin{array}{r}10.2 \\
9.1 \\
8.2 \\
6.5 \\
6.1\end{array}$ & $\begin{array}{l}5.0 \\
5.0 \\
5.5 \\
5.6 \\
5.4\end{array}$ & $\begin{array}{l}6.5 \\
5.9 \\
4.9 \\
4.8 \\
4.7\end{array}$ & $\begin{array}{l}4.9 \\
4.5 \\
4.4 \\
4.2 \\
4.2\end{array}$ & $\begin{array}{l}4.55 \\
3.75 \\
4.05 \\
5.45 \\
4.75\end{array}$ & $\begin{array}{l}3.75 \\
4.75 \\
4.65 \\
4.05 \\
4.05\end{array}$ & $\begin{array}{l}3.8 \\
3.7 \\
3.65 \\
3.5 \\
3.5\end{array}$ & $\begin{array}{l}3.7 \\
3.7 \\
3.8 \\
3.8 \\
3.8\end{array}$ & $\begin{array}{l}3.8 \\
3.8 \\
3.9 \\
3.9 \\
3.9\end{array}$ & $\begin{array}{l}3.9 \\
4.0 \\
4.0 \\
4.1 \\
4.1\end{array}$ \\
\hline $\begin{array}{l}26 \ldots \\
27 \ldots \\
28 \ldots \\
29 \ldots \\
30 \ldots \\
31 \ldots \\
\end{array}$ & $\begin{array}{l}5.5 \\
5.1 \\
4.9 \\
4.6 \\
4.5 \\
4.5\end{array}$ & \begin{tabular}{|c}
7.1 \\
6.5 \\
6.5 \\
$\ldots \ldots$
\end{tabular} & $\begin{array}{l}6.0 \\
5.9 \\
5.7 \\
5.5 \\
5.5 \\
6.5\end{array}$ & $\begin{array}{l}4.9 \\
4.8 \\
4.8 \\
4.8 \\
4.7 \\
\ldots \ldots\end{array}$ & $\begin{array}{l}4.6 \\
4.5 \\
4.5 \\
4.5 \\
4.5 \\
4.5\end{array}$ & $\begin{array}{c}4.2 \\
4.2 \\
4.1 \\
4.1 \\
4.0 \\
\ldots . .\end{array}$ & $\begin{array}{l}4.05 \\
3.95 \\
3.95 \\
3.95\end{array}$ & $\begin{array}{l}3.95 \\
3.85 \\
5.45 \\
5.25 \\
4.25 \\
3.95\end{array}$ & $\begin{array}{c}3.5 \\
3.5 \\
3.6 \\
\ldots \ldots \\
4.0 \\
\ldots \ldots . .\end{array}$ & $\begin{array}{l}3.7 \\
3.7 \\
3.7 \\
3.7 \\
3.7 \\
3.7\end{array}$ & $\begin{array}{c}9.2 \\
7.3 \\
7.1 \\
6.9 \\
6.3 \\
\cdots . . .\end{array}$ & $\begin{array}{l}4.1 \\
4.1 \\
4.1 \\
4.0 \\
4.0 \\
4.5\end{array}$ \\
\hline $\begin{array}{l} \\
19 \\
1 \ldots \ldots \\
2 \ldots \ldots \\
3 \ldots \ldots \\
4 \ldots \ldots \\
5 \ldots \ldots\end{array}$ & $\begin{array}{l}4.4 \\
4.2 \\
4.2 \\
4.3 \\
4.3\end{array}$ & $\begin{array}{l}4.6 \\
4.4 \\
4.4 \\
4.4 \\
4.8\end{array}$ & $\begin{array}{l}4.5 \\
4.5 \\
4.4 \\
5.0 \\
5.5\end{array}$ & $\begin{array}{r}5.7 \\
5.6 \\
18.0 \\
15.5 \\
10.5\end{array}$ & $\begin{array}{l}5.9 \\
5.8 \\
5.6 \\
5.4 \\
5.3\end{array}$ & $\begin{array}{l}6.0 \\
5.6 \\
5.4 \\
5.3 \\
5.3\end{array}$ & $\begin{array}{l}4.3 \\
4.3 \\
4.2 \\
4.2 \\
4.2\end{array}$ & $\begin{array}{l}4.1 \\
4.0 \\
4.0 \\
4.0 \\
4.0\end{array}$ & $\begin{array}{l}4.3 \\
4.9 \\
4.5 \\
4.4 \\
4.3\end{array}$ & $\begin{array}{l}5.2 \\
5.1 \\
4.9 \\
4.8 \\
4.6\end{array}$ & $\begin{array}{l}4.2 \\
4.2 \\
4.2 \\
4.2 \\
4.2\end{array}$ & $\begin{array}{l}4.9 \\
4.7 \\
4.9 \\
4.9 \\
4.9\end{array}$ \\
\hline $\begin{array}{r}6 \ldots \\
7 \ldots \\
8 \ldots \\
9 \ldots \\
10 \ldots\end{array}$ & $\begin{array}{l}4.2 \\
4.1 \\
4.0 \\
4.1 \\
4.1\end{array}$ & $\begin{array}{l}4.8 \\
4.7 \\
4.6 \\
4.5 \\
4.5\end{array}$ & $\begin{array}{l}5.3 \\
5.0 \\
5.0 \\
5.1 \\
5.5\end{array}$ & $\begin{array}{r}7.9 \\
8.3 \\
7.2 \\
6.5 \\
6.2\end{array}$ & $\begin{array}{l}5.2 \\
5.1 \\
5.1 \\
5.1 \\
5.5\end{array}$ & $\begin{array}{l}5.0 \\
5.5 \\
7.1 \\
5.4 \\
5.2\end{array}$ & $\begin{array}{l}4.7 \\
4.3 \\
4.2 \\
4.1\end{array}$ & $\begin{array}{l}7.1 \\
6.0 \\
4.7 \\
4.6\end{array}$ & $\begin{array}{l}4.3 \\
4.3 \\
4.3 \\
4.3 \\
4.2\end{array}$ & $\begin{array}{l}4.4 \\
4.3 \\
4.3 \\
4.2 \\
4.2\end{array}$ & $\begin{array}{l}4.3 \\
4.3\end{array}$ & $\begin{array}{l}4.9 \\
4.8 \\
4.8 \\
4.8 \\
7.5\end{array}$ \\
\hline & $\begin{array}{l}5.5 \\
7.6 \\
6.9 \\
6.4 \\
5.9\end{array}$ & $\begin{array}{l}4.5 \\
4.5 \\
4.4 \\
4.3 \\
4.3\end{array}$ & $\begin{array}{r}20.5 \\
11.9 \\
8.0 \\
7.3 \\
6.9\end{array}$ & $\begin{array}{l}5.9 \\
5.6 \\
5.6 \\
5.4 \\
6.9\end{array}$ & $\begin{array}{l}5.5 \\
5.4 \\
5.3 \\
5.1 \\
4.9\end{array}$ & \begin{tabular}{l|}
4.9 \\
4.9 \\
8.0 \\
6.4 \\
6.75
\end{tabular} & $\begin{array}{l}4.1 \\
5.1 \\
5.5 \\
4.8 \\
4.8\end{array}$ & $\begin{array}{l}4.2 \\
4.1 \\
4.6 \\
4.4 \\
4.3\end{array}$ & $\begin{array}{l}4.2 \\
4.2 \\
4.2 \\
4.2 \\
4.2\end{array}$ & $\begin{array}{l}4.1 \\
4.8 \\
5.0\end{array}$ & $\begin{array}{l}4.3 \\
4.3 \\
4.3\end{array}$ & $\begin{array}{c}6.5 \\
6.1 \\
5.3 \\
5.4 \\
19.75\end{array}$ \\
\hline & $\begin{array}{l}4.9 \\
4.8 \\
4.7 \\
4.7 \\
4.5\end{array}$ & $\begin{array}{l}4.3 \\
4.3 \\
4.3 \\
4.4 \\
4.9\end{array}$ & $\begin{array}{l}6.1 \\
5.9 \\
5.8 \\
5.6 \\
5.3\end{array}$ & $\begin{array}{l}6.5 \\
6.3 \\
5.9 \\
5.5 \\
9.6\end{array}$ & $\begin{array}{l}4.9 \\
4.9 \\
4.9 \\
4.85 \\
4.8\end{array}$ & $\begin{array}{l}7.45 \\
7.2 \\
6.1 \\
5.9 \\
5.4\end{array}$ & $\begin{array}{l}4.9 \\
5.0 \\
4.9 \\
5.2 \\
5.1\end{array}$ & $\begin{array}{l}5.6 \\
4.9 \\
5.1 \\
4.5 \\
6.4\end{array}$ & $\begin{array}{l}5.5 \\
4.7 \\
4.5 \\
4.5 \\
4.4\end{array}$ & $\begin{array}{l}4.9 \\
4.8 \\
4.4 \\
4.2 \\
4.2\end{array}$ & $\begin{array}{l}4.2 \\
4.2 \\
4.2\end{array}$ & $\begin{array}{l}9.5 \\
7.1 \\
6.9 \\
6.5 \\
6.2\end{array}$ \\
\hline $\begin{array}{l}21 \ldots \\
22 \ldots \\
23 \ldots \\
24 \ldots \\
25 \ldots \\
\end{array}$ & $\begin{array}{l}4.3 \\
4.3 \\
4.2 \\
4.2 \\
4.2\end{array}$ & $\begin{array}{l}5.0 \\
5.1 \\
5.0 \\
5.0 \\
4.9\end{array}$ & $\begin{array}{r}10.5 \\
7.6 \\
7.1 \\
6.0 \\
6.0\end{array}$ & $\begin{array}{r}14.6 \\
9.6 \\
8.1 \\
8.5 \\
7.9\end{array}$ & $\begin{array}{r}4.8 \\
4.8 \\
11.3 \\
9.1 \\
7.9\end{array}$ & $\begin{array}{l}5.2 \\
5.2 \\
5.1 \\
4.9 \\
4.7\end{array}$ & $\begin{array}{l}5.1 \\
4.9 \\
4.5 \\
4.4 \\
4.2\end{array}$ & $\begin{array}{l}6.1 \\
5.2 \\
4.5 \\
4.6 \\
5.0\end{array}$ & $\begin{array}{l}4.3 \\
4.3 \\
4.2 \\
4.1 \\
4.1\end{array}$ & $\begin{array}{l}4.1 \\
4.1 \\
4.1 \\
4.1 \\
4.1\end{array}$ & $\begin{array}{l}4.2 \\
4.2 \\
4.5 \\
9.4 \\
7.5\end{array}$ & $\begin{array}{l}6.0 \\
5.9 \\
5.9 \\
5.7 \\
5.5\end{array}$ \\
\hline $\begin{array}{l}26 . . \\
27 . \\
28 . \\
29 . \\
30 . \\
31 .\end{array}$ & $\begin{array}{l}4.3 \\
4.3 \\
4.3 \\
4.8 \\
4.7 \\
4.7\end{array}$ & $\begin{array}{l}4.8 \\
4.6 \\
4.5\end{array}$ & $\begin{array}{c}6.1 \\
13.55 \\
10.4 \\
7.4 \\
5.9 \\
5.8\end{array}$ & $\begin{array}{l}7.3 \\
6.9 \\
6.4 \\
6.1 \\
6.0\end{array}$ & $\begin{array}{r}7.1 \\
9.0 \\
9.2 \\
11.5 \\
9.1 \\
8.6\end{array}$ & $\begin{array}{l}4.6 \\
4.5 \\
4.5 \\
4.5 \\
4.5\end{array}$ & $\begin{array}{l}4.2 \\
4.5 \\
4.3 \\
4.2 \\
4.2 \\
4.1\end{array}$ & $\begin{array}{l}4.9 \\
4.5 \\
4.3 \\
4.2 \\
4.2 \\
4.2\end{array}$ & $\begin{array}{l}4.1 \\
4.1 \\
4.1 \\
4.7 \\
6.7\end{array}$ & $\begin{array}{l}4.2 \\
4.2 \\
4.2 \\
4.2 \\
4.2 \\
4.2\end{array}$ & $\begin{array}{l}5.9 \\
5.5 \\
5.3 \\
5.1 \\
5.0\end{array}$ & $\begin{array}{c}5.5 \\
7.35 \\
6.5 \\
13.25 \\
18.5 \\
12.5\end{array}$ \\
\hline
\end{tabular}

$a$ River frozen at the gage January 1-6 and February 1-4, 1900.

$b$ No gage weight on the wire June 1-4, 1901; gage heights estimated by the observer. 
Daily gage height, in feet, of Monocacy River near Frederick, Md.-Continued.

\begin{tabular}{|c|c|c|c|c|c|c|c|c|c|c|c|c|}
\hline Day. & Jan. & Feb. & Mar. & Apr. & May. & June. & July. & Aug. & Sept. & Oct. & Nov. & Dec. \\
\hline $\begin{array}{l}1 \\
1 \ldots . \\
2 \ldots \\
3 \ldots \\
4 \ldots \\
5 \ldots\end{array}$ & $\begin{array}{l}8.5 \\
8.2 \\
7.1 \\
6.0 \\
7.9\end{array}$ & $\begin{array}{l}5.9 \\
5.9 \\
5.9 \\
8.5 \\
8.5\end{array}$ & $\begin{array}{r}25.2 \\
14.5 \\
10.9 \\
10.2 \\
9.6\end{array}$ & $\begin{array}{r}7.0 \\
6.9 \\
10.5 \\
8.5 \\
8.3\end{array}$ & $\begin{array}{l}5.2 \\
5.1 \\
5.1 \\
5.0 \\
4.9\end{array}$ & $\begin{array}{l}4.3 \\
4.3 \\
4.3 \\
4.2 \\
4.2\end{array}$ & $\begin{array}{l}6.9 \\
6.4 \\
4.9 \\
4.8 \\
4.8\end{array}$ & $\begin{array}{l}5.2 \\
4.5 \\
4.2 \\
4.1 \\
4.1\end{array}$ & $\begin{array}{l}3.7 \\
3.7 \\
3.7 \\
3.7 \\
3.9\end{array}$ & $\begin{array}{l}4.6 \\
6.0 \\
5.1 \\
4.2 \\
4.5\end{array}$ & $\begin{array}{l}5.1 \\
5.0 \\
4.9 \\
4.7 \\
4.6\end{array}$ & $\begin{array}{r}9.5 \\
7.9 \\
16.0 \\
8.6 \\
8.2\end{array}$ \\
\hline $\begin{array}{r}6 . . \\
7 . \\
8 . \\
9 . \\
10 .\end{array}$ & $\begin{array}{l}8.2 \\
8.1 \\
7.5 \\
\mathbf{5} .9 \\
\mathbf{5 . 5}\end{array}$ & $\begin{array}{l}8.1 \\
7.9 \\
7.8 \\
7.4 \\
6.9\end{array}$ & $\begin{array}{r}9.2 \\
9.1 \\
8.9 \\
9.9 \\
15.2\end{array}$ & $\begin{array}{r}8.0 \\
7.9 \\
7.5 \\
17.9 \\
16.9\end{array}$ & $\begin{array}{l}4.9 \\
4.8 \\
4.8 \\
4.7 \\
4.7\end{array}$ & $\begin{array}{l}4.1 \\
4.1 \\
4.1 \\
4.1 \\
4.1\end{array}$ & $\begin{array}{l}4.8 \\
4.8 \\
4.6 \\
4.5 \\
4.4\end{array}$ & $\begin{array}{l}4.2 \\
4.2 \\
4.1 \\
4.0 \\
4.0\end{array}$ & $\begin{array}{l}3.7 \\
3.6 \\
3.6 \\
3.6 \\
3.6\end{array}$ & $\begin{array}{l}7.1 \\
5.2 \\
5.1 \\
5.0 \\
4.9\end{array}$ & $\begin{array}{l}4.5 \\
4.4 \\
4.6 \\
4.5 \\
4.5\end{array}$ & $\begin{array}{l}7.9 \\
7.8 \\
7.8 \\
7.5 \\
7.4\end{array}$ \\
\hline & $\begin{array}{l}5.4 \\
5.4 \\
5.3 \\
5.3 \\
5.2\end{array}$ & $\begin{array}{l}6.5 \\
6.5 \\
6.5 \\
6.3 \\
6.1\end{array}$ & $\begin{array}{l}15.5 \\
12.2 \\
14.5 \\
10.1 \\
10.0\end{array}$ & $\begin{array}{l}8.2 \\
7.5 \\
7.3 \\
6.9 \\
6.5\end{array}$ & $\begin{array}{l}4.7 \\
4.6 \\
4.6 \\
4.6 \\
4.6\end{array}$ & $\begin{array}{l}4.1 \\
4.0 \\
7.1 \\
5.5 \\
4.2\end{array}$ & $\begin{array}{l}4.3 \\
4.2 \\
4.1 \\
4.0 \\
4.0\end{array}$ & $\begin{array}{l}4.0 \\
4.0 \\
3.9 \\
3.9 \\
3.8\end{array}$ & $\begin{array}{l}3.6 \\
3.55 \\
3.5 \\
3.5 \\
3.5\end{array}$ & $\begin{array}{r}5.5 \\
12.8 \\
8.1 \\
6.5 \\
5.1\end{array}$ & $\begin{array}{l}4.5 \\
4.4 \\
4.4 \\
4.4 \\
4.3\end{array}$ & $\begin{array}{r}7.9 \\
9.2 \\
11.5 \\
9.5 \\
8.2\end{array}$ \\
\hline & $\begin{array}{l}5.1 \\
4.9 \\
4.9 \\
4.9 \\
4.9\end{array}$ & $\begin{array}{l}5.9 \\
5.9 \\
5.9 \\
5.9 \\
6.1\end{array}$ & $\begin{array}{r}9.5 \\
14.6 \\
10.2 \\
8.4 \\
8.1\end{array}$ & $\begin{array}{l}6.3 \\
6.1 \\
5.9 \\
5.8 \\
5.7\end{array}$ & $\begin{array}{l}4.6 \\
4.5 \\
4.5 \\
4.5 \\
4.5\end{array}$ & $\begin{array}{l}4.2 \\
4.2 \\
4.1 \\
4.1 \\
4.1\end{array}$ & $\begin{array}{l}3.9 \\
3.9 \\
3.9 \\
4.1 \\
4.1\end{array}$ & $\begin{array}{l}3.8 \\
3.8 \\
3.8 \\
3.8 \\
3.8\end{array}$ & $\begin{array}{l}3.5 \\
3.5 \\
3.5 \\
3.5 \\
3.6\end{array}$ & $\begin{array}{l}4.9 \\
4.7 \\
4.6 \\
4.5 \\
4.4\end{array}$ & $\begin{array}{l}4.2 \\
4.2 \\
4.2 \\
4.6 \\
4.5\end{array}$ & $\begin{array}{r}15.3 \\
14.5 \\
8.5 \\
8.2 \\
9.5\end{array}$ \\
\hline & $\begin{array}{c}7.2 \\
18.05 \\
7.1 \\
6.9 \\
6.1\end{array}$ & $\begin{array}{r}10.2 \\
14.4 \\
9.6 \\
9.4 \\
10.5\end{array}$ & $\begin{array}{l}7.9 \\
7.4 \\
7.1 \\
6.9 \\
6.5\end{array}$ & $\begin{array}{l}5.6 \\
5.5 \\
5.4 \\
5.3 \\
5.3\end{array}$ & $\begin{array}{l}4.5 \\
4.5 \\
4.5 \\
4.4 \\
4.4\end{array}$ & $\begin{array}{l}4.2 \\
4.2 \\
4.1 \\
4.1 \\
4.1\end{array}$ & $\begin{array}{l}5.2 \\
4.3 \\
4.2 \\
4.1 \\
4.1\end{array}$ & $\begin{array}{l}3.7 \\
3.7 \\
3.7 \\
3.6 \\
3.6\end{array}$ & $\begin{array}{l}3.6 \\
3.6 \\
3.5 \\
3.5 \\
3.8\end{array}$ & $\begin{array}{l}4.3 \\
4.2 \\
4.2 \\
4.2 \\
4.1\end{array}$ & $\begin{array}{l}4.4 \\
4.3 \\
4.2 \\
4.2 \\
4.2\end{array}$ & $\begin{array}{r}10.5 \\
17.1 \\
14.2 \\
8.4 \\
7.5\end{array}$ \\
\hline $\begin{array}{l}26 . \\
27 . \\
28\end{array}$ & $\begin{array}{c}6.9 \\
11.15 \\
7.5 \\
6.9 \\
6.5 \\
6.2\end{array}$ & \begin{tabular}{|l|}
24.0 \\
21.4 \\
19.35
\end{tabular} & $\begin{array}{l}6.3 \\
6.0 \\
6.0 \\
5.9 \\
5.6 \\
6.2\end{array}$ & $\begin{array}{l}5.2 \\
5.1 \\
5.0 \\
5.2 \\
5.2\end{array}$ & $\begin{array}{l}4.4 \\
4.5 \\
4.5 \\
4.4 \\
4.3 \\
4.3\end{array}$ & $\begin{array}{l}5.9 \\
5.6 \\
5.1 \\
4.5 \\
4.5\end{array}$ & $\begin{array}{l}4.0 \\
4.0 \\
3.9 \\
3.9 \\
4.5 \\
5.5\end{array}$ & $\begin{array}{l}3.6 \\
3.6 \\
4.1 \\
3.9 \\
3.7 \\
3.7\end{array}$ & $\begin{array}{l}4.0 \\
9.1 \\
5.1 \\
4.9 \\
4.8\end{array}$ & $\begin{array}{r}4.1 \\
4.1 \\
13.7 \\
9.5 \\
5.5 \\
5.3\end{array}$ & $\begin{array}{l}5.9 \\
9.4 \\
7.9 \\
5.9 \\
5.9\end{array}$ & $\begin{array}{l}7.2 \\
6.9 \\
6.5 \\
6.4 \\
6.2 \\
5.5\end{array}$ \\
\hline $\begin{array}{r}1903 . \\
1 \ldots \ldots \\
2 \ldots \ldots \ldots \\
3 \ldots \ldots \ldots \\
4 \ldots \ldots \\
5 \ldots \ldots \ldots\end{array}$ & $\begin{array}{c}5.5 \\
15.8 \\
13.35 \\
10.5 \\
9.5\end{array}$ & \begin{tabular}{|c|}
7.6 \\
7.3 \\
8.3 \\
16.35 \\
10.5
\end{tabular} & $\begin{array}{r}21.4 \\
9.5 \\
8.1 \\
7.9 \\
7.5\end{array}$ & $\begin{array}{l}7.9 \\
7.4 \\
6.9 \\
7.9 \\
7.2\end{array}$ & $\begin{array}{l}5.6 \\
5.5 \\
5.5 \\
5.5 \\
5.4\end{array}$ & $\begin{array}{l}6.5 \\
6.4 \\
6.1 \\
5.1 \\
4.9\end{array}$ & $\begin{array}{r}9.5 \\
8.1 \\
7.5 \\
8.1 \\
11.5\end{array}$ & $\begin{array}{l}5.3 \\
5.1 \\
4.9 \\
4.9 \\
4.9\end{array}$ & $\begin{array}{l}6.5 \\
5.9 \\
5.5 \\
5.4 \\
5.2\end{array}$ & $\begin{array}{l}4.4 \\
4.4 \\
4.3 \\
4.3 \\
4.3\end{array}$ & $\begin{array}{l}4.3 \\
4.3 \\
4.3 \\
4.3 \\
4.3\end{array}$ & $\begin{array}{l}4.3 \\
4.2 \\
4.2 \\
4.2 \\
4.1\end{array}$ \\
\hline & $\begin{array}{l}8.2 \\
7.3 \\
6.9 \\
6.3 \\
6.9\end{array}$ & $\begin{array}{l}7.9 \\
7.5 \\
7.3 \\
7.5 \\
\mathbf{6 . 9}\end{array}$ & $\begin{array}{l}7.2 \\
6.9 \\
7.5 \\
8.6 \\
7.4\end{array}$ & $\begin{array}{r}6.5 \\
6.9 \\
7.5 \\
12.2 \\
7.9\end{array}$ & $\begin{array}{l}5.3 \\
5.3 \\
5.3 \\
5.2 \\
5.1\end{array}$ & $\begin{array}{l}4.9 \\
5.8 \\
5.8 \\
5.4 \\
5.1\end{array}$ & $\begin{array}{l}\mathbf{9 . 5} \\
7.3 \\
6.2 \\
\mathbf{6 . 1} \\
\mathbf{5 . 9}\end{array}$ & $\begin{array}{l}4.9 \\
5.94 \\
5.2 \\
5.1 \\
4.9\end{array}$ & $\begin{array}{l}5.0 \\
4.9 \\
4.8 \\
5.4 \\
7.2\end{array}$ & $\begin{array}{l}4.3 \\
4.4 \\
5.2 \\
7.2 \\
5.2\end{array}$ & $\begin{array}{l}4.3 \\
4.3 \\
4.3 \\
4.3 \\
4.3\end{array}$ & $\begin{array}{l}4.1 \\
4.6 \\
4.2 \\
4.2 \\
4.2\end{array}$ \\
\hline & $\begin{array}{l}9.6 \\
9.3 \\
8.6 \\
8.4 \\
8.2\end{array}$ & \begin{tabular}{|r}
7.1 \\
10.5 \\
7.5 \\
7.3 \\
7.8
\end{tabular} & $\begin{array}{l}7.2 \\
7.1 \\
6.3 \\
6.2 \\
6.2\end{array}$ & $\begin{array}{r}7.2 \\
6.7 \\
7.4 \\
19.3 \\
21.6\end{array}$ & $\begin{array}{l}5.0 \\
4.9 \\
4.9 \\
4.8 \\
4.8\end{array}$ & $\begin{array}{l}5.8 \\
8.1 \\
6.5 \\
6.2 \\
5.8\end{array}$ & $\begin{array}{c}5.8 \\
9.15 \\
18.8 \\
8.5 \\
7.5\end{array}$ & $\begin{array}{l}4.9 \\
4.8 \\
4.8 \\
4.7 \\
4.7\end{array}$ & $\begin{array}{l}6.9 \\
5.5 \\
4.9 \\
4.7 \\
4.6\end{array}$ & $\begin{array}{l}6.1 \\
5.9 \\
5.6 \\
5.2 \\
5.1\end{array}$ & $\begin{array}{l}4.3 \\
4.3 \\
4.3 \\
4.3 \\
4.3\end{array}$ & $\begin{array}{l}4.2 \\
4.1 \\
4.1 \\
4.2 \\
4.5\end{array}$ \\
\hline & $\begin{array}{l}8.2 \\
7.9 \\
7.3 \\
7.3 \\
7.2\end{array}$ & \begin{tabular}{|r}
11.2 \\
7.9 \\
7.4 \\
7.1 \\
6.9
\end{tabular} & $\begin{array}{l}6.2 \\
6.1 \\
5.9 \\
5.9 \\
5.8\end{array}$ & $\begin{array}{r}20.4 \\
11.4 \\
9.4 \\
8.4 \\
7.9,\end{array}$ & $\begin{array}{l}4.7 \\
4.7 \\
4.8 \\
4.8 \\
4.7\end{array}$ & $\begin{array}{l}5.4 \\
5.2 \\
5.6 \\
5.4 \\
6.65\end{array}$ & $\begin{array}{r}6.8 \\
6.5 \\
8.2 \\
11.5 \\
6.9\end{array}$ & $\begin{array}{l}7.5 \\
7.1 \\
6.2 \\
5.9 \\
5.7\end{array}$ & $\begin{array}{r}4.6 \\
6.5 \\
11.1 \\
5.8 \\
5.2\end{array}$ & $\begin{array}{l}4.9 \\
4.9 \\
5.5 \\
5.4 \\
5.2\end{array}$ & $\begin{array}{l}4.3 \\
4.4 \\
4.9 \\
4.7 \\
4.5\end{array}$ & $\begin{array}{l}4.6 \\
4.8 \\
4.6 \\
4.2 \\
6.5\end{array}$ \\
\hline & \begin{tabular}{|c|}
8.55 \\
11.7 \\
8.6 \\
7.1 \\
6.5
\end{tabular} & $\begin{array}{l}6.7 \\
6.4 \\
6.2 \\
6.2 \\
6.2\end{array}$ & $\begin{array}{r}5.8 \\
9.4 \\
17.1 \\
18.2 \\
8.6\end{array}$ & $\begin{array}{l}7.4 \\
7.1 \\
6.7 \\
6.5 \\
6.4\end{array}$ & $\begin{array}{l}4.7 \\
4.7 \\
4.7 \\
6.5 \\
6.1\end{array}$ & $\begin{array}{l}7.5 \\
6.4 \\
5.7 \\
6.3 \\
5.9\end{array}$ & $\begin{array}{l}6.4 \\
6.2 \\
\mathbf{5 . 6} \\
\mathbf{5 . 4} \\
\mathbf{5 . 3}\end{array}$ & $\begin{array}{l}4.9 \\
4.7 \\
4.6 \\
4.5 \\
4.5\end{array}$ & $\begin{array}{l}4.9 \\
4.8 \\
4.7 \\
4.7 \\
4.6\end{array}$ & $\begin{array}{l}4.9 \\
4.7 \\
4.6 \\
4.6 \\
4.5\end{array}$ & $\begin{array}{l}4.4 \\
4.3 \\
4.3 \\
4.3 \\
4.3\end{array}$ & $\begin{array}{c}12.05 \\
6.5 \\
4.9 \\
4.8 \\
4.7\end{array}$ \\
\hline & $\begin{array}{r}6.3 \\
6.1 \\
9.8 \\
10.6 \\
11.2 \\
8.1\end{array}$ & $\begin{array}{r}6.2 \\
7.3 \\
21.2\end{array}$ & $\begin{array}{r}7.9 \\
7.5 \\
7.1 \\
6.5 \\
9.5 \\
13.9\end{array}$ & $\begin{array}{l}6.2 \\
6.1 \\
5.9 \\
5.7 \\
5.7\end{array}$ & $\begin{array}{r}5.4 \\
4.9 \\
4.9 \\
4.8 \\
14.8 \\
7.5\end{array}$ & $\begin{array}{r}5.6 \\
5.6 \\
5.6 \\
21.2 \\
14.9\end{array}$ & $\begin{array}{l}\mathbf{5 . 2} \\
\mathbf{5 . 2} \\
\mathbf{5 . 1} \\
\mathbf{5 . 1} \\
\mathbf{5 . 4} \\
\mathbf{5 . 4}\end{array}$ & $\begin{array}{r}4.8 \\
4.7 \\
10.5 \\
12.1 \\
7.9 \\
7.4\end{array}$ & $\begin{array}{l}4.5 \\
4.5 \\
4.4 \\
4.4 \\
4.4\end{array}$ & $\begin{array}{l}4.5 \\
4.4 \\
4.4 \\
4.4 \\
4.3 \\
4.3\end{array}$ & $\begin{array}{l}4.3 \\
4.3 \\
4.2 \\
4.2 \\
4.2\end{array}$ & $\begin{array}{l}4.6 \\
4.5 \\
4.5 \\
4.6 \\
4.5 \\
4.5\end{array}$ \\
\hline
\end{tabular}

$a$ River frozen at gage February 4-19, 1902. Observer estimated gage heights March 20 to April 8 1902 the gage being broken.

$b$ River frozen at gage January 12-20, 1903. 
Daily gage height, in feet, of Monocacy River near Fredernck, Md.-Continued.

\begin{tabular}{|c|c|c|c|c|c|c|c|c|c|c|c|c|}
\hline Day. & Jan. & Feb. & Mar. & Apr. & May. & June. & July. & Aug. & Sept. & Oct. & Nov. & Dec. \\
\hline & $\begin{array}{l}4.6 \\
4.9 \\
4.8 \\
5.5 \\
5.1\end{array}$ & $\begin{array}{l}5.0 \\
4.9 \\
5.4 \\
4.9 \\
4.6\end{array}$ & $\begin{array}{l}8.1 \\
7.5 \\
6.2 \\
6.2 \\
5.9\end{array}$ & $\begin{array}{l}5.9 \\
7.2 \\
6.4 \\
5.5 \\
5.4\end{array}$ & $\begin{array}{l}4.9 \\
4.8 \\
4.7 \\
4.8 \\
4.7\end{array}$ & $\begin{array}{l}7.5 \\
6.4 \\
6.0 \\
5.3 \\
7.4\end{array}$ & $\begin{array}{l}4.5 \\
4.3 \\
4.3 \\
4.2 \\
4.2\end{array}$ & $\begin{array}{l}4.2 \\
4.9 \\
4.7 \\
4.5 \\
4.3\end{array}$ & $\begin{array}{l}3.8 \\
3.8 \\
3.8 \\
3.8 \\
3.8\end{array}$ & $\begin{array}{l}4.0 \\
4.0 \\
3.8 \\
3.8 \\
3.8\end{array}$ & $\begin{array}{l}4.0 \\
4.0 \\
4.0 \\
4.0 \\
4.0\end{array}$ & $\begin{array}{l}4.0 \\
4.0 \\
4.0 \\
4.0 \\
4.0\end{array}$ \\
\hline & $\begin{array}{l}4.9 \\
4.7 \\
4.6 \\
4.6 \\
4.6\end{array}$ & \begin{tabular}{|c}
4.7 \\
8.2 \\
14.15 \\
6.4 \\
6.1
\end{tabular} & $\begin{array}{r}6.0 \\
17.0 \\
17.2 \\
8.2 \\
6.5\end{array}$ & $\begin{array}{l}5.5 \\
5.4 \\
5.6 \\
7.8 \\
7.2\end{array}$ & $\begin{array}{l}4.8 \\
4.7 \\
5.0 \\
4.9 \\
5.7\end{array}$ & $\begin{array}{l}7.0 \\
6.8 \\
5.7 \\
5.4 \\
5.2\end{array}$ & $\begin{array}{l}4.2 \\
4.4 \\
6.2 \\
7.1 \\
8.2\end{array}$ & $\begin{array}{l}4.0 \\
4.0 \\
4.2 \\
4.5 \\
4.5\end{array}$ & $\begin{array}{l}3.9 \\
3.9 \\
3.9 \\
3.9 \\
4.9\end{array}$ & $\begin{array}{l}3.8 \\
3.8 \\
3.8 \\
3.8 \\
3.8\end{array}$ & $\begin{array}{l}4.0 \\
4.0 \\
4.0 \\
4.0 \\
4.2\end{array}$ & $\begin{array}{l}4.0 \\
4.0 \\
4.0 \\
4.0 \\
4.2\end{array}$ \\
\hline 5.. & $\begin{array}{l}4.6 \\
4.6 \\
4.6 \\
4.6 \\
4.6\end{array}$ & $\begin{array}{l}6.0 \\
5.4 \\
5.1 \\
4.9 \\
4.9\end{array}$ & $\begin{array}{l}6.6 \\
7.0 \\
6.2 \\
5.9 \\
5.8\end{array}$ & $\begin{array}{l}6.4 \\
5.9 \\
5.6 \\
5.4 \\
5.1\end{array}$ & $\begin{array}{l}5.4 \\
4.8 \\
4.7 \\
4.8 \\
5.0\end{array}$ & $\begin{array}{l}5.4 \\
5.1 \\
5.0 \\
4.7 \\
4.6\end{array}$ & $\begin{array}{l}7.3 \\
6.1 \\
7.4 \\
6.4 \\
5.2\end{array}$ & $\begin{array}{r}12.6 \\
5.6 \\
4.6 \\
4.2 \\
4.2\end{array}$ & $\begin{array}{l}5.2 \\
4.9 \\
4.8 \\
4.8 \\
4.7\end{array}$ & $\begin{array}{l}3.8 \\
3.8 \\
6.0 \\
5.4 \\
4.8\end{array}$ & $\begin{array}{l}4.0 \\
4.0 \\
4.2 \\
4.4 \\
4.4\end{array}$ & $\begin{array}{l}4.2 \\
4.2 \\
4.2 \\
4.2 \\
4.2\end{array}$ \\
\hline $\begin{array}{l}16 . . \\
17 . \\
18 . . \\
19 . . \\
20 . .\end{array}$ & $\begin{array}{l}4.6 \\
4.5 \\
4.5 \\
4.5 \\
4.5\end{array}$ & $\begin{array}{l}5.0 \\
4.9 \\
4.9 \\
4.9 \\
4.9\end{array}$ & $\begin{array}{l}5.6 \\
5.5 \\
5.6 \\
5.5 \\
5.8\end{array}$ & $\begin{array}{l}5.2 \\
5.0 \\
4.9 \\
4.9 \\
4.8\end{array}$ & $\begin{array}{l}5.0 \\
4.8 \\
4.9 \\
5.2 \\
5.2\end{array}$ & $\begin{array}{l}4.6 \\
4.5 \\
4.6 \\
4.5 \\
9.8\end{array}$ & $\begin{array}{l}4.2 \\
4.1 \\
4.1 \\
4.1 \\
4.0\end{array}$ & $\begin{array}{l}4.1 \\
4.1 \\
4.0 \\
4.0 \\
4.2\end{array}$ & $\begin{array}{l}4.7 \\
4.6 \\
4.6 \\
4.6 \\
5.6\end{array}$ & $\begin{array}{l}4.0 \\
4.0 \\
3.8 \\
3.8 \\
3.8\end{array}$ & $\begin{array}{l}4.2 \\
4.2 \\
4.2 \\
4.2 \\
4.2\end{array}$ & $\begin{array}{l}4.2 \\
4.2 \\
4.2 \\
4.2 \\
4.2\end{array}$ \\
\hline .. & $\begin{array}{r}4.5 \\
4.5 \\
19.9 \\
11.5 \\
6.1\end{array}$ & \begin{tabular}{|c}
5.0 \\
13.75 \\
12.2 \\
9.0 \\
6.8
\end{tabular} & $\begin{array}{l}6.2 \\
6.5 \\
6.8 \\
6.2 \\
5.9\end{array}$ & $\begin{array}{l}4.8 \\
4.6 \\
4.6 \\
4.7 \\
4.6\end{array}$ & $\begin{array}{l}4.9 \\
4.8 \\
4.6 \\
4.6 \\
4.4\end{array}$ & $\begin{array}{l}7.4 \\
5.9 \\
4.9 \\
4.5 \\
4.4\end{array}$ & $\begin{array}{l}4.0 \\
4.0 \\
4.0 \\
5.1 \\
5.1\end{array}$ & $\begin{array}{l}4.7 \\
4.7 \\
4.6 \\
4.5 \\
4.2\end{array}$ & $\begin{array}{l}5.2 \\
4.9 \\
4.6 \\
4.4 \\
3.9\end{array}$ & $\begin{array}{l}4.0 \\
5.0 \\
4.6 \\
4.4 \\
4.2\end{array}$ & $\begin{array}{l}4.0 \\
4.0 \\
4.0 \\
4.0 \\
4.0\end{array}$ & $\begin{array}{l}4.2 \\
4.2 \\
4.2 \\
4.4 \\
4.6\end{array}$ \\
\hline $1 \ldots \ldots$ & $\begin{array}{l}5.9 \\
4.6 \\
4.6 \\
5.5 \\
6.1 \\
5.9\end{array}$ & $\begin{array}{r}5.2 \\
4.9 \\
4.8 \\
4.8 \\
\ldots \ldots \ldots \\
\ldots \ldots \ldots .\end{array}$ & $\begin{array}{l}5.7 \\
5.7 \\
5.4 \\
5.2 \\
5.2 \\
5.4\end{array}$ & $\begin{array}{l}4.8 \\
4.9 \\
5.3 \\
5.3 \\
5.1\end{array}$ & $\begin{array}{l}4.5 \\
4.4 \\
4.5 \\
4.3 \\
4.4 \\
4.5\end{array}$ & $\begin{array}{l}4.3 \\
4.1 \\
4.2 \\
4.2 \\
4.3\end{array}$ & $\begin{array}{l}5.1 \\
5.1 \\
5.0 \\
4.9 \\
4.4 \\
4.2\end{array}$ & $\begin{array}{l}4.2 \\
4.1 \\
3.9 \\
3.8 \\
3.8 \\
3.8\end{array}$ & $\begin{array}{l}4.0 \\
4.2 \\
4.4 \\
4.2 \\
4.0\end{array}$ & $\begin{array}{l}4.0 \\
4.0 \\
4.0 \\
4.0 \\
4.0 \\
4.0\end{array}$ & $\begin{array}{l}4.0 \\
4.0 \\
4.0 \\
4.0 \\
4.0\end{array}$ & $\begin{array}{l}5.4 \\
6.8 \\
9.4 \\
9.2 \\
7.8 \\
5.8\end{array}$ \\
\hline & $\begin{array}{l}6.0 \\
6.4 \\
6.8 \\
6.4 \\
6.2\end{array}$ & $\begin{array}{l}5.2 \\
5.0 \\
5.0 \\
4.8 \\
4.8\end{array}$ & $\begin{array}{l}5.8 \\
5.8 \\
5.4 \\
5.2 \\
6.6\end{array}$ & $\begin{array}{l}6.0 \\
5.8 \\
5.6 \\
5.4 \\
6.4\end{array}$ & $\begin{array}{l}4.8 \\
4.8 \\
4.8 \\
4.6 \\
4.6\end{array}$ & $\begin{array}{l}4.6 \\
4.4 \\
4.4 \\
4.4 \\
4.2\end{array}$ & $\begin{array}{l}4.5 \\
5.2 \\
4.9 \\
4.6 \\
4.5\end{array}$ & $\begin{array}{l}5.7 \\
5.1 \\
4.9 \\
4.7 \\
4.6\end{array}$ & $\begin{array}{l}5.0 \\
4.9 \\
7.5 \\
6.4 \\
6.1\end{array}$ & $\begin{array}{l}4.2 \\
4.1 \\
4.2 \\
5.0 \\
4.5\end{array}$ & $\begin{array}{l}4.7 \\
4.7 \\
4.7 \\
4.6 \\
4.6\end{array}$ & $\begin{array}{l}8.1 \\
7.2 \\
9.5 \\
9 . \\
8.3\end{array}$ \\
\hline $\begin{array}{l}6 . \\
7 . \\
8 . \\
9 .\end{array}$ & $\begin{array}{r}6.2 \\
17.4 \\
9.8 \\
7.2 \\
6.8\end{array}$ & $\begin{array}{l}4.8 \\
4.8 \\
4.8 \\
4.8 \\
4.8\end{array}$ & $\begin{array}{r}6.8 \\
7.4 \\
7.4 \\
10.9 \\
14.6\end{array}$ & $\begin{array}{l}6.4 \\
6.2 \\
5.8 \\
5.6 \\
5.4\end{array}$ & $\begin{array}{l}4.6 \\
4.6 \\
4.6 \\
4.6 \\
4.6\end{array}$ & $\begin{array}{l}4.2 \\
4.6 \\
7.4 \\
7.2 \\
5.6\end{array}$ & $\begin{array}{l}8.0 \\
7.9 \\
7.6 \\
6.5 \\
5.6\end{array}$ & $\begin{array}{l}4.5 \\
4.4 \\
4.5 \\
4.5 \\
4.5\end{array}$ & $\begin{array}{l}5.9 \\
5.1 \\
5.1 \\
4.9 \\
4.8\end{array}$ & $\begin{array}{l}4.3 \\
4.4 \\
4.2 \\
4.3 \\
4.3\end{array}$ & $\begin{array}{l}4.6 \\
4.6 \\
4.5 \\
4.5 \\
4.4\end{array}$ & $\begin{array}{l}6.1 \\
5.6 \\
5.5 \\
5.4 \\
5.3\end{array}$ \\
\hline $15 \ldots$. & $\begin{array}{l}6.6 \\
6.4 \\
6.4 \\
6.2 \\
5.8\end{array}$ & $\begin{array}{l}4.8 \\
4.8 \\
4.8 \\
4.8 \\
4.8\end{array}$ & $\begin{array}{r}11.8 \\
9.8 \\
9.0 \\
8.8 \\
8.2\end{array}$ & $\begin{array}{l}7.6 \\
6.8 \\
6.4 \\
6.0 \\
5.8\end{array}$ & $\begin{array}{l}4.4 \\
4.4 \\
4.4 \\
4.8 \\
5.0\end{array}$ & $\begin{array}{l}4.4 \\
4.9 \\
8.3 \\
6.0 \\
5.2\end{array}$ & $\begin{array}{r}4.9 \\
4.9 \\
4.9 \\
6.1 \\
10.6\end{array}$ & $\begin{array}{l}4.5 \\
4.5 \\
5.8 \\
6.1 \\
5.5\end{array}$ & $\begin{array}{l}4.9 \\
7.2 \\
5.5 \\
5.1 \\
4.9\end{array}$ & $\begin{array}{l}4.4 \\
6.7 \\
6.1 \\
5.9 \\
5.3\end{array}$ & $\begin{array}{l}4.4 \\
4.4 \\
4.4 \\
4.4 \\
4.3\end{array}$ & $\begin{array}{l}5.1 \\
5.0 \\
5.0 \\
5.0 \\
4.9\end{array}$ \\
\hline$\cdots$ & $\begin{array}{l}5.8 \\
6.0 \\
6.2 \\
6.4 \\
6.4\end{array}$ & $\begin{array}{l}4.8 \\
4.8 \\
4.8 \\
4.8 \\
4.8\end{array}$ & $\begin{array}{l}8.2 \\
9.0 \\
9.2 \\
8.6 \\
9.4\end{array}$ & $\begin{array}{l}5.6 \\
5.4 \\
5.4 \\
5.2 \\
5.2\end{array}$ & $\begin{array}{l}4.8 \\
4.8 \\
4.8 \\
4.8 \\
4.6\end{array}$ & $\begin{array}{l}4.8 \\
4.6 \\
4.4 \\
4.4 \\
4.4\end{array}$ & $\begin{array}{l}7.9 \\
5.9 \\
4.9 \\
4.6 \\
4.6\end{array}$ & $\begin{array}{l}5.4 \\
5.3 \\
4.6 \\
4.4 \\
4.5\end{array}$ & $\begin{array}{l}4.6 \\
4.7 \\
5.1 \\
5.2 \\
5.1\end{array}$ & $\begin{array}{l}4.9 \\
4.4 \\
4.4 \\
4.9 \\
7.4\end{array}$ & $\begin{array}{l}4.3 \\
4.3 \\
4.3 \\
4.3 \\
4.3\end{array}$ & $\begin{array}{l}4.9 \\
5.0 \\
5.0 \\
5.0 \\
5.75\end{array}$ \\
\hline 23. & $\begin{array}{l}5.8 \\
5.6 \\
5.4 \\
5.2 \\
5.4\end{array}$ & $\begin{array}{l}4.8 \\
4.8 \\
4.8 \\
4.8 \\
4.8\end{array}$ & \begin{tabular}{|r|}
14.8 \\
10.4 \\
8.8 \\
8.2 \\
8.0
\end{tabular} & $\begin{array}{l}5.2 \\
5.0 \\
5.0 \\
5.0 \\
4.8\end{array}$ & $\begin{array}{l}4.6 \\
4.6 \\
4.4 \\
4.4 \\
4.2\end{array}$ & $\begin{array}{l}4.1 \\
4.3 \\
6.3 \\
7.4 \\
7.5\end{array}$ & $\begin{array}{r}4.6 \\
4.6 \\
5.9 \\
12.1 \\
7.9\end{array}$ & $\begin{array}{r}4.5 \\
4.5 \\
4.4 \\
4.4 \\
16.0\end{array}$ & $\begin{array}{l}4.9 \\
4.6 \\
4.6 \\
4.5 \\
4.4\end{array}$ & $\begin{array}{l}7.0 \\
6.9 \\
6.1 \\
5.1 \\
4.7\end{array}$ & $\begin{array}{l}4.3 \\
4.3 \\
4.3 \\
4.3 \\
4.3\end{array}$ & $\begin{array}{c}18.65 \\
12.6 \\
10.5 \\
7.5 \\
6.9\end{array}$ \\
\hline 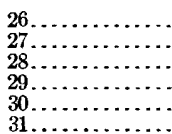 & $\begin{array}{l}6.2 \\
5.8 \\
5.6 \\
5.6 \\
5.6 \\
5.6\end{array}$ & $\begin{array}{l}5.0 \\
5.6 \\
5.6\end{array}$ & $\begin{array}{l}8.0 \\
7.6 \\
6.8 \\
6.4 \\
6.4 \\
6.2\end{array}$ & $\begin{array}{l}4.8 \\
4.8 \\
5.8 \\
5.2 \\
5.0\end{array}$ & $\begin{array}{l}4.2 \\
4.2 \\
4.2 \\
4.2 \\
4.2 \\
4.2\end{array}$ & $\begin{array}{l}6.9 \\
6.5 \\
5.1 \\
4.9 \\
4.6\end{array}$ & $\begin{array}{l}6.9 \\
5.1 \\
4.9 \\
4.8 \\
7.6 \\
6.9\end{array}$ & $\begin{array}{r}18.8 \\
12.5 \\
6.4 \\
5.7 \\
5.4 \\
5.3\end{array}$ & $\begin{array}{l}4.4 \\
4.3 \\
4.2 \\
4.2 \\
4.2\end{array}$ & $\begin{array}{l}5.6 \\
5.7 \\
5.3 \\
4.8 \\
4.8 \\
4.7\end{array}$ & $\begin{array}{l}4.3 \\
4.3 \\
4.3 \\
7.4 \\
8.5\end{array}$ & $\begin{array}{c}6.5 \\
5.9 \\
5.7 \\
10.65 \\
8.7 \\
6.7\end{array}$ \\
\hline
\end{tabular}

$a$ River frozen at the gage Jan. 4-22 and Feb, 15-22, 1904

${ }_{b}$ From $\bar{J}$ an. 27 to Feb. 28,1905 , the river was frozen entirely across except for a narrow channel in the middle. Gage heights are to the surface of the water in a hole in the ice. Thickness of ice about 1 foot. 
Daily gage height, in feet, of Monocacy River near Frederick, Md.--Continued.

\begin{tabular}{|c|c|c|c|c|c|c|c|c|c|c|c|c|}
\hline Day. & Jan. & Feb. & Mar. & Apr. & May. & June. & July. & Aug. & Sept. & Oct. & Nov. & Dec. \\
\hline $\begin{array}{c}1906 . a \\
1 \ldots \ldots \\
2 \ldots \\
3 \ldots \ldots \ldots \\
4 \ldots \ldots\end{array}$ & $\begin{array}{l}6.2 \\
5.7 \\
7.5 \\
18.35 \\
12.6\end{array}$ & $\begin{array}{l}4.9 \\
4.8 \\
4.5 \\
4.5 \\
5.1\end{array}$ & $\begin{array}{c}5.3 \\
5.5 \\
9.1 \\
16.65 \\
9.1\end{array}$ & $\begin{array}{l}9.7 \\
7.8 \\
7.4 \\
6.9 \\
6.5\end{array}$ & $\begin{array}{l}5.4 \\
5.4 \\
5.3 \\
5.3 \\
5.2\end{array}$ & $\begin{array}{l}\text { 4. } 7 \\
\text { 4. } 7 \\
\text { 4. } 6 \\
\text { 4. } 5 \\
\text { 4. } 4\end{array}$ & $\begin{array}{l}4.5 \\
4.5 \\
4.5 \\
5.3 \\
5.1\end{array}$ & $\begin{array}{r}4.5 \\
9.1 \\
16.1 \\
10.1 \\
5.5\end{array}$ & $\begin{array}{l}5.5 \\
5.2 \\
5.1 \\
4.9 \\
4.9\end{array}$ & $\begin{array}{l}\text { 4. } 3 \\
4.3 \\
4.3 \\
4.8 \\
7.1\end{array}$ & $\begin{array}{l}5.6 \\
5.5 \\
5.3 \\
5.2 \\
5.1\end{array}$ & $\begin{array}{l}\text { 4. } 5 \\
\text { 4. } 5 \\
\text { 4. } 5 \\
\text { 4. } 5 \\
\text { 4. } 5\end{array}$ \\
\hline $\begin{array}{r}6 \ldots \\
7 \ldots \\
8 \ldots \\
9 \ldots \\
10 \ldots\end{array}$ & $\begin{array}{l}7.6 \\
6.7 \\
6.6 \\
6.2 \\
5.9\end{array}$ & $\begin{array}{l}5.0 \\
4.9 \\
4.8 \\
5.2 \\
5.1\end{array}$ & $\begin{array}{l}6.9 \\
6.5 \\
6.4 \\
6.3 \\
6.1\end{array}$ & $\begin{array}{r}6.3 \\
6.2 \\
6.1 \\
7.5 \\
16.8\end{array}$ & $\begin{array}{l}5.3 \\
5.3 \\
5.3 \\
5.2 \\
5.0\end{array}$ & $\begin{array}{l}\text { 4. } 4 \\
\text { 4. } 4 \\
\text { 4. } 4 \\
\text { 4. } 4 \\
\text { 5. } 5\end{array}$ & $\begin{array}{l}\text { 4. } 6 \\
4.6 \\
4.5 \\
4.5 \\
4.4\end{array}$ & $\begin{array}{l}5.3 \\
5.1 \\
4.9 \\
4.8 \\
4.8\end{array}$ & $\begin{array}{l}\text { 4. } 8 \\
\text { 4. } 7 \\
\text { 4. } 6 \\
\text { 4. } 6 \\
\text { 4. } 5\end{array}$ & $\begin{array}{l}6.1 \\
5.8 \\
4.9 \\
4.7 \\
4.6\end{array}$ & $\begin{array}{l}5.0 \\
4.9 \\
4.8 \\
4.8 \\
4.8\end{array}$ & $\begin{array}{l}4.7 \\
4.8 \\
4.8 \\
4.7 \\
5.9\end{array}$ \\
\hline $\begin{array}{l}11 . \ldots \ldots \\
12 . \ldots \\
13 . \ldots \\
14 . \ldots \\
15 . \ldots \ldots\end{array}$ & $\begin{array}{l}5.7 \\
6.9 \\
6.7 \\
6.2 \\
6.5\end{array}$ & $\begin{array}{l}5.0 \\
4.9 \\
4.8 \\
5.9 \\
8.1\end{array}$ & $\begin{array}{l}5.9 \\
5.8 \\
5.8 \\
5.8 \\
5.8\end{array}$ & $\begin{array}{r}9.1 \\
8.5 \\
7.1 \\
6.9 \\
21.8\end{array}$ & $\begin{array}{l}5.0 \\
4.9 \\
4.9 \\
4.9 \\
4.7\end{array}$ & $\begin{array}{l}5.1 \\
\text { 4. } 5 \\
\text { 4. } 5 \\
\text { 4. } 4 \\
4.4\end{array}$ & $\begin{array}{l}4.4 \\
4.9 \\
4.5 \\
4.4 \\
4.4\end{array}$ & $\begin{array}{l}6.1 \\
5.1 \\
6.1 \\
4.8 \\
47\end{array}$ & $\begin{array}{l}4.4 \\
4.4 \\
4.4 \\
4.4 \\
4.4\end{array}$ & $\begin{array}{l}4.5 \\
4.4 \\
43 \\
4.3 \\
43\end{array}$ & $\begin{array}{l}4.8 \\
4.7 \\
4.7 \\
4.7 \\
48\end{array}$ & $\begin{array}{l}6.8 \\
6.4 \\
5.3 \\
5.2 \\
5.2\end{array}$ \\
\hline $\begin{array}{l}16 \ldots \ldots \\
17{ }_{1} \ldots \ldots \\
19 \ldots \ldots \\
20 \ldots \ldots\end{array}$ & $\begin{array}{l}7.1 \\
6.9 \\
6.2 \\
5.9 \\
5.7\end{array}$ & $\begin{array}{l}5.4 \\
4.9 \\
4.8 \\
5.0 \\
5.0\end{array}$ & $\begin{array}{l}5.8 \\
5.7 \\
5.7 \\
5.6 \\
5.6\end{array}$ & $\begin{array}{r}15.5 \\
10.2 \\
8.1 \\
7.5 \\
7.1\end{array}$ & $\begin{array}{l}4.8 \\
4.8 \\
4.7 \\
4.7 \\
4.5\end{array}$ & $\begin{array}{ll}4 & 5 \\
4 & 6 \\
7 . & 1 \\
9.7 \\
9 . & 5\end{array}$ & $\begin{array}{l}4.4 \\
4.5 \\
4.5 \\
4.4 \\
4.4\end{array}$ & $\begin{array}{l}\text { 4. } 6 \\
\text { 4. } 6 \\
\text { 4. } 5 \\
8.5 \\
7.5\end{array}$ & $\begin{array}{l}4.4 \\
4.4 \\
4.4 \\
4.4 \\
4.4\end{array}$ & $\begin{array}{r}4.3 \\
4.3 \\
4.3 \\
9.5 \\
13.2\end{array}$ & $\begin{array}{r}4.8 \\
-4.8 \\
4.9 \\
5.2 \\
5.5\end{array}$ & $\begin{array}{r}7.6 \\
9.8 \\
12.1 \\
8.5 \\
6.5\end{array}$ \\
\hline $\begin{array}{l}21 \ldots \ldots \\
22 \ldots \ldots \ldots \\
23 \ldots \ldots \cdots \\
24 \ldots \ldots \\
25 \ldots \ldots\end{array}$ & $\begin{array}{l}5.7 \\
5.7 \\
5.6 \\
5.6 \\
5.6\end{array}$ & $\begin{array}{l}5.4 \\
9.4 \\
6.5 \\
6.1 \\
6.7\end{array}$ & $\begin{array}{l}5.5 \\
5.5 \\
5.5 \\
5.6 \\
5.6\end{array}$ & $\begin{array}{l}6.7 \\
6.4 \\
6.3 \\
6.1 \\
5.9\end{array}$ & $\begin{array}{l}4.5 \\
4.5 \\
4.5 \\
4.5 \\
4.4\end{array}$ & $\begin{array}{r}10.5 \\
10.7 \\
6.7 \\
6.1 \\
5.9\end{array}$ & $\begin{array}{l}4.3 \\
4.4 \\
4.8 \\
5.1 \\
4.8\end{array}$ & \begin{tabular}{l|}
6.5 \\
5.4 \\
7.35 \\
6.5 \\
7.5
\end{tabular} & $\begin{array}{l}\text { 4. } 8 \\
\text { 4. } 6 \\
4.4 \\
4.4 \\
4.4\end{array}$ & $\begin{array}{r}9.5 \\
11.5 \\
8.5 \\
7.5 \\
8.5\end{array}$ & $\begin{array}{l}5.1 \\
5.0 \\
4.9 \\
4.8 \\
4.7\end{array}$ & $\begin{array}{l}9.5 \\
7.5 \\
6.9 \\
5.8 \\
5.7\end{array}$ \\
\hline $\begin{array}{l}26 \ldots \ldots \\
27 \ldots \ldots \\
28 \ldots \ldots \\
29 \ldots \ldots \\
30 \ldots \ldots \\
31 \ldots \ldots\end{array}$ & $\begin{array}{l}5.5 \\
5.5 \\
5.55 \\
5.3 \\
5.2 \\
5.1\end{array}$ & $\begin{array}{r}6.9 \\
5.9 \\
6.1 \\
\cdots \\
\cdots\end{array}$ & $\begin{array}{c}5.5 \\
9.0 \\
17.45 \\
12.5 \\
10.5 \\
9.6\end{array}$ & $\begin{array}{r}5.8 \\
5.7 \\
5.5 \\
5.4 \\
5.4 \\
\hdashline . . .\end{array}$ & $\begin{array}{l}4.5 \\
4.5 \\
5.6 \\
5.1 \\
4.8 \\
4.7\end{array}$ & $\begin{array}{l}5.8 \\
5.1 \\
4.9 \\
4.8 \\
4.7 \\
\cdots\end{array}$ & $\begin{array}{l}\text { 4. } 6 \\
4.4 \\
4.35 \\
4.5 \\
4.3 \\
4.3\end{array}$ & $\begin{array}{r}7.8 \\
11.0 \\
9.8 \\
7.5 \\
6.1 \\
5.8\end{array}$ & $\begin{array}{l}4.3 \\
4.3 \\
4.3 \\
4.3 \\
4.3\end{array}$ & $\begin{array}{l}8.1 \\
7.5 \\
6.7 \\
6.2 \\
5.8 \\
5.7\end{array}$ & $\begin{array}{l}4.6 \\
\text { 4. } 6 \\
4.6 \\
4.5 \\
4.5\end{array}$ & $\begin{array}{l}5.6 \\
7.8 \\
6.9 \\
5.8 \\
5.7 \\
8.4\end{array}$ \\
\hline
\end{tabular}

Rating tables for Monocacy River near Frederick, $M d$.

- AUGUST 4, 1896, TO DECEMBER 31, 1903.o.

\begin{tabular}{|c|c|c|c|c|c|c|c|}
\hline $\begin{array}{l}\text { Gage } \\
\text { height. }\end{array}$ & Discharge. & $\begin{array}{c}\text { Gage } \\
\text { height. }\end{array}$ & Discharge. & $\begin{array}{c}\text { Gage } \\
\text { height. }\end{array}$ & Discharge. & $\begin{array}{c}\text { Gage } \\
\text { height. }\end{array}$ & Discharge. \\
\hline $\begin{aligned} \text { Feet. } \\
3.40 \\
3.50 \\
\text { 3. } 60 \\
3.70 \\
3.80 \\
\text { 3. } 90 \\
4.00 \\
4.10 \\
4.20 \\
4.30 \\
\text { 4. } 40 \\
4.50 \\
4.60 \\
\text { 4. } 70 \\
\text { 4. } 80 \\
\text { 4. } 90 \\
\text { 5. } 00\end{aligned}$ & \begin{tabular}{|c|} 
second-feer. \\
43 \\
55 \\
69 \\
85 \\
103 \\
124 \\
147 \\
172 \\
198 \\
226 \\
256 \\
288 \\
322 \\
358 \\
396 \\
437 \\
480
\end{tabular} & $\begin{array}{l}\text { Feer. } \\
5.10 \\
5.20 \\
5.30 \\
5.40 \\
5.50 \\
5.60 \\
5.70 \\
5.80 \\
5.90 \\
6.00 \\
6.10 \\
6.20 \\
6.30 \\
6.40 \\
6.50 \\
6.60 \\
6.70\end{array}$ & \begin{tabular}{|c|} 
Second-feet. \\
525 \\
575 \\
625 \\
675 \\
730 \\
785 \\
840 \\
900 \\
960 \\
1,025 \\
1,090 \\
1,155 \\
1,225 \\
1,295 \\
1,370 \\
1,445 \\
1,520
\end{tabular} & $\begin{array}{l}\text { Feet. } \\
6.80 \\
6.90 \\
7.00 \\
7.20 \\
7.40 \\
7.60 \\
7.80 \\
8.00 \\
8.20 \\
8.40 \\
8.60 \\
8.80 \\
9.00 \\
9.20 \\
9.40 \\
9.60 \\
9.80\end{array}$ & \begin{tabular}{|c|} 
Second-feet. \\
1,600 \\
1,680 \\
1,760 \\
1,930 \\
2,105 \\
2,285 \\
2,465 \\
2,650 \\
2,840 \\
3,030 \\
3,220 \\
3,410 \\
3,600 \\
3,800 \\
4,000 \\
4,200 \\
4,400
\end{tabular} & $\begin{array}{l}\text { Feet. } \\
10.00 \\
10.50 \\
11.00 \\
11.50 \\
12.00 \\
13.00 \\
14.00 \\
15.00 \\
16.00 \\
17.00 \\
18.00 \\
19.00 \\
20.00 \\
22.00 \\
24.00 \\
26.00\end{array}$ & $\begin{array}{c}\text { Second-feet. } \\
4,600 \\
5,100 \\
5,600 \\
6,100 \\
6,600 \\
7,650 \\
8,700 \\
9,750 \\
10,800 \\
11,850 \\
12,900 \\
13,950 \\
15,000 \\
17,100 \\
19,200 \\
21,300\end{array}$ \\
\hline
\end{tabular}

$a$ This table is strictly applicable only for open-channel conditions. It is based on discharge measurements made during 1896-1903. It is fairly well defined between gage heights 4.0 feet and 9.0 feet. Above gage height 12.0 feet the rating curve is a tangent, the difference being 105 per tenth 
Rating tables for Monocacy River near Frederick, Md.-Continued.

JANUARY 1, 1904, TO DECEMBER 31,1906.a

\begin{tabular}{|c|c|c|c|c|c|c|c|}
\hline $\begin{array}{c}\text { Gage } \\
\text { height. }\end{array}$ & Discharge. & $\begin{array}{c}\text { Gage } \\
\text { height. }\end{array}$ & Discharge. & $\begin{array}{c}\text { Gage } \\
\text { height. }\end{array}$ & Discharge. & $\begin{array}{c}\text { Gage } \\
\text { height. }\end{array}$ & Discharge. \\
\hline Feet. & Second-feet. & Feet. & Second-feet. & Feet. & Second-feet. & Feet & Second feet. \\
\hline 3.80 & 80 & 5.30 & 584 & 6.80 & 1,545 & 9.60 & 4,100 \\
\hline 3.90 & 99 & 5.40 & 632 & 6.90 & 1,625 & 9.80 & 4,300 \\
\hline 4.00 & 120 & 5.50 & 682 & 7.00 & 1,705 & 10.00 & 4,500 \\
\hline 4. 10 & 144 & 5. 60 & 734 & 7.20 & 1,875 & 10. 50 & 5,000 \\
\hline 4. 20 & 170 & 5. 70 & 789 & 7.40 & 2,045 & 11.00 & 5,500 \\
\hline 4. 30 & 198 & 5. 80 & 846 & 7.60 & 2,220 & 11.50 & 6,000 \\
\hline 4. 40 & 228 & 5.90 & 906 & 7.80 & 2,400 & 12.00 & 6,500 \\
\hline 4. 50 & 260 & 6.00 & 969 & 8.00 & 2,580 & 13.00 & 7,550 \\
\hline 4. 60 & 294 & 6.10 & 1,034 & 8.20 & 2,765 & 14.00 & 8,600 \\
\hline 4. 70 & 330 & 6.20 & 1,102 & 8.40 & 2,955 & 15.00 & 9,650 \\
\hline 4. 80 & 368 & 6.30 & 1,172 & 8.60 & 3,145 & 16.00 & 10,700 \\
\hline 4. 90 & 408 & 6.40 & 1,242 & 8.80 & 3,335 & 17.00 & 11,750 \\
\hline 5. 00 & 450 & 6.50 & 1,315 & 9.00 & 3,525 & 18.00 & 12,800 \\
\hline 5. 1 & 493 & 6.60 & 1,390 & 9.20 & 3,715 & 19.00 & 13,850 \\
\hline 5. & 538 & & & 9.40 & 05 & 20.00 & 14,900 \\
\hline
\end{tabular}

$a$ This table is strictly applicable only for open-channel conditions. It is based on discharge measurements made during 1904-1906. It is fairly well defined between gage heights 4.0 feet and 10.0 feet. Above gage height 12.0 feet the rating curve is a tangent, the difference being 105 per tenth.

Estimated monthly duscharge of Monocacy River near Frederick, Md.

[D rainage area, 660 square miles.a]

\begin{tabular}{|c|c|c|c|c|c|c|c|c|}
\hline \multirow[b]{2}{*}{ Month } & \multicolumn{3}{|c|}{ Discharge in second-feet. } & \multicolumn{3}{|c|}{ Run-off. } & \multicolumn{2}{|c|}{ Precipitation. } \\
\hline & Maximum. & Minimum. & Mean. & $\begin{array}{l}\text { Second- } \\
\text { feet per } \\
\text { square } \\
\text { mile. }\end{array}$ & $\begin{array}{l}\text { Depth } \\
\text { in } \\
\text { inches. }\end{array}$ & $\begin{array}{l}\text { Per cent } \\
\text { of pre- } \\
\text { cipita- } \\
\text { tion. }\end{array}$ & $\begin{array}{c}\text { In } \\
\text { inches. }\end{array}$ & $\begin{array}{l}\text { Loss in } \\
\text { inches. }\end{array}$ \\
\hline $\begin{array}{r}1896 . \\
\text { January..... }\end{array}$ & & & & & & & 1.48 & \\
\hline February. & & & & & & & 5.83 & \\
\hline Mareh. & & & & & & & 5.32 & \\
\hline Apr & & & & & & & $\begin{array}{l}1.43 \\
1.43\end{array}$ & \\
\hline $\mathrm{Ma}$ & & & & & & & 2.04 & \\
\hline Jui & & & & & & & 4.31 & 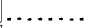 \\
\hline Aug & 256 & 69 & 125 & $189^{\circ}$ & 197 & & $\begin{array}{r}5.05 \\
6.44\end{array}$ & .......... \\
\hline $\begin{array}{l}\text { August 4-3 } \\
\text { September }\end{array}$ & $\begin{array}{l}2300 \\
480\end{array}$ & $\begin{array}{l}69 \\
69\end{array}$ & $\begin{array}{l}120 \\
115\end{array}$ & .189 & .194 & $\ddot{6}$ & $\begin{array}{r}01.44 \\
2.94\end{array}$ & $\cdots$ \\
\hline October.. & 396 & 103 & 144 & .218 & .251 & 19 & 1.31 & 1.06 \\
\hline Nover & 2,195 & 124 & 306 & .464 & .518 & 14 & 3.82 & 3.30 \\
\hline December & 396 & 124 & 187 & .283 & .326 & 41 & .81 & .48 \\
\hline The year.. & . & $\cdots$ & & $\cdots$ & & .. & 35.78 & $\ldots \ldots \ldots$ \\
\hline & & & & & & & & \\
\hline January & 730 & 147 & 307 & .465 & .536 & 28 & 1.95 & 1.41 \\
\hline Feb & 9,750 & 256 & 2,062 & 3.12 & 3.25 & 63 & 5.16 & 1.91 \\
\hline Marcl & 4,400 & 575 & 1,384 & 2.10 & 2.42 & 84 & 2.88 & .46 \\
\hline Apri & 3,900 & 322 & 907 & 1.3 & 1.53 & 53 & & 1.33 \\
\hline May. & 10,380 & 322 & 1,650 & 2.50 & 2.88 & 50 & 5.73 & 2.85 \\
\hline & 2,1 & 19 & 492 & .745 & .831 & 27 & 3. 10 & 2.27 \\
\hline July d. & 4,500 & 19 & 71 & 1.0 & 1.24 & 18 & & 5.57 \\
\hline August $d$. & 11,010 & 172 & 968 & 1.47 & 1.70 & 46 & 3.70 & 2.00 \\
\hline Septe & & 114 & 19 & .289 & .322 & 15 & 2.12 & 1.80 \\
\hline Octol & 241 & 94 & 137 & .208 & .240 & 12 & 1.96 & 1.72 \\
\hline Nove & 9,592 & 185 & 1,008 & 1.53 & 1.71 & 26 & 6.53 & 4.82 \\
\hline December & 9,330 & 550 & 1,980 & 3.00 & 3.46 & 99 & 3.48 & .02 \\
\hline The year & 11,010 & 94 & 983 & 1.49 & 20.12 & 43 & 46.28 & 26.16 \\
\hline
\end{tabular}

$a 660$ squares miles used for alt years except 1904 and 1905 , when 665 was used.

$b$ Precipitation for complete month August, 1896.

$c$ River frozen at the gage January 25 to February 2, 1897; no correction made in estimates.

$d$ Estimates July 3 to December 31,1897 , liable to some error owing to uncertainty of wire length during that period. 
Estimated monthıy discharge of Monocacy River near Frederick, MI.-Continued.

\begin{tabular}{|c|c|c|c|c|c|c|c|c|}
\hline \multirow[b]{2}{*}{ Month } & \multicolumn{3}{|c|}{ Discharge in second-feet. } & \multicolumn{3}{|c|}{ Run-off. } & \multicolumn{2}{|c|}{ Precipitation. } \\
\hline & Maximum. & Minimum. & Means. & $\begin{array}{c}\text { Second- } \\
\text { feet per } \\
\text { square } \\
\text { mile. }\end{array}$ & $\begin{array}{l}\text { Depth } \\
\text { in } \\
\text { inches. }\end{array}$ & $\begin{array}{c}\text { Per cent } \\
\text { of pre- } \\
\text { cipita- } \\
\text { tion. }\end{array}$ & $\begin{array}{c}\text { In } \\
\text { inches. }\end{array}$ & $\begin{array}{l}\text { Loss in } \\
\text { inches. }\end{array}$ \\
\hline $\begin{array}{l}\quad 1898 . \\
\text { January..... } \\
\text { February } a . . \\
\text { March ....... } \\
\text { April........ } \\
\text { May .......... } \\
\text { June......... } \\
\text { July ......... } \\
\text { August ..... } \\
\text { September... } \\
\text { October..... } \\
\text { November... } \\
\text { December... }\end{array}$ & $\begin{array}{r}7,020 \\
9,540 \\
5,350 \\
1,845 \\
8,070 \\
730 \\
675 \\
7,020 \\
198 \\
6,150 \\
6,968 \\
12,850 \\
\end{array}$ & $\begin{array}{r}416 \\
480 \\
437 \\
396 \\
322 \\
256 \\
124 \\
147 \\
103 \\
103 \\
256 \\
480 \\
\end{array}$ & $\begin{array}{r}2,099 \\
1,479 \\
1,319 \\
692 \\
1,575 \\
408 \\
223 \\
926 \\
128 \\
693 \\
1,132 \\
1,943 \\
\end{array}$ & $\begin{array}{l}3.18 \\
2.24 \\
2.00 \\
1.05 \\
2.39 \\
.618 \\
.338 \\
1.40 \\
.194 \\
1.05 \\
1.72 \\
2.94 \\
\end{array}$ & $\begin{array}{l}3.67 \\
2.33 \\
2.31 \\
1.17 \\
2.76 \\
.690 \\
.390 \\
1.61 \\
.216 \\
1.21 \\
1.92 \\
3.39 \\
\end{array}$ & $\begin{array}{r}95 \\
146 \\
59 \\
45 \\
38 \\
58 \\
12 \\
24 \\
15 \\
20 \\
43 \\
81 \\
\end{array}$ & $\begin{array}{l}3.86 \\
1.60 \\
3.94 \\
2.61 \\
7.34 \\
1.18 \\
3.14 \\
6.75 \\
1.46 \\
5.97 \\
4.42 \\
4.17\end{array}$ & $\begin{array}{r}0.19 \\
-.73 \\
1.63 \\
1.44 \\
4.58 \\
.49 \\
2.75 \\
5.14 \\
1.24 \\
4.76 \\
2.50 \\
.78\end{array}$ \\
\hline The yea & 12,850 & 103 & 1,051 & 1.59 & 21.67 & 47 & 46.44 & 24.77 \\
\hline 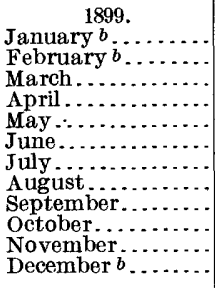 & $\begin{array}{r}9,592 \\
12,690 \\
12,060 \\
7,230 \\
2,375 \\
5,700 \\
288 \\
525 \\
1,025 \\
480 \\
2,015 \\
1,845\end{array}$ & $\begin{array}{r}480 \\
575 \\
1,370 \\
396 \\
322 \\
198 \\
124 \\
103 \\
85 \\
103 \\
172 \\
124\end{array}$ & $\begin{array}{r}1,971 \\
2,939 \\
3,428 \\
1,179 \\
680 \\
759 \\
196 \\
208 \\
309 \\
133 \\
401 \\
449\end{array}$ & $\begin{array}{c}2.99 \\
4.45 \\
5.19 \\
1.79 \\
1.03 \\
1.15 \\
.297 \\
.315 \\
.468 \\
.202 \\
.608 \\
.680\end{array}$ & $\begin{array}{c}3.45 \\
4.63 \\
5.98 \\
2.00 \\
1.19 \\
1.28 \\
.342 \\
.363 \\
.522 \\
.233 \\
.678 \\
.784\end{array}$ & $\begin{array}{r}109 \\
88 \\
115 \\
115 \\
25 \\
41 \\
11 \\
9 \\
10 \\
9 \\
30 \\
33\end{array}$ & $\begin{array}{l}3.16 \\
5.27 \\
5.19 \\
1.74 \\
4.74 \\
3.09 \\
3.17 \\
4.02 \\
5.18 \\
2.64 \\
2.23 \\
2.35\end{array}$ & $\begin{array}{r}-.29 \\
.64 \\
-.79 \\
-\quad .26 \\
3.55 \\
1.81 \\
2.83 \\
3.66 \\
4.66 \\
2.41 \\
1.55 \\
1.57\end{array}$ \\
\hline The year. & 12,690 & 85 & 054 & 1.59 & 21.44 & 50 & 42.78 & 21.34 \\
\hline 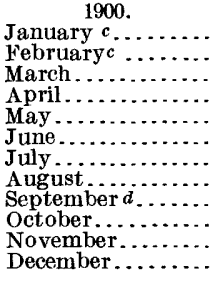 & $\begin{array}{r}4,800 \\
15,840 \\
8,175 \\
1,295 \\
1,760 \\
1,025 \\
702 \\
702 \\
147 \\
172 \\
3,800 \\
6,705 \\
\end{array}$ & $\begin{array}{r}288 \\
525 \\
675 \\
358 \\
226 \\
147 \\
69 \\
49 \\
55 \\
85 \\
85 \\
124\end{array}$ & $\begin{array}{r}1,030 \\
2,641 \\
2,188 \\
576 \\
438 \\
294 \\
154 \\
150 \\
82.1 \\
102 \\
454 \\
520\end{array}$ & $\begin{array}{l}1.56 \\
4.00 \\
3.32 \\
.873 \\
.664 \\
.445 \\
.233 \\
.227 \\
.124 \\
.155 \\
.688 \\
.788\end{array}$ & $\begin{array}{c}1.80 \\
4.16 \\
3.83 \\
.974 \\
.766 \\
.496 \\
.269 \\
.262 \\
.138 \\
.179 \\
.768 \\
.908\end{array}$ & $\begin{array}{r}81 \\
73 \\
111 \\
68 \\
41 \\
11 \\
8 \\
8 \\
6 \\
7 \\
21 \\
30\end{array}$ & $\begin{array}{l}2.22 \\
5.69 \\
3.44 \\
1.43 \\
1.89 \\
4.51 \\
3.34 \\
3.16 \\
2.31 \\
2.59 \\
3.64 \\
3.02\end{array}$ & $\begin{array}{r}.42 \\
1.53 \\
-\quad .39 \\
.46 \\
1.12 \\
4.01 \\
3.07 \\
2.90 \\
2.17 \\
2.41 \\
2.87 \\
2.11\end{array}$ \\
\hline The year & 15,840 & 49 & 719 & 1.09 & 14.56 & 39 & 37.24 & 22.68 \\
\hline 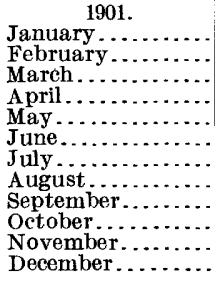 & $\begin{array}{r}2,285 \\
525 \\
15,530 \\
12,900 \\
6,100 \\
2,650 \\
730 \\
1,845 \\
1,520 \\
575 \\
4,000 \\
14,740\end{array}$ & $\begin{array}{l}147 \\
226 \\
256 \\
675 \\
396 \\
288 \\
172 \\
147 \\
172 \\
172 \\
198 \\
358\end{array}$ & $\begin{array}{r}438 \\
329 \\
2,167 \\
2,742 \\
1,521 \\
839 \\
316 \\
445 \\
296 \\
268 \\
480 \\
2,313\end{array}$ & $\begin{array}{c}.664 \\
.498 \\
3.28 \\
4.15 \\
2.30 \\
1.27 \\
.479 \\
.674 \\
.448 \\
.406 \\
.727 \\
3.50\end{array}$ & $\begin{array}{l}.766 \\
.519 \\
3.78 \\
4.63 \\
2.65 \\
1.42 \\
.552 \\
.777 \\
.500 \\
.468 \\
.811 \\
4.04\end{array}$ & $\begin{array}{l}27 \\
69 \\
69 \\
72 \\
49 \\
33 \\
11 \\
13 \\
13 \\
34 \\
31 \\
52\end{array}$ & $\begin{array}{l}2.82 \\
.75 \\
5.48 \\
6.47 \\
5.36 \\
4.26 \\
5.04 \\
6.22 \\
3.77 \\
1.38 \\
2.59 \\
7.76\end{array}$ & $\begin{array}{r}2.05 \\
.23 \\
1.70 \\
1.84 \\
2.71 \\
2.84 \\
4.49 \\
5.44 \\
3.27 \\
.91 \\
1.78 \\
3.72\end{array}$ \\
\hline The year. . & 15,530 & 147 & 1,013 & 1.53 & 20.92 & 40 & 51.90 & 30.98 \\
\hline
\end{tabular}

$a$ River frozen February $2-9,1898$; no correction made in estimates.

$b$ River frozen at the gage January 2, February 9-21, and December 28-31, 1899 ; no correction made in the estimates.

$c$ River frozen at the gage January 1-6 and February 1-4, 1900; no correction made in the estimates. a Discharge interpolated September 29,1900 . 
Estimated monthly discharge of Monocacy River near Frederick, Md.-Continued.

\begin{tabular}{|c|c|c|c|c|c|c|c|c|}
\hline \multirow[b]{2}{*}{ Month. } & \multicolumn{3}{|c|}{ Discharge in second-feet. } & \multicolumn{3}{|c|}{ Run-offi. } & \multicolumn{2}{|c|}{ Precipitation. } \\
\hline & Maximum. & Mininium. & Means. & $\begin{array}{l}\text { Second- } \\
\text { feet per } \\
\text { square } \\
\text { mile. }\end{array}$ & $\begin{array}{l}\text { Depth } \\
\text { in } \\
\text { inches. }\end{array}$ & $\begin{array}{l}\text { Per cent } \\
\text { of pre- } \\
\text { cipita- } \\
\text { tion. }\end{array}$ & $\begin{array}{c}\text { In } \\
\text { inches. }\end{array}$ & $\begin{array}{l}\text { Loss in } \\
\text { inches. }\end{array}$ \\
\hline 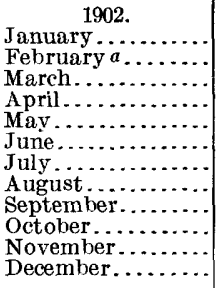 & $\begin{array}{r}12,950 \\
19,200 \\
20,460 \\
12,800 \\
575 \\
1,845 \\
1,680 \\
575 \\
3,700 \\
8,385 \\
4,000 \\
11,960\end{array}$ & $\begin{array}{r}437 \\
960 \\
785 \\
480 \\
226 \\
147 \\
124 \\
69 \\
55 \\
172 \\
198 \\
730\end{array}$ & $\begin{array}{r}1,924 \\
3,902 \\
4,677 \\
2,261 \\
339 \\
323 \\
335 \\
143 \\
232 \\
1,157 \\
552 \\
3,981\end{array}$ & $\begin{array}{l}2.92 \\
5.91 \\
7.09 \\
3.43 \\
.514 \\
.489 \\
.508 \\
.217 \\
.352 \\
1.75 \\
.836 \\
6.03\end{array}$ & $\begin{array}{l}3.37 \\
6.15 \\
8.17 \\
3.83 \\
.593 \\
.546 \\
.586 \\
.250 \\
.393 \\
2.02 \\
.933 \\
6.95\end{array}$ & $\begin{array}{r}93 \\
127 \\
173 \\
134 \\
55 \\
12 \\
16 \\
15 \\
9 \\
28 \\
28 \\
110\end{array}$ & $\begin{array}{l}3.61 \\
4.85 \\
4.72 \\
2.85 \\
1.08 \\
4.44 \\
3.74 \\
1.67 \\
4.52 \\
7.20 \\
3.28 \\
6.33\end{array}$ & $\begin{array}{r}0.24 \\
-1.30 \\
-3.45 \\
-\quad .98 \\
.49 \\
3.89 \\
3.15 \\
1.42 \\
4.13 \\
5.18 \\
2.35 \\
-.62\end{array}$ \\
\hline The year ... & 20,460 & 55 & 1,652 & 2.50 & 33.79 & 70 & 48.29 & 14.50 \\
\hline 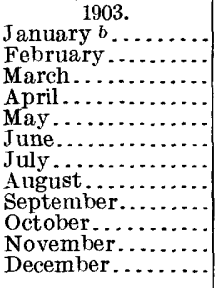 & $\begin{array}{r}10,590 \\
16,260 \\
16,470 \\
16,680 \\
9,540 \\
16,260 \\
13,740 \\
6,705 \\
5,700 \\
1,930 \\
437 \\
6,652 \\
\end{array}$ & $\begin{array}{r}730 \\
1,155 \\
900 \\
840 \\
358 \\
437 \\
525 \\
288 \\
256 \\
226 \\
198 \\
172 \\
\end{array}$ & $\begin{array}{r}3,319 \\
3,124 \\
3,400 \\
3,532 \\
898 \\
1,806 \\
2,342 \\
1,063 \\
795 \\
477 \\
239 \\
540\end{array}$ & $\begin{array}{l}5.03 \\
4.73 \\
5.15 \\
5.35 \\
1.36 \\
2.74 \\
3.55 \\
1.61 \\
1.20 \\
.723 \\
.362 \\
.818\end{array}$ & $\begin{array}{l}5.80 \\
4.92 \\
5.94 \\
5.97 \\
1.57 \\
3.06 \\
4.09 \\
1.86 \\
1.34 \\
.834 \\
.404 \\
.943\end{array}$ & $\begin{array}{r}123 \\
85 \\
105 \\
105 \\
42 \\
35 \\
76 \\
28 \\
47 \\
26 \\
40 \\
42 \\
\end{array}$ & $\begin{array}{l}4.72 \\
5.78 \\
5.66 \\
5.69 \\
3.78 \\
8.68 \\
5.36 \\
6.74 \\
2.84 \\
3.16 \\
.99 \\
2.26\end{array}$ & $\begin{array}{r}-1.08 \\
.86 \\
-.28 \\
-.28 \\
2.21 \\
5.62 \\
1.27 \\
4.88 \\
1.50 \\
2.33 \\
.59 \\
1.32 \\
\end{array}$ \\
\hline The year ... & 16,680 & 172 & 1,795 & 2.72 & 36.72 & 66 & 55.66 & 18.94 \\
\hline 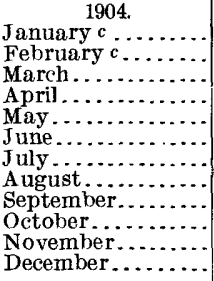 & $\begin{array}{r}14,800 \\
8,758 \\
11,960 \\
2,400 \\
789 \\
4,300 \\
2,765 \\
7,130 \\
734 \\
969 \\
228 \\
3,905\end{array}$ & $\begin{array}{r}260 \\
294 \\
538 \\
294 \\
198 \\
144 \\
120 \\
80 \\
80 \\
80 \\
120 \\
120\end{array}$ & $\begin{array}{r}1,069 \\
1,502 \\
1,812 \\
729 \\
375 \\
817 \\
586 \\
436 \\
251 \\
209 \\
138 \\
549\end{array}$ & $\begin{array}{l}1.61 \\
2.26 \\
2.72 \\
1.10 \\
.561 \\
1.23 \\
.881 \\
.656 \\
.377 \\
.314 \\
.208 \\
826\end{array}$ & $\begin{array}{l}1.86 \\
2.44 \\
3.14 \\
1.23 \\
.647 \\
1.37 \\
1.02 \\
.756 \\
.421 \\
.362 \\
.232 \\
.952\end{array}$ & $\begin{array}{r}54 \\
122 \\
97 \\
56 \\
22 \\
29 \\
17 \\
18 \\
17 \\
17 \\
17 \\
36\end{array}$ & $\begin{array}{l}3.46 \\
2.00 \\
3.23 \\
2.21 \\
2.98 \\
4.80 \\
5.88 \\
4.32 \\
2.49 \\
2.12 \\
1.37 \\
2.63\end{array}$ & $\begin{array}{r}1.60 \\
-. .44 \\
.09 \\
.98 \\
2.33 \\
3.43 \\
4.86 \\
3.56 \\
2.07 \\
1.76 \\
1.14 \\
1.68\end{array}$ \\
\hline The year .... & 14,800 & 80 & 706 & 1.06 & 14.43 & 38 & 37.49 & 23.06 \\
\hline 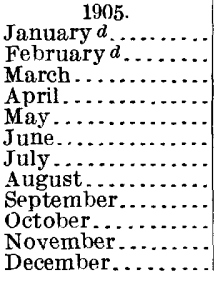 & $\begin{array}{r}12,170 \\
734 \\
9,440 \\
2,220 \\
450 \\
2,860 \\
6,605 \\
13,640 \\
2,130 \\
2,045 \\
3,050 \\
13,480\end{array}$ & $\begin{array}{l}538 \\
368 \\
538 \\
368 \\
170 \\
144 \\
260 \\
228 \\
170 \\
144 \\
198 \\
468\end{array}$ & $\begin{array}{r}1,503 \\
409 \\
3,056 \\
790 \\
279 \\
748 \\
1,296 \\
1,427 \\
546 \\
583 \\
390 \\
2,124\end{array}$ & $\begin{array}{l}2.26 \\
.615 \\
4.60 \\
1.19 \\
.420 \\
1.12 \\
1.95 \\
2.15 \\
.821 \\
.877 \\
.586 \\
3.19\end{array}$ & $\begin{array}{l}2.61 \\
. .640 \\
5.30 \\
1.33 \\
.484 \\
1.25 \\
2.25 \\
2.48 \\
.916 \\
1.01 \\
.654 \\
.368\end{array}$ & $\begin{array}{r}63 \\
35 \\
129 \\
49 \\
26 \\
21 \\
27 \\
46 \\
29 \\
23 \\
32 \\
77\end{array}$ & $\begin{array}{l}4.15 \\
1.83 \\
4.11 \\
2.71 \\
1.84 \\
5.88 \\
8.32 \\
5.40 \\
3.17 \\
4.39 \\
2.00 \\
4.75\end{array}$ & $\begin{array}{r}1.54 \\
1.19 \\
-1.19 \\
1.38 \\
1.36 \\
4.63 \\
6.07 \\
2.92 \\
2.25 \\
3.38 \\
1.35 \\
1.07\end{array}$ \\
\hline The year. .... & 13,640 & 144 & 1,096 & 1.65 & 22.60 & 47 & 48.55 & 25.95 \\
\hline
\end{tabular}

$a$ River frozen at the gage February 4-19, 1902; no correction made in the estimates. $b$ River frozen at the gage January 12-20, 1903; no correction made in the estimates.

$c$ River frozen January 4-22 and Fehruary 15-22, 1904; no correction made in the estimates.

$d$ River frozen January 27 to February 28, 1905; no correction made in the estimates.

IRR $192-07-12$ 
Estimated monthly discharge of Monocacy River near Frederick, Ma.--Continued.

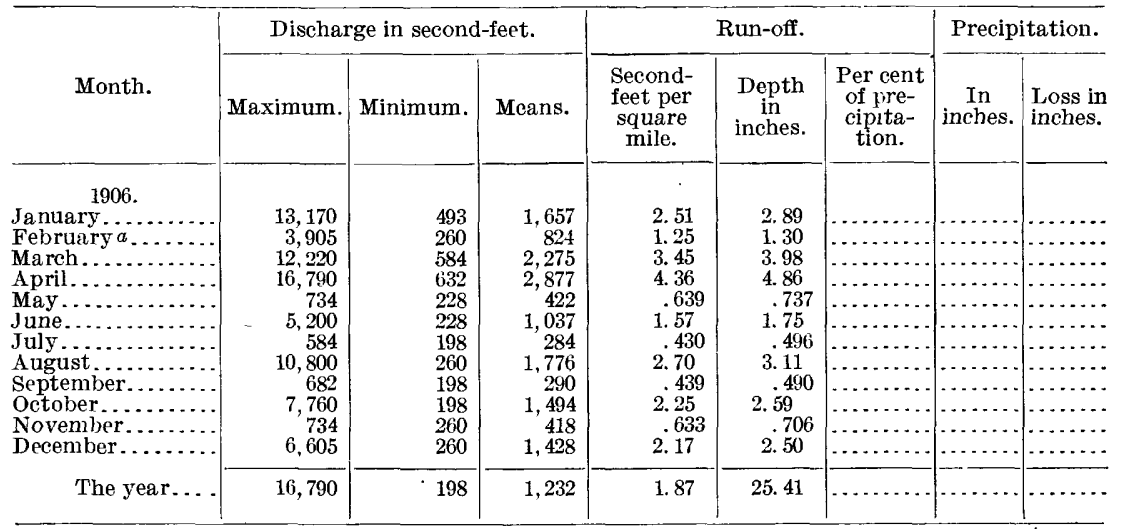

$a$ River frozen at the gage February $6-9,1906$; no correction made in the estimates.

The following table gives the horsepower, 80 per cent efficiency per foot of fall, that may be developed at different rates of discharge, and shows the number of days on which the flow and the corresponding horsepower were respectively less than the amounts given in the columns for "discharge" and "horsepower."

Discharge and horsepower table for Monacacy River near Frederick, Md., from 1896 to 1906.

\begin{tabular}{|c|c|c|c|c|c|c|c|c|c|c|c|c|}
\hline \multirow{2}{*}{$\begin{array}{l}\text { Dis- } \\
\text { charge } \\
\text { in sec- } \\
\text { ond-feet. }\end{array}$} & \multirow{2}{*}{$\begin{array}{l}\text { Horse- } \\
\text { power, } \\
80 \text { percent } \\
\text { efficiency, } \\
\text { per foot } \\
\text { fall. }\end{array}$} & \multicolumn{11}{|c|}{ Days of deficient flow. } \\
\hline & & 1896. $a$ & 1897. & 1898. & 1899. & 1900. & 1901. & 1902. & 1903. & 1904. & 1905. & 1906. \\
\hline 55 & 5 & & & & & 4 & & & & & & \\
\hline 66 & 6 & . & & & & 16 & & 10 & & & & $\cdots$ \\
\hline 88 & 8 & 16 & & & 4 & 63 & & 22 & & 21 & $\ldots$ & - . \\
\hline 110 & 10 & 32 & 6 & 22 & 29 & 89 & & 29 & & 27 & & $\cdots$ \\
\hline 132 & 12 & 74 & 19 & 37 & 56 & 119 & & 38 & & 78 & & \\
\hline 154 & 14 & 98 & 45 & 48 & 75 & 142 & 1 & 48 & & 85 & 2 & $\cdots$ \\
\hline 176 & 16 & 114 & 56 & 58 & 100 & 163 & 25 & 72 & 4 & 123 & 17 & $\ldots$ \\
\hline 198 & 18 & 115 & 63 & 59 & 100 & 165 & 25 & 72 & 4 & 123 & 17 & $\ldots$ \\
\hline 220 & 20 & 128 & 86 & 68 & 118 & 174 & 74 & 94 & 15 & 129 & 36 & 18 \\
\hline 275 & 25 & 136 & 115 & 99 & 149 & 196 & 131 & 115 & 56 & 152 & 78 & 82 \\
\hline 330 & 30 & 142 & 130 & 116 & 163 & 213 & 155 & 147 & 78 & 187 & 106 & 96 \\
\hline 385 & 35 & 142 & 149 & 123 & 169 & 224 & 165 & 152 & 94 & 216 & 151 & 138 \\
\hline 440 & 40 & 145 & 171 & 138 & 187 & 244 & 204 & 170 & 128 & 240 & 169 & 154 \\
\hline 495 & 45 & 147 & 192 & 157 & 206 & 254 & 216 & 176 & 130 & 259 & 195 & 175 \\
\hline 550 & -50 & 147 & 199 & 169 & 213 & 265 & 229 & 185 & 138 & 269 & 205 & 184 \\
\hline 660 & -60 & 148 & 222 & 206 & 231 & 275 & 243 & 207 & 154 & 285 & 220 & 202 \\
\hline 770 & 70 & 149 & 243 & 234 & 243 & 299 & 264 & 217 & 173 & 297 & 237 & 226 \\
\hline
\end{tabular}

a A ugust 4 to December 31, 1896.

NOTE.-The minimum flow during the period covered by the above table was 49 second-feet, giving. 4.5 horsepower per foot of fall for four consecutive days during August, 1900 . 
POTOMAC RIVER AT GREAT FALIS, MD., AND AT CHAIN BRIDGE, D. C.

Estimates of the flow of Potomac River at Chain Bridge and at Great Falls are not considered sufficiently accurate for republication owing to poor conditions at the Chain Bridge section and the effect on the flow of the river of a daily range of tide of about 3 feet. Estimates at Great Falls, as previously published, were based on the discharge at Chain Bridge, and hence are subject to the same errors. The flood discharges as determined at these two stations are probably accurate enough for all practical purposes, but for medium and low stages the estimates are considered too high and likely to be very misleading if used.

ROCK CREEK AT LYON'S MILL AND ZOOLOGICAL PARK, D. C.

Rock Creek rises south of Laytonsville, in the central part of Montgomery County, Md., and flows southward into Potomac River at Washington, D. C. The total drainage area at the mouth is 86 square miles.

A study of the discharge of Rock Creek was begun in July, 1892, at the request of the Commissioners of the District of Columbia. August 18, 1892, a self-registering gage was placed at Lyon's Mill Road Bridge, at the east corner of Oak Hill Cemetery, Georgetown, by C. C. Babb. The gage-height record was continued until November 30,1894 .

January 18, 1897, a new station was established by E. G. Paul at the Park Bridge, near the eastern entrance of the National Zoological Park, Washington, D. C. The staff gage was above and within the influence of a dam. Measurements were made at various cross sections. The bridge was rebuilt and the gage destroyed in November, 1900.

The estimated discharge of Rock Creek at Lyon's mill, which is published below for the first time, is probably within 5 per cent of the true discharge above gage height, -0.3 foot. Below gage height, -0.3 foot, the probable error may vary from 5 to 15 per cent. The effect of ice conditions is not known.

No estimates are possible for Rock Creek at Zoological Park, owing to the insufficient range of stage at which measurements were made.

Discharge measurements of Rock Creek, at Lyon's mill, Washington, D. $C$.

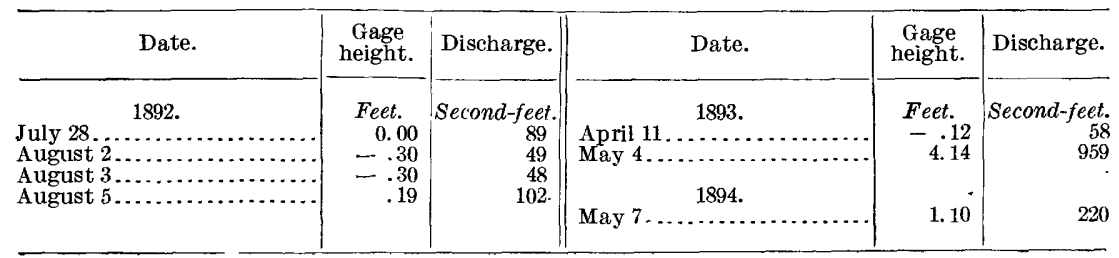


Discharge measurements of Rock Creek referred to Zoological Park Bridge gage, Distruct oj Columbia.

\begin{tabular}{|c|c|c|c|c|c|}
\hline Date. & $\begin{array}{l}\text { Gage. } \\
\text { height. }\end{array}$ & Discharge. & Date. & $\begin{array}{l}\text { Gage } \\
\text { height. }\end{array}$ & Discharge \\
\hline $\begin{array}{c}1897 . \\
\text { August } 18 a_{2} \ldots \ldots \ldots \ldots \ldots \\
\text { November } 21 b \ldots \ldots \ldots \ldots \ldots\end{array}$ & $\begin{array}{l}\text { Feet. } \\
2.90 \\
3.00\end{array}$ & $\mid \begin{array}{r}\text { Second-feet. } \\
26 \\
37\end{array}$ & $\begin{array}{r}1900 . \\
\text { April } 24 d . \ldots .\end{array}$ & $\begin{array}{l}\text { Feet. } \\
\quad 2.75\end{array}$ & Second-feet \\
\hline $\begin{array}{c}1898 . \\
\text { January } 28 c_{\ldots} \ldots \ldots \ldots \ldots \ldots \\
\text { May } 18 c \ldots \ldots \ldots \ldots \ldots \ldots\end{array}$ & $\begin{array}{l}3.23 \\
3.20\end{array}$ & $\begin{array}{l}89 \\
95\end{array}$ & $\begin{array}{r}1902 . \\
\text { February } 27 d \ldots\end{array}$ & & $1,81 \mathrm{I}$ \\
\hline
\end{tabular}

$a$ Measured at Woodley Bridge.

$b$ Measured at Klingle Ford. $c$ Measured at Lyon's mill.

$d$ Measured at Rustic Bridge.

Daily gage height, in feet, of Rock Creek at Lyon's mill, Washington, D. C.

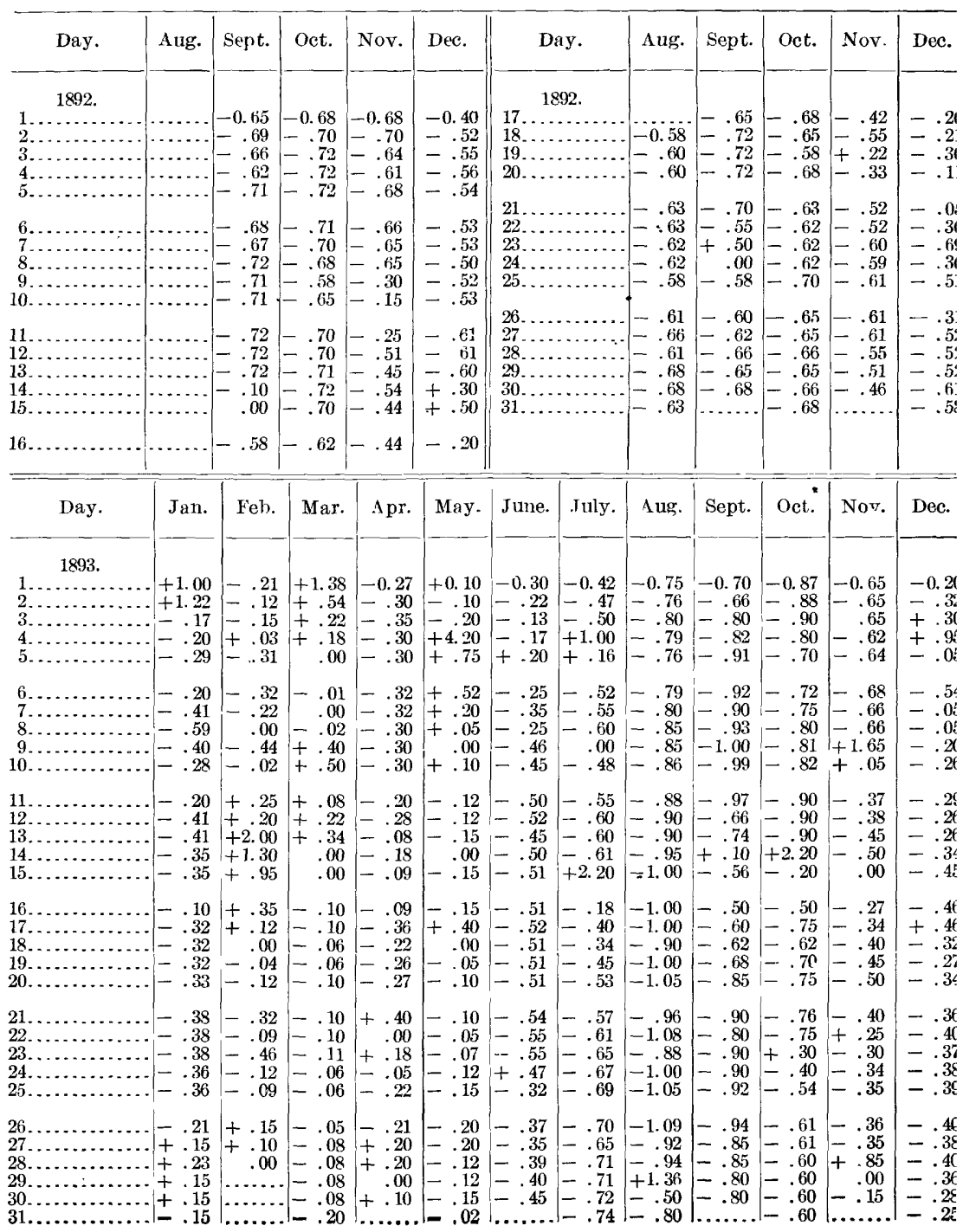


Daily gage height, in feet, of Rock Creek at Lyon's mill, Washington, D. C.-Continued.

\begin{tabular}{|c|c|c|c|c|c|c|c|c|c|c|c|c|}
\hline Day. & Jan. & Feb. & Mar. & Apr. & May. & June. & July. & Aug. & Sept. & Oet. & Nov. & Dec. \\
\hline $\begin{array}{r}189 \\
1 \ldots \ldots \\
2 \ldots \ldots \\
3 \ldots \ldots \\
4 \ldots \ldots \\
5 \ldots \ldots\end{array}$ & \begin{tabular}{l}
-0.22 \\
\hdashline .32 \\
$=.34$ \\
-.31 \\
-.33
\end{tabular} & $\begin{array}{l}-0.14 \\
-.14 \\
-.28 \\
-.30 \\
-.36\end{array}$ & $\begin{array}{l}-0.20 \\
-.12 \\
+1.00 \\
+.62 \\
+.12\end{array}$ & $\begin{array}{l}-0.24 \\
-.24 \\
=.26 \\
=.25 \\
=.30\end{array}$ & $\begin{array}{l}-0.45 \\
-.41 \\
=.39 \\
-.34 \\
-.20\end{array}$ & $\begin{array}{l}-0.40 \\
=.41 \\
=.44 \\
=.50 \\
=.51\end{array}$ & & & $\begin{array}{r}-0.85 \\
-.80 \\
-.80 \\
-.82\end{array}$ & $\begin{array}{l}-0.60 \\
-.60 \\
-.50 \\
-.60 \\
-.40\end{array}$ & $\begin{array}{r}-0.10 \\
-.50 \\
-.10 \\
-.00 \\
-.45\end{array}$ & \\
\hline $\begin{array}{r}6 \ldots \\
7 \ldots \ldots \\
8 \ldots \\
9 \ldots \\
10 \ldots\end{array}$ & $\begin{array}{l}\mid .30 \\
.31 \\
-.33 \\
-.33\end{array}$ & $\begin{array}{l}-.30 \\
=.30 \\
=.31 \\
=.32 \\
-.30\end{array}$ & $\begin{array}{l}+.04 \\
+.05 \\
+.05 \\
-.12 \\
-.21\end{array}$ & $\begin{array}{r}-.36 \\
-.36 \\
-.28 \\
-.28 \\
-.18\end{array}$ & $\begin{array}{l}+.21 \\
+.60 \\
+.03 \\
-.20 \\
-.26\end{array}$ & \begin{tabular}{|l}
-.51 \\
-.52 \\
-.55 \\
-.64 \\
-.64
\end{tabular} & $=.85$ & & $\begin{array}{l}-.85 \\
-.85 \\
-.80 \\
-.80 \\
-.60\end{array}$ & $\begin{array}{l}-.50 \\
-.60 \\
-.70 \\
-.80 \\
-.50\end{array}$ & $\begin{array}{l}-.50 \\
-.55 \\
-.60 \\
-.30 \\
-\quad 45\end{array}$ & \\
\hline $\begin{array}{l}15 \ldots \\
4.4 \ldots \\
5\end{array}$ & $\begin{array}{l}-.33 \\
-.28 \\
-.31 \\
-.24 \\
-.24\end{array}$ & $\begin{array}{l}-.27 \\
-.28 \\
-.32 \\
-.34 \\
-.20\end{array}$ & $\begin{array}{l}-.20 \\
-.20 \\
-.22 \\
-.25 \\
-.24\end{array}$ & $\begin{array}{r}+1.18 \\
+.69 \\
+.65 \\
+.21 \\
+.00\end{array}$ & $\begin{array}{l}-.28 \\
-.28 \\
-.29 \\
-.31 \\
-.31\end{array}$ & $\begin{array}{l}-.66 \\
=.68 \\
=.69 \\
=.71 \\
-.73\end{array}$ & -.92 & & $\begin{array}{r}-.55 \\
-.65 \\
=.70 \\
-.65 \\
-.65\end{array}$ & $\begin{array}{r}-.20 \\
-.60 \\
-.70 \\
-.70 \\
-.70\end{array}$ & $\begin{array}{l}-.50 \\
-.60 \\
-.60 \\
-.65 \\
-.70\end{array}$ & \\
\hline $20 \ldots \ldots$ & $\begin{array}{l}-.24 \\
=.25 \\
-.26 \\
-. .29 \\
-. .30\end{array}$ & $\begin{array}{r}.00 \\
+.12 \\
+.42 \\
+.65 \\
.00\end{array}$ & $\begin{array}{l}-.24 \\
=.22 \\
=.23 \\
=.23 \\
-.23\end{array}$ & $\begin{array}{l}-.07 \\
=.11 \\
-.15 \\
=.18 \\
-.19\end{array}$ & $\begin{array}{l}-.32 \\
-.48 \\
-.32 \\
-.28 \\
+.65\end{array}$ & & & -1.10 & $\begin{array}{l}-.65 \\
-.65 \\
-.70 \\
-.70 \\
-.70\end{array}$ & $\begin{array}{l}-.70 \\
-.70 \\
-.70 \\
-.70 \\
-.75\end{array}$ & $\begin{array}{l}-.70 \\
-.70 \\
-.70 \\
-.65 \\
-.70\end{array}$ & \\
\hline $\begin{array}{l}21 \ldots \ldots \\
22 \ldots \ldots \\
23 \ldots \\
24 \ldots \\
25 \ldots\end{array}$ & $\begin{array}{l}-.26 \\
-.24 \\
-.20 \\
-.21 \\
-.20\end{array}$ & $\begin{array}{l}-.12 \\
-.20 \\
=.20 \\
-.20 \\
-.20\end{array}$ & $\begin{array}{l}-.21 \\
=.20 \\
=.18 \\
=.20 \\
-.20\end{array}$ & $\left\{\begin{array}{l}-.22 \\
-.25 \\
-.30 \\
-.33 \\
-.37\end{array}\right.$ & $\begin{array}{l}+.23 \\
-.10 \\
-.27 \\
-.30 \\
-.33\end{array}$ & & & $\begin{array}{l}-1.10 \\
-1.10 \\
-1.00 \\
-1.10 \\
-1.20\end{array}$ & $\begin{array}{l}-.70 \\
-.70 \\
\end{array}$ & $\begin{array}{l}-.80 \\
-.80 \\
-.75 \\
-.75 \\
-.75\end{array}$ & $=\begin{array}{l}-.70 \\
=.70 \\
=.70 \\
=.70 \\
-.70\end{array}$ & \\
\hline $\begin{array}{l}26 \ldots \\
27 \ldots \\
28 \ldots \\
29 \ldots \\
30\end{array}$ & $\begin{array}{r}-.20 \\
-.20 \\
. .21 \\
1.00 \\
.00\end{array}$ & {$\left[\begin{array}{l}-.20 \\
-.26 \\
-.20\end{array}\right.$} & $\begin{array}{l}-.17 \\
-.20 \\
-.20 \\
-.22 \\
-.21 \\
-.22\end{array}$ & \begin{tabular}{|l}
-.31 \\
-.39 \\
-.34 \\
-.40 \\
-.42 \\
\hdashline. .3 \\
\end{tabular} & $\begin{array}{l}-.30 \\
-.30 \\
-.32 \\
-.36 \\
-.36 \\
-.36\end{array}$ & & & $\begin{array}{l}-1.25 \\
-.90 \\
-1.20 \\
-1.10\end{array}$ & 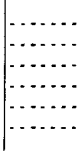 & $\begin{array}{l}-.75 \\
-.75 \\
-.75 \\
-.72 \\
-.70 \\
-.70\end{array}$ & $\begin{array}{l}-.70 \\
=.65 \\
=.70 \\
=.70 \\
-.70\end{array}$ & \\
\hline
\end{tabular}

Daily gage height, in feet, of Rock Creek at Zoological Park, D. C.

\begin{tabular}{|c|c|c|c|c|c|c|c|c|c|c|c|c|}
\hline Das & Jan. & Feb. & Mar. & Apr. & May. & June. & July. & Aug. & Sept. & Oct. & Nov. & Dec. \\
\hline $1 \ldots$ & & $\begin{array}{l}2.9 \\
3.5 \\
4.15 \\
\text { 3. } 45 \\
3.15\end{array}$ & $\begin{array}{l}2.35 \\
2.35 \\
2.35 \\
2.4 \\
2.35\end{array}$ & $\begin{array}{l}2.3 \\
2.25 \\
2.25 \\
2.25 \\
2.5\end{array}$ & $\begin{array}{l}2.3 \\
2.4 \\
2.45 \\
2.55 \\
2.5\end{array}$ & $\begin{array}{l}2.3 \\
2.5 \\
2.65 \\
2.95 \\
2.15\end{array}$ & $\begin{array}{l}3.1 \\
3.1 \\
3.1 \\
3.05 \\
3.05\end{array}$ & $\begin{array}{l}3.05 \\
3.0 \\
3.0 \\
2.95 \\
2.95\end{array}$ & $\begin{array}{l}2.85 \\
2.85 \\
2.85 \\
2.85 \\
2.85\end{array}$ & $\begin{array}{l}2.8 \\
2.85 \\
2.85 \\
2.85 \\
2.85\end{array}$ & $\begin{array}{l}\text { 3.1 } \\
3.5 \\
3.3 \\
3.1 \\
\text { 3. } 05\end{array}$ & $\begin{array}{l}3.05 \\
3.05 \\
3.05 \\
\text { o. } 25 \\
3.9\end{array}$ \\
\hline & & $\begin{array}{l}3.25 \\
4.25 \\
2.7 \\
2.45 \\
2.4\end{array}$ & $\begin{array}{l}2.35 \\
2.4 \\
2.35 \\
2.35 \\
2.35\end{array}$ & $\begin{array}{l}2.65 \\
2.45 \\
2.4 \\
3.15 \\
2.9\end{array}$ & $\begin{array}{l}2.35 \\
2.3 \\
2.2 \\
2.2 \\
2.15\end{array}$ & & & & $\begin{array}{l}2.85 \\
2.8 \\
2.8 \\
2.8 \\
2.8\end{array}$ & & & $\begin{array}{l}3.35 \\
3.2 \\
3.15 \\
3.1 \\
3.1\end{array}$ \\
\hline & & $\begin{array}{l}2.3 \\
2.45 \\
2.6 \\
2.6 \\
2.7\end{array}$ & $\begin{array}{l}2.3 \\
2.3 \\
2.35 \\
2.45 \\
2.6\end{array}$ & & $\begin{array}{l}2.2 \\
2.5 \\
3.7 \\
2.65 \\
2.95\end{array}$ & & & & & & & $\begin{array}{l}3.1 \\
3.1 \\
3.1 \\
3.25 \\
4.3\end{array}$ \\
\hline & $\begin{array}{l}3.0 \\
2.95 \\
2.9\end{array}$ & $\begin{array}{l}2.45 \\
2.35 \\
2.3 \\
2.3 \\
2.25\end{array}$ & $\begin{array}{l}2.4 \\
2.35 \\
2.95 \\
2.85 \\
3.05\end{array}$ & $\begin{array}{l}2.45 \\
2.45 \\
2.4 \\
2.35 \\
2.3\end{array}$ & $\begin{array}{l}2.6 \\
2.5 \\
2.4 \\
2.4 \\
2.35\end{array}$ & & $\begin{array}{l}3.0 \\
3.1 \\
3.1 \\
3.45 \\
3.25\end{array}$ & $\begin{array}{l}2.95 \\
3.0 \\
2.9 \\
2.9 \\
2.9\end{array}$ & $\begin{array}{l}2.8 \\
2.8 \\
2.9 \\
2.85 \\
2.8\end{array}$ & $\begin{array}{l}2.85 \\
2.85 \\
2.9 \\
2.9 \\
3.0\end{array}$ & & $\begin{array}{l}3.35 \\
3.2 \\
3.15 \\
3.1 \\
3.1\end{array}$ \\
\hline & $\begin{array}{l}3.15 \\
3.3 \\
3.35 \\
3.05 \\
2.85\end{array}$ & $\begin{array}{l}2.4 \\
3.75 \\
4.1 \\
2.9 \\
2.6\end{array}$ & & $\begin{array}{r}2.3 \\
2.3 \\
2.3 \\
2.3 \\
2.3\end{array}$ & $\begin{array}{l}2.3 \\
2.25 \\
2.6 \\
3.45\end{array}$ & & $\begin{array}{l}\text { 4. } 0 \\
3.4 \\
3.1 \\
3.05\end{array}$ & $\begin{array}{l}2.9 \\
2.9 \\
2.95 \\
2.9\end{array}$ & $\begin{array}{l}2.8 \\
2.8 \\
2.9 \\
3.2 \\
2.9\end{array}$ & & & $\begin{array}{l}3.15 \\
3.2 \\
3.2 \\
3.3 \\
3.05\end{array}$ \\
\hline & $\begin{array}{l}3.0 \\
2.9 \\
2.9 \\
2.95 \\
2.9 \\
2.9\end{array}$ & $\begin{array}{l}2.5 \\
2.45 \\
2.45\end{array}$ & $\begin{array}{l}2.4 \\
2.4 \\
2.35 \\
2.3 \\
2.3 \\
2.3\end{array}$ & $\begin{array}{l}2.25 \\
2.25 \\
2.2 \\
2.25 \\
2.25\end{array}$ & $\begin{array}{l}2.55 \\
2.4 \\
2.35 \\
2.3\end{array}$ & $\begin{array}{l}3.15 \\
3.1 \\
3.1 \\
3.1 \\
3.1\end{array}$ & $\begin{array}{l}3.0 \\
3.45 \\
4.3 \\
3.1 \\
3.1 \\
3.1\end{array}$ & $\begin{array}{l}2.9 \\
2.9 \\
2.85 \\
2.85 \\
2.85 \\
2.85\end{array}$ & $\begin{array}{l}2.8 \\
2.8 \\
2.8 \\
2.8 \\
2.8\end{array}$ & $\begin{array}{l}3.1 \\
3.0 \\
2.95 \\
2.9 \\
2.9 \\
2.9\end{array}$ & $\begin{array}{l}3 . \\
3 . \\
3 . \\
3 \\
3 .\end{array}$ & $\begin{array}{l}3.1 \\
3.1 \\
3.05 \\
3.0 \\
3.1 \\
3.1\end{array}$ \\
\hline
\end{tabular}


Daily gage height, in feet, of Rock Creek at Zoological Park, D. C.-Continued.

\begin{tabular}{|c|c|c|c|c|c|c|c|c|c|c|c|c|}
\hline D & Jan. & Feb. & Mar. & Apr. & May. & June. & July. & Aug. & Sep & Oct. & Nov. & Dec. \\
\hline $\begin{array}{l}1 \ldots \\
2 \ldots \\
3 \ldots \\
4 \ldots \\
5 \ldots \\
5 \ldots\end{array}$ & $\begin{array}{l}3.1 \\
2.95 \\
3.1 \\
3.05 \\
3.05\end{array}$ & $\begin{array}{l}3.15 \\
3.15 \\
3.1 \\
3.1 \\
3.05\end{array}$ & $\begin{array}{l}3.1 \\
3.1 \\
3.1 \\
3.15 \\
3.15\end{array}$ & $\begin{array}{l}3.3 \\
3.2 \\
3.2 \\
3.1 \\
3.2\end{array}$ & $\begin{array}{l}3.5 \\
3.5 \\
3.5 \\
3.0 \\
3.5\end{array}$ & $\begin{array}{l}2.95 \\
2.95 \\
2.95 \\
2.9 \\
2.9\end{array}$ & $\begin{array}{l}2.85 \\
2.8 \\
2.8 \\
2.8 \\
2.85\end{array}$ & $\begin{array}{l}2.8 \\
2.8 \\
2.8 \\
2.8 \\
3.9\end{array}$ & $\begin{array}{l}2.45 \\
2.4 \\
2.4 \\
2.4 \\
2.4\end{array}$ & $\begin{array}{l}2.3 \\
2.35 \\
2.35 \\
2.4 \\
2.4\end{array}$ & $\begin{array}{l}2.8 \\
2.65 \\
2.6 \\
2.6 \\
2.6\end{array}$ & $\begin{array}{l}2.95 \\
2.8 \\
2.8 \\
3.15 \\
4.45\end{array}$ \\
\hline $\begin{array}{r}7 . \\
8 . \\
9 . \\
10 .\end{array}$ & $\begin{array}{l}3.0 \\
3.05 \\
3.05 \\
3.05 \\
3.9\end{array}$ & $\begin{array}{l}3.05 \\
\text { 3. } 05 \\
3.05 \\
3.1 \\
3.1\end{array}$ & $\begin{array}{l}3.1 \\
3.1 \\
3.1 \\
3.05 \\
3.05\end{array}$ & $\begin{array}{l}3.15 \\
3.1 \\
3.1 \\
3.1 \\
3.1\end{array}$ & $\begin{array}{l}\text { 3.2 } \\
\text { 3. } 95 \\
\text { 3. } 4 \\
\text { 3. } 3 \\
\text { 3. } 0\end{array}$ & $\begin{array}{l}2.9 \\
2.9 \\
2.9 \\
2.9 \\
2.9\end{array}$ & $\begin{array}{l}2.85 \\
2.8 \\
2.8 \\
2.8 \\
2.8\end{array}$ & $\begin{array}{l}2.85 \\
2.8 \\
2.8 \\
2.9 \\
3.45\end{array}$ & $\begin{array}{l}2.4 \\
2.4 \\
2.35 \\
2.35 \\
2.35\end{array}$ & $\begin{array}{l}2.4 \\
2.4 \\
2.35 \\
2.35 \\
2.35\end{array}$ & $\begin{array}{l}2.65 \\
2.65 \\
2.6 \\
2.6 \\
2.65\end{array}$ & $\begin{array}{l}3.15 \\
3.0 \\
2.9 \\
2.85 \\
2.85\end{array}$ \\
\hline & $\begin{array}{l}3.35 \\
3.2 \\
3.3 \\
3.2 \\
3.4\end{array}$ & $\begin{array}{l}\text { 3. } 1 \\
\text { 3.1 } \\
\text { 3. } 1 \\
\text { 3. } 05 \\
\text { 3. } 05\end{array}$ & $\begin{array}{l}3.05 \\
3.05 \\
3.05 \\
3.05 \\
3.05\end{array}$ & $\begin{array}{l}3.05 \\
3.15 \\
3.15 \\
3.15 \\
3.15\end{array}$ & $\begin{array}{l}3.0 \\
\text { 3. } 15 \\
\text { 3. } 15 \\
\text { 3. } 15 \\
3.1\end{array}$ & $\begin{array}{l}2.9 \\
2.9 \\
2.9 \\
3.2 \\
2.9\end{array}$ & $\begin{array}{l}2.8 \\
2.75 \\
2.75 \\
2.75 \\
2.8\end{array}$ & $\begin{array}{l}3.7 \\
4.0 \\
\text { 4. } 15 \\
3.95 \\
2.75\end{array}$ & $\begin{array}{l}2.35 \\
2.3 \\
2.3 \\
2.3 \\
2.3\end{array}$ & $\begin{array}{l}2.35 \\
2.4 \\
2.4 \\
2.45 \\
2.4\end{array}$ & $\begin{array}{l}3.4 \\
3.85 \\
2.7 \\
2.65 \\
2.65\end{array}$ & $\begin{array}{l}2.75 \\
2.8 \\
2.85 \\
2.8 \\
2.8\end{array}$ \\
\hline & $\begin{array}{l}4.1 \\
3.3 \\
3.25 \\
3.15 \\
3.2\end{array}$ & $\begin{array}{l}3.05 \\
3.0 \\
3.05 \\
3.25 \\
3.5\end{array}$ & $\begin{array}{l}3.05 \\
3.25 \\
3.2 \\
3.1 \\
3.1\end{array}$ & $\begin{array}{l}3.1 \\
3.1 \\
3.1 \\
3.6 \\
3.2\end{array}$ & $\begin{array}{l}3.1 \\
3.9 \\
3.1 \\
3.1 \\
3.1\end{array}$ & $\begin{array}{l}2.85 \\
2.85 \\
2.85 \\
2.9 \\
2.85\end{array}$ & $\begin{array}{l}2.8 \\
2.75 \\
2.75 \\
2.95 \\
2.9\end{array}$ & $\begin{array}{l}2.8 \\
2.55 \\
\text { 2. } 55 \\
3.15 \\
2.75\end{array}$ & $\begin{array}{l}2.3 \\
2.3 \\
2.3 \\
2.3 \\
2.3 \\
2.3\end{array}$ & $\begin{array}{l}2.45 \\
2.45 \\
2.4 \\
2.9 \\
2.75\end{array}$ & $\begin{array}{l}2.65 \\
2.8 \\
3.25 \\
4.05 \\
3.1\end{array}$ & $\begin{array}{l}2.8 \\
2.75 \\
2.75 \\
2.8 \\
3.35\end{array}$ \\
\hline & $\begin{array}{l}3.35 \\
3.2 \\
3.25 \\
3.3 \\
3.15\end{array}$ & $\begin{array}{l}3.75 \\
\text { 3.4 } \\
3.25 \\
3.2 \\
3.15\end{array}$ & $\begin{array}{l}3.05 \\
3.05 \\
3.05 \\
3.1 \\
3.5\end{array}$ & $\begin{array}{l}3.3 \\
3.3 \\
3.3 \\
3.1 \\
3.15\end{array}$ & 3. 3 & $\begin{array}{l}2.85 \\
2.85 \\
2.8 \\
2.8 \\
2.8\end{array}$ & $\begin{array}{l}2.9 \\
2.8 \\
2.75 \\
2.75 \\
2.75\end{array}$ & $\begin{array}{l}2.75 \\
2.7 \\
2.6 \\
2.6 \\
2.55\end{array}$ & $\begin{array}{l}2.25 \\
2.3 \\
2.45 \\
2.6 \\
2.35\end{array}$ & $\begin{array}{l}2.6 \\
2.75 \\
2.9 \\
2.7 \\
2.65\end{array}$ & $\begin{array}{l}2.85 \\
2.8 \\
2.8 \\
2.8 \\
2.75\end{array}$ & $\begin{array}{l}3.35 \\
2.9 \\
3.6 \\
3.05 \\
2.9\end{array}$ \\
\hline $\begin{array}{l}30 \ldots \ldots \ldots \\
31 \ldots \ldots \ldots\end{array}$ & $\begin{array}{l}3.65 \\
3.4 \\
3.25 \\
3.15 \\
3.15 \\
3.15\end{array}$ & $\begin{array}{l}3.1 \\
3.1 \\
3.1\end{array}$ & $\begin{array}{l}3.2 \\
3.1 \\
3.1 \\
3.15 \\
3.95 \\
3.45\end{array}$ & $\begin{array}{l}3.1 \\
3.65 \\
3.1 \\
3.1 \\
3.1\end{array}$ & $\begin{array}{l}3.5 \\
3.5 \\
3.0 \\
3.0 \\
2.95 \\
2.95\end{array}$ & $\begin{array}{l}2.8 \\
2.8 \\
3.1 \\
2.95 \\
2.9\end{array}$ & $\begin{array}{l}2.75 \\
3.1 \\
3.1 \\
3.0 \\
2.8 \\
2.8\end{array}$ & $\begin{array}{l}2.55 \\
2.5 \\
2.5 \\
2.5 \\
2.5 \\
2.45\end{array}$ & $\begin{array}{l}2.35 \\
2.3 \\
2.3 \\
2.3 \\
2.3\end{array}$ & $\begin{array}{l}2.55 \\
2.6 \\
2.6 \\
2.6 \\
2.85 \\
2.95\end{array}$ & $\begin{array}{l}2.8 \\
2.75 \\
2.75 \\
2.8 \\
2.85\end{array}$ & $\begin{array}{l}2.8 \\
2.8 \\
2.8 \\
2.7 \\
2.75 \\
2.85\end{array}$ \\
\hline $\begin{array}{r} \\
1 \ldots \\
2 \ldots \\
3 \ldots \ldots \\
4 \ldots\end{array}$ & $\begin{array}{l}3.05 \\
2.75 \\
2.88 \\
2.9 \\
3.33\end{array}$ & $\begin{array}{l}2.78 \\
2.88 \\
2.88 \\
2.95 \\
\text { 3. } 13\end{array}$ & $\begin{array}{l}3.3 \\
3.23 \\
3.13 \\
.1 \\
4.5\end{array}$ & $\begin{array}{l}3.0 \\
2.93 \\
2.9 \\
2.9 \\
2.9\end{array}$ & $\begin{array}{l}2.75 \\
2.7 \\
2.73 \\
2.7 \\
2.7\end{array}$ & $\begin{array}{l}2.88 \\
2.68 \\
2.63 \\
2.6 \\
2.55\end{array}$ & $\begin{array}{l}2.4 \\
2.4 \\
2.35 \\
2.35 \\
2.6\end{array}$ & $\begin{array}{l}2.35 \\
2.35 \\
3.9 \\
3.2 \\
2.65\end{array}$ & $\begin{array}{l}2.4 \\
2.4 \\
2.4 \\
2.4 \\
2.38\end{array}$ & $\begin{array}{l}2.58 \\
2.5 \\
2.5 \\
2.5 \\
2.5\end{array}$ & $\begin{array}{l}3.65 \\
2.83 \\
2.8 \\
3.1 \\
2.8\end{array}$ & $\begin{array}{l}2.53 \\
2.58 \\
2.53 \\
2.5 \\
2.5\end{array}$ \\
\hline $\begin{array}{c}6 \ldots \\
7 \ldots \\
8 \ldots \\
9 \ldots \\
10 \ldots\end{array}$ & $\begin{array}{l}3.65 \\
4.25 \\
3.23 \\
3.05 \\
3.0\end{array}$ & $\begin{array}{l}3.0 \\
3.0 \\
2.93 \\
2.63 \\
2.93\end{array}$ & $\begin{array}{l}3.4 \\
\text { 3. } 18 \\
3.1 \\
3.1 \\
3.08\end{array}$ & $\begin{array}{l}2.9 \\
2.95 \\
3.8 \\
3.08 \\
3.0\end{array}$ & $\begin{array}{l}2.7 \\
2.75 \\
2.75 \\
3.23 \\
2.83\end{array}$ & $\begin{array}{l}2.55 \\
2.55 \\
2.55 \\
2.5 \\
3.63\end{array}$ & $\begin{array}{l}2.58 \\
2.7 \\
2.5 \\
2.78 \\
2.48\end{array}$ & $\begin{array}{l}2.9 \\
2.55 \\
2.5 \\
2.48 \\
2.53\end{array}$ & $\begin{array}{l}2.3 \\
2.3 \\
2.3 \\
2.3 \\
2.3 \\
2.3\end{array}$ & $\begin{array}{l}2.75 \\
2.7 \\
2.58 \\
2.6 \\
2.58\end{array}$ & $\begin{array}{l}2.7 \\
2.63 \\
2.6 \\
2.6 \\
2.6\end{array}$ & $\begin{array}{l}2.5 \\
2.5 \\
2.5 \\
2.5 \\
2.5\end{array}$ \\
\hline & $\begin{array}{l}3.0 \\
3.1 \\
2.9 \\
3.0 \\
3.0\end{array}$ & $\begin{array}{l}2.78 \\
2.78 \\
2.78 \\
2.78 \\
2.95\end{array}$ & $\begin{array}{l}3.23 \\
3.18 \\
3.13 \\
3.0 \\
3.1\end{array}$ & $\begin{array}{l}2.9 \\
2.9 \\
2.9 \\
2,9 \\
2.85\end{array}$ & $\begin{array}{l}2.83 \\
2.98 \\
2.78 \\
2.73 \\
2.7\end{array}$ & $\begin{array}{l}\text { 2. } 98 \\
\text { 2. } 73 \\
2.65 \\
\text { 2. } 63 \\
\text { 2. } 55\end{array}$ & $\begin{array}{l}2.43 \\
2.4 \\
2.5 \\
2.7 \\
2.45\end{array}$ & $\begin{array}{l}2.5 \\
2.48 \\
2.45 \\
2.75 \\
2.5\end{array}$ & $\begin{array}{l}2.4 \\
2.43 \\
2.3 \\
2.3 \\
2.3\end{array}$ & $\begin{array}{l}2.55 \\
2.5 \\
2.5 \\
2.5 \\
2.5\end{array}$ & $\begin{array}{l}2.6 \\
2.58 \\
2.55 \\
2.55 \\
2.55\end{array}$ & $\begin{array}{l}3.75 \\
2.63 \\
2.53\end{array}$ \\
\hline & $\begin{array}{l}3.0 \\
3.1 \\
3.08 \\
2.98 \\
2.93\end{array}$ & $\begin{array}{l}2.95 \\
4.0 \\
\text { 4. } 25 \\
\text { 4. } 43 \\
4.05\end{array}$ & $\begin{array}{l}3.3 \\
3.0 \\
3.05 \\
3.9 \\
3.33\end{array}$ & $\begin{array}{l}3.05 \\
3.0 \\
2.9 \\
2.85 \\
2.85\end{array}$ & $\begin{array}{l}2.68 \\
2.73 \\
\text { 3. } 25 \\
2.05 \\
2.78\end{array}$ & $\begin{array}{l}2.5 \\
2.5 \\
2.5 \\
2.5 \\
2.5\end{array}$ & $\begin{array}{l}2.45 \\
2.9 \\
\text { 2. } 58 \\
\text { 2. } 45 \\
2.4\end{array}$ & $\begin{array}{l}2.7 \\
2.5 \\
2.45 \\
2.43 \\
2.4\end{array}$ & $\begin{array}{l}2.25 \\
2.25 \\
2.3 \\
2.43 \\
3.0\end{array}$ & $\begin{array}{l}2.5 \\
2.5 \\
2.5 \\
2.5 \\
2.5\end{array}$ & $\begin{array}{l}2.55 \\
2.55 \\
2.55 \\
2.58 \\
2.55\end{array}$ & $\begin{array}{l}2.5 \\
2.5 \\
2.55 \\
2.6 \\
2.6\end{array}$ \\
\hline & $\begin{array}{l}3.13 \\
2.85 \\
2.85 \\
2.85 \\
3.68\end{array}$ & $\begin{array}{l}3.88 \\
4.18 \\
4.1 \\
3.5 \\
3.2\end{array}$ & $\begin{array}{l}3.1 \\
\text { 3. } 18 \\
3.18 \\
3.03 \\
3.0\end{array}$ & $\begin{array}{l}2.8 \\
2.8 \\
2.8 \\
2.8 \\
2.8\end{array}$ & $\begin{array}{l}2.7 \\
2.7 \\
2.68 \\
2.65 \\
2.6\end{array}$ & $\begin{array}{l}2.48 \\
2.45 \\
2.45 \\
2.45 \\
2.48\end{array}$ & $\begin{array}{l}2.4 \\
2.4 \\
2.4 \\
2.4 \\
2.4\end{array}$ & $\begin{array}{l}2.4 \\
2.73 \\
2.45 \\
2.35 \\
2.3\end{array}$ & $\begin{array}{l}2.63 \\
2.55 \\
2.4 \\
2.35 \\
2.65\end{array}$ & $\begin{array}{l}2.5 \\
2.45 \\
2.45 \\
2.48 \\
2.45\end{array}$ & $\begin{array}{l}2.55 \\
2.55 \\
2.6 \\
2.63 \\
2.6\end{array}$ & $\begin{array}{l}2.6 \\
2.55 \\
2.5 \\
2.93 \\
2.95\end{array}$ \\
\hline $27 .$. & $\begin{array}{l}3.08 \\
3.0 \\
2.88 \\
2.85 \\
2.83 \\
2.85\end{array}$ & $\begin{array}{l}3.15 \\
\text { 3. } \\
\text { 3. } 33\end{array}$ & $\begin{array}{l}3.0 \\
3.0 \\
\text { 3. } 23 \\
3.45 \\
3.15 \\
3.03\end{array}$ & $\begin{array}{l}2.8 \\
2.83 \\
2.78 \\
2.75 \\
2.75\end{array}$ & $\begin{array}{l}2.6 \\
2.6 \\
2.6 \\
2.65 \\
2.6 \\
2.6\end{array}$ & $\begin{array}{l}2.53 \\
2.5 \\
2.48 \\
2.45 \\
2.43\end{array}$ & $\begin{array}{l}2.5 \\
2.5 \\
2.4 \\
2.4 \\
2.4 \\
2.38\end{array}$ & $\begin{array}{l}2.3 \\
2.55 \\
2.75 \\
2.48 \\
2.4 \\
2.4\end{array}$ & $\begin{array}{l}5.25 \\
3.75 \\
2.85 \\
2.6 \\
2.6\end{array}$ & $\begin{array}{l}2.45 \\
2.5 \\
2.5 \\
2.5 \\
2.5 \\
2.63\end{array}$ & $\begin{array}{l}2.6 \\
2.55 \\
2.55 \\
2.55 \\
2.55\end{array}$ & $\begin{array}{l}2.68 \\
2.53 \\
2.6 \\
2.53 \\
2.53 \\
2.5\end{array}$ \\
\hline
\end{tabular}


STREAM FLOW: ROCK CREEK.

Daily gage height, in feet, of Rock Creek at Zoological Park, D. C.-Continued.

\begin{tabular}{|c|c|c|c|c|c|c|c|c|c|c|c|c|}
\hline Day. & Jan. & Feb. & Mar. & Apr. & May. & June. & Tuly. & Aug. & Sept. & Oct. & Nov. & Dec. \\
\hline 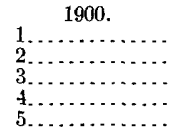 & $\begin{array}{l}\text { 2. } 45 \\
\text { 2. } 43 \\
2.43 \\
2.4 \\
2.38\end{array}$ & $\begin{array}{l}2.43 \\
2.43 \\
2.38 \\
2.4 \\
3.03\end{array}$ & $\begin{array}{l}3.3 \\
3.25 \\
2.88 \\
2.78 \\
2.75\end{array}$ & $\begin{array}{l}2.75 \\
2.7 \\
2.7 \\
2.7 \\
2.68\end{array}$ & $\begin{array}{l}2.6 \\
2.6 \\
2.6 \\
2.6 \\
2.6\end{array}$ & $\begin{array}{l}2.45 \\
2.73 \\
2.6 \\
2.55 \\
2.5\end{array}$ & $\begin{array}{l}2.35 \\
2.35 \\
2.35 \\
2.4 \\
2.38\end{array}$ & $\begin{array}{l}\text { 2. } 35 \\
2.28 \\
2.3 \\
2.28 \\
2.25\end{array}$ & $\begin{array}{l}2.15 \\
2.13 \\
2.1 \\
2.1 \\
2.1\end{array}$ & $\begin{array}{l}2.4 \\
2.3 \\
2.2 \\
2.2 \\
2.2\end{array}$ & $\begin{array}{l}2.1 \\
2.15 \\
2.15 \\
2.28 \\
2.35\end{array}$ & \\
\hline $\begin{array}{r}6 \\
7 \\
8 \\
9 \\
10 \ldots\end{array}$ & $\begin{array}{l}2.4 \\
2.4 \\
2.4 \\
2.4 \\
2.4\end{array}$ & $\begin{array}{l}2.6 \\
2.53 \\
2.83 \\
2.95 \\
2.68\end{array}$ & $\begin{array}{l}2.8 \\
2.85 \\
2.73 \\
2.7 \\
2.68\end{array}$ & $\begin{array}{l}2.65 \\
2.65 \\
2.65 \\
2.63 \\
2.6\end{array}$ & $\begin{array}{l}2.55 \\
2.55 \\
2.55 \\
2.6 \\
2.63\end{array}$ & $\begin{array}{l}2.45 \\
2.45 \\
2.58 \\
2.45 \\
2.45\end{array}$ & $\begin{array}{l}2.38 \\
2.38 \\
2.35 \\
2.33 \\
2.3\end{array}$ & $\begin{array}{l}2.25 \\
2.23 \\
2.2 \\
2.2 \\
2.2\end{array}$ & $\begin{array}{l}2.1 \\
2.1 \\
2.1 \\
2.1 \\
2.1\end{array}$ & $\begin{array}{l}2.2 \\
2.15 \\
2.25 \\
2.25 \\
2.2\end{array}$ & $\begin{array}{l}2.28 \\
2.25 \\
2.4 \\
2.48 \\
2.43\end{array}$ & \\
\hline $\begin{array}{l}11 \ldots \\
12 \ldots \\
13 \ldots \\
14 \ldots \\
15 \ldots\end{array}$ & $\begin{array}{l}2.43 \\
3.6 \\
3.03 \\
2.88 \\
2.58\end{array}$ & $\begin{array}{l}2.6 \\
2.68 \\
3.88 \\
3.0 \\
2.8\end{array}$ & $\begin{array}{l}2.65 \\
2.7 \\
2.7 \\
2.7 \\
2.68\end{array}$ & $\begin{array}{l}2.6 \\
2.8 \\
2.75 \\
2.68 \\
2.65\end{array}$ & $\begin{array}{l}2.58 \\
2.55 \\
2.53 \\
2.5 \\
2.5\end{array}$ & $\begin{array}{l}2.4 \\
2.35 \\
2.45 \\
2.48 \\
2.68\end{array}$ & $\begin{array}{l}2.3 \\
2.3 \\
2.3 \\
2.3 \\
2.3\end{array}$ & $\begin{array}{l}2.2 \\
2.15 \\
2.18 \\
2.15 \\
2.15\end{array}$ & $\begin{array}{l}2.1 \\
2.1 \\
2.1 \\
2.1 \\
2.13\end{array}$ & $\begin{array}{l}2.2 \\
2.2 \\
2.23 \\
2.35 \\
2.35\end{array}$ & & \\
\hline $\begin{array}{l}16 \ldots \\
17 \ldots \\
18 \ldots \\
19 \ldots \\
20 \ldots\end{array}$ & $\begin{array}{l}2.53 \\
2.8 \\
2.8 \\
2.83 \\
3.08\end{array}$ & $\begin{array}{l}2.75 \\
2.7 \\
2.7 \\
2.7 \\
2.78\end{array}$ & $\begin{array}{l}2.7 \\
2.78 \\
2.8 \\
2.8 \\
3.75\end{array}$ & $\begin{array}{l}2.65 \\
2.7 \\
2.7 \\
3.1 \\
2.8\end{array}$ & $\begin{array}{l}2.5 \\
2.5 \\
2.5 \\
2.9 \\
2.73\end{array}$ & $\begin{array}{l}2.65 \\
4.3 \\
3.45 \\
2.78 \\
2.7\end{array}$ & $\begin{array}{l}2.3 \\
2.3 \\
2.3 \\
2.3 \\
2.3 \\
2.4\end{array}$ & $\begin{array}{l}2.15 \\
2.15 \\
2.15 \\
2.1 \\
2.1\end{array}$ & $\begin{array}{l}2.65 \\
2.4 \\
2.2 \\
2.17 \\
2.15\end{array}$ & $\begin{array}{l}2.3 \\
2.25 \\
2.2 \\
2.2 \\
2.2\end{array}$ & & \\
\hline $\begin{array}{l}21 . . \\
22 . \\
23 . \\
24 . \\
25 . \\
\end{array}$ & $\begin{array}{l}3.13 \\
2.88 \\
2.8 \\
2.75 \\
2.68\end{array}$ & $\begin{array}{l}2.73 \\
5.2 \\
3.35 \\
2.9 \\
2.9\end{array}$ & $\begin{array}{l}3.18 \\
2.85 \\
2.8 \\
2.78 \\
2.73\end{array}$ & $\begin{array}{l}2.73 \\
2.8 \\
2.8 \\
2.75 \\
2.7\end{array}$ & $\begin{array}{l}2.55 \\
2.5 \\
2.5 \\
2.45 \\
2.5\end{array}$ & $\begin{array}{l}2.58 \\
2.55 \\
2.5 \\
2.5 \\
2.5\end{array}$ & $\begin{array}{l}2.4 \\
2.48 \\
2.4 \\
2.73 \\
2.48\end{array}$ & $\begin{array}{l}2.18 \\
2.28 \\
2.23 \\
2.25 \\
2.48\end{array}$ & $\begin{array}{l}2.15 \\
2.15 \\
2.15 \\
2.15 \\
2.15\end{array}$ & $\begin{array}{l}2.2 \\
2.2 \\
2.2 \\
2.25 \\
2.23\end{array}$ & & \\
\hline $\begin{array}{l}26 \ldots \\
27 \ldots \\
28 \ldots \\
29 \ldots \\
30 \ldots \\
31 \ldots \\
\end{array}$ & $\begin{array}{l}2.6 \\
2.58 \\
2.68 \\
2.48 \\
2.49 \\
2.55\end{array}$ & $\begin{array}{l}3.08 \\
3.05 \\
2.78\end{array}$ & $\begin{array}{l}2.75 \\
2.8 \\
2.8 \\
2.8 \\
2.8 \\
2.8\end{array}$ & $\begin{array}{l}2.7 \\
2.7 \\
2.65 \\
2.6 \\
2.6 \\
\cdots . . .\end{array}$ & $\begin{array}{l}2.5 \\
2.45 \\
2.45 \\
2.45 \\
2.45 \\
2.4\end{array}$ & $\begin{array}{r}2.48 \\
2.48 \\
2.43 \\
2.35 \\
2.35 \\
\text {..... }\end{array}$ & $\begin{array}{l}2.35 \\
2.38 \\
2.3 \\
2.3 \\
2.3 \\
2.5\end{array}$ & $\begin{array}{l}2.28 \\
2.2 \\
2.2 \\
2.2 \\
2.2 \\
2.15\end{array}$ & $\begin{array}{l}2.15 \\
2.15 \\
2.45 \\
2.4 \\
2.6 \\
\ldots . . .\end{array}$ & $\begin{array}{l}2.18 \\
2.15 \\
2.15 \\
2.1 \\
2.1 \\
2.1\end{array}$ & & \\
\hline
\end{tabular}

'Rating table for Rock Creek at Lyon's mill, Washington, D. C., from August 18, 1892, to November 30,1894 . a

\begin{tabular}{|c|c|c|c|c|c|c|c|}
\hline $\begin{array}{c}\text { Gage } \\
\text { height. }\end{array}$ & Discharge. & $\begin{array}{c}\text { Gage } \\
\text { height. }\end{array}$ & Discharge. & $\begin{array}{c}\text { Gage } \\
\text { height. }\end{array}$ & Discharge. & $\begin{array}{c}\text { Gage } \\
\text { height. }\end{array}$ & Discharge. \\
\hline $\begin{array}{l}\text { Feet. } \\
-1.30 \\
-1.20 \\
-1.10 \\
-1.00 \\
=.90 \\
=.80 \\
=.70 \\
=.60 \\
=.40 \\
=.30 \\
-.20\end{array}$ & \begin{tabular}{|c|} 
Second-feet. \\
5 \\
6 \\
8 \\
10 \\
13 \\
17 \\
22 \\
28 \\
34 \\
41 \\
49 \\
58
\end{tabular} & $\begin{array}{r}\text { Feet. } \\
-0.10 \\
.00 \\
.10 \\
.20 \\
.30 \\
.40 \\
.50 \\
.60 \\
.70 \\
.80 \\
.90 \\
1.00\end{array}$ & \begin{tabular}{|c|} 
Second-feet. \\
68 \\
78 \\
89 \\
100 \\
112 \\
124 \\
137 \\
150 \\
163 \\
177 \\
191 \\
205
\end{tabular} & $\begin{array}{l}\text { Feet. } \\
1.10 \\
1.20 \\
1.30 \\
1.40 \\
1.50 \\
1.60 \\
1.70 \\
1.80 \\
1.90 \\
2.00 \\
2.20 \\
2.40\end{array}$ & \begin{tabular}{|c|} 
Second-feet. \\
220 \\
235 \\
251 \\
268 \\
286 \\
305 \\
325 \\
346 \\
368 \\
390 \\
436 \\
484
\end{tabular} & $\begin{array}{l}\text { Feet. } \\
2.60 \\
2.80 \\
3.00 \\
3.20 \\
3.40 \\
3.60 \\
3.80 \\
4.00 \\
4.20\end{array}$ & \begin{tabular}{|c} 
Second-feet. \\
533 \\
583 \\
635 \\
689 \\
744 \\
800 \\
858 \\
918 \\
980
\end{tabular} \\
\hline
\end{tabular}

$a$ This table is strictly applicable only for open-channel conditions. It is based on seven discharge measurements made during 1892-1894. It is well defined between gage heights -0.3 foot and 4.2 feet. 
Estimated monthly discharge of Rock Creek at Lyon's mill, Washington, D. C.

[Drainage area, 85 square miles.]

\begin{tabular}{|c|c|c|c|c|c|}
\hline \multirow[b]{2}{*}{ Month. } & \multicolumn{3}{|c|}{ Discharge in second-feet. } & \multicolumn{2}{|c|}{ Run-off. } \\
\hline & Maximum. & Minimum. & Mean. & $\begin{array}{c}\text { Second-feet } \\
\text { per square } \\
\text { mile. }\end{array}$ & $\begin{array}{l}\text { Depth in } \\
\text { inches. }\end{array}$ \\
\hline 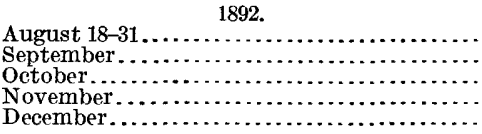 & $\begin{array}{r}29 \\
137 \\
29 \\
102 \\
137\end{array}$ & $\begin{array}{l}23 \\
21 \\
21 \\
22 \\
27\end{array}$ & $\begin{array}{l}26.4 \\
32.8 \\
23.7 \\
35.4 \\
44.5\end{array}$ & $\begin{array}{r}0.311 \\
.386 \\
.279 \\
.416 \\
.524\end{array}$ & $\begin{array}{r}0.162 \\
.431 \\
.322 \\
.464 \\
.604\end{array}$ \\
\hline 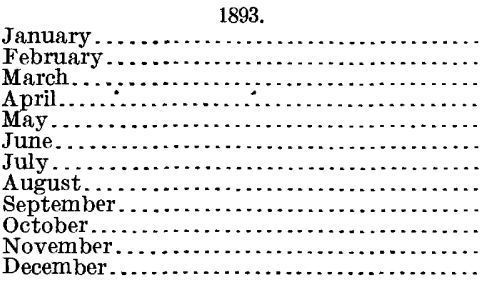 & $\begin{array}{r}238 \\
390 \\
265 \\
124 \\
980 \\
133 \\
436 \\
261 \\
89 \\
436 \\
315 \\
198\end{array}$ & $\begin{array}{r}29 \\
37 \\
58 \\
44 \\
58 \\
31 \\
20 \\
8 \\
10 \\
13 \\
23 \\
32\end{array}$ & $\begin{array}{r}65.7 \\
94.0 \\
88.5 \\
65.9 \\
107 \\
46.0 \\
53.0 \\
22.0 \\
19.8 \\
39.3 \\
56.5 \\
58.6\end{array}$ & $\begin{array}{l}.773 \\
1.11 \\
1.04 \\
.775 \\
1.26 \\
.541 \\
.624 \\
.259 \\
.233 \\
.462 \\
.665 \\
.689\end{array}$ & $\begin{array}{l}.891 \\
1.16 \\
1.20 \\
.865 \\
1.45 \\
.604 \\
.719 \\
.299 \\
.260 \\
.533 \\
.742 \\
.794\end{array}$ \\
\hline The year. & 980 & 8 & 59.7 & .703 & 9.52 \\
\hline 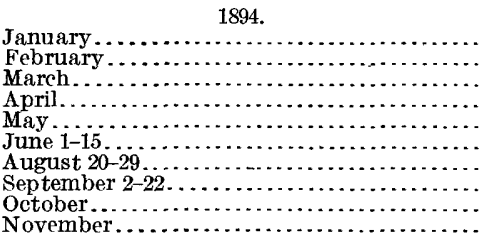 & $\begin{array}{r}205 \\
156 \\
205 \\
232 \\
156 \\
41 \\
13 \\
31 \\
58 \\
78\end{array}$ & $\begin{array}{r}46 \\
44 \\
54 \\
40 \\
35 \\
20 \\
6 \\
15 \\
17 \\
22\end{array}$ & $\begin{array}{r}56.0 \\
63.0 \\
68.6 \\
67.8 \\
59.6 \\
29.8 \\
8.1 \\
21.4 \\
24.9 \\
31.3\end{array}$ & $\begin{array}{l}.659 \\
.741 \\
.807 \\
.798 \\
.701 \\
.351 \\
.095 \\
.252 \\
.293 \\
.368\end{array}$ & $\begin{array}{l}.760 \\
.772 \\
.930 \\
.890 \\
.808 \\
.196 \\
.035 \\
.197 \\
.338 \\
.411\end{array}$ \\
\hline
\end{tabular}

MISCELLANEOUS MEASUREMENTS IN POTOMAC RIVER DRAINAGE BASIN BELOW SHENANDOAH RIVER.

William Rich Hutton, of New York, states that in the summer of 1856 he made a careful examination of the flow of Potomac River a short distance below Great Falls, using loaded poles reaching as near as possible to the bottom and placed at 5-foot intervals across the width of the river. The water was then at the lowest stage known to. persons who had observed the river for many years. The discharge was 1,063 second-feet. Mr. Hutton was of the opinion that the river was as low in 1862 , but no measurements were made in that year.

In 1839 a civil engineer, M. C. Ewing, assistant to Major Turnbull, United States topographic engineer, during the construction of the Alexandria Aqueduct above Georgetown, reported the discharge below Little Falls to be 1,904 second-feet. Thomas L. Patterson, of Cumberland, is reported to have found the discharge of North Branch of the Potomac at that point in the low water of 1838 to be 24 second-feet, and at Patterson Creek, about 12 miles below, 48 second-feet. Figures of discharge of Potomac River are given in the statement regarding the extension of the Chesapeake and Ohio Canal in House Ex. Doc. No. 208, Forty-third Congress, first session; also in House Ex. Doc. No. 137, Forty-fourth Congress, first session. 
The following miscellaneous discharge measurements were made in the Potomac River drainage basin by hydrographers in the United States Geological Survey. Measurements in the fall of 1897 were made in connection with a study of the sources of pollution in the Potomac drainage basin. They were made at a time when the flow throughout the basin was considered generally lower than usual, aithough a few measurements were affected by local rains which preceded them.

Miscollaneous discharge measurements in Potomac River basin below Shenandoah River.

\begin{tabular}{|c|c|c|c|c|c|c|}
\hline Date. & Stream. & Locality. & Width & $\begin{array}{l}\text { Area of } \\
\text { section. }\end{array}$ & \begin{tabular}{|c|} 
Mean \\
veloc- \\
ity.
\end{tabular} & $\begin{array}{l}\text { Dis- } \\
\text { charge. }\end{array}$ \\
\hline $\begin{array}{c}1897 . \\
\text { October } 14 .\end{array}$ & $\begin{array}{l}\text { Carroll Creek (tribu- } \\
\text { tary to Monocacy } \\
\text { River). }\end{array}$ & $\begin{array}{l}\text { Zentz's mill race, Freder- } \\
\text { ick, Md. }\end{array}$ & Feet. & $\begin{array}{c}\text { Square } \\
\text { feet. }\end{array}$ & $\begin{array}{l}\text { Feet per } \\
\text { second. } \\
\quad 1.40\end{array}$ & $\begin{array}{l}\text { Second- } \\
\text { feet. } \\
5.6\end{array}$ \\
\hline $\begin{array}{l}\text { Do.... } \\
\text { Do.... }\end{array}$ & Goose Creek................. & At Frederick, Md...th, near & $\begin{array}{l}11 \\
34\end{array}$ & $\begin{array}{r}6 \\
38\end{array}$ & $\begin{array}{r}1.00 \\
.50\end{array}$ & $\begin{array}{r}6 \\
19\end{array}$ \\
\hline Do... & $\begin{array}{l}\text { Catoctin Creek, in } \\
\text { Maryland. }\end{array}$ & $\begin{array}{l}\text { Edwards Ferry, Md. } \\
\text { A bove B zltimore and ohio } \\
\text { R. R. bridge near Catoctin } \\
\text { station, Md. }\end{array}$ & ${ }^{21}$ & 9 & 2.22 & 20 \\
\hline $\begin{array}{l}\text { Do...... } \\
\text { October } 15 . .\end{array}$ & 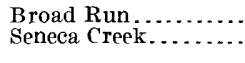 & $\begin{array}{l}\text { Near Edwards Ferry, Md. } \\
\text { In Seneca mill race, Seneca, }\end{array}$ & 11 & 20 & 1.25 & $\begin{array}{r}1 \\
25\end{array}$ \\
\hline & 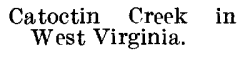 & $\begin{array}{l}\frac{1}{2} \text { mile above mouth and } 1 \\
\text { mile above Point of Rocks, } \\
\text { Md. }\end{array}$ & 16 & 6 & 1. 17 & 7 \\
\hline Do. & $\begin{array}{l}\text { South Treek. } \\
\text { Tuscarora }\end{array}$ & $\begin{array}{l}\text { Near Baltimore and Ohio } \\
\text { R. R. bridge near Licks- }\end{array}$ & 5 & 2.5 & 1.24 & 3.1 \\
\hline Do... & Monocacy River...... & $\begin{array}{l}\text { Between } \\
\text { Ohio } \mathrm{R} \text {. R. bridge and } \\
\text { canal aqueduct near Dick- } \\
\text { erson, Md. }\end{array}$ & 203 & 184 & 1. 19 & $a 219$ \\
\hline
\end{tabular}

$a$ Gage height on Monocacy River at Freclerick, Md., 4.4 feet.

FLOODS NEAR WASHINGTON, D. C.

FLOOD OF FEBRUARY, 1881. $a$

February 12 and 13, 1881, there occurred in the vicinity of Washington the greatest flood, with the exception of that of June 2, 1889, hereinafter described, of which there is any record.

- This flood was caused by the gorging effect of an ice jam, and the discharge did not equal that of the freshet of 1877 or 1889 , but the amount of damage inflicted on shipping, wharf property, and private property was far greater. The low portion of the city along the Mall and extending across Pennsylvania avenue was flooded, and a large amount of damage was caused by the flooding of cellars and first floors. The area of the flooded district was about 254 acres.

The winter had been unusually severe in the long continuance and intensity of its period of cold, during which 23.2 inches of snow had fallen and unusually thick ice had formed over the river. A rise of only a few feet in the river from rains and melted snow caused a 
breaking up of this ice, which gorged and so obstructed the waterway that although the freshet above Georgetown did not attain the height of 1877 by 3 feet, the water rose to a height of 12.25 feet above low water at Long Bridge, or 3.19 feet higher than the freshet of 1877 .

The-íce broke at Georgetown about 1 a. m. on Saturday, February 12,1881 , the stage of the river being then 2 to 3 feet above high tide, but rapidly rising as the ice passed down and added to the gorge below, until the highest water was reached at $7 \mathrm{p}$. m., when it was about 0.8 foot higher than the flood of 1877 .

At Georgetown comparatively little damage was done, owing chiefly to the precaution taken to secure shipping and movable property on the wharves. Along the Washington wharves the ice began to break about $2 \mathrm{a}$. m., the water being 4 to 5 feet above high tide, and moved off rapidly with the current, the channel at times being nearly clear of ice.

The gorge did not at first form at Long Bridge, so far at least as concerned the Washington Channel, as is proved from the fact that between 3 and 4 a. m. on Saturday, February 12, a long boat and several scows were swept away from the Seventeenth street wharf and carried through the Washington draw, and the long boat was found later in the day lodged in the gorge below Arsenal Point, the scows having been carried still farther down the river.

The complete gorge was formed below Arsenal Point, commencing about 7.30 a. m., and the successive additions of floating ice soon caused it to extend to Long Bridge.

Whether a gorge was complete across Georgetown Channel at this point before the lower gorge extended to the bridge was not determined, but subsequently the gorge became complete for the whole length of the bridge. It is probable, however, from the fact that large quantities of ice came across the flats above Long Bridge and passed through the Washington Channel, that a gorge independent of that below was formed across Georgetown Channel at Long Bridge at or about the time of its formation below.

By 9 a. m. the entire waterway of Long Bridge was choked. An occasional movement of short duration served only to jam the ice more closely and raise it higher, until the water passed over the causeway and 2.2 feet above it. The pressure on the bridge became greater than it could withstand, and about $8.30 \mathrm{p} . \mathrm{m}$. three spans of the north end gave way and were swung around on the flats below. This movement, together with a break in the railroad embankment between the river and Fort Runyon (which is one-third of a mile from the Virginia end of Long Bridge, on the railroad to Alexandria) 1,006 feet in length, so far relieved the pressure that no further damage was done to the bridge. 


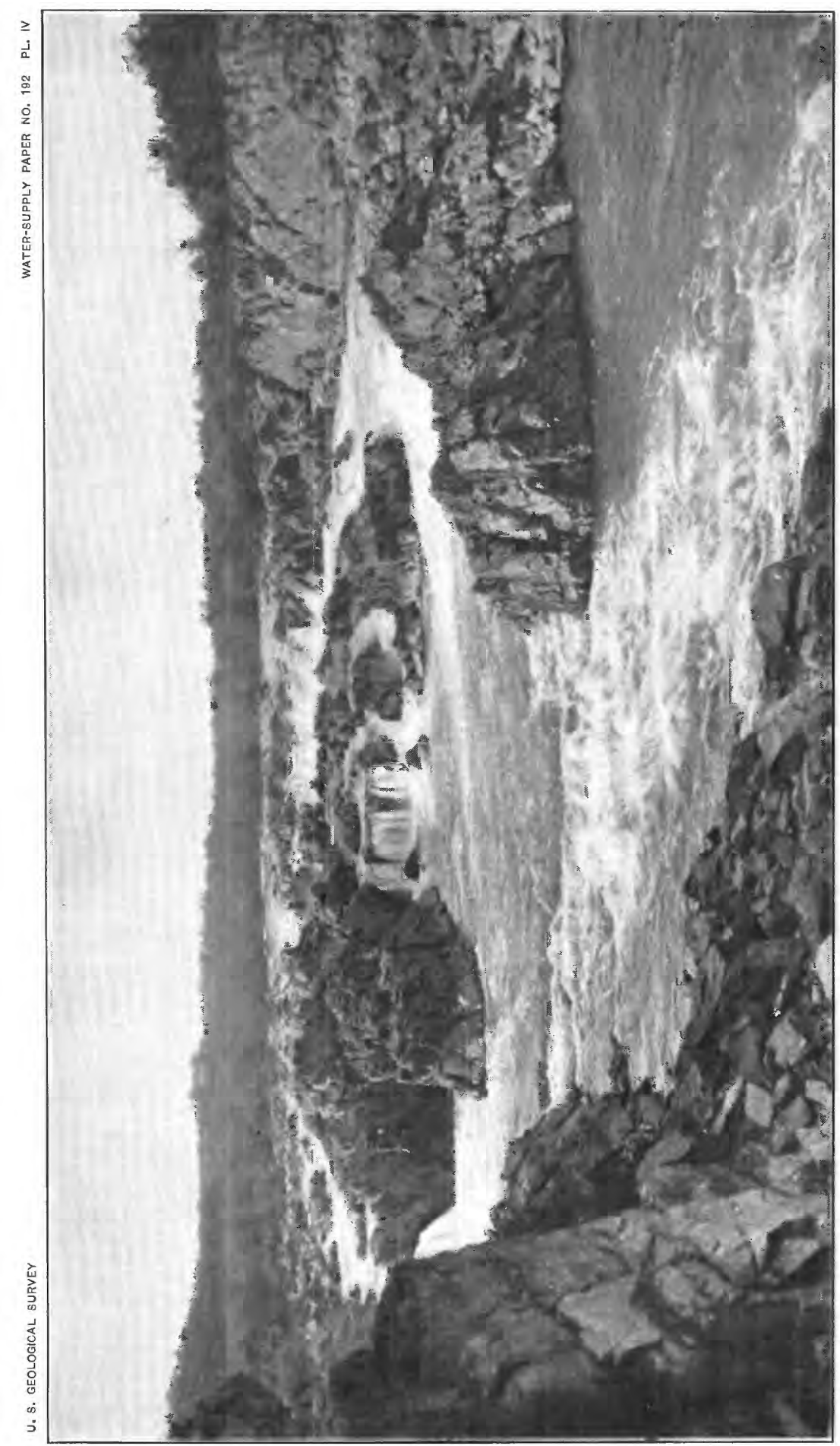

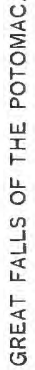



By Sunday morning the water, which had been $2 \frac{1}{2}$ feet higher on the Washington wharves than in the freshet of 1877 , was off the wharves, but large piles of ice remained upon them, and many which escaped serious injury from lateral pressure while the ice was running were crushed when the water, by its subsidence, withdrew its buoyant support.

The following are the heights above low water of the freshets of 1877 and 1881 at the several points named:

Height of Potomac River above low water in floods of 1877 and 1881.

\begin{tabular}{|c|c|c|c|c|c|}
\hline Locality. & 1877. & 1881. & Locality. & 1877. & 1881. \\
\hline $\begin{array}{l}\text { Outlet lock above Georgetown } \\
\text { Aqueduct Bridge } \ldots \\
\text { Rock Creek } \\
\text { Easbys Point } \\
\text { A bove Long Bridge }\end{array}$ & $\begin{array}{r}\text { Feet. } \\
19.72 \\
15.96 \\
13.35 \\
11.99 \\
9.06\end{array}$ & $\begin{array}{l}\text { Feet. } \\
16.73 \\
14.29 \\
13.77 \\
13.54 \\
12.26\end{array}$ & 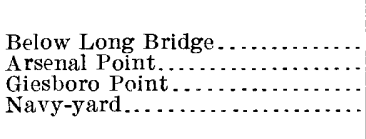 & $\begin{array}{l}\text { Feet. } \\
8.44 \\
7.66 \\
7.73\end{array}$ & $\begin{array}{l}\text { Feet. } \\
11.20 \\
7.01 \\
4.93 \\
5.01\end{array}$ \\
\hline
\end{tabular}

FLOOD OF JUNE, 1889. $a$

June 2, 1889, there occurred the greatest freshet in Potomac River of which there is any authoritative record. The Potomac at Harpers Ferry rose to the height of 34 feet above the low stage. The water was at one time 2.8 feet above the rails of the Baltimore and Ohio Railroad on the bridge and 6.8 feet higher than the freshet of 1877 . At Great Falls the maximum height was 16 feet above the top surface of the coping of the dam, 4 feet higher than in the freshet of 1877 . At Chain Bridge it was 43.3 feet above tide level. The freshet was preceded by strong southeasterly winds, which made the tides at this point unusually high.

Observations were taken while the freshet was rumming to determine the levels above low water at various points. from Chain Bridge down to Arsenal Point. The freshet attained its maximum height at about 10 a. $\mathrm{m}$. on June 2. At that time the height of the water surface above low water at Chain Bridge was 43.3 feet, at Aqueduct Bridge 19.5 feet, at Easby's wharf and at the sewer canal 13.3 feet, at Long Bridge 12.7 feet, and at Arsenal Point 11.1 feet. It was within 3 feet of its maximum height for a period of about twenty-four hours and within 6 feet of it for about thirty hours. After the river began to fall it fell rapidly, but for more than twenty-four hours it was too high for the sewers in the low part of the city to discharge their contents. Hence if a heavy rainfall had occurred during this period the sewers could not have carried off the rain water at all, and a still larger area of the city would have been flooded. The highest point reached by the water at the sewer canal at the foot of Seventeenth street was 13.26 feet. Before this height was reached the 
water backed up in the $B$ street sewer and came into the streets from the sewer outlets. After the water rose above the level of B street it came into the city from the sewers and from the river direct. Great damage was done to private property in the city, as the water on B street was in many places over 4 feet deep, and reached to the store doors on the north side of Pennsylvania avenue, between Ninth and Tenth streets. At B and Fifteenth streets the high water of the 1889 freshet was 3.2 feet above that of 1877 . and 0.7 foot higher than in 1881 .

The discharge area of the river at Aqueduct Bridge when the freshet was at its maximum was about 38,000 square feet. No velocity observations were taken, but on the assumption that C. C. Babb's estimate of 470,000 second-feet discharge at Chain Bridge was correct, the mean velocity at Aqueduct Bridge was about 12.4 feet per second.

Rock Creek, which enters Potomac River 4,000 feet below Aqueduct Bridge, was estimated to have a maximum discharge of 20,000 to 25,000 second-feet during the night of May 31 to June 1, 1889.

\section{SLOPE OF POTOMAC RIVER.}

The fall of the Potomac below Harpers Ferry is about 245 feet. Of this about 90 feet occurs in a short distance at Great Falls (Pl. IV); and if this be subtracted the fall in the remaining distance is found to average about 2.5 feet per mile.

As a water-power stream the principal disadvantage of the Potomac is the great variability of its flow. Good rock foundations for dams can generally be found at small depth, the banks are as a rule favorable, and there are several sites where large falls could be rendered available. Building materials are generally obtainable, and facilities for transportation are excellent. A very insignificant amount of power has been developed.

Slope of the Potomac River.

[PIs. V and VI.]

\begin{tabular}{|c|c|c|c|c|c|}
\hline Locality. & $\begin{array}{l}\text { Distance } \\
\text { from } \\
\text { mouth. }\end{array}$ & $\begin{array}{l}\text { Eleva- } \\
\text { tion } \\
\text { above } \\
\text { tide. }\end{array}$ & $\begin{array}{l}\text { Distance } \\
\text { between } \\
\text { points. }\end{array}$ & $\begin{array}{l}\text { Fall be- } \\
\text { tween } \\
\text { points. }\end{array}$ & $\begin{array}{l}\text { Fall per } \\
\text { mile be- } \\
\text { tween } \\
\text { points. }\end{array}$ \\
\hline Georgetown. & $\begin{array}{l}\text { Miles. } \\
0.0\end{array}$ & Feet. ${ }_{0}$ & Miles. & Feet. & Feet. \\
\hline Harpers Ferry. & 61.5 & 245 & 61.5 & 245 & 4.0 \\
\hline Shepherdstown. & 71.0 & 280 & 9.5 & 35 & 3.7 \\
\hline Dam No. 4..... & 85.0 & 319 & 14.0 & 39 & 2.8 \\
\hline Dam No. 5. & 107.0 & 357 & 22.0 & 38 & 1.7 \\
\hline Cumberland...... & 185.0 & 610 & 78.0 & 253 & 3.2 \\
\hline
\end{tabular}




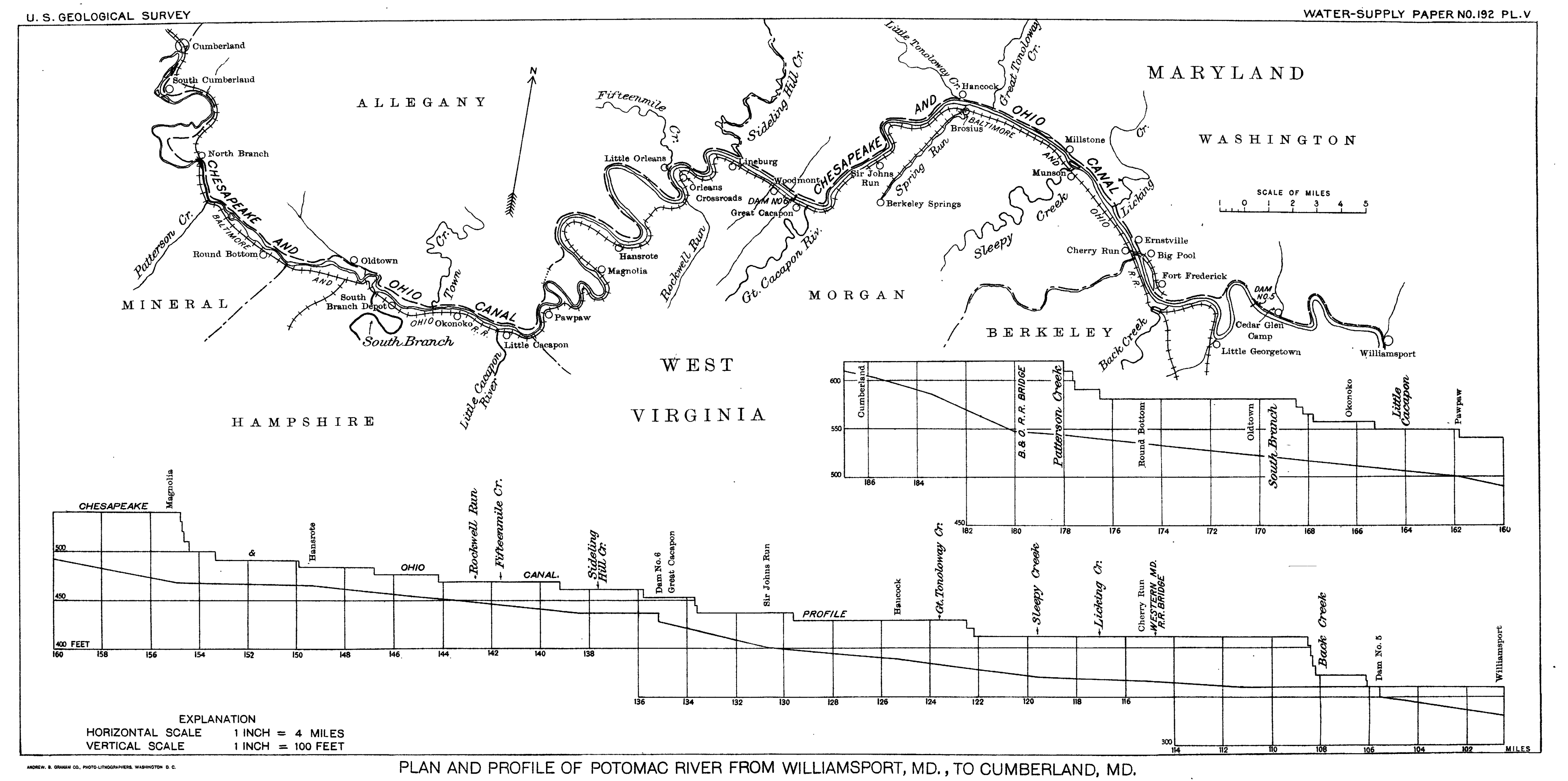




\section{THE CHESAPEAKE AND OHIO CANAL. $a$}

By Horatio N. Parker.

The idea of building a chain of internal improvements to connect the Potomac with the headwaters of the Ohio was conceived by George Washington, whose life had made him thoroughly familiar with the country. In 1748 he was active in the organization of the Ohio Company, which was formed to trade with the Indians west of the Alleghenies, and whose charter made it incumbent on the stockholders to settle a certain number of families in the Ohio Valley. His interest in that company, his expedition to Fort Duquesne in 1753, and, finally, his connection with the Braddock expedition of 1754 convinced him that it was imperative to establish water communication with the West. Accordingly, in the fall of 1774 , he procured the passage of a law by the general assembly of Virginia, empowering such individuals as were disposed to embark in the enterprise to open the Potomac so as to render it navigable to Wills Creek. In a letter to Jefferson dated March, 1774, he said that the project was in good train and would have been in an excellent way had it not been for the opposition of the Maryland assembly, which was supposed to be instigated by Baltimore merchants, who feared the consequences of water transportation to Georgetown. The outbreak of the Revolution interrupted his plans, though he seems always to have had this problem on his mind, for a little before the close of the war he left the Continental camp at Newburgh and made a long and perilous journey up the Mohawk Valley with a view of determining the practicability of a water route to the West through that valley, returning convinced that the great rival route to the Potomac would be through New York. With the close of the Revolution, the problem became more acute than ever, for it seemed likely that unless quick and cheap communication could be established with the territory west of the Alleghenies it would ally itself with the Mississippi Valley interests and be forever lost to the eastern colonies. Washington strove to rouse Virginia and Maryland to meet the problem, with the result that the Potomac Company, incorporated by Virginia and confirmed by Maryland, was organized May 17, 1785. So far as known, the first minutes extant are now in the possession of the Chesapeake and Ohio Canal Company and are of the meeting held May 30, 1785. George Washington was its first president, but his services were required by his country and he was compelled to resign to accept the Presidency of the United States. The Potomac Company was only moderately successful under the immediate presidency of Washington, and when it was deprived of his counsels it became markedly less

$a$ Ward, G. W., Early development of the Chesapeake and Ohio Canal project: Johns Hopkins Univ. Studies in Historical and Political Science, series 17, Nos. 9-10 and 11, 1899. 
efficient, and finally with his death lost the confidence of the public. However, it accomplished much work during its existence. At the time it was organized little more was expected of it than to render the Potomac navigable from Georgetown to Cumberland, from which point it was thought to be an easy matter to connect with the waters of the Ohio by means of a well-built road. The chief obstacles encountered in descending the Potomac were five in number. The first of these was at House Falls, 5 miles above Harpers Ferry, where a canal 50 yards long, with a total fall of 3 feet, was built. The second difficulty was at Shenandoah Falls, immediately above Harpers Ferry, and was overcome by a canal 1 mile long, with a total fall of 15 feet. At Seneca Falls a third canal, three-fourths of a mile long, with a total fall of 17 feet, was constructed. To this point no locks were found necessary, but at Great Falls they were used in connection with a canal 6,000 feet long on the Virginia shore. The fifth and last canal, built for passing Little Falls, was on the Maryland shore, and was over 2 miles long, with a total fall of 37 feet.

The charter of the company originally provided that the work for which it was organized should be completed in three years. It soon became evident that it would be impossible to finish the work in that period, and the general assemblies of Virginia and Maryland passed an act extending the time. Five such acts were passed by the Maryland legislature and ten by that of Virginia. Finally, in 1819, after existing thirty-five years and spending $\$ 700,000$, the Potomac Company applied to the board of public works of Virginia for relief. The board ordered Thomas Moore, its chief engineer, to make a survey of the river with a view of locating a canal in its valley, a policy suggested by the board to the general assembly of Virginia in 1816 .

'Moore's work was begun on June 30,1820, and in his report dated December 27,1820 , he stated that the construction of the canal was practicable, and estimated the cost at $\$ 1,114,300$. The board transmitted this report to the governor of Virginia and he sent it to the general assembly, which adopted a resolution authorizing the governor to appoint a conmittee to cooperate with a similar one appointed by the governor of Maryland to examine into the affairs of the Potomac Company. The committee met in Georgetown July 2, 1821, and found the Potomac Company bankrupt. Having satisfied themselves that there were no means available for opening the Potomac River to navigation, they believed the time had come to recommend a continuous canal from tidewater to Cumberland, so they proceeded to that city July 15, and spent the rest of the month examining the river westward to Savage River. Having completed this work, under the guidance of Moore's survey of 1820, the commissioners began the location of a canal whose construction they believed 
would at once be undertaken by the States of Maryland and Virginia. Sickness among their engineers and finally that of Moore himself, September 18, compelled the abandonment of the work after it had proceeded 157 miles eastward from its beginning. Moore died and was succeeded by Isaac Briggs, who rapidly completed the work. For the basis of their estimate the commissioners adopted a canal 30 feet wide at the surface, 20 feet wide at the bottom, and deep enough for 3 feet of water. Its cost was estimated at $\$ 1,574,954$. The general assembly of Virginia passed a bill incorporating the Potomac Canal Company in February, 1823, but the Maryland assembly under pressure from Baltimore failed to do so; hence the project was held up for a time. The failure of the Maryland legislature aroused the friends of the canal and popular meetings were held in Virginia, Maryland, and Pennsylvania during the spring and summer of 1823 . The sentiment in favor of the canal was found to be so strong that it was determined to hold a convention in Washington during the autumn for the purpose of uniting counsels, proposing legislation, and enlisting the cooperation of Maryland, Pennsylvania, and Virginia and especially that of the Federal Government; which up to this time had steadfastly refused to inaugurate a system of internal improvements. The convention met at Washington November 6, 7, and 8, 1823, and declared in favor of a canal. It appointed committees in each of the States interested to advance legislation and a central committee to give direction to all the various forces at work on behalf of the canal. Among other things, the central committee was empowered to memorialize Congress, gather all information possible, hasten the surveys, have commissioners appointed, open books for the subscription of stock, and, if occasion required, to call another meeting of the convention. This convention is a landmark in the history of internal improvements in the United States, for the Federal Government had up to this time held it to be unconstitutional for it to construct such works, but it now unshackled itself from this impediment and entered into the canal project heartily. The Virginia assembly passed an act incorporating the Canal Company in 1824. Maryland passed one, too, after the jealousies of the Baltimore merchants had been allayed by an amendment permitting them to tap the canal with a branch canal at some convenient point in Maryland or the District of Columbia. The United States confirmed the incorporation and President Monroe signed the bill March 3, 1825. Finally, Pennsylvania passed an act sanctioning the canal.

The United States engineers, under the immediate direction of Gen. S. Bernard, made an elaborate and detailed report, which was not ready for publication until October, 1826. They proposed a canal divided into the Chesapeake and Ohio Canal proper from tidewater to 
Pittsburg, and the Ohio and Erie Canal from Pittsburg to Lake Erie. The Chesapeake and Ohio Canal proper was divided into three sections-the eastern section, from tidewater to the mouth of Savage River; the middle section, from Savage River to the Youghiogheny at the mouth of Bear Creek; and the western section, from the mouth of Bear Creek through the valley of the Youghiogheny to Pittsburg. The work done in preparing this report was very thorough and elaborate and necessarily consumed much time. The friends of the canal grew restive and in March, 1826, induced General Bernard to give out his estimate for the eastern section. It was, exclusive of the item of contingencies, $\$ 8,085,000$, which was, of course, for those days, prohibitive. The central committee called another convention, which met in Washington December 6, 1826. This body decided to their own satisfaction that when the errors of the United States engineers in estimating labor and materials were corrected, the canal could be built for less than $\$ 5,000,000$. At the instance of twenty members of Congress, President Adams ordered a new survey by James Geddes and Nathan S. Roberts, who reported that the eastern section of the canal could be built for $\$ 4,479,346.93$. Subscription books were opened October 1, 1827. The beginning of work on the canal was an event. A party was formed in Washington and went to Little Falls, where, on the Fourth of July, 1828, President Adams removed the first spadeful of earth, and speeches were made by prominent men to the crowd which had assembled to see the ceremony.

The company's charter required 100 miles of canal to be built in three years. Contracts were closed for 43 miles of the canal before March, 1829, and soon the whole 48 miles between Georgetown and Point of Rocks was under contract. By May 1, 1829, work had been done on all five "residences" into which the section had been divided. However, labor proved scarce, and it was found necessary to import it from Europe. Sickness broke out among the men, who contracted fever in the valley, and some of the contractors were compelled to suspend operations. It was late in October before the various gangs were all in good condition, but the winter proved an open one, and it was possible to continue the work far into it. The men proved disorderly, and the laws of those days were not stringent enough to deal with them promptly, so that ultimately the importation of foreign labor by the company was abandoned. The canal from Seneca Creek to a point within sight of Georgetown was completed in 1831 . In the meantime, about January, 1828, the canal company became involved in a suit with the Baltimore and Ohio Railroad. This railroad had the active support of the Baltimore merchants, and the first spadeful of earth which marked the inauguration of work on it was removed by James Carroll, of Carrollton, on the very day and only a few miles from the spot where President Adams celebrated the beginning of work on the Chesapeake and Ohio Canal. By its charter the Chesapeake 
and Ohio Canal Company had obtained a right of way for a canal on the Maryland bank of the Potomac from Washington to Cumberland. By its surveys the railroad was compelled to gain the Potomac at Point of Rocks, 12 miles below Harpers Ferry, and follow the river to the latter point; otherwise a tunnel would have to be built under the mountain spurs, a financially impossible alternative. The canal company now sought to exclude the railroad from laying tracks between Point of Rocks and Harpers Ferry on lands to which it claimed prior rights. The suit was finally decided in favor of the canal company in 1832, but by that tume it was bankrupt and Federal aid had been withdrawn. Such a predicament offered an opportunity which was successfully utilized by the Baltimore and Ohio Rallroad to complete its tracks to Harpers Ferry. After a series of compromise proposals by the railroad to the canal company had been refused, the Maryland legislature took up the matter. May 9, 1833, a compromise was effected by the passage of a law calling for the joint construction of a canal and railroad through the disputed territory. The compromise cost the railroad heavily, for it was compelled to subscribe for 2,500 shares of the canal company's stock $(\$ 266,000)$, and the canal company built the road through the disputed territory. (Point of Rocks). In 1834 the railroad completed the road to the Maryland side of the Potomac, opposite Harpers Ferry, where it was compelled to stop, for the agreement signed by the two companies demanded that the railroad should not be built across the Potomac untrl the canal should have been completed to Cumberland, if that was done within the time named in the charter-1840. At the time of the compromise, in 1834, Maryland came to the rescue of the canal with a loan of $\$ 2,000,000$. This was soon exhausted, and $\$ 3,000,000$ more was appropriated by the State June 4, 1836, but on condition that the.Baltimore and Ohio Railroad be allowed equal rights between Harpers Ferry and Cumberland. This enabled the railroad to complete its line before the canal was. finished. The summer of 1837 found the canal completed only to Dam No. 5, 127 miles from Georgetown. The next 27 miles of the canal, to Dam No. 6 at Great Cacapon, were in process of construction, and the last 50 miles, from Great Cacapon to Cumberland, were under contract. More funds were needed, and the general assembly of Maryland in 1838 advanced an additional amount of $\$ 1,375,000$. By 1841 the waterway was completed only to Dam No. 6, and both the canal and the State of Maryland were in financial difficulties, so that further aid was not given until 1844, when the State of Maryland permitted the company, under certain conditions, to issue bonds to the amount of $\$ 1,700,000$. With the funds thus raised the canal was completed to Cumberland in October, 1850.

Since its completion the canal has not been prosperous. In 1877 IRR $192-07-13$ 
the works of the canal were seriously damaged by a freshet, and this provided an excuse to burden the canal with more debt by issuing additional bonds, which was done with the consent of the State of Maryland. In 1889 another freshet did even greater damage; and as those in charge of the canal had no funds with which to pay for repairs, it was left in this condition for two and one-half years, during which time the elements added to the damage and increased the cost of reconstruction.

At this time a movement was begun to dispose of the canal to certain railroad interests, but the bondholders of 1844 asserted their rights and began proceedings to get possession of the canal. As trustees they were given control for a certain number of years on condition that they would repair it, retire the bonds of 1878 , and carry out other conditions imposed by the court. Apparently the trustees have satisfied the court of the feasibility of their plans, for the period given them to operate the canal has been extended from time to time and it is still in their possession.

To-day the canal between Georgetown and Cumberland lies on the Maryland side of the Potomac and pursues the immediate valley of the river to a point 1 mile below Pawpaw, where it passes through a spur of Town Hill by means of a tunnel 3,636 feet long and of circular cross section 27 feet across. This tunnel saves about 6 miles. The total rise from the level of midtide at Georgetown to the Cumberland basin is 609.7 feet. This ascent is broken by 74 lift locks and a tide lock that connects Rock Creek basin with the Potomac. The canal has a depth of 6 feet throughout, and from Georgetown to Harpers Ferry, 60 miles, it is 65 feet wide at the surface and 41 feet at the bottom. From Harpers Ferry to Dam No. 6, 47 miles, the width at the surface is 60 feet and at the bottom 36 feet. From Dam No. 6 to Cumberland, 50 miles, the surface width is 55 feet and the bottom width 31 feet. The average lift of the locks is a little in excess of 8 feet, though there are thirteen 10-foot locks and four 6-foot locks. The locks are 100 feet long and 15 feet in the clear and pass boats carrying 122 tons of 2,240 pounds. The canal is fed with water at eight different points. The first is at the Beal mill race in Cumberland, which is connected by gates with Wills Creek at the dam near the tannery of the United States Leather Company. The enormous amount of sewage which this race receives is, to a large extent, deposited as sludge in the head basin of the canal. This action is probably facilitated by the nature of the water received at the second supply point, the head-gates of the canal. This water is, as a rule, largely from Wills Creek, mixed with considerable water from North Branch, though in times of low water the entire flow of both North Branch, above Dam No. 7, and Wills Creek goes down the canal. The Wills Creek water is heavily polluted by mine waters, and therefore con- 


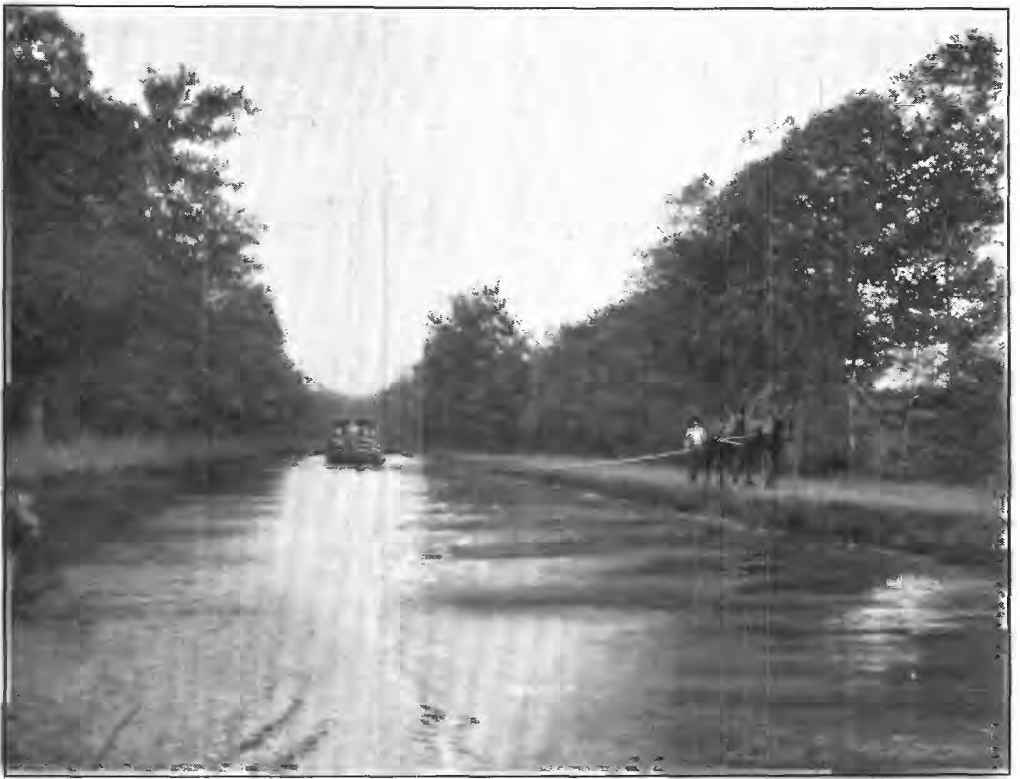

A. CHESAPEAKE AND OHIO CANAL ABOVE WILLIAMSPORT, MD.

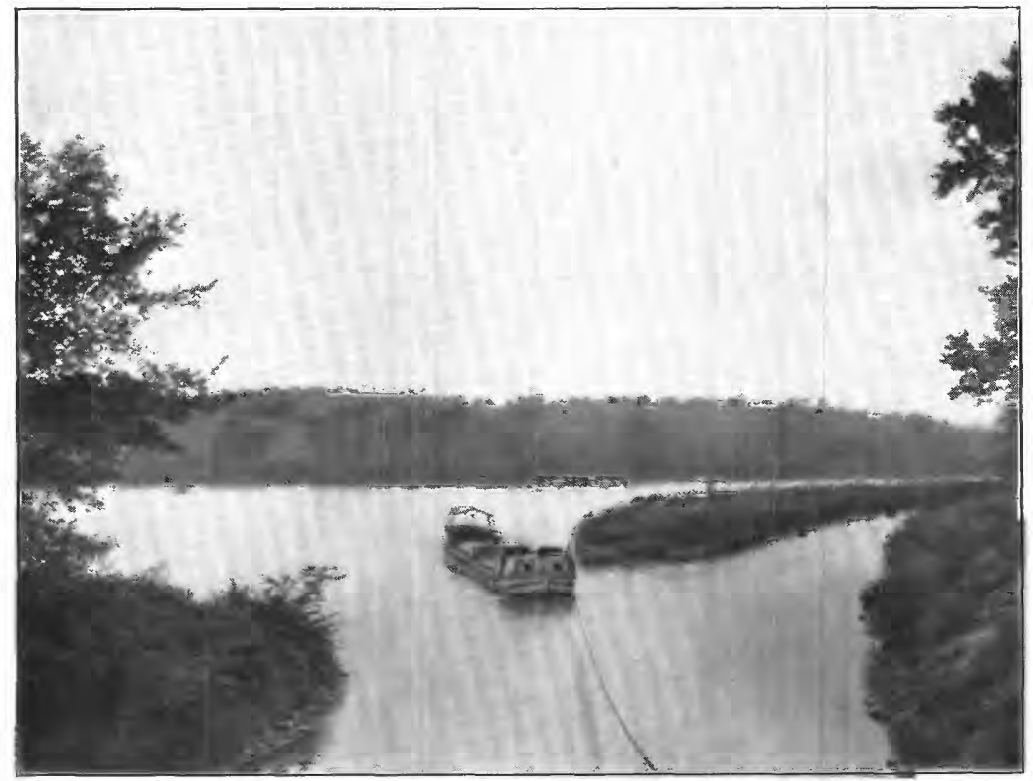

B. POTOMAC RIVER AND CHESAPEAKE AND OHIO CANAL AT DAM NO. 5. 

tains considerable quantities of iron and sulphuric acid, which are precipitating elements, as is the lime of North Branch. So great is the accumulation of precipitated sewage in the head basin that the canal company finds it necessary to dredge it out every other spring. When this is done the sludge is distributed over the towpath and river side of the embankment. It becomes very hard and most of it remains in place, though some of it is carried off by the river. In the winter, after the water is drawn off from the canal, the contents of the race discharge into the head basin as usual, but instead of continuing down the canal, as when the canal is in use, they return to the river near the foot of the basin and below Dam No. 7. This first supply is not properly a part of the works of the canal. The precipitating action continues for 51 miles down the canal to the third supply point, Dam No. 6 , where the water in the canal is said to be much clearer than at Cumberland. Dam No. 6, below Greenspring, admits river water, which to a large extent comes from South Branch, and hence is usually of better quality than the river water at Cumberland and by dilution greatly improves the water in the canal. From Dam No. 6 to Dam No. 5 the distance is 29 miles. About 6 miles above Dam No 5 the canal passes through what are known as Little and Big pools. These were originally high-water channels of the river, which have been incorporated into the canal. Big Pool is 2 miles long. The occurrence of these pools is noted, because the current through them must necessarily be very small and so gives opportunity for sedimentation of material suspended in the canal water and also because they must add to the time it takes the water to complete its passage from Cumberland to the river. The canal and the river unite half a mile above Dam No. 5 (Pl. VII, $B$ ), and in the half-mile stretch above the dam the canal and river waters are thoroughly mixed. A.t Dam No. 5 the canal resumes its separate course and a fourth point of water supply is established.

- From Dam No. 5 to Dam No. 4, the fifth point where water is received by the canal, the distance is $21 \frac{1}{2}$ miles. About $4 \frac{1}{2}$ miles above Dam No. 4 the canal reenters the river and continues united with it for 3.3 miles to a point 6,000 feet above the dam. In this distance the waters of the canal and river have become thoroughly mixed, and it is well to remember that this dam is but $67 \frac{1}{2}$ miles above Great Falls. From Dam No. 4 the canal continues 23 miles to Dam No. 3, at Harpers Ferry, the sixth point of water supply. Dam No. 2. half a mile east of Seneca Falls and 40 miles below Harpers Ferry, is the seventh and last point of water supply until Dam No. 1 is reached at Little Falls.

Throughout its length the canal is built on the surface of the land and so receives little ground water; in fact, the water has a tendency to seep out through the canal banks and in places to make the farm 
lands too wet to be successfully cultivated. The current in the canal is very variable, changing in its different sections and with the stage of the river. Perhaps it would be correct to assume a current of 1 mile an hour in normal sections. At times of flood portions of the canal are under water.

Two expensive aqueducts carry the canal over Conococheague Creek and Monocacy River. The myriad little runs that come down from the hillsides to the river are excluded from the canal by means. of passages made for them beneath the canal through masonry culverts. Thus these streams find direct entrance to the Potomac, except three, which enter Big Pool, and a few dry runs, the most important of which are one a little east of Monocacy River, one just above Harpers Ferry, and one 2 miles below Dam No. 6 .

Every winter the water is drained off from the canal and its service discontimued. The length of the canal season is usually eight to nine months, and repairs are made every spring before the canal is reopened. It is not found necessary to clean out the canal on account of suspended matter deposited during the season's use, but certain points on the canal need rather frequent dredging because of earthy material that is brought in over its banks by water flowing over cultivated ground and in other ways. The canal company has no sanitary regulations and the crews of canal boats use the canal as a receptacle for feces and for kitchen offal. A few of the privies of the lock keepers overhang the canal, but the feces do not get into the river except in times of freshet. The canal company has succeeded in abolishing all but two or three of the other privies which overhang its line, and these, it is expected, will soon be removed. During the season of 1905 but one man employed on the canal boats was known by the officials of the company to have suffered from typhoid fever.

The canal company claims that by the terms of its charter it is entitled to all the water power of the river from Washington to Cumberland, but it has never developed it for manufacturing purposes, one reason being that a survey made to determine the amount of power which could be made available indicated that the outlook in this direction was not promising. A small mill at Williamsport and one at Seneca are the only ones above Washington to which power is sold by the company. At Dam No. 5 surplus water is sold for power purposes to a plant on the West Virginia side of the river, and the same people are contemplating the development of works at Dam No. 4. At Georgetown about 1,000 horsepower is sold. The water of the canal is not furnished to any community for domestic supply, though a few individuals here and there along the line use it for washing purposes. 


\title{
STREAM POLLUTION, OCCURRENCE OF TYPHOID FEVER, AND CHARACTER OF SURFACE WATERS IN PO'TOMAC BASIN.
}

\author{
By Horatio N. Parker. \\ STREAM POLLUTION.
}

GENERAL ASPECTS.

The prosperity of the industries of the Potomac Valley, with its attendant increase of population, is justly a cause of congratulation to the several States within which the basin lies. Yet this success brings responsibilities that can not be shirked, but must be wisely met if growth is to continue and health and happiness prevail. In dealing with the new problems it should be borne in mind that the wealth of every region is limited. Resources which were extravagantly wasted when there were few to claim them have to be carefully husbanded when there is hardly enough to go around. Acts which may be viewed with indifference in a sparsely settled country become crimes in densely populated communities. No resource will be more seriously affected by changed conditions than water. It is strictly limited in amount, and therefore to injure any source which is valuable for manufacturing or domestic use is a spendthrift deed, sure in the end to increase the cost of living. For, one by one, the sources of pure water which are not too expensive to utilize will be preempted, and then will come the time when the supplies that have been ruthlessly damaged must be purified. It can be done, but it costs money. Water rates will rise, and then every man will have to decide for himself whether it will be more profitable for him to remain where he has built up his business or to migrate to some place where the supply of water is yet unpolluted, or where the citizens have been forehanded enough to keep, by well-considered legislation, the cost of purifying their water supply within reasonable limits.

Often before industry is crippled by bad water a town or city experiences epidemics of water-borne disease that result from an impure water supply. This condition is brought about by contamination of the water by the feces of persons sick with Asiatic cholera or typhoid fever. Happily in this country outbreaks have generally been 
limited to the latter disease. At first the policy of concealment is adopted by the afflicted community, and "malaria" is said to be present. Later, when suspicion arises that the trouble is something more serious, the terms "fever" or "typhoid malaria" are used, and when the outside world can be no longer deceived by euphuistic names it is admitted that "there was some typhoid last year." By this time the degree of contamination in the supply has either become sufficiently great to produce typical cases of typhoid fever, or so many cases develop at one time that an epidemic is said to be present. Public opinion is aroused, the town is bonded for the construction of a new water supply or for purification works, and the tribute of human lives demanded by pollution is stopped.

Both sewage and industrial pollution may inflict great loss in the community by making its streams unfit for harvesting ice. The late Prof. Thomas M. Drown ${ }^{a}$ showed that ice in freezing has a decided tendency to exclude impurities, but he found this action to be most marked in the layers that are formed by the slow growth of the ice downward, because the surface in its rapid congealing entangles suspended matter, particularly if the water is stirred up by the wind. Moreover, if the ice is frozen after surface flooding, which is frequently brought about intentionally by the harvesters, it will contain all the impurities of the water so added. In making artificial ice in the ordinary way the entire body of the water is frozen and all the impurities are concentrated in the last part of the cake to freeze. Hence an impure water is particularly undesirable for ice manufacture. Likewise, when shallow ponds freeze solid, the impurities are concentrated in the bottom ice.

The fact that ice forms in greater purity than its surrounding medium undoubtedly has permitted it to be obtained from questionable sources and still to be used with impunity. But outbreaks of typhoid fever have been attributed to polluted ice, and it is certain that a stained, or ill-smelling ice is unsalable, so that consumer and producer alike suffer from river pollution. Indeed, probably most readers know of ice ponds which have had to be abandoned because the ice from them was no longer marketable.

The question how best to get rid of the wastes of human life and of the industries which are a part of it is a very pressing one. Every health officer is familiar with the subterfuges adopted to avoid the expense attendant on the proper disposal of these wastes. Very naturally, rivers have been seized on as the easiest way to solve the problem, because they passively accept all burdens given to their charge and carry them away. Indeed, water has been called the great scavenger, and not a few manufacturers regard the streams as natural sewers created by Providence for their use. 
Natural beauty has a distinct value, and those who mar it impair the resources of a country, for a discolored repulsive stream will not be utilized for recreation purposes, and a stinking one will not be featured in realty advertisements. The monetary loss to communities arising from severe pollution of the streams on which they are located is often very heavy, and the damage is reparable only at great expense.

The constantly increasing cost of food has become a serious factor in the development of the country. Dishes that were common on the tables of our fathers have become rarities to many and are entirely foregone by not a few. In spite of this we are carelessly curtailing our fish supply, as the filth poured into our.streams and rivers drives the fish from their spawning grounds. Some streams they have quite abandoned; others they visit in decreasing numbers. It is true that we place large quantities of fry in various streams every year, but some fish-the sturgeon, for example-can not be perpetuated in this way.

It should be remembered that some one always pays for water pollution. If the laws are such that they relieve one corporation of caring for the wastes it creates, it is likely that they entail great expense on another. Thus a railroad which is compelled to use polluted water in its locomotives will in consequence have many repairs to make, and the increased cost of mileage caused thereby will have to be borne by those who travel and transport goods over the road. Similarly, if one man is unmolested in turning the effluent from his mill into a stream, it may drive his neighbor downstream to drill deep wells in order to introduce a new water supply into his works. Instances of this sort of thing might be multiplied, but it is enough to recognize the fact that though a river basin may lie in several States, as does the Potomac, its people are more closely bound together by its waters than are the inhabitants of a single State by the arbitrary boundary lines established for them. The silver river threads are direct lines of communication between each individual and every other below him on the stream. The offenses that he commits against the water are paid for by his fellow countrymen in the basin, and the bill is large or small according to the gravity of the transgressions.

\section{INDUSTRIES DISCHARGING WASTES INTO THE STREAMS.}

In order that a clear understanding may be had of the various wastes which enter the Potomac, a description of the manufacture of the principal products of the Potomac basin is subjoined.

\section{LEATHER TANNING.}

Pelts are divided into three classes, according to their size. Hides are skins from large and fully grown animals, such as the cow, ox, and 
horse, and make heavy leather, such as shoe soles, belting, and trunk leather. Kips are the skins from yearlings of the above species or from undersized animals. Skins are obtained from small animals, such as calves, goats, sheep, and dogs, and yield a lighter leather, suitable for a great variety of purposes. The thickest and heaviest hides come from sparsely settled countries, and a hide varies in thickness and texture in different parts, being thicker on the neck and butt than on the flank and belly. The skin of the animal is made up of three layers. The first or outer layer is known as the epidermis, and consists of a dead layer which is continually being worn off and of a live layer which renews the dead layer as it wears away. To this epidermis the hairs of the animal are attached. The second layer, or corium, is the true skin, and the only part of the hide which is valuable in making leather. It consists of connective tissue composed of bundles of fibers which interlace somewhat closely on the under side, but are closely matted on the epidermal side. The third and inmost layer is a loose network of connective tissue, containing muscular fibers, fat cells, blood vessels, and sudorific glands, whose ducts pass through the corium and epidermis.

The pelts come to the tanner "green" (fresh from the slaughterhouse), wet, or dry salted (with the salt rubbed on the flesh side), or as dry hides. The dry hides are mostly imported from South America. Green pelts are usually washed in clear water to free them from blood and dirt; salted pelts, if not dried, are washed in several changes of water to remove the salt, which retards the action of the lime in the unhairing processes and also induces efflorescence (spewing) on the finished leather. Dried hides must be softened by soaking them in lukewarn water or in the liquor drawn from the soaking of a previous lot. The water dissolves a part of the hide substance and putrefaction soon begins, the liquor developing an alkaline reaction, owing to the formation of amines and ammonia, which give it a much more rapid softening action on the skin. Great care is necessary in using this "putrid soak," for it is likely to attack the hide itself. The time of soaking varies from "two or three days to as many weeks, depending on the thickness and dryness of the hide and the age and temperature of the soak liquors. When the hide is soft enough to bend in a short turn without cracking, it is put into the "stocks," where it is pounded and rolled under heavy wooden mallets and rolls.

The character of the water used in a tannery is important. Soft water makes the skins thin and slim, which is desirable in light leather. Water containing calcium or magnesium sulphate "plumps" or swells the hide, thus exposing a larger surface to the action of the tan liquors, which is desirable for heavy hides. Water bearing carbonates of lime or magnesia gives trouble, because in the tan pits these salts are transmuted into tannates of lime and magnesia, and these rapidly oxidize in 
the air into tan-oxalic and tan-melanic acids, which impart a reddishbrown color to the leather. Ferruginous waters can not be used in tanning, for the ferric salts combine with the tannin and produce a black coloration of tannate of iron. Chlorides cause the hides to "fall;" that is, to become thin and flabby. Perhaps this is due to the greater solubility of the coriin in saline liquors. If used for washing after the liming, water having temporary hardness tends to fix the lime among the fibers in an insoluble form, thus causing the leather to be harsh on the grain and producing color spots because of unequal deposits of tannin and coloring matters while the skin is in the tan pits. If the water contains organic impurities it may have an acid nature and cause the hides to "fall" after liming. Highly polluted waters can not be used in tanning, because the putrefactive bacteria attack and destroy the hides.

The "putrid soak" and the liquor from the washings of the green and salted hides are uniformly discharged into the nearest creek, being regarded by the tanner as so much dirty water and nothing more. As a matter of fact, this liquor contains much salt and organic matter. The putrid soak may also contain cresol or other preservatives, which are sometimes used in conserving the dried hides. The soak liquors may be the means of spreading anthrax. An outbreak of this disease occurred in Pennsylvania in the spring and autumn of 1897 and was investigated by Ravinel. Three tanneries operated by the United States Leather Company were involved. It was found that each of them had received a part of a cargo of hides from Chicago, and it was believed that they were the fomites which spread the disease. In one of the tanneries twenty-four cases of "malignant pustule" developed, one of which proved fatal. Below this tannery 12 head of cattle died, some of the deaths occurring as much as 10 miles downstream. In all, 12 men and 60 head of cattle died near the tanneries. The men were without exception operatives, while the cattle were on pastures watered by the streams carrying tannery effluents.

Bacteriological experiments were conducted which led to the conclusion that the process of tanning as ordinarily carried out does not in any way protect the operative from infection by the anthrax germ. ${ }^{a}$

The second step in tanning is the "unhairing" of the hides, and this is accomplished in two ways-either by sweating or liming. Green hides are usually sweated by allowing them to hang in a room where the temperature is $70^{\circ} \mathrm{F}$., and permitting disintegration to advance to the point at which the hair can be readily removed mechanically, but beyond which the hide is injured by being itself attacked. Before passing to the next stage, treating with tannin, sweated hides must be "plumped" by immersion in dilute acid, such as hydrochloric acid. In liming, the skins are laid in a vat or pit with milk of lime, which 
loosens the epidermis and forms a soap with the fatty matter. It also dissolves the coriin-the intercellular substance which fills the spaces between the bundles of fibers-loosening the fibers, which swell and plump the hides. Lime is used in excess in amounts varying from half a pound for a small, light skin, to 4 pounds for a heavy one. The vats or pits when prepared to receive the skins are called limes. The skins are turned over and worked about at intervals while in the vats. Heavy hides, which are to form a stiff, hard leather are limed for only few days, but when a soft, elastic, and pliable product is desired the process is continued for fifteen to twenty days or longer. Warming the limes to $85^{\circ}$ or $90^{\circ} \mathrm{F}$. hastens the action very much, but causes the skins to "fall." Dried hides are often treated by adding sodium sulphide to a thick cream of lime, forming a paste which is spread on the hair side of the skin, after which the hide is folded together, when the hair can be easily removed after a few hours. This process depends on the formation of calcium sulphide, which dissolves the hair, in contrast to the process employing lime alone, which dissolves the hair sheath as well as the intercellular substances and softens the epidermis, which comes off when the hair is scraped. Sometimes the sodium sulphide is added in solution to the milk of lime instead of being applied as a paste. Still another process for unhairing dried hides is the adding of arsenic sulphides to the amount of 10 per cent of the weight of lime. This forms calcium sulfarsenite $\left(\mathrm{HCaAsS}_{3}\right)$, which is a very rapid depilatory. The waste from the lime pit is frequently turned directly into the nearest stream, but the best tanners utilize it as a fertilizer by running it into pools, where it is allowed to evaporate. The accumulated material is applied to the land, on which it seems to have a very good effect, probably on account of the large amount of organic matter derived from the hides, though the lime itself may in some cases be valuable to neutralize acid soils. It is not likely that those "limes" which contain arsenic could be used in this manner, and they cause much trouble in those places where entire tannery wastes are treated by intermittent filtration, because they kill the bacteria, which, of course, destroys the action of the filter bed. It is wasteful and unnecessary to dispose of the lime waste by running it into a stream. It should always be recovered as a fertilizer.

When the hide has been unhaired by one of these processes, it is washed, and the washings are added to the liquors wasted from the lime vats; it is then ready for the "beam," a peculiar sloping bench or table on which the hides can be spread out and conveniently worked. On the beam the hair and epidermis are scraped off with a blunt knife, and the fatty tissues are removed with a sharper one. After trimming off the waste parts of the skin it is thoroughly washed and is usually again scraped on the beam ("scudded"), to remove as much of the lime as possible. The hair is sold for cheap cloth 
and blanket manufacture, or most usually is disposed of to plasterers. The flesh and partially saponified fat which are removed from the inside of the hide are collectively known as "fleshings," and are either dried and sold to glue manufacturers or are rendered and the fat sold for the manufacture of dégras. The liquor which results from rendering the fleshings is turned into the nearest watercourse, but it should go to the lime pool. From the beam house the hides which are not to be made soft and pliable go directly to the tanning liquors, while those skins to which it is desired to impart these qualities are "bated" or "puered." Bating consists in soaking hides in a mixture of $\operatorname{dog}$ or bird dung in warm water. This quickly becomes putrid and evolves hydrogen sulphide. By some it is claimed that the bate merely removes the lime from the pores of the hide, while others assert that it also takes away some of the coriin, thus leaving the fibers looser and allowing more perfect action of the tan liquors. The latter view seems to be more probable, for there is little doubt that the bacteria in the bate do feed on the hide substances. Moreover, the forms of tripepsin, pancreatin, etc., present undoubtedly have some function, for when used alone they will cause a "plump" skin to "fall." Also the ammonia salts formed probably assist in the solution of the lime and the skin. The process lasts from two to four days, according to the thickness of the skin and the temperature. It is largely dependent on atmospheric conditions, being much more rapid in warm, sultry weather; such as precedes a thunderstorm, when a few hours are often sufficient to injure the skin. At all times great care must be used and the skins stirred about frequently to prevent too great local action, which would produce thin places or holes in the leather. Many proposals have been made to replace the offensive bate with pure solutions of weak mineral and organic acids, but such substitutes. have not been successful, the objection being that they make the leather harsh and of a bad grain.

To effect a complete removal of the lime the skins are passed from the bate into the "bran drench," an infusion of bran and water at a temperature of about $89.5^{\circ} \mathrm{F}$. Acetic and butyric acids are developed and neutralize the lime. The wastes from the bate and drench are not large and should be utilized for fertilizer in the same way, as the spent lime liquors. This, however, is rarely done, the custom being to turn them loose into some stream. Both the light and the heavy hides are now ready for the tanning process. There are three kinds of tanning-with tannin in any form (vegetable tannage), with metallic salts (mineral tannage or tawning), and with oils or fats (oil tannage). As only the first process is used by Potomac valley tanneries it alone will be described. 
The tanning liquors are usually made by extracting finely ground chestnut-oak or hemlock bark with water. Hides treated with infusions of the former make a tough, durable leather known as oaktanned; infusions of hemlock bark make a hard, stiff leather known as hemlock-tanned; a combination of the two produces union-tanned goods. Hemlock-tanned leather is of two varieties-acid and nonacid. In oak, nonacid, and union tanning the processes are essentially the same, and so only oak tanning and acid hemlock tanning will be described.

Good tanning material yields other extractive matters than tannic acid when treated with water. They are nontannins and consist mainly of sugars, gums, resins, and coloring matters, which assist in tanning in several ways. Some of them are directly absorbed by the skin, increasing its weight and solubility; others set up fermentation in the tan pit, producing organic acids, which assist in the formation of a leather of good body and weight. Tannins derived from gallic acid cause a white efflorescence (ellagic acid) on the leather, while those of the protocatechuic-acid group deposit red coloring matters (phlobaphenenes). The tan liquors are prepared by systematic lixiviation of the ground tan stuffs. Warm water is generally used for extracting the ground bark, and the process is usually carried on at the top of the tannery, so that the liquor can be readily distributed about the works, either to pits which are to be strengthened up or to those which are to be filled anew. Prepared tanning extracts are often used, either alone or in conjunction with the liquor obtained by extracting the bark at the tannery. These prepared extracts are frequently adulterated with glucose or molasses, so that tests with the barkometer-a special form of hydrometer used for determining the strength of tan liquors-are of no value. In tanning, the hides ("butts") are first hung from frames in vats ("suspenders") containing weak or nearly spent tan liquors from a previous lot. There they are mechanically agitated in order that they may take up the tannin evenly. Weak liquors are used at first, because strong ones would harden the surface of the butt and prevent the thorough penetration into the interior of the hide. This partial tannage strengthens the hides sufficiently for them to withstand the rough usage which they receive when transferred to the "handlers"- vats where the hides lie flat in a pile and are worked over ("handled") once or twice a day for a month or six weeks. There are several of these vats, and the hides are treated systematically first with weak and then with stronger liquors, after which they are put into the "layers"-pits filled with alternate layers of hides and ground bark and velonia, etc. Strong liquor ("ooze") of $35^{\circ}$ barkometer is run in until the hides are submerged, and the pit well is then covered with ground bark to exclude the air. After eight or ten days the hides are taken out, 
rubbed clean, and "laid away" again in fresh tan and stronger liquor, in which they remain a longer time. This process is often repeated, the time consumed being on an average from eight to ten months. The process may be hastened by keeping the liquor in the tan pit in constant circulation, or by using pressure to force the liquor into the skins, or by using strong extracts and continually moving the skins. As a rule, however, rapid tannage makes the hides lacking in substance ("hungry") or brittle. In oak tanning, of sole leather, usually one hundred and twenty to two hundred days are consumed. When the hide comes from the lay-away vats it is covered with a "bloom" of ellagic acid, which sole-leather and harness-leather tanners remove with "scouring machines" to improve the appearance of the goods. Scouring requires considerable water, which is stained red by the process. The waste is uniformly turned into the nearest stream and of course discolors it.

In acid hemlock tanning, the hides are first colored with a weak solution of tannin, after which they are abnormally swelled by being put in a 10 to 30 per cent bath of sulphuric acid, where they remain for twenty-four to forty-eight hours. They are then put in a stronger tannin solution and are finished in one hundred and sixty days.

The waste tan liquors or. sour bark liquors, as they are usually termed, are usually the most voluminous wastes from a tannery and consist of a small percentage of tannic and gallic acids, with considerable organic matter in suspension. They are usually discharged into a stream, either constantly or in large volumes, at regular intervals, and are always strong enough to color the water a deep red and to stain the banks and bottom of the stream. The volume is so large that this waste is difficult to treat. The most successful way of dealing with it known at present is that devised by the Massachusetts State board of health; in this process it is mixed with city sewage and treated by intermittent filtration. Such a method of treatment could not be applied in the Potomac drainage area, because the tanneries are located in places where city sewage is unavailable. Some tanners claim that they have practically no waste of sour bark liquor, because they maintain a constant circulation of the liquor through their works; that is, when the liquor is so depleted as to be of no further use for tanning it is run back onto fresh ground bark, where its strength is renewed. In tanneries of this type it is generally found necessary to waste the tan liquor which first receives the hide after it comes from the "beam," as the lime salts extracted at that time are thought to be sufficient in amount to affect large volumes of tan liquor, making it turn out a brittle leather. Many tanners refuse to adopt this continuous-circulation process, claiming that they can not get the kind of leather they are in the habit of producing by its use. 
After hides are tanned they are washed in clear liquor, and when they have dried somewhat they are oiled on the grain and hung on poles in the drying loft. When about half-dry they are laid in piles ("sammed") and allowed to sweat, so as to facilitate the "striking" operation which follows; this is done by hand or machinery. They are then allowed to dry a little more, and finally they are rolled again, after which they are ready for the market, though in some factories the hides are colored on the grain side by a mixture of ocher with size and oil to give the leather gloss.

In the final finishing up of the leather-that is, in cases where hides are oiled or stuffed at the tannery-considerable oil escapes into the nearest creek, making it unsightly.

The spent tan bark, which formerly caused much complaint when it was discharged into the stream and allowed to accumulate about the tannery, is now disposed of without trouble, because it has been discovered to be a valuable fuel and is used for such both in extract plants and tanneries. But a single instance was found in the course of this investigation where any trouble was caused by spent tan bark. This was at Moorefield, where a tanner had accommodated the proprietor of a gristmill by furnishing him spent tan bark to stop the many leaks in the old dam which furnished power to the mill. It was said that the stream was considerably discolored in consequence.

To sum up the wastes from the processes of tanning, most of them are putrefactive and therefore add materials to the stream which make it capable of sustaining bacterial life, and for this reason their presence is objectionable. Moreover, when all the wastes are turned into a stream, unless it is a very large one, they discolor it, making it unsightly and also cause a great nuisance by the odors of decomposition which rise in the stream and in the vicinity of the tannery. Where tannery effluents are discharged into streams polluted with mine waters large quantities of tannate of iron are formed, thus literally converting the waters into rivers of ink. It is needless to say that this condition is vigorously resented by all who are unfortunate enough to be brought into contact with it.

\section{MANUFACTURE OF TANNING EXTRACTS.}

Tanning extracts are made by leaching various finely ground tannin-bearing materials, such as sumac and chestnut-oak wood, in water and then evaporating the solution to a thick sirup. It is perfectly possible to manufacture the extracts without offense, but leaky vats produce extensive discoloration of the streams into which they discharge, and in certain places this causes much complaint. An entirely negligible amount of tannin escapes in the condenser water from all extract factories. Carbonate waters are unsuitable for the manufacture of extracts for use in dyeing, as they form "lakes" with 
the dyes, and consequently loss of coloring matter ensues. The concentration of large volumes of the tannic juice made with carbonate waters causes the precipitation of normal carbonates of lime and magnesium, which are harmful in the tan pits. Iron in the water combines with the tannic and other acids extracted, forming ferric compounds, which give dark-blue, olive, and green precipitates that not only discolor hides, but waste the tannin-forming materials from which the extract is made. Moreover, compounds of the tannic acids with salts of the alkaline earths result when water containing appreciable quantities of these salts is used in extracting. Thus the extracts produced are poorer, and the process of manufacture is slower, because the deposits prevent the water from penetrating into the woody fiber.

\section{MANUFACTURE OF WOOD PULP.}

There are three common ways of manufacturing wood pulp, known as the mechanical, the soda, and the sulphite processes. The first two of these are used in the Potomac Valley and will be briefly described.

Mechanical wood pulp.-The bark is first shaved off the wood and then the knots are removed, after which it is cut up into blocks, gaged to the width of the stones used in grinding. These stones are usually imported from Scotland and are of sandstone, covered over three quadrants with an iron casing, the fourth being left bare. The faces of the stones are kept rough, as they revolve, by a steel roller studded with points, which is pressed against them. In addition to this, two sets of channels, which cross each other in the center of the stones, are cut into them about one-quarter of an inch deep at distances of 2 to 3 inches. They carry the pulp to the sides of the stones and give them increased grinding surface.

The wood is forced against the revolving stones, over which a water jet plays, and by means of screws worked by a suitable gearing is held at the steady pressure necessary to insure a pulp of uniform character. The water jet carries away the pulp as fast as it forms, first to the rake, which catches the splinters that have escaped grinding; then the stream of pulp passes through the sorters, cylinders 3 feet long and 2 feet wide, of wide-meshed wire cloth, which separate the insufficiently disintegrated fragments. The fibers that are retained are subsequently reduced to the proper size in the "refiners," which are cylinders of sandstone, one above the other, the upper one of which revolves. The material that passes the refiners is again screened and returned to the pulp stream, from which the sorter is conducted through a series of screens of gradually increasing fineness, and thus graded into different qualities. The ground and sorted pulp, mixed with water, flows into a tank in which revolves a cylinder covered with wire gauze. The water passes through the cylinder, 
while the pulp adheres and is delivered to an endless belt, which carries it to a pair of squeeze rollers. The pulp is compacted in passing through these rolls and sticks to the upper one, from which it can be readily removed when it has become sufficiently thick. Finally, it is cut into sheets, which are pressed into boards of convenient size for transportation. Mechanical wood pulp is suitable only for the cheapest kind of paper, such as newspapers, because the fibers are short and do not felt together well, hence the paper lacks strength;. moreover, it yellows readily, as the lignin and resin which the pulp contains predominate. Mechanical wood pulp is much used as a filler in manufacturing certain kinds of paper.

No chemicals are used in this method of pulp production, so that there are no liquid wastes to produce noxious effects on streams and lakes, but the shavings and the finest fiber that escape cause bitter complaint. (See Pl. VIII, B, p. 222.) They soon become water-logged and sink, accumulating rapidly on the bottom of the stream, with the result, as one old fisherman aptly put it, "that the bedding, feeding, and breeding grounds of the fish are destroyed." The effect on the spawn is particularly bad, for the motion of the water rolls the material over the eggs and smothers them. Besides, in time the mass begins to rot and then certain gases are liberated, which also may have a harmful effect on fish life.

It is wholly unnecessary to dispose of the shavings in the streams, for they can be burned at no great expense, and the laws which prohibit sawmill pollution should be extended to cover mechanical pulp mills.

Soda wood pulp. a-In the manufacture of soda wood pulp the logs are cut to thin shavings and are treated for about eight hours in digesters with a caustic-soda solution at a pressure of about 100 pounds of steam. After being washed the pulp is bleached by a solution of chloride of lime, the process consuming from six to eight hours. When the bleaching is complete the mass is reduced to a more fluid state by the addition of water and is pumped into large vats with porous bottoms through which the water runs. When the bleached pulp is thoroughly drained it is pumped into a large storage vat, from which it is taken to a cylinder machine and is felted in the usual manner.

The soda solution drained from the wash pans is treated in order to recover the soda. It is evaporated and the concentrate is turned into rotary furnaces where the lignin and other organic materials are burned off. The residue is composed almost entirely of carbon and sodium carbonate and is known as black ash. This substance is then passed through a bleaching process, the liquid or recovered soda

a For a full description of the processes used in the manufacture of soda wood pulp see Water-Sup. and Irr. Paper No. 121, U.S. Geol. Survey, 1905, pp. 24-33. 
(sodium carbonate) being conducted to the causticizing plant, while the sludge, known as black-ash sludge, remains in the tanks and is subsequently washed out with water and carried away as waste.

The soda recovered in the form of carbonate is converted to a hydrate or caustic soda by the application of caustic lime. The result is a sodium hydrate or caustic soda in the solution, while the calcium carbonate, or common lime, remains in the bottom of the container as a heavy sludge. The solution is drawn off for use in the digestion process. Considerable soda remains in the lime sludge after causticization, and repeated washings are necessary to recover all the soda. When the washings are completed the lime sludge is discharged.

The solution used to bleach the pulp is prepared by the treatment of chloride of lime in solution tanks fitted with rotary agitators. The bleach is drawn off from the tank and the sludge is again treated in order to utilize all the bleaching solution, and the lime sludge left after the second treatment is discharged as waste.

The important wastes are (1) the black-ash sludge, which is mainly carbon, with a small percentage of undissolved carbonate of soda; (2) the lime sludge $\left(\mathrm{CaCO}_{3}\right)$ from the causticizing process, which also contains a little soda; (3) the lime sludge $\left(\mathrm{CaCO}_{3}\right)$ from the bleaching process, which contains a very appreciable amount of chlorine. To these should be added the considerable amount of wood fiber that escapes from the mill, and the bits of wood from the chipper. In some factories the various sludges are sedimented with good results, large quantities of suspended matter being thus kept out of the streams.

At Luke, Md., there are no sedimentation beds and North Branch receives the entire waste of the West Virginia Pulp and Paper Company, which, in addition to the effuents above enumerated, contains alum, kaolin, a little ultramarine blue, and size. The effect of this pollution on North Branch is to increase the chlorine and alkalinity of the water. But these elements serve to neutralize the acid waters of Georges Creek, with good results.

\section{MANUFACTURE OF ILLUMINĄTING GAS.}

Coal gas.-Coal gas is made by the destructive distillation of bituminous coal. The by-products of the process are ammoniacal liquors, tar, and coke. In large plants the first of these is sold to ammonia works; the second to works for recovering coal-tar products, and the third is used for fuel in the plant or sold for domestic consumption. Small works are compelled to waste the coal-tar products and the ammoniacal liquors into the nearest watercourse, unless they are sufficiently near to recovery plants to make it profitable to ship them,

IRR $192-07-14$ 
The retorts in which the coal is distilled are of fire clay, and in the various works are differently designed. The gas is led from the mouth of the retorts by vertical pipes to a pipe dipping downward into a hydraulic main and extending beneath the surface of the water which partially fills the main, the back flow of gas to the retorts being thus prevented. The hydraulic main is a long covered trough to which all the retorts are connected. In it most of the tar and oily products condense beneath the water and flow to the tar wells, while the water itself dissolves most of the ammonium salts out of the gas.

The hydraulic main leads the gas to the condenser, which is constructed in several ways, but always with the purpose of cooling the gas in long iron pipes, whose lower ends dip beneath the surface of water held in an iron tank. The lowering of temperature effects a condensation of those constituents of the coal gas which at ordinary temperatures are not volatile, and forms a tar of them, which sinks to the bottom of the tank and flows to the tar well. At the end of the condenser is an exhauster, which keeps under suction the gas in its passage from the retorts to the end of the condenser and puts the rest of the plant under pressure.

From the condenser the gas is passed by the exhauster into the tar extractor, a short tower filled with many horizontal, perforated plates, in passing through which the gas is relieved by friction of its last traces of tar. It then goes to the scrubber, where it comes into contact with thin films of ammoniacal liquid from the hydraulic main or condenser, which trickles over pebbles, coke, etc. This liquid absorbs from the gas some of the carbon dioxide and hydrogen sulphide, which combine with the ammonia. From the scrubber the gas goes to the washer, where clean running water removes the ammonia.

The gas next flows to the purifiers, where its sulphur compounds are taken out. The purifiers are covered iron boxes which hold on a grating either slacked lime or hydrated ferric oxide. The gas enters from below and passes through the lime or iron, yielding up its sulphur as it does so. Usually the gas passes through four purifiers in succession. The lime in addition to removing the sulphur compounds combines with the carbon dioxide which the gas carries and which is an undesirable constituent. The iron extracts only the sulphur. In time both iron and lime become exhausted, being converted by the sulphur into very ill smelling sulphides. The iron can be renewed two or three times by exposing it to the air, which oxidizes it. In the end, both substances have to be disposed of, which is done by dumping them into the nearest stream, or sometimes by selling them. From the purifiers the gas goes through the meter house into the holders, from which it is delivered to the mains. The wastes in the manufacture of coal gas in works of considerable size favorably situ- 
ated for shipment are all recovered, because the by-productsobtained from them are valuable. Many small plants scattered about the country find it impossible to ship these products at a profit and therefore they turn them into the nearest watercourse, where they produce a highly objectionable state of affairs. The light tars float as an oily iridescent film on the surface of the water, giving it a disgusting appearance. The heavy tars sink to the bottom and foul the bed of the stream. The effect of the ammoniacal wastes is not visible, but ammonium salts are good food material for bacteria and microscopic organisms, and therefore the tendency of such wastes would be to foster their development. Moreover, gas wastes are believed to be very fatal to fish, and even when present in quantities insufficient to kill the hardy varieties, they impart a flavor of gas to the flesh which compels their abandonment as food.

Water gas.-Water gas is produced by the action of steam on incandescent carbon and is composed mainly of hydrogen and carbon monoxide. It has a high heat value, but is a poor illuminant, and therefore it has to be enriched with various hydrocarbons, such as ethane, ethelyne, acetylene, and benzene, which impart to it the necessary light-giving quality. As these substances are yielded by petroleum, it is used to carburet the gas.

The manufacture of water gas is carried on in three chambers. The first is called "the generator," the second "the carburetor," and the third "the superheater." The chambers are circular, the superheater being the tallest. Both it and the carburetor are lined with fire brick and are filled with a checker work of the same material.

Anthracite is put into the generator and brought to incandescence by a blast of air which is introduced at the bottom. Thus is liberated a gas known as "producer gas," which consists mostly of nitrogen, carbon dioxide, and a little carbon monoxide. This producer gas escapes into the top of the carburetor, and in circulating down through it is partly burned by a blast of air introduced near the top of the chamber. This combustion makes the brickwork hot. From the bottom of the carburetor the gas is conducted to the bottom of the superheater, where another blast accomplishes the complete combustion and raises the checkerwork to red heat, the gases finally escaping through a hood at the top of the chambers. When both the carburetor and superheater have reached the desired temperature the air blasts are shut off and superheated steam is blown in at the bottom of the generator. This forms carbon monoxide, or water. gas, which passes into the carburetor, where it meets a stream of oil introduced at the top. The oil is decomposed into illuminants which mix with the water gas and pass into the superheater, where they are completely fixed as incombustible gases. 
From the superheater the gas is led to a holder, from which it passes through a purifying apparatus involving principles identical to those that obtain in the manufacture of coal gas.

Water gas is very poisonous owing to the large percentage of carbon monoxide that it carries, and much of the harm that is commonly attributed to so-called sewer gas comes from defective gas piping and inefficient burners in conjunction with the water-gas service.

The tar from the manufacture of water gas is of less value than that from coal-gas production. It can be recovered and used as fuel in the plant, thus obviating the objectionable effects, similar to those of coal-gas tar, which result when the waste is turned directly into a stream.

\section{MANUFACTURE OF AMMONIA.}

The chief source of ammonia is the gas liquor from the hydraulic mains and scrubbers of coal-gas works. By destructive distillation the nitrogen contained in coal is largely converted into ammonium salts, the principal of which are the carbonate, sulphide, and sulphohydrate, which are volatile in steam, and the sulphate, thiosulphate, sulphite, sulphocyanide, and ferrocyanide, which are not. All of these compounds, together with free ammonia, are found in gas liquor.

The gas liquor received at the ammonia works is allowed to stand in order that the tar may settle out, and then clear liquor is distilled to separate the ammonia. There are several forms of apparatus for this purpose. In the simplest of them the liquor is heated in one still until all the volatile salts are expelled, after which it is drawn into another, where milk of lime is added. Then heat is again applied until the fixed salts are decomposed and the ammonia is driven off. The ammonia and volatile salts are condensed in a chamber containing sulphuric or hydrochloric acid. Some hydrogen sulphide and other foul-smelling gases pass out of the absorption vessel and are led into the chimney or otherwise disposed of.

The only liquid waste from ammonia works is the lime sludge from the exhausted milk of lime. It should be sedimented and the solid portion sold as fertilizer, for which it is well suited on account of the traces of ammonia salts that it contains. The supernatant liquid on the sedimentation beds contains lime salts in solution and is usually disposed of by running it into a convenient stream.

WOOL SCOURING.

Before raw wool can be manufactured it must be freed from the impurities which constitute 30 to 80 per cent. of its total weight. They consist of the "yolk" and suint; which exude from the body of 
the animal with the perspiration, and the dirt and dung mechanically mixed with them. The yolk is made up of fatty or waxlike bodies which are not easily saponified with alkali, but which can be emulsified with soap solutions and thus easily removed from the fiber. The suint consists mainly of potassium salts of acetic and fatty acids, together with sulphates, chlorides, phosphates, and nitrogenous bodies. All these substances are generally removed by washing the raw wool in especially designed machines. It was formerly a common practice to wash wool in stale urine, which was effective because of the ammonium carbonate it contained. This has been largely replaced by ammonia, soaps, etc. The wool is first immersed in a soap solution which contains more or less impurities from its previous use. From this machine it passes into a second containing cleaner water and finally into a third which contains clear water or fresh soap liquor.

The wool is then taken out and dried, while the foul-smelling, dirty-brown liquor from the first machine is sometimes drawn off, evaporated, and calcined to recover the potassium salts, which amount to 1 to 8 per cent of weight. In some countries the liquor in which the wool is washed is treated to recover the wool grease. T.o do this the liquor from the third tank is settled to remove the coarse dirt, and then sulphuric acid is added to decompose the salts and set free the fatty acids, which rise to the surface and carry the wool grease with them. The water is drawn off from the magma, which is placed in canvas bags. The grease is kept in liquid condition until all sediment is deposited, when it is turned into casks, in which it solidifies on cooling. It is used as a lubricant and in leather dressing.

In this country the potassium salts are rarely recovered, it being customary to run the entire waste to the stream. Some of the largest mills now remove the fats by a gasoline process and afterwards wash the wool to free it from dirt. The fats thus saved are valuable by-products. The amount of water used in scouring wool is usually 100 gallons to the pound, though.in some works it rises to 200 gallons. In a modern process of washing now coming into use the amount of water is reduced to 50 gallons. The liquid resulting from scouring wool is large in volume and rich in organic rnatter and in mineral matter in suspension and solution, and is not readily acted on by the agents of decomposition, putrefaction, and ritrification, so it is a most undesirable addition to the waters of a stream. It is well to distinguish between the scouring liquor proper, which is small in volume and contains most of the impurities, and the rinse water, which is of great volume and is relatively clean. The Massachusetts State board of health has found that wool scourings 
can be purified by sand filtration, after being mixed with considerable quantities of city sewage. So far as Potomac River is concerned, however, this is not important, for city sewage in many places is not to be had.

\section{WASHING WOOLEN CLOTH.}

In the process of washing woolen cloth large quantities of water are used, and the water is grossly polluted thereby, for it becomes charged with organic matter. When the rinse from the finished goods is discharged into streams, it adds considerably to the putrescible material they contain and is therefore exceedingly undesirable.

Waste from shoddy mills consists of dirt and organic matter washed from the rags and stock. Large quantities of acid are often used and the wastes are decidedly deleterious in their effect.

DYEING.

General discussion.-The art of dyeing consists of imparting to various substances, mostly fabrics, a color of considerable permanence. Dyes are distinguished from pigments by their solubility in water, and a pure supply of that element is a necessity to the trade. The solution of the colors requires care, those derived from coal tar being particularly prone to spot and streak the goods, if this part of the process is carelessly performed. The dyeing is done in iron, wooden, or stone vessels, whose shape and capacity are adapted to the amount and quantity of goods handled. Some dyes require a high temperature, while others are injured by much heat. In any case, direct heating is always avoided, it having been found best to heat the vats by steam coils.

The phenomena of dyeing are explained by the mechanical and the chemical theories. The former assumes a mechanical absorption of the coloring matter into the pores of the fiber, while the latter regards the process as a chemical combination between the dye and some or all of the constituents of the fiber. The chemical theory seems the better adapted to animal fibers, but the mechanical theory is perhaps more applicable to vegetable fibers.

Thorp groups dyes into five classes, according to the method of their application-(1) direct dyes, which yield full colors with the use of a mordant; (2) basic dyes, which form insoluble tannates and require a mordant on vegetable fibers, but which dye animal fibers without a mordant; (3) acid dyes, which do not require a mordant on animal fibers, but which have only a limited use on vegetable fibers; (4) mordant dyes, which require a mordant on both animal and vegetable fibers; and (5) special dyes, which can be applied to the fiber only by special processes. 
The trade distinguishes between substantive and adjective dyeing. In substantive dyeing the colors are applied to all fibers without a mordant, but " assistants," such as Glauber's salt, sodium phosphate, borax, soda, and soap are used with them to insure an even disposition of the color, thus avoiding streaky results. The assistants do not combine with the color. As direct dyes are very soluble, the goods are likely to bleed when washed, but silks take them very well, giving brilliant shades and fast colors. A little acetic acid makes them fast milling. Adjective dyeing requires the use of a mordant. Mordanting is of prime importance, and has for its object the precipitation on the fiber of some substance which has an affinity for and which will effect a more or less complete fixing of the coloring matter used for dyeing. The nature of the mordant depends on the character of the fiber, the kind of dye, and the effect sought. Wool is usually mordanted by boiling it in a solution of a metallic salt in the presence of some acid; bichromate of potassium and sulphuric acid are often used. Silk can be mordanted in this way, but it is usually done at lower temperatures. Cotton has little affinity for coloring matters and has to be specially prepared. It has an affinity for tannic acid and so is commonly steeped in a solution of sumac or catechu, after which it is washed and worked in a bath of some soluble metallic salt. An insoluble compound results which then has the property of uniting with the dye. It is not always necessary to treat cotton with tannin, for an immersion in the mordant followed by oxidation or ageing is sometimes sufficient. The operations of dyeing are multifarious, and it would be out of place to detail them here. Only the enumeration of some of the chief mordants will be attempted, with the description of a few common processes, in order to give an idea of some of the substances that may be met in dye-house effluents. The mordants are both mineral and organic. They are as follows:

\section{Mineral mordants:}

Aluminum acetate.

Aluminum sulpho-acetate.

Ferrous sulphate.

Ferrous acetate:

Nitrates of iron.

Nitro-sulphates of iron.

Potassium bichromate.

Copper sulphate.

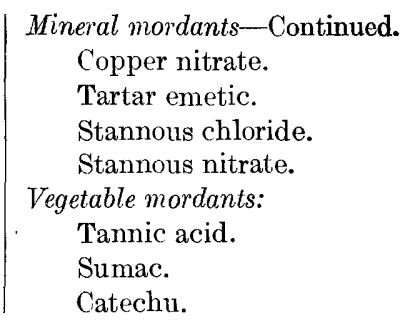

As assistants the common mineral acids are used, also acetic, oxalic, tartaric, and citric acids.

Cotton dyeing.-Indigo requires no mordant and is always applied cold. A common method is to make a mixture in definite proportions of water, lime, copperas, and indigo, and apply it in a series of vats. Zinc powder often replaces the copperas. 
Methylene blue is used to produce indigo shades. The cotton is mordanted with sumac at $160^{\circ} \mathrm{F}$., given several turns, and allowed to steep for ten hours, after which it is wrung out and worked for twenty minutes in a $2 \frac{1}{2}$ per cent solution of tartar emetic. Then it is washed and dyed in a bath prepared with 3 per cent acetic acid at $75^{\circ} \mathrm{F}$., the temperature being gradually raised to $160^{\circ} \mathrm{F}$.

Aniline black is a dye of unknown constitution, and the color is produced directly on cotton by means of aniline oil in the presence of oxidizing agents. Two methods of procedure are used-the warm and the cold. In the warm method 75 parts of water, 32 of hydrochloric acid, 16 of potassium bichromate, and 8 of aniline oil are taken. The acid and aniline are each diluted with water and carefully mixed. The solution thus obtained is then added to the main volume of the water. The potassium bichromate is previously dissolved and added after the aniline. The cotton is immersed and worked for three-fourths of an hour in the cold, after which the temperature is gradually run up to $140^{\circ}$ or $150^{\circ} \mathrm{F}$. In the cold method 18 parts of hydrochloric acid, 8 to 10 of aniline oil, 20 of sulphuric acid at $66^{\circ}$ B., 14 to 20 of potassium bichromate, and 10 of copperas are compounded as in the warm process, except that much less water is used. The goods are worked in one-half of the material for an hour or so, after which the rest is added and the operation continued for about one and one-half hours longer, when the goods are washed and boiled in a soap solution. In both processes the cotton is subjected to further oxidation with potassium bichromate, copperas, and sulphuric acid, which tends to prevent greening. Chlorate of soda is frequently used as an oxidizing agent in the dye bath, in which case the replacing of the potassium bichromate with vanadium chloride or vanadate of ammonium has been recommended.

Linen dyeing.-The uses to which linen is commonly put make it necessary that the colors it is dyed be fast. Accordingly, alizarin and indigo are generally used.

Wool dyeing.-Indigo is easily applied and extensively used to produce light and dark shades on wool by simply boiling the goods in a bath of the dye, sulphuric acid, and sodium sulphate. Loose wool is dyed in the so-called fermentation vat by keeping the wool below the surface of the liquid and working it with long rakes. After a sufficient time it is taken out and put in cord bags or upon rope screens and drained and oxidized, being then dipped into a dilute acid to remove the soluble impurities. Finally it is washed and dried.

Logwood is the real base of the blacks on wool, and is used with potassium bichromate as a mordant. As a rule the coal-tar colors are applied to wool without special treatment at boiling temperatures in a bath of 10 per cent sodium sulphate and 2 to 4 per cent of sulphuric acid. 
Sitk dyeing.-Silk has a great affinity to coal-tar colors, and can be dyed without a mordant, though soap is commonly used to prevent spotting and streaking. Heavily weighted goods are obtained by dipping them in an iron solution and then in liquids containing tannin, a process that is often repeated several times.

Besides the dyes mentioned in the above processes the following are in common use: Fuchsine, safranine, methyl-violet, and methylgreen.

Résumé.- The amount of organic matter contained in dye wastes is as a rule small, so that apparently they hurt the streams chiefly by discoloring the water. In places this may be a serious nuisance, while elsewhere it may be a trifling matter. The extent of the injury is determined by the use to which the water that receives these dyes is put. In some processes of dyeing much organic matter is washed from the cloth, and then the pollution assumes a new character and becomes important.

\section{MANUFACTURE OF WHISKY.}

The first step in whisky manufacture is the making of the mash, which is done by mixing in the mash tub raw and malted grains, the former largely predominating, with water at $150^{\circ} \mathrm{F}$., and agitating the mixture. The first malting requires about fourteen hours. Successive additions of water at $190^{\circ}-200^{\circ}$ maintains the temperature, the object being to convert all of the starch into maltose, which is directly fermentable by the action of the yeast, and to accomplish this the mashing must be done at a temperature of about $146^{\circ}$, for above it maltose production begins to decrease. By keeping within this limit of temperature the diastase from the small admixture of malt will not only greatly change the starch, but will bring about a hydration of the residual dextrine, converting it into maltose. When the wort has attained its maximum density, as found by the saccharimeter, it is drained off and a quantity of water at $190^{\circ} \mathrm{F}$. is run upon the residue in the mash tub and allowed to infuse with it for one or two hours. The second wort is then added to the first. A third weak wort is often obtained and used to infuse new lots of grain. It is stated that in this direct mashing 10 per cent of the starch escapes decomposition, even though the grain may be finely ground. Hence a little preliminary warming with the water, to which has been added a little ground malt, followed by heating with water under a pressure of several atmospheres, often precedes the addition of the main quantity of malt, which is to complete the conversion of the starch and dextrine into maltose. Reduction of the loss by 5 to 10 per cent is reported by this method.

The second step in the manufacture of whisky is the fermentation of the wort. This is done by adding to the wort, which is always cooled before beginning the process, yeast (usually fresh brewer's or compressed 
yeast) that has previously been softened in water. For 100 liters of grain, 8 to 10 liters of liquid yeast or $1 \frac{1}{2}$ kilograms of compressed yeast are used, and the best results are obtained when the temperature through fermentation rises to $94^{\circ} \mathrm{F}$. Three stages of fermentation are recognized-a preliminary fermentation, during which the yeast cells grow without producing much alcohol; a main fermentation, during which the maltose is fermented; and an after fermentation, during which the dextrine is largely changed into maltose, which in turn is changed into alcohol. The time of fermentation varies from three to nine days, and the process is carried on until the density of the liquor as determined by the saccharimeter ceases to lessen.

The third step in the manufacture is the distillation of the fermented wort, which is done in stills varying from great simplicity to extreme complexity in construction.

The refining of spirits consists of the redistillation of the "low wines," as the product of the first distillation is known. The low wines have a specific gravity of 0.975 , and the first product of the redistillation is a milky-white spirit abounding in oil. This is followed by a clear spirit that is caught separately, and the remaining weak spirit, known as "faints," is mixed with the low wines for another distillation.

After distillation the whisky is put in barrels, the inside of which has been charred. This is done to give the well-known amber color to the whisky and more particularly to assist in its aging, for the charred wood brings about a very complete oxidation of certain constituents of raw whisky, thereby imparting a delicate bouquet to it, and also relieves it of the injurious constituents. The barreled liquor is stored under bond for various periods and then is marketed.

The waste in the manufacture of whisky is the exhausted mash and residue from the stills. In many cases it is disposed of by feeding it to hogs and cattle. These animals are often herded under the most disgusting conditions, and the nuisance they create is almost greater than that which would result from turning this highly putrescible waste into a small water course. Sometimes the animals raised on the waste are disposed of to packers at favorable prices, and as often they are sold at a loss, so that from both a sanitary and a financial standpoint this method of disposal may be considered a failure.

The exhausted mash is a valuable food material when properly dried, and many works are recovering it and placing it on the market as a cattle food. The large volume of liquor treated makes the process an expensive one, and the profits are not always sure. A profitable method of disposing of this waste has been devised, and the reader is referred to Water-Supply and Irrigation Paper No. 179 for information concerning it. 


\section{POLLUTION IN NORTH BRANCH OF POTOMAC RIVER BASIN.}

\section{GENERAL DESCRIPTION.}

North Branch of Potomac River rises at the Fairfax Stone, the extreme southwestern point of Maryland, at the eastern edge of the high plateau which divides the waters of Potomac and Blackwater rivers. The stream is the boundary line between Maryland on the north and West Virginia on the south. From its source to the towns of Piedmont, W. Va., and Westemport, Md., the river flows in a general northeasterly direction in the narrow valley it has carved for itself between Backbone Mountain, Maryland, and New Creek Mountain, West Virginia. The course of the river is tortuous and its current is swift. It has high steep banks, and the bed is filled with stones and bowlders. The channel lies nearer Backbone than New Creek Mountain, with the result that the northern or Maryland streams, with the single exception of Savage River, which has cut its way across the northern valley wall, are shorter than those on the West Virginia side. Both the northern and southern tributaries are torrential in character, being typical bright, tumbling mountain brooks. The West Virginia feeders are most important, the largest of them taking on the dignity of rivers, while most of the others are locally known as creeks, in contradistinction to the little runs that enter from Maryland. Both sets of streams have precipitous, quickspilling watersheds; consequently in winter and spring the river and its tributaries are high and even impassible; in summer the feeders shrink and become relatively less important, and the main stream dwindles away to a thread which winds its hurried way through the stony bed. This region is sparsely settled, but mining and lumbering, the chief industries, have concentrated the population in about twenty hamlets, situated on the railroad, which closely follows the river as it winds in and out among the mountains.

\section{NORTH BRANCH OF POTOMAC RIVER FROM WILSONIA TO GEORGES CREEK.}

Wilsonia, W. Va., was formerly the westernmost of these settlements and had a sawmill which supported a small population; now it is abandoned, and Henry, W. Va., the station next below it, is the first important place in the valley. A coal mine here discharges a considerable amount of mine water into a little stream to the west of Elk Run. This is the first important pollution which North Branch receives, and the amount is large as compared to the total volume of the river at this point. The houses set well back from the river and such pollution as is received from them is indirect.

At Dobbin, W. Va., 2 miles below Henry, a dam is built across the river to furnish power for a sawmill, which is the life of the settlement. As the company uses the sawdust for fuel, it is disposed of 
without injury to the river. The houses of the operatives are on both sides of the river and most of them are at some distance from it, consequently only a few of the privies pollute the river directly. A primitive water supply is provided, the source being several springs in the neighborhood. The water is piped through the main streets on the southern side of the river only.

At Bayard, W. Va., 4 miles below Dobbin, is a tannery of the American Hide and Leather Company, employing 50 hands. The waste, which consists of leakage from the bark-liquor vats and water from the boiled fleshings, together with a little lime water, enters North Branch a short distance below Buffalo Creek. At the time of the last inspection lime sludge was piled dangerously near the edge of the river bank. At no time during these inspections have any large amounts of waste liquors been observed discharging from the tannery into the river, but the fact that the bed of the stream is stained a deep brown color would indicate either that such has been the case in former times or that it occurs now at infrequent intervals. It is said that recently, when the tops of the bark-liquor vats were shortened 18 inches, a considerable pollution took place. There is no public water supply in Bayard, and the tannery takes its water from Buffalo Creek, which flows through the town and is somewhat polluted with mine water. The water from this stream is said to be less damaging to boilers than that of North Branch at this point. The pollution from privies in Bayard is mostly indirect, but there are a few dangerously near Buffalo Creek.

At Gormania, W. Va., 2 miles below Bayard, the A. G. Hoffman \& Sons Company maintains a tannery, with 75 employees. The only wastes that enter the river directly are the weak lime liquor and that from the scouring machines. The company has built a settling pool at the edge of the stream near the factory and into it drain the heavy lime liquor, sewage from the employees' houses, and the small quantity of bark liquor that is wasted. The liquors from the pool seep slowly through the earth into North Branch, and as occasion requires the sludge at the bottom is removed to farms to be used as a fertilizer. This arrangement is a great improvement on the common practice of turning all tannery wastes directly into the river. The appearance of the river above the tannery is clear and it has some color, but more is acquired as it flows by the Hoffman tannery. ' As the current is somewhat slackened by a bend in the stream below the tannery the river has a tendency to deposit such material as it has in suspension. The water supply of the town consists of private cisterns, wells, and springs. Some of the privies in the town are too near the river and all of them furnish indirect pollution. On the whole, however, Gormania is an unusually clean town for this section of the country.

Stoyer, Md., is the next town downstream. It has no public water 
supply and the use of privies is universal. A coal mine on the heights above the town discharges its mine water into a run which enters North Branch at this point.

Wallman, Md., owes its existence to a sawmill which is polluting the river with sawdust. To escape the Maryland authorities it dumps the sawdust within 10 feet of the West Virginia shore, and by this subterfuge works continuous injury to the stream without molestation.

Opposite Hubbard, W. Va., is a coal mine, and a short distance above a sawmill, with a huge pile of sawdust not far from the river.

At Harrison, W. Va., Abram Creek empties its waters, polluted by mine waste; into North Branch. On the Maryland side of the river, somewhat below the town, is Wolfden Run, which is likewise polluted by mine water. Below this run, at the edge of the river, are 23 privies, besides those belonging to the houses at intervals all the way to Blaine.

Blaine is an unincorporated town situated on both the Maryland and West Virginia banks, 1 mile below Harrison. Here a woolen mill which employs 10 men turns into the river spent dyes and rinse water from the finished goods to the amount of 750 gallons a day, besides the sewage of its employees. The water supply at Blaine is from private wells and cisterns. The use of latrines is universal and some of them pollute the river and the race which supplies power to the woolen mill. Others are located upon low ground in the center of the town and are scoured out by the river in times of high freshets. The privies are not cleaned, but are moved to new trenches when it becomes necessary to fill the old vaults. Thus the indirect pollution of the river is considerable.

Three Fork Run enters North Branch at Chaffee, W. Va., 4 miles below Blaine. This stream carries its quota of mine water and is likely to become more polluted in the future than it is now, because of the probable extension of mining in its valley.

At Shaw, W. Va., 4 miles farther downstream, the river receives - Deep Run, which is heavily polluted with mine water and with the wastes of Atlantic and Elk Garden, two mining towns high up among the hills far back from North Branch. Elk Garden is the more important of the two, having, in fact, a considerable population. Its water supply is from private wells and cisterns, and it has no sewerage, the use of privies being general. When these are cleaned, the soil is removed to the commons on a tributary of Deep Run and buried. Elk Garden has no sewers, but there is one drain for surface water only, which reaches North Branch by way of Deep Run.

Savage River, whose watershed supports no mines and is given over to lumbering, joins North Branch near Bloomington. As its drainage area is practically uninhabited, the water is relatively pure and, being 
of nearly equal volume to that of North Branch at this point, serves greatly to improve its character by diluting the impurities. ${ }^{a}$

Between Bloomington and West Virginia Central Junction, North Branch receives a considerable amount of mine water. The town of West Virgimia Central Junction contributes its share of indirect pollution. The river passes over a dam here and with its fall changes its enviromment and character. Up to this point it is a turbulent mountain stream to whose immediate banks the population is confined. - Back from the river the Maryland watershed is umimhabited save for a few isolated farm houses here and there, and the population supported on the upland drained by its West Virginia tributaries is scattered, except that in some places about the country stores a few homes are clustered. From this point on, North Branch becomes less wild and on its banks appear populous towns with varied industries and in some cases sewerage systems. The influences of nature are less manifest and the artificial conditions imposed by man are everywhere visible.

A little below the dam the town of Luke, Md., has been built up by the West Virginia Pulp and Paper Company. Seven hundred men are employed in the factory, and the sewage which they create is turned straight into North Branch, as is also the waste from the factory, which is the largest industrial plant in the whole Potomac basin. Soda pulp, mechanical wood pulp, and paper are made here, and enormous quantities of alum, soda bleach, and lime are used, besides kaolin, glue, size, and ultramarine blue. The discharge of the waste chemicals and the shreds of wood fiber radically alter the character of the river at this point.

From Luke it is but a short distance to Westernport, Md., and Piedmont, W. Va., two towns on opposite sides of the river, which have identical interests and form one community. Piedmont has a sewerage system and pollutes North Branch both directly and indirectly. ${ }^{b}$ The town owns its water supply, which, by arrangement with the West Virginia Pulp and Paper Company, is pumped from Savage River. Though the water undergoes no process of purification, it has* so far been used with good results, being only slightly polluted, owing to the almost entire absence of population on the watershed from whence it is derived. Westernport for the last three years has bought its water from Piedmont. Its use is general in the town, for the wells along Georges Creek have been destroyed by infiltrating waters from the creek, so that the only wells in use are those in the western part of town, which is higher than the other parts. The town has two sewers. One of them has 50 connections, runs parallel with the main street, and is 600 feet long and 30 inches in diameter. The other has 500

$a$ The results of measurements of Savage River at Bloomington are given on pp. 43-46.

$b$ The results of measurements of North Branch of Potomac River at Piedmont are given on pp. 46-54. 
connections, is partly open, and is 500 feet long and 36 inches in diameter. Both discharge into Georges Creek near its mouth. Besides these a few private sewers empty into the creek and North Branch.

\section{GEORGES CREEK, 0}

Georges Creek has its source on Big Savage Mountain, and drains the heart of the soft-coal region of Maryland. At its very head at New Shaft a large stream of mine water is constantly running, and thence all along its course accessions of mine water are received at short intervals, so that by the time Westernport is reached the waters are surcharged with mine drainage, and are lethal instead of life giving in character. They destroy all vegetable and animal life in the channel, and stain the rocks on which they flow yellow with a deposit of iron hydrate.

At the head of the creek on a high peak of Big Savage Mountain is the city of Frostburg. It has a good public water supply from springs on the mountain, and the Frostburg Water Company furnishes an auxiliary supply derived from a well and springs. The city for the last three years has had about 15 cases of typhoid fever each year. Some of the cases are of outside origin. There is no sewerage system in Frostburg nor is there need of any, for the coal mines beneath the town drain all the cesspools and care for some of the surface water as well.

Below Frostburg are Borden Shaft, Ocean, and Midland, all mining communities in which crude sanitary conditions exist.

Lonaconing, about midway between .Frostburg and Westernport, is also a mining town. It has developed a public water supply on Jackson Run for a reservoir fed by two mountain streams upon whose watersheds but two or three families live. The water is at present delivered to 523 families, but the available supply is $5,000,000$ gallons a day. The fact that but nine cases of typhoid appeared in the city during eleven months of 1904 points to the comparative purity of the water. The death rate in the town is about 10 per thousand and is said to be augmented by the high birth rate, for the local statistics seem to show that a large number of children die before reaching 5 years of age. There are some sewers which serve a few houses, stores, and hotels and which empty into Georges Creek, as do the many privies that directly overhang it. Besides these, four shambles exist, one of which is far from the town, and buries its offal. The three others are in the middle of the town and are uscd by six butchers, who throw much of the offal into the creek, though some of it is fed to hogs. There is one steam laundry which drains into Koontz Run. 


\section{NORTH BRANCH OF POTOMAC RIVER FROM GEORGES CREEK TO WILLS CREEK.}

At Westernport North Branch turns and flows in a southeasterly direction across the foothills of New Creek Mountain until it reaches Keyser. At this place the most obvious contamination is from the mill of the Patchett Worsted Company, which scours 1,800 pounds of wool a day and uses 100 pounds of aniline dyes a week. The wastes are turned directly into the river. This company has had some trouble with its water supply. The Potomac water, it is claimed, makes the wool which is washed in it harsh, while the waters of New Creek stain the wool badly.

On New Creek, 6 miles above Keyser, a plant of the United States Leather Company is located. Above this plant the water is colorless, or nearly so, while below the place where the tannery discharges liquors the stream becomes red or almost black. Though the color is reduced in the course of flow to the river, the water is still tawny at the creek's mouth. The tannery employs 99 men. In the summer of 1904 there was an outbreak of typhoid among them, due, it is thought, to contaminated springs and wells in the neighborhood. New Creek is polluted by sewage also, for while the use of privies is common in Keyser a part of the town is pretty thoroughly sewered, and the sewer empties into the creek, as does that of the Baltimore and Ohio Railroad shop, with 300 employees. The latter connection is indirect, reaching the creek through Longs Gut. Typhoid fever is said to be rare in Keyser, by reason of the purity of the water supply in general use. The source is a mountain spring 4 miles east of the town. The reservoir has a capacity of $6,750,000$ gallons and affords a pressure of 160 pounds per square inch in the lowest part of the town.

WILLS CREEK AND CUMBERLAND. $a$

At Keyser the Potomac resumes its northeasterly course and flows - between Dans Mountain on the north and Knobly Mountain on the south to Cumberland, where it receives the waters of Wills Creek. The drainage area of this creek must be carefully studied in order to understand sanitary conditions in Cumberland.

Wills Creek rises on the western slope of Savage Mountain, Pennsylvania, and runs northwestward to Mance, where it turns and flows eastward to Hyndman. The population on its entire watershed above Hyndman is not more than 1,000, and not many of the small villages need especial notice.

Foley is a railroad station, with a few houses huddled about it. Most of these are provided with privies that pollute the creek, which runs on to Fairhope, where there is a brick factory, and where the stream is further contaminated by overhanging latrines. Identical

$a$ The results of measurements of Wills Creek and North Branch of Potomac River at Cumberland are given on pp. 58-64, 
conditions exist at Williams, the next town below, and at Hoblitzell, a small agricultural community, a few more houses add their filth to the water course.

Hyndman is the largest town on Wills Creek. Its public water supply comes from a spring on the west side of Wills Mountain, $1 \frac{1}{2}$ miles from town, and its purity is evidenced by the fact that there is rarely any typhoid fever in the place. There is one sewer, about a quarter of a mile in length, which takes slops from the houses on the line and soil from a few of them and from the bank and hotels. The rest of the inhabitants use privy vaults, which seem to be well cared for by the local board of health. The tannery of the Elk Tanning Company, which employs 55 men and makes oak-tanned sole leather, on the eastern bank of Wills Creek, when first visited poured all its wastes directly into the creek. Since then conditions have been bettered, for the company has constructed at the edge of the stream near the plant a settling pool with a capacity of 5,500 cubic feet, and all wastes are turned into it. At the time it was inspected it was full and overflowing a little, and it was observed that a sluice with a gate had been provided by which direct communication with the creek could be established in case the capacity of the pool was overtaxed. In spite of these defects the new arrangement is commendable, and has done a great deal toward clearing up the waters of the stream. A privy which is used by the employees overhangs the creek and a water closet in the office of the company is also connected with it. The capacity of the plant is 105 hides a day, and the total wastes are estimated at 2,000 gallons a day.

Some distance below Hyndman is Ellerslie, where there is a planing mill and a pumping station of the Standard Oil Company. At Corriganville Jennings Run joins the creek. This stream heads at Frostburg, and both near that town and at other places in its narrow valley receives large quantities of mine water. At Mount Savage it is joined by Mount Savage Run, which is polluted by many overhanging privies, and upstream by the acid waters of a fire-clay mine. Mount Savage is located on the steep slopes of high hills, so that the run-off through the town is very quick, and in times of heavy rains a great deal of the refuse of the town is washed into Jennings Run or its tributary, Mount Savage Run. At Barrelville a small tributary enters Jennings Run from Wellersburg, and that too is contaminated by mine water from the coal mines which are being opened up in its valley; but from Barrelville to its mouth the run is befouled but little more.

Below Corriganville there is no notable pollution until Braddock Run is reached. This stream heads at Eckhart Mines, a thriving mining town that is supplied with water by the Frostburg Water Company. Privies and domestic wastes defile the run, but it is free I̦RR $192-07-15$ 
from mine water, owing to the fact that the waters of the mines beneath the town are pumped into Georges Creek near Borden Shaft. The completion near Clarysville of a tunnel, now far advanced in construction, 11,000 feet long and 7 by 8 feet in cross section, will divert the drainage of the Eckhart and Hoffman mines from Georges Creek to Braddock Run, into which it was formerly pumped. The effects of the consummation of this project will be far-reaching. Braddock Run, whose waters are now comparatively soft and suitable for boiler purposes, will be converted into a stream with the characteristics of Georges Creek at the present time, while the water of Georges Creek will become less acid and so less potent in its effect on the limy waters of North Branch at Westernport. Moreover, with the reduction of its acidity the Georges Creek water is likely to become less noxious to bacteria, so that the sanitary conditions which prevail along the creek will be vastly more important than they now are to the towns below it, notably to the city of Cumberland, whose waterworks intake is at Ridgely, on North Branch, one-half mile above Wills Creek. The Cumberland water now receives the benefit of the clarification and germicidal action which the united waters of Georges Creek and North Branch accomplish about 30 miles above it, and should this action become less effectual it is conceivable that Cumberland might find its water supply less pure than it is now. The acid waters of Braddock Run after this change is made effective will increase the acidity of Wills Creek, and that in turn will work its effect below. At present Braddock Run flows from Eckhart Mines to Allegany Grove, a camping and picnic resort where many large privies pollute it badly. Somewhat below Allegany Grove is the plant of the James Clark Distilling Company, whose wastes, consisting of slops from the distilled grains, are conducted through a 10-inch terra-cotta pipe along the sides of the run to the Narrows, where they are discharged into Wills Creek. The concern mashes 300 bushels of grain a day, but the amount of waste disgorged into Wills Creek is problematical, as a farmer below the distillery taps the pipe line and withdraws what he needs for his cattle, while another one hauls away enough to supply a herd of forty. The fact that the distillery is closed down in summer is undoubtedly of benefit, for the waste, which is putrescible, is thus usually discharged only in times of comparatively high water, which tends to dilute it. Moreover, at summer temperatures a local nuisance would be created about the Narrows by rapid decomposition of the distillery slops, whereas under present conditions, at the lower temperatures of autumn and winter, decomposition is more gradual, as it takes place at a comparatively slow rate and over a considerable distance.

Below the Narrows of Wills Creek is a tannery of the United States Leather Company, where the course of the stream varies with its 
stage. In times of low water practically the entire stream is diverted by the dam at the tannery into the Beal mill race, which flows through the most populous part of Cumberland into the Chesapeake and Ohio Canal. When the creek is high, so much of its waters as do not go through the race continue over the dam and flow through the business section of Cumberland to the head of the Chesapeake and Ohio Canal, into which, when the gates are open, some of the water goes, while the rest joins North Branch. At a point a little below the confluence Dam No. 7 has been built. Its purpose, like that of the other dams on the Potomac, is to hold back the waters of the river and make them available for feeding the canal; its effect is to check the current of Wills Creek and to create slack water for 3 miles up North Branch. It can be easily understood that in times of drought, when no water passes over the dam at the tannery on Wills Creek and none over Dam No. 7, there is formed a bow-shaped pool of practically stagnant water, one arm of which extends from the Wills Creek dam to North Branch and the other from Dam No. 7 up North Branch for 3 miles. The river arm is but little polluted, while the creek arm throughout its length of a mile through the center of Cumberland is very much so. Into it slaughter barns, privies, steam laundries, breweries, and some of the sewers of the city discharge, producing a thoroughly regrettable condition of affairs. In time of high water this corruption is carried into the canal or river, but whenever there is a drought it lies festering in the midst of the city.

Cumberland is the largest city on the Potomac watershed above Washington. It is a vigorous, growing place, but its rapid increase in population has outstripped its development in sanitary matters. The water of the city is not free from contamination. As private wells are commonly polluted, most of them have been closed up. Cisterns are used to some extent, but the city supply is generally relied on by the citizens. It is pumped directly out of North Branch opposite Ridgely, 1 mile above Wills Creek, and is consumed without purification of any kind. This would probably be more disastrous than it now is were it not for the beneficial purification which, as explained elsewhere, takes place about 30 miles above the city. There is no system of sewage purification, though there is a sewerage system which serves about. 75 per cent of the city. Soil, slops, and some surface drainage are taken by the sewers, though about 95 per cent of the system carries no storm water, as most of the surface drainage goes directly to the race, canal, creek, and river. Six of the city sewers discharge into the race; two of these are 36 inches in diameter, one 12 inches, one 10 , one 8 , and one 6 . Besides these the race receives the droppings from many overhanging privies and is polluted by many private sewers. The dye works of Thomas Footer \& Son discharge wastes consisting of rinse water and spent dyes, estimated 
at 10,000 gallons a day, into the race, together with the sewage of its 310 employees. Moreover, the race is somewhat obstructed by ashes which are thrown into it. Besides the sewers discharging into the race, the following empty into the river: One 24-inch sewer, whose full capacity is probably never required, entering at Valley street; one 6 -inch private sewer for sewage only, entering at a point about opposite the Town Hall; one 12-inch city sewer, and the 8 or 12 inch sewer owned by Allegany County. In South Cumberland a sewer empties into a brook that flows into the Chesapeake and Ohio Canal near the Queen City glass factory. In winter, when the canal is drained off, the stream and sewage flow over the canal banks directly into North Branch. About 5 per cent of the houses in South Cumberland are connected to this sewer.

Many people along Wills Creek find it to their advantage to sewer directly into it. Above the Market Street Bridge the United States Leather Company, which employs 100 men, discharges its tannery wastes, amounting to 10,000 gallons in twenty-four hours, together with the sewage of its employees, into the creek. Below this tannery the Cumberland Brewing Company, employing 50 men, empties the washings from its barrels and sewage from its employees. At the Market Street Bridge, on the west bank, the German Brewing Company pours out the washings from its barrels, amounting to 1,500 gallons per day, together with the sewage of its 35 employees. On the opposite bank a gas company lets some of its waste liquors escape. From this point down, on the eastern side of Wills Creek, there is a succession of overhanging privies, interspersed by the sewers of two steam laundries and by a few slaughterhouses, whose floors drain the blood of the animals and with it a small amount of offal into the creek (Pl. VIII, $A$ ).

Throughout the southwest part of that section of Cumberland lying west of Wills Creek flows a small run that is undoubtedly the recipient of much promiscuous refuse, which it empties into North Branch about one-fourth of a mile below the waterworks intake at Ridgeley. Whether this imperils the city water supply or not can not be stated, but the possibility of its doing so should be borne in mind.

The Chesapeake and Ohio Canal receives most of the irregular pollution of South Cumberland. The Baltimore and Ohio Railroad shops, employing $600 \mathrm{men}$, sewer into it, and so does the N. \& G. Taylor Company, a concern which employs 300 men and which makes steel and rolls it for manufacture into tin plate. In the process of manufacture large quantities of sulphate of iron are used, and this when pretty well exhausted finds its way into the canal.

There are many other industries in Cumberland, but none of them have liquid wastes. That other manufacturers will come to the city is more than likely, for Cumberland is advantageously situated and 


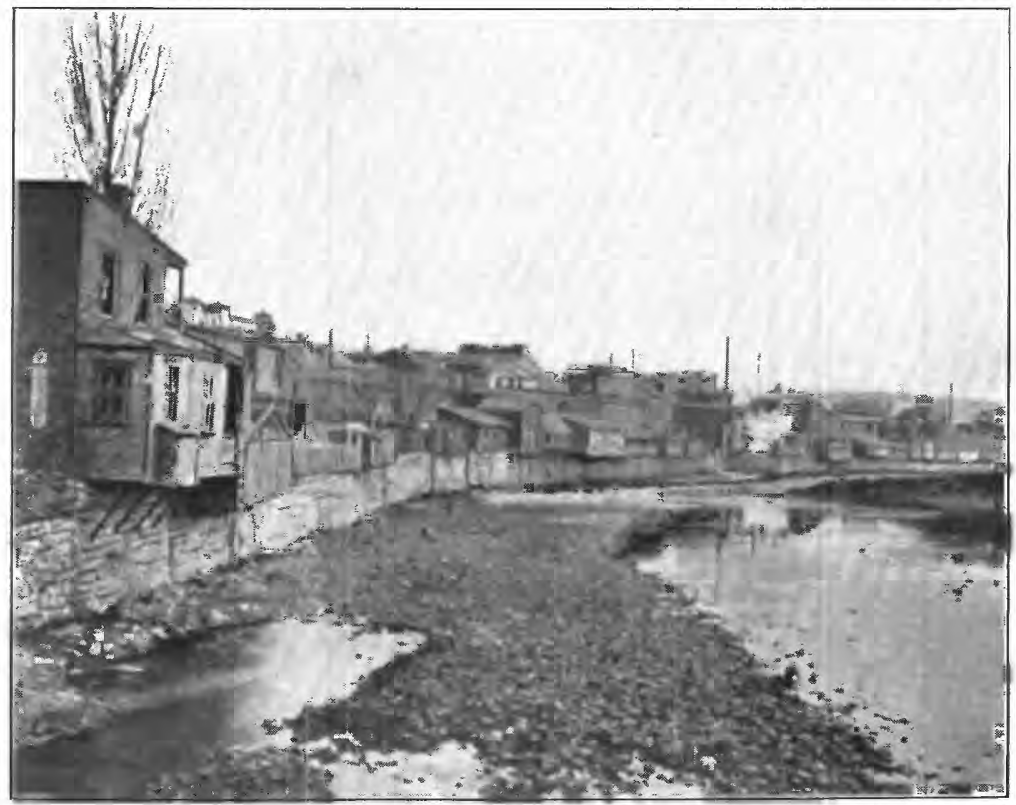

A. WILLS CREEK FROM MARKET STREET BRIDGE, CUMBERLAND, MD.

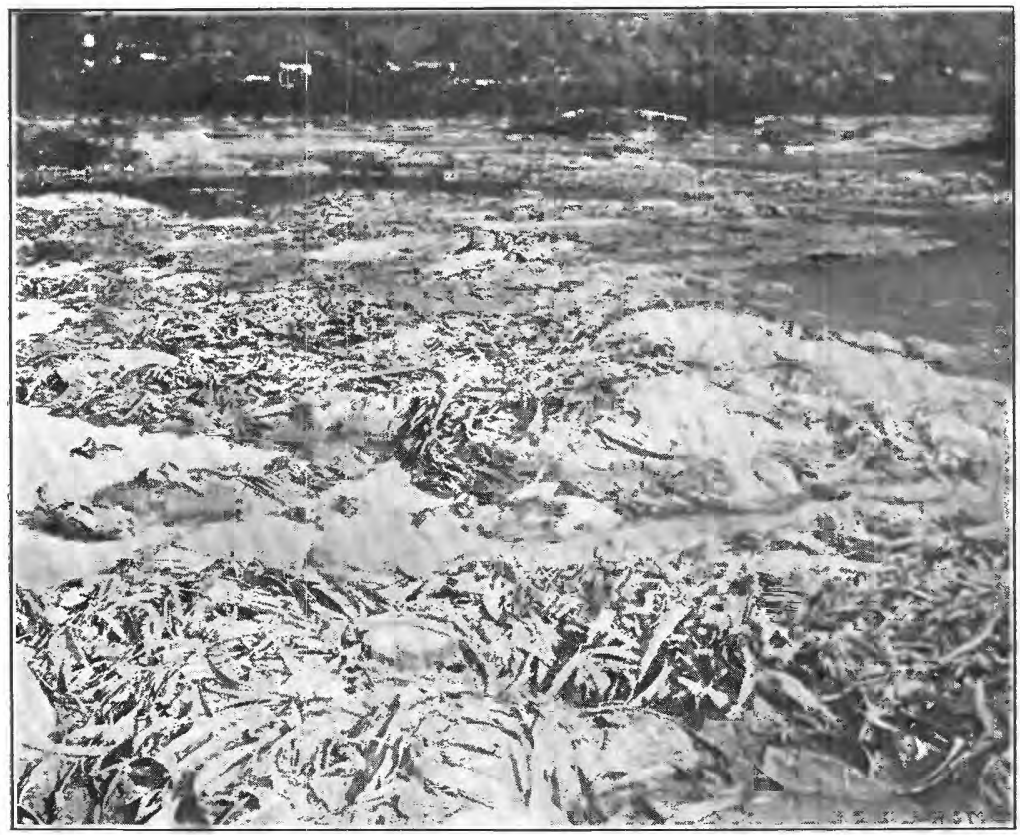

B. POLLUTION OF POTOMAC RIVER BY WASTES FROM THE MECHANICAL WOOD-PULP MILL AT HARPERS FERRY. 
i 
seems destined to enjoy much greater prosperity. This, however, will be still further increased by bettering the conditions above described. The city should adopt some method of disposing of its wastes which will relieve the river of doing so, and its water supply should be placed above criticism.

NORTH BRANCH OF POTOMAC RIVER BELOW WILLS CREEK.

Below Cumberland North Branch enters the Greater Valley of the Appalachian Plateau in that part known as the Allegheny Ridges, from the fact that mountains trending northeast and southwest cross it at frequent intervals. In the valleys between these ridges flow many streams, and the most prominent of these will be taken up in the order in which they empty into North Branch.

Evitts Creek, which drains the thinly settled country between Shriver Ridge and Evitts Mountain, enters North Branch from the north a short distance below South Cumberland, where its water is used for boiler purposes in the Baltimore and Ohio Railroad roundhouse. At one time it was advocated as a source of public water supply for Cumberland, but was rejected partly because of the prevalence of typhoid in its drainage area. Patterson Creek, the next important tributary, comes in from the south at Patterson Depot. Burlington, with a population of 250 , is the largest town in the Patterson Creek Valley; its inhabitants gain their livelihood by farming and logging. From Patterson Depot North Branch flows on to a point 2 miles east of Greenspring and then loses its identity, being joined by South Branch of the Potomac to form the main stream, whose conditions will be described after taking up South Branch.

\section{POLLUTION IN SOUTH BRANCH OF POTOMAC RIVER BASIN. $a$}

South Branch of the Potomac rises in Highland County, Va., at Hightown, on the divide separating the headwaters of James and Potomac rivers. The stream flows northeastward to a point 6 miles west of Petersburg, W. Va., where it receives North Fork of South Branch, which is formed at the north end of Middle Mountain by the confluence of Laurel Fork with Straight Fork and flows northeastward to its junction with South Branch. The stream continues in a northeasterly direction to Moorefield, where it receives the waters of - Moorefield River, a stream that flows in a northeasterly course from its source on Shaw Ridge north of Palo Alto, Highland County, Va., near the head of Cow Pasture River, a tributary of the James. The entire country drained by South Branch of the Potomac is very rugged. Several of the mountains rise to the height of 3,000 feet

$a$ The results of stream measurements on South Branch of Potomac River at Springfield, W. Va., are given on pp. 66-77. 
and there are many less lofty peaks. The watershed is much cut up by the multitude of creeks and runs that are tributary to the main stream, and these waters fall precipitously into the river from the mountains, making it rise and fall suddenly. In spring the melting of the snow produces very high water, which recedes quickly.

South Branch from its source to a point 6 miles west of Petersburg flows between North Fork and South Fork mountains, and lies between North Fork of South Branch and Moorefield River, both of which are parallel to it, all three streams flowing in a northeasterly direction. North Fork Valley has been eroded between Spruce and North Fork mountains. Its population of woodsmen and mountaineers is very sparse, so that the stream may be dismissed with the statement that it reaches South Branch almost unsullied. Moorefield River has cut its valley between Shenandoah and South Fork mountains; the region is unsettled and, like North Fork, the stream is but little polluted. The only source of contamination is a tanning-extract factory located in Brandywine, Pendleton County, W. Va., a place where it is said there has been much typhoid. When this plant was started the stream was discolored greatly by the leakage from the vats where the chestnut-oak wood was steeped. At the present time this leakage is insignificant and such coloring matter as enters the stream comes from the condenser water, which absorbs some of the abstract in the process of manufacture.

South Branch itself in its upmost reaches is polluted only by the wastes at Monterey, Highland County, Va., and by those of a few scattered settlements. At Franklin, however, where the stream has attained considerable size, the pollution is important. This town has a water supply from springs on Entry Mountain, but about one-third of the people use wells. The sewage must reach the river very indirectly, for the houses are a long way from the river bank and there is no public sewerage. One or two private sewers lead from the main streets of the town to the bottoms, but most of the people use privies, and the soil removed from these, when they are cleaned, is deposited in boxes near the river, which are scoured out by occasional floods. So far as information goes typhoid is rare in Franklin, though once in a while a case comes into town from the outside. The most extensive pollution of the river here is accomplished by the Franklin Tannery Company, whose wastes amount to practically 10,000 gallons a day. The factory is located in the southwestern part of the town, and its effluents are carried 1,100 feet through a 6 -inch terra-cotta cementjointed pipe line to a settling pool 83 yards from the river. Here the solid matters sediment out, and the liquids usually filter slowly through the ground to the river. In times of high water the earth becomes saturated, causing the pool to discharge at its south end 
directly into the stream, while in times of flood the entire settling basin is invaded by the river. This is considered unavoidable by the tannery company, because in its opinion the best available place has been utilized for the disposal of the waste. The company claims that this refuse, which must ultimately go into the river, is best discharged at such times, for in the huge volume of water that rushes downstream it is hardly noticed and presumably does comparatively little harm.

The country between Franklin and Petersburg is given over to grazing and farming and is very beautiful. The pollution received by the river in this interval is very small and is such as comes from scattered farmhouses and the domestic animals about them. At Upper Tract there is a country store and something of a settlement. Petersburg is a small town at which there is a tannery of the United States Leather Company that employs 30 hands. The wastes from this plant amount to 3,000 gallons daily and are discharged into Lunice Creek very near the point where it enters South Branch. At the time of inspection South Branch was in flood, so that the effects of the tannery wastes were not discernible, but it is entirely probable that in low water they discolor the stream, for there is no attempt to purify them. Petersburg has neither public water supply nor sewerage, and the only receptacles for soil are privies. The next town below Petersburg is Moorefield, which takes its water from Moorefield River, a short distance above the tannery dam on that stream. The daily consumption is 36,000 gallons. Besides this, nearly every house has its well, and these are locally supposed to furnish water of good quality. There are no sewers, but there are one or two drains for surface water. The tannery at Moorefield at the time of the inspection was owned by the Cover \& Drayton Company, but has now passed into the hands of the United States Leather Company. It is situated well back from the bank of Moorefield River, about 2 miles from the center of the town. The plant has a capacity of 50 hides a day, and its wastes, which amount to 10,000 gallons a day, are carried to a settling pool near the edge of Moorefield River. This pool is believed to be large enough to take care of the tannery wastes at all times, but in case it is not the liquors will be discharged directly into the stream. Like the settling pool of the tannery at Franklin this one is flushed out by floods.

At Old Field, a short distance below Moorefield, South Branch flows between Mill Creek Mountain and South Branch Mountain in a precipitous valley locally known as the Trough, while the highway turns around the southwest end of Mill Creek Mountain and follows the valley of Mill Creek to Romney, passing on the way the settlements of Purgitsville and Moorefield Junction. The inhabitants of these places are farmers and the population is very small. 
- Romney is situated on a high hill well back from South Branch, where it emerges from the Trough. It has a public water supply from springs, but no public sewerage. The State asylum for the deaf and blind located here has a private sewer that discharges into Big Run, and this rivulet carries the sewage into South Branch one-half mile below the town limits. From Romney to its mouth the valley of South Branch is peopled by farmers and there is no further important pollution.

The waters of this large stream are remarkably pure and should be protected from contamination. Its beautiful valley, with its rugged mountains, interesting streams, and fair intervales, so near the populous cities of the seacoast, is likely to yield a richer return as a wisely developed region of summer resorts than under exploitation by industrial interests.

\section{POLLUTION IN POTOMAC RIVER BASIN BETWEEN MOUTH OF, SOUTH BRANCH AND SHENANDOAH_RIVER.}

POTOMAC RIVER FROM MOUTH OF SOUTH BRANCH TO PAWPAW, $a$

The first important stream entering Potomac River below the confluence of North and South branches is Little Cacapon River, a southern tributary. A few miles below this stream is Pawpaw, W. Va., a small town at which there is a tannery that employs 140 men. The tannery wastes amount to 15,000 gallons a day. Part of them are sedimented near the river's edge, but the rest are discharged directly into the river and markedly discolor it.

From Pawpaw the river takes a meandering course between Town Hill and Sideling Hill. At the hamlet of Little Orleans Fifteenmile Creek enters from the north, and 4 miles farther downstream is the mouth of Sideling Hill Creek. The Potomac then cuts across Sideling Hill and Tonoloway Ridge, on the eastern side of which lies the village of Great Cacapon.

GREAT CACAPON RIVER.

A short distance east of Great Cacapon is the mouth of Great Cacapon River, which is formed at Forks of Capon by the junction of North River and Cacapon River.

North River rises in South Branch Mountain, Hardy County, W. Va. Two tanning-extract factories are located in this stream, one at Inkerman and the other at Rio. Cacapon River, or Lost River, as it is known above Wardensville, on account of its sinking beneath its bed for a short distance, rises in North Mountain, Hardy County, W. Va. There are two tanneries in its basin, one at Lost City and the other at Capon Bridge. Each employs 25 men and turns out 50 hides a day. The wastes are said to be sedimented before being turned into the river. 
Great Cacapon River is less than 75 miles long. For a considerab distance above its mouth it is about 150 feet wide. It is made up of a succession of rapids and pools. In some of the pools the water is deep, but the rapids are shallow. In ordinary stages the river is fordable at almost any place except in the pools. The lower part of the river has a considerable fall per mile and is full of large rocks and bowlders.

\section{POTOMAC RIVER FROM GREAT CACAPON RIVER TO CONOCOCHEAGUE OREEK.}

The next important tributary below the Great Cacapon is Warm Spring Run, which enters the Potomac from the south at Brosius. This stream is polluted by sewage from the town of Berkeley Springs, which is 6 miles from the Potomac and which owes its existence to thermal springs that supply the community with water and are held in high esteem. The hotels and leading houses are served by a sewer which discharges into the run about one-half mile north of the town.

Opposite Brosius is Hancock, one of the oldest towns in Maryland. It is a small place without factories, and as it is located well back from the river the trivial refuse that is created there must reach the stream either very indirectly or by the two runs which flow along the extreme east and west ends of the town. The pollution at Hancock may become more important in the future if the stimulus recently received from the advent of the Wabash Railroad is sufficient to develop steady growth. From Hancock the Potomac continues its way eastward. From the north, a little east of Hancock, it receives Great Tonoloway Creek; from the south, a considerable distance farther on, Sleepy Creek; and beyond that, from the north, Licking Creek, a stream which rises in Bedford County, Pa., and receives the indirect pollution of McConnellsburg, a sizable country town, where there is a tannery having a capacity of about a hide a day. A few miles below Licking Creek, Back Creek joins the river from the south. Above Dam No. 5 the Chesapeake and Ohio Canal enters the Potomac and becomes one with it for miles, not resuming its separate course until it reaches Dam No. 5, 7 miles above Williamsport, Md., where Conococheague Creek enters the river.

\section{CONOCOCHEAGUE OREEK.}

Conococheague Creek has intrenched itself in the eastern edge of Cumberland Valley and drains a well-populated, prosperous region. The stream rises in South Mountain, Adams County, Pa., and flows in a westerly direction by many villages. At Scotland there is a large industrial school, which disposes of its sewage by the Waring system, a matter of importance to Chambersburg, the city next below it. The water supply of this flourishing city is pumped from Conococheague Creek at a point 2 miles upstream and is delivered without purification to the citizens, nearly all of whom are dependent on it, 
for the well water is too hard to be used satisfactorily in boilers and is commonly so polluted that the wells have fallen into disuse. Chambersburg has no public sewerage system, probably because the seams of the much fractured limestone on which the city is built act as conduits for the sewage of the cesspools that are as a matter of course provided for every house and carry it away, presumably to the creek. There are two private 10-inch sewers, to one of which are connected three hotels and fifteen private dwellings and to the other a hotel, the trust company, and the court-house. There is also a private 8-inch sewer which is used by 25 families and another for the 300 employees of the Wolf Company. All these sewers empty into Conococheague Creek, which is further corrupted by a part of the sewage of the Wilson College for Girls, an institution having 400 students, by the washings of a creamery and of a beer-bottling establishment, by the spent lye of J. G. Gerbig \& Sons' soap factory, by the residues of the Chambersburg Gas Company, and by the spent dyes of a small dyehouse which maintains its own connection to the creek. Moreover, three slaughterhouses utilize the stream for the disposal of their offal. Its tributary, Falling Spring Run, increases the pollution, for to this little stream are connected the urinals of the Cumberland Valley Railroad shops, which are used by 230 men, and the five water-closets of its main office building, where there are 50 employes. The run is also the recipient of the sewage of the 90 hands of the Chambersburg Shoe Company. The Chambersburg Woolen Company voids into it the sewage of the 95 employees, together with wastes consisting of spent dyes and rinse water from the finished goods; and, finally, a steam laundry adds its affluent. From Chambersburg Conococheague Creek runs southwestward for 15 miles to the point where it receives the waters of Back Creek, a stream on which is located Williamson, a small town, where a large dairy and butter factory pollutes the water.

Four miles below its confluence with Back Creek, Conococheague Creek is joined by Moss Bank Run. This little stream probably receives practically all of the sewage which leaks away from the cesspools in the limestone that underlies the city of Greencastle. The run at the eastern edge of the city receives the washwater and dyes of a little woolen mill and then disappears below ground, apparently to reappear on the western side of the city, from which point it flows eastward 1 mile to Conococheague Creek. Greencastle is supplied with water by the Greencastle Water Company from Eshleman and Spangler springs, 2 miles east of the city. It is a gravity supply and was put in at a cost of $\$ 30,000$.

From Moss Bank Run Conococheague Creek flows southward for 5 miles, and then receives the waters of its West Branch, which rises on the divide between Cumberland and Franklin counties and- flows southward until it reaches the main stream. The first contamination 
of West Branch is received a short distance above Mercersburg Junction, where the waters of Trout Run come in. This stream heads in Cove Mountain a little to the west of Foltz, in Cove Gap, and passes through the hog yard of a small distillery. The animals are fed on the slops, and while they doubtless dispose of most of it probably a part escapes, and with the excrement of the swine contaminates the stream.

From Mercersburg Junction, West Branch continues to a point about 2 miles east of Mercersburg. This city has a public water supply derived from Trout Run, before mentioned, at a point above the distillery known as Buchanan's birthplace, where there is a dam across the run, the slackened waters being piped to an equalizing reservoir and thence to the city. The supply is probably very satistory, because the waters are, tapped in the Pennsylvania Forest Reserve, which insures their freedom from contamination. The water is used generally in the city, but is supplemented by six public wells, which are on the main street. The sewerage system consists of two sewers, one a 6 -inch pipe 2,500 feet long, laid through the main street, and the other an 8-inch pipe 800 feet long, known as the Church street sewer. Besides these, Mercersburg College, which has 300 students, has a private sewer. All this sewage, together with the effluent of the tannery, is discharged into a little rivulet known locally as Dickeys Run, heading back of the town. When inspected at the point-where it crosses the main street this run was simply a brooklet of blood owing to the discharge of a slaughterhouse near by. Below the slaughterhouses is located the tannery of W. D. Bryon \& Sons. The capacity of the plant at present is 75 hides a day, but it is being increased. The wastes now amount to 2,500 gallons a day, and by agreement with the property owners below are discharged only at night. The length of the run to West Branch is 2 miles. Near its mouth, at the time of inspection, it did not show evidence of the gross pollution which it receives, though as it was inspected in daytime the tannery effluent was not a factor.

West Branch joins Conococheague Creek 10 miles below Dickeys Run, and from the confluence the creek continues without conspicuous pollution to Williamsport, Md., where it joins the Potomac. This town was at one time visited by General Washington to determine its advantages as the future capital of the United States. At present it is a large, beautifully located village without a public water supply and without sewerage, though authority to construct the former was given at the last session of the legislature. There is a little manufacturing done, but the industries do not create liquid wastes, with the single exception of the tannery of W. D. Bryon \& Sons. This firm employs 250 men, and its wastes, amounting to 16,000 gallons a day, are discharged into Conococheague Creek but a short distance above its mouth. 
OPEQUON CREEK.

Opequon Creek rises east of Stephens City, Frederick County, Va., and flows northeastward, emptying into the Potomac River $2 \frac{1}{2}$ miles east of Martinsburg, W. Va. In general its course is straight, but from Middleway to Martinsburg it is somewhat meandering. Although this stream is small, and through most of its course flows in a farming region in which it receives but little pollution and that mostly indirect and derived from enriched land, domestic animals, and a score of scattered hamlets, yet from the sanitary standpoint it is important because of the nature and amount of sewage received at three different places.

Winchester, the county seat of Frederick County, Va., is situated on Abrams Creek, 5 miles from Opequon Creek. It has a public water supply which consists of what are known as the old and the new waterworks. The old works date from colonial times and derive water from a spring three-fourths of a mile west of the courthouse; the new works, installed in 1894 at a cost of $\$ 55,000$, are supplied from a spring three-fourths of a mile southwest of the courthouse and afford 2,500,000 gallons in twenty-four hours. There is no sewerage system and the use of cesspools and privy vaults is universal; they care for themselves by draining away through the crevices of the limestone. Probably much of the pollution ultimately enters Town Run, which passes through the center of the town and joins Abrams Creek. Besides the indirect contamination, Town Run receives waste from a.steam laundry and gas house; the latter makes the run foul and ill smelling. Near the gas house. W Graichen's tannery discharges its effluent, made up of lime, sourbark liquor, and water from wool scourings, all of which amount to 200 gallons a day; the Winchester creamery contributes the washings of its floors, vats, and cans; the Lewis Jones Knitting Company adds spent dyes and run-down lime bleach; and the Virginia Woolen Company discharges its waste, consisting of 1,000 gallons a day of scouring liquors and spent dyes. This unsavory burden is delivered by Town Run to Abrams Creek on the southeastern outskirts of Winchester. West of Winchester, nearly 3 miles above the mouth of Town Run, Abrams Creek receives the waste of the American Strawboard Company's factory, which makes paper board from old newspapers. The effluent is dirty water full of shreds of paper and is run into a settling pool from which it flows into Abrams Creek. The sediment is occasionally taken out of the pool by farmers for use as a fertilizer. A. C. Williamson \& Brothers, manufacturers of woolen goods for public institutions, pour the rinse from their manufactured goods and some spent dyes into the creek, after which it flows 7 miles to Opequon Creek without further industrial pollution other than that delivered by Town Run, as already mentioned. 
Two miles below the mouth of Abrams Creek, Opequon Creek receives Lick Run, which, at Jordan Springs, is polluted from June to September by the sewage of a summer hotel and its cottages, planned to accommodate 300 guests.

Martinsburg, W. Va., is on Tuscarora Creek, 1 mile from Opequon Creek. $^{a}$ In past years the city suffered seriously from typhoid, but the citizens finally delivered themselves from the scourge by closing up the wells and abandoning the old water supply, both of which were considered to be polluted. A new and satisfactory water supply from Kilmer Spring, 1 mile west of the city, was installed in 1903 , at a cost of $\$ 30,000$. There is no sewerage other than four drains, aggregating 2,000 feet in length; these are for surface water only, which they discharge into a tributary of Tuscarora Creek. About 5 per cent. of the houses have water-closets, and these empty into cesspools; the rest of the people are dependent on privies. Both cesspools and privies are well looked after, owing to the city's experience with typhoid, but they undoubtedly furnish Tuscarora Creek with considerable indirect pollution. Only one privy, that of the electric-light company, was noticed to overhang the creek directly.

Tuscarora Creek is greatly polluted by industrial wastes. The first and most noticeable of these is that of the Hannis Distilling Company, amounting to 8,000 gallons in twenty-four hours. Inasmuch as it consists of slops from the mash, which is a good cattle feed, much thought has been spent by the company in devising means to separate the solid portion from the liquid in which it is suspended. By means of a somewhat elaborate system of settling and filtering, all but the finest impalpable material is saved and put on the market. The part that is discharged into the creek is finely subdivided matter in suspension in weakly acid liquor, and has defied every device' of the company to recover it. Once in the stream it collects in long undulating streamers, which adhere to the banks and bottom, giving the creek an unsightly appearance. As this effluent is undoubtedly good food for algæ and bacteria, it favors their development.

A considerable distance from the Hannis distillery, at the south end of the city, the gas company discharges ammoniacal liquors into the stream. Opposite the gas works a little run enters which carries most of the manufacturing effluents received by Tuscarora Creek. The Kilbourn Knitting Machine Company's waste amounts to 10,000 gallons a day, and consists of wool scourings, spent dyes, and rinse waters from the manufactured goods. These liquors are run into a sedimenting pool, whence they overflow into the run. The solid matter is pumped out from time to time and allowed to stand in the vicinity of the pool. The Crawford Woolen Company discharges directly into the

\footnotetext{
$a$ Results ef stram measurements on Opequon and Tuscarora creeks near Martinsburg are given on pp. 78-82.
} 
run 10,000 gallons of spent dyes and 5,000 gallons of wool scourings in twenty-four hours. The Martinsburg Worsted and Cassimere Company utilizes the run for its spent-dye liquors, which amount to 5,000 gallons in twenty-four hours.

No other important contamination taints Tuscarora Creek below this little run. Before leaving the discussion of Martinsburg, however, it should be noted that the ice consumed in the city, though mainly of local manufacture, is in part cut on the Tuscarora Creek above the city and in part on Opequon Creek àbove Tuscarora Creek. The latter source is undoubtedly unsafe on account of the pollution of Opequon Creek by the city of Winchester, and the practice of harvesting ice on it should be prohibited. From the mouth of Tuscarora Creek, Opequon Creek flows for a few miles through a farming country to the Potomac.

\section{POTOMAC RIVER FROM OPEQUON GREEK TO ANTIETAM CREEK.}

On the Potomac, 20 miles below Opequon Creek, is Shepherdstown, a village with no public water supply and but few wells, most of the inhabitants using cistern water. There is no sewerage system, but a small run which courses through the town performs the functions of one. It is lined with privies and hogpens, which pollute it badly. The town is too small to contaminate the Potomac greatly, but its contribution of excrement should not be overlooked, for small outbreaks of typhoid fever are very likely to occur in places without water supply and sewerage, and the disease can easily be disseminated by means of infected feces through the agency of the river. Six miles below Shepherdstown is the mouth of Antietam Creek.

\section{ANTIETAM CREEK, $a$}

Antietam Creek rises in the Green Ridge Mountains, Franklin County, Pa., and discharges into the Potomac 5 miles below Sharpsburg. On Little Antietam Creek is the town of Mont Alto, which furnishes some indirect pollution to the stream. Between East Branch and Little Antietam Creek, in Franklin County, is the borough of Waynesboro, which contains several large industrial plants, but none of them have liquid wastes. The Frick Company, however, runs the sewage of its 700 employees into Little Antietam Creek through an open sewer. The water supply of Waynesboro is well adapted to boiler use, and gives general satisfaction. It is derived from two sources. One of them, Bailey Spring Run, is 4 miles northeast of the town. The reservoir was constructed in 1885, and is now rarely used, being simply a reserve which can be called on in case of emergency. The . main supply of the town is derived from Rattlesnake Run, across which a dam has been built at its confluence with East Branch of Antietam Creek in the Pennsylvania Forest Reserve. From this point the

$a$ Results of stream measurements on Antietam Creek at Sharpsburg are given o 1 pp. 82-90. 
water is carried 7 miles southward and served to the town. There is no sewerage other than an irregular system of drains, which are supposed to exclude soil. The cesspools, which are universally used, drain through the broken limestone, presumably to one or the other of the branches of the Antietam. The result is that practically all the wells of the town are polluted, and have been abandoned both on this account and because the water is hard and not adapted to domestic or manufacturing use.

The next town of importance below Waynesboro is Hagerstown, a large industrial center of western Maryland. Its public water supply is furnished by the Washington County Water Company, and is derived from runs on South Mountain, 11 miles from the city. The present waterworks were started in 1896 and completed in 1903. Their capacity is $1,500,000$ gallons in twenty-four hours; that of the old works, established in 1881, was 400,000 gallons. In 1902, owing to the depleted condition of the reservoirs, it was found necessary to establish a pumping station on Antietam Creek at Bridgeport. Water was pumped for thirty days only, and since that time this source has never been used, but it is still available. Hagerstown has no regular sewerage system, but is pretty thoroughly drained by terra-cotta pipes, which are supposed to exclude all soil. Slops, however, undoubtedly enter these pipes. Fifty per cent of the houses have water-closets; the rest depend on privies. Cesspools which serve the water-closets are in many cases abandoned wells, and estimates have been made that 40 per cent of them are so. Privy-vault matter is sometimes buried in the town, but more commonly it is carried outside of the city limits and disposed of on farms. Owing to the fact that cesspool drainage and other pollution finds its way through the crevices and seams in the rocks that underlie the town to the general water level, most of the wells have become polluted, and consequently many of them have been closed up. The fact that a considerable number of typhoid cases occur every year in Hagerstown and that these are as a rule confined to users of well water seems to indicate that the remaining wells should be abandoned. The indirect pollution is probably largely carried off by Town Run, a tributary of Marsh Run, which in turn enters Antietam Creek. Town Run receives the condenser water and washings from beer barrels of the Hagerstown Brewery Company, which claims that it is this flux of water alone that keeps the run from stagnating and becoming very foul and offensive in summer time. The Blue Ridge Knitting Company does considerable dyeing not only for itself, but for other firms in Maryland and Pennsylvania. Its wastes are spent dyes and rinse water from the finished goods and amount to 2,400 to 3,600 gallons in twenty-four hours. They are disposed of in the Town Run. J. C. Roulette \& Co. bleach their goods, and the rinse water, amounting 
to 3,500 gallons in twenty-four hours, and the wasted spent bleach enter Marsh Run. The Antietam Creek Paper Company is located outside of Hagerstown, and makes paper from wood, rags, and paper by the soda process. The most important waste is spent lime bleach, which is sedimented near the mill and applied to a farm owned by the company.

Below its confluence with Marsh Run, Antietam Creek receives important pollution from but one other source--the town of Sharpsburg, Md., located on a little run a short distance from the creek. The town is a small one, but it is near the battlefield of Antietam, and therefore is periodically visited by crowds who seyerely tax its limited sanitary facilities. The run on which it is located heads in a spring which is in common use as a water supply, though the inhabitants have to go to it for water. In the summer of 1904 there was a typhoid epidemic which some persons attributed to the use of the spring water. At that time there was a general cleaning out of the privy vaults and hog pens that line the run, and now very few of the hog pens directly overhang it, though it undoubtedly receives much fecal matter of both man and animals. While the amount must be small in proportion to pollution from other sources, it is perhaps important because Sharpsburg is but 60 miles above Washington, where the Potomac is used as a public water supply, and pollution from Sharpsburg would probably reach Washington in a day or two.

POTOMAC RIVER FROM ANTIETAM CREEK TO SHENANDOAH RIVER.

Ëlk Branch, which enters the Potomac a little above Harpers Ferry, receives the sewage of Shenandoah Junction, W. Va., where a considerable number of travelers find victuals and temporary lodging. Ten miles below the mouth of Antietam Creek, at Harpers Ferry, the Potomac is joined by the Shenandoah. The town is situated on the tongue of land between the rivers and is unsewered, so that various ways are adopted to get the soil into the Potomac, where practically all the refuse of the town goes. The Baltimore and Ohio station is right on the river, into which its water-closets conveniently discharge beneath the railroad bridge. The subject of the sanitation of the railroads in the Potomac basin is not an unimportant one, for besides the stations, which are, of course, fixed and can always be carefully provided for, there are 1,200 miles of track over which pass every day many hundreds of people from all parts of the Union, many of them from cities and towns in which typhoid fever is common. The usual method of caring for the excreta of these travelers is to let it drop along the tracks. It has generally been assumed that this is the best way of handling this material, but it seems a fair question whether it is or not. Undoubtedly the method was originally adopted because of its simplicity, and it has received general acceptance without much 
thought as to its possibilities for spreading infection. Perhaps in the future this problem will receive more careful attention and some other means of disposing of the feces, such as collecting them and leaving them at definite points for disposal, may be adopted.

One of the hotels in Harpers Ferry has its fixtures connected to a cesspool, the overflow of which is piped to the river. Another is connected by a sewer to the old arsenal yard, where abandoned raceways conduct the sewage to the river. It is said that not a few private houses dispose of their sewage in the same way. Privies are common in Harpers Ferry, and the vault matter is disposed of as suits the owners; it is believed that some of them throw the soil into the rivers. There is no public water supply, the people for the most part relying on cisterns, though a few take water from the brewery, which brings its water from springs in the Blue Ridge Mountains across the bed of the Shenandoah. The Hill Top Hotel pumps some of its water from Potomac River. The only industrial wastes in Harpers Ferry are those of the brewery and two pulp mills, one of which is on the Potomac and the other on the Shenandoah. Both mills manufacture mechanical wood pulp, in which process the logs are freed from their bark before being ground (PI. VIII, $B$, p. 222). The shavings were formerly disposed of by being put into the rivers, and at the time of the inspection evidences of this pollution were very manifest. Since that time both mills have installed apparatus for burning the shavings and the nuisance is abated. The brewery discharges the washings from its beer kegs into the Shenandoah.

POLLUTION IN SHENANDOAH RIVER BASIN.

Shenandoah River is formed at Riverton, Va., by the confluence of North and South forks. South Fork in turn is formed at Port Republic by the junction of North and South rivers.

\section{SOUTH FORK OF SHENANDOAH RIVER BASIN.}

SOUTH RIVER.a

South River rises west of Greenville, Augusta County, W. Va., and flows eastward past Basic City and Waynesboro, towns on opposite banks of the river, forming a considerable center of population. Here the stream receives its initial industrial pollution. Basic City has a small blanket factory and a factory for the manufacture of tanning extract from chestnut-oak wood. The pollution furnished by the former consists of rinse water from the finished goods, and from the latter, at the present time, such amounts of extract as are absorbed by the condenser water. When the plant was new the leaching vats leaked and caused much complaint because the extract stained the river

$a$ The results of measurements of South River at Basic City and Port Republic are given on pp. 91-98.

IRR $192-07-16$ 
badly. Several factories have located at different times in Basic City, but their existence has been short. A large summer hotel anmually attracts many visitors. The city is supplied with water from a spring close to the river. There is no sewerage system, but one of the hotels has a private sewer which discharges into a run that enters North River below the waterworks.

Waynesboro is supplied with water from Bakers Spring, which is owned by private parties, and for the use of which the town pays a small sum every year. The mains and service pipes, together with a storage tank of 50,000 gallons capacity, are owned by the town, and were installed in 1897 at a cost of $\$ 10,000$. The introduction of this supply reduced the typhoid rate and has led to the general abandonment of wells. A stove factory is the largest industrial plant in Waynesboro. It has no liquid wastes, but the sewage from its 100 employees is discharged into a race which enters the river. Brunswick Inn is also reported as sewering into the river, and it is said that the material from the many privies in town is thrown into it. The slaughterhouses in both Waynesboro and Basic City dispose of their offal in the stream.

From Basic City South River flows in a northerly direction. At Crimora a small run enters, which is very turbid owing to the washing of manganese ore by the Crimora Manganese Company near its head. This concern employs 100 men, who are established in small huts scattered over the watershed of the run. A long tumnel is being driven in the mines which will drain them more thoroughly and deliver the water on lands of the company instead of to the run. It is proposed to construct settling basins to clarify the water, which will then be led, in some way not determined, to the river.

\section{NORTH RIVER. $a$}

North River rises in the Shenandoah Mountains in the northwest corner of Augusta County, on the slopes of Briery Branch Knob. The upper part of its watershed is given over to lumbering, and at Stokesville, just east of Narrow Back Mountain, are located a sawmill and a tanning-extract factory. The latter is still uncompleted and unless umusual precautions are taken when the plant is started, the leaking vats may discolor the river. In the summer of 1905 this town was the seat of an outbreak of typhoid fever. The first case, which was probably imported, was in a family whose spring was in common use in the town. About two weeks afterwards two other users of the waters of this spring were taken sick. One of them was removed to the west end of the town, where he remained for a few days, after which he was taken to Harrisonburg. In this interval he apparently infected a privy which was used by several families, 
situated directly opposite the house where he was confined. Later, typhoid germs from this source must have been disseminated in the neighborhood by flies, for 4 cases appeared close by and they were the only ones in the whole town among those who did not use the spring water. Other cases, 13 in number, occurred among the users of the spring and people who lived along the run which rises in it, and who either had access to the spring itself or used the water of the run for one purpose or another in their homes. This epidemic illustrates very well how typhoid fever originates and spreads in the basin of the Potomac. As a rule, the outbreaks occur in small towns where there are no facilities for disposing of ordure and where the limited water supply is in general use and is derived from some source surrounded by dwellings. Sanitary precautions, through the ignorance of the people, are entirely neglected and twelve months in the year conditions are ripe for an outbreak of typhoid. All that is needed is to have the initial case imported. Once that is accomplished, the disease spreads rapidly. In many towns where repeated outbreaks have occurred, the disease may be said to have established itself permanently, a case or two being present all the time. This can be readily understood, for the stools are not sterilized, and it needs only a transportation of privy matter or some other infectious material to start fresh cases.

The next town below Stokesville is Bridgewater, which also has been subject to outbreaks of typhoid fever. There is no public water supply, the people being dependent on cisterns and wells. On the river somewhat west of the town is a woolen factory where blankets are made. It is run only part of the year and the wastes, consisting of a small quantity of wool scourings, spent dyes, and rinse from the finished goods, are emptied directly into North River.

Below Bridgewater is Mount Crawford, located on North River somewhat to the west of Cooks Creek. ${ }^{a}$ Typhoid has been prevalent here, which may perhaps be partly accounted for by the fact that the citizens have cut and used ice from Cooks Creek, a stream receiving the sewage of Harrisonburg.

Harrisonburg is about 8 miles northeast of Mount Crawford. It is one of the largest cities in the valley and seems to be growing steadily. It has a public water supply derived from Dry River, 13 miles farther west. It is said that since the supply was introduced typhoid, which was formerly very common, has been materially reduced. The sewerage system is a combined one and is available in nearly all parts of the city. The flow, which is estimated at 250,000 gallons in twenty-four hours, is discharged a quarter of a mile south of the city into Blacks Run, a tributary of Cooks Creek. This sewage is augmented by the waste from the plant of the J. P. Houck 
Tanning Company, estimated at 2,500 gallons a day. The waste from the lime vats is sedimented and the liquor run into the sewer; as is the waste sour bark liquor. The liquor from the scouring machines and a waste made up of various small leakages that occur in various parts of the tannery are turned directly into the rum. Together they make the water very foul, especially in summer, when the city authorities have occasionally been obliged to flush out the stream on account of the bad odors arising from it. Whenever observed, the run has appeared to be taxed to its utmost, and it is reported that in summer the waste from the tannery has decidedly discolored North River as far as Mount Crawford railroad station. No other factories in Harrisonburg have liquid wastes.

Four miles above Port Republic North River receives Middle River, which rises in Little North Mountain southwest of Staunton. Middle River flows northeastward to Long Glade, then southeastward to Laurel Hill, where Lewis Creek enters. ${ }^{a}$ 'This creek is heavily polluted by the sewage of Staunton, an energetic, prosperous city estimated to have 12,000 buildings, 50 per cent of which are conmected with the sewer. There are no manufacturing plants having liquid wastes; therefore the sewage is almost entirely domestic. The system, which is a combined one, was established twenty-five years ago and has grown slowly and irregularly ever since. The first water supply was introduced in 1849 and is still in use, but the main supply was put into service in 1876, at a cost of $\$ 100,000$, and comes from springs on the bank of a stream one-half mile west of the city. The wells of the city are badly polluted and most of them have been closed up by the local authorities. The typhoid rate of the city is very low, only one death being recorded in 1904.

The water supply of Staunton was at one time unpleasantly affected by fresh-water sponge which grew in one of the reservoirs. The trouble was investigated by Prof. J. W. Mallet, whose report is on file with the city authorities. The sponge was killed and the trouble completely overcome by fluctuations of the water level.

\section{SOUTH FORK OF SHENANDOAH RIVER BELOW PORT REPUBLIC.}

Below Port Republic, South Fork of Shenandoah River flows northeastward through the Page Valley, being separated by Massanutten Mountain from North Fork, which joins it at Riverton.

Keezletown is located on the west side of the mountain, but discharges its refuse into South Fork through Cub Run. It is a small town, but the run receives some pollution there from the tannery of A. D. Bertram, which has a capacity of 700 hides a year. 
A little below the inouth of Cub Run South Fork receives Stony Run, which rises on the east side of the mountain and flows through McGaheysville, where it is contaminated by a tannery having a capacity of about a hide a day.

At Elkton South Fork is much befouled by the waters of Elk Run, which rises in the Blue Ridge Mountains and flows westward to the river $^{a}$ Elkton has a public water supply which is furnished from springs by the Wampole Lithia Company. There is no sewerage; the inhabitants depend entirely on outhouses, the scil of which is said to be thrown into Elk Run when they are cleaned. . One of the hotels has a trench for its urinal and slops which leads directly to the run. The stream also receives pollution from the tannery of $J$. R. Cover \& Sons. This plant employs 25 hands, and has a privy directly overhanging a ditch that leads to the run. Through this ditch are discharged the -wastes, which amount to 1,500 gallons a day. Inspection makes it quite evident that this amount is too large for the little run to carry off promptly and easily. Indeed, the effect of the pollution on the river itself must be considerable. In the sumner a hotel is opened in Elkton which is said to accommodate many guests. While such places add to the wealth of the towns that are fortunate enough to possess them, they contain an element of danger, for their patrons come from many different places and may carry in their persons germs of disease contracted in their homes. In due time these unfortunates sicken and then the infection elaborated by them is likely to lay hold of the community they are visiting and make it a center from which-contagion spreads.

Below Elkton is Shenandoah, a prosperous town which can pollute the river but indirectly. Thirty miles below is the mouth of Hawksbill Creek, on which is located, in Page county, the town of Luray. ${ }^{b}$ The creek divides the town into very nearly equal portions which rise somewhat abruptly from it; consequently the run-off through the town is quick and carries with it considerable refuse. A water supply was installed in 1900. Typhoid fever is said to have been prevalent prior to its introduction, but such is not now the case. There is no regular sewerage system in town, and cesspools are commonly used. As a rule they do not need cleaning, because they leak away through the underlying limestone. A number of water-closets and one of the hotels discharge directly into Haw ksbill Creek. At one time there was some agitation against permitting them to do so, but it died out, and now the suggestion has beer made that the town establish a sewerage system and utilize the creek for an outlet. Luray has usually a large transient population, attracted thither by

$a$ Results of measurements of Elk Run at Elkton are given on pp. 110-112.

$b$ Results of measurements of Hawksbill Creek at Luray a re given on pp. 112-115. 
the wonderful caverns. Thus the town is considerably exposed to the danger of infection being imported from outside. The chief pollution of Hawksbill Creek is by the Luray tannery, owned by the Deford Company, of Baltimore. This plant employs 170 hands, and the waste poured into the creek amounts to 10,000 gallons a day. The waste from the beam house and the scouring liquor are run into two cesspools, where they are allowed to evaporate. The residue is used for a fertilizer on a farm owned by the company. The volume of sour bark liquor, which with the soil of six water-closets is carried in a long trench to the creek, is so large in proportion to that of the stream that the latter is colored a deep red and maintains the hue to its mouth, 5 miles below the factory. An acetylene-gas company in the center of the town piles its lime sludge on the banks of the Creek.

\section{NORTH FORK OF SHENANDOAH RIVER BASIN.}

North Fork of the Shenandoah rises in the northern part of Rockingham County and flows southeastward to Broadway, where it turns to the northeast. North Fork is not much polluted until it reaches New Market, where, on a tributary of Smiths Creek, there is a small tannery owned by F. M. Tusing. The next town below is Mount Jackson, which is on Mill Creek a little way from its mouth. It has neither public water supply nor sewerage, the people relying almost wholly on cistern water, as there are but a few wells in town. The pollution here is inconsiderable and very indirect. From Edinburg to Strasburg the course of North Fork is decidedly meandering. At Tomsbrook a creamery pollutes North Fork. At Woodstock a creamery pollutes Hollow Run, but the rest of the refuse of the town is insignificant. There is no public water supply nor sewerage, and the river receives only a little very indirect pollution from the town.

Strasburg is the largest town in the valley of North Fork of the Shenandoah. It is traversed by Hupp Spring Run, and this stream carries off most of the waste from the town, and is also polluted by the sludge from a small acetylene-gas plant and by the effluent from O. F. Chandler's tannery, which has a capacity of about a hide a day. At the time of inspection Strasburg was depending on springs and wells, but as the well water is very hard a public water supply was being installed which it was expected to follow up with a sewerage system.

The only other town to be noticed in the discussion of North Fork is Middletown, a place without water supply and sewerage, located on Marsh Run; a tributary of Cedar Creek, which flows into North Fork below Strasburg. The pollution from Middletown is only slight and very indirect. 


\section{SHENANDOAH RIVER BASIN BELOW NORTH AND SOUTH FORKS.}

Riverton lies between North and South forks of Shemandoah River and pollutes the rivers but little, as it is a small place, with most of the houses some distance from the two streams. The town purchases its water from Front Royal, and therefore enjoys the same immunity from typhoid as that town. There is a large duck farm which drains into the North Fork of the Shenarıdoah. No other pollution here is worthy of note.

A short distance below Riverton, Shenandoah River receives the draimage of Front Royal, which is on Happy Creek, 3 miles from its mouth. Most of the pollution of the creek by the town is indirect, for there are few water-closets, most of the people using outhouses, though some of the privies are dangerously naar the stream. One of the hotels has a cesspool from which the overflow is carried in a private sewer toward the creek. The town is a clean one, and is free from typhoid fever. The chief source of pollution on the creek is a slaughter barn. The offal is fed to swine kept in a yard that reaches down to the creek in order that the animals may drink there. When observed, no slaughtering was being done, but that considerable offal and blood goes into the creek when killing is in progress seems to be the general opinion. Formerly there used to be many cases of typhoid fever in Front Royal every year, bu.t this stopped twelve years ago, when a public water supply was introduced from Chester Gap, 4 miles to the southeast. The source of supply is from springs whose flow is collected in a reservoir of 1,500,000 gallons capacity and run by gravity into the town. The cost of the works was $\$ 40,000$.

Twenty-four miles below Riverton is the nouth of Lewis Run, which drains the town of Berryville, the county seat of Clarke County. There is no mamufacturing in Berryville, but the run is polluted by hogpens and privies which are near it. Moreover, many of the houses have sewers for their slops which empty into the run. The town has a public water supply which is said to be excellent. Previous to its introduction, typhoid was rampant in the town and now it is of rare occurrence.

Fifteen miles below Berryville Evitt Run ex.ters the Shenandoah. This is a rather attractive stream of clear water, which does not. show ocular evidence of pollution, though it carries the indirect pollution of Charles Town, the county seat of Jeferson County. This city has no sewerage system and has not been compelled to construct one because of the fact that if there are not seams enough in the limestone to carry away the leakage of the numerous cesspools, the explosion of a dynamite cartridge in the rock at the bottom of the cesspool is sufficient to insure its caring for itself. The water supply of the 
town was originally obtained from several town pumps, all but two of which have been given up because the above-mentioned method of caring for sewage has allowed cesspool matter to enter the wells.

The present water supply is sufficient and satisfactory: It is furnished by the Charles Town Water and Manufacturing Company, which brings it from a spring 1 mile west of town.

Between Evitt Run and Harpers Ferry Flowing Run enters the river. This stream is contaminated by the paper box board factory of Eister \& Sons at Halltown, W. Va. The firm makes box board from old paper, and the effluent, consisting of dirty water with much paper and fiber in suspension, is turned directly into the run and muddies it. Additional pollution is furnished by the privies of the concern, which overhang the stream for the convenience of its 35 employees.

\section{POLLUTION IN POTOMAC RIVER BASIN BELOW SHENANDOAH RIVER.}

POTOMAC RIVER FROM SHENANDOAH RIVER TO MONOCACY RIVER.

At Harpers Ferry the Potomac cuts through the Blue Ridge Mountains between Loudoun Heights on the south and Maryland Heights on the north, and enters the upper Coastal Plain region. Along the northern bank there is a scattered population, most of whom are densely ignorant of sanitary laws. The privy vaults and hogpens are near the houses except where there is a little stream, in which case the structures are located over it, and thus the offal is carried to the river. At Weverton and Knoxville, Md., this population is somewhat concentrated, and at Knoxville the privies and hogpens which overhang the creek that passes through the town are more numerous than is usual in this region. The migratory character of the population in these towns and the accompanying crude sanitary conditions constitute a decided menace to the river. During the summer of 1905 typhoid fever prevailed in these settlements, and probably if records could be obtained for the past dozen years it would be found that it had done so every year. They are not more than 35 miles from Washington, and should be subjected to rigid sanitary supervision.

Below Knoxville is Brunswick, Md., a town which has sprung into existence on account of the location of the Baltimore and Ohio Railroad shops there. These shops employ 400 men, and while there are no liquid wastes the sewage of the employees furnishes the Potomac with considerable indirect pollution. The privy which is used by the roundhouse force is located close to the canal and is. nearly surrounded by a green pool caused by seepage from it; about 100 yards distant is another privy in a large cinder bed. The town is on a high, steep hill and is crossed by four different runs along which are a number of privies, some of them actually overhanging the streams. 
Second Culvert Run furnishes the most important pollution, for into it is discharged the sewage of the railroad bunk house, which is fitted with water-closets, bath tubs, and urinals. This sewage is created by a roving population, and consequently by one more exposed to disease than a settled one would be, and the excrement is correspondingly more dangerous. It is expected that a large Y. M. C. A. building will be erected alongside the bunk-house, and that too will discharge into the run. There are a few water-closets in the town, some of which are connected to the runs, but in general privies are used. For water the town is dependent on wells and cisterns, though at the east end of town there is a private supply which comes from the Potomac. The peculiar topography and population of Brunswick make it very necessary that sanitary matters be thoroughly looked after there. The fact that it is on the steep sides of a high hill insures all the offal finding its way promptly. to the river, and the shifting population, however well intentioned it may be, is more or less likely to bring disease into the community from without. Finally, the town is near Washington, and therefore typhoid germs will be transported by the Potomac to that city in a short time. In the summer of 1905 , at the time the inspection was made, it was reported that there were five cases of typhoid fever in Brunswick, but what precautions were taken to sterilize the stools and otherwise protect the public were not ascertained.

A few miles below Brunswick the Potomac receives Catoctin Creek of Virginia and Catoctin Creek of Maryland, after which it cuts through Catoctin Mountains and emerges at Point of Rocks, in the lower Coastal Plain region. Point of Rocks and Washington Junction form a single settlement skirting the Chesapeake and Ohio Canal. ${ }^{a}$ Like the other places below Harpers Ferry, they are lacking in sanitary arrangements. At Washington Junction the watercloset of the railroad station discharges into the river. From Point of Rocks it is 3 miles to the mouth of Tuscarora Creek, on which at Adamstown there is a small cannery. The creek is also polluted at Doubs.

\section{MONOCACY RIVER BASIN.}

Two miles east of South Tuscarora Creek Monocacy River enters the Potomac. It has three large feeders that rise in Adams CountyMarsh, Rock, and Middle creeks. Between the two former, on the watershed of Rock Creek, is the borough of Gettysburg, and on Middle Creek is the town of Emmitsburg. Gettysburg derives most of its water supply from Marsh Creek, southwest of the town. The watershed above the intake is not heavily polluted, though at McKnightstown there is a tannery with a capacity of 100 hides a week. The water is filtered through a Warren filter that was installed

a Results of measurements of Potomac River at Point of Rocks are given on pp. 148-161. 
twelve years ago. Coagulent is used only when the creek is very turbid. This supply is supplemented and mixed with water obtained from two driven wells on Cemetery Hill. These are pumped by wind power and are constantly in use. The borough is now installing a sewerage system for soil only which when finished will have 7 miles of pipe line and it is expected will be generally utilized by the citizens. The sewage will be treated in two septic tanks, and the effluent from them will be filtered through brick walls and then run into Rock Creek into which the private sewers of the town now discharge. It is to be hoped that the treatment of the sewage will be effective, for Gettysburg is almost daily visited by large numbers of sight-seers. The throng of pilgrims is variable, sometimes being small and sometimes rising to 10,000 in a single day. The people come to see the beautiful battlefield, which has been carefully preserved by the National Government. As the park is a permanent one, there will probably never be any diminution in the number of visitors. In a throng of this kind there are apt to be many who seize the opportunity for a rest because they are out of condition, run down, or sick, and think that a little excursion will restore them. Among such people there are likely to be some in one stage or another of typhoid fever, so that the transient population that visits Gettysburg may be something of a menace to the waters of the Potomac; hence the desirability of carefully purifying the sewage before it is discharged into the creek.

Stevens Run, a tributary of Rock Creek, is polluted by the effluent of a gas works. The waste makes the run ill smelling and repulsive in appearance. Moreover, it is said to injure fish life, and a suit has been brought by the Fish Commission against the company. Culps Run, another tributary of Rock Creek, receives the sewage and effluents from W. S. Duttera's tannery, which turns out 50 hides a week, and the wastes from which are similar to those discharged from other tanneries.

Emmitsburg, on Middle Creek, is the site of Mount St. Mary's Academy, St. Joseph's Academy, and the mother house in the United States of the Sisters of Charity. The population of the town, including the sisterhood, is 2,000 . There is neither public water supply nor sewerage.

On Double Pipe Creek is Westminster, a large town without public water supply or sewerage.

From the junction of Marsh and Middle creeks to the mouth of Carroll Creek the distance is 25 miles. Carroll Creek rises in Catoctin Mountain, northwest of Frederick, Frederick County, Md., and flows southeastward through the southern part of the city to the Monocacy. The Frederick water supply is obtained from Big Tuscarora Creek, Little Tuscarora Creek, and Fishing Creek and is said to be wholesome, a statement that is borne out by the low typhoid rate of the city. 
Each of the two main streets has a single drain, which was put in primarily with the objeat of carrying off the water from cellars of abutting houses. They have developed into sewers from which soil is excluded, but which take in slops and surface water and discharge into Carroll Creek. In many of the streets of the city the slops run through the gutters and ultimately reach the creek.

The indirect industrial pollution of Carroll Creek consists of an insignificant amount of waste from a cannery and from the scrubbers of a gas house. It is directly contaminated by the scouring liquor from the Brierly tannery, together with some lime sludge that is piled on the banks of the creek; by the effluent from a steam laundry, by the sewage of the Carroll County almshouse, and, most obviously, by the pollution from the works of the Union Knitting Company, consisting of the sewage of 200 employees and a large quantity of spent dyes.

The pollution received by the Monocacy from the mouth of Carroll Creek to the Potomac is, for the most part, very insignificant, being only that incident to the life of widely separated farms. At Frederick Junction the closets of the Baltimore and Ohio Railroad station empty into the river. Buckeystown is the most considerable settlement below Frederick on the Monocacy. It is drained by a little run which receives the washings of a cannery and a small creamery, besides the discharge of water-closets. The unvaulted privies of a large brick works in the town are about 20 feet from the stream. The water used in Buckeystown is derived from cisterns.

POTOMAC RIVER FROM MONOCACY RIVER TO GREAT FALLS.

The largest and most important tributary of the Potomac between the mouth of the Monocacy and Great Falls is Goose Creek. It has . a large drainage area, which is on the south side of the river and lies east of the Blue Ridge Mountains. The watershed is given over to agriculture and contains but one town of much size. Hence the proximity of the basin to Washington is not nearly so important as it would be if the population was large and concentrated at a few points. The development of this drainage area will be watched with interest, for should it become the home of a large summer population, or should other considerable towns spring up in it, the creek might become a serious menace to Washington, inasmuch as disease germs from it could be transported to the city in a few hours.

Leesburg, the important town alluded to, is on Tuscarora Creek, a tributary of Goose Creek. There is no sewerage system in the town, but 800 feet of 2-foot terra-cotta drains, which are supposed to carry surface water only, discharge into Tuscarora Creek. Therefore the pollution caused by Leesburg is indirect save for the few privies that overhang Tuscarora Creek. The water supply of the town is inadequate and was suspected at one time of being contaminated, for in the 
summer of 1904 there was considerable typhoid fever, and this impelled the citizens to bond the town for $\$ 30,000$ to enable it to increase its water supply. The new system will furnish an abundant supply and will probably result in the construction of a complete sewerage system, which will have its outlet in Goose Creek.

Eight miles above Great Falls is Seneca Creek, whose watershed is given over to small farming and which does not contain a single important center of population. The creek would not be worth mentioning here did not its proximity to Washington make it possible for disease germs originating in its basin to be transmitted to the capital in a very short time.

\section{POPULATION AND DRAINAGE AREAS.}

The following tables show the population and the drainage areas of the smaller basins that make up the Potomac watershed:

Population of the Potomac Rvver basin.

[According to the census of 1900.]

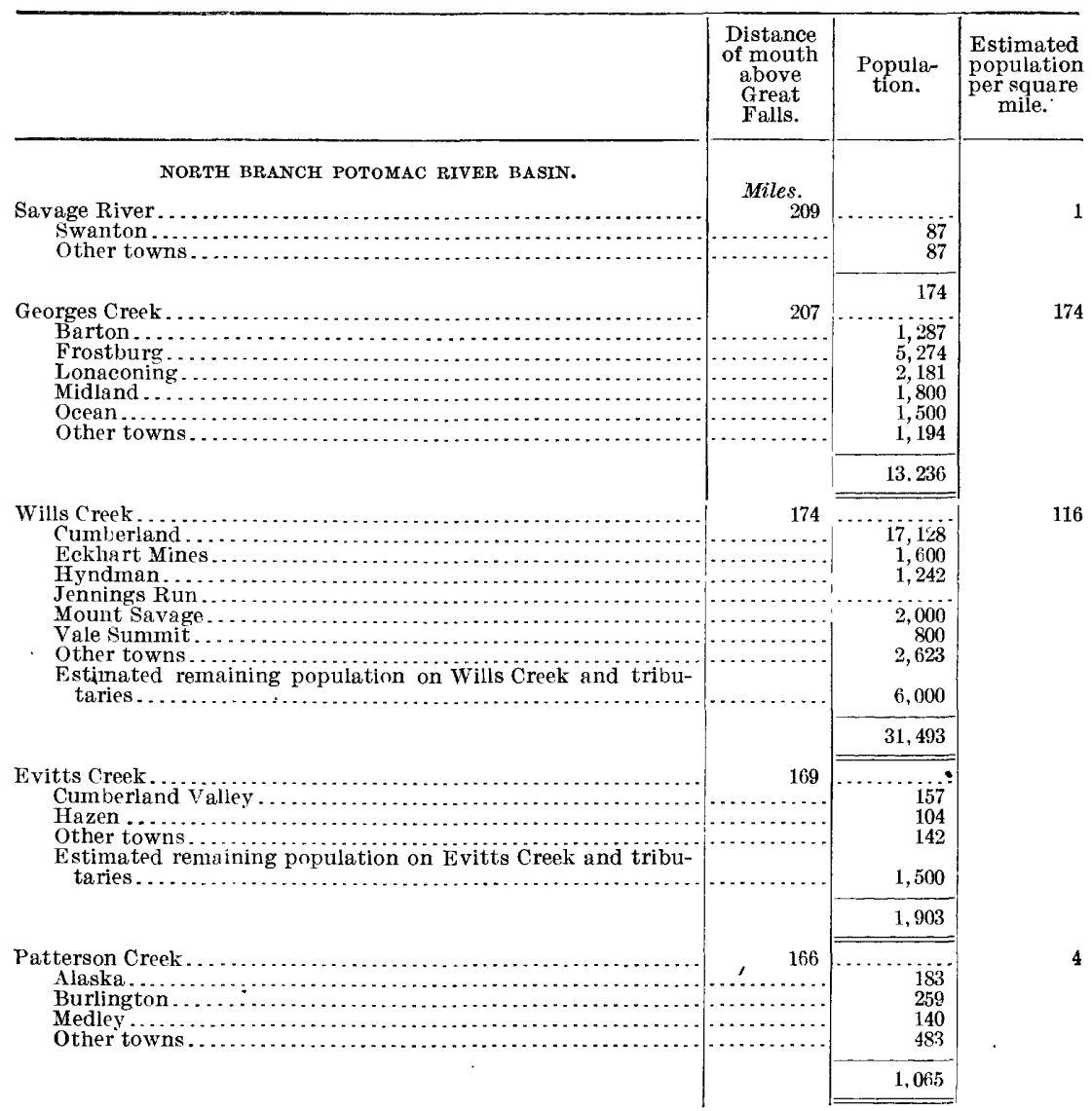


Population of the Potomac River basin-Continued.

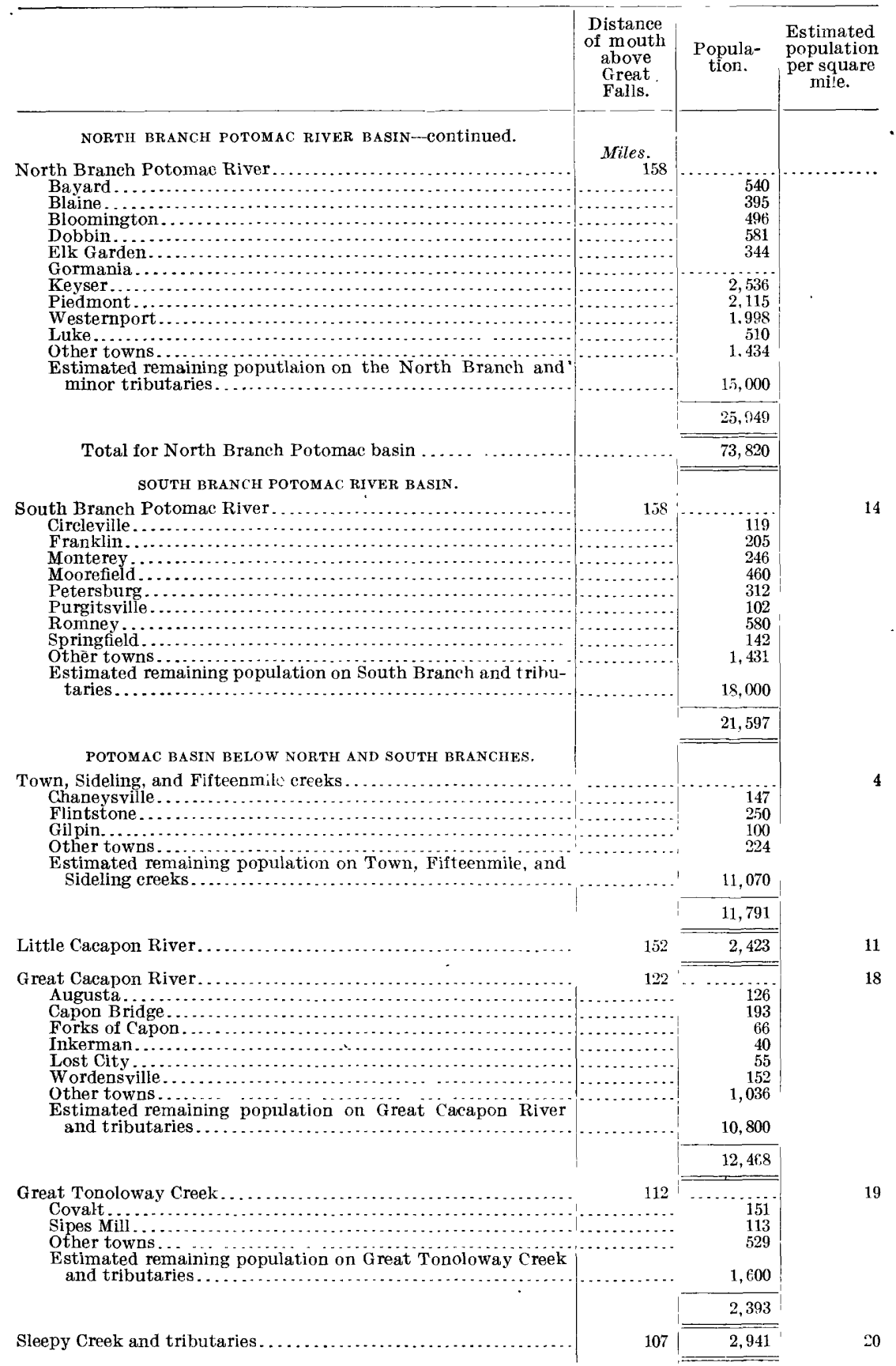


Population of the Potomac River basin-Continued.

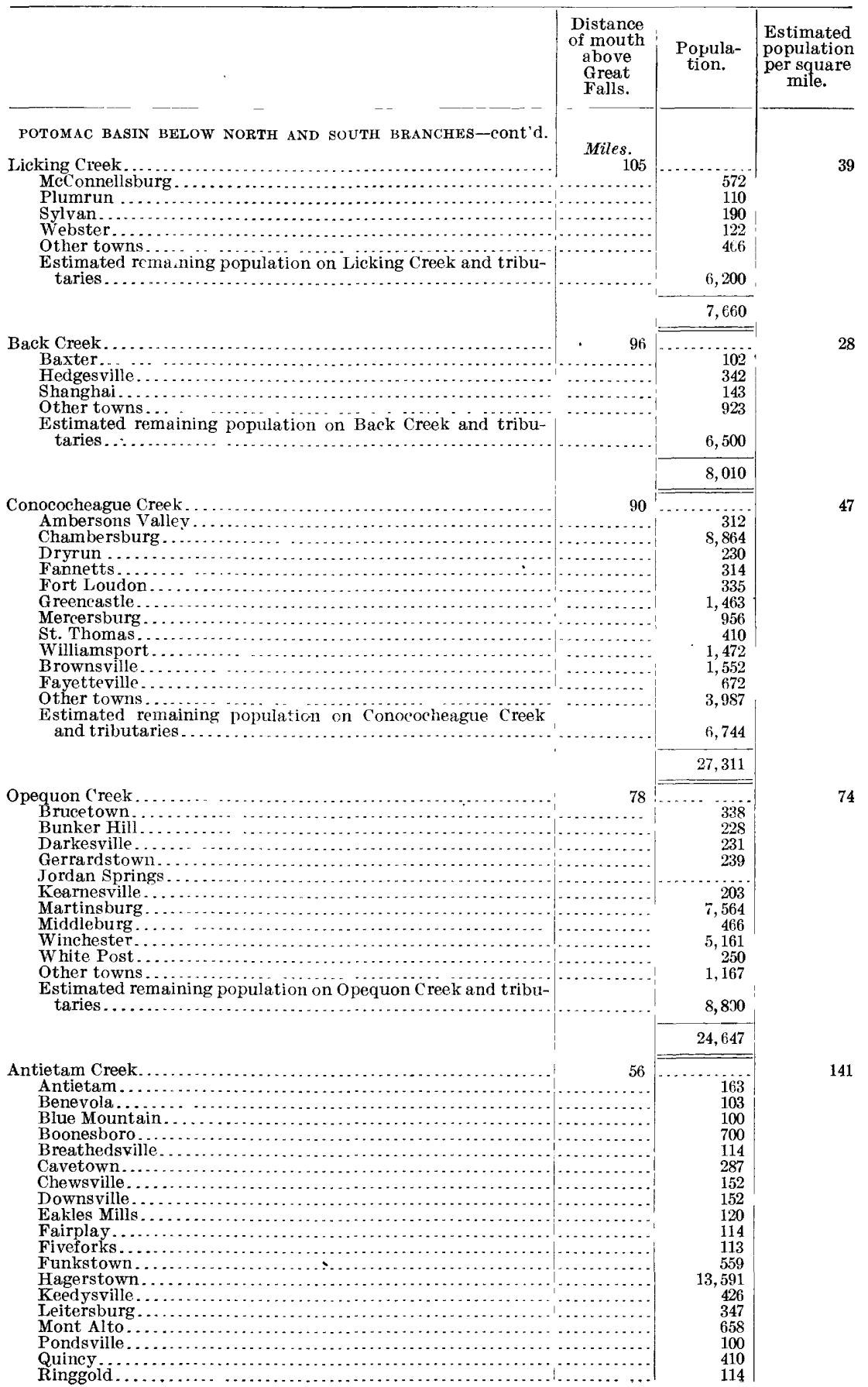


Population of the Potomac River basin-Continued.

\begin{tabular}{|c|c|c|c|}
\hline 4 & $\begin{array}{c}\text { Distance } \\
\text { of mouth } \\
\text { above } \\
\text { Great } \\
\text { Falls. }\end{array}$ & $\begin{array}{l}\text { Popula- } \\
\text { tion. }\end{array}$ & $\begin{array}{c}\text { Estimated } \\
\text { population } \\
\text { per square } \\
\text { mile. }\end{array}$ \\
\hline \multicolumn{4}{|l|}{ POTOMAC BASIN BELOW NORTH AND SOUTH BRANCHES-CONT'd. } \\
\hline \multirow{2}{*}{\multicolumn{4}{|c|}{$\begin{array}{l}\text { Antietam Creek-Continue } 1 . \\
\text { Shad ygrove }\end{array}$}} \\
\hline & $\ldots . . . . .$. & 186 & \\
\hline \multicolumn{4}{|c|}{$\begin{array}{l}\text { Shadygrove } \ldots \ldots \ldots \\
\text { Sharpsburg }{ }_{n} \ldots \ldots\end{array}$} \\
\hline \multicolumn{4}{|l|}{ 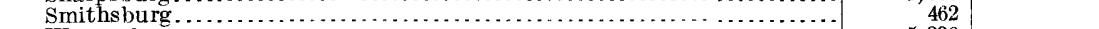 } \\
\hline \multirow{2}{*}{\multicolumn{2}{|c|}{$\begin{array}{l}\text { Waynesboro } \ldots \ldots \ldots \\
\text { Other towns.... }\end{array}$}} & 5,396 & \\
\hline & & 1,201 & \\
\hline \multicolumn{4}{|l|}{ Estimated remaining population on Ant etam Creek and trib- } \\
\hline \multicolumn{2}{|c|}{ 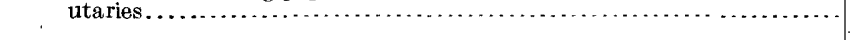 } & 16,342 & \\
\hline & & 42,940 & \\
\hline \multicolumn{4}{|l|}{ Shenandoah River basin: } \\
\hline \multicolumn{4}{|l|}{ 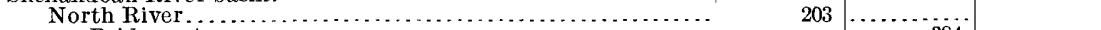 } \\
\hline \multicolumn{4}{|c|}{ Bridgewater . . . . } \\
\hline \multicolumn{4}{|c|}{ Dayton } \\
\hline \multicolumn{4}{|l|}{ 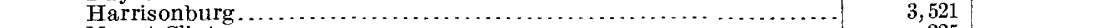 } \\
\hline \multicolumn{2}{|c|}{ 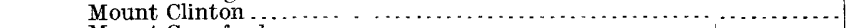 } & 225 & \\
\hline \multicolumn{2}{|c|}{ 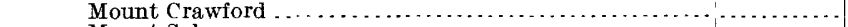 } & 330 & \\
\hline 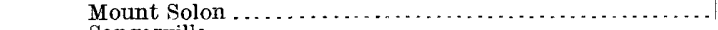 & ............ & 172 & \\
\hline 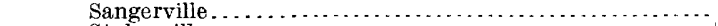 & $\ldots .$. & 175 & \\
\hline 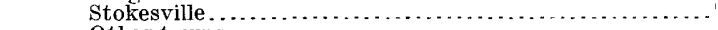 & $\ldots . .$. & 284 & \\
\hline 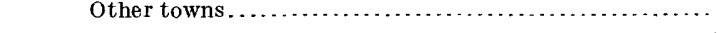 & $\cdots$. & 1,026 & \\
\hline & & 6,542 & \\
\hline 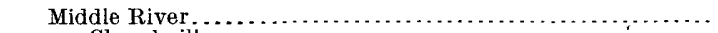 & 208 & & \\
\hline Churchville. . . . & ..... & 239 & \\
\hline Longglade $\ldots \ldots \ldots \ldots \ldots \ldots \ldots \ldots \ldots \ldots \ldots \ldots \ldots \ldots$ & $\ldots .$. & 120 & \\
\hline 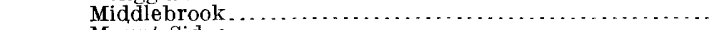 & $\ldots$. & 300 & \\
\hline 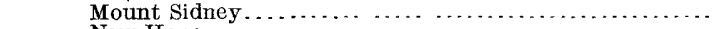 & $\ldots$. & 197 & \\
\hline 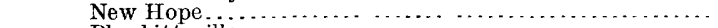 & .... & 124 & . \\
\hline Plunkittsville $\ldots \ldots \ldots \ldots \ldots \ldots \ldots \ldots \ldots \ldots \ldots \ldots \ldots \ldots \ldots$ & $\ldots$. & 100 & \\
\hline 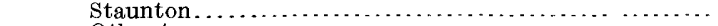 & ..... & 7,289 & \\
\hline 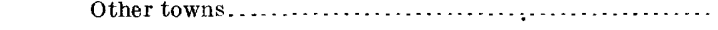 & $\ldots$ & 936 & \\
\hline & & 9,305 & \\
\hline South River.............. & 203 & & \\
\hline 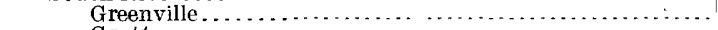 & $\ldots .$. & 328 & \\
\hline 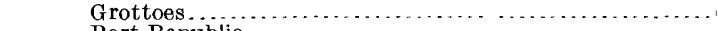 & ..... & 381 & \\
\hline Port Republic . . . . . . . . . . . . . . . . . . . . . . . & $\ldots . .$. & 200 & \\
\hline 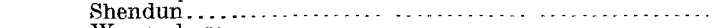 & $\ldots$. & 381 & \\
\hline Waynesboro......................... & ..... & $\begin{array}{r}856 \\
749\end{array}$ & \\
\hline Other towns $\ldots \ldots \ldots \ldots \ldots \ldots \ldots \ldots \ldots$ & & 1,749 & \\
\hline . & & 3,895 & \\
\hline South Fork of Shenandoah River................... & 102 & & \\
\hline 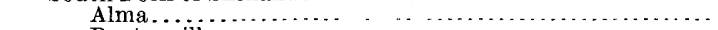 & $\ldots . . . .$. & 127 & \\
\hline Bentonville. . . . . . . . . . . . . & $\ldots \ldots \ldots$. & 197 & \\
\hline 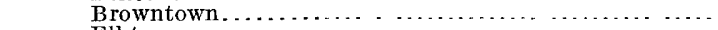 & $\ldots \ldots \ldots$ & 143 & \\
\hline 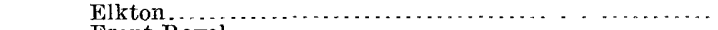 & $\ldots . . . . . .$. & 511 & \\
\hline 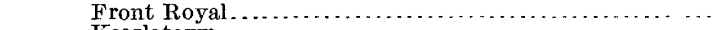 & $\cdots$ & 1,005 & \\
\hline 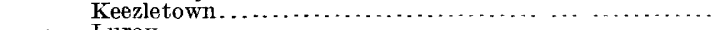 & $\ldots . . . . . .$. & 225 & \\
\hline 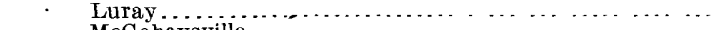 & $\cdots$ & 1,147 & \\
\hline McGaheysville $\ldots \ldots \ldots \ldots \ldots \ldots \ldots \ldots$ & $\ldots \ldots . . . . .$. & 375 & \\
\hline Shenandoah $\ldots \ldots \ldots \ldots \ldots \ldots$ & ............ & $\begin{array}{r}1,220 \\
225\end{array}$ & \\
\hline $\begin{array}{l}\text { Stanleyton } \ldots \ldots \ldots \ldots \ldots \ldots \ldots \\
\text { Other towns } \ldots \ldots \ldots \ldots \ldots \ldots \ldots \ldots\end{array}$ & (n....... & 1,353 & \\
\hline ' & & 6,528 & \\
\hline Passage Creek. & 108 & 205 & \\
\hline North Fork of Shenandoah River $\ldots \ldots \ldots \ldots \ldots \ldots \ldots \ldots$ & 102 & & \\
\hline Broadway .................. & $\ldots \ldots . . . .$. & 400 & \\
\hline 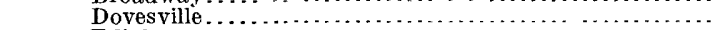 & (............ & 512 & \\
\hline 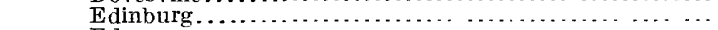 & ........... & 143 & \\
\hline$\ldots \ldots \ldots \ldots \ldots \ldots \ldots \ldots$ & & 176 & \\
\hline 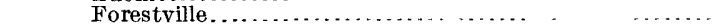 & & 104 & \\
\hline Laceys Spring . . . . . . . . . . . . . . . . & $\ldots . . .$. & 105 & \\
\hline Lantz Mill . . . . . . . . . . . . . . . & & 250 & \\
\hline 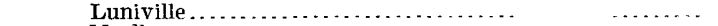 & $\ldots . . .$. & 125 & \\
\hline 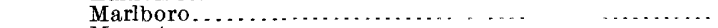 & $\ldots \ldots \ldots . .$. & 100 & \\
\hline 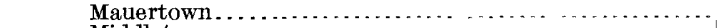 & .............. & 423 & \\
\hline 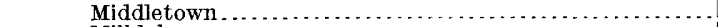 & $\ldots . . . . . .$. & 153 & \\
\hline 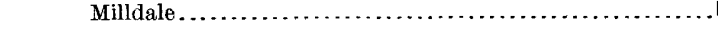 & & 153 & \\
\hline
\end{tabular}


Population of the Potomac River basin-Continued.

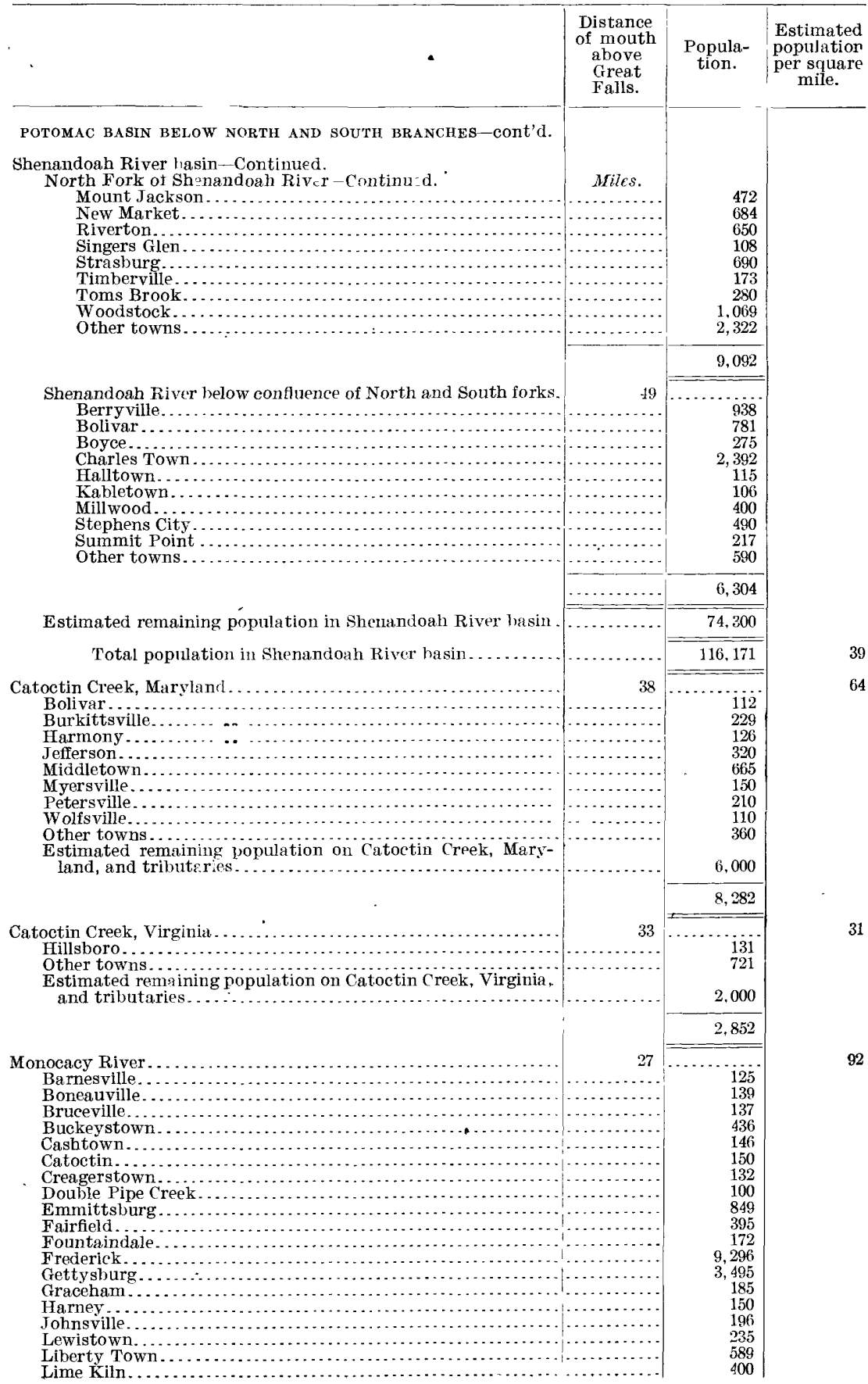


Population of the Potomac River basin-Continued.

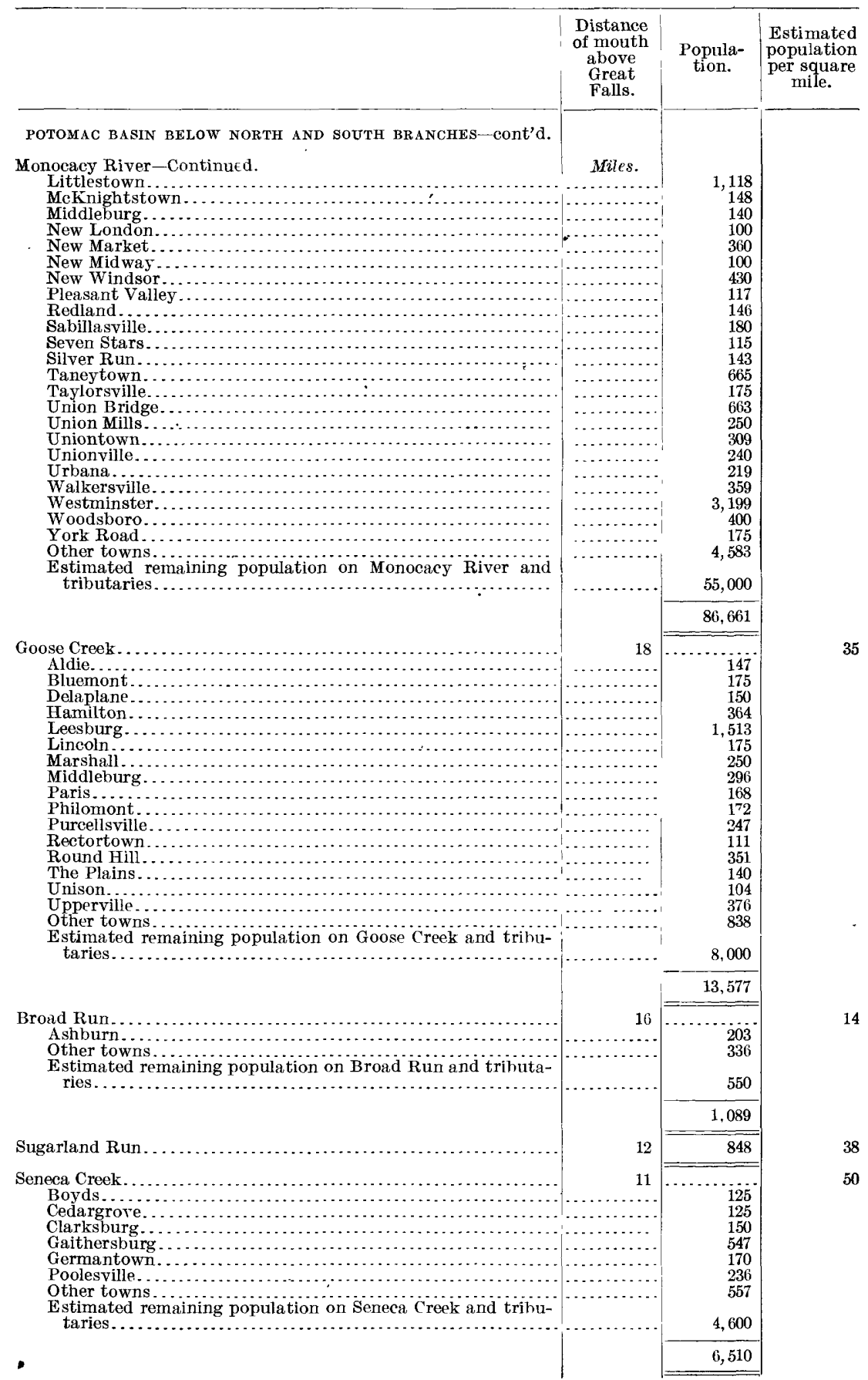

IRR $192-07-17$ 
Population of the Potomac River basin-Continued.

\begin{tabular}{|c|c|c|c|}
\hline & $\begin{array}{l}\text { Distance } \\
\text { of mouth } \\
\text { above } \\
\text { Great } \\
\text { Falls. }\end{array}$ & $\begin{array}{l}\text { Popula- } \\
\text { tion. }\end{array}$ & $\begin{array}{c}\text { Estimated } \\
\text { population } \\
\text { per square } \\
\text { mile. }\end{array}$ \\
\hline POTOMAC BASTN BELOW NORTH AND SOUTH BRANCHES-cont'd. & & & \\
\hline $\begin{array}{c}\text { Potomac River and minor tributaries below North and South } \\
\text { branches } \ldots \ldots \ldots \ldots \ldots \ldots \ldots\end{array}$ & Miles. & & \\
\hline Adamstown & & $256^{\circ}$ & \\
\hline Berkeley Springs. & & 781 & \\
\hline Brunswick........ & $\ldots \ldots$ & 2,471 & \\
\hline $\begin{array}{l}\text { Clear Spring... } \\
\text { Darnestown... }\end{array}$ & ........ & $\begin{array}{l}474 \\
148\end{array}$ & \\
\hline Great Cacapon. & .... & 131 & \\
\hline Hancock....... & & 824 & \\
\hline Harpers Ferry. & & 896 & \\
\hline Knoxville....... & & 363 & \\
\hline Magnolia......... & -1 & 153 & \\
\hline $\begin{array}{l}\text { Oldtown.......... } \\
\text { Paw Paw......... }\end{array}$ & & $\begin{array}{l}112 \\
693\end{array}$ & \\
\hline Point of Rocks... & & 364 & \\
\hline Potomac.......... & & 127 & \\
\hline Shenandoah Junction. & & 318 & \\
\hline Shepherdstown.. & & 1,184 & \\
\hline Sir Johns Run................ & & 239 & \\
\hline Weverton....................... & & 136 & \\
\hline Other towns. . $\ldots$ & & 2,186 & \\
\hline \multirow[t]{2}{*}{$\begin{array}{l}\text { Estimated remaining population on Potomac River and minor } \\
\text { tributaries below North and South branches.............. }\end{array}$} & & 15,800 & \\
\hline & & 27,656 & \\
\hline 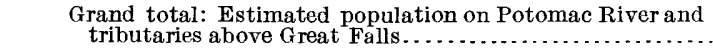 & & 501,647 & 44 \\
\hline
\end{tabular}

Drainage areas of Potomac River and of its principal tributaries at several points in the river basin.

NORTH BRANCH POTOMAC RIVER BASIN.

Squar miles.

North Branch Potomac River ahove Bloomington, Md . . . . . . . . . . $\quad 290$

Savage River at mouth . . . . . . . . . . . . . . . . . . . . . . . . . 120

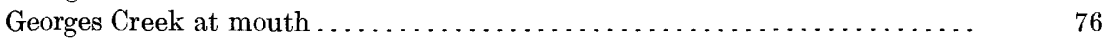

North Branch Potomac River at Piedmont, W. Va., including Savage River and Georges Creek................................ 490

North Branch Potomac River at United States Geological Survey gaging

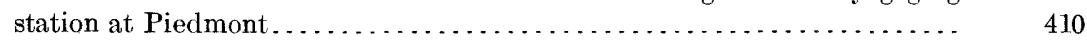

New Creek at Keyser, W. Va . . . . . . . . . . . . . . . . . $\ldots \ldots \ldots \ldots$

Wills Creek at mouth . . . . . . . . . . . . . . . . . . . . . . . . . . . $\quad 270$

North Branch Potomac River at Cumberland, Md., including New ('reek and Wills Creek . . . . . . . . . . . . . . . . . . . . . . . . . . . . 890

Patterson Creek below Mill Creek at Burlington . . . . . . . . . . . . . . 155

Patterson Creek at mouth . . . . . . . . . . . . . . . . . . . . . . . $\quad 280$

North Branch Potomac River at junction with South Branch . . . . . . $\quad 1,360$

SOUTH BRANCH POTOMAC RIVER BASIN.

South Branch Potomac River at Franklin, W. Va . . . . . . . . . . . . . . 190

South Branch Potomac River at junction with North Fork of South Branch. $\quad 320$

North Fork of South Branch Potomac River below Seneca Creek . . . . . . . 240

North Fork of South Branch Potomac River at mouth .... . . . . . . . . . . $\quad 320$

South Branch Potomac River, including North Fork of South Branch . .... $\quad 640$

South Branch Potomac River at Moorefield, W. Va . . . . . . . . . . . . . 900

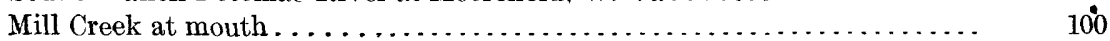


Moorefield River at mouth at Moorefield, W. Va . . . . . . . . . . . . . .

South Branch Potomac River below Moorefield, including Moorefield River. 1,200

South Branch Potomac River at Romney, W. Va . ................ 1,410

South Branch Potomac River at United States Geological Survey gaging

station near Springfield . . . . . . . . . . . . . . . $4 \ldots \ldots \ldots \ldots \ldots \ldots . \ldots \ldots$

South Branch Potomac River at mouth . . . . . . . . . . . . . . . 490

POTOMAC RIVER BASIN BETWEEN SHENANDOAH RIVER AND JUNCTION OF NORTH AND SOUTH BRANCHES.

Potomac River at junction of North and South branches............ 2,850

Little Cacapon River at mouth . . . . . . . . . . . . . . . . . . . . . . . . . 115

Town Creek ................................................ 190

Sideling Creek . . . . . . . . . . . . . . . . . . . . . . . . . . . . . 120

Potomac River above Great Cacapon River, including Little Cacapon River. $\quad 3,390$

Great Cacapon River at mouth . . . . . . . . . . . . . . . . . . . . $\quad 670$

North River at mouth . . . . . . . . . . . . . . . . . . . . . . . . . . . . . 205

Lost River at mouth . . . . . . . . . . . . . . . . . . . . . . . . . . . 190

Cacapon Riv๋er above North River ........................... 405

Potomac River below Great Cacapon River . . . . . . . . . . . . . 4, 060

Potomac River at Hancock, Md . . . . . . . . . . . . . . . . . . . 4, 100

Warm Spring Run at mouth ................................. 16

Sleepy Creek at mouth......................................... 145

Licking Creek at mouth . . . . . . . . . . . . . . . . . . . . . . . . . . . . 195

Back Creek at mouth . . . . . . . . . . . . . . . . . . . . . . . . . . . . 290

Great Tonoloway Creek at mouth . . . . . . . . . . . . . . . . . . . . 125

Conococheague Creek at mouth . . . . . . . . . . . . . . . . . . . . . . 580

Potomac River at Williamsport, Md., including Warm Spring Run, Great

Tonoloway Creek, Sleepy Creek, Licking Creek, Back Creek, and Conococheague Creek .

5,560

335

305

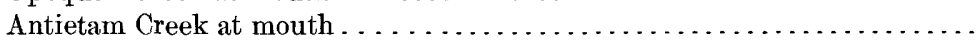

Antietam Creek at United States Geological Survey gaging station near Sharpsburg......................................... 295

Potomac River at Harpers Ferry above Shenandoah ............. 6, 350

SHENANDOAH BASIN.

North River above Bridgewater . . . . . . . . . . . . . . . . . . . 295

Cooks Creek at mouth.................................... 42

North River to Middle River . . . . . . . . . . . . . . . . . . . . . . . . . 420

North River to South River. .............................. 805

Middle River to North River. . . . . . . . . . . . . . . . . . . . . . . . 365

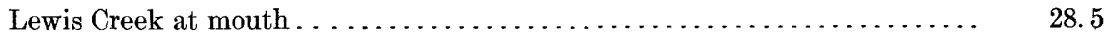

South River to North River . . . . . . . . . . . . . . . . . . . . . . . . 245

North, South, and Middle rivers above Port Republic . . . . . . . . 1, 050

South Fork of Shenandoah River at Shenandoah, Va . . . . . . . . . . . 1,290

Hawksbill Creek at mouth . . . . . . . . . . . . . . . . . . . . . . . . 94

Hawksbill Creek at United States Geological Survey gaging station....... 52

South Fork of Shenandoah River at Overall . . . . . . . . . . . . . . . . 1,490

South Fork of Shenandoah River at mouth at Riverton . . . . . . . . . . . 1,590

North Fork of Shenandoah River at Brocks Gap . . . . . . . . . . . . . . . 215

North Fork of Shenandoah River at Mount Jackson . . . . . . . . . . . . . 510

North Fork of Shenandoah River at mouth at Riverton............. 1,040 
South and North forks of Shenandoah River at Riverton .

Square miles.

Shenandoah River at United States Geological Survey gaging station above

Millville.

2,630

Shenandoah River at Harpen Ferry . . . . . . . . . . . . . . . . .

3,000

3,010

POTOMAC BASIN BELOW SHENANDOAH RIVER.

Potomac River below Harpers Ferry, including the Shenandoah . . ........

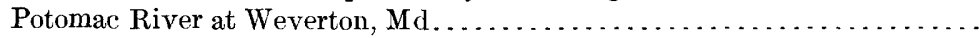

Potomac River at Point of Rocks, Md

9,370

Catoctin Creek, Maryland, at mouth.

9,650

Monocacy River at United States Geological Survey gaging station .......

Monocacy River at mouth . . . . . . . . . . . . . . . . . . . . . . . . .

Goose Creek . . . . . . . . . . . . . . . . . . . . . . . . . . . . .

Broad Run, Virginia ........................................

Sugarland Run . . . . . . . . . . . . . . . . . . . . . . . . . . .

Seneca Creek............................................

Rock Creek.

Potomac River at Edwards Ferry . . . . . . . . . . . . . . . . . . . . .

Potomac River at Great Falls. . . . . . . . . . . . . . . . . . . . . . . . . . .

\section{OCCLRRENCE OF TYPHO D FEVER.}

\section{CAUSES OF TYPHOID FEVER.}

It is now the generally accepted view that typhoid fever is caused by the typhoid bacillus and that only by the multiplication of this organism in the human body is a person brought down with the disease. The bacillus is readily demonstrated in the spleen of those who die from typhoid and is eliminated in the saliva, feces, and urine of the sick. In the latter excretion it is known to be very persistent, so much so that one who has recovered may for a long time thereafter scatter the specific bacteria through this discharge. The evacuations of those not suffering from typhoid never contain the organism. Thus it is that a well or other water supply may be long polluted by dejecta without causing this disease, and may be quickly converted to a plague center by the advent of one stricken with it. Such a person is a menace to the public health, and the infection from him may be diffused in several ways. The simplest of these is by direct contact. Cases are common where those nursing the patient are brought low as a result of handling the discharges or soiled linen of the sick room, and perhaps by using the knives and forks or drinking cups of the sick. Sometines the infected objects are not confined to the domicile of the patient. Thus in Montclair, N. J., an outbreak was brought about on one milk route by infection spread from an unreported case. The milk bottles were brought into the sick room, where they became infected, and were then returned to the dealer, who used them without sterilizing them. In consequence 
typhoid broke out among those of his customers who took their milk in pint bottles, the only kind left at the house in question, none of the other customers being attacked. With the withdrawal of the supply the epidemic ended. It is possible for the disease to be even more widely disseminated by objects handled by the sick. In collecting facts in the Potomac watershed, it was reported that an epidemic had prevailed among the pickers in a certain peach orchard, and it is quite conceivable that the unclean fingers of the laborers may have infected the fruit, which transmitted the disease to the cities where it was marketed.

Unhappily the sickness is not always severe enough to compel its victim to take to bed. He may go about and ease himself not at a single privy, but at the one nearest at hand when necessity is on him. Thus several unsuspected infection centers may be established. Even when the patient is shut up his stools may be thrown, through ignorance or carelessmess, without disinfection into a cesspool or privy or, still worse, on the bare ground.

Typhoid is most frequently spread from the ordure of those afflicted, and this is accomplished by several agencies. The common house fly visits such matter to feed and occasionally to deposit its eggs, and, having accomplished the purpose, returns, with its legs and proboscis bedraggled with filth, to the kitchen or pantry and tracks the infection through the food, which then becomes the vehicle of the disease.

This matter was thoroughly investigated by the typhoid-fever commission of the United States Army in 1898. It was found that the flies alternately visited and fed on the infected stools of the soldiers and the food in the mess tents. More than once, when lime had been scattered over the fecal matter in the pits, flies with their feet covered with lime were seen walking over the food. Moreover, typhoid fever was much less frequent among members of messes who had their tents screened than it was among those who did not take this precaution. The disease gradually died out in the fall of 1898 in Camp Knoxville and Camp Meade with the disappearance of the flies, and this occurred at a time of year when in civil practice typhoid fever is generally on the increase. This was not due to the fact that all those susceptible to the disease had developed it, because the army at these places was considerably recruited at this time. It was also found that flies may carry infected material from soiled bedding and bed pans spattered with the discharge of patients. So this commission pointed out that the typhoid patient should be protected from the annoyance of this insect, not only because he will be more comfortable, but in order to prevent the spread of the disease through its agency.

The fly has also the habit of biting the mouth of man, who frequently moistens his lips with his tongue, and may so infect himself. 
L. O. Howard ${ }^{a}$ enumerates thirteen different flies that are commonly found in houses. Of these, the common house fly he finds to be far the most numerous. In 1900 he made a collection of flies in dining rooms in different parts of the country, and out of a total of 23,087 flies, 22,808 were Musca domestica L.- that is, 98.8 per cent of the whole number captured. The remainder, 1.2 per cent of the whole, comprised various species. The next most abundant fly is Stomoxys calcitrans L., which is vulgarly called the stable fly. It differs from the house fly in that its mouth parts are formed for piercing the skin, which the house fly's are not. It is a common impression that the house fly bites. This is not true, the impression being given by the presence of stable flies. in houses.

Musca domestica commonly lays its eggs upon horse manure. This substance seems to be its favorite larval food. It will deposit upon cow manure, but it has been impossible to rear it in this substance. It will also breed in human excrement and will lay its eggs upon other decaying vegetable and animal matter, but of the flies that infest dwelling houses, both in cities and on farms, the vast proportion come from horse manure. Packard ${ }^{b}$. states that at Salem, Mass., he bred a generation in fourteen days in horse manure. The duration of the egg state was twenty-four hours, the larval state from five to seven days, and the pupal state from five to seven days. At Washington, Howard found that in midsummer each female lays about 120 eggs, which hatch in eight hours, the larval period lasting five days and the pupal period five days, making the total time for the development of the generation ten days. This was at the end of June. The periods of development vary with the climate and with the season, and the insect hibernates in the puparium condition in manure or at the surface of the ground under a manure heap. The adult also hibernates in houses, hiding in the crevices. The Washington observations indicate that the larvæ molt twice, and the insect averages, thus, three distinct larval stages. The periods of development were found to be about as follows: Egg from deposition to hatching, one-third of a day; hatching of larva to first molt, one day; first to second molt, one day; second molt to pupation, three days; pupation to issuing of the adult, five days; total life round, approximately ten days. There is thus abundant time for the development of twelve or thirteen generations in the climate of Washington every summer.

The number of eggs laid by individual flies is undoubtedly large, averaging about 120 , and the enormous numbers in which the insects occur are thus plainly accounted for, especially in view of the abundance and universal occurrence of appropriate larval food. 
Howard says that people living in agricultural communities will probably never be rid of the pest, but that in cities, with better methods of disposal of garbage and with the lessening of the numbers of horses and horse stables due to the advent of automobiles and electric street railways, the time may come when window screens may be discarded. The prompt gathering of horse manure, which may be variously treated or kept in a specially prepared receptacle, would greatly abate the nuisance, and city ordinances compelling horse owners to follow some such course are desirable.

During the summer of 1897 a series of experiments was carried out by Howard with the intention of showing whether it would be possible to treat a manure pile in such a way as to stop the breeding of flies. It was found to be impracticable to use air-slacked lime, land plaster, or gas lime with good results. Few or no larvæ were killed by a thorough mixing of the manure with either of these substances. Chloride of lime, however, was found to be an excellent maggot killer. Where 1 pound of chloride of lime was mixed with 8 quarts of horse manure, 90 per cent of the maggots were killed in less than twenty-four hours. However, chloride of lime costs at least $3 \frac{1}{2}$ cents a pound in large quantities, so that frequent treatment of a large manure pile with this substance would be out of the question in actual practice.

Kerosene was also tested. Hundreds of thousands of flies were destroyed by its use in the experiments, but it was found far from perfect, since if used at an economical rate the kerosene could not be made to penetrate through the whole pile, so that a considerable portion of the house-fly larvæ in the manure escaped injury in this treatment, which was found to be so laborious that hardly anyone could be induced to adopt it.

The most efficient preventive measure was found to be the preparation of a special receptacle for the manure, which was very readily accomplished. A closet was built in a corner of the stable nearest the manure pile. It had a door opening into the stable proper and also a window. A door was built in the outside wall of this closet and the hostlers were directed to abolish the outside manure pile, and in future to throw all the manure collected each morning into this closet, the window of which was furnished with a wire screen. A barrel of chloride of lime was put in the corner of the closet. Since that time every morning the manure of the stable is thrown into the closet and a small shovelful of chloride of lime is scattered over it. At the expiration of ten days or two weeks the gardeners open the outside door, shovel the manure into a cart, and carry it off to be thrown upon the grounds. This treatment has proved very successful at the stable of the United States Department of Agriculture. 
The experiments described above have reference only to the prevention of the breeding of flies in horse manure. Somewhat different measures are necessary to prevent them from breeding in human excrement. The box privy is always a nuisance from any point of view, and is undoubtedly dangerous as a breeder of flies. Hence box privies should not be permitted to exist unless they are conducted on the earth-closet principle. With a proper vault or other receptacle, closed except from above, the breeding of house flies can be prevented. Covering the refuse with lime, however, is more certain than the use of earth. The privy-vault nuisance is a real one, and no community can hope to stamp out typhoid entirely without either exercising the most unremitting, rigid supervision of the latrines or abolishing them altogether.

Wind may also affect the distribution of infected feces. Cases are on record where pits have been dug for the accommodation of considerable numbers, such as soldiers in camp, and the material has become dried and been blown by a violent wind into food which was exposed. Perhaps at the same time persons were infected by inhaling the swirling dust through the mouth.

It is known that typhoid is distributed by vegetables commonly eaten raw, which have been cultivated with contaminated night soil. Often infected privy vault or cesspool matter is used as a fertilizer with disastrous results. An epidemic of typhoid fever in a Massachusetts institution was traced to the use of celery which was enriched with manure which was known to contain enteric feces.

About the 1st of August, 1892, there appeared at Springfield, Mass., a typhoid-fever epidemic that was confined to the customers of one milkman, who, it was found, purchased his milk from several dairymen. On one of these farms had occurred a case of "bilious typhoid fever" and others of the household had been obliged to go to bed with "slow fever." . The stools of the patients went into the privy, it is believed, without disinfection. It was the practice on this place to cool the milk by submerging the cans in a well in such a manner that they rested on the bottom and were covered by 2 to 4 feet of water. As the stoppers leaked, it followed that if the water was polluted with typhoid germs the milk would become infected. It was at first difficult to account for the pollution of the water, for the well was at some distance from the house and the water was not used therein. However, the construction of the well, which was an ordinary dug one of unusually large diameter, admitted of its being readily polluted from the top. The mouth was covered with a platform of old and badly worn planks, loosely laid on, with rounded edges bordering on wide cracks. Furthermore, the planks were separate in order that they might be readily lifted to put the milk in the well to cool. The spout of the pump overhung the platform, so that 
careless pumping easily washed matter on the planks through the wide cracks into the well. Lumps of manure, evidently from the dirty boots of men, were on the platform, and it was found that ordinary stepping about upon the boards shook this dung into the well and also that a little pumping washed it in. Shortly before the typhoid fever broke out in Springfield, the contents of the privy vault before'mentioned were spread upon a tobacco field, and from this field the laborers frequently passed to get water and to work about the milk. Thus fecal matter originally from the privy clung to their boots as they worked in the field and was scraped off and shaken into the well as the men performed their duties about it. So the well water and milk were polluted and the epidemic was established. ${ }^{a}$

Milk is a medium in which the typhoid bacillus multiplies with great rapidity and contaminated milk supplies have repeatedly propagated the disease. The infection is usually brought about by a case of "walking typhoid" (i. e., where the patient is not confined to his bed) in the dairy, or the washing of the utensils or adulteration of the milk with polluted water. The infective matter is circulated about a dairy with such facility that the milk ordinances of cities forbid in the most positive terms the delivery of milk from farms where the disease exists.

In late years it has been discovered that shellfish, such as oysters and clams, which live in sewage-polluted water are apt to convey typhoid. Several occurrences of the disease have been found to be due to this cause. This source of typhoid should arouse the solicitude not only of sanitarians but also of fish dealers, clam diggers, and oyster dredgers, who have a right to demand protection in their business.

While all these factors according to circumstances play a more or less important rôle in the dissemination of typhoid, it is generally recognized that impure water is the principal agent in the transmission of the disease. Every one of the bad epidemics has been indubitably traced back to polluted water, and there is not a State in the Union or $\boldsymbol{a}^{\dot{a}}$ country in the world that does not pay its monthly toll of lives to contaminated water.

Augusta, Me.; Lowell, Lawrence, and Newburyport, Mass.; New Haven, Conn.; Ithaca, N. Y.; Butler, Plymouth, and Pittsburg, Pa.; Cumberland, Md.; Ashland, Wis.; Chicago, Ill.; Washington, D. C., and many other places have passed through the ordeal of a waterborne typhoid epidemic. The supplies are always contaminated by the introduction of infected feces, usually suddenly, but sometimes for considerable lengths of time. Typhoid-fever epidemics due to impure water are characterized by being very limited or very widespread, according to whether the water is used by few or many. The former 
type pertains to well waters and the latter to city supplies of considerable size. In many well-water epidemics it is possible to draw a circle of short radius, with the well for a center, which includes nearly all of the cases. That is, the victims are among those who habitually use the well. The onset of such epidemics is usually sudden, their period of extreme virulence is brief, and they have a tendency to disappear.

When large supplies are infected the cases are distributed throughout the community and appear either in a desultory fashion, as the result of slight pollution, very likely at different times and places, or in great numbers at one time, owing to the sudden introduction of a large quantity of pollution, which is usually effected by heavy rains or rapid thaws. In the case of epidemics from large supplies the period of malignancy is somewhat less sharply marked than in well-water outbreaks, but the proclivity to die out is as noticeable.

This tendency of epidemics to run out brings us to a consideration of the typhoid bacillus itself, for it is manifest that if this organism is the cause of the disease something must have happened to it or the outbreak would continue indefinitely. At the outset it must be frankly admitted that we have not so certain a knowledge of the history of the bacillus in water as is desirable. Great ingenuity and much work have been spent in the effort to determine the longevity of the typhoid bacillus in water. The difficulty has been to reproduce in experiments the conditions that surround the germ in flowing streams, wells, and reservoirs. Such environments appear to have been most nearly approximated in the methods introduced by Jordan and Zeit ${ }^{a}$ and developed by Russell and Fuller. ${ }^{b}$ The results of the latter investigations are as a whole concordant with those of the former, and show that the typhoid bacillus lives five days in sewage and eight to ten in water. They indicate also that the life of the bacilli is shorter when they are in direct contact with sewage bacteria than it is when they are exposed in sewage but protected from the bacteria therein. The experiments are so perfect that it seems justifiable to assume a life of ten days for the typhoid bacillus in water, but great caution should be exercised in generalizing further, though the results may tempt one to infer that the existence of the germ is but little, if at all, prolonged beyond the maximum period observed in the experiments. Whipple and Mayer ${ }^{c}$ have by experiments suggested that the amount of oxygen dissolved in the water may have an important influence on the longevity of the typhoid bacillus and query whether the rapid disappearance of the germ in sewage is not in part due to the absence of this element. It seems to be the prevalent belief that the various mineral salts commonly held in solution by natural waters neither

a Jour. Infectious Diseases, vol 1, no. 4; Engineering Record, December 24, 1904.

$b$ Reports and Papers, Am. Pub. Health Assoc., vol. 21, pt. 2, p. 40.

cReports and Papers, Am. Pub. Health Assoc., vol. 21, pt. 2, p. 76. 
favor nor inhibit the growth of the typhoid organism. The literature on this subject appears to be scanty, and it is suggested that here is a field for investigation. Thus it appears that before definite opinions as to the longevity of the typhoid bacillus can be formulated it will be necessary to prosecute every feasible bacteriological experiment and to carefully collate and study the facts gleaned from inquiries into epidemics.

Although the science of bacteriology has not determined the length of life of the typhoid bacillus in water, it has adduced certain facts in regard to bacteria in general that it seems reasonable to suppose apply to that germ. These are:

1. That all bacteria multiply rapidly in their natural habitat, and conversely that multiplication is checked elsewhere.

2. That an abundant food supply and an optimum temperature are prime factors in bacterial development.

3. That insolation has an injurious effect on bacteria.

4. That they are slightly heavier than water and so tend to sink in it.

5. That certain microscopic organisms, namely, algæ and infusoria, feed on them and so reduce their numbers.

The typhoid bacillus thrives best in the human intestine, where food is abundant and the temperature is high, so that its invironment becomes unfavorable when it is brought into water. There it has to compete for food with the bacteria that live in that element. The low temperature, the sunlight, the tendency to sink, and microscopic organisms all work to exterminate it. These are the natural factors that kill the germ and end epidemics without the intervention of human aid.

In regard to the temperature of rivers, it is to be regretted that no measurements are available, nor have any very extended observations been made on any American river with a delicate instrument, such as the thermophone. Kofoid, ${ }^{a}$ however, did some careful work with thermometers on Illinois River from August, 1892, to March, 1899. According to his observations, the temperature of river water follows an annual cycle of the same general character year after year, with ever present minor variations of local origin. In winter the water is coldest and varies least in temperature; the minimum observed was $32^{\circ} \mathrm{F}$., the average was $32.75^{\circ}$, and the maximum was -rarely higher than $34^{\circ}$. This constancy is doubtless due to the ice which normally covers the stream and especially its backwaters. When the temperature is below $39.2^{\circ} \mathrm{F}$., the point of greatest density of water, the colder waters are at the surface, though there is usually very little difference of temperature at different levels during this season. In spring the temperature of the water commences to rise 
very early, the beginning and rate of increase depending on the peculiarities of individual years; the observations made indicate a gradual increase from an average of $40.45^{\circ} \mathrm{F}$. in March to $60.46^{\circ}$ in April and to $68.27^{\circ}$ in May, which is a month not only of marked rise but also of considerable fluctuation in temperature. In summer the water is warmest and varies greatly in temperature; the average maximum rose from $77.75^{\circ}$ in June to $81.49^{\circ}$ in August and fell to $74.21^{\circ}$ in September. The average fluctuation during this season was less than $10^{\circ} \mathrm{F}$., though the absolute range for August in five years was from $74.3^{\circ}$ to $89^{\circ}$. These rises and falls, combined with the effects of the wind and diurnal changes in temperature, cause considerable vertical circulation of the water, because the surface waters, especially on still hot days, are from a fraction of a degree to $5^{\circ}$ warmer than the deeper waters. In autumn the fall of temperature begins late in September and becomes practically complete in November; like the rise in spring, it is subject to irregularities in its rate and permanence. The maximum and minimum observed in the river during this season were respectively $96^{\circ}$ and $32^{\circ} \mathrm{F}$. When the temperature of the air falls in autumn below that of the water the consequent convection of the surface and bottom layers of the river causes the water temperature to become practically uniform at all points in the vertical section. These well-defined periods of minimum, increasing, maximum, and decreasing temperature doubtless cause in the minute organisms in the water corresponding seasonal changes as fundamental and as extensive as those that affect the plant and the animal life of aerial environment.

In addition to the influence of season there are several minor causes of variation in temperature, such as the entrance of water from tributary streams, springs, and impounded backwaters, the local shallowness of the water, and the diurnal range of temperature in the air. The changes produced by the entrance of water are of course individual and variable. The depth of the stream determines the effect of the temperature of the bed, though the difference between the surface and bottom temperatures of stream water is, as a rule, very small. The daily variations in temperature depend essentially on those of the air. On August 5, 1898, the temperature of the water near the surface varied from $79.5^{\circ} \mathrm{F}$. at $5 \mathrm{p}$. m. to $74^{\circ}$ at 2 a. m., while that of the water near the bottom (depth 8 feet) varied from $74^{\circ}$ at $8 \mathrm{a} . \mathrm{m}$. to $76^{\circ}$ at $11 \mathrm{a}$. m., a fluctuation of only $2^{\circ} \mathrm{F}$.

Some of the conclusions reached by Kofoid are particularly pertinent to the relation of river temperature to typhoid fever. The average temperature of Illinois River in August was $81.49^{\circ} \mathrm{F}$, , only $16.7^{\circ}$ below body temperature, and the maximum observed in that month was $89^{\circ} \mathrm{F}$, only $9.2^{\circ}$ below body temperature. Accordingly it would appear that at times the bacillus of typhoid fever may be in 
water at a temperature not far from the optimum. The fact that in summer the temperature causes a vertical circulation in the river water should be borne in mind in deciding on the efficacy of sedimentation in removing bacteria. The fact that the seasonal variations in temperature cause corresponding variations in the vitality of micro-organisms indicates that decrease of heat is of unequal moment at different seasons of the year in destroying bacterial life. Finally, it would be interesting to know whether, on account of the small difference noted between surface and bottom temperatures, it is ever possible for sewage to flow a considerable distance in a river without mixing with the stream water. If it is, then sewage might in some cases flow from one citv to another and arrive in a fairly concentrated condition.

With regard to the Potomac itself, it may be remarked that the large quantity of water received from ground flow from mountain tributaries and from mines all tend to influence its temperature.

The destruction of food material in rivers apparently bears an intimate relation to temperature, as was shown by investigations on Illinois River and the Chicago drainage canal between Bridgeport and Joliet. " Ordinarily in the warm weather there is a marked oxidation here with evident destruction of organic matter. This was shown in the investigations of 1888 and in striking degree in those of the summer of 1886 . In the cold winter and spring months of 1889 , on the contrary, the oxidation was slight between Bridgeport and Joliet, and this is probably the normal low-temperature condition."'a

It is an interesting fact that the period of minimum temperature, as defined by Kofoid, coincides with the months of the minimum prevalence of typhoid fever in Washington, and that the influence of the period of maximum temperature extends over all the months during which the disease is at its height except only the second half of October. If it be admitted that by reason of the geographic situation of the Potomac basin the temperature of the stream remains at the maximum a few weeks longer than it does in Illinois River, the months of the maximum occurrence of the disease fall quite within the period of maximum temperature. Furthermore, the decline of typhoid fever in autumn seems to coincide very closely with the fall of temperature, and the increase of typhoid fever that succeeds its period of minimum prevalence seems to occur near the end of the period of increase of temperature. This apparently indicates that it is necessary for the water to attain a rather high temperature before it reaches its most favorable condition for transporting the germs of typhoid fever. It is true that it has not been uncommon for water-borne epidemics of the disease to occur in early spring and

a Egan, J. A., Pollution of the Illinois River: Sanitary Investigations of the Illinois River and its Tributaries, Illinois State Board of Health. 1901, p. xxiii. 
also in early winter. However, it is believed that in these cases the time that elapsed between the introduction of infected feces into the water and the lodgment of the bacilli in their victims was brief, and that therefore the germs were able to withstand untoward temperature conditions to which they would have succumbed had the interval of exposure been longer.

It should be remembered that, as Kofoid has determined, the temperature of a river responds very quickly to fluctuations in the temperature of the air abeve it. Sedgwick and Winslow ${ }^{a}$ have pointed out that "in communities with reasonably pure water supplies the typhoid fever follows the curve of seasonal temperature with extraordinary regularity. If the monthly deaths from the disease be plotted and compared with the monthly temperatures, it will be found that the curves are almost parallel, the typhoid rising with the temperature after about two months, an interval representing the incubation period of the disease and the time which elapses before death." So, in a measure, these river-temperature periods but reflect typhoid conditions which prevail under like temperature conditions on land; and it is felt that the river temperatures act in the same direction as the air temperatures and play a considerable part in fostering or restraining the dissemination of the disease. In this connection the statement made by Clark and Gage ${ }^{b}$ that in a river as polluted as the Merrimac the numbers of Bacillus coli, the common intestinal bacillus, are more numerous in warm than in cold weather is possibly significant.

How much importance should be attributed to microscopic organisms in reducing the bacteria in water is a moot question. 'There seems to be a concurrence of opinion that they play only a minor part, but the matter will have to be investigated more carefully than it has been before the possibility of their having a considerable beneficial influence in this direction can be positively denied. However, it is certain that at some seasons of the year they are present in the water in very small numbers and therefore that their action is not constant.

As water is not the normal habitat of typhoid bacilli, it is universally admitted that their detention in that medium is of the utmost importance in their elimination. By giving the factors of insolation, unfavorable temperature, and reduction of their food supply an opportunity to act through considerable time the vitality of the germ is reduced and its final destruction is brought about. The quicker the journey from intestine to intestine is accomplished the better is the chance for the survival of the bacillus. Now, sedimentation is recognized as potent in delaying the germ in its travels. ${ }^{c}$ The process is

$b$ Rept. Massachusetts State Board of Health, 1892, p. 279.

$c$ Whipple, G. C., The Microscopy of Drinking Water, 1899. 
a simple one in reservoirs and lakes, for it is interfered with only by the convectional currents established by the heating of the water and by the very considerable circulation of the water brought about by the wind. But in rivers it is further complicated by the phenomena of transportation. ${ }^{a}$ Everyone knows that the solid material brought to a river by various agencies does not sink to the bottom as soon as it reaches the stream, but is carried forward by the current. By careful experimentation some of the laws that govern this action have been discovered. It is well known that the power of flowing water to transport débris increases with increasing velocity, and that the size, specific gravity, and form of the loose material determine whether or not it will be moved by a current of given velocity. The smaller the divisions of any mass the larger the ratio of surface to weight, and the force which a current of given velocity exerts against objects in its path varies as the area of the opposing surface. Therefore the finer the particles the more easily will they be transported. But the ability of streams to carry débris in suspension is dependent not only on these factors but on the presence of secondary and especially of upward currents, which tend to lift up the particles brought within their influence. Were it not for these minor currents particles would sink in a regular curve, whereas the path actually traveled by them is a very broken line, sometimes tending toward the bottom, at others toward the banks of the stream, and again upward toward the surface. When the particles finally reach the bottom they remain there only so long. as they do not come within the influence of a current strong enough to lift them into circulation, which may be for either a long or a short period. It has been found that the change in the velocity of streams in horizontal planes is greatest near the shore and least near the thread of maximum current; and that in vertical planes it is greatest near the bottom and surface and least at about one-third of the depth of the stream - that is, where the absolute velocity is greatest. If, then, the water be either charged to its maximum capacity or overcharged with sediment, the highest percentage of material will be found near the banks, surface, and bottom, and the least amount at a depth of about one-third that of the stream. If, however, the water is undercharged with suspended material, as is the case with most streams, the distribution will not follow any law, the amount at any locality being dependent on the chance swirls and boils. It is an important fact that the transporting power of running water increases in a greater ratio than the increase in velocity. It has been found that if the surface of an object remains constant the force of a current striking it varies as the square of its velocity; also that the transporting power of a current, or the weight of the largest fragment it can carry, 
va ries as the sixth power of the velocity. It will be seen that under this law doubling the velocity of a current increases its transporting power 64 times. An increase in the volume of a stream increases its velocity; hence floods multiply the transporting power of water and by so doing acquire a destructive power which is accounted for by the foregoing law.

Besides the material which a stream carries in suspension, there is that finely divided matter which it rolls along over its bed. This does not travel in sheets, but moves along in wave-like forms which have a long, gentle slope in the direction of the current and an abrupt drop on the downstream end. Thus particles which reach the bottom of a stream do not come to rest unless they are too heavy for the stream to drag along.

From this discussion it is evident that sedimentation is profoundly affected by any factor which tends to quicken or retard stream flow, and that it is exceedingly difficult to follow material after it is once committed to a stream. At one time it will be in suspension, at another at rest. Now it may travel near the bottom, again at the surface, or at another time it may be deflected against the banks of the river. But it is evident that all these migrations take time, and this is what is desired to accomplish bacterial purification. Thus food material may become exhausted before it has traveled far downstream, or some other agent may have worked considerable change in a few miles of river flow.

It should be noted that sedimentation means more than the mere settling out of bacteria by their own weight, for other and heavier materials in sinking mechanically entrain the germs and drag them toward the bottom. This action is most effective when large quantities of silt are being rapidly deposited - that is, when the action is taking place in turbid waters. In relatively clear water it loses much of its importance.

Rapid sedimentation is often brought about by chemical means. Thus, when highly acid mine waters are discharged into rivers whose waters contain notable amounts of lime, insoluble salts are formed, which sink rapidly to the bottom. As they do so they carry a large proportion of the bacteria with them, so that mine wastes are not always an unmixed evil.

Though the Potomac is as a whole a swift river, sedimentation is undoubtedly active in much of its course. Even in its upper reaches this force is at work, as anyone can test for himself by walking along the banks from Westernport to Keyser, where large quantities of salts resulting from the action of the waters of Georges Creek on the limy wastes of the West Virginia Pulp and Paper Company have been deposited on the rocks and in the shallows. In the many pools in the bed of the river the current must necessarily be checked and deposition. 
accelerated. Particularly important are the seven dams, which cause slack water for miles. In low stages of the river the water must move toward the dams very slowly, and they undoubtedly afford the forces of purification by deposition the greatest play. Some question has been raised whether the protection against typhoid accorded in this way does not contain an element of danger. Thus Sedgwick has noted that in Lowell and Lawrence, Mass., the summer typhoid occurred after sudden rises in Merrimac River caused by thunderstorms and the like, and has suggested that the increase of the disease might be partly accounted for by the fact that considerable infected material had collected behind the dams on the river and had been carried downstream when the water flowed over the dams in considerable quantities.

This raisès the important question as to what becomes of germs after they are deposited at the bottom of streams. If they simply rest there until they are again brought into circulation by the scouring of the beds in floods and by other forces, much more importance has been attached to sedimentation than it deserves. But it would seem that the environment of the bottom of a stream must be hostile to germs which live in the human body. Jordan has pointed out that a careful examination of the bed of Illinois River failed to reveal any accumulation of sludge from the large amounts of sewage it receives, and therefore it may be concluded that the amount of available food material for bacteria at the bottom of streams can not be very great. It is conceivable that the sewer of a small village might be made to discharge into a pool of a stream, instead of into the current, and so convert the pool in times of low water into a place favorable for the multiplication of germs, which would be carried into circulation by a sudden flood, with disastrous results. But these factors are not generally considered of serious import as affecting the belief that sedimentation is one of the most powerful factors in purifying water. Rather, it is held that they operate only under special conditions, at rare intervals, if at all. However, the sediment of sewage-polluted streams would undoubtedly be a fruitful field of investigation.

There has been much quibbling over the question whether the dilution of a severely polluted stream with the waters of a pure one should be considered as purification or not. It would seem that the result achieved is clear-namely, the chance of drinking typhoid germs has been lessened. In that sense there has been purification, but it does not necessarily mean that there has been any destruction of food material nor that any germs have been killed.

The deaths from typhoid fever in the principal cities of the world are shown in the following table: 


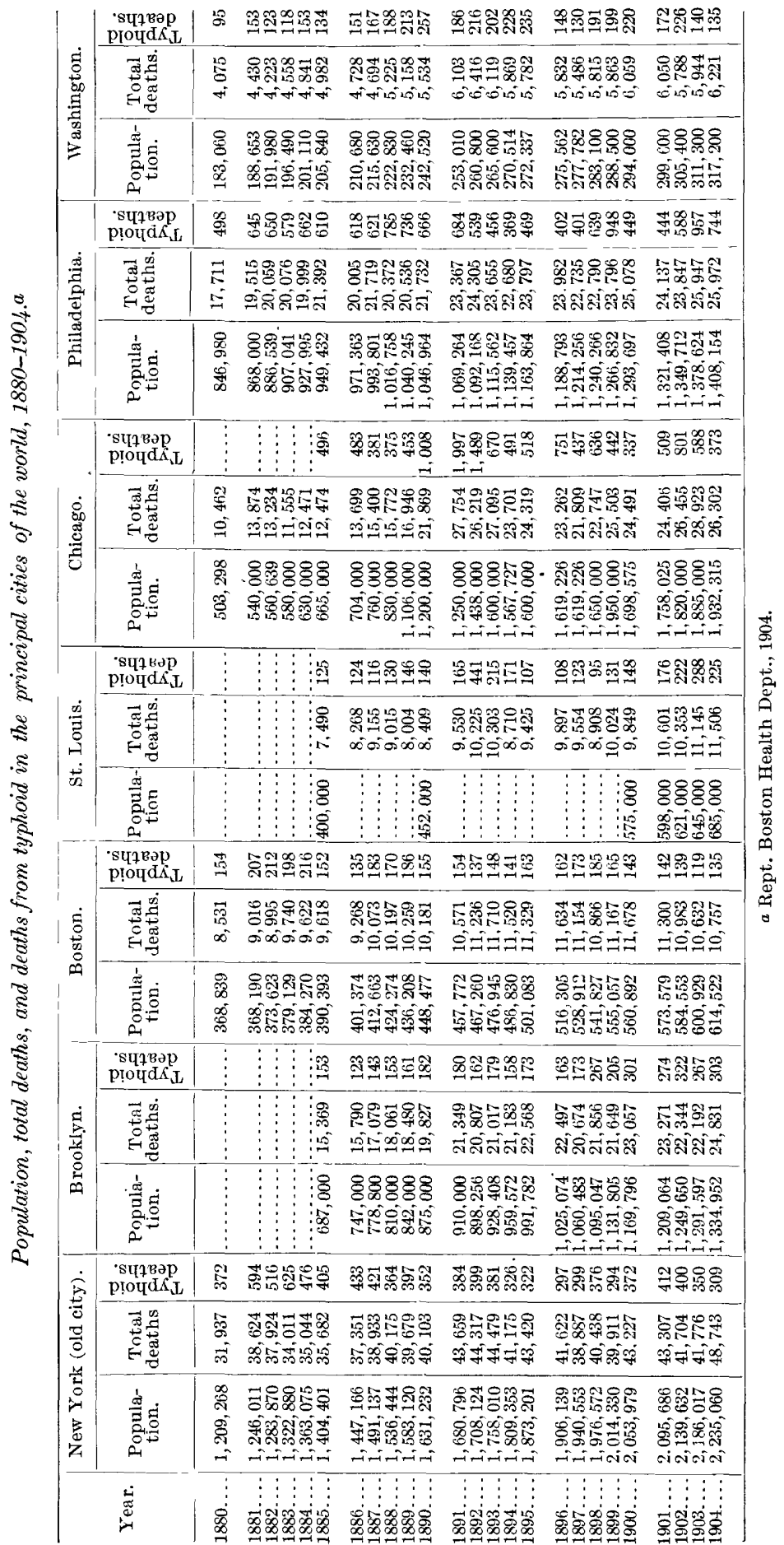


TYPHOID FEVER, DEATHS FROM.

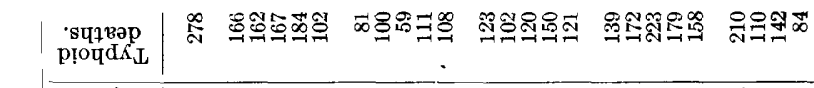

官 矛弯

若

-

苛品

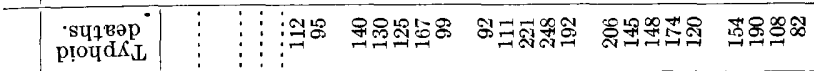

- $\overrightarrow{0}$ 西

F苋

莺

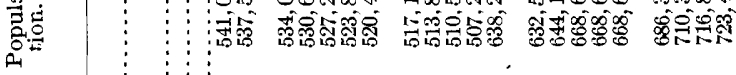

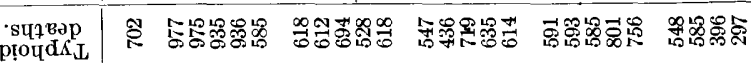

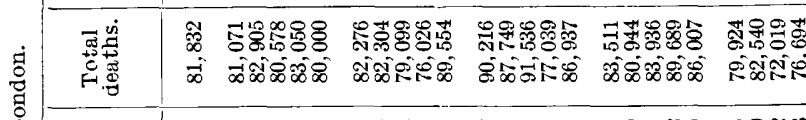

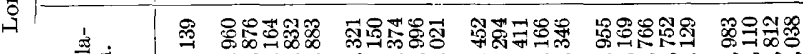

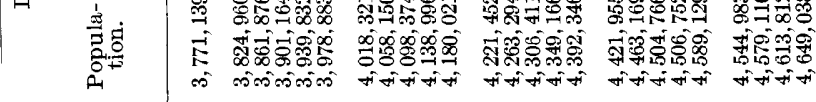

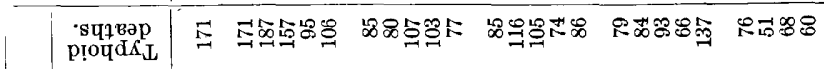

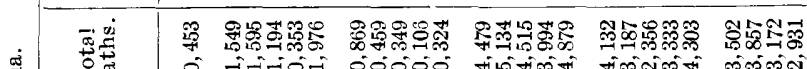

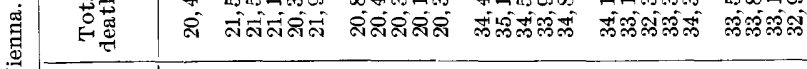

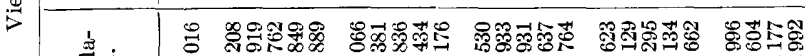

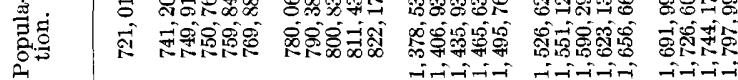

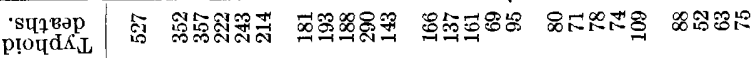

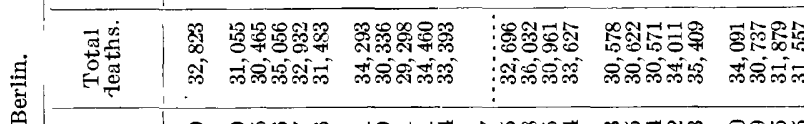

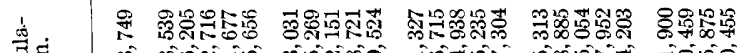

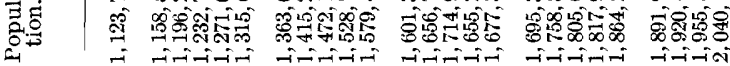

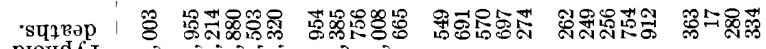

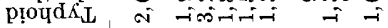

\begin{tabular}{|c|c|c|c|c|c|c|}
\hline 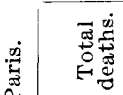 & है & 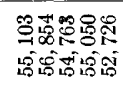 & 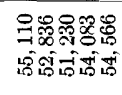 & 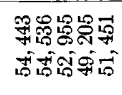 & 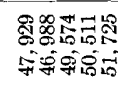 & 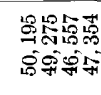 \\
\hline 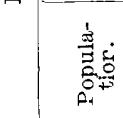 & & 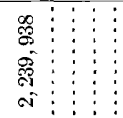 & 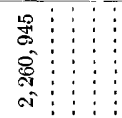 & $\begin{array}{l}\text { 용 } \\
\text { ज़ } \\
\text { هi }\end{array}$ & 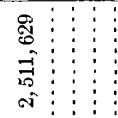 & 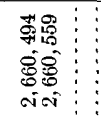 \\
\hline 恋 & & 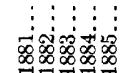 & $\begin{array}{l}\infty \\
\infty \\
\infty \\
\infty \\
\infty \\
\infty\end{array}$ & 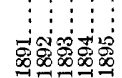 & $\begin{array}{l} \\
\& \\
\infty\end{array}$ & 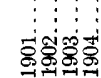 \\
\hline
\end{tabular}


TYPHOID FEVER AT WASHINGTON, CUMBERLAND, AND MOUNT SAVAGE.

When this inquiry was begun a thorough investigation of the typhoid-fever mortality was contemplated, for the ravages of this disease are in general caused by the contamination of water supplies by privies, cesspools, and sewers, and it was thought that no better measure of financial loss through river pollution could be had than the destruction of human life it caused. But it was discovered that none of the four States in which the basin lies had registration laws. Accordingly this study had to be confined to the District of Columbia, where the records extend over thirty-one years. The law has been administered with great fidelity by the health department, which has assembled the results with intelligence and discrimination. It is to be regretted that the statistics do not cover the entire period through which the Potomac has been the water supply of Washington, but they were not collected from the troublous times of the civil war until August, 1874. Effective registration really began in January, 1875 , and only from that date on are the results of value. Recently Pennsylvania has enacted an excellent registration law and Maryand has adopted one which it is hoped may prove serviceable, but Virginia and West Virginia have none. This is to be regretted, for the faithful registration of births and deaths more than repays its cost by the aid it gives in establishing property rights and in suppressing child murder. Moreover, by the lack of vital statistics sanitary science is deprived of a great help in the fight against communicable disease. Unless the health of a community is a matter of knowledge, of record, and not of hearsay, it is impossible to tell whether its condition is normal or whether its water supply, its milk supply, or some other function is deranged and diseased. It is to be sincerely hoped that this defect will soon be cured by wise legislation.

At Washington the undue prevalence of typhoid fever has been caused by the public water supply, which has been taken from Potomac River since December, 1863, and which was consumed without purification until October 5,1905.

Some of the other factors which have been mentioned as producing typhoid undoubtedly are responsible for a part of the cases. There are many privies in the city, and they have in all probability been at times foci from which contagion spread. There are polluted wells which must have played their part. Cases of auto-infection were probably numerous. Small epidemics caused by infected milk have been traced to their sources, and the disease has been brought in from outside. But the typhoid has been too constant and too generally distributed in the city to lay more than a small part of it to the door of these sporadic outbreaks. It can be accounted for only by 
an impure water supply, and it now remains to consider what scope the natural and artificial forces at work on the Potomac give to the several factors that favor or militate against bacterial life. Food supply for the germs must be plentiful, being furnished by the numerous sewers and privies that discharge into the river, the distillery wastes, the rinse water from the finished goods of several factories, and most of the organic wastes received by the stream. The action of sunlight is regarded as only mildly inhibitory, but it has full play on the Potomac, for the water is light colored and is clear except during rather short periods of freshets. Moreover, much of the river is shallow, so that the light rays penetrate quite to the bottom over considerable stretches.

In the early days of Washington the feasibility of supplying the city with water from the Potomac was considered, but it was not till 1859 that water was turned into the aqueduct. It came from Dalecarlia Reservoir, then wholly supplied by Little Falls Branch. This reservoir was built with the idea of affording an opportunity for clarification of the water by sedimentation as it passed through. The same year the distributing reservoir in Georgetown was built. In the early days these reservoirs may have afforded considerable protection, but with the present daily consumption of $65,000,000$ gallons the former gives a storage of only sixteen hours and the latter of only twenty-three hours. In 1902 the Washington city reservoir was put in use and gives an additional storage of forty-five hours. In December, 1863, water from the Potomac at Great Falls, 14 miles above Washington, was received, and it has been the main supply of the city ever since. As there were no vital statistics kept at that time, it is impossible to say what effect the introduction of the supply had on the public health. Indeed, had such records been kept their interpretation would demand great caution, for it was at the time of the civil war and the soldiers of both armies suffered terribly from typhoid, as may be seen from the subjoined figures taken from the Medical and Surgical History of the War of the Rebellion. The figures do not include typhoid malaria, and it is to be remembered that the troops did not operate in the Potomac basin all the time. The table does not include all the troops operating in the basin, and some of the cases were of men who belonged to the armies cited, but who were temporarily detached therefrom at the time of sickness. However, the figures it is believed give an idea of the prevalence of the disease, which is all that is desired. The armies of the Confederacy are believed to have suffered more heavily than did those of the Union. 
Typhoid fever in Federal Army durinq civil war.

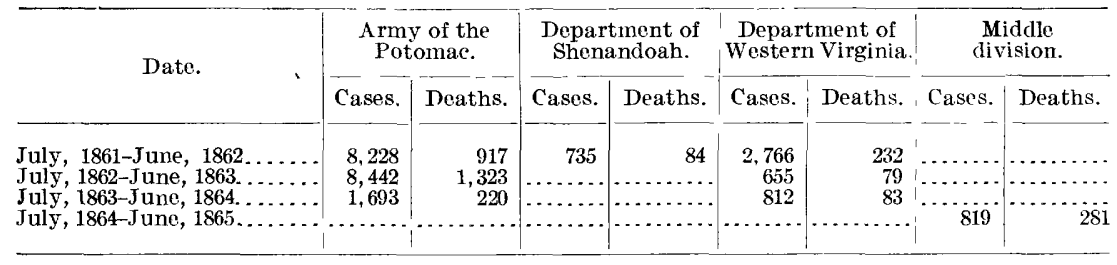

It is evident that the whole Potomac drainage area was thoroughly seeded with typhoid during the war.

Other events have had an intimate relation to the prevalence of typhoid fever in Washington. ${ }^{a}$ In 1888 there were many complaints against the character of the water, both on account of its turbidity and its supposed pollution. Accordingly at that time Dalecarlia Reservoir, which was still fed by Little Falls Branch, was cut out of the supply and used only on the rare occasions when repairs to the aqueduct made it necessary. This expedient does not appear to have had any effect on the typhoid death rate of the city. In 1893 it was decided to divert the waters of Little Falls Branch, Mill Creek, and East Creek, together with other waters, from Dalecarlia Reservoir, into which they fed. The work was completed May 27, 1895. The typhoid death rate of that year was 53.7, as against 86.3 in 1894 . The following year it was still lower, but it rose again in the three succeeding years, yet it never has attained the figures of the years immediately preceding the execution of this work.

There are probably mild outbreaks of typhoid fever at one or more places on the Potomac watershed every year, but two well-marked epidemics are on record which produced decided effects in the District. $^{b}$

The first of these was at Cumberland, Md., and was brought about by typhoid dejecta finding their way into Sulphur Run, which empties into the Potomac somewhat above the waterworks intake. The trouble began about December 10, 1889, and was not quelled until the beginning of June, 1890. In all 485 cases and 97 deaths of the disease were reported in Cumberland. The river was said to be in middle stage at the time, and soon after the outbreak at Cumberland typhoid appeared at Hancock, 60 miles downstream. Captain Gaillard ${ }^{c}$ estimates the time of flow of water from Cumberland to Washington as from four to seven days, but the disease did not appear in unusual measure in Washington until March, 1890. The number of typhoid deaths contimued to be abnormally high after that until August, when it was not much above the usual number for the city. The following table gives an idea of the sequence of the disease: 
Deaths from typhoid fever at Cumberland and Washington, January to July, 1890.

\begin{tabular}{|c|c|c|c|c|c|}
\hline \multirow[b]{2}{*}{ Month. } & \multicolumn{2}{|c|}{ Deaths. } & \multirow[b]{2}{*}{ Month. } & \multicolumn{2}{|c|}{ Deaths. } \\
\hline & $\begin{array}{c}\text { Cumber- } \\
\text { land. }\end{array}$ & $\begin{array}{l}\text { Wash- } \\
\text { ington. }\end{array}$ & & $\begin{array}{l}\text { Cumber- } \\
\text { land }\end{array}$ & $\begin{array}{l}\text { Wash- } \\
\text { ington. }\end{array}$ \\
\hline $\begin{array}{l}\text { January } \ldots \ldots \ldots \\
\text { February } \ldots \ldots \\
\text { March } \ldots \ldots \\
\text { April } \ldots \ldots \ldots\end{array}$ & $\begin{array}{r}18 \\
27 \\
39 \\
8\end{array}$ & $\begin{array}{r}11 \\
6 \\
19 \\
11\end{array}$ & $\begin{array}{l}\text { May ......... } \\
\text { June ......... } \\
\text { July ..... }\end{array}$ & \begin{tabular}{|r}
\multicolumn{1}{|c}{5} \\
$\ldots \ldots \ldots$ \\
\hdashline$\ldots \ldots . .6$
\end{tabular} & $\begin{array}{l}13 \\
24 \\
36\end{array}$ \\
\hline
\end{tabular}

The second epidemic which is known to have produced calamitous results in Washington occurred at Mount Savage, Md., in 1904, and was studied by Dr. M. L. Price, whose report, by the courtesy of Dr. John S. Fulton, secretary of the Maryland State board of health, is made the basis of this account. Mount Savage is situated on Jennings Run, about 10 miles west of Cumberland and 185 miles above Washington. The town lies at an elevation of about 1,200 feet, on a mountain whose steep sides descend abruptly to Jennings Run and Mount Savage Run, both of which flow through the town. The inhabitants are mostly employees of the Union Mining Company and of the Cumberland and Piedmont Railroad. They live largely in tenement houses or rows, boarding houses, and cottages, which are scattered along the side of the mountain up to an elevation of several hundred feet above the streams. The occurrence of house epidemics among the Union Mining Company's laborers is favored by the general lack of household furnishings, which makes it necessary for one utensil or set of utensils to serve a whole family. In the majority of families one dipper is used for drinking purposes, water being taken from a common bucket. Cheaply constructed privies are in universal use and receive very little care, for every heavy rain washes the excess of excrement into the streams. Jennings Run is at all times grossly polluted by human sewage, and contains a miscellaneous collection of offal, dead animals, and refuse. The odor during the day is usually offensive, but becomes sweet whenever a heavy rain flushes the run and carries its filth into Wills Creek, which in turn delivers it to the Potomac. The water of Jennings Run is unpotable, because it is very heavily polluted by mine drainage, which contains free sulphuric acid and large quantities of iron salts, besides sulphates and carbonates of lime.

The water supply of Mount Savage was obtained from 15 private springs and 3 artesian wells. After the epidemics broke out several of these were found to be contaminated and were closed by the county board of health. The roundhouse of the railroad company has an artesian well. Only one of its 125 employees had typhoid fever, and it is certain that he did not confine himself to the company's well. The brickyard spring was the only source of water known to have been 
specifically polluted by typhoid dejecta, and the victims of the epidemic were confined to those who drank from it.

The outbreak at Mount Savage is interesting, for we have here a story which is probably repeated yearly at one place or another on the Potomac watershed. - A number of the wells were polluted by human excreta, no care whatever was given to the privy vaults, and the stream that ran through the town was abominably defiled, yet year after year went by and Mount Savage was not scourged by any pestilence; but the penalty for ignorance and neglect was paid in full when the germs of disease were introduced into the midst of such conditions.

July 4, Mrs. - who occupied a small house about 300 feet above the brickyard, on a rather steep incline forming the north bank of Jennings Run, and who had just returned from nursing her brother, a typhoid-fever patient, at Luke, Md., was taken ill with the same

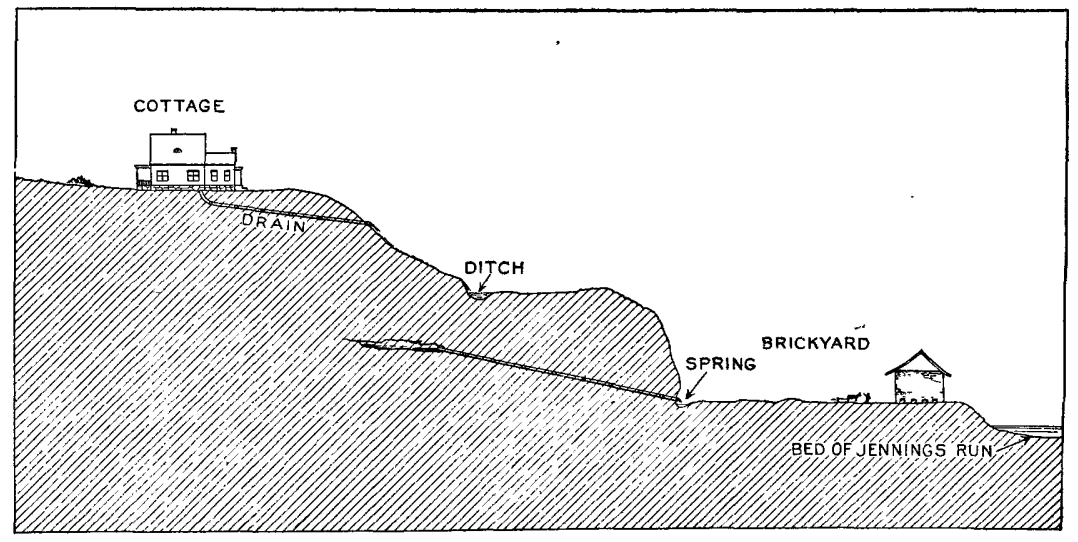

FIG. 2.-Elevation of north bank of Jennings Run, showing course of drainage.

disease. The brother probably contracted the disease in Piedmont, W. Va. Mrs. - ' ' infection was evidently contracted at the same place or from her brother. All of the drainage from this house was conveyed through a 4 or 6 inch iron pipe which emerges from the ground and ends 40 or 50 feet below on the side of the hill (fig. 2). This mixed drainage found its way down the hill, a small portion of it reaching an open drain. A road runs along the side of the open drain, over a bank of fire clay above the brickyard. At the bottom of the fire-clay bank, a short distance from the open drain, was a large flowing spring, which furnished an abundant supply of clear water of pleasant taste and appearance and of agreeable coolness. The water was drunk by all of the brickyard employees, about 200 in number. During the early part of July heavy rains occurred, washing surface impurities down the side of the mountain upon which the 
cottage was located into the spring and Jennings Run. July 11, about one week after Mrs. ___ s arrival, 20 workmen from the brickyard reported to Doctor Murray, the company doctor, complaining of headache, backache, lassitude, and digestive disorders. The strict limitation of these cases to the brickyard employees and the similarity of their symptoms suggested a common source of infection. Accordingly, on the following day, July 12, Doctor Murray posted a notice declaring the water bad and directing the discontinuance of its use. Five additional men reported on this day with typhoid prodromata. From the railroad and other shops supplied with artesian water no cases appeared. The brickyard men were again enjoined against the use of the spring, but a certain number continued to use it during the succeeding twelve or thirteen days. Additional cases were now appearing at the rate of five or six daily, and some of the original cases were showing unmistakable evidences of enteric fever. July 25 Doctor Murray effectually previented further use of the water by the destruction of the spring, mineral ashes and fire clay being thrown into it till it was buried several feet deep. The chemical and bacteriological reports of water samples drawn by Doctor Murray were received by this time and indicated that the brickyard spring was badly polluted and that other wells and springs in the town were polluted to a greater or less degree. Cases of typhoid fever continued to develop until August 10, sixteen days after the last of the brickyard spring water was drunk. The destruction of this spring by Doctor Murray effectually removed the source of infection and ended the epidemic, but with the arrival of Doctor Price, August 11, other wise measures were taken. The most important of these had for their object the prevention of a secondary epidemic that might be caused by the spreading of contagion from the infected stools broadcast through the community by means of flies. To accomplish this, the privy vaults were thoroughly cleaned through the cooperation of the Union Mining Company, which furnished horses and men to carry on the work. The method adopted was the digging of pits and the burying of the vault contents. Those contaminated springs which could be sealed were closed up and the water of the others was made undrinkable by the use of a harmless emetic, such as copper sulphate or alum. In this work the Allegany County board of health was active, as well as in posting notices requiring the boiling of water, the policing of front yards, and the use of earth ashes or lime in the privies to cover evacuations as soon as they were passed, and in the appointing of district nurses to teach and secure personal prophylaxis in the homes. Disinfectants were used in the stools and urine of the sick. The extermination of the epidemic was due to the effective cooperation of the local physicians, Doctors Murray and Quarles, 
with officials of the county and State boards of health. The results of this epidemic up to August 17 were as follows:

Statsstics of typhoid-fever epidemic at Mount Savage, Md., July \& to August 17, 1904.

Total number of cases . . . . . . . . . . . . . . . . . . . . . . . . . . . . 115

Bed cases . . . . . . . . . . . . . . . . . . . . . . . . . . . . . . . . . 80

"Walking cases" . . . . . . . . . . . . . . . . . . . .

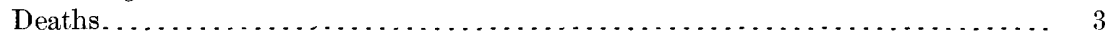

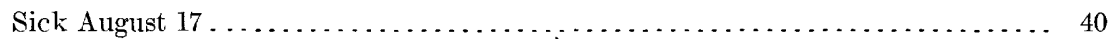

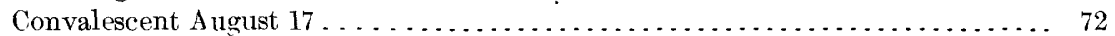

By August 22 five more cases had appeared, raising the total to 120.

The health department of Washington did not learn of the epidemic until August 20, when it sent out warnings to boil the Potomac water. The step was a commendable one, but as the epidemic in Mount Savage was practically over at the time, it was impossible to avert the consequences of the early cases. The effect of the epidemic on Washington may be seen in the following table:

Typhoid cases and deaths in Washington, D. C., five months of 1902-1904.

\begin{tabular}{|c|c|c|c|c|c|c|}
\hline \multirow{2}{*}{ Month. } & \multicolumn{2}{|c|}{1902.} & \multicolumn{2}{|c|}{1903.} & \multicolumn{2}{|c|}{1904.} \\
\hline & Cases. & Deaths. & Cases. & Deaths. & Cases. & Deaths. \\
\hline $\begin{array}{l}\text { July ......... } \\
\text { August } \\
\text { September... } \\
\text { October.... } \\
\text { November... }\end{array}$ & $\begin{array}{l}130 \\
328 \\
290 \\
247 \\
156\end{array}$ & $\begin{array}{l}21 \\
39 \\
25 \\
32 \\
19\end{array}$ & $\begin{array}{r}121 \\
188 \\
138 \\
148 \\
88\end{array}$ & $\begin{array}{r}17 \\
26 \\
18 \\
19 \\
8\end{array}$ & $\begin{array}{l}101 \\
226 \\
212 \\
138 \\
104\end{array}$ & $\begin{array}{l}16 \\
22 \\
25 \\
14 \\
11\end{array}$ \\
\hline Total. & 1,151 & 136 & 683 & 88 & 781 & 88 \\
\hline
\end{tabular}

As in 1902 and 1903, the number of cases and deaths made a sudden jump in August, 1904, but the September figures, unlike those of the two other years, remained as high as they were in August. In October, 1904, there was a quick drop in both cases and deaths, which was not the case in 1902 and 1903 . It is evident, therefore, that the specific cause of typhoid fever in Washington in 1904 was discovered and removed. Moreover, the prevalence of the disease was synchronous with that at Mount Savage, which it is known was stamped out. Hence it is concluded that the typhoid at Mount Savage caused that in Washington.

It is interesting to note that the germs at Mount Savage must have gone pretty directly into the highly acid waters of Jennings Run and then into the acid waters of Wills Creek before reaching the Potomac. From this it would seem that mine waters can not be relied on to kill the typhoid bacillus. On the other hand it should be remembered that the supply of food material was probably abundant in the initial stages of the journey of the germs to Washington, for there are two tanneries on Wills Creek, and the sewage of Cumberland must also have been available. 
Another factor which has influenced the degree of prevalence of typhoid fever in Washington is that the cities and towns in the basin have been procuring new and pure water supplies. At many places the information was given during this investigation that typhoid fever is not common at present, but was so before the new water-supply system was installed. In protecting themselves these communities have protected others, because if typhoid fever is reduced to a minimum the privies and sewers must necessarily turn fewer of the bacilli into the streams. The sources of the water supplies of the most important places in the Potomac basin are given in the following table:

Public water supplies in the Potomac basin.

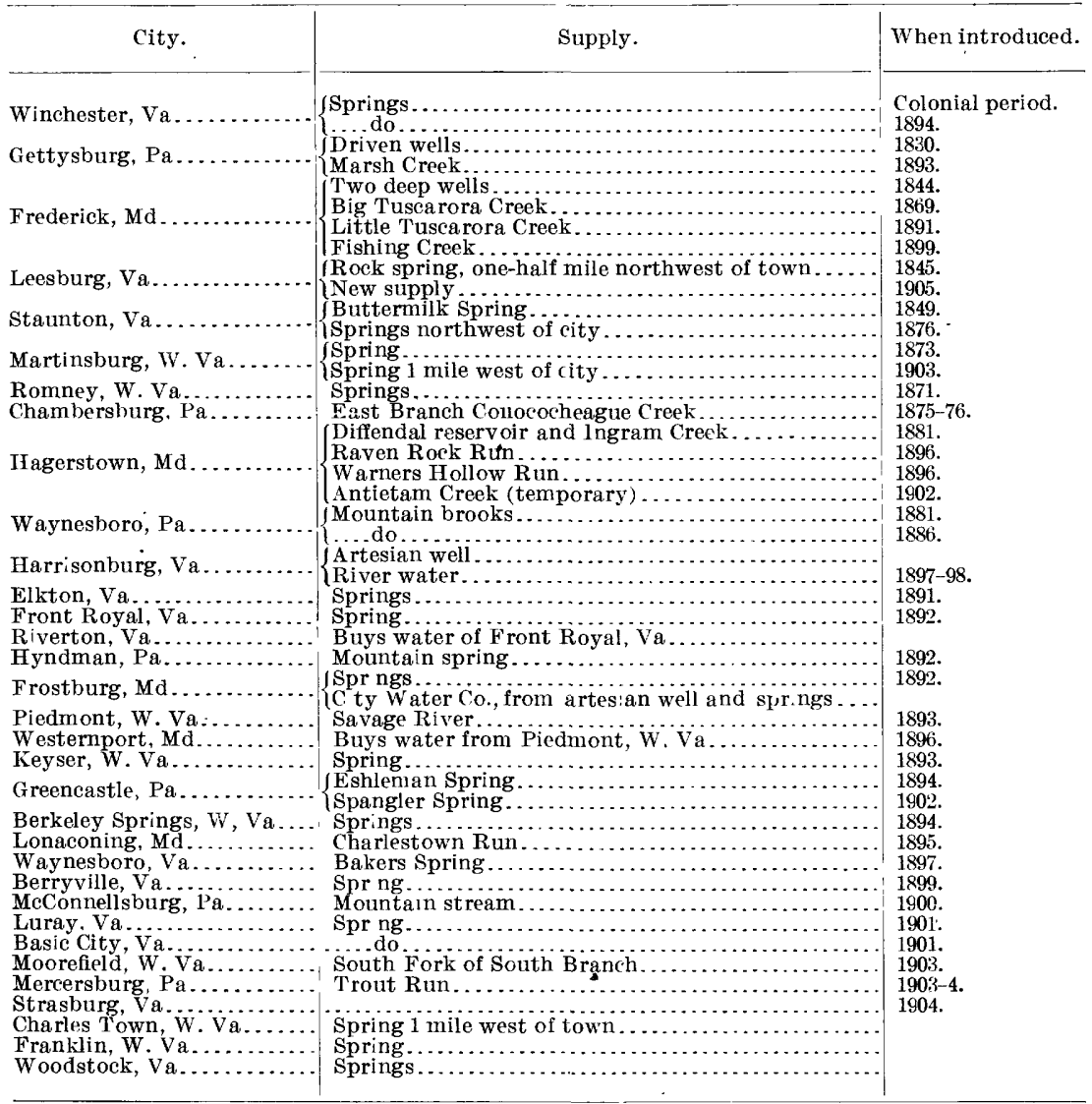

The only places of considerable size, aside from Washington, that use Potomac River or any of its large tributaries for a water supply are Moorefield, W. Va., Cumberland, Md., and Brunswick, Md. Of these Cumberland is the most important. In Brunswick the Potomac water is served to only a few and in Moorefield more people use wells than the water of South Fork. 
The minimum flow of the Potomac at Great Falls occurred in 1856 and was 1,063 second feet, and the maximun, 470,000 second-feet, was in 1889 ; but there are long periods every year when the flow is 3,000 second-feet or less. Several observers have noted that it is in the times of low flow that typhoid fever is rampant in Washington. This investigation points to the same conclusion, as is shown by the figures obtained from the hydrograph of the river at Point of Rocks. (See Pl. IX.) It is to be regretted that the gaging station is above Monocacy River, but it is unlikely that the addition of the waters of that stream would materially alter the results obtained and it is considered singularly fortunate that it was possible to secure both stream gagings and vital statistics for the same river covering a long period.

Pl. IX shows for the years 1902, 1903, 1904, and 1905 the daily flow of Potomac River at Point of Rocks, the daily deaths from typhoid fever in the District of Columbia, and the number of cases of typhoid fever reported daily at the health office of the District. The total deaths and the deaths from typhoid fever in the District from August, 1874 , to December, 1905, inclusive, are shown in the table on pages $281-282$.

Pl. IX shows that typhoid fever is prevalent during periods of low water, while there are comparatively few cases in the portions of the year when the water is high. At first thought these results seem surprising, for it would perhaps be natural to expect that the periods of excessive typhoid would occur in flood seasons, when a great deal of filth and excrement are washed into the river. But one who is thoroughly familiar with the river realizes that it is the constant recipient of large amounts of fecal matter from the many sewers and multitude of privies that line its banks and that the scourings intermittently washed into the stream are small, indeed, compared to the excrement that is every day dropped into it. Therefore it is perfectly natural that when this everyday defilement is concentrated in a small stream flow the results should be disastrous, for at such times, when typhoid germs are present, the chances of one's drinking a considerable dose of them are many times multiplied. Typhoid fever is known to be a disease of summer and autumn, and therefore the bacilli are most likely to be in the stools at the very time the river is low. If the germs are not present, there will not be typhoid in times of low water. It can be seen from an inspection of the diagram (Pl. IX) that low water has occurred in winter, but that there has not been a large increase of typhoid following. It would be foolish to maintain that a rain never brings about an increase of typhoid among the users of Potomac water. It may do so, provided it washes in a large quantity of bacilli and does not at the same time bring about such a dilution of the water containing them that the possibility of drinking them is no greater than it was before. There is an ever- 


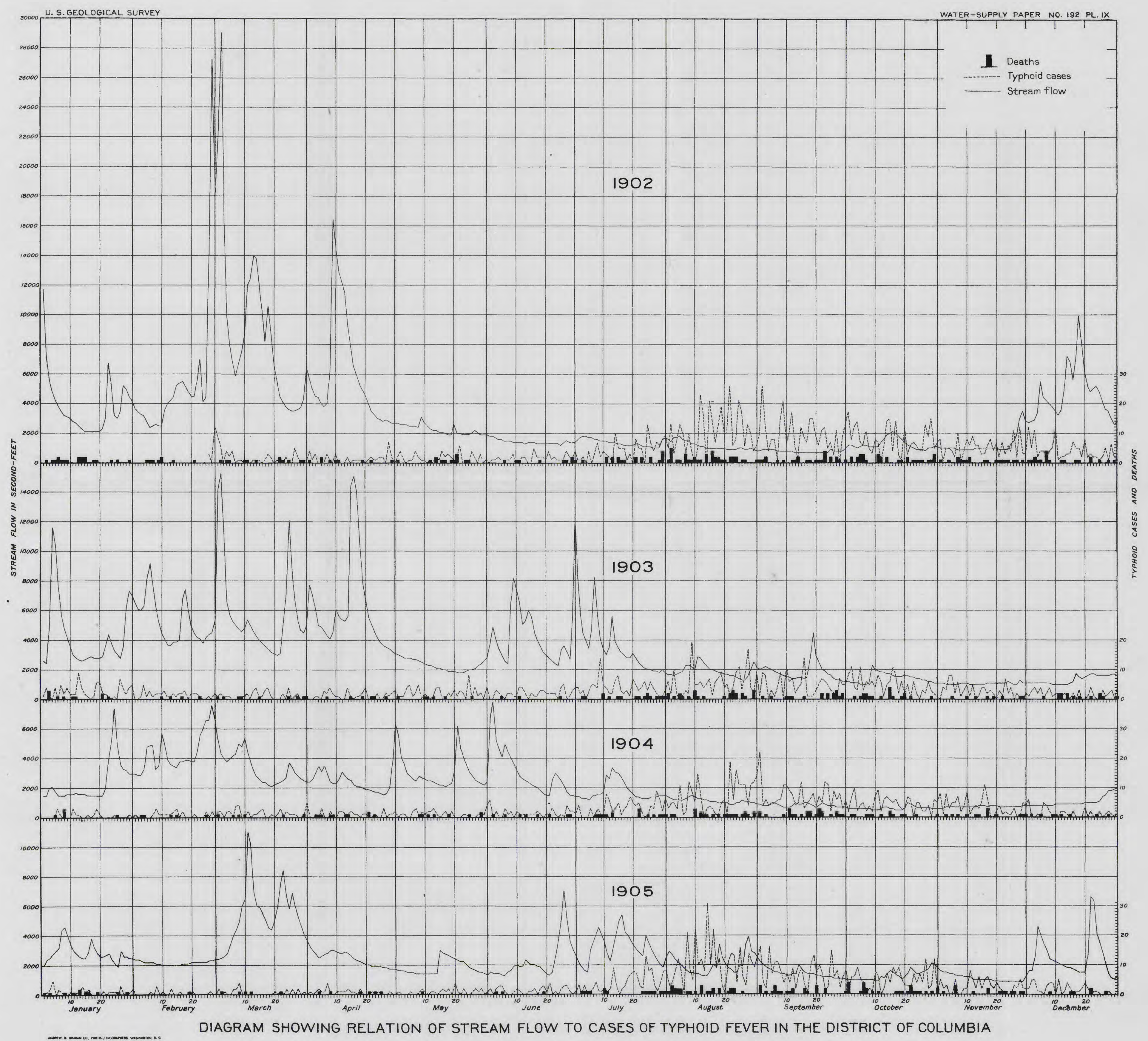


balancing adjustment between dilution and concentration. Whenever dilution is in the ascendant, the chances of drinking typhoid germs are reduced; whenever concentration prevails, the reverse is true. It is conceivable that in the case of a city pumping water from a river there might be an opportunity for a small quantity of excreta deposited on the bank to be carried rapidly past the intake by the very flood that washed it into the stream, at a time when the pumps were not in service or were pumping at a low rate. Thus the city would escape infection entirely or would feel the effects thereof only slightly. So it is that no rigid law can be laid down concerning the time at which typhoid fever may prevail at Washington, for it depends principally on the play of three factors--the presence of the bacilli in the water, the dilution of the water containing them, and their concentration in it.

There are two distinct types of water-borne typhoid epidemics. One is illustrated in the case of New Haven, Conn., which for years enjoyed a pure supply, but was suddenly overwhelmed by an epidemic caused by the careless disposal of typhoid defecations on the watershed and their sudden precipitation into the water thereafter. That is, the city for years enjoyed a considerable degree of immunity, suddenly experienced an epidemic, and then returned to another period of immunity with the passing of accidental conditions. To this type the disease in Washington does not show a resemblance. It is more like the type described by Whipple and Levy ${ }^{a}$ in their investigation of typhoid fever in the Kennebec Valley in 1902-3. Here the towns which used the Kennebec water experienced a certain amount of typhoid for some time previous to the epidemic, which was severe enough to impel the citizens to an investigation of the conditions that surrounded them. In one respect, however, the prevalence of typhoid in Washington differs from that in the Kennebec Valley. There several large towns, one below another, drew their water supply from the river and sewered into it as well. The result was that one city passed the contagion on to another and that the excessive prevalence of the disease was extended from the normal period of three months (August to October) into nine months (August to April). From the danger of such a position Washington is happily relieved at present, for Cumberland, Md., is the only other large city in the basin that uses the polluted river as a public water supply. If Brunswick, Md., increases its present limited use of Potomac water, such increase may be fraught with serious consequences to Washington. With the growth of the cities in the Potomac basin the time is bound to come when the springs and mountain brooks that now supply them will become inadequate. Then they will have to turn to the river, and then conditions will be like those on the Kennebec. 


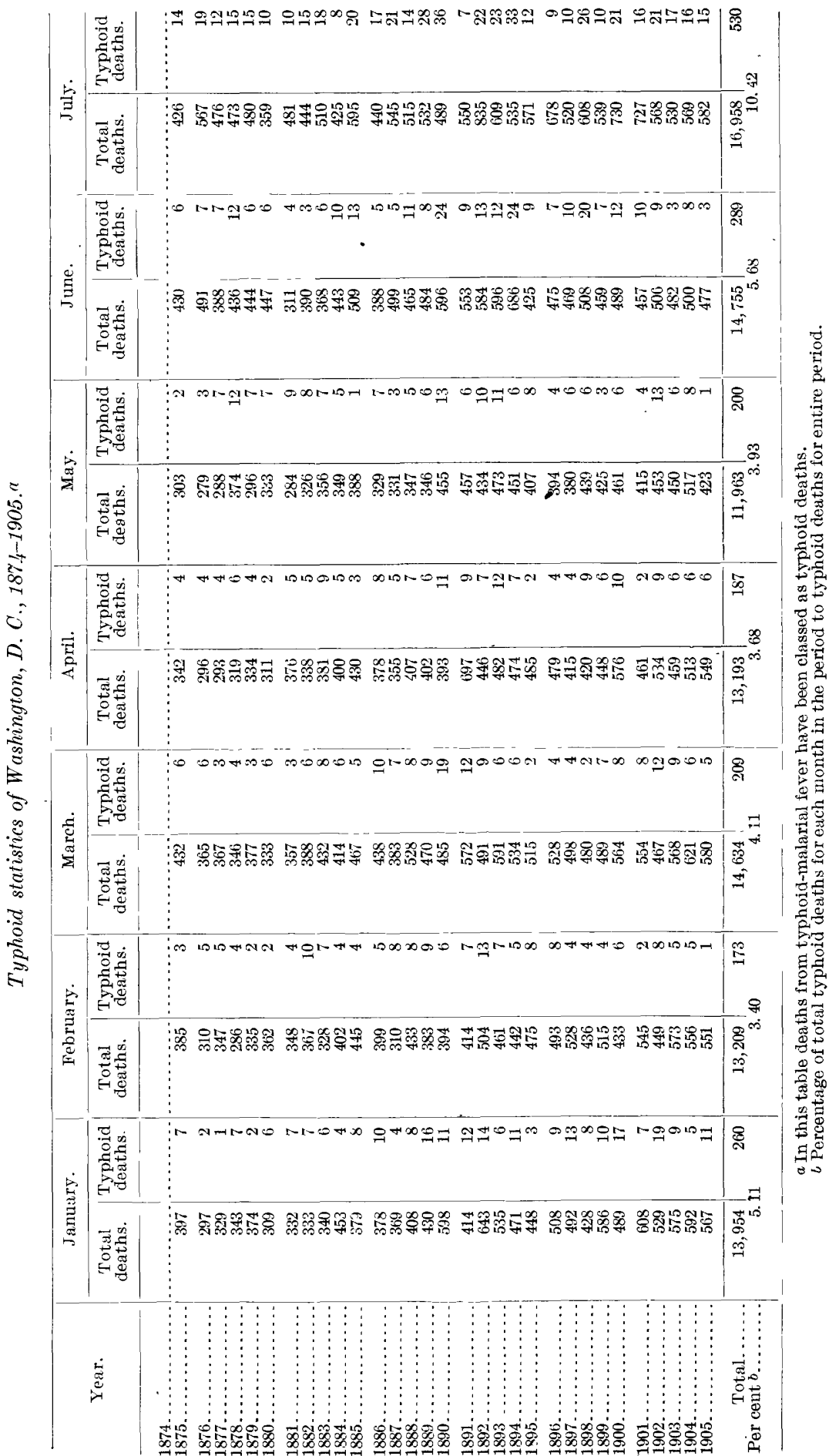




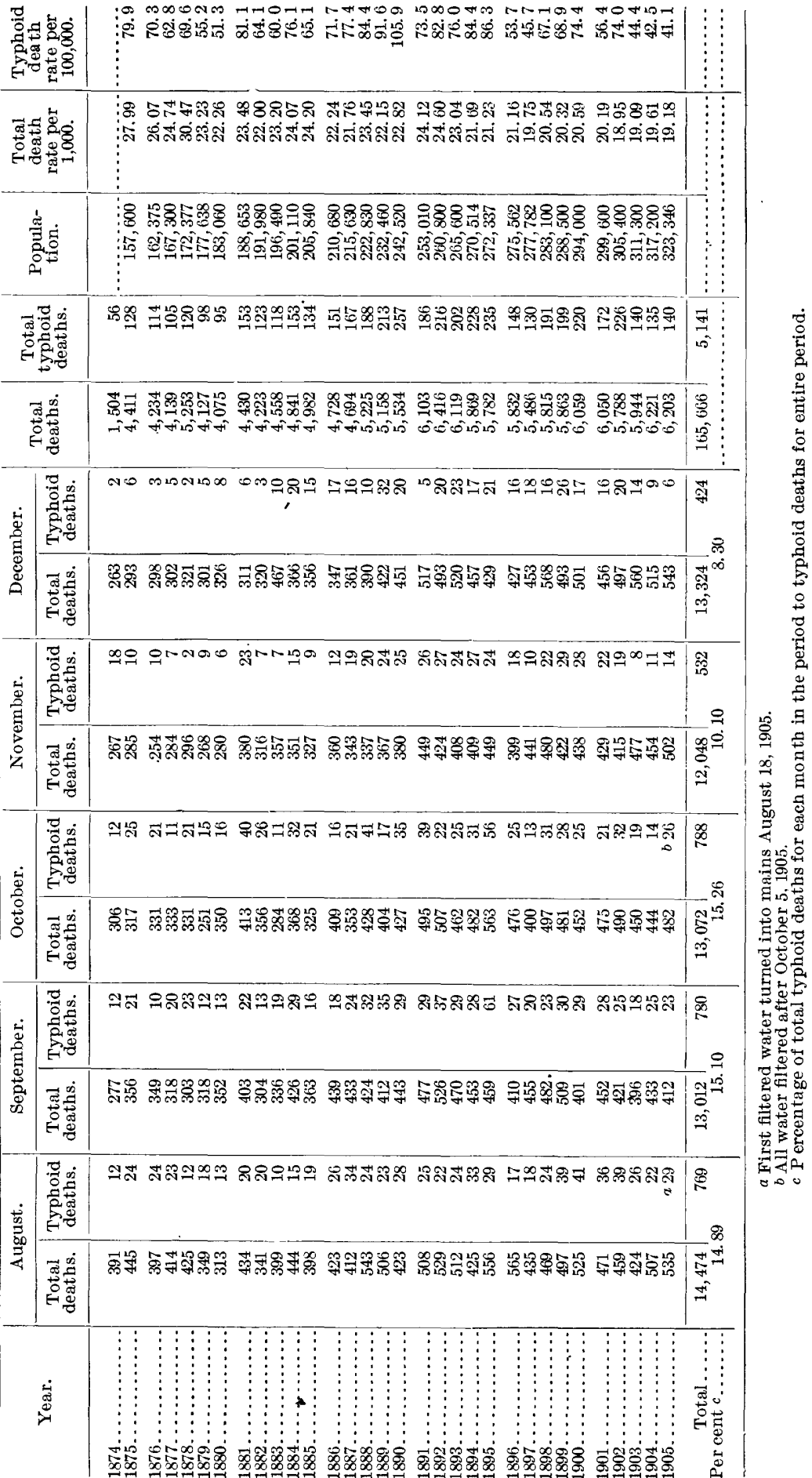


In the last thirty-one years there have been 5,085 deaths in Washington from typhoid fever, or an average of 164 a year. If the deaths during this time have been 10 per cent of the cases, as experience in many places shows that they usually are, there have been 50,000 cases of typhoid fever, or an average of 1,600 a year. The table on pages 280 and 281 shows to what degree typhoid fever has been present in Washington since the disease has been reported.

Cases of typhoid fever in the District of Columbia, 1902-1905.

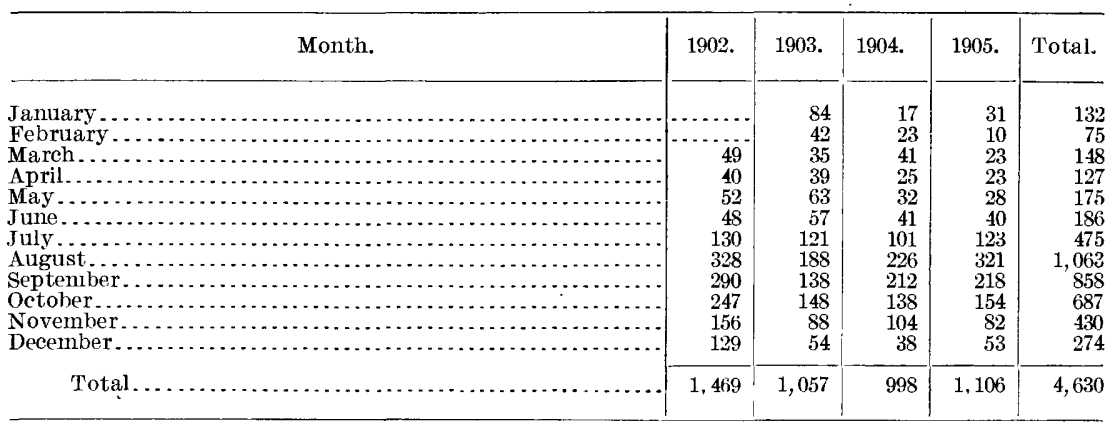

To protect the District of Columbia against so serious a menace a filtration plant has been built, at a cost of $\$ 3,500,000$, which it is estimated will cost $\$ 100,000$ a year to run. Undoubtedly the expenditure is a wise one, but it is an impressive lesson in pollution. Moreover, the end is not yet, for if the pollution is unrestrained the evil results therefrom will make the water more difficult to purify. This means that the present rates of filtration can not be maintained, which will add to the cost of treating the same amount of water and necessitate a larger plant. Common sense demands, therefore, that some check be placed on the extravagance of needlessly fouling the river, for what Washington has been compelled to do will become necessary for other cities in the basin. 


\section{QUALITY OF SURFACE WATERS.}

FIELD ASSAYS.

Inasmuch as large quantities of coal-mine water are poured into North Branch at sundry points along its course from Henry to Piedmont, W. Va., it was deemed advisable to investigate its effect on the river, and the study also covered the changes wrought by the large quantities of lime sludge and spent lime bleach from the mill of the West Virginia Pulp and Paper Company at Luke, Md.

Mine waters are characterized by large amounts of free sulphuric acid, sulphates of lime, magnesia, and alumina, and ferrous sulphate of iron. The protosulphate of iron on exposure to the air breaks up, with the formation of ferric hydrate and ferric sulphate. The hydrate precipitates; the sulphate remains in solution. The protosulphate of iron has a very destructive corrosive action on steam boilers and the sulphates of lime and magnesia form hard incrustations on them which can be removed only at considerable expense and not without damaging the plates. The appearance of a stream contaminated by mine water is striking and somewhat uncanny, for all vegetable and animal life is destroyed, and the bright, clear waters splash forbiddingly over the bed, which is stained yellow by the iron.

Field assays were made from September 20 to 23 and from October 4 to 14,1905 . The first trip covered the river from Dam No. 5 , above Williamsport, to West Virginia Central Junction, and was not interfered with at all by the weather. The second extended from Wilsonia to Piedmont, W. Va., and was very satisfactory, for there was no rainfall until the 11 th to increase the stream flow and thereby change the concentration of the water, which was at low stage.

North Branch above Henry contains but a trace of sulphates and is of low alkalinity. The mine water that enters at Henry through a little nameless run increases the sulphates in the river to such a point that the lumber company at Dobbin has been compelled to abandon the river water for boiler use.

Between Dobbin and Wilson the tributary streams have no sulphates, so that by the time Wilson is reached the sulphates have been diluted to 35 parts per million, and at Bayard the sulphates have all but disappeared and the water is suitable for boiler use. Buffalo Creek, which enters at Bayard, is said to be polluted by mine wastes, but the amount received must be small, because the water is nearly normal for the region. For some reason which could not be ascertained the sulphates in the river between Bayard and Gormania rise to 30 parts per million. Notwithstanding the fact that the run at Stoyer adds more mine water, North Branch at Stony River contains but a trace of sulphates.

IRR $192-07-19$ 
It is interesting to follow the chlorine from Bayard to Schcll. The tanneries wash out large quantities of salt from the hides and discharge it in the soaks. Above Bayard the river contained but 6 parts per million of chlorine. At Gormania it rose to 34 parts; inflowing waters diluted this to 19 parts at Stony River and to 14 parts at Schell. Samples taken below this point were collected after a rain, so that the results are not comparable, but this process of dilution undoubtedly would have continued regularly. This is an excellent example of the way factory effluents may be traced in a river by selecting some characteristic, readily detectable salt, and following it downstream.

At Schell the water of North Branch is very soft, owing to the fact that it has received the waters of Stony River, which are remarkably low in dissolved mineral substances. There is no change by the time it reaches a point a little above Harrison, but at this town the waters of Abram Creek, slightly polluted by mine water, fall in. From Harrison downstream, Wolfden Run, Three Fork Run, and Deep Run, in order, join North Branch. All of them are polluted by mine water, and Deep Run, which drains the extensive Elk Garden coal regions, carries a heavier quota of mine waste than any other stream tributary to North Branch, with the exception of Georges Creek. Despite these additions, the river above Elk Lick Run, near Shaw, W. Va., does not contain an objectionable amount of incrusting constituents, though the total hardness is considerably increased. Above Savage River the total hardness becomes low again and the river is in good condition. The entrance of Savage River and of a small volume of mine water below it does not change the water very much, and it soon after flows over the dam of the West Virginia Pulp and Paper Company.

In the entire distance from Henry to West Virginia Central Junction the tributary streams are normally low in objectionable mineral ingredients and by dilution counteract the mine waters which are poured into the river, so that the water arrives at the paper company's dam with practically the same mineral content that it has above Henry. This is somewhat surprising, for prior to the investigations it was believed that the mine water was so considerable as to have a decidedly deleterious effect on the stream.

A short distance below the dam the factory of the West Virginia Pulp and Paper Company discharges its effluent, consisting of carbonate of lime, chloride of lime, and sulphate of soda, into North Branch. The quantity is so great that it converts the clear stream into a turbid, milky-white one, and the tests applied to the water showed the marked effect of the chemicals on it. October 14, 1905, the lime rose from 26 parts per million above the dam to 105 at a point opposite the Baltimore and Ohio roundhouse in Piedmont, the 
alkalinity rose from 31 parts per million to 44 , the sulphates from a trace to 56, and the chlorine from 9 to 29 . Thus the character of the stream is radically altered by this effluent, and it is still further changed by the influx of the waters of Georges Creek, which enter at Westernport and which are heavily charged with mine waters. The waters of the creek when they unite with those of North Branch react with the matter which it contains in suspension and solution. Free sulphuric acid combines with the carbonate of lime, producing a precipitate which makes the water roily and may be observed all the way from Westernport to Keyser. The iron salts, including the ferric sulphate, are precipitated in the form of ferric oxide.

September 23 there were in the waters of North Branch above the dam at West Virginia Central Junction 26 parts per million of lime, 18 of alkalinity, a trace of sulphates, and 9 parts of chlorine. At the Baltimore and Ohio roundhouse there were 130 parts of lime, 47 of alkalinity, 117 of sulphates, 24 of chlorine, and 1 of iron. Georges Creek contained 288 parts of calcium, 522 of sulphates, and 54 of iron. One mile below Westernport, North Branch contained 130 parts of calcium, 6 parts of alkalinity, 185 parts of sulphates, and 2.8 parts of iron. It is evident from these results that the sulphuric acid combined with the carbonates represented in the test by alkalinity to the extent of nearly eliminating them. The sulphate of lime formed thereby was considerable, and the iron for the most part precipitated out or became obscured by dilution. By following the tests in the table on pages 287-290, it becomes apparent that from a point 1 mile below Westernport the alkalinity steadily increased in amount to Dam No. 5, being 38 parts per million 2 miles below Keyser, 46 at the Cumberland waterworks intake, and 73 at Dam No. 5, at Williamsport. That is, after partial elimination the carbonates tended to return to the normal for the region. Moreover, the sulphates dropped steadily, being 181 parts per million 2 miles below Keyser, 140 at Cumberland, and but a trace at Dam No. 5 .

This extensive precipitation has a potent influence on the bacterial content of North Branch, for the sulphates in settling out entangle the germs and drag them to the bottom, thus decidedly improving the water. In Georges Creek the acid waters are undoubtedly inimical to the bacteria and tend to destroy the organic, matter which is so abundantly supplied to the stream at the numerous thriving mining towns in its valley. This reduces the effect of the pollution of North Branch at Westernport. Finally, as has been shown by experiments described elsewhere, ${ }^{a}$ the waste discharged by a soda pulp mill is a powerful germicide and an excellent precipitant of sewage.

$a$ Leighton, M. O., Preliminary report on the pollution of Lake Champlain; Water-Sup, and Irr. Paper No. 121, U. S. Geol. Survey, 1905. 
Together, these several influences effect so considerable a reduction of the bacteria in North Branch that its water is used raw by the city of Cumberland with results much less evil than the gross pollution of the watershed would lead one to expect. While this is fortunate, it is to be remembered that the purification is effected by a number of factors acting independently of each other, without intent of improving the water. A diminution of the output of the paper mill or of the flow from the pumps at the mines would at once interfere with the purification and might lead to serious results. Fortunately, the stream flow is usually low at that season of the year when typhoid fever is most prevalent, so that the proportion of chemicals in the water is greatest when it is most needed. It is manifest that this quasi purification must be uncertain and that it is dangerous to depend on it. Therefore it is obvious that the city of Cumberland should install proper water-purification works without delay. As it is a manufacturing city, and as the sulphates in the North Branch water at this point are sufficient to cause some trouble by the formation of hard scale in boilers, it might be profitable to establish also a water-softening plant. The amount of iron in the surface and subsoil waters of North Branch above Cumberland is usually great enough to compel the use of casings to exclude it from wells which are driven for industrial enterprises.

The field tests made on the waters of Wills Creek indicate that above Jennings Run the water is low in incrustants. The run is heavily polluted by mine water, and consequently raises the sulphates in Wills Creek sufficiently to make the water harmful for steam-producing purposes. The assays of various springs and wells in the Wills Creek valley show them to differ considerably, the water in some being soft and in others very hard.

The tests applied to Conococheague Creek show that a small amount of sulphates is usually present and that the water has a varying amount of temporary hardness, which is more marked in the waters of West Branch than in those of the main stream.

The headwaters of the Monocacy about Gettysburg, Pa., to wit, Rock Creek, Marsh Creek, Stevens Run, and Willoughby Run, show different characteristics by the field tests. Marsh Creek water is the softest and gives more satisfaction in Gettysburg for boiler use than those from the deep wells, all of which are corrosive and high in incrustants. Some of these wells will very likely soon be abandoned on account of these bad qualities. 
Field assays of waters in Potomac River basin.

[Parts per million.]

\begin{tabular}{|c|c|c|c|c|c|c|c|c|}
\hline Stream. & Date. & 害 & 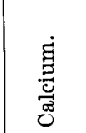 & $\begin{array}{l}\text { 点 } \\
\text { 穿 } \\
\text { 总 }\end{array}$ & 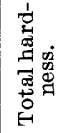 & 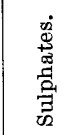 & 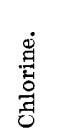 & 焗 \\
\hline Silcott Fiun at Wilsonia, W. & Oct. 4,1905 & 8 & Trace. & 259 & & 0 & 9 & $\mathbf{1}$ \\
\hline $\begin{array}{l}\text { North Branch Potomac River } \\
\text { above Henry and above creek } b . .\end{array}$ & & 8 & Trace. & 24 & 22 & Trace. & 6 & 2 \\
\hline Creek at Henry, W. Va.c.... & .....do. & 20 & 119 & 38 & 90 & 155 & 9 & 4 \\
\hline $\begin{array}{l}\text { Elk Run near mouth................ } \\
\text { North Branch Potomac }\end{array}$ & .....do.. & 5 & 0 & 15 & 12 & 0 & 6 & 0 \\
\hline above dam at Dobbin, W. Va... & do & 8 & 67 & 22 & 51 & 77 & 6 & 2 \\
\hline 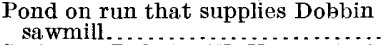 & . $\mathrm{d}$ & 15 & Trace. & 8 & 12 & 0 & 4 & 1 \\
\hline $\begin{array}{l}\text { Spring at Dobbin, W Va., east of } \\
\text { sawmill and West Virginia Cen- } \\
\text { tral arid Pittsburg R. R. track.. }\end{array}$ & do & 45 & Trace. & 8 & 22 & 0 & 6 & 0 \\
\hline $\begin{array}{l}\text { Laurel Creek northwest of Dobbin, } \\
\text { W. Va }\end{array}$ & & 10 & Trace. & 16 & 12 & 0 & 4 & 2.2 \\
\hline $\begin{array}{l}\text { Red Oak Run at Wilson, W. Va.d } \\
\text { North Franch Potomac River at }\end{array}$ & $\therefore d$ & 5 & Trace. & 16 & 18 & 0 & 6 & 0 \\
\hline 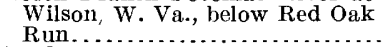 & & 8 & 32 & 17 & & 29 & 6 & 2.2 \\
\hline $\begin{array}{l}\text { Sand Rum, Md., opposite Wilson, } \\
\text { W. Va.......... }\end{array}$ & & 10 & Trace. & 17 & 18 & 0 & 6 & 2 \\
\hline $\begin{array}{l}\text { North Franch Potomac River at } \\
\text { Bayard, W. Va., above Buffalo } \\
\text { Creek........................... }\end{array}$ & & 5 & 24 & 18 & & Trace. & 6 & 1.5 \\
\hline Buffalo oreek at Bayard, w. Va... & …...d & 5 & Trace. & 8 & 18 & Trace. & 6 & 0 \\
\hline $\begin{array}{l}\text { North Firanch Potomac River at } \\
\text { Bayard, below Buffalo Creek and } \\
\text { tannery }\end{array}$ & & 5 & 28 & 19 & 27 & Trace. & 6 & 1.5 \\
\hline $\begin{array}{l}\text { J. G. Hcffman \& Sons co.'s drilled } \\
\text { well at, Gormania, W. Va.d }\end{array}$ & Oct. & 12 & 47 & 143 & 51 & Hove. & 8 & .5 \\
\hline 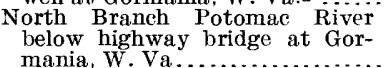 & Oct. & 15 & 55 & 35 & 49 & 33 & 34 & 2 \\
\hline North Branch Potomac River $e_{-}$ & Oct. & & 41.04 & & 128.2 & 22 & 28.7 & 2.86 \\
\hline Nydegger Run at Gorman, Md &, 1905 & $\ddot{8}$ & 26 & 36 & 27 & Trace. & 4 & 1 \\
\hline Glade Run at mouth. & Oct. 10,1905 & 5 & 26 & 34 & 22 & Trace. & 4 & .5 \\
\hline Run & Oct. 8,1905 & 5 & 39 & 2 & 32 & Trace. & 6 & .5 \\
\hline Difficult Run & .....do....... & 5 & Trace. & 24 & 32 & & 9 & 0 \\
\hline $\begin{array}{l}\text { North Branch Potomac River } \\
\text { short distance above Stony River. }\end{array}$ & & 5 & 26 & 24 & 27 & Trace. & 19 & 1 \\
\hline Stony River, W. Va., at mouth... & $\cdots . . \mathrm{d}$ & 5 & 0 & 9 & 12 & 0 & 9 & 1 \\
\hline $\begin{array}{l}\text { North Branch Potomac River } \\
\text { above Laurel Run and opposite }\end{array}$ & & 5 & Trace & 21 & 12 & Trace. & 14 & 1 \\
\hline Ilun at mouth. & & $\begin{array}{l}5 \\
5\end{array}$ & $\begin{array}{l}\text { Trace. } \\
\text { Trace. }\end{array}$ & $\begin{array}{l}21 \\
18\end{array}$ & $\begin{array}{l}12 \\
18\end{array}$ & & $\begin{array}{r}14 \\
4\end{array}$ & $\begin{array}{l}1 \\
0\end{array}$ \\
\hline Lostland Run near mouth & 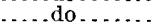 & 5 & 0 & 14 & 10 & Trace. & 9 & 0 \\
\hline $\begin{array}{c}\text { North Branch Potomac River } \\
\text { above Abram Creek............... }\end{array}$ & Oct. 1 & 10 & Trace. & 13 & 18 & Trace. & 9 & 1.5 \\
\hline Abram Creek at mouth. & Geve 1 & 10 & Trace. & & 32 & Trace. & 9 & 1.5 \\
\hline Wolfden Run, Md. $f$............... & $\ldots \mathrm{d}$ & 60 & 48 & Acid. & 66 & & 9 & 7 \\
\hline $\begin{array}{l}\text { Three Fork Run, Md., northeast of } \\
\text { Harrison, W. Va................. }\end{array}$ & & 10 & Trace. & 24 & 22 & Trace. & 4 & 0 \\
\hline W. Va.f. & & 140 & 119 & Acid. & & 273 & 19 & 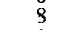 \\
\hline Howell Run a & d & 5 & Trace. & 28 & 22 & Trace. & 4 & 1 \\
\hline $\begin{array}{l}\text { North Branch Potomac River } \\
\text { above Elk Lick Run, Md......... }\end{array}$ & & 15 & Trace. & 17 & 61 & Trace. & 14 & 2.2 \\
\hline 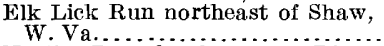 & & 5 & 23 & 40 & & Trace. & 9 & \\
\hline $\begin{array}{l}\text { North Branch Potomae River } \\
\text { above Savage River...................... }\end{array}$ & Oct. 14,1905 & 10 & 24 & 20 & 27 & Trace. & 9 & 1.7 \\
\hline Savage River near mouth $g . \ldots \ldots$. & Apr. 5,1905 & & 0 & 25 & & 0 & 9 & Trace. \\
\hline $\begin{array}{l}\text { Savage River, tap in Kenny House, } \\
\text { Piedmont, W. Va............... }\end{array}$ & Oct. 3, & 5 & 27 & 44 & & Trace. & 9 & 0 \\
\hline Savage River near mouth & Oct. 14,1905 & 5 & Trace. & 26 & is & Trace. & 9 & 0 \\
\hline $\begin{array}{l}\text { North Branch Potomac River } \\
\text { above West Virginia end of dain } \\
\text { at Luke, Md.................................... }\end{array}$ & Sept. 23, 1905 & & 26 & 18 & 32 & Trace. & 9 & 1 \\
\hline Do. & Oct. 14,1905 & & 26 & 31 & 27 & Trace. & 9 & 1 \\
\hline
\end{tabular}

$a$ Partis per million of $\mathrm{CaCO}^{3}$ required to neutralize the acidity.

$b$ Cont.xminated by mine water.

$c$ Reces ves much mine water.

$d$ Cased 30 feet to keep out iron-bearing water, which is said to be found 10 to 20 feet below surface of the ground in North Branch of Potomac valley above Cumberland, Md.

$e$ Analysis by Prof. J. W. Mallet.

$f$ Polluted by mine water.

$g$ Assay by S. J. Lewis. 
Field assays of waters in Potomac River basin-Continued.

\begin{tabular}{|c|c|c|c|c|c|c|c|c|}
\hline Stream. & Date. & 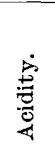 & 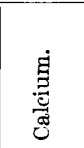 & 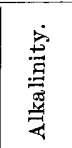 & 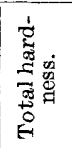 & 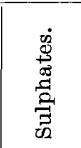 & 兽 & Б्ं \\
\hline $\begin{array}{l}\text { North Branch Potomac River } \\
\text { above pulp mill at Luke,Md, }{ }^{2} . . .\end{array}$ & July 14,1899 & & 6.93 & & 24.7 & 17 & 4.6 & 1.92 \\
\hline $\begin{array}{l}\text { North Branch Potomac River } \\
\text { opposite Baltimore and Ohio } \\
\text { roundhouse, Piedmont, W. Va... }\end{array}$ & Sept. 23,1905 & & & 47 & & 117 & 24 & \\
\hline 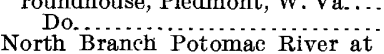 & $\begin{array}{l}\text { Sept. } 23,1905 \\
\text { Oct. } 14,1905\end{array}$ & 5 & 105 & 44 & 90 & 56 & $\begin{array}{l}24 \\
29\end{array}$ & 1.5 \\
\hline $\begin{array}{l}\text { Piedmont, W. Va.b } \\
\text { Georges Creek at mouth. }\end{array}$ & Apr. ${ }_{\text {do }} 4,1905$ & & 73 & $\begin{array}{l}40 \\
\text { Acid. }\end{array}$ & & $\begin{array}{r}10 \\
410\end{array}$ & & $\begin{array}{r}1 \\
28\end{array}$ \\
\hline 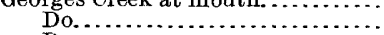 & Sept. 23,1905 & & 288 & & & 522 & & 54 \\
\hline Do... & Oct. 14,1905 & 462 & 228 & & & 359 & 19 & 36 \\
\hline Do.a & July 14,1899 & & 129.54 & & 439.1 & 502 & 10 & 191.44 \\
\hline $\begin{array}{l}\text { North Branch Potomac River, } \\
\text { West Virginia side, } 1 \text { mile below } \\
\text { Westernport, Md................ }\end{array}$ & Sept. 23,1905 & & 130 & 6 & & 18.5 & 19 & 2.8 \\
\hline $\begin{array}{l}\text { North Branch Potomac River, } \\
\text { Maryland side, } 1 \text { mile below West- }\end{array}$ & sept. 23,1903 & & 130 & 6 & $\cdots \cdots$ & 185 & 19 & 2.8 \\
\hline $\begin{array}{l}\text { ernport, Md, } \\
\text { North Branch Potomac River, }\end{array}$ & Oct. 14,1905 & 10 & 130 & 26 & 100 & 115 & 14 & .5 \\
\hline $\begin{array}{l}\text { West Virginia side, } 2 \frac{1}{2} \text { miles below } \\
\text { Westernport, Md } . \ldots \ldots \ldots \ldots \ldots \ldots\end{array}$ & Sept. 23,1905 & & 126 & 8.5 & & 185 & 19 & 3 \\
\hline 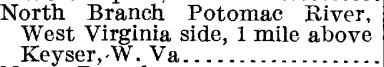 & & & 133 & 19 & & 164 & 19 & 1.2 \\
\hline $\begin{array}{l}\text { North Branch Potomac River at } \\
\text { Keyser, W. Va.a.................... }\end{array}$ & Apr. 27,1905 & & 73 & 10 & 104 & 64 & 9 & 4 \\
\hline $\begin{array}{r}\text { Rey } \\
\text { Do........ }\end{array}$ & $\begin{array}{l}\text { Apr. } 27,1905 \\
\text { Sept. } 23,1905\end{array}$ & & 130 & 17 & 104 & $\begin{array}{r}04 \\
159\end{array}$ & $\begin{array}{r}y \\
19\end{array}$ & 4 \\
\hline New Creek $a \frac{1}{4} \mathrm{n}$ & July 14,1899 & & 45.08 & & 113.7 & 20 & 32.4 & 1.97 \\
\hline $\begin{array}{l}\text { New Creek at mouth........... } \\
\text { Keyser city supply (impou }\end{array}$ & Sept. 23,1905 & & 123 & 128 & $\cdots \cdots$ & Trace. & 39 & 0.5 \\
\hline 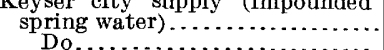 & Apr. 27,1905 & & 261 & 171 & & 15 & 9 & Trace. \\
\hline North Branch Fotomac River be- & 1905 & & 179 & 211 & $\ldots$ & Trace. & 4 & 0.0 \\
\hline $\begin{array}{r}\text { low Keyser, W. Va } a, \ldots \ldots \\
\text { Do } a \ldots \ldots\end{array}$ & {$\left[\begin{array}{l}1899 \\
-1899\end{array}\right.$} & & $\begin{array}{l}48 \\
78.6\end{array}$ & & $\begin{array}{l}156 \\
200\end{array}$ & $\begin{array}{l}202 \\
406\end{array}$ & $\begin{array}{l}11.9 \\
22.6\end{array}$ & $\begin{array}{l}29 \\
47.7\end{array}$ \\
\hline $\begin{array}{l}\text { Dorth Branch Potomac River at } \\
\text { Baltimore and Ohio } R \text {. R. bridge } \\
\text { 21, Maryland side, } 2 \text { miles below }\end{array}$ & $\begin{array}{l}-1899 \\
-1899\end{array}$ & & 63.1 & & 156 & $\begin{array}{l}400 \\
290\end{array}$ & 20.3 & 20.5 \\
\hline $\begin{array}{l}\text { Keyser........................... } \\
\text { North Branch Potomac }\end{array}$ & Sept. 23, 1905 & & 130 & 38 & & 151 & 19 & 1.0 \\
\hline $\begin{array}{l}\text { above Cumberland, Md.......... } \\
\text { North Branch Potomac River (hy- }\end{array}$ & $-\quad, 1899$ & & 56 & & 171 & 206 & 12.1 & 7.0 \\
\hline drant in Cumberland, Md.) $a . .$. & $\longrightarrow$ & & 31.12 & & 128.5 & 110 & 13.6 & 11. 79 \\
\hline $\begin{array}{l}\text { North Branch Potomac River, at } \\
\text { Ridgely above Cumberland wa- } \\
\text { terworks intake } b . . . . . . . . . . . . .\end{array}$ & Apr. & & 55 & 20 & 118 & 78 & 9 & 2.5 \\
\hline $\begin{array}{l}\text { North Branch, Potomac } \\
\text { Cumberland waterworks }\end{array}$ & & & & & & & & 10 \\
\hline $\begin{array}{l}\text { Cumberland waterworks intake } \\
\text { North Branch Potomac River, } 300 \\
\text { feet below dam at Cumberland, } \\
\text { Md.b.... }\end{array}$ & Sept. 22,1905 & & 123 & 46 & & 117 & 14 & 1.0 \\
\hline $\begin{array}{l}\text { North Branch Potomac Kiver } \\
\text { above sewage outfall at South } \\
\text { Cumberland } b . . . \ldots\end{array}$ & Apr. 3,1905 & & 96 & 25 & & 84 & 9 & 5.0 \\
\hline $\begin{array}{l}\text { Chesapeake and Ohio Canal at lock } \\
\text { above Dam No. 5, where canal en- } \\
\text { ters river.... }\end{array}$ & Sept. 20,1905 & & 69 & 62 & 61 & 33 & 15 & Trace. \\
\hline $\begin{array}{l}\text { North Branch Potomac Kiver be- } \\
\text { low Dam No. } 5 . \ldots \ldots \ldots \ldots \ldots \ldots\end{array}$ & $d$ & & 70 & 73 & & Trace. & 9 & 0 \\
\hline $\begin{array}{l}\text { Kilmer Spring, public water sup- } \\
\text { ply, Martinsburg, W. Va.b....... }\end{array}$ & & & 126 & 205 & & 0 & 27 & race. \\
\hline Public wat & Apr. $\quad 7,1905$ & & 0 & 7 & & 0 & 7 & 0 \\
\hline $\begin{array}{l}\text { Wills Creek west of Hyndman, Pa.b } \\
\text { Wills Creek, } 100 \text { yards below tan- }\end{array}$ & Apr. 8,1905 & & 0 & 10 & 35 & Trace. & 9 & 0 \\
\hline $\begin{array}{l}\text { nery } b \\
\text { Wills Creek }\end{array}$ & Apr. $\quad 9,1$ & & 0 & 15 & & Trace. & 9 & Trace. \\
\hline $\begin{array}{l}\text { Wills Creek at Corri } \\
\text { Jennings Run at mo }\end{array}$ & Apr. 4,1905 & & Trace. & 25 & - & 10 & $\begin{array}{r}9 \\
79\end{array}$ & Trace. \\
\hline ck & & & $\begin{array}{l}63 \\
30\end{array}$ & 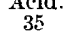 & & $\begin{array}{r}239 \\
10\end{array}$ & $\begin{array}{r}79 \\
9\end{array}$ & \\
\hline Wills & Apr. 3,1905 & & 110 & & & 99 & & 5.5 \\
\hline $\begin{array}{l}\text { Wills Creek, west side, } 2,000 \text { feet } \\
\text { above mouth } a \ldots \ldots \ldots \ldots \ldots \ldots \ldots\end{array}$ & July 15,1899 & & 48.6 & & 168.6 & 245 & 41.7 & 6.9 \\
\hline
\end{tabular}

a Analysis by Prof. J. W. Mallet.

b Assay by S. J. Lewis. 
Field assays of waters in Potomac River basin--Continued.

\begin{tabular}{|c|c|c|c|c|c|c|c|c|}
\hline Stream. & INate. & 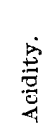 & $\frac{\vec{E}}{\stackrel{B}{\vec{C}}}$ & 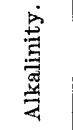 & 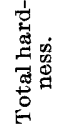 & 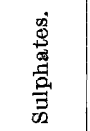 & 总 & 总 \\
\hline $\begin{array}{l}\text { Bradigans well, } 20 \text { feet deep, } 1 \text { mile } \\
\text { northeast of Foley, Pa.a. }\end{array}$ & Apr. 8.1905 & & 55 & 44 & & 18 & 19 & Trace. \\
\hline $\begin{array}{l}\text { Becks spring } \frac{7}{8} \text { mile east of Foley, } \\
\text { Pa. } a \ldots \ldots\end{array}$ & & & 0 & 12 & 28 & .0 & 9 & 0 \\
\hline $\begin{array}{l}\text { Shaffers Run at Fairhope, Pa., } \\
\text { mile above wills Creek } a . . . . . .\end{array}$ &.$d$ & & 0 & 13 & 21 & 0 & 9 & Trace. \\
\hline $\begin{array}{l}\text { Spring at Fairhope, Pa., south side } \\
\text { of road } 30 \text { feet above Wills Creek } a .\end{array}$ & .4 & & $\mathbf{0}$ & 17 & 35 & 0 & 9 & 0 \\
\hline 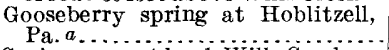 & & & 82 & 62 & 97 & Trace. & 9 & 0 \\
\hline $\begin{array}{l}\text { Spring on west bank wills creek op } \\
\text { posite brick factory }{ }^{a}\end{array}$ & 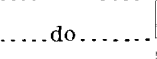 & & Trace. & 14 & 28 & Trace. & 9 & Trace. \\
\hline $\begin{array}{l}\text { Spring on top Wills Mountain, } \\
\text { piped to Shaffer's house, } 2 \text { miles } \\
\text { east of Hyndman, Pa.a. }\end{array}$ & Apr. 7,1905 & & 0 & 20 & & 0 & 9 & 0 \\
\hline $\begin{array}{l}\text { Anthony Shaffer's well, } 1 \frac{1}{2} \text { miles } \\
\text { east of Hyndman, Pa., at String- } \\
\text { town } \text { a }\end{array}$ & 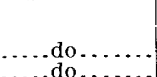 & & $\begin{array}{r}228 \\
20\end{array}$ & $\begin{array}{r}152 \\
29\end{array}$ & & $\begin{array}{r}24 \\
6\end{array}$ & $\begin{array}{r}80 \\
9\end{array}$ & $\begin{array}{l}0.5 \\
0\end{array}$ \\
\hline $\begin{array}{l}\text { Spring }{ }_{3}^{1} \text { mile from Cooks Mills, Pa. } a \\
\text { Emrich's well at Cooks Mills, Pa... }\end{array}$ & $\begin{array}{ll}. d \\
. . d\end{array}$ & & $\begin{array}{r}20 \\
146\end{array}$ & $\begin{array}{l}29 \\
44\end{array}$ & 63 & $\begin{array}{r}6 \\
30\end{array}$ & 22 & \\
\hline Spring 1 mile west of Cooks Mills, & $\ldots d$ & & 173 & 187 & & 6 & 9 & \\
\hline $\begin{array}{l}\text { Well } 112 \text { feet deep at Spangler Hotel, } \\
\text { Hancock, Md................... } \\
\text { Great Tonoloway Creek at Na- }\end{array}$ & Apr. 18,1906 & & 142 & 115 & & 108 & 129 & 0 \\
\hline $\begin{array}{l}\text { Great Tonoloway Creek at Na- } \\
\text { tional Road between Hancock } \\
\text { and Millstone, Md............. } \\
\text { Iicking Creek at National Road be- }\end{array}$ & $\ldots \mathrm{dc}$ & & Trace. & 14 & 21 & Trace. & 4.5 & 0 \\
\hline $\begin{array}{l}\text { tween Millstone, Md., a1 } \\
\text { Pool, Md................... }\end{array}$ & $d$ & & 34 & 45 & 35 & Trace. & 45 & 0 \\
\hline $\begin{array}{l}\text { Conococheague Creek above Wolf } \\
\text { Co.'s dam, Chambersburg, Pa ... } \\
\text { Falling Spring Run above mouth.. }\end{array}$ & Sept. 15,1905 & & $\begin{array}{r}46 \\
186\end{array}$ & $\begin{array}{r}58 \\
b 178\end{array}$ & 42 & $\begin{array}{r}0 \\
\text { Trace. }\end{array}$ & $\begin{array}{l}4 \\
4\end{array}$ & $\begin{aligned} 0.0 \\
\text { Trace. }\end{aligned}$ \\
\hline $\begin{array}{l}\text { Conococheague Creek below Ger- } \\
\text { big's soap factory, Chambers- } \\
\text { burg, Pa......................... }\end{array}$ & & & 96 & 96 & 80 & Trace. & 4 & 0.0 \\
\hline $\begin{array}{l}\text { Conococheague creek, tap in wash- } \\
\text { ington Hotel, city supply....... }\end{array}$ & Sept. 16,1905 & & 35 & 56 & & 0 & 9 & Trace. \\
\hline 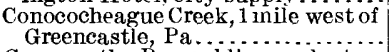 & Sept. 19,1905 & & 110 & 118 & 80 & Trace. & 9 & 0.0 \\
\hline $\begin{array}{c}\text { Greencastle, Pa., public supply, tap } \\
\text { in National Iotel, supply from } 3 \\
\text { springs } \ldots \ldots\end{array}$ & & & 0 & 202 & & Trace. & 9 & 0.0 \\
\hline $\begin{array}{l}\text { Trout Run at Mercersburg Water } \\
\text { Co.'s Reservoir } 1 \text { mile north of } \\
\text { Foltz, } 2 \frac{1}{2} \text { miles east of Mercers- } \\
\text { burg, and above Dickys Run..... }\end{array}$ & Sept.17,1905 & & Trace. & 26 & & 0 & 9 & 0.5 \\
\hline $\begin{array}{l}\text { West Branch of Conococheague } \\
\text { Creek } . . \ldots \ldots\end{array}$ & Sept & & & & 90 & & 8 & \\
\hline $\begin{array}{l}\text { Dickys Run near mouth. } \\
\text { West Branch of Conoc }\end{array}$ & ...... & & 71 & 208 & & ace. & 14 & 0. \\
\hline $\begin{array}{l}\text { West Branch of } \mathrm{C} \\
\text { Creek } 3 \text { miles sout } \\
\text { cersburg, Pa., belov }\end{array}$ & $\ldots . . . d s$ & & 90 & b 98 & 71 & Trace. & 9 & Trace. \\
\hline Licki & & & 94 & & 80 & & 9 & Trace. \\
\hline & Sept. 20,1905 & & 96 & $b 108$ & 80 & Trace. & 14 & Trace. \\
\hline $\begin{array}{l}\text { Stevens Rum at Gett } \\
\text { Rock Creek below Cu }\end{array}$ & Sept. 9,1905 & & 200 & & & & & \\
\hline $\begin{array}{l}\text { tyshurg, Pa.................. } \\
\text { Marsh Creek below Gettysurg Wa- }\end{array}$ & do & & 84 & 91 & & Trace. & 14 & 2.5 \\
\hline $\begin{array}{l}\text { Marsh Creek below Gettysburg Wa- } \\
\text { ter Co.'s intake } \\
\text { Willoughby Run at Gettysburg, } \mathrm{Pa} \text {. }\end{array}$ & & & 100 & $\begin{array}{l}b 41 \\
106\end{array}$ & & Trace. & 9 & Trace. \\
\hline $\begin{array}{l}\text { Willoughby Run } \\
\text { Mountain strean }\end{array}$ & & & & 106 & 71 & Trace. & 4 & 0.0 \\
\hline $\begin{array}{l}\text { le west of Mc- } \\
\text { ublic supply.- } \\
\text { nd of McCon- }\end{array}$ & Sept. 17,1905 & & Trace. & 27 & & Trace. & 4 & 0.0 \\
\hline $\begin{array}{l}\text { Cove Creek at west end of McCon- } \\
\text { nellsburg, Pa.... }\end{array}$ & do & & 137 & 158 & & Trace. & 4 & 0.0 \\
\hline $\begin{array}{l}\text { Litte Antiotam Creek west of } \\
\text { Waynesboro, } \mathrm{Pa} \ldots \ldots \ldots \ldots\end{array}$ & Sept. 12,1905 & & 137 & b 194 & & Trace. & 9 & 0.0 \\
\hline $\begin{array}{l}\text { Waynesboro, Pa., public supply } \\
\text { above dam of Waynesboro water } \\
\text { Co., at conduence of Rattlesnake } \\
\text { Run and East Branch Antietam } \\
\text { Creek }\end{array}$ & & & . & b 21 & & 0 & 4 & Trace. \\
\hline
\end{tabular}

$a$ Assay by $\mathrm{S}$. J. Lewis.

$b$ Alkaline carbonates, 21 parts per million. 
Field assays of waters in Potomac River basin-Continued.

\begin{tabular}{|c|c|c|c|c|c|c|c|c|}
\hline Stream. & Date. & 总 & 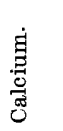 & 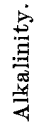 & 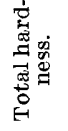 & 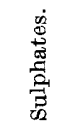 & $\begin{array}{l}\stackrel{\Xi}{\Xi} \\
\stackrel{\Xi}{\Xi} \\
\stackrel{\Xi}{\Xi}\end{array}$ & 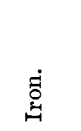 \\
\hline $\begin{array}{l}\text { East Branch Little Antietam } \\
\text { Creek east of Waynesboro, Pa... }\end{array}$ & Sept. 12,1905 & & 28 & a. 51 & & 0 & 4 & Trace. \\
\hline Spanglers Spring at Gettysburg, & Sept. 9,1905 & & 39 & 85 & 42 & 0 & 9 & 0.0 \\
\hline $\begin{array}{l}\text { Well of Gettysburg Brick co. at } \\
\text { Gettysburg, Pa.................... }\end{array}$ & & & 218 & 250 & & 0 & 14 & 0.0 \\
\hline $\begin{array}{l}\text { Well of Gettysburg Transit Co. at } \\
\text { Gettysburg, Pa................. }\end{array}$ & do & & 200 & 173 & & Trace. & 29 & 0.0 \\
\hline $\begin{array}{l}\text { Well of Western Maryland R. R. at } \\
\text { Gettysburg, } \mathrm{Pa} b . . .\end{array}$ & do & & 123 & 291 & & 110 & 258 & 0.5 \\
\hline $\begin{array}{l}\text { Steam laundry dug well at Gettys- } \\
\text { burg, Pa...... }\end{array}$ & Sept. 10,1905 & & 72 & 79 & & Trace. & 29 & 0.0 \\
\hline $\begin{array}{l}\text { Gettysburg Water Co's Well No. } \\
\text { 1, Gettyshurg, Pa....................... }\end{array}$ & Sept. 9,1905 & & 23 & 41 & 51 & Trace. & 9 & 0.0 \\
\hline
\end{tabular}

a Alkaline carbonates, 21 parts per million. $b$ Magnesium present.

\section{SANITARY AND MINERAL ANALYSES.}

By Raymond Outwater.

Numerous chemical analyses of the waters of the Potomac have been made during the course of this investigation, and the results are given in the accompanying table. It is very difficult to draw definite conclusions of a general nature from these sanitary analyses. Inspection of the various places on the river and its tributaries points to a considerable pollution at practically all of the places examined and the chemical analyses confirm the inspections. Evidences of pollution can, in some instances; be seen in the main stream after pollution has entered it; yet chemical analysis does not indicate that the water is much more polluted at Great Falls than at many points farther up the river and on its tributaries.

The tap water was collected in the McKinley School of Manual Training. The water supplied to this building flows from the river to the Dalecarlia Reservoir, thence to the distributing reservoir in Georgetown, and thence to the Washington City Reservoir, from which it flows by gravity through the street mains to the laboratory. None of the water supplied to Washington during the progress of the chemical examination was filtered.

A study of the analyses leads to the following conclusions:

1. The variations in the amount of each component have been considerable, and these are to a certain extent unaccounted for.

2. A certain proportion of the variations can possibly be accounted for by the variation in the rainfall.

3. If these analyses are of accuracy equal to those obtained by previous investigations they indicate that the river has become more impure, the figure for required oxygen being the only one which shows a decrease. 
The mineral analyses which were made represent the main stream and all of the principal tributaries, and it is believed that they give an insight into the chemical denudation of the basin. The purpose of these analyses was to determine the amount and the nature of the dissolved mineral matter. The water was filtered through a PasteurChamberland filter; measured quantities were then evaporated nearly to dryness in porcelain vessels, after which the evaporation was completed in platinum dishes. The residue thus obtained was treated with hydrochloric acid and twice evaporated to dryness, after which it was redissolved in hydrochloric acid and the silica was separated by filtration, ignited, and weighed. The residue left after treatment with hydrofluoric acid was added to the "iron and alumina." The filtrate from the silica was diluted to 200 c. c. and one portion of $100 \mathrm{c.c}$. was taken for the estimation of iron and alumina, calcium, and magnesium, and the other portion for the estimation of sulphuric acid, sodium, and potassium. This method of determining sodium and potassium is preferable to the common one of separating sodium and potassium in the filtrate from the magnesium. It is unnecessary to detail the methods, which may be found in standard text-books on quantitative chemical analysis.

Some interesting facts are brought out by comparing the flow of the Potomac with the analytical results. For this purpose, the gage readings at Point of Rocks, Md., 7 miles above the Monocacy River, were used, no gage being maintained by the United States Geological Survey below this point. As most of the analyses were of samples from the Washington city taps, which are fed with water diverted from the river at Great Falls, the results are not strictly accurate, but they are approximately so.

The average flow of the Potomac at Point of Rocks for 1905 was calculated from observations made by the United States Geological Survey and was found to be 7,534 second-feet. As the weight of a cubic foot of water is 28.3 kilograms (62.4 pounds), this is equal to 213,212 kilograms per second. Calculating the total amount of water flowing past this point from the average, we have for the year $6,724,000,000,000$ kilograms or liters. From the figures on analyses we have: Total solids in the unfiltered water, 146.1 parts per million; total solids in solution, 114.6 parts; in suspension, 31.5 parts; from which we find that the following amount of material is carried past this point in the course of a year: Total solicis, 982,000,000 kilograms; in solution, $771,000,000$ kilograms; in suspension, $212,000,000$ kilograms; or, in solution, 850,000 tons; in suspension, 234,000 tons.

The drainage area of the Potomac above Great Falls is 11,400 square miles. By comparing this area with the amount of material being carried down in suspension and in solution, the following figures, showing the average amount of solid material being carried off annually 
from each square mile of territory in the Potomac basin, are obtained: In solution, 74.4 tons; in suspension, 20.5 tons; total, 94.9 tons.

\section{Sanitary analyses of surface water in the Potomac basin.}

[Parts per million.]

\begin{tabular}{|c|c|c|c|c|c|c|c|c|c|c|}
\hline \multirow[b]{2}{*}{ Stream. } & \multirow[b]{2}{*}{ Date. } & \multirow[b]{2}{*}{$\begin{array}{c}\text { Re- } \\
\text { quired } \\
\text { oxy- } \\
\text { gen. }\end{array}$} & \multicolumn{4}{|c|}{ Nitrogen as- } & \multirow[b]{2}{*}{$\begin{array}{l}\text { Chlo- } \\
\text { rine. }\end{array}$} & \multicolumn{3}{|c|}{ Unfiltered water. } \\
\hline & & & $\begin{array}{l}\text { Albu- } \\
\text { minoid } \\
\text { am- } \\
\text { monia. }\end{array}$ & $\begin{array}{c}\text { Free } \\
\text { am- } \\
\text { monia. }\end{array}$ & $\begin{array}{c}\mathrm{Ni}- \\
\text { trites. }\end{array}$ & $\begin{array}{c}\mathrm{Ni}- \\
\text { trates. }\end{array}$ & & $\begin{array}{l}\text { Total } \\
\text { resi- } \\
\text { due. }\end{array}$ & $\begin{array}{l}\text { Loss } \\
\text { on jg- } \\
\text { ni- } \\
\text { tion. }\end{array}$ & $\begin{array}{l}\text { Total } \\
\text { hard- } \\
\text { ness. }\end{array}$ \\
\hline $\begin{array}{l}\text { North Branch Potomac } \\
\text { River above Bayard.. }\end{array}$ & Feb, 27,190 & 1.65 & 0.16 & 0.12 & Trace. & 3.00 & 6 & $2 b$ & 19 & 29. \\
\hline Buffalo Creek at mouth. & .... do..... & 1.70 & .06 & .05 & Trace. & 1.50 & 9 & 39 & 15 & $\begin{array}{l}24.4 \\
39.7\end{array}$ \\
\hline $\begin{array}{l}\text { North Branch Potomac } \\
\text { River above Gormania. } \\
\text { North Branch Potomac }\end{array}$ & Feh. 25, 1905 & 2.15 & .11 & .07 & .002 & 1.25 & 4 & 30 & 15 & 42.6 \\
\hline River below Gor- & & & & & & & & & & \\
\hline$\underset{D}{\operatorname{mania}} a \ldots \ldots \ldots \ldots \ldots$ & Oct. $\quad 6,1899$ & 26.42 & .676 & .182 & .023 & .261 & 28.7 & 311.6 & 140.7 & 128.2 \\
\hline Abram Creek at mouth. & $\begin{array}{l}\text { Feb. } 25,1905 \\
\text { Feb. } 27,1905\end{array}$ & 1.95 & .13 & $\begin{array}{l}.06 \\
19\end{array}$ & Trace. & $\begin{array}{l}2.00 \\
2.50\end{array}$ & $\begin{array}{l}9 \\
5\end{array}$ & $\begin{array}{l}46 \\
47\end{array}$ & $\begin{array}{l}17 \\
26\end{array}$ & $\begin{array}{l}55.9 \\
51 \cdot 5\end{array}$ \\
\hline $\begin{array}{l}\text { North Branch Potomac } \\
\text { River above Savage }\end{array}$ & Fen. $2 \pi$, & & & & 1race. & & & & & \\
\hline River.................. & Feb. 25,1905 & 1.80 & .15 & .02 & 0 & 3.00 & 11 & 23 & 9 & 50 \\
\hline $\begin{array}{l}\text { Savage River........... } \\
\text { North Branch Potomae }\end{array}$ & ... do........ & .80 & .06 & Trace. & 0 & 3.00 & $\mathbf{5}$ & 32 & 15 & 33.8 \\
\hline $\begin{array}{c}\text { River above mill of } \\
\text { West Virginia Pulp } \\
\text { and Paper Co. at Luke. } \\
\text { Do............................ }\end{array}$ & $\begin{array}{l}\text { July } \quad 14,1899 \\
\text { Feb. }\end{array}$ & $\begin{array}{l}7.76 \\
1.45\end{array}$ & $\begin{array}{l}.234 \\
.18\end{array}$ & $\begin{array}{l}.154 \\
.09\end{array}$ & $\begin{array}{l}\text { Trace. } \\
0\end{array}$ & $\begin{array}{l}.171 \\
2.50\end{array}$ & 15 & $\begin{array}{l}59.4 \\
57\end{array}$ & $\begin{array}{l}25.5 \\
18\end{array}$ & $\begin{array}{l}24.7 \\
50\end{array}$ \\
\hline $\begin{array}{l}\text { North Branch Potomac } \\
\text { River below mill of } \\
\text { West Virginia Pulp }\end{array}$ & & & & & & & & & & \\
\hline $\begin{array}{l}\text { and Paper Co. at Luke- } \\
\text { Georges Creek at West- }\end{array}$ & ...do. & 3.20 & .09 & .05 & Trace. & 3.00 & 25 & 448 & 177 & 120.5 \\
\hline 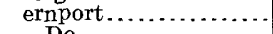 & July 14,1899 & 13.05 & .446 & .591 & .011 & 1. 422 & 10.1 & 1013 & 6 & 439 \\
\hline Dorth Branch Potomac & Feb. 25,1905 & 2.35 & .06 & .51 & Trace. & 2.50 & 17 & 1057 & 442 & 524 \\
\hline $\begin{array}{l}\text { River between Keyser } \\
\text { and Piedmont.......... }\end{array}$ & .0 & 1.80 & .06 & .06 & 0 & 2.5 & 10 & 145 & 50 & 63.2 \\
\hline $\begin{array}{l}\text { North Branch Potomac } \\
\text { River below mill at } \\
\text { Keyser.................. }\end{array}$ & & 2.35 & .09 & .05 & Trace. & 2.50 & 11.0 & 189 & 60 & 97.0 \\
\hline $\begin{array}{l}\text { New Creek } \frac{1}{4} \text { mile above } \\
\text { mouth } a\end{array}$ & Julv & 11.26 & 371 & 137 & 069 & 933 & & & & \\
\hline $\begin{array}{l}\text { New } \\
\text { Nortl }\end{array}$ & Fel. 25,1905 & 2.80 & .46 & .30 & .004 & 2.50 & 22 & 233 & 8 & $\begin{array}{l}115.7 \\
105.8\end{array}$ \\
\hline River belo & , 1899 & & .380 & $.05-2>$ & .16 & 2.31 & 11.9 & 452 & 19 & 100.6 \\
\hline $\begin{array}{l}\text { Do } \\
\text { North Bran }\end{array}$ & 1899 & & .483 & .08 & .11 & .74 & 22.6 & & & 140.3 \\
\hline land. & 1899 & & .295 & .075 & & 2.86 & 1 & 491.8 & 22 & 110.5 \\
\hline & 1899 & & .397 & & & & & & & Non \\
\hline Hydrant in & July 15,1899 & 23.689 & .374 & .163 & .005 & .219 & 13.6 & 276.5 & 104.9 & 128.5 \\
\hline $\begin{array}{l}\text { Cumberland water sup- } \\
\text { ply } \\
\text { wills Creek above Jen- }\end{array}$ & Dec. 29,1904 & 7.45 & .32 & .37 & Trace. & 1.20 & 5.0 & 126 & 47 & 73.3 \\
\hline Gek Run at Alle- & Dec. 29,1904 & 1.7 & .00 & & thace. & 1.00 & 14 & 115 & $\because 33$ & 78 \\
\hline $\begin{array}{l}\text { Wills Creek above dam } \\
\text { of United States } \\
\text { Leother Co., Cumber- } \\
\text { land...................... }\end{array}$ & Dec. 27,1904 & 1.70 & .28 & .06 & Trace. & 1.00 & 14 & 115 & 33 & 78 \\
\hline $\begin{array}{l}\text { Wills Creek above Baiti- } \\
\text { more Street Bridge, } \\
\text { Cumberland........... }\end{array}$ & ...do. & 13.95 & .66 & .40 & .004 & .90 & 17 & 224 & 60 & 137.3 \\
\hline Wills Creek 100 yards & July 15,1899 & 32.476 & .909 & .827 & .008 & .257 & 41.7 & 372.5 & 135.2 & 168.6 \\
\hline $\begin{array}{l}\text { Patterson Creek at } \\
\text { mouth.................. }\end{array}$ & Feb. 13,1905 & .45 & .05 & Trace. & 0 & 1.00 & 8.0 & 142 & 55 & 129.5 \\
\hline $\begin{array}{l}\text { North Branch Potomac } \\
\text { River at Greenspring. } \\
\text { South Branch.Potomac }\end{array}$ & Feb. 10,1905 & 2.10 & .35 & .27 & .008 & 2.00 & 14.5 & 219 & 63 & 152.9 \\
\hline $\begin{array}{l}\text { South Branch. Potomac } \\
\text { River at Franklin } \\
\text { above tannery }\end{array}$ & Mar. 11,1905 & 1.70 & .29 & Trace. & 0 & 1.12 & 3.50 & 85 & 35 & 80.9 \\
\hline $\begin{array}{l}\text { Branch Potomac River } \\
\text { above South Branch.. }\end{array}$ & Mar. 12,1905 & 1.75 & .17 & .01 & 0 & 1.00 & 2.5 & 83 & 25 & 42.6 \\
\hline
\end{tabular}

a Analysis by Prof. J. W. Mallet. 
Sanitary analyses of surface water in the Potomac basin-Continued.

\begin{tabular}{|c|c|c|c|c|c|c|c|c|c|c|}
\hline \multirow[b]{2}{*}{ Stream. } & \multirow[b]{2}{*}{ Date. } & \multirow[b]{2}{*}{$\begin{array}{c}\text { Re- } \\
\text { quired } \\
\text { oxy- } \\
\text { gen. }\end{array}$} & \multicolumn{4}{|c|}{ Nitrogen as- } & \multirow[b]{2}{*}{$\begin{array}{l}\text { Chlo- } \\
\text { rine. }\end{array}$} & \multicolumn{3}{|c|}{ Unfiltered water. } \\
\hline & & & $\begin{array}{c}\text { Albu- } \\
\text { minoid } \\
\text { am- } \\
\text { monia. }\end{array}$ & $\begin{array}{c}\text { Free } \\
\text { am- } \\
\text { monia. }\end{array}$ & $\underset{\text { Nites. }}{\mathrm{Ni}}$ & Ni- & & $\begin{array}{l}\text { Total } \\
\text { resi- } \\
\text { due. }\end{array}$ & $\mid \begin{array}{c}\text { Loss } \\
\text { on ig- } \\
\text { ni- } \\
\text { tion. }\end{array}$ & $\begin{array}{l}\text { Total } \\
\text { hard- } \\
\text { ness. }\end{array}$ \\
\hline $\begin{array}{l}\text { outh } \mathrm{F} \\
\text { River }\end{array}$ & & & & & & & & & & \\
\hline $\begin{array}{r}\text { River abc } \\
\text { burg } . . . \\
\text { Mill Creek. }\end{array}$ & Mar. 10,1905 & 1.85 & 0.31 & 0.03 & 0 & 1.25 & 4.0 & 171 & 26 & 55.9 \\
\hline $\begin{array}{l}\text { Mill Creek, Hardy Coun- } \\
\text { ty, W. Va.............. }\end{array}$ & do & 2.10 & .17 & .03 & 0 & 1.30 & 3.5 & 199 & 48 & 66.2 \\
\hline $\begin{array}{l}\text { South Branch Potomac } \\
\text { River below Peters- } \\
\text { burg }\end{array}$ & $\ldots u$ & 1.95 & .22 & .07 & 0 & 1.25 & 3.0 & 220 & 41 & 50 \\
\hline $\begin{array}{l}\text { South Branch Potomac } \\
\text { River above Moore- } \\
\text { field......................... }\end{array}$ & $\cdots$ & 2.00 & .13 & .03 & 0 & 1.25 & 2.5 & 276 & 32 & 00 \\
\hline $\begin{array}{l}\text { Moorefield River above } \\
\text { tannery at Moorefield. }\end{array}$ & & 90 & 12 & 09 & 0 & & & & & \\
\hline $\begin{array}{l}\text { Moorefield River below } \\
\text { tannery at Moorefield. }\end{array}$ & Evis & 1.90 & .12 & .02 & 0 & 1.50 & 4.5 & 258 & 29 & 47.0 \\
\hline $\begin{array}{l}\text { tannery at } \\
\text { South Branc }\end{array}$ & $\ldots . . d$ & 2.40 & .21 & .01 & 0 & 1.25 & 4.0 & 471 & 44 & 44.1 \\
\hline $\begin{array}{l}\text { River } \\
\text { feld }\end{array}$ & Mar & 2.10 & .13 & .03 & 0 & 1.50 & 3.5 & 498 & 77 & 60. \\
\hline $\begin{array}{l}\text { Mill Creek at Romney... } \\
\text { South Branch Potomac }\end{array}$ & Mar. & 1.65 & .12 & .03 & 0 & 1.25 & 4.5 & 119 & 32 & 50 \\
\hline River : & $\ldots$. & 1.30 & .14 & .03 & Trace. & 1.25 & 3.0 & 98 & 28 & 55.4 \\
\hline $\begin{array}{l}\text { Cherry Run } \\
\text { South Bran }\end{array}$ & $\ldots$ & 1.70 & .36 & .03 & $\mathrm{Tr}$ & 1.40 & 7.5 & 93 & 29 & 35 \\
\hline & Feb: 10,1905 & 1.15 & .11 & Trace. & Trace. & 2.00 & 7 & 120 & 43 & 123.2 \\
\hline $\begin{array}{l}\text { Potomac River below } \\
\text { South Branch }\end{array}$ & $\cdots$ & 1.15 & .13 & Trace. & Trace. & 00 & 1 & 127 & 48 & \\
\hline Potomac River above & & & & & & & 0.1 & & 48 & 121.7 \\
\hline $\begin{array}{c}\text { Pawpaw } \ldots \ldots . . . \ldots \\
\text { Potomac }\end{array}$ & & 1.30 & .22 & .09 & .004 & 2.00 & 8 & 156 & 49 & 127.9 \\
\hline River above & do & 2.20 & .24 & .10 & .004 & 2.00 & 14 & 176 & 57 & 157.6 \\
\hline Poto & Feb. 12,1905 & .90 & .10 & .01 & Trace. & .88 & 8 & 162 & 54 & 135.7 \\
\hline $\begin{array}{l}\text { Great Cacapon River at } \\
\text { mouth }\end{array}$ & & 1.35 & .11 & .08 & & 1.00 & 5 & 108 & 37 & $\begin{array}{r}103.4 \\
82.7\end{array}$ \\
\hline $\begin{array}{l}\text { Potomac River below } \\
\text { Great Cacapon River. }\end{array}$ & & .70 & .03 & Trace. & 0 & 1.00 & 0 & & 35 & \\
\hline Warm Spring Run ...... & Feb. 10,1905 & .50 & .03 & .08 & .012 & 2.25 & 6 & $\begin{array}{r}94 \\
181\end{array}$ & $\begin{array}{l}35 \\
53\end{array}$ & $\begin{array}{r}93.6 \\
165.4\end{array}$ \\
\hline $\begin{array}{l}\text { Conococheague Creek } \\
\text { above Chambersburg. } \\
\text { Conococheague Creek }\end{array}$ & Mar. 31.1905 & .40 & .04 & .03 & 0 & 1.00 & 3.5 & 135 & 93 & 47.0 \\
\hline $\begin{array}{l}\text { Conococheague Creek } \\
\text { below Chambersburg. }\end{array}$ & & & & & & & & & & \\
\hline $\begin{array}{l}\text { below Chambersburg. } \\
\text { West Branch of Conoco- } \\
\text { cheague Creek above }\end{array}$ & & .50 & .08 & .03 & Trace. & 4.00 & 8 & 233 & 147 & 91.0 \\
\hline of Conoco- & Apr. 1,1905 & .65 & .14 & .02 & Trace. & 3.00 & 4.5 & 159 & 31 & 130.8 \\
\hline & $\ldots . . \mathbf{u}$ & .70 & .08 & .01 & Trace. & 3.50 & 5.0 & 127 & 43 & 105.8 \\
\hline $\begin{array}{l}\text { Back Creek above Wil- } \\
\text { liamson............... }\end{array}$ & Mar & & & & & & & & & \\
\hline Wil- & Mar & .25 & .22 & .02 & Trace. & 2.00 & 4.5 & 101 & 40 & 126.4 \\
\hline $\begin{array}{l}\text { liar } \\
\text { Cono }\end{array}$ & de & 2.10 & .16 & .04 & Trace. & 3.50 & 5.5 & 77 & 66 & 129.4 \\
\hline $\begin{array}{l}\text { low Bac } \\
\text { Conococh }\end{array}$ & do & .60 & .13 & .05 & Trace. & 5.00 & 5.5 & 222 & 66 & 192.6 \\
\hline $\begin{array}{l}\text { low junc } \\
\text { West Bran }\end{array}$ & & & & & & & & & & \\
\hline $\begin{array}{l}\text { West Bran } \\
\text { Potomac Ri }\end{array}$ & Apr. 1,1905 & .55 & .12 & .02 & Trace. & 2.50 & 5.0 & 115 & 80 & 137.6 \\
\hline Con & & .50 & .12 & .02 & Trace. & 2.25 & 4.0 & 104 & 16 & 47 \\
\hline $\begin{array}{l}\text { Potomac Ri } \\
\text { Conocochea }\end{array}$ & & & & & & & & & & \\
\hline Opec & & .50 & .17 & .03 & Trace. & 2.75 & 7.0 & 165 & 33 & 125 \\
\hline & Feb. 18,1905 & .40 & .10 & .01 & .0 & 2.5 & 9 & & 121 & \\
\hline $\begin{array}{l}\text { Abra } \\
\text { Opeq }\end{array}$ & $d x$ & 925 & 10 & .03 & .0 & 5.00 & 17 & & 140 & 224 \\
\hline $\begin{array}{l}\text { Apeq } \\
\text { Opeq }\end{array}$ & 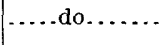 & 2.05 & .17 & .04 & .012 & 2.75 & 15 & 305 & 25 & 157.3 \\
\hline Tuscarora Creek...... & Feb. 17 & $\boldsymbol{W}$ & .04 & .03 & .0 & 5.0 & 19 & & 62 & \\
\hline $\begin{array}{l}\text { Tusce } \\
\text { Opeq }\end{array}$ & & 1.50 & a & .07 & .128 & 5.00 & 17 & 328 & 129 & 305.8 \\
\hline $\begin{array}{l}\text { Opequon Creek near } \\
\text { mouth }\end{array}$ & $\ldots$ & .70 & .11 & 03 & .024 & 5.50 & 12 & 284 & 56 & \\
\hline $\begin{array}{l}\text { Potomac Riv } \\
\text { Opequon Cre }\end{array}$ & & & & & & & & & & 266 \\
\hline $\begin{array}{l}\text { uon Cre } \\
\text { Riv }\end{array}$ & $d$ & .40 & .12 & .10 & .004 & 2.50 & 13 & 178 & 87 & 123.5 \\
\hline $\begin{array}{l}\text { Op } \\
\text { Potor }\end{array}$ & do. & .90 & $: 10$ & .03 & .024 & 2.75 & 20 & 164 & 22 & 210.2 \\
\hline $\begin{array}{l}\text { Potomac River ab } \\
\text { Antietam Creek.. }\end{array}$ & Jan. 17,1905 & 3.33 & .25 & .08 & Trace. & 1.00 & 8.5 & 185 & 22 & 93.3 \\
\hline
\end{tabular}


Sanitary analyses of surface water in the Potomac basin-Continued.

\begin{tabular}{|c|c|c|c|c|c|c|c|c|c|c|}
\hline \multirow[b]{2}{*}{ Stream. } & \multirow[b]{2}{*}{ Date. } & \multirow[b]{2}{*}{$\begin{array}{l}\text { quired } \\
\text { oxy- } \\
\text { gen. }\end{array}$} & \multicolumn{4}{|c|}{ Nitrogen as- } & \multirow[b]{2}{*}{$\begin{array}{l}\text { Chlo- } \\
\text { rine. }\end{array}$} & \multicolumn{3}{|c|}{ Unfiltered water. } \\
\hline & & & $\begin{array}{c}\text { Albu- } \\
\text { minoid } \\
\text { am- } \\
\text { monia. }\end{array}$ & $\begin{array}{c}\text { Free } \\
\text { am- } \\
\text { monia. }\end{array}$ & $\begin{array}{c}\mathrm{Ni}- \\
\text { trites. }\end{array}$ & $\mid \begin{array}{c}\mathrm{Ni}- \\
\text { trates. }\end{array}$ & & $\begin{array}{l}\text { Total } \\
\text { resi- } \\
\text { due. }\end{array}$ & $\begin{array}{c}\text { Loss } \\
\text { on ig- } \\
\text { ni- } \\
\text { tion. }\end{array}$ & $\begin{array}{l}\text { Total } \\
\text { hard- } \\
\text { ness. }\end{array}$ \\
\hline $\begin{array}{c}\text { Antietam } \\
\text { mouth }\end{array}$ & Jan. 17,1905 & 1.95 & 0.15 & Trace. & 0.004 & 2.50 & 10 & 216 & 46 & 148.8 \\
\hline $\begin{array}{l}\text { Potomac River below } \\
\text { Antietam Creek...... }\end{array}$ & & & & & & & & & & \\
\hline Antietam Creek ....... & & 2.65 & .41 & 0.03 & .004 & 2.50 & 2.5 & 238 & 67 & 150.1 \\
\hline $\begin{array}{r}\text { Cooks Creek } . . . \\
\text { Blacks Creek. } . . .\end{array}$ & $\begin{array}{c}\text { Jan. } 28,1905 \\
\ldots \ldots \text { do } \ldots \ldots\end{array}$ & $\begin{array}{l}1.35 \\
4.35\end{array}$ & .16 & Trace. & $\begin{array}{r}\text { Trace. } \\
.032\end{array}$ & $\begin{array}{l}1.00 \\
2.50\end{array}$ & $\frac{11}{33}$ & 137 & $\begin{array}{l}39 \\
81\end{array}$ & 137. 3 \\
\hline North River below & & & & & & & & & & \\
\hline $\begin{array}{l}\text { Cooks Creek. } \\
\text { Middle River above }\end{array}$ & ..... & 1.05 & .16 & .02 & Trace. & 1.25 & 15 & 147 & 39 & 170 \\
\hline Lewis Creek.............. & ... & 1.15 & .07 & .12 & 0 & 1.25 & 4 & 219 & 42 & 218. 4 \\
\hline $\begin{array}{l}\text { mouth. } \\
\text { Basic. }\end{array}$ & $\operatorname{Jan}$ & 2.25 & .47 & 1.52 & .064 & 3. 00 & 16 & 365 & 67 & 230.9 \\
\hline $\begin{array}{l}\text { South River above Basic. } \\
\text { South River below Basic. }\end{array}$ & Jan. 2 & 1.25 & .17 & .01 & Trace. & & 6 & 10 & 36 & 117 \\
\hline $\begin{array}{l}\text { South Fork of Shenan- } \\
\text { doah River above Elk }\end{array}$ & -.....do & 1.90 & .13 & .03 & .002 & 1.00 & 4 & 112 & 35 & 109.2 \\
\hline $\begin{aligned} \text { Ru } \\
\text { Elk } 1\end{aligned}$ & Feb. 4,1905 & $\begin{array}{r}1.15 \\
21.45\end{array}$ & $\begin{array}{r}.14 \\
1.12\end{array}$ & .02 & $\begin{array}{r}.003 \\
\text { Trace. }\end{array}$ & $\begin{array}{l}3.50 \\
4.00\end{array}$ & $\stackrel{6}{67.0}$ & $\begin{array}{l}152 \\
214\end{array}$ & $\begin{array}{r}52 \\
145\end{array}$ & $\begin{array}{r}105.3 \\
55.4\end{array}$ \\
\hline $\begin{array}{l}\text { South Fork of Shenan- } \\
\text { doah River below Elk }\end{array}$ & & & & & & & & & & \\
\hline $\begin{array}{l}\text { Run } \\
\text { South Fork of Shenan- }\end{array}$ & .....do....... & 3.85 & .14 & .02 & .002 & 4. 00 & 12 & 157 & 66 & 145. \\
\hline $\begin{array}{l}\text { doah River above } \\
\text { Hawksbill Creek....... }\end{array}$ & Jan. & 1.15 & .14 & .01 & Trace. & 1.75 & 7 & 143 & 42 & 137.3 \\
\hline $\begin{array}{r}\text { Hawksbill Creek above } \\
\text { Luray ..................... }\end{array}$ & & 1.00 & .09 & .01 & & 00 & 9 & 124 & 45 & - \\
\hline $\begin{array}{l}\text { Do } \\
\text { Yawksbill Creek bel }\end{array}$ & Feb. 3,1905 & 1.05 & .8 & Trace. & .001 & 4.00 & 15 & 138 & 44 & 77 \\
\hline ...................... & ....do & 4. 35 & .48 & .03 & .002 & 3. 50 & 24 & 175 & 85 & 94.5 \\
\hline $\begin{array}{l}\text { South Fork of Shenan- } \\
\text { doah River below } \\
\text { Hawksbill Creek...... }\end{array}$ & Jan. & 2.35 & .21 & .05 & Trace. & 50 & 10 & & 47 & - \\
\hline $\begin{array}{l}\text { South Fork of Shenan- } \\
\text { doah River at Riverton }\end{array}$ & Jant. & 1.15 & .08 & .30 & Trace. & 1.200 & 13 & 143 & 29 & 21.5 \\
\hline $\begin{array}{l}\text { North Fork of Shenan- } \\
\text { doah River above Pas- } \\
\text { sage Creek................ }\end{array}$ & Feb. & 1.70 & .14 & .09 & Trace. & 2.00 & 10 & 200 & 82 & 180.1 \\
\hline Fork of Shenar- & 20 & 1. 50 & .10 & .01 & 0 & 1.00 & 5 & 78 & 37 & 89.1 \\
\hline & & 1.95 & .16 & .24 & Trace. & 25 & 6 & 189 & 81 & 160.6 \\
\hline River below & Feb. $\quad 3,1905$ & 1.00 & .24 & .03 & Trace. & 1 & 8 & 71 & 12 & 75.6 \\
\hline North & & & & & & & & & & \\
\hline $\begin{array}{l}\text { Evi } \\
\text { Flo }\end{array}$ & Jan. & 300 & .06 & .01 & .004 & & & & 10 & \\
\hline ing Run .... & Jan. & 3.00 & .72 & .21 & .024 & b. 00 & 9. & 3 & 123 & 277.7 \\
\hline mo & July & 1.55 & 32 & .02 & .004 & 50 & & 243 & 55 & 11 \\
\hline & Dec. 1 & & .10 & .25 & Traet & & & & & \\
\hline & Dee. 20 & 1.20 & Trace. & Trace. & .001 & .50 & 4.0 & 241 & 51 & 172. \\
\hline $\begin{array}{l}\text { above } \\
\text { ver..... }\end{array}$ & & & & & & & & & & \\
\hline ver. $\ldots$. & $\begin{array}{l}\text { July } 4, \\
\text { Dec. } 12,\end{array}$ & $\begin{array}{l}1.45 \\
3.35\end{array}$ & $\begin{array}{l}.33 \\
.24\end{array}$ & $\begin{array}{l}.01 \\
.01\end{array}$ & $\begin{array}{l}.004 \\
\text { Trace }\end{array}$ & .50 & & 195 & 59 & 9.7 \\
\hline & Dec. 20,1904 & 2.15 & .12 & Trace. & .004 & .50 & 8.0 & 229 & 55 & 158.7 \\
\hline elow & & & & & & & & & & \\
\hline She & Dec. & 75 & .22 & 01 & Trac & 2.00 & & & 20 & \\
\hline & $\mathrm{J}$ & & .70 & .25 & & & & & & \\
\hline & Jan. 1 & 1. .4 & .14 & Trace. & Trace. & & & 260 & 13 & 42 \\
\hline & Jan. 18,1905 & .95 & .01 & .05 & Trace. & 1.50 & 6. & 71 & 26 & 40.2 \\
\hline $\begin{array}{l}\text { lcy River at } \\
\text { ll Creek..... }\end{array}$ & Jan. 12,1905 & 1. 88 & .26 & .22 & .008 & 1.50 & 6.0 & 111 & 21 & \\
\hline Creek above & & & & & & & & & & \\
\hline $\mathrm{Fr}$ & Jan. 1 & 2.05 & .14 & .20 & 008 & 1.50 & 9. & 182 & 40 & 129 \\
\hline $\begin{array}{l}r \text { mouth } \\
\text { below }\end{array}$ & & & & .48 & Trace. & 50 & 10.0 & 220 & 76 & 127 \\
\hline icreek......... & Jan. 12,1905 & 2.00 & .18 & .13 & Trace. & 1. 35 & 8.0 & 100 & 28 & 62 \\
\hline $\begin{array}{c}\text { Monocacy River at } \\
\text { mouth....................... }\end{array}$ & Jan. 16,1905 & 1.40 & .13 & .02 & Trace. & 1.50 & 3.0 & 115 & 42 & 82.8 \\
\hline above & & & & & & & & & & \\
\hline acy River...... & Jan. 1 & 20 & .17 & .10 & & & & 219 & 47 & 13 \\
\hline below & Jan. & 1.5 & & .02 & $\mathrm{~T}$ & 2. & 6. & 222 & 64 & 138 \\
\hline cy $R$ & $\mathrm{~J}$ & 1.5 & .3 & .0 & & & & 1. & 39 & 89 \\
\hline & Ta & & 1 & .10 & & & & 121 & & \\
\hline 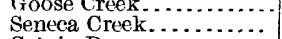 & $\begin{array}{l}\text { Jan. } 3 \\
\text { Mar. } 2\end{array}$ & $\begin{array}{r}1.50 \\
.20\end{array}$ & .16 & .92 & $\begin{array}{r}.002 \\
\text { Trace. }\end{array}$ & 1 . & & $\begin{array}{l}61 \\
74\end{array}$ & $\begin{array}{l}14 \\
13\end{array}$ & $\begin{array}{l}43.7 \\
36.8\end{array}$ \\
\hline Calvin Run.. & Mar. 10,1905 & 2.40 & .18 & .09 & 0 & 2.25 & 5. 0 & 73 & 24 & 211. 7 \\
\hline
\end{tabular}


SANITARY ANALYSES OF SURFACE WATERS.

Sanitary analyses of tap water at Washington.

[ Parts per million.]

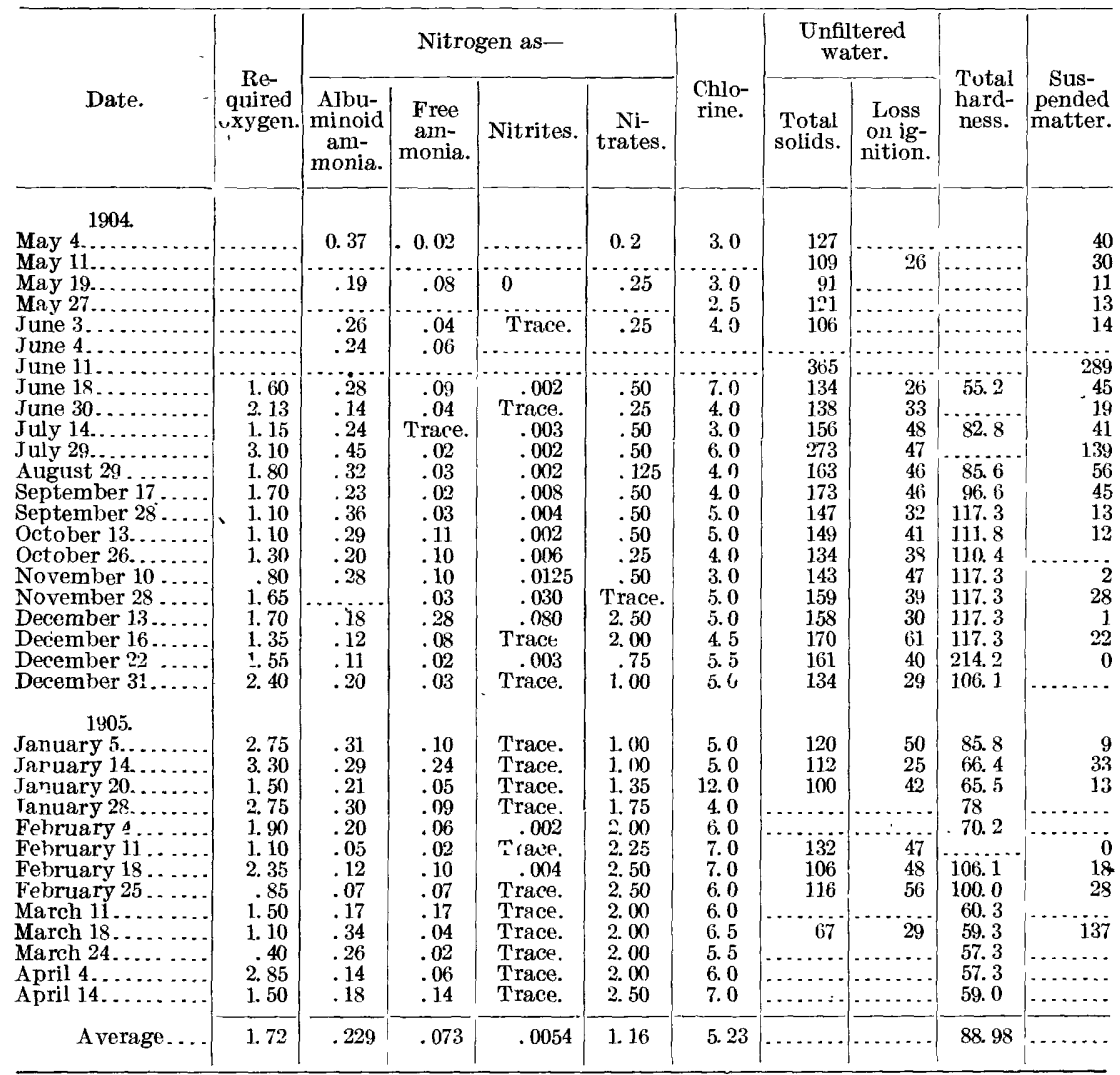

Comparison of analysis of Potomae water by several investigators.

[Parts per million.]

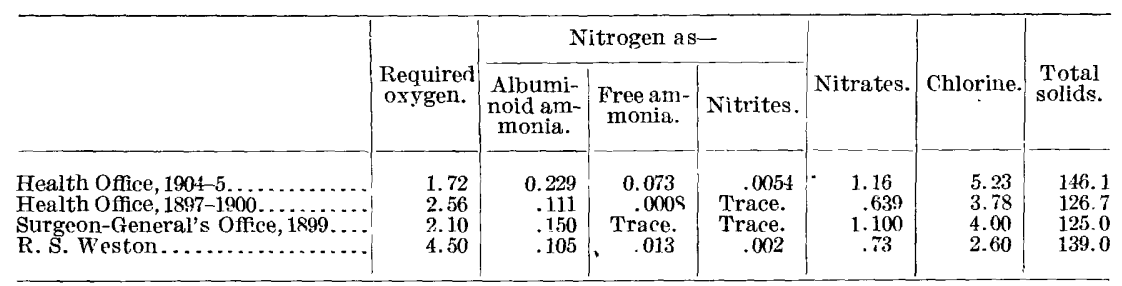


Mineral analyses of surface waters in Potomac basin.

[Parts per million.]

\begin{tabular}{|c|c|c|c|c|c|c|c|}
\hline & $\begin{array}{c}\text { North } \\
\text { Branch } \\
\text { Potomac } \\
\text { River } \\
\text { above } \\
\text { Bayard, } \\
\text { Feb. 27, } \\
1905 .\end{array}$ & $\begin{array}{c}\text { Buffalo } \\
\text { Creek } \\
\text { at } \\
\text { mouth, } \\
\text { Feb. 27, } \\
1005 .\end{array}$ & $\begin{array}{l}\text { Abram } \\
\text { Creek } \\
\text { at } \\
\text { mouth, } \\
\text { Feb. 27, } \\
1905 .\end{array}$ & $\begin{array}{c}\text { Savage } \\
\text { River, } \\
\text { Feb. 25, } \\
1905 .\end{array}$ & $\begin{array}{l}\text { Georges } \\
\text { Creek } \\
\text { at } \\
\text { mouth, } \\
\text { Feb. 25, } \\
1905 .\end{array}$ & $\begin{array}{c}\text { North } \\
\text { Branch } \\
\text { Potomac } \\
\text { River } \\
\text { above } \\
\text { Savage } \\
\text { River } \\
\text { Feb. 25, } \\
1905 .\end{array}$ & $\begin{array}{c}\text { North } \\
\text { Branch } \\
\text { Potomac } \\
\text { River } \\
\text { above } \\
\text { mill at } \\
\text { Luke, } \\
\text { Feb. 25, } \\
1905 .\end{array}$ \\
\hline \multirow[t]{2}{*}{ 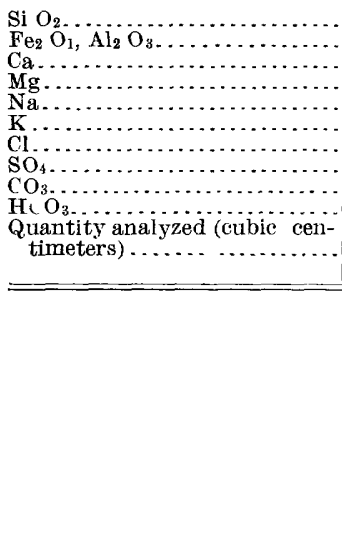 } & $\begin{array}{c}6.30 \\
5.20 \\
6.29 \\
1.33 \\
\text { Trace. } \\
\text { Trace. } \\
6.0 \\
95.69 \\
\text { None. } \\
33.3 \\
2,000\end{array}$ & $\begin{array}{c}6.10 \\
3.19 \\
8.21 \\
1.62 \\
5.22 \\
\text { Trace. } \\
9.0 \\
5.42 \\
\text { Non e. } \\
27.3 \\
2,000\end{array}$ & \begin{tabular}{|c|}
5.75 \\
4.40 \\
1.44 \\
Trace. \\
Trace. \\
5.0 \\
113.65 \\
None. \\
30.3 \\
2,000
\end{tabular} & $\begin{array}{c}9.46 \\
6.00 \\
9.65 \\
1.07 \\
\text { Trace. } \\
\text { Trace. } \\
5.0 \\
60.94 \\
\text { None. } \\
30.3 \\
1.500\end{array}$ & $\begin{array}{r}17.40 \\
127.60 \\
138.96 \\
33.66 \\
6.97 \\
\text { Trace. } \\
17.0 \\
577.08 \\
\text { None. } \\
\text { None. } \\
1,500\end{array}$ & $\begin{array}{r}4.95 \\
4.90 \\
10.00 \\
1.44 \\
\text { Trace. } \\
\text { None. } \\
11.0 \\
\text { None. } \\
36.3 \\
2,000\end{array}$ & $\begin{array}{r}9.55 \\
6.30 \\
9.15 \\
1.86 \\
\text { Trace. } \\
\text { Trace. } \\
15.0 \\
81.10 \\
\text { None. } \\
27.3 \\
2,000\end{array}$ \\
\hline & $\begin{array}{l}\text { North } \\
\text { Branch } \\
\text { Potomac } \\
\text { River } \\
\text { below } \\
\text { mill at } \\
\text { 5luke, } \\
\text { Feb. 25, } \\
1905 .\end{array}$ & $\begin{array}{c}\text { North } \\
\text { Branch } \\
\text { Potomac } \\
\text { River } \\
\text { between } \\
\text { Keyser } \\
\text { and } \\
\text { Pied- } \\
\text { mont, } \\
\text { Feb. 25, } \\
1905 .\end{array}$ & \begin{tabular}{|c|} 
North \\
Branch \\
Potomac \\
River \\
above \\
New \\
Creek, \\
Feb. 25, \\
1905.
\end{tabular} & $\begin{array}{c}\text { Wills } \\
\text { Creek } \\
\text { above } \\
\text { Kreig- } \\
\text { baum, } \\
\text { Dec. 29, } \\
1904 .\end{array}$ & $\begin{array}{l}\text { Jennings } \\
\text { Run at } \\
\text { mouth, } \\
\text { Dec. } 29 \text {, } \\
1904 .\end{array}$ & $\mid \begin{array}{c}\text { Braddock } \\
\text { Run at } \\
\text { Alle- } \\
\text { gheny } \\
\text { Grove, } \\
\text { Dec. } 29, \\
1904 .\end{array}$ & $\begin{array}{c}\text { Wills } \\
\text { Creek at } \\
\text { Cumber- } \\
\text { land, } \\
\text { Dec. 30, } \\
1904 .\end{array}$ \\
\hline \multirow[t]{2}{*}{ 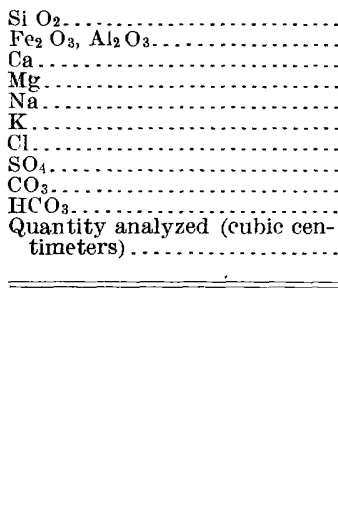 } & $\begin{array}{c}5.00 \\
78.92 \\
2.52 \\
1.75 \\
\cdots \\
75.0 \\
73.95 \\
\text { Nonp. } \\
78.7 \\
2,000\end{array}$ & $\begin{array}{c}6.06 \\
40.39 \\
8.45 \\
4.61 \\
4.12 \\
\text { Trace. } \\
10.0 \\
14.42 \\
\text { None. } \\
36.3\end{array}$ & $\begin{array}{c}5.09 \\
26.79 \\
40.02 \\
4.07 \\
\text { Trace. } \\
\text { Trace. } \\
11.0 \\
15.93 \\
11.92 \\
39.4\end{array}$ & $\begin{array}{r}4.67 \\
69.84 \\
22.24 \\
2.28 \\
6.59 \\
\text { Trace. } \\
10.0 \\
22.81 \\
\text { None. } \\
42.4 \\
\\
2,000\end{array}$ & $\begin{array}{c}11.1 \\
28.49 \\
85.49 \\
1.51 \\
.32 \\
1.77 \\
15.0 \\
274.44 \\
\text { None. } \\
9.1 \\
2,000\end{array}$ & $\begin{array}{r}5.13 \\
2.17 \\
24.88 \\
4.53 \\
\text { Trace. } \\
\text { Trace. } \\
9.0 \\
33.41 \\
\text { None. } \\
54.5 \\
\\
1,500\end{array}$ & $\begin{array}{r}5.70 \\
1.47 \\
40.18 \\
5.11 \\
5.89 \\
\text { Trace. } \\
12.0 \\
59.46 \\
\text { None. } \\
59.6 \\
7,000\end{array}$ \\
\hline & $\begin{array}{c}\text { North } \\
\text { Branch } \\
\text { Potomac } \\
\text { River } \\
\text { at Cum- } \\
\text { berland, } \\
\text { Dec. } 30 . \\
1904 .\end{array}$ & $\begin{array}{c}\text { Potomac } \\
\text { River } \\
\text { above } \\
\text { Pawpaw, } \\
\text { Feb. 10, } \\
1905 .\end{array}$ & $\begin{array}{c}\text { Potornac } \\
\text { River } \\
\text { below } \\
\text { Creat } \\
\text { Cacapon, } \\
\text { Feb. 12, } \\
1905 .\end{array}$ & $\begin{array}{l}\text { Potoniac } \\
\text { River } \\
\text { at } \\
\text { Opequon } \\
\text { Creek, } \\
\text { Feb 1\%, } \\
1905 .\end{array}$ & $\begin{array}{l}\text { Potomac } \\
\text { River } \\
\text { above } \\
\text { Harpers } \\
\text { Ferry, } \\
\text { July 4, } \\
1904 .\end{array}$ & $\begin{array}{l}\text { North } \\
\text { Fork of } \\
\text { Shenan- } \\
\text { doah } \\
\text { River } \\
\text { above } \\
\text { Passage } \\
\text { Creek, } \\
\text { Feb.2, } \\
1905 .\end{array}$ & $\begin{array}{l}\text { Passage } \\
\text { Creek at } \\
\text { mouth, } \\
\text { Feb. 2, } \\
1905 .\end{array}$ \\
\hline 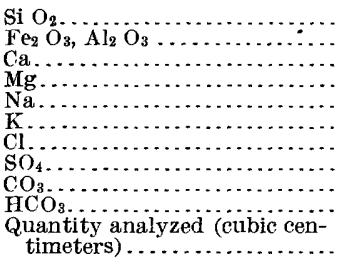 & $\begin{array}{c}7.31 \\
3.52 \\
38.04 \\
5.52 \\
2.97 \\
1.19 \\
5.0 \\
42.16 \\
\text { None. } \\
45.4 \\
4,500\end{array}$ & $\begin{array}{c}6.14 \\
7.20 \\
52.33 \\
4.92 \\
2.47 \\
\text { None. } \\
8.0 \\
140.54 \\
\text { None. } \\
215.0 \\
1,500\end{array}$ & $\begin{array}{c}8.63 \\
43.87 \\
3.01 \\
4.10 \\
1.94 \\
\text { None. } \\
4.0 \\
3.95 \\
\text { None. } \\
\text { 106.0 } \\
1,900\end{array}$ & $\begin{array}{r}7.47 \\
5.66 \\
47.76 \\
4.78 \\
1.17 \\
\text { Trace. } \\
11.5 \\
48.36 \\
\text { None. } \\
125.7 \\
3,000\end{array}$ & $\begin{array}{r}6.48 \\
32.75 \\
40.26 \\
3.95 \\
1.00 \\
\text { Trace. } \\
5.0 \\
18.37 \\
14.89 \\
42.39 \\
5,000\end{array}$ & $\begin{array}{r}6.66 \\
94.92 \\
.38 \\
9.90 \\
\text { None. } \\
\text { None. } \\
12.5 \\
4.71 \\
\text { None. } \\
202.9 \\
3,000\end{array}$ & $\begin{array}{r}9.56 \\
43.90 \\
1.99 \\
3.13 \\
2.11 \\
\text { Trace. } \\
10.0 \\
3.42 \\
\text { None. } \\
70.6 \\
2,500\end{array}$ \\
\hline
\end{tabular}


Mineral analyses of sùrface waters in Potomac basin-Continued.

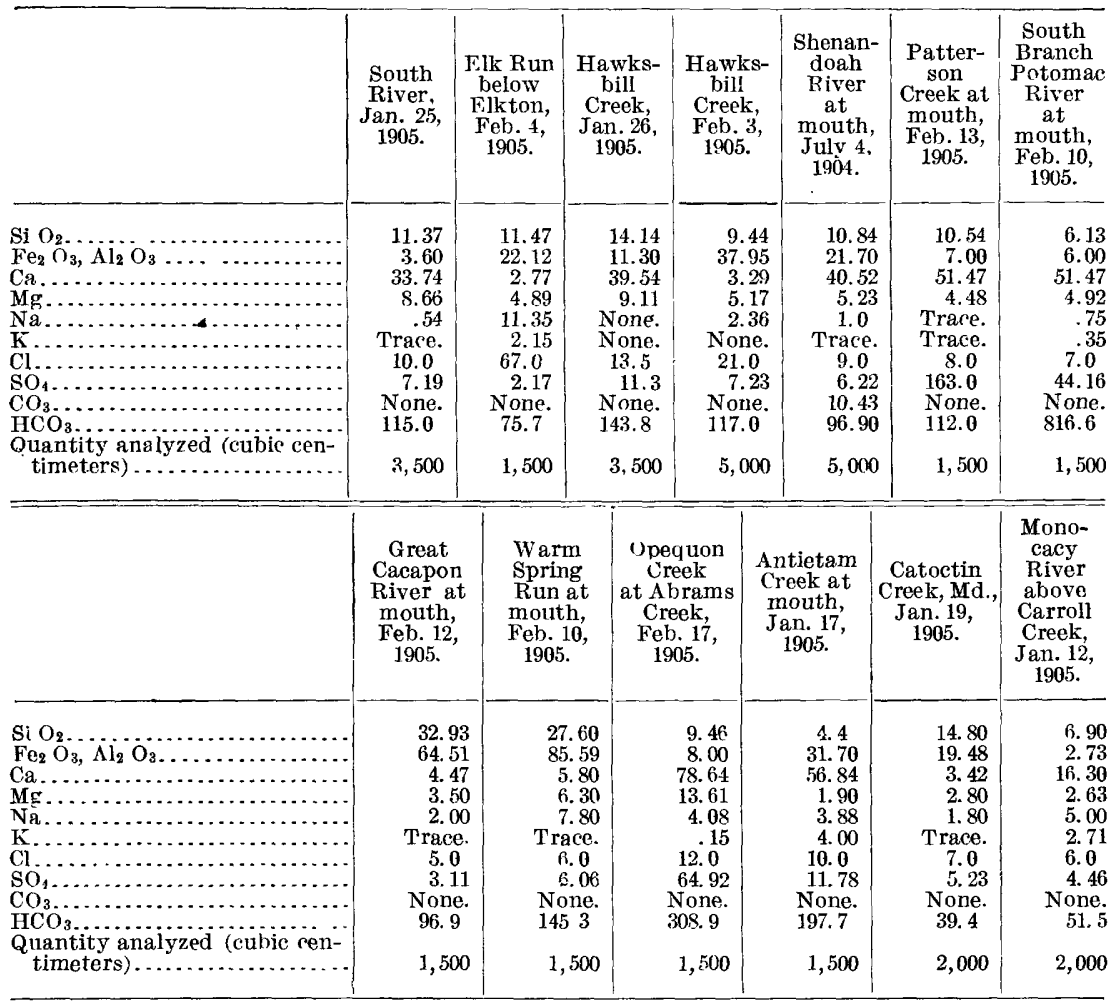

Monthly analyses of dissolved mineral matter in tap water at Washington, D. C.

[Parts per milion.]

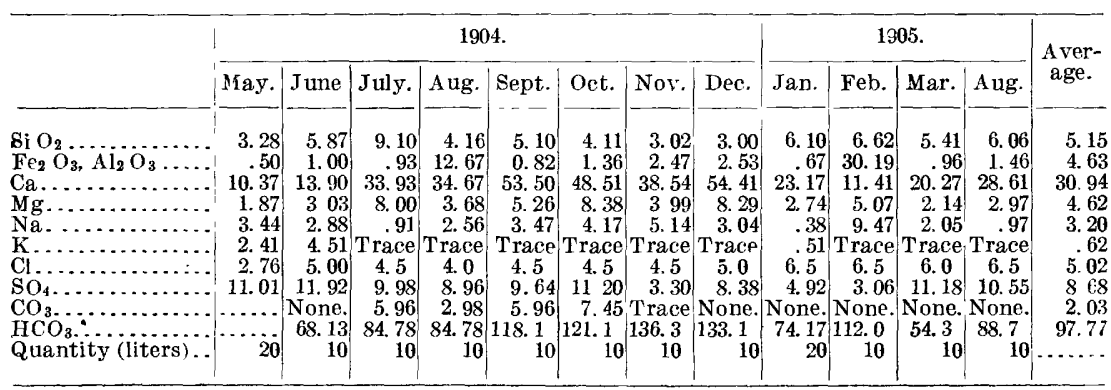


Determination of the different forms of carbon dioxide in the tap water at Washington, D.C.

\begin{tabular}{|c|c|c|c|c|c|}
\hline \multirow[b]{2}{*}{ Date. } & \multirow{2}{*}{$\begin{array}{c}\mathrm{CO}_{3} \\
\text { (parts } \\
\text { per } \\
\text { million). }\end{array}$} & \multirow{2}{*}{$\begin{array}{c}\mathrm{HCO}_{3} \\
\text { (parts } \\
\text { per } \\
\text { million). }\end{array}$} & \multicolumn{3}{|c|}{$\mathrm{CO}_{2}$ (cubic centimeter). } \\
\hline & & & $\begin{array}{l}\text { Bicar- } \\
\text { bonate. }\end{array}$ & Excess. & Free. \\
\hline 1904. & & & & & \\
\hline August $29 \ldots$. & 2.98 & 84.8 & 15.5 & 14.9 & None. \\
\hline September 17 . & 5.96. & 109.0 & 19.9 & 23.1 & 3.2 \\
\hline September 28 . & 5. 96 & 109.0 & 19.9 & 24. 2 & 4. 3 \\
\hline$\ldots \ldots \ldots \ldots \ldots \ldots \ldots \ldots \ldots$ & 5.96 & 121. 1 & 22.1 & 27.9 & 5.8 \\
\hline$\ldots \ldots \ldots \ldots \ldots \ldots \ldots \ldots$ & 5.96 & 130.2 & 23.8 & 27.9 & 4. 1 \\
\hline$\ldots \ldots \ldots \ldots \ldots \ldots$ & None. & 139.3 & 25.4 & 39.5 & 14. 1 \\
\hline$\ldots \ldots \ldots \ldots$ & None. & 142.3 & 25.9 & 42.3 & 16.4 \\
\hline . & None. & 142.0 & 26.0 & 32.0 & 6.0 \\
\hline December $31 \ldots . . .$. & None. & 109.0 & 19.9 & 26.7 & 6.8 \\
\hline January $5 \ldots$ & None. & 87.8 & 16.1 & 26.6 & 15. 1 \\
\hline January $14 \ldots$ & None. & 63.6 & 11. 5 & 16.6 & 5.1 \\
\hline 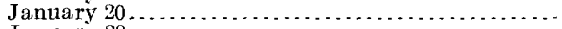 & None. & 69.6 & 12.7 & 7.0 & None. \\
\hline January $28 \ldots \ldots \ldots$ & None. & 75.7 & 13.9 & 3.0 & None \\
\hline February $4 \ldots$ & None. & 87.8 & 16.0 & 30.1 & 14.1 \\
\hline$\ldots \ldots \ldots \ldots \ldots \ldots \ldots \ldots$ & None. & 181.7 & 55.7 & 18.9 & None. \\
\hline 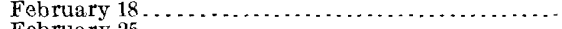 & None. & 105.9 & 19.3 & 22.2 & 2.9 \\
\hline February $25 \ldots \ldots \ldots$ & None. & 99.9 & 18. 2 & 18.9 & .7 \\
\hline
\end{tabular}




\title{
RELATION OF SOILS AND FOREST COVER TO QUALITY AND QUAN'TITY OF SURFACE WATER IN THE PO'TOMAC BASIN.
}

\author{
By W. W. Ashe. \\ EFFECT OF SOILS ON TURBIDITY OF WATER. \\ GENERAL DISCUSSION.
}

The turbidity or muddiness of the Potomac River water, which to the majority of the users is considered its most objectionable quality, is derived from no one section of the basin and from no single geologic formation or type of soil, and comes in part from land of gentle gradient and in part from sections where the topographic features are stronger. The farming land furnishes the largest amount, but small amounts are due to the wash from woodland on certain types of soil. Certain parts are washed from roads, and some comes from the cutting away of their banks by streams during freshets. The turbidity during freshets is unduly increased above the normal by the fact that the more rapid current again takes up the sediment deposited as silt beds and sand bars at points of slack water during periods of slower flow, and by the additional fact that on very few of the tributaries are there flood plains to form natural settling basins for some of the heaviest silt.

Turbidity of the Potomac is not a recent phenomenon. The silt and clay which the older geologic formations west of the Blue Ridge have contributed to the building of the Coastal Plain were washed in muddy streams from their valleys of shale and limestone. The Piedmont region has also contributed its share. The deep gorges and ravines which, starting at the river, ramify through the soft schists, shales, and sandstones that form this portion of the Piedmont region, indicate that natural erosion of the friable soils has been proceeding at a rapid rate.

The fertile red soils are not entirely responsible for the turbidity; the broken topography, the long, warm summer, and heavy intermittent rainfall are also active factors, and when these occur together turbidity is an inalienable accompaniment of a rapid stream. 
In a humid climate farther north the growing season is short, the nitrifying and oxidizing capacity of the soil lower; humus rapidly accumulates, both in woodland and in tilled land; the porosity of the soil is maintained, and granulation, even in a heavy soil, seems to be almost a natural condition. The heavy humus content, with the concomitant porosity, whether in a forest soil or in tilled land, promotes absorption and retention of rainfall and minimizes erosion.

Similarly, sod, which is maintained with difficulty in the desiccating autumn climate of the South, naturally sets in a cooler climate in ditches, on stream banks, and in waste places, and forms nearly as perfect a protection against erosion as a forest cover. In those sections where thick sod does not form, a forest cover is the best protection against erosion on steep land.

The conditions which surround the upper headwaters of North Branch of Potomac River above Cumberland are largely those which determine the clearness of northern streams; and until it reaches Cumberland it is a clear stream. Below Cumberland there is a rapid change; both the soil and climatic conditions become more favorable for increased muddiness, reaching the optimum conditions just above Washington and in the Shenandoah and Cumberland valkeys.

The present turbidity, however, is excessive. The washed-out beds of the smaller streams, extending in places from hill to hill without banks, and the many-gullied and thin-soiled slopes all indicate that erosion is taking place now more rapidly than formerly and that the turbidity is greater. Its further increase from certain sources can be checked, and it can undoubtedly be considerably lessened from other sources, to the general benefit of the valleys, as well as to the improvement of the potability of the water.

While the turbidity is from many different sources, the greater part of it is from the wash from steep or badly tilled cleared land. Where it is the fault of the manner of tillage, more rational cultural methods can eliminate or reduce it. Where it is from the erosion of steep land and can not be prevented by better methods of culture, and it is evident that the amount and rapidity of the erosion are such as to jeopardize the future earning power of the land, this land and other areas of the same character that are yet in timber should be regarded as forest land and nonagricultural. Far higher and more potent reasons than the clarification of the water demand the withdrawal of the land from a use which means its ultimate loss of earning power, to be preserved by applying it to a different use as an active factor in the nation's future wealth. 


\section{SOILS EAST OF THE ALLEGHENY FRONT.}

SOIL FORMATIONS.

The important soil formations ${ }^{a}$ of the Potomac River basin, east of the Allegheny Mountains, are the Cecil soils, marked by stiff, heary, usually red subsoils, heterogeneous in texture, underlying more friable and looser surface soils; the Chester series, until recently ${ }^{b}$ included in the Cecil, distinguished by lighter, less coherent, and usually more micaceous soils, which are more subject to erosion than either the Cecil or Penn soils, represented on the Potomac watershed by only two soil types, the Chester mica loam and Chester loam; the Penn series, dark-red soils and subsoils, more homogeneous in texture than the Cecil soils and resembling them in cohesion, but far less friable than the Chester soils; the Hagerstown series, generally heavy limestone soils; the yellow shale soils, compact yellow or reddish clays or leachy gravel; the Upshur series, dark-red shallow loams and sandy loams, and the Dekalb series, gray somewhat sandy soils, usually shallow, stony, and coarse grained.

The first five are, as a rule, valley types, and when not on steep slopes are generally farmed. The Upshur soils are partly cleared, but are little farmed. The Dekalb series are largely mountain types. They are but little cleared and where cleared are largely in grass.

The Cecil and Penn soils are largely drained by the streams east of the Blue Ridge, only small areas of these types lying on its western side. The other soils very largely lie to the west of the Blue Ridge: The Cecil and Chester soils are responsible for most of the turbidity of the Piedmont streams of the South Atlantic States.

The streams east of the Blue Ridge contribute very largely to the turbidity of the Potomac, and it is probable that they add a large, if not the largest, part of the coarser silt that is brought down during seasons of medium heavy rain, with $1 \frac{1}{2}$ to 2 inches of rainfall distributed over a period of twenty-four to thirty-six hours. The prevailing soil types are of the Penn and Cecil series, which are usually of sufficient depth for cultivation, but in many of their phases loose and incoherent, and, if denuded, eroding under moderate rains. Their tendency to wash is increased, especially near the river, by their situation on steep slopes.

\section{CECIL AND CHESTER SOILS.}

Cecil silt loam is a gray soil of fine texture derived from the decay of partly metamorphosed sandy shales. On surfaces which are at all steep it erodes badly, forming deep gullies down to the undecom-

$a$ The classification and nomenclature of soils used in this paper are those adopted by the Bureau of of Soils, Department of Agriculture. In no sense are they geologic, and the names should not be confused with the names of geologic formations.

$b$ Soil Survey Field Book, U. S. Department of Agriculture, 1906, p. 108. 
posed rock from which it was formed, and entirely destroying the value of the land for farming purposes. Its composition is such that its washings add a very fine and undesirable matter to the water. The mechanical analyses ${ }^{a}$ of this soil made by the Bureau of Soils from a sample 3 miles east of Leesburg, Va., give it the following percentage of silt and clay: Silt $(0.05$ to $0.005 \mathrm{~mm}$.), 60.06 per cent; clay ( 0.005 to $0.001 \mathrm{~mm}$.), 22.80 per cent.

Most of the areas of this soil on the basin are badly situated to prevent washing. Some of the largest are on the south side of the river at the mouth of Goose Creek, where the rough topographythe river hills being steep and 200 or more feet high-is unfavorable for clean tillage. Other areas lie on the steep upper slopes of Broad Run and below that stream, all close to the river, and frequently showing, in spite of attempts at careful cultivation, gullying and washing. The agricultural limits of the soil are partly recognized, and less than one-half of these areas are cleared. It does not hold a grazing sod, and when the slope becomes at all steep it ceases to be an agricultural soil and its earning capacity in such situations can be perpetuated only by retaining it in timber. The forests are largely of black oak, chestnut oak, scarlet oak, and pitch pine. Its situation so close to the river and the relatively short distance above the intake of the Washington water supply render the protection of this soil from further erosion important. The humus formed is not deep except in the bottom of the deep hollows, and in spite of it some erosion takes place, the run-off from wooded areas of this soil being slightly turbid.

The Chester mica loam is a loose, incoherent, red soil, derived from mica schist. On steep surfaces it washes badly and considerable soil transportation habitually takes place. On account of its composition, 25 per cent being clay and about 30 per cent fine silt, such washing adds an objectionable element to the water. This soil occurs in small areas along the eastern base of the Catoctin Mountains, in Virginia; in larger areas in the eastern part of Frederick County, Md., on Monocacy River; in Montgomery County, Md., on the upper waters of Seneca Creek; and in the lower portion of Fairfax County, Va. On Seneca Creek it is extensively farmed and contributes much to the objectionable turbidity of that stream. Where level and undulating it does not erode so rapidly, but on steep slopes a great deal of badly gullied land can be seen, and in such situations it ceases to be a farming soil and its preservation is possible only by keeping it in forest. It is not a good grazing soil, becoming too dry in the autumn. The characteristic forest growth on this soil is composed of chestnut and - chestnut oak on the better phases, and scarlet oak and pitch or Jersey 
pine on the drier portions, usually with an underwood of mountain laurel. The humus varies from good to thin. When this soil is in forest there is little washing. A large area of this type has been cleared, and much of it has deteriorated appreciably. The roads on this soil wash very badly, often being several feet below the surface level. The beds of streams also are much washed, and they are often without banks; turbidity comes from both sources.

The Cecil clay loam is a dark-red soil having an extensive distribution on Monocacy River in Adams County, Pa., and Frederick County, Md., and is extensively cleared and used for farming. Soil transportation steadily takes place, and while gullies are not formed, there is a constant and uniform removal of soil from even moderate slopes. Fortunately, it is in few places deeply dissected, and careful methods of tillage can do much to reduce turbidity from these soils. It grasses only moderately well. The remaining forest is of oak and hickory and forms a sufficient humus to insure protection.

The Chester loam and Cecil clay form large areas on Monocacy River in Carroll and Frederick counties, Md., on Seneca Creek in Montgomery County, Md., and on Broad Run and other small streams in Loudoun and Fairfax counties, Va., between Broad Run and Great Falls. They also form extensive areas in upper Loudoun County and in western Frederick County, on the two Catoctin creeks and at the head of Goose Creek. These soils are derived largely from the Catoctin schist and granite and from other mica schists. They are light brown or red in color, deep, and rather porous, but the lighter phases are often incoherent and when dry much resemble the mica loams in texture and behavior toward water. While they form large areas of productive and well-farmed land, considerable washing takes place, especially in the spring, when raw-plowed surfaces, already saturated, are exposed to heavy rains, and this washing increases with the gradient. The surface of these soils, especially near the mountains and along the river, is deeply carved into high, rolling-topped hills, the stream valleys in many places being 200 to 300 feet deep. Only an insignificant portion of these soils remains uncleared, and while they generally grass well, there is on steep land considerable gullying. In Virginia, except on Catoctin Creek, these lands are mostly held in large estates and are used for grazing, some of the sod not having been turned for sixty years. In Maryland they are divided into small farms and tillage is the rule. Roads wash badly on these soils, and the beds of the smaller streams, especially on the Maryland areas, are badly eroded and much enlarged, the banks being steep, naked, and cut back to the base of the hill on either side. On account of the fertility of these soils, clearings have been extended to slopes which are much too steep even for grazing, 
and several thousand acres of such slopes lying in deep hollows can have the soil retained only by reforesting. The mechanical analyses of the Bureau of Soils show that from 50 to 70 per cent of these soils consist of clay and fine silt. On account of their extensive area and situation on steep slopes close to the river and only a short distance above the Great Falls intake, they contribute much to the turbidity of the Washington water. The forests are of oak and hickory on the heavier phases; chestnut on the lighter. Humus on both types is good, and where forested there is no appreciable erosion, except on the steepest slopes during very heavy rains.

\section{PENN SOILS.}

The Penn soils cover extensive areas in the valley of Monocacy River and also on the lower part of Goose Creek. The heavier members have usually a rolling surface, and there is sufficient cohesion to prevent excessive erosion, which is also reduced by the fact that the soils grass very well and are excellently tilled. Many of the areas lie close to the larger streams, and some steep banks have been cleared which would have held better in timber. Roads wash somewhat; stream beds only slightly. These soils are largely cleared. The forests were of oak, hickory, walnut, and ash.

The Penn shale loam, on the other hand, which is a dark-red soil, extending southwest from Gettysburg, $\mathrm{Pa}$., in a broad belt, undergoes continuqus erosion. A great portion of it is gently rolling, but near the larger streams it is much broken, and since it does not grass well much erosion takes place from cultivated land in such situations. Erosion is usually in the manner of uniform soil transportation, resulting in the gradual thinning of the entire slope and not in the formation of gullies. Some of the cleared slopes are too steep to justify tillage. This soil has from 50 to 80 per cent of fine silt and clay, and undoubtedly the steeper gradients contribute to the turbidity of the Potomac. The smaller stream valleys are shallow and broad, and there is little erosion from them. Pitch and Jersey pines, with scarlet, black, and chestnut oaks, and in some places chestnut, form the forest. There is considerable old field pine. This type of forest forms only a thin humus, but there seems to be very little washing from beneath it. The greater part of this soil is cleared.

LIMESTONE SOILS.

The limestone soils of the Hagerstown series of the classification of the Bureau of Soils constitute the most sought for, best-farmed, and highest-priced soils of the Potomac basin. Together with some calcareous and argillaceous shale soils they form more than threefourths of the cleared land in the valley of Virginia and the Cumber- 
land Valley. The Hagerstown clay is a residual soil from the weathering of the purer limestones, and represents the less soluble portion of the rock. The loamy and sandy phases are from limestone that is less pure and has more quartz veins in it, and more and coarser insoluble residue has been left to form the soil; or they are derived from shales. On gentle slopes there is very little erosion of these soils. There is more, however, on the heavy types, where the compact texture of the clay greatly impedes absorption, than on the looser, more porous types. On steeper slopes there is, at least on the heavier soils, continual though often slight transportation, which reaches its minimum when the soil is in permanent sod or in timber. On account of the fertility of these soils, and especially their high productivity in wheat, they are in constant cultivation under a short rotation, including both corn and wheat as well as hay.

While the gradients are usually slight on these soils in the Cumberland Valley, in the valley of Virginia, and in the smaller areas on Monocacy River in Adams County, Pa., and Frederick County, Md., yet there are local areas, especially contiguous to the larger streams, where the slopes are very steep, and many badly eroded fields are to be seen in such places. Even when deep plowing and careful tillage has prevented excessive gullying on steep slopes, rapid soil transportation takes place, though it is largely distributed over the entire surface, and the soil after each heavy rain is left thimner than before, and this unchecked waste portends its ultimate depauperation. $^{a}$ In fact, this has already taken place on extensive areas of limestone soil, which are closely similar to the heavier types of the Hagerstown soils. These are limestone soils in the Martin Mountains in Bedford county, Pa., in the Patterson Creek Mountains in Grant and Mineral counties, W. Va., in a portion of the Tonoloway Ridge in Hampshire County, W. Va., in the elevated valleys of the Knobly Mountains, and in the high steep limestone slopes which lie just beneath the crest of North Fork Mountain and in nearly similar situations on South Fork Mountain just east of -Franklin, Pendleton County, W. Va. There are, moreover, many fields in each of the soil areas just mentioned so badly gullied that grassing has become impossible. The soils have become so shallow, and transportation of soil from the raw surfaces takes place so constantly, that the blue-grass sod, which is the natural covering of these lands, can not spread and hold, and reforestation will be the only means of again giving them a permanent earning power. The difficulty with soils of this character is that they can not be manured, on account of the impossibility of getting a wagon and team on the steep

\footnotetext{
$a$ In the southern Piedmont region such long-continued partial erosion gives rise to sandy surface soils underlain by heavy subsoils.
} 
slopes, and every crop of grain or grass which is removed robs them of fertility, while nothing except mineral fertilizers of the acid-phosphate type are added to them in compensation. When these soils are kept in grass there is very little washing, but on account of their productivity, as compared with either the shale soil of the valleys or the surrounding thin soils of the sandstones, their cultivation by the small landholder is a necessity until their final ruin precludes cultivation of any kind.

The well-known case of the soil-denuded limestones of the Alps of Bosnia and southern Austria, the Karst region, is being reproduced here under almost the same conditions. Both are regions of steep slopes and naturally rather shallow but very fertile limestone soils, which have by the exhaustion of humus through constant tillage lost their granulation and had their absorption capacity for rainfall so reduced that extensive washing has taken place, leading to the destruction of the agricultural value of the soils. The highest earning power which such soils now have is in timber production. Their natural forests are of hickory, white oak, walnut, locust, red oak, and, on dry knolls, black oak, and since all these except the black oak yield high-priced woods, and rapid growth is made by timber on the limestome, reforestation could be regarded as a financially profitable undertaking. When forested there is a heavy humus on even the steepest limestone land and a heavy undergrowth of weeds, bushes, and vines. The thick humas not only holds a great deal of water, but the heavy clays of the limestone have a.higher capacity for absorption than any other soil on the Potomac, and the humus allows those soils to become fully saturated. On account of the heavy humus there is almost no turbid run-off from even the steepest limestone soils when in timber. In addition to the areas already mentioned, other heavy limestone soils cover several thousand acres in Highland County, Va., between Monterey and Hightown, and areas of less extent in the "Hunting Ground" in Pendleton County, W. Va., on the upper waters of North Fork of the Potomac; on Evitts Creek, Bedford County, Pa., in the Little Cumberland Valley; and in the Cove Valley about McConnellsburg, Fulton County, Pa. In these areas the topography is rolling or the hills have long, moderately gentle slopes, except along the larger streams, where the slopes are steeper, as they are in the valley of Virginia, and erosion takes place in the same manner and under the same conditions as on the Hagerstown soils farther east. The soils are worked in short rotations, more than two-thirds of the land being habitually in tillage and the rest in grass.

Mechanical analyses ${ }^{a}$ of the Hagerstown clay, which will cover the composition of the heaviest limestone soils in the Potomac basin, 
show it to contain from 34 to 38 . per cent of clay less than 0.005 $\mathrm{mm}$. in size, and from 40 to 46 per cent of fine silt between 0.05 and $0.005 \mathrm{~mm}$. More than three-fourths of its composition is matter sufficiently divided to be transported on the most quietly moving portions of Potomac River, and practically none of the wash from these lands settles above the Great Falls intake.

The more sandy members of the calcareous soils show a lower percentage of clay content and a higher of silt, the clay forming from 14 to 36 per cent, ${ }^{a}$ while the silt forms from 31 to 50 per cent. While the relative proportions of the light and heavy soils are not known, at least one-half of the limestone soils are referable to the heavy. members. The above analyses of texture are of the top soils. On the eroded land on the mountains, where gullying is taking place, the material is largely from the subsoil, and this shows a far higher proportion of fine, transportable clay, the amount being from 50 to 63 per cent ${ }^{a}$ of the entire soil. The proportion of fine silt, however, is slightly smaller.

So great is the erosion from steep limestone lands that during heary summer storms the turbid streams which bear their wash strongly exhale the argillaceous odor characteristic of a freshly cut surface of heavy clay. This is especially noticeable with the water of Town Creek, which bears the wash from Martin Mountain; Big Cove Creek, which has the wash from the limestones in the vicinity of McConnellsburg; Conococheague Creek, which drains the Cumberland Valley; and the two branches of Shenandoah River, which drain the valley of Virginia and the Page $\mathrm{Nalley}$; and there are other streams in which it is probably at times as noticeable as in these.

In addition to the wash from the agricultural lands a small amount takes place from the roads, but most of the roads through the limestone are surfaced with crushed stone and have been graded or washed to a level before being surfaced.

Near the banks of streams the limestone soils grass well, and there is very slight erosion of banks on any of the smaller streams.

It is evident, on account of the constant erosion that takes place on the limestone soils, which rapidly increases with the gradient, and the large proportions of transportable clay that these soils contain, that they contribute a large proportion of the turbidity to the river, probably even a larger proportion than the soils of the Cecil and Chester groups; but with this difference in respect to the Washington water supply, that local showers on the Cecil soils, on account of their nearness to the Washington intake, and especially rains on the lower part of Monocacy River and on Seneca Creek, which empty into the Potomac on the same side on which the intake is situated, produce high 
turbidity for short periods, especially during summer thunderstorms. The turbidity produced by similar storms higher up on the watershed is largely reduced by dilution, opportunity being given for thorough mixing with the water across the entire channel of the river, and also by distribution, a portion being carried far ahead in the main current of the river, while those parts which mixed with the water near the bank, where friction is greatest, get farther and farther behind. The total effect on the water by the time the intake is reached is a slight rise in turbidity, which is distributed, however, over a day's flow or more. Such local rains on the Cecil soils in Maryland, as has been explained, cause high turbidity along the north bank, and there is neither time for further dilution nor opportunity for distribution. The rapidity and unexpectedness with which this condition is produced occasionally cause a considerable amount of highly turbid water to enter the reservoirs which supply Washington.

About one-tenth of the mountain land between the Allegheny Front and Shenandoah Mountain is limestone or limestone shales. The largest part of the land lies in Highland County, Va., and Pendleton, Grant, Hardy, and Mineral counties, W. Va. The average slope of this land is more than 1 foot in 5 . Probably one-half of it is at present cleared, and the rest is being cleared rapidly. The limestone soils, including both the valley and mountain lands, constitute about onesixth of the total area of the Potomac River basin, or about 2,000 square miles.

There are very few springs on the limestone soils, in spite of their very great water-storage capacity, butsuch as there are show great constancy and boldness. Many of them are undoubtedly streams which permeate fissures or caverns in the limestone.

SHALE SOILS.

Shale soils are of two different series-those derived from massive or fissile argillaceous shales, known throughout the basin as "yellowslate soils,"'largely the product of weathering of the Romney shale and the shales of the Jennings formation; and those derived from the weathering of the sandy shales and sandstone of the Hampshire formation, which are known as "red-slate soils." The yellow-slate soils are soft, erode rapidly, and constitute essentially a valley type of soil; the red-slate soils form plateaus and low mountains.

The yellow-shale soils are extensively distributed, and some of their phases are the source of considerable turbidity. The first of the two extreme types is the typical "slate soil," largely formed of small, sharp-angled pieces of argillaceous shale. Occasionally it is called gravelly soil. Only a small amount of fine earth is associated with it. It is extremely porous and leachy, and transportation takes place so 
rapidly that very little finely divided earth accumulates. It is essentially a thin, light soil, and its condition is due not only to the fact that the shale decomposes slowly, but also to the erosion of the finer material proceeding practically at the same rate at which it is formed, and to its situation on steep slopes high above the base-level of erosion; or it is in places the wornout shale clay of the type described in the next paragraph, from which the fine soil has been removed by washing when cultivated.

The second type embraces yellow-shale clay and its loamy phases. It is the residual product from the weathering of the argillaceous and moderately sandy shales, where there has been but little soil transportation. On broad flats near the heads of streams and where the valley hills have been eroded nearly to stream level, soil has accumulated to the depth of several feet, but as the slope becomes steeper the soil rapidly becomes shallower and "slate" chips more abundant, a rapid transition taking place with increased slope into the "slate-soil" type. These soils do not hold grass well except at high elevations, and constant tillage rapidly changes their character and lessens their earning value, as the fine soil is eroded. Above 1,500 feet altitude and in cool situations along moist slopes sheltered in part from the heat of the sun, especially on the heavier and more calcareous shales of the Rockwood formation, and some in the Romney formation, a permanent grazing sod can be maintained. In other situations the grass dies in a few years and a scant growth of pennyroyal and spring and early summer weeds takes its place. When the shale soils are on high slopes the characteristic topographic features are the small size of the hills and the number and depth of the fissures which indent them, the surface features being in strong contrast to the rolling summits and broad, flat slopes, even where steep, which mark the limestone. This broken surface of the shale hills is another drawback to general farming on them, making the fields small and their cultivation difficult, and causing some inequality in the maturing of the crops. A crop makes on the typical leachy slate soil only when there is a wet growing season, and for this reason wheat, growing largely during the wet spring, is more successfully raised on it than corn. The soils are not, however, adapted to farming, and large areas which have been cleared are turned out nominally as pasture, but in fact are practically waste. Where the soils are deep and the slopes gentle the soils are very productive in corn, hay, small grain, and apples.

Mechanical analyses of these soils show that the earthy portion includes from 22 to 40 per cent of clay. They are responsible for a considerable portion of the turbidity of South Branch of the Potomac, and of Patterson, Back, Sleepy, Sideling Hill, Great Tonoloway, and Licking creeks. While a large part of the wash comes from cleared land, the washing of the banks of small streams, especially when the 
trees and bushes have been entirely cut off, is a notable feature. Some also comes from roads, which are inclined to wash, especially on the compact clay soils. A small amount comes from the woodland, as the streams which drain forested watersheds becomes conspicuously clouded during heavy and especially prolonged rains.

The forests on the shale soils vary with the drainage of the soil. Where the topography is gentle and the clay soil is deep, white oak, a small amount of chestnut oak, and in the hollows red oak form the timber, the white oak always being the aggressive species. With increased dryness, due either to the thinness of the decomposed clay soil or to greater inclination of slope, Jersey pine and chestnut oak become the dominant species, white oak being of minor importance. A moderate humus accumulates under the red and white oaks, but elsewhere the humus is very light on this soil. The springs in the shales are numerous, but small and irregular in their flow, many of them going dry during the summer and autumn. Probably one-half of the total area of shale soils has been cleared, but a large part of it is idle. On steep slopes and in its leachy phases the shale soils are not agricultural, and while they have a low earning power in forest they are best preserved by being wooded. On gentle slopes the shale soils can be made highly productive. A feature about them is the rapidity with which the bits of shale disintegrate when exposed to the weather; for this reason these soils can not be completely exhausted.

The red shales and sandstones of the Hampshire formation west of Shenandoah Mountain yield soils which are referred by the Bureau of Soils to the Upshur series. On the Potomac watershed the country rock of this formation is largely a sandy dark-red shale. It occurs usually in elevated valleys, as Long Hollow, in Washington County, Md., or in broken plateaus, as the "Levels," in Hampshire County, W. Va., and Timber Ridge, in Fulton County, Pa.; or it forms low, flat-topped mountains, as Town Hill and the foothills of Sideling Hill, in West Virginia, Maryland, and Pennsylvania. The larger streams have in most places cut deep gorges through it, with steep, precipitous sides. The gently rolling surface, which is generally farmed, is in sharp contrast with the much broken surface of the yellow shales, and when the two occur side by side the red shale is usually at a higher elevation. The soils derived from it east of the Allegheny Front are usually loams and sandy loams, in few places passing into stiff phases; they are shallow, rarely more than 2 feet deep, with much broken shale and bits of sandstone disseminated through them, and dark-red or chocolate in color. The porosity is excellent and, the underlying shale being much fissured, there is comparatively little erosion except on steep slopes, where considerable sand and even coarse gravel is transported. The sandy members are too light, thin, and leachy for successful production of corn and wheat, the staple crops of the region, 
and large areas which have been cleared, amounting probably to more than 10,000 acres, have been abandoned and are offered for sale at prices between $\$ 5$ and $\$ 8$ an acre. Other extensive areas, which are held in connection with operated farms as pasture land, are practically waste. The lighter phases will not hold a grazing sod, and hay grass lasts only a season or two on it. Except on the heavier phases or the most gentle slopes, as about Three Churches and the middle portion of Timber Ridge, this soil yields, if worked in grain and grass, only the scantiest living to the tiller. Under the current system of tillage' it is not a productive general farming soil. Recently, however, extensive areas of this soil on the "Levels" and elsewhere have been planted in peaches, to which it is well adapted. The soil can be used profitably for this purpose and similar intensive farming, to which it seems to be adapted, only where transportation is available, which excludes at present the largest areas. It is, when shallow and on steep slopes, a forest soil and must ultimately be regarded as such unless planted in peaches, small fruit, or truck. More than one-half of this soil is yet in woodland. As would be expected from its lightness, the timber is neither tall nor the stand heavy. On the heavier members shortleaf pine, black oak, and pigmut hickory form the growth, which changes to scarlet oak and pitch pine as the soil becomes more sandy and shallower. The humus is light.

Not so much turbidity originates from these soils as would be supposed from their red color, although the run-off from tilled land is always muddy. The waters which come from wooded areas, however, seem always to be clear.

SANDSTONE SOILS.

Sandstones form the greater part of the mountains, the limestones and smaller areas of shale and granite forming the rest; and since the sandstone yields a sandy soil, this is the prevailing and characteristic mountain type. The soils of the sandstone, forming the sandy members of the Dekalb series, are all very sandy, some very shallow, and most of them stony, their final form being determined by the hardness of the rock from which they originated and the steepness of the slope on which they are formed.

The deepest sandy soils and those best suited to tillage come from the "Monterey sandstone" and the sandy beds of the Jennings and Hampshire formations. These are loams and sandy loams, and while they are deep in but few places they are usually found at the higher elevations and are well supplied with organic matter. Even in the case of some of these formations which rapidly decompose, there is, where they lie on steep slopes, constant soil transportation, and the residual soil is shallow. On account of this constant loss there 
is around the base of many of the sandstone mountains a heavy talus of sand washed from the heights, forming a mantle over the valley formations of shale and limestone. This is especially noticeable in the Cumberland Valley, in the valley of Virginia, and, to a less extent, elsewhere.

The more indurated sandstones and the quartzite yield only the thinnest kinds of mountain soils, which are strewn with large and angular fragments of stone. Such stony land is suitable only for forest growth, and no attempt has yet been made to clear it. At least one-half of the mountain land east of the Allegheny Mountains is of this character. The sandy soils of the valleys, as well as those of the level benches of the mountains, except where light and leachy, are farming and fruit-raising soils. Considerable areas of sandy land on the mountains, with soil of some depth, were cleared originally for grass land. It is well suited for sod land, and so long as the turf is not turned it holds very well, its porosity being sufficient to absorb heavy rains readily without washing. When cultivated, especially in corn, it washes very badly; less so when in oats and buckwheat.

While these soils contribute very little to the turbidity of the river, their preservation on the mountains is necessary on other grounds. They and the underlying sandstone, which is in most places well fissured, form the natural reservoirs at the head of nearly all the streams of the Potomac basin. The springs from the sandstone are numerous and constant in their flow, though few of them have the volume and boldness of the limestone springs.

The forests on the sandy soils vary widely in composition and quality, according to the depth of the soil, its drainage, and the aspect. Where the soil is deep and mellow, especially at high elevations or on cool slopes, chestnut and red oak are the characteristic trees, and the humus naturally formed is excellent. In somewhat drier situations white oak is the dominant tree, chestnut oak also becoming abundant, the proportion of the latter increasing with better drainage. The humus becomes thinner as the amount of chestnut oak increases. On cool western slopes, especially in elevated valleys, white pine was a factor in the original growth, but very few trees are now seen, even of young growth. On ridges, especially those that are warm and sunny, pitch and table-mountain pine form groves in many places, almost unmixed with other trees, and in such groves the humus is very thin and poor.

\section{SOILS WEST OF THE ALLEGHENY FRONT.}

The soils of the Allegheny Mountains are loams and sandy loams from conglomerates, sandstone, and sandy shales, with smaller areas of clay from more argillaceous shalès and from limestone. They are generally gray or yellow, in a few places red or brown, and for the most 
part are stony. Large areas are so thin soiled and stony as to be entirely worthless for agriculture of any kind. These include especially the soils from the harder conglomerates and sandstone of the Blackwater and Pocono formations and the sandstone of the Bayard formation. These are extensively developed on Spruce Mountain, along Backbone, Savage, and Dans mountains, and on Stony River, where many acres at some places are covered with large angular stones, such places being locally known as rock bars.

The humus content of the soil is large for the several reasons which have been pointed out, and their absorptive capacity for water is high. The humus, as well as the general looseness, permits rapid percolation of water through the soil to the subsoil. Unless subject to clean tillage on the slopes there is very little erosion of cleared land. Fields were seen, however, on Wills Creek and on Georges Creek, which from the cultivation of corn or potatoes had washed badly and are now too thin to form a good sod. On the slopes of Red Oak Mountain also, and along the Allegheny Mountain farther north, the grazing land on the steep southern slopes has become thin and the sod is scant. Some of the clearings, however, are old, situated along the early pikes and trails which crossed the Allegheny Mountains to the fertile lands of the Ohio Valley. They clearly indicate, though, that much of this land which grows well when fresh gradually becomes exhausted even in sod on the steep slopes and must eventually again be timbered to hold the soil.

Very little turbidity comes from North Branch of Potomac River above Cumberland, and the same is true of the headwaters of Wills Creek, in Somerset County, Pa., and of Seneca Creek, Laurel Fork, and the intermediate streams in Pendleton County, W. Va. Only the heaviest sunmer rains, or spring rains after the loosening of fallplowed ground by frost, cause evident washing of farmed land, except on the steepest slopes. In spite of the fact that so large a part of the cleared land is in sod, the number and constancy of the springs are noteworthy.

The forests in the Alleghenies vary widely in composition; chestnut, red oak, and chestnut oak are the prevailing species in the warmer and drier situations. The chestnut forms a good humus, and the quantity of humus is largely determined by the proportion of this tree.

On northern slopes and in the moister situations birch, sugar maple, basswood, and beech form most of the timber, while hemlock occurs with these in many of the ravines. This type of forest occurs in many places on very rough and stony land. The humus is very deep, having accumulated locally to a depth of 8 to 10 inches. Above 4,000 feet elevation, on the headwaters of Stony River, Difficult Creek, and Buffalo Creek, in Grant County, and on Seneca Creek and Laurel Fork, in Pendleton County, W. Va., the forests are largely of spruce. 
The soil is of the thinnest character, much of it only beds of stone. In forest which has been unlumbered or unburnt the moss and humus are deep and largely replace the soil for supplying the necessary moisture. There is no discernible erosion of the forest soils where the humus has not been destroyed or lessened. The smaller streams are at times discolored by the leaching of the humus, but to an insignificant degree.

More than one-half of the area of the Allegheny Mountains is forest covered, including nearly all of the steepest slopes.

It is evident that the soils which most largely contribute to the turbidity of Potomac River are those that are most valuable for farming, which are largely cleared. Considerable forest, however, is yet situated on the steeper slopes of some of these soils, especially on certain members of the Cecil series and on much of the limestone on the mountains west of North Mountain. When these areas are cleared the wash from them will augment the already high turbidity of the river. As a matter of eivic policy it would be advisable to retain these lands in forest and to reforest extensive areas of similar soils which have been pauperized by erosion incidental to culture or whose permanence is threatened by tillage, since the erosion from them when in forest is insignificant.

\section{EROSION OF FARM LAND.}

The primary cause of erosion is the failure of the soil to absorb the rain water which falls upon it. If the rainfall is all absorbed, as by a coarse, sandy soil, there is no run-off and no erosion. As the soil becomes finer in texture, more compact, and correspondingly less pervious, the rain is not absorbed as fast as it falls, and the very smallness of the grains which form the soil facilitates its transportation whenever the attitudinal factors are favorable. The impact of the raindrops loosens the fine particles of soil, and unless absorption takes place the drops gather into small streams and rivulets, transporting with them, by a system of natural elutriation, the finest particles of soil and leaving behind the larger and heavier grains. At first this is entirely due to the hydraulic action of the impinging raindrops, but no sooner do the rivulets gather power, either by the added volume of water or by increased gradient, than they likewise begin cutting loose and transporting the soil. The eroding and transporting action of water is increased by the increase in its volume; it is also increased four times by doubling the gradient. For this reason the steepest land erodes most easily, and since the slope of a rain-formed hill is an ogee and steepest in the middle the most rapid erosion takes place on the middle slope, while the heaviest transported material is deposited by the slackened current on the more gently sloping base. 
The capacity of a stiff soil for water is in practice 35 to 50 per cent of its volume, or for ordinary farmed soils 4 to 5 inches of rainfall to the surface foot. In spite of this capacity, the greater part of a heavy shower will usually not be absorbed. The coarse structure of a sandy soil permits the rainfall to be absorbed as rapidly as it falls. In a clay soil, unless in a high state of tilth, the pores are smaller and there is less open cellular communication between them, and absorption must largely take place through cracks, worm holes, and root holes, and when there are few of these absorption is largely retarded until the air can be expelled. In the extreme case, that of a raw clay soil with its surface puddled by a previous heavy rain, the result is, as King ${ }^{a}$ points out, "that when a heavy rain falls, the close structure and feeble granulation result in the surface pores of the soil becoming so quickly closed that the soil air has little opportunity to escape, and yet only so fast as it does escape can rain enter the soil, and hence during heavy rains the water accumulates quickly and extensively upon the surface."

The greater portion of the tilled soils of the Potomac basin, especially of the lower part, are of heavy type and close texture, and the run-off from them indicates failure to absorb. But were they well granulated and in good tilth they, as well as the more permeable sandy soils, could readily absorb, without undue accumulation of surface water, a much heavier rain than commonly falls at one time on the Potomac basin.

The porous condition or granulation of a heavy soil necessary to effect absorption is best procured by the addition of humus.

There is over a greater portion of the tilled area a deficiency in the organic content or humus. The soils of the great valley and the region to the east lie well within the southern field of long, hot summers and heavy, irregularly distributed rainfall. The organic content of typical tilled soils of this region, as given by the Bureau of Soils, is for the Iredell clay loam, 1.43 per cent; Penn loam, 1.70 per cent; Penn clay, 1.92 to 3.04 per cent; Hagerstown clay, 1.51 per cent; Cecil loam, 1.07 per cent; Cecil clay, 1.63 per cent. ${ }^{b}$ These are from typical areas in Virginia, West Virginia, and Maryland, in a well-farmed section, and without doubt adequately represent the humous content of these and similar soils throughout the lower cultivated portion of the Potomac River basin.

For the same or similar soils in the more southern Atlantic States, the organic matter is given ${ }^{c}$ as from 5 to 7 per cent for the Cecil clay, 2.4 to 4 per cent for the Cecil sandy loam, and 4.15 to 4.75 for the Iredell clay, and it is recognized that even these large amounts are generally too small ${ }^{d}$ to maintain soils in that latitude in good tilth and sufficiently porous and strongly granulated to prevent washing.

$a$ Yearbook U. S. Dept. Agric., 1903, p. 164.

$b$ Field Operations Bureau of Soils, $\cdot 1903$, pp. 210 et seq.

c Idem, 1901, pp. 280 et seq.

$a$ Idem, 1901, p. 281; Yearbook U. S. Dept. Agric., 1903, p. 168.

IRR 192-07-21 
The growing season in the lower part of the Potomac basin is hotter and longer than in the basin of the Susquehanna and the other streams to the northeast. Except near the coast, where the relative. humidity is high; on certain types of soil, which are very well adapted to grass; and at high elevations, a permanent sod is with difficulty maintained or even established. Many of the soils have high oxidizing power, and humus, either in the shape of leaf mold in the forest, as manure in farming land, or litter in grass land, is rapidly oxidized. The result is that a low soil porosity is maintained, with small absorptive capacity, saturation quickly taking place and the heavy run-off from the steep slopes causing erosion.

Spillman ${ }^{a}$ has shown in actual practice that the large increase in the organic content of a yellow-shale soil in eastern Pennsylvania produced a soil structure more largely independent of weather conditions than any soil he had ever seen. "Torrential rains are soaked up," he says, "in a very short time, so that the soil may be handled after a rain much sooner than that of adjacent forms."

On account of their inability to absorb the necessary rain, the moisture content of the heavy clays is often insufficient in the autumn for the proper maturing of the corn crop and causes the grass to "burn badly."

On clay soils, such as the heavy phases of the limestone, tile drainage also increases the thickness of the permeable layer of top soil and its water-storage capacity. Such drainage would undoubtedly be beneficial over a large area of the nearly level or gently rolling clay lands of the limestone, shale, and Penn series in lessening erosion from saturated soils.

The run-off can also be reduced by terracing, which is especially adapted to the deep-soiled Penn, Cecil, and Chester series east of the Blue Ridge and to the shale soils west of it. This permits the absorption and the subsoil storage of the heavy spring and summer rains, which are so badly needed for plant growth and which are so largely lost to the crop by the run-off. Terracing has been done, in isolated cases, in more southern States, and the increased yield and greater immunity from drought have more than compensated for the reduced area and increased cost of tillage.

Another element in the run-off from farming land which is fully as important as the loss of the water is the loss of the solid mineral elements which form the turbidity as well as the soluble matter which is washed off. Mr. Outwater states in another part of this report that the soluble matter removed, if derived largely from the agricultural land, amounts to about 400 pounds per acre per year, and the plant food in it is about equal to that removed by a crop. While the soluble material lost by leaching is replaced with about the same 


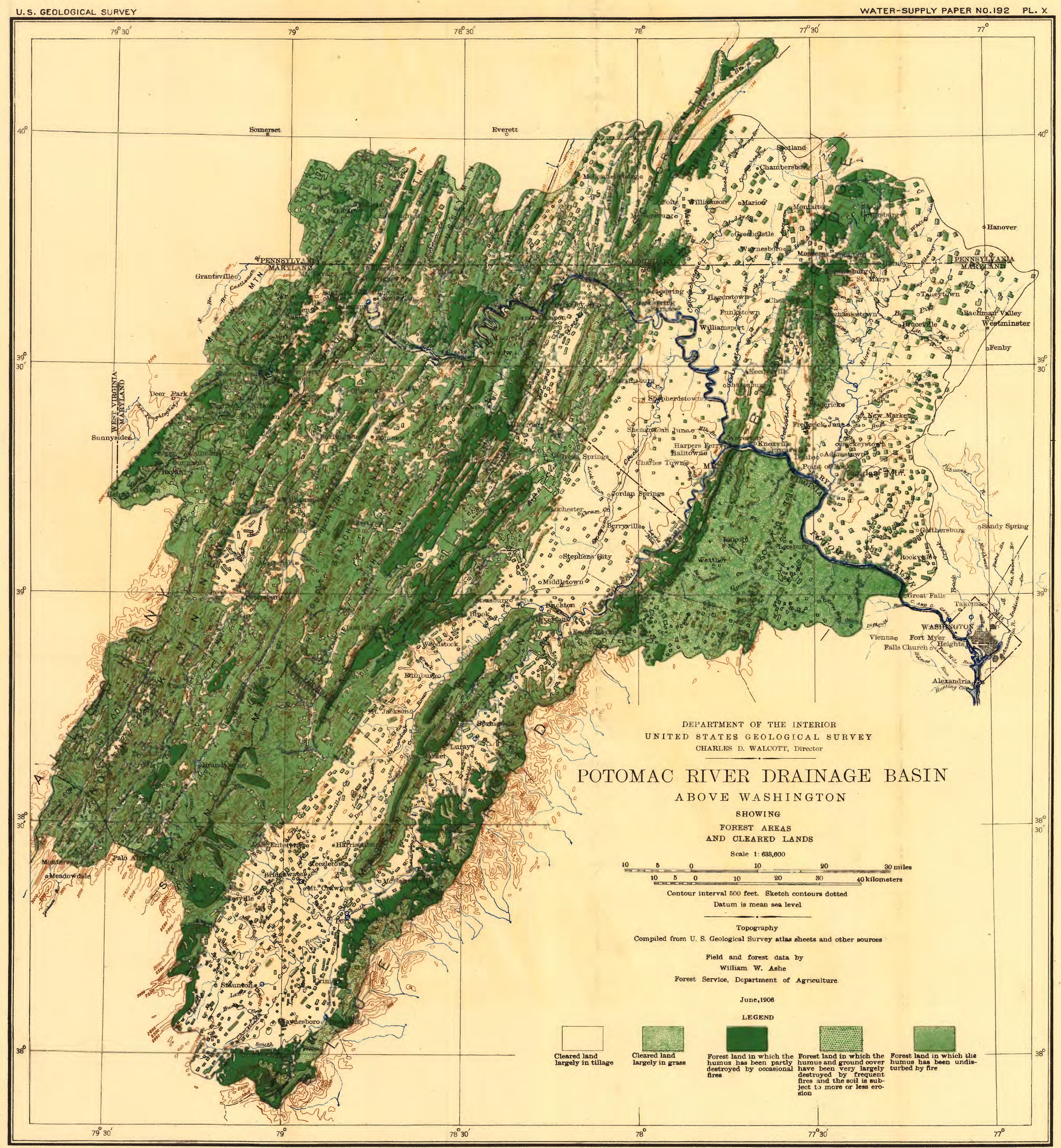


rapidity as it is removed, the fine particles of silt and clay are replaced very slowly. This fine solid matter represents the most valuable portion of the mineral soil on account of its favorable influence on the retention of soil moisture and the large surface it offers for root: absorption. This indicates the necessity from an agricultural standpoint of storing in the soil the heavy spring and summer rains, that the water may be available for the growing crops and that at the same time the fertility of the soil may be maintained by retaining in it the silt and clay which form the turbidity and the soluble plant food, which is also washed away.

\section{EFFECT OF FOREST COVER ON STREAM FLOW.}

\section{EXTENT AND INFLUENCE OF FOREST COVER.}

Originally the greater portion of the Potomac basin was wooded, although the Indians kept much of the Shenandoah and Cumberland. valleys burned off, as well as certain areas on Monocacy River in Adams County, Pa., which even now, on account of this, are known. as the "Barrens," and smaller areas elsewhere, as at Petersburg, Grant County, W. Va., and below Moorefield, Hardy County, W. Va. It is also probable that they regularly burned over portions of the mountains to facilitate hunting, although they do not seem to have kept the timber suppressed, as was the case in the "Barrens" and elsewhere in the valleys.

Woodland at present covers about one-third of the area of the basin. (Pl. X.) It has been very largely removed from the Great Valley, from the Blue Ridge and Catoctin Mountains, and from the Piedmont area east of the Blue Ridge. The Shenandoah and Tuscarora mountains are yet well wooded, and there is a more or less continuous strip of forest on the upper slopes of many of the Appalachian ridges. In places it is continuous for several miles and will cover a great portion of the slope; elsewhere it is a very narrow strip along the rocky crest, or it is entirely segmented into wood lots and covers less than one-fourth of the mountain slope.

In the Allegheny Mountains the forest covers most of the very stony and rough land and many of the steep slopes, especially in Maryland and West Virginia; but in Somerset County, Pa., large areas of even, steep land have been cleared and put into grass, and there are no extensive bodies of forest. From this it is evident that the forests have not yet been cleared from a large part of the steep land of the basin, and the facts as brought out go to show that they are sufficient in extent to play an important rôle in oonnection with the quality of the Potomac water, although their influence is not as potent as formerly and must contimue to decrease as their area becomes smaller. The forests are influential in improving the potability of the Potomac 
water in three ways: They prevent greater erosion from certain soil types on steep slopes which are yet partly in forest and which wash badly when deforested, and lessen in this way the very high turbidity of the water. They maintain the volume, already very low, of the summer and autumn run-off, which by dilution adds to the real purity of the water, although this is of relatively slight value. They also steady and equalize the flow of the smaller streams and lessen the erosion of their banks.

The water of the smaller streams which flow from forest-covered mountain watersheds is clear and pure. The water of such mountain streams is being used by several towns in the Potomac basin, which obtain their water supply from streams in the Pennsylvania forest reserves, this water being less open to pollution than that of springs and wells. The protection of such forested watersheds in other States than Pennsylvania, to insure their permanence and the general utilization of these streams in place of springs and wells, which are now in general use, or in place of the polluted river water which is used by Cumberland, will, by furnishing a pure supply, largely eliminate these towns as foci of infection of typhoid fever and other waterborne diseases.

One of the tributaries of the Potomac, Great Cacapon River, affords clear and reasonably pure water which would be sufficient for the requirements of Washington, D. C. It drains a valley whose soils are, for the most part, loam and sandy loam, largely nonagricultural and forest covered. Three small villages are the chief sources of contamination. Its distance, 117 miles above the Great Falls intake, is probably too great to render it available as a present source of supply.

There is, as has been pointed out, room for a large storage of storm water in farming soils, and more rational farming methods must ultimately lead to this. The effect of such storage would be reflected in a diminished run-off of flood water, especially of heavy midsummer rains. Little of the water thus accumulated would normally pass off as seepage to spring and river flow. The improved growth of the crops would utilize such stored water, and, given a more constant and a vailable amount of soil moisture, two crops a year would be the rule and not the exception as at present over much of the warmer part of the basin, and in place of the crops transpiring from 4 to 6 inches of the rainfall, as at present, they would use about double that amount.

Any additional storage, either transient, tending to prolong or distribute a flood crest, or deep seated, tending to increase the amount of the dry-season flow, can be secured only in the forest soil. Storage in forest soil, except in sandy phases, takes place very largely through the medium of humus. The amount of water which is actually retained by a thin humus is small, in fact, a thin humus is usually 
far more litter than humus and has a very low retentive capacity; but the chief functions of humus, except where it has accumulated to a great depth, are (1) to maintain the volume and depth of the soil by preventing erosion; (2) to secure the porosity of the soil; (3) to promote the absorption of rainfall by the soil in retarding the runoff of heavy showers until the water can be absorbed, as the air is gradually expelled from the soil; (4) to act as a mulch and lessen the evaporation of soil moisture.

On coarse, deep sands of low gradient the office of humus is at a minimum. There is no surface flow of storm water and no soil transportation; evaporation of soil moisture is low both from the surface of the soil and from transpiration by the xerophytic flora, and the larger part of the rainfall passes as percolation. With an increase in the clay component, greater gradient, and a decrease in soil depth, humus becomes more essential in supplementing the water-absorbing and water-carrying capacity of the soil, its functions in this respect attaining a maximum on heavy, shallow clays of mountain slopes. Much of the mountain area of the Potomac River basin affords such conditions; the slopes are steep and the soils are shallow, in part loams and in part clays of limestone and shale derivation.

Where the humus is thick, however, it possesses a high storage capacity. While there is some doubt in regard to the exact amount of water humus is capable of holding, the quantity is relatively large. The lowest estimates by Ebermayer place it at considerably more than its own weight, and Wiley's investigations of Florida mucks give it about the same capacity, while Wollny, as quoted by Henry, ${ }^{a}$ places it at about four times its weight, and Henry's laboratory experiments tend to confirm Wollny's high limit. The capacity of humus must vary, however, not only with the state of decomposition, but with its origin as well, since the pore space is the final determinant of its water-bearing capacity. The undecomposed litter, which covers the humus and from which humus is formed, does not exhibit the characters of humus toward water. It protects the humus, as humus does the underlying soil, from excessive transportation by surface water and in addition from excessive evaporation, acting as a mulch.

The accumulation of humus on forest soil depends, if it has neither been disturbed nor destroyed, on the kind of species forming the forest and to some extent on the soil, the destruction of humus proceeding rapidly on loose, porous soils, which permit freer circulation of air and afford the condition best suited for bacteriological activity, and since many species of trees, especially the white oak and chestnut, have a wide range of soil adaptability, their capacity to accumulate humus is modified both by their rate of growth on the soil and by the oxidizing capacity of the soil on which they happen to be growing. 


\section{FOREST TYPES.}

The forest types can be arranged in respect to their humus-forming capacity about in the following order, those forming the smallest amounts being named first and those beneath which humus and absorbent material accumulate to the greatest depth last: Pine type, chestnut oak-white oak type, chestnut type, birch-linden-red oak type, beech-maple-hemlock type, and spruce type.

\section{PINE TYPE.}

Beneath the pine the humus is not only shallow, but seldom accumulating to a greater depth than 2 inches. It is destroyed so rapidly that in the main it is barely replaced annually by the decomposition of litter. Where conditions are normal and the humus has not been destroyed or lessened, the top soil contains for a depth of 2 to 4 inches a varying amount of organic material which adds greatly to its absorptive eapacity. On account of the open character of most of the pine forests and their situation, which is usually on exposed ridges, especially with southern aspects, and on crests, it is much exposed to desiccation by both insolation and wind. Such humus is largely protective. Forests of this character are situated on both sandy and clay soils. The pitch pine and table-mountain pine, with their associated trees, are on the sandiest phases. The soils are usually shallow or porous. They include members of the Dekalb and Upshur soil series. Shortleaf pine, with its associated trees, occurs on the driest aspects of loams and clays both of the limestone and of the yellow-shale and Upshur series. Jersey pine occurs on crests and more leachy phases of clays from yellow shale, as well as on many of the friable and easily eroded Cecil and Chester soils east of the Blue Ridge. Close-textured soils with low absorptive capacity probably form one-half of the pine type, the total area of which amounts to more than 600 square miles. Nearly all of this area has a highly dissected topography, many of the slopes being both long and steep, and offers, when denuded of humus, favorable opportunity for excessive erosion. Under the conditions of this type the protective value of humus is high in preventing erosion and increase of turbidity, as well as in lessening evaporation of soil moisture during the summer and autumn, to which this type of forest soil is much exposed on account of the thin and incomplete canopy of the crown of the pines and associated intolerant species.

Unfortunately, in much of this forest the humus has been badly burned and its accumulation prevented by repeated, in some places almost annual, fires. This condition exists over large areas. The summit of Tussey Mountain, a broad, flat-topped ridge, has been badly burned. It now forms a part of the Pennsylvania State Forest 
Reserve, and it is probable that fires will hereafter be less frequent on it. The humus has also been burned from large areas on the west slope of Town Hill, the burned areas extending for several miles on bath sides of the Maryland-Pennsylvania State line; from extensive areas on Sideling Hill, in both Maryland and Pennsylvania; from the Pratt woods on Tenmile Creek, in Allegany County, Md.; from the broken region below Buck Valley, in the southwest corner of Fulton County, Pa., and the eastern part of Allegany County, Md.; from several long, narrow strips on the western slope of Tonoloway Ridge in both Maryland and Pennsylvania; from large areas on the lower part of Fourmile Creek, and from smaller areas on Scrub Ridge and on the west side of Tuscarora Mountain, in Fulton County, $\mathrm{Pa}$. The accumulation of humus is prevented on large areas of pine forest on both slopes, but especially on the western, of Shenandoah Mountain in Virginia and West Virginia. Sandy Ridge, in Hardy County, W. Va., is frequently burned, as are smaller areas in Hardy, Hampshire, Mineral, Grant, and Pendleton counties, W. Va.; larger areas of pine forest on the western slope of the Blue Ridge in the valley of Virginia, and on the interior slope of the west ridge of Massanutten Mountain, and many smaller areas in other places. On many of the slopes with little or no humus soil transportation and erosion freely take place, especially where the soil from long exposure has entirely lost its humus content. Much of the forest has been reduced to sprout wood or scrub oak, and since the pines when fire killed do not sprout, they are gradually being eliminated. Owing to the suppression of young timber by fire the forest density on other areas is far too low to check the wind, and the soil, already too dry, is exposed to excessive evaporation. There are more than 200 square miles of the pine type on which the humus is too thin to perform its functions adequately and on which the soil has undergone evident deterioration in absorptive capacity. The results from this condition must be reflected in excessive run-off during heavy storms and in increased evaporation during the summer.

\section{CHESTNUT OAK-WHITE OAK TYPE.}

Humus beneath the chestnut oak-white oak type of forest seldom exceeds 3 inches in depth. This forest, like the pine type, is largely situated on slopes and ridges and occupies loams and clay soils on both the mountains and valleys. It is well represented east of the Blue Ridge, and west of that range it is the prevailing type on the dry phases of the valley clays, on all the thin-soiled, loamy slopes of the mountains below an elevation of 3,000 feet and above that elevation in numerous crests and ridges, especially those with southern aspects. On account of its situation on slopes, especially where on 
heavier soils, as on the mountain limestones and the heavy shaleclay phases, the protective function of its humus is important in preventing erosion, and it is likewise important in promoting absorption by these close soils, as well as in preventing the excessive evaporation which takes place from the clays, three or four times that from the sandy soils. This type covers more than 2,000 square miles, of which about 700 square miles are clay soils, the remainder being sands, loams, and clay loams, largely thin and in many places extremely stony or strewn with rock bars.

The humus of this type has a small water-carrying capacity, but its chief function is protective. It has been reduced in thickness over more than one-half of the area of this forest type, and over more than 300 square miles it has been destroyed and its reaccumulation prevented. The reduction in thickness is due to the pasturage of sheep, occasional fires, and the desiccation incidental to thorough opening of the forest cover in lumbering or peeling bark, which industries are being extensively prosecuted in the area of this type. Where the humus is subjected to a part or all of these deteriorating influences simultaneously, the result is usually its complete destruction. This has happened on large areas on the east slope of Sideling Hill west of Needmore, Fulton County, Pa., where the destruction is so effectual that the soil has washed badly on many of the steeper wooded slopes. The same conditions exist over larger areas on the west slope of the Blue Ridge, in Rockingham County, Va., and to a less extent in Augusta County, on the same range, while many of the foothills of Shenandoah and Great North mountains in these counties and in Frederick County, Va., and in Hardy and Hampshire counties, W. Va., have been repeatedly burned, and the forest cover has been so thinned that large areas of mineral soil are exposed and washing. Injury of a less degree exists over areas of this type throughout Grant, Pendleton, and Mineral counties, W. Va., Bedford and Fulton counties, Pa., and Allegany County, Md. It is worse in the oldest settled commumities and near the routes of transportation, and gradually diminishes as distance from these destructive elements increases. Since the causes which lead to the destruction of humus are cumulative in their effects, the continued activity in lumbering, with the inevitable fires and pasturage, is gradually enlarging the area of sprout wood, scrub oak, and brush land, on which there is little humus or none at all.

\section{CHESTNUT TYPE.}

The chestnut, with which usually red oak is associated at higher elevations and white oak at lower, produces a better humus than either of the types already described. Its humus frequently attains a depth of 4 inches and is best when the chestnut is associated with 
red oak or beech. Chestnut requires a deep soil, preferring a loam or loamy clay or lighter soil, and for this reason on the mountains it grows in many places on benches where there has been little soil transportation and on lower slopes where transported material has accumulated. In the latter situation it is partly sheltered from wind and excessive evaporation of soil moisture, but on benches it is much exposed. Humus of the depth formed by chestnut has an appreciable water-carrying capacity and exerts a highly beneficial influence on the soil. There are more than 700 square miles of this type, nearly all of it on mountains. In very little of it has the humus been so completely destroyed as in the types already discussed, but there are large badly burned areas on which accumulation is seriously checked. Such areas occur on the Blue Ridge in many places, especially on the upper slopes in Page and the adjoining counties in Virginia; in many situations in the South Mountains, Tuscarora Mountains, and Scrub Ridge, in Adams, Franklin, and Fulton counties, $\mathrm{Pa}$; on the east face and fore knobs of the Allegheny Mountains in Grant and Pendleton counties, W. Va.; and on Savage Mountain and Backbone Ridge, in Allegany County, Md., and Somerset County, $\mathrm{Pa}$. The total area on which the humus shows the effects of recent fires is more than one-third of the entire area of the chestnut type.

\section{BIRCH-BASSWOOD-RED OAK TYPE.}

The birch-basswood-red oak type. often yields humus 5 inches deep. It is normally confined to hollows or northern slopes and sheltered situations, mostly at high elevations, and even on extremely shallow soils in such situations the humus frequently attains a depih of 6 inches, besides filling large and deep crevices between the rocks. Water can be wrung from such humus for several days after a rain, and it becomes dry and crumbly only during periods of long drought. Many of the birch and hemlock roots lie within the humus, and it undoubtedly-furnishes some transpiration moisture to these species in spite of the low absorptive power they have in acid humus. 'This type covers a considerable area on the mountains of the watershed, being represented near the crests on northern slopes and in the hollows. The proportion of it increases with the altitude. It is especially well represented on the Allegheny Mountains and is least abundant east of the Blue Ridge and in the Shenandoah River valley. The total area probably amounts to less than 300 square miles. On account of the moist floor, only small and inconsiderable areas have been burned, and in these the burning was limited to the upper layers of humus. Heavy cutting in lumbering has caused deterioration of the humus in many places, and cattle have done some damage. 


\section{BEECH-HARD MAPLE-HEMLOCK TYPE.}

Humus accumulates beneath the beech-hard maple-hemlock type to depths of 6 inches or more, and is of very fine quality, fine grained, and always moist. On very rocky land it is in places deep enough to fill the crevices between the large rocks and seldom becomes at all dry except on top. This type is best developed on the Allegheny Mountains at high elevations, especially above 2,000 feet, on moist slopes, and in deep hollows and in similar situations at the head of North Fork of Potomac River and on the mountains drained by Seneca Creek, Laurel Fork, and adjoining streams. Locally on very rocky soil the humus seems to be almost the only soil there is. Here and there this type is well developed on good soil, especially on limestone at high elevations, as at the head of North Fork of Potomac River. On limestore soils the humus and litter are in places a foot or more deep and the weeds and small undergrowth are extremely rank and thick. Where there has been no lumbering the forest cover is dense and there is low soil evaporation. This low evaporation is partly offset, however, by high transpiration from the deciduous trees of this type. Humus of this type has a high storage capacity. It is burned but rarely, and then the fire is usually confined to the top layers. Considerable areas have been badly burned, after lumbering, on Stony River, Difficult Creek, and Buffalo Creek, in Grant County, W. Va., in the south end of Garrett County, Md., and smaller areas elsewhere. Unless burnings are frequently repeated, which is rarely the case except where cutting in lumbering is severe, and unless brambles and such shrubs as make large amounts of dry brush become thick, the humus is replaced in a few years. This type of forest covers about 300 square miles. In 60 square miles of this area the humus is shallow on account of fires.

\section{SPRUCE TYPE.}

Humus accumulates to a depth of several inches beneath spruce forests, and in addition there is a deep moss, the lower layers of which hold a large amount of water. In the Potomac basin spruce is confined to the thinnest soiled, stoniest land at elevations above 3,000 feet. Much of the land has no soil at all, the loose stone lying on the undecomposed sandstone; in other places the soil is only a few inches deep, or, if deeper, is so coarse as to be scarcely more than gravel. There are 69 square miles of such spruce land situated on Stony River, Difficult Creek, and Buffalo Creek, in Grant County, W. Va., and on Seneca Creek, Laurel Fork, Spruce Mountain, and Tamarack Ridge, in Pendleton County, W. Va., and Highland County, Va., on the very headwaters of North Branch and North Fork of the Potomac. This is the region of heaviest rainfall in the 
basin (pp. 34-40) and the region where the best humus is needed. Of the total area of spruce land, 48 square miles, or considerably more than half, has been burned either before or after lumbering, and the humus and forest soil have been entirely destroyed. The burned areas are southwest of Bismarck, at the head of Stony River, and at the head of Buffalo Creek, in Grant County, W. Va., and on Spruce Mountain and along the face of the Allegheny Mountains, in Pendleton County, W. Va. Most of this is burned bare or has a scant cover of blueberry and brambles, which have gradually spread. Over other areas, especially where the soil is best, birch and popple and fire cherry are colonizing and reestablishing forest conditions. The area of this burned land, however, is rapidly increasing as lumbering proceeds. The absence of the absorbent humus and moss over these thin-soiled areas, where it is most necessary, must have an appreciable influence on the movement of storm waters in the streams which drain these areas. No turbidity comes directly from this land.

While the reestablishment of normal humus conditions would undoubtedly have a beneficial effect in alleviating the danger from high floods, the division of the forest into so large a number of narrow strips on the mountains, its diverse ownership, its location in four States, and the rapid progress of lumbering assure a progressive accentuation of the unfavorable conditions.

\section{MELTING OF SNOW.}

High turbidity usually accompanies the spring floods of April and May caused by the melting of the snow on the mountains at the head of the river, especially on the Allegheny Mountains. The longer the process of melting the lower is the flood crest and the lower the accompanying turbidity. The coniferous forests are instrumental in prolonging the melting of the snow. Near Hancock, Md., at an elevation of 600 feet above sea level, a 20 -inch snow which fell during March, 1906, was nine days longer in melting beneath the cover of Jersey pines than on adjacent field land with the same soil, slope, and aspect. At the head of the river the spruce forest, with its more dense foliage, must exert a greater effect than Jersey pine in prolonging melting. Some difference, though much less marked, was noticed in the melting of snow in the deciduous forest of oak and in the adjoining fields. The total area of coniferous forest in the Potomac basin is more than 700 square miles, and its influence must be considerable in distributing the crest of floods from melting snow, by retarding melting in the forest land and by promoting absorption and subterranean drainage. 


\section{PROTECTIVE FORESTS.}

The State of Pennsylvania, realizing the inability of private owners to supply the technical knowledge necessary to manage small forest areas in such a manner as to make them permanent earning investments, as well as the necessity of properly protecting by forests the headwaters of the large rivers of the State, has purchased extensive areas of mountain forest land within its borders. Portions of this land, lying on Tussey Mountain, Tuscarora and its associate mountains, and Jack and Green mountains, are on the Potomac watershed, although the greater portion is on the Susquehanna, which is-essentially a Pennsylvania stream. The further extension of this protective forest to the south and southwest, to include the most important mountain areas in the Potomac basin in Maryland, West Virginia, and Virginia, is desirable. Where the States are unable to assume either the cost or the responsibility of acquiring and managing such large areas for the protection of interstate streams, this duty devolves on the Federal Government, whenever the States concerned grant the Government the right to acquire and hold such property. This right has already been granted by the legislatures of two of the States under consideration-Virginia and West Virginia-in connection with the establishment of the proposed Southern Appalachian Forest Reserve, and it would doubtless be extended by them to include the Potomac drainage basin. On account of the importance of the Potomac as a source of domestic water supply, an Appalachian forest-reserve system could fittingly be inaugurated by the acquisition of the important forested mountain lands in its basin, and, with the already extensive Pennsylvania system, two important wateisheds of the Middle Atlantic drainage, the Susquehanna and Potomac, would be protected.

The control of the waters of the East by the Federal Government for the benefit of the people is as important as the control of those of the West for extending irrigation, and the principle which underlies the withholding of public money by not selling forest land is the same as that which underlies the acquiring of forest land by purchase for similar purposes.

One consideration in the selection of such forests on the Potomac basin should have reference to their protecting small mountain streams which can be utilized as sources of water supply for near-by towns and cities. The water from such forest-protected basins is of great purity, clearness, and softness, and its general use by the towns is desirable, not only on their own account, since it furnishes them a pure supply, but because by purifying their supply these towns are largely eliminated as sources of typhoid fever.

The limestone springs which are used by many towns are more 
exposed to contamination than freestone springs. The latter are open only to local surface contamination, while many sink holes on farms, which are the dumping places for waste, communicate with underground streams that are in numerous places the sources of limestone springs. Many limestone springs are also streams which have sunk and reappeared on the surface at the spring. Numbers of such springs are easily traceable to streams which have sunk in the Cumberland Valley, in the valley of Virginia, and on South Branch of the Potomac.

When the towns using springs or water from small streams which are open to pollution, such as that used by Chambersburg, $\mathrm{Pa}$., find it necessary to obtain new sources of supply, either because of inadequacy of their present supply or on account of its known pollution or questionable purity, as is the case with Leesburg, Va., no purer water can be obtained than that from streams of forest-protected watersheds. Two towns on the Potomac watershed in Pennsylvania are already taking advantage of the permanent purity which the Pennsylvania foresi reserves guarantee and have made use of streams in forest reserves as sources of supply. These towns are Mercersburg, which uses Trout Run in the Tuscarora Mountains, and Waynesboro, which uses streams in the South Mountain Forest Reserve. The near-by city of Hagerstown, Md., has recently sought a purer supply from the streams of South Mountain in Maryland, a few miles south of the streams which supply Waynesboro. There is assurance neither of permanency or purity to its supply, however, since the watershed is privately owned and the number of farms is increasing, with the constant danger of contamination.

Such a system of protective forests as has been suggested need not be designed to include the headwaters of all the streams in the basin, but should, primarily at least, be planned to include all those which now afford a water supply to towns of the basin or which by their situation will, in the future, be necessary as sources of water supply. With many of these towns the amount of the supply will be a matter for consideration as well as the purity, and the protection of the humus and the forest floor will be beneficial, while the regular cutting which would be carried on under the pollicy of protection forests would in no way jeopardize the amount of regularity of the stream flow.

\section{EXTENSION OF THE CLEARED AREA.}

While for a mountain region a large proportion of the land in the Potomac basin has been cleared, the limit has by no means been reached. During the period between 1880 and 1890 the uninterrupted emigration to the West checked further clearing of rough land for small farms, and on account of the agricultural depression in the 
following decade there was little incentive for further extension of the farmed area. In fact, much land was abandoned in Franklin and Bedford counties, Pa., and in Allegany and Garrett counties, Md., and some in Hardy, Hampshire, Mineral, and Grant counties, W. Va. The present feeling in the East that the West no longer offers any better opportunity for farming for the man of limited means than the East has checked emigration and given a new impetus to farming in this section. In addition to the fact that the man of small means, who twenty years ago would have gone West, is now buying a small farm at home, usually in timber which he clears, two other causes have stimulated additional clearing. (1) One of these is the release for farming purposes of recently logged timber land or recently opened coal land. Much of this land is of good quality for farming, and as rapidly as it is lumbered it is cleared and put in cultivation, or if too rough for tillage the large trees are killed by girdling, the ground is burned over to kill the brush, and the land is put in grass. The high price of sheep has made this profitable, and large areas of rough land, some of it extremely steep and thin soiled, are being sodded in this way on and along the Allegheny Mountains. Extensive areas are being opened for grass land on Laurel and Straight forks of North Fork of the Potomac, following the lumbering of hemlock and hardwoods, and for general farming on Savage River, following the lumbering of hardwoods, while over the entire basin there is at present a steady addition to the farming area of recently lumbered land. (2) The extension of fruit and especially of peach culture during the past decade has led to the clearing of a large amount of steep, sandy mountain land. Some of the largest orchard districts which have been cleared in the past few years are on the south slope of Tonoloway Ridge, in Washington and Franklin counties, Pa., where a heavy limestone soil has been selected for apple culture. Large areas on the southern slopes of Town Hill, in Allegheny County, Md., and on Patterson Creek Mountain, Knobly Mountain, North Mountain, Jersey Mountain, and the Allegheny Front, near Keyser, have been cleared for peaches. Much of this land would seem to be too steep and the slopes too long for permanent profitable cultivation. While the soil beneath an orchard is protected by a cover crop during the winter, clean culture is given during the summer, subjecting steep slopes to the erosion of heavy summer rains. It is possible that with the lessening of the profits which are now obtained and which are due to the freshness of the land and the immunity from insect pests the portion of this land which is least favorably situated will be abandoned.

Further contraction of the forest area tends to increase the already large disparity between maximum and minimum flows of the streams, with the concomitant influences on the potability of the water. 


\section{TURBIDITY IN' RESERVOIRS AT WASHINGTON, D. C.}

The water supply for Washington, D. C., before going to the filter beds usually passes through three settling reservoirs. From the intake at Great Falls it is piped to the Dalecarlia Reservoir, about eight hours being required for the journey. The water remains in the Dalecarlia Reservoir about one day and is then pumped to the Georgetown Reservoir. Thence it passes to the Washington Reservoir, which is located at the filtration plant, about 5 miles distant. About two days are consumed in the passage from the Georgetown Reservoir through the Washington Reservoir and through the filter beds. From these beds the water passes to a distributing reservoir. The conduits at the reservoirs are so arranged that any reservoir can be cut out of service without interrupting the flow from the other reservoirs or from the river.

The river water which enters the Dalecarlia Reservoir is usually turbid, the turbidity varying, according to the standard of the United States Geological Survey, from 3,000 or more during periods of flood in the river to 15 or 20 after long periods of little or no rainfall on the Potomac watershed above the intake. In general, it may be said that the turbidity will be in excess of 300 for about forty-three days in the year, above 50 and less than 300 for about one hundred and twentythree days, and less than 50 for the remaining period. It will exceed 1,000 for probably less than eighteen days. The first settling to lessen the turbidity takes place in the Dalecarlia Reservoir and removes the greater portion of the heaviest silt and a large part of the clay from the water, but the clearness of the water when it leaves this reservoir. depends largely on its initial turbidity. Further clarification by settling, though slight compared with that which takes place in the Dalecarlia Reservoir, occurs in both the Georgetown and Washington reservoirs. The finer material which occasions most of the turbidity after the water leaves the Dalecarlia Reservoir settles very slowly. Sedimentation usually proceeds with a certain expected uniformity, a high percentage of turbidity being eliminated from water of a high turbidity and a smaller percentage from that of a lower turbidity, owing to the slower settling of the finer particles which cause the low turbidity. The greater proportion of clarification in water of high turbidity is suggested by Mason as being due to the well-known tendency of larger falling particles to drag down with them very fine particles, and even matter in solution. The subsiding, heavier silt drags down not only much of the finest clay, but bacteria as well. It is important, therefore, that the sedimentation in the Dalecarlia Reservoir be as thorough and uninterrupted as possible, since after the settling of the heavier particles the finer material which is left, having failed to be carried down by the coarse material, remains in suspension a great while and is with difficulty eliminated from the water by further 
sedimentation. Only a certain proportion of it is removed by filtration, and it follows that the lower the turbidity of the water as it goes to the filter beds the more pellucid the effluent.

At different times, but usually during the winter and spring, on account of wave action produced by wind, sedimentation in the reservoirs not only takes place very slowly and irregularly or not at all, but additional turbidity is acquired by the water from the scouring of the sides or, at shallow places, the bottoms of the reservoirs, a portion of the thin coating of silt and clay that has previously been deposited being again taken up in suspension. With this increase in turbidity a portion of the recently deposited bacteria are also redistributed through the water.

The period of wind agitation of water in small lakes and reservoirs; as has been pointed out by Birge, is largely limited to the winter and early spring months, after the water has become homothermous. During the summer and early fall the temperature of the surface water becomes much higher than that of the water at the bottom of the reservoir, and the surface water is consequently much lighter, there being a warm, superficial stratum of water, the colder bottom stratum, and a thin stratum between them in which the temperature rapidly falls, called by Birge the thermocline.

Wind agitation of water during the summer and autumn, when the stratified condition is present, is largely confined to the lighter superficial layer, which does not readily mix with the dense layer at the bottom, and there is little churning of this bottom layer to cause added turbidity. The depth of the water, however, has some influence, the stratification being most marked and the difference in temperature and density greatest in deep water. The upper and lower layers become more uniform in thermal and physical properties with lessened depth, and the susceptibility to wind agitation during the summer increases. For this reason the deep reservoir is least disturbed by wave action.

During winter and spring, however, when the temperature of the water is about the same throughout, constant wind action, though light, from any one direction, will easily cause a complete overturning of the water in the reservoir. The thermal resistance to wind action which is offered by water is greatest in summer. In the winter it is greater in the day than at night and greater during sunny days than during cloudy weather. For this reason wind agitation is at its maximum during cloudy winter weather. The situation of Washington favors the existence of a long period in which the thermal conditions are conducive to wave action. The winter and spring period is long; there are a large number of cloudy, windy days, and on comparatively few days is the weather so cold that the reservoirs are frozen and protected against wind influences. 
March, 1906, was a windy, month, and, notwithstanding the low turbidity of the raw river water, except for a few periods of short duration, the turbidity of the water in the reservoirs remained higher than that in the river, exhibiting constantly the influence of wind action. The turbidity of the water in the Dalecarlia Reservoir remained so high that it was temporarily dropped from service and water from the river diverted direct into the Georgetown Reservoir; and during a short period of very high turbidity in the Georgetown Reservoir and lower turbidity in the river, March 9 and 10, the river water was pumped direct to the Washington Reservoir and thence to the filter beds. There was scarcely a day between March 13 and March 22 when the turbidity of the water in each of the reservoirs was not higher than that of the river water.

After filtration the water is passed into the distributing reservoir. Under low wind conditions water turned into this reservoir at a turbidity of 8 or 9 will become during the day slightly clearer by sedimentation, but during this period no subsequent clarification whatever took place. The following table shows the influence of the wind in raising the turbidity in each of the reservoirs for seventeen days in March:

Turbidity record of Washington water supply, March 6-22, 1906.

\begin{tabular}{|c|c|c|c|c|c|c|c|c|c|}
\hline \multirow[b]{2}{*}{ Day. } & \multicolumn{4}{|c|}{ Turbidity of water at- } & \multirow{2}{*}{$\begin{array}{l}\text { Tur- } \\
\text { bidity of } \\
\text { effluent } \\
\text { after } \\
\text { filtra- } \\
\text { tion. }\end{array}$} & \multirow{2}{*}{$\begin{array}{l}\text { Tur- } \\
\text { bidity of } \\
\text { water in } \\
\text { the dis- } \\
\text { tributing } \\
\text { reser- } \\
\text { voir. }\end{array}$} & \multirow{2}{*}{$\begin{array}{l}\text { Maxi- } \\
\text { mum } \\
\text { wind } \\
\text { velocity } \\
\text { (miles } \\
\text { per } \\
\text { hour). } a\end{array}$} & \multirow{2}{*}{$\begin{array}{c}\text { Total } \\
\text { wind } \\
\text { during } \\
24 \text { hours } \\
\text { (miles). }\end{array}$} & \multirow{2}{*}{$\begin{array}{l}\text { Direction } \\
\text { of wind at } \\
\text { time of } \\
\text { maximum } \\
\text { velocity. } a\end{array}$} \\
\hline & $\begin{array}{c}\text { Great } \\
\text { Falls } \\
\text { intake. }\end{array}$ & $\begin{array}{c}\text { Dale- } \\
\text { carlia } \\
\text { Reser- } \\
\text { voir. }\end{array}$ & $\begin{array}{c}\text { George- } \\
\text { town } \\
\text { Reser- } \\
\text { voir. }\end{array}$ & $\begin{array}{c}\text { Wash- } \\
\text { ington } \\
\text { Reser- } \\
\text { voir. }\end{array}$ & & & & & \\
\hline & 820 & 250 & 70 & 35 & 5 & & 14 & 146 & South. \\
\hline & 180 & b 350 & 110 & 40 & 5 & & 11 & 77 & South. \\
\hline & 120 & $b 250$ & b 120 & 45 & 5 & & 9 & 110 & North. \\
\hline & 110 & $b 250$ & b 120 & 65 & 5 & & 24 & 233 & West. \\
\hline 10. & 95 & b 350 & b 110 & 80 & 5 & - & 31 & 327 & West. \\
\hline & 130 & $b 350$ & b 100 & 80 & & & 15 & 154 & South. \\
\hline 12 & 50 & b 300 & 100 & 80 & 6 & & 26 & 278 & Northwest. \\
\hline 13. & 35 & b 180 & 90 & 6 & 7 & 7 & 12 & 168 & Northeast. \\
\hline 14. & 26 & $b 180$ & 70 & 80 & $\therefore$ & 7 & 11 & 16 & Northeast. \\
\hline 15. & 20 & $b 250$ & 55 & 90 & 0 & 8 & 21 & 26 & Northeast. \\
\hline 16. & 65 & b 350 & 45 & 60 & 0 & 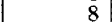 & 18 & 183 & Northwest. \\
\hline 17. & 40 & $b 250$ & 50 & 55 & 9 & 8 & 24 & 274 & Northwest. \\
\hline 18. & 28 & b 250 & 45 & 50 & & & 11 & 138 & Northwest. \\
\hline 19. & 22 & $b 180$ & 35 & 53 & 9 & & 13 & 141 & Southeast. \\
\hline & 22 & b 180 & & oe & 0 & 0 & 31 & 380 & Northwest. \\
\hline & 35 & & 28 & 1 & & & 18 & 248 & South. \\
\hline & 40 & $b 180$ & 26 & $?$ & & & 22 & 302 & Northwest. \\
\hline
\end{tabular}

$a$ Wind velocity and direction taken from records of the U. S. Weather Bureau. $b$ Reservoir not in service on account of high turbidity.

During January, 1906, for a period of more than a week, the turbidity, both in the river and in the Dalecarlia Reservoir, was low, but there was a high increase in that of both the Georgetown and Washington reservoirs. The wind velocities were low, but the wind was constant, the prevailing directions being from the west and northwest. The high turbidity of the effluent after filtration is due to only a portion of the filter being in operation. 
Turbidity record of Washington water supply, January 13-19, 1906.

\begin{tabular}{|c|c|c|c|c|c|c|c|c|}
\hline \multirow[b]{2}{*}{ Day. } & \multicolumn{4}{|c|}{ Turbidity of water at- } & \multirow{2}{*}{$\begin{array}{l}\text { Turbidity } \\
\text { of effluent } \\
\text { after } \\
\text { filtration. }\end{array}$} & \multirow{2}{*}{$\begin{array}{l}\text { Maxi- } \\
\text { mum } \\
\text { wind } \\
\text { velocity } \\
\text { (miles } \\
\text { per } \\
\text { hour). }\end{array}$} & \multirow{2}{*}{$\begin{array}{c}\text { Total } \\
\text { wind } \\
\text { during } \\
24 \text { hours } \\
\text { (miles). }\end{array}$} & \multirow{2}{*}{$\begin{array}{l}\text { Direction } \\
\text { of wind at } \\
\text { time of } \\
\text { maximum } \\
\text { velocity. }\end{array}$} \\
\hline & $\begin{array}{l}\text { Great } \\
\text { Falls } \\
\text { intake. }\end{array}$ & $\begin{array}{l}\text { Dale- } \\
\text { carlia } \\
\text { Reser- } \\
\text { voir. }\end{array}$ & $\begin{array}{l}\text { George- } \\
\text { town } \\
\text { Reser- } \\
\text { voir. }\end{array}$ & $\begin{array}{l}\text { Wash- } \\
\text { ington } \\
\text { Reser- } \\
\text { voir. }\end{array}$ & & & & \\
\hline $\begin{array}{l}12 \ldots \ldots \\
13 \ldots \ldots \\
14 \ldots \ldots \\
15 \ldots \ldots \\
16 \ldots \ldots \\
17 \ldots \ldots \\
18 \ldots \ldots\end{array}$ & $\begin{array}{l}26 \\
35 \\
28 \\
35 \\
35 \\
30 \\
30\end{array}$ & $\begin{array}{l}45 \\
28 \\
28 \\
28 \\
35 \\
35 \\
30\end{array}$ & $\begin{array}{l}50 \\
55 \\
85 \\
80 \\
70 \\
60 \\
45\end{array}$ & $\begin{array}{l}70 \\
65 \\
60 \\
60 \\
60 \\
60 \\
55\end{array}$ & $\begin{array}{l}20 \\
20 \\
715 \\
12 \\
10 \\
11\end{array}$ & $\begin{array}{l}15 \\
16 \\
10 \\
17 \\
28 \\
20 \\
17\end{array}$ & $\begin{array}{c}170 \\
248 \\
122 \\
203 \\
226 \\
183 \\
203\end{array}$ & $\begin{array}{l}\text { West. } \\
\text { North. } \\
\text { West. } \\
\text { South. } \\
\text { Northwest. } \\
\text { West. } \\
\text { Northwest. }\end{array}$ \\
\hline
\end{tabular}

During the summer and autumn, when the water is stratified, even wind velocities of 15 to 25 miles an hour usually have little effect if the weather is bright and warm. A five-day period of high windMay 6-10, 1906, during bright, warm weather-was accompanied by constant decrease in the turbidity of water in the reservoirs. A period of cloudy and rainy weather, very cool for the season, two weeks later, May 24-26, so far equalized the temperature of the water in the reservoirs that even lighter but steady winds showed an appreciable influence in preventing sedimentation, and the effluent after filtration, which normally should have shown a turbidity of 1 or less, rose to 3 . The same effects, and usually to a greater degree, are apparent on windy, cloudy days, especially when cold, throughout every month in the year. The comparatively low velocity of the wind which occasionally, especially during the winter months, causes high turbidity, suggests the use of wind-breaks of heavy-foliaged, coniferous trees around the reservoirs, or at least on those sides from which the prevailing winter and spring winds blow, which are now unprotected.

To be most efficient a wind-break must begin a few feet bàck of the water level of the reservoir. The shelter power of a wind-break is variously estimated, but for winds of low velocity such as prevail here, even a low wind-break would lessen wind action for a considerable distance from the edge of the reservoir. The overturning of the water would require higher winds and would be less complete. Libocedrus or a mixture of Libocedrus and Norway spruce would probably be sufficiently tolerant not to become open below until 50 feet high, when they could be underplanted with a more tolerant species, or rows of young trees could be planted behind them. These species will also probably stand the warm summers and autumns of Washington, on account of the high humidity. Hemlock would be an ideal tree but for its slow growth, while the native red cedar has a desirable shape, but is too small and too intolerant.

The Dalecarlia Reservoir, where the raw water is received from the Potomac; and where the first settling of the coarsest matter in 
suspension takes place, is an irregularly shaped basin with an extreme length, including the narrow arms, of more than half a mile. The depth of the water in many of the arms and over a considerable portion of the body of the reservoir, owing to settling up by the sediment which has been deposited from the water for many years, is less than 12 feet, and at the end of one of the arms it is even more shallow, the bottom sloping up from a depth of about 9 feet to the shore. This reservoir occupies a deep valley, with partly wooded hills rising more or less abruptly from the edges of the reservoir to heights of 50 to 200 feet, the summits of the hills lying from one-eighth to one-half mile distant from the reservoir. The reservoir valley is entirely open toward the south end, through which it drains into the river, and at the northwest end, where it is fed by a small stream. There are several other deep diverging gorges, two of which are sufficiently deep to hold arms of the reservoir.

The highest hills, rising more than 200 feet above the surface of the water in the reservoir, lie to the north and east, but their summits are about half a mile distant from the reservoir, and probably only the steep lower slopes, with a height not exceeding 100 feet, afford any protection to the reservoir as a wind barrier. On the south and west sides the hills are only about 65 feet above the surface of the water, and on account of the great length of the reservoir they protect less than half of it from southwesterly and westerly winds. The greater portion of the west end of the reservoir is exposed to winds which come up the open south valley. A portion of the west end of the reservoir is likewise unprotected from northerly winds, which would follow the north prong of the valley, or from northeasterly winds, which would follow the east prong of the valley.

The rebound of the wind from the steep slopes on the eastern side of the reservoir and the wind currents which follow the gorge-like valleys possibly explain the quick response of the water to winds of even low velocity. The period when this agitation is most constant and most pronounced is the season of defoliation of the hardwood timber on the surrounding slopes, when these trees are of least efficiency as a windbreak. It is pointed out by Professor Bigelow, of the Weather Bureau, that local windstorms frequently follow these narrow, gorge-like valleys, either in converging toward Potomac River or in spreading from it.

This constant wind action during the spring at the Dalecarlia Reservoir can be reduced by the construction of breakwaters, one across the northwestern arm and one across the southern arm. The tops of the breakwaters should be 20 feet wide, and al double row of evergreen trees should be planted alternately along each, which will still further decrease the amount of water surface exposed to the 
full sweep of the wind, by protecting parts of the body of the reservoir from winds which come through the southern and northern gorges. Similar breakwaters could be placed across the other arms and one across the middle of the reservoir, the tops of all being planted with a double row of evergreen trees. Every contraction of the large unbroken water surface, which is now offered for wave action, as well as any diversion of the wind from the surface of the water, will be beneficial. It is desirable to have the settling which takes place in this reservoir as perfect as possible irrespective of whether the sides of the reservoir are paved or not, since the clearness with which the water leaves this reservoir in large measure controls the condition of the effluent of the filter. Undisturbed subsidence of the coarser silt is very desirable, as has been previously explained, since when this takes place much of the very fine suspended matter, including bacteria as well as the clay particles, is carried to the bottom by the silt as it descends.

The Georgetown Reservoir is situated about 200 yards north of Potomac River, on the south slope of the river hills. It is about 75 feet above the river, while to the north the hill gradually rises, within the distance of a mile, about 200 feet higher than the reservoir. It is entirely exposed to winds from the northwest and west, which come down the river gorge, and to those from the east, southeast, and south, which come up the river. On account of the gentle slope of the hills to the north the shelter afforded by them is slight. A breakwater separates the reservoir, which is nearly a third of a mile long, into two lagoons and lessens the violence of wind action, especially under high northwest winds. The southern and southeastern sides of the reservoir are formed by high banks, which have been built up and are rather steep on the outside. Except at a few points along these banks, where some additional banking to broaden the crest might be necessary, there is room around the entire reservoir for a double row of trees for a windbreak, and in places for three or more rows. Protection against wind is especially desirable on the west, northwest, and southwest sides of the reservoir, since these are the directions of the prevailing winds during winter and spring, when the water is in an unstable condition and lacks adequate thermal stratification to prevent overturning. The planting of the breakwater is also desirable.

The Washington City Reservoir is situated at the northeastern edge of the city, on an abrupt rise about 120 feet above the river and more than 2 miles distant from it. To the southwest and south the country is lower than the reservoir, and the wind has no check when coming from these directions. To the north and west nearby buildings and the gently sloping hills on which Petworth and the Soldiers' Home are situated rise much above the reservoir. The slope of 
these hills, however, is so gradual that their protective value is doubtful. The reservoir, which has a surface of about 45 acres, is about one-fourth mile long and crescent shaped, and since its longer dimension lies northwest and southeast, both the high northwesterly winds of winter and the southeasterly winds of early spring have unchecked play upon the surface. At times when the water is unstratified a breakwater in the center would lessen churning from wind. Its sides should be paved and its top sufficiently widened for two rows of trees to be planted. Windbreaks of three rows of trees should be planted on the north, west, and south sides of the reservoir. On the northeast side the filter beds are so close to the reservoir that not more than two rows can be planted. 



\section{THE EFFECT OF SOME INDUSTRIAL WASTES ON FISHES.}

By M. C. Marsh.

\section{INTRODUCTION.}

At the instance of the United States Geological Survey, through which most of the samples were furnished, the experiments herein detailed were made by the United States Bureau of Fisheries for the purpose of learning the approximate concentrations at which the various industrial and other pollutions flowing into the Potomac basin are fatal to the food and game fishes of the region. The sources of the industrial wastes with which experiments were made are discussed on pages 191-246.

The species on which the observations were chiefly made are the large-mouthed black bass (Micropterus salmoides Lacépède) and the yellow perch (Perca flavescens Mitchill). These were selected as typical species of the Potomac fresh-water fishes, comparatively hardy and prolific, and valuable both as game and food. The perch were young and vigorous individuals, about $15 \mathrm{~cm}$. in length, taken from the fish lakes of the Bureau of Fisheries in Washington. They were hatched naturally in Potomac River and had strayed through the screens of the ponds when small. The black bass were young fish of a length of 7 to $12 \mathrm{~cm}$. and were hatched and reared in these fish lakes.

The bass and perch were brought from the lakes in small lots at short intervals and held in flowing tap water, and may be considered equal in vigor and resisting powers to normal wild fish not affected by aquarium conditions. When any material difference of temperature existed between the water from which the fish were taken and the pollution or dilution into which they were transferred the change of temperature was gradually made.

In addition to these two species a few salmon and trout fry and others were used as indicated in the discussion. The "shiner" is Notropis hudsonius.

The reaction of water which will support fish life must be slightly alkaline. When the water becomes even slightly acid fishes can not live in it, and in experimenting with acid pollutions the alkalinity of 
the water used as a diluent of course affects the results. In all the present trials Potomac service water was used for diluting the pollutions and for the controls. Its alkalinity was determined at intervals during the winter by titration with decinormal sulphuric acid, methyl orange indicator, and varied from 46 to 52 parts per million of calcium carbonate equivalent.

\section{ME'THODS.}

The conditions under which foreign substances exercise an effect on fishes in the streams can not for the most part be exactly duplicated in the laboratory. A continual flow of water impregnated constantly with a known amount of the foreign matter under observation has not been practicable in these tests. The method followed consisted in making a known dilution of the pollution with Potomac tap water, introducing a small number of fish into the dilution held in open glass jars at the room temperature and leaving them until they died or until the experiment was otherwise ended.

All pollutions were tried with no aeration of the dilution save that occurring spontaneously at the surface, and many of them were in addition tried with artificial aeration by introducing at the bottom of the jar a continuous current of air broken into minute bubbles by passing it through linden plugs, as is usual in maintaining large aquaria. The volume of the diluted pollution used in a single trial was usually 10 liters, but varied from 5 to 30 liters. The fish were not fed while subjected to the trials. In the cases whereadilution failed to kill the trial was continued as long as circumstances allowed, in some instances two or three months. Controls were carefully carried and are separately discussed.

It is obvious that these laboratory conditions differ in some important particulars from those prevailing in polluted streams. In the first place, the fishes can not be subjected for very long periods to the influence of the pollution, for other causes will terminate the experiment, and thus the long-contimued influence of very diluted pollutions which are not fatal for short periods are not covered. The aeration which occurs in flowing streams is greater than in standing dilutions and less than in dilutions artificially aerated as described, and aeration im many cases modifies considerably the polluting agent. The fish in jars are of course held under very unnatural conditions as to their immediate surroundings, particularly in the matter of space, but this does not complicate the matter much, since in aquaria with flowing water they may be held almost indefinitely.

Notwithstanding these differences between the experimental pollution and that naturally occurring, which obviously impair the accuracy of the results in the one case as applied to the other, it is equally obvious that information of value may be obtained and that reliable 
inferences may be drawn from such experiments. Standing dilutions without artificial aeration, which are quickly fatal, will be likewise fatal in natural waters, and standing dilutions copiously aerated, which are uniformly fatal even after considerable time, would be practically certain to kill in the same or shorter period in streams. Where unaerated dilutions fail to kill during exposures which terminate after a few days, it is not unreasonable to make some inference respecting the further dilution necessary to render the pollution harmless, remembering that an acclimating process will tend to offset the influence of the more prolonged exposure.

The temperature of the dilutions was substantially that of the controls.

A check jar in Potomac water was not set for every trial, but a series of checks was started at different times covering the whole series of pollution experiments. The results from the fish in the 10liter samples of Potomac water are given in the accompanying table and show that one perch and one bass will live for weeks in that amount of water standing without other than the natural surface aeration, without food and at a temperature between $15^{\circ}$ and $20^{\circ} \mathrm{C}$. They may be summed up briefly, as follows:

In three trials one small bass lived an average of 72 days in 10 liters of water, and none less than 50 days. In two trials a perch lived 44 and 46 days, respectively, in the former case nearly half the water evaporating, owing to the greater surface area. In two trials with two bass in each, one died after 55 days and the other in the same jar after 71 days. In the other jar one died after 69 days, while the other was alive at the close of this record, on the seventy-fourth day.

In eight trials with one bass and one perch together in 10 liters both survived an average period of 24 days. There is considerable individual variation, for among these eight trials, the fish being selected by chance from presumably healthy individuals in the supply tank, in one case the bass died on the third day, while in another neither fish died until the forty-third day. Some unnoticed injury may possibly account for the death of this bass within 3 days.

One bass 3 inches long in 5 liters of water in a McDonald hatching jar, with occasional aeration, lived 102 days, the surface area of water exposed to the air being $215 \mathrm{sq}$. $\mathrm{cm}$.

In general the fish in Potomac water survived much longer than the fatal periods for fish in polluted water and fulfilled their office as controls for the latter.

In the few cases where the pollution trial was carried on in a volume less than 10 liters accompanying controls were set of which no further statement is made. The control is implied if not expressed, and no uncontrolled results are included in the statements of fatal concentrations. 
Each pollution or refuse matter which is customarily thrown into the waters of the Potomac or its tributaries will bedescribed briefly, and the quantity or concentration necessary to kill stated as nearly as the experiments permit. The concentration is expressed as parts of the pollution sample in parts of the dilution. For example, " 1 in 100 " means 1 part of the pollution as received, plus 99 parts of water by volume or a 1 per cent solution.

The following table shows the results of experiments in 10-liter samples of Potomac water in glass jars; temperature of water, $4.5^{\circ}$ to $8^{\circ} \mathrm{C}$. at beginning, fluctuating with the room temperature, and never higher than $20^{\circ}$ C.; area of exposed surface of water, $500 \mathrm{sq} . \mathrm{cm}$. unless otherwise stated:

Results of experiments on fish in Potomac service water.

\begin{tabular}{|c|c|c|c|c|c|c|}
\hline \multirow{2}{*}{ Date of beginning. } & \multicolumn{2}{|c|}{ Number of-- } & \multirow{2}{*}{$\begin{array}{l}\text { Length } \\
\text { of bass } \\
\text { (cm.).a }\end{array}$} & \multirow{2}{*}{$\begin{array}{l}\text { Perch } \\
\text { lived } \\
\text { (days). }\end{array}$} & \multirow{2}{*}{$\begin{array}{c}\text { Bass } \\
\text { lived } \\
\text { (days). }\end{array}$} & \multirow{2}{*}{ Remarks. } \\
\hline & Perch. & Bass. & & & & \\
\hline January 9 . & & 1 & 7.5 & & 50 & \\
\hline Do.. & & 1 & 7.5 & & 92 & \\
\hline Do..... & 1 & & & $\ddot{44}$ & & $\begin{array}{l}967 \text { sq. cm. water surface exposed; } 4.3 \\
\text { liters evaporated. }\end{array}$ \\
\hline Do.. & 1 & & & 46 & & 2 liters evaporated; 4 c. c. of oxygen per \\
\hline $\begin{array}{l}\text { January } 21 \ldots \ldots \\
\text { January } 31 \ldots \ldots\end{array}$ & $\begin{array}{l}1 \\
1\end{array}$ & $\begin{array}{l}1 \\
1\end{array}$ & $\begin{array}{l}11 \\
11\end{array}$ & ii & $\begin{array}{l}3 \\
7\end{array}$ & A bandoned on third day. \\
\hline February 9. & & 2 & 11 & & $\begin{array}{l}55 \\
71\end{array}$ & \\
\hline Do.. & & 2 & 11 & & $\begin{array}{r}69 \\
b 78\end{array}$ & \\
\hline Do... & $\cdots$ & 1 & 10 & & b 78 & \\
\hline February 15. & 1 & 1 & $11-12$ & 13 & 13 & $\cdot$ \\
\hline Do....... & 1 & 1 & 16 & 17 & 50 & \\
\hline Do.... & 1 & 1 & $11-12$ & 34 & 34 & Perch spawned after thirty-first day. \\
\hline Do... & 1 & 1 & $11-12$ & 37 & 37 & \\
\hline Do.. & 1 & 1 & 9.5 & 41 & 55 & \\
\hline Do.. & 1 & 1 & $11-12$ & 43 & 66 & \\
\hline
\end{tabular}

$a$ The length of the individual perch was not taken, as they were all of nearly the same size-from 12.5 to $15 \mathrm{~cm}$.

$b$ Alive at close of record.

\section{PAPER AND PULP MILL WASTES.}

Spruce fiber.-This consists of coarse short fibers of spruce wood which do not pass through the screen that separates the finer fibers from the pulp made by grinding the logs. It was received from mills at Harpers Ferry, W. Va., in damp balls which lost about 38 per cent in weight by air drying.

One kilogram of the damp fibers in 28 liters of tap water failed to kill or apparently harm black bass during 10 days. During the first 4 days the fibers were held in a cheese-cloth bag, and during the remainder of the time they were free in the water without causing any mechanical injury, though the fish were annoyed when the fibers were occasionally distributed by stirring. The bass were about 6 inches in length, and the water was constantly aerated.

Spruce strips or shavings, partly bark.-These are sliverings from the outer portion of the log and include both wood and bark. 
Two hundred and fifty grams of the shavings, in 28 liters of water, with continuous aeration, was fatal to bass within 24 hours. Fifty grams was not fatal during 7 days, though the solution became very dark brown. A small constant flow of water prevents any fatal effect. Three hundred c. c. per minute passing through 2 kilograms of the shavings held in a 30 -liter jar failed to kill bass during 7 days, the brown tinge of extracted bark disappearing from the effluent after the first day.

Spruce bark.-One hundred grams of the bark stripped or cut from the above-mentioned shavings, in 28 liters of water, with aeration, killed bass within 19 hours. Fifty grams failed to kill during 3 days. The woody portion of the shavings without the bark has no effect.

Poplar chips and dust.-When logs are prepared for digesting to pulp by cutting instead of grinding, the product of the cutter is screened. That which passes the screen is the dust referred to and the larger pieces the chips. The two portions differ only in the size of the pieces, the dust consisting of particles larger than coarse sawdust, while the chips are much larger. This material was from the mill at Luke, Md.

The aqueous extract from both chips and dust is fatal, the latter more rapidly so, since it extracts more readily. One kilogram of the chips, free and floating in 28 liters of water, with aeration, killed 10 quinnat salmon fry within 17 hours, the solution being colored slightly brown. Five hundred grams killed 2 out of 10 fry within 22 hours, 8 within 30 hours, and all within 50 hours. Three hundred grams of the dust, wrapped in cheese cloth, killed 10 fry within 22 hours, the water taking on a brown tinge within one-half hour. One hundred grams killed 10 fry between the third and fourth days.

Five hundred grams of the chips were placed in 28 liters, with aeration, and 1 mummichog, 1 sunfish, 1 goldfish, and 1 shiner were introduced. Four days later 1 kilogram of chips was added, but after 10 days all were alive. Three hundred grams of dust, wrapped in cheese cloth, were placed in 28 liters, with aeration, and 1 each of the species named added. They soon showed symptoms of being affected, but did not succumb, and on the fourth day 500 grams of dust were added. On the seventh day the mummichog and shiner were dead, but after 10 days no more deaths had occurred.

The action of fish in poplar-wood extract was unusual and seemed to be characteristic. They came to the surface when its effects began to be felt, but not for lack of oxygen, since the water was constantly aerated. Later they swam about in a slanting position, and the salmon fry assumed the perpendicular, some of them whirling slowly or rapidly on a vertical axis before succumbing. 
Sulphite liquor. ${ }^{a-T h i s}$ is the spent liquor from the digesters in paper-pulp manufacture by the sulphite process, and is a dark-brown liquid of peculiar odor, markedly acid in reaction, and of 1.028 specific gravity at $11^{\circ} \mathrm{C}$. It is a complex of imperfectly known substances designated technically as "sulphonated lignone bisulphite compounds," and is a waste of considerable importance occurring in large amounts in some regions. These samples were from the West Virginia Pulp and Paper Company, Covington, Va. The following table gives the results in detail:

Effect of sulphite liquor on perch, bass, and brook-trout fry.

[10-liter dilutions for perch and bass; 1 to 3 liters for trout fry. Perch and bass trials with 1 individual of each species.]

\begin{tabular}{|c|c|c|c|}
\hline \multirow{2}{*}{ Dilution. } & \multicolumn{3}{|c|}{ Time (hours) required to kill一 } \\
\hline & Perch. & Bass. & Brook-trout fry. \\
\hline 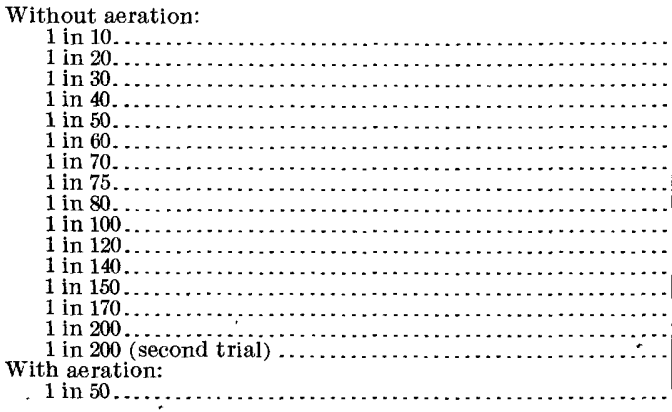 & 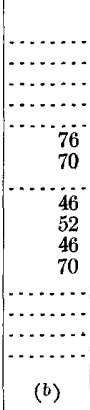 & 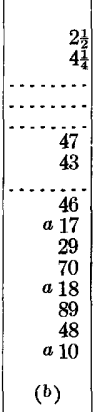 & $\begin{array}{l}22 \text { (all of } 12) \\
22-41 \text { (all of } 12) \\
48(9 \text { of } 12) \\
113(10 \text { of } 12)\end{array}$ \\
\hline
\end{tabular}

a Days

$b$ Not killed in 27 days.

The marked irregularity in the reaction of the fishes to the sulphite liquor is presumably due to their individual variation. Even with the rather extended series of trials the dilution which is certainly harmless to bass and perch can not be stated, but may be inferred to be slightly weaker than 1 in 200 . Brook-trout fry in the sac stage are more resistant to the sulphite than either bass or perch. Aeration of the dilutions lessens their toxicity very markedly, 1 in 50 with continuous artificial aeration failing to kill either bass or perch during 27 days, when the trial was abandoned. It has, in fact, been suggested that the harmful effects of sulphite pollution in streams were due to the abstraction of dissolved oxygen from the water by the oxidizable sulphite and the consequent suffocation of fishes. Determinations of the oxygen on sulphite dilutions protected from the air appear to show that no loss of dissolved oxygen sufficient to account for the death of fishes occurs. A long, narrow-necked flask, holding about 1,300 c. c., was filled to the brim with a 1 in 100 sulphite dilution at $3^{\circ}$

$a$ One sample of this was furnished by Dr. E. C. Levy, director of the laboratory of the city water department, Richmond, Va. 
C. (tap water) and kept for 23 hours at $14^{\circ} \mathrm{C}$. The dissolved gases were then boiled off and $6.5 \mathrm{c}$. c. of oxygen per liter were found, which is not much short of air saturation. Since there was a slight exposure to the atmosphere the experiment was repeated, using a 1 in 25 dilution at $14.5^{\circ}$ completely sealed and held for 19 hours, at the end of which period its temperature was $18^{\circ} \mathrm{C}$. and it contained 4 c. c. of oxygen per liter. A 1 in 25 dilution causes the death of all ordinary fishes in a few hours, but this could not occur from suffocation in water thus oxygenated.

Bleach sludge.-A clear, colorless, strongly alkaline liquid of 1.006 specific gravity at $9^{\circ} \mathrm{C}$. and containing a heavy white sediment. The clear liquor alone was used. Chloride of lime is the basis of the bleaching liquor. This waste comes from paper-pulp manufacture by the soda-lime process.

In unaerated dilutions 1 in 300 killed perch within 23 hours, and 1 in 400 on the ninth day; 1 in 500 was abandoned as not fatal after 14 days. In aerated dilutions 1 in 300 killed perch in 20 to 44 hours and 1 in 400 was not fatal during 5 days. Aeration does not materially affect the toxicity.

\section{TANNERY WASTES.}

"Sour bark liquor." a_A dark-colored acid waste containing small amounts of tannic acid from the aqueous extraction of oak bark; specific gravity 1.005 at $17^{\circ} \mathrm{C}$.

In unaerated dilutions 1 in 2 killed sunfish in 30 minutes; 1 in 10 killed bass, perch, and shiners in 16 hours, 1 in 20 within 24 hours, 1 in 30 within 30 hours, and 1 in 40 within 24 hours; 1 in 50 failed to kill bass during 65 hours. 1 in 30, aerated, failed to kill bass and shiners during 47 hours. The dilutions darken in color very rapidly with aeration and more slowly when standing without artificial aeration.

"Rocker sour bark liquor." a_A brownish, cloudy, acid liquor labeled "valueless in tannic acid ;" specific gravity 1.007 at $10^{\circ} \mathrm{C}$.

Unaerated, 1 in 30 fatal to bass in 41 to 68 hours; 1 in 60 failed to kill bass during 9 days, but was fatal to perch in 20 hours; 1 in 100 killed perch in 22 hours, 1 in 120 in 3 days, and 1 in 140 failed to kill perch in 4 days. Aerated, 1 in 20 fatal to bass in 18 hours; 1 in 30 not fatal during 6 days, but killed perch in 16 hours; 1 in 40 killed perch in 17 hours. Perch are much more susceptible to this liquor than bass. Like the other bark liquiors, the dilutions grow very dark by exposure to air.

"Dye-house liquor." a-A greenish liquid with some dark sediment about neutral in reaction; specific gravity 1.003 at $16^{\circ} \mathrm{C}$.; without pronounced taste. Dilutions have a yellow color and the undiluted 
liquor tinges the stock bottle a yellow color, which does not readily wash out.

Undiluted and unaerated, bass are killed in $1 \frac{1}{2}$ hours. Unaerated, 1 in 2 was fatal to bass and perch in 19 hours; 1 in 5 to bass in 24 hours; 1 in 6 to perch and bass in 43 hours; 1 in 8 to bass in 20 hours, to perch in 23 to 42 hours; 1 in 10 to bass in 11 days, but failed to kill perch in 12 days; 1 in 15 failed to kill perch in 31 days, but killed bass in 9 days. The fish killed by the stronger dilutions were dyed yellow in color.

"Bate." a-A straw-colored liquor; specific gravity 1.004 at $9^{\circ} \mathrm{C}$; alkalinity 400 parts per million ( $\mathrm{Ca} \mathrm{CO}_{3}$ equivalent); contains caustic lime.

The undiluted liquor killed a bass in 20 minutes. Unaerated, 1 in 5 killed bass in 16 hours, 1 in 6 in 4 days; 1 in 7 and 1 in 10 failed to kill during 4 days.

-) "Soak liquor" from hides. ${ }^{b}$-A colorless neutral liquid, of 1.002 specific gravity. It contains merely small amounts of sodium chloride soaked from the hides preserved in salt and appears to be harmless, the aerated undiluted liquor failing to affect bass during 4 days.

A sample of only 1 liter from Cumberland, Md., labeled "sample from soaks" is apparently of a similar nature, but was too small for extended trials. Undiluted and aerated, a bass was killed within 18 - hours, but the fish was anemic and unhealthy at the beginning. A perch died in a 1 in 3 aerated dilution after 15 days, but since this fish had survived previous experiments with other pollutions, and the dilution was less than 3 liters in volume, the result tends rather to show that the soak has no toxic properties.

Bass changed directly from fresh Potomac water into a solution of common commercial salt of 1.025 specific gravity, which is approximately the density of sea water, are killed within a few minutes. Bass were killed in less than 16 hours, perch within 42 hours, by transfer from fresh water to a 1.015 solution. The change from fresh water to a 1.010 solution failed to kill either during 14 days.

"Sour liquor tail handler."-An acid liquor of pinkish color and foul odor, extracted originally from hemlock bark; specific gravity, 1.013.

Unaerated, 1 in 15 killed bass in 4 hours; 1 in 20 in 6 hours; 1 in 30 bass, perch, and mummichogs in 18 hours; sunfish in 24 hours; 1 in 40 killed bass and perch in 17 hours; 1 in 50 killed bass in 24 hours, perch in 29 hours; 1 in 60 killed bass in 29 hours and failed to kill perch in 54 hours. Aerated, 1 in 10 killed a shiner in 30 minutes, bass in 2 hours; 1 in 30 killed a mummichog in 41 hours, perch in 19 hours; 1 in 35 and 1 in 40 killed perch and bass in 17 hours; 1 in 50 failed to kill perch and bass during 5 days. The dilutions 
are darkened by exposure to air, this taking place more rapidly when they are artificially aerated, which turns them grayish black.

"Lime liquor." a-A small sample of liquor showing caustic lime alkalinity.

Unaerated, 1 in 20 killed perch in $5 \frac{1}{2}$ hours, while 1 in 50 was not fatal during a trial of 30 days.

"Sample from hair washer." a-A cloudy grayish liquid, of 1.004 specific gravity at $14^{\circ} \mathrm{C}$., and alkalinity 630 parts per million.

Unaerated, 1 in 10 killed perch in less than 17 hours, 1 in 20 in less than 23 hours, and 1 in 50 failed to kill during 13 days.

Lime and sodium sulphide. ${ }^{b}-\mathrm{A}$ dirty-yellowish, strongly alkaline $\left(4,000\right.$ parts per million) liquor, specific gravity 1.015 at $7^{\circ} \mathrm{C}$.

Unaerated, with bass, 1 in 5 fatal in 30 minutes, 1 in 120 in 70 hours, 1 in 150 failed to kill during 43 days, 1 in 180 fatal in 90 hours; with perch, 1 in 10 fatal in less than 20 hours, 1 in 40 in 19 hours, 1 in 100 in 21 hours, 1 in 160 in 20 hours, 1 in 180 not fatal during 4 days, and 1 in 200 not fatal during 44 days. Aerated, 1 in 100 killed perch in 17 hours, 1 in 150 killed perch in one case in 24 hours, in another only after 8 days. Brook-trout fry in the sac stage are more resistant than either perch or bass. With unaerated dilutions 1 liter in volume, at $10^{\circ} \mathrm{C}$, 1 in 40 killed all of 12 fry in less than 17 hours, 1 in 50 killed 9 of 15 in 40 hours, and 1 in 80 killed 7 of 12 in 40 hours.

This liquor is the most toxic of all the tannery pollutions examined.

\section{DYE WASTES FROM KNITTING MILLS. $c$}

Spent chrome liquor--A brownish, transparent, strongly acid liquor, of 1.005 specific gravity at $10^{\circ} \mathrm{C}$.

Undiluted, the liquor killed bass in 1 hour. Unaerated, 1 in 10 killed bass in 3 hours, 1 in 20 in 20 hours, 1 in 30 in 16 hours, 1 in 40 in 45 hours, 1 in 50 in 44 hours, 1 in 60 not fatal in 45 hours; 1 in 30 fatal to perch in 24 hours, and 1 in 50 not fatal during 4 days. Aerated, 1 in 30 killed bass in 25 hours, 1 in 40 in 43 hours, 1 in 50 in 4 days, 1 in 60 in 43 hours, 1 in 70 in 48 hours; 1 in 30 and 1 in 60 failed to kill perch in 14 days.

This liquor acts as a coagulant of the turbidity in Potomac water.

Spent dye liquor.-A brownish, nearly neutral liquid, specific gravity 1.010 at $10^{\circ} \mathrm{C}$.

The undiluted and unaerated liquor was fatal to bass in 3 hours. Unaerated, 1 in 2 was fatal to bass in 20 hours, 1 in 5 in 17 hours, 1 in 7 in 3 days, and 1 in 10 only after 88 days. The bass in 1 in 10 received no food and its death was no doubt hastened by starvation."

a From tannery of United States Leather Company, Cumberland, Md.

$b$ From J. R. Cover \& Sons, Elkton, Md.

- F rom mill of Blue Ridge Knitting Company, Hagerstown, $\mathrm{Md}$. 
This dilution, though of a marked brownish-yellow color, is probably entirely harmless, and the result may be regarded as a control. Unaerated, 1 in 8.5 failed to kill perch during 8 days.

\section{SEW AGE.}

Sewage from human habitations is fatal to fishes on account of the exhaustion of the dissolved oxygen caused by the luxuriant growth of aerobic bacteria. Ten liters from the Seventeenth street canal in Washington killed bass and perch in less than 17 hours when the sewage was not aerated. Another portion aerated artificially failed to kill during the 53 hours in which the fish were kept under observation. A sample from the James Creek canal, unaerated, killed perch and bass at the end of 16 hours. With aeration no deaths or distress occurred during 48 hours. In the unaerated samples the fish give evidence of suffocation, leaping about spasmodically and then sinking weakly to the bottom as if exhausted. Oxygen determinations after the death of the fish showed about 1 c. c. per liter, and a sample in which no fishes had been held contained scarcely more.

\section{WASTES FROM MANUFACTURE OF ILLUMINATING GAS.}

Illuminating gas is itself markedly fatal to fishes. Gas from the service pipes was allowed to bubble into 20 liters of tap water near the surface for 3 to 4 minutes, and the resulting solution killed a perch within 20 hours.

\section{WASTES FROM THE WATER-GAS PROCESS.}

Filter effuent.-A cloudy grayish liquid with a moderate odor of gas; specific gravity 1.00 at $24^{\circ} \mathrm{C}$. This is the effluent from filter beds which remove the tarry oils at the plant of the Washington Gas Light Company.

Undiluted and unaerated, it was fatal to bass within 6 minutes. Unaerated, 1 in 10 killed bass in 22 hours, 1 in 20 killed perch in 68 hours, 1 in 30 failed to kill perch during 32 days, and in 1 in 50 a perch spawned after 24 days and died the next day. Control perch usually died after spawning.

Tar from wells.- This sample consisted of two parts, a floating black tarry liquor and a grayish watery liquid beneath.

The lighter liquid, which floats diluted 1 in 1,000, unaerated, killed a perch in a few minutes, and 1 in 100,000 caused evident distress within 9 hours, but did not kill until the fifth day.

The heavier grayish liquid, diluted to 1 in 40, unaerated, was fatal to perch within a few hours, while 1 in 80 failed to kill during 34 days and 1 in 100 had no effect during a trial of 21 days.

Tarry liquor.-A black, tarry, strongly aromatic liquor, lighter than water; specific gravity 0.95 at $21^{\circ} \mathrm{C}$. It has the highest toxicity 
of all the wastes of whatever nature with which experiments were made. It does not visibly mix with water, but spreads out in a film on the surface. The dilutions were made by volume, as in other cases, though evidently only a small portion of the liquor attains solution in the water.

Eight solutions stronger than 1 in 100,000 were first tried, but all were fatal in a few minutes or hours. Unaerated, 20 liters of 1 in 100,000 killed perch in 102 to 117 hours; 1 in 200,000, in 100 to 115 hours; 1 in 300,000, in 52 to 67 hours, and 1 in 400,000 killed a perch in 12 days, but failed to kill bass during 41 days. A solution of 1 in 500,000 was made up by weighing off 40 milligrams of the liquor in a watch glass and placing it with the glass in 20 liters of water. Two perch lived in this for 24 days, when one spawned and both died the next day. The weather had become warm and the temperature of the dilution reached $19^{\circ} \mathrm{C}$. A dilution of 1 in 500,000 may be considered practically harmless to perch and bass.

Aeration reduces markedly the poisonous effect. Aerated, 1 in 60,000 killed one perch in 24 hours, another after 3 days; 1 in 80,000 failed to kill during 11 days, and 1 in 100,000 during 9 days.

The sealing of the water from contact with the air, by means of the surface film, may possibly contribute slightly to the harmful effects in the higher dilutions not artificially aerated. That the substance is tremendously poisonous, however, is evident from the fact that even dilutions as weak as 1 in 40,000 kill in a very few hours, long before the exhaustion of oxygen could play a part. Moreover, the symptoms at death are manifestly not those of suffocation. Nearly all the fishes dying from gas wastes in the higher dilutions display characteristic movements. There is a rapid nervous fluttering of the fins, particularly the pectorals, with rapid respiration, and the body may assume the perpendicular. They sometimes appear to be dying for days before they finally succumb.

\section{WASTES FROM THE COAL-GAS PROCESS.}

Tar from wells.-This is ordinary coal tar, a thick black liquid with the typical odor. When dropped into water, the main portion of the drop sinks, while a lesser part separates and spreads gradually into a surface film.

The dilutions were not made volumetrically. The amount desired was weighed in drops on a strip of bristol board and then smeared in a thin layer and the strip placed in the measured quantity of water, which was stirred thoroughly. Only unaerated dilutions were used. One of 1 to 4,000 (5 grams of tar in 20 liters of water) was fatal to perch within less than 19 hours, 1 to 66,666 was fatal to both bass and perch in 4 days, and 1 to 200,000 failed to kill perch during a trial of 34 days. 
Ammoniacal liquor.a-A nearly clear pink liquid of marked ammoniacal odor, specific gravity 1.029 at $14^{\circ} \mathrm{C}$.

Unaerated, 1 in 100 killed perch in 5 minutes; 1 in 1,000 , in 40 minutes; 1 in 2,000, in less than 18 hours, and 1 in 3,000 failed to kill during 24 days.

Effluent from ammonia sludge bed. ${ }^{b-\mathrm{A}}$ clear watery liquid with no very marked odor, specific gravity about 1.00 at $12^{\circ} \mathrm{C}$.

Undiluted and unaerated, the effluent killed bass in 18 minutes. Unaerated, 1 in 10 killed perch in 20 hours, and 1 in 100 was not fatal during 34 days.

\section{WASTES FROM BOTH WATER AND COAL-GAS PROCESSES.}

Lime from "purifiers."-This is a coarse gray powder consisting originally of quicklime and having a strong odor of illuminating gas. The gas is passed through large tanks of the substance in order to remove 'carbon dioxide.

Five grams in 10 liters of unaerated tap water caused distress to perch in a few hours and was fatal in less than 21 hours; 1 gram in 10 liters, unaerated, killed a bass within about 69 hours; 1 gram in 20 liters, unaerated, failed to kill bass during 41 days.

Calcium oxide alone is fatal to trout fry at about 18 parts per million.

Iron oxide from "purifiers."-Iron rust is used to purify the gas of sulphur compounds. Iron filings and small pieces are mixed with wet wood shavings or thin chips and allowed to rust. The material is held in large purifiers, through which the unrefined gas is passed. The sample received for the tests was of a dark-brown color, with a strong odor of gas.

Twenty grams in 10 liters of water, unaerated, killed a perch in less than 20 hours; 5 grams in 10 liters killed a perch in 29 hours; 4 grams in 20 liters was fatal to perch in 56 hours, and 2 grams in 20 liters failed to kill during 9 days.

\section{SUMMARY.}

In reviewing the effects of the various wastes of industrial processes in the Potomac watershed, it appears that a wide gap in poisonous properties exists between the liquid wastes which come from the manufacture of illuminating gas and those from all other sources. The most toxic of the latter are made harmless by the addition of a few hundred parts of water, while the tarry by-produsts from the gas works require hundreds of thousands parts of water before they are diluted to the point of safety.

$a$ From the Clapp Ammonia Company, Washington, D. C. It comes originally from the ammonia well of the gas-manufacturing company.

$b$ From works of the Clapp Ammonia Company, which recovers ammonia from the waste products of the coal process of gas manufacture. 


\section{N D E X .}

A.

Abram Creek, W. Va., measurements on, near Harrison

pollution of water of, field assay of mechanical analysis of .... sanitary analysis of

Abrams Creek, Va., pollution of _._. sanitary analysis of

Acknowledgments to those aiding -Adamstown, Md., stream pollution at. Adjusted drainage, meaning of -...Agriculture, turbidity of streams due to

See also Farm land.

Allegany Grove, Ma., stream pollution at

water at, mineral analysis of..sanitary analysis of

Alps, denudation in Ammonia, manufacture of, description of -....

manufacture of, pollution from Analyses, mineral, results of _-__- 290

See also particular places and 291, 296-298 streams.

Analyses, sanitary, results of --_-- 290-295

See also particular analyses.

Anthrax, dissemination of, by tannery wastes ..........

Antietam Creek, Md., basin of, population and area of _._. and area of $\ldots-248-249,253$ measurements on, at Hagerstown, Md

pollution on $\ldots$

station on, near Sharpsburg, Md., description of _-_ $82-83$ measurements at _..._._. 83-90 water of, mineral analysis of _-_ $\quad 297$ sanitary analysis of _____ $\quad 294$

Appalachian Mountains, age of _.... 11 geologic history of _._._._. 12-14

Area curves, construction and use of _ $\quad 21$ figure showing _._._._._. 25

Army, typhoid in $\ldots \ldots \ldots \ldots$ 255, 270-271

Ashe, W. W., on relation of soils and forest cover to Potomac water _._.

Assays, field, results of _._._____ 283-290 See also particular places and streams.
Atlantic, W. Va., stream pollution from Autogenous drainage, meaning of - -

Page. 215

B.

Bachman Valley, Md, precipitation at -...

Bacillus, typhoid. See Typhoid bacillus; Bacteria.

Bacillus coli in rivers, effect of temperature on

Back Creek, W. Va., basin of, population and area of _._ 248,253 measurements of, near North Mountain _... 91

pollution of _.. 228

settlement on

soils on _._. 309

water of, sanitary analysis of _- $\quad 293$

Backbone Ridge, Md., fires on_-_--- 323 soils on

Bacteria, facts concerning _-_ 261-267, $271,285-286239$ See also Typhoid bacillus.

Bailey Spring Run, Pa, water of _-- 232 Bakers Spring, Va., water from____ $\quad 236$

Baltimore and Ohio Railroad, beginning of relations of Chesapeake a $\mathbf{n} \mathbf{d}$ Ohio Canal and _._. 186-187

Barrelville, Md., stream pollution at $\quad 219$

Basic City, Va., station on South River at, description of siation on South River at, measurements at._._. $9:-94$

stream pollution at_._______ 235 -236 water at, sanitary analysis of __ $\quad 294$ water supply of _._._._._. 277

Bass, black, experiments on _._._ $337-348$

Basswood, distribution of _._._. 313 humus from

Battles, sites of

Bayard, W. Va., measurements of Buffalo Creek at _..__- 65

precipitation at stream pollution at__._._ $214,283,284$ water at, field assays of _.___ $\quad 287$ mineral analysis of _.___ $\quad 296$ sanitary analysis of _._._. 292

Bayard formation, soils from 
Bedington, W. Va., measurements on Opequon Creek near.-.

Beech, distribution of.

bumis from

Berkeley Springs, W. Va., stream pollution at.....-..-

water supply of

Berlin, typhoid in, deaths from

Bernard, S., report of, on Chesapeake and Ohio Canal____ 185-186

Berryville, Va., measurements on Crystal Run near....-

stream pollution at_._-_._._water supply of

Big Pool, Md., water near, field assay of

Big Run, W. Va., pollution of

Big Springs Run, Md., measurements on, at Charles Mills_.-

Birch, distribution of humus from

Bismarck, W. Va., fires near._._.

Blacks Run, Va., pollution of water of, sanitary analysis of

Black water formation, soils from

Blaine, Md. and W. Va., stream pollution at

Bleacheries, stream pollution from wastes from, effect of, on fishes_.

Bloomington, Md., measurements on North Branch of Potomac near

station on Savage River at, description of -_._-_- 43-44 measurements at Boettcherville, Md., precipitation at $\quad 35$

Bolster, R. H., on stream flow in Potomac basin

Borden Shaft. Md., conditions at.--_ 217

Boston, typhoid in, deaths from_-_- 268

Braddock Run, Md., pollution of_ 219-220 water of. field assay of _._.__- 288 mineral analysis of _._._._ $\quad 296$ sanitary analysis of

Brandywine, W. Va., stream pollution at... $2: 4$

Brewery wastes, pollution from 222 , 233,235

Bridgewater, Va., stream pollution

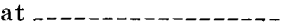

Briggs, Isaac, work of

Broad Run, Md., basin of, population and area of --- 251,254 measurements on, near Edwards Ferry _....... 179 soils on

Brooklyn, typhoid in, deaths from

Brunswick, Md., stream pollution

at _._._. $242-243$

water supply of -_-___-__-_ 277, 279

Buck Creek, Pa., fires on______ 321

Buckeystown, Md., stream pollution at
Buckton, Va., station on Passage Creek at, description of Passage Creek at,

station on Passage Creek at, measurements at

Buffalo Creek, W. Va., fires on _- 324,325 measurements on pollution of

timber on

water of, field assay of _.____-_ 287 mineral analysis of _..._._ 296 sanitary analysis of

water supply from

Bullskin Run, measurements on, near Kabletown, W. Va _._. 147

Burlington, W. Va., precipitation at_

\section{C.}

Cacapon River, W. Va., pollution of settlement on

Calvin Run, Md., water of, sanitary analysis of .......-

Capon Bridge, W. Va., stream pollution at

Carroll Creek, Md., measurements on, at Frederick

pollution of _._._...... 244-245

water of, sanitary analysis of _- $\quad 294$

Catoctin, Md., measurements on Catoctin Creek near.--

Catoctin Creek. Md., basin of, population and area of 250,254 measurements on, near Catoctin, $M d_{-}$

near Point of Rocks_____ 197

settlement on

soils on

water of, mineral analysis of _... 297 sanitary analyses of

Catoctin Creek, Va., basin of, population and area of

soils on

Catoctin Mountains. Va., soils at base of _...........

Cecil soils, character and distribution of _-_ $301,303-304,315$

timber on _______._. 304,320

turbidity from _._._._._._. $307-308$

Cedar Creek, Va., measurements on, near Strasburg. 135

settlement on

Chaffee, W. Va., stream pollution at -215

Chambersburg, Pa., precipitation at- 35

steam pollution at_______. 228

water at, field assays of _..... 289

mineral analyses of _._._- 296

sanitaly analyses of

water supply of______- $227-228,277$

Charles Mills, Md., measurements on Big Spring Run at_.--measurements on Little Conococheague Creek at 
Charles Town, W. Va., measurements on Evitt Run near.--

stream pollution at water supply of

Cherry Run, W. Va., sanitary analysis of

Chesapeake and Ohio Canal, history and status of

pollution on_.______.. 190, 222

view on

water of, field assay of _.___._. 288

Chester Gap, Va,, water from____. 241 Chester soils, character and distribution of

mechanical analysis of _._._. 302,304 timber on . . turbidity due to

Chestnut, distribution of _._._._ 302,304 , $313,322-323$

humus of _._._._._._._. $322-323$

Chewsville, Md., precipitation at_.. $\quad 35$

Chicago, typhoid in, deaths from _-- 268

Chloride of lime, use of, for destroying files

Cholera, transportation of, by water Civil war. See War.

Clark, H. W., and Gage, S. De MI., on occurrence of bacilli.

Clearspring, Md., precipitation at Coal, discovery and use of Coal gas. See Gas, illuminating. Coal mines, stream pollution from $215,217,219$

waters from, precipitation by -- 266 quality of ________ 283-286

Columbia formation, occurrence of -Conococheague Creek, Md., basin of, population and area of _... 248,253

measurements on, near williamsport _. _...... 91

pollution of

settlement on

soils on

water of, field assays of sanitary analyses of

West Branch of, pollution of _ 228-229 water of, field assays of _-_ $\quad 289$ sanitary analyses of _._._. 293

Consequent streams, definition of

Cooks Creek, Va., drainage area of $\quad 253$

pollution of

station on, at Mount Crawford, description of _._- 98-99 measurements at__._._._. 99-101

Cooks Mill, Pa., water at, field assays of

Corriganville, Md., water at, field assay of

Cotton dyeing, methods of 288

Cove Creek, Pa., soils on

water of, field assay of

306,307

Cresap, Thomas, settlement by Crimora, Va., stream pollution at.-

Crooked Run, Va., measurements on, near Riverton (3)
Crystal Run, Va., measurements on, near Berryville........

Cub Run, pollution of

C'ulps Run, Pa., pollution of

Cumberland, Md., measurements on

Evitts Creek near.-- 65

precipitation at_._._._._. settlement of

station at, on North Branch of Potomac, description of $-42,60-61$ measurements at_._._ 61-64 on Wills Creek, description of $\ldots$ measurements at - 58-60.65 view at 220 stream pollution at typhoid fever at water at and near, field assays

of _._. 288

sanitary analyses of water supply of Cumberland Valley, Md., soils of _... 304 305,312

Current meter, use of 23

\section{D.}

Dale Enterprise, Va,, precipitation at _..._. 35

Dalecarlia Reservoir, description of 332-333 history of _._._________ 271-272 sedimentation in _.......... 320

wind action in _._._. 331, $183-334$ Dams, sedimentation and, relations of -

Dans Mountain, Md., soils on _._._. 313

Davis, W. M., on Potomac River___ 16

Deep Run, W. Va., pollution in_-- 215-284 water of, field assay of ______ 287 Deer Park, Md., precipitation at ___- 36 Definitions of terms________ 26 Dekalb soils, character and distribution of - _ $301,311-312$

timber on

Dickerson, Md., measurements on Monocacy River near _- 179

Dickeys Run. Pa., pollution of $\ldots \ldots 229$ water of, field assay of _... _.. 289

Difficult Creek, W. Va., fires on measurements on, near Gor. mania, W. Va_..... 65

timber on

Difficult Run, water of, field assay of $\quad 287$ Discharge, computation of _._. . 23-24 Discharge of Potomac and of tributaries, comparison of _ 30-33

Discharge curves, construction and use of

figure showing

Distillery waste, stream pollution by _.___. 212, 220, 229, 231
Distributing reservoir, D. C., precipitation at 
District of Columbia, vital statistics Washington.

see also Washington.

Dobbin, W. Va., stream pollution at_-_._- 213-214 water at, field assay of ______- 287

Doubs, Md., stream pollution at__._ 242

Drainage, infiuence of rocks on map showing

Drainage, trellised, arrangement of Drown, T. M., on polluted ice

Dry River, water of ________ 237 Dyeing. discussion of _._._._. 208-209 methods of - _._._-_-_ 209-211 polution from wastes of _._. 209 $211,-221-222,228,230-233,245$ wastes from, effect of, on fishes_-_._-

E.

East Creek, D. C., diversion of Eckhart mines, coal from stream pollution from

Edwards Ferry, Md., measurements on Broad Run near...measurements on Goose Creek near...............

Elk Garden, W. Va., stream pollution at._..._..........

Elk Lick Run, W. Va., water of, field assay of

Elk Run, Va., measurements on, near Elkton

pollution of

station on, at Elkton, description of measurements at 110-112

water of, field assay of _._._. 287 mineral analysis of _._.__- 297 sanitary analysis of _._._._. 294

Elkton, Va., measurements on Elk Run near

measurements on South Fork of Shenandoah near.-..-

station on Elk Run at, description of .

123

123

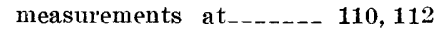

stream pollution at_._._. 239

water near, mineral analyses of _ $\quad 297$

water power at_._._._._._. 109

water supply of

Ellerslie, Md., stream pollution at.-- 219

Epidemics, typhoid, occurrence of_ 259-282

Ernstville, Md., Licking Creek near, measurements on _... 91

Erosion, river, process of _... 10-13, 15-16 results of _-______ $299-300,314-317$ See also Turbidity.

Evitt Run, W. Va., measurements on, near Charles Town

pollution of ...._... water of, sanitary analysis of _-

Evitts Creek, Md., basin of, population and area of

measurements on, near Cumberland.
Evitts Creek, Md., pollution of soils near _...

$$
\text { F. }
$$

Fairfax, Lord, possessions of, on Potomac _...-.

Fairfax Stone, Va., location of _._- 4

Fairhope, Pa., stream pollution at -- 218 water at, field assays of _._._. 289

Falling Spring Run, Pa., pollution of

water of, field assay of _-___- 289

Farm land, erosion of _._._. 300, 314-317

Fertilizer, use of wastes for 196 ,

$197,206,230$

Field assays, results of _._._._._. 283-290

Fifteenmile Creek, Md., measurements on, near Little Orleans

Filtration, effect of

Fires, forest, occurrence of ____ 320-321, $322,323,324$

Fish, experiments on _._._._- 340-348 experiments on, methods of - 338-340 injury to, by industrial pollution -- 193, 202, 205, 337-348

Fisheries, Bureau of, cooperation of 1,337

Flies, data on _._. 256-257

insecticides for $\ldots \ldots \ldots \ldots$ 257-258

typhoid spread by

Flint Run, Va., measurements on, near Front Royal

123

Floods near Washington, descriptions of _._____ 179-182

Flowing Run, W. Va., measurements on, near Millville _.-- 147

Flowing Run, Va., pollution of _-_- 242

Flowing Spring Iiun, Va., water of, sanitary analysis of _- 294

Foley, Pa., stream pollution at_... 218

water at, field assay of

Forestry, Bureau of, cooperation of $\quad 1$

l'orestry map of Potomac basin_._- 316

Forests, character and distribution of _..._. $302,304,306,310$, $312,313-311,317-328$

clearings in, extension of _._ $327-328$ distribution of, map showing-- 316 effect of, on melting snow on stream fiow fires in _._._. $320-321,322,323,324$ protective character of _._._. $326-327$ types of, description and disiri-

bution of ._.

water supplies from

Fourmile Creek, Pa., fires on_-__- 321

Franklin, W. Va., soils near.

stream pollution at_______ 224 water at, sanitary analysis of_ 292 water supply of

Frederick, Md., measurements on Carroll Creek at_._.- 179

precipitation at__._. 36

settlement of 
Frederick, Md., station on Monocacy River near, description of Monocacy River near,

station on Monocacy River near, measurements at_-- 162-172 water power at_._._._._ 172

water supply of

Frederick Junction, Md., stream pollution at.......

Freezing, effect of, on flow

Front Royal, Va., measurements on Flint Run near..--

measurements on Gooneys Creek near

station on South Fork Shenandoah at, description of

$115-116$ measurements at _...... 116-123 stream pollution at__-___._- 241 water supply of _._._._. 277

Frostburg, Md, coal mines at_._- 6 typhoid fever at 217 water supply of Fruit, typhoid fever transmitted by $\quad 255$ Fuller, G. W., and Russell, H. L., on typhoid germs

G.

Gage, s. De M, and Clark, H. W., on typhoid bacilli Gages, description and use of _._._ 23,29 Gaging stations, list of _._._._-_ 42 Gas, illuminating, effect of, on fishes _... $346-348$ manufacture of, description of 203-206 pollution from $206,222,228,230,231,240,245$

wastes from, effect of, on fishes -

Geographic history of Potomac-_- 7-22 Geologic history in Potomac basin _- 11-12 Georges rreek, Md., basin of, population and area of _-_- 246,252 pollution of _-_ $203,216-217,220,285$ settlement on soils on
station on, at Westernport, description of 55 measurements at_- 55-57,65 trough of water of, fleld assays of _...mineral analysis of sanitary analysis of Georges Creek Coal and Iron Co., de velopment by

Georgetown Reservoir, description of history of sedimentation in

wind action in

Germs. See Bacteria.

Gerstell, Md., station on North Branch Potomac near, measurements at

Gettysburg, Pa., precipitation at soils near. stream pollution at
Gettysburg, Pa., water at, field assays of _-______ 289-290

water supply of _._._._._. 277

Glade Run, Md., water of, field assay of -

Glasgow, typhoid in, deaths from Gooneys Creek, Va., measurements on, near Front Royal..

Goose Creek, Md., basin of, population and area of measurements on, near Edwards Ferry, Md 179

pollution of _-_._-_._-_-_-_ 245-246 soils on _._._._._._ $302,303,304$

water of, sanitary analysis of -- 294

Gorman, Md., water at, field assay of

Gormania, W. Va., measurements on Difficult Creek at measurements on North Branch of Potomac at._...measurements on Stony River near -

stream pollution at_-_-_-_-_ 214, 284 water at, field assays of ___ 287 sanitary analysis of

Graded stream, definition of _... 10 Grantsville, Md., precipitation at__. $\quad 36$ Great Cacapon, - W. Va., measurements of Great Cacapon River near....-

station on Potomac River at, description of ...-...measurements at 7

10

Great Cacapon River, W. Va., basin of, population and area of p 247,253 description of _._._._._. 226-227 measurements on, near Great Cacapon _._. 90 pollution of water of, mechanical analysis of __- 297 quality of 318 sanitary analysis of

Great Falls, Md., low-water fiow at - 278

pollution at_. 290

precipitation at_._._._. 36

view of 182

Great North Mountain, Va., flres on. 322 Great Tonoloway Creek, Ma., basin

$$
\begin{aligned}
& \text { of, population and area } \\
& \text { of } \\
& \text { measurements on, near Han- } \\
& \text { cock }
\end{aligned}
$$$$
\text { soils on }
$$$$
\text { water of, field assay of }
$$

Greencastle, Pa., water at and near, field assays of 289

water supply of __._._. 277

Greenspring, W. Va., water at, san- . itary analysis of _..._ 292

Greenspring Furnace, Md., precipitation at.......

Grove Hill, Va., water power at_.--

91
309
289

289
277

292

36
109


H.

Page.

Hagerstown, Md., measurements on Antietam Creek at measurements of Marsh Run at

precipitation at

stream pollution at _..._._. 233-234

typhoid fever at

water supply of _._._._._._. 233,277

Hagerstown clay, character and distribution of

$305-307,315$

erosion of 307

mechanical analysis of _-_._ 306-307

Hampshire formation, soils from _- 311

Hancock, Md., measurements at, on Great Tonoloway Creek measurements at, on Potomac River -

measurements near, on Tonoloway Creek

on Warm Spring Run ___ 91

precipitation at__._._._. 37

stream pollution at________ 227

typhoid fever at__________ 272

water at and near, field assay of $\quad 289$

Happy Creek, Va., pollution at_.....

water of, sanitary analysis of

Harney, Md., precipitation at

Harper, Robert, settlement by

Harper's Ferry, W. Va., measurements of Shenandoah River at

precipitation at

settlement at_................

station on Potomac River at. description of

measurements at

stream pollution at $--2-0-2-234-235$

view of

water near, mineral analysis of.- $\quad \mathbf{2 9 6}$

Harrisburg peneplain, character of..

Harrison, w. Va, measurements on Abram Creek, near.--

stream pollution at

water near, field assay of ___ $\quad 287$

Harrisonburg, Va., stream pollution

$$
\text { at _. }
$$

water supply of _._.__.___. 237, 277

Hawksbill Creek, Va., drainage area

$$
\text { of }
$$

pollution of -

station on, near Luray, Va., de-

scription of

measurements at $\ldots$ 113-115, 123

water of, mineral analysis of - 297

sanitary analysis of _._.- 294

Headwaters, description of _..... 7

Hemlock, distribution of 313

humus of

use of, in wind-breaks _._. 332

Henry, W. Va., stream pollution at 213 ,

water at and near, field assays

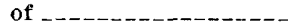

Hides. see Pelts.
Hightown, Va., soils near._... 306 History of Potomac basin, outline of $\quad 2-22$ Hite, Joist, settlement by Hoblitzel, Pa., water at, field assay

$$
\text { of _- } 289
$$

Hollow Run, Md., pollution of --_-- 240

Horse manure, fly breeding in_._._. 256

fly breeding in, prevention of _ $257-258$

Howard, John, exploration by _._ 4

Howard, L. O., flies investigated by 256-258

Howell Run, W. Va., water of, fleld assay of

Hubbard, W. Va., stream pollution at _._. 215

Humus, character of prevention of erosion by_._._ 300,306 water storage by _._._._._ 318-319 Hupp Spring Run, Md., pollution of_ 240 Hutton, W. R., measurements by-- 178 Hyndman, Pa., stream pollution at_ 219 water at, field assays of _... $\quad 288$ water supply of

I.

Ice, effect of, on flow pollution of, danger from

Illinois River, sewage in _._._._. 267 temperature observations on - 261-263 Indians, hostility of _._._. 3 Industrial wastes, effect of, on fishes _..._.

pollution from, by streams_.- 213-254 sources and character of _-_- 193-212 Iredell clay loam, character of ____ $\quad \mathbf{3 1 5}$ Isohyet, definition of

\section{J.}

Jackson Run, water supply from

James River, loss of, to Potomac, reason of _._._. Jennings formation, soils from _-_ 308,311 Jennings Run, Md., drainage to, figure showing pollution of ______ $219,273-275,286$ water of, field assay of _._._._. 288 mineral analysis of _.__ $\quad 296$ sanitary analysis of Jordan, E. O., on Illinois River_-_- 267 Jordan, E. O., and Zeit, F. R., experiments by, on typhold germ

Jordan Springs, Va., stream pollution at

260

\section{K.}

Kabletown, w. Va., measurements on Bullskin Run near. Keezleton, Va., stream pollution at Kemple Falls. Va., water power at.Kennebec Valley, typhoid in Kerosene, use of, in killing flies...Keyser, W. Va., measurements on New Creek near 
Page.

Keyser,W. Va., stream pollution'at_ 218, 285 water at and near, field assays of _................... sanitary analysis of water supply of

Kilmer Spring, W. Va., water from_ water of, field assay of

King, F. H., on porosity Kips. See Pelts.

Knitting mills, dye wastes from, effect of, on fishes

Knobly Mountains, W. Va., soils of Knoxville, Md., stream pollution at_ Kofoid, C. A., on water temperatures _..

Koontz Run, Md., pollution of _.__ $\quad 217$ Kreigbaum, Md., water near, mineral analyses of

L.

Lafayette formation, deposition of -Latrines, danger from

Laundries, stream pollution from_- 217 , $228,230,245$

Jaurel Creek, W. Va., water of, field assay of

Iaurel Fork, W. Va., timber on _- 313,324

Laurel Run, water of, field assay of _ 287

Leather tanning, fat from, use of -- 197 hair from, use of

lime in, use of _._._._._. 196-197

lime wastes from, use of

liquors for._._.

oil from__._._. 200

processes and wastes of _._._ 195-201

tan bark, spent, disposition of _- 200

water for, character of _____ 194-195

Leesburg, Va., soil at, analysis of $\quad 302$

stream pollution at_______ 245-246

water supply of _.______. 277

Levels, The, W. Va., soils of __- 310,311

Lewis, John, settlement by _..._._. 4

Lewis Creek, Va., drainage area of _- 253 pollution of 238

station on, near Staunton, Va., description of - 101

measurements at_.... 102-103

water of, sanitary analysis of $-\quad 294$

Lewis Run, Va., pollution of _._._. 241

Libocedrus, use of, for wind-breaks_ 332

Lick Run, W. Va., pollution of _-_-- 231

Iicking Creek, Md., basin of, population and area of -_- 248,253

measurements on, near Ernstville, $M a_{-}$

pollution of

soils on

water of, field assay of

Licksville, Md., South Tuscarora Creek near...............

Lime, chloride of. See Chloride of lime.

Iime, use of, in tanning _._._._. 196-197

Lime sludge, stream pollution from 206,283

Limestone soils, character and dis-

tribution of -
Lincoln, Va., precipitation at_....-

Page.

Iinden, Va., measurements on Wap pan Run near...-.--

Lineburg, W. Va., measurements on Sideling Creek near--

Linen dyeing, methods of

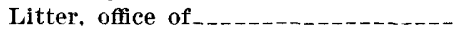

Little Antietam Creek, Ma., pollution of

water of, field assays of

Little Cacapon, W. Va., measurements on Little Cacapon River near

Little Cacapon River, W. Va.. basin of, population and area of _.

measurements on, near Little Cacapon

Iittle Conococheague Creek, Md, measurements on, at Charles Mills_.......

Little Cumberland Valley, soils in -Little Falls Branch, Md,, diversion of -

Little Orleans, Md., measurements on Fifteenmile Creek near _........

Liverpool, typhoid in, deaths from Lonaconing, Ma., stream pollution at typhoid fever at

water supply of

London, typhoid in, deaths from ... Long Bridge, Washington, D. C., tidal statistics at

Long Hollow, Md., soils in Lost City, W. Va., stream pollution at

90

269

217

217

277

269

43

310

226

253

Lost River, W. Ya., drainage area of settlement of

See also Moorfield River.

Lostland Run, W. Va., water of, field assay of

Luke, Md., stream pollution at $203,216,283$ water at, field assays of _._- 287-288 mineral analyses of _..._. $\quad 296$ sanitary analysis of _-_._-_ 292

Luray, Va., station on Hawksbill Creek near, description of _.

station on Hawksbill Creek near, measurements at _... 113-

115,123

$\begin{array}{lr}\text { stream pollution at } & \\ \text { water at, sanitary analyses of }-- & 294\end{array}$ water supply of

Lyon's mill, D. C., station on Rock Creek at, description of 173 station on Rock Creek at, measurements at $173-175,177-178$

M.

McConnellsburg, Pa., soils of stream pollution at water at, field assay of _....... water supply of -...--_-_McGaheysville, Va., stream pollution at _...-... 
McKnightstown, Pa., stream pollution at

Mallett, J. W., on Staunton water supply

Man, geologic action of

Manganese mining, pollution from --

Map, drainage, of Potomac system- Pocket.

Map, forestry, of Potomac system -_ 316

Map, rainfall, of Potomac system_ Pocket.

Map, topographic, of Potomac system _._._._._. Pocket.

Maple, distribution of humus of

Marion, Pa., precipitation at

Marsh, M. C., on effect of industrial wastes on fishes

Marsh Creek, Pa., pollution of _-___ 286

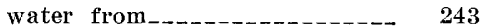
field assay of _-___-__-_ $\quad 289$

Marsh Run, Md., measurements on, at Hagerstown pollution of Martin Mountains, Pa., soils of Martinsburg, W. Va., precipitation at -...-

station at, on Opequon Creek. description of ..... measurements at

on Tuscarora Creek, description of measurements at

stream pollution at typhoid fever at water at, field assay of water supply of Maryland, vital statistics in, lack of Mash, whisky, pollution from Massachusetts, treatment of tannery wastes in

Massachusetts state board of health, on purification of wool scouring waste

Massanutten Mountain, Va., fires on Mechanical wood pulp. See Wood pulp.

Mercersburg, Pa, stream pollution at

water near, field assay of ____- 289 water supply of _________ 277,327

Merrimac River, Mass., occurrence of typhoid on

Micropterus salmoides, experiments on _.___- 337-348

Middle River, Va., basin of, poptlation and area of $--249,253$ measurements on, near Mount Meridian, Va_._......

pollution of -...-water of, sanitary analysis of Middleton, Va., stream pollution at_ Midland, Md., sanitary conditions at Milk typhoid fever spread by $258-259,270$

Mill Creek, D. C., diversion of _-_Mill Creek, W. Va., drainage area of measurements on, near Romney. water of, sanitary analyses of
Page.

Milldale, Va., measurements on Stonebridge Run at ---

Millstone, Md, water near, field assay of -

Millville, W. Va., measurements on Flowing Run at_..-station on Shenandoah River at, description of $\ldots$ 135-136 measurements at _... $31,136-146$ water power at_________ 147

Millwood, Va., measurements on Parker Creek at

Mine waters, coagulation and precipitation by

effect of, on Potomac River-- 283-286 Mineral analyses, results of _._. 290-291, 296-298

See also particular places.

Mines. See Coal mines.

Monocacy River, Md., basin of, population and area of -250 251,254

Moorefield, W. Va., measurements on South Fork of South Branch of Potomac at 179 pollution of settlement on soils on station on, near Frederick, description of _._._._. 161-162 measurements at_..___._ 162-172 water power at__._._._. 172 water of, mineral analysis of _- $\quad 297$ sanitary analyses of._._.- $\quad 294$

Mont Alto, Pa., stream pollution at 232 Monterey, Va., soils near._._._. 306 stream pollution at______._- 224 Morgan, Richard, settlement by Moss Bank Run, Md., pollution of _- 228 Mount Crawford, Va., station on Cooks Creek at, description of

station on Cooks Creek at, measurements at _.. 99-101

typhoid fever at__________ 237

Mount Meridian, Va., Middle River near, measurements on_ 108

Mount St. Mary College, Md., precipitation at

Mount Savage, Md., stream pollution at_._. 219

typhoid fever at_._._._._. 273-276 Mount Savage Run, Md., pollution of _. 
Muddiness. See Turbidity.

Munson, W. Va., measurements on Sleepy Creek near.

Murray, Doctor, on typhoid at Mount Savage, Md_--

Musca domestica. See Flies.

\section{N.}

Naked Creek, Va., measurements on, near Verbena

New Creek, $\mathrm{Md}_{\mathrm{s}}$, drainage area of measurements on, near Keyser. pollution of water of, field assays of sanitary analyses of -

New Haven, Conn., typhoid at _..

New Market, Md., precipitation at_ stream pollution at

New York, typhoid fever at Newport, Va, water power at North Branch of Potomac. See Potomac River, North Branch.

North Fork of Shenandoah. See Shenandoah, Nor t b Fork.

North Mountain, W. Va., measurements on Back Creek near -

North River (of Great Cacapon), W. Va., description of pollution of

North River (of Shenandoah), Va., basin of, population and area of _..._. 249,253

basin of, stream fiow in _._._. 98-108 measurements on, near Mount Meridian _... 108 pollution of station on, at Port Republic, de-

scription of - 103-104

measurements at _._._- 104-107

water of, sanitary analyses of _- $\quad 294$ water powers on

Notropis hudsonius, experiments on.. Nydegger Run, Md., water of, field

assay of

\section{o.}

Oaks, distribution of _.. $302,304,306,310$, $311,312,313,321-322$ humus from

Ocean, Md., sanitary conditions at _- 217

old Field, W. Va., precipitation at. stream pollution at

Opequon Creek, W. Va., basin of, population and area of $-248,253$

- measurements on, near Bedington pollution of settlement on station on, near Martinsburg, description of _-_._-_- $78-79$
Obequon Creek, W. Va., station on,

Page. near Martinsburg, measurements at _.____ $79-81$ water of, mineral analysis of _- 297 sanitary analyses of _._._. 293

Ordure, typhoid fever spread by__- 255

Organisms, microscopic, relation of bacteria and__.____ 264

Organic movements, course of $\ldots$

Outwater, Raymond, on loss by erosion

Oysters, typhoid spread by

$$
\text { P. }
$$

Packard, A. S., on fiy breeding ---

Paper-mill wastes. See Wood pulp.

Paris, typnoid in, deaths from_... 268

Parker, H. N., on Chesapeake and Ohio Canal_____. 183-190

on Potomac basin

on stream pollution, typhoid fever, and character of surface water_._._. 191-298

Farker Creek, Va., measurements on, near Millwood........

Passage Creek, Va., basin of, population of

measurements on, near Riverton

station on, at Buckton, descrip tion of.

measurements at

water of, mineral analysis of _- 296 sanitary analysis of _..... 294

Patterson, W. Va., measurements on Patterson Creek near.

Patterson Creek, W. Va., basin of, population and area of _._.

measurements on, at Patterson $\quad 65$

pollution of 223

settlement on

soils on 309

water of, mechanical analysis of $\quad 297$

Patterson Creek Mountains, W. Va., soils of _._. 305

Pawpaw, W. Va., measurements of Purslane Run near.--

stream pollution at $\quad 20$

water at, mineral analysis of -- 296 sanitary analyses of _.__ $\quad 293$

Pelts, classification and description of _-

Feneplain, definition of

Penn soils, character and distribution of _.___ $301, \because 04,315$

Pennsylvania, anthrax in $\ldots$

vital statistics in, inadequacy of 270

Pennsylvania Forest Reserve, establishment of _....... 326

waters of 227

Perca flavescens, experiments on $337-348$ Perch, yellow, experiments on Petersburg, W. Va., measurements on North Fork of South Branch of Potomac near 
Petersburg, W. Va., measurements on South Branch of Potomac near.

stream pollution at water at, sanitary analyses of -Philadelphia, Pa., typhoid in, deaths from

Fiedmont, w. Va., station on North Branch Potomac at, description of _._._._. 46-47

station on North Branch Potomac at, measurements at _...... 31, 47-54 stream pollution at________ 216 water at, field assays of ___ 287-288 water near, mineral analyses of 296 water supply of _._._._._._. 277

Pine, distribution of _.___._._- 302-303, $304,310,311,312,320-321$

effect of, on melting snow 325

humus from__._. 320-321

Point of Rocks, Md., precipitation

$$
\text { at _....... }
$$

Pocono formation, soils from

station on Potomac River at, curves at, plate showing description of ...._._. 148-149 measurements at $31,150-161,291$ water power at_..._._- 160-161

Pollution in Potomac River sys: tem basin _..._... 191-298

See also names of places, streams, manufactures, etc.

Poplars, wastes from, effect of, on fishes _.............

Population of basins tributary to Potomac _._. 246-252

Porosity, data on__________ 314-315

Port Republic, Va., station on North River at, description of _-_._-_ 103-104

station on North River at, measurements at_- 104-107

station on South River at, description of _... 94

measurements at _._._._._ 95-98

water power at_____ 109

Potomac River (main stream), basin of, description of _._- 7-9 basin of, pollution in _..._. 226-235, $242-246$

population and area of - - 247-256 stream flow in _- 78-91, 148-179 water supplies in floods on flow of, connection of typhoid fever and__._._._._ 278-279 chart showing Great Falls of, view of _.____ 182 measurements of, at Chain Bridge, D. C. at Great Falls, Mdd...... at Hancock, Md.
Potomac River (main stream), obstacles to navigation on pollution of _._._._._. 226,227 , $232,234,242-243,245-246$ view of _..... 222 profile of _... 9

plate showing _......... 182 sedimentation in station on, at Great Cacapon,

w. Va., description of _- 78

measurements at _..-- 78

at Harpers Ferry, W. Va., description of .....measurements at ...--

at Long Bridge, D. C., description of

at Point of Rocks, Md., curves at, ptate showing _...... 25 description of _____ 148-149 measurements at $31,150-160^{-}$ water power at___- $160-161$ valley of, description of _._._ 9 view on 188 water of, sanitary analyses of _. temperature of, relation of typhoid fever and _-_ 263-264 turbidity of use of, for drinking _.___ $\quad 277$ in fish experiments $339-340$ typhoid fever from_-_- 270

Potomac River (North Branch), basin of, description of _._.

basin of, population and area of _... 246-247, 252

stream flow in ......... 43-65 measurements of, near Bloomington, Md__._._. 65 ,

near Gerstell, Md______ 65

near Gormania, W. Va _-_ $\quad 65$

near Schell, W. Va_______ 65

near Twenty-first, Md__._ $\quad 65$ pollution of _._-_..._._. 203, 213-223 profile of, plate showing______ 182 settlement on soils on station on, at Cumberland, Md., description of $-42,60-61$ measurements at_.... 61-64 at Piedmont, W. Va., descrip-

tion of _. measurements at_- $31,47-58$ timber on water of, field assays of _.___ 287-288 mineral analyses of quality of _-_-_- $283-288,292,296$ sanitary analyses of _...- 292 turbidity of

I'otomac River (South Branch), basin of, description of _. $66,223-224$ 
Potomac River (South Branch), basin of, pollution in $223-226$ basin of, population and area of 247 , $252-253$

stream flow in $66-67$

measurements on, near Petersburg, W. Va near Romney, W. Va

North Fork of, drainage area of measurements on, near Petersburg, W. Va_...water of, sanitary analysis of

profile of

settlement on soils on

South Fork of, measurements on, near Moorefield, W. Va

station on, near Springfield, W. Va., description of _._ $66-68$ measurements of _____ 31, 68-77 water of, mineral analysis of _- $\quad 297$ sanitary analyses of _-_- 292-293

Potomac River system, age of _._._. 11 basin of, description of _._._. 7-9 development of _......._. 9-22

drainage map of _._._._._._. 8

forestry map of pollution in _._._._._. 191-298 population and area of $--246-252,291$ rainfall map of _._._.____- Pocket. stream flow in ........ 23-190 topographic map of ${ }^{\circ}$._._._. Pocket. water of, quality of _..._. 283-298 water supplies in

Precipitation. See Rainfall; Sediinentation.

Price, L. M., on typhoid at Mount Savage, Md_____- 273-276

Privies, proper construction of 258

Profile, ideal, description of

Profle of Potomac River, description of

plates showing

of Shenandoah River, plate showing - .

Pulp, wood. See Wood pulp.

Purslane Run, W. Va., measurements on, near Pawpaw-

Q.

Quality of surface waters $283-298$

\section{R.}

Railroads, sanitation of -

Rainfall, in Potomac basin, comparison of run-off and _._. $40-41$ data on

map showing construction of

Rating tables, construction and use of

Rattlesnake Run, Pa., water of
Receiving reservoir, Ma., precipitation at

Red Oak Mountain, W. Va, soils on $\quad 313$ Red Oak Run, W. Va., water of, field assay of -

Red-shale soils. See Upshur soils.

Reservoirs, sedimentation and wind action in

Rivers. See Streams.

Riverton, Va., measurements at_..measurements at, on Crooked Run _......... 135

on Happy Creek

on Passage Creek precipitation at station at, on North Fork of Shenandoah, deseription of _... 125-127 measurements at _-- 127-135

on Shenandoah River, description of

stream pollution at_..._..... 241 water at, sanitary analysis of _- $\quad 294$ water power at_-_._-_._- 109 water supply of $\quad 277$

Rock Creek (of Potomac), I. C., drainage area of _...- 254 station on, at Lyons Mill, description of _._._. 173 measurements at $\quad 173-175$, $177-178$

at Zoological Park, description of measurements at $174-177$

water of, fleld assay of Rock Creek (of Monocacy River), Md., pollution of _-- 243-244

Rocks, character of, influence of, on drainage _..._._. $8,15-16$

Romney, W. Va., measurements on Mill Creek near

precipitation at settlement of South Branch Potomac near, measurements on_..._ $\mathbf{7 7}$

stream pollution at

water of, sanitary analyses of $\quad 293$

water supply of

Romney slrale, soils from

Run-off, comparison of rainfall and_ 40-41 definition of See also particular drainage basins.

Russell, H. L., and Fuller, G. W., on typhoid germ

S.

St. Louis, typhoid in, deaths from -268 Salmon, experiments on Sand Run, Md., water of, field assay of Sandstone soils, character and distribution of _-_-_-_ 311-312 Sandy Ridge, W. Va, fires on _.... 321 
Page.

Sanitary analyses, results of _--- 290-295

See also particular places.

Savage Mountain, Ma., fires on soils on -

Savage River, Md., basin of, population and area of -- 246,252 station on, at Bloomington, description of

$43-44$ measurement of _._._-_-_ 44-46

water of, quality of _._._. 215-216

field assay of _._._._._. 287

mineral analysis of _._._-_ 296

sanitary analysis of -._-_- 292

Sawdust, stream pollution from

Schell, W. Va., measurements on North Branch Potomac near .......

measurements on Stony River near - -

stream pollution at

water at, field assay of

Schooley peneplain, character and history of

Schuler, Va., water power at__._- 109

Scope of paper_._._. 1

Scotland, Pa., stream pollution at $\quad 227$

Scrub Ridge, Pa., fires on

Second Culvert Run, Md., pollution

$$
\text { of - }
$$

Second-foot, definition of

Sedgwick, W. T., and Winslow, C.-E. A., on typhoid fever

Sedimentation, effect of, on germs 267,285 process of use of in industrial wastes_... 196, 203,206

Seneca, Md., measurements on Seneca Creek near._..._- 179

Seneca Creek, Md., basin of, population and area of _.._ 251,254 measurements on, near Seneca, Md

179

pollution of 246 soils on 302,303

timber on

turbidity of _._._._._._._. 307,313

water of, sanitary analysis of -- 294

Sewage, effect of, on typhoid germ_ 260 effect of, on fishes_._._._._. 346

Shaffers Run, Pa., water of, field assay of

Shale soils, character and distribution of _._.

Shenandoah, Va., precipitation at station on Antietam Creek near, description of _._._. $82-83$ measurements at ._._._- 83-90 stream pollution at_______ 234

Shaw, W. Va., stream pollution at _..._. 215,284

water at and near, field assays of _. 287

Shellfish, typhoid fever spread by_-- $\quad 259$
Page.

Shenandoah, Va., precipitation at_- 38

stream pollution at _._._._. 230

water power at

Shenandoah Junction, W. Va., stream pollution at____ 234

Shenandoah Mountain, fires on

Shenandoah River, basin of, pollution in _.

basin of, population and area of ------- 249-250, 253-254

stream flow in _._._._. 91-147

length of, reason for measurements on, at Harpers Ferry, W. Va_____ 147

pollution on

profile of _._._... 135

plate showing

station on, at Millville, W. Va.,

description of -_-_-_ 135-136 measurements at_ 31, 136-146 water power at

at Riverton, Va., description of ._._. 41-43

valley of, description of

water of, mineral anaylsis of _.. $\quad 297$ sanitary analysis of __._.__ 294

Shenandoah River (North Fork), basin of, pollution in.-

basin of, population and area of -stream flow in pollution in settlement on solls on station on, near Riverton, Va., description of ----- 125-127 measurements at trough of _._._._._._. 12 water of, mechanical analysis of $\quad 296$

sanitary analyses of _._._- 294

Shenandoah River (South Fork), basin of, description of _..... 108

basin of, pollution in $91-123,231-238$ population and area of 253-254

stream flow in $110-123$

water powers in

measurements on pollution of profile of, plate showing_____. 134 settlement on soils on 307 station on, near Front Royal,

Va., description of -- 115-116 measurements at__-_- 116-123

water of, sanitary analysis of - $\quad 294$ Shenandoah plain, description of _- 20-21 Shendun, Va., water power at____ 109 Shepherdstown, Pa., stream pollution at__._. 232

Shriver, Joseph, map of _....... Sideling Creek, W. Va., basin of, population and area of _. 
Page.

322

321

fires on. 309,310

station on, near Lineburg, measurements at

Silcott Run, W. Va., water of, field assay of

Silk dyeing, methods of

Sir Johns Run, W. Va., measurements on, near Sir Johns Run

Sir Johns Run, W. Va., measurements on Sir Johns Run near.

Slaughterhouses, pollution from_._ 217 , $222,228,229,236,241$

Sleepy Creek, W. Va., basin of, population and area of _- 247,253 measurements on, near Munson. soils on

Smith, John, exploration on Potomac by

Snow, melting of, turbidity due to Soap making, stream pollution by Sod, prevention of erosion by - Soda, recovery of _._. Soda wood pulp. See Wood pulp.

Soldiers, typhoid among_-_-_ 255, 271-272

Soils, classification and descriptions of _...

effect of, on turbidjty_____ 299-317

Somerset, Pa., precipitation at__.-- 39

Somerville peneplain, description of

South Branch of the Potomac. See Potomac River, South Branch.

South Fork of Shenandoah River. See Shenandoah River, South Fork.

South Mountain, Va., fires on

South River, Va., basin of, population and area of _-_ 249-253 stream flow in 91-98 pollution of station on, at Basic City, description of _._._._. 91-92 measurements at__... 92-94

at Port Republic, Va., description of measurements at._._. 95-98

water of, mineral analysis of sanitary analyses of water power's on

South Tuscarora Creek, Md., measurements on, near Licksville

Southern Appalachian Forest Reserve, establishment of

Spillman, W. J., on yellow-slate soil _-

Spottswood, Governor, expedition of Springfield, Mass., typhoid fever at _._.

Springfield, W. Va., station on South Branch Potomac at, description of
Springfield, W. Va., station on South

Page.

Branch Potomac at, measurements 'at - - 31, 68-77

Springs, contamination of _-_._- 326-327

Spruce, distribution of ________ 313,324

effect of, on melting snow_-_- 325

humus from

use of, in wind-breaks_____._ 332

wastes from, effect of, on

fishes -

Spruce Mountain, W. Va., fires on timber on

Staunton, Va., precipitation at_-_- $\quad 39$ settlement of station on Lewis Creek near, description of 101

measurements at_._._._._ 102-103

stream pollution at

water supply of

Steel mills, stream pollution from_ 222

Stephens City, Va., precipitation at $\quad 39$

Stephens Run, Pa., pollution of _._ $\quad 244$

Stevens Run, Pa., water of, field assay of

Stokesville, Va., stream pollution at -

typhoid fever at

Stomoxys calcitrans. See Flies.

Stonebridge Run, Va., measurements on, near Milldale_-_- 147

Stony River, W. Va., fires on $\ldots \ldots 324,325$ measurements on, near Gormania, W. Va______ 65

near Schell, W. Va_____. 65

pollution of 284

soils on _._- 313

timber on

water of, field assay of

Stony Run, Va., pollution of _._-_ 239

Stover, Jacob, settlement by _..._ 3

Stoyer, Md., stream pollution at_._- 214-

215,283

water at, field assay of _-_-- 287

Strasburg, Va., measurements on Cedar Creek near

settlement of

stream pollution at______- 240

water supply of

Strawboard wastes, stream pollution by _... 230, 242

Stream pollution in Potomac River basin _._.

See also particular places, streams, manufactures, etc.

Streams, alkalinity of, necessity of, for fish life character and location of 7-8 dilution of disease borne by _- 191-192, 259-282 fiow of, connection of typhoid

fever and _.____ 278-279

measurements of accuracy of comparisons of 
Steams, flow of, measurements of, curves used in, plate showing

oxidation in

263
details of _..._... 213-298 indnstries and, relations of - purification of, cost of _..._ 191, 193 sedimentation in _._._._._._. 261-266 temperature of relation of typhoid $f$ e $v$ e $r$ and _._._-_-_-_- 262-263

transportation of solid matter by _-_._.

turbidity of, effect of soils on -

velocity of

Stringtown, Pa., water at, field assay of -.......

Sugarland Run, Md., basin of, population and area of _- 251, 254

Sulphite pulp, wastes from, effect of, on fishes___._._. 342-343

Sulphur Run, Md., pollution of _._ 272 Sunnyside, Md., precipitation at _- $\quad 39$ Surface waters, quality of ______ 283-298 Susquehanna, gain of, from Potomac, explanation of

\section{T.}

Tables, explanations of _._.___._. 27-28

Takoma Park, Md., precipitation at Tamarack Ridge, W. Va., timber on Tan bark, disposal of Taneytown, Md., precipitation at_- 39 Tanneries, pollution from _-- 195-200, 214, $218,219,221-222,224$ $225,226,229,230,238$, $239-240, \quad 243-245, \quad 284$

wastes from, effect of, on fishes .............. 343-345

Tanning. See Leather tanning.

Tanning extracts, pollution from_ 200-201, $224,226,235,236$

Tar from gas manufacture, effect of, on fish $\ldots$

pollution by__________ 205-206

Temperature of streams, observations of _._._._. 261-264

Tenmile Creek, Md., fires on _._._ 321

Terracing, prevention of erosion by $\quad 316$

Three Churches, W. Va., soils near

Three Fork Run, W. Va., pollution of _...

water of, field assay of

Timber Ridge, Pa., soils of _.____ 310,311

Tomsbrook, stream pollution at_-_- 240

Tonoloway Creek., Md., measurements on, near Hancock

Tonoloway Ridge, W. Va., fires on soils of
Town Creek, Va., measurements on, near forks of Potomac $\quad 90$ soils near

'own Hill, fires on

soils of

Train sewage, disposal of _-____- 234-235

Trellised drainage, meaning of _-_-_ 8

Tributaries, character and location of

discharge of, ratio of, to $\mathrm{Po}$ tomac

Trout, experiments on

Trout Run, Pa., pollution of _-__- 229 water of, field assay of _._._._. $\quad 289$

water supply from__._._._. 327

T'urbidity, effect of soils on____- 299-317 melting snow and, relation of _- 325

Turbidity in Washington reservoirs, lessening of, method of 329

Tuscarora Creek (of Goose), Md., pollution of _..._- 245-246

Tuscarora Creek (of Opequon), W. Va., pollution of _-_ $231-232$ station on, at Martinsburg, description of measurements at_._._._. 82

Tuscarora Creek (of Potomac), Md., pollution of

Tuscarora Mountain, Pa., fires on 321,323 Tussey Mountain, Pa., fires on_-- 320-321 Twenty-first, Md., measurements on North Branch Potomac near._-_._- 65

Typhoid bacillus, life history of - 260-261 Typhoid fever, causes of _.____ 254-269 causes of, at Washington___ 270-276 connection of, with low-water flow of Potomac___ 278-279 chart showing - 278 deaths from, in cities of the world..._._._._._- 268-269

in Washington__.___- 273-276 epidemics of, types of _..._._. 279 occurrence of, in Potomac ba$\sin$ 223, 224, 231, 233, 234, 236, $237,241,242-243,254-282$ transportation of, by water._._ 191 $192,236-237$ Typhoid fever commission, U. $\mathbf{S}$. Army, facts found by 255

U.

Uplift, history of $17-21$

Uppertract, W. Va., precipitation at

Upshur soils, character and distribution of _-_-_- $301,310-311$

timber on Urine, vitality of typhoid germs in_ 254

V.
Valley, river, ideal, description of. Van Metre, John, exploration by 
Vegetables, raw, typhoid fever spread by

Velocity curves, construction and use of _..._._. 24, 26

figure showing .

Verbena, Va., measurements on Naked Creek near._...-

Vienna, typhoid in, deaths from Virginia, valley of, character of -... settlement of soils of -

vital statistics in, lack of _...Vital statistics, lack of

$$
\text { W. }
$$

Wallman, Md., stream pollution at Wappan Run, Va., measurements on

$\begin{array}{rr}\text { near Linden } & 147 \\ \text { War, typhoid fever in } & \end{array}$ Warm Spring Run, Md,, drainage area of

measurements on, near Hancock

pollution of _............... water of, mineral analysis of _sanitary analysis of

Washington, D. C., filtration at floods near precipitation at reservoirs of, history of sedimentation and wind action in _. _. typhoid at $\ldots \ldots \ldots 263,268,270-282$ relation of Cumberland typhoid and___._-_- 272-273 relation of Mount Savage typhoid and _._._. 273-276 relation of low-water flow of Potomac and chart showing _...... 278 statistics of vital statistics at _.____ 270 water at, mineral analyses of_ $297-298$ sanitary analyses of _._._. 295 water supply of analyses of _._.___. 295, 297-298 character of 290 turbidity of _.___._____ 331-332

Washington, George, connection of, with Chesapeake and Ohio Canal

explorations by

Washington City Reservoir, description of

sedimentation in

wind action in

Washington Junction, Md., stream pollution at

Water. see Streams.

Water gas. See Gas, illuminating.

Water power, occurrence and character of

$147,160-161,172$

Water supplies of towns in Potomac basin, sources of ......
Waynesboro, Va., stream pollution at_water at, field assays of _-_- 289-290 water supply of _-_-_232-233, 277,327 Weather Bureau, gaging records of 42 rainfall records of _._______ $\quad 33$ Wells, pollution of, by sewage _._.- 233 , 242,259

pollution of, typhoid due to $.260,270$

West Virginia, vital statistics in, lack of _... 270

West Virginia Central Junction, stream pollution at 216,285

West rirginia Pulp and Paper Co., stream pollution by 216, 283, 284-285

Westernport, Md., precipitation at_- $\quad 39$ station on Georges Creek at, description of measurements at_..... $55-57,65$ streain pollution at____- 216-217, 285 water near, field assays of _.._- 288 sanitary analysis of _.____ $\quad 292$ water supply of Weverton, Md., stream pollution at_ 242 Whiffle and Moyer, on typhoid germ_ $\quad 260$ Whisky manufacture, description of -

stream pollution from_-- $212,220,231$ Will, life and property of _....... 5 Williams, Pa., stream pollution at_- 219 Williamson, Pa., stream pollution at 228 water at, sanitary analyses of 293

Williamsport, Md., measurements on Conococheague Creek near _............. 91

stream pollution at Willis, Bailey, on geographic history of Potomac basin

Willoughby Run, Pa., water of, field assay of

Wills Creek, Md, basin of, population and area of $-246,252$ pollution of _-__-__ 218-222, 273,286 soils on station on, at Cumberland, Md., description of -..... 58 measurements at_..._. 58-60,65 view at_..._._._._._._. 222 . turbidity of _._._. 313 water of, fleld assay of _._.___ $\quad 288$ mineral analyses of _..._. 296 sanitary analyses of _..._. 292

Wilson, W. Va., water at, field assays of _..._. 287

Wilsonia, W. Va., water at, field assay of _._._._. 287

Winchester, Va., settlement of _-_- 4 steam pollution at_______ 230,232 water supply of _________ 230,277

Wind, disease spread by turbidity due to Wind-breaks, creation of _._._._- 332-335 Wind gaps, character and location of - 
Winslow, C.-E. A., and Sedgwick, Page. W. 'T., on typhoid fever 264

Wolfden Run, Md., pollution of--- 215, 284 water of, field assay of _..__._. 287

Wood pulp, manufacture of, descrip-

tion of -

stream pollution from

$203,216,234,235,283,285$

view of

wastes from, effect of, on

fishes _..._... 340-343

Woodstock, Va., precipitation at___- $\quad 39$

streasu pollution at__.____ 240

water supply of

Wool dyeing, methods of ..._... 210

Wool scouring, methods of _-___ 206-208 pollution from

$208,218,230,231-232$ waste from, purification of-. 207-208
Woolens, washing of, pollution from 208 $215,235,237$

Y.

Yellow-slate soils, character and distribution of _.-. $308-310,316$ mechanical analyses of _._._._ 309

\section{Z.}

Zeit, F. R., and Jordan, E. O., experiments by, on typhoid germs

Zoological Park, D. C., station on Rock Creek at, description of _.......

station on Rock Creek at, measurements at_-_-_-_ 174-177 


\title{
CLASSIFICATION OF THE PUBLICATIONS OF THE UNITED STATES GEOLOGICAL SURVEY.
}

\author{
[Water-Supply Paper No. 192.]
}

The serial publications of the United States Geological Survey consist of (1) Annual Reports, (2) Monographs, (3) Professional Papers, (4) Bulletins, (5) Mineral Resources, (6) Water-Supply and Irrigation Papers, (7) Topographic Atlas of United States-folios and separate sheets thereof, (8) Geologic Atlas of United Statesfolios thereof. The classes numbered 2, 7, and 8 are sold at cost of publication; the others are distributed free. A circular giving complete lists can be had on application.

Most of the above publications can be obtained or consulted in the following ways:

1. A limited number are delivered to the Director of the Survey, from whom they can be obtained, free of charge (except classes 2,7 , and 8), on application.

2. A certain number are delivered to Senators and Representatives in Congress, for distribution.

3. Other copies are deposited with the Superintendent of Documents, Washington, D. C., from whom they can be had at practically cost.

4. Copies of all Government publications are furnished to the principal public libraries in the large cities throughout the United States, where they can be consulted by those interested.

The Professional Papers, Bulletins, and Water-Supply Papers treat of a variety of subjects, and the total number issued is large. They have therefore been classified into the following series: A, Economic geology; B, Descriptive geology; C, Systematic geology and paleontology; D, Petrography and mineralogy; E, Chemistry and physies; F, Geography; G, Miscellaneous; H, Forestry; I, Irrigation; J, Water storage; K, Pumping water; L, Quality of water; M, General hydrographic investigations; N, Water power; O, Underground waters; $\mathrm{P}$, Hydrographic progress reports. This paper is the fourteenth in Series $\mathrm{H}$, the eighteenth in Series $\mathrm{L}$, and the twentieth in Series M, the complete lists of which follow ( $\mathrm{PP}=$ Professional Paper; WS $=$ WaterSupply Paper):

\section{SERIES H-FORESTRY.}

PP 4. The forests of Oregon, by Henry Gannett. $1902.36 \mathrm{pp} ., 7 \mathrm{pls}$.

PP 5. The forests of Washington, a revision of estimates, by Henry Gannett. $1902.38 \mathrm{pp} ., 1 \mathrm{pl}$.

PP 6. Forest conditions in the Cascade Range, Washington, between the Washington and Mount Rainier forest reserves, by F. G. Plummer. 1902. 42 pp., 11 pls.

PP 7. Forest conditions in Olympic Forest Reserve, Washington, from notes by A. Dodwell and T. F. Rixon. 1902. 110 pp., 20 pls.

PP 8. Forest conditions in the northern Sierra Nevada, California, by J. B. Leiberg. 1902.194 pp., 12 pls.

PP 9. Forest conditions in Cascade Range Forest Reserve, by A. D, Langille, F. G. Plummer, A. Dodwell, T. F. Rixon,-and J. B. Leiberg, with introduction by. Henry Gannett. 1903. 298 pp., 41 pls.

PP 22. Forest conditions in the San Francisco Mountains Forest Reserve, Arizona, by J. B. Leiberg, T. F. Rixon, and A. Dodwell, with an introduction by F. G. Plummer. 1904. 95 pp., 7 pls.

PP 23. Forest conditions in the Black Mesa Forest Reserve, Arizona, prepared by F. G. Plummer, from notes by T. F. Rixon and Arthur Dodwell. $1904.62 \mathrm{pp} ., 7 \mathrm{pls}$.

PP 29. Forest conditions in the Absaroka division of the Yellowstone Forest Reserve, Montana, and the Livingston and Big Timber quadrangles, by J. B. Leiberg. 1905.48 pp., 3 pls.

PP 30. Forest conditions in the Little Belt Mountains Forest Reserve, Montana, and the Little Belt Mountains quadrangle, by J. B. Leiberg. 1901. 75 pp., 2 pls. 
PP 33. Forest conditions in the Lincoln Forest Reserve, New Mexico, by F. G. Plummer and M. G. Gowsell. 1804. 47 pp., 12 pls.

PP 37. The Southern Appalachian forests, by H. B. Ayres and W. W. Ashe. 1905.291 pp., 37 pls.

PP 39. Forest conditions in the Gila River Forest Reserve, New Mexico, by T. F. Rixon. 1905. 89 pp., 2 pls.

WS 192. The Potomac River basin: Geographic history-rainfall and stream flow-pollution, typhoid fever, and character of water-relation of soils and forest cover to quality and quantity of surface water-effect of industrial wastes on fishes, by H. N. Parker, Bailey Willis, R. H. Bolster, W. W. Ashe, and M. C. Marsh. 1907. 364 pp., 10 pls.

\section{SERIES L, QUALITY OF WATER.}

WS 3. Sewage irrigation, by G. W. Rafter. 1897. 100 pp., 4 pls. (Out of stock.) WS 22. Sewage irrigation, Pt. II, by G. W. Rafter. 1899.100 pp., 7 pls. (Out of stock.) WS 72. Sewage pollution near New York City, by M. O. Leighton. 1902.75 pp., 8 pls. WS 76. Flow of rivers near New York City, by H. A. Pressey. 1903. 108 pp., 13 pls.

WS 79. Normal and polluted waters in northeastern United States, by M. O. Leighton. $1903.192 \mathrm{pp.}$ $15 \mathrm{pls}$.

Ws 103. Review of the laws forbidding pollution of inland waters in the United States, by $\mathbf{E}$. B. Goodell. 1904. $120 \mathrm{pp}$.

WS 108. Quality of water in the Susquehanna River drainage basin, by M. O. Leighton, with an introductory chapter on physiographic features, by G. B. Hollister. 1904.76 pp., 4 pls.

WS 113. Strawboard and oil wastes, by R. L. Sackett and Isaiah Bowman. 1905.52 pp., 4 pls.

WS 121. Preliminary report on the pollution of Lake Champlain, by M. O. Leighton. 1905. 119 pp., 13 pis.

WS 144. The normal distribution of chlorine in the natural waters of New York and New England, by D. D. Jackson. $1905.31 \mathrm{pp} ., 5 \mathrm{pls}$.

Ws 151. Field assay of water, by M. O. Leighton. 1905. 77 pp., 4 pls. (Out of stock.)

Ws 152. A review of the laws forbidding pollution of inland waters in the United States, second edition, by E. B. Goodell. 1905. 149 pp.

Ws 161. Quality of water in upper Ohio River basin and at Erie, Pa., by S. J.'Lewis. 1906. 114 pp., 6 pls.

WS 179. Prevention of stream pollution by distillery refuse, based on investigations at Lynchburg, Ohio, by Herman Stabler. 1906. 34 pp., 1 pl.

Ws 185. Investigations on the purification of Boston sewage, by C. E. A. Winslow and Earle B. Phelps. $1906.163 \mathrm{pp}$.

WS 186. Stream pollution by acid-iron wastes, a report based on investigations made at Shelby, ohio. by Herman Stabler. 1906. 36 pp., 1 pl.

WS 189. The prevention of stream pollution by strawboard waste, by Earle Bernard Phelps. 1906. 29 pp., 2 pls.

WS 192. The Potomac River basin: Geographic history-rainfall and stream fiow-pollution, typhoid fever, and character of water-relation of soils and forest cover to quality and quantity of surface water-effect of industrial wastes on fishes, by H. N. Parker, Bailey Willis, R. H. Bolster, W. W. Ashe, and M. C. Marsh. 1907. 364 pp., 10 pls.

\section{SERIES M-GENERAL HYDROGRAPHIC INVESTIGATIONS.}

WS 56. Methods of stream measurement. 1901. $51 \mathrm{pp} ., 12 \mathrm{pls}$.

WS 64. Accuracy of stream nieasurements, by E. C. Murphy. $1902.99 \mathrm{pp} ., 4 \mathrm{pls}$.

WS 76. Observations on the flow of rivers in the vicinity of New York City, by H. A. Pressey. 1902, 108 pp., 13 pls.

WS 80. The relation of rainfall to run-off, by G. W. Rafter. 1903. $104 \mathrm{pp}$

WS 81 . California lyydrography, by J. B. Lippincott. 1903.488 pp., 1 pl.

WS 88. The Passaic flood of 1902, by G. B. Hollister and M. O. Leighton. 1903.56 pp., 15 pls.

WS 91. Natural features and economic development of the Sandusky, Maumee, Muskingum, and Miami drainage areas in Ohio, by B. H. Flynn and M. S. Flynn. $1904.130 \mathrm{pp}$.

WS 92. The Passaic flood of 1903 , by M. O. Leighton. $1904.48 \mathrm{pp}$., $7 \mathrm{pls}$.

WS 94. Hydrographic manual of the United States Geological Survey prepared by E. C. Murphy, J. C. Hoyt, and G. B. Hollister. 1904.76 pp., 3 pls.

WS 95. Accuracy of stream measurements (second edition), by E. C. Murphy. 1904.169 pp., 6 pls. WS 96. Destructive floods in the Lnited States in 1903, by E. C. Murpliy. $1904.81 \mathrm{pp} ., 13 \mathrm{pls}$.

WS 106. Water resources of the Philadelphia district, by Florence Baserim. 1904.75 pp., 4 pls.

WS 109. Hydrography of the Susquehanna River drainage basin, by J. C. Hoyt and R. H. Anderson. 1904. 215 pp., 28 pls.

WS 116. Water resotarces near Santa Barbara, California, by J. B. Lippincott. 1904.99 pp., 8 pls.

WS 147. Destructive floods in the Cnited States in 1904, by E. C. Murphy and others. 1905.206 pl', $18 \mathrm{pls}$. 
WS 150. Weir experiments, coefficients, and formulas, by R. E. Horton. 1906.189 pp., 38 pls.

WS 162. Destructive floods in the United States in 1905, by F. C. Murphy and others. 1906. 105 pp., 4 pls.

WS 180. Turbine water-wheel tests and power tables, by Robert E. Horton. 1906. 13-1 pp., 2 pls.

Ws 187, Determination of stream flow during the frozen season, by H. K. Barrows and Robert $\mathrm{E}$. Horton. 1907. $93 \mathrm{pp}, 1 \mathrm{pl}$.

WS 192. The Potomac River basin: Geographic history-rainfall and stream flow-pollution, typhoid fever, and character of water-relation of soils and forest cover to quality and quantity of surface water-effect of industrial wastes on flshes, by H. N. Parker, Bailey Willis, R. H. Bolster, W. W. Ashe, and M. C. Marsh. 1907. 364 pp., 10 pls.

Correspondence should be addressed to

The Director,

UNiTED STATEN (

Washington, D. C.

MaRCH, 1907 . 




